\title{
ALPHABETICAL LIST OF MEMBERS
}

\section{A U n World Wide Web}

News relevant to the IAU community, among which the individual membership of the Union, are available electronically via Internet to all Members who have access to Internet and a WWW client programme such as Mosaic. If you use an $\mathrm{X}$-window terminal under UNIX (probably the most common case) with X-Mosaic installed, you can connect to the service by typing in:

xmosaic http://WWW.lsw.uni-heidelberg.de/iau.html

If you use a different system, please consult your system experts about access possibilities.

Abbreviations in use under the heading Commission(s):

Executive Committee:

EC Member of the Executive Committee

Commissions:

P President of a Commission

VP Vice-President of a Commission

OC Member of the organizing Committee of a Commission

$S$ Secretary of a Commission

Executive Committee Working Group Presidents:

WGDAA Working Group for the Development of Antarctic Astronomy

WGPSN Working from for Planetary System Nomenclature

WGWWDA Working Group for the World Wide Development of Astronomy 
AANNESTAD PER ARNE DR, DPT OF PHYSICS, ARIZONA STATE UNIVERSITY, TEMPE AZ 85287, USA

Telcphone: (1) 6029653644

Facsimile: (1)

Telex:

Email:

Commission(s): 34

AARSETH SVERRE J DR, INSTITUTE OF ASTRONOMY, THE OBSERVATORIES, MADINGLEY RD, CAMBRIDGE CB3 OHA, UK

Telephone: (44) 1223337548

Facsimile: (4) 1223337523

Telex: 817297 astron $\mathrm{g}$

Email:

Commission(s): 33,37C

ABAD ALBERTO J DR, DPT FISICA TEORICA, UNIVERSIDAD DE ZARAGOZA, E 50009 ZARAGOZA, SPAIN

Telephone: (34) 76357011

Facsimile: (34)

Telex: 58198

Email:

Commission(s): 07

ABADI MARIO, OBSERVATORIO ASTRONOMICO, LAPRIDA 85 4,5000 CORDOBA, ARGENTINA

Telcphone: (54) 51214059

Email: abadi@uncbob.cdu.ar

Facsimile: (54) 51210613 Telex:

Commission(s):

ABALAKIN VICTOR K DR, INST THEORETIC ASTRONOMY, ACADEMY OF SCIENCES, 10 KUTUZOV QUAY 196140 ST PETERSBURG, RUSSIA

Telephone: (7) 8121233392

Facsimile: (7)

Telex: 12261 feniks

Email: vicabal@gao.spb.su

Commission(s): (44C,05,07,20

ABBASOV ALIK R DR, SCIENTIF \& INDUSTRIAL ASS, OF COSMIC RESEARCH, LENIN PROSPEKT 159 370106 BAKU, AZERBAIDZHAN

Telcphonc: (994)

Facsimile: (994)

Telex:

Email:

Commission(s): 10

ABBOTT DAVID C DR, PLANETARY SCIENCE, UNIVERSITY OF COLORADO, FACULTY

BOULDER CO 80309 0391, USA

Telephone: (1)

Facsimile: (1)

Telex:

Email:

Commission(s): 36

ABBOTT WILLIAM N DR, UNIVERSITY OF ATHENS, MICHALACOPOULOU 42, GR 11528 ATHENS, GREECE

Telephone: (30) 17213352

Facsimile: (30)

Telex:

Email:

Commission(s): 22

ABD EL HAMID RABAB, NTL RESEARCH INSTITUTE, OF ASTRONOMY \& GEOPHYSICS, CAIRO 02, EGYPT

Telephone: (20) $2780046 / 0645$

Facsimile; (20) 2782683

Telex: 93070 hiag vn

Email:

Commission(s):

ABDEL HADY AHMED DR, CAIRO, EGYPT

Telephone: (20)

Facsimile: (20)

Telex:

Email:

Commission(s):

ABDELATIF TOUFIK, CRAAG, OBSERVATOIRE D'ALGER, BP 63, BOUZAREAH, ALGERIA

Telephone: (213) 2941606

Facsimile: (213) $29+1157$ Telex:

Email:

Commission(s): 11

ABDELKAWI M ABUBAKR DR, ACADEMY OF SCIENTIFIC, RESEARCH AND TECHNOLOGY

101 KASR EL-EINI STREET, CAIRO, EGYPT

Telephone: (20)

Facsimile: (20)

Commission(s):

Telex:

Email:

ABDULla SHAKER ABDUL AZIZ, SARC, SCIENTIFIC RES COUNCIL, BOX 2441, JADIRIYAH BAGHDAD, IRAQ

Telephone: (964) $17765127 \quad$ Facsimile: $(964)$

Email:

Commission(s): 40

Telex: 2187 bathilmi ik

ABELE MARIS K DR, ASTRONOMICAL OBSERVATORY, LATVIAN STATE UNIVERSITY, RAINIS BUL 19

226098 RIGA, LATVIA

Telephone: (371)

Facsimile: (371)

Telex:

Email:

Commission(s): 31

ABHYANKAR KRISHNA D PROF, DPT OF ASTRONOMY, UNIVERSITY OF OSMANIA, HYDERABAD 500 O07, INDIA

Telephone: (91) 851672

Facsimile: (91)

Telex:

Email:

Commission(s): $24,29,36,+2$ 
$\mathrm{M} 402$

ABLES HAROLD D DR, US NAVAL OBSERVATORY, FLAGSTAFF STATION, BOX 1149, FLAGSTAFF AZ 86002, USA

Telephone: (1) 6027795132

Facsimile: (1)

Telex: 26230 astro

Email:

Commission(s): 09,25,28

ABLES JOHN G DR, CSIRO, DIV OF RADIOPHYSICS, BOX 76, EPPING NSW 2121, AUSTRALIA

Telephone: (61) 28680222

Facsimile: (61) 28680310

Telex:

Email:

Commission(s): 40

ABOUDARHAM JEAN DR, OBSERVATOIRE DE PARIS, SECTION DE MEUDON, F 92195 MEUDON PPL CDX, FRANCE

Telephone: (33) 145077784

Facsimile: (33) 145077469

Telex: 201571

Email: mesioa::abou

Commission(s): 10,12

ABOU-EL-ELLA MOHAMED S DR, HELWAN OBSERVATORY, HELWAN, EGYPT

Telephonc: (20) 78 0645/2683

Facsimile: (20)

Commission(s): 37

Telex: 93070 hiag un

Email:

ABRAHAM ZULEMA DR, IAG, UNIVERSIDADE DE SAO PAULO, CP 9638, 01065 SAO PAULO SP, BRAZIL

Telephone: (55) 115778599

Facsimile: (55) 112763848

Telex: 1156735 iagm br

Email: span 47550::iagusp

Commission(s):

ABRAMI ALBERTO PROF, OAT, BOX SUCC TRIESTE 5, VIA TIEPOLO $11,13+131$ TRIESTE, ITALY

Telephone: (39)

Facsimile: (39)

Telex:

Email:

Commission(s): 10,40

ABRAMIAN HAMLET, BYURAKAN OBSERVATORY, 378+33 BYURAKAN, ARMENIA

Telephone: (7) $8852284077 \quad$ Facsimilc: (7)

Telex:

Email: byurzakan(a)adonis.ias.msk.su

Commission(s): 28

ABRAMOWICZ MAREK DR, SISSA, ST COSTIERA 11, MIRAMARE, I 34014 TRIESTE, ITALY

Telephone: (39) 40224281

Facsimile: (39)

Telex: 460392 ictp

Email:

Commission(s): 44

ABT HELMUT A DR, KITT PEAK NTL OBS, BOX 26732,950 N CHERRY AVE, TUCSON AZ 85726 6732, USA

Telephone: (1) 5203259215

Facsimile: (1) $520323+183$

Telex: 0666484 aura noao

Email:

Commission(s): 05C,26C,29,30

ABULAZM MOHAMED SAMIR DR, HELWAN OBSERVATORY, HELWAN, EGYPT

Telephone: (20) 78 06+5/2683

Facsimilc: (20)

Commission(s):

Telex: 93070 hiag un

Email:

ACHARYA BANNANJE S DR, TIFR/COSMIC RAY GROUP, HOMI BHABHA RD, COLABA, BOMBAY 400 005, INDLA

Telephone: (91) 222152971

Facsimile: (91) 222152110

Telex: 0113009 tifr in

Email: acharya(a)tifrvax.bitnet

Commission(s): +4

ACHTERBERG ABRAHAM DR, STERREKUNDIG INSTITUTE, BOX 80000, NL 3508 TA UTRECHT, NETHERLANDS

Telephone: (31) $3053 \$ 213 \quad$ Facsimile: (31)

Email: bitnet:wnmacht(ahutruu0 Commission(s):

Tclex: 40048 fylut $\mathrm{nl}$

ACKER AGNES PROF DR, OBS DE STRASBOURG, 11 RUE UNIVERSITE, F 67000 STRASBOURG, FRANCE

Telephone: (33) 88358200

Facsimile: (33) 88250160

Telex: 890506 starobs

Email:

Commission(s): 34,46

ACTON LOREN W DR, DPT OF PHYSICS, MONTANA STATE UNIVERSITY, BOZEMAN MT 59717, USA

Telephone: (1) 4069946072

Facsimile: (1) 4069944452

Telex:

Email: 24707::acton

Commission(s): 12,4t

ADAM MADGE G DR, DPT OF ASTROPHYSICS, UNIVERSITY OF OXFORD, SOUTH PARKS RD

OXFORD OX1 3RQ, UK

Telephone: (44)

Facsimile: (4t)

Telex:

Email:

Commission(s): 12

ADAMS DAVID J DR, DPT OF ASTRONOMY, UNIVERSITY OF LEICESTER, UNIVERSITY RD

LEICESTER LE1 7RH, UK

Telephone: (44) 1162554455

Email:

Facsimile: (4+)

Telex: $3+1198$

Commission(s): 44 
ADAMS FRED DR, DPT OF PHYSICS, UNIVERSITY OF MICHIGAN, ANN ARBOR MI 48109 1090, USA

Telephone: (1) 3137474320

Facsimilc: (1)

Telex:

Email: 47509::fadams/fadams(a)umiphys Commission(s): 34

ADAMS JAMES H JR DR, NAVAL RESEARCH LABORATORY, CODE 41542,4555 OVERLOOK AVE SW

WASHINGTON DC 20375 5000, USA

Telephone: (1) 2027672747

Facsimile: (1)

Telex:

Email: span::11335::adams

Commission(s):

ADAMS THOMAS F DR, LOS ALAMOS SCIENTIFIC LAB, MS 329 G 7, BOX 1663, LOS ALAMOS NM 87545, USA

Telephone: (1) 5056676384

Facsimile: (1)

Telcx:

Email:

Commission(s):

ADAMSON ANDREW DR, SCHOOL OF PHYSICS \& ASTRO, LANCASHIRE POLYTECHNIC, CORPORATION ST PRESTON PR1 2TQ, UK

Telephone: (44)

Facsimile: (4t)

Telex:

Email:

Commission(s): 33

ADE PETER A R DR, DPT OF PHYSICS, QUEEN MARY/WESTFIELD COLL, MILE END RD, LONDON E1 4NS, UK Telephone: (44) 1719804811

Facsimile: (44)

Telex: 893750

Email:

Commission(s): 40

ADEL ARTHUR F PROF EMER, BOX 942, FLAGSTAFF AZ 860012, USA

Telephone: (1) 6027746597

Facsimile: (1)

Email:

Commission(s):

Telex:

ADELMAN SAUL J DR, DPT OF PHYSICS, THE CITADEL, CHARLESTON SC 29409, USA

Telephone: (1) 8037926943

Facsimile: (1)

Telex:

Email: adclmans@acitadel.bitnct

Commission(s): $14 \mathrm{C}, 25 \mathrm{C}, 29$

ADJABSHIRIZADEH ALI, CTR FOR ASTRON RESEARCH, UNIVERSITY OF TABRIZ, TABRIZ 51664, IRAN

Telephone: (98) $\$ 13256+\quad$ Facsimile: (98)

Telex:

Email:

Commission(s):

ADLER DAVID SCOTT, NRAO, BOX 0, SOCORRO NM 87801 0387, USA

Telephone: (1) $5058357000 \quad$ Facsimile: (1) $5058357027 \quad$ Telex:

Email: dadler(a)nrao.edu

Commission(s): 28

ADOLFSSON TORD DR, KRAGEHOLMSGATAN 12, S 21619 MALMOE, SWEDEN

Telephone: (46) 40157586

Facsimile: (46)

Telex:

Email:

Commission(s):

AFANASJEVA PRASKOVYA M DR, PULKOVO OBSERVATORY, ACADEMY OF SCIENCES, 10 KUTUZOV QUAY 196140 ST PETERSBURG, RUSSIA

Telephone: (7) 2982242

Facsimile: (7)

Telex:

Email:

Commission(s): 31

AFANAS'EV VIKTOR L DR, SPECIAL ASTROPHYSICAL OBS, ACADEMY OF SCIENCES, NIZHNIJ ARKHYZ 357147 STAVROPOLSKIJ, RUSSIA

Telephone: (7)

Facsimile: (7)

Telex:

Email:

Commission(s): 28,33

AGEKJAN TATEOS A PROF, ASTRONOMICAL OBSERVATORY, ST PETERSBURG UNIVERSITY BIBLIOTECHNAJA PL 2, 199178 ST PETERSBURG, RUSSIA

Telephone: (7)

Facsimile: (7)

Telex:

Email:

Commission(s): 33,37

AGRAWAL P C DR, TIFR, HOMI BHABHA RD, COLABA, BOMBAY 400 005, INDIA

Telephone: (91) $224952311 * 393$

Facsimile: (91)

Telex: 0113009 tifr in

Email:

Commission(s): 4

AGRINIER BERNARD L MR, CEA CEN, DAPNIA/SAP, BP 2, F 91191 GIF/YVETTE CDX, FRANCE

Telephone: (33)

Facsimile: (33)

Telex:

Email:

Commission(s): 
M 404

AGUERO ESTELA L DR, OBSERVATORIO ASTRONOMICO, DE CORDOBA, LAPRIDA 854, 5000 CORDOBA, ARGENTINA

Telephone: (54) 5123 0491/236876

Email: aguero@astro.edu.ar
Facsimile: (54) 51210613

Commission(s): 28
Telex: 51822 bucor ar

AGUIAR ODYLIO DENYS DR, INPE, CP 515, 12201 S JOSE DOS CAMPOS, BRAZIL

Telephone: (55) $123418977^{\star} 689$

Facsimile: (55) 123218743

Commission(s): 44

Emait: inpedas@brfapesp.bitnet

Telex: 1233530 inpe br

AGUILAR CHIU LUIS A DR, NTL ASTRONOMY OBSERVATORY, APDO 877, ENSENDA BC 22800, MEXICO

Telephone: (52) 61744580

Facsimile: (52) 61744607

Telex:

Email: aguilar@bufadora.astroscu.unam.mx

Commission(s): $\mathbf{2 8 , 3 3}$

AGUILAR MARIA LUISA, UNSM, FAC CIENCIAS FISICAS, AV ARICA 830, LIMA 5, PERU

Telephone: (51) $243961 / 521343$

Facsimile: (51)

Telex:

Email: mlah(a)unmsm.pe

Commission(s): 46

AHLUWALIA HARJIT SINGH DR, DPT PHYSICS \& ASTRONOMY, UNIVERSITY OF NEW MEXICO 800 YALE BLVD NE, ALBUQUEROUE NM 87131, USA

Telephone: (1) $5052772941 \quad$ Facsimilc: (1)

Telex: 660461

Email:

Commission(s): $10,44,49$

AHMAD FAROOQ DR, DPT OF PHYSICS, UNIVERSITY OF KASHMIR, SRINAGAR 190 006, INDLA

Telephone: (91) 71559

Facsimile: (91)

Telex:

Email:

Commission(s): 28

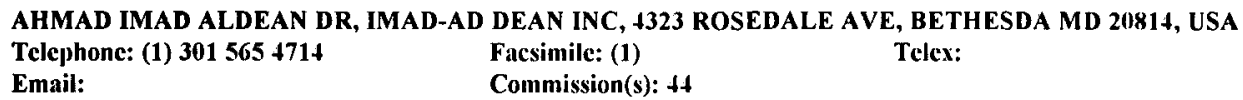

Email:

Commission(s): +4

AHMED IMAM IBRAHIM PROF, DPT OF ASTRONOMY, FACULTY OF SCIENCES, CAIRO UNIVERSITY, GEZA EGYPT

Telephone: (20)

Facsimile: (20)

Telex:

Email:

Commission(s):

AHMED MOSTAFA DR, DPT OF ASTRONOMY, FACULTY OF SCIENCES, CAIRO UNIVERSITY, GEZA, EGYPT

Telephone: (20)

Facsimile: (20)

Telex:

Email:

Commission(s): 07

AI GUOXIANG, BEIJING ASTRONOMICAL OBS, CAS, W SUBURB, BEIJING 100080, CHINA PR

Facsimile: (86) 12561085 Telex:

Email: bmabao@ica.beijing.canet.cn

Commission(s): 09,10VP,12

AIAD A PROF, DPT OF ASTRONOMY, FACULTY OF SCIENCES, CAIRO UNIVERSITY, GEZA, EGYPT

Telephone: (20) 869538

Facsimilc: (20)

Telex:

Email:

Commission(s): $34,35,37,46$

AIELLO SANTI DR, DPT DI FISICA, UNIVERSTIA DI FIRENZE, VIA L PANCALDO 3/45, I 50125 FIRENZE, ITALY

Telephone: (39) 554378540

Facsimile: (39) $55+35939$

Telex:

Email:

Commission(s):

AIKMAN G CHRIS L, HERZBERG INST ASTROPHYS, DOMINION ASTROPHYS OBS, 5071 W SAANICH RD VICTORIA BC V8X 4M6, CANADA

Telephonc: (1) 6043883975

Facsimile: (1) $60+3630045$

Telex: 0497295

Email: aikman@dao.nrc.ca

Commission(s): 29

AIME C DR, DPT ASTROPHYSIQUE, UNIVERSITE DE NICE, PARC VALROSE, F 0603 + NICE CDX, FRANCE

Telephone: (33) 93519100

Facsimile: (33)

Telex:

Email:

Commission(s): 09,12

AITKEN DAVID K DR, 5 DEWSBURY COTTAGES, BISHOPHILL, YORK YO1 1 HB, UK

Telephone: $(44)$

Email: dave@phadfa.ph.adfa.oz.au
Facsimile: $(4+)$

Commission(s): 34
Telex: 
AIZENMAN MORRIS L DR, NTL SCIENCE FOUNDATION, DIV ASTRONOMICAL SCIENCES, 1800 G ST NW WASHINGTON DC 20550, USA

Telephone: (1) 2023577643

Facsimile: (1)

Telex:

Email:

Commission(s): $05,27,35$

AIZU KO PROF, 3-24-3 KATAHIRA, ASAO KU, KAWASAKI, JAPAN

Telephone: (81)

Facsimile: (81)

Email:

Commission(s): $33,40,+7,44$

Telex:

AKABANE KENJI A PROF, TOKYO ASTRONOMICAL OBS, NAOJ, OSAWA MITAKA, TOKYO 181, JAPAN

Telephone: (81) 267982831

Facsimile: (81)

Telex: 3329005 tao nro j

Email:

Commission(s): 34,40

AKABANE TOKUHIDE DR, HIDA OBSERVATORY, UNIVERSITY OF KYOTO, KAMITAKARA, GIFU 506 13, JAPAN

Telephone: (81) 57862311

Facsimile: (81)

Telex:

Email:

Commission(s): 16

AKAN MUSTAFA CAN, DPT ASTRONOMY \& SPACE SCI, EGE UNIVERSITY OBS, SCIENCE FACULTY BORNOVA IZMIR 35100, TURKEY

Telephonc: (90) $2323880110 / 2322$

Email: efeast04(a) trean.bitnet

Facsimile: (90)

Telex:

Commission(s):

AKCAYLI MELEK M A DR, FACULTY OF SCIENCE, EGE UNIVERSITY, BOX 21, 35100 BORNOVA IZMIR, TURKEY Telephone: (90)

Facsimile: (90)

Telex:

Email:

Commission(s):

AKE THOMAS BELLIS, GSFC, CODE 681/CSC, GREENBELT MD 20771, USA

Telephone: (1) 3012863924

Email: hrsake(âhrs.dsfic.nasa.gov

Facsimile: (1) 3012861752 Telex:

Commission(s): 29

AKIM EFRAIM L DR, INST OF APPLIED MATHS, ACADEMY OF SCIENCES, MIUSSKAJA SQ 4, 125047 MOSCOW RUSSIA

Telephone: (7) 2513739

Facsimile: (7)

Telex:

Email:

Commission(s): 07

AKSNES KAARE DR, INST THEORET ASTROPHYSICS, UNIVERSITY OF OSLO, BOX 1029 N 0315 BLINDERN OSLO 3, NORWAY

Facsimile: (47) $22856505 \quad$ Telex: 72705 astro $n$

Email: kaare.aksnes $(\hat{a}$ astro.uio.no

Commission(s): $06,07,20 \mathrm{C}$, WGPSN

AKUJOR CHIDI E, DPT OF PHYSICS \& ASTRON, UNIVERSITY OF NIGERIA, NSUKKA ANAMBRA STATE, NIGERIA

Telephone: (234) $\$ 2771532 \quad$ Facsimile: (234)

Email:

Commission(s): 40

Telex: 51496u lion ng

AKYOL MUSTAFA UNAL PROF, FACULTY OF EDUCATION, SELCUK UNIVERSITY, 42090 KONYA, TURKEY

Telephone: (90)

Facsimile: (90)

Telex:

Email:

Commission(s):

ALBERS HENRY PROF, 62 PROSPECT ST, FALMOUTH MA 02540 , USA

Telephone: (1) $5085400978 \quad$ Facsimile: (1) Telex:

Email:

Commission(s): 45

ALBINSON JAMES DR, DPT OF PHYSICS, UNIVERSITY OF KEELE, KEELE ST5 5BG, UK

$\begin{array}{ll}\text { Telephone: (44) } 1782 \text { 621 } 111 & \text { Facsimile: }(44) \\ \text { Email: janct:jsa(Guk.ac.kl,ph.star } & \text { Commission(s): } 27\end{array}$

Telex: 36113 unklib $g$

ALBRECHT MIGUEL A DR, ESO, KARL SCHWARZSCHILDSTR 2, D 85740 GARCHING MUENCHEN, GERMANY

Telephone: (49) 89 $320063+6$

Facsimile: (49) 8932006480

Telex: 5282820 co d

Email: matbrecht(a)eso.ory

Commission(s): 05

ALBRECHT RUDOLF DR, SPACE TELESCOPE EUROPEAN, COORDINATING FACILITY, KARL

SCHWARZSCHILDSTR 2, D 85740 GARCHING MUENCHEN, GERMANY

Telephone: (49) 8932006287

Facsimile: (\$9)

Conumission(s): (19,25

Telex: $52828222 \mathrm{cod}$

Email: 
ALCAINO GONZALO DR, INSTITUTO ISAAC NEWTON, CASILLA 8-9, SANTIAGO 9, CHILE
Telephone: (56) 2472013
Facsimile: $(56)$
Telex: c/o eso 240853 esogo

Commission(s): 28,37

Email:

ALCOLEA JAVIER DR, CTR ASTRON DE YEBES, OAN, APD 148, E 19090 GUADALAJARA, SPAIN

Telephone: (34) 11290311

Facsimile: (34) $11290063 \quad$ Telex:

Email: alcolca@cay.es

Commission(s): 34

ALDROVANDI RUBEN DR, INST DI FISICA TEORICA, RUA PAMPLONA 15, 01405 SAO PAULO SP, BRAZIL

Telephone: (55) 112885643

Facsimile: (55)

Telex:

Email:

Commission(s):

ALDROVANDI S M VIEGAS DR, IAG, UNIVERSIDADE DE SAO PAULO, CP 9638, 01065 SAO PAULO SP, BRAZIL

Telephone: (55) 115778599

Facsimilc: (55) 112763848

Telex: 115 6735 iagm br

Email: iagusp\%brfapesp.ansp

Commission(s): 34

ALECLAN GEORGES DR, OBSERVATOIRE DE PARIS, SECTION DE MEUDON, DAF, F 92195 MEUDON PPL CDX FRANCE

Telephone: (33) $1453+7420$

Facsimile: (33)

Telex: 201571

Email:

Commission(s): 29

ALEXANDER DAVID R, PHYSICS DPT, WICHITA STATE UNIVERSITY, WICHITA KS 67260, USA

Telephone: (1) 3166893190

Facsimile: (1) $3166893770 \quad$ Telex:

Email: dra@twsuvm.uc.twsu.edu

Commission(s):

ALEXANDER JOSEPH K, NASA HEADQUARTERS, CODE P; OFFICE OF CHIEF SCIENTIST

WASHINGTON DC 20546, USA

Telephonc: (1)

Facsimile: (1)

Telex:

Email:

Commission(s): 40,44

ALEXANDER MURRAY E DR, NTL RESEARCH COUNCIL, INST FOR BIODIAGNOSTICS, 435 ELLICE AVE WINNIPEG MB R3B 1YG, CANADA

Telephone: (1)

Email:

Facsimile: (1)

Telex:

Commission(s): 07

ALEXANDER PAUL DR, MULLARD RADIO ASTRON OBS, CAVENDISH LABORATORY, MADINGLEY RD CAMBRIDGE CB3 OHE, UK

Telephone: (44) 1223337294

Email: pa25@uk.ac.cam.phx

Facsimile: (44) $122335 \$ 599 \quad$ Telex: 81292 cavlab g

Commission(s): 40

ALEXANDROV YURI V PROF, STR GROZNENSKAYA 32, APT 117, 31012 4 KHARKOV, UKRAINE

Telephonc: (7) $0572457537 / 432428$

Facsimile: (7)

Telex:

Email: alex@astron.kharkov.ua

Commission(s): 16,46

ALFARO EMILIO JAVIER, INST ASTROFISICA, DE ANDALUCIA APD 3004, C/SANCHO PANZA S/N

E 18080 GRANADA, SPAIN

Telephone: $(3$

Email: 16+88::cmilio/cmilio@aiaa.es

Facsimilc: $(3 t)$

Commission(s): 27,37

Telex: 78573 ialag e

ALFVEN HANNES PROF, DPT OF PLASMA PHYSICS, ROYAL INST OF TECHNOLOGY, S 100 \&4 STOCKHOLM 70 SWEDEN

Telephone: (46) 87877000

Facsimilc: $(46)$

Commission(s): 44,47

Telex: 10389 kthl

Email:

ALISSANDRAKIS C PH D, DPT OF ASTROPHYSICS, NTL UNIVERSITY OF ATHENS, PANEPISTIMIOPOLIS GR $1578+$ ATHENS, GREECE

Telephone: (30) 17235122

Facsimile: (30)

Telex:

Email:

Commission(s): 10,12

ALKSNIS ANDREJS DR, RADIOASTROPHYSICAL OBS, LATVIAN ACAD OF SCIENCES, TURGENEVA 19 LV 1524 RIGA, LATVIA

Telephone: (371) 132932088

Facsimile: (371) 132228784

Telex:

Email: astra\%lza.riga.lv

Commission(s): 37 
ALLADIN SALEH MOHAMED DR, DPT OF ASTRONOMY, UNIVERSITY OF OSMANIA, HYDERABAD 500 O07, INDIA Tclephone: (91) 71116

Facsimile: (91)

Telex:

Email:

Commission(s): 28

ALLAN DAVID W MR, BOX 66, FOUNTAIN GREEN UT 84632, USA

Telephone: (1)

Facsimile: (1) 8014453215

Email:

Commission(s): 31C

Telex:

ALLAN PETER M, DPT OF ASTRONOMY, UNIVERSITY OF MANCHESTER, MANCHESTER M13 9PL, UK

Telephone: (44) 1612737121

Facsimile: (44)

Telex:

Email:

Commission(s): 47

ALLARD NICOLE DR, OBSERVATOIRE DE PARIS, SECTION DE MEUDON, F 92195 MEUDON PPL CDX, FRANCE

Telephone: (33) 145077449

Facsimile: (33) $145077469 \quad$ Telex: 201571

Email: 17670::allard

Commission(s): 14

ALLEGRE CLAUde PROF, INST PHYSIQUE DU GLOBE, 4 PLACE JUSSIEU, F 75005 PARIS, FRANCE

Telephone: (33)

Facsimilc: (33)

Telex:

Email:

Commission(s): 15

ALLEN ANTHONY JOHN DR, ASTRONOMY UNIT, OUEEN MARY/WESTFIELD COLL, MILE END RD

LONDON E1 4NS, UK

Telephone: (44) $1719804811 \quad$ Facsimile: (4t)

Email: allen@uk.ac.qme.maths Commission(s):

Telex: 893750 qmeuol g

ALLEN CHRISTINE, INSTITUTO DE ASTRONOMIA, UNAM, APDO POSTAL 70-264, (04510 MEXICO DF, MEXICO

Telephone: (52)

Facsimile: (52)

Telex:

Email:

Comnission(s): 26C,37

ALLEN DAVID A DR, AAO, OBSERVATORY, BOX 296, EPPING NSW 2121, AUSTRALIA

Telephone: (61) 28681666

Facsimile: (61) 28768536

Telex: 23999 osyd aa

Email:

Commission(s):

ALLEN RONALD J DR, STSCI, HOMEWOOD CAMPUS, 3700 SAN MARTIN DR, BALTIMORE MD 21218, USA

Tclephonc: (1) $301338+574$

Facsimile: (1) $3013385090 \quad$ Telex:

Email: vjallen(a)stsci.edu

Commission(s): 28,40

ALLEN WILLIAM, ADAMS LANE OBSERVATORY, 46 ADAMS LANE, BLENHEIM, NEW ZEALAND

Telejphone: (64) 05787258

Facsimile: (64)

Telex:

Email:

Commission(s):

ALLER HUGH D DR, DPT OF ASTRONOMY, UNIVERSITY OF MICHIGAN, DENNISON BLDG

ANN ARBOR MI 481091090 , USA

Telephone: (1) $31376+3466$

Facsimile: (1)

Telcx:

Email:

Commission(s): 40

ALLER LAWRENCE HUGH, DPT OF ASTRONOMY, UNIVERSITY OF CALIFORNIA, MATH SCIENCES BLDG

LOS ANGELES CA 9002 $\downarrow$, USA

Tclephonc: (1) 2138253515

Facsimile: (1)

Commission(s): 29,34,36

Telex: 910-342-7597

Email:

ALLER MARGO F DR, DPT OF ASTRONOMY, UNIVERSITY OF MICHIGAN, DENNISON BLDG

ANN ARBOR MI 48109 1090, USA

Telephone: (1) $31376+3465$

Facsimile: (1)

Telex: 810-223-6056

Email:

Commission(s): 40

ALLEY CARROL O DR, ASTRONOMY PROGRAM, UNIVERSITY OF MARYLAND, COLLEGE PARK MD 20742, USA

Telephonc: (1) $30145+3405$

Facsimile: (1)

Telex: 908787

Email:

Commission(s): 31

ALLOIN DANIELLE DR, OBSERVATOIRE DE PARIS, SECTION DE MEUDON, DAEC, F 92195 MEUDON PPL CDX FRANCE

Telephone: (33) 145077404

Facsimile: (33) 145077469

Telex: 201571

Email: alloin@afrmeus1

Commission(s): 28 
M 408

ALMAR IVAN PROF, KONKOLY OBSERVATORY, THEGE U 13/17, BOX 67, H 1525 BUDAPEST, HUNGARY

Telephone: (36) 1175 5866/175 4122

Facsimile: (36) 11569640

Telex: 227460

Email:

Commission(s): 51C

ALMLEAKY YASSEEN, ASTRONOMY DPT, BOX 9028, KAAU, JEDDAH 21413, SAUDI ARABIA

Telephone: (966) 26952285

Email: scf3017@sakaau03

Facsimile: (966) $26400736 \quad$ Telex:

Commission(s):

ALPAR ALI DR, DPT OF PHYSICS, MIDDLE EAST TECH UNIV, 06531 ANKARA, TURKEY

Facsimile: (90) 412236945

Telex: 42761 olltk tr

Email: alpar@trmetu

Commission(s): 27

ALPHER RALPH ASHER, DPT OF PHYSICS, UNION COLLEGE, SCHENECTADY NY 12308, USA

Telephone: (1) 5183886345

Facsimile: (1) $51838869+7$

Telex:

Email: alpherr@gar.union.edu

Commission(s):

ALTAMORE ALDO, ISTITUTO ASTRONOMICO, UNIVERSITA DI ROMA, VIA G M LANCISI 29, I 00161 ROMA, ITALY

Telephone: (39) $68442977 \quad$ Facsimile: (39)

Email:

Commission(s):

Telex: 613255 infro

ALTAVISTA CARLOS A DR, OBSERVATORIO ASTRONOMICO, PASEO DEL BOSQUE S/N, 1900 LA PLATA (BS AS)

ARGENTINA

Telephone: (54) 21217308

Facsimile: (54) 21211761

Commission(s): 07

Telex: 31151 bulap

Email:

ER RADIOASTRONOMIE, AUF DEM HUEGEL 69, D 53121 BONN, GERMANY

ALTENHOFF WILHELM J DR, MPI FUER RADIOASTRONOMIE, AUF DEM HUEGEL
Tclcphone: (49) 228525293

Email: $\quad$ Commission(s): $33,34,40$

ALTROCK RICHARD C DR, AIR FORCE GEOPHYSICS LAB, NTL SOLAR OBSERVATORY, BOX 62

SUNSPOT NM 88349, USA

Telcphone: (1) 5054347016

Email: raltrock(a)sunspot.noao.cdu

Facsimile: (1) 5054347029

Telex: 1561030

Commission(s): 10,12,36

ALTSCHULER DANIEL R, ARECIBO OBSERVATORY, BOX 995, ARECIBO PR 00613, USA

Telephone: (1) 8098782612 Facsimile: (1) 8098781861 Telex:

Email: danicl@naic.cdu Commission(s):

ALTSCHULER MARTIN D PROF, DPT RAD THERAPY BOX 522, HOSP UNIV OF PENNSYLVANIA, 3400 SPRUCE ST PHILADELPHIA PA 19104, USA

Telephone: (1) 2156626472

Facsimile: (1)

Telex:

Email:

Commission(s): 10,12

ALURKAR S K DR, PHYSICAL RESEARCH LAB, NAVRANGPURA, AHMEDABAD 380 009, INDIA

Telcphone: (91) 272462129

Facsimilc: (91) $272+45292$

Telex: $121-397 \mathrm{prl}$ in

Email:

Commission(s):

ALVAREZ HECTOR DR, DPT DE ASTRONOMIA, UNIVERSIDAD DE CHILE, CASILLA 3G D, SANTIAGO, CHILE

Telephone: (56) $22294101 \quad$ Facsimile: (56)

Telex: $4+0001$

Email:

Commission(s):

ALVAREZ MANUEL DR, OBS ASTRONOMICO NACIONAL, BOX 439(27, SAN YSIDRO CA 92027, USA

Telephone: (1) 7066744580

Facsimile: (1) 7066674607

Telex: 56.539 ciceme

Email: alvarez(a)alfa.astroscu.unam.mx Commission(s):

ALVAREZ PEDRO DR, INST DE ASTROFISICA, DE CANARIAS, OBS DEL TEIDE, E 38071 LA LAGUNA, SPAIN

Telephone: (34)

Facsimile: (34)

Telex: 92640 iace e

Email:

Commission(s): $105,(19$

ALVAREZ POMARES A O DR, INST GEOPHYS \& ASTRONOMY, CALLE 212 N 2906/29 Y 31, LISA, LA HABANA, CUBA

Telephone: (53) 218416

Email: biotec(a)ceniai.cu

Facsimile: (53)

Tclex: (05112+0

Commission(s):

ALY JEAN JACQUES DR, CEA CEN, DAPNIA/SAP, BP 2, F 91191 GIF/YVETTE CDX, FRANCE

Telephone: (33) 169084030

Facsimile: (33) 169089266

Telex: 604860

Email: jjaly@asolar

Commission(s): 10 
AL-NAIMY HAMID M K DR, SARC, SCIENTIFIC RES COUNCIL, BOX 2441, JADIRIYAH BAGHDAD, IRAQ

Telephone: (964) 17765127

Facsimile: (964)

Telex: 212187

Email:

Commission(s): 42,51

AL-SABTI ABDUL ADIM DR, DPT OF PHYSICS, UNIVERSITY OF BAGHDAD, SCIENCE COLLEGE JADIRIYAH BAGHDAD, IRAQ

Telephone: (964) 15552340

Facsimile: (964)

Telex:

Email:

Commission(s): 38,51

AL-MALKI M B, DPT OF ASTRONOMY, KING SUAD UNIVERSITY, BOX 2455, RIYADH 11451, SAUDI ARABIA

Telephone: (966) $14676314 / 23 / 12$

Facsimile: (966) 14674253

Telex: $401019 \mathrm{k}$ s u sj

Email: f40a004(a)saksu00.bitnet

Commission(s):

AMAR MAKHLOUF, 14 RUE ZIGHOUD YOUCEF, DREAN, ALTARF 36, ALGERIA

Telephone: (213) 08680792

Facsimile: (213)

Telex:

Email:

Commission(s): 07

AMARI TAHAR DR, OBSERVATOIRE DE PARIS, SECTION DE MEUDON, SECTION D'ASTROPHYSIQUE F 92195 MEUDON PPL CDX, FRANCE

Telephone: (33) 145077760

Facsimile: (33) $1 \$ 5077469$

Telex: 270912

Email: amari@frmeu51

Commission(s): 10

AMBARTSUMIAN V A PROF DR, BYURAKAN ASTROPHYSICAL, OBSERVATORY, 378433 BYURAKAN, ARMENIA

Telephone: (7) 8852284580

Facsimile: (7) 8852284142

Telex: 412623

Email:

Commission(s): $28,33,51$

AMBASTHA A K DR, UDAIPUR SOLAR OBSERVATORY, 11 VIDYA MARG, UDAIPUR 313 001, INDIA

Telephone: (91) 25626

Facsimile: (O1)

Telex:

Email:

Commission(s): 10,33

AMBROZ PAVEL DR, ASTRONOMICAL INSTITUTE, CZECH ACADEMY OF SCIENCES, ONDREJOV OBSERVATORY CZ 25165 ONDREJOV, CZECH R

Telephone: (42) 20485201

Facsimile: (42) 20485314

Telex: 121579 astr $\mathrm{c}$

Email: astsun@ecsearn

Commission(s): 10

AMBRUSTER CAROL DR, DPT OF ASTRONOMY, VILLANOVA UNIVERSITY, MENDEL HALL

VILLANOVA PA 19085, USA

Telephone: (1) 2156454822

Email: ambrusterâvuvaxcom

Facsimile: (1) $2156457465 \quad$ Telex:

Commission(s): 29)

ANAND S P S DR, APPLIED RESEARCH CORP, 8201 CORPORATE DRIVE, SUITE 920, LANDOVER MD 20785, USA

Telephone: (1) 3014598442

Facsimile: (1)

Telex:

Email:

Commission(s): 35

ANANTHAKRISHNAN S, TATA INST OF FUNDAMENTAL, RESEARCH, POONA UNIVERSITY CAMPUS

PUNE 411 007, INDIA

Telephone: (91) 212336105

Email: uunet!shakti!gmrt!ananth

Facsimile: (91) $212335760 \quad$ Telex: 0145658 gmrt in

Commission(s): 49

ANANTHARAMAIAH K R DR, RAMAN RESEARCH INSTITUTE, SADASHIVANAGAR, SADASHIVANAGAR PO BANGALORE 560080 , INDIA

Telephone: (91) $8033+0122$

Facsimile: (91) $8033+0492$

Telex: 8452671 rri in

Email: anantha(a)rri.ernet.in

Commission(s): $34,40 \mathrm{C}$

ANDERNACH HEINZ DR, ESA IUE OBSERVATORY, VILLAFRANCA, APD 50727, E 28080 MADRID, SPAIN

Telephonc: (34) 18131 100/102

Facsimile: (34) 18131 139/119 Telex:

Email: hja@ avilspa.csa.es

Conmmission(s): $05,28,40$

ANDERSEN BO NYBORG DR, NORWEGIAN SPACE CENTRE, BOX 85, SMESTAD, N 0309 OSLO 3, NORWAY

Telephone: (47) 2523800

Facsimile: (47) 472522397

Telex: 005678174 spacen

Email; 21813::bandersen

Commission(s): 10,12,44

ANDERSEN JOHANNES, COPENHAGEN UNIVERSITY OBS, BRORFELDEVEJ 23, DK 4340 TOLLOSE, DENMARK Telephone: (45) 53488195

Facsinile: (45) 53488755

Email: ja@astro.ku.dk

Commission(s): 30,33,42,EC

Telex: 
M 410

ANDERSEN TORBEN BRENDER, LOCKHEED PALO ALTO RES LB, OPTICAL DESIGN GR B254 E 3251 HANOVER ST, PALO ALTO CA 94304, USA

Telephone: (1)

Facsimile: (1)

Telex:

Email:

Commission(s):

ANDERSON BRYAN DR, NRAL, JODRELL BANK, MACCLESFIELD SK11 9DL, UK

Telephone: (44) Facsimile: (44) Telex:

Email: $\quad$ Commission(s):

ANDERSON CHRISTOPHER M DR, WASHBURN OBSERVATORY, UNIVERSITY OF WISCONSIN 475 N CHARTER ST, MADISON WI 53706, USA

Telephone: (1) $6082620492 \quad$ Facsimile: (1) Telex:

Email:

Commission(s):

ANDERSON KINSEY A PROF, SPACE SCIENCES LABORATORY, UNIVERSITY OF CALIFORNIA BERKELEY CA 94720, USA

Telephone: (1) 4156421313

Email:

Facsimile: (1)

Telex: 910-3667945 uc space

ANDERSON KURT S, DPT OF ASTRONOMY, NEW MEXICO STATE UNIV, LAS CRUCES NM 88003, USA

Telephone: (1) 5056461032

Facsimile: (1)

Telex: 210-983-0549 nmsuci

Email:

Commission(s):

ANDO HIROYASU DR, TOKYO ASTRONOMICAL OBS, NAOJ, OSAWA MITAKA, TOKYO 181, JAPAN

Telephone: (81) 422325111

Facsimile: (81)

Telex: 2822307 taomk j

Email:

Commission(s): $12,27,51$

ANDREANI PAOLA MICHELA DR, DPT DI ASTRONOMIA, UNIVERSITA DI PADOVA, VIC DELL OSSERVATORIO 5 I 35122 PADOVA, ITALY

Telephone: (39) 498293442

Email: 39003::andreani span

Facsimile: (39) $498759840 \quad$ Telex: 432071 astros i

Commission(s): 47

ANDREEV VLADIMIR, ENGELHARDT ASTRONOMICAL, OBSERVATORY, KAZAN 422526, RUSSIA

Telephone: (7) 8432324827

Facsimile: (7) $8432380924 \quad$ Telex:

Email: eao a astro.kazan.su

Commission(s):

ANDREW BRYAN H DR, CHIEF PROGRAM SERVICES, NTL RESEARCH COUNCIL, 100 SUSSEX DR OTTAWA ON K1A 0R6, CANADA

Telephone: (1) 6139933731

Facsimile: (1) $6139526602 \quad$ Telex: 0533145

Email:

Commission(s): 34,40

ANDREW KENNETH L PROF, 1637 MAY 10(2), WICHITA KS 67213, USA

Telephone: (1) 316243838

Facsimile: (1)

Telex:

Email:

Commission(s): 14

ANDREWS DAVID A DR, ARMAGH OBSERVATORY, COLLEGE HILL, ARMAGH BTGI ODG, UK

Telephone: (44)

Facsimile: $(44)$

Telex:

Email:

Commission(s):

ANDREWS FRANK, CARTER OBSERVATORY, BOX 2909, WELLINGTON, NEW ZEALAND

Telephone: (64) 44728167

Facsimile: $(64)+4728320 \quad$ Telex:

Email:

Commission(s): 46

ANDREWS PETER J DR, ROYAL GREENWICH OBS, MADLINLRY RD, CAMBRIDGE CB3 OEZ, UK Telephone: (44) 1223374000

Facsimile: (44) 1223374700

Tclex: 817235 rgostr g

Email:

Commission(s):

ANDRIENKO DMITRY A DR, ASTRONOMICAL OBSERVATORY, KIEV STATE UNIVERSITY OBSERVATORNAYA UL 3, 252053 KIEV, UKRAINE

Telephone: (7) 250775 Facsimile: (7)

Email:

Commission(s): 15

Telex: 132201

ANDRIESSE CORNELIS D DR, POGGENBECSKSTRAAT 31, NL 6813 KD ARNHEM, NETHERLANDS

Telephone: (31)

Facsimilc: (31)

Telex:

Email:

Commission(s): 34 
ANDRILLAT HENRI L PROF, LAB ASTRONOMIE, USTL II, PLACE EUGENE BATAILLON F 34095 MONTPELLIER CDX 5, FRANCE

Telephone: (33) 67143415

Facsimile: (33) 67543079

Telex: $\mathbf{4 9 0 9 4 4}$ ustmont $f$

Email:

Commission(s): 29,34,46,47

ANDRILLAT YVETTE DR, LAB ASTRONOMIE, USTL II, PLACE EUGENE BATAILLON F 34095 MONTPELLIER CDX 5, FRANCE

Telephone: (33) 67143412

Facsimile: (33) 67543079

Telex: 490944 ustmont $f$

Email:

Commission(s): 28,29,34

ANDRONOV IVAN DR, DPT OF ASTRONOMY, ODESSA STATE UNIVERSITY, T G SHEVCHENKO PARK 270014 ODESSA, UKRAINE

Telephone: (7)

Facsimile: (7)

Telex:

Email: $\operatorname{root}(\boldsymbol{a})$ astro.odessa.ua

Commission(s): 34

ANGEL J ROGER P PROF, STEWARD OBSERVATORY, UNIVERSITY OF ARIZONA, TUCSON AZ 85721, USA

Telephonc: (1) $52062165+1 \quad$ Facsimile: (1)

Email:

Commission(s): 25

Telex: 467175

ANGELETTI LUCIO DR, OAR, VIA DEL PARCO MELLINI 84, I 00136 ROMA, ITALY

Telephone: (39) 63452794

Facsimile: (39)

Telex:

Email:

Commission(s):

ANGELOV TRAJKO, INSTITUTE OF ASTRONOMY, UNIVERSITY OF BELGRADE, STUDENTSKI TRG 16

YU 11000 BEOGRAD, YUGOSLAVIA-SERBIA \& MONTENEGRO

Telephone: (38) $11638715 \quad$ Facsimile: (38)

Commission(s): 35

Telex:

Email:

TRONOMY, SAN DIEGO STATE UNIV, SAN DIEGO CA 92182, USA

ANGIONE RONALD J DR, DPT OF ASTRONOMY, SAN DIEGO STATE UNIV, SAN
Telephone: (1) 6192656183
Facsimile: (1)

Email: angione(a)mintaka.sdsu.cdu Commission(s): 21,25

ANGLADA GUILLEM DR, DPT ASTRON I METEOROLOG, UNIVERSIDAD DE BARCELONA, AVD DIAGONAL 647 E 08028 BARCELONA, SPAIN

Telephone: (34) 34021121

Facsimile: (34) 34110873

Telex:

Email: 16488::50179::guillem

Commission(s): 34

ANGUITA CLAUDIO A DR, DPT DE ASTRONOMIA, UNIVERSIDAD DE CHILE, CASILLA 36 D, SANTIAGO, CHILE

Telephone: (56) 22294002

Facsimile: (56) 22294101

Telex: 440001

Email:

Commission(s): 08,EC

ANILE ANGELO M, DPT DI MATEMATICA, CITTA UNIVERSITARIA, VIA A DORIA 6, I 95125 CATANIA, ITALY

Telephone: (39) 95330533

Facsimile: (39)

Commission(s):

Telex:

Email:

ANN HONG BAE DR, DPT EARTH SCIENCES, PUSAN NTL UNIVERSITY, KUM JONG KU, PUSAN 609 735, KOREA R Telephone: (82) 515102705 Facsimile: (82) 515137495 Telex:

Email: Commission(s): 28

ANOSOVA JOANNA, NTL ASTRONOMICAL OBS, 211 OSAWA, MITAKA, TOKYO 181, JAPAN

Telephone: (81) 442343634

Facsimile: $(81) 4+2343746$

Telex:

Email: anosova(aferio.mtk.nao.ac.jp

Commission(s): 07,26,28

ANSARI S M RAZAULLAH PROF, DPT OF PHYSICS, ALIGARH MUSLIM UNIVERSITY, ALIGARH UP 202 002, INDIA Telephone: (91) 57129001

Facsimile: (91)

Commission(s): $12,41 \mathrm{P}, 40$

Telex: $56+230$ amu-in

Email:

ANTALOVA ANNA, ASTRONOMICAL INSTITUTE, SLOVAK ACADEMY SCIENCES, SK 05960 TATRANSKA LOMNI SLOVAK R

Telephone: (42) $969967866 / 7 / 8$

Facsimile: (42) 969967656

Telex:

Email:

Commission(s): 10 
ANTHONY-TWAROG BARBARA J, DPT PHYSICS \& ASTRONOMY, UNIVERSITY OF KANSAS LAWRENCE KS 66045, USA

Telephone: (1) 9138644933

Facsimile: (1)

Telex:

Email:

Commission(s): 25

ANTIA H M DR, TIFR, HOMI BHABHA RD, COLABA, BOMBAY 400 005, INDIA

$\begin{array}{lll}\text { Telephone: (91) } 224952311 & \text { Facsimile: (91) } & \text { Telex: } 0113009 \text { tifr in } \\ \text { Email: antia@tifrvax.bitnet } & \text { Commission(s): 12,35 } & \end{array}$

ANTIOCHOS SPIRO KOSTA, NAVAL RESEARCH LABORATORY, CODE 7675, 4555 OVERLOOK AVE SW WASHINGTON DC 20375 5352, USA

Telephone: (1) 2027676199

Facsimile: (1) $2024047997 \quad$ Telex:

Email: santiochos@solar.stanford.edu

Commission(s): 10

ANTIPOVA LYUDMILA DR, INST OF ASTRONOMY, ACADEMY OF SCIENCES, PYATNITSKAYA UL 48 109017 MOSCOW, RUSSIA

Telephone: (7) 952310680

Facsimile: (7)

Telex: 411576 ascon su

Email:

Commission(s): 27,42

ANTONA D' FRANCESCA DR, OAR, MONTE PORZIO, I 00040 MONTEPORZIO, ITALY

Tclephone: (39) 69449019

Facsimile: (39)

Telex:

Email:

Commission(s): $35,37,42$

ANTONACOPOULOS GREG PROF, DPT OF ASTRONOMY, UNIVERSITY OF PATRAS, GR 26110 RION, GREECE Telephone: (30) 61991145

Facsimile: (30)

Commission(s): 07

Telex:

Email:

ANTONELLO ELIO, OSS ASTRONOMICO DI MILANO, VIA E BIANCHI 46, I 22055 MERATE, ITALY

Telephone: (39) 592035

Facsimile: (39)

Commission(s): 27

Telex:

Email:

ANTONOPOULOU E DR, DPT OF ASTRONOMY, NTL UNIVERSITY OF ATHENS, PANEPISTIMIOPOLIS GR 15771 ZOGRAFOS, GREECE

Telephone: (30)

Facsimile: (30) Telex:

Email:

Commission(s): 42

ANTONOV VADIM A DR, INST OF THEORET ASTRONOMY, ACADEMY OF SCIENCES, N KUTUZOVA 10 191187 ST PETERSBURG, RUSSIA

Telephone: (7) 8122788835

Email: ita@iipah.spb.su

Facsimile: (7) 8122727968

Telex: 121578 ita su

ANTONUCCI ESTER DR, IST DI FISICA, UNIVERSITA DI TORINO, CORSO D AZEGLIO 46, I 10125 TORINO, ITALY

Telephonc: (39) 11657694

Facsimile: (39)

Telex: $2110+1$ infnto $i$

Email:

Commission(s): 10

ANTONUCCIO-DELOGU V, OSS ASTROFISICO, CITTA UNIVERSITARIA, VIA A DORIA 6, I 95125 CATANIA, ITALY

Telephone: (39) 957332244

Facsimile: (39) 95330592

Telex: 970359 astrcti

Email: vantonuccio@astrct.ct.astro.it

Commission(s):

ANTOV ALEXAFDAR, DPT OF ASTRONOMY, TSARIGRADSKO SHOSE BLV 72, PARCHEVICH STR 38 BG 1784 SOFIA, BULGARIA

Telephone: (359) 2758927

Email: belogr@b)bearn.bitnet

Facsimilc: (359) 2758927

Telex:

Commission(s): 27

ANZER ULRICH DR, MPI F PHYSIK \& ASTROPHYS, KARL SCHWARZSCHILDSTR 1

D 85740 GARCHING MUENCHEN, GERMANY

Telephone: (49) $8932990 \quad$ Facsimile: (49)

Email:

Commission(s): 10

Telex: $52+629$ astro d

AOKI SHINKO PROF, TOKYO ASTRONOMICAL OBS, NAOJ, OSAWA MITAKA, TOKYO 181, JAPAN

Telephone: (81) 422325111

Facsimile: (81)

Telex: 2822307 taomtk j

Email:

Commission(s): $04,07,31,33,41$

APARICI JUAN DR, DPT DE ASTRONOMIA, UNIVERSIDAD DE CHILE, CASILLA 36 D, SANTIAGO, CHILE

Telephone: (56) 22294101

Facsimile: (56)

Commission(s): 09,40

Telex: 440005

Email: 
APARICIO ANTONIO DR, INST DE ASTROFISICA, DE CANARIAS, OBS DEL TEIDE, E 38200 LA LAGUNA, SPAIN Telephone: (34) 22262211 Facsimile: (34) 22263005

Telex: 92640 iac e

Email:

Commission(s): 37

APPARAO K M V DR, TIFR, HOMI BHABHA RD, COLABA, BOMBAY 400005 , INDIA

Telephone: (91) $22219111 * 3+1$

Facsimile: (91)

Telex: 011-3009 tifr in

Email:

Commission(s): 4
APPENZELLER IMMO PROF, LANDESSTERNWARTE, KOENIGSTUHL, D 69117 HEIDELBERG, GERMANY
Telephone: (49) 6221509292
Facsimile: (49) 6221509202
Telex:
Email: iappenze@hp2.Isw.uni-heidellerg.de
Commission(s): 29,35,EC

APPLEBY JOHN F, JPL CALTECH, MS 183 301, 4800 OAK GROVE DR, PASADENA CA 91109, USA

Telephone: (1) $81835439+3$

Facsimile: (1)

Telex:

Email:

Commission(s): 16

APPLETON PHILIP NOEL DR, DPT OF PHYSICS, IOWA STATE UNIVERSITY, AMES IA 50011, USA

Telephone: (1) 5152943667

Facsimilc: (1)

Telex:

Email: bitnet:s1.pna@isumvs

Commission(s):

AQUILANO ROBERTO OSCAR DR, INSTITUTO DE FISICA, ROSARIO CONICET UNR, BV 27 DE FEBRERO 210 BIS 2000 ROSARIO, ARGENTINA

Telcphone: (54) 4182 1769/72

Facsimile: (54) 41257164

Email: usuarios\%ifir.edu.ar(âuunct.uu Commission(s): 42

Telex: $\mathbf{4 1 8 1 7}$ ciros ar

ARABELOS DIMITRIOS DR, DPT GEODESY \& SURVEYING, UNIVERSITY THESSALONIKI, UNIV BOX 503

GR 54006 THESSALONIKI, GREECE

Telephone: (30) $31992693 \quad$ Facsimile: (30)

Email:

Commission(s): 19

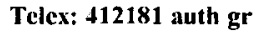

ARAFUNE JIRO DR, INST COSMIC RAY RESEARCH, UNIVERSITY OF TOKYO, MIDORICHO TANASHI

TOKYO 188, JAPAN

Telephone: (81) 424614131

Facsimilc: (81) 424681438

Telex: 2822371 icrtuj

Email:

Commission(s): 44

ARAI KENZO DR, DPT OF PHYSICS, KUMAMOTO UNIVERSITY, 2-39-1 KUROKAMI, KUMAMOTO 860, JAPAN

Telephone: (81) 963442111

Facsimile: (81)

Telex:

Email:

Commission(s): 35

ARAUJO FRANCISCO X DE DR, OBSERVATORIO NACIONAL, RUA GL BRUCE 586, SAO CRISTOVAO 20921 RIO DE JANEIRO RJ, BRAZIL

Telephone: (55) 215800235

Email: userfxa@ilncc.bitnct
Facsimile: (55) 215800332

Commission(s): 29
Telex: 21288

ARDAVAN HOUSHANG DR, INSTITUTE OF ASTRONOMY, THE OBSERVATORIES, MADINGLEY RD

CAMBRIDGE CB3 OHA, UK

Telephone: (44) 1223337548

Email:

Facsinile: (44) 1223337523

Commission(s):

Telex: 817297 astron $\mathrm{g}$

ARDEBERG ARNE L PROF, LUND OBSERVATORY, BOX 43, S 22100 LUND, SWEDEN

Telephone: (46) 46107290

Facsimile: $(46)$

Commission(s): $28,33,45,50 \mathrm{C}$

Telex: 33199 obsnot s

Email:

ARDEBILI M REZA DR, BOX $\$ 7+153+1$, BABOLSAR, IRAN

Telephone: (98)

Facsimile: (08)

Commission(s):

Telex:
2 PLACE MAURICE QUENTIN, F 75039 PARIS CDX 01, FRANCE $\begin{array}{ll}\text { ARDUINI-MALINOVSKY M DR, CNES, } 2 \text { PLACE MAURICE QUENTIN, F } 75039 \\ \text { Telephone: (33) } & \text { Facsimile: (33) } \\ \text { Email: } & \text { Commission(s): } 14\end{array}$

$\begin{array}{ll}\text { ARDUINI-MALINOVSKY M DR, CNES, } & \text { PLACE MAURICE QUENTIN, F } 75039 \\ \text { Telephone: (33) } & \text { Facsimile: (33) } \\ \text { Email: } & \text { Commission(s): } 14\end{array}$

Email:

ARELLANO FERRO ARMANDO, INSTITUTO DE ASTRONOMIA, UNAM, APDO POSTAL 70-264 04510 MEXICO DF, MEXICO

Telephone: (52) 9055485305

Email:
Telex: 01760155 cicme
Facsimile: (52)

Commission(s): 27,45 
M 414

ARENOU FREDERIC, OBSERVATOIRE DE MEUDON, BAT 11, 5 PL JULES JANSSEN

F 92195 MEUDON PPL CDX, FRANCE

$\begin{array}{lll}\text { Telephone: (33) } 145077849 & \text { Facsimile: (33) } 145077878 & \text { Telex: 201571 flam } \\ \text { Email: arenou@a)mehipa.obspm.fr } & \text { Commission(s): } & \end{array}$

ARGUE A NOEL MR, INSTITUTE OF ASTRONOMY, THE OBSERVATORIES, MADINGLEY RD

CAMBRIDGE CB3 0HA, UK

Telephone: (44) $1223337548 \quad$ Facsimile: (44) $1223337523 \quad$ Telex: 817297 astron g

Email:

Commission(s): $24,25,26$

ARGYLE P E DR, SUITE 401, 5880 HAMPTON PLACE, VANCOUVER BC VGT 2E9, CANADA

Tclephone: (1)

Facsimile: (1)

Telex:

Email:

Commission(s): 26

ARGYLE ROBERT WILLIAM MR, ROYAL GREENWICH OBS, MADINGLEY RD, CAMBRIDGE CB3 OEZ, UK

Telephone: (44) 1223374783

Email: nva@uk.ac.rgo.srf

Facsimile: (4) 1223374700

Telex: 817235 rgostr $\mathrm{g}$

ARGYRAKOS JEAN PROF DR, 193 PATISSON ST, GR 11253 ATHENS, GREECE

Telephone: (30) $18677000 \quad$ Facsimile: (30) Telex:

Email:

Commission(s): $08,+1$

ARIAS DE GREIFF J PROF, OBSERVATORIO NACIONAL, APDO 2584, BOGOTA 1 DE, COLOMBIA

Telephone: (57)

Facsimile: (57)

Telex:

Email:

Commission(s): $04,08,50$

ARIAS ELISA FELICITAS DR, OBSERVATORIO ASTRONOMICO, PASEO DEL BOSQUE S/N

1900 LA PLATA (BS AS), ARGENTINA

Telephone: (54) 21217308

Facsimile: (54) 21211761

Telex:

Email: felicitas@fcaglp.edu.ar

Commission(s): 19C

ARIMOTO NOBUO DR, OBSERVATOIRE DE PARIS, SECTION DE MEUDON, LAM

F 92195 MEUDON PPL CDX, FRANCE

Telephone: (33) $1453+7570 \quad$ Facsimile: (33)

Email: $\quad$ Commission(s): 35

Telex: 207571

ARKHIPOVA V P DR, STERNBERG STATE ASTR INST, UNIVERSITETSKIJ PROSP 13, 119899 MOSCOW, RUSSIA

Telephone: (7) 951392657

Facsimile: (7)

Telex:

Email:

Commission(s): $27,28,34$

ARLOT JEAN-EUDES, BUREAU DES LONGITUDES, 77 AVE DENFERT ROCHEREAU, F 75014 PARIS, FRANCE

Telephone: (33) 140512267

Facsimile: (33) 1 t6 332834

Telex:

Email: arlot@bdl.fr

Commission(s): $0+\mathrm{C}, 20 \mathrm{C}$

ARMANDROFF TAFT E DR, KITT PEAK NTL OBS, BOX 26732, 950 N CHERRY AVE, TUCSON AZ 85726 6732, USA

Telephone: (1) 5203259382

Facsimile: (1) 5203259360

Telex: $066648+$

Email: armand@noao.edu

Commission(s): 37

ARMSTRONG JOHN THOMAS DR, US NAVAL OBSERVATORY, ASTROMETRY DIV AD 5

3450 MASSACHUSETTS AVE NW, WASHINGTON DC 203925100 , USA

Telephone: (1) 2026531769

Facsimile: (1)

Telex:

Email: atlas!armstr@interf.com

Commission(s): 26

ARNAL MARCELO EDMUNDO DR, IAR, CC 5, 189+ VILLA ELISA (BS AS), ARGENTINA

Telephone: (54) 2143793

Facsimile: (54) $21211761 \quad$ Telex: 31216

Email: arnal@irma.cdu.ar

Commission(s): 40

ARNAUD JEAN PAUL, OBS MIDI PYRENEES, $1+$ AVE E BELIN, F 31400 TOULOUSE CDX, FRANCE

Telephone: (33) 61332929

Facsimile: (33) 61536722

Telex: $530776 \mathrm{f}$

Email:

Commission(s): $09,12,25$

ARNAUD MONIQUE, CEA CEN, DAPNIA/SAP, BP 2, F 91191 GIF/YVETTE CDX, FRANCE

Telephone: (33) 169087017

Facsimile: (33)

Telex: 604860

Email: span:32779::arnaud

Commission(s): $\$ 4$ 
ARNETT W DAVID PROF, ENRICO FERMI INSTITUTE, UNIVERSITY OF CHICAGO, 933 E 56TH ST CHICAGO IL 60637, USA

Telephone: (1) 3129628208

Facsimile: (1)

Commission(s): 35

Telex: 9102215617

Email:

ARNOLD JAMES R DR, DPT OF CHEMSTRY, UCSD, B 017, LA JOLLA CA 92093 0216, USA
Telephone: (1) 6195342908
Facsimile: (1)

Email: bitnet:jarnold@ucsd

Commission(s): 15

ARNOULD MARCEL L DR, UNIV LIBRE DE BRUXELLES, CP 226, B 1050 BRUSSELS, BELGIUM

Telephonc: (32) 26490030

Facsimile: (32)

Telex: 23069 unilib

Email:

Commission(s): 35,44

ARNY THOMAS T DR, DPT PHYSICS \& ASTRONOMY, UNIV OF MASSACHUSETTS, GRC B

AMHERST MA 01003, USA

Telephone: (1) 4135452194

Email:

Facsimile: (1)

Telex:

Commission(s): 34

ARONS JONATHAN, ASTRONOMY DPT, UNIVERSITY OF CALIFORNIA, 601 CAMPBELL HALL BERKELEY CA 94720, USA

Telephone: (1) 4156424730

Email:

Facsimile: (1)

Commission(s): 44

Telex: 820181 ucb ast ral

ARP HALTON DR, MPI F PHYSIK \& ASTROPHYS, KARL SCHWARZSCHILDSTR 1

D 85740 GARCHING MUENCHEN, GERMANY

Telephone: (49)

Facsimile: (49)

Telex:

Email:

Commission(s): 9

ARPIGNY CLAUDE PROF, INSTITUT D'ASTROPHYSIQUE, UNIVERSITE DE LIEGE, AVE COINTE 5

B 4000 COINTE-LIEGE, BELGIUM

Telephone: (32) $41529980 * 263$

Facsimile: (32) $4152747 \downarrow$

Telex:

Email:

Commission(s): 15,36

ARRIBAS SANTIAGO DR, INST DE ASTROFISICA, DE CANARIAS, OBS DEL TEIDE, E 38200 LA LAGUNA, SPAIN

Telephonc: (34) 22262211

Facsimile: (34)

Commission(s):

Telex: $926+0$

Email: span:iac:sam

Cominission(s):

ARSENIJEVIC JELISAVETA, ASTRONOMICAL OBSERVATORY, VOLGINA 7, YU 11050 BEOGRAD YUGOSLAVIA-SERBIA \& MONTENEGRO

Telephone: (38)

Email:

Facsimile: (38)

Commission(s): 25,27,50

Telex:

ARSHAKIAN T G, BYURAKAN ASTROPHYSICAL OB, ACADEMY OF SCIENCES, 378433 BYURAKAN, ARMENIA Telephone: (7) $8852283+53 / 284142$ Facsimile: (7) 8852523640 Telex:

Email:

Commission(s):

ARTHUR DAVID W G, US GEOLOGICAL SURVEY, BRANCH OF ASTROGEOLOGY, 2255 N GEMINI DR

FLAGSTAFF AZ 86001, USA

Telephone: (1)

Facsimile: (1)

Commission(s): 16

Telex:

Email:

ARTRU MARIE-CHRISTINE DR, OBSERVATOIRE DE LYON, AVE CHARLES ANDRE

F 69561 S GENIS LAVAL CDX, FRANCE

Telephone: (33) 78560705

Facsimile: (33) 72399791

Telex: 310926

Email:

Commission(s): 14,29

ARTYMOWICZ PAWEL, STOCKHOLM OBSERVATORY, S 13336 SALTSJOEBADEN, SWEDEN

Telephone: (46) 8164461

Email: pawel@astro.su.se

Facsimile: (46) 87174719

Telex:

Commission(s):

ARTYUKH VADIM, LEBEDEV PHYSICAL INST, ACAEDMY OF SCIENCES, LEMINSKY PROSPEKT 53 117924 MOSCOW, RUSSIA

Telephone: (7) 0959233558

Facsimile: (7) 0951357880

Telex:

Email: artyukh@rasfian.serpukhov.su

Commission(s): 
ARTZNER GUY, IAS, BAT 121, UNIVERSITE PARIS XI, F 91405 ORSAY CEDEX, FRANCE

Telephone: (33) $169858584 / 8525 \quad$ Facsimile: (33) 169858675 Telex: 600252

Email: iaslab::artzner

Commission(s):

ASCHENBACH BERND PH D, MPI F PHYSIK \& ASTROPHYS, INST F EXTRATERR PHYSIK

KARL SCHWARZSCHLDSTR 1, D 85740 GARCHING MUENCHEN, GERMANY

Telephone: (49) Facsimile: (49) Telex:

Email:

Commission(s): 44

ASCHWANDEN MARKUS DR, ASTRONOMY PROGRAM, UNIVERSITY OF MARYLAND

COLLEGE PARK MD 20742, USA

Telephone: (1) 3014051525

Email: markus@astro.umd.cdu

Facsimile: (1) $3013149067 \quad$ Telex:

Commission(s): 10,40

ASFLEY MICHAEL, SCHOOL OF PHYSICS, UNIV OF NEW SOUTH WALES, SYDNEY NSW 2052, AUSTRALIA

Telephone: (61) 23855465

Facsimile: (61) 26633420

Telex:

Email: meba@newt.phys.unsw.edu.au Commission(s): 9

ASHOK N M DR, PHYSICAL RESEARCH LAB, NAVRANGPURA, AHMEDABAD 380 009, INDIA

Telephone: (91) 272462129

Facsimile: (91) 272445292

Telex: 121397

Email:

Commission(s): 09,25

ASLAN ZEKI DR, 8 SOKAK, BAHGELI EVLER, ANKARA, TURKEY

Telephone: $(90)$

Facsimilc: (90)

Commission(s):

Telex:

Email:

ASLANOV I A DR, SHEMAKHA ASTROPHYSICAL, OBSERVATORY, AZER ACADEMY OF SCIENCES 373243 SHEMAKHA, AZERBAIDZHAN

Telephone: (994)

Facsimile: (994)

Telex:

Email:

Commission(s):

ASPIN COLIN DR, JOINT ASTRONOMY CENTER, 665 KOMOHANA ST, HILO HI 96720, USA

Telephonc: (1) 8089613756

Email: caa@jach.hawaii.edu

Facsimile: (1) $8089616516 \quad$ Telex:

Commission(s): 25

ASSEO ESTELLE DR, CENTRE PHYSIQUE THEORIQUE, ECOLE POLYTECHNIQUE, F 91128 PALAISEAU CDX FRANCE

Telephone: (33) 169418200

Facsimilc: (33)

Telex: 691596

Email:

Commission(s): 44

ASSOUSA GEORGE ELIAS DR, 545 BOYLSTON ST, SUITE 901, BOSTON MA 02116, USA

Telephone: (1)

Facsimile: (1)

Telex:

Email:

Commission(s): 40

ASSUS PIERRE DR, OCA OBSERV DE NICE, BP 229, F 06304 NICE CDX 4 , FRANCE

Telephone: (33) 92003086

Facsimile: (33) 92003033 Telex:

Email:

Commission(s): 09

ASTERIADIS GEORGIOS DR, DPT GEODESY \& SURVEYING, UNIVERSITY THESSALONIKI, UNIV BOX 503 GR 54006 THESSALONIKI, GREECE

Telephone: (30) 31992693

Email:

Facsimilc: $(30)$

Commission(s): 27,33

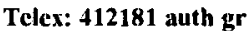

ATAC TAMER, KANDILLI OBSERVATORY, BOGAZICI UNIVERSITY, CENGELKOY, 81220 ISTANBUL, TURKEY

Telephone: (90) 13320240

Facsimile: (90)

Commission(s): 10,29

Telex: $26+11$ boun tr

Email:

ATANASIJEVIC IVAN DR, FACULTY OF SCIENCES, NL 6500 GL NIJMEGEN, NETHERLANDS

Telephone: (31)

Telex:

Email:

Commission(s):

ATHANASSOULA EVANGELIE DR, OBSERVATOIRE DE MARSEILLE, 2 PLACE LE VERRIER F 13248 MARSEILLE CDX 04, FRANCE

Telephonc: (33) 91959088

Facsimile: (33)

Telex: $42012+1 \mathrm{f}$

Email:

Commission(s): 28,33 
ATHAY R GRANT DR, BOX 343, BRUNSWICH NC 28424, USA

Telephone: (1)

Facsimile: (1)

Telex:

Email:

Commission(s): 10,12,36

ATHERTON PAUL DAVID, ASTROPHYSICS GROUP, IMPERIAL COLLEGE, BLACKETT LABORATORY

LONDON SW7 2BZ, UK

Telephone: (44)

Facsimile: (44)

Telex:

Email:

Commission(s): 09

ATREYA SUSHIL K, DPT ATM \& OCEANIC SCIENCE, UNIVERSITY OF MICHIGAN, SPACE RESEARCH BLDG ANN ARBOR MI 48109 2143, USA

Telephone: (1) 3137643335

Facsimile: (1)

Telex: 8102236056

Email:

Commission(s): 16

AUBIER MONIQUE G DR, OBSERVATOIRE DE PARIS, SECTION DE MEUDON

F 92195 MEUDON PPL CDX, FRANCE

Telephone: (33) 145347755

Facsimile: (33)

Telex: 270912

Email:

Commission(s):

AUDOUZE JEAN PROF, INSTITUT D'ASTROPHYSIQUE, 98BIS BD ARAGO, F 75014 PARIS, FRANCE

Telephone: (33) 144328133

Facsimilc: (33) 14432800

Telex:

Email:

Commission(s): $35,44,47$

AUER LAWRENCE H DR, LOS ALAMOS NATIONAL LAB, MS F665, ESS 5, LOS ALAMOS NM 87545, USA

Telephone: (1) 5056675824

Facsimile: (1)

Telex:

Email:

Commission(s): 36

AUGARDE RENEE DR, OBSERVATOIRE DE MARSEILLE, 2 PLACE LE VERRIER

F 13248 MARSEILLE CDX 04, FRANCE

Telephonc: (33) 91959088

Facsimile: (33)

Telex: $4202+1$

Email:

Commission(s):

AUGASON GORDON C DR, NASA AMES RESEARCH CTR, MS 2456 , MOFFETT FIELD CA 94035, USA

Telephone: (1) 4156944156

Facsimile: (1)

Telex:

Email:

Commission(s):

AULUCK FAOIR CHAND PROF, DPT PHYSICS \& ASTROPHYS, UNIVERSITY OF DELHI, NEW DELHI 110 007, INDIA

Telephone: (91) 112918993

Facsimile: (91)

Commission(s): 47

Telex:

Email:

AUMAN JASON R PROF, DPT GEOPHYS \& ASTRONOMY, UNIV OF BRITISH COLUMBIA, 2075 WESBROOK PL VANCOUVER BC VGT 1WS, CANADA

Telephone: (1) 6042282892

Facsimile: (1) $60+22860+7 \quad$ Telex:

Email:

Commission(s): 36

AUNER GERHARD DR, INSTITUT FUER ASTRONOMIE, UNIVERSITAET WIEN, TUERKENSCHANZSTR 17

A 1180 WIEN, AUSTRIA

Telephone: (43) 13453600

Email:

Facsimile: (43)

Commission(s):

Telex:

AURASS HENRY DR, ZNTRLINST F ASTROPHYSIK, AKADEMIE DER WISSENSCHAFT

D 14473 TREMSDORF, GERMANY

Telephone: (49) 2261

Facsimile: (49)

Telex: $15+20$

Email:

Commission(s): 10,40

AURIEMMA GIULIO DR, DPT DI FISICA, UNIVERSITA DI ROMA, PA MORO 2, I 00187 ROMA, ITALY

Telephone: (39) $64976336 \quad$ Facsimile: (39)

Telex: 613255 infnro

Email:

Commission(s):

AURIERE MICHEL, OBS MIDI PYRENEES, 14 AVE E BELIN, F 31400 TOULOUSE CDX, FRANCE

Telephone: (33) 62951969

Facsimile: (33)

Telex:

Email:

Commission(s): 37

AUVERGNE MICHEL, OCA OBSERV DE NICE, BP 229, F 06304 NICE CDX 4, FRANCE

Telephone: (33) 93890420

Facsimile: (33) 92003033

Telex: 460004 obsnice $f$

Email:

Commission(s): 
AVCIOGLU KAMURAN PROF DR, UNIVERSITY OBSERVATORY, UNIVERSITY OF ISTANBUL, 34452 ISTANBUL TURKEY

Telephone: (90) 15223597

Facsimile: (90)

Commission(s):

Telex:

Email:

AVERY LORNE W DR, HERZBERG INST ASTROPHYS, NTL RESEARCH COUNCIL, 100 SUSSEX DR OTTAWA ON K1A 0R6, CANADA

Telephone: (1) 6139936060

Facsimile: (1) 6139526602

Telex: 0533715

Email:

Commission(s): 34,40

AVGOLOUPIS STAVROS DR, DPT OF ASTROPHYSICS, UNIVERSITY THESSALONIKI GR 54006 THESSALONIKI, GREECE

Telephone: (30) 31991357

Facsimile: (30)

Commission(s): 27

Telex: 0412181 auth $\mathrm{gr}$

Email:

AYIGNON YVETTE DR, OBSERVATOIRE DE PARIS, SECTION DE MEUDON, F 92195 MEUDON PPL CDX, FRANCE Telephone: (33) 145347771

Facsimile: (33)

Commission(s): 10,40

Telex: 200590

Email:

AVRETT EUGENE H DR, CFA, HCO/SAO, 60 GARDEN ST, CAMBRIDGE MA 02138, USA

$\begin{array}{lll}\text { Telephone: (1) } 6174957+23 & \text { Facsimile: (1) } & \text { Telex: } 921428 \text { satellite cam } \\ \text { Email: } & \text { Commission(s): } 36 & \end{array}$

AWAD MERVAT EL-SAID DR, DPT OF ASTRONOMY, FACULTY OF SCIENCES, CAIRO UNIVERSITY, GEZA

EGYPT

Telephone: $(20) \quad$ Facsimile: (20) T Telex:

Email:

Commission(s):

AWADALLA NABIL SHOUKRY DR, DPT OF PHYSICS, UNIVERSITY OF BAHRAIN, BOX 32038, BAHRAIN STATE OF BAHRAIN

Telephone: (973) 681234

Facsimile: (973) 682582

Commission(s): 42

Telex: 9552 jamea bn

Email:

ONOMIE, POSTF

AXFORD W IAN PROF, MPI FUER AERONOMIE, POSTFACH 20, D 37189 KATLENBURG LINDAU, GERMANY Telephone: (49) 555641414

Facsimile: (49)

Commission(s): $15,34,44$

Telex: 965527

Email:

AXON DAVID, NRAL, JODRELL BANK, MACCLESFIELD SK11 9DL, UK

$\begin{array}{ll}\text { Telephone: (44) } 147771321 & \text { Facsimile: (44) } \\ \text { Email: } & \text { Commission(s): 25,40 }\end{array}$

Telex: 36149

AYANI KAZUYA, BISEI ASTRONOMCAL OBS, OHKURA, BISEI, OKAYAMA 714 14, JAPAN

Telephone: (81) $866874222 \quad$ Facsimile: (81) $866874224 \quad$ Telex:

Email: ayani@bao.go.jp

Commission(s): 28

AYDIN CEMAL PROF DR, DPT OF ASTRONOMY, UNIVERSITY OF ANKARA, FEN FAKULTESI 06100 BESEVLER, TURKEY

Telephone: (90) $41232105 * 94$

Email:

Facsimile: $(90)$

Commission(s):

Telex:

AYRES THOMAS R, CASA, UNIVERSTTY OF COLORADO, BOX 391, BOULDER CO 803090391 , USA

Telephone: (1) 3034925320

Facsimile: (1)

Telex: $7558+2$ jila

Email:

Commission(s): 12,44

AZCARATE ISMAEL N DR, IAR, VILLA ELISA 1984, CC 5, 1894 VILLA ELISA (BS AS), ARGENTINA

Telephone: (54) 2143793

Email: azcarate@irma.edu.ar

Facsimile: (54) 21254909

Telex: 18052 cicyt ar

Commission(s): 34

AZUMA TAKAHIRO DR, DOKKYO UNIVERSITY, SOKA, SAITAMA 340, JAPAN

Telephone: (81) $489+21111$

Facsimile: (81)

Telex: 2972005

Email:

Commission(s): 47

AZZOPARDI MARC DR, CANADA FRANCE HAWAII, TELESCOPE CORP, BOX 1597, KAMUELA HI 96743, USA

Telephone: (1)

Facsimile: (1)

Telex:

Email: marca(a)cfht.hawaii.edu

Commission(s): 28 
A'HEARN MICHAEL F DR, DPT OF ASTRONOMY, UNIVERSITY OF MARYLAND, COLLEGE PARK MD 20742, USA

Telcphonc: (1) 3014056076

Facsimile: (1) $3013149067 \quad$ Telex:

Email: ma@astro.umd.edu

Commission(s): 05,15P,20

BAADE DIETRICH DR, ESO, ST/ECF, KARL SCHWARZSCHILDSTR 2, D 85740 GARCHING MUENCHEN, GERMANY

Telephone: (49) 8932006388

Facsimile: (49)

Telex: 05282820 co d

Email: dbaade@dgaeso51.bitnet

Commission(s): $27,29,36$

BAAN WILLEM A, ARECIBO OBSERVATORY, BOX 995, ARECIBO PR 00613, USA

Telephone: (1) 8098782612

Facsimilc: (1)

Telex: 385638

Email:

Commission(s): 44

BAARS JACOB W M DR, STEWARD OBSERVATORY, UNIVERSITY OF ARIZONA, TUCSON AZ 85721, USA

Telephone: (1) 5206211515

Facsimile: (1) 5206211532

Telex: 467175

Email: baars@tucsmt.as.arizona.edu

Commission(s): 34,40

BAART EDWARD E PROF, DPT OF PHYSICS, RHODES UNIVERSITY, BOX 94, GRAHAMSTOWN 6140 SOUTH AFRIC Telephonc: (27) 0461-7128

Facsimile: (27)

Telex: $24+226$

Email:

Commission(s): $34,40 \mathrm{C}$

BAATH LARS B DR, BOX $8+81, \mathrm{~S}+3900$ ONSALA, SWEDEN

Telephone: (46) 317725500

Facsimile: (46) 317725550

Telex: 2400 onspace s

Email:

Commission(s): $40 \mathrm{C}$

BABA NAOSHI DR, DPT OF PHYSICS, HOKKAIDO UNIVERSITY, KITA 10 NISHI 8, SAPPORO 060, JAPAN

Telephone: (81) 117162111

Facsimile: (81) 0117264336

Telex: 932302 hokuen-j

Email: a10156@jpnac.bitnet

Commission(s): 09

BABADZHANIANC MICHAIL DR, ASTRONOMICAL OBSERVATORY, ST PETERSBURG UNIVERSITY

194904 ST PETERSBURG, RUSSIA

$\begin{array}{ll}\text { Telephone: (7) } & \text { Facsimile: (7) } \\ \text { Email: } & \text { Commission(s): }\end{array}$

BABADZHANOV PULAT B DR, ASTROPHYSICAL INSTITUTE, TADJIK ACAD OF SCIENCES

734670 DUSHANBE, TAJIKISTAN

Telephone: (7)

Email:

Facsimile: (7)

Telex:

Commission(s): $15,20,22 \mathrm{C}$

BABCOCK HORACE W DR, CARNEGIE OBSERVATORIES, 813 SANTA BARBARA ST, PASADENA CA 91101, USA

Telephone: (1) 8185771122

Facsimile: (1) 8187958136

Telex: 1561318 ociv ut

Email:

Commission(s): 09

BABEL JACQUES, INST FUER ASTRONOMIE UND, ASTROPHYSIK, UNIVERSITAET KIEL, D 24098 KIEL, GERMANY

Telephone: (49) 4318804101

Facsimile: (49) $4318804100 \quad$ Telex:

Email: babel@astrophysik.uni-kicl.de Commission(s):

BABIN ARTHUR DR, CRIMEAN ASTROPHYS OBS, UKRAINIAN ACAD OF SCIENCE, NAUCHNY

334413 CRIMEA, UKRAINE

Telephone: (7)

Facsimile: (7)

Telex:

Email:

Commission(s): 10

BABIN V G DR, SIBIZMIR, ACADEMY OF SCIENCES, 664697 IRKUTSK 33, RUSSIA

Telephone: (7) 629383

Facsimile: (7)

Telex: 142407

Email:

Commission(s): 10

BABU G S D, INDIAN INSTITU.TE OF, ASTROPHYSICS, KORAMANGALA, BANGALORE 560 034, INDIA

Telephone: (91) 803569179

Facsimile: (91)

Commission(s): 45

Telex: 8452763 iiab in

Email:

BACCHUS PIERRE PROF, 40 RUE HAUTE, F 77130 LA GDE PAROISSE, FRANCE

Telephone: (33) 64321315

Facsimile: (33)

Telex:

Email:

Commission(s): 08,26

BACHILLER RAFAEL DR, CTR ASTRON DE YEBES, OAN, APD 148, E 19080 GUADALAJARA, SPAIN

Telephonc: (34) $11290311 \quad$ Facsimilc: (34)

Email:

Commission(s): 34,40

Telex: $23+65$ igc e 
M 420

BACKER DONALD CH DR, RADIO ASTRONOMY LAB, UNIVERSITY OF CALIFORNIA, GO1 CAMPBELL HALL BERKELEY CA 94720, USA

Telephone: (1) 4156425128

Facsimile: (1)

Commission(s): $08,31 \mathrm{C}, 40$

Tclex: 820181 ucb ast ral

Email:

BADIALI MASSIMO, IAS, CNR, VIA ENRICO FERMI 21, I 00044 FRASCATI, ITALY

Telephone: (39) 69425655

Facsimile: (39) 69416847

Telex: $610261 \mathrm{cnr}$ fra

Email: massimo@irmias.bitnet

Commission(s): 44

BADOLATI ENNIO, VIA GIUSEPPE COTRONEI 11, I 80129 NAPOLI, ITALY

Telephone: (39) 81243245 Facsimile: (39) Telex:

Email:

Commission(s): 41

BAECK NICOLE A L DR, STERREKUNDIG OBSERV, RIJKSUNIVERSITEIT GENT, KRIJGSLAAN 281

B 9000 GENT, BELGIUM

Telephone: (32) 92644764

Facsimile: (32) 91644989

Telex: 12754 rugent b

Email: nicole.baeck(a)rug.ac.be

Commission(s):

BAEK CHANG RYONG, DPT OF PHYSICS, KIM IL SUNG UNIVERSITY, TAESONG DISTRICT

PYONGYANG, KOREA DPR

Telephone: (850)

Facsimilc: (850)

Telex:

Email:

Commission(s):

BAERENTZEN JORN, ELMEHOJVEJ 66, DK 8270 HOJBJERG, DENMARK

Telephonc: (45) 86272428

Facsimile: (45)

Commission(s):

Telex:

Email:

BAESSGEN MARTIN DR, ASTRONOMISCHES INSTITUT, UNIVERSITAET TUEBINGEN, WALDHAEUSERSTR GA D 72076 TUEBINGEN, GERMANY

Telephone: (49) 7071295470

Email: aitmvx::baessgen

Facsimile: (49) $7071293+58$

Telex: 7262714

BAFFA CARLO DR, OSS ASTROFISICO, DI ARCETRI, LARGO E FERMI 5, I 50125 FIRENZE, ITALY

Telephone: (39) 554378540

Email: baffa@astrfi.cineca.it

Facsimile: (39) 55435939

Telex: 572268 arcetr $i$

BAGARE S P DR, INDIAN INSTITUTE OF, ASTROPHYSICS, KORAMANGALA, BANGALORE 560 034, INDIA

Telephone: (91) $803566585 / 497$

Facsimile: (91)

Telex: 845763 iiab in

Email:

Commission(s): 10

BAGGALEY WILLIAM J PROF, DPT PHYSICS \& ASTRONOMY, UNIVERSITY OF CANTERBURY

PRIVATE BAG 4800, CHRISTCHURCH 1, NEW ZEALAND

Telephone: (64) $33667001 * 7559 \quad$ Facsimile: (64) $33642469 \quad$ Telex

Email: phys051(a)csc.canterbury.ac.nz Commission(s): 21,22VP

BAGHOS BALEGH B DR, HELWAN OBSERVATORY, HELWAN, EGYPT

Telcphone: (20) 78 0645/2683 Facsimile: (20) Telex: 93070 hiag

Email:

Commission(s): 07

BAGILDINSKIJ BRONISLAV K, PULKOVO OBSERVATORY, ACADEMY OF SCIENCES, 10 KUTUZOV QUAY 196140 ST PETERSBURG, RUSSIA

Telephone: (7)

Facsimile: (7)

Telex:

Email:

Commission(s): 08

BAGLIN ANNIE DR, OBSERVATOIRE DE PARIS, SECTION DE MEUDON, DASGAL

F 92195 MEUDON PPL CDX, FRANCE

Telephone: (33) 147057855

Facsimile: (33)

Telex:

Email:

Commission(s): $27,35,45$

BAGNUOLO WILLIAM G JR DR, CHARA, GEORGIA STATE UNIVERSITY, ATLANTA GA 30303 3083, USA

Telephone: (1) 4046512932

Facsimile: (1)

Telex:

Email:

Commission(s): 26 
BAGOLY ZSOLT, DPT ATOMIC PHYSICS, EOTVOS UNIVERSITY, PUSHKIN U 5-7, H 1088 BUDAPEST, HUNGARY

Telêphone: (36)

Facsimile: (36)

Telex:

Email;

Commission(s):

BAGRI DURGADAS S, NRAO, BOX 0, SOCORRO NM 87801 0387, USA

$\begin{array}{ll}\text { Telephone: (1) } 5057724011 & \text { Facsimile: (1) } \\ \text { Email: } & \text { Commission(s): } 40\end{array}$

Telex: 910-988-1710

BAHCALL JOHN N PROF, INST FOR ADVANCED STUDY, SCHOOL OF NATURAL SCIENCE, OLDEN LN BLDG E PRINCETON NJ 08540, USA

Telephone: (1) 6097348054

Email:

Facsimile: (1) 6099247592

Telex: 837680

BAHNER KLAUS DR, MPI FUER ASTRONOMIE, ADOLF-KOLPING-STR 5, D 6903 NECKARGEMUEND, GERMANY Telephone: (49) 62233735

Facsimile: (49)

Telex:

Email:

Commission(s):

BAIER FRANK, POTSDAM UNIVERSITY, SITZ IM AIP, AN DER STERNWARTE 16, D 14482 POTSDAM, GERMANY

Tclephone: (49) 3317499208

Email: fbaicr@aip.de Facsimile: (49) 3317499309 Telex: 361231 sthbg d

Commission(s): 33

BAILEY JEREMY A, AAO, BOX 296, EPPING NSW 2121, AUSTRALIA

Telephone: (61) 28681666

Facsimile: (61) 28768536

Telex: 23999 aaosyd aa

Email:

Commission(s):

BAILEY MARK EDWARD, COMPUTING \& MATHES SC, BYROM ST, LIVERPOOL L3 3AF, UK

Telephone: (44) 1512312092

Facsimile: (44) 1512074594 Telex:

Email: istmbailey@uk.ac.livjm.vax

Commission(s): $15 C, 20,28$

BAIRD SCOTT R, DPT PHYSICS \& ASTRONOMY, BENEDICTINE COLLEGE, N 14, ATCHISON KS 66002 1499, USA

Telephone: (1) 9133675340

Facsimile: (1)

Telex:

Email:

Commission(s): 36

BAIZE PAUL DR, ROUTE DE CONTANGES, F 50560 GOUVILLE SUR MER, FRANCE

Telephone: (33)

Email:

Facsimile: (33)

Commission(s): 26

Telex:

BAJAJA E DR, IAR, CC 5, 1894 VILLA ELISA (BS AS), ARGENTINA

Tclephonc: (54) 2143793

Email:

Facsimile: (54) 21211761

Commission(s): 28

Telex:

BAKER JAMES GILBERT DR, 14 FRENCH DR, BEDFORD NH 03102, USA

Telephone: (1) 6034725860

Facsimile: (1)

Commission(s):

Telex:

Email:

BAKER NORMAN H PROF, DPT OF ASTRONOMY, COLUMBIA UNIVERSITY, PUPIN HALL 538 W 120 TH ST NEW YORK NY 10027, USA

Telephone: (1) 2122803280

Email:

Facsimile: (1)

Commission(s): $\mathbf{0 5 , 2 7 , 3 5}$

Telex: 220094 colu ur

BAKRY ABDEL-AZIZ, EL AZHAR UNIVERSITY, FACULTY OF SCIENCES, ASTRONOMY DPT, CAIRO, EGYPT Telephone: (20) $2022629357 / 58$

Email: Facsimile: (20) 2022611404 Telex:

Commission(s): 08

BALACHANDRAN SUCHITRA C, DPT OF ASTRONOMY, OHIO STATE UNIVERSITY, 174 W 18TH AVE

COLUMBUS OH 432101106 , USA

Telephone: (1) 6142926925

Facsimile: (1) 6142922928

Telex:

Email: suchitra@payne.mps.ohio-statc.edu

Commission(s):

BALASUBRAMANIAN V DR, TIFR/RADIO ASTRONOMY CTR, BOX 8, UDHAGAMANDALAM 643 001, INDIA

Telephone: (91) 4232032

Facsimile: (91)

Telex: 8504208 rac in

Email:

Commission(s): 40

BALAZS BELA A PROF DR, COLLEGIUM HUNGARICUM DIR, HOLLANDSTR 4, A 1020 WIEN 2, AUSTRIA

Telephone: (43) 1240581

Email:

Facsimile: (43) 16296575

Telex:

Commission(s): 37,51 
BALAZS LAJOS G DR, KONKOLY OBSERVATORY, THEGE U 13/17, BOX 67, H 1525 BUDAPEST, HUNGARY Telephone: (36) 1175 5866/175 4122 Facsimile: (36) 11569640

Telex: 227460 konob $h$

Email: balazs@ogyalla.konkoly.hu

Commission(s): 33

BALBUS STEVEN A DR, UNIVERSITY STATION, UNIVERSITY OF VIRGINIA, BOX 3818

CHARLOTTESVILLE VA 22903 , USA

Telephone: (1) 8049244897

Facsimile: (1)

Telex:

Email: sb(avirginia.edu

Commission(s): 33

BALDINELLI LUIGI DR, CP 1630, I 40100 BOLOGNA, ITALY

Telephone: (39) 51227002

Facsimile: (39)

Telex:

Email:

Commission(s): 25

BALDWIN JACK A DR, CERRO TOLOLO, INTERAMERICAN OBSERVATORY, CASILLA 603, LA SERENA, CHILE Telephone: (56) 51213352

Email:

Facsimile: (56) $51212466 * 342 \quad$ Telex: 359620301 aura ct

Commission(s): 28

BALDWIN JOHN E DR, MULLARD RADIO ASTRON OBS, CAVENDISH LABORATORY, MADINGLEY RD CAMBRIDGE CB3 OHE, UK

Telephonc: (44) 1223337294

Email:

Facsimile: (44) 1223354599

Telex: 81292 cavlab g

BALDWIN RALPH B, 6190 GATEHOUSE DR SE, GRAND RAPIDS MI 49506, USA

Telephone: (1) 6199496190

Facsimile: (1)

Commission(s):

Telex:

Email:

BALEGA YURI YU DR, SPECIAL ASTROPHYSICAL OBS, ACADEMY OF SCIENCES, NIZHNIJ ARKHYZ 357147 STAVROPOLSKIJ, RUSSIA

Telephone: (7) 8657892501

Facsimile: (7)

Telex: 123244 zenit su

Email:

Commission(s): $26 \mathrm{C}$

BALICK BRUCE PROF, DPT OF ASTRONOMY, UNIVERSITY OF WASHINGTON, FM 20, SEATTLE WA 98195, USA

Telephone: (1) 2065437683

Facsimile: (1)

Commission(s):

Telex: 4740096 uw ui

Email:

BALIUNAS SALLIE L, CFA, HCO/SAO, 60 GARDEN ST, CAMBRIDGE MA 02138, USA
Telephone: (1) 6174957415
Facsimile: (1)

$\begin{array}{ll}\text { Telephone: (1) } 6174957415 & \text { Facsimile: (1) } \\ \text { Email: } & \text { Commission(s): } 12,29,36,44\end{array}$

Telex:

BALKLAVS A E DR, RADIOASTROPHYSICAL OBS, LATVIAN ACAD OF SCIENCES, TURGENEVA 19 226524 RIGA, LATVIA

Telephone: (371) Facsimile: (371) Telex:

Email:

Commission(s): 40

BALKOWSKI-MAUGER CH DR, OBSERVATOIRE DE PARIS, SECTION DE MEUDON, DAF

F 92195 MEUDON PPL CDX, FRANCE

Telephone: (33) 145347556

Facsimile: (33)

Telex: 201571

Email:

Commission(s): 28

BALL JOHN A DR, HAYSTACK OBSERVATORY, WESTFORD MA 01886, USA

Telephone: (1) 6176924764 Facsimile: (1) Telex:

Email:

Commission(s): 51

BALL LEWIS DR, THEORETICAL ASTROPHYSICS, UNIVERSITY OF SYDNEY, SYDNEY NSW 2006, AUSTRALIA

Telephone: (61) 26923241

Facsimile: (61) $26602903 \quad$ Telex:

Email: ball@aphysics.su.oz.au

Commission(s): 40

BALLABH G M DR, DPT OF ASTRONOMY, UNIVERSITY OF OSMANIA, HYDERABAD 500 007, INDIA

Telephone: (91) 71 951*247

Facsimile: (91)

Telex:

Email:

Commission(s): 24,28

BALLARIO M C PROF, OSS ASTRONOMICO, DI ARCETRI, VIA S LEONARDO, I 50100 FIRENZE, ITALY

Telephone: (39)

Facsimile: (39)

Telex:

Email:

Commission(s): 
BALLEREAU DOMINIQUE DR, OBSERVATOIRE DE PARIS, SECTION DE MEUDON, SECTION D'ASTROPHYSIQUE F 92195 MEUDON PPL CDX, FRANCE

Telephone: (33) 145077854

Facsimile: (33) $145077878 \quad$ Telex: 631987

Email: mesioa: ballcreau

Commission(s): 29

BALLESTER JOSE LUIS DR, DPT FISICA, UNIVERSIDAD DE LAS ISLAS, BALEARES E 07071 PALMA DE MALLORCA, SPAIN

$\begin{array}{lll}\text { Telephone: (34) } 71173228 & \text { Facsimile: (34) } 71173426 & \text { Tclex: } 69121 \text { unpm e } \\ \text { Email: dfsulbo(a)ps.uib.es } & \text { Commission(s): } 10 & \end{array}$

BALLI EDIBE PROF, UNIVERSITY RASATHANESI, ISTANBUL, TURKEY

Telephone: (90) Facsimile: (90) Telex:

Email:

Commission(s): 10

BALLY JOHN DR, CASA, UNIVERSITY OF COLORADO, CAMPUS BOX 389, BOULDER CO 80309, USA

Teléphone: (1) 3034925786

Email: bally@janos.colorado.edu

Facsimile: (1) 3034927178 Telex:

Commission(s): 40

BALMINO GEORGES G DR, CNES/GRGS/BGI, 18 AVE E BELIN, F 31055 TOULOUSE CDX, FRANCE

Telephone: (33) $61274427 \quad$ Facsimile: (33) Telex: 531081 cnest b f

Email:

Commission(s): 07

BALONA LUIS ANTERO DR, SAAO, BOX 9, OBSERVATORY 7935, SOUTH AFRICA

Telephone: (27) $21470025 \quad$ Facsimile: (27) $21473639 \quad$ Telex: 20309

Email: lab(a)saao.ac.za Commission(s): $25,27 \mathrm{C,30,36}$

BALONEK THOMAS J DR, DPT OF PHYSICS \& ASTRON, COLGATE UNIVERSITY, HAMILTON NY 13346, USA

Telephone: (1) 3158241000

Facsimile: (1)

Telex:

Email: bitnet:tbalonek(a) colgateu

Commission(s): 40

BALTHASAR HORST DR, KIEPENHEUER INSTITUT, FUER SONNENPHYSIK, SCHOENECKSTR 6

D 79104 FREIBURG BREISGAU, GERMANY

Telephone: (49) $7613198208 \quad$ Facsimile: (49) $7613198111 \quad$ Telex: 7721552 kis d

Email: hoba(a)kis.uni-freiburg.de Commission(s): 12

BALUTEAU JEAN-PAUL DR, OBSERVATOIRE DE MARSEILLE, 2 PLACE LE VERRIER

F 13248 MARSEILLE CDX 04, FRANCE

Telephone: (33) 91959088

Facsimile: (33)

Commission(s): 34

Telex: $420241 \mathrm{f}$

Email: obsmrs@fromrs51

BANDERMANN L W DR, 21131 GRENOLA DR, CUPERTINO CA 95014, USA

Telephone: (1)

Facsimile: (1)

Commission(s):

Telex:

Email:

CO, DI ARCETRI, LARGO E FERMI 5, I 50125 FIRENZE, ITALY

Email: bandiera@astrfi.infn.it Commission(s):

BANDYOPADHYAY A PROF, RESEARCH DIVISION, BIRLA PLANETARIUM, 96 JAWAHARLAL NEHRU RD CALCUTTA 700 071, INDIA

Telephone: (91) 33281515

Facsimile: (91)

Commission(s): $04,+1$

Telex:

Email:

YSICS, UNIVERSITY OF BURDWAN, GOLOPBAG, BURDWAN 713 104, INDIA

BANERJI SRIRANJAN DR, DPT OF PHYSICS, UNIVERSITY OF BURDWAN, GOL
Telephone: (91)
Telex:

Email:

Commission(s): 47

BANG YONG GOL, PYONGYANG ASTRON OBS, ACADEMY OF SCIENCES DPRK, TAESONG DISTRICT PYONGYANG, KOREA DPR

Telephonc: (850)

Email:

Facsimile: $(\mathbf{8 5 0})$

Telex:

Commission(s): 19

BANHATTI DILIP GOPAL DR, SCHOOL OF PHYSICS, MADURAI KAMARAJ UNIVERS, PALKALAINAGAR MADURAI 625021, INDIA

Telephone: (91) 85252

Facsimile: (91)

Email:

Commission(s): $28,33,40,47$

Telex: $445308 \mathrm{mku}$ in 
BANIA THOMAS MICHAEL, DPT OF ASTRONOMY, BOSTON UNIVERSITY, 725 COMMONWEALTH AVE BOSTON MA 02215, USA

Telephone: (1) 6173533652

Email:

\author{
Facsimile: (1) \\ Commission(s): 34,51
}

Telex: 95129 bos univ bsn

BANNI ALDO DR, STAZIONE ASTRONOMICA, VIA OSPEDALE 72, I 09124 CAGLIARI, ITALY

Telephone: (39) 70663544

Facsimile: (39) 70657657 Telex

Email: vaxga2::laser

Commission(s): 19

BANOS COSMAS J DR, ASTRONOMICAL INSTITUTE, NTL OBSERVATORY OF ATHENS, BOX 20048

GR 11810 ATHENS, GREECE

Telephone: (30) 13461191

Facsimile: (30)

Commission(s): 21

Telex: 215530 obsa gr

Email:

BANOS GEORGE J PROF, DPT OF PHYSICS/DAG, UNIVERSITY OF IOANNINA, GR 45332 IOANNINA, GREECE

Telephone: (30) $65191697 \quad$ Facsimile: (30)

Email:

Commission(s):

Telex: 322160

BAO KEREN, NANJING ASTRONOMICAL, INSTRUMENT FACTORY, NANJING, CHINA PR

Telephone: (86) $2546191 \quad$ Facsimilc: (86) Telex: 34136 glynj c/o naif

Email:

Commission(s): 09

BARANNE A DR, OBSERVATOIRE DE MARSEILLE, 2 PLACE LE VERRIER, F 13248 MARSEILLE CDX 04, FRANCE Telephone: (33) 91959088

Facsimile: (33)

Commission(s): 09

Telex:

Email:

BARANOVSKY EDWARD A, CRIMEAN ASTROPHYS OBS, UKRAINIAN ACAD OF SCIENCE, NAUCHNY 334413 CRIMEA, UKRAINE

Telephone: (7)

Facsimilc: (7)

Telex:

Email:

Commission(s): 10

BARATTA GIOVANNI BATTISTA, OAR, VIA DEL PARCO MELLINI 84, I 00136 ROMA, ITALY

Telephone: (39) 6347056

Facsimile: (39)

Telex:

Email:

Commission(s): 29

BARATTA GIUSEPPE ANTONIO, OSS ASTROFISICO, CITTA UNIVERSITARIA, VIA A DORIA 6 I 95125 CATANIA, ITALY

Telephone: (39)

Facsimile: (39)

Telex:

Email:

Commission(s):

BARBA RODOLFO HECTOR DR, OBSERVATORIO ASTRONOMICO, PASEO DEL BOSQUE S/N 1900 LA PLATA (BS AS), ARGENTINA

Telephone: (54) 21217308

Email: rbarba@fcaglp.edu.ar

Facsimile: (54) $21211761 \quad$ Telex:

Commission(s):

BARBANIS BASIL PROF, DPT OF ASTRONOMY, UNIVERSITY THESSALONIKI, GR 5†006 THESSALONIKI, GREECE Telephone: (30) 31991357

Facsimile: (30)

Telex: $\mathbf{4 1 2 1 8 1}$

Email:

Commission(s): 33

BARBARO G DR, OSS ASTRONOMICO DI PADOVA, VIC DELL OSSERVATORIO 5, I 35122 PADOVA, ITALY

Telephone: (39) $49661499 \quad$ Facsimile: (39)

Email:

Commission(s):

Telex: $\mathbf{4 3 0 1 7 6}$ unpadu $i$

BARBERIS BRUNO, IST DI FISICA MATEMATICA, UNIVERSITA DI TORINO, VIA C ALBERTO 10

I 10123 TORINO ITALY

Telephone: (39) 11539214

Facsimile: (39)

Telex:

Email:

Commission(s): $07,33,47$

BARBIERI CESARE PROF, DPT DI ASTRONOMIA, UNIVERSITA DI PADOVA, VIC DELL OSSERVATORIO 5 I 35122 PADOVA, ITALY

Telephone: (39) 49661499

Facsimile: (39)

Telex: 430176 unpadu $i$

Email:

Commission(s): 5 
BARBIER-BROSSAT M DR, OBSERVATOIRE DE MARSEILLE, 2 PLACE LE VERRIER F 13248 MARSEILLE CDX 04, FRANCE

Tclephone: (33) 91959088

Facsimilc: (33)

Commission(s): 30,45

Telex: $\mathbf{4 2 0 2 4 1 \mathrm { f }}$

Email:

TORIO ASTROFISICO, VIA DELL

BARBON ROBERTO PROF, OSSERVATORIO ASTROFI
Telephone: (39) $42462665 \quad$ Facsimile: (39)

Email:

Commission(s): 28

Telex: 430110 seturist

BARBUY BEATRIZ DR, IAG, UNIVERSIDADE DE SAO PAULO, CP 9638, 01065970 SAO PAULO SP, BRAZIL

Telephone: (55) 115778599

Facsimile: (55) $112763848 \quad$ Telex: 1156735 iagm br

Email: 47556::barbuy/barbuy(a)iag.ansp.br

Commission(s): 29VP

BARCIA ALBERTO DR, CTR ASTRON DE YEBES, OAN, APD 148, E 19080 GUADALAJARA, SPAIN

Telephone: (34) 11290311

Facsimile: (34) 11290063 Telex:

Email: barcia cay.es

Commission(s): 09

BARCONS XAVIER DR, DPT DE FISICA MODERNA, UNIVERSIDAD DE CANTABRIA, AVD LOS CASTROS S/N

E 39005 SANTANDER, SPAIN

Telephone: (34) 42201461

Facsimile: (34) 42201402

Telex: 35861 educi c

Email: barcons 2 ccucvx.unican.es

Commission(s): 28

BARCZA SZABOLCS DR, KONKOLY OBSERVATORY, THEGE U 13/17, BOX 67, H 1525 BUDAPEST, HUNGARY

Telephone: (36) $1175 \$ 866 / 1754122$

Facsimile: (36) 11569640

Telex: 227+60 konob h

Email:

Commission(s):

BARDEEN JAMES M PROF, DPT OF PHYSICS, UNIVERSITY OF WASHINGTON, FM 15, SEATTLE WA 98195, USA

Telephone: (1) 2065452394

Facsimile: (1)

Commission(s): 47

Telex: $\$ 740096$ uw ui

Email:

BARGE PIERRE, LABO ASTRONOMIE SPATIALE, BP 8, F 13376 MARSEILLE CDX 12, FRANCE

Telephone: (33) 91055900

Email: barge(a)astrsp-mis.fr

Facsimile: (33) 91661855

Telex:

Commission(s):

BARKAT ZALMAN PROF, RACAH INST OF PHYSICS, HEBREW UNIV OF JERUSALEM, JERUSALEM 91904, ISRAEL Telephone: (972) $2584 \$ 90$

Facsimile: (972)

Telex: 25391

Email:

Commission(s):

BARKER EDWIN S DR, MCDONALD OBSERVATORY, UNIVERSITY OF TEXAS, BOX 1337

FT DAVIS TX $7973+1337$, USA

Telcphone: (1) $915 \$ 263263$

Email: esb(a)astro.as.utexas.cdu

Facsimile: (1) $915+2636+1 \quad$ Telex:

Commission(s): 15

BARKER PAUL K DR, DPT OF ELECTROPHYSICS, BOX 1143 STATION B, LONDON ON NGA 5K2, CANADA

Telephonc: (1) 5196682871

Facsimile: (1) 5196682871

Telex: $06.52+736$

Email:

Commission(s):

BARKER TIMOTHY DR, DPT PHYSICS \& ASTRONOMY, WHEATON COLLEGE, NORTON MA (12766, USA

Telephone: (1) 5082857722

Facsimile: (1)

Telex:

Email:

Commission(s):

BARLAI KATALIN DR, KONKOLY OBSERVATORY, THEGE U 13/17, BOX 67, H 1525 BUDAPEST, HUNGARY

Telephone: (36) $11755866 / 175+122$

Facsimilc: (36) 11569640

Telex: 227460 konob h

Email:

Commission(s):

BARLETTI RAFFAELE ENG, OSS ASTROFISCIO, DI ARCETRI, LARGO E FERMI 5, I 50125 FIRENZE, ITALY

Telephone: (39) $55+378540$

Facsimile: (39) $55+35939$

Telex: $\mathbf{5 7 2 2 6 8}$

Email:

Commission(s):

BARLIER FRANCOIS E DR, OCA CERGA, AVE COPERNIC, F 06130 GRASSE, FRANCE

Telephone: (33) 93365849

Facsimile: (33)

Telex: 470865

Email:

Commission(s): 19 
BARLOW MICHAEL J DR, DPT PHYSICS \& ASTRONOMY, UNIVERSITY COLLEGE LONDON, GOWER ST LONDON WC1E 6BT, UK

Telephone: (44) 1713877050

Email:
Facsimile: (44)

Commission(s): $3 \downarrow$
Telex: 28722 ucphys g

BARNES AARON DR, NASA AMES RESEARCH CTR, MS 2453 , MOFFETT FIELD CA 94035 , USA

Telephone: (1) 4156945506

Facsimile: (1)

Telex:

Commission(s): $34,+9$

BARNES III THOMAS G DR, MCDONALD OBSERVATORY, UNIVERSITY OF TEXAS, MCDONALD OBSERVATORY AUSTIN TX 78712 1083, USA

Telephone: (1) 5124711301

Email: tgh@astro.as.utexas.cdu
Facsimile: (1)

Commission(s): $25,27 \mathrm{C}$
Telex: 910-874-1351

BARNOTHY JENO DR PROF, 833 LINCOLN ST, EVANSTON IL 60201, USA

Telephonc: (1) 3129356299

Facsimile: (1)

Telex:

Email:

BAROCAS VINICIO PROF, 11 YEWLANDS AVE, FULWORD, PRESTON PR2 4QR, UK

Telephone: (44) $1772719249 \quad$ Facsimile: (44)

Telex:

Email:

Commission(s):

BARONE FABRIZIO DR, DPT DI SCIENZE FISICHE, UNIVERSITA DI NAPOLI, MOSTRA D OLTREMARE PAD 19 I 80125 NAPOLI, ITALY

Telephone: (39) 817253447

Email: fbaronc $(\bar{a}$ napoli.infn.it

Facsimile: (39) $8161+508$

Telex: 720320

BARRETO LUIZ MUNIZ PROF, OBSERVATORIO NACIONAL, RUA GL BRUCE 586, SAO CRISTOVAO 20921 RIO DE JANEIRO RJ, BRAZIL

Telephone: (55) 215807313

Facsimile: (55) 215800332

Telex: 02121288 obsn

Email:

Commission(s): 50

BARRETT PAUL EVERETT DR, NASA GSFC, CODE 668.1, GREENBELT MD 20771, USA

Telephone: (1)

Facsimile: (1)

Telex:

Email: barrett@heasrc.gsfe.nasa.gov

Commission(s): 25

BARROSO JR JAIR, OBSERVATORIO NACIONAL, RUA GL J CRISTINO 77, SAO CRISTOVAO 20921 RIO DE JANEIRO RJ, BRAZIL

Telephone: (55) $215807313 * 273$

Facsimile: (55) 215800332

Telex: 021-21288

Email:

Commission(s): 09

BARROW COLIN H DR, MPI FUER AERONOMIE, MAX PLANCK STR 2, POSTFACH 20

D 37189 KATLENBURG LINDAU, GERMANY

Telephone: (49) 55564011

Facsimile: (49) 5556401240

Telex:

Email:

Commission(s): $10,16,40,49$

BARROW JOHN DAVID, ASTRONOMY CENTRE, UNIVERSITY OF SUSSEX, FALMER, BRIGHTON BN1 9QH, UK Telephone: (44) 1273606755

Email:

BARROW RICHARD F DR, PHYSICAL CHEMISTRY LAB, UNIVERSITY OF OXFORD, SOUTH PARKS RD OXFORD OX1 3QZ, UK

Telephone: (44) 186553322

Email:

\author{
Facsimile: (4) Telex: \\ Commission(s): 14
}

BARSONY MARY, DPT OF PHYSICS, UNIVERSITY OF CALIFORNIA, RIVERSIDE, RIVERSIDE CA 92521, USA

Telephone: (1) 9097873984

Facsimile: (1) $909787+529 \quad$ Telex:

Email: fun(a)nusun.ucr.edu

Commission(s):

BARSTOW MARTIN ADRIAN DR, DPT OF PHYSICS, UNIVERSITY OF LEICESTER, UNIVERSITY RD

LEICESTER LE1 7RH, UK

Telephone: (44) $1162523+92$

Email: mab(auk.ac.lc.star/19838::mab

Facsimile: (44) $1162550182 \quad$ Telex:

Commission(s): 4 
BARTASIUTE STANISLAVA, ASTRONOMICAL OBSERVATORY, CIURLIONIO 29, VILNIUS 2009, LITHUANIA

Telephone: (370) 2633343

Facsimile: (370) 2223563 Telex:

Email: bartasiute@iff.vu.It

Commission(s): 33

BARTAYA R A DR, ABASTUMANI ASTROPHYSICAL, OBSERVATORY, GEORGIAN ACAD OF SCIENCES 383762 ABASTUMANI, GEORGIA

Telephone: (7) 237 ABASTUMANI

Facsimile: (7)

Telex: 327\$199

Email:

Commission(s): $45 \mathrm{C}$

BARTEL NORBERT HARALD DR, DPT OF PHYSICS, YORK UNIVERSITY, 4700 KEELE ST NORTH YORK ON M3J 1P3, CANADA

Telephone: (1) 4167365424

Facsimile: (1) $4167365516 \quad$ Telex:

Email:

Commission(s): 40

BARTH CHARLES A PROF, LASP, UNIVERSITY OF COLORADO, BOX 392, BOULDER CO 80309 0392, USA

Telephone: (1) 3034927502

Facsimile: (1)

Telex:

Email:

Commission(s): 49

BARTHEL PETER DR, KAPTEYN ASTRONOMICAL INST, UNIVERSITY OF GRONINGEN, BOX 800

NL 9700 AV GRONINGEN, NETHERLANDS

Telephone: (31) $50634073 \quad$ Facsimile: (31)

Email:

Commission(s): $28,40,47$

Telex: $\mathbf{5 3 5 7 2}$ stars $\mathrm{nl}$

BARTHOLDI PAUL DR, OBSERVATOIRE DE GENEVE, CHEMIN DES MAILLETTES 51

CH 1290 SAUVERNY, SWITZERLAND

Tclephone: (41) 227552611

Email: bartho@obs.unige.ch

Facsimile: (41) $227553983 \quad$ Telex: $419209 \mathrm{obs}$ ch

Commission(s):

BARTKEVICIUS ANTANAS, INST THEORETICAL PHYSICS, \& ASTRONOMY, GOSTAUTO 12

VILNIUS 2600, LITHUANIA

Telephone: (370) $2613440 \quad$ Facsimile: (370) $222+69+\quad$ Telex:

Email: barta itpa.fi.lt

Commission(s):

BARTOLINI CORRADO, DPT DI ASTRONOMIA, UNIVERSITA DI BOLOGNA, VIA ZAMBONI 33

I 40126 BOLOGNA, ITALY

Telephone: (39) 51226677

Facsimile: (39)

Commission(s): 27,42

Telex: 211664

Email:

IRE DE PARIS, SECTION DE MEUDON, F 92195 MEUDON PPL CDX, FRANCE

BARUCCI MARIA A DR, OBSERVATOIRE DE PARIS, SECTION DE MEUDON, F
Telephone: (33) 14507 7539)

Email: mesioa: :barucci/17670::barucci Commission(s): $15 \mathrm{C}$

BARUCH JOHN DR, DPT OF ELECTRICAL ENGIN, UNIVERSITY OF BRADFORD, BRADFORD BD7 IDP, UK

Telephone: $(\$ 4) 127 \$ 38+024$

Facsimile: (4) $127+391521 \quad$ Telex: 51309 unibfd g

Email: uk.ac.bradford.cleceng::jefb $\quad$ Commission(s): 09

BARVAINIS RICHARD DR, HAYSTACK OBSERVATORY, WESTFORD MA 01886, USA

Telephone: (1) $617692+764$ Facsimile: (1) Telex: 948149

Email:

Commission(s): 40

BARWIG HEINZ, INST ASTRON \& ASTROPHYSIK, UNIVERSITAETS STERNWARTE, SCHEINERSTR 1 D 81679) MUENCHEN, GERMANY

Telephone: (49) $89989021 \quad$ Facsimile: (49) $8992209427 \quad$ Telex: 529815 univm d

Email:

Commission(s): 09,27

BARYSHEV YURI, ASTRONOMICAL INSTITUTE, ST PETERSBURG UNIVERSITY, BYBLYOTECHNAYA PL 2 ST PETERSBURG 198904, RUSSIA

Telephone: (7) $812+287129$

Email: yuba(a)aispbu.spb.su

Facsimile: (7) $812+28+259 \quad$ Telex:

Commission(s):

BASART JOHN P, DPT OF PHYSICS, IOWA STATE UNIVERSITY, AMES IA 50011, USA

Telephone: (1) $51529+2663$

Facsimile: (1)

Telex:

Email:

Comnission(s): 
BATH GEOFFREY T DR, DPT OF ASTROPHYSICS, UNIVERSITY OF OXFORD, SOUTH PARKS RD OXFORD OX1 3RQ, UK

Telephone: (4) 1865511336

Email:

\author{
Facsimile: (4t) \\ Commission(s): 27,42
}

Telex: 83295

BATRAKOV YU V DR, INST OF THEORET ASTRONOMY, ACADEMY OF SCIENCES, N KUTUZOVA 10 191187 ST PETERSBURG, RUSSIA

Telephone: (7) 2724023

Facsimile: (7)

Telex: 121578 ita su

Email:

Conmission(s): 07,20

BATSON RAYMOND MILNER DR, US GEOLOGICAL SURVEY, BRANCH OF ASTROGEOLOGY, 2255 N GEMINI DR FLAGSTAFF AZ 86001, USA

Telephone: (1) 6025567260

Email: nasamailra\%batson

Facsimile: (1) $6025567090 \quad$ Telex:

Commission(s): 16

BATTANER EDUARDO DR, DPT FIS TEORICA \& COSMOS, FAC DE CIENCIAS, AVD FUENTENUEVA, E 18002 GRANADA, SPAIN

Telephone: (34) 202212306

Facsimile: (34)

Telex:

Email:

Commission(s): 16,28

BATTEN ALAN H DR, HERZBERG INST ASTROPHYS, DOMINION ASTROPHYS OBS, 5071 W SAANICH RD VICTORIA BC V8X +M6, CANADA

Telephonc: (1) $60+363$ (00109

Email: batten(a)dao.nrc.ca

Facsimile: (1) $60+36300+5 \quad$ Telex:

Conumission(s): $26,30,+2,46 \mathrm{C}$, WGWWDA

BATTINELLI PAOLO DR, OAR, VIA DEL PARCO MELLINI 84, I 00136 ROMA, ITALY

Telephone: (39) $63+7056$

Facsimile: (39) $63+7802$

Telex: 626326 daroma $i$

Email: 40061::battinclli

Commission(s): 28

BATTISTINI PIERLUIGI DR, OSS ASTRONOMICO, UNIVERSITA DI BOLOGNA, VIA ZAMBONI 33

I 40126 BOLOGNA, ITALY

Telephone: (39) 51519593

Email:

Facsimile: (39)

Commission(s):

Telex:

BATTY MICHAEL DR, SCIOOL OF MATHS PHYSICS, COMPUTING \& ELECTRONONICS

MACQUARIE UNIVERSITY, NSW 2109, AUSTRALIA

Telephone: (61)

Email:

Facsimile: (61)

Telex:

Commission(s): 40

BAUD BOUDEWIJN DR, FOKKER BV, SPACE DIVISION, BOX 760\%, NL 1117 ZJ SHIPHOL, NETHERLANDS

Telephone: (31) $2054+9111$

Facsimile: (31)

Commission(s): 33

Telex:

Email:

BAUDRY ALAIN DR, OBSERVATOIRE DE BORDEAUX, BP 89, F 33270 FLOIRAC, FRANCE

Telephone: (33) $5686+331$

Facsimile: (33) $56+10+251$ Telex:

Email:

Commission(s): 34,40

BAUER CARL A DR, DPT OF ASTRONOMY, PENNSYLVANIA STATE UNIV, 50G DAVEY LAB UNIVERSITY PARK PA 16802 , USA

Telephone: (1)

Facsimile: (1)

Telex:

Email:

Commission(s):

BAUER WENDY HAGEN, WHITIN OBSERVATORY, WELLESLEY COLLEGE, WELLESLEY MA 02181, USA

Telephone: (1) 617235 (1320)

Facsimile: (1)

Telex:

Email: w baucralucy.wellesley.edu

Commission(s): 27,29

BAUM STEF ALISON, STSCI, IOMEWOOD CAMPUS, 3700 SAN MARTIN DR, BALTIMORE MO 21218, USA

Telephone: (1) $410338+797$

Facsimile: (1)

Telex:

Email: sbaum(âstsci.edu

Commission(s): 28,40

BAUM WILLIAM A DR, 2124 NE PARK RD, SEATTLE WA 98105, USA

Telephone: (1)

Facsimile: (1)

Commission(s): 09,16,28,51

Telex:

Email: 
M 430

BAUSTIAN W W MR, KITT PEAK NTL OBS, BOX 26732, 950 N CHERRY AVE, TUCSON AZ 85726 6732, USA

Telephone: (1)

Facsimile: (1)

Telex:

Email:

Commission(s):

BAUTZ LAURA P DR, NTL SCIENCE FOUNDATION, DIV ASTRONOMICAL SCIENCES, 1800 G ST NW

WASHINGTON DC 20550, USA

Telephonc: (1) 2023579488

Facsimile: (i)

Telex:

Email:

Commission(s):

BAYM GORDON ALAN DR, DPT OF PHYSICS, UNIVERSITY OF ILLINOIS, 1110 W GREEN ST, URBANA IL 61801, USA

Telephone: (1) 2173334363

Facsimile: (1)

Telex: 910-830-6599 physics

Email:

Commission(s): 35,44

BAZILEVSKY ALEXANDR T, VERNADSKY INST GEOCHEM \&, ANALYTICAL CHEMISTRY, KOSYGIN STR 19 117334 MOSCOW, RUSSIA

Telephone: $(7)$

Facsimile: (7)

Telex:

Email:

Commission(s): 16

BEALE JOHN S DR, 231 MARLBOROUGH RD, SWINDON SN3 1NN, UK

Telephone: (44) $179334725 \quad$ Facsimile: (4)

Email: $\quad$ Commission(s):

Telex:

BEARD DAVID B DR, GORHAM HOUSE, 50 NEW PORTLAND RD, GORHAM ME 04(138, USA

Telephone: (1)

Facsimile: (1)

Telex:

Email:

Commission(s): $12,15,22$

BEAUDET GILLES DR, DPT DE PHYSIQUE, UNIVERSITE DE MONTREAL, CP 6128 SUCC A MONTREAL QC H3C 3J7, CANADA

Telephonc: (1) 5143436669

Facsimilc: (1) $51+3+32071$

Telex: $05562+25$

Email:

Commission(s): 35,51

BEAVERS WILLET I DR, ERWIN W FICK OBSERVATORY, IOWA STATE UNIVERSITY, AMES IA 50011, USA

Telephonc: (1) $51529+3667$

Facsimilc: (1)

Telex:

Email:

Commission(s): 26,30

BECHTOLD JILL DR, STEWARD OBSERVATORY, UNIVERSITY OF ARIZONA, TUCSON AZ 85721, USA

Telephone: (1) 5206216533

Facsimile: (1) $520+282854$

Telex:

Email:

Commission(s): 47

BECK H G, CARL ZEISS, FORSCHUNGSZENTRUM, CARL-ZEISS STR 1, D 07740 JENA, GERMANY

Telephone: (49)

Facsimile: (49)

Telex:

Email:

Commission(s):

BECK RAINER, MPI FUER RADIOASTRONOMIE, AUF DEM HUEGEL 69, D 53121 BONN, GERMANY

Telephone: (49) 228525320

Facsimile: (49)

Telex: $886+40$

Email: $\operatorname{rbeck}(\hat{a})$ mpifr-bonn.mpg.de

Commission(s): 25,28,40

BECKER ROBERT A DR, BOX 460\%, CARMEL CA 93921, USA

Telephone: (1)

Facsimile: (1)

Commission(s):

Telex:

Email:

BECKER ROBERT HOWARD, DPT OF PHYSICS, UNIVERSITY OF CALIFORNIA, DAVIS CA 95616, USA

Telephone: (1) 9167526921

Facsimile: (1)

Telex: 910-531-(0785 uc davs

Email:

Commission(s): 44

BECKER STEPHEN A, LOS ALAMOS NATIONAL LAB, MS B220, BOX 1663, LOS ALAMOS NM 87545, USA

Tclephonc: (1) 5056678931

Facsimilc: (1)

Telex: 660495

Email:

Commission(s): 35

BECKER SYLVIA, UNIVERSITAETS STERNWARTE, MUENCHEN, SCHEINERSTR 1, D 81679 MUENCHEN

GERMANY

Telephone: (49) 8992209439

Email: becker(a)usm.uni-muenchen.de
Facsimile: (49) $89922(19+27 \quad$ Telex:

Commission(s): 36 
BECKER WILHELM PROF, ASTRONOMISCHES INSTITUT, UNIVERSITAET BASEL, VENUSSTRASSE 7 CH 4102 BINNINGEN, SWITZERLAND

Telephone: (41) $612717711 / 12$

Facsimile: (41)

Telex:

Email:

Commission(s): $25,33,37$

BECKERS JACQUES M DR, ESO, KARL SCHWARZSCHILDSTR 2, D 85740 GARCHING MUENCHEN, GERMANY Telephone: (49) 89320060

Facsimile: (49) 893202362

Telex: $052828222 \mathrm{co} \mathrm{d}$

Email:

Commission(s): 10,12

BECKLIN ERIC E DR, DPT OF ASTRONOMY, UNIVERSITY OF CALIFORNIA, LOS ANGELES CA 90024, USA

Telephonc: (1) 2132060208

Facsimile: (1)

Telex:

Email: becklin@uclastro.bitnet

Commission(s): 09,3+

BECKMAN JOHN E PROF, INST DE ASTROFISICA, DE CANARIAS, OBS DEL TEIDE, E 38071 LA LAGUNA, SPAIN

Telephone: (34)

Facsimile: (3+)

Telex:

Email: iac::jeb/jeb(aiac.es

Commission(s): 12,29,34,47,51

BECKWITH STEVEN V W, MPI FUER ASTRONOMIE, KOENIGSTUHL, D 69117 HEIDELBERG, GERMANY

Telephone: (49) 6221528211

Facsimile: (49) $6221528246 \quad$ Telex:

Email: swwb@mpia-hd.mpg.de

Commission(s): 34,51

BEC-BORSENBERGER ANNICK, BUREAU DES LONGITUDES, 77 AVE DENFERT ROCHEREAU, F 75014 PARIS, FRANCE

Telephone: (33) 140512273

Facsimile: (33) 146332834

Telex:

Email: span:iapc08::borsenberger

Commission(s): $04,07,20$

BEDOGNI ROBERTO, DPT DI ASTRONOMIA, UNIVERSITA DI BOLOGNA, CP 596, I 40100 BOLOGNA, ITALY

Telephone: (39) 51222956

Facsimile: (39)

Telex: $21166+$ infnbo i

Email:

Commission(s): 27,34

BEEBE HERBERT A, DPT OF ASTRONOMY, NEW MEXICO STATE UNIV, LAS CRUCES NM 88003, USA

Telephone: (1) $505646+438$

Facsimile: (1)

Telex: 910-983-0549 nmsuc

Email:

Commission(s): 10,12

BEEBE RETA FAYE DR, DPT OF ASTRONOMY, NEW MEXICO STATE UNIV, BOX 4500, LAS CRUCES NM 88003, USA

Telephone: (1) 5056461938

Facsimile: (1)

Telex:

Email:

Commission(s): 16,51

BEER REINHARD DR, JPL CALTECH, MS 183 301, 4800 OAK GROVE DR, PASADENA CA 91109, USA

Telephone: (1) $81835+4748$

Facsimile: (1)

Telex:

Email:

Commission(s): (09,16

BEERS TIMOTHY C DR, PHYSICS \& ASTRONOMY DPT, MICHIGAN STATE UNIVERSITY

EAST LANSING MI $4882 \downarrow$, USA

Telephone: (1) $517353+541$

Facsimilc: (1)

Telex:

Email: beers $a$ msupa.bitnet

Commission(s): 30

BEGELMAN MITCHELL CRAIG, JILA, UNIVERSITY OF COLORADO, BOX 440, BOULDER CO 80309 0440, USA

Telephone: (1) $303+927856$

Facsimile: (1)

Telex: 755842 jila

Email:

Commission(s): 4

BEGEMAN KOR G DR, KAPTEYN ASTRONOMICAL INST, BOX 800, NL 9700 AV GRONINGEN, NETHERLANDS

Telephonc: (31) $5063+073$

Facsimile: (31) 50 636100

Telex: $\mathbf{5 3 5 7 2}$

Email: kgb@rugfxt.rug.nI

Commission(s): 28

BEGGS DENIS W MR, INSTITUTE OF ASTRONOMY, THE OBSERVATORIES, MADINGLEY RD

CAMBRIDGE CB3 OHA, UK

Telcphone: (44) 1223337548

Email:
Facsimile: (4) 1223337523

Commission(s):
Telex: 817297 astron

BEHR ALFRED PROF EMERITUS, ESCHENWEG 3, D 3406 BOVENDEN, GERMANY
Telephonc: (49) 5518897

Facsimile: (49)

Commission(s): 25

Talex:

Email: 
BEINTEMA DOUWE A DR, SPACE RESEARCH DPT, UNIVERSITY OF GRONINGEN, BOX 800 NL 9700 AV GRONINGEN, NETHERLANDS
Telephonc: (31) 50116631
Facsimile: (31)
Telex: 53572
Email:
Commission(s):

BEKENSTEIN JACOB D DR, DPT OF PHYSICS, BEN GURION UNIVERSITY, BOX 653, BEERSHEVA 84105, ISRAEL Telephone: (972) 57664271

Facsimile: (972)

Telex: 5253 unasi il

Email:

Commission(s):

BEL NICOLE J DR, OBSERVATOIRE DE PARIS, SECTION DE MEUDON, F 92195 MEUDON PPL CDX, FRANCE

Telephone: (33) $145347412 \quad$ Facsimile: (33) Telex: 201571

Email:

Commission(s): $12,34,47$

BELINSKY VLADIMIR DR, LANDAU INST THEOR PHYSICS, ACADEMY OF SCIENCES, 117940 MOSCOW, RUSSIA Telephone: (7) 951373244

Facsimile: (7) Telex:

Email:

Commission(s): 47

BELKOVICH O I DR, ENGELHARDT ASTRONOMICAL, OBSERVATORY, OBSERVATORIA STATION 422526 KAZAN, RUSSIA

Telephone: (7) 324827

Email: cao(a)astro.kazan.su

Facsimile: (7)

Commission(s): 21,22

BELL BARBARA DR, CFA, HCO/SAO, 60 GARDEN ST, CAMBRIDGE MA 02138, USA

Telephone: (1) 6174952688 Facsimile: (1) Telex:

Email: $\quad$ Commission(s): 10

BELL BURNELL S JOCELYN DR, DPT OF PHYSICS, THE OPEN UNIVERSITY, WALTON HALL

MILTON KEYNES MK7 6AA, UK

Telephone: (4t) $190827+066 \quad$ Facsimile: (4t) 1908653744 Telex:

Email: sjb_burnell(âk.ac.open.acs.vax Commission(s):

BELL JEFFREY F, PLANETARY GEOSCIENCES DIV, HAWAII INST OF GEOPHYSICS, 2525 CORREA RD HONOLULU HI 96822, USA

Telephone: (1) 8089563136

Facsimile: (1) $8089566322 \quad$ Telex:

Email: bell(akahana.pgd.hawaii.clu

Commission(s): 15

BELL KENNETH LLOYD DR, DPT OF APPLIED MATHS, \& THEORETICAL PHYSICS, QUEEN'S UNIVERSITY BELFAST BT7 1NN, UK

Telephone: (44) $1232245133 \quad$ Facsimile: (44) Telex: 74487 qubadm

Email:

Commission(s):

BELL MORLEY B, HERZBERG INST ASTROPHYS, NTL RESEARCH COUNCIL, 100 SUSSEX DR

OTTAWA ON K1A ORG, CANADA

Telephone: (1) 6139936060

Email:

Facsimile: (1) 61395266012 Telex: 0533715

Commission(s):

BELL ROGER A DR, ASTRONOMY PROGRAM, UNIVERSITY OF MARYLAND, COLLEGE PARK MD 20742, USA Telephone: (1) $301+5+6282$

Facsimile: (1)

Tclex: 887294

Email:

Commission(s): $36,37,45$

BELL STEVEN DR, ROYAL GREENWICH OBS, MADINGLEY RD, CAMBRIDGE CB3 OEZ, UK

Telephone: (4) $122337+774$

Facsimile: (44) $122337+700$

Telex: 265451 monref $g$

Email: sab(a)uk.ac.cambridge.astronomy. Commission(s): 42

BELLAS-VELIDIS IOANNIS DR, ASTRONOMICAL INSTITUTE, NTL OBSERVATORY OF ATHENS, BOX 20048

GR 11810 ATHENS, GREECE

Telephone: (30) 13461191

Facsimile: (30) $13463803 \quad$ Telex:

Email: ybellas(a)grathun 1

Commission(s): 29

BELMONTE AVILES J A DR, INST DE ASTROFISICA, DE CANARIAS, OBS DEL TEIDE, E 38200 LA LAGUNA, SPAIN Telephone: (34) 22605200

Facsimilc: (34) 22 26,3005

Telex: $926+10$ iac e

Email: iac::jba

Commission(s): 27,35 
BELSERENE EMILIA P, 421 E AHLVERS RD, PORT ANGELES WA 98362, USA

Telephone: (1) 2064573806 Facsimile: (1) Telex:

Email:

Commission(s): 27

BELTON MICHAEL J S DR, NOAO/KPNO, BOX 26732, 950 N CHERRY AVE, TUCSON AZ 85726 6732, USA

Telephone: (1) 5203188000

Facsimile: (1) $5203188360 \quad$ Telex:

Email: mbelton@noao.edu

Commission(s): 15,16

\author{
BELVEDERE GAETANO DR, IST DI ASTRONOMIA, CITTA UNIVERSITARIA, VIA A DORIA 6 \\ I 95125 CATANIA, ITALY \\ Telephone: (39) 95330533 \\ Facsimile: (39) \\ Commission(s): 10,27 \\ Telex: 970359 astret $i$ \\ Email:
}

BELYAEV NIKOLAJ A DR, INST OF THEORET ASTRONOMY, ACADEMY OF SCIENCES, N KUTUZOVA 10 191187 ST PETERSBURG, RUSSIA

Telephone: (7) 2790667

Facsimile: (7)

Commission(s):

Telex: 121578 ita su

Email:

BELY-DUBAU FRANCOISE, OCA OBSERV DE NICE, BP 229, F 06304 NICE CDX 4, FRANCE
Telephone: (33) $93890420 \quad$ Facsimile: (33) $92003033 \quad$ Telex: 460004 obsnice

Email: $\quad$ Commission(s): 14

BEM JERZY DR, ASTRONOMICAL INSTITUTE, WROCLAW UNIVERSITY, UL KOPERNIKA 11 PL 51622 WROCLAW, POLAND

Telephone: (48)

Facsimile: (48)

Telex:

Email:

Commission(s): 08

BENACCHIO LEOPOLDO, OSS ASTRONOMICO DI PADOVA, VIC DELL OSSERVATORIO 5, I 35122 PADOVA, ITALY

Telephone: (39) 49661499

Facsimilc: (39)

Commission(s): 05

Telex: 430176 unpadu i

Email: astrpd::benacchio

LAS REDES, OCEANO ALANTICO 11, E 11500 PUERTO SANTA MARI, SPAIN

BENAVENTE JOSE, URBANIZACION LAS REDES, OCEANO ALANTICO 11, E
Telephonc: (3t)
Facsimile: (34) Telex:

Email:

Commission(s): 31

BENAYDOUN JEAN-JACQUES DR, OBSERVATOIRE DE GRENOBLE, CERMO/ASTROPHYSIQUE, BP 53X F 38041 GRENOBLE CEDEX, FRANCE

$\begin{array}{lll}\text { Telephone: (33) } 76514914 & \text { Facsimile: (33) } 764+8821 & \text { Telex: usmg } 980134 \\ \text { Email: benayoun(a)frgagsi } & \text { Commission(s): } 34\end{array}$

BENDER PETER L DR, JILA, UNIVERSITY OF COLORADO, BOX $4+0$, BOULDER CO 803090440 , USA

Telephone: (1) $3034926793 \quad$ Facsimile: (1)

Email: $\quad$ Commission(s): 16,19,31

Telex: 755842 jila

BENDER RALF DR, UNIVERSITAETS STERNWARTE, SCHEINERSTR 1, D 81679 MUENCHEN, GERMANY

Telephone: (49) 8992209426

Facsimile: (49) $8992209427 \quad$ Telex:

Email: bender@usm.uni-muenchen.de Commission(s): 28

BENDINELLI ORAZIO, DPT DI ASTRONOMIA, UNIVERSITA DI BOLOGNA, VIA ZAMBONI 33

I 40126 BOLOGNA, ITALY

Telephone: (39) $51226677 * 956$

Facsimile: (39)

Email:

Commission(s): 28

Telex: $21166+$ infnbo i

BENEDICT GEORGE F DR, ASTRONOMY DPT, UNIVERSITY OF TEXAS, RLM 15 308, AUSTIN TX 78712 1083, USA

Telephone: (1) 5124714461

Facsimile: (1)

Telex:

Email:

Commission(s): $24,28,44$

BENEST DANIEL DR, OCA OBSERV DE NICE, BP 229, F O6304 NICE CDX 4, FRANCE

Telephone: (33) 92003108

Facsimile: (33) 92003033

Telex: $46000+$ obsnice $f$

Email: benest(a)obs-nice.fr

Commission(s): 07,20,51

BENEVIDES SOARES P DR, IAG, UNIVERSIDADE DE SAO PAULO, CP 9638, 01065 SAO PAULO SP, BRAZIL

Telephone: (55์) 112753720

Facsimile: (55) 112763848

Telex: 1156735 iagm br

Email:

Commission(s): 08C 
BENFORD GREGORY DR, DPT OF PHYSICS, UNIVERSITY OF CALIFORNIA, IRVINE CA 92717, USA

Telephone: (1) 7148565147

Facsimile: (1)

Telex:

Email:

Commission(s): 12,44

BENN CHRIS R DR, ROYAL GREENWICH OBS, ISAAC NEWTON GP TELESCOPE, APT 321

E 38780 SANTA CRUZ, SPAIN

Telephone: (34)

Facsimile: (34)

Telex:

Email:

Commission(s): 05,40

BENNETT CHARLES L DR, NASA GSFC, CODE 685, GREENBELT MD 20771, USA

Telephone: (1) 3012863902

Facsimile: (1)

Telex:

Email: champ: :bennett

Commission(s): $40,44,47$

BENNETT KEVIN DR, ESA/ESTEC, SSD, BOX 299, NL 2200 AG NOORDWIJK, NETHERLANDS

Telephone: (31) $171983559 \quad$ Facsimile: (31)

Commission(s): 44

Telex: 39098

Email: kbennett@estec

BENSAMMAR SLIMANE DR, OBSERVATOIRE DE PARIS, SECTION DE MEUDON F 92195 MEUDON PPL CDX, FRANCE

Telephone: (33) 145347835

Facsimile: (33)

Telex: 270912

Email:

Commission(s): 09,50

BENSON PRISCILLA J DR, WHITIN OBSERVATORY, WELLESLEY COLLEGE, WELLESLEY MA 02181, USA

Telephone: (1) 6172350320

Facsimile: (1)

Telex:

Email: pbenson@lucy.wcllesley.edu

Commission(s): $27,40,41,46$

BENVENUTI PIERO DR, ESO, ST/ECF, KARL SCHWARZSCHILDSTR 2, D 85740 GARCHING MUENCHEN, GERMANY

Telephone: (49) $8932006291 \quad$ Facsimile: (49)

Telex: 52828222 co d

Email:

Commission(s):

BENVENUTO OMAR DR, OBSERVATORIO ASTRONOMICO, PASEO DEL BOSQUE S/N 1900 LA PLATA (BS AS), ARGENTINA

Telephone: (54) 21217308

Facsimile: (54) 21255004

Telex: 31151 bulap ar

Email:

Commission(s): 44

BENZ ARNOLD DR, INSTITUT FUER ASTRONOMIE, ETH ZENTRUM, CH 8092 ZUERICH, SWITZERLAND

Telephone: (41) 12564223

Facsimile: (41) 12520192

Telex: 53178 ethbi ch

Email:

Commission(s): 10C,40,49C

BENZ WILLY, CENTER FOR ASTROPHYSICS, HCO/SAO, 60 GARDEN ST, CAMBRIDGE MA 02138, USA

Telephone: (1) 6174959889

Facsimile: (1)

Telex:

Email:

Commission(s): 35

BERENDZEN RICHARD DR, DPT OF PHYSICS, THE AMERICAN UNIVERSITY, , WASHINGTON DC 20016-8058, USA

Telcphone: (1) 2028852121

Facsimile: (1)

Telex:

Email:

Commission(s): 41,51

BERG RICHARD A DR, HQ DEFENSE MAPPING AGENCY, STOP A 10, 8613 LEE HIGHWAY, FAIRFAX VA 22031, USA

Telephone: (1) 7032859222

Facsimile: (1) 7032859396 Telex:

Email: wk02017@worldlink.com

Commission(s):

BERGE GLENN L DR, CALTECH, MS 170 25, OWENS VALLEY RADIO OBS, PASADENA CA 91125, USA

Telephone: (1) 8183566969

Facsimile: (1)

Telex: $675+25$

Email:

Commission(s): 16,40

BERGEAT JACQUES G DR, OBSERVATOIRE DE LYON, AVE CHARLES ANDRE

F 69561 S GENIS LAVAL CDX, FRANCE

Telephone: (33) 78560705

Email:

Facsimile: (33) 72399791

Telex:

Commission(s):

BERGER CHRISTIANE DR, OCA CERGA, AVE COPERNIC, , F 06130 GRASSE, FRANCE

Telephone: (33) 93405389

Facsimile: (33) 93405353

Telex: $470865 \mathrm{f}$

Email: berger@ocar01.obs-azur.fr

Commission(s): 
BERGER JACQUES G DR, OBSERVATOIRE DE PARIS, 61 AVE OBSERVATOIRE, F 75014 PARIS, FRANCE

Telephone: (33) 140512247

Facsimile: (33)

Commission(s): 29

Telex: 270776 obs $f$

Email:

BERGER MITCHELL DR, DPT OF APPLIED MATHS, UNIVERSITY OF ST ANDREWS, NORTH HAUGH

ST ANDREWS FIFE KY16 9SS, UK

Telephone: (44) $133476161 \quad$ Facsimile: (44)

Email: earn\%solar::mberger

Commission(s): 10

Telex: 76213 saulib gb

BERGERON JACQUELINE A DR, ESO, KARL SCHWARZSCHILDSTR 2, D 85740 GARCHING MUENCHEN, GERMANY

Telephone: (49) 89320060

Facsimile: (49) 89320 2362/32006480 Telex:

Email: jbergero@eso.org

Commission(s): $28,34,44,47, \mathrm{EC}$

BERGSTRALH JAY T DR, NASA, MC SLC, WASHINGTON DC 20546, USA

Telephone: (1) 2023580313

Facsimile: (1) 2023583097 Telex:

Email: jbergstr@nhqvax.hq.nasa.gov

Commission(s): 16

BERGVALL NILS AKE SIGVARD, ASTRONOMICAL OBSERVATORY, BOX 515, S 75120 UPPSALA, SWEDEN

Telephone: (46)

Facsimile: (46)

Telex:

Email:

Commission(s): 28

BERKHUIJSEN ELLY M DR, MPI FUER RADIOASTRONOMIE, AUF DEM HUEGEL 69, D 53121 BONN, GERMANY

Telephonc: (49)

Email:

Facsimile: (49)

Commission(s): $28,33,34,40 \mathrm{C}$

Telex: 886440

BERMAN MARCELO S DR, RUA CANDIDO HARTMAN 575, AP 17 ED RENOIR, 80430 CURITIBA PR, BRAZIL

Telephonc: (55) $\$ 12246426$

Facsimile: (55) $412261679 \quad$ Telex:

Email:

Commission(s): 47

BERMAN ROBERT HIRAM DR, DPT OF PHYSICS, MIT, BOX 165, CAMBRIDGE MA 02139, USA

Telephone: (1) 6172531000

Facsimile: (1)

Telex:

Email:

Commission(s):

BERMAN VLADIMIR, STSCI, CSC SPST, 3700 SAN MARTIN DR, BALTIMORE MD 21218, USA

Telephone: (1) 4104863518

Facsimile: (1) $\$ 10338+767 \quad$ Telex:

Email: berman@stsci.edu

Commission(s): 28

BERNACCA P L PROF, OSSERVATORIO ASTROFISICO, VIA DELL OSSERVATORIO 8, I 36012 ASIAGO, ITALY

Telephone: (39) $42462505 \quad$ Facsimile: (39)

Email:

Commission(s): $26,44,51$

Telex: $\mathbf{4 3 0 1 1 0}$ setour

BERNAT ANDREW PLOUS DR, DPT OF COMPUTER SCIENCE, UNIVERSITY OF TEXAS, EL PASO TX 79968, USA

Telephone: (1) $9157+75494$

Facsimile: (1)

Telex:

Email:

Commission(s): 34,36

BERNSTEIN HANS HEINRICH, ASTRON RECHEN INSTITUTE, MOENCHHOFSTR 12-14

D 69120 HEIDELBERG, GERMANY

Telephone: (49) 6221405252

Facsimile: (49) $6221405297 \quad$ Telex:

Email: s03@mvs.urz.uni-heidelberg.de

Commission(s): 08

BERRINGTON KEITH ADRIAN, DPT OF APPLIED MATHS \& THEORETICAL PHYSICS, QUEEN'S UNIVERSITY BELFAST BT7 1NN, UK

Telephone: (44)

Facsimile: (44)

Telex:

Email:

Commission(s): 14

BERRUYER-DESIROTTE N DR, OCA OBSERV DE NICE, BP 229, F 06304 NICE CDX 4, FRANCE

Telephonc: (33) 92003011

Facsimile: (33) 92 (00) 3033

Telex: 460004 obsnice $f$

Email:

Commission(s):

BERTAUX J L DR, SERVICE D'AERONOMIE, BP 3, F 91371 VERRIERES BUISSON, FRANCE

Telephone: (33) 169203116

Facsimile: (33)

Telex: 602400

Email:

Commission(s): 16,49 
M 436

BERTELLI GIANPAOLO DR, DPT DI ASTRONOMIA, UNIVERSITA DI PADOVA, VIC DELL OSSERVATORIO 5 I 35122 PADOVA, ITALY

Telcphone: (39) 49661499

Facsimilc: (39)

Commission(s):

Telex: $\mathbf{4 3 2 0 7 1}$ astros $i$

Email:

BERTHET STEPHANE, OFFICE FEDERAL DE L'EDUCA, DE LA SCIENCE, BOX 5675, CH 3001 BERN, SWITZERLAND Telephone: (41) 313229967 Facsimile: (41) 313227854 Telex: 912981 bbw ch

Email: stephane.berthet@r.bbw0.adwin-ch.adminCommission(s):

BERTHOMIEU GABRIELLE DR, OCA OBSERV DE NICE, BP 229, F 06304 NICE CDX 4, FRANCE

Telcphone: (33) 93890420

Facsimile: (33) 92003033

Telex: $\mathbf{4 6 0 0 0 4}$ obsnice $f$

Email:

Commission(s): 27,35

BERTIAU FLOR C PROF, WAVERSEBAAN 220, B 3030 HEVERLEE, BELGIUM

Telephone: (32)

Email:

Facsimile: (32)

Telex:

BERTIN GIUSEPPE PROF, SCUOLA NORMALE SUPERIORE, PIAZZA DEI CAVALIERI, I S6100 PISA, ITALY

Telephone: (39) $50597265 \quad$ Facsimile: (39)

Email: bertin@ipisnsva Commission(s):

Telex: $\mathbf{5 9 0 5 4 8}$ snspi i

BERTOLA FRANCESCO PROF, DPT DI ASTRONOMLA, VIC DELL OSSERVATORIO 5, I 35122 PADOVA, ITALY

Telephone: (39) 498293436

Facsimile: (39) $\$ 98759840 \quad$ Telex:

Email: bertola@astrpd.pd.astro.it

Commission(s): 28VP,47

BERTOUT CLAUDE, INSTITUT D'ASTROPHYSIQUE, 98BIS BD ARAGO, F 75014 PARIS, FRANCE

Telephone: (33) 144328000

Facsimile: (33) $1+4328001 \quad$ Telex:

Email:

Commission(s): 34,36

BERTSCHINGER EDMUND DR, DPT OF PHYSICS, MIT RM 6 207, BOX 165, CAMBRIDGE MA 02139, USA

Telcphone: (1) 6172535083

Facsimile: (1) $6172539798 \quad$ Telex:

Email: edbert@arcturus.mit.edu

Commission(s): 47

BESSELL MICHAEL S DR, MOUNT STROMLO \& SIDING, SPRING OBSERVATORIES, PRIVATE BAG

WESTON CREEK PO ACT 2611, AUSTRALIA

Telephone: (61) 62490268

Facsimile: (61) 62490233

Commission(s): 05C,25,27,29P

Telex: 62270 canopus aa

Email: bessell@mso.anu.edu.au

BETANCORT-RIJO JUAN DR, INST DE ASTROFISICA, DE CANARIAS, OBS DEL TEIDE, E 38200 LA LAGUNA, SPAIN

Telephone: (34) 22262211

Facsimile: (34) 22263005

Telex: 92640 iac e

Email:

Comnission(s): 47

BETTIS DALE G PROF, TICOM, UNIVERSITY OF TEXAS, AUSTIN TX 78712 1083, USA

Telephone: (1)

Facsimile: (1)

Commission(s): 07

Telex:

Email:

BETTONI DANIELA DR, OSS ASTRONOMICO DI PADOVA, VIC DELl OSSERVA TORIO 5, I 35122 PADOVA, ITALY

Telephone: (39) $49661499 \quad$ Facsimile: (39)

Email:

Commission(s): 28

Telex: $\mathbf{4 0 1 7 6}$ unpadu $i$

BEUERMANN KLAUS P PROF, UNIVERSITAETS STERNWARTE, GOETTINGEN, GEISMARLANDSTR 11 D 37083 GOETTINGEN, GERMANY

Telephone: (49) 551395041

Email: beucrmann(a) uswo50.dnet?gwdg.de

Facsimile: (49) $551395043 \quad$ Telex:

Commission(s):

BEUTLER GERHARD PROF, ASTRONOMISCHES INSTITUT, UNIVERSITAET BERN, SIDLERSTRASSE 5 CH 3012 BERN, SWITZERLAND

Telephone: (41) 31658596

Email: bcutler@ajub.unibe.ch

Facsimile: (41) 31653869

Telex: 912643 pibe ch

BHANDARI N DR, PHYSICAL RESEARCH LAB, NAVRANGPURA, AHMEDABAD 380 009, INDIA

Telephone: (91) 272462129

Facsimilc: (91) 272 +\5292 Telex: 0121397

Email:

Commission(s): 22 
BHANDARI RAJENDRA DR, RAMAN RESEARCH INSTITUTE, SADASHIVANAGAR, CV RAMAN AVE BANGALORE 560 080, INDIA

Telephone: (91) 803360122

Facsimile: (91) 803340492

Telex: $\mathbf{8 4 5 2 6 7 1}$ rri in

Email:

Commission(s): 40

BHAT CHAMAN LAL DR, HEAD NRL HARL C 7, BHABHA ATOMIC RES CENTRE, SHASTRI NAGAR JAMMU 180 004, INDIA

Telephone: (91)

Facsimile: (91)

Email:

Commission(s):

Telex:

BHAT NARAYANA P DR, TIFR, HOMI BHABHA RD, COLABA, BOMBAY 400 005, INDIA

Telephone: (91) $224952311 \quad$ Facsimile: (91)

Telex: 0113009 tifr in

Email:

Commission(s):

BHATIA PREM K DR, DPT OF MATHEMATICS, UNIVERSITY OF JODHPUR, JODHPUR 342 O01, INDIA

Telephone: (91)

Facsimile: (91)

Telex:

Email:

Commission(s):

BHATIA R K DR, DPT OF ASTRONOMY, UNIVERSITY OF OSMANIA, HYDERABAD 500 007, INDIA

Telephone: (91) 71951

Facsimile: (91)

Telex:

Email:

Commission(s): 16

BHATIA V B DR, DPT PHYSICS \& ASTROPHYS, UNIVERSITY OF DELHI, NEW DELHI 110 007, INDIA

Telephone: (91) $112918993 \quad$ Facsimile: (91)

Telex:

Email:

Commission(s):

BHATNAGAR ARVIND DR, UDAIPUR SOLAR OBSERVATORY, 11 VIDYA MARG, UDAIPUR 313 001, INDIA

Telephone: (91) 25 626/23 861

Facsimile: (91)

Telex:

Email:

Commission(s): 10C,12

BHATNAGAR ASHOK KUMAR, POSITIONAL ASTR CTR, P 546 BLOCK N 1ST FL, NEW ALIPORE

CALCUTTA 700 053, INDIA

Telephonc: (91) $33450321 / 493541 \quad$ Facsimile: (91) Telex:

Email:

Commission(s): 04

BHATNAGAR K B DR, ZAKIR HUSSAIN COLLEGE, UNIVERSITY OF DELHI, AJMERI GATE

NEW DELHI 110 006, INDIA

Telephone: (91) 11522802

Facsimile: (91) 117234544

Telex: 3162442,3162431

Email:

Commission(s): 07

BHATT H C DR, INDIAN INSTITUTE OF, ASTROPHYSICS, KORAMANGALA, BANGALORE 560 034, INDIA Telephone: (91) 803566585

Facsimile: (91)

Commission(s): 34

Telex: 845763 iiab in

Email:

BHATTACHARYA DIPANKAR, RAMAN RESEARCH INSTITUTE, SADASHIVANAGAR, CV RAMAN AVE

BANGALORE 560 080, INDIA

Telephone: (91) 803340122

Facsimile: (91) 803340492

Telex: 8452671

Email: dipankar@rri.ernet.in

Commission(s):

BHATTACHARYYA J C PROF, INDIAN INSTITUTE OF, ASTROPHYSICS, KORAMANGALA

BANGALORE 560034 , INDIA

Telephone: (91) 80356 6583/6585

Facsimile: (91) 805534043

Telex: 845763 iiab in

Email:

Commission(s): 09C,12,50C

BHATTACHARYYA TARA DR, JOGAMAYA DEVI COLLEGE, 92 SYAMAPRADAD MUKERJEE

CALCUTTA 700 026, INDIA

Telephone: (91)

Facsimile: (91)

Telex:

Email:

Commission(s): 28

BHAVSAR SUKETU P, DPT PHYSICS \& ASTRONOMY, UNIVERSITY OF KENTUCKY

LEXINGTON KX 405060055 , USA

Telephone: (1) 6062576722

Facsimile: (1)

Telex:

Email:

Commission(s): 47 
BHONSLE RAJARAM V PROF, PHYSICAL RESEARCH LAB, NAVRANGPURA, AHMEDABAD 380 009, INDIA Telephone: (91) 272462129 Facsimile: (91) 272445292 Telex: 121397 prl in

Email: Commission(s): 40

BIAN YULIN, BEIJING ASTRONOMICAL OBS, CAS, W SUBURB, BEIJING 100080, CHINA PR Telephone: (86) Facsimile: (86) 12561085 Telex: 22040 baoas cn Email: bmabao@ica.beijing.canet.cn Commission(s): 28

BIANCHI LUCIANA, OSS ASTRONOMICO DI TORINO, ST OSSERVATORIO 20, I 10025 PINO TORINESE, ITALY Telephone: (39) 11842040 Facsimile: (39) Email: Commission(s): 34,44 Telex: 213236 to astr $i$

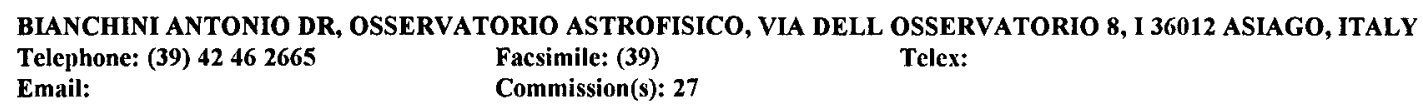

BICAK JIRI DR, DPT OF MATH PHYSICS, CHARLES UNIVERSITY, HOLESOVICKACH 2 CZ 18000 PRAHA 8, CZECH R

Telephone: (42) 2849951

Facsimile: (42) 2847688

Telex: $121673 \mathrm{mff}$

Email: bicak@cspunil2

Commission(s):

BICKNELL GEOFFREY V DR, MOUNT STROMLO \& SIDING, SPRING OBSERVATORIES, PRIVATE BAG WODEN PO ACT 2606, AUSTRALIA

Telephone: (61) 62881111

Email: nssdca::psi\%mssso::geoff

Facsimile: (61) 62490233

Telex: 62270 aa

Commission(s): 44,47

BIDELMAN WILLIAM P PROF, WARNER \& SWASEY OBS, CASE WESTERN RESERVE UNIV CLEVELAND OH 44106, USA

Telephone: (1) 2163686699

Facsimile: (1)

Telex:

Email:

Commission(s): 45

BIEGING JOHN HAROLD DR, STEWARD OBSERVATORY, UNIVERSITY OF ARIZONA, TUCSON AZ 85721, USA

Telephone: (1) 5206214878

Facsimile: (1)

Telex:

Email: jbieging@as.arizona.edu

Commission(s): 34,40

BIEMONT EMILE DR, INSTITUT D'ASTROPHYSIQUE, UNIVERSITE DE LIEGE, AVE COINTE 5

B 4000 COINTE-LIEGE, BELGIUM

Telephone: (32) $41529980 \quad$ Facsimile: (32) $41527474 \quad$ Telex:

Email:

Commission(s): 14

BIEN REINHOLD DR, ASTRON RECHEN INSTITUTE, MOENCHHOFSTR 12-14, D 69120 HEIDELBERG, GERMANY

Telephone: (49) 622149026

Facsimile: (49)

Telex: 461336 arihd d

Email:

Commission(s): 08,20

BIENAYME OLIVIER DR, OBSERVATOIRE DE BESANCON, BP 1615, F 25010 BESANCON CDX, FRANCE

Telcphone: (33) 81666900

Email: bienayme@efrobes51

Facsimile: (33) 81666944

Telex: 361144

Commission(s): 33

BIERMANN PETER L DR, MPI FUER RADIOASTRONOMIE, AUF DEM HUEGEL 69, D 53121 BONN, GERMANY

Telephone: (49) 228525279

Facsimile: (49)

Email:

Commission(s): $28,40,44$

Telex: 8864+0

BIGNAMI GIOVANNI F, IST DI FISICA COSMICA, CNR, 15/A VIA BASSINI, I 20133 MILANO, ITALY

Telephone: (39) 22367587

Facsimile: (39)

Email:

Commission(s): 44,47

Telex: 313839 muacnr $i$ 
BIGNELL R CARL DR, NRAO, VLA, BOX 0, SOCORRO NM 87801 0387, USA

Telephone: (1) 5057724242

Facsimile: (1)

Telex: 910-988-1710

Email:

Commission(s): 34,40

BIJAOUI ALBERT DR, OCA OBSERV DE NICE, BP 229, F 06304 NICE CDX 4, FRANCE

Telephone: (33) 93890420

Facsimile: (33) 92003033

Telex: 460004 obsnice $f$

Email:

Commission(s): 28,37

BILLAUD GERARD J, OCA CERGA, AVE COPERNIC, F 06130 GRASSE, FRANCE

Telephone: (33) 93365849

Facsimile: (33)

Telex: $\mathbf{4 7 0 8 6 5}$

Email:

Commission(s): 08

BILLINGHAM JOHN, SETI INSTITUTE, 2035 LANDINGS DR, MOUNTAIN VIEW CA 94043, USA

Telephone: (1) 4159616633

Facsimile: (1) 4159617099

Telex: 348408 nasa ames mof

Email:

Commission(s): 51

BILLINGS DONALD E PROF, 3E MOORE STREET, STATESBORO CA 30458, USA

Telephone: (1) 9127647625

Facsimile: (1)

Telex:

Email:

Commission(s): 12

BINETTE LUC, CITA MCLENNAN LABS, UNIVERSITY OF TORONTO, 60 ST GEORGE ST TORONTO ON MSS 1A1, CANADA

Telephone: (1) 4169788497

Email:

Facsimile: (1) $4169783921 \quad$ Telex:

Commission(s): 28,34

BINGGELI BRUNO, ASTRONOMISCHES INSTITUT, UNIVERSITAET BASEL, VENUSSTRASSE 7

CH 4102 BINNINGEN, SWITZERLAND

Telephone: (41) $612717711 / 12 \quad$ Facsimile: (41) Telex:

Email:

Commission(s): 28

BINGHAM RICHARD G DR, ROYAL GREENWICH OBS, MADINGLEY RD, CAMBRIDGE CB3 OEZ, UK

Telephone: (44) 1223374000

Facsimile: (44) $1223374700 \quad$ Telex:

Email: rgb@uk.ac.ro-greenwich.srf

Commission(s): 09

BINNEY JAMES J DR, DPT OF PHYSICS, UNIVERSITY OF OXFORD, 1 KEBLE RD, OXFORD OX1 3NP, UK

Telcphone: (44) 1865273979

Facsimile: (44) 1865273974

Telex: 83295 nuclox $g$

Email: binncy@thphys.ox.ac.uk

Commission(s): 28,33P

BINZEL RICHARD P DR, DPT OF EARTH SCIENCE, MIT RM 54 426, BOX 165, CAMBRIDGE MA 02139, USA

Telephone: (1) 6172536486

Facsimile: (1)

Telex:

Email: rpb@astron.mit.edu

Commission(s): 15C,16

BIRAUD FRANCOIS DR, OBSERVATOIRE DE PARIS, SECTION DE MEUDON, F 92195 MEUDON PPL CDX, FRANCE

Telephone: (33) 145077602

Facsimile: (33)

Commission(s): 06,40,51

Telex: 270912

Email:

BIRCH PETER MR, PERTH OBSERVATORY, BICKLEY WA 6076, AUSTRALIA
Telephone: (61) 92938255
Facsimile: (61)

Email:

Commission(s): 15

BIRETTA JOHN ANTHONY DR, STSCI, HOMEWOOD CAMPUS, 3700 SAN MARTIN DR, BALTIMORE MD 21218, USA

Telephone: (1) 4103384917

Email: birctta@astsci.edu

Facsimile: (1) $4103384767 \quad$ Telex:

Commission(s): 28,40

BIRKINSHAW MARK, DPT OF ASTRONOMY, HARVARD UNIVERSITY, 60 GARDEN ST, CAMBRIDGE MA 02138, USA

Telephone: (1) 6174959092

Facsimilc: (1)

Telex: 921428

Email:

Commission(s): $28,40,47$

BIRKLE KURT PH D, MPI FUER ASTRONOMIE, KOENIGSTUHL, D 69117 HEIDELBERG, GERMANY

Telephone: (49)

Facsimile: (49)

Commission(s): 34

Telex:

Email:

BISHOP ROY L DR, AVONPORT NS BOP 1X0, CANADA

Telephone: (1)

Facsimile: (1)

Commission(s): 41

Telex:

Email: 
M 440

BISIACCHI GIANFRANCO DR, INSTITUTO DE ASTRONOMIA, UNAM, APDO POSTAL 70-264 04510 MEXICO DF, MEXICO

Telephone: (52) 9055484537

Facsimile: (52)

Email:

Commission(s):

Telex: 1760155 cicme

BISIKALO DMITRIJ, INSTITUTE OF ASTRONOMY, ACADEMY OF SCIENCES, 48 PYATNITSKAYA ST MOSCOW 109017, RUSSIA

Telephone: (7) 0952317375

Email: biskalo@inasan.rssi.ru
Facsimile: (7) 0952302081

Commission(s):
Telex: 411576 ascon su

BISNOVATYI-KOGAN G S DR, SPACE RESEARCH INSTITUTE, ACADEMY OF SCIENCES PROFSOJUZNAYA UL 84/32, 117810 MOSCOW, RUSSIA

Telephone: (7) 953333122

Facsimile: (7)

Commission(s): 35

Email:

RAY GROUP, HOMI BHABHA RD, COLABA, BOMBAY 400 005, INDIA

Telephone: (91) $22219111 \quad$ Facsimile: (91)
Email:

Telex: 113009 tifr in

BJOERNSSON GUNNLAUGUR, SCIENCE INSTITUTE, DUNHAGI 3, REYKJAVIK ISS 107, ICELAND

Telephone: (354) 1694800

Facsimile: (354) 128911

Telex: isinfo 2307

Email: gulli@raunvis.hi.is

Commission(s):

BJORNSSON CLAES-INGVAR, STOCKHOLM OBSERVATORY, S 133 36 SALTSJOEBADEN, SWEDEN

Telephonc: (46) 87170195

Facsimile: (46) 87174719

Telex: 12972 sobserv s

Email: bjornsson(a)astro.su.se

Commission(s):

BLAAUW ADRIAAN PROF DR, KAPTEYN ASTRONOMICAL INST, BOX 800

NL 9700 AV GRONINGEN, NETHERLANDS

Telephone: (31) 50634084

Facsimile: (31)

Commission(s): 24,33,37

Telex: $\mathbf{5 3 5 7 2}$ stars nl

Email:

BLACK ADAM ROBERT S, CAMBRIDGE UNIVERSITY PRES, STM THE EDINBURGH BLDG

SHAFTESBURY ROAD, CAMBRIDGE CB2 2RU, UK

Telephone: (44) 1223325766

Email: ablack@cup.cam.ac.uk

Facsimilc: (44) 1223315052

Telcx: $\mathbf{8 1 7 2 5 6}$

BLACK JOHN HARRY DR, STEWARD OBSERVATORY, UNIVERSITY OF ARIZONA, TUCSON AZ 85721, USA Telephone: (1) 5206216531

Facsimile: (1)

Commission(s): 14,34

Telex: 467175

Email:

BLACKMAN CLINTON PAUL DR, CARLSTON LODGE, CAMPSIE RD, TORRANCE, GLASGOW G64 4HD, UK Telephone: (44)

Facsimile: (44)

Email:

Commission(s):

Telex:

BLACKWELL DONALD E PROF, DPT OF ASTROPHYSICS, UNIVERSITY OF OXFORD, SOUTH PARKS RD OXFORD OX1 3RQ, UK

Telcphonc: (44) 1865511336

Email:

Facsimile: (44)

Telex:

Commission(s): 12,49

BLADES JOHN CHRIS DR, STSCI, HOMEWOOD CAMPUS, 3700 SAN MARTIN DR, BALTIMORE MD 21218, USA

Telephone: (1) 3013384805

Facsimile: (1)

Commission(s): $3 \sharp$

Telex: $68+9101$ stsci uw

Email: stscic::blades

BLAHA MILAN DR, NAVAL RESEARCH LABORATORY, CODE 4720,4555 OVERLOOK AVE SW, WASHINGTON DC 203755000 , USA

Telephone: (1)

Facsimile: (1)

Telex:

Email:

Commission(s): 14

BLAIR DAVID GERALD, DPT OF PHYSICS, UNIVERSITY W AUSTRALIA, NEDLANDS WA G009, AUSTRALIA

Telephone: (61)

Facsimile: (61)

Commission(s): 40

Telex: 92992 aa

Email:

BLAIR GUY NORMAN DR, MS 63 196, BOX 1000, WILSONVILLE OR 97070, USA
Telephone: (1)
Facsimile: (1)

Email: guy_blair@ecm.hf.intel.com Commission(s): 34 
BLAIR WILLIAM P DR, STSCI, HOMEWOOD CAMPUS, CHARLES \& 34TH ST, BALTIMORE MD 21218, USA

Telephone: (1) 3013388447

Facsimile: (1)

Telex: 9102400225

Email: span:scivax::wblair

Commissien(s): 42

BLAMONT JACQUES E PROF, CNES, 2 PLACE MAURICE QUENTIN, F 75039 PARIS CDX 01, FRANCE

Telephone: (33) 145087612

Facsimile: (33)

Telex: 214674

Email:

Commission(s): $12,15,16,21,44$

BLANCHARD ALAIN, OBS DE STRASBOURG, 11 RUE UNIVERSITE, F 67000 STRASBOURG, FRANCE

Telephone: (33) 88358220

Facsimile: (33) 88250160

Telex:

Email: blanchard@edsxb6.u-strasbu.fr Commission(s): 47

BLANCO CARLO DR, IST DI ASTRONOMIA, CITTA UNIVERSITARIA, VLA A DORIA 6, I 95125 CATANIA, ITALY

Telephone: (39) 95330533

Facsimile: (39)

Telex: 970359 astret i

Email:

Commission(s): $16 \mathrm{C}, 20,36,50$

BLANCO VICTOR M DR, 104 STANDISH DR, CHAPEL HILL NC 27514, USA

Tclephone: (56)

Email:

Facsimile: $(56)$

Telex:

Commission(s): $25,33,45,50 \mathrm{C}$

BLANDFORD ROGER DAVID DR, CALTECH, MS 130 33, THEROETICAL ASTROPHYSICS, PASADENA CA 91125 , USA

Telephone: (1) 2133564200

Facsimile: (1)

Telex: 675429

Email:

Commission(s): $40,44,49$

BLAND-HAWTHORN JONATHAN, ANGLO AUSTRALIAN OBS, BOX 296, EPPING NSW 2121, AUSTRALIA

Telephone: (61) 23724851

Facsimile: (61) $23724880 \quad$ Telex:

Email:

Commission(s): 28

BLASIUS KARL RICHARD DR, SANTA BARBARA RESEARCH CT, B 31/40, 75 COROMAR DRIVE

GOLETA CA 93117, USA

Telephone: (1) 8059683511

Email:

Facsimile: (1)

Telex:

Commission(s):

BLAZIT ALAIN DR, OCA OBSERV DU CALERN, CAUSSOLS, F 06460 S VALLIER THIEY, FRANCE

Telephone: (33) $93426270 \quad$ Facsimile: (33)

Email:

Commission(s):

Telex: 461402

BLECHA ANDRE BORIS G DR, OBSERVATOIRE DE GENEVE, CHEMIN DES MAILLETTES 51

CH 1290 SAUVERNY, SWITZERLAND

Telephone: (41) 227552611

Email: blecha@obs.unige.ch

Facsimile: (41) $227553983 \quad$ Telex:

Commission(s): 25

BLEEKER JOHAN A M DR IR, SPACE RESEARCH LABORATORY, SRON, SORBONNELAAN 2

NL 3584 CA UTRECHT, NETHERLANDS

Telephone: (31) 30535600

Facsimile: (31) 30540860

Email:

Commission(s): 44

Telex: 47224 astro $\mathrm{nl}$

BLESS ROBERT C PROF, DPT OF ASTRONOMY, UNIVERSITY OF WISCONSIN, 475 N CHARTER ST

MADISON WI 53706, USA

Telephone: (1) 6082621715

Facsimile: (1)

Telex:

Email:

Commission(s): $34,36,44,51$

BLEYER ULRICH, WIP GRAVITATION PROJEKT, UNIVERSITY OF POTSDAM, AN DER STERNWARTE 16 D 14482 POTSDAM, GERMANY

Telephone: (49) 3317499322

Email: ubleyer(a)aip.de

Facsimile: (49) $3317499203 \quad$ Telex:

Commission(s): 47

BLINOV N S DR, STERNBERG STATE ASTR INST, UNIVERSITETSKIJ PROSP 13, 119899 MOSCOW, RUSSIA

Telephone: (7) 951391049

Facsimile: (7)

Telex:

Email:

Commission(s): 19,31

BLITZ LEO DR, ASTRONOMY PROGRAM, UNIVERSITY OF MARYLAND, COLLEGE PARK MD 20742, USA

Telephone: (1) 3014056650

Facsimile: (1) 3013149067

Telex: 7108260352

Email: blitz@astro.umd.edu

Commission(s): 28,33C, 34 
BLITZSTEIN WILLIAM DR, DPT ASTRON \& ASTROPHYS, UNIV OF PENNSYLVANIA DAVID RITTENHOUSE LAB E1, PHILADELPHIA PA 19104, USA

Telephone: (1) 2158987899

Facsimile: (1)

Telex: 834621

Email:

Commission(s): 09,42

BLOCK DAVID LAZAR PROF, DPT COMPUT \& APPL MATHS, WITWATERSRAND UNIVERSITY PRIVATE BAG 3, WITS 2050, SOUTH AFRICA

Telephone: (27) 27117163761

Facsimile: (27) 27117163000

Telex: 427125 sa

Email: 076blok@witsvma.wits.ac.za

Commission(s): 28

BLOEMEN JOHANNES B G M DR, STERREWACHT, BOX 9513, NL 2300 RA LEIDEN, NETHERLANDS

Telephone: (31) $71275818 \quad$ Facsimile: (31)

Email:

Commission(s): 33C

Telex: 39058 astro $\mathrm{nl}$

BLOEMHOF ERIC E DR, CENTER FOR ASTROPHYSICS, HCO/SAO, 60 GARDEN ST, CAMBRIDGE MA 02138, USA

Telephone: (1) 6174957314

Facsimile: (1)

Telex:

Email: bloemhof@ecfa

Commission(s): 40

BLOMME RONNY DR, OBSERVATOIRE ROYAL DE, BELGIOUE, AVE CIRCULAIRE 3, B 1180 BRUSSELS, BELGIUM Telephone: (32) 23752484

Email: ronny@astro.oma.be Facsimile: (32) 23749822

Telex: 21565

BLONDIN JOHN M DR, DPT PHYSICS \& ASTRONOMY, UNIVERSITY NORTH CORALINA, PHILLIPS HALL CB 3255 CHAPEL HILL NC 275993255 , USA

Telephone: (1) 9199623018

Facsimile: (1) 9199620480

Telex:

Email: blondin@physics.unc.edu

Commission(s): 44

BLOW GRAHAM L, CARTER OBSERVATORY, BOX 2909, WELLINGTON, NEW ZEALAND

Telephone: (64) 4728167

Facsimile: $(64)$

Telex: nz 30172 natobs

Email:

Commission(s): 20

BLUDMAN SIDNEY A PROF, DPT OF PHYSICS, UNIV OF PENNSYLVANIA, PHILADELPHIA PA 19104, USA

Telephone: (1) 2158988151

Facsimile: (1)

Telex: 831908

Email:

Commission(s): $35,44,47$

BLUM PETER PROF, INSTITUT FUER ASTROPHYSIK, UNIVERSITAET BONN, AUF DEM HUEGEL 71 D 53121 BONN, GERMANY

Telephone: (49) 228733665

Email:

Facsimile: (49)

Telex: 886440

Commission(s): 49

BLUMENTHAL GEORGE R DR, LICK OBSERVATORY, UNIVERSITY OF CALIFORNIA, SANTA CRUZ CA 95064, USA

Telephone: (1) 4084292005

Email: bitnet:george@portal

Facsimile: (1)

Telex:

Commission(s): 28

BO SHU-REN, INST HISTORY NAT SCIENCE, 137 CAO NEISTREEL, BEIJING 100080, CHINA PR

Telephone: (86) 1557180

Facsimile: (86)

Tclex:

Email:

Commission(s): 41

BOBROWSKY MATTHEW, CTA INCORPORATED, 6116 EXECUTIVE BLVD, SUITE 800, ROCKVILLE MD 20852, USA

Telephone: (1) 3018161281

Facsimile: (1) 3018161429

Telex:

Email: mattb@cta.com

Commission(s): 34,46

BOCCHIA ROMEO DR, OBSERVATOIRE DE BORDEAUX, BP 89, F 33270 FLOIRAC, FRANCE

Telephone: (33) 56864330

Facsimile: (33) 56404251

Tclex:

Email:

Commission(s): $10,12,35$

BOCHKAREV NIKOLAI G DR, STERNBERG STATE ASTR INST, UNIVERSITETSKIJ PROSP 13 119899 MOSCOW, RUSSIA

Telephone: (7) $959391672 / 9328844$

Facsimile: (7) 959390126

Telex: $\mathbf{4 1 1 4 8 3} \mathbf{m g u}$ su

Email: boch@sai.msk.su

Commission(s): 34

BOCHONKO D RICHARD DR, DPT OF MATHS \& ASTRONOMY, UNIVERSITY OF MANITOBA WINNIPEG MB R3T 2M8, CANADA

Telephone: (1) 2044749501

Facsimile: (1)

Telex:

Email:

Commission(s): 27,46 
BOCHSLER PETER, PHYSIKALISCHES INSTITUT, UNIVERSITAET BERN, SIDLERSTRASSE 5 CH 3012 BERN, SWITZERLAND

Telephone: (41) 31654429

Facsimile: (41)

Email:

Commission(s): 49

Telex: 32320 phybe ch

BOCKELEE-MORVAN DOMINIQUE, OBSERVATOIRE DE PARIS, SECTION DE MEUDON F 92195 MEUDON PPL CDX, FRANCE

Telephone: (33) 145077605

Facsimile: (33)

Telex: 270912

Email:

Commission(s): 15,40

BODDAPATI G ANANDARAO DR, PHYSICAL RESEARCH LAB, NAVRANGPURA, ROOM 760 AHMEDABAD 380 009, INDLA

Telephone: (91) 272462129

Facsimile: (91) 272445292

Telex: 121397

Email:

Commission(s):

BODE MICHAEL F, SCHOOL SCI \& TECHNOLOGY, LIVERPOOL POLYTECHNIC, BYROM ST LIVERPOOL L3 3AF, UK

Telephone: (44)

Facsimile: (44)

Telex:

Email:

Commission(s): 34

BODENHEIMER PETER PROF, LICK OBSERVATORY, UNIVERSITY OF CALIFORNIA, SANTA CRUZ CA 95064, USA Telephone: (1) 4084292064

Facsimilc: (1)

Telex:

Email:

Commission(s): $\mathbf{3 4 , 3 5}$

BODO GIANLUIGI DR, OSS ASTRONOMICO DI TORINO, ST OSSERVATORIO 20, I 10025 PINO TORINESE, ITALY

Telephone: (39) $1184 ' 1067$

Facsimile: (39)

Commission(s): 36,39

Telex: 213236 to astri

Email:

BOEHM KARL-HEINZ PROF, DPT OF ASTRONOMY, UNIVERSITY OF WASHINGTON, FM 20

SEATTLE WA 98195, USA

Telephone: (1) 2065432888

Facsimile: (1)

Telex: $\mathbf{4 7 4 0 0 9 6}$

Email:

Commission(s): 12,35,36

BOEHM-VITENSE ERIKA PROF, DPT OF ASTRONOMY, UNIVERSITY OF WASHINGTON, FM 20

SEATTLE WA 98195 , USA

Telephone: (1) 2065434858

Facsimile: (1)

Telex:

Email:

Commission(s): 12,36

BOEHNHARDT HERMANN DR, INST ASTRON \& ASTROPHYSIK, UNIVERSITAETS STERNWARTE

SCHEINERSTR 1, D 81679 MUENCHEN, GERMANY

Telephone: (49) 899229446

Facsimile: (49) 899229427

Telex:

Email: hermann@vlt.usm.uni-muenchen.d

Commission(s): 15

BOERNER GERHARD DR, MPI F PHYSIK \& ASTROPHYS, FOEHRINGER RING 6, D 8000 MUENCHEN, GERMANY Telephone: (49)

Facsimile: (49)

Telex:

Email:

Commission(s):

BOERNGEN FREIMUT DR PH, ZNTRLINST F ASTROPHYSIK, KARL-SCHWARZSCHILD-OBS

D 6901 TAUTENBURG, GERMANY

Telephone: (49) 7823530

Email:

Facsimile: (49)

Telex:

Commission(s): 20,28

BOESGAARD ANN M PROF, INSTITUTE FOR ASTRONOMY, UNIVERSITY OF HAWAII, 2680 WOODLAWN DR HONOLULU HI 96822, USA

Telephone: (1) 8089568756

Facsimile: (1) 8089882790

Telex: $\mathbf{7 2 3 - 8 4 5 9}$

Email:

Commission(s): 29C,36

BOESHAAR GREGORY ORTH DR, STSCI, HOMEWOOD CAMPUS, 3700 SAN MARTIN DR

BALTIMORE MD 21218, USA

Tclephone: (1)

Facsimile: (1)

Telex:

Email:

Commission(s): 28,34

BOGDANOVICIUS PAVELAS, INST THEORETICAL PHYSICS, \& ASTRONOMY, GOSTAUTO 12

VILNIUS 2600 , LITHUANIA

Telephone: (370) 2620949

Facsimile: (370) 2225361

Telex:

Email: bogd@itpafi.lt

Commission(s): 
BOGGESS ALBERT DR, NASA GSFC, CODE 685, GREENBELT MD 20771, USA

Telephone: (1) $3012865975 \quad$ Facsimile: (1) Telex:

Email: $\quad$ Commission(s): 29,34,44

BOGGESS NANCY W DR, 319 STONINGTON RD, SILVER SPRING MD 20902, USA

Telephone: (1) Facsimile: (1) Telex:

Email: stars::nboggess Commission(s): 44

BOGOD VLADIMIR, SAO OF RUSSIAN AS, PULKOVO, ST PETERSBURG 196140, RUSSIA

Telephone: (7) $8121234200 \quad$ Facsimile: (7) $8123151701 \quad$ Telex: 121351 pulob su

Email: vbog@saoran.spb.su Commission(s):

BOHANNAN BRUCE EDWARD, KITT PEAK NTL OBS, BOX 26732, 950 N CHERRY AVE, TUCSON AZ 85726 6732, USA

Telephone: (1) $5203275511 \quad$ Facsimile: (1)

Telex: 0666484 aaura noao

Email:

Commission(s):

BOHLIN J DAVID DR, NASA HEADQUARTERS, CODE EZ, 600 INDEPENDENCE AVE SW

WASHINGTON DC 20546, USA

$\begin{array}{lll}\text { Telephone: (1) } 2024531466 & \text { Facsimile: (1) } & \text { Telex: } 89530 \\ \text { Email: } & \text { Commission(s): } & \end{array}$

BOHLIN RALPH C DR, STSCI, HOMEWOOD CAMPUS, 3700 SAN MARTIN DR, BALTIMORE MD 21218, USA

Telephone: (1) $3013384804 \quad$ Facsimile: (1) Telex: 6849101 stsci uwi

Email: $\quad$ Commission(s): 34,44

BOHN HORST-ULRICH, ELZERBERG 21, D 82541 MUENSING-AMMERLAN, GERMANY

Telephone: (49) $81778624 / 1714403745$ Facsimile: (49) Telex:

Email:

Commission(s): 10,12

BOHRMANN ALFRED PROF, SCHAERSTR 23, D 21029 HAMBURG, GERMANY

Telephone: (49) $7399800 \quad$ Facsimile: (49) Telex:

Email: $\quad$ Commission(s):

BOICE DANIEL CRAIG, SOUTHWEST RESEARCH INST, DIV 15, 6220 CULEBRA RD

SAN ANTONIO TX 782280551 , USA

Telephone: (1) 2105223782 Facsimile: (1) 2106474325 Telex:

Email: boice@swri.space.swri.edu Commission(s): 15

BOIGEY FRANCOISE, IMTA LAB MECAN CELESTE, UNIVERSITE PARIS VI, 4 PLACE JUSSIEU TOUR 66 F 75230 PARIS CDX 05, FRANCE

Telephone: (33)

Email:

Facsimile: (33)

Commission(s): 07

Telex:

BOISCHOT ANDRE DR, OBSERVATOIRE DE PARIS, SECTION DE MEUDON, F 92195 MEUDON PPL CDX, FRANCE

Telephone: (33) 145077774

Facsimile: (33)

Commission(s): 40

Telex: 200590

Email:

BOISSE PATRICK DR, RADIOASTRONOMIE ENS, 24 RUE LHOMOND, F 75231 PARIS CDX 05, FRANCE

Telephone: (33) 143291225

Facsimile: (33) 145873489

Tclex: 202601

Email: 29233::boisse

Commission(s): 34

BOISSON CATHERINE DR, OBSERVATOIRE DE PARIS, SECTION DE MEUDON, DAEC F 92195 MEUDON PPL CDX, FRANCE

Telephone: (33) 145077436

Facsimile: (33) $145077469 \quad$ Telex: 201571

Email: 17733::boisson/hoisson@frmeus1 Commission(s): 28

BOKSENBERG ALEC PROF, ROYAL GREENWICH OBS, MADINGLEY RD, CAMBRIDGE CB3 OEZ, UK

Telephone: (44) 1323374000

Facsimile: (44) 1223374700

Telex: 87451 rgobsy $g$

Email:

Commission(s): $28,44,47$

BOLAND WILFRIED, NFRA, BOX 2, NL 7990 AA DWINGELOO, NETHERLANDS

Telephone: (31) 52197244

Facsimile: (31)

Commission(s): 34

Email:

Telex: 
BOLCAL CETIN DR, DPT OF PHYSICS, UNIVERSITY OF ISTANBUL, 34459 VEZNECILER, TURKEY

$\begin{array}{lll}\text { Telephone: (90) } 13320240 & \text { Facsimile: (90) } & \text { Telex: } 26401 \text { bountr } \\ \text { Email: } & \text { Commission(s): }\end{array}$

Email:

Commission(s):

BOLDT ELIHU DR, NASA GSFC, CODE 661, GREENBELT MD 20771, USA

$\begin{array}{ll}\text { Telephone: (1) } 3012865853 & \text { Facsimile: (1) } \\ \text { Email: } & \text { Commission(s): }\end{array}$

BOLEY FORREST I, DPT OF PHYSICS \& ASTRON, DARTMOUTH COLLEGE, WILDER LABORATORY

HANOVER NH 03755, USA

Telephone: (1) 6036462966

Facsimile: (1)

Telex:

Email:

Commission(s):

BOLOIX RAFAEL DR, REAL INST Y OBSERVATORIO, DE LA ARMADA, CECILIO PUJAZON S/N

E 11110 SAN FERNANDO, SPAIN

Telephone: (34)

Facsimile: (34)

Telex:

Email:

Commission(s): 31

BOLTON C THOMAS PROF, DAVID DUNLAP OBSERVATORY, UNIVERSITY OF TORONTO, BOX 360 RICHMOND HILL ON LAC 4Y6, CANADA

Telephone: (1) 4168849562

Facsimile: (1) 4169783921

Telex: 06986766 tor

Email:

Commission(s): 27,42

BOMMIER VERONIQUE DR, OBSERVATOIRE DE PARIS, SECTION DE MEUDON, DAMAP

F 92195 MEUDON PPL CDX, FRANCE

Telephone: (33) 145077454

Facsimile: (33) Telex: 201571

Email: 28726::bommier

Commission(s): $10,12,14$

BONACCINI DOMENICO DR, OSS ASTROFISICO, DI ARCETRI, LARGO E FERMI 5, I S0125 FIRENZE, ITALY

Telephone: (39) 554378540

Facsimile: (39) $55435939 \quad$ Telex:

Email: 38954::bonaccini

Commission(s):

BONANNO GIOVANNI DR, OSS ASTROFISICO, CITTA UNIVERSITARIA, VIA A DORIA 6, I 95125 CATANIA, ITALY

Telephone: (39) 95330533

Facsimile: (39) 95330592 Telex:

Email: 40297::giovanni

Commission(s): 09

BONAZZOLA SILVANO DR, OBSERVATOIRE DE PARIS, SECTION DE MEUDON

F 92195 MEUDON PPL CDX, FRANCE

Telephone: (33) 145077429

Facsimilc: (33)

Telex: 201571

Email:

Commission(s): 42,44

BOND HOWARD E DR, STSCI, HOMEWOOD CAMPUS, 3700 SAN MARTIN DR, BALTIMORE MD 21218, USA

Telephone: (1) 3013384718

Facsimilc: (1)

Telex: 6849101

Email:

Commission(s): 27,29

BOND JOHN RICHARD, CITA MCLENNAN LABS, UNIVERSITY OF TORONTO, 60 ST GEORGE ST

TORONTO ON MSS 1A1, CANADA

Telephone: (1) 4169786874

Email: bond@utorphys.bitnet

Facsimile: (1) $4169783921 \quad$ Telex:

Commission(s): 47C

BONDAL KRISHNA RAJ DR, UTTAR PRADESH STATE, OBSERVATORY, PO MANORA PEAK 263129

NAINITAL 263 129, INDIA

Telephone: (91) 2136/2583

Email: astronomy nainital

Facsimile: (91)

Commission(s): 10

Telex:

BONDARENKO L N DR, STERNBERG STATE ASTR INST, UNIVERSITETSKIJ PROSP 13, 119899 MOSCOW, RUSSIA

Telephone: (7) 951393721

Facsimile: (7)

Telex:

Email:

Commission(s): 16

BONDI HERMANN PROF SIR, 69 MILL LANE, IMPINGTON CAMBS CB4 4XN, UK

Telephone: (44) 1223235075

Facsimile: (4) $1223336180 \quad$ Telex:

Email:

Commission(s): 35,47

BONET JOSE A, INST DE ASTROFISICA, DE CANARIAS, OBS DEL TEIDE, E 38071 LA LAGUNA, SPAIN

Telephone: (34)

Facsimile: (34)

Email:

Commission(s):

Tclex: 
BONIFAZI ANGELO DR, OSS ASTRONOMICO, UNIVERSITA DI BOLOGNA, CP 596, I 40100 BOLOGNA, ITALY Telephone: (39)

Facsimile: (39)

Telex:

Email:

Commission(s):

BONNEAU DANIEL, OCA OBSERV DU CALERN, CAUSSOLS, F 06460 S VALLIER THIEY, FRANCE

Telephone: (33) 93426270

Facsimile: (33)

Telex: 461402

Email:

Commission(s): 09,26

BONNET ROGER M DR, ESA, 8-10 RUE MARIO NIKIS, F 75738 PARIS CDX 15, FRANCE

Telephone: (33) $153697654 \quad$ Facsimile: (33) $153697236 \quad$ Telex: 202746

Email:

Commission(s): $12,44,49$

BONNET-BIDAUD J M DR, CEA CEN, DAPNIA/SAP, BP 2, F 91191 GIF/YVETTE CDX, FRANCE

Telephone: (33) 169089259

Facsimile: (33) 169086577

Telex: 604860

Email: 32779::bobi bobi at frsac11

Commission(s): 44

BONNOR W B PROF, 1 SOUTH BANK TERRACE, SURBITON SURREY KT6 6DG, UK

Telephone: (44) 13991103

Facsimile: (44)

Telex:

Email:

Commission(s): 47

BONO GIUSEPPE DR, OAT, BOX SUCC TRIESTE 5, VIA TIEPOLO II, I 34131 TRIESTE, ITALY

Telephone: (39) 403199233

Facsimile: (39) 40309418

Telex: $\$ 61137$ oat $i$

Email: astrts::bono/38439::bono

Commission(s): 35

BONOLI FABRIZIO, OSS ASTRONOMICO, UNIVERSITA DI BOLOGNA, CP 596, I 40100 BOLOGNA, ITALY

Telephone: (39) 51222956

Facsimile: (39)

Commission(s): 41

Telex: 211664 infnbo $i$

Email:

BONOMETTO SILVIO A DR, DPT DI FISICA G GALILEI, UNIVERSITA DI PADOVA, VIA MARZOLO 8

I 35131 PADOVA, ITALY

Telephone: (39) 49844111

Facsimile: (39) $4984+245$

Telex:

Email:

Commission(s): 44

BONSACK WALTER K PROF, SUITE 298, 5100 1B CLAYTON ROAD, CONCORD CA 94521, USA

Telephone: (1)

Facsimile: (1)

Telex:

Email:

Commission(s): 29

BONTEKOE ROMKE DR, ESA/ESTEC, SSD, BOX 299, NL 2200 AG NOORDWIJK, NETHERLANDS

Telephone: (31) 171985160 Facsimile: (31) $31171984690 \quad$ Telex:

Email: romke@guspace.rug.nI

Commission(s): 28

BOOK DAVID L, NAVAL RESEARCH LABORATORY, CODE 4040, 4555 OVERLOOK AVE SW WASHINGTON DC 20375 5000, USA

Telephone: (1)

Facsimile: (1)

Telex:

Email:

Commission(s): 12

BOOKBINDER JAY A DR, CENTER FOR ASTROPHYSICS, HCO/SAO MS 58, 60 GARDEN ST CAMBRIDGE MA 02138, USA

Telephone: (1) 6174957058

Facsimile: (1)

Email: bookbind@cfa227.harvard.cdu Commission(s):

Telex: 921428 satellite cam

BOOKMYER BEVERLY B DR, DPT PHYSICS \& ASTRONOMY, CLEMSON UNIVERSITY

CLEMSON SC 296341911 , USA

Telephone: (1) 8036563417

Facsimile: (1)

Telex:

Email:

Commission(s): 25,42

BOOTH ANDREW J, SCHOOL OF PHYSICS, UNIVERSITY OF SYDNEY, SYDNEY NSW 2006, AUSTRALIA

Telephone: (61) 26922222

Facsimile: (61)

Telex:

Email:

Commission(s):

BOOTH ROY S PROF, ONSALA SPACE OBSERVATORY, CHALMERS UNIV OF TECH, S 439 92 ONSALA, SWEDEN

Telephone: (46) 317725500

Facsimile: (46) 317725550

Telex: 2400 onspace s

Email:

Commission(s): 40 
TOLEDO OH 43606, USA

Telephone: (1) 4195372274

Email:
Facsimile: (1)

Commission(s): 27,29,36,42
Telex:

\section{BORCHKHADZE TENGIZ M DR, ABASTUMANI ASTROPHYSICAL, OBSERVATORY GEORGLAN ACAD OF SCIENCES, 383762 ABASTUMANI, GEORGIA}

Telephone: (7)

Email:
Facsimile: (7)

Telex:

Commission(s): 28

BORD DONALD JOHN, DPT OF NATURAL SCIENCES, UNIVERSITY OF MICHIGAN

DEARBORN MI 48128, USA

Telephone: (1) 3135935483

Facsimile: (1)

Telex:

Email:

Commission(s):

BORDERIES NICOLE, JPL, MS 301 150, 4800 OAK GROVE DR, PASADENA CA 91109, USA

Telephone: (1) $8183548211 \quad$ Facsimile: (1) Telex: 675429

Email: $\quad$ Commission(s): 07

BORGEEST ULF DR, HAMBURGER STERNWARTE, GOJENSBERGSWEG 112, D 21029 HAMBURG, GERMANY

Telcphone: (49) 4072524121

Facsimile: (49) 4072524198

Telex: 217884

Email: st40010@dhhuni4.bitnct

Commission(s): 47

BORGMAN JAN DR PROF, KAPTEYN OBSERVATORY, WERKGROEP, MENSINGHEWEG 20 NL 9301 KA RODEN, NETHERLANDS

Telephone: (31)

Facsimile: (31)

Commission(s): 25,34

Telex:

Email:

BORGNINO JULIEN DR, DPT ASTROPHYSIQUE, UNIVERSITE DE NICE, PARC VALROSE

F 06034 NICE CDX, FRANCE

Telephone: (33) 93519100

Facsimile: (33)

Commission(s): 09

Telex:

Email:

BORIAKOFF VALENTIN, NAIC, CORNELL UNIVERSITY, 420 SPACE SCIENCES BLDG, ITHACA NY 14853 6801, USA

Telephone: (1) 6072563734

Facsimile: (1)

Telex: 932454

Email:

Commission(s): 40

BORISOVA JORDANKA, BLVD TSARIGGRADSKO 72, BG 1784 SOFIA, BULGARIA

Telephone: (359) 2758927

Facsimile: (359) $2758927 \quad$ Telex:

Email: borisova@abgearn.bitnet

Commission(s):

BORKOWSKI KAZIMIERZ M DR, INST OF RADIO ASTRONOMY, N COPERNICUS UNIVERSITY, UL CHOPINA 12/18 PL 87100 TORUN, POLAND

Telephone: (48) 4856783327

Facsimile: (48) 485611651

Telex: 552324 trao pl

Email: kazik@pltumk11

Commission(s):

BORNMANN PATRICIA L DR, NOAA ERL R/E/SE, SPACE ENVIRONMENT LAB, 325 BROADWAY BOULDER CO 80303, USA

Telephone: (1) 3034973532

Email: span:selvax:pbornmann

Facsimilc: (1)

Telex: 45897 solterwarn bdr

BOROVICKA JIRI, ASTRONOMICAL INSTITUTE, CZ 25165 ONDREJOV, CZECH R

Telephone: (42) 204857153

Facsimile: (42) 204851611

Telex:

Email: borovic@asu.cas.cz

Commission(s): 22

BORRA ERMANNO F DR, DPT DE PHYSIQUE, UNIVERSITE DE LAVAL, STE FOY QC G1K 7P4, CANADA

Telephone: (1) 4186567405

Facsimile: (1) 4186562040

Telex: 05131621

Email:

Commission(s): 25

BOS ALBERT DR, NFRA, BOX 2, NL 7990 AA DWINGELOO, NETHERLANDS

Telephone: (31) 52197244

Facsimile: (31) 3152197332

Telex:

Email: psi-02041521004::system

Commission(s): 40 
BOSCHAN PETER DR, ASTRONOMISCHES INSTITUT, UNIVERSITAET MUENSTER, WILHELM KLEMM STR 10 D 48149 MUENSTER, GERMANY

Telephone: (49) $251833561 \quad$ Facsimile: (49) $251833669 \quad$ Telex: 892529

Email: boschan@cygnus.uni-muenster.de Commission(s):

BOSMA ALBERT DR, OBSERVATOIRE DE MARSEILLE, 2 PLACE LE VERRIER

F 13248 MARSEILLE CDX 04, FRANCE

Telephone: (33) $91959088 \quad$ Facsimile: (33)

Commission(s): 28

Telex: 420241

Email:

BOSMA PIETER B DR, DPT PHYSICS \& ASTRONOMY, FREE UNIVERSITY, DE BOELELAAN 1081 NL 1081 HV AMSTERDAM, NETHERLANDS

Telephone: (31) $205484139 \quad$ Facsimile: (31) $20461459 \quad$ Telex:

Imail: $\quad$ Commission(s): 16

BOSMAN-CRESPIN DENISE, BVD D AVROY 68, BOX 093, B 4000 COINTE-LIEGE, BELGIUM

Telephone: (32) $41237486 \quad$ Facsimile: (32)

Email:

Commission(s)

Telex:

BOSS ALAN P DR, DPT TERRESTR MAGNETISM, CARNEGIE INST WASHINGTON, 5241 BROAD BRANCH RD NW WASHINGTON DC 20015, USA

Telephone: (1) 2026844370

Facsimile: (1) 2023648726

Telex: 440427

Email: boss(a)ciw.ciw.edu

Commission(s): 07,16,35

BOTEZ ELVIRA DR, ASTRONOMICAL OBSERVATORY, STR CIRESILOR 19, R 3400 CLUJ NAPOCA, RUMANIA

Telephone: (40) 64194592

Facsimilc: (40)

Telex:

Email:

$\operatorname{Commission}(s): 41,46$

BOTTINELLI LUCETTE DR, OBSERVATOIRE DE PARIS, SECTION DE MEUDON, RADIOASTRONOMIE F 92195 MEUDON PPL CDX, FRANCE

Telephone: (33) 145077604

Facsimile: (33)

Telex: 270912

Email:

Commission(s): $28,40,46$

BOUCHER CLAUDE DR, INSTITUT GEOGRAPHIQUE NTL, 2 AVE PASTEUR, F 94160 SAINT MANDE, FRANCE

Telephone: (33) 143988000

Facsimile: (33)

Telex:

Email: boucher(a)ign.fr

Commission(s): 19

BOUCHET FRANCOIS R DR, INSTITUT D'ASTROPHYSIQUE, 98BIS BD ARAGO, F 75014 PARIS, FRANCE

Telephone: (33) 144328095

Facsimile: (33) 144328001

Telex:

Email: bouchet@friap51

Commission(s): 47

BOUCHET PATRICE DR, ESO, CASILLA 19001, SANTIAGO 19, CHILE

Telephone: (56) $26988757 \quad$ Facsimile: (56)

Email: pbouchet@dgaeso51/esomci::pato Commission(s): 28

Telex: 240881

BOUGEARD MIREILLE L DR, OBSERVATOIRE DE PARIS, 61 AVE OBSERVATOIRE, F 75014 PARIS, FRANCE

Telcphone: (33) 140512226

Facsimile: (33) 140512232

Telex: 270775 obs f

Email:

Commission(s): 08

BOUGERET J L DR, OBSERVATOIRE DE PARIS, SECTION DE MEUDON, DESPA

F 92195 MEUDON PPL CDX, FRANCE

Telephone: (33) 14507770

Facsimilc: (33)

Telcx: $20446+$

Email:

Commission(s): 10,12,4

BOUIGUE R, 14 AVE VICTOR HUGO, F 09500 MIREPOIX, FRANCE

Telephone: (33)

Facsimilc: (33)

Commission(s): 24

Telex:

Email:

BOULANGER FRANCOIS, RADIOASTRONOMIE ENS, 24 RUE LHOMOND, F 75231 PARIS CDX 05, FRANCE

Telephone: (33)

Facsimile: (33)

Telex:

Email:

Commission(s): 34

BOULESTEIX JACQUES, OBSERVATOIRE DE MARSEILLE, 2 PLACE LE VERRIER F 13248 MARSEILLE CDX 04, FRANCE

Telephone: (33) 91959088

Facsimile: (33)

Telex: $420241 f$

Email:

Commission(s): 
BOULON JACQUES J DR, OBSERVATOIRE DE PARIS, 61 AVE OBSERVATOIRE, F 75014 PARIS, FRANCE

Telephone: (33) 140512253

Facsimile: (33)

Commission(s): $27,30,33$

Telex: 270776 obs $f$

Email:

BOUSKA JIRI DR, DPT OF ASTRONOMY, CHARLES UNIVERSITY, SVEDSKA 8, CZ 150 00 PRAHA 5, CZECH R
Telephone: (42) 2540395
Facsimile: (42) 2299272

Email:

Commission(s): 05,15

BOUVIER JEROME, OBSERVATOIRE DE GRENOBLE, CERMO/ASTROPHYSIQUE, BP 68 F 38402 S MARTIN HERES CD, FRANCE

Telephone: (33) $76514790 \quad$ Facsimile: (33) $76448821 \quad$ Telex:

Email: bouvier@frgag51.bitnet Commission(s): 29,34

BOUVIER PIERRE PROF, OBSERVATOIRE DE GENEVE, CHEMIN DES MAILLETTES 51

CH 1290 SAUVERNY, SWITZERLAND

Telephone: (41) $227552611 \quad$ Facsimile: (41) $227553983 \quad$ Telex: 419209 obs ch

Email:

Commission(s): 29,37

BOWELL EDWARD L G DR, LOWELL OBSERVATORY, 1400 W MARS HILL RD, FLAGSTAFF AZ 86001, USA

Telephone: (1) 6027743358

Email: elgb@lowell.edu

Facsimilc: (1)

Telex:

BOWEN GEORGE H DR, DPT OF PHYSICS, IOWA STATE UNIVERSITY, AMES IA 50011, USA

Telephone: (1) 5152947659

Facsimile: (1)

Telex:

Email: bitnct:s1.ghb@isumvs

Commission(s): 27,36

BOWERS PHILLIP F, NAVAL RESEARCH LABORATORY, CODE 4134, 4555 OVERLOOK AVE SW

WASHINGTON DC 20375 5000, USA

Telephone: (1) 2027672495

Email:

Facsimile: (1)

Telex:

Commission(s): 40

BOWYER C STUART PROF, ASTRONOMY DPT, UNIVERSITY OF CALIFORNIA, 601 CAMPBELL HALL

BERKELEY CA 94720, USA

Telephone: (1) 5106421648

Facsimile: (1) 5106438303

Telex: 9103667945

Email: sbowyer@cea.berkelcy.edu

Commission(s): $21 \mathrm{VP}, \mathbf{4 4 , 5 1}$

BOYARCHUK A A DR, INST OF ASTRONOMY, ACADEMY OF SCIENCES, PYATNITSKAYA UL 48

109017 MOSCOW, RUSSIA

Telephone: (7) 0952302081

Facsimile: (7) 0952302081

Telex: $\mathbf{4 1 1 5 7 6}$ ascon su

Email: aboyar@inasan.rssi.ru

Commission(s): $27,29,38,44, E C$

BOYARCHUK MARGARITA E DR, INST OF ASTRONOMY, ACADEMY OF SCIENCES, PYATNITSKAYA UL 48 109017 MOSCOW, RUSSIA

Telcphone: (7)

Facsimile: (7)

Telex:

Email:

Commission(s): 27

BOYCE PETER B DR, AMERICAN ASTRON SOCIETY, 2000 FLORIDA AVE NW, SUITE 400

WASHINGTON DC 20009, USA

Telephone: (1) 2023282010

Facsimile: (1) $2022342560 \quad$ Telex:

Email: pboyce@aas.org

Commission(s): 09,16,51

BOYD ROBERT L F PROF SIR, 41 CHURCH ST, LITTLEHAMPTON BN17 SPU, UK

Telephone: (44)

Email:

Facsimile: (44)

Telex:

Commission(s): 44

BOYDAG-YILDIZDOGDU F S DR, KING SAUD UNIVERSITY, COLLEGE OF SCIENCE, BOX 2452

RIYADH 11495, SAUDI ARABIA

Telephone: (966)

Facsimile: (966)

Telex:

Email:

Commission(s):

BOYER RENE, OBSERVATOIRE DE PARIS, SECTION DE MEUDON, DASOP, F 92195 MEUDON PPL CDX, FRANCE

Telephone: (33) 145077741

Facsimile: (33)

Commission(s): 10

Telex: 201571

Email: 
M 450

BOYLE BRIAN DR, AAO, BOX 296, EPPING NSW 2121, AUSTRALIA

Telephone: (61) $28681666 \quad$ Facsimile: (61) 28768536

Telex: 23999 aaosyd aa

Email:

Commission(s): 47

BOYLE RICHARD P DR, VATICAN OBSERVATORY, I 00120 VATICAN CITY, VATICAN CITY STATE

Telephone: (39) $66985266 \quad$ Facsimile: (39)

Email: boyle@arizrvax.bitnet

Commission(s):

Telex: 5042020 vatobs va

BOYLE STEPHEN, DPT PHYSICS \& ASTRONOMY, UNIVERSITY COLLEGE LONDON, 553 WATFORD WAY LONDON NW7 2QS, UK

Telephone: (44) 1819590421

Facsimile: (44) 1813881450

Telex: 28722 ucphys g

Email: sjb@uk.ac.ucl.star

Commission(s): 42

BOYNTON PAUL EDWARD DR, DPT OF ASTRONOMY, UNIVERSITY OF WASHINGTON, FM 20

SEATTLE WA 98195, USA

Telephone: (1)

Facsimile: (1)

Telcx:

Email:

Commission(s):

BOYTEL JORGE DEL PINO DR, EST DE RAST DE SATELITES, ACADEMIA DE CIENCLAS, MANDULEY 308 VISTA ALEGRE 90400, CUBA

Telephone: (53) 741623

Facsimile: (53)

Telex: $061254 \mathrm{cmict}$ cu

Email: cmstgo(a)ceniai.cu

Commission(s): 19

BOZIS GEORGE PROF, DPT THEORET MECHANICS, UNIVERSITY THESSALONIKI GR 54006 THESSALONIKI, GREECE

Telephone: (30) 31992845

Facsimile: (30)

Telex:

Email:

Commission(s): 07

BOZKURT SUKRU DR, OBSERVATORY, EGE UNIVERSITY, BOX 21, 35100 BORNOVA IZMIR, TURKEY

Telephone: (90) 51180306

Facsimile: (90)

Telex:

Email:

Commission(s):

BRACCESI ALESSANDRO PROF, DPT DI ASTRONOMIA, UNIVERSITA DI BOLOGNA, VIA ZAMBONI 33

I 40126 BOLOGNA, ITALY

Telephone: (39) 51222956

Facsimile: (39)

Commission(s): 28

Telex: 211664 infnboi

Email:

BRACEWELL RONALD N PROF, DURAND 329 A, STANFORD UNIVERSITY, STANFORD CA 94305, USA

Telephone: (1) 4154973545

Facsimile: (1)

Telex:

Email:

Commission(s): 40,51

BRADSTREET DAVID H DR, DPT OF PHYSICAL SCIENCE, EASTERN COLLEGE, ST DAVIDS PA 19087, USA

Telephone: (1) 2153415945

Facsimile: (1)

Telex:

Email:

Commission(s): 42

BRAES L L E DR, STERREWACHT, BOX 9513, NL 2300 RA LEIDEN, NETHERLANDS

Telephone: (31) 71272727

Facsimile: (31)

Telex:

Email:

Commission(s): 46

BRAGA JOAO DR, INPE, CP 515, 12201 S JOSE DOS CAMPOS, BRAZIL

Telephone: (55) $123418977^{\star} 679$

Facsimile: (55) 123218743

Commission(s): 44

Telex: 123353 inpe br

Email: inpedas@brfapesr.bitnet

BRAHDE ROLF, INST THEORET ASTROPHYSICS, UNIVERSITY OF OSLO, BOX 1029

N 0315 BLINDERN OSLO 3, NORWAY

Telephone: (47) 2456508

Facsimile: (47)

Telex:

Email:

Commission(s):

BRAHIC ANDRE DR, OBSERVATOIRE DE PARIS, SECTION DE MEUDON, F 92195 MEUDON PPL CDX, FRANCE Telephonc: (33) 145077402

Facsimile: (33)

Telex: 201571

Email:

Commission(s): 16

BRANCH DAVID R DR, DPT PHYSICS \& ASTRONOMY, UNIVERSITY OF OKLAHOMA, NORMAN OK 73019, USA

Telephone: (1) 4053253961

Facsimile: (1)

Telex: 9108306521

Email:

Commission(s): 
BRAND JAN, IST DI RADIOASTRONOMIA, C N R, VIA GOBETTI 101, I 40129 BOLOGNA, ITALY

Telephone: (39) 516399372

Facsimile: (39) $516399341 \quad$ Telex:

Email: brand@astbo1.bo.cnr.it

Commission(s): 33,34

BRAND PETER W J L DR, ROYAL OBSERVATORY, BLACKFORD HILL, EDINBURGH EH9 3HJ, UK

Telephone: (44) 1316673321

Facsimile: (44)

Commission(s): 34

Telex: 72383 roe edin $g$

Email:

BRANDENBURG AXEL DR, HIGH ALTITUDE OBSERVATORY, NCAR, BOX 3000, BOULDER CO 80307 3000, USA

Telcphone: (1) 3034971589

Facsimile: (1) $3034971586 \quad$ Telex:

Email: brandenb@hao.ucar.edu

Commission(s): 10

BRANDI ELISANDE ESTELA DR, OBSERVATORIO ASTRONOMICO, PASEO DEL BOSQUE S/N 1900 LA PLATA (BS AS), ARGENTINA

Telephone: (54) 21217308

Facsimile: (54) $21211761 \quad$ Telex:

Email:

Commission(s): 29,42

BRANDIE GEORGE W DR, ENVIRONMENTAL ENGINEERING, QUEEN'S UNIVERSITY

KINGSTON ON K7L 3N6, CANADA

Telephone: (1)

Facsimile: (1)

Telex:

Email:

Commission(s):

BRANDT JOHN C DR, LASP, UNIVERSITY OF COLORADO, BOX 392, BOULDER CO 80309 0392, USA

Telephone: (1) $3034923215 \quad$ Facsimile: (1)

Telex: 9109403441

Email:

Commission(s): $15,44,49$

BRANDT PETER N, KIEPENHEUER INSTITUT, FUER SONNENPHYSIK, SCHOENECKSTR 6

D 79104 FREIBURG BREISGAU, GERMANY

$\begin{array}{lll}\text { Telcphone: (49) } 76132864 & \text { Facsimile: }(49) & \text { Telcx: } 7721552 \\ \text { Email: } & \text { Commission(s): } 10,12 & \end{array}$

BRANDUARDI-RAYMONT G, MULLARD SPACE SCIENCE LAB, UNIVERSITY COLLEGE LONDON

HOLMBURY ST MARY, DORKING SURREY RHS 6NT, UK

Telephone: (44) $130670292 \quad$ Facsimile: $(44)$

Telex: 859185

Email:

Commission(s):

BRANHAM RICHARD L JR, CENTRO REGIONAL DE INVEST, CIENTIFICAS Y TECNOL, CC 131

500 MENDOZA, ARGENTINA

$\begin{array}{lll}\text { Telephone: (54) } 612411794 & \text { Facsimile: (54) } & \text { Telex: } 55438 \text { cytme ar } \\ \text { Email: } & \text { Commission(s): 07,20,24 } & \end{array}$

BRANSCOMB L M DR, NTL BUREAU OF STANDARDS, WASHINGTON DC 20025, USA

Telephone: (1)

Facsimile: (1)

Telex:

Email:

Commission(s): 14

BRANSON NICHOLAS J B A DR, UNIVERSITY REGISTRY, THE OLD SCHOOLS, CAMBRIDGE CB2 1TN, UK

Telephone: (44) 1223332250

Email:

Facsimile: (4) $1223332332 \quad$ Telex: 81240 camspl g

Commission(s):

BRATIJCHUK MATRONA V, SATELITE OBSERVATORY, UZHGOROD STATE UNIVERSITY, HORKIY 46 294000 UZHGOROD, UKRAINE

Telephone: (7) 36065

Facsimile: (7)

Commission(s):

Telex: 274155 kniga

Email:

BRAUDE SEMION YA PROF AG, INSTITUTE OF RADIO ASTRON, UKRAINIAN ACAD OF SCIENCE KRASNOZNAMENNAYA UL 4, 310002 KHARKOV, UKRAINE

Telephone: (7) 0441092

Facsimile: (7)

Telex:

Email:

Commission(s): 40

BRAULT JAMES W DR, NTL SOLAR OBSERVATORY, BOX 26732, 950 N CHERRY AVE, TUCSON AZ 85726 6732, USA

Telephone: (1) 5203259363

Facsimile: (1)

Telex: 666484 aura noao tuc

Email:

Commission(s): $09,12,14$

BRAUN ARIE, RACAH INST OF PHYSICS, HEBREW UNIV OF JERUSALEM, JERUSALEM 91904, ISRAEL

Telephone: (972) $2584521 \quad$ Facsimile: (972)

Email:

Commission(s):

Telex: 25391 hu il 
M 452

BRAUN ROBERT DR, NFRA, BOX 2, NL 7990 AA DWINGELOO, NETHERLANDS

Telephone: (31) $52197244 \quad$ Facsimile: (31) $52197332 \quad$ Telex:

Email: rbraun@nfra.nl Commission(s): 28

BRAUNINGER HEINRICH DR, MPI F EXTRATERRESTRISCHE, PHYSIK, KARL SCHWARZSCHILDSTR 1 D 85740 GARCHING MUENCHEN, GERMANY

Telephone: (49) $893299566 \quad$ Facsimile: (49)

Telex:

Email:

Commission(s):

BRAUNSFURTH EDWARD PH D, IM HAARMANNSBOCH 99 A, D 4630 BOCHUM 1, GERMANY

Telephone: (49)

Facsimile: (49)

Telex:

Email:

Commission(s): 34

BRAVO EDUARDO, DPT FISICA I ENG NUCLEAR, UNI POLITECNICA CATALUNYA, AVE DIAGONAL 647 E 08028 BARCELONA, SPAIN

Telcphone: (34) 34016565

Email: bravo@fen.upc.es

Facsimile: (34) 34016600

Telex:

Commission(s): 35

BRAY ROBERT J DR, 31/126 CRIMEA ROAD, MARSFIELD NSW 2122, AUSTRALIA

Telephone: (61)

Facsimile: (61)

Telex:

Email:

Commission(s): 10,12

BREAKIRON LEE ALLEN DR, US NAVAL OBSERVATORY, TIME SERVICE DPT, 3450 MASSACHUSETTS AVE NW WASHINGTON DC 203925100 , USA

Telephone: (1) 2026531888

Facsimile: (1)

Telex:

Email: lab@tycho.usno.navy.mil

Commission(s): 24,31

BRECHER AVIVA DR PROF, 35 MADISON ST, BELMONT MA 02178, USA

Telephone: (1) 6174891386

Facsimile: (1)

Telex:

Email:

Commission(s): 15,16

BRECHER KENNETH PROF, DPT OF ASTRONOMY, BOSTON UNIVERSITY, 725 COMMONWEALTH AVE BOSTON MA 02215, USA

Telephone: (1) 6173533423

Email:

Facsimile: (1)

Commission(s): 28,44,47

Telex: 95-1289 bis univ bsn

BRECKINRIDGE JAMES B DR, JPL CALTECH, MS 183 301, 4800 OAK GROVE DR, PASADENA CA 91109, USA

Telephone: (1) 2133546785

Facsimile: (1)

Telex: 675429

Email:

Commission(s): 09,12

BREGER MICHEL PROF DR, INSTITUT FUER ASTRONOMIE, UNIVERSITAET WIEN, TUERKENSCHANZSTR 17

A 1180 WIEN, AUSTRIA

Telephone: (43) 13453605

Email: breger(avia.una.ac.at

Facsimile: (43) 134536015

Telex: 133099 viast a

BREGMAN JACOB D IR, NFRA, BOX 2, NL 7990 AA DWINGELOO, NETHERLANDS

Telephone: (31) 52197244

Facsimile: (31)

Telex:

Email:

Commission(s): 40

BREGMAN JOEL N, DPT OF ASTRONOMY, UNIVERSITY OF MICHIGAN, DENNISON BLDG ANN ARBOR MI 48109 1090, USA

Facsimile: (1)

Telex:

Email: jbregman(Gastro.Isa.umich.edu

Commission(s):

BREINHORST ROBERT A DR, ASTRONOMISCHES INSTITUT, UNIVERSITAET BONN, AUF DEM HUEGEL 71 D 53121 BONN, GERMANY

Telephone: (49) 228733660

Email:

Facsimile: (49)

Telex:

Commission(s): 42

BREITSCHWERDT DIETER DR, MPI FUER KERNPHYSIK, POSTFACH 103 980, D 69029 HEIDELBERG, GERMANY

Telephone: (49) 6221516471

Facsimile: (49) 6221516324

Telex: 461666

Email: winds@dhdmpi5v (bitnet)

Commission(s): 34 
BREJDO IZABELLA I DR, PULKOVO OBSERVATORY, ACADEMY OF SCIENCES, 10 KUTUZOV QUAY 196140 ST PETERSBURG, RUSSIA

Telephone: (7) 2979459

Email:

Facsimile: (7)

Commission(s): 09

Telex:

BREKKE PAL ORDING LIE, INST THEORET ASTROPHYSICS, UNIVERSITY OF OSLO, BOX 1029 N 0315 BLINDERN OSLO 3, NORWAY

Telephone: (47) 22856508

Email: paalb@astro.uio.no

Facsimile: (47) $22856505 \quad$ Telex:

Commission(s):

BRETAGNON PIERRE DR, BUREAU DES LONGITUDES, 77 AVE DENFERT ROCHEREAU, F 75014 PARIS, FRANCE Telephone: (33) 140512269

Facsimile: (33)

Telex:

Email:

Commission(s): 04,07

BREUKERS R J L H DR, STERREWACHT, BOX 9513, NL 2300 RA LEIDEN, NETHERLANDS

Telephone: (31)

Facsimile: (31)

Commission(s):

Telex:

Email:

SCHWARZSCHILDSTR 2, D 85740 GARCHING MUENCHEN, GERMANY

Telephone: (49) 8932006224

Facsimile: (49) 893202362

Telex: $\mathbf{5 2 8 2 8 2 0}$

Email:

Commission(s): 29

BRIDGELAND MICHAEL DR, INSTITUTE OF ASTRONOMY, THE OBSERVATORIES, MADINGLEY RD

CAMBRIDGE CB3 OHA, UK

Telephone: (44) 1223337524

Email:

Facsimile: (44) 1223337523

Telex: 817297 astron' $\mathrm{g}$

Commission(s): 09

BRIDLE ALAN H PROF, NRAO, 520 EDGEMONT RD, CHARLOTTESVILLE VA 22903, USA

Telcphone: (1) 8042960375

Facsimile: (1)

Telex:

Email:

Commission(s): 40

BRIEVA EDUARDO PROF, OBSERVATORIO NACIONAL, APDO 2584, BOGOTA 1 DE, COLOMBIA

Telephone: (57) 1423786

Facsimile: (57)

Commission(s): 07,46

Telex:

Email:

BRIHAYE CHARLES C A DR, LAAG, VRIJE UNIV BRUSSELS, CP 165, B 1050 BRUSSELS, BELGIUM

Telephone: (32) 26876928

Facsimile: (32)

Commission(s):

Telex:

Email:

BRINI DOMENICO PROF, INST DI FISICA, UNIVERSITA DI BOLOGNA, VIA IRNERIO 46, I 40126 BOLOGNA, ITALY
Telephone: (39)

Telephone: (39)

Commission(s):

Telex:

Email:

BRINKMAN BERT C DR, SPACE RESEARCH LABORATORY, SRON, SORBONNELAAN 2 NL 3584 CA UTRECHT, NETHERLANDS

Telephone: (31) 30535600

Facsimile: (31) 30540860

Telex: 47224 sron $n]$

Email:

Commission(s): 44

BRINKMANN WOLFGANG, MPI F EXTRATERRESTRISCHE, PHYSIK, KARL SCHWARZSCHILDSTR 1 D 85740 GARCHING MUENCHEN, GERMANY
Telephone: (49) 893299877
Facsimile: (49)
Telex: 05215845 xter d
Email:
Commission(s): 28,34

BRINKS ELIAS DR, NRAO, BOX 0, SOCORRO NM 87801 0387, USA

Facsimile: (1) 5058357027

Email: cbrinks@nrao Commission(s): 28,40

Telex: 9109981710

BRIOT DANIELLE DR, OBSERVATOIRE DE PARIS, 61 AVE OBSERVATOIRE, F 75014 PARIS, FRANCE

Telephone: (33) $140512239 \quad$ Facsimile: (33)

Email:

Commission(s):

Telex: 270776 obs $f$

BROADFOOT A LYLE DR, LUNAR \& PLANETARY LAB, UNIVERSITY OF ARIZONA, 901 GOULD SIMPSON BLDG TUCSON AZ 85721, USA

Telephone: (1) 5206214301

Facsimile: (1)

Telex: 910-952-1143

Email:

Commission(s): 16,21 
M 454

BROCATO ENZO DR, OSS ASTRONOMICO COLLURANI, TERAMO, VIALE MAGGINI, I 64100 TERAMO, ITALY Telephone: (39) 861210490

Facsimile: (39) 861210492

Telex:

Email: 40018::brocato

Commission(s):

BRODERICK JOHN DR, PHYSICS DPT, VIRGINIA TECH, BLACKSBURG VA 24061, USA

Telephone: (1) 703231532

Facsimile: (1)

Telex: 9103331861 vpibks

Email:

Commission(s): 40,51

BRODIE JEAN P, LICK OBSERVATORY, UNIVERSITY OF CALIFORNIA, SANTA CRUZ CA 95064, USA

Telephone: (1) 4084592987

Facsimile: (1)

Commission(s): 28

Telex:

Email: brodie@lick.ucsc.edu

BROGLIA PIETRO DR, OSS ASTRONOMICO DI MILANO, VIA E BIANCHI 46, I 22055 MERATE, ITALY

Telephone: (39) 592035

Facsimile: (39)

Telex:

Email:

Commission(s): 42

BROMAGE GORDON E DR, RUTHERFORD APPLETON LAB, SPACE \& ASTROPHYSICS DIV, BLDG R25/R68, CHILTON DIDCOT OX11 0QX, UK

Telephone: (44) 123521900

Facsimile: (44)

Telex: 83159

Email:

Commission(s): 14,34

BRONFMAN LEONARDO DR, DPT DE ASTRONOMIA, UNIVERSIDAD DE CHILE, CASILLA 36 D, SANTIAGO, CHILE

Telephone: (56) 22281941

Facsimile: (56)

Telex: 440001

Email:

Commission(s): 33,40

BRONNIKOVA NINA M, PULKOVO OBSERVATORY, ACADEMY OF SCIENCES, 10 KUTUZOV QUAY 196140 ST PETERSBURG, RUSSIA

Telephone: (7)

Facsimile: (7)

Telex:

Email:

Commission(s): 24

BROOKES CLIVE J DR, DPT OF MATHEMATICS, EARTH/SATELLITE RES UNIT, ASTON UNIVERSITY BIRMINGHAM B4 7ET, UK

Telephone: (44) 1213593611

Email:

Facsimile: (44)

Telex: 335787

Commission(s): 07

BROOKS RANDALL C DR, PHYSICS SCIENCES, NTL MUSEUM SCIENCE \& TECH, BOX 9724

OTTAWA ONT K1G 5A3, CANADA

Telephone: (1) 6139902804

Facsimile: (1) $6139913636 \quad$ Telex:

Email: brooks@husky1.stmarys.ca

Commission(s): 41

BROSCH NOAH DR, WISE OBSERVATORY, TEL AVIV UNIVERSITY, RAMAT AVIV, TEL AVIV 69978, ISRAEL

Telephone: (972) 3413788

Email: bitnet:h38@ataunos.

Facsimile: (972)

Commission(s): 28,46

Telex: 342171 versy il

BROSCHE PETER PROF, OBSERVATORIUM HOHER LIST, UNIV STERNWARTE BONN, D 5568 DAUN, GERMANY

Telephone: (49) 65922150

Facsimile: (49)

Telex:

Email:

Commission(s): 19,24,26,28

BROSIUS JEFFREY WILLIAM, NASA GSFC, CODE 682 1, GREENBELT MD 20771, USA

Telephone: (1) 3012866200

Facsimile: (1) $3012861617 \quad$ Telex:

Email: stars::brosius

Commission(s): 10,12

BROSTERHUS E B F DR, LOCKHEED CITY, BOX 6308, JEDDAH, SAUDI ARABIA

Telephone: $(966) 26562501 * 355 \quad$ Facsimile: $(966)$

Telex:

Email:

Commission(s):

BROTEN NORMAN W, HERZBERG INST ASTROPHYS, NTL RESEARCH COUNCIL, 100 SUSSEX DR

OTTAWA ON K1A 0R6, CANADA

Telephone: (1) 6139936060

Facsimile: (1) 6139526602

Telex: 0533715

Email:

Commission(s): 40

BROUCKE ROGER DR, 7203 RUNNING ROPE CIRCLE, AUSTIN TX 78731, USA

Telephone: (1) 5123456435

Facsimile: (1)

Commission(s): 07

Telex:

Email: 
BROUILLET NATHALIE, OBSERVATOIRE DE BORDEAUX, BP 89, F 33270 FLOIRAC, FRANCE

Telephone: (33) $5686+330$

Facsimila: (33) $56+04251$

Telex:

Email: nathalie $\bar{a}$ observ.u-bordeaux.fi. Commission(s): 34

BROUW W N DR, CSIRO, ATNF, BOX 76, EPPING NSW 2121, AUSTRALIA

Telephone: (61) $23724316 \quad$ Facsimile: (61) 23724400 Telex:

Email: wbrouw(a) atnf.csiro.au Commission(s): 08,40

BROWN ALEXANDER, JILA, UNIVERSITY OF COLORADO, BOX 440, BOULDER CO 80309 0440, USA

Telephone: (1) $3034928962 \quad$ Facsimile: (1) Telex: 755842 jila

Email: $\quad$ Commission(s): $36,+4$

BROWN DOUGLAS.NASON, DPT OF ASTRONOMY, UNIVERSITY OF WASHINGTON, FM 20

SEATTLE WA 98195 , USA

Telcphone: (1) $206543+313 * 2888$

Email:

Facsimile: (1)

Telex:

Commission(s): $25,27,29,36$

BROWN JOHN C PROF, DPT PHYSCIS \& ASTRONOMY, UNIVERSITY OF GLASGOW, GLASGOW G12 8QQ, UK

Telephone: (44) 1413305182

Facsimile: (4)

Email:

Commission(s): 10

Telex: 777070 unigla

BROWN ROBERT HAMILTON, JPL CALTECH, MS 183 501, 4800 OAK GROVE DR, PASADENA CA 91109, USA

Telephone: (1) $81835+2517$

Facsimile: (1)

Telex:

Email:

Commission(s): $15,16,50$

BROWN ROBERT L DR, NRAO, 520 EDGEMONT RD, CHARLOTTESVILLE VA 22903, USA

Telephonc: (1) 8042960222

Facsimile: (1) $80+2960385 \quad$ Telex:

Email: rbrown(a) nrao.edu

Commission(s):

BROWN RONALD D PROF, DPT OF CHEMISTRY, MONASH UNIVERSITY, WELLINGTON RD

CLAYTON VIC 3168, AUSTRALIA

Telephone: (61) $35654550 \quad$ Facsimile: (61) $35654597 \quad$ Telex: at 32691

Email: rdbrown(a)vaxc.cc.monash.celu.au Commission(s): $34,51 \mathrm{C}$

BROWNE IAN W A DR, NRAL, JODRELL BANK, MACCLESFIELD SK11 9DL, UK

Telephone: (44) $147771321 \quad$ Facsimile: (4) $\quad$ Telex: 36149

Email: $\quad$ Commission(s): 40

BROWNING PHILIPPA DR, DPT OF PURE APPLIED PHY, UMIST, BOX 88, MANCHESTER MG0 1QD, UK

Telephone: $(\$ 4) 1612363311 \quad$ Facsimile: $(4+)$

Email: mecppb(auk.ac.umrcc.cms Commission(s): 10

Telex: 666094

BROWNLEE DONALD E PROF, DPT OF ASTRONOMY, UNIVERSITY OF WASHINGTON, FM 20

SEATTLE WA 98195, USA

Telcphone: (1) $2065+32888$

Facsimile: (1)

Telex:

Email: brownlec $(\hat{a}$ astro.washington.cdu Commission(s): 15,22

BROWNLEE ROBERT R DR, 4879 FRANKLIN AVE, LOVELAND CO 80538, USA

Telephone: (1) $30366306+6 \quad$ Facsimile: (1) Telex:

Email: $\quad$ Commission(s): 35,42

BRUCATO ROBERT J, CALTECH, MS 115 24, PALOMAR OBS, PASADENA CA 91125, USA

Telephone: (1) 8183564035

Email:

Commission(s):

Telex: $675+25$ or 188192

BRUCH ALBERT, ASTRONOMISCHES INSTITUT, UNIVERSITAET MUENSTER, WILHELM KLEMM STR 10 D 48149 MUENSTER, GERMANY

Telephonc: (40)

Facsimile: (49) 251833669

Telex:

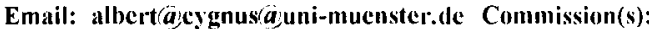

BRUCK HERMANN A PROF, CRAIGOWER, PENICUIK EH26 9LA, UK

Telephone: (4) $\left.196875^{\circ}\right) 18$

Facsimile: (4)

Email:

Commission(s): 25

Telex: 
M 456

BRUCK MARY T DR, CRAIGOWER, PENICUIK, MIDLOTGIAN EH26, 9LA, UK

Telephonc: (44) 1968675918 Facsimile: (4) Telex:

Email:

Commission(s): to

BRUECKNER GUENTER E DR, NAVAL RESEARCH LABORATORY, CODE 4160, +555 OVERLOOK AVE SW WASHINGTON DC 20375 5000, USA

Telephone: (1) 2027673287

Facsimile: (1)

Telex:

Email:

Commission(s): $10,12,44$

BRUGEL EDWARD W DR, CASA, UNIVERSITY OF COLORADO, BOX 391, BOULDER CO 80309 0391, USA

Telephonc: (1) $303 \$ 924054 \quad$ Facsimile: (1)

Telex:

Email: brugelôcygnus.colorado.edu

Commission(s):

BRUHWEILER FRED C JR, CATHOLIC UNIV AMERICA, ASTROPHYSICS PROGRAM, DPT OF PHYSICS WASHINGTON DC 20064, USA

Telephonc: (1) 2023195315

Facsimile: (1) $202319+4+8$

Telex:

Email: bruhweiler(a)cua.edu

Commission(s): $29,3+\mathrm{C},+2,+4$

BRUMBERG VICTOR A DR, INST OF APPLIED ASTRONOMY, ACADEMY OF SCIENCES, ZDANOVSKAYA UL 8 197042 ST PETERSBURG, RUSSIA

Telephone: (7)

Email:
Facsimile: (7)

Commission(s): (14,07,31C
Telex:

BRUNER MARILYN E DR, LOCKHEED PALO ALTO RES LB, DPT 9120 BLDG 255, 3251 HANOVER ST

PALO ALTO CA 9430 4 , USA

Telephone: (1) $415858+023$

Email:

Facsimile: (1)

Commission(s): 10,12,4t

Telex: 346409 Imsc

BRUNET JEAN-PIERRE DR, OBS MIDI PYRENEES, 14 AVE E BELIN, F 31400 TOULOUSE CDX, FRANCE

Telephone: (33) 61252101

Facsimile: (33)

Telex: $\mathbf{5 0 3 7 7 6}$

Email:

Commission(s): 4

BRUNING DAVID H DR, STELLAR RESEARCH \& EDU, BOX 1223, WAUKESHA WI 53187, USA

Telephone: (1)

Facsimile: (1)

Telex:

Email: dhbrun01(a)ulkyvx

BRUNINI ADRIAN DR, IAG, UNIVERSIDADE DE SAO PAULO, AV MIGUEL STEFANO $\$ 200$

04301 SAO PAULO SP, BRAZIL

Telephone: (55) 115778599

Facsimile: (55) 11276,3848

Telex: 1156735 iagm br

Email: iagusp\% \% psp.hepnet $(\vec{a}$ llo.bitnet

Commission(s): 07

BRUNK WILLIAM E DR, NASA HEADQUARTERS, CODE SL OFF SPACE SCIENCE, 400 MARYLAND AVE S W WASHINGTON DC 20546, USA

Telephone: (1) 2024531596

Facsimile: (1)

Telex:

Email:

Commission(s): 15,16

BRUNS ANDREY V, CRIMEAN ASTROPHYS OBS, UKRAINIAN ACAD OF SCIENCE, NAUCHNY 33+413 CRIMEA, UKRAINE

Telephone: (7) $655+71119$

Facsimile: (7) $655+40704$

Telex:

Email: bruns $a$ crao.crimea.ua

Commission(s): 10

BRUSTON PAUL DR, EPCOS/LPCE URA CNRS 1404, UNIV PARIS VAL DE MARNE, AVE DU GENERAL DE GAULLE F 94010 CRETEIL CEDEX, FRANCE

Telephone: (33) 142071285

Email:

Facsimile: (33) $142077012 \quad$ Telex:

Commission(s):

BRUZEK ANTON DR, SCHWAIGHOFSTR 7, D 7910 4 FREIBURG BREISGAU, GERMANY

Telephone: (49) 76178522

Email:

Facsimilc: (49)

Telex:

BRUZUAL GUSTAVO, CIDA MRD 1726, BOX 02 8537, MIAMI FL 33102 8537, USA

Telephone: (1)

Facsimile: (1)

Telex:

Email:

Commission(s): $28 \mathrm{C}$ 
BRYANT JOHN DR, 47 AVE FELIX FAURE, F 75015 PARIS, FRANCE

Telephone: (33) 145577647

Email:
Facsimile: (33)

Commission(s):
Telex:

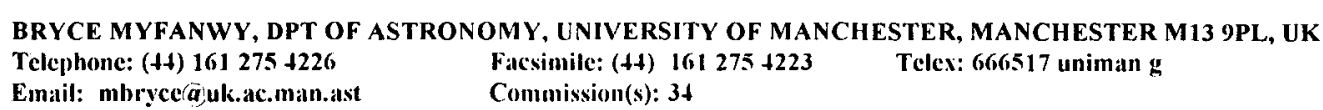

BRZEZINSKI ALEKSANDER DR, SPACE RESEARCH CENTER, POLISH ACAD OF SCIENCES, UL BARTYCKA 1

PL 00 716 WARSAW, POLAND

Telephone: (48) $39121273 \quad$ Facsimile: (48) $22403766 \quad$ Telex: $825670 \mathrm{cblk}$ pl

Email: alek(a)clok.waw.pl

Commission(s): 19

BUCCIARELLI BEATRICE DR, STSCI, HOMEWOOD CAMPUS, 3700 SAN MARTIN DR, BALTIMORE MD 21218, USA

Telephone: (1) 301338 4570

Facsimile: (1) 3013382617

Telex: 684910178724

Email: 6559::bucc/bucc (ajstsci.edu

Commission(s): 08

BUCHLER J ROBERT PROF, DPT OF PHYSICS, UNIVERSITY OF FLORIDA, GAINESVILLE FL 32611, USA

Telephone: (1) 9043739942

Facsimile: (1)

Telex:

Email:

Commission(s): 35

BUCKLEY DAVID, SAAO, BOX 9, OBSERVATORY 7935, CAPE TOWN 7935, SOUTH AFRICA

Telephone: (27) $21+70$ (125

Ficsimile: (27) $21+73639 \quad$ Telex:

Email: dibnob/äsano.ac.»a

Commission(s):

BUDDING EDWIN DR, CARTER OBSERVATORY, BOX 2909, WELLINGTON 1, NEW ZEALAND

Telephone: $(64)+4728167$

Facsimile: $(64)+\$ 728320$ Telex:

Email: buddingämataivuw.ac, n\%

Commission(s): 42,46

BUECHER ALAIN DR, OBS MIDI PYRENEES, 9 R PONT DE LA MOUETTE, F 65200 BAGNERES BIGORRE, FRANCE Telephone: (33) 62951969 Facsimile: (33) 62951070

Commission(s): 10

Telex: 531625 obspic i

Email:

BUECHNER JOREG DR, MPI FUER EXTRATERRESTISCH, AUSS BERLIN GEB 1616/1625, RUDOWER CHAUSSEE 5 D 12489 BERLIN ADLERSHOF, GERMANY

Telephone: (49) 3063923937

Fanimile: (49) $3063923937 \quad$ Telex: 302542

Email: jb(a)mpe.fta-berlin.dc

Commission(s): 111,49

BUES IRMELA D DR, DR REMEIS STERNWARTE, STERNWARTSTR 7, D 96149 BAMBERG, GERMANY

Telephone: (49) 95157708

Facsimile: (49)

Telex:

Email: buesásternwalte.uni-crlangen.d Commission(s): 29,36

BUFF JAMES S DR, DPT OF PHYSICS \& ASTRON, DARTMOUTH COLLEGE, HANOVER NH 03755, USA

Telephone: (1)

Fitcsimile: (1)

Telex:

Enail:

Commission(s):

BUHL DAVID DR, NASA GSFC, CODE 693, INFRARED \& RADIO ASTRO BR, GREENBELT MD 20771, USA

Telephone: (1) 3012868810

Ficsimile: (1)

Telex:

Email:

Commission(s):

BUIE MARC W, LOWELL OBSERVATORY, 1400 W MARS HILL RD, FLAGSTAFF AZ 86001, USA

Telephone: (1) $601277+3358$

Falesimile: (1) 6112 77f6296 Telex:

Email: buic $a$ low cll.edu

Commission(s): 15,16

BUITRAGO JESUS, INST DE ASTROFISICA, DE CANARIAS, OBS DEL TEIDE, E 38071 LA LAGUNA, SPAIN

Tclephone: (34) 22262211

Facsimile: (34)

Telcx: $926+0$

Email:

Commission(s):

BUJARRABAL VALENTIN, CTR ASTRON DE YEBES, OAN, APD 148, E 19080 GUADALAJARA, SPAIN

Telephone: (34) 11223358

Facsimile: $\{3+)$

Telex:

Email:

Commission(s): 34,40

BUMBA VACLAV DR, ASTRONOMICAL INSTITUTE, CZECH ACADEMY OF SCIENCES ONDREJOV OBSERVATORY, CZ 25I 6.5 ONDREJOV, CZECH R

Telephonc: (\$2) $20+857157 \quad$ Facsimile: (42) 204857111

Email: astsun(ä)csearn

Commission(s): 111,12,4t

Telex: 121579 astr c 
BUNCLARK PETER STEPHEN DR, INSTITUTE OF ASTRONOMY, THE OBSERVATORIES, MADINGLEY RD CAMBRIDGE CB3 OHA, UK

Telephone: (44) 1223337548

Facsimile: (44) 1223337523

Telex: 817297 astron g

Email:

Commission(s): 24

BUNNER ALAN N DR, PERKIN ELMER CORP, MS 897, 100 WOOSTER HEIGHTS RD, DANBURY CT 06810 7859, USA Telephone: (1) 2037976339

Facsimile: (1)

Telex:

Email:

Commission(s): 42,44

BUONANNO ROBERTO, OAR, VIA DEL PARCO MELLINI 84, I 00136 ROMA, ITALY

Telephone: (39) 63452656

Facsimile: (39) $6347802 \quad$ Telex:

Email:

Commission(s): $37 \mathrm{C}$

BURATTI BONNIE J DR, JPL, MS 183 501, 4800 OAK GROVE DR, PASADENA CA 91109, USA

Telephone: (1) 8183547427

Facsimile: (1)

Telex:

Email:

Commission(s): 15,16

BURBIDGE E MARGARET PROF, CASS, UCSD, C 011, LA JOLLA CA 92093 0216, USA

Telephone: (1) 6194524477

Facsimile: (1)

Telex:

Email:

Commission(s): 28

BURBIDGE GEOFFREY R PROF, CASS, UCSD, C 011, LA JOLLA CA 92093 0216, USA

Telephone: (1) 6194526626

Facsimile: (1)

Telex:

Email:

Commission(s): $28,35,40,44,47$

BURDYUZHA VLADIMIR, SPACE RESEARCH INSTITUTE, ACADEMY OF SCIENCES, PROFSOJUZNAYA UL 84/32 117810 MOSCOW, RUSSIA

Telephone: (7) 0953333366

Facsimile: (7) 0953107023

Telex:

Email: burdyuzh(a)dpc.asc.rssi.ru

Commission(s):

BURGER J J DR IR, ESA/ESTEC, SSD, BOX 299, NL 2200 AG NOORDWIJK, NETHERLANDS

Telephone: (31) $171984404 \quad$ Facsimile: (31)

Email:

Commission(s):

Telex: 39098

BURGER MARIJKE DR, OBSERVATOIRE ROYAL DE, BELGIQUE, AVE CIRCULAIRE 3

B 1180 BRUSSELS, BELGIUM

Telephone: (32) $23752484 \quad$ Facsimile: (32)

Tclex: 21565 obsbel b

Email: marijke@astro.ome.be

Commission(s): 4

BURGESS ALAN DR, DPT APPLIED MATHS, \& THEORETICAL PHYSICS, SILVER STREET

CAMBRIDGE CB3 9EW, UK

Telephone: (44) 1223337900

Email:

Facsimile: (44) $1223337918 \quad$ Telex:

Commission(s): 14,34

BURGESS DAVID D PROF, ASTROPHYSICS GROUP, IMPERIAL COLLEGE, BLACKETT LABORATORY

LONDON SW7 2BZ, UK

Telephone: (44) $171589511{ }^{*} 6931$

Facsimile: (4)

Telex:

Email:

Commission(s):

BURKE BERNARD F DR, DPT OF PHYSICS, MIT RM 26335 , BOX 165, CAMBRIDGE MA 02139, USA

Telephone: (1) 6172532572

Facsimile: (1)

Telex: 92-1+73

Email:

Commission(s): $33,34,40,44,51$

BURKE J ANTHONY DR, DPT OF PHYSICS, UNIVERSITY OF VICTORIA, BOX 1700

ICTORIA BC V8W 2Y2, CANADA

Telephone: (1) $60472177+3$

Facsimile: (1) $6047217715 \quad$ Telex:

Email:

Commission(s):

BURKHART CLAUDE DR, OBSERVATOIRE DE LYON, AVE CHARLES ANDRE F 69561 S GENIS LAVAL CDX

FRANCE

Telephone: (33) 78560705

Facsimile: (33) 72399791

Telex: 310-926

Email:

Commission(s): 29

BURKHEAD MARTIN S, ASTRONOMY DPT, INDIANA UNIVERSITY, SWAIN WEST 3, BLOOMINGTON IN 47405, USA

Telephone: (1) 8123356917

Facsimile: (1)

Telex:

Email:

Commission(s): 37 
BURKI GILBERT PROF, OBSERVATOIRE DE GENEVE, CHEMIN DES MAILLETTES 51 CH 1290 SAUVERNY, SWITZERLAND

Telephone: (41) 227552611

Facsimile: (41) 227553983

Telex: 419209 obs ch

Email: burki(a)obs.unige.ch

Commission(s): $27,30 \mathrm{C}$

BURLAGA LEONARD F DR, NASA GSFC, CODE 692, GREENBELT MD 20771, USA

Telephone: (1)

Email:

Facsimile: (1) $3012863271 \quad$ Telex:

Commission(s): $15,49 \mathrm{C}$

BURNAGE ROBERT, OHP, F 04870 S MICHEL OBS, FRANCE

Telephone: (33) 92766368

Facsimile: (33)

Commission(s): 30

Telex: $410690 \mathrm{ohp} f$

Email:

BURNS JACK O'NEAL JR, DPT PHYSICS \& ASTRONOMY, UNIVERSITY OF NEW MEXICO, 800 YALE BLVD NE ALBUQUERQUE NM 87131, USA

Telephone: (1) 5052772705

Email:

Facsimile: (1)

Telex:

Commission(s): 28

BURNS JOSEPH A PROF, CORNELL UNIVERSITY, THURSTON HALL, ITHACA NY 14850, USA

Telephone: (1) 6072564875

Facsimile: (1)

Telex: 937478

Email:

Commission(s): $15,16,20$

BURROWS ADAM SETH, DPT OF PHYSICS, STONY BROOK, STONY BROOK NY 11794, USA

Telephone: (1) 5162466810

Facsimile: (1)

Telex:

Email:

Commission(s): +4

BURROWS DAVID NELSON, DPT OF ASTRONOMY, PENNSYLVANIA STATE UNIV, 525 DAVEY LAB UNIVERSITY PARK PA 16802, USA

Telephone: (1) $81+8632466$

Facsimile: (1) $81+8633399 \quad$ Telex:

Email: burrows(a)astro.psu.edu

Commission(s):

BURS LUCIAN, ASTRONOMICAL OBSERVATORY, P TA AXENTE SEVER 1, TIMISOARA 1900, RUMANIA

Telephone: (40) 56162838

Facsimile: (40)

Telex:

Email:

Commission(s):

BURSA MILAN DR, ASTRONOMICAL INSTITUTE, CZECH ACADEMY SCIENCES, BOCNI II 1401

CZ 141 31 PRAHA 4, CZECH R

Telephone: (42) $2250551 / 8757$

Facsimile: $(42) 2255010$

Telex: $122+86$

Email: astdss(a)esearn

Commission(s):

BURSTEIN DAVID, DPT OF PHYSICS, ARIZONA STATE UNIVERSITY, TEMPE AZ 85287, USA

Telephone: (1)

Facsimile: (1)

Telex:

Email:

Commission(s): 28,50

BURTON MICHAEL G DR, SCHOOL OF PHYSICS, UNIVERSITY OF NEW SOUTH, WALES SYDNEY NSW 2025, AUSTRALIA

Telephone: (61) 23855618

Facsimile: (61) 26633420

Telex:

Email: mgbancwt.phys.unsw.edu.au

Commission(s): 34,WGDAA

BURTON W BUTLER DR, STERREWACHT, BOX 9513, NL 2300 RA LEIDEN, NETHERLANDS

Telephone: (31) 71272727

Facsimile: (31)

Telex: 39058 astronl

Email:

Commission(s): $09,33,34$

BURTON WILLIAM M, RUTHERFORD APPLETON LAB, SPACE \& ASTROPHYSICS DIV, BLDG R25/R68 CHILTON DIDCOT OX11 0QX, UK

Telephone: (4) 123521900

Facsimile: $(4)$

Telex: 83159

Email:

Conmission(s): 4

BUSCOMBE WILLIAM PROF, 1231 ASBURY AVE, EVANSTON IL 60202-1101, USA

Telephone: (1)

Facsimile: (1) $708+919982$ Telex:

Email: buscomberanvacvm.bitnet

Commission(s): 45,46

BUSER ROLAND DR, ASTRONOMISCHES INSTITUT, UNIVERSITAET BASEL, VENUSSTRASSE 7

CH 4102 BINNINGEN, SWITZERLAND

Telephone: (41) $612717711 / 12$

Facsimile: $(+1)$

Telex:

Email:

Commission(s): 25,45 
BUSKO IVO C DR, INPE, CP 515, 12210970 S JOSE DOS CAMPO, BRAZIL

Telephone: (55) $123228977 * 353 \quad$ Facsimile: (55) $123218743 \quad$ Telex: 011-33530 inpe br

Email: busko@uas.inpe.br

Commission(s): 27

BUSON LUCIO M DR, OSS ASTRONOMICO DI PADOVA, VIC DELL OSSERVATORIO 5, I 35122 PADOVA, ITALY

Telephone: (39) $49661499 \quad$ Facsimile: (39)

Email: buson@astrpd.infnet Commission(s):

Telex: 432071 astros $i$

BUSSO MAURIZIO, OSS ASTRONOMICO DI TORINO, ST OSSERVATORIO 20, I 10025 PINO TORINESE, ITALY

Telephone: (39) $11841067 \quad$ Facsimile: (39) Telex: 213239 to astr $\mathrm{i}$

Email:

Commission(s): $\mathbf{4 2}$

BUTA RONALD J DR, DPT PHYSICS \& ASTRONOMY, UNIVERSITY OF ALABAMA, BOX 870324

TUSCALOOSA AL 35487 0324, USA

Telcphone: (1) 2053483792

Email: buta@sarah.astr.ua.cdu

Facsimile: (1) $2053+85051 \quad$ Telex:

Commission(s): 28

BUTCHER HARVEY R PROF DR, KAPTEYN ASTRONOMICAL INST, BOX 800 NL 9700 AV GRONINGEN, NETHERLANDS

$\begin{array}{ll}\text { Telephone: (31) } 590819631 & \text { Facsimile: (31) } \\ \text { Email: } & \text { Commission(s): } 28\end{array}$

Telex: 53767 kswro n!

BUTCHINS SYDNEY ADAIR, DPT OF CIVIL AVIATION STU, FAC OF MATHS, THE MINORIES TOWER HILL EC3N 1JY, UK

Telephone: (44) 1717227344

Email:

Facsimile: (44)

Telex:

BUTI BIMLA PROF, NATIONAL PHYSICAL LAB, DR K S KRISHNAN ROAD, NEW DEHLI 110012, INDIA

Telephone: (91) 116899903

Facsimile: (91) 115752678

Telex: $03177099 \mathrm{npl}$ in

Email: buti\%npl@sirnetd.ernet.in

Commission(s): 49C

BUTLER C JOHN DR, ARMAGH OBSERVATORY, COLLEGE HILL, ARMAGH BT61 9DG, UK

Telephone: (44) $1861522928 \quad$ Facsimile: (4) Telex: 747937 armobs g

Email:

Commission(s): $27 \mathrm{C},+4$

BUTLER DENNIS DR, YALE UNIVERSITY OBS, YALE STATION, BOX 2023, NEW HAVEN CT 06520, USA

Telephone: (1)

Facsimile: (1)

Telex:

Email:

Commission(s): 27,37

BUTLER KEITH DR, INST ASTRON \& ASTROPHYSIK, UNIVERSITAETS STERNWARTE, SCHEINERSTR 1 D 81679 MUENCHEN, GERMANY

Telephonc: (49) 89989021

Facsimile: (49) $8992209+27$

Telex:

Email:

Commission(s): 29

BUTTERWORTH PAUL, NASA GSFC, CODE 664, GREENBELT MD 20770, USA

Telcphonc: (1) 3013454310

Email: heavax::butterworth

Facsimile: (1) $3012861629 \quad$ Telex:

Commission(s): 44

BUYUKGIEV GEORGI DR, NTL ASTRONOMICAL OBS, BULGARIAN ACAD SCIENCES, BOX 136 BG 4700 SMOLJAN, BULGARLA

Telephone: (359) 7341599

Email:

Facsimile: (359)

Telex: $\mathbf{4 8 + 4 6}$ sst renhg

Commission(s): 10

BYARD PAUL L DR, DPT OF ASTRONOMY, OHIO STATE UNIVERSITY, 174 W 18TH AVE

COLUMBUS OH 432101106 , USA

Telephonc: (1) 6144221773

Facsimile: (1)

Telex:

Email:

Commission(s):

BYKOV MIKLE F DR, ASTRONOMICAL INSTITUTE, UZBEK ACADEMY OF SCIENCES 700000 TASHKENT, UZBEKISTAN

Telephone: (7)

Facsimile: (7)

Telex:

Email:

Commission(s): 08 
BYLEVELD WILLEM DR, OMNIVERSUM SPACE THEATRE, PRES KENNEDYLAAN 5

NL 2517 JK THE HAGUE, NETHERLANDS

Tclephone: (31) 70547479

Facsimile: (31) 70524280

Telex:

Email:

Commission(s):

BYRD GENE G DR, DPT OF PHYSICS \& ASTRON, UNIVERSITY OF ALABAMA, BOX 1921 TUSCALOOSA AL 35487 0324, USA

Telephone: (1) 2053485050

Facsimile: (1)

Telex:

Email:

Commission(s): 28,37

BYRNE PATRICK B DR, ARMAGH OBSERVATORY, COLLEGE HILL, ARMAGH BT61 9DG, UK

Telephone: (44) $1861522928 \quad$ Facsimile: (44)

Email: pbb@star.arm.gub.ac.uk.

Commission(s): 27

Telex: 747937 armobs g

BYSTROVA NATALIJA V DR, ST PETERSBURG BRANCH, STERNBERG ASTRON OBSERV

196140 ST PETERSBURG, RUSSIA

Telephone: (7) 812123 4038/4372

Email: bnv@sai.sob.su

Facsimile: (7) 8123151701

Telex: 64121986 at 321262

Commission(s): 34

CABRITA EZEQUIEL DR, OBS ASTRONOMICO DE LISBOA, TAPADA DA LISBOA, P 1300 LISBOA, PORTUGAL
Telephone: (351) 637351-634669

Telephone: (351) 637351-634669 Facsimile: (351)

Email:

Commission(s): 26

CACCIANI ALESSANDRO PROF, DPT DI FISICA, UNIVERSITA DI ROMA, PA MORO 2, I 00185 ROMA, ITALY

Telephonc: (39) 64976265

Facsimile: (39)

Telex: 613255 infuro

Email:

Commission(s):

CACCIARI CARLA DR, OSS ASTRONOMICO, UNIVERSITA DI BOLOGNA, CP 596, 140100 BOLOGNA, ITALY

Telephone: (39) $51259301 * 9401$

Facsimile: (39) 51259407

Telex: $52063+$ infnbo $i$

Email: caccuaru@astbo1.infnet

Commission(s):

CACCIN BRUNO, DPT DI FISICA, VIA RAIMONDO SNC, UNIVERSITA TOR VERGATA, I 00173 ROMA, ITALY

Telephone: (39) 679792323

Facsimile: (39)

Commission(s): 38

Telex: 626382 fiuntv

Email:

S, UNIVERSITY OF LJUBLJANA, JADRANSKA 19, LJUBLJANA, SLOVENIA

CADEZ ANDREJ DR, DPT OF PHYSICS, UNIVERSITY OF LJUBLJANA, JADRANS
Telephone: (38) 61265061
Facsimile: (38) 61217281

Email: andrej.cadez@uni-lj.ac.mail.yu Commission(s):

CADEZ VLADIMIR, INSTITUTE OF PHYSICS, BOX 57, YU 11001 BEOGRAD, YUGOSLAVIA-SERBIA \& MONTENEGRO

Telephone: (38) 11212219

Email:
Facsimile: (38)

Commission(s): 10,12
Telex: 11002 infiz yu

CAHN JULIUS H PROF, DPT OF ASTRONOMY, UNIVERSITY OF ILLINOIS, BOX 755, TESUQUE NM 87574, USA

Telephone: (1) $5059830691 \quad$ Facsimile: (1)

Commission(s):

Telex:

Email:
CAILLAULT JEAN PIERR

CAILlaULT JeAN PIERR

Telephone: (1) $4045+22883$

Email: jpc $(\underline{a}$ jove.physast.uga.edu

Facsimilc: (1)

Commission(s):

CALAMAI GIOVANNI, OSS DI ARCETRI, L E FERMI 5, I 50125 FIRENZE, ITALY

$\begin{array}{lll}\text { Telephone: (39) } 552752310 & \text { Facsimile: (39) } 55220039 & \text { Telex: } 572268 \text { arcetr i } \\ \text { Email: vanni@arcetri.astro.it } & \text { Commission(s): } & \end{array}$

CALAME ODILE DR, OCA CERGA, AVE COPERNIC, F 06130 GRASSE, FRANCE

Telephone: (33) 93365849

Facsimile: (33)

Commission(s): 07,16,20

Telex: $\mathbf{4 7 0 8 6 5}$

Email:

CALDWELL JOHN A R, SAAO, BOX 9, OBSERVATORY 7935, SOUTH AFRICA

Telephone: (27) $021+70025$

Facsimile: (27)

Commission(s): 27,33

Telex: 57-20309 
CALDWELL JOHN JAMES, DPT OF PHYSICS, YORK UNIVERSITY, 4700 KEELE ST NORTH YORK ON M3J 1P3, CANADA

Telephone: (1) 4167362100

Facsimile: (1) 4167365386

Telex: 06524736

Email:

Commission(s): 16

CALLANAN PAUL DR, DPT OF ASTROPHYSICS, UNIVERSITY OF OXFORD, KEBLE RD, OXFORD OX1 3RH, UK

Telephone: (44) 1865273293

Facsimile: (44) 1865273418

Telex: 83295 nuclox g

Email: uk.ac.ox.astro (janet)

Commission(s): 42

CALLEBAUT DIRK K DR, DPT OF PHYSICS, UNIVERSITY OD ANTWERPEN, UNIVERSITEITSPLEIN 1 B 2610 ANTWERPEN WILRIJK, BELGIUM

Telephone: (32) 38202457

Facsimile: (32) 38202245

Telex: 33646

Email:

Commission(s): 35,37

CALLY PAUL S DR, DPT OF MATHEMATICS, MONASH UNIVERSITY, WELLINGTON RD CLAYTON VIC 3168, AUSTRALIA

Telephone: (61) $35654471 \quad$ Facsimile: (61)

Telex: monash aa 32691

Email: apm150f(a)vaxc.cc.monash.edu.au Commission(s): 10

CALOI VITTORIA DR, LAS, CNR, CP 67, I 000 $4+$ FRASCATI, ITALY

Telephone: (39) $69+25654$

Email:

Facsimile: (39) $694168+7$

Telex: $610261 \mathrm{cnr}$ fra

CALVANI MASSIMO DR, DPT DI ASTRONOMIA, UNIVERSITA DI PADOVA, VIC DELL OSSERVATORIO 5 I 35122 PADOVA, ITALY

Telephone: (39) 49661499

Email: calviani(a) astrpd.astro.it

Facsimile: (39)

Commission(s): 47

Telex: $\mathbf{4 3 0 1 7 6}$ unjadu $i$

CALVET NURIA DR, CIDA, BOX 264, MERIDA 5101 A, VENEZUELA

Telephone: (58) $74639930 \quad$ Facsimile: (58)

Email:

Commission(s): 46

Telex: $7+17+$ cida ve

CALVIN WILLLAM H DR, DPT OF PHYSICS, UNIVERSITY OF WASHINGTON, FM 15, SEATTLE WA 98195, USA Telephone: (1) 2063281192 Facsimile: (1) $2065+33041$ Telex:

Email: wealvin a)u.washington.cdu

Commission(s): 51

CALVO MANUEL, DPT DE ASTRONOMIA, UNIVERSIDAD DE ZARAGOZA, E 50009 ZARAGOZA, SPAIN

Telephone: (34) 357011

Facsimile: (34)

Email:

Commission(s):

Telex:

CAMARENA BADIA VICENTE PR, DPT MATEMATICA APLICADA, UNIVERSIDAD DE ZARAGOZA

E 50009 ZARAGOZA, SPAIN

Telephone: (34)

Facsimile: (34)

Commission(s):

Telex:

Email:

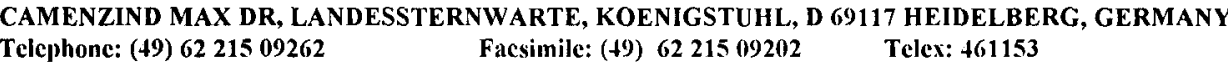

Email: ab+adhdurz1.bitnet

Commission(s): 44

CAMERON ALASTAIR G W PROF, CENTER FOR ASTROPHYSICS, HCO/SAO, 60 GARDEN STAMBRIDGE MA 02138, USA

Telephone: (1) 6174955374

Facsimile: (1)

Commission(s): $35,4+$

Telex:

Email:

CAMERON ANDREW COLLIER DR, ASTRONOMY CENTRE, UNIVERSITY OF SUSSEX, FALMER

BRIGHTON BN1 9QH, UK

Telephone: (44) 1273678117

Email:

Facsimile: (4t)

Commission(s): 27

Telex: 877159 bhvtxs $g$

CAMERON LUZIUS MARTIN, ASTRONOMISCHES INSTITUT, UNIVERSITAET BASEL, VENUSSTRASSE ?

CH 4102 BINNINGEN, SWITZERLAND

Telephone: (\$1) $612717711 / 12$

Facsimile: ( +1$)$

Telex:

Email:

Commission(s): 28 
CAMERON WINIFRED S MRS, LA RANEHITA DE LA LUNA, BLDG 26, 200 ROJO DR, SEDONA AZ 86336, USA

Telephone: (1)

Facsimile: (1)

Telex:

Email:

Commission(s): 16

CAMICHEL HENRI DR, 24 AVE C FLAMMARION, F 31500 TOULOUSE, FRANCE

Telephone: (33) 61489691

Facsimile: (33)

Telex:

Email:

Commission(s): 16

CAMPBELL ALISON DR, DPT PHYSICS \& ASTRONOMY, JOHNS HOPKINS UNIVERSITY, CHARLES \& 34TH ST BALTIMORE MD 21218, USA

Telephone: (1) 3013385186

Email: awc@stsci.bitnet/scivax::awc

Facsimile: (1) $3013388260 \quad$ Telex:

Commission(s): 26

CAMPBELL BELVA G S DR, DPT PHYSICS \& ASTRONOMY, UNIVERSITY OF NEW MEXICO, 800 YALE BLVD N ALBUQUERQUE NM 87131, U

Telephone: (1) 5052775148

Facsimile: (1)

Telex:

Email: bitnet:bel@unmb

Commission(s):

CAMPBELL DONALD B, CORNELL UNIVERSITY, SPACE SCIENCES BLDG, ITHACA NY 14853, USA

Telephone: (1) 6072555274

Facsimile: (1)

Telex: 932454

Email:

Commission(s): 16

CAMPBELL JAMES W, ROYAL OBSERVATORY, BLACKFORD HILL, EDINBURGH EH9 3HJ, UK

Telephone: (44)

Facsimile: (44)

Telex:

Email:

Commission(s):

CAMPBELL MURRAY F, DPT PHYSICS \& ASTRONOMY, COLBY COLLEGE, WATERVILLE ME 04901, USA

Telephone: (1) 2078723251

Email:

Facsimile: (1)

Telex:

Commission(s): +4

CAMPINS HUMBERTO DR, DPT OF ASTRONOMY, UNIVERSITY OF FLORIDA, 211 SSRB

GAINESVILLE FL 32611, USA

Telephone: (1) 9043923066

Facsimile: (1)

Telex:

Email: campins@astro.ufl.cdu.

Commission(s): 15

CAMPOS L M BRAGA DA COSTA, INST SUPERIOR TECNICO, AVE ROVISCO PAIS P 1096 LISBOA CODEX, PORTUGAL

Telephone: (351) 800525

Facsimile: (351)

Telex: $63+23$ istutl p

Email:

Commission(s):

CAMPUSANO LUIS E, DPT DE ASTRONOMIA, UNIVERSIDAD DE CHILE, CASILLA 36 D, SANTIAGO, CHILE

Telephone: (56) 22294101

Facsimile: (56)

Telex: 440001

Email:

Commission(s): 28,51

CANAL RAMON M DR, DPT FISICA DE ATMOSFERA, UNIVERSIDAD DE BARCELONA, AVD DIAGONAL 645 E 08028 BARCELONA, SPAIN

Telephone: (34)

Email:

Facsimile: (34)

Commission(s): $35 \mathrm{C}$

Telex:

CANAVAGGIA RENĘE DR, OBSERVATOIRE DE PARIS, 61 AVE OBSERVATOIRE, F 75014 PARIS, FRANCE

Telephone: (33) $143201210 \quad$ Facsimile: (33)

Telex: 270776 obs $f$

Email:

Commission(s):

CANDY MICHAEL P MR, PERTH OBSERVATORY, BICKLEY WA 6076, AUSTRALIA

Telephone: (61) 92938255

Facsimile: (61)

Telex:

Email:

Commission(s): $06,07,15,20$

CANE HILARY VIVIEN, DPT OF PHYSICS, UNIVERSITY OF TASMANIA, GPO BOX 252C

HOBART TAS 7001, AUSTRALIA

Telephone: (61) 2202401

Facsimile: (61) 2202186

Telex: aas8150

Email: hilary.cane(aphys.utas.edu.au

Commission(s): 10,33

CANFIELD RICHARD C DR, INSTITUTE FOR ASTRONOMY, UNIVERSITY OF HAWAII, 2680 WOODLAWN DR, HONOLULU HI $96822, \mathrm{U}$

Telephone: (1)

Facsimile: (1)

Telex:

Email:

Commission(s): 
M 464

CANIZARES CLAUDE R PROF, CENTER FOR SPACE RESEARCH, MIT RM 37241 , BOX 165

CAMBRIDGE MA 02139, USA

Telephone: (1) 6172537480

Email: brendap(a)space.mit.edu

Facsimile: (1) $6172530861 \quad$ Telex: 921473 mitcam

Commission(s):

CANNON RUSSELL D DR, AAO, BOX 296, EPPING NSW 2121, AUSTRALIA

Telephone: (61) $28681666 \quad$ Facsimile: (61) $28768536 \quad$ Telex: 23999 aaosyd

Email: $\quad$ Commission(s): 28,37

CANNON WAYNE H DR, DPT PHYS/EARTH \& ATM SCI, YORK UNIVERSITY, 4700 KEELE ST, DOWNSVIEW ON M3J 1P3, CANADA

Telephone: (1) $4166676410 \quad$ Facsimile: (1)

Email: $\quad$ Commission(s):

Telex: $0652+736$

CANTO JORGE DR, INSTITUTO DE ASTRONOMIA, UNAM, APDO POSTAL 70-264, 04510 MEXICO DF, MEXICO

Telephone: (52) $9055485305 \quad$ Facsimile: (52) Telex: 1760155 cic me

Email:

Commission(s): 34

CANTU ALBERTO M DR, IST CIBERNETICA/BIOFISICA, CNR, I 16032 CAMOGLI, ITALY

Telephone: (39) $185770646 \quad$ Facsimile: (39) Telex:

Email: $\quad$ Commission(s):

CAO CHANGXIN, NANJING ASTRONOMICAL, INSTRUMENT FACTORY, NANJING, CHINA PR

Telephone: (86) 2546191 Facsimile: (86) Telex: 34136 glynj cn

Email:

Commission(s): 09

CAO LIHONG, PURPLE MOUNTAIN OBSERV, CAS, NANJING, CHINA PR

Telephone: (86) $2546700 \quad$ Facsimile: (86) $25301459 \quad$ Telex: 34144 pmonj cn

Email: $\quad$ Commission(s):

CAO SHENGLIN, DPT OF ASTRONOMY, BEIJING NORMAL UNIVERSITY, BEIJING 100875, CHINA PR

Telephone: (86) 12012255

Facsimile: $(86)$

Telex: 222701

Email:

Commission(s): 28

CAPACCIOLI MASSIMO DR, DPT DI ASTRONOMIA, UNIVERSITA DI PADOVA, VIC DELL OSSERVATORIO 5 I 35122 PADOVA, ITALY

Telephone: (39) 49661499

Email:

Facsimile: (39)

Telex: $\$ 30176$ undapu $i$

Commission(s): 28

CAPACCIONI FABRIZIO DR, IAS, REPORTO DI PLANETOLOGIA, VIA DELL'UNIVERSITA 11, I O0185 ROMA, ITALY

Telephonc: (39) 64456951

Facsimile: (39) $6+454969$

Telex:

Email:

Commission(s): 15

CAPELATO HUGO VICENTE DR, INPE, CP 515, 12201 S JOSE DOS CAMPOS, BRAZIL

$\begin{array}{ll}\text { Telephone: (55) } 123229977 & \text { Facsimile: (55) } \\ \text { Email: } & \text { Commission(s): }\end{array}$

Email:

CAPEN CHARLES F, SOLIS LACUS OBSERVATORY, RT 2, BOX 262 E, CUBA MO 65453, USA

Telephone: (1)

Facsimile: (1) Telex:

Email:

Commission(s):

CAPITAINE NICOLE, OBSERVATOIRE DE PARIS, 61 AVE OBSERVATOIRE, F 75014 PARIS, FRANCE

Telephone: (33) 140512231

Facsimile: (33)

Telex: 270776 obs $f$

Email:

Commission(s): 04,19C

CAPLAN JAMES, OBSERVATOIRE DE MARSEILLE, 2 PLACE LE VERRIER, F 13248 MARSEILLE CDX 04, FRANCE

Telephone: (33) 91959088

Facsimile: (33)

Telex:

Email:

Commission(s): $3 \downarrow$

CAPPA DE NICOLAU CRISTINA, IAR, CC 5, 189+ VILLA ELISA (BS AS), ARGENTINA

Telephone: (54) $2125+909$

Facsimile: (54) 21254909

Telex: 31216 cesla ar

Email: ccappa@irma.edu.ar

Commission(s): 34 
CAPPELLARO ENRICO DR, OSS ASTRONOMICO DI PADOVA, VIC DELL OSSERVATORIO 5

I 35122 PADOVA, ITALY

Telephone: (39) 49661499

Email: span:astrpd::cappellaro

Facsimile: (39)

Telex: $\mathbf{4 3 2 0 7 1}$ astros $i$

CAPPI ALBERTO, OSSERVATORIO ASTRONOMICO, DI BOLOGNA, VIA ZAMBONI 33, I 40126 BOLOGNA, ITALY

Telephone: (39) 51259406

Facsimile: (39) $51259407 \quad$ Telex:

Email: cappi@astbo3.bo.astro.it

Commission(s): 47

CAPRIA MARIA TERESA DR, IAS, REPARTO DI PLANETOLOGIA, VIA DELL'UNIVERSITA 11, I 00185 ROMA, ITALY
Telephone: (39) 64456951
Facsimile: (39) 64454969

Email: teresa@irmias Commission(s): 15,16

CAPRIOLI GIUSEPPE PROF, OAR, VIA TRIONFALE 204, I 00136 ROMA, ITALY

Telephone: (39) $63+7050 \quad$ Facsimile: (39) Telex:

Email: $\quad$ Commission(s): 31

CAPRIOTTI EUGENE R DR, DPT OF ASTRONOMY, OHIO STATE UNIVERSITY, S058 ALPHEUS SMITH LAB

COLUMBUS OH 432101106 , USA

Telephone: (1) 6144221773

Facsimile: (1)

Telex:

Email:

Commission(s): 34

CAPUTO FILIPPINA DR, IAS, CNR, CP 67, I (00044 FRASCATI, ITALY

Telephone: (39) $69+25651 \quad$ Facsimile: (39) $69416847 \quad$ Telex: 610261 cnr fra

Email: $\quad$ Commission(s): 35,37

CAPUZZO DOLCETTA ROBERTO, ISTITUTO ASTRONOMICO, UNIVERSITA DI ROMA, VIA G M LANCISI 29 I 00161 ROMA, ITALY

Telephone: (39) 6867525

Email:

Facsimile: (39) Telex:

Commission(s): 34,37

CARANICOLAS NICHOLAS DR, DPT OF ASTRONOMY, UNIVERSITY THESSALONIKI, GR 540 O6 THESSALONIKI, GREECE

Telephonc: (30) $31991357 / 59$

Facsimile: (30)

Telex:

Email:

Commission(s): 07

CARBON DUANE F DR, NASA AMES RESEARCH CTR, MS 258 5, MOFFETT FIELD CA 94035, USA

Telephonc: (1) 4156044413

Facsimilc: (1)

Telex:

Email: dcarbon@anas.nasa.gov

Commission(s): 14,36

CARDELLI JASON A DR, DPT OF ASTRONOMY, VILLANOVA UNIVERSITY, 800 LANCASTER AVE

VILLANOVA PA 19085, USA

Telephonc: (1) 6105196396

Email: jcardelliâuucis.vill.edu

Facsimile: (1)

Telex:

Commission(s): 34

CARDINI DANIELA DR, IAS, CNR, CP 67, I 000 +4 FRASCATI, ITALY

Tclephone: (39) $69+25655$

Email: daniela(a)irmias

Facsimilc: (39) 69416847

Telex: $610261 \mathrm{cnr}$ fra

CARdona octavio dr, inAOe, tonantzintlaz, APdo POSTAL 216 y 51, 72000 PUEbla PUE, MEXICO

Telephonc: (52) $9122470500 \quad$ Facsimile: (52)

Telex:

Email:

Commission(s):

CARDUS ALMEDA J O MR, OBSERVATORIO DEL EBRO, E 43520 ROQUETES, SPAIN

Telephonc: (34) 77500511 Facsimile: (34) Telex:

Email:

Commission(s):

CARGILL PETER J DR, NAVAL RESEARCH LABORATORY, CODE 4790,4555 OVERLOOK AVE SW

WASHINGTON DC 20375 5000, USA

Telephone: (1) $202767+978$

Facsimile: (1) $2027670631 \quad$ Telex:

Email: cargillappdpi1.nrl.navy.mil

Commission(s): 10

CARIGNAN CLAUDE DR, DPT DE PHYSIQUE, UNIVERSITE DE MONTREAL, CP 6128 SUCC A MONTREAL QC H3C 3J7, CANADA

Telephone: (1) 5143437355

Facsimile: (1) $51+3+32071$

Telex: 05562425

Email: carignangec.umontreal.ca

Commission(s): 28 
CARLBERG RAYMOND GARY DR, DPT OF PHYSICS, YORK UNIVERSITY, 4700 KEELE ST NORTH YORK ON M3J 1P3, CANADA

Telephone: (1) 4166673851

Facsimile: (1)

Telex: 06524736

Email:

Commission(s):

CARLETON NATHANIEL P DR, CENTER FOR ASTROPHYSICS, HCO/SAO, 60 GARDEN ST CAMBRIDGE MA 02138, USA

Telephone: (1) 6174957405

Facsimilc: (1)

Telex: $921+28$ satellite cam

Email:

Commission(s):

CARLQVIST PER A DR, DPT OF PLASMA PHYSICS, ROYAL INST OF TECHNOLOGY

S 10044 STOCKHOLM 70, SWEDEN

Telephone: (46) 87877697

Facsimile: (46)

Telex:

Email:

Commission(s): 10

CARLSON JOHN B, ARCHEOASTRONOMY CENTER, BOX X, COLLEGE PARK MD 20740, USA

Telephone: (1) 3018646637

Facsimile: (1)

Telex:

Email:

Commission(s): 41,51

CARLSSON MATS DR, INST THEORET ASTROPHYSICS, UNIVERSITY OF OSLO, BOX 1029 N 0315 BLINDERN OSLO 3, NORWAY

Telephone: (47)

Facsimile: (47)

Telex:

Email:

Commission(s): 36

CARNEY BRUCE WILLIAM, DPT PHYSICS \& ASTRONOMY, UNIVERSITY NORTH CAROLINA 204 PHILLIPS HALL 039A, CHAPEL HILL NC 27514, USA

Telephone: (1) 9199623023

Facsimile: (1)

Telex:

Email:

Commission(s): $25,29,30,37$

CAROFF LAWRENCE J, NASA AMES RESEARCH CTR, MS 245 6, SPACE SCIENCE DIV MOFFETT FIELD CA 94035, USA

Telephone: (1) 4156945523

Facsimilc: (1)

Telex:

Email:

Commission(s):

CAROUBALOS C A PROF, LAB ELECTRONIC PHYSICS, NTL UNIVERSITY OF ATHENS, KTHRLA TYPA-ILISSIA GR 15701 ATHENS 144, GREECE

Telephone: (30) 17244 096/11119

Email:

Facsimile: (30)

Telex: 215530 obsa gr

Commission(s): 40

CARPENTER KENNETH G DR, NASA GSFC, CODE 681, GREENBELT MD 20771, USA

Telephone: (1) 3012863453

Facsimile: (1)

Telex: 89675 k carpenter

Email: span:6172::hrscarpenter

Commission(s): 29,4t

CARPENTER LLOYD DR, 13902 RESIN CT, BOWIE MD 20720, USA

Telephone: (1)

Facsimile: (1)

Telex:

Email:

Commission(s):

CARPINO MARIO DR, OSS ASTRONOMICO DI BRERA, VIA BRERA 28, 120121 MILANO, ITALY

Telephone: (39) 2874444

Email: carpino(astmib.astro.it

Facsimile: (39) $272001600 \quad$ Telex:

Commission(s): 07

CARQUILLAT JEAN-MICHEL, OBS MIDI PYRENEES, $1+$ AVE E BELIN, F 31400 TOULOUSE CDX, FRANCE

Telephone: (33) 61252101

Facsimilc: (33)

Telex: $\mathbf{5 3 0 7 7 6}$ obstlse

Email:

Commission(s): 30

CARR BERNARD JOHN, SCHOOL OF MATHEMATICAL SC, QUEEN MARY/WESTFIELD COLL, MILE END RD LONDON E1 4NS, UK

Telephone: (44) 1719804811

Facsimilc: ( +4$)$

Telex:

Email:

Commission(s): 47

CARR JOHN SHERMAN, DPR OF ASTRONOMY, OHIO STATE UNIVERSITY, 17 $\downarrow$ W 18TH AVE

COLUMBUS OH 432101106 , USA

Telephone: (1) 6142926925

Facsimile: (1) $61+2922928$

Telex:

Email: carr@payne.mps.ohio-statc.edu

Commission(s): 
CARR THOMAS D PROF, DPT OF ASTRONOMY, UNIVERSITY OF FLORIDA, 211 SSRB

GAINESVILLE FL 32611, USA

Telephone: (1) 9043922066

Facsimile: (1) Telex:

Email: tacarr@uffsc.bitnet

Commission(s): 40,51

CARRAMinana Alberto, INAOE, AP 51/216, PUEbla PUE 72000, MEXICO

Telephone: (52) $22472011 \quad$ Facsimile: (52) $22472231 \quad$ Telex:

Email: alberto(a)tonali,inaocp.mx Commission(s):

CARRANZA GUSTAVO J DR, IBSERVATORIO ASTRONOMICO, DE CORDOBA, LAPRIDA 854

5000 CORDOBA, ARGENTINA

Telephone: (54) 51230491

Facsimile: (54) 51210613

Telex: 51822 bucor ar

Email: carranza(a) astro.edu.ar

Commission(s): 28

CARRASCO BERTH ESPERANZA, INAOE, AP 51/216, PUEBLA PUE 72000, MEXICO

Telephone: (52)

Facsimile: (52)

Commission(s):

Telex:

Email:

CARRASCO GUILLERMO DR, DPT DE ASTRONOMIA, UNIVERSIDAD DE CHILE, CASILLA 36 D, SANTIAGO, CHILE

Telephone: (56) 22294002

Facsimile: (56)

Commission(s): 08

Telex: 440001

Email:

ASTRONOMIA, UNAM, APDO POSTAL 70-264, 04510 MEXICO DF, MEXICO

CARRASCO LUIS DR, INSTITUTO DE ASTRONOMIA, UNAM, APDO POSTAL 70-2
Telephone: (52) 9055485305
Facsimile: (52)

Email: $\quad$ Commission(s): 33

CARROLL P KEVIN PROF, DPT OF PHYSICS, UNIVERSITY COLLEGE, BELFIELD, DUBLIN 4, IRELAND

Telephone: (353) 1693244

Facsimile: (353)

Telex:

Email:

Commission(s): 14,44

CARRUTHERS GEORGE R DR, NAVAL RESEARCH LABORATORY, CODE 7123, SPACE SCIENCE DIVISION WASHINGTON DC 20375 5000, USA

Telephone: (1) 2027672764

Facsimile: (1)

Telex:

Email:

Commission(s): 15,34

CARSENTY URI DR, DFVLR, NE OE PE, OBERPFAFFENHOFEN, D 8031 WESSLING, GERMANY

Telephone: (49) 8153281328

Facsimile: (49) $81532+76 \quad$ Telex:

Email: span:ooepes::carsenty

Commission(s): 15

CARSMARU MARIA M DR, ASTRONOMICAL INSTITUTE, CUTITUL DE ARGINT 5, BOX 28 R 75212 BUCHAREST, RUMANIA

Telephone: (40) 16236892

Facsimile: (40) 13123391

Telex: 11882 astro $r$

Email: mcirsmaru(a)imar.ro

Commission(s): 16

CARSON T R DR, DPT PHYSICS \& ASTRONOMY, UNIVERSITY OF ST ANDREWS, NORTH HAUGH

ST ANDREWS FIFE KY16 9SS, UK

Telephone: (44) $133+76161$

Facsimile: (\$4) $13347+487 \quad$ Telex:

Email:

Commission(s): 35,36

CARSWELL ROBERT F DR, INSTITUTE OF ASTRONOMY, THE OBSERVATORIES, MADINGLEY RD

CAMBRIDGE CB3 OHA, UK

Telephone: (44) 1223337548

Facsimile: (44) $1223337523 \quad$ Telex: 817297 astron g

Email:

Commission(s): 28

CARTER BRAD, SCHOOL OF PHYSICS, UNIV OF NEW SOUTH WALES, SYDNEY NSW 2052, AUSTRALIA

Telephone: (61) 26974553

Facsimile: (61) $26633420 \quad$ Telex:

Email: bdc(a)newt.phys.unsw,edu.au Commission(s): 09,29

CARTER DAVID DR, MOUNT STROMLO \& SIDING, SPRING OBSERVATORIES, PRIVATE BAG WODEN PO ACT 2606, AUSTRALIA

Telephone: (61) 62881111

Facsimile: (61) $62 \$ 90233$

Telex: $62270 \mathrm{aa}$

Email:

Commission(s): 28

CARTER WILLIAM EUGENE, NGS, N/CG 114, ROCKVILLE MD 20852, USA

Telephone: (1) $301+438+23$

Facsimile: (1)

Email:

Commission(s): 19,31 
CARUSI ANDREA, IAS, REPARTO DI PLANETOLOGLA, VIA DELL'UNIVERSITA 11, I 00185 ROMA, ITALY

Telephone: (39) $64456951 \quad$ Facsimile: (39) 64454969

Telex: 610261 cnrfra

Email: carusi@itcaspur.caspur.it

Commission(s): 15,20C,22

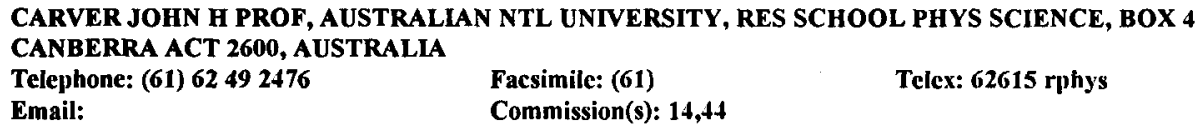

CASERTANO STEFANO DR, DPT OF ASTRONOMY, UNIVERSITY OF ILLINOIS, 1011 W SPRINGFIELD AVE URBANA IL 61801, USA

Telephone: (1) $2173339380 \quad$ Facsimile: (1) Telex:

Email: stefano@rigel.astro.uluc.edu Commission(s): 28
CASH WEBSTER C JR, LASP, UNIVERSITY OF COLORADO, BOX 392, BOULDER CO 80309 0392, USA
Telephone: (1) $303 \$ 928208$ Facsimile: (1) Telex:
Email: $\quad$ Commission(s): 44

CASOLI FABIENNE DR, RADIOASTRONOMIE ENS, 24 RUE LHOMOND, F 75231 PARIS CDX 05, FRANCE

Telephone: (33) 143291225 Facsimile: (33) Telex: 202601

Email: casoli@frulm11 Commission(s):

CASSATELLA ANGELO DR, IAS, CNR, CP 67, $10004+$ FRASCATI, ITALY

Telephone: (39) 69425655 Facsimile: (39) 69416847 Telex:

Email: $\quad$ Commission(s): 29

CASSE MICHEL DR, CEA CEN, DAPNIA/SAP, BP 2, F 91191 GIF/YVETTE CDX, FRANCE

Telephone: (33) Facsimile: (33) Telex:

Email: $\quad$ Commission(s): 44

CASSINELLI JOSEPH P DR, DPT OF ASTRONOMY, UNIVERSITY OF WISCONSIN, 475 N CHARTER ST MADISON WI 53706, USA

Telephone: (1) 6082621752

Email:

Facsimile: (1)

Commission(s): 36

Telex: $265+52$ uofwisc mds

CASTAGNINO MARIO DR, IAFE, CC 67 SUC 28, 1428 BUENOS AIRES, ARGENTINA

$\begin{array}{lll}\text { Telephone: (54) } 17816755 & \text { Facsimile: (54) } 181+4299 & \text { Telex: } 17181 \text { verba } \\ \text { Email: castagnino@iafe edu.ar } & \text { Commission(s): } 47\end{array}$

CASTANEDA HECTOR, INST DE ASTROFISICA, DE CANARIAS, OBS DEL TEIDE, E 38200 LA LAGUNA, SPAIN

Telephone: (34) $22262211 \quad$ Facsimile: (34) 22263005 Telex: 92640 iac e

Email: $\quad$ Commission(s): 34

CASTELAZ MICHEAL W DR, DPT OF PHYSICS, E TENNESSEE STATE UNIV, BOX, JOHNSON CITY TN 37614, USA

Telephone: (1) 6154617064

Facsimile: (1)

Telex:

Email: r29castz@etsu.bitnet

Commission(s): 25

CASTELLANI VITTORIO PROF, DPT DI FISICA, UNIVERSITA DI PISA, PIAZZA TORRICELLI 2, I 56127 PISA, ITALY

Telephone: (39) $50443343 \quad$ Facsimile: (39) 5048277

Telex: 500319 psafis i

Email:

Commission(s): $35,37 \mathrm{C}$

CASTELLI FIORELLA DR, OAT, BOX SUCC TRIESTE 5, VIA TIEPOLO 11, I 34131 TRIESTE, ITALY

Telephone: (39) 40793921

Facsimile: (39)

Commission(s): 29

Telex: $\$ 61137$ oat $i$

Email:

CASTELLI JOHN P, 125 HILLSIDE AVE, ARLINGTON MA 02174, USA

Telephone: (1)

Facsimile: (1)

Email:

Commission(s): 
CASTETS ALAIN DR, OBSERVATOIRE DE GRENOBLE, CERMO/ASTROPHYSIQUE, BP 53X F 38041 S MARTIN HERES CD, FRANCE

Telephone: (33) 76514786

Email: earn:castets@frags1

Facsimile: (33) $76448821 \quad$ Telex:

Commission(s): 40

CASTOR JOHN I DR, LAWRENCE LIVERMORE LAB, L 58, BOX 808, LIVERMORE CA 94550, USA

Telephone: (1) 5104224664

Email: castor1(a)Inl.gov

Facsimile: (1) 5104225102

Commission(s): 35,36

Telex: 9103868339 IInllvm

CASWELL JAMES L DR, CSIRO, DIV OF RADIOPHYSICS, BOX 76, EPPING NSW 2121, AUSTRALIA

Telephone: (61) 28680222

Facsimile: (61) 28680310

Telex: 26230 astro aa

Email: jcaswell@atnf.csiro.au

Commission(s): $33,34,40$

CATALA CLAUDE DR, OBSERVATOIRE DE PARIS, SECTION DE MEUDON, F 92195 MEUDON PPL CDX, FRANCE

Telephone: (33) 145077663

Facsimile: (33)

Commission(s): 29,36

Telex: 204464

Email:

CATALA POCH M A, M ASUNCION, MUNTANER 83 B 3/3, E 08011 BARCELONA, SPAIN
Telephone: (34) 4532569
Facsimile: (34)

Email:

Commission(s): 46

CATALAN MANUEL DR, REAL INST Y OBSERVATORIO, DE LA ARMADA, CECILIO PUJAZON S/N

E 11110 SAN FERNANDO, SPAIN

Telephone: (34) 56883548

Facsimile: (34)

Commission(s): $04,08,31$

Telex: 76108

Email:

CATALANO FRANCESCO A DR, IST DI ASTRONOMIA, CITTA UNIVERSITARIA, VIA A DORIA 6 I 95125 CATANIA, ITALY

Telephone: (39) 95330533

Email:

Facsimile: (39)

Telex: 970359 astret $\mathbf{i}$

CATALANO SANTO DR, IST DI ASTRONOMIA, CITTA UNIVERSITARIA, VIA A DORIA G, I 95125 CATANIA, ITALY

Telephone: (39) 95330533

Facsimile: (39)

Telex: 970359 astret $i$

Email:

Commission(s): $16,29,36,42$

CATARZI MARCO DR, OSS ASTROPISICO, DI ARCETRI, LARGO E FERMI 5, I 50125 FIRENZE, ITALY

Telephone: (39) 554378540

Facsimile: (39)

Telex: 572268 arcetr $i$

Email: 38954::catarzi

Commission(s): 40

CATCHPOLE ROBIN M DR, ROYAL GREENWICH OBS, MADINGLEY RD, CAMBRIDGE CB3 OEZ, UK

Telephone: (44) 1223374000

Facsimile: (44) 1223374700

Telex: $26541 / 265871$

Email: cathpole@uk.ac.cam.ast-star

Commission(s): 27,29

CATO B TORGNY DR, NORDISK TELESATELLITSTAT, BOX 107, 5457 00 TANUMSHEDE, SWEDEN

Telephone: (46) $52529155 \quad$ Facsimile: (46)

Email:

Commission(s):

Telex: 20164 nordsat s

CATON DANIEL B DR, ASSISTANT PROFESSOR, PHYSICS AND ASTRONOMY, APPALACHIAN STATE UNIV BOONE NC 28608, USA

Telephone: (1) 7042622446

Facsimile: (1)

Telex: 888370 or 62671500

Email: bitnet:catondb@appstate

Commission(s):

CATURA RICHARD C DR, LOCKHEED PALO ALTO RES LB, DPT 9120 BLDG 255, 3251 HANOVER ST

PALO ALTO CA 94304, USA

Telephone: (1) 4158584066

Facsimile: (1)

Commission(s): 44

Telex: 346409 Imsc suvl

Email:

CAUGHLAN GEORGEANNE R, DPT OF PHYSICS, MONTANA STATE UNIVERSITY, BOZEMAN MT 59717, USA

Telephonc: (1) 4069946170

Facsimile: (1)

Commission(s): 35,44

Telex:

Email:

CAVALIERE ALFONSO G PROF, DPT DI ASTROFISICA, II UNIVERSITA DI ROMA, VIA ORAZIO RAIMONDO I 00173 ROMA, ITALY

Telephone: (39)

Facsimile: (39)

Telex:

Email:

Commission(s): 44,47 
CAVALLINI FABIO, OSS ASTROFISICO, DI ARCETRI, LARGO E FERMI 5, I 50125 FIRENZE, ITALY

Telephone: (39) 554378540

Facsimile: (39) 55435939

Telex: 572268

Email:

Commission(s): 12

CAWLEY MICHAEL DR, DPT OF PHYSICS, ST PATRICK'S COLLEGE, MAYNOOTH, CO KILDARE, IRELAND
$\begin{array}{ll}\text { Telephone: (353) } 1285 \text { 222*X499 } & \text { Facsimile: (353) } \\ \text { Email: bitnet:mcawley@vax1.may.ie } & \text { Commission(s): }\end{array}$

CAYREL DE STROBEL GIUSA, OBSERVATOIRE DE PARIS, SECTION DE MEUDON F 92195 MEUDON PPL CDX, FRANCE

Telephone: (33) 145077863

Facsimile: (33) Telex:

Email:

Commission(s): 29,36

CAYREL ROGER DR, OBSERVATOIRE DE PARIS, 61 AVE OBSERVATOIRE, F 75014 PARIS, FRANCE

Telephone: (33) 140512251

Facsimile: (33)

Commission(s): $29,36,50$

Telex: 270776 obs f

Email:

CAZENAVE ANNY DR, CNES/GRGS, 18 AVE E BELIN, F 31055 TOULOUSE CDX, FRANCE

Telephone: (33) $61274011 \quad$ Facsimile: (33)

Email:

Commission(s):

Telex: $\mathbf{5 3 1 0 8 1}$

CAZZOLA PAOLO DR, OSS ASTRONOMICO DI PADOVA, VIC DELL OSSERVATORIO S, I 35122 PADOVA, ITALY Telephone: (39) 49661499

Facsimile: (39)

Telex:

Email:

Commission(s):

CEFOLA PAUL J DR, MAIl STATION 64, C S DRAPER LAB, 555 TECHNOLOGY SQ, CAMBRIDGE MA 02139, USA

Telephone: (1) 6172581787

Facsimile: (1)

Telex:

Email:

Commission(s): 07

CELIS LEOPOLDO DR, DPT DE ASTRONOMIA, UNIVERSIDAD CATOLICA, CASILLA 6014, SANTIAGO, CHILE

Telcphone: (56) 25522375

Facsimile: (56) 25525692

Telex: $\mathbf{4 4 0 0 0 1}$

Email:

Commission(s): 25,45

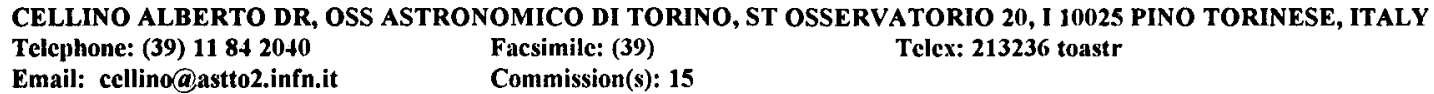

CELNIKIER LUDWIK DR, OBSERVATOIRE DE PARIS, SECTION DE MEUDON, F 92195 MEUDON PPL CDX, FRANCE

Telephone: (33) $145077410 \quad$ Facsimile: (33)

Email:

Commission(s):

Telex: 201571

CENTRELLA JOAN M DR, DPT OF PHYSICS, DREXEL UNIVERSITY, PHILADELPHIA PA 19104, USA

Telephonc: (1) $2158952715 \quad$ Facsimile: (1)

Telex:

Email:

Commission(s):

CENTURION MARTIN MIRIAM, IAC, C/ VIA LACTEA S/N, E 38200 LA LAGUNA, SPAIN

Telephone: (34) 22605200

Email: mcm@iac.es

Facsimile: (34) $22605210 \quad$ Telex: 92640 iac c

Commission(s): 34

CEPA JORDI DR, INST DE ASTROFISICA, DE CANARIAS, OBS DEL TEIDE, E 38200 LA LAGUNA, SPAIN

Telephone: (34) 22262211

Facsimile: (34) 22263005

Telex: $926+0$ iac c

Email: iac::jen

Commission(s): 28

CEPLECHA ZDENEK DR, ASTRONOMICAL INSTITUTE, CZECH ACADEMY OF SCIENCES

ONDREJOV OBSERVATORY, CZ 251 65 ONDREJOV, CZECH R

Telephone: (42) 204 857155/7111

Email: ceplecha@asu.cas.cz Commission(s): 15,22C

Telex: 121579 astr c

CEPPATELLI GUIDO DR, OSS ASTROFISICO, DI ARCETRI, LARGO E FERMI 5 , I 50125 FIRENZE, ITALY

Telephone: (39) 554378540

Facsimile: (39) $55+35939$

Telex: 572268

Email:

Commission(s): 12

CERNICHARO JOSE DR, CTR ASTRON DE YEBES, OAN, APD 148, E 19080 GUADALAJARA, SPAIN

Telephone: (34) 11290311

Facsimile: (34)

Telex: 58279508 iram

Email:

Commission(s): 34,40 
CERNIS KAZIMIERAS, INST THEORETICAL PHYSICS, \& ASTRONOMY, GOSTAUTO 12, VILNIUS 2600, LITHUANIA Telephone: (370) 2613440 Facsimile: (370) 2224694 Telex:

Email: astro@itpa.fi.lt Commission(s):

CERRONI PRISCILLA DR, IAS, REPARTO DI PLANETOLOGIA, VIA DELL'UNIVERSITA 11, I 00185 ROMA, ITALY
$\begin{aligned} & \text { Telephone: (39) } 64956951 \\ & \text { Facsimile: (39) } 64454969\end{aligned}$ Telex: $610261 \mathrm{cnr}$ fra

Email: bitnet:carusi@irmunisa

Commission(s): 15

CERRUTI-SOLA MONICA, OSS ASTROFISICO, DI ARCETRI, LARGO E FERMI 5, I 50125 FIRENZE, ITALY

Tclephone: (39) 554378540

Facsimile: (39) 55435939

Telex: 572268 arcetr $i$

Email:

Commission(s): 34

CERSOSIMO JUAN CARLOS DR, DPT OF PHYSICS/ELECTRONIC, UNIVERSITY OF PUERTO RICO, CUH STATION

HUMACAO PR 00791, USA

Telcphone: (1) 8098509381

Facsimile: (1) $8098524638 \quad$ Telex:

Email:

Commission(s): 34

CESARSKY CATHERINE J DR, CEA CEN, DPHG/SAP, BP 2, F 91191 GIF/YVETTE CDX, FRANCE
Telephone: (33) 169083912
Facsimile: (33)

Email:

Commission(s): $34,33 C, 44 C$

CESARSKY DIEGO A DR, INSTITUT D'ASTROPHYSIQUE, 98BIS BD ARAGO, F 75014 PARIS, FRANCE

Telephone: (33) 144328000

Facsimile: (33) 144328001 Telex:

Email:

Commission(s): 33,34

CESTER BRUNO PROF, OAT, BOX SUCC TRIESTE 5, VIA TIEPOLO 11, I 34131 TRIESTE, ITALY

Telephone: (39) $40793921 * 221 \quad$ Facsimile: (39) Telex: 461137 oat i

Email:

Commission(s): $26,42,45$

CEVOLANI GIORDANO, FISBAT, CNR, VIA CASTAGNOLI 1, I 40126 BOLOGNA, ITALY

Telephone: (39) 5123 9593/94 Facsimile: (39) Telex: 511350

Email:

Commission(s): 22

CHA DU JIN, PYONGYANG ASTRON OBS, ACADEMY OF SCIENCES DPRK, TAESONG DISTRICT, PYONGYANG, KOREA DPR

Telephone: (850)

Facsimile: (850)

Telex:

Email:

Commission(s): 08

CHA GI UNG, PYONGYANG ASTRON OBS, ACADEMY OF SCIENCES DPRK, TAESONG DISTRIC

PYONGYANG, KOREA DPR

Telephone: (850)

Facsimile: (850)

Telex:

Email:

Commission(s):

CHAFFEE FREDERIC H DR, MULT MIRROR TELESCOPE OBS, UNIVERSITY OF ARIZONA, TUCSON AZ 85721, USA

Telephone: (1)

Facsimile: (1)

Telex:

Email:

Commission(s):

CHAISSON ERIC J PROF, STSCI, HOMEWOOD CAMPUS, 3700 SAN MARTIN DR, BALTIMORE MD 21218, USA

Telephone: (1) 3013384757

Facsimile: (1)

Telex: 6849101

Email:

Commission(s): $\mathbf{5 1}$

CHAKRABARTI SANDIP K DR, TIFR, HOMI BHABHA RD, COLABA, BOMBAY 400 005, INDIA

Telephonc: (91) $222152971 * 305$

Facsimile: (91) 222152110

Telex: 01183009 tifr in

Email: chakraba@tifrvax.bitnet

Commission(s): 28C,44

CHAKRABORTY DEO K DR, DPT OF PHYSICS, UNIVERSITY OF RAVISHANKAR, RAIPUR 492 010, INDIA

Telephonc: (91) 27064

Facsimile: (91)

Tclex:

Email:

Commission(s): 44

CHALABAEV ALMAS DR, OHP, F 04870 S MICHEL OBS, FRANCE

Telephone: (33) 92766368

Facsimilc: (33)

Email: chalabacv@froni51

Commission(s): 28

Telex: $\mathbf{4 1 0 6 9 0 ~ o h p ~ f ~}$

CHAMARAUX PIERRE DR, OBSERVATOIRE DE PARIS, SECTION DE MEUDON, F 92195 MEUDON PPL CDX, FRANC

Telephonc: (33) 145077594

Facsimile: (33)

Email:

Commission(s): 28

Tclex: 270912 
CHAMBE GILBERT, OBSERVATOIRE DE PARIS, SECTION DE MEUDON, DASOP F 92195 MEUDON PPL CDX, FRANCE

Telephone: (33) 145347793

Facsimile: (33) Telex:

Email:

Commission(s): 10,12

CHAMBERLAIN JOSEPH M DR, ADLER PLANETARIUM, 1300 S LAKE SHORE DR, CHICAGO IL 60605, USA

Telephone: (1) 3123220325

Facsimile: (1)

Telex:

Email:

Commission(s): $08,31,46$

CHAMBERLAIN JOSEPH W PROF, DPT SPACE PHYS \& ASTRON, RICE UNIVERSITY, HOUSTON TX 77001, USA

Telephone: (1) 7135278101

Facsimile: (1)

Telex: 556457

Email:

Commission(s): $16,21,49$

GHAMBLISS CARLSON R DR, DPT PHYSICAL SCIENCES, KUTZTOWN UNIVERSITY, KUTZTOWN PA 19530, USA

Telephone: (1) 2156834439

Facsimile: (1)

Telex:

Email:

Commission(s): 42

CHAN KWING LAM, APPLIED RESEARCH CORP, 8201 CORPORATE DR, SUITE 920, LANDOVER MD 20785, USA Telephone: (1) 3014598442

Facsimile: (1)

Telex:

Email:

Commission(s): $12,35,36,40$

CHAN ROBERTO DR, OBSERVATORIO NACIONAL, RUA GL J CRISTINO 77, SAO CRISTOVAO 20921 RIO DE JANEIRO RJ, BRAZIL

Telephone: (55) 215807313

Facsimile: (55) 215800332

Telex: 02121288

Email: userchan@inccvm

Commission(s): 35

CHAN SIU KUEN JOSPHINE, MAX PLANCK GESELLSCHAFT, AG STAUB IN SEG, SCHILLERGAESSCHEN 3 D 07745 JENA, GERMANY

Telephone: (49) 364155593

Email: sjchan@fred.astro-uni-jena.de

Facsimilc: (49) $36+155594$

Telex: 331506 uni d

CHANCE KELLY V, ATOMIC \& MOLECULAR PHYS D, HARVARD SMITHSONIAN CTR, 60 GARDEN ST CAMBRIDGE MA 02138, USA

Telephone: (1) 6174957389

Facsimile: (1) $6174957389 \quad$ Telex:

Email: kelly@ecfa.harvard.cdu

Commission(s): 14

CHANDRA SUBHASH, MIS PHILIPS LABS, 345 SCARBOROUGH RD, BRIAR CLIFF NY 10510, USA

Telephone: (1)

Facsimile: (1)

Telex:

Email:

Commission(s):

CHANDRA SURESH DR, DPT OF PHYSICS, UNIVERSITY OF GORAKHPUR, GORAKHPUR 273 009, INDIA

Telephone: (91)

Facsimile: (91)

Telex:

Email:

Commission(s): 10,3t

CHANDRASEKHAR S PROF, ASTROPHYS \& SPACE RES LAB, UNIVERSITY OF CHICAGO, 933 E 56TH ST

CHICAGO IL 60637, USA

Telephone: (1) 3129627860

Email:

Facsimile: (1)

Telex:

Commission(s): 35,44

CHANDRASEKHAR T DR, PHYSICAL RESEARCH LAB, NAVRANGPURA, AHMEDABAD 380 009, INDIA

Telephone: (91) 272462129

Email:

Facsimile: (91) $272+45292 \quad$ Telex: 121397 prl in

Commission(s): 15

CHANG KYONGAE DR, DPT PHYSICS \& OPTICS, CHUNGJU UNIVERSITY, CHUNGJU, KOREA R

Telephone: (82)

Facsimile: (82)

Telex:

Email:

Commission(s): 47

CHANMUGAM GANESAR PROF, DPT PHYSICS \& ASTRONOMY, LOUISIANA STATE UNIY

BATON ROUGE LA 70803 4001, USA

Telephone: (1) 5043886894

Facsimile: (1)

Telex:

Email:

Commission(s): 42

CHAOUDHURI ARNAB DR, DPT OF PHYSICS, INDIAN INSTITUTE SCIENCE, BANGALORE 560012, INDIA

Telephone: (91)

Facsimile: (91)

Telex:

Email:

Commission(s): 10 
CHAPMAN CLARK R DR, PLANETARY SCIENCE INST, 620 N SLXTH AVE, TUCSON AZ 857058331 , USA

Telephone: (1) 5206626300

Facsimile: (1) 5206228060

Telex:

Email:

Commission(s): 15,16

CHAPMAN GARY A DR, DPT PHYSICS \& ASTRONOMY, SAN FERNANDO OBSERVATORY

CALIFORNIA STATE UNIV., NORTHRIDGE CA 91330, USA

Telephone: (1) 8188852775

Facsimile: (1)

Telex:

Email:

Commission(s): 10,12

CHAPMAN JESSICA DR, CSIRO, AUSTRALIAN TELESCOPE, BOX 296, EPPING NSW 2121, AUSTRALIA

Telephone: (61) 28680222

Email: jchapman@atnf.csiro.au

Facsimile: (61) 28680310

Telex: 26230 astro aa

CHAPMAN ROBERT D DR, 10976 SWANFIELDS RD, COLUMBIA MD 21044, USA

Telephone: (1)

Facsimile: (1) Telex:

Email: champ::chapman

Commission(s): $15, \mathbf{4 2 , 4 4}$

CHAPRONT JEAN DR, BUREAU DES LONGITUDES, 77 AVE DENFERT ROCHEREAU, F 75014 PARIS, FRANCE

Telephonc: (33) 140512271

Facsimile: (33)

Commission(s): 04C,07

Telex:

Email:

mission(s): $04 \mathrm{C}, 07$

CHAPRONT-TOUZE MICHELLE, BUREAU DES LONGITUDES, 77 AVE DENFERT ROCHEREAU F 75014 PARIS, FRANCE

Telephone: (33) 140512266

Facsimile: (33)

Telex:

Email:

Commission(s): 04,07,20

CHARLES PHILIP ALLAN, DPT OF ASTROPHYSICS, UNIVERSITY OF OXFORD, SOUTH PARKS RD OXFORD OX1 3RQ, UK

Telephone: (44) 1865511336 \$506

Email:

Facsimile: (44)

Commission(s): 44

Telex: 83295 nuclox

CHARLOT PATRICK, FRANCE

Telephone: (33)

Email:

Facsimile: (33) Telex:

Commission(s):

CHASSEFIERE ERIC, SERVICE D'AERONOMIE, BP 3, F 91371 VERRIERES BUISSON, FRANCE

Telephone: (33) $164474211 \quad$ Facsimile: (33)

Email: $\quad$ Commission(s): 49

Telex: 602400

CHATTERJEE TAPAN K DR, FAC DE CIENCIAS, UNIVERISDAD A PUEBLA, APDO POSTAL 1316

72000 PUEBLA PUE, MEXICO

Telephone: (52) 9122330455

Email:

Facsimile: (52) 9122448947 Telex:

Commission(s): 28

CHAU WAI Y PROF, DPT OF PHYSICS, QUEEN'S UNIVERSITY, KINGSTON ON K7L 3N6, CANADA

Telephone: (1) 6135473526

Facsimile: (1) $6135456463 \quad$ Telex:

Email:

Commission(s):

CHAUBEY UMA SHANKAR DR, UTTAR PRADESH STATE, OBSERVATORY, PO MANORA PEAK 263 129NAINITAL 26 129, INDIA

Telephone: (91) 2136/2583

Facsimile: (91)

Telex:

Email:

Commission(s): 42

CHAUVINEAU BERTRAND, OCA CERGA, AVE COPERNIC, F 06130 GRASSE, FRANCE

Telephone: (33)

Email:

Facsimile: (33)

Telex:

CHAVARRIA-K CARLOS, INSTITUTO DE ASTRONOMIA, UNAM, APDO POSTAL 70-264, 04510 MEXICO DF, MEXICO

Telephone: (52)

Email:

Facsimile: (52)

Commission(s): 37

Telex:

ChAVIRA ENRIQUE SR, INAOE, TONANTZINTLAZ, APDO POSTAL 216 y 51, 72000 PUEBLA PUE, MEXICO

Telephone: (52) 9122470500

Facsimile: (52)

Email:

Commission(s): 27

Telex: 
CHECHETKIN VALERIJ M DR, INST OF APPLIED MATHS, ACADEMY OF SCIENCES, MIUSSKAJA SQ 4 125047 MOSCOW, RUSSIA

Telephone: (7) 2513739

Facsimile: (7)

Telex:

Email:

Commission(s): 35,44

CHELLI ALAIN, INSTITUTO DE ASTRONOMLA, UNAM, APDO POSTAL 70-264, 04510 MEXICO DF, MEXICO

Telephone: (52) $9055483712 * 5306$

Facsimile: (52)

Email:

Commission(s): 09

Telex: 1760155

CHEN BIAO, PURPLE MOUNTAIN OBSERV, CAS, NANJING, CHINA PR

Telephone: (86) 2546700

Facsimile: (86) 25301459

Telex: $3 \$ 144$ pmonj cn

Email:

Commission(s): 10,12,49

CHEN DAO-HAN, PURPLE MOUNTAIN OBSERV, CAS, NANJING, CHINA PR

Telephone: (86) 2531096

Facsimile: (86) 25301459

Telex: $3+144$ pmonj cn

Email:

Commission(s): 15,16

CHEN HONGSHENG, BEIJING ASTRONOMICAL OBS, CAS, W SUBURB, BEIJING 100080, CHINA PR

Telephone: (86)

Facsimile: (86)

Telex: 9053

Email:

Commission(s): 40

CHEN JIAN-SHENG, BEIJING ASTRONOMICAL OBS, CAS, W SUBURB, BEIJING 100080, CHINA PR

Telephone: (86)

Email:

Facsimile: (86)

Commission(s): $28 \mathrm{C}, 47$

Telex: 22040 baoas en

CHEN KWAN-YU PROF, DPT OF ASTRONOMY, UNIVERSITY OF FLORIDA, 211 SSRB, GAINESVILLE FL 32611, USA

Telephone: (1) 9043922055

Facsimile: (1)

Telex:

Email:

Commission(s): 42

CHEN PEISHENG, YUNNAN OBSERVATORY, CAS, BOX 110, KUNMING 72946 YUNNAN, CHINA PR

Telephone: (86) 8712035

Facsimile: (86)

Commission(s): 36

Telex:

Email:

CHEN WEN PING, INST PHYSICS \& ASTRONOMY, NTL CENTRAL UNIVERSITY, CHUNG LI TAIWAN, CHINA R

Telephone: (886) 34223424

Facsimile: (886) 34262304

Telex:

Email: when@phyast.phy.ncu.edu.tw Commission(s):

CHEN XIAO-ZHONG, BEIJING PLANETARIUM, 138 XI WAI ST, BEIJING, CHINA PR

Telephone: (86) 1893003

Facsimile: (86)

Telex:

Email:

Commission(s):

CHEN ZHEN, PURPLE MOUNTAIN OBSERV, CAS, NANJING, CHINA PR

Telephone: (86) 2546700

Facsimile: (86) 25301450

Telex: 34144 pmonj cn

Email:

Commission(s): 07,26,33

CHEN ZHENCHENG, BEIJING ASTRONOMICAL OBS, CAS, W SUBURB, BEIJING 100080, CHINA PR

Telephone: (86) 1281698

Facsimile: (86)

Commission(s): 10,28

Telex: 9053

Email:

CHENG CHUNG-CHIEH DR, NAVAL RESEARCH LABORATORY, CODE 4175CC, 4555 OVERLOOK AVE SW, WASHINGTON DC 20375 5000, USA

Telephone: (1) 2027672350 .

Facsimile: (1)

Telex:

Email:

Commission(s): 10,12

CHENG FU-HUA, CFA, UNIV SCIENCE \& TECHNOLOGY, HEFEI 230026 ANHUI, CHINA PR

Telephone: (86) 551331134 *526 Facsimile: (86)

Email:

Commission(s): 47

Telex: 90028 ustc $\mathrm{cn}$

CHENG FU-ZHEN, ASTROPHYSICS DIVISION, UNIV SCIENCE \& TECHNOLOGY, HEFEI 230026 ANHUI, CHINA PR

Telephone: (86) $551331134 * 987$

Facsimile: (86)

Commission(s): 47

Telex: 90028 uste cn

Email:

CHENG KWANG PING, 14204 CASTLE BLVD, SILVER SPTING MD 20904, USA
Telephone: (1) 3012863019
Facsimile: (1) 3012861753

Email: cheng(astars.gsfc.nasa.gov

Commission(s): 34,37 
CHENLI, DPT OF ASTRONOMY, BEIJING NORMAL UNIVERSITY, BEIJING 100875, CHINA PR

Telephone: (86) 1201 2288-2918

Facsimile: (86)

Telex: 222701 bnu cn

Email: zhouzf@bnu.ihep.ac.cn

Commission(s):

CHEREDNICHENKO V I DR, KIEV POLYTECHNICAL INST, 252056 KIEV, UKRAINE

Telephone: (7)

Facsimile: (7)

Telex:

Email:

Commission(s): 15

CHEREPASHCHUK A M PROF, STERNBERG STATE ASTR INST, UNIVERSITETSKIJ PROSP 13 119899 MOSCOW, RUSSIA

Telephone: (7) 951393838

Facsimile: (7)

Telex:

Email:

Commission(s): $27,42,45$

CHERNEGA N A A DR, ASTRONOMICAL OBSERVATORY, KIEV SYAYE UNIVERSITY, OBSERVATORNAYA UL 3 252053 KIEV, UKRAINE

Telephone: (7) 044262391

Email:

Facsimile: (7)

Telex:

Commission(s): 08

$\begin{array}{ll}\text { CHERNIN ARTHUR D, STERNBERG } & \text { STATE ASTR INST, UNIVERSITETSKIJ PROSP 13 } \\ \text { 119899 MOSCOW, RUSSIA } & \text { Facsimile: (7) } \\ \text { Telephone: (7) } & \text { Commission(s): } \\ \text { Email: } & \text { Telex: }\end{array}$

CHERNOV GENNADIJ DR, IZMIRAN, ACADEMY OF SCIENCES, 142092 TROITSK, RUSSIA

Telephone: (7) 3340902

Facsimile: (7) 3340124

Telex: $\mathbf{4 1 2 6 2 3}$ sestp su

Email:

Commission(s): 10

CHERNYKH N S DR, CRIMEAN ASTROPHYS OBS, UKRAINIAN ACAD OF SCIENCE, NAUCHNY 334413 CRIMEA, UKRAINE

Telephone: (7) 432945

Facsimile: (7)

Telex:

Email:

Commission(s): 15,20

CHERTOK ILIA, IZMIRAN, ACADEMY OF SCIENCES, TROITSK, MOSCOW 142092, RUSSIA

Telephone: (7) 0953340902

Facsimile: (7) $0953340124 \quad$ Telex: $412623 \mathrm{scst}$ )

Email: ichertok@lars.izmiran.troitsk.su Commission(s): 10,12

CHERTOPRUD V E DR, HYDROMETEOROLOGICAL CTR, 123376 MOSCOW, RUSSIA

Telephone: (7)

Facsimile: (7)

Telex:

Email:

Commission(s): 10

CHEVALIER CLAUDE DR, OHP, F 04870 S MICHEL OBS, FRANCE

Telephone: (33) $92766368 \quad$ Facsimile: (33)

Email:

Commission(s): 35

Telex: 410690 ohp f

CHEVALIER ROGER A DR, UNIVERSITY STATION, UNIVERSITY OF VIRGINIA, BOX 3818 CHARLOTTESVILLE VA 22903, USA

Telephone: (1) 8049244889

Facsimile: (1) Telex:

Email: rac5x@virginia.edu

Commission(s): 34

CHE-BOHNENSTENGEL ANNE, SUELZBRACKRING 39A, D 21029 HAMBURG, GERMANY

Telephone: (49) $407238550 \quad$ Facsimile: (49)

Telex:

Email:

Commission(s):

CHIAN ABRAHAM CHIAN-LONG, INPE, CP 515, 12200 S JOSE DOS CAMPOS, BRAZIL

Telephone: (55) $123229977 \quad$ Facsimile: (55) $123218743 \quad$ Telex: 011-33530

Email:

Commission(s): 44

CHIKADA YOSHIHIRO DR, NOBEYAMA RADIO OBS, NAOJ, MINAMIMAKI MURA, NAGANO 384 13, JAPAN

Telephone: (81) 267982831

Facsimile: (81)

Telex: 3329005 taonro

Email:

Commission(s): 40

CHINCARINI GUIDO L DR, OSS ASTRONOMICO DI MILANO, VIA E BIANCHI 46, I 22055 MERATE, ITALY

Telephone: (39) 596412

Facsimile: (39)

Telex:

Email:

Commission(s): 28,47 
CHINI ROLF, MPI FUER RADIOASTRONOMIE, AUF DEM HUEGEL 69, D 53121 BONN, GERMANY

Telephone: (49)

Facsimile: (49)

Telex: 886440

Email:

Commission(s): $\mathbf{3 4 , 4 0}$

CHIO CHOL ZONG, PYONGYANG ASTRON OBS, ACADEMY OF SCIENCES DPRK, TAESONG DISTRICT

PYONGYANG, KOREA DPR

Telephone: (850)

Facsimile: (850)

Telex:

Email:

Commission(s): 20

CHIOSI CESARE S DR, DPT DI ASTRONOMIA, UNIVERSITA DI PADOVA, VIC DELL OSSERVATORIO 5

I 35122 PADOVA, ITALY

Telephone: (39) $498293422 / 8293411$

Facsimile: (39) $498759840 \quad$ Telex:

Email: chiosi@astrpd.pd.astro.it

Commission(s): 35P,37

CHIS GHEORGHE DORIN, ASTRONOMICAL OBSERVATORY, STR CIRESILOR 19, R 3400 CLUJ NAPOCA, RUMANIA

Telephone: (40) 64194592

Facsimile: (40) 64194592

Telex:

Email:

Commission(s):

CHISTYAKOV VLADIMIR E DR, USSURIISK SOLAR STATION, PRIMORSKY KRAY 692533 GORNOTAEZHNOE, RUSSIA

Telephone: (7) 91121

Facsimile: (7)

Telex: $21395+$ solnze

Email:

Commission(s): 12

CHITRE DATTAKUMAR M DR, COMPUTER SCIENCES CORP, SYSTEM SCIENCES DIV, 8728 COLESVILLE RD SILVER SPRING MD 20910, USA

Telephone: (1)

Facsimile: (1)

Telex:

Email:

Commission(s): $38 \mathrm{C}, \mathbf{4 7 , 4 9}$

CHITRE SHASHIKUMAR M DR, TIFR, HOMI BHABHA RD, COLABA, BOMBAY 400 005, INDIA

Telephone: (91) 22219111

Facsimile: (91)

Telex: 011-3009 tifr in

Email:

Commission(s): $35,44,49$

CHIU HONG YEE, GSFC, CODE 914, GREENBELT MD 20771, USA

Telephone: (1) 3012868256

Facsimile: (1) 3012861663

Telex:

Email: chiu@oz.gsfc.nasa.gov

Commission(s):

CHIU HONG-YEE DR, MUDD BLDG RM 828, COLUMBIA UNIVERSITY, NEW YORK NY 10027, USA

Telephone: (1)

Facsimile: (1)

Telex:

Email:

Commission(s): 35

CHIU LIANG-TAI GEORGE, IBM, THOMAS J WATSON RES CTR, BOX 218, YORKTOWN HEIGHTS NY 10598, USA

Telephone: (1) 9149452436

Facsimile: (1)

Telex:

Email:

Commission(s): 24

CHIUDERI CLAUDIO PROF, DPT DI ASTRONOMIA, UNIVERSITA DI FIRENZE, LARGO E FERMI 5

I 50125 FIRENZE, ITALY

Telephone: (39) 5527521

Facsimile: (39) 55220039

Telex: 572268 arcetr $i$

Email:

Commission(s):

CHIUDERI-DRAGO FRANCA PR, OSS ASTROFISICO, DI ARCETRI, LARGO E FERMI 5, I 50125 FIRENZE, ITALY

Telephone: (39) 554378540

Email:

Facsimile: (39) 55435939

Telex: 572268 arcetr $i$

CHIUEH TZIHONG DR, INST PHYSICS \& ASTRONOMY, NTL CENTRAL UNIVERSITY, CHUNG LI TAIWAN, CHINA

Telephone: (886) 34227151 *5341 Facsimile: (886) 34251175 Telex:

Email: chiuch@phyast.dnet.ncu.edu.tw Commission(s): 10

CHIUMIENTO GIUSEPPE, OSS ASTRONOMICO DI TORINO, ST OSSERVATORIO 20, 1 10025 PINO TORINESE, ITALY

Telephone: (39) 11841067

Facsimile: (39)

Telex: 213236 toastr i

Email:

Commission(s): 08,19

CHKHIKVADZE IAKOB N, ABASTUMANI ASTROPHYSICAL, OBSERVATORY, GEORGIAN ACAD OF SCIENCES 383762 ABASTUMANI, GEORGIA

Telephone: (7) 278

Facsimile: (7)

Telex: 327409

Emait:

Commission(s): 35 
CHLISTOVSKY FRANCA DR, OSS ASTRONOMICO DI BRERA, VIA BRERA 28, I 20121 MILANO, ITALY

Telephone: (39) 2874444

Facsimile: (39) 272001600

Telex:

Email:

Commission(s): 08

CHMIELEWSKI YVES DR, OBSERVATOIRE DE GENEVE, CHEMIN DES MAILLETTES 51

CH 1290 SAUVERNY, SWITZERLAND

Telephone: (41) 227552611

Email: igor(a)obs.unige.ch

Facsimile: (41) $227553983 \quad$ Telex: 419209 obs ch

Commission(s):

CHO SE HYUNG DR, ISSA, YOOSUNG KOON, DAEJEON 305 348, KOREA R

Tclephone: (82) 428511281

Email:

Facsimile: (82) 428615610 Telex:

Commission(s): 40

CHOCHOL DRAHOMIR, ASTRONOMICAL INSTITUTE, SLOVAK ACADEMY SCIENCES

SK 05960 TATRANSKA LOMNI, SLOVAK R

Telephone: (42) $969967866 / 7 / 8 \quad$ Facsimile: (42) $969967656 \quad$ Telex: 78277

Email:

Commission(s): 42,44

CHOE SEUNG URN DR, DPT OF EARTH SCIENCE \& ED, SEOUL NTL UNIVERSITY, SINLIM DONG GWANG GU SEOUL 151 742, KOREA R

Telephone: (82)

Facsimile: $(\mathbf{8 2})$

Commission(s):

Telex:

Email:

METEOROLOGY, YONSEI UNIVERSITY, SUDAEMUN, SEOUL 120749 , KOREA R

CHOI KYU-HONG, DPT ASTRON \& METEOROLOGY,
Telephone: (82) 23920131 Facsimile: (82) Telex:

Email: $\quad$ Commission(s): 07,42

CHOI WON CHOL, PYONGYANG ASTRON OBS, ACADEMY OF SCIENCES DPRK, TAESONG DISTRICT

PYONGYANG, KOREA DPR

Telephone: (850) $53134 / 5 \& 53239 \quad$ Facsimile: $(850)$

Telex:

Email:

Commission(s):

CHOLLET FERNAND DR, OBSERVATOIRE DE PARIS, 61 AVE OBSERVATOIRE, F 75014 PARIS, FRANCE

Telephone: (33) 140512205

Facsimile: (33)

Telex: 270776 obs f

Email:

Commission(s): 04,08

CHOLONIEWSSKI JACEK DR, ASTRONOMICAL OBSERVATORY, WARSAW UNIVERSITY, AL UJAZDOWSKIE 4

PL 00478 WARSAW, POLAND

Telephone: (48) $294011 \quad$ Facsimile: (48)

Email: jch@plwauw61.bitnet

Commission(s):

Telex: 817063 oauw pl

CHOPINET MARGUERITE DR, 57 RUE THIERS, F 92100 BOULOGNE, FRANCE

Telephone: (33) $147611144 \quad$ Facsimile: (33) Telex:

Email:

Commission(s): 34

CHOU CHIH-KANG DR, INST PHYSICS \& ASTRONOMY, NTL CENTRAL UNIVERSITY, CHUNG LI TAIWAN

CHINA R

Telephone: (886) 34251175

Facsimile: (886)

Commission(s): 28,49

Telex:

Email:

CHOU DEAN-YI DR, DPT OF PHYSICS, NTL TSING HUA UNIVERSITY, HSIN CHU 300043, CHINA R

Telephone: (886)

Facsimile: (886)

Telex:

Email: dychou@twnctu01

Commission(s):

CHOU KYONG CHOL PROF, KOREAN SPACE \& ENVIRONM, 1402 LIFE OFFICETEL BLDG

TOIDO DONG YONGDONGPO KU, SEOUL 150 010, KOREA R

Telephone: (82) 27610031

Facsimile: (82) $276 \mathbf{1 0 0 3 2}$

Telex:

Email:

Commission(s): $\mathbf{5 1}$

CHOUDHARY DEBI PRASAD, UDAIPUR SOLAR OBSERVATORY, 11 VIDYA MARG, UDAIPUR 313 001, INDIA

Telephone: (91) 294560626

Facsimile: (91) 294525959

Telex: 0335223 uso in

Email: uso@prl.ernet.in

Commission(s): 09,10

CHRISTENSEN PER R DR, NIELS BOHR INSTITUTE, BLEGDAMSVEJ 17, DK 2100 COPENHAGEN 0, DENMARK

Telephone: (45) 31421616

Facsimile: (45) 31389157

Telex: $15216 \mathrm{nbi} \mathrm{dk}$

Email: perrex@nbivax.nbi.dk

Commission(s): 
M 478

CHRISTENSEN-DALSGAARD J, INST OF PHYSICS \& ASTRON, UNIVERSITY OF AARHUS, NY MUNKEGADE DK 8000 AARHUS C, DENMARK

Telephone: (45) 86128899

Facsimile: (45) 86202711

Email:

Commission(s): 12,27C,35

Telex: $6+767$ aausci $\mathrm{dk}$

CHRISTIAN CAROL ANN, CTR FOR EUV ASTROPHYSICS, UNIVERSITY OF CALIFORNIA, 2150 KITTREDGE ST BERKELEY CA 94720, USA

Telephone: (1)

Facsimile: (1)

Telex:

Email:

Commission(s): 37

CHRISTIANSEN WAYNE A, DPT PHYSICS \& ASTRONOMY, UNIVERSITY NORTH CAROLINA

204 PHILLIPS HALL O39A, CHAPEL HILL NC 27514, USA

Tclephone: (1) 9199623011

Facsimile: (1)

Telex:

Email:

Commission(s): 40

CHRISTIANSEN WILBUR PROF, 42 THE GRANGE, 67 MAC GREGOR ST, DEAKIN ACT 2600, AUSTRALIA

Tclephone: (61) 062815576

Facsimile: (61)

Telex:

Email:

Commission(s): 40

CHRISTODOULOU DMITRIS DR, STEWARD OBSERVATORY, UNIVERSITY OF ARIZONA, TUCSON AZ 85721, USA

Telephone: (1) 5206212288

Email: bitnet:phchri@Isumvs

Facsimile: (1) 5206211532

Telex: 467175

Commission(s): 33

CHRISTY JAMES WALTER DR, HUGHES AIRCRAFT CO, 1720 W NIONA PL, TUCSON AZ 85704, USA

Telephone: (1) 5202971377

Facsimile: (1)

Telex:

Email:

Commission(s): $09,24,45$

CHRISTY ROBERT F DR, CALTECH, MS 105 24, PALOMAR OBS, PASADENA CA 91125, USA

Telephone: (1) 2137956811

Facsimilc: (1)

Telex:

Email:

Commission(s): 27,35

CHU HAN-SHU PROF, PURPLE MOUNTAIN OBSERV, CAS, NANJING, CHINA PR

Telephone: (86) 25301096

Facsimile: (86) 25301459

Telex: 34144 pmonj en

Email:

Commission(s): 40

CHU YAOQUAN, ASTROPHYSICS DIVISION, UNIV SCIENCE \& TECHNOLOGY, HEFEI 230026 ANHUI, CHINA PR

Telephone: (86) 551331134

Facsimile: (86) 551331760

Telex: 90028 ustc cn

Email:

Commission(s): $05,28,47$

CHU YOU-HUA, DPT OF ASTRONOMY, UNIVERSITY OF ILLINOIS, 1011 W SPRINGFIELD AVE

URBANA IL 61801, USA

Telephone: (1) 2173335535

Facsimile: (1)

Telex:

Email:

Commission(s): 34

CHUBB TALBOT A DR, 5023 N 38TH ST, ARLINGTON VA 22207, USA

Telephone: (1)

Facsimile: (1)

Commission(s): 44

Telex:

Email:

CHUGAI NIKOLAI N DR, INST OF ASTRONOMY, ACADEMY OF SCIENCES, PYATNITSKAYA UL 48, 109017 MOSCOW, RUSSIA

Telephone: (7) 952312129

Facsimile: (7)

Telex: 411576 ascon su

Email:

Commission(s): 28,36

CHUGAJNOV P F DR, CRIMEAN ASTROPHYS OBS, UKRAINIAN ACAD OF SCIENCE, NAUCHNY

334413 CRIMEA, UKRAINE

Tclephone: (7) 432945

Facsimile: (7)

Telex:

Email:

Commission(s): 25,27

CHUN MUN-SUK DR, DPT ASTRON \& METEOROLOGY, YONSEI UNIVERSITY, SUDAEMUN

SEOUL 120749 , KOREA R

Telephone: (82) 23920131

Facsimile: (82)

Commission(s): 37

Telex:

Email:

CHUNG HYUN SOO, KOREA ASTRONOMY OBS, 361 WHAAMDONG, YUSEONGGU, TAEJON 305 348, KOREA R

Telephone: (82)

Facsimilc: (82)

Email: hschung@hanul.issa.re.kr

Commission(s):

Telex: 
CHUPP EDWARD L DR, DPT OF PHYSICS, UNIV OF NEW HAMPSHIRE, DEMERITT HALL, DURHAM NH 03824, USA

Telephone: (1) 6038622750

Facsimile: (1)

Telex: 950030

Email: solmax::chupp

Commission(s): 10,44

CHURCHWELL EDWARD B DR, WASHBURN OBSERVATORY, UNIVERSITY OF WISCONSIN, 475 N CHARTER ST MADISON WI 53706, USA

Telephone: (1) 6082627857

Facsimile: (1)

Telex: 265452 uofwisc mds

Email:

Commission(s): 33,34

CHUVAEV K K DR, CRIMEAN ASTROPHYS OBS, UKRAINLAN ACAD OF SCIENCE, NAUCHNY

334413 CRIMEA, UKRAINE

Telephone: (7) 432945

Facsimile: (7)

Commission(s): 28

Telex:

Email:

\section{Comich INSTIT)}

CHVOJKOVA WOYK E DR, ASTRONOMICAL INSTITUTE, CZECH ACADEMY SCIENCES, BOCNI II 1401

CZ 14131 PRAHA 4, CZECH R

Telephone: (42)

Email:

Facsimile: (42)

Commission(s): 12

Telex:

CIATTI FRANCO DR, OSSERVATORIO ASTROFISICO, VIA DELL OSSERVATORIO 8, I 36012 ASIAGO, ITALY

Telephone: (39) $42462665 \quad$ Facsimilc: (39)

Email:

Commission(s):

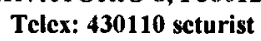

CID PALACIOS RAFAEL PROF, DPT DE ASTRONOMLA, UNIVERSIDAD DE ZARAGOZA, E 50009 ZARAGOZA, SPAIN Telephone: (34) 357011

Facsimile: (34)

Email:

Commission(s): 07

Telex:

CIDALE Lydia SONLA, OBSERVATORIO ASTRONOMICO, PASEO DEL BOSQUE S/N

1900 LA PLATA (BS AS), ARGENTINA

Telephone: (54) 21217308

Email: lydia@fcaglp.cdu.ar

Facsimile: (54) $21211761 \quad$ Telex:

Commission(s): 29,36

CILLIE G G PROF, 4 MINSERIE ST, STELLENBOSCH 7600, SOUTH AFRICA

$\begin{array}{lll}\text { Telcphone: (27) 02231-3515 } & \text { Facsimile: (27) } & \text { Telex: } \\ \text { Email: } & \text { Commission(s): } 42\end{array}$

CIURLA TADEUSZ, ASTRONOMICAL INSTITUTE, WROCLAW UNIVERSITY, UL KOPERNIKA 11

PL 51622 WROCLAW, POLAND

Telephone: (48)

Facsimilc: (48)

Commission(s): 33

Telex:

Email:

Commission(s). 33

CLAIREMIDI JACQUES DR, OBSERVATOIRE DE BESANCON, 41 BIS AVE OBSERVATOIRE

F 25000 BESANCON CDX, FRANCE

Telephone: (33) 81666900

Email: clairemi@frobes51

Facsimile: (33)

Commission(s): $15,16,21$

Telex: $361144 \mathrm{f}$

CLARIA JUAN DR, OBSERVATORIO ASTRONOMICO, DE CORDOBA, LAPRIDA 854, 5000 CORDOBA, ARGENTINA

Telc|phone: (54) 51230491

Email: claria@astro.cdu.ar

Facsimile: (54) 51210613

Commission(s): $37,42,45,47$

Telex: 51822 bucor ar

CLARK ALFRED JR PROF, DPT MECH \& AEROSPACE SCI, UNIVERSITY OF ROCHESTER ROCHESTER NY 14627, USA

Telephone: (1)

Facsimile: (1)

Commission(s):

Telex:

Email:

CLARK BARRY G DR, NRAO, VLA, BOX 0, SOCORRO NM 87801 0387, USA
Telephone: (1) 5057724011
Email:
Facsimile: (1)
Commission(s): 40
Telex: 9109881710

CLARK DAVID H DR, SCIENCE DIV, SCI \& ENGINEER RES COUNCIL, NORTH STAR AVE, SWINDON SN2 1ET, UK Telephone: (44) 179326222 Facsimile: (44)

Email:

Commission(s): 40

Telex: 449466 
M 480

CLARK FRANK OLIVER DR, DPT PHYSICS \& ASTRONOMY, UNIVERSITY OF KENTUCKY

LEXINGTON KY 40506, USA

Telephone: (1) 6062573376

Facsimile: (1)

Telex:

Email:

Commission(s): 34,40

CLARK GEORGE W PROF, CENTER FOR SPACE RESEARCH, MIT RM 37 611, BOX 165, CAMBRIDGE MA 02139, USA

Tclephone: (1) 6172535842

Facsimile: (1)

Telex:

Email:

Commission(s): 44

CLARK THOMAS A DR, NASA GSFC, CODE 974, GREENBELT MD 20771, USA

Telephone: (1) 3012865957

Facsimile: (1)

Telex:

Email:

Commission(s): 51

CLARK THOMAS ALAN DR, DPT OF PHYSICS, UNIVERSITY OF CALGARY, 2500 UNIVERSITY DR NW

CALGARY AB T2N 1N4, CANADA

Telephone: (1) 4032845392

Facsimile: (1) 4032893331

Telex:

Email:

Commission(s): 12,4

CLARKE CATHERINE, INSTITUTE OF ASTRONOMY, THE OBSERVATORIES, MADINGLEY RD

CAMBRIDGE CB3 OHA, UK

Telephone: (44) 1223337535

Facsimile: (44) 1223337523 Telex:

Email: cclarke@)castr.ast.cam.ac.uk

Commission(s):

CLARKE DAVID DR, DPT OF ASTRONOMY, UNIVERSITY OF GLASGOW, GLASGOW G12 8QQ, UK

Telephone: (44) 1413398855

Facsimile: (44)

Telex: 778421

Email:

Commission(s): 09

CLARKE JOHN T, NASA GSFC, CODE 681, HUBBLE SPACE TELESCOPE, GREENBELT MD 20771, USA

Telephone: (1) 3012865781

Facsimile: (1)

Commission(s):

Telex: $710-828-9716$

Email:

CLARKE THOMAS R DR, MCLAUGHLIN PLANETARIUM, ROYAL ONTARIO MUSEUM

100 QUEENS PARK CRESCENT, TORONTO ON M5S 2C6, CANADA

Telephone: (1) $4169988551 \quad$ Facsimile: (1)

Telex:

Email:

Commission(s):

CLAUSEN JENS VIGGO LEKTOR, COPENHAGEN UNIVERSITY OBS, BRORFELDEVEJ 23 DK 4340 TOLLOSE, DENMARK

Telephone: (45) $53488195 \quad$ Facsimile: $(45) 58488755 \quad$ Telex: 44155 danast

Email:

Commission(s): $42 \mathrm{C}$

CLAUSSEN MARK J DR, NAVAL RESEARCH LABORATORY, CODE 4210, 4555 OVERLOOK AVE SW WASHINGTON DC 20375 5000, USA

Telephone: (1) 2027670670

Facsimile: (1)

Commission(s):

Telex:

Email:

CLAVEL JEAN, ESA/ESTEC, SSD, BOX 299, NL 2200 AG NOORDWIJK, NETHERLANDS
Telephonc: (31)

Email:

Commission(s): 28

CLAYTON DONALD D PROF, DPT PHYSICS \& ASTRONOMY, CLEMSON UNIVERSITY, CLEMSON SC 29634 1911, US

Telephone: (1) 8036565299

Facsimile: (1)

Telex:

Email: clayton(a)clemson

Commission(s):

CLAYTON GEOFFREY C DR, CASA, UNIVERSITY OF COLORADO, CAMPUS BOX 389, BOULDER CO 80309, USA

Telephone: (1) 3034924057

Facsimile: (1) $3034927178 \quad$ Telex:

Email: gelayton(a)fenway.colorado.edu Commission(s): 15

CLAYTON ROBERT N DR, ENRICO FERMI INSTITUTE, UNIVERSITY OF CHICAGO, 5640 S ELLIS AVE

CHICAGO IL 60637, USA

Telephone: (1) 3127027777

Email:

Facsimile: (1)

Telex:

Commission(s): 15

CLEGG PETER E DR, DPT OF PHYSICS, QUEEN MARY/WESTFIELD COLL, MILE END RD, LONDON E1 4NS, UK

Telephone: (44) 1719755038

Facsimile: (44) 1719819465

Telex: 893750

Email: pec@star.qmw.ac.uk/19764::pec Commission(s): 
CLEGG ROBIN E S DR, ROYAL GREENWICH OBS, MADINGLEY RD, CAMBRIDGE CB3 OEZ, UK

Telephone: (44) 1223374000

Facsimile: (4) $122337+700$

Telex:

Email:

Commission(s): 34

CLEMENS DAN P DR, DPT OF ASTRONOMY, BOSTON UNIVERSITY, 725 COMMONWEALTH AVE

BOSTON MA 02215, USA

Telephone: (1) 6173536140

Facsimile: (1)

Telex:

Email:

Commission(s): 33,40

CLEMENT MAURICE J PROF, DPT OF ASTRONOMY, UNIVERSITY OF TORONTO, 60 ST GEORGE ST

TORONTO ON MSS 1A1, CANADA

Telephone: (1) 4169784833

Facsimile: (1) 4169783921

Telex: 06986766

Email:

Commission(s):

CLIFTON KENNETH ST, NASA/MSFC, CODE ES 63, HUNTSVILLE AL 35812, USA

Telephone: (1) 2054532305

Facsimile: (1)

Telex: 594416

Email:

Commission(s): 22

CLIMENHAGA JOHN L PROF, DPT PHYSICS \& ASTRONOMY, UNIVERSITY OF VICTORIA, BOX 3055 VICTORIA BC V8W 3P6, CANADA

Telephone: (1) 6047217741

Facsimile: (1) $60+7217715 \quad$ Telex:

Email:

Commission(s): 29

CLINE THOMAS L DR, NASA GSFC, CODE 661, LHEA, GREENBELT MD 20771, USA

Telephone: (1) $3012868375 \quad$ Facsimile: (1)

Email: span:6197::cline Commission(s):

Telex: 89675 nascom gblt

CLIVER EDWARD W, A F GEOPHYSICS LABORATORY, SPACE PHYSICS DIV, HANSCOM AFB BEDFORD MA 01731, USA

Telephone: (1) 6178613975

Email:

Facsimile: (1)

Telex: 928123 afgl hanscom

Commission(s): 10

CLUBE S V M DR, DPT OF PHYSICS, PARTICLE \& NUCLEAR PHYS L, KEBLE RD, OXFORD OX1 3RH, UK

Telephone: (44)

Facsimile: (44)

Telex:

Email:

Commission(s): $15,22,24,33$

CLUTTON-BROCK MARTIN DR, DPT OF MATHS \& ASTRONOMY, UNIVERSITY OF MANITOBA

WINNIPEG MB R3T 2N2, CANADA

Telephone: (1) 2042619255

Facsimile: (1)

Telex:

Email:

Commission(s):

COCHRAN ANITA L DR, ASTRONOMY DPT, UNIVERSITY OF TEXAS, RLM 15 308, AUSTIN TX 78712 1083, USA

Telephone: (1) 5124711471

Facsimile: (1)

Telex: 9108741351

Email: anita@zinfandel.as.utexas.edu

Commission(s): 15,16

COCHRAN WILLIAM DAVID DR, ASTRONOMY DPT, UNIVERSITY OF TEXAS, RLM 15308

AUSTIN TX 78712 1083, USA

Telephone: (1) 5124714461

Email:

Facsimilc: (1)

Telex:

Commission(s): 15,30C

COCKE WILLIAM JOHN PROF, STEWARD OBSERVATORY, UNIVERSITY OF ARIZONA, TUCSON AZ 85721, USA

Telephonc: (1) 5206216540

Facsimile: (1)

Commission(s): 47

Telex:

Email:

CODE ARTHUR D, WASHBURN OBSERVATORY, UNIVERSITY OF WISCONSIN, 475 N CHARTER ST MADISON WI 53706, USA

Telephone: (1) 6082629594

Facsimile: (1)

Telex:

Email:

Commission(s): 34,44

CODINA LANDABERRY SAYD J, OBSERVATORIO NACIONAL, RUA GL BRUCE 586, SAO CRISTOVAO 20921 RIO DE JANEIRO RJ, BRAZIL

Telephone: (55) $215807313 * 267$

Facsimile: (55) 215800332

Tclex: 21288

Email:

Commission(s): 46 
M 482

CODINA VIDAL J M DR, FABRA OBSERVATORY, GRAN VIA DE LOS CORTES, CATALANES 679

E 08013 BARCELONA, SPAIN

Telephone: (34) 32454766

Facsimile: (3t)

Telex:

Email:

Commission(s):

COE MALCOLM, DPT OF PHYSICS, SOUTHAMPTON UNIVERSITY, THE UNIVERSITY

SOUTHAMPTON SO9 5NH, UK

Telephone: (44) 1703592108

Facsimile: (44) $1703585813 \quad$ Telex:

Email: mjc@phastr.soton.ac.uk

Commission(s):

COELHO BALSA MARIO C DR, RUA TRINDADE COELHO 21, 20 DTO, P 3000 COIMBRA, PORTUGAL

Telephone: (351)

Facsimile: (351)

Telex:

Email:

Commission(s):

COFFEEN DAVID L DR, BOX 151, HASTINGS HUDSON NY 10706, USA

Telephone: (1) $9144782594 \quad$ Facsimile: (1) Telex:

Email:

Commission(s):

COFFEY HELEN E MS, NOAA, NGDC E/GC2, 325 BROADWAY, BOULDER CO 80303, USA

Telephone: (1) $3034976223 \quad$ Facsimile: (1)

Email: $\quad$ Commission(s): 10

Telex: 592811 noaa masc bdr

COGAN BRUCE C DR, MOUNT STROMLO \& SIDING, SPRING OBSERVATORIES, PRIVATE BAG WODEN PO ACT 2606, AUSTRALIA

Telcphone: (61) 62881111

Facsimile: (61) 62490233

Telcx: 62270

Email:

Commission(s): 05

COHEN JEFFREY M DR, DPT OF PHYSICS, UNIV OF PENNSYLVANIA, PHILADELPHLA PA 19104, USA

Telephone: (1)

Facsimilc: (1)

Telex:

Email:

Commission(s): $35,44,47$

COHEN JUDITH DR, CALTECH, MS 105 24, PALOMAR OBS, PASADENA CA 91125, USA

Telephone: (1) 8183564005

Facsimile: (1)

Telex:

Email: jlc@deimos.caltech.edu

Commission(s):

COHEN LEON PROF, DPT OF PHYSICS, HUNTERS COLLEGE, 695 PARK AVE, NEW YORK NY 10021, USA

Telephone: (1) 2125705696

Facsimile: (1)

Telex:

Email:

Commission(s):

COHEN MARSHALL H PROF, CALTECH, MS 105 24, PALOMAR OBS, PASADENA CA 91125, USA

Telephone: (1) 2133564000

Facsimile: (1)

Telex: $675+25$

Email:

Commission(s): 34,40

COHEN MARTIN DR, RADIO ASTRONOMY LAB, UNIVERSITY OF CALIFORNIA, GO1 CAMPBELL HALL BERKELEY CA 94720, USA

Telephone: (1) 4156422833

Email:

Facsimile: (1)

Telex: $\mathbf{8 2 0 1 8 1}$ ucb ast ralud

Commission(s): 27

COHEN RAYMOND J DR, NRAL, JODRELL BANK, MACCLESFIELD SK11 9DL, UK

Telephone: (44) $147771321 \quad$ Facsimile: (44) Telex: 36149

Email:

Commission(s): 40

COHEN RICHARD S, INST FOR SPACE STUDIES, 2880 BROADWAY, NEW YORK NY 10025, USA

Telephone: (1) 2126785611

Facsimile: (1)

Telex:

Email:

Commission(s): 09,33,40

COHEN ROSS D DR, CASS, UCSD, C 011, LA JOLLA CA 92093 0216, USA

Telephone: (1) 6195342664

Facsimile: (1)

Telex:

Email:

Commission(s): 28,47

COHN HALDAN N, ASTRONOMY DPT, INDIANA UNIVERSITY, SWAIN WEST 319, BLOOMINGTON IN 47405, USA

Telephone: (1) 8123354174

Facsimile: (1)

Telex:

Email:

Commission(s): 
COLBURN DAVID S DR, $19+4$ WAVERLEY STREET, PALO ALTO CA 9 4301 , USA

Telephone: (1) Facsimile: (1) Telcx:

Email:

Commission(s):

COLE TREVOR WILLIAM PROF, SCHOOL OF ELECTRICAL ENG, UNIVERSITY OF SYDNEY

SYDNEY NSW 2006, AUSTRALIA

Telephone: (61) 26922682

Facsimile: (61)

Telex:

Email:

Commission(s): 40

COLEMAN PAUL HENRY DR, KAPTEYN ASTRONOMICAL INST, BOX 800

NL 9700 AV GRONINGEN, NETHERLANDS

Telejohone: (31) 5063064

Email: bitnet:gruff(a)hgrrugs

Facsimile: (31)

Commission(s): 40

Telex: 53572 stars nl

COLES PETER DR, ASTRONOMY UNIT, QUEEN MARY/WESTFIELD COLL, MILE END RD, LONDON E1 4AS, UK

Telephone: (+4) $1719755+81$ Facsimile: (4t) 1819819587

Telex: 893750

Email: pcolesauk.ac.qmw.starlink Commission(s): 47

COLGATE STIRLING A DR, LOS ALAMOS SCIENTIFIC LAB, MS B275, THEORETICAL DIV

LOS ALAMOS NM 87545, USA

Telephone: (1) 5056672897

Facsimile: (1)

Telex:

Email:

Commission(s):

COLIN JACQUES DR, OBSERVATOIRE DE BORDEAUX, BP 89, F 33270 FLOIRAC, FRANCE

Telephone: (33) 56864330

Facsimile: (33) $56+10+251 \quad$ Telex:

Email:

Commission(s): $28,37,33$

COLIN PEDRO, UNIV NTL AUTONOMA DE MEXI, INSTITUTO DE ASTRONOMIA, AP 70 264 CU

MEXICO CITY (4510, MEXICO

Telephone: (52)

Facsimile: (52)

Telex:

Email:

Commission(s):

COLLADOS MANUEL DR, INST DE ASTROFISICA, DE CANARIAS, OBS DEL TEIDE, E 38200 LA LAGUNA, SPAIN

Telephone: (34) 22262211

Facsimile: (34)

Commission(s): 10,12C

Telex: 92640

Email:

COLLINS GEORGE W II PROF, DPT OF ASTRONOMY, OHIO STATE UNIVERSITY, 174 W 18TH AVE

COLUMBUS OH $\$ 321011106$, USA

Telephone: (1) $61++225467$

Facsimile: (1)

Telex:

Email:

Commission(s): 42

COLLIN-SOUFFRIN SUZY DR, INSTITUT D'ASTROPHYSIQUE, 98BIS BD ARAGO, F 75014 PARIS, FRANCE

Telephone: (33) $14+328078$

Facsimile: (33) 1 \$4328001 Telex:

Email:

Commission(s): 34,44

COLOMB FERNANDO R DR, IAR, CC 5,1894 VILLA ELISA (BS AS), ARGENTINA

Telephone: (54) $2125+909$

Facsimile: (54) 21254909

Telex:

Email: rcolombräirmu.clu.ar

Commission(s): 34,40C,51VP

COLOMBO G PROF DR, IST MECCANICA APPL, UNIVERSITA DI PADOVA, VIA F MARZOLO?

I 35122 PADOVA, ITALY

Telephone: (39) 4966 1499

Email:

Facsimile: (39)

Telex:

Commission(s): 16

COLUZZI REGINA DR, OAR, VIA DEL PARCO MELLINI 84, I 00136 ROMA, ITALY

Telephone: (39) 6 34 7156, Facsimile: (39)

Telex: 626226 oa roma i

Enail:

Commission(s): $(15,29,45$

COMA JUAN CARLOS, REAL INST Y OBSERVATORIO, DE LA ARMADA, CECILIO PUJAZON S/N

E 11110 SAN FERNANDO, SPAIN

Telephone: (34)

Facsimile: $(34)$

Telex:

Email:

Commission(s): 04 
COMBES FRANCOISE DR, OBSERVATOIRE DE PARIS, SECTION DE MEUDON, DEMIRM F 92195 MEUDON PPL CDX, FRANCE

Telephone: (33) $145077898 \quad$ Facsimile: (33)

Commission(s): 34

Telex: 270912

COMBES MICHEL, OBSERVATOIRE DE PARIS, SECTION DE MEUDON, F 92195 MEUDON PPL CDX, FRANCE Telephone: (33) $140512157 \quad$ Facsimile: (33)

Email:

Commission(s): Telex: 201571

COMBI MICHAEL R DR, SPACE PHYSICS RESEARCH LB, UNIVERSITY OF MICHIGAN, 2455 HAYWARD ST ANN ARBOR MI 48109 2143, USA

Telephone: (1) $31376+7226$

Facsimile: (1) $3137+73083$

Telex:

Email: combiassprlc.spl.umich.edu

Commission(s): 15,16

COMINS NEIL FRANCIS, DPT PHYSICS \& ASTRONOMY, UNIVERSITY OF MAINE, BENNETT HALL ORONO ME 04469, USA

Telephone: (1) 2075811037

Facsimile: (1)

Telex:

Email:

Commission(s): 33

COMORETTO GIOVANNI, OSS ASTROFISICO, DI ARCETRI, LARGO E FERMI 5, I 50125 FIRENZE, ITALY

Telephone: (39) 554378540

Facsimile: (39) 55435939

Telex: 572268 arcetr $i$

Email:

Commission(s):

COMTE GEORGES DR, OBSERVATOIRE DE MARSEILLE, 2 PLACE LE VERRIER

F 13248 MARSEILLE CDX 14, FRANCE

Telephone: (33) $91959088 \quad$ Facsimile: (33)

Email: carn::"comte(afronirs51" Commission(s): 28

Telex: $\$ 202+1$ f

CONCONI PAOLO DR, OSS ASTRONOMICO DI MILANO, VIA E BIANCHI 46, I 22055 MERATE, ITALY

Telephone: (39) $592035 \quad$ Facsimile: (39)

Telex:

Email:

Commission(s):

CONDON JAMES J DR, NRAO, 520 EDGEMONT RD, CHARLOTTESVILLE VA 22903, USA

Telephone: (1) 804296021

Facsimile: (1)

Telex:

Email:

Commission(s): $40,44,47$

CONKLIN EDWARD K, FORTH INC, 111 N SEPULVEDA BLVD 300, MANHATTAN BEACH CA 90266, USA

Telcphone: (1)

Facsimilc: (1)

Telex: 275182 fort ur

Email:

Commission(s): 40

CONNES JANINE DR, CIRCE, BP 63, F 91400 ORSAY, FRANCE

Telephone: (33) 169287675

Facsimile: (33)

Telex: 692166

Email:

Commission(s): 16

CONNES PIERRE DR, SERVICE D'AERONOMIE, BP 3, F 91371 VERRIERES BUISSON, FRANCE

Telephone: (33) $164+7+277$

Facsimile: (33)

Telex: $6112+00$

Email:

Commission(s): 24,51

CONNOLLY LEO PAUL, DPT OF PHYSICS, CALIFORNIA STATE UNIV, 5500 UNIVERSITY PARKWAY SAN BERNARDINO CA 92407 , USA

Telephone: (1) $71+8805+100$

Facsimile: (1)

Telex:

Email:

Commission(s): $25,27,35$

CONSOLMAGNO GUY JOSEPH, VATICAN OBS RESEARCH GP, STEWARD OBSERVATORY

UNIVERSITY OF ARIZONA, TUCSON AZ 85721, USA

Telephone: (1) 5206217855

Facsimile: (1) $5206211532 \quad$ Telex:

Email: gic(a)as.arizona.edu

Commission(s): $15,16 \mathrm{C}$

CONTADAKIS MICHAEL E DR, DPT GEODESY \& SURVEYING, UNIVERSITY THESSALONIKI, UNIV BOX 503 GR 540 06 THESSALONIKI, GREECE

Telephonc: (30) 31992693

Facsimile: (31)

Commission(s): 27

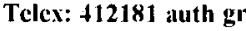

Email:

CONTI PETER S DR, JILA, UNIVERSITY OF COLORADO, BOX +40, BOULDER CO 80309 (1440, USA

Telephone: (1) 3034927789

Facsimile: (1) $303+925235$

Email: 33833::pconti/pconti/äjila

Commission(s): 29,30

Telex: 755842 jila 
CONTOPOULOS GEORGE PROF, DPT OF ASTRONOMY, NTL UNIVERSITY OF ATHENS, PANEPISTIMIOPOLIS GR 15783 ATHENS, GREECE

Telephone: (30) 17243211

Facsimile: (30)

Telex:

Email:

Commission(s): $07,28,33$

CONWAY ROBIN G DR, NRAL, JODRELL BANK, MACCLESFIELD SK11 9DL, UK

Telephone: (44) 147771321

Facsimile: (44)

Telex: 36149

Email:

Commission(s): 40

COOK ALAN H PROF, DPT PHYS/UNIV CAMBRIDGE, THE MASTER'S LODGE, SELWYN COLLEGE

CAMBRIDGE CB3 9DQ, UK

Telephone: (44) 1223335889

Facsimile: (44) $1223335837 \quad$ Telex: 81292 cavlab

Email:

Commission(s): 07,14

COOK JOHN W, 8032 SLEEPY VIEW LN, SPRINGFIELD VA 22153, USA

Telephone: (1) 2027672161

Facsimile: (1)

Telex:

Email:

Commission(s): 10,12

COOK KEM HOLLAND DR, LAWRENCE LIVERMORE LAB, L 401, BOX 808, LIVERMORE CA 94550, USA

Telephone: (1) 5104234634

Facsimile: (1) 5102945512

Telex:

Email: kcook@imager.IInl.gov

Commission(s): 27,28

COOKE B A DR, DPT OF PHYSICS, UNIVERSITY OF LEICESTER, X-RAY ASTRONOMY GROUP

LEICESTER LE 1 7RH, UK

Telephone: (44) $1533554455 * 188$

Facsimile: (44)

Telex: 341664

Email:

Commission(s):

COOKE JOHN ALAN, ROYAL OBSERVATORY, BLACKFORD HILL, EDINBURGH EH9 3HJ, UK

Telephone: (44) $1316673221 \quad$ Facsimile: (4) Telex: 72383 roedin g

Email:

Commission(s): 09

CORADINI ANGIOLETTA, IAS, REPARTO DI PLANETOLOGIA, VIA DELL'UNIVERSITA 11, I 00185 ROMA, ITALY

Telephone: (39) $64956951 \quad$ Facsimile: (39)

Email:

Commission(s):

Telex: $680489 \mathrm{cnr}$ fra

CORBALLY CHRISTOPHER, STEWARD OBSERVATORY, UNIVERSITY OF ARIZONA, VATICAN OBS RESEARCH G TUCSON AZ 85721, USA

Telephone: (1) 5206213225

Facsimile: (1)

Telex: 467175

Email: corbally@as.arizona.edu

Commission(s): 29,45

CORBELLI EDVIGE DR, OSS ASTROFISICO, DI ARCETRI, LARGO E FERMI 5 , I 50125 FIRENZE, ITALY

Telephone: (39) 554378540

Facsimíle: (39) 55435939

Telex:

Email: edvige@sisifo.arcetri.astro.it Commission(s): 34

CORBIN THOMAS ELBERT DR, US NAVAL OBSERVATORY, ASTROMETRY DPT, 3450 MASSACHUSETTS AVE NW WASHINGTON DC 203925420 , USA

Telephone: (1) 2026531557

Facsimilc: (1) $2026530944 \quad$ Telex:

Email: tec@sicon.usno.nay.mil

Commission(s): 08P,24

CORDES JAMES M, CORNELL UNIVERSITY, SPACE SCIENCES BLDG, ITHACA NY 14853, USA

Telephone: (1) 6072563734

Facsimilc: (1)

Telex: 932458

Email:

Commission(s): 40

CORDOVA FRANCE A D, DPT OF ASTRONOMY, PENNSYLVANIA STATE UNIV, 525 DAVEY LAB UNIVERSITY PARK PA 16802, USA

Telephone: (1)

Facsimile: (1)

Telex:

Email:

Commission(s): 44

CORLISS C H DR, FOREST HILLS LABORATORY, 2955 ALBEMARLE STREET NW, WASHINGTON DC 20008, USA

Telephone: (1) 2023626085

Facsimilc: (1)

Commission(s): 14

Telex:

Email:

, TONANTZINTLAZ, APDO POSTAL 216 y 51, 72000 PUEBLA PUE, MEXICO

CORNEJO ALEJANDRO A DR, INAOE, TONANTZINTLAZ, APDO POSTAL 216
Telephone: (52) 9122470500
Facsimile: (52)

Email:

Commission(s): 09,41 
CORNIDE MANUEL, DPT DE ASTROFISICA, FAC DE FISICA, UNIVERSIDAD COMPLUTENSE E 28040 MADRID, SPAIN

Telephone: (34) 14495316

Email:
Facsimile: (34)

Commission(s): 29
Telex: $\mathbf{4 7 2 7 3}$ ffuc

CORNILLE MARGUERITE DR, OBSERVATOIRE DE PARIS, SECTION DE MEUDON, UA 812 F 92195 MEUDON PPL CDX, FRANCE

Telephone: (33) $145077455 \quad$ Facsimile: (33)

Email: bitnet:frors31

Telex: 201571

Commission(s): 14

CORREIA EMILIA DR, CRAAE/ESCOLA POLITECNICA, UNIVERSIDADE DE SAO PAULO, CP 8174 O5508 SAO PAULO SP, BRAZIL

Telephone: (55) 118156289

Facsimile: (55) 118154272

Telex: 1180127 inpe br

Email: ecorreia@brusp.ansp.br

Commission(s): 10

CORWIN HAROLD G JR, CALTECH, MS 100 22, IPAC, PASADENA CA 91125, USA

Telephone: (1) 8183979537

Email: hgcjr@ipac.caltech.edu

Facsimile: (1) $8183979600 \quad$ Telex:

Commission(s): 28

COSMOVICI BATALLI C DR, IAS, CNR, I 000 4 FRACASTI, ITALY

Telephone: (39) 69423801

Facsimile: (39)

Telex: $610261 \mathrm{i}$

Email:

Commission(s): 15

COSTA ANDREA, IAFE, CC 67 SUC 28, 1428 BUENOS AIRES, ARGENTINA

Tclephone: (54) 17816755

Email: costa@iafe.edu.ar

Facsimile: (54) 17868114 Telex:

Commission(s):

COSTA EDGARDO DR, DPT DE ASTRONOMIA, UNIVERSIDAD DE CHILE, CASILLA 36 D, SANTIAGO, CHILE

Tclephone: (56) 22294101

Facsimile: (56) 22712799

Telex: 440001

Email:

Commission(s): $\mathbf{0 8 , 3 3}$

COSTA ENRICO, IAS, CNR, CP 67, I 00044 FRASCATI, ITALY

Telephonc: (39) $69425655 \quad$ Facsimile: (39) 69416847

Email:

Commission(s):

Telex: $610261 \mathrm{i}$

COSTA JOAQUIM E R DR, CRAAE/PTE ESCOLA POLI USP, CP 8174 CEP, 01051 SAO PAULO SP, BRAZIL

Telephone: (55) 118156289

Email: jercosta@brusp.ansp.br

Facsimilc: (55) $11815+272$

Telex: 1180127 inpe br

COSTA VICTOR DR, INST ASTROFISICA, DE ANDALUCIA APD 3004, C/SANCHO PANZA S/N

E 18080 GRANADA, SPAIN

Telephone: (34) 58121311

Facsimilc: (34)

Email:

Commission(s):

Telex: 78573 iagg e

COSTERO RAFAEL, INSTITUTO DE ASTRONOMIA, UNAM, APDO POSTAL 70-264, 04510 MEXICO DF, MEXICO

Telephone: (52) 9055485305

Facsimile: (52)

Email:

Commission(s): $34,50 \mathrm{C}$

Telex: 1760155 cicme

COTTON WILLIAM D Jr, NRAO, 520 EDGEMONT RD, CHARLOTTESVILLE VA 22903, USA

Telephone: (1) 8042960319

Facsimile: (1)

Telex: $5105875+82$

Email:

Commission(s): 40

COTTRELL PETER LEDSAM, DPT PHYSICS \& ASTRONOMY, UNIVERSITY OF CANTERBURY

PRIVATE BAG, CHRISTCHURCH 1, NEW ZEALAND

Telephone: (64) $03482009 \quad$ Facsimile: (6t)

Email:

Commission(s): $27,29 \mathrm{C}$

Tclex: $41+4 \mathrm{nz}$

COUCH WARRICK DR, SCHOOL, OF PHYSICS, UNIV NEW SOUTH WALES, BOX 1

KENSINGRON NSW 2033, AUSTRALIA

Telephone: (61) 23854578

Facsimile: (61)

Telex:

Email: wjc@newt.phys.unsw.edu.au

Commission(s): 28

COUCHMAN HUGH M P, DPT OF ASTRONOMY, UNIV OF WESTERN ONTARIO, LONDON ON N6A 3K7, CANADA

Telcphonc: (1) 5196613183

Facsimile: (1) 5196612009

Telex:

Email:

Commission(s): 
COULSON IAIN M DR, JOINT ASTRONOMY CENTER, 600 N A'OHOKO PL, UNIVERSITY PARK, HILO HI 96720 , USA

Telephone: (1)

Facsimile: (1)

Telex:

Email:

Commission(s): 27

COUNSELMAN CHARLES C PROF, DPT OF EARTH PLANET \& SCI, MIT RM 54 620, BOX 165

CAMBRIDGE MA 02139, USA

Telephone: (1) $6172537902 \quad$ Facsimile: (1) Telex: 921473 mit cam

Email:

Commission(s): 07,08,16

COUPER HEATHER MISS, COLLINS COTTAGE, LOWER RD, LOOSLEY ROW, BUCKS HP17 OPF, UK

Telephone: (44)

Facsimile: (44)

Telex:

Email:

Commission(s): 46,51

COUPINOT GERARD DR, OBS MIDI PYRENEES, 9 R PONT DE LA MOUETTE

F 65200 BAGNERES BIGORRE, FRANCE

Telephone: (33) $62951969 \quad$ Facsimile: (33) $62951969 \quad$ Telex:

Emait:

Commission(s):

COURTES G PROF, LAS, TRAVERSE DU SIPHON, LES TROIS LUCS, F 13012 MARSEILLE, FRANCE

Telephone: (33) $91055900 \quad$ Facsimile: (33) $91661855 \quad$ Telex: 410584

Email:

Commission(s): $28,33,34,44$

COURTIN REGIS, OBSERVATOIRE DE PARIS, DPT DE RECHERCHE SPATIALE, 5 PL JULES JANSSEN

F 92195 MEUDON PPL CDX, FRANCE

Telephone: (33) 145077729

Facsimile: (33) $145077469 \quad$ Telex:

Email: courtin@mesioa.obspm.fr

Commission(s):

COURVOISIER THIERRY J-L, OBSERVATOIRE DE GENEVE, CHEMIN DES MAILLETTES 51

CH 1290 SAUVERNY, SWITZERLAND

Telephone: (41) 227552611

Facsimilc: (41) 227553983

Telex: 419209 obs ch

Email: courvois@obs.unige.ch

Commission(s): 28,44

COUSINS A W J DR, SAAO, BOX 9, OBSERVATORY 7935, SOUTH AFRICA

Telephone: (27) 021-47-0025 Facsimile: (27)

Email:

Commission(s): 25

Tclex: $\mathbf{5 7 2 0 3 0 9}$

COUTEAU PAUL PROF, OCA OBSERV DE NICE, BP 229, F 0630 + NICE CDX 4, FRANCE

Telephone: (33) $93890420 \quad$ Facsimile: (33) $92003033 \quad$ Telex: 460004 obsnice $f$

Email:

Commission(s): 26

COUTREZ RAYMOND A J PROF, 6 RUE EGIDE BOUVIER, B 1160 BRUSSELS, BELGIUM

Telcphone: (32)

Facsimile: (32)

Commission(s): 40

Telex:

Email:

COUTTS-CLEMENT CHRISTINE, DPT OF ASTRONOMY, UNIVERSITY OF TORONTO, 60 ST GEORGE ST TORONTO ON MSS 1A1, CANADA

Telephone: (1) $4169785186 \quad$ Facsimilc: (1) $4169783921 \quad$ Telex: 06986766

Email:

Commission(s): 27

COUTURIER PIERRE, INSU, 77 AVE DENFERT ROCHEREAU, F 75014 PARIS, FRANCE

Telephone: (33) 140512004 Facsimile: (33) 140512101 Telex:

Email: iapobs::couturier

Commission(s): 49

COVINGTON ARTHUR E, 131 COLLEGE STREET, KINGSTON ON K7L 4L7, CANADA

Telcphone: (1)

Facsimile: (1)

Telex:

Email:

Commission(s): 10,40

COWAN JOHN J DR, DPT PHYSICS \& ASTRONOMY, UNIVERSITY OF OKLAHOMA, NORMAN OK 73019, USA

Telephone: (1) 4053253961

Facsimile: (1)

Telex:

Email:

Commission(s): 35

COWIE LENNOX LAUCHLAN DR, INSTITUTE FOR ASTRONOMY, UNIVERSITY OF HAWAII, 2680 WOODLAWN DR HONOLULU HI 96822, USA

Telcphone: (1)

Facsimile: (1)

Telex:

Email:

Commission(s): 34,44 
M 488

COWLEY ANNE P DR, DPT OF PHYSICS, ARIZONA STATE UNIVERSITY, TEMPE AZ 85287, USA

Telephone: (1) 6029652919

Facsimile: (1)

Telex:

Email:

Commission(s): 29,42,45

COWLEY CHARLES R PROF, DPT OF ASTRONOMY, UNIVERSITY OF MICHIGAN, DENNISON BLDG

ANN ARBOR MI 48109 1090, USA

Telephone: (1) 3137643437

Facsimile: (1)

Telex: 810-2236056

Email:

Commission(s): 29,36

COWSIK RAMANATH, TIFR, HOMI BHABHA RD, COLABA, BOMBAY 400005 , INDIA

Telephone: (91) 22219111

Facsimile: (91)

Telex:

Email:

Commission(s): 28,44

COX ARTHUR N DR, LOS ALAMOS NATIONAL LAB, BOX 1663, LOS ALAMOS NM 87545, USA

Telephone: (1) $5056677648 \quad$ Facsimile: (1) Telex: 910-988-1773

Email: $\quad$ Commission(s): 12,27

COX DONALD P PROF, DPT OF ASTRONOMY, UNIVERSITY OF WISCONSIN, 475 N CHARTER ST, MADISON WI 53706, USA

Telcphone: (1) 6082625916

Facsimile: (1)

Telex:

Email:

Commission(s): 34

GOX PIERRE DR, OBSERVATOIRE DE MARSEILLE, 2 PLACE LE VERRIER, F 13248 MARSEILLE CDX 04, FRANCE Telephone: (33) 91959088 Facsimile: (33) 91621190 Telex:

Email: $\operatorname{cox} @$ fromrs51

Commission(s): 34

COYNE GEORGE V DR, DIRETTORE DELLA SPECOLA, VATICANA, I 00120 VATICAN CITY, VATICAN CITY STATE

Telephone: (39) 66983411

Facsimile: (39)

Commission(s): $25,34,50$

Telex: 2020 vat obs va

Email:

CRABTREE DENNIS DR, HERZBERG INST ASTROPHYS, DOMINION ASTROPHYS OBS, 5071 W SAANICH RD VICTORIA BC V8X 4M6, CANADA

Tclephone: (1) 6043880025

Email: crabtree@dao.nrc.ca
Facsimile: (1) 6043630045

Commission(s):
Telex: 0497295

CRAIG IAN DR, DPT OF MATHEMATICS, UNIVERSITY OF WAIKATO, PRIVATE BAG, HAMILTON, NEW ZEALAND
Telephone: (64)

Email:

Facsimile: $(6+)$

Commission(s): 10

Telex:

CRAIG IAN JONATHAN D DR, DPT APPLIED MATHEMATICS, UNIVERSITY OF WAIKATO

HAMILTON, NEW ZEALAND

Telephone: (64) 62889

Email:

Facsimile: (64)

Telex:

Commission(s): 12
CRAINE ERIC RICHARD DR, WESTERN RESEARCH CO, 2127 E SPEEDWAY, SUITE 209, TUCSON AZ 85719, USA Tclephone: (1) 5203254505

Email: craine@ noao.edu
Telex:

Commission(s):

CRAM LAWRENCE EDWARD PROF, SCHOOL OF PHYSICS, UNIVERSITY OF SYDNEY

SYDNEY NSW 2006, AUSTRALIA

Telephone: (61) 23512537

Email: lc@astrop.physics.su.oz.au

Facsimilc: (61) 26602903

Telex: aa26169 unisyd

CRAMER NEIL DR, SCHOOL OF PHYSICS, UNIVERSITY OF SYDNEY, SYDNEY NSW 2006, AUSTRALIA

Telephone: (61) 26923162

Facsimile: (61) 26602903

Telex: aa 26169

Email:

Commission(s): 10,12,49

CRAMPTON DAVID DR, HERZBERG INST ASTROPHYS, DOMINION ASTROPHYS OBS, 5071 W SAANICH RD VICTORIA BC V8X 4M6, CANADA

Telephonc: (1) 6043883900

Facsimile: (1) $60436300+5$

Telex: 0497295

Email:

Commission(s): 30,33

CRANE PATRICK C, INTERFEROMETRICS INC, 8150 LEESBURG PIKE, VIENNA VA 221182, USA

Telephone: (1) 2024048941

Facsimile: (1)

Telex:

Email: crane@susim.nrl.navy.mil

Commission(s): 40,47 
CRANE PHILIPPE, ESO, KARL SCHWARZSCHILDSTR 2, D 85740 GARCHING MUENCHEN, GERMANY

Telephonc: (49) 89792098

Facsimile: (49)

Telex: 528-28222 co d

Email:

Commission(s): $28,34,47$

CRANNELL CAROL JO DR, NASA GSFC, CODE 682, GREENBELT MD 20771, USA

Telephone: (1) 3012865007

Facsimile: (1)

Telex: 89675

Email:

Commission(s): 10, 44

CRAWFORD CAROLIN, INSTITUTE OF ASTRONOMY, THE OBSERVATORIES, MADINGLEY RD

CAMBRIDGE CB3 OHA, UK

Telephone: (44) 1223337510

Email: csc@mail.ast.cam.ac.uk

Facsimile: (44) $1223337523 \quad$ Telex:

Commission(s):

CRAWFORD DAVID L DR, KITT PEAK NTL OBS, BOX 26732, 950 N CHERRY AVE, TUCSON AZ 85726, USA

Telephone: (1) 5203259346

Facsimile: (1) 5203259360

Telex:

Email: derawford(a)noao.edu

Commission(s): 09, 25,33,45,50C

CRAWFORD IAN ANDREW DR, DPT PHYSICS \& ASTRONOMY, UNIVERSITY COLLEGE LONDON, GOWER ST

LONDON WC1E 6BT, UK

Telephone: (44) $1713877050 \star 3498$

Facsimile: (44) 1713807145

Telex: 28722

Email: iac(a)star.ucl.ac.uk

Commission(s): 34

CRENSHAW DANIEL MICHAEL, NASA GSFC, CODE 681, COMPUTER SCIENCES CORP, GREENBELT MD 20771, USA

Telephone: (1) 3012860871

Facsimile: (1) 3012861752

Telex:

Email: iue:crenshaw

Commission(s):

CREZE MICHEL DR, OBS DE STRASBOURG, 11 RUE UNIVERSITE, F 67000 STRASBOURG, FRANCE

Telephone: (33) 88358216

Facsimile: (33) 88250160

Telex: obsbes $361144 \mathrm{f}$

Email: creze@simb̨ad.u-strasbg.fr

Commission(s): $05 \mathrm{C}, 24,33$

CRIFO FRANCOISE DR, OBSERVATOIRE DE PARIS, SECTION DE MEUDON, DEPEG

F 92195 MEUDON PPL CDX, FRANCE

Telephone: (33) 145077834

Facsimilc: (33)

Telex: 201571

Email:

Commission(s): 08,24

CRISTALDI SALVATORE DR, OSS ASTROFISICO, CITTA UNIVERSITARIA, VIA ARTALE ALAGONA 75 I 95125 CATANIA, ITALY

Telcphonc: (39) 330734

Facsimile: (39)

Commission(s): 42

Telex: 970359 astret $i$

Email:

CRISTESCU CORNELIA G DR, ASTRONOMICAL INSTITUTE, CUTITUL DE ARGINT 5, BOX 28

R 75212 BUCHAREST, RUMANIA

Telephone: (40) 16236892

Facsimile: (40) 13123391

Telex: 11882 astro $r$

Email:

Commission(s): 15,20

CRISTIANI STEFANO DR, DPT DI ASTRONOMIA, UNIVERSITA DI PADOVA, VIC DELL OSSERVATORIO 5

I 35122 PADOVA, ITALY

Telephone: (39) 49661499

Email: span:39003::cristiani

Facsimile: (39)

Commission(s): 47

Telex: $\mathbf{4 2 0 7 1}$

CRIVELLARI LUCIO, OAT, BOX SUCC TRIESTE 5, VIA TIEPOLO 11, I 3+131 TRIESTE, ITALY

Telephonc: (39) $40793221 \quad$ Facsimile: (39)

Email:

Commission(s): 36

Telex: 461137 oat i

CROCKER DEBORAH ANN DR, DPT OF PHYSICS \& ASTRON, UNIVERSITY OF ALABAMA, BOX 870324 TUSCALOOSA AL 35387 0324, USA

Telcphone: (1) 2053483758

Facsimile: (1)

Telex:

Email: crock@kudzu.astr.ua.edu

Commission(s):

CROOM DAVID L DR, RUTHERFORD APPLETON LAB, SPACE \& ASTROPHYSICS DIV, BLDG R25/R68 CHILTON DIDCOT OX11 0QX, UK
Telephone: (4) 123521900
Facsimilc: (4t)
Email:
Commission(s): 40
Telex: 83159 
M 490

CROTTS ARLIN PINK, DPT OF ASTRONOMY, COLUMBIA UNIVERSITY, 538 W 120TH ST, NEW YORK NY 10027, US

Telephone: (1) 2128547899

Facsimilc: (1) 2123169504

Telex:

Email: arlin@eurcka.columbia.edu

Commission(s):

CROVISIER JACQUES, OBSERVATOIRE DE PARIS, SECTION DE MEUDON, F 92195 MEUDON PPL CDX, FRANCE

Telephone: (33) 145077599

Facsimilc: (33)

Telex: 270912

Email:

Commission(s): $15,34,40$

CROWTHER PAUL, DPT PHYSICS \& ASTRONOMY, UNIVERSITY COLLEGE LONDON, GOWER ST

LONDON WC1E 6BT, UK

$\begin{array}{ll}\text { Telephone: (44) } 1713877050 \star 3474 & \text { Facsimile: }(44) 17138071+5 \quad \text { Tetex: } \\ \text { Email: pac@star.ucl.ac.uk } & \text { Commission(s): } 29\end{array}$

CRUIKSHANK DALE P DR, NASA AMES RESEARCH CTR, MS 245 6, MOFFETT FIELD CA 94035, USA

Telephone: (1)

Facsimile: (1)

Telex:

Email: cruikshank@ssa1.arc.nasa.gov

Commission(s): 15,16C

CRUISE ADRIAN MICHAEL DR, RUTHERFORD APPLETON LAB, CHILBOLTON OBSERVATORY, DITTON PARK SLOUGH SL3 9JX, UK

Telephone: (44)

Facsimile: (44)

Email:

Commission(s): 4

Telex:

CRUTCHER RICHARD M DR, DPT OF ASTRONOMY, UNIVERSITY OF ILLINOIS, 1011 W SPRINGFIELD AVE URBANA IL 61801, USA

Telephone: (1) 2173339581

Facsimile: (1)

Telex:

Email:

Commission(s):

CRUVELLIER PAUL E DR, LAS, TRAVERSE DU SIPHON, LES TROIS LUCS, F 13012 MARSEILLE, FRANCE

Telephone: (33) 91055900

Email: cruvecofrlasm51

Facsimile: (33) 91661855

Telex: $+2058+$

CRUZALEBES PIERRE, OCA, DPT FRESNEL, F 06460 CAUSSOLS, FRANCE

Telcphone: (33)

Commission(s): 34

Email:

Facsimile: (33)

Commission(s):

Telex:

CRUZ-GONZALEZ IRENE, INSTITUTO DE ASTRONOMIA, UNAM, APDO POSTAL 70-264, 04510 MEXICO DF, MEXIC

Tclephone: (52) 9055485306

Facsimile: (52)

Telex:

Email:

Commission(s):

CSADA IMRE K DR, KONKOLY OBSERVATORY, THEGE U 13/17, BOX 67, H 1525 BUDAPEST, HUNGARY

Tclephone: (36) $166+26$

Facsimilc: (36)

Telex: 227460

Email:

Commission(s): 10

CUBARSI RAFAEL DR, DPT MATEMATICA APLICADA, UNIV POLITEC DE CATALUNYA, BOX 30002

E 08080 BARCELONA, SPAIN

Telephone: (34) 34016799

Email: matrcmômat.upc.es

Facsimile: (34) 34016801

Telex: 52821 upc-c.

Commission(s): 33

CUDABACK DAVID D DR, RADIO ASTRONOMY LAB, UNIVERSITY OF CALIFORNIA, 601 CAMPBELL HALL, BERKELEY CA 94720, USA

Tclephone: (1) $4156+25724$

Facsimile: (1)

Telex: 820181 ucb ast

Email: cudaback@abkyast.berkerley.edu Commission(s): 34,41)

CUDWORTH KYLE MCCABE DR, YERKES OBSERVATORY, UNIVERSITY OF CHICAGO, BOX 258

WILLIAMS BAY WI 53191, USA

Telephone: (1) $41+2455555$

Facsimile: (1)

Telex:

Email:

Commission(s): $24,33,37 \mathrm{C}$

CUFFEY J MR, DPT OF EARTH SCI \& ASTRON, NEW MEXICO STATE UNIV, UNIVERSITY PARK NM 88001, USA

Telephone: (1)

Facsimile: (1)

Telex:

Email:

Commission(s): 37

CUGIER HENRYK DR, ASTRONOMICAL INSTITUTE, WROCLAW UNIVERSITY, UL KOPERNIKA 11

PL 51622 WROCLAW, POLAND

Telephone: (48) 7148 243+

Facsimile: $(48)$

Telex: 0712791 uwr pl

Email:

Commission(s): 36 
CUGNON PIERRE DR, OBSERVATOIRE ROYAL DE, BELGIQUE, AVE CIRCULAIRE 3, B 1180 BRUSSELS, BELGIUM

Telephone: (32) 23752484

Facsimile: (32)

Commission(s): $3+$

Telex: 21565 obsbel

Email: pierrec@astro.ome.be

CUGUSI LEONINO DR, DPT DI SCIENZE FISICHE, UNIVERSITA DI CAGLIARI, VIA OSPEDALE 72

I 09100 CAGLIARI, ITALY

Telephone: (39) 70664770

Facsimile: (39)

Telex:

Email:

Commission(s):

CUI DOU-XING, CHANGCHUN ARTIFICIAL, SATELLITE OBSERVATORY, BOX 1067, CHANGCHUN, CHINA PR Telephone: (86) 42859

Email:

Telex: $2+21$ changchun

Commission(s): 07

CUI ZHEN-HUA, BEIJING PLANETARIUM, 138 XI WAI ST, BEIJING, CHINA PR

Telephone: (86) 1893003

Facsimile: (86)

Telex:

Email:

Commission(s): 41,46

CULHANE LEONARD PROF, MULLARD SPACE SCIENCE LAB, UNIVERSITY COLLEGE LONDON HOLMBURY ST MARY, DORKING SURREY RH5 6NT, UK

Telephone: (44) $130670292 \quad$ Facsimile: (4t)

Telex: 859185 ucmssl g

Email:

Commission(s): 10,4t

CULLUM MARTIN DR, ESO, KARL SCHWARZSCHILDSTR 2, D 85740 GARCHING MUENCHEN, GERMANY

Telephone: (49) 89320060

Facsimile: (49) 893202362

Telex: $\mathbf{5 2 8 2 8 2 0}$ eo d

Email: carn:cullum(a)dgacso51

Commission(s): 09C

CULVER ROGER BRUCE DR, DPT OF PHYISCS, COLORADO STATE UNIVERSITY, FT COLLINS CO 80523, USA

Telcphone: (1) 3034916206

Facsimile: (1)

Telex: 9109309000 engresuft

Email:

Commission(s): 26

CUNTZ MANFRED DR, JILA, UNIVERSITY OF COLORADO, BOX 440 , BOULDER CO 80309 0440, USA

Telephone: (1)

Facsimile: (1)

Telex:

Email:

Commission(s): 36

CUNY YVETTE J DR, OBSERVATOIRE DE PARIS, SECTION DE MEUDON, F 92195 MEUDON PPL CDX, FRANCE

Telephone: (33) 145077838

Facsimile: (33)

Telex:

Email:

Commission(s): 36

CUPERMAN SAMI PROF, DPT OF PHYSICS \& ASTRON, TEL AVIV UNIVERSITY, RAMAT AVIV

TEL AVIV 69978, ISRAEL

$\begin{array}{lll}\text { Telephone: (972) } 3 \$ 20 ~ 21 / 425697 & \text { Facsimile: }(972) & \text { Telex: } 3 \$ 2171 \text { versy ii } \\ \text { Email: } & \text { Commission(s): } 33,49 & \end{array}$

CURIR ANNA, OSS ASTRONOMICO DI TORINO, ST OSSERVATORIO 20, 10025 PINO TORINESE, ITALY

Telephone: (39) 11841067

Facsimile: (39)

Telex: 213236 to astr i

Email: astto2::curir

Commission(s): 44

CURRIE DOUGLAS G DR, ASTRONOMY PROGRAM, UNIVERSITY OF MARYLAND, COLLEGE PARK MD 20742, USA

Telephone: (1) $301+5+3405$

Facsimile: (1)

Telex:

Email:

Commission(s): 09,51

CUTISPOTO GIUSEPPE DR, OSS ASTROFISICO, CITTA UNIVERSITARIA, VIA A DORIA 6, I 95I25 CATANIA, ITALY

Telephone: (39) 95337241

Facsimile: (39) 95330592

Telex: 970359 astret $i$

Email: 40297::giuseppe

Commission(s): 27

CUYPERS JAN DR, OBSERVATOIRE ROYAL DE, BELGIQUE, AVE CIRCULAIRE 3, B 1180 BRUSSELS, BELGIUM

Telephonc: (32) 23730234

Facsimile: (32) $237+9822$

Telex:

Email: jan@astro.ome.he

Commission(s): $15,25,27$

CZERNY BOZENA DR, COPERNICUS ASTRON CENTER, POLISH ACAD OF SCIENCES, UL BARTYCKA 18

PL 00716 WARSAW, POLAND

Telephone: (48) WARSAW 410041

Facsimile: $(48)$

Email: bcz@camk.edu.pl

Commission(s):

Telex: 
M 492

CZERNY MICHAL DR, DPT OF ASTRONOMY, UNIVERSITY OF LEICESTER, UNIVERSITY RD LEICESTER LE1 7RH, UK

Telephone: (44) 1533522073

Email:

Facsimile: (44)

Commission(s):

Telex: 347250 leicun

CZYZAK STANLEY J DR, 800 NORTH MAPLE AVE, FAIRBORN OH 45324, USA

Telephone: (1)

Facsimile: (1) Telex:

Email:

Commission(s): 14,34

DA COSTA ANTONIO A DR, COMPLEXO INTERDISCIPLINAR, INSTITUTO SUPERIOR TECNIC P 1096 LISBOA CODEX, PORTUGAL

Telephone: (351) $35113524303 \quad$ Facsimile: (351) $35113524372 \quad$ Telex:

Email:

Commission(s): 44

DA COSTA GARY STEWART DR, MOUNT STROMLO OBSERVATORY, PRIVATE BAG, WESTERN CREEK PO CANBERRA ACT 2611, AUSTRALIA

Telephone: (61) 62490236

Facsimile: (61) $62490233 \quad$ Telex:

Email: gdc@mso.anu.edu.au

Commission(s): 37VP

DA COSTA JOSE MARQUES DR, INPE, CP 515, 12200 S JOSE DOS CAMPOS, BRAZIL

Tclephone: (55) 123229977

Facsimile: (55) 123218743

Telex: 1133530 inpebr

Email:

Commission(s): 4

DA COSTA L.A. NICOLACI, OBSERVATORIO NACIONAL, RUA GL BRUCE 586, SAO CRISTOVAO 20921 RIO DE JANEIRO RJ, BRAZIL

Telephonc: (55) 215807181

Email: Indc@alnccvm

Facsimile: (55) $215800332 \quad$ Telex:

Commission(s): $28,30 \mathrm{C}$

DA ROCHA VIEIRA E DR, INSTITUTO DE FISICA, UFRGS, CP 15051, 90000 PORTO ALEGRE RS, BRAZIL

Telcphone: (55) $512217666 \quad$ Facsimile: (55)

Email:

Commission(s):

Telex: 0511055 ufrsbr

DA SILVA A V C S, OBSERVATORIO ASTRONOMICO, UNIVERSIDADE SANTA CLARA

P 3000 COIMBRA, PORTUGAL

Telephone: (351)

Facsimile: (351)

Telex:

Email:

Commission(s):

DA SILVA LICIO DR, OBSERVATORIO NACIONAL, RUA GL BRUCE 586, SAO CRISTOVAO 20921 RIO DE JANEIRO RJ, BRAZIL

Telephone: (55) 215807313

Facsimile: (55) 215800332

Telex: 21288

Email:

Commission(s):

DACHS JOACHIM PROF DR, ASTRONOMISCHES INSTITUT, RUHR UNIVERSITAET BOCHUM, POSTFACH 102148 D 44780 BOCHUM, GERMANY
Telephone: (49) $23+7003+5+$
Facsimile: (49)
Telex: 825860

Commission(s): 25

Email:

DADAEV ALEKSANDR N DR, PULKOVO OBSERVATORY, ACADEMY OF SCIENCES, 10 KUTUZOV QUAY 196140 ST PETERSBURG, RUSSIA

Telephone: (7)

Facsimile: (7)

Telex:

Email:

Commission(s): 26,42

DADHICH NARESH DR, IUCAA, PO BOX 4, GANESHKHIND, PUNE $\$ 11$ 007, INDIA

Telephone: (91) 212336415

Facsimilc: (91) 212335760

Telex: $01+5658 \mathrm{gmrt}$ in

Email: root@iucaa.crnet.in

Commission(s): 44,47

DADIC ZARKO DR, ZAVOD ZA POVIJEST, ZNANOSTI JAZU, ANTE KOVACICA 5, ZAGREB, CROATIA

Telephone: (38) 41440124

Facsimile: (38)

Commission(s): 41

Telex:

Email:

DAGKESAMANSKY RUSTAM D DR, LEBEDEV PHYSICAL INST, ACADEMY OF SCIENCES, LENINSKY PROSPEKT 5 117924 MOSCOW, RUSSIA

Telephone: (7) 951351429

Facsimile: (7)

Commission(s): 40

Telex: $\mathbf{4 1 1 4 7 9}$ ncod su

Email: 
DAHN CONRAD DURTIS DR, US NAVAL OBSERVATORY, FLAGSTAFF STATION, BOX 1149

FLAGSTAFF AZ 86002 1149, USA

Telephone: (1) 6027795132

Facsimile: (1) Telex:

Email: dahn@nofs.navy.mil

Commission(s): $24,25,34$

DAIGNE GERARD, OBSERVATOIRE DE BORDEAUX, BP 89, F 33270 FLOIRAC, FRANCE

Telephone: (33) 56864330

Facsimile: (33) $56404251 \quad$ Telex:

Email:

Commission(s): $\mathbf{5 1}$

DAINTREE EDWARD J DR, NRAL, JODRELL BANK, MACCLESFIELD SK11 9DL, UK

Telephone: (44) $147771321 \quad$ Facsimile: (44)

Email:

Commission(s): 40

Telex: 36149

DAISHIDO TSUNEAKI PROF, DPT OF SCIENCE, WASEDA UNIVERSITY, SHINJUKU KU, TOKYO 160, JAPAN
Tclephonc: (81) 22034141
Facsimile: (81)
Telex: 2323280 waseda $\mathbf{j}$

Commission(s): 40

Email:

DALGARNO ALEXANDER PROF, CENTER FOR ASTROPHYSICS, HCO/SAO, 60 GARDEN ST

CAMBRIDGE MA 02138, USA

Telephone: (1) 6174954403

Facsimile: (1)

Telex: $921+28$

Email: dalgarno@efa7

Commission(s): 14,34

DALLAPORTA N PROF, DPT DI ASTRONOMIA, UNIVERSITA DI PADOVA, VIC DELL OSSERVATORIO 5

I 35122 PADOVA, ITALY

Telephone: (39) 49661499

Facsimile: (39)

Telex:

Email:

Commission(s):

DALL'OGLIO GIORGIO DR, DPT DI FISICS, UNIVERSITA DI ROMA, PA MORO 2, I 00185 ROMA, ITALY

Telephone: (39) 649914271

Email: vaxma::dallaglio

Facsimile: (39) 64957697

Telex: 613255 infn ro

Commission(s): 09

DALTABUIT ENRIQUE DR, INSTITUTO DE ASTRONOMIA, UNAM, APDO POSTAL 70-264

04510 MEXICO DF, MEXICO

Telephone: (52)

Email:

Facsimile: (52)
Commission(s):

DALY RUTH AGNES PROF, DPT OF PHYSICS, PRINCETON UNIVERSITY, PRINCETON NJ 08544, USA

Telephone: (1) 6092584413 Facsimile: (1) 6092586853

Telex:

Email: daly@pupgg.princeton.cdu/4\$117::daly

Commission(s): 47

DAMINELI NETO AUGUSTO DR, IAG, UNIVERSIDADE DE SAO PAULO, CP 9638, 01065 SAO PAULO SP, BRAZIL Telephone: (55) 115778599 Facsimile: (55) 112763848 Telex: 1156735 iagm br

Email: Commission(s): 29

DAMLE S V DR, TIFR, HOMI BHABHA RD, COLABA, BOMBAY 400 005, INDIA

$\begin{array}{lll}\text { Telephone: (91) } 22219111 & \text { Facsimile: (91) } & \text { Telex: 0113009 tifr in } \\ \text { Email: } & \text { Commission(s): } 4+ & \end{array}$

DAN XHI-XIANG, SHANGHAI OBSERVATORY, CAS, 80 NANDAN RD, SHANGHAI 200030, CHINA PR

$\begin{array}{ll}\text { Telephonc: (86) } 21386191 & \text { Facsinile: (86) } \\ \text { Email: } & \text { Commission(s): 09 }\end{array}$

Telex: 33164 shao cn

DANBY J M ANTHONY DR, DPT OF MATHEMATICS, N CAROLINA STATE UNIV, RALEIGH NC 27695 8205, USA

Telephone: (1) 9197373210

Facsimilc: (1)

Telex:

Email:

Commission(s): 07

DANESE LUIGI DR, OSS ASTRONOMICO DI PADOVA, VIC DELL OSSERVATORIO 5, I 35122 PADOVA, ITALY

Telephone: (39) $49661499 \quad$ Facsimile: (39)

Email:

Commission(s): 47

Telex: +30176 unpadu-i

DANEZIS EMMANUEL DR, DPT OF ASTROPHYSICS, NTL UNIVERSITY OF ATHENS, PANEPISTIMIOPOLIS

GR 15783 ZOGRAFOS, GREECE

Telephone: (30) $172+3414$

Facsimile: (30)

Commission(s):

Telex:

Email: 
DANFORD STEPHEN C DR, DPT OF PHYSICS \& ASTRON, UNIVERSITY OF N CAROLINA, AT GREENSBORO GREENBORO NC 27412, USA

Telephone: (1) 9193345669

Email: danford@uncg.bitnet

Facsimile: (1) Telex:

Commission(s): $25,27,37$

DANIEL JEAN YVES, INSTITUT D'ASTROPHYSIQUE, 98BIS BD ARAGO, F 7501+ PARIS, FRANCE

Telephone: (33) 144328147

Facsimile: (33) 144328001 Telex:

Email:

Commission(s):

DANILOV VLADIMIR M DR, DPT OF ASTRONOMY, URALSKIJ STATE UNIVERSITY, 629983 SVERDLOVSK, RUSSIA

Telephonc: (7) 223386

Facsimile: (7)

Telex:

Email:

Commission(s):

DANKS ANTHONY C DR, 1315 PEACHTREE CT, BOWIE MD 20721, USA

Telephone: (1) 3012498206

Facsimile: (1)

Telex:

Email:

Commission(s): $15,28,3+$

DANLY LAURA DR, STSCI, HOMEWOOD CAMPUS, 3700 SAN MARTIN DR, BALTIMORE MD 21218, USA

Telephonc: (1) 3013384422

Facsimilc: (1) 3013385090

Telex: 6839101

Email: scivax::danly/danly@stsci

Commission(s): $3+$

DANZIGER I JOHN DR, ESO, KARL SCHWARZSCHILDSTR 2, D 85740 GARCHING MUENCHEN, GERMANY

Telephone: (49) 89320060

Facsimile: (49) 893202362

Telex:

Email:

Cómmission(s):

DAPERGOLAS A DR, ASTRONOMICAL INSTITUTE, NTL OBSERVATORY OF ATHENS, BOX 20048

GR 11810 ATHENS, GREECE

Telephone: (30) 13461191

Facsimile: (30)

Telex:

Email:

Commission(s): 37

DAPPEN WERNER, DPT PHYSICS \& ASTRONOMY, UNIV SOUTHERN CALIFORNIA

LOS ANGELES CA 900891342 , USA

Telephone: (1) $2137 \not 01316$

Facsimile: (1) $2137 \$ 06653$

Telex:

Email: wdappen 9 solar.stanford.cdu

Commission(s):

DARA HELEN DR, RES CENTER FOR ASTRONOMY, ACADEMY OF ATHENS, 14 ANAGNOSTOPOULOU ST GR 10673 ATHENS, GREECE

Telephone: (30) 13613589

Facsimile: (30)

Telex:

Email: cxakazo@grathun]

Commission(s): 12

DAS MRINAL KANTI, DPT PHYSICS/DELHI UNIV, SRI VENKATESWARA COLLEGE, DHAULA KUAN NEW DELHI 110 021, INDIA

Telephone: (91)

Facsimile: (91)

Telex:

Email:

Commission(s): 35

DAS P K DR, INDIAN INSTITUTE OF, ASTROPHYSICS, KORAMANGALA, BANGALORE 560 034, INDIA

Telephone: (91) 803566585

Facsimile: (91)

Tclex: $8+5763$ iiab in

Email:

Commission(s): 47

DATLOWE DAYTON DR, LOCKHEED PALO ALTO RES LB, DPT 9120 BLDG 255, 3251 HANOVER ST

PALO ALTO CA $9+304$, USA

Telephone: (1) $415858+074$

Facsimile: (1)

Tclex:

Email:

Commission(s): 10

DATTA BHASKAR DR, INDIAN INSTITUTE OF, ASTROPHYSICS, KORAMANGALA, BANGALORE 560 034, INDIA Telephonc: (91) 80356 6585/6497 Facsimile: (91)

Email:

Commission(s): 47

Telex: $8+5763$ iiab in

DAUBE-KURZEMNIECE I A DR, RADIOASTROPHYSICAL OBS, LATVIAN ACAD OF SCIENCES, TURGENEVA 19 $22652+$ RIGA, LATVIA

Telephone: (371) 226796

Email:

Facsimile: (371)

Telex:

Commission(s): 37

DAUTCOURT G DR, WIP-KAI BERLIN, HANS OTTO STR 17, D 1055 BERLIN, GERMANY

Telephone: (\$9) $30+296181$

Facsimile: (49)

Telex:

Email: 1000+1.273(a)compuserve.com

Commission(s): 4 
DAVID LAURENCE P DR, CENTER FOR ASTROPHYSICS, MS 4, 60 GARDEN ST, CAMBRIDGE MA 02138, USA

Telephone: (1) 6174957245

Facsimile: (1) 6174957356

Telex:

Email: david@cfa

Commission(s):

DAVID MARC, DPT MATH \& COMP SCI, GROENENBORGERLAAN 171, B 2020 ANTWERPEN, BELGIUM
$\begin{array}{lll}\text { Telephone: (32) } 32180355 & \text { Facsimile: (32) } & \text { Telex: } \\ \text { Email: david@ruca(g)ua.ac.be } & \text { Commission(s): }\end{array}$

DAVIDGE TIMOTHY J DR, HERZBERG INST ASTROPHYS, DOMINION ASTROPHYS OBS, 5071 W SAANICH RD VICTORIA BC V8X 4M6, CANADA

Telephone: (1) 6043630047

Facsimile: (1)

Telex:

Email: davidge@dao.nrc.ca

Commission(s): 28

DAVIDSEN ARTHUR FALNES DR, DPT PHYSICS \& ASTRONOMY, JOHNS HOPKINS UNIVERSITY CHARLES \& 3+TH ST, BALTIMORE MD 21218, USA

Telephone: (1) 3013387370

Facsimile: (1)

Email:

Commission(s): 28,44

Telex:

DAVIDSON KRIS DR, SCHOOL OF PHYS \& ASTRON, UNIVERSITY OF MINNESOTA, 116 CHURCH ST SE MINNEAPOLIS MN 55455, USA

Telephone: (1) 6123737795

Email: kd@aps2.spa.umn.cdu

Facsimile: (1)

Commission(s):

Telex:

DAVIDSON WILLIAM PROF, 80 WEST CLOSE, FERNHURST, HASLEMERE, SURREY GU27 3JT, UK

Telephone: $(\$+)$

Facsimile: $(4+4)$

Telex:

Email:

Commission(s): 44,47

DAVIES MERTON E MR, THE RAND CORPORATION, 1700 MAIN ST, SANTA MONICA CA 90406, USA

Telephone: (1) $3103930+11 * 7+28$

Facsimile: (1) $310+516960 \quad$ Telex:

Email: davies(ähyrax.span.nasa.gov

Commission(s): $04,16 \mathrm{C}$

DAVIES PAUL CHARLES W, SCHOOL OF PHYSICS, UNIVERSITY OF NEWCASTLE, NEWCASTLE/TYNE NE1 7RU, U

Telephone: (4)

Facsimile: (44)

Commission(s): +7

Telex:

Email:

DAVIES RODNEY D PROF, NRAL, JODRELL BANK, MACCLESFIELD SK11 9DL, UK

Telephonc: (44) 1\$77571321

Facsimile: (4t) 1477571618

Telex: $361+9$

Email: rdd@jb.man.ac.uk

Commission(s): $28,33,34,40$

DAVIES ROGER L DR, KITT PEAK NTL OBS, BOX 26732, 950 N CHERRY AVE, TUCSON AZ 85726 6732, USA

Telephonc: (1) 5203259353

Facsimilc: (1)

Commission(s): 47

Telex: 0666 484 aura noa

Email:

DAVILA JOSEPH DR, NASA GSFC, CODE 682.1, GREENBELT MD 20771, USA

Email: solar::idavila Commission(s): 10

DAVIS CECIL G JR, LOS ALAMOS NATIONAL LAB, MS D406, GROUP P-15, LOS ALAMOS NM 87545, USA

Trlephone: (1) 5056675908

Facsimilc: (1)

Telex:

Email:

Commission(s): 35,36

DAVIS JOHN PROF, SCHOOL OF PHYSICS, UNIVERSITY OF SYDNEY, A28, NSW 2006, AUSTRALIA

Telephone: (61) 26922544

Facsimile: (61) 26602903

Telex:

Email: davis@physics.su.oz.au

Commission(s): 09,50C

DAVIS JONATHAN IVOR, DPT PHYSICS \& ASTRONOMY, UNIV WALES COLLEGE, BOX 913, CARDIFF CF2 3YB, UK Telephone: (4) $122287+458$

Email: jid@astro.cf.ac.uk

Facsimile: (4t) $122287+056$ Telex:

Commission(s): 28,34

DAVIS LEVERETT JR PROF, 1772 N GRAND OAKS AVE, ALTADENA CA 91001, USA

Telcphonc: (1)

Facsimilc: (1)

Telex:

Email:

Commission(s): 44 
DAVIS MARC DR, ASTRONOMY DPT, UNIVERSITY OF CALIFORNIA, 601 CAMPBELL HALL BERKELEY CA 94720, USA

Telephone: (1) 4156425156

Facsimilc: (1)

Telex: 820181 ucb ast

Email:

Commission(s): 28,30,47

DAVIS MICHAEL M DR, ARECIBO OBSERVATORY, BOX 995, ARECIBO PR 00613, USA

Telephone: (1) $8098782612 \quad$ Facsimile: (1) Telex: 385638

Email: Commission(s): $40,44,47,51$

DAVIS MORRIS S PROF, DPT PHYSICS \& ASTRONOMY, UNIVERSITY NORTH CAROLINA, 204 PHILLIPS HALL 039 CHAPEL HILL NC 27514, USA

Telephone: (1) 9199623011

Facsimile: (1)

Telex:

Email:

Commission(s): 05,07

DAVIS RICHARD J DR, NRAL, JODRELL BANK, MACCLESFIELD SK11 9DL, UK

Telephone: (44)

Facsimile: $(4+)$

Telex:

Email:

Commission(s): 40C

DAVIS ROBERT J DR, CENTER FOR ASTROPHYSICS, HCO/SAO MS 20, G0 GARDEN ST, CAMBRIDGE MA 02138, USA

Telephone: (1) 6164957335

Facsimile: (1)

Commission(s): $05,30,40,44$

Telex: 921428 satellite cam

Email:

UNIVERSITY OF CALIFORNIA, BERKELEY CA 94720, USA

DAVIS SUMNER P DR, PHYSICS DPT, UNIVERSITY OF CALIFORNIA, BERKELE:
Telephone: (1) $4156+24857$
Facsimile: (1)

Email:

Commission(s): 14

DAVOUST EMMANUEL, OBS MIDI PYRENEES, $1+$ AVE E BELIN, F 31400 TOULOUSE CDX, FRANCE

Telephonc: (33) 61252101

Facsimilc: (33)

Telex: 530776

Email:

Commission(s):

DAWANAS DJONI N DR, DPT OF ASTRONOMY, BANDUNG INSTITUTE OF TECH, JL GANESHA 10 BANDUNG 40132, INDONESIA

Telephonc: (62) 6222440252

Facsimilc: (62)

Telex: $2832+$ itb bd

Email:

Commission(s): 29

DAWE JOHN ALAN DR, ANU, SIDING SPRING OBSERVATORY, PRIVATE BAG COONABARABRAN NSW 2857, AUSTRALIA

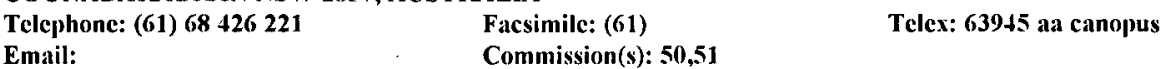

DE ALMEIDA AMAURY A DR, IAG, UNIVERSIDADE DE SAO PAULO, CP 9638, 01065 SAO PAULO SP, BRAZIL

Telephone: (55) $115778599 * 38 \quad$ Facsimile: (55) $112763848 \quad$ Telex: 1156735 iagm br

Email: 47556::amaury

Commission(s): 15

DE BERGH CATHERINE DR, OBSERVATOIRE DE PARIS, SECTION DE MEUDON, DPT DE RECHERCHE SPATIALE F 92195 MEUDON PPL CDX, FRANCE

Telephone: (33) $145077666 \quad$ Facsimile: (33) 145072806

Telex: 201571

Email: mesioa::debergh/deberghrämesiob.obspm.fr

Commission(s): 16VP

DE BIASE GIUSEPPE A DR, OAR, VIA DEL PARCO MELLINI 84, 100136 ROMA, ITALY

Telephonc: (39) $63+7056$

Facsimile: (39)

Telex:

Email:

Commission(s):

DE BOER KLAAS SJOERDS DR, ASTRONOMISCHES INSTITUT, UNIVERSITAET BONN, AUF DEM HUEGEL 71 D 53121 BONN, GERMANY

Telephone: (49) 228733656

Facsimile: (49)

Telex: $886+40$

Email:

Commission(s): $05,28,34$

DE BRUYN A GER DR, NFRA, BOX 2, NL 7990 AA DWINGELOO, NETHERLANDS

Telephone: (31) 52197244

Facsimilc: (31)

Telex:

Email:

Commission(s): 28

DE CARVALHO REINALDO DR, OBSERVATORIO NACIONAL, RUA GL BRUCE 586, SAO CRISTOVAO 20921 RIO DE JANEIRO RJ, BRAZIL

Telephone: (55) 215807181

Email: $47556:$ :rcinaldo
Facsimile: (55) 215800332

Commission(s): 28
Telex: 21288 


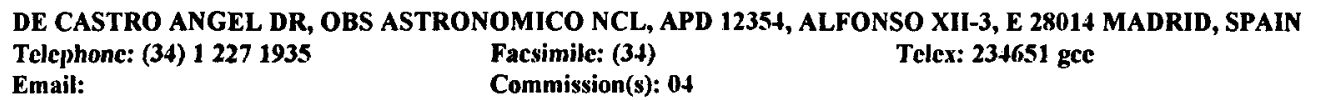

Email:

Commission(s): 04

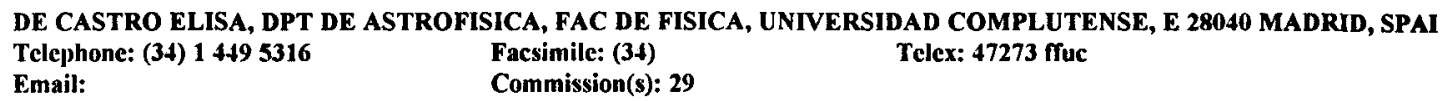

DE CUYPER JEAN-PIERRE M, STRERRENWACHT VAN BELIGIE, RINGLAAN 3, B 1180 BRUSSELS, BELGIUM

Telephonc: (32) 2373 0206:0211

Facsimilc: (32) $237+9822$

Telex: 21565 obsbel

Email: jeanpicras astro.oma.bcl

Commission(s):

DE FELICE FERNANDO DR, DPT DI FISICA G GALILEI, UNIVERSITA DI PADOVA, VIA MARZOLO 8

I 35131 PADOVA, ITALY

Telephone: (39) 49844278

Email:

Facsimile: (39)

Commission(s): 44

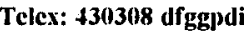

DE FREES DOUGLAS J DR, IBM ALMADEN RESEARCH CENT, DPT K 84/801, 650 HARRY ROAD

SAN JOSE CA 95120-6099, USA

Telephone: (1) 4089272854

Email: defrees(a)almvma

Facsimile: (1) $4089272100 \quad$ Telex:

Commission(s): 14

DE FREITAS PACHECO J A DR, IAG, UNIVERSIDADE DE SAO PAULO, CP 9638, 01065 SAO PAULO SP, BRAZIL

Telephonc: (55) 217173518

Facsimilc: (55) $1127638+8$

Telex: 1156735 iagm br

Email:

Commission(s):

DE GEUS EUGENE, CALTECH, MS 105 24, PASADENA CA 91125, USA

Telephone: (1) 8183956378

Email: edg(a)astro.caltcch.cdu

Facsimile: (1) $8185689352 \quad$ Telex:

Commission(s):

DE GOUVEIA DAL PINO E M, IAG, UNIVERSIDADE DE SAO PAULO, AV MIGUEL STEFANO 4200 04301904 SAO PAULO SP, BRAZIL

Tclephonc: (55) 115778599

Facsimile: (55) $1127638+8 \quad$ Telex: 1156735 iagmbr

Email: dalpino@astro2.iagusp.usp).br

Commission(s): 34

DE GRAAF T DR, INSTITUUT VOOR FONETISCHE, WETENSCHAPPEN, GROTE ROZENSTRAAT 31 NL 9712 TG GRONINGEN, NETHERLANDS

Telephone: (31)

Facsimile: (31)

Commission(s): 44

Telex:

Email:

DE GRAAFF W DR, APPELGAARDE 117, NL 3992 JD HOUTEN, NETHERLANDS
Telephonc: (31)
Facsimile: (31)

$\begin{array}{ll}\text { Telephonc: (31) } & \text { Commission(s): } 51\end{array}$

DE GRAAUW TH DR, SRON GRONINGEN, LANDLEVEN 12, BOX 800, NL 9700 AV GRONINGEN, NETHERLANDS

Telephone: (31) $5063+074$

Facsimile: (31) $5063+033 \quad$ Telex:

Email:

Commission(s):

DE GREVE JEAN-PIERRE DR, IAAG, VRIJE UNIV BRUSSELS, CP 165, B 1050 BRUSSELS, BELGIUM

Telephone: (32) 26413498

Facsimile: (32)

Telex: 61051 vubco

Email:

Commission(s): $35,+2$

DE GROOT MART DR, ARMAGH OBSERVATORY, COLLEGE HILL, ARMAGH BTG1 9DG, UK

Telephone: (44) 1861522928

Facsimile: $(+4)$

Telex: $7 \$ 7937$ armobs $g$

Email:

Commission(s): $27,29,42$

DE GROOT T DR, STERREKUNDIG INSTITUTE, BOX 80000, NL 3508 TA UTRECHT, NETHERLANDS

Telephone: (31) 30535200

Facsimile: (31)

Telex: 47224 astro

Email:

Commission(s): 40

DE JAGER CORNELIS PROF, SPACE RESEARCH LABORATORY, SRON, SORBONNELAAN 2

NL 358 4 CA UTRECHT, NETHERLANDS

Telephone: (31) 30535723

Facsimile: (31) 30540860

Telex: $\$ 7224$ sron $\mathbf{n l}$

Email: xmkecsjahutruuo.bitnet

Commission(s): $12,35,+0,44,49,51$ 
DE JAGER GERHARD PROF, DPT ELECTRICAL ENG, UNIVERSITY OF CAPE TOWN, PRIVATE BAG RONDEBOSCH 7700, SOUTH AFRICA

Telephone: (27) 216502791

Email: gerhard@eleceng.uct.ac.za

Facsimile: (27) $216503465 \quad$ Telex:

DE JAGER OCKER C DR, DPT OF PHYSICS, POTCHEFSTROOM UNIVERSITY POTCHEFSTROOM 2520, SOUTH AFRICA

Telephone: (27) $148992+18 \quad$ Facsimile: (27)

Email:

Commission(s):

Telex: $3+6019$

DE JONG TEIJE DR, ASTRONOMICAL INSTITUTE, UNIVERSITY OF AMSTERDAM, KRUISLAAN 403 NL 1098 SJ AMSTERDAM, NETHERLANDS

Telephone: (31) 205257491

Facsimile: (31) 205257484

Telex: 10262 hef $\mathrm{nl}$

Email:

Commission(s): 33,3+

DE JONGE J K DR, DPT OF ASTRONOMY, UNIVERSITY OF PITTSBURGH, RIVERVIEW PARK, PITTSBURGH PA 15214, USA

Tclephone: (1)

Facsimile: (1)

Telex:

Email:

Commission(s): 30,51

DE KOOL MARTHIJN, MPA, KARL SCHWARZSCHILDSTR 1, D $85+78$ GARCHING MUENCHEN, GERMANY

Facsimile: (49) $8932993235 \quad$ Telex:

Email: dekool@mpa-garching.mpg.de Commission(s):

DE KORT JULES J DR, HOUTLAAN 4, NL 6500 GV NIJMEGEN, NETHERLANDS

Telephone: (31) Facsimile: (31) Telex:

Email:

Commission(s): 42

DE KORTE PIETER A J DR, SPACE RESEARCH LABORATORY, SRON, SORBONNELAAN 2

NL 358 4 CA UTRECHT, NETHERLANDS

Telephone: (31) 30535600

Facsimile: (31) 30540860

Telex: $\$ 7224$

Email:

Commission(s):

DE LA HERRAN V JOSE ENG, INSTITUTO DE ASTRONOMIA, UNAM, APDO POSTAL 971

04510 MEXICO DF, MEXICO

Telephone: (52)

Facsimile: (52)

Telex:

Email:

Commission(s):

DE LA NOE JEROME DR, OBSERVATOIRE DE BORDEAUX, BP 89, F 33270 FLOIRAC, FRANCE

Telephone: (33) 56864330

Facsimile: (33) $5640+251$

Telex:

Email:

Commission(s): $28,34,40$

DE LA REZA RAMIRO DR, OBSERVATORIO NACIONAL, RUA GL BRUCE 586, SAO CRISTOVAO 20921 RIO DE JANEIRO RJ, BRAZIL

Telephone: (55) 215807313

Facsimile: (55) 215800332

Telex: 21288

Email:

Commission(s):

DE LAPPARENT-GURRIET V DR, INSTITUT D'ASTROPHYSIQUE, 98BIS BD ARAGO, F 75014 PARIS, FRANCE Telephone: (33) 144328000

Email: lapparen@friap51 Facsimile: (33) 1 \$4 $328001 \quad$ Telex:

Commission(s): 47

DE LOORE CAMIEL PROF, IAAG, VRIJE UNIV BRUSSELS, CP 165, B 1050 BRUSSELS, BELGIUM

Telephone: (32) $26+13496$

Facsimile: (32)

Telex: 61051 vubco b

Email:

Commission(s): $35,+2,51$

DE MEDEIROS JOSE RENAN DR, DPT DE FISICA, UNIVERSIDADE FEDERAL, DO RIO GRANDE DO NORTE 59072 970 NATAL, BRAZIL

Telephone: (55) 8+2319586

Facsimile: (55) $8+23197+9 \quad$ Telex:

Email: renan@dfte.ufrn.br

Commission(s): 35

DE PASCUAL MARTINEZ M DR, OBS ASTRONOMICO NCL, ALFONSO XII $3 \&$ 5 , E 2801+ MADRID, SPAIN

Telephone: (34) 12270107

Facsimile: (34)

Telex: 23475 igc

Email:

Commission(s): 20 
DE PATER IMKE, ASTRONOMY DPT, UNIVERSITY OF CALIFORNIA, GO1 CAMPBELL HALL BERKELEY CA 9\$720, USA

Telephone: (1) 4156421947

Email:

Facsimile: (1) Telex:

Commission(s): 15,16

DE ROBERTIS M M DR, DPT OF PHYSICS, YORK UNIVERSITY, 4700 KEELE ST

NORTH YORK ON M3J 1P3, CANADA

$\begin{array}{lll}\text { Telcphonc: (1) } 4167362100 * 7761 & \text { Facsimile: (1) } 4167365386 & \text { Telex: } 06524736 \\ \text { Email: fs300141 } Q \text { yosol.bitnet } & \text { Commission(s): } 28 & \end{array}$

DE ROP YVES, INSTITUT D'ASTROPHYSIQUE, 5 AVE DE COINTE, B 4000 LIEGE, BELGIUM

Telephone: (32) Facsimile: (32) Telex:

Email:

Commission(s):

DE RUITER HANS RUDOLF, IST DI RADIOASTRONOMIA, CNR, VIA IRNERIO 46, I 40126 BOLOGNA, ITALY

Telephone: (39) $51232856 \quad$ Facsimile: (39) Telex: 211664 infn bo i

Email:

Commission(s): 40,47

DE SABBATA V PROF DR, IST DI FISICA, UNIVERSITA DI BOLOGNA, VIA IRNERIO 46, I 40100 BOLOGNA, ITALY

Telephone: (39) $51260991{ }^{*} 051 \quad$ Facsimile: (39) Telex:

Email:

Commission(s):

DE SANCTIS GIOVANNI, OSS ASTRONOMICO DI TORINO, ST OSSERVATORIO 20, I 10025 PINO TORINESE, ITALY

Telephonc: (39) $118+1067 \quad$ Facsimile: (39)

Email:

Commission(s): 15,20

Telex: 213236 toastr $i$

DE SILVA L N K DR, DPT OF MATHEMATICS, UNIVERSITY OF COLOMBO, COLOMBO 03, SRI LANKA

Telephone: (94)

Facsimile: (94)

Commission(s): 28

Telex:

Email:

DE SOUZA RONALDO DR, IAG, UNIVERSIDADE DE SAO PAULO, CP 9638, 01065 SAO PAULO SP, BRAZIL

Telephone: (55) $115778599 \quad$ Facsimile: (55) $112763848 \quad$ Telex: 1156735 iagm br

Email:

Commission(s):

DE VAUCOULEURS GERARD PR, ASTRONOMY DPT, UNIVERSITY OF TEXAS, RLM 15212

AUSTIN TX 78712 1083, USA

Telephone: (1) 5124714461

Facsimile: (1)

Commission(s): $28,30,47$

Telex:

Email:

DE VEGT CH PROF DR, HAMBURGER STERNWARTE, GOJENSBERGSWEG 112, D 21029 HAMBURG, GERMANY

Telephone: (49) $\$ 072524128 / 4112$

Facsimile: (49) 4072524198

Telex: $\mathbf{2 1 7 8 8}$

Email:

Commission(s): 08,24C

DE VINCENZI DONALD DR, NASA AMES RESEARCH CTR, MS 245 1, MOFFETT FIELD CA 94035, USA

Telephone: (1)

Facsimile: (1)

Telex:

Email:

Commission(s): 51

DE VRIES CORNNELIS DR, SRL, HUYGENS LAB, BOX 9504, NL 2300 RA LEIDEN, NETHERLANDS

Telephone: (31) 71275816

Facsimile: (31)

Telex:

Email: sroncgihlerul2

Commission(s):

DE YOUNG DAVID S DR, KITT PEAK NTL OBS, BOX 26732, 950 N CHERRY AVE, TUCSON AZ 85726 6732, USA

Telephonc: (1) 5203275511

Facsimile: (1)

Telex:

Emait:

Commission(s): $40,+4$

DE ZEEUW PIETER T DR, SRL, HUYGENS LAB, BOX 9504, NL 2300 RA LEIDEN, NETHERLANDS

Telephonc: (31) $71275879 / 5832$

Facsimile: (31) 71275819

Tclex: 38058 astro $\mathrm{nl}$

Email: tim@arulhsy.Icidenuniv.nl

Commission(s): 28

DE ZOTTI GIANFRANCO DR, DPT DI ASTRONOMIA, UNIVERSITA DI PADOVA, VIC DELL OSSERVATORIO 5 I 35122 PADOVA, ITALY

Tclephone: (39) 49661499

Email:

Facsimile: (39)

Commission(s): 47

Telex: 430176 unpadu i 
DEARBORN DAVID PAUL S DR, LAWRENCE LIVERMORE LAB, L 23, BOX 808, LIVERMORE CA 94550, USA Telephone: (1) Facsimile: (1) Telex:

Email:

Commission(s): 35

DEBARBAT SUZANNE V DR, OBSERVATOIRE DE PARIS, G1 AVE OBSERVATOIRE, F 75014 PARIS, FRANCE

Telephone: (33) 140512209 Facsimile: (33) 143541804

Telex: 270776 obs of

Email: mesioaa::danof/danof@frmeu51

Commission(s): 08,19,41C

DEBEHOGNE HENRI DR SC, OBSERVATOIRE ROYAL DE, BELGIQUE, AVE CIRCULAIRE 3

B 1180 BRUSSELS, BELGIUM

Telephone: (32) 23743801

Facsimile: (32) $237+9822$

Telex: 21565 b

Email: henri@astro.ome.be

Commission(s): 15,20

DEBRUNNER HERMANN DR, PHYSIKALISCHES INSTITUT, UNIVERSITAET BERN, SIDLERSTRASSE 5

CH 3000 BERN, SWITZERLAND

Telephone: (41) 31654051

Facsimile: (41)

Telex: $32320 \mathrm{ch}$

Email:

Commission(s): 44

DEEMING TERENCE J DR, ICARUS RESEARCH, BOX 540205, HOUSTON TX 77254, USA

Telephone: (1) 7137728414

Facsimile: (1)

Telex:

Email:

Commission(s): 41

DEGAONKAR S S DR, PHYSICAL RESEARCH LAB, NAVRANGPURA, AHMEDABAD 380 009, INDIA

Telcphone: (91) 272462129

Facsimite: (91) $272+45292$

Telex: $121397 \mathrm{prl}$ in

Email:

Commission(s): 40

DEGENHARDT DETLEV, RECHENZENTRUM DER UNIV, HERMANN-HERDER STR 10

D 79104 FREIBURG, GERMANY

Tclephone: (49) 7612034648

Facsimile: (49) $761203+643 \quad$ Telex:

Email: degenhar@ruf.de

Commission(s): 12

DEGEWIJ JOHAN DR, FAHRENHEITSTRAAT 56 I, NL 1097 PT AMSTERDAM, NETHERLANDS

Telephone: (31)

Facsimile: (31)

Telex:

Email:

Commission(s): 15,16

DEGUCHI SHUJI DR, NOBEYAMA RADIO OBS, NAOJ, MINAMIMAKI MURA, NAGANO 38+ 13, JAPAN

Telephone: (81) 267982831

Facsimile: (81)

Telex: 3329005 taonro j

Email:

Commission(s): 3-

DEHANT VERONIQUE DR, OBSERVATOIRE ROYAL DE, BELGIQUE, AVE CIRCULAIRE 3 B 1180 BRUSSELS, BELGIUM

Email: veroniq(a)astro.oma.be/jupiter.oma.be

Telex: 21565 obshel b

Commission(s): 19C,31

DEHARVENG JEAN-MICHEL DR, LAS, TRAVERSE DU SIPHON, LES TROIS LUCS, F 13012 MARSEILLE, FRANCE

Telephone: (33) 91055900

Facsimile: (33) 91661855

Telex: $42058+f$

Email: jmd@frlasm51

Commission(s):

DEHARVENG LISE DR, OBSERVATOIRE DE MARSEILLE, 2 PLACE LE VERRIER F 13248 MARSEILLE CDX 04, FRANCE

Telephone: (33) 91959088

Facsimile: (33)

Email:

Commission(s): 34

Telex:

DEINZER W PROF DR, UNIVERSITAETS STERNWARTE, GOETTINGEN, GEISMARLANDSTR 11

D 37083 GOETTINGEN, GERMANY

Telephone: (49) $551395044 \quad$ Facsimile: (49) Telex: 96753

Email:

Commission(s): 35

DEISS BRUNO M DR, INST THEORETISCHE PHYSIK, UNIVERSITAET FRANKFURT, ROBERT MAYER STR 8-10 D 6005 4 FRANKFURT A M, GERMANY
Telephone: (49) 697982636
Facsimile: (49) 697988350
Telex:

Email: dciss@astro.uni-frankfurt.d400.de Commission(s): 3+

DEJAIFFE RENE J DR, OBSERVATOIRE ROYAL DE, BELGIQUE, AVE CIRCULAIRE 3, B 1180 BRUSSELS, BELGIUM

Telephone: (32) 2375 248-

Email: rjd@astro.ome.be
Facsimile: (32) $237+9822$

Commission(s): 08,19 
DEJONGHE HERWIG BERT DR, STERREKUNDIG OBSERV, RIJKSUNIVERSITEIT GENT, KRIJGSLJAN 281 B 9000 GENT, BELGIUM

Telephone: (32) 92644761

Email: herwig.dejonghe@rug.ac.be
Facsimile: (32) 91644989

Commission(s): $28,33,37$
Telex: 12754 rugent b

DEKEL AVISHAI, DPT OF PHYSICS, HEBREW UNIV OF JERUSALEM, JERUSALEM 91904, ISRAEL

Telephone: (972) 2584605

Facsimile: (972)

Telex:

Commission(s): $28,33,47$

DEKKER E DR, MEIDOORNLAAN 13, NL 3461 ES LINSCHOTEN, NETHERLANDS

Telephone: (31) $348015406 \quad$ Facsimile: (31) Telex:

Email: $\quad$ Commission(s): 41

DEL OLMO OROZCO A DR, INST ASTROFISICA, DE ANDALUCIA APD 3004, C/SANCHO PANZA S/N E 18080 GRANADA, SPAIN

$\begin{array}{lll}\text { Telephone: (34) } 58121311 & \text { Facsimile: (34) } 58814530 & \text { Telex: } 78573 \text { iaag c } \\ \text { Email: chony@iaa.es/16488::chony } & \text { Commission(s): } 28\end{array}$

DEL RIO GERARDO DR, OBS ASTRONOMICO NCL, ALFONSO XII-3, E 28014 MADRID, SPAIN

Telephone: (34) $12270107 / 1935$ Facsimilc: (34) Telex: 23465 igce

Email:

Commission(s):

DEL TORO INIESTA JOSE DR, INST DE ASTROFISICA, DE CANARIAS, OBS DEL TEIDE

E 38200 LA LAGUNA, SPAIN

Telephone: (34) $22262211 \quad$ Facsimile: (34) $922263005 \quad$ Telex: 92640 iace

Email: span:iac::jti

Commission(s): 10,12

DELABOUDINIERE J.-P., IAS, BAT 121, UNIVERSITE PARIS XI, F 91405 ORSAY CEDEX, FRANCE

Telephone: (33) $169858625 / 8425 \quad$ Facsimile: (33) 169858675 Telex: 600252

Email: iaslab::boudine

Commission(s):

DELANNOY JEAN DR, IRAM, 300 RUE DE LA PISCINE, F 38406 S MARTIN HERES CD, FRANCE

Telejhone: (33) $76423383 \quad$ Facsimilc: (33) Telex: 980753

Email:

Commission(s): 40

DELBOUILLE LUC PROF, INSTITUT D'ASTROPHYSIQUE, UNIVERSITE DE LIEGE, AVE COINTE 5

B 4000 COINTE-LIEGE, BELGIUM

Telephone: (32) 41529980

Email:

Facsimile: (32) 41527474

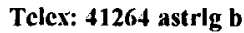

DELCROIX ANDRE J S DR, 19A RUE E VANDERVELDE, B 7230 FRAMERIES, BELGIUM

Telephone: (32)

Facsimile: (32)

Telex:

Email:

Commission(s):

DELGADO ANTONIO JESUS, INST ASTROFISICA, DE ANDALUCIA APD 3004, PROFESOR ALBAREDA 1 E 18080 GRANADA, SPAIN

$\begin{array}{lll}\text { Telephone: (34) } 58121300 & \text { Facsimile: (34) } & \text { Telex: } 78573 \text { iaag e } \\ \text { Email: } & \text { Commission(s): } 27,42 & \end{array}$

DELHAYE JEAN PROF, 2 RUE DE LA PLEIADE, F $9 \$ 240$ L'HAY LES ROSES, FRANCE

Telephone: (33) $1466+5771$ Facsimile: (33) Telex:

Email: $\quad$ Commission(s): 24,33

DELIYANNIS JEAN DR, DPT OF ASTROPHYSICS, NTL UNIVERSITY OF ATHENS, PANEPISTIMIOPOLIS GR 15783 ZOGRAFOS, GREECE

$\begin{array}{lll}\text { Telephone: (30) } 17243414 & \text { Facsimile: (30) } 17228981 & \text { Telex: } \\ \text { Email: yannis(g)grathun1 } & \text { Commission(s): } 12\end{array}$

DELLA VALLE MASSIMO DR, DPT DI ASTRONOMIA, UNIVERSITA DI PADOVA, VIC DELL OSSERVATORIO 5 I 35122 PADOVA, ITALY

Telephone: (39) 49661499

Email: infnct:39003::dellavalle

Facsimile: (39)

Commission(s):

Telex: $\mathbf{4 3 2 0 7 1}$

DELLI SANTI SAVERIO, OSS ASTRONOMICO, UNIVERSITA DI BOLOGNA, CP 596, I 40100 BOLOGNA, ITALY

Telephone: (39) 51222956

Facsimile: (39)

Email:

Commission(s):

Telex: $21166+$ infnbo $i$ 
M 502

DENNIS BRIAN ROY DR, NASA GSFC, CODE 682, GREENBELT MD 20771, USA

Telephone: (1) 3012866604

Facsimile: (1)

Telex: 89675

Email:

Commission(s): 10,44

DENNISON EDWIN W DR, 985 CYNTHIA AVE, PASADENA CA 91107, USA

Telephone: (1) $8183518751 \quad$ Facsimile: (1) Telex:

Email:

Commission(s):

DENNISON P A DR, TRINITY HALL, UNIVERSITY OF CAMBRIDGE, CAMBRIDGE CB3, UK

Telephone: (44)

Facsimile: (44)

Telex;

Email:

Commission(s):

DENOYELLE JOZEF KIC, OBSERVATOIRE ROYAL DE, BELGIQUE, AVE CIRCULAIRE 3, B 1180 BRUSSELS, BELGIUM

Telephone: (32) 23752484

Facsimile: (32) 23749822

Telex: 21565 obsbel

Email: jozef@astro.ome.be

Commission(s): 25,33

DENT WILLIAM A PROF, DPT PHYSICS \& ASTRONOMY, UNIV OF MASSACHUSETTS, GRC B, AMHERST MA 01003, USA

Telephone: (1) 4135453665

Facsimile: (1)

Telex:

Email:

Commission(s): 40

DEPRIT ANDRE PROF, NTL BUREAU OF STANDARDS, APPLIED MATHEMATICS CTR, GAITHERSBURG MD 20899, USA

Telephone: (1) 3019212631

Facsimile: (1)

Telex:

Email:

Commission(s): 04,07

DERE KENNETH PAUL, NAVAL RESEARCH LABORATORY, CODE $\$ 163,4555$ OVERLOOK AVE SW, WASHINGTON DC 20375 5000, USA

Telephone: (1) $2027672517 \quad$ Facsimile: (1) Telex:

Email: $\quad$ Commission(s): 10

DERMAN I ETHEM DR, DPT OF ASTRONOMY, UNIVERSITY OF ANKARA, FEN FAKULTESI, OG100 BESEVLER, TURKEY

Telephone: (90) 4123 6550/0109 Tacsimile: (90) Telex:

Email:

Commission(s):

DERMENDJIEV VLADIMIR DR, DPT OF ASTRONOMY, BULGARIAN ACAD SCIENCES, 72 LENIN BLVD, BG 1784 SOFLA, BULGARIA

Telephone: (359) 2758927

Email:

Facsimile: (359)

Telex: 23561 ecf ban bg

Commission(s): 10

DERMOTT STANLEY F, DPT OF ASTRONOMY, UNIVERSITY OF FLORIDA, 224 SSRB, GAINESVILLE FL 32611, USA

Telephone: (1) 9043922361

Facsimile: (1) $9043929605 \quad$ Telex:

Email: dermott@jupiter.astro.ufl.edu

Commission(s): 15,16,21

DESAI JYOTINDRA N, PHYSICAL RESEARCH LAB, NAVRANGPURA, ROOM 763, AHMEDABAD 380 009, INDIA

Telephone: (91) 272462129

Facsimile: (91) $272445292 \quad$ Telex: 121397

Email:

Commission(s): 09

DESESQUELLES JEAN DR, UNIVERSITE LYON 1, CAMPUS LA DOUA, 43 BD DU 11 NOVEMBRE, F 69621

VILLEURBANNE, FRANCE

Telephone: (33) $78898124 \quad$ Facsimile: (33)

Email:

Commission(s): 14

Telex: 380273 ipn f

DESHPANDE AVINASH, RAMAN RESEARCH INSTITUTE, SADASHIVANAGAR, CV RAMAN AVE, BANGALORE S60 080, INDIA

Telephone: (91) $803360122 \quad$ Facsimile: (91) $803340492 \quad$ Telex: 8452671 rri in

Email: desh@rri.ernet.in

Commission(s): 40

DESHPANDE M R DR, PHYSICAL RESEARCH LAB, NAVRANGPURA, AHMEDABAD 380 009, INDIA

Tclephone: (91) 272462129

Facsimilc: (91) $2724+5292$

Telex: $121397 \mathrm{prl}$ in

Email:

Commission(s): 25 
DESPAIN KEITH HOWARD DR, LOS ALAMOS NATIONAL LAB, MS B220 X 2, BOX 1663, LOS ALAMOS NM 87545, US Telęphone: (1) 5056672388

Facsimile: (1)

Telex:

Email:

Commission(s): 35

DESPOIS DIDIER DR, OBSERVATOIRE DE BORDEAUX, BP 89, F 33270 FLOIRAC, FRANCE

Telephone: (33) 56864330

Facsimile: (33) $56404251 \quad$ Telex:

Email: earn:despois@rrobor51

Commission(s): 40

DETTMAR RALF-JUERGEN DR, ASTRONOMISCHES INSTITUT, RUHR UNIVERSITAET BOCHUM, POSTFACH 102148 , D44780 BOCHUM, GERMANY

$\begin{array}{lll}\text { Telephone: (49) } 2347003454 & \text { Facsimile: (49) } 2347094169 & \text { Telex: } \\ \text { Email: dettmar@astro.uni-bonn.de } & \text { Commission(s): } 28 & \end{array}$

DEUBNER FRANZ-LUDWIG DR, ASTRONOMISCHES INSTITUT, DER UNIVER WUERZBURG, AM HUBLAND, D 9707 WUERZBURG, GERMANY

Telephone: (49) 9318885030

Facsimile: (49) 9318884603

Telex: 68671 uniwbg d

Email: deubner@astro.uni-wuerzburg.de Commission(s): 10,12P

DEUL ERIK DR, STERREWACHT, BOX 9513, NL 2300 RA LEIDEN, NETHERLANDS

Telephone: (31) 71275880

Facsimile: (31) 71275819

Telex: 39058 astro $\mathrm{nl}$

Email: duel@hleruls1/rulhl1::deul

Commission(s):

DEUPREE ROBERT G DR, LOS ALAMOS NATIONAL LAB, MS F665 ESS 5, BOX 1663, LOS ALAMOS NM 87545, USA

Telephone: (1) 5056678215

Facsimile: (1)

Telex:

Email:

Commission(s): 27,35

DEUTSCHMAN WILLIAM A DR, OREGON LASER CONSULTANTS, 455 HILLSIDE AVE, KLAMATH FALLS OR 97601, USA

Telephone: (1) 5038823295

Facsimile: (1)

Telex:

Email:

Commission(s): 15

DEVINNEY EDWARD J DR, 100 UNION AVE, DELANCO NJ 08075, USA

Telephone: (1) $6097641250 \quad$ Facsimile: (1) Tclex:

Email: $\quad$ Commission(s):

DEVORKIN DAVID H, NTL AIR \& SPACE MUSEUM, SMITHSONIAN INSTITUTION, ROOM 3530, WASHINGTON DC 20560, USA

Telephone: (1) 2023572828

Facsimile: (1)

Telex:

Email:

Commission(s): 41

DEWDNEY PETER E F DR, DOMINION RADIO ASTROPHYS, OBSERVATORY, BOX 248, PENTICTON BC V2A 6K3, CANADA

Telephonc: (1) 6044932277

Facsimile: (1) $6044937767 \quad$ Telex: 04888127

Email:

Commission(s): 34,40

DEWEY RACHEL J, DPT OF PHYSICS, PRINCETON UNIVERSITY, JADWIN HALL, PRINCETON NJ 08544, USA

Telephone: (1) 6092584365

Facsimile: (1) $6092586853 \quad$ Telex:

Email: rachel@pulsar.princeton.edu Commission(s):

DEWHIRST DAVID W DR, INSTITUTE OF ASTRONOMY, THE OBSERVATORIES, MADINGLEY RD, CAMBRIDGE

CB3 OHA, UK

Telephone: (44) 1233337548

Facsimile: (44) $1223337523 \quad$ Telex: 817297 astron g

Email:

Commission(s): 05,41

DEWITT BRYCE S DR, ASTRONOMY DPT, UNIVERSITY OF TEXAS, RLM 15308 , AUSTIN TX 78712 1083, USA

Telephone: (1) 5124715055

Facsimilc: (1)

Telex: 9108741305

Email:

Commission(s): 44

DEWITT JOHN H JR, 3602 HOODS HILL RD, NASHVILLE TN 37215, USA

Telephone: (1) 6153838272

Facsimile: (1)

Commission(s):

Telex:

Email:

DEWITT-MORETTE CECILE PR, DPT OF PHYSICS, UNIVERSITY OF TEXAS, RLM 15 308, AUSTIN TX 78712 1083, US

Telephone: (1) 5124711052

Facsimile: (1)

Telex: 910-8741305

Email:

Commission(s): 
DESPAIN KEITH HOWARD DR, LOS ALAMOS NTL LAB, MS B220 X 2, BOX 1663, LOS ALAMOS NM 87545, USA Telephone: (1) 5056672388 Facsimile: (1) Telex:

Email:

Commission(s): 35

DESPOIS DIDIER DR, OBSERVATOIRE DE BORDEAUX, BP 89, F 33270 FLOIRAC, FRANCE

Telephone: (33) 56864330

Facsimile: (33) $5640+251 \quad$ Telex:

Email: earn:despois@frobor51

Commission(s): 40

DETTMAR RALF-JUERGEN DR, ASTRONOMISCHES INSTITUT, RUHR UNIVERSITAET BOCHUM
$\begin{array}{lll}\text { POSTFACH 102148, D } 44780 \text { BOCHUM, GERMANY } & \\ \text { Telephone: (49) } 2347003454 & \text { Facsimile: (49) } 2347094169 & \text { Telex: } \\ \text { Email: dettmar@astro.uni-bonn.de } & \text { Commission(s): } 28 & \end{array}$

DEUBNER FRANZ-LUDWIG DR, ASTRONOMISCHES INSTITUT, DER UNIVER WUERZBURG, AM HUBLAND D 97074 WUERZBURG, GERMANY

Telephone: (49) $9318885030 \quad$ Facsimile: (49) $9318884603 \quad$ Telex: 68671 uniwbg d

Email: deubner@astro.uni-wuerzburg.de Commission(s): 10,12P

DEUL ERIK DR, STERREWACHT, BOX 9513, NL 2300 RA LEIDEN, NETHERLANDS

$\begin{array}{lll}\text { Telephone: (31) } 71275880 & \text { Facsimile: (31) } 71275819 & \text { Telex: 39058 astro nl } \\ \text { Email: duel@hlerul51/rulhl1::deul } & \text { Commission(s): }\end{array}$

DEUPREE ROBERT G DR, LOS ALAMOS NATIONAL LAB, MS F665 ESS 5, BOX 1663, LOS ALAMOS NM 87545, USA Telephone: (1) 5056678215 Facsimile: (1) Telex:

Email: Commission(s): 27,35

DEUTSCHMAN WILLIAM A DR, OREGON LASER CONSULTANTS, 455 HILLSIDE AVE

KLAMATH FALLS OR 97601, USA

$\begin{array}{ll}\text { Telephone: (1) } 5038823295 & \text { Facsimile: (1) } \\ \text { Email: } & \text { Commission(s): } 15\end{array}$

DEVINNEY EDWARD J DR, 100 UNION AVE, DELANCO NJ 08075 , USA

$\begin{array}{lll}\text { Telephone: (1) } 6097641250 & \text { Facsimile: (1) } & \text { Telex: } \\ \text { Email: } & \text { Commission(s): }\end{array}$

DEVORKIN DAVID H, NTL AIR \& SPACE MUSEUM, SMITHSONIAN INSTITUTION, ROOM 3530 WASHINGTON DC 20560, USA

Telephone: (1) $2023572828 \quad$ Facsimile: (1) Telex:

Email:

Commission(s): 41

DEWDNEY PETER E F DR, DOMINION RADIO ASTROPHYS, OBSERVATORY, BOX 248

PENTICTON BC V2A 6K3, CANADA

Telephone: (1) $6044932277 \quad$ Facsimile: (1) $60+4937767 \quad$ Telex: 04888127

Email:

Commission(s): 34,40

DEWEY RACHEL J, DPT OF PHYSICS, PRINCETON UNIVERSITY, JADWIN HALL, PRINCETON NJ 08544, USA

Telephone: (1) 6092584365 Facsimile: (1) $6092586853 \quad$ Telex:

Email: rachel@pulsar.princeton.edu Commission(s):

DEWHIRST DAVID W DR, INSTITUTE OF ASTRONOMY, THE OBSERVATORIES, MADINGLEY RD

CAMBRIDGE CB3 OHA, UK

Telephone: (44) 1233337548

Email:

Facsimile: (4+) 1223337523

Telex: 817297 astron $g$

DEWITT BRYCE S DR, ASTRONOMY DPT, UNIVERSITY OF TEXAS, RLM 15308 , AUSTIN TX 78712 1083, USA

Telephone: (1) 5124715055

Facsimile: (1)

Telex: $91087+1305$

Email:

Commission(s): tt

DEWITT JOHN H JR, 3602 HOODS HILL RD, NASHVILLE TN 37215, USA

Telephone: (1) 6153838272

Facsimile: (1)

Commission(s):

Telex:

Email:

DEWITT-MORETTE CECILE PR, PHYSICS DPT, UNIVERSITY OF TEXAS, RLM 15 308, AUSTIN TX 78712 1083, USA Telephone: (1) 5124711052

Facsimile: (1)

Email:

Commission(s)

Telex: $910-87+1305$ 
DEZSO LORANT PROF, HELIOPHYSICAL OBSERVATORY, BOC 30, H 4010 DEBRECEN, HUNGARY

Telephone: (36) 52311015

Facsimile: (36)

Telex: 72517 deobs I

Email:

Commission(s): 10,12

DHAWAN VIVEK DR, NRAO, BOX 0, SOCORRO NM 87801, USA

Telephone: (1) $5058357378 \quad$ Facsimile: (1) 5058357027

Email: vdhawan@inrao.edu

Commission(s): 40

Telex:

DHILLON VIKRAM SINGH, ROYAL GREENWICH OBS, APDO 321, SANTA CRUZ DE LA PALMA

E 38780 TENERIFE, SPAIN

Telephone: (34) 22405500

Email: vsd@ing.iac.es

Facsimile: (34) $22405646 \quad$ Telex:

Commission(s):

DI COCCO GUIDO, IST TESRE, CNR, VIA DE CASTAGNOLI 1, I 40126 BOLOGNA, ITALY

Telephone: (39) $51519593 \quad$ Facsimile: (39) Telex: $511350 \mathrm{cnr}$ bo $\mathrm{i}$

Email:

Commission(s): 44

DI FAZIO ALBERTO, OAR, VIA DEL PARCO MELLINI 84, I 00136 ROMA, ITALY

Telephone: (39) 6347056

Facsimile: (39)

Telex: 613103 pprmt i

Email:

Commission(s): 28,34,37

DI MARTINO MARIO, OSS ASTRONOMICO DI TORINO, ST OSSERVATORIO 20, I 10025 PINO TORINESE, ITALY

Telephone: (39) 11841067

Facsimile: (39)

Commission(s): 15

Telex: 213236 to astr $i$

Email:

DI SEREGO ALIGHIERI S DR, OSS ASTROFISICO, DI ARCETRI, LARGO E FERMI 5, I 50125 FIRENZE, ITALY

Telephone: (39) 554378540

Facsimile: (39) 55435939

Telex:

Email: astrfi::sperello

Commission(s): 28

DI TULLIO GRAZIELLA DR, OSS ASTRONOMICO DI PADOVA, VIC DELL OSSERVATORIO 5

I 35122 PADOVA, ITALY

Telephone: (39) 49661499

Email:

Facsimile: (39)

Commission(s):

Telex:

DI XIAO-HUA, PURPLE MOUNTAIN OBSERV, CAS, NANJING, CHINA PR

Telephone: (86) $2537609 \quad$ Facsimile: (86) $25301459 \quad$ Telex: 34144 pmonj cn

Email:

Commission(s): 04

DLALETIS DIMITRIS DR, ASTRONOMICAL INSTITUTE, NTL OBSERVATORY OF ATHENS, BOX 20048

GR 11810 ATHENS, GREECE

Telejhone: (30) 13461191

Facsimile: (30)

Commission(s): 10

Telex: 215530 obsa gr

Email:

DIAMOND PHILIP JOHN DR, NRAO, BOX 0, SOCORRO NM 87801 0387, USA
$\begin{array}{llll}\text { Telephone: (1) } 5058357900 & \text { Facsimile: (1) } & \text { Telex: } 9109881710\end{array}$

Email: pdiamond@nrao.edu Commission(s):

DIAZ ANGELES ISABEL DR, DPT DE FISICA TEORICA, C-XI UNIVERSIDAD AUTONOMA DE MADRID CANTOBLANCO, E 28049 MADRID, SPAIN

Telephone: (34) $13974223 \quad$ Facsimile: (34)

Telex: 27810

Email: bitnet:adiaz@emduam11 Commission(s): 28

DICK STEVEN $J$, US NAVAL OBSERVATORY, 3450 MASSACHUSETTS AVE NW, WASHINGTON DC 20392 5100, USA

Telephone: (1) 2026531641

Facsimile: (1) 2026531497

Telex:

Email: dick@aricl.usno.navy.mil

Commission(s): $08,41 \mathrm{VP}, 51$

DICK WOLFGANG DR, INST F ANGEW GEODAESIE, AUSSENSTELLE POTSDAM, POSTFACH 600808

D 14408 POTSDAM, GERMANY

Telephone: (49) 331316619

Email: wdi@potsdam.ifag.de

Facsimile: (49) $331316602 \quad$ Telex:

Commission(s): 24,41

DICKE ROBERT H PROF, DPT OF PHYSICS, PRINCETON UNIVERSITY, JOSEPH HENRY LABS

PRINCETON NJ 08544, USA

Telephone: (1) 6094524317

Facsimile: (1)

Telex:

Email:

Commission(s): 44,47 
DICKEL HELENE R DR, 103 UI ASTRONOMICAL BLDG, 1002 WEST GREEN ST, URBANA IL 61801, USA Telephone: (1) 2172447044 Facsimile: (1) 2172447638 Telex:

Email: Ianie@astro.uiuc.edu Commission(s): 05,33,34,40C

DICKEL JOHN R, 103 UI ASTRONOMICAL BLDG, 1002 WEST GREEN ST, URBANA IL 61801, USA Telephone: (1) 2173335532

Email: johnd@astro.uiuc.edu Facsimile: (1) 2172447638 Telex: 9102452434 purch

DICKENS ROBERT J DR, RUTHERFORD APPLETON LAB, SPACE \& ASTROPHYSICS DIV, BLDG R25/R68 CHILTON DIDCOT OX11 0QX, UK

Telephone: (44) 123521900

Facsimile: (44)

Telex: 83159

Email:

Commission(s): $27,28,37$

DICKEY JEAN O'BRIEN, JPL CALTECH, MS 138 208, 4800 OAK GROVE DR, PASADENA CA 91109, USA Telephone: (1) 8183543235 Facsimile: (1) 8183936980 Telex: 675429

Email:

Commission(s): 04,16,19C,31

DICKEY JOHN M, DPT OF ASTRONOMY, UNIVERSITY OF MINNESOTA, 116 CHURCH ST SE MINNEAPOLIS MN 55455, USA

Telephone: (1) 6123733308

Facsimile: (1)

Telex:

Email: john@ast1.spa.umn.edu

Commission(s): $28,34,40$

DICKINSON DALE F DR, LOCKHEED PALO ALTO RES LB, DPT 9220 BLDG 205, 3251 HANOVER ST PALO ALTO CA 94304, USA

Telephone: (1) 4154242701

Facsimile: (1)

Telex:

Email:

Commission(s):

DICKMAN ROBERT L DR, US NTL SCIENCE FOUNDATION, DIV OF ASTRONOMICAL SCI, 1800 G ST NW WASHINGTON DC 20550, USA

Telephone: (1)

Facsimile: (1)

Telex:

Email:

Commission(s): 33,40

DICKMAN STEVEN R, DPT GEOLOGICAL SCIENCES, STATE UNIV OF NEW YORK, BINGHAMTON NY 13901, USA

Telephone: (1) 6077774378

Facsimile: (1)

Telex:

Email:

Commission(s): 19C

DIEGO FRANCISCO DR, DPT PHYSICS \& ASTRONOMY, UNIVERSITY COLLEGE LONDON, GOWER ST

LONDON WC1E 6BT, UK

Telephone: (44) $1713877050 * 3512$

Facsimile: (44) 1713807145

Telex: 28722

Email: fd@uk.ac.ucl.starlink

Commission(s): 09

DJERCKSEN GEERD H F PH D, MPI F PHYSIK \& ASTROPHYS, KARL SCHWARZSCHILDSTR 1

D 85740 GARCHING MUENCHEN, GERMANY

Telephone: (49) $8932990 \quad$ Facsimile: (49)

Email:

Commission(s): 14

Telex: 524629 astro d

DIETER NANNIELOU H DR, CLAY RD, N THERFORD VT 05054, USA

Telepbone: (1) 8023334079

Facsimile: (1)

Telex:

Email:

Commission(s): 33,40

DIKOVA SMILIANA D, DPT OF ASTRONOMY, BULGARIAN ACAD SCIENCES, 72 LENIN BLVD BG 1784 SOFIA, BULGARIA

Telephone: (359) 2758927

Email: dinblag@dbearn.bitnet
Facsimile: (359)

Commission(s): 07
Tclex: 23561 ecf ban

DIMITRIJEVIC MILAN, ASTRONOMICAL OBSERVATORY, VOLGINA 7 YU 11050 BEOGRAD YUGOSLAVIA-SERBIA \& MONTENEGRO

Telephone: (38) 11419 357/421 $875 \quad$ Facsimile: (38) Telex:

Email: eaop021@yubgss21.bitnet Commission(s): 14,36

DINERSTEIN HARRIET L, ASTRONOMY DPT, UNIVERSITY OF TEXAS, RLM 15 308, AUSTIN TX 78712 1083, USA Telephone: (1) 5124713449

Facsimile: (1) Tclex: 910-874-1351

Email: 
DINESCU A DR, ASTRONOMICAL INSTITUTE, CUTITUL DE ARGINT 5, BOX 28, R 75212 BUCHAREST, RUMANIA

Telephone: (40) 16236892

Facsimile: (40) 13123391

Telex: 11882 astro $r$

Email:

Commission(s):

DING MING DE, DPT OF ASTRONOMY, NANJING UNIVERSITY, NANJING 210008, CHINA PR

Telephone: (86) $2566375512882 \quad$ Facsimile: (86) $253302728 \quad$ Telex: 34151 prenu cn

Email:

Commission(s): 10,12

DING YOU-JI, YUNNAN OBSERVATORY, CAS, BOX 110, KUNMING 72946 YUNNAN, CHINA PR

Telephone: (86) $8712035 \quad$ Facsimile: (86)

Email:

Commission(s): 10

Telex: 64040 yuobs $\mathrm{cn}$

DINTINJANA BOJAN DR, ASTRONOMICAL OBSERVATORY, UNIVERSITY OF E KARDELJ, JADRANSKA 19

LJUBLJANA, SLOVENIA

Telephone: (38) 61265061

Facsimile: (38) $61217281 \quad$ Telex:

Email: bojan.dintinjana@uni-lj.ac.mai

Commission(s):

DINULESCU SIMONA, CALEA 13 SEPT 126, BL P34 SC L ET 3 AP 11, R 76125 BUCHAREST S5, RUMANIA

Telephone: (40)

Facsimile: (40)

Telex:

Email:

Commission(s): 10,49

DIONYSIOU DEMETRIOS PROF, HELLENIC AIR-FORCE ACAD, DEKELIA-ATTICA, 18 AMASSIAS STR

GR 11634 ATHENS, GREECE

$\begin{array}{lll}\text { Telephone: (30) } 17238436 & \text { Facsimile: (30) } & \text { Telex: }\end{array}$

Email:

Commission(s): 47

DISNEY MICHAEL J PROF, DPT OF PHYSICS, UNIV WALES COLLEGE, BOX 913, CARDIFF CF1 3TH, UK

Telephone: (44) 1222874785

Facsimile: (44) $1222371921 \quad$ Telex: 498635

Email:

Commission(s): 34,44

DIVAN LUCIENNE DR, INSTITUT D'ASTROPHYSIQUE, 98BIS BD ARAGO, F 75014 PARIS, FRANCE

Telephone: (33) 144328000

Facsimile: (33) 144328001

Telex:

Email:

Commission(s): 29,45

DIVARI N B DR, ODESSA POLYTECHNICAL INST, 270044 ODESSA, UKRAINE

Telephone: (7) Facsimile: (7) Telex:

Email: $\quad$ Commission(s):

DIVER DECLAN ANDREW, DPT PHYSICS \& ASTRONOMY, UNIVERSITY OF GLASGOW, GLASGOW G12 8QQ, UK Telephone: (44) 1413398855 *5686 Facsimile: (44) 1413349029 Telex: 777070 unigla

Email: diver@astro.gla.ac.uk Commission(s): 12

DIXON ROBERT S DR, RADIO OBSERVATORY, OHIO STATE UNIVERSITY, 2015 NEIL AVE

COLUMBUS OH 43210, USA

Telephone: (1) 6144226789

Facsimile: (1)

Telex:

Email:

Commission(s): $05,40,51$

DIZER MUAMMER PROF, KANDILLI OBSERVATORY, BOGAZICI UNIVERSITY, CENGELKOV

81220 ISTANBUL, TURKEY

Telephone: (90) 13320277

Email:

Facsimile: (90)

Telex:

Commission(s): 10

DJORGOVSKI STANISLAV DR, CALTECH, MS 105 24, PASADENA CA 91125, USA

Telephone: (1) $8183564415 \quad$ Facsimile: (1)

Telex: 675425

Email: george@deimos.caltech.edu Commission(s): 22,33

DJURASEVIC GOJKO, ASTRONOMICAL OBSERVATORY, VOLGINA 7, YU 11050 BEOGKAD YUGOSLAVIA-SERBLA \& MONTENEGRO

Telephone: (38) 11419553

Facsimile: (38)

Telex:

Email:

Commission(s):

DJUROVIC DRAGUTIN M DR, DPT OF ASTRONOMY, FACULTY OF SCIENCES, STUDENTSKI TRG 16 YU 11000 BEOGRAD, YUGOSLAVIA-SERBIA \& MONTENEGRO

Telephone: (38) 11420221

Facsimile: (38)

Telex:

Email:

Commission(s): 08,19 
DLUZHNEVSKAYA O B DR, INST OF ASTRONOMY, ACADEMY OF SCIENCES, PYATNITSKAYA UL 48 109017 MOSCOW, RUSSIA

Telephone: (7) 0952315461

Facsimile: (7) 0952302081

Telex: $\mathbf{4 1 1 5 7 6}$ ascon su

Email: olgad@inasan.rssi.ru/olga(@astro.free.net

Commission(s): 05VP,35,37

DOAZAN VERA DR, OBSERVATOIRE DE PARIS, 61 AVE OBSERVATOIRE, F 75014 PARIS, FRANCE

Telephone: (33) 140512235

Facsimile: (33)

Commission(s): 29,36

Telex: 270776 obs $f$

Email:

DOBRONRAVIN PETER DR, CRIMEAN ASTROPHYS OBS, UKRAINIAN ACAD OF SCIENCE, NAUCHNY 334413 CRIMEA, UKRAINE

Telephone: (7) 432945

Facsimile: (7)

Telex:

Email:

Commission(s): 09,29

DOBRZYCKI JERZY PROF, HISTORY OF SCIENCE, POJISH ACAD OF SCIENCES, GWIAZDZISTA 27/169 PL 01814 WARSAW, POLAND

Telephone: (48) 332203

Facsimile: (48)

Telex:

Email:

Commission(s): 41

DOCOBO DURANTEZ JOSE A, OBSERVATORIO ASTRONOMICO, RAMON MARIA ALLER AVD DE LAS CIENCIAS S/N, E SANTIAGO DE COMPOSTELA, SPAIN

Telephone: (34)

Facsimile: (34)

Telex:

Email:

Commission(s): 26

DODD RICHARD J DR, CARTER OBSERVATORY, BOX 2909, WELLINGTON 1, NEW ZEALAND

Telephone: (64) 728-167 Facsimile: (64)

Email:

Commission(s):

Telex: 30172 natobs nz

DOGAN NADIR PROF, DPT OF ASTRONOMY, UNIVERSITY OF ANKARA, FEN FAKULTESI, 06100 BESEVLER TURKEY

Telephone: (90)

Facsimile: (90)

Telex:

Email:

Commission(s): 12

DOGGETT LEROY E DR, US NAVAL OBSERVATORY, NAUTICAL ALMANAC OFFICE

3450 MASSACHUSETTS AVE NW, WASHINGTON DC 203925100 , USA

Facsimile: (1) Telex:

Email: doggett@ariel.usno.navy.mil Commission(s): 04,07,41

DOHERTY LORNE H DR, HERZBERG INST ASTROPHYS, NTL RESEARCH COUNCIL, 100 SUSSEX DR OTTAWA ON K1A 0R6, CANADA

Telephone: (1) 6139936060

Facsimile: (1) 6139526602

Telex: 0533715

Email:

Commission(s):

'DOHERTY LOWELL R PROF, DPT OF ASTRONOMY, UNIVERSITY OF WISCONSIN, 475 N CHARTER ST MADISON WI 53706, USA

Telephone: (1) 6082621249

Email:

Facsimile: (1)

Telex:

Commission(s):

DOKUCHAEV VYACHESLAV DR, INST NUCLEAR RESEARCH, ACADEMY OF SCIENCES 6OTH ANIV OCT PROSPEKT 7A, 117312 MOSCOW, RUSSIA

$\begin{array}{lll}\text { Telephone: (7) } 953827678 & \text { Facsimile: (7) } 0952926511 & \text { Telex: } 411051 \text { inr su } \\ \text { Email: dokuchacv@inucres:msk.su } & \text { Commission(s): 28,34,44 } & \end{array}$

` DOKUCHAEVA OLGA D DR, STERNBERG STATE ASTR INST, UNIVERSITETSKIJ PROSP 13

Telephone: (7)

Facsimile: (7)

Telex:

Email:

Commission(s): 09,34

DOLAN JOSEPH F DR, NASA GSFC, CODE 681, GREENBELT MD 20771, USA

Telephone: (1) 3012865920

Facsimile: (1)

Telex: 89675

Email:

Commission(s): 25,44

DOLEZ NOEL DR, OBS MIDI PYRENEES, 14 AVE E BELIN, F 31400 TOULOUSE CDX, FRANCE

Telephone: (33)

Facsimile: (33)

Telex:

Email:

Commission(s): 
DOLGINOV ARKADY Z PROF DR, IOFFE PHYSICAL TECH INST, ACADEMY OF SCIENCES POLYTECHNICHESKAYA UL 26, 194021 ST PETERSBURG, RUSSIA

Telephone: (7)

Facsimile: (7)

Telex:

Email:

Commission(s): 49

DOLIDZE MADONA V DR, ABASTUMANI ASTROPHYSICAL, OBSERVATORY, GEORGIAN ACAD OF SCIENCES 383762 ABASTUMANI, GEORGIA

Telephone: (7)

Facsimile: (7)

Telex:

Email:

Commission(s): 29

DOLLFUS AUDOUIN PROF, OBSERVATOIRE DE PARIS, SECTION MEUDON, F 92195 MEUDON PPL CDX, FRANCE

Telephone: (33) 145347530

Facsimile: (33)

Telex:

Email:

Commission(s): 10,16,20

DOMINGO VICENTE DR, ESAESTEC, SSD, BOX 299, NL 2200 AG NOORDWIJK, NETHERLANDS

Telephone: (31) $171983576 \quad$ Facsimile: (31) $171984698 \quad$ Telex: 39098

Email: estc1::vdomingo/vdomingo(a)estec Commission(s): 44

DOMINKO FRAN PROF DR, SARANOVICEVA 11, LJUBLJANA, SLOVENIA

Telephone: (38) $61322210 \quad$ Facsimile: (38) Telex:

Email: $\quad$ Commission(s):

DOMKE HELMUT PH D, ZNTRLINST F ASTROPHYSIK, STERNWARTE BABELSBERG, ROSA LUXEMBURG STR 17A D 14473 POTSDAM, GERMANY

Telephone: (49)

Facsimile: (49)

Telex:

Email:

Commission(s): 36

DOMMANGET J DR, OBSERVATOIRE ROYAL DE, BELGIQUE, AVE CIRCULAIRE 3, B 1180 BRUSSELS, BELGIUM

Telephone: (32) 223752484

Facsimile: (32) 23749822

Telex: 21565

Email: omer@astro.ome.be

Commission(s): $24,26,50$

DONAS JOSE DR, LAS, TRAVERSE DU SIPHON, LES TROIS LUCS, F 13012 MARSEILLE, FRANCE

Telephone: (33) 91055900

Facsimile: (33) $91661855 \quad$ Telex: $420584 \mathrm{f}$

Email: donas@frlasm51

Commission(s): 28

DONG IL ZUN, PYONGYANG ASTRON OBS, ACADEMY OF SCIENCES DPRK, TAESONG DISTRICT, PYONGYANG KOREA DPR

Telephone: (850)

Facsimile: (850)

Telex:

Email:

Commission(s):

DONN BERTRAM D, NASA GSFC, CODE 691, GREENBELT MD 20771, USA

Telephone: (1) 3012866859

Facsimile: (1)

Telex: 89675

Email:

Commission(s): 15,34

DONNER KARL JOHAN, OBS \& ASTROPHYSICS LAB, UNIVERSITY OF HELSINKI, TAEHTITORNINMAKI

SF 00130 HELSINKI 13, FINLAND

Telephone: (358)

Facsimile: (358)

Telex:

Email:

Commission(s): 28

DONNISON JOHN RICHARD DR, DPT OF MATH SCIENCES, GOLDSMITHS' COLLEGE, NEW CROSS LONDON SE14 6NW, UK

Telephone: (44) 181716927171

Facsimile: (44)

Telex:

Email:

Commission(s): 20

DOPITA MICHAEL A PROF, MOUNT STROMLO \& SIDING, SPRING OBSERVATORIES, PRIVATE BAG WESTON CREEK PO ACT 2611, AUSTRALIA
Telephone: (61) 62490212
Facsimile: (61) 62490233
Telex:
Email: michael.dopita@anu.cdu.au
Commission(s): 34VP

DORENWENDT KLAUS DR, PHYSIKALISCH-TECHNISCHES, BUNDESANSTALT, BUNDESALLEE 100 D 3300 BRAUNSCHWEIG, GERMANY
Telephone: (49) 5315921210
Facsimile: (49)
Email:
Commission(s): 31

Telex: 952822 
M 510

DORFI ERNST ANTON DR, INSTITUT FUER ASTRONOMIE, UNIVERSITAET WIEN, TUERKENSCHANZSTR 17 A 1180 WIEN, AUSTRIA

Telephone: (43) 13453600

Facsimile: (43)

Telex: 133099

Email:

Commission(s): 42

DORMAND JOHN RICHARD DR, DPT OF MATHEMATICS, TEESSIDE POLYTECHNIC, MIDDLESBROUGH CLEVELAND TS1 3BA, UK

$\begin{array}{ll}\text { Telephone: (44) } 1642218121 * 4365 & \text { Facsimile: }(44) \\ \text { Email: } & \text { Commission(s): } 07\end{array}$

DOROSHKEVICH ANDREI G DR, INST OF APPLIED MATHS, ACADEMY OF SCIENCES, MIUSSKAJA SQ 4 125047 MOSCOW, RUSSLA

Telephone: (7)

Facsimile: (7)

Telex:

Email:

Commission(s): 47

DORSCHNER JOHANN DR, UNIV STERNWARTE JENA, SCHILLERGAESSCHEN 2, D 07745 JENA, GERMANY

Telcphone: (49) 788222637

Facsimile: (49)

Email:

Commission(s): $\mathbf{3 4 , 5 1}$

Telex: $\mathbf{5 8 8 6 1 3 4}$

DOSCHEK GEORGE A DR, NAVAL RESEARCH LABORATORY, CODE 4170, 4555 OVERLOOK AVE SW

WASHINGTON DC 203755000 , USA

Telephone: (1) 2027676473

Facsimile: (1)

Telex:

Email:

Commission(s):

DOSSIN F DR, INSTITUT D'ASTROPHYSIAUE, UNIVERSITE DE LIEGE, AVE COINTE 5

B 4000 COINTE-LIEGE BELGIUM

Telephone: (32) 41529980

Facsimile: (32) 41527474

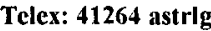

Email:

Commission(s): 15

DOTANI TADAYASU DR, ISAS, 3-1-1 YOSHINODAI, SAGAMIHARA, KANAGAWA 229, JAPAN

Telephone: (81) 427513911

Facsimile: (81) 427594253

Telex: j27758 isas cro

Email: dotani@astro.isas.ac.jp

Commission(s): 44

DOTTORI HORACIO A DR, INSTITUTO DE FISICA, UFRGS, CP 15051, 900+9 PORTO ALEGRE RS, BRAZIL

Telephone: (55) 512364677

Facsimile: (55)

Commission(s): 28,34

Telex: 511055 ufrs br

Email:

DOUBINSKIJ B A DR, INST OF RADIO \& ELECTRON, ACADEMY OF SCIENCES, 103907 MOSCOW, RUSSIA

Telephone: (7)

Facsimile: (7)

Telex:

Email:

Commission(s): 40,51

DOUGHERTY SEAN, CHEMICAL \& PHYSICAL SCI, LIVERPOOL JOHN MOORES UNI, BYROM ST

LIVERPOOL L3 3AF, UK

Telephone: (44) 1512314103

Email: smd@staru1.livjm.ac.uk

Facsimile: (44) $1512312337 \quad$ Telex:

Commission(s): 42

DOUGHTY NOEL A DR, DPT PHYSICS \& ASTRONOMY, UNIVERSITY OF CANTERBURY, PRIVATE BAG CHRISTCHURCH 1, NEW ZEALAND

Telephone: (64)

Facsimile: (64)

Telex:

Email:

Commission(s): 42

DOUGLAS JAMES N PROF, ASTRONOMY DPT, UNIVERSITY OF TEXAS, RLM 15 308, AUSTIN TX 78712 1083, USA

Telephone: (1) 5124714461

Facsimile: (1)

Telex: 910874-1351

Email:

Commission(s): 40

DOUGLAS NIGEL DR, KAPTEYN ASTRONOMICAL INST, UNIVERSITY OF GRONINGEN, BOX 800

NL 9700 AV GRONINGEN, NETHERLANDS

Telephone: (31) 590828818

Facsimile: (31) $590828800 \quad$ Telex:

Email: ndouglas@hrdksw5

Commission(s): 09

DOUGLAS R J MR, PHYSICS DIVISION (M-36), NTL RESEARCH COUNCIL, 100 SUSSEX DR

OTTAWA ON K1A OS1, CANADA

Telephone: (1) 6139936060

Facsimile: (1) 6139526602

Telex:

Email:

Commission(s): 31 
DOUGLASS GEOFFREY G, US NAVAL OBSERVATORY, ASTROMETRY DPT, 3450 MASSACHUSETTS AVE NW WASHINGTON DC 203925400 , USA

Telephone: (1) 2026531438

Facsimile: (1) 2026530944 Telex:

Email: ggd@cruz.usno.navy.mil

Commission(s): 24,26

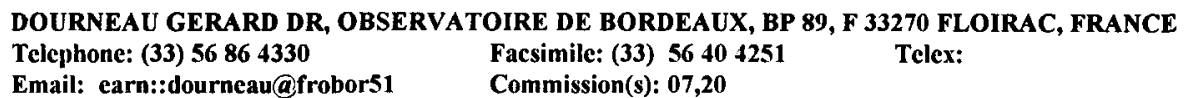

Email: earn::dourneau $(a$ frobor51

Commission(s): 07,20

DOVAL JORGE PEREZ DR, INST GEOPHYS \& ASTRONOMY, CALLE 212 N 2906/29 Y 31, RPTO COROMELA/LA LISA

LA HABANA, CUBA

Telephone: (53) $218435 / 0644$

Facsimile: (53)

Commission(s): 20

Telex: 511240 geoas cu

Email:

DOWNES ANN JULIET B, MULLARD RADIO ASTRON OBS, CAVENDISH LABORATORY, MADINGLEY RD

CAMBRIDGE CB3 OHE, UK

Telephone: (44) 1233337294

Facsimile: (44) $1223354599 \quad$ Telex: 81292

Email:

Commission(s):

DOWNES DENNIS DR, IRAM, 300 RUE DE LA PISCINE, F 38406 S MARTIN HERES CD, FRANCE

Telephone: (33) $176824900 \quad$ Facsimile: (33) 176515938

Telex: 980753

Email: downes@iram.grenct.fr

Commission(s): $33,34,40$

DOWNES RONALD A DR, STSCI, C/O USER SUPPORT BRANCH, 3700 SAN MARTIN DR, BALTIMORE MD 21218, USA

Telephone: (1) 3013384700

Facsimile: (1)

Telex:

Email: scivax::downes

Commission(s): 27

DOWNS GEORGE S DR, MIT LINCOLN LABORATORY, ROOM B285, BOX 73, LEXINGTON MA 02173, USA

Telephone: (1)

Facsimile: (1)

Telex:

Email:

Commission(s): 40,51

DOYLE JOHN GERARD, ARMAGH OBSERVATORY, COLLEGE HILL, ARMAGH BT61 90G, UK

Telephone: (44) $1861522928 \quad$ Facsimile: (44)

Commission(s): 36

Telex: 747937

Email:

DOYLE LAURANCE R DR, SETI INSTITUTE 245 7, NASA AMES RESEARCH CENTER

MOFFETT FIELD CA 94035 , USA

Telephone: (1) 4156043372

Facsimile: (1)

Telex:

Email: 24609::doyle span

Commission(s):

DRAINE BRUCE T, PRINCETON UNIVERSITY OBS, PEYTON HALL, PRINCETON NJ 08544, USA

Telephone: (1) 6094523574

Facsimile: (1)

Telex:

Email:

Commission(s): $3+$

DRAKE FRANK D PROF, DIV OF NATURAL SCIENCES, UNIVERSITY OF CALIFORNIA

SANTA CRUZ CA 95064, USA

Telephone: (1) $4084292931 \quad$ Facsimile: (1) Telex: 7607936 ucsc uc

Email:

Commission(s): $16,40,44,51$

DRAKE JEREMY, CTR FOR EUV ASTROPHYSICS, UNIVERSITY OF CALIFORNIA, 2150 KITTREDGE ST

BERKELEY CA 94720, USA

Telephone: (1) 5106422397

Email: jurake@cea.berkelcy.edu

Facsimile: (1) $5106435660 \quad$ Telex:

Commission(s):

DRAKE STEPHEN A, NASA GSFC, CODE 668, GREENBELT MD 20771, USA

$\begin{array}{ll}\text { Telephone: (1) } 3012866962 & \text { Facsimile: (1) } \\ \text { Email: } & \text { Commission(s): 36,40 }\end{array}$

DRAMBA C PROF, ASTRONOMICAL INSTITUTE, CUTITUL DE ARGINT 5, BOX 28, R 75212 BUCHAREST, RUMANIA Telephone: (40) 16753998 Facsimile: (40) 16193407 Telex:

Email:

Commission(s):

DRAPATZ SIEGFRIED W DR, MPI F EXTRATERRESTRISCHE, PHYSIK, KARL SCHWARZSCHILDSTR 1

D 85740 GARCHING MUENCHEN, GERMANY

Telephonc: (49) 893299880

Facsimile: (49)

Email:

Commission(s): 34

Telex: 05215845 
M 512

DRAVINS DAINIS PROF, LUND OBSERVATORY, BOX 43, S 22100 LUND, SWEDEN

Telephone: (46) $46107297 \quad$ Facsimile: (46) $46104614 \quad$ Telex: 33199 obsnot s

Email: dainis@astro.lu.se (internet) Commission(s): 09,12,29,36C

DRAVSKIKH A F DR, PULKOVO OBSERVATORY, ACADEMY OF SCIENCES, 10 KUTUZOV QUAY

196140 ST PETERSBURG, RUSSIA

Telcphone: (7) 2979452

Facsimile: (7)

Commission(s): 08,40

Telex:

Email:

STERNWARTE, ASTR INST UNIV ERLANGEN-N, STERNWARTSTR 7

DRECHSEL HORST DR, DR REN
D 96049 BAMBERG, GERMANY

Telephone: (49) $95157708 \quad$ Facsimile: (49)

Email: drechsel@sternwarte.uni-eriang Commission(s): 42C

Telex: 629830 unier d

DREHER JOHN W, DPT OF PHYSICS, MIT RM 26 315, BOX 165, CAMBRIDGE MA 02139, USA

Telephone: (1) 6172538519

Facsimile: (1)

Telex: 921473

Email:

Commission(s): $09,34,40$

DRESSEL LINDA L, APPLIED RESEARCH CORP, 8201 CORPORATE DR, SUITE 920, LANDOVER MD 20785, USA

Telephone: (1)

Facsimile: (1)

Telex:

Email:

Commission(s): 28

DRESSLER ALAN, CARNEGIE OBSERVATORIES, 813 SANTA BARBARA ST, PASADENA CA 91101 1292, USA

Telephone: (1) 8185771122

Facsimile: (1) 8187958136

Telex: 1561318 ociw ut

Email:

Commission(s): $28,47 \mathrm{C}$

DRESSLER KURT PROF, LAB PHYSIK CHEMIE, ETH ZENTRUM, CH 8092 ZUERICH, SWITZERLAND

Telephone: (41) $12564441 \quad$ Facsimile: (41)

Telex: 53178 ethbi ch

Email:

Commission(s): 14

DREVER RONALd W P DR, CALTECh, 130 33, PASADENA CA 91125, USA

Telephone: (1)

Facsimile: (1) 8187951547

Telex: 675425 caltech psd

Email:

Commission(s):

DREW JANET, DPT OF ASTROPHYSICS, UNIVERSITY OF OXFORD, KEBLE RD, OXFORD OX1 3RH, UK

Telephone: (44)

Facsimile: (44)

Telex:

Email:

Commission(s):

DRILLING JOHN S, DPT PHYSICS \& ASTRONOMY, LOUISIANA STATE UNIV, BATON ROUGE LA 70803 4001, USA

Telephone: (1) 5043886795

Facsimile: (1)

Telex:

Email:

Commission(s): 33

DRINKWATER MICHAEL, ANGLO AUSTRALIAN, OBSERVATORY, COONABARABRAN NSW 2357, AUSTRALIA

Telephone: (61) 68426315

Facsimile: (61) 68422288

Telex:

Email: mjd@aaochn.aao.gov.au

Commission(s): $\mathbf{2 8 , 4 7}$

DROSSART PIERRE DR, OBSERVATOIRE DE PARIS, SECTION DE MEUDON, DESPA F 92195 MEUDON PPL CDX, FRANCE

Telephone: (33) $145077664 \quad$ Facsimile: (33) $145072806 \quad$ Telex: 204464

Email: frmeu51@drossart Commission(s): 16

DROZYNER ANDRZEJ, INSTITUTE OF ASTRONOMY, N COPERNICUS UNIVERSITY, UL CHOPINA 12/1

PL 87100 TORUN, POLAND

Telcphone: (48) 260 17/260 37

Email:

Facsimile: (48)

Telex: 0552234 astr pl

Commission(s): 07

DRURY LUKE O'CONNOR DR, DIAS, SCHOOL OF COSMIC PHYSICS, 5 MERRION SQ, DUBLIN 2, IRELAND

Telephone: (353) 1774321

Email: Id@diascp.uucp

Facsimile: (353)

Telex: 31687 dias

Commission(s): 44

DRYER MURRAY DR, NOAO ERL R/E/SE, SPACE ENVIRONMENT LAB, 325 BROADWAY, BOULDER CO 80303, USA

Telephone: (1) 3034973978

Facsimile: (1)

Telex: 592811 noaa masc bdr

Email: mdryer@selvax.scl.bldrdoc.gov

Commission(s): 10,15,49C 
DUBATH PIERRE, LICK OBSERVATORY, UNIVERSITY OF CALIFORNIA, NATURAL SCIENCES II SANTA CRUZ CA 95064, USA

Telephone: (1) 4084593771

Email: pdubath@lick.ucsc.edu

Facsimile: (1) $4084263115 \quad$ Telex:

Commission(s):

DUBAU JACQUES DR, OBSERVATOIRE DE PARIS, SECTION DE MEUDON, F 92195 MEUDON PPL CDX, FRANCE

Telephone: (33) $145077456 \quad$ Facsimile: (33)

Email:

Commission(s): $10,14 \mathrm{C}$

Telex: 270912

DUBIN MAURICE DR, NASA GSFC, CODE 616, LAB FOR ATMOSPHERES, GREENBELT MD 20771, USA

Telephone: (1) 3012865475

Facsimile: (1) $3012862630 \quad$ Telex:

Email:

Commission(s): 21,22

DUBNER GLORIA DR, IAR, CC 5, $189+$ VILLA ELISA (BS AS), ARGENTINA

Telephone: (54) $2143793 \quad$ Facsimile: (54) $21211761 \quad$ Telex: 22414 cedoc ar

Email:

Commission(s): 34

DUBOIS MARC A, CEA CEN, DRFC, BP 6, F 92260 FONTENAY AUX ROSE, FRANCE

Telcphone: (33) $146547881 \quad$ Facsimile: (33) Telex:

Email: $\quad$ Commission(s): 10

DUBOIS PASCAL DR, OBS DE STRASBOURG, 11 RUE UNIVERSITE, F 67000 STRASBOURG, FRANCE

Telephone: (33) $88358200 \quad$ Facsimile: (33) $88250160 \quad$ Telex:

Email: $\quad$ Commission(s): 05,28

DUBOUT RENEE, OBSERVATOIRE DE LYON, AVE CHARLES ANDRE, F 69561 S GENIS LAVAL CDX, FRANCE

Telephone: (33) 78560705

Email: Facsimile: (33) $72399791 \quad$ Telex: 310926

Commission(s): 25,34

DUCATI JORGE RICARDO DR, INSTITUTO DE FISICA, UFRGS, CP 15051, 90000 PORTO ALEGRE RS, BRAZIL

Telephonc: (55) 512364677

Facsimile: (55) 513361762

Telex: 0511055 ufrs br

Email: ducati@if.1.ufrgs-anrs.br

Commission(s): 05,25,33,38C

DUCHESNE MAURICE DR, OBSERVATOIRE DE PARIS, 61 AVE OBSERVATOIRE, F 75014 PARIS, FRANCE

Telephone: (33) 143201210

Facsimile: (33)

Telex:

Email:

Commission(s): 09

DUCOURANT CHRISTINE, OBSERVATOIRE DE BORDEAUX, BP 89, F 33270 FLOIRAC, FRANCE

Telephone: (33) 56864330

Facsimile: (33) 56404251

Telex:

Email: ducourant(a)obscrv.u-bordeaux.fr Commission(s): 33

DUERBECK HILMAR W DR, HEMBRICH 16, D 54552 SCHALKENMEHREN, GERMANY
$\begin{array}{ll}\text { Telephonc: (49) } & \text { Facsimile: (49) } \\ \text { Email: } & \text { Commission(s): } 42\end{array}$

Email:

Commission(s): 42

DUERST JOHANNES DR, LANGWIES, CH 8824 SCHOENENBERG, SWITZERLAND

Telephone: (41) 17881785 Facsimile: (41) Telex:

Email:

Commission(s):

DUFAY MAURICE PROF, UNIVERSITE LYON 1, CAMPUS LA DOUA, 43 BD DU 11 NOVEMBRE

F 69621 VILLEURBANNE, FRANCE

Tclephone: (33) 78898124

Facsimile: (33)

Telex:

Email:

Commission(s): 14,21

DUFFETT-SMITH PETER JAMES, MULLARD RADIO ASTRON OBS, CAVENDISH LABORATORY, MADINGLEY RD CAMBRIDGE CB3 OHE, UK

Telephone: (44) 1223337294

Email: pjos@mrao.cam.ac.uk

Facsimile: (44) 1223354599

Telex: 81292 cavlab g

Commission(s): 40

DUFLOT MARCELLE DR, OBSERVATOIRE DE MARSEILLE, 2 PLACE LE VERRIER F 13248 MARSEILLE CDX 04, FRANCE

Telephone: (33) 91959088

Email:

Facsimile: (33)

Commission(s): 30,45

Telex: 420241 
DUFOUR REGINALD JAMES, DPT SPACE PHYS \& ASTRON, RICE UNIVERSITY, 204K SPACE SCIENCE BLDG HOUSTON TX 77001, USA

Telephone: (1) 7135278101

Email:

\author{
Facsimilc: (1) \\ Telex: \\ Commission(s): 28,34
}

DUFTON PHILIP L DR, DPT OF PURE \& APPL PHYS, QUEEN'S UNIVERSITY, BELFAST BT7 1NN, UK Telephone: (44) 1232245133

Facsimile: (44)

Commission(s): 36

Telex: 74487

Email:

DULDIG MARCUS LESLIE DR, DPT OF PHYSICS, UNIVERSITY OF TASMANIA, GPO BOX 252C

HOBART TAS 7001, AUSTRALIA

Facsimile: (61) 2202410

Telex: aa 58150 cosant

Email: dulgig@physvax.phys.utas.edu.a Commission(s): 10,49

DULEY WALTER W PROF, DPT OF PHYSICS, UNIVERSITY OF WATERLOO, WATERLOO ON N2L 3G1, CANADA

Telephone: (1) $5198851211 * 3108$

Email: duley@physics.watstar.uwaterloo.ca

Facsimile: (1) $5197468115 \quad$ Telex:

Commission(s): 34

DULK GEORGE A PROF, CASA, UNIVERSITY OF COLORADO, BOX 391, BOULDER CO 80309 0391, USA

Telephone: (1) 3034928788

Facsimile: (1)

Telex:

Email:

Commission(s): 10,40

DULTZIN-HACYAN D DR, INST ASTROFISICA, DE ANDALUCIA APD 3004, PROFESOR ALBAREDA 1

E 18080 GRANADA, SPAIN

Telephone: (34) 58121300

Facsimile: $(34)$

Commission(s): 28,47

Telex: 78573 iaage

Email:

DUMA DMITRIJ P DR, MAIN ASTRONOMICAL OBS, UKRAINIAN ACAD OF SCIENCE, GOLOSEEVO 252127 KIEV, UKRAINE

Telephone: (7) 663110

Facsimile: (7)

Telex: 131406 sky su

Email:

Commission(s): 08

DUMITRACHE CHRISTANA, ASTRONOMICAL INSTITUTE, CUTITUL DE ARGINT 5, BOX 28 R 75212 BUCHAREST, RUMANIA

Telephone: (40) 16413686

Facsimile: (40) 13123391

Email: crisd@imar.ro

Commission(s): 10

Telex: 11882 astro $r$

DUMITRESCU ALEXANDRU, ASTRONOMICAL INSTITUTE, CUTITUL DE ARGINT 5, BOX 28

R 75212 BUCHAREST, RUMANIA

Telephone: (40) 16236892

Facsimile: (40) $13123391 \quad$ Telex:

Email: adumitrescu@imar.ro

Commission(s):

DUMONT RENE DR, OBSERVATOIRE DE BORDEAUX, BP 89, F 33270 FLOIRAC, FRANCE

Telephone: (33) 56864330

Facsimile: (33) $56404251 \quad$ Telex:

Email:

Commission(s): 21

DUMONT SIMONE DR, INSTITUT D'ASTROPHYSIQUE, 98BIS BD ARAGO, F 75014 PARIS, FRANCE

Telephone: (33) 144328000

Facsimile: (33) 144328001

Telex:

Email:

Commission(s): 12,41

DUNCAN DOUGLAS KEVIN DR, ASTRONOMY \& ASTROPHYS CTR, UNIVERSITY OF CHICAGO, 5640 S ELLIS AVE CHICAGO IL 60637, USA

Telephone: (1) 3127028207

Email: duncan@oddjob.uchicago.edu

Facsimile: (1)

Telex:

Commission(s): 29

DUNCAN ROBERT A PROF, CSIRO, DIV OF RADIOPHYSICS, BOX 76, EPPING NSW 2121, AUSTRALIA

Telephone: (61) 28680222

Email: rduncan@atnf.csiro.au

Facsimile: (61) 28680310 Telex:

Commission(s): 10

DUNCOMBE RAYNOR L DR, AEROSPACE ENGINEERING DPT, UNIVERSITY OF TEXAS, WRW 414, AUSTIN TX 78712, USA

Telephone: (1) 5124714250

Facsimile: (1)

Telex: 704265 csrutx ud

Email:

Commission(s): $04,05,07,08$ 
DUNHAM DAVID W, COMPUTER SCIENCES CORP, SYSTEM SCIENCES DIV, 8728 COLESVILLE RD

SILVER SPRING MD 20910, USA

Telephone: (1)

Facsimile: (1)

Telex:

Email:

Commission(s): $04,20,26$

DUNKELMAN LAWRENCE, INST FOR SCIENTIFIC \&, SPACE RESEARCH, 4925 N CALLE BOSQUE TUCSON AZ 95718 6335, USA

Telephone: (1)

Email: dunk2@aol.com

Facsimile: (1) 5202934588

Telex:

Commission(s): 09,12,21,44,50

DUNLOP JAMES, CHEMICAL \& PHSYICAL SCI, LIVERPOOL JOHN MOORES UNI, BYROM ST LIVERPOOL L3 3AF, UK

Telephone: (44) 1512312039

Emaii: jsd@uk.ac.livjm.staru1

Facsimile: (44) $1512312337 \quad$ Telex:

Commission(s): 47

DUNLOP STORM, 140 STOCKS LANE, EAST WITTERING, CHICHESTER, WEST SUSSEX PO20 8NT, UK

Telephone: (44) 1243670354

Facsimile: (44) $1243670400 \quad$ Telex:

Email: sdunlop@starlink.sussex.ac.uk Commission(s): 27

DUNN RICHARD B DR, AIR FORCE GEOPHYSICS LAB, NTL SOLAR OBSERVATORY, SUNSPOT NM 88349, USA

Telephone: (1) 5054341390

Facsimile: (1)

Telex:

Email:

Commission(s): 10,12

DUORAH HIRA LAL DR, DPT OF PHYSICS, UNIVERSITY OF GAUHATI, GUWAHATI 781014, INDIA

Telephone: (91) 88531

Facsimile: (91)

Commission(s): 44

Telex:

Email:

DUPREE ANDREA K DR, CENTER FOR ASTROPHYSICS, SOLAR \& STELLAR DIVISION, 60 GARDEN ST CAMBRIDGE MA 02138, USA

Telephone: (1) 6174957489

Email: duprec@efa.harvard.cdu

Facsimile: (1)

Commission(s): $34,36,44$

Telex: 921428 satellite cam

DUPUY DAVID L DR, DPT OF PHYSICS, VIRGINIA MILITARY INST, LEXINGTON VA 24450, USA

Telephone: (1) $7034647504 \quad$ Facsimile: (1)

Telex:

Email: fpydupuy\%faculty\%vmi@ist.vmi.c

Commission(s): 27,46

DURGAPRASAD N DR, TIFR, HOMI BHABHA RD, COLABA, BOMBAY 400 005, INDIA

$\begin{array}{ll}\text { Telephone: (91) } 22219111 * 342 & \text { Facsimile: (91) } \\ \text { Email: } & \text { Commission(s): }\end{array}$

DURIEZ LUC DR, LABORATOIRE D'ASTRONOMIE, 1 IMPASSE OBSERVATOIRE, F S9000 LILLE, FRANCE

Telephone: (33) 20524424

Facsimile: (33)

Commission(s): 07

Telex:

Email: carn:duriez@frcitl71

DURISEN RICHARD H DR, ASTRONOMY DPT, INDIANA UNIVERSITY, SWAIN WEST 319
BLOOMINGTON IN 47405, USA

BLOOMINGTON IN 47405, USA

Telephone: (1) 8123356921

Facsimile: (1)

Telex:

Email:

Commission(s): 35,42

DURNEY BERNARD DR, NTL SOLAR OBSERVATORY, BOX 26732, 950 N CHERRY AVE, TUCSON AZ 85726 6732, USA

Telephone: (1) 5203275511

Facsimile: (1)

Commission(s): 49

Telex:

Email:

DUROUCHOUX PHILIPPE, CEA CEN, DAPNIA/SAP, BP
Telephone: (33) 169083376
$\begin{array}{ll}\text { Email: } & \text { Facsimile: (33) } \\ \end{array}$

2, F 91191 GIF/YVETTE CDX, FRANCE

Email:

Commission(s): 44

Telex: 690860

DURRANCE SAMUEL T DR, DPT PHYSICS \& ASTRONOMY, JOHNS HOPKINS UNIVERSITY, CHARLES \& 34TH ST BALTIMORE MD 21218, USA

Telephone: (1) 3013388707

Email:

Facsimile: (1)

Commission(s): 16

Telex: 9102400225 jhu cas

DURRANT CHRISTOPHER J DR, DPT OF APPLIED MATHS, UNIVERSITY OF SYDNEY, SYDNEY NSW 2006, AUSTRALIA

Telephone: (61) 26923373

Eacsimile: (61) 26602903

Commission(s):

Telex: 20056 fishlib aa

Email: 
M 516

DURRET FLORENCE DR, INSTITUT D'ASTROPHYSIQUE, 98BIS BD ARAGO, F 75014 PARIS, FRANCE

Telephone: (33) 144328093

Facsimile: (33) $144328001 \quad$ Telex:

Email: iapobs::florence

Commission(s): 28

DUSCHL WOLFGANG J DR, INST F THEOR ASTROPHYSIK, DER UNIVERSITAET, IM NEUENHEIMER FELD S61 D 69120 HEIDELBERG, GERMANY

Telephone: (49) $6221562967 \quad$ Facsimile: (49)

Email: bitnet:cj0@dhdurz1 Commission(s): 42

Telex: 46515 unihd $d$

DUTHIE JOSEPH G PROF, DPT PHYSICS \& ASTRONOMY, UNIVERSITY OF ROCHESTER ROCHESTER NY 14627, USA

Telephone: (1)

Facsimile: (1)

Telex:

Email:

Commission(s): 44

DUVAL MARIE-FRANCE, OBSERVATOIRE DE MARSEILLE, 2 PLACE LE VERRIER

F 13248 MARSEILLE CDX 04, FRANCE

Telephone: (33) 91959088

Facsimile: (33)

Telex:

Email:

Commission(s): 28,46

DUVALL THOMAS L JR, NTL SOLAR OBSERVATORY, BOX 26732, 950 N CHERRY AVE, TUCSON AZ 85726 6732, USA

Telephone: (1) 5203259338

Facsimile: (1)

Commission(s): 12

Telex: $666-484$ aura-kjono-

Email:

DUVERT GILLES DR, OBSERVATOIRE DE GRENOBLE, CERMO/ASTROPHYSIQUE, BP S3X

F 38041 S MARTIN HERES CD, FRANCE

Telephone: (33) 76514885

Email: bitnet:duvert@frgag51

Facsimile: (33) 76448821

Telex:

DVORAK RUDOLF DR, INSTITUT FUER ASTRONOMIE, UNIVERSITAET WIEN, TUERKENSCHANZSTR 17

A 1180 WIEN, AUSTRIA

Telephone: (43) 13453600

Facsimile: (43)

Telex:

Email:

Commission(s): $07 \mathrm{C}, 20$

DWARAKANATH K S, RAMAN RESEARCH INSTITUTE, SADASHIVANAGAR, CV RAMAN AVE BANGALORE 560080 , INDIA

Telephone: (91) 803340122

Facsimile: (91) $8033+0492$

Telex:

Email:

Commission(s): 40

DWEK ELI, NASA GSFC, CODE 697, LEP, GREENBELT MD 20771, USA

Telephone: (1) 3012866209

Facsimilc: (1)

Telex:

Email:

Commission(s): 21,34

DWIVEDI BHOLA NATH DR, DPT OF APPLIED PHYSICS IT, UNIVERSITY BANARAS HINDU VARANASI 221 005, INDHA

Telephone: (91)

Email:
Facsimilc: (91) 542312059

Commission(s): 10
Telex: 0545304 bhu in

DWORETSKY MICHAEL M DR, DPT PHYSICS \& ASTRONOMY, UNIVERSITY COLLEGE LONDON, GOWER ST LONDON WC1E 6BT, UK

Telephone: (44) 1713877050

Email:

Facsimile: (44)

Telex: 28722

Commission(s): 29,46

DYCK M DR, DPT PHYSICS \& ASTRONOMY, UNIVERSITY OF WYOMING, BOX 3905, LARAMIE WY 82071, USA

Telephone: (1) 3077666150

Facsimilc: (1)

Telex:

Email: meldyck@corral.uwyo.edu

Commission(s):

DYER CHARLES CHESTER DR, SCARBOROUGH COLLEGE, UNIVERSITY OF TORONTO, PHYS SCS GR RM S 650 TORONTO ON M1C 1A4, CANADA

Telephone: (1) 4162843318

Facsimile: (1)

Telex:

Email:

Commission(s): 47

DYER EDWARD R DR, 3626 DAVIS ST NW, WASHINGTON DC 20007, USA

Telephone: (1)

Facsimile: (1)

Commission(s):

Telex:

Email: 
DYSON F J DR, INST FOR ADVANCED STUDY, SCHOOL OF NATURAL SCIENCE, PRINCETON NJ 08540, USA Telephone: (1) 6097348055 Facsimile: (1) Telex:

Email:

Commission(s): 40,51

DYSON JOHN E DR, DPT OF ASTRONOMY, UNIVERSITY OF MANCHESTER, MANCHESTER M13 9PL, UK

Telephone: (44) 1612754235

Facsimile: (44)

Telex: 668932

Email:

Commission(s): 34,49

DZHAPLASHVILI VICTOR P DR, ABASTUMANI ASTROPHYSICAL, OBSERVATORY GEORGIAN ACAD OF SCIENCES, 383762 ABASTUMANI, GEORGIA

Telephone: (7)

Facsimile: (7)

Telex:

Email:

Commission(s): 15,16

DZIEMBOWSKI WOJCIECH PROF, COPERNICUS ASTRON CENTER, POLISH ACAD OF SCIENCES

UL BARTYCKA 18, PL 00716 WARSAW, POLAND

Telephone: (48)

Facsimile: (48)

Telex:

Email:

Commission(s): 27,35

DZIFCAKOVA ELENA, DPT ASTRON \& ASTROPHYSICS, KOMENSKY UNIVERSITY, MLYNSKA DOLINA 1 SK 84215 BRATISLAVA, SLOVAK R

Telephone: (42) 7720 003/684

Email: dzifcakova@fmph.uniba.sk

Facsimile: (42) 7725882

Telex:

Commission(s):

DZIGVASHVILI R M DR, ABATSUMANI ASTROPHYSICAL, OBSERVATORY, GEORGIAN ACAD OF SCIENCES 3383762 ABASTUMANI, GEORGIA

Telephone: (7)

Facsimile: (7)

Telex:

Email:

Commission(s): 33

DZUBENKO NIKOLAI, DPT OF ASTRONOMY, UNIVERSITY OF KIEV, ACAD GLUSHKOV STR 6 252022 KIEV, UKRAINE

Telephone: (7) 0442664457

Facsimile: (7) $0442664507 \quad$ Telex:

Email: kotsarenko@univ.kicv.ua

Commission(s): 10,12

D'ODORICO SANDRO DR, ESO, KARL SCHWARZSCHILDSTR 2, D 85740 GARCHING MUENCHEN, GERMANY

Telephone: (49) $89320060 \quad$ Facsimile: (49) 893202362 Telex: 52828222

Email:

Commission(s): 28,34

EATON JOEL A DR, CTR EXECLLENCE INFORM SYS, TENNESSEE STATE UNIV, 265G/330 TENTH AVE NR

NASHVILL TN 372033401 , USA

Telephone: (1) 8123354176

Facsimile: (1)

Telex:

Email:

Commission(s): 42

EBISUZAKI TOSHIKAZU DR, KOMABA MEGURO-KU, TOKYO 153, JAPAN

Telephone: (81) $334671171 * 665$

Facsimile: (81) 334653925

Telex:

Email:

Commission(s): 28

ECCLES MICHAEL J DR, BALLENCRIEFF TOLL, SUNNYSIDE, BATHGATE EH48 4LD, UK

Telephone: (44) 150653989

Facsimile: (44)

Telex: $\mathbf{7 2 7 4 8 4}$

Email:

Commission(s): 51

ECHEVERRIA ROMAN JUAN M, OBS ASTRONOMICO NACIONAL, UNAM, APDO POSTAL 877

22860 ENSENADA B CALIF, MEXICO

Telephone: (52)

Facsimile: (52)

Telex:

Email:

Commission(s)

EDDY JOHN A DR, 3460 ASH AVE, BOULDER CO 80303, USA

Telephone: (1) 3034971680

Facsimile: (1)

Telex: 45694

Email:

Commission(s): 10,41

EDELMAN COLETTE DR, BUREAY DES LONGITUDES, 77 AVE DENFERT ROCHEREAU, F 75014 PARIS, FRANCE

Telephone: (33) 140512272

Facsimile: (33)

Telex:

Email:

Commission(s): 07,20

EDELSON RICK DR, CASA, UNIVERSITY OF COLORADO, BOX 391, BOULDER CO 80309 0391, USA

Telephone: (1) 3034926784

Facsimile: (1)

Email: elroy::rick span

Commission(s): 28,40,44 
EDGE ALASTAIR, INSTITUTE OF ASTRONOMY, THE OBSERVATORIES, MADINGLEY RD

CAMBRIDGE CB3 OHA, UK

Telephone: (44) 1223330803

Email: ace@mail.ast.cam.ac.uk

Facsimile: (44) $1223337523 \quad$ Telex:

Commission(s):

EDLEN BENGT PROF, DPT OF PHYSICS, UNIVERSITY OF LUND, SOELVEGATAN 14, S 223 62 LUND, SWEDEN

Telephone: (46) 46107730

Facsimile: (46)

Telex:

Email:

Commission(s): 14

EDMONDSON FRANK K PROF, ASTRONOMY DPT, INDIANA UNIVERSITY, SWAIN WEST 319 , BLOOMINGTON IN 47405, USA

Telephone: (1) 8123356918

Facsimile: (1)

Telex:

Email:

Commission(s): $20,30,33,41$

EDMUNDS MICHAEL GEOFFREY, DPT OF PHYSICS, UNIV WALES COLLEGE, BOX 913, CARDIFF CF1 3TH, UK Telcphone: (44) 1222874785

Facsimile: (44) 1222371921

Telex:

Email:

Commission(s): 28

EDVARDSSON BENGT DR, ASTRONOMICAL OBSERVATORY, BOX 515, S 75120 UPPSALA, SWEDEN

Telephone: (46) 18512488

Facsimile: (46) 18527583

Telex: 76024 univups s

Email: astbe@seudac21 bitnet

Commission(s): 36

EDWARDS ALAN CH DR, M/S 238-600, JET PROPULSION LABS, 4800 OAK GROVE DR, PASADENA CA 91109, USA

Telephone: (1) 8183544321

Facsimile: (1) 8183934965 Telex:

Email: chad@logos.jpl.nasa.gov

Commission(s): 35

EDWARDS PAUL J DR, MOUNT STROMLO \& SIDING, SPRING OBSERVATORIES, PRIVATE BAG

WODEN PO ACT 2606, AUSTRALIA

Telephone: (61) 6288111

Email:

Facsimile: (61) 62490233

Commission(s): $25,27,44,50$

Telex: 68270 aa

EDWARDS TERRY W, DPT PHYSICS \& ASTRONOMY, UNIVERSITY OF MISSOURI, COLUMBIA MO 65211, USA

Telephone: (1) 3148823036

Facsimile: (1)

Telex:

Email:

Commission(s): 35

EDWIN PATRICIA, DPT OF MATHEMATICS, UNIVERSITY OF ST ANDREWS, ST ANDREWS, FIFE KY16 9SS, UK

Telephone: (44) 133476161

Facsimile: (44) 133463748

Telex: $9312110846 \mathrm{sag}$

Email: p.m.edwin@st-andrews.ac.uk

Commission(s):

EDWIN ROGER P, DPT PHYSICS \& ASTRONOMY, UNIVERSITY OF ST ANDREWS, NORTH HAUGH

ST ANDREWS FIFE KY16 9SS, UK

Telephone: (44) 133476161

Facsimile: (44) 133474487 Telex:

Email:

Commission(s): 09

EELSALU HEINO DR, TARTU ASTROPHYSICAL OBS, ESTONIAN ACAD OF SCIENCES, EE 202444 TARTU, ESTONIA

Telephone: (372) 143428163

Facsimilc: (372) 143410205

Telex:

Email:

Commission(s): $25,30,41$

EFBEMOV YU I DR, INST OF APPLIED MATHS, ACADEMY OF SCIENCES, MIUSSKAJA SQ 4

125047 MOSCOW, RUSSIA

Telephone: (7)

Facsimile: (7)

Telex:

Email:

Commission(s):

EFREMOV YURI N DR, STERNBERG STATE ASTR INST, UNIVERSITETSKIJ PROSP 13, 119899 MOSCOW, RUSSIA

Telephone: (7) 951392657

Facsimile: (7)

Commission(s): 27,33,37

Telex:

Email:

EFSTATHIOU GEORGE, INSTITUTE OF ASTRONOMY, THE OBSERVATORIES, MADINGLEY RD

CAMBRIDGE CB3 OHA, UK

Telephone: (44) 1223337548

Email:

Facsimile: (44) 1223337523

Commission(s): 28,47
Telex: 817297 astron g 
EGGLETON PETER P DR, INSTITUTE OF ASTRONOMY, THE OBSERVATORIES, MADINGLEY RD

CAMBRIDGE CB3 OHA, UK

Telephone: (44) 1223337548

Facsimile: (44) 1223337523

Telex: 817297 astron $\mathrm{g}$

Email:

Commission(s): $35,42 C$

EGRET DANIEL DR, OBS DE STRASBOURG, 11 RUE UNIVERSITE, F 67000 STRASBOURG, FRANCE

Telephone: (33) 88358200

Facsimile: (33) $88250160 \quad$ Telex: 890506 starobs

Email: grenon@cgeuge54

Commission(s): $05,33,45$

EHGAMBERDIEV SHURAT DR, ASTRONOMICAL INSTITUTE, UZBEK ACADEMY OF SCIENCES

ASTRONOMICHESKAYA 33, 700052 TASHKENT, UZBEKISTAN

Telephone: (7) $3712358102 \quad$ Facsimile: (7) 3712327789 Telex:

Email: shuhrat@kumbel.silk.glas.apc.org Commission(s): 12

EHLERS JURGEN PROF, MPI F PHYSIK \& ASTROPHYS, KARL SCHWARZSCHILDSTR 1

D 85740 GARCHING MUENCHEN, GERMANY

Telephone: (49) $8932999444 \quad$ Facsimile: (49) Telex: 524629 astro d

Email:

Commission(s): 47

EICHHORN HEINRICH K DR, BOX 112055, GAINESVILLE FL 32611 2055, USA

Telephonc: (1) $9043927745 \quad$ Facsimile: (1) Telex:

Email: eichhorn@astro.ufl.edu Commission(s): 07,08,24,26

EICHLER DAVID DR, ASTRONOMY PROGRAM, UNIVERSITY OF MARYLAND, COLLEGE PARK MD 20742, USA

Telephone: (1) $3014546448 \quad$ Facsimile: (1)

Telex: 7108260352

Email:

Commission(s): 44

EIDELSBERG MICHELE DR, OBSERVATOIRE DE PARIS, SECTION DE MEUDON

F 92195 MEUDON PPL CDX, FRANCE

Telephone: (33) 145077562

Facsimile: (33) $145077469 \quad$ Telex:

Email: frmeu051

Commission(s): 14

EILEK JEAN, DPT OF PHYSICS, NEW MEXICO TECH, CAMPUS STATION, SOCORRO NM 87801, USA

Telephone: (1) 5058355433

Facsimile: (1)

Telex:

Email:

Commission(s): 44

EINASTO JAAN DR, TARTU ASTROPHYSICAL OBS, ESTONIAN ACAD OF SCIENCES, TORAVERE

EE 2444 TARTUMAA, ESTONIA

Telephone: (372) 7410151

Facsimile: (372) 7410205 Telex:

Email: maret@jupiter.ani.ee

Commission(s): $28,33,37,47$

EINASTO MARET, TARTU ASTROPHYSICAL OBS, EE 2444 TORAVERE, ESTONIA

Telephone: (372) 3410450

Facsimile: (372) $3+10205$ Telex:

Email:

Commission(s):

EINAUDI GIORGIO, DPT DI ASTRONOMIA, UNIVERSITA DI FIRENZE, LARGO E FERMI 5, I SO125 FIRENZE, ITALY

Telephone: (39) 5527521

Email:

Facsimile: (39) 55220039

Telex: 572268 arcetr $i$

EINICKE OLE H DR, COPENHAGEN UNIVERSITY OBS, BRORFELDEVEJ 23, DK 4340 TOLLOSE, DENMARK

Telephone: (45) 53488195

Facsimile: (45) 58488755

Telex:

Email:

Commission(s): 08

EIROA DE SAN FRANCISCO C, OBS ASTRONOMICO NCL, ALFONSO XII-3, E 28014 MADRID, SPAIN

Telephone: (34) $12270107 \quad$ Facsimile: (34)

Telex: 49880 oanm

Email:

Commission(s):

EKER ZEKI, KING SAUD UNIVERSITY, COLEEGE OF SCIENCE, BOX 2455, RIYADH 11451, SAUDI ARABIA

Telephone: (966) 14676315

Facsimile: (966)

Telex:

Email: f40a010@saksu00

Commission(s):

EKERS RONALD D DR, CSIRO, DIV OF RADIOPHYSICS, BOX 76, EPPING NSW 2121, AUSTRALIA

Telephone: (61) 28680222

Facsimile: (61) 28680310

Telex: 26230

Email: rekers@atnf.csiro.au

Commission(s): 28,40 
ELMEGREEN DEBRA MELOY, DPT PHYSICS \& ASTRONOMY, VASSAR COLLEGE, POUGHKEEPSIE, NY 12601, USA

Telephone: (1) 9144377356

Email: elmegreen@vassar.edu
Facsimile: (1)

Commission(s): 28,33,34
Telex:

ELSAESSER HANS PROF, MPI FUER ASTRONOMIE, KOENIGSTUHL, D 69117 HEIDELBERG, GERMANY
Telephone: (49) 6221528200

Facsimile: (49)

Commission(s): 21,33

Telex:

Email:

ELSMORE BRUCE DR, MULLARD RADIO ASTRON OBS, CAVENDISH LABORATORY, MADINGLEY RD CAMBRIDGE CB3 OHE, UK

Telephone: (44) 1223337294

Email:

Facsimile: (44) $1223354599 \quad$ Telex: 81292

Commission(s): $19,24,40$

ELSON REBECCA ANNE WOOD, INSTITUTE OF ASTRONOMY, THE OBSERVATORIES, MADINGLEY RD CAMBRIDGE CB3 OHA, UK

Telephone: (44) 1223337541

Email: elson(a)mail.ast.cam.ac.uk

Facsimile: (44) $1223337523 \quad$ Telex: 817297 astron $\mathrm{g}$

Commission(s):

ELST ERIC WALTER DR, OBSERVATOIRE ROYAL DE, BELGIQUE, AVE CIRCULAIRE 3

B 1180 BRUSSELS, BELGIUM

Telephone: (32) 23752484

Facsimile: (32) $23749822 \quad$ Telex:

Email: elst@atmos.ome.be

Commission(s): 20

ELSTE GUNTHER H DR, DPT OF ASTRONOMY, UNIVERSITY OF MICHIGAN, DENNISON BLDG

ANN ARBOR MI 48109 1090, USA

$\begin{array}{ll}\text { Telephone: (1) } 3137643444 & \text { Facsimile: (1) } \\ \text { Email: } & \text { Commission(s): 10,12,36 }\end{array}$

ELSTON WOLFGANG E PROF, DPT OF GEOLOGY, UNIVERSITY OF NEW MEXICO, 800 YALE BLVD NE

ALBUQUERQUE NM 87131, USA

Telephone: (1) $5052775339 \quad$ Facsimile: (1) Telex: 660461

Email:

Commission(s): 16

ELSWORTH YVONNE, DPT PHYSICS \& ASTRONOMY, UNIVERSITY OF ST ANDREWS, NORTH HAUGH

ST ANDREWS FIFE KY16 9SS, UK

Telephone: (44)

Facsimile: (44)

Telex:

Email:

Commission(s):

ELVIS MARTIN S DR, CENTER FOR ASTROPHYSICS, HCO/SAO, 60 GARDEN ST, CAMBRIDGE MA 02138, USA

Telephone: (1) 6174957442

Facsimile: (1)

Telex: 921428

Email:

Commission(s): $28,44,47$

ELVIUS AINA M PROF, STOCKHOLM OBSERVATORY, S 133 36 SALTSJOEBADEN, SWEDEN

Telcphonc: (46) $87170195 \quad$ Facsimile: (46) $87174719 \quad$ Telex: 12972 sobbserv s

Email: clvius@astro.su.se Commission(s): 28,34

ELWERT GERHARD PROF, LEHRSTUHL F THEORET ASTRO, PHYSIK DER UNIV TUEBINGEN AUF DER MORGENSTELLE 12 C, D 72076 TUEBINGEN, GERMANY

Telephone: (49) $7071296483 \quad$ Facsimilc: (49)

Email:

Commission(s): 10,40

Telex: $7-262714$ alt d

EL-BASSUNY ALAWY A A DR, HELWAN OBSERVATORY, HELWAN, EGYPT

Telephone: (20) 78 0645/2683

Facsimile: (20)

Telex: 9703 hiag un

Email:

Commission(s): 27,37

EL-BAZ FAROUK DR, ITEK OPTICAL SYSTEMS, 10 MAGUIRE RD, LEXINGTON MA 02173, USA

Telephone: (1) $6172762532 \quad$ Facsimile: (1)

Commission(s): 16

Telex: 923456

Email:

EL-RAEY MOHAMED E PROF, DPT ENVIRONMENT STUDIES, INST GRADUATE STUD \& RES UNIVERSITY OF ALEXANDRIA, ALEXANDRIA, EGYPT

Telephone: (20) 20

Facsimile: (20)

Telex:

Email:

Commission(s): 44 
EL-SHAHAWY MOHAMAD PROF, DPT ASTRON \& METEOROLOGY, FACULTY OF SCIENCES, CAIRO UNIVERSITY GEZA, EGYPT

Telcphone: (20) 272022

Email:

Facsimile: (20)

Commission(s): 19

Telex: 94372 uncai

EMANUELE ALESSANDRO DR, IAS, CNR, CP 67, I 0(6)4+ FRASCATI, ITALY

Teleplione: (39) $69+25655$

Facsimile: (39) 69416847

Telex: 6111261

Email: polifemoajirmias

Commission(s): $4 \downarrow$

EMELLANOV NIKOLAJ V DR, STERNBERG STATE ASTR INST, UNIVERSITETSKIJ PROSP 13

119899 MOSCOW, RUSSIA

Telephone: (7) 951393764

Email:

Facsimile: (7)

Commission(s): 07

Telex:

EMERSON DAVID, ROYAL OBSERVATORY, BLACKFORD HILL, EDINBURGH EH9 3HJ, UK

Telephone: (4)

Facsimile: (4)

Telex:

Email:

Commission(s): $28,40,46$

EMERSON JAMES P, DPT OF PHYSICS, QUEEN MARY/WESTFIELD COLL, MILE END RD, LONDON E1 4NS, UK Telejohonc: (44) $171980+811$

Email:

EMINZADE T A DR, SHEMAKHA ASTROPHYSICAL, OBSERVATORY, AZER ACADEMY OF SCIENCES $3732+3$ SHEMAKHA, AZERBAIDZHAN

Telephone: (994)

Facsimile: (994)

Telex:

Email:

Commission(s): 35

EMSLIE A GORDON, DPT OF PHYSICS, UNIVERSITY OF ALABALA, HUNTSVILLE AL 35899, USA

Telephone: (1) 2058956167

Facsimile: (1)

Telex:

Email:

Commission(s): 10

ENARD DANIEL DR, ESO, KARL SCHWARZSCHILDSTR 2, D 85741 GARCHING MUENCHEN, GERMANY

Telephone: (49) 8932066251

Facsimile: (49) 893202362

Telex:

Email:

Commission(s):

ENCRENAZ PIERRE J DR, RADIOASTRONOMIE ENS, 24 RUE LHOMOND, F 75231 PARIS CDX 05, FRANCE

Telephone: (33) $1+3291235 \quad$ Facsimile: (33)

Email:

Commission(s): 34

Telex:

ENCRENAZ THERESE DR, OBSERVATOIRE DE PARIS, SECTION DE MEUDON, GROUPE PLANETES, F 92195

MEUTON PPL CDX, FRANCE

Telephone: (33) $145(177691$

Facsimile: (33)

Telex: 204464

Email:

Commission(s): 15,16

ENDAL ANDREW S DR, APPLIED RESEARCH CORP, 8201 CORPORATE DR, SUITE 920, LANDOVER MD 20785, USA

Telephone: (1) $301+508+42$

Facsimile: (1)

Commission(s): 35

Telex:

Email:

ENGELBRECHT CHRISTIAN DR, UNIVERSITY OF STELLENBOSC, PRETORIA, SOUTH AFRICA

Telephone: (27)

Facsimile: (27)

Telex:

Enaili:

Commission(s):

ENGELS DIETER DR, HAMBURGER STERNWARTE, GOJENSBERGSWEG 112, D 21029 HAMBURG, GERMANY

Telephonc: (49) t0 $7252+136$

Facsimile: $(49)+107252+198$

Telex: 217884

Email: dengelsálos.uni-hamburgode

Commission(s): (19)

ENGIN SEMANUR PROF, DPT OF ASTRONOMY, UNIVERISTY OF ANKARA, FEN FAKULTESI, OG100 BESEVLER, TURKEY

$\begin{array}{ll}\text { Telephonc: (90) } & \text { Facsimile: (90) } \\ \text { Email: } & \text { Commission(s): }\end{array}$

ENGINOL TURAN B DR, INST FOR GRADUATE STUDIES, IN SCIENCE \& ENGINEERING, BOGAZICI UNIVERSITY TURKEY

Telephone: (90) 11631500

Facsimile: (90) 11658480

Commission(s): 47

Telex: $26+11$ boun tr

Email: enginol f tr boun 
M 522

N 0315 BLINDERN OSLO 3, NORWAY

Telephone: (47) 22856521

Facsimile: (47) 22856505

Telex:

Email: oddbjorn.engvold@astro.uio.no

Commission(s): $09,10 \mathrm{P}$

ENOME SHINZO PROF, NOBEYAMA RADIO OBS, NAOJ, MINAMIMAKI MURA, NAGANO 384 13, JAPAN

Telephone: (81) 267982831

Facsimile: (81) 267982884

Telex: 3329005 naonro j

Email:

Commission(s): 10,40

ENSLIN HEINZ DR, ALSTEDDERSTR $1805, \mathrm{D}+453+$ LUENEN, GERMANY

Telephone: (49)

Email:

Facsimile: (49)

Telex:

Commission(s): 19,31

EPISHEV VITALI P, LAB OF COSMIC INVESTIGAT, UNIVERSITY, DALYOKAYA STR 2A 294000 UZHGOROD, UKRAINE

Telephone: (7) 312236065

Facsimile: (7) 312236136

Telex: $27+155$ kniga

Email:

Commission(s): 16,20

EPPS HARLAND WARREN PROF, DPT OF ASTRONOMY, UNIVERSITY OF CALIFORNIA, MATH SCI RM 8983 LOS ANGELES CA 90024, USA

Telephone: (1) 2138253025

Facsimile: (1)

Telex: 910-3427597

Email:

Commission(s):

EPSTEIN EUGENE E DR, AEROSPACE CORPORATION, 2118 PATRICIA AVE, LOS ANGELES CA 90009, USA

Telephone: (1) 2136486798

Facsimile: (1)

Telex: 664460

Email:

Commission(s): 40,51

EPSTEIN GABRIEL LEO DR, NASA GSFC, CODE 682, GREENBELT MD 20771, USA

Telephone: (1)

Facsimilc: (1) Tclex:

Email:

Commission(s): 12,14

EPSTEIN ISADORE PROF, DPT OF ASTRONOMY, COLUMBIA UNIVERSITY, PUPIN HALL 538 W 120 TH ST NEW YORK NY 10027, USA

Telephone: (1) 2122803280

Email:

Facsimile: (1)

Telex: 125953 columbia

Commission(s): 35

EPSTEIN RICHARD I DR, LOS ALAMOS NATIONAL LAB, MS 436, BOX 1663, LOS ALAMOS NM 87545, USA

Telephone: (1) 5056679595

Facsimile: (1)

Telex:

Email:

Commission(s):

ERCAN E NIHAL, KANDILLI OBSERVATORY, BOGAZICI UNIVERSITY, CENGELKOY, 81220 ISTANBUL, TURKEY

Telephonc: (90) $13320240 / 41$

Facsimile: (90) 12657131

Telex: 26411 boun tr

Email:

Commission(s):

ERDI B DR, ASTRONOMY DPT, EOTVOS UNIVERSITY, KUN BELA TER 2, H 1083 BUDAPEST, HUNGARY

Tclephone: (36) $111+1019$

Facsimile: (36)

Telex:

Ematil:

Commission(s): 07

ERGMA E V DR, DPT THEORET PHYS \& ASTOPH, TARTU UNIVERSITY, UELIKOOLI 18, EE 202400 TARTU, ESTONIA

$\begin{array}{lll}\text { Telephone: (372) } 73775 & \text { Facsimile: }(372) & \text { Telex: } 412623 \text { scstp su } \\ \text { Email: } & \text { Commission(s): } 35,49 & \end{array}$

ERICKSON WILLIAM C DR, DPT OF PHYSICS, UNIVERSITY OF TASMANIA, GPO BOX 252C, HOBART TAS 7001, AUSTRALIA

Telephone: (61) 2202401

Facsimile: (61) 2202186

Commission(s): 40

Telex: aas8150

Email:

ERIGUCHI YOSHIHARU DR, DPT EARTH SCI \& ASTRONOMY, UNIVERSITY OF TOKYO, MEGURO KU

TOKYO 153, JAPAN

Telephone: (81) 346 71 171*439 Facsimile: (81)

Email:

Commission(s): 35

Telex: $25 \$ 10$ unitokyo 
ERIKSEN GUNNAR PROF, INST THEORET ASTROPHYSICS, UNIVERSITY OF OSLO, BOX 1029 N 0315 BLINDERN OSLO 3, NORWAY

Telephone: (47) 02-45-65-15

Email:

Facsinile: (47)

Telex:

Commission(s): 40

ERIKSSON KJELL DR, ASTRONOMICAL OBSERVATORY, BOX 515, S 75120 UPPSALA, SWEDEN

Telephone: (46) 18112488

Facsimile: (46)

Commission(s): 36

Telex: 76024 univups s

Email:

ERPYLEV N P DR, INST OF ASTRONOMY, ACADEMY OF SCIENCES, PYATNITSKAYA UL 48 109017 MOSCOW, RUSSIA

Telephone: (7) 952315461

Facsimile: (7)

Commission(s): 41

Telex: 412623 sestp su

Email:

ERSH KOVICH ALEXANDER PROF, DPT GEOPHYS \& PLANET SCI, TEL AVIV UNIVERSITY, TEL AVIV, ISRAEL

Telephone: (972) 3413505

Facsimile: (972)

Telex: $3 \$ 2171$ versy il

Email:

Commission(s): 15

ERTAN A YENER DR, FACULTY OF SCIENCE, EGE UNIVERSITY, BOX 21, 35100 BORNOVA IZMIR, TURKEY

Telcphone: (90)

Facsimile: (90)

Telex:

Email:

Commission(s):

ESCALANTE VLADIMIR DR, INSTITUTO DE ASTRONOMIA, UNAM, APDO POSTAL 70-264

04510 MEXICO DF, MEXICO

Email: vadimir@astroscu.unam.mx

Facsimile: (52) 56160653

Telex:

Commission(s): 34

ESHLEMAN VON R PROF, DURAND 221, STANFORD UNIVERSITY, STANFORD CA 94305, USA

Telephone: (1) 4154973531

Facsimile: (1)

Telex:

Email:

Commission(s): $16,40,49$

ESIPOV VALENTIN F DR, STERNBERG STATE ASTR INST, 11723 4 MOSCOW, RUSSIA

Telephone: (7)

Facsimile: (7)

Telex:

Email:

Commission(s): 34

ESKIOGLU A NIHAT, ARS GOR METIN SALTIK, SAKARYA FAKULTESI, OZANLAR ASTROPHYS LAB ADAPAZARI, TURKEY

Telephone: $(90)$

Facsimile: $(\mathcal{O} 0)$

Commission(s): 27

Telex:

Email:

ESKRIDGE PAUL B DR, CENTER FOR ASTROPHYSICS, MS 81, 60 GARDEN ST, CAMBRIDGE MA 02138, USA

Telephone: (1) 617 f96 7585

Facsimile: (1)

Telex:

Email: eskridge(a)cfa.harvard.edu

Commission(s):

ESPOSITO F PAUL PROF, DPT OF PHYSICS, UNIVERSITY OF CINCINNATI, 210 BRAUNSTEIN ML 11

CINCINNATI OH 452210111 , USA

Telephone: (1) 5134752233

Facsimile: (1)

Commission(s):

Telex:

Email:

ESPOSITO LARRY W, LASP, UNIVERSITY OF COLORADO, BOX 392, BOULDER CO 80309 0392, USA

Telephone: (1) 3034927325

Facsimile: (1)

Telex:

Email:

Commission(s): 16

ESSER RUTH DR, INST MATHS \& PHYSICAL SCI, UNIVERSITY OF TROMSO, BOX 953, N 9000 TROMSO, NORWAY

Telephone: $(47)+783+5164$ Facsimile: (47) +78389852

Telex: $6+124$ surob $n$

Email: ruth-esser a esuit.uit.no

Commission(s): 12

ESTALELLA ROBERT, DPT FISICA DE ATMOSFERA, UNIVERSIDAD DE BARCELONA, AVD DIAGONAL 645, E 08028 BARCELONA, SPAIN

Telephone: (34) $33307311 / 298$

Facsimilc: (34)

Telex:

Email:

Commission(s):

ESTEBAN LOPEZ CESARA, IAC, C/ VIA LACTEA S/N, E 38200 LA LAGUNA, SPAIN

Telephonc: (34) 22605200

Facsimilc: (3t) 22605210

Telex:

Email:

Commission(s): $34,+1$ 
ETZEL PAUL B DR, DPT OF ASTRONOMY, SAN DIEGO STATE UNIV, SAN DIEGO CA 92182, USA

Tclephone: (1) 6194956169

Facsimile: (1) $6195945485 \quad$ Telex:

Email: etzel@mintaka.sdsu.edu

Commission(s): 42

EUBANKS THOMAS M DR, 7243 ARCHLAW DR, CLIFTON VA 22024 2126, USA

Telephone: (1) Facsimile: (1) Telex:

Email:

Commission(s):

EVANGELIDIS E DR, PLASMA PHYSICS DIVISION, ATOMIC ENERGY CORP, PO BOX 582

PRETORIA 0001, SOUTH AFRICA

Telephonc: (27) 2712213311

Facsimilc: (27)

Telex: 30253 sa

Email:

Commission(s): 33,36

EVANS ANEURIN, DPT OF PHYSICS, UNIVERSITY OF KEELE, KEELE STS 5BG, UK

Facsimile: (44)

Tclex: 36113 unklib g

Email:

Commission(s): 27,34

EVANS DAFYDD WYN, ROYAL GREENWICH OBS, MADINGLEY RD, CAMBRIDGE CB3 OEZ, UK

Telephone: (44) 1223374000

Facsimile: (44) $1223374700 \quad$ Telex:

Email: dwe (a)mail.ast.cam.ac.uli

Commission(s):

EVANS IAN NIGEL, STSCI, HOMEWOOD CAMPUS, 3700 SAN MARTIN DR, BALTIMORE MD 21218, USA

Telephone: (1) 4103384756

Facsimile: (1) $\$ 103384767 \quad$ Telex:

Email: evans(a)fos.stsci.cdu

Commission(s):

EVANS J V DR, COMSAT LABORATORIES, 22300 COMSAT DR, CLARKSBURG MD 20871, USA

Telephone: (1) $301+28+422$

Facsimile: (1)

Telex: 908753

Email:

Commission(s): 12

EVANS JOHN W DR, 1 BAYA RD, ELDORADO, SANTA FE NM 87503, USA

Telephone: (1)

Facsimile: (1)

Telex:

Email:

Commission(s):

EVANS KENTON DOWER DR, DPT OF PHYSICS, UNIVERSITY OF LEICESTER, UNIVERSITY RD

LEICESTER LE 1 7RN, UK

Telephone: (44) 1533554455

Email:

Facsimile: (4t)

Telex: 341664

Commission(s): 40

EVANS NANCY REMAGE DR, SAL/ISTS, YORK UNIVERSITY, 4700 KEELE ST, NORTH YORK ON LAK 3C8, CANADA

Tclephonc: (1)

Facsimile: (1) $\$ 167365386 \quad$ Telex:

Email:

Commission(s): 27

EVANS NEAL J II ASS PROF, ASTRONOMY DPT, UNIVERSITY OF TEXAS, RLM 15 308, AUSTIN TX 78712 1083, USA

Telephone: (1) $512471+461$

Facsimile: (1)

Telex:

Email:

Commission(s): 34,51

EVANS ROBERT REV, WARRUMBUNGLE UNITING, CHURCH PARISH, 63 CASSILIS ST

COONABARABRAN NSW 2357, AUSTRALIA

Telephone: (61) $68+21325$

Facsimilc: (61)

Telex:

Enail:

Commission(s): 28

EVANS ROGER G DR, RUTHERFORD APPLETON LAB, SPACE \& ASTROPHYSICS DIV, BLDG R25/R68 CHILTON DIDCOT OX11 (OQX, UK

Telephone: (4) 123521900

Facsimile: (44)

Commission(s): 28

Telex: 83159 ruthlb g

Email:

\section{OS ALAMOS NM 87544, USA}

EVANS W DOYLE, 390 EL
Telephone: (1) $50566736+4$

Facsimile: (1)

Telex:

Email:

Commission(s): $4+$

EVANS WYN, OXFORD, UK

Telephone: (4)

Facsimile: (4t)

Telex:

Email:

Commission(s): 33 
EVIATAR AHARON PROF, DPT GEOPHYS \& PLANET SCI, TEL AVIV UNIVERSITY, TEL AVIV, ISRAEL

Telephone: (972) 3420620

Facsimile: (972)

Commission(s): 15,49

Telex: 342171 versy il

Email:

EVREN SERDAR DR, FACULTY OF SCIENCE, EGE UNIVERSITY, BOX 21, 35100 BORNOVA IZMIR, TURKEY

Telephone: (90) $51180110^{\star 2322}$

Facsimile: (90)

Telex:

Email: efeast01@ trearn.bitnet

Commission(s): 27

EWEN HAROLD I DR, HILLCREST DRIVE 60, BEAVER, SOUTH DEERFIELD MA 01373, USA

Telephone: (1)

Facsimile: (1)

Telex:

Email:

Commission(s):

EWING MARTIN S, YALE UNIVERSITY SECF, BOX 208267, NEW HAVEN CT 06520 8267, USA

Telephone: (1) $20343242+3$

Email: martin.cwing@yalc.edu

Facsimile: (1) 2034322797

Telex: 9102508365 yale

Commission(s): 40

EZER-ERYURT DILHAN PROF, DPT OF PHYSICS, MIDDLE EAST TECH UNIV, 06531 ANKARA, TURKEY

Telephone: $(90) 41237100 * 3255$

Facsimile: (90)

Commission(s): 35

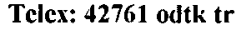

Email:

FABBIANO GIUSEPPINA, CENTER FOR ASTROPHYSICS, HCO/SAO, 60 GARDEN ST, CAMBRIDGE MA 02138, USA

Telephone: (1) 6174957204

Facsimile: (1)

Telex: 921428 satellite cam

Email:

Commission(s): 28

FABER SANDRA M PROF, LICK OBSERVATORY, UNIVERSITY OF CALIFORNIA, SANTA CRUZ CA 95064, USA

Telephone: (1) $408+292944$

Facsimile: (1)

Telex:

Email:

Commission(s): $28,33,47$

FABIAN ANDREW C DR, INSTITUTE OF ASTRONOMY, THE OBSERVATORIES, MADINGLEY RD

CAMBRIDGE CB3 OHA, UK

Tclephone: (4t) 1223337548

Facsimile: (4) $1223337523 \quad$ Telex:

Email:

Commission(s): 4

FABREGAT JUAN DR, DPT MATEMATICA Y ASTRON, UNIVERSIDAD DE VALENCIA, BURJASOT, E 46100

VALENCIA, SPAIN

Telephonc: (34) $6386+573$

Email: 16+44::fabregat

Facsimile: (34) 63864735

Telex:

Commission(s): 25

FABRICANT DANIEL G, CENTER FOR ASTROPHYSICS, HCO/SAO, 60 GARDEN ST, CAMBRIDGE MA 02138, USA

Telephone: (1) 6174957398

Facsimile: (1)

Telex: 921428 sattellite cam

Email:

Commission(s): $09,28,44$

FABRICIUS CLAUS V, COPENHAGEN UNIVERSITY OBS, BRORFELDEVEJ 23, DK 4340 TOLLOSE, DENMARK

Telephone: (45) 53488195

Facsimile: (45) 58488755

Telex: $\$+155$ danast dk

Email:

Commission(s): 08

FACONDI SILVIA ROSA DR, IST DI RADIOASTRONOMIA, CNR, VIA IRNERIO 46, I 40126 BOLOGNA, ITALY

Telephone: (39) 51287835

Email: sfacondiagastbo1.infin.it

Facsimile: (39) $512+3130$

Telex: 520634 infn.bo

FADEYEV YURI A, INST OF ASTRONOMY, ACADEMY OF SCIENCES, PYATNITSKAYA UL 48 109017 MOSCOW, RUSSIA

Telephone: (7) 952315461

Email:
Facsimile: (7)

Commission(s): 27,35
Telex: +12623 scstp su

FAELTHAMMAR CARL GUNNE PR, DPT OF PLASMA PHYSICS, ROYAL INST OF TECHNOLOGY S 100 + + STOCKHOLM 70, SWEDEN

Telephone: (46) $86877685 \quad$ Facsimile: (46)

Email:

Commission(s):

Telex: 10389 kthb

FAHEY RICHARD P, GSFC, CODE 684 , GREENBELT MD 20771, USA

Telephone: (1) 3012869877

Email: fahey/astars.gsfc.nasa.gov

Facsimile: (1) 3012861753

Commission(s):
Telex: 
FAHLMAN GREGORY G DR, DPT GEOPHYS \& ASTRONOMY, UNIV OF BRITISH COLUMBIA, 2075 WESBROOK PL VANCOUVER BC V6T 1W5, CANADA

Telcphonc: (1) 6042284891

Facsimile: (1) $60+2286047 \quad$ Telex:

Email:

Commission(s):

FAHR HANS JOERG PROF DR, INSTITUT FUER ASTROPHYSIK, UNIVERSITAET BONN, AUF DEM HUEGEL 71 D 53121 BONN, GERMANY

Telephone: (49) 228733677

Facsimile: (49)

Commission(s): 49C

Telex: $886+40$

Email:

FAIRALL ANTHONY P PROF, DPT OF ASTRONOMY, UNIVERSITY OF CAPE TOWN, RONDEBOSCH 7700 SOUTH AFRICA

Telephone: (27) $21698531 \times 629$

Facsimile: (27)

Telex: $5721+39$

Email:

Commission(s): $28,30 \mathrm{C}, 46$

FALCHI AMBRETTA, OSS ASTROFISICO, DI ARCETRI, LARGO E FERMI 5, I 50125 FIRENZE, ITALY

Telephone: (39) 552752236

Facsimile: (39)

Telex: 572268 arcetr $i$

Email:

Commission(s): 10

FALCIANI ROBERTO DR, DPT DI ASTRONOMIA, UNIVERSITA DI FIRENZE, LARGO E FERMI 5 I 50125 FIRENZE, ITALY

Telephone: (39) 5527521

Email:

Facsimile: (39) 55220039

Telex: $\mathbf{5 7 2 2 6 8}$ arcetr i

FALCO-ACOSTA EMILIO E DR, CENTER FOR ASTROPHYSICS, HCO/SAO MS 19, 60 GARDEN ST CAMBRIDGE MA 02138, USA

Telephone: (1) 6174957131

Email: falco@cfa.harvard.cdu
Facsimile: (1) $617+957467$

Commission(s): 28
Telex: 921428 satellite cam

FALGARONE EDITH, CALTECH, MS 40547 , DOWNES LAB OF PHYSICS, PASADENA CA 91125 , USA

Telephone: (1) 8182432438

Email:

Facsimile: (1)

Telex:

Commission(s): 34C

FALK SYDNEY W JR DR, 1011 SHELLEY AVE, AUSTIN TX 787(13, USA

Telephone: (1)

Facsimile: (1)

Telex:

Email:

Commission(s): 34,47

FALL S MICHAEL DR, STSCI, HOMEWOOD CAMPUS, 3700 SAN MARTIN DR, BALTIMORE MD 21218, USA

Telephone: (1)

Facsimile: (1)

Telex:

Email:

Commission(s): $28,33,37,47$

FALLE SAMUEL A DR, DPT OF APPL MATHEMATICS, UNIVERSITY OF LEEDS, LEEDS LS2 9JT, UK

Telephone: (4) $1135+31751$

Facsimile: (44)

Telex:

Email:

Commission(s): 34

FALLER JAMES E PROF, JILA/NBS, UNIVERSITY OF COLORADO, BOX 400 , BOULDER CO 80309 0440, USA

Telephonc: (1) 3034928509

Facsimile: (1)

Commission(s):

Telex: $7558+2$ jila

Email:

FALLON FREDERICK W DR, NGS, N/CG 11 NOAA, 6010 EXECUTIVE BLVD, ROCKVILLE MD 20852, USA

Telcphonc: (1) $301+438+24$

Facsimilc: (1)

Telex:

Email:

Commission(s): 24,31

FALOMO RENATO DR, OSS ASTRONOMICO DI PADOVA, VIC DELL OSSERVATORIO S, I 35122 PADOVA, ITALY

Telephone: (39) 49661499

Facsimile: (39)

Commission(s):

Telex: $\$ 32071$ astros $i$

Email: astrpd::falomo

FAN YING, DPT OF ASTRONOMY, BEIJING NORMAL UNIVERSITY, BEIJING 100875, CHINA PR

Telephone: (86) $1653531 * 6285 \quad$ Facsimile: $(86)$

Telex:

Email:

Commission(s): 34

FANG CHENG, DPT OF ASTRONOMY, NANJING UNIVERSITY, NANJING, CHINA PR

Telephone: (86) $2534651 * 2882$

Facsimile: (86)

Commission(s): 10,12C

Telex: $3+151$ prenu en 
FANG LI-ZHI, DPT OF PHYSICS, UNIVERSITY OF ARIZONA, TUCSON AZ 85721, USA

Telephone: (1)

Facsimile: (1)

Telex:

Email:

Commission(s): 44,47

FANSELOW JOHN LYMAN, JPL, MS 169 315, 4800 OAK GROVE DR, PASADENA CA 91109, USA

Telephone: (1) 2133546323 Facsimile: (1) Telex: $675+29$

Email:

Commission(s): 24

FANTI CARLA GIOVANNINI, IST DI RADIOASTRONOMIA, CNR, VIA IRNERIO 46, I 40126 BOLOGNA, ITALY

Telephone: (39) 5123 2856/57

Facsimile: (39)

Telex: 211664 infin bo

Email:

Commission(s):

FANTI ROBERTO, IST DI FISICA, UNIVERSITA DI BOLOGNA, VIA IRNERIO 46, I 40126 BOLOGNA, ITALY

Telephone: (39) $51232856 / 57$

Facsimile: (39)

Telex: $21166+$ infn bo

Email:

Commission(s): 40

FARAGGIANA ROSANNA PROF, OAT, BOX SUCC TRIESTE 5, VIA TIEPOLO 11, I 34131 TRIESTE, ITALY

Telephone: (39) 40793921

Facsimilc: (39)

Telex: 461137 oat $\mathrm{i}$

Email:

Commission(s): $29,36,44,45$

FARINELLA PAOLO DR, IST DI MATEMATICA, UNIVERSITA DI PISA, VIA BUONARROTI 2, I 56127 PISA, ITALY

Telephone: (39) $5059952 t$

Facsimile: (39) 50599524

Telex: 500371 enuce $i$

Email: twin2@aicnucerm.bitnet

Commission(s): $07,15,16$

FARNIK FRANTISEK, ASTRONOMICAL INSTITUTE, CZECH ACADEMY OF SCIENCES, ONDREJOV OBSERVATORY CZ 251 65 ONDREJOV, CZECH R

Telephone: $(+2) 20+857329 / 7111$

Email: astsunacscarn
Facsimile: $(+2) 204851611$

Commission(s): 10
Telex: 121579 astr c

FAUCHER PAUL DR, OCA OBSERV DE NICE, BP 229, F 06304 NICE CDX 4, FRANCE

Telephone: (33) 93890420

Email:

Facsimile: (33) 92003033

Telex: $\$ 60004$ obsnice $f$

Commission(s): 14

FAULKNER DONALD J DR, MOUNT STROMLO \& SIDING, SPRING OBSERVATORIES, PRIVATE BAG WODEN PO ACT 2606, AUSTRALIA

Telephone: (61) 62881111

Facsimile: (61) $62+90233$

Telex: 62270

Email:

Commission(s): 34,35

FAULKNER JOHN PROF, LICK OBSERVATORY, UNIVERSITY OF CALIFORNIA, SANTA CRUZ CA 95064, USA Telephone: (1) $408+292815$

Facsimile: (1)

Telex:

Email:

Commission(s): 35,42

FAUNDEZ-ABANS M DR, LAB NAC DE ASTROFISICA, CAIXA POSTAL 21, 37500 000 ITAJUBA MG, BRAZIL.

Telephone: (56)

Facsimile: (56)

Telex:

Email: $\ln a(a)(u . a n s p) . b r$

Commission(s):

FAUROBERT-SCHOLL M DR, OCA OBSERV DE NICE, BP 229, F (0630 4 NICE CDX 4 , FRANCE

Telephone: (33) 92003011

Facsimile: (33) 92003033

Telex: $\$ 60004$ obsnice $f$

Email: faurob(âfronis1.bitnct

Commission(s): 36

FAWELL DEREK R DR, UNIVERSITY OF LONDON OBS, MILL HILL PARK, LONDON NW7 2QS, UK

Telephone: $(+H)$

Facsimile: $(+4)$

Telex:

Email:

Commission(s):

FAY THEODORE D DR, TELEDYNE BROWN ENG, CUMMINGS RES PARK, MS 19, HUNTSVILLE AL 35807, USA

Telephone: (1)

Facsimile: (1)

Email:

Commission(s):

Telex:

FAZIO GIOVANNI G DR, HARVARD SMITHSONIAN CFA, G0 GARDEN ST, CAMBRIDGE MA 02138, USA

Telephone: (1) 6174957458

Facsimilc: (1) 6174957490

Telex:

Email: gfaziơäcfa.harvard.edu

Commission(s): 4+P,51

FEAST MICHAEL W PROF, SAAO, BOX 9, OBSERVATORY 7935, SOUTH AFRICA

Tclephone: (27) $2721+70025$

Facsimile: (27) $2721+73639$ Telex: 520309

Email:

Commission(s): 27,28C,29,33,37,45 
FEAUTRIER NICOLE DR, OBSERVATOIRE DE PARIS, SECTION DE MEUDON, F 92195 MEUDON PPL CDX, FRANCE Telephone: (33) 145077552

FECHTIG HUGO DR, SANSERWEG 3, D 6906 LEIMEN, GERMANY

Telephone: (49)

Facsimile: (49)

Email:

Commission(s): $15,21,22$

Telex:

FEDERICI LUCIANA, DPT DI ASTRONOMIA, UNIVERSITA DI BOLOGNA, CP 596, I 40100 BOLOGNA, ITALY

Telephonc: (39) 51222956

Facsimile: (39)

Telex: 211664 infn bo $\mathrm{i}$

Email:

Commission(s): 14

FEDERMAN STEVEN ROBERT, DPT PHYSICS \& ASTRONOMY, UNIVERSITY OF TOLEDO, 2801 W BANCROFT ST TOLEDO OH 43606, USA

Telephone: (1) 4195372652

Email:

Facsimile: (1) Telex:

Commission(s): $1+, 3+$

FEDOROV PETRO, NIKOLAEV OBSERVATORY, UKRAINIAN ACAD OF SCIENCE, 327030 NIKOLAEV, UKRAINE

Tclephonc: (7) $247853 / 362037 \quad$ Facsimile: (7)

Telex:

Email: nao(a)asor.nikolacv.ua

Commission(s):

FEDOROVA RIMMA T DR, NIKOLAEV OBSERVATORY, UKRAINIAN ACAD OF SCIENCE

327000 NIKOLAEV, UKRAINE

Tclephonc: (7) 375714

Facsinile: (7)

Telex:

Email: root@mao.nikolacv.ua

Commission(s): 08

FEGAN DAVID J DR, DPT OF PHYSICS, UNIVERSITY COLLEGE, BELFIELD, DUBLIN 4, IRELAND

Telephone: (353) $1693244 \quad$ Facsimile: (353)

Telex: 32693 ued ei

Email:

Commission(s):

FEHRENBACH CHARLES PROF, LES MAGNANARELLES, LOURMARIN, F 81160 CADENET, FRANCE

Telephone: (33) 90680028

Facsimile: (33)

Telex:

Email:

Commission(s): $(09,30,33,45$

FEIBELMAN WALTER A DR, NASA GSFC, CODE 685 , GREENBELT MD 20771, USA

Telephone: (1) 3012865272

Facsimile: (1)

Telex:

Email:

Commission(s): 10,27,34

FEIGELSON ERIC D DR, DPT OF ASTRONOMY, PENNSYLVANIA STATE UNIV, 525 DAVEY LAB

UNIVERSITY PARK PA 16802, USA

Telephone: (1) $81+8650162$

Facsimile: (1)

Telex: $8+2510$

Email: internet:edfáastro.psu.edu

Commission(s): 40

FEINSTEIN ALEJANDRO DR, OBSERVATORIO ASTRONOMICO, PASEO DEL BOSQUE S/N 1900 LA PLATA (BS AS), ARGENTINA

Telephone: (54) 21258985

Facsimile: (54) 21211761

Telex:

Email: afcinstein(äfcaglp.edu.ar

Commission(s): 25,37P

FEINSTEIN CARLOS DR, OBSERVATORIO ASTRONOMICO, PASEO DEL BOSQUE S/N 1900 LA PLATA (BS AS), ARGENTINA

Telephone: (54) 21217308

Facsimile: (54) $21258985 \quad$ Telex:

Email: cfeinstein(âf fcaglp.edu.ar

Commission(s): 28

FEISSEL MARTINE DR, OBSERVATOIRE DE PARIS, 61 AVE OBSERVATOIRE, F 7501+ PARIS, FRANCE

Telephonc: (33) 140512226

Facsimilc: (33) 140512232

Telex: 270776 OBS F

Email: feisseláobspm.fr

Commission(s): $08,19 \mathrm{C}, 31$

FEITZINGER JOHANNES PROF, ASTRONOMISCHES INSTITUT, RUHR UNIVERSITAET BOCHUM POSTFACH 102148, D +4780 BOCHUM, GERMANY

Telephone: (49) $23470(13+50 \quad$ Facsimile: (49)

Email:

Commission(s): $28,33,3+$

Telex: 825860

FEIX GERHARD DR, DPT XII, RUHR UNIVERSITAET BOCHUM, POSTFACH 111214, D 46047 BOCHUM, GERMANY

Telephone: (49) $23+7002051$

Email:
Facsimile: (4)

Commission(s): 40
Telex: 0825860 
FEJES ISTVAN DR, FOMI SATELLITE, GEODETIC OBSERVATORY, BOX 546, H 1373 BUDAPEST, HUNGARY

Telephone: (36)

Facsimile: (36)

Telex:

Email:

Commission(s): $\mathbf{5 1}$

FEKEL FRANCIS C, DYER OBSERVATORY, VANDERBILT UNIVERSITY, NASHVILLE TN 37235, USA

Telephone: (1) 6153222804

Facsimile: (1)

Telex:

Email:

Commission(s): $26 \mathrm{C}, 30 \mathrm{C}, 42$

FELDMAN PAUL A DR, HERZBERG INST ASTROPHYS, NTL RESEARCH COUNCIL, 100 SUSSEX DR OTTAWA ON K1A 0R6, CANADA

Telephone: (1) 6139936060

Facsimile: (1) 6139526602

Telex: 0533715

Email:

Commission(s): 40,51

FELDMAN PAUL DONALD DR, DPT PHYSICS \& ASTRONOMY, JOHNS HOPKINS UNIVERSITY, CHARLES \& 34TH S BALTIMORE MD 21218, USA

Telephone: (1) 3013387339

Facsimile: (1)

Telex: $710-234-1090$

Email:

Commission(s): $15 \mathrm{C}, 21,44$

FELDMAN URI, NAVAL RESEARCH LABORATORY, HOLBURT CTR FOR SPACES RE, 4555 OVERLOOK AVE SW WASHINGTON DC 20375 5000, USA

Telephone: (1) 2027673286

Facsimile: (1)

Telex:

Email:

Commission(s): 12

FELDMAN URI DR, NAVAL RESEARCH LABORATORY, CODE $+174,4555$ OVERLOOK AVE SW WASHINGTON DC 20375 50(0), USA

Telcphone: (1)

Facsimile: (1)

Telex:

Email:

Commission(s):

FELENBOK PAUL DR, OBSERVATOIRE DE PARIS, SECTION DE MEUDON, F 92195 MEUDON PPL CDX, FRANCE

Telephone: (33) 145077523

Facsimile: (33)

Telex: 201571

Email: felenboka $\operatorname{agin.obspm.fr}$

Commission(s): (09,29

FELLI MARCELLO DR, OSS ASTROFISICO, DI ARCETRI, LARGO E FERMI 5, I 50125 FIRENZE, ITALY

Telephone: (39) 552752240

Facsimile: (39)

Telex: $\mathbf{5 7 2 2 6 8}$

Email:

Commission(s): $3+, 40$

FELTEN JAMES E DR, NASA GSFC, CODE 685, GREENBELT MD 20771, USA

Telephone: (1) 3015521526

Facsimile: (1)

Telex:

Email:

Commission(s): $34,40,44,47$

FENG HESHENG, YUNNAN OBSERVATORY, CAS, BOX 110, KUNMING 72946 YUNNAN, CHINA PR

Telephone: (86) 8712035

Facsimile: (86)

Telex:

Email:

Commission(s):

FENG KE-JIA, DPT OF ASTRONOMY, BEIJING NORMAL UNIVERSITY, BEIJING 100875, CHINA PR

Telephone: (86) $1653531 * 6967$

Facsimile: (86)

Telex:

Email:

Commission(s): 10,46

FENKART ROLF P PROF DR, ASTRONOMISCHES INSTITUT, UNIVERSITAET BASEL, VENUSSTRASSE 7 CH +102 BINNINGEN, SWITZERLAND

Telephonc: (\$1) $612717711 / 12$

Facsimile: ( +1$)$

Tclex:

Email:

Commission(s): 33

FENTON K B DR, DPT OF PHYSICS, UNIVERSITY OF TASMANIA, GPO BOX 252C, HOBART TAS 7001, AUSTRALIA

Telephone: (61) $2202+11$

Facsimile: (61) $2202+10$

Telex: $\mathbf{5 8 1 5 0}$ aa

Email:

Commission(s): +4

FERETTI LUIGINA, IST DI RADIOASTRONOMIA, CNR, VIA IRNERIO +6, 1 +0126 BOLOGNA, ITALY

Telephone: (39) 51232856

Facsimile: (39)

Telex: 520634 infnbo $i$

Email:

Commission(s): 40

FERLAND GARY JOSEPH, DPT PHYSICS \& ASTRONOMY, UNIVERSITY OF KENTUCKY

LEXINGTON KY $\$ 0506$, USA

Telephone: (1)

Facsimile: (1)

Telex:

Email: garyajasta.pa.uky.edu

Commission(s): 27,28 
M 530

FERLET ROGER DR, INSTITUT D'ASTROPHYSIQUE, 98BIS BD ARAGO, F 75014 PARIS, FRANCE

Telephone: (33) 144328074

Facsimile: (33) $144328001 \quad$ Telex:

Email: ferlet@iap.fr

Commission(s): 34

FERLUGA STENO DR, DPT DI ASTRONOMIA, UNIVERSITA DI TRIESTE, VIA TIEPOLO 11, I 34131 TRIESTE, ITALY

Telephone: (39) 40763912

Facsimile: (39) 40309418

Telex: 461137 oat $i$

Email: astronet:astrts: :ferluga

Commission(s): 42

FERNANDEZ JEAN-CLAUDE DR, OCA OBSERV DE NICE, BP 229, F 06304 NICE CDX 4, FRANCE

Telephone: (33) 93890420

Facsimile: (33) 92003033

Telex: $46000+$ obsnice $f$

Email:

Commission(s):

FERNANDEZ JULIO A DR, DPT DE ASTRONOMIA, FAC HUMANIDADES Y CIENCIA, TRISTAN NARAJA 1674

MONTEVIDEO, URUGUAY

Telephone: (598) 5982419 087/089

Facsimile: (598) 5982409973

Telex: udelar uy 26692

Email:

Commission(s): 15C,20,46

FERNANDEZ SILVIA M DR, OBSERVATORIO ASTRONOMICO, DE CORDOBA, LAPRIDA 85† 5000 CORDOBA, ARGENTINA

Telephone: (54) 51230491

Facsimile: (54) 51210613

Telex: $\mathbf{5 1 8 2 2}$ bucor ar

Email: silfer(a) astro.cdu.ar

Commission(s): 07

FERNANDEZ-FIGUEROA M J DR, DPT DE ASTROFISICA, FAC DE FISICA, UNIVERSIDAD COMPLUTENSE E 28040 MADRID, SPAIN

Telephonc: (34) 14495316

Email:

Facsimile: (34)

Telex: $\mathbf{4 7 2 7 3} \mathrm{ff}$ uc

Commission(s): 29,46

FERNANZ MARGARITA DR, CSIC, CAMI DE STA BARABARA, E 17300 BLANES GIRONA, SPAIN

Telephone: (34) 7233 6101/02

Facsimile: (34) 972337806

Telex: 56372

Email: carn::marga(a)ceab.es

Commission(s): 35

FERNIE J DONALD PROF, DAVID DUNLAP OBSERVATORY, UNIVERSITY OF TORONTO, BOX 360 RICHMOND HILL ON L4C 4Y6, CANADA

Telephone: (1) $41688+9562 \quad$ Faesimile: (1) $+169783921 \quad$ Telex: 06986766 telexperts

Enmail: fernic@centaur,astro.utoronto. Commission(s): 25,27,41

FERRANDIZ JOSE MANUEL DR, ETS, INGENIEROS INDUSTRIALES, PASEO DEL CAUCE S/N

E +7011 VALLADOLID, SPAIN

Telephone: (34) 83304700

Email:

Facsimile: (34) $83392026 \quad$ Telex:

Commission(s):

FERRANDO PHILIPPE DR, CEA CEN, DPHG/SAP, BP 2, F 91191 GIF/YVETTE CDX, FRANCE

Telephone: (33) $169082020 \quad$ Facsimile: (33) Telex: 604860

Email: bitnet:ferrando(a 32779 Commission(s):

FERRARI ATTILIO DR, IST DI FISICA, UNIVERSITA DI TORINO, CORSO D AZEGLIO 46, I 10125 TORINO, ITALY

Telephone: (39) $11657694 \quad$ Facsimile: (39)

Email:

Commission(s): $40,+4$

Telex: 211041 infn to i

FERRARI D'OCCHIEPPO K DR, OESTERREICHISCHE AKADEMIE, DER WISSENSCHAFTEN

DR IGNAZ-SEIPEL-PLATZ 2, A 1010 WIEN, AUSTRIA

Telephone: (43) $1522281991 \quad$ Facsimile: (43)

Email: Commission(s): $41,+2$

Telex: 01-12628

FERRARI TONIOLO MARCO, IAS, CNR, CP 67,1 (0004+ FRASCATI, ITALY

Telephone: (39) 69425651

Facsimile: (39) $694168+7$

Telex: 610261 cnr-fra i

Email:

Commission(s): $4+$

FERRAZ-MELLO S PROF DR, IAG, UNIVERSIDADE DE SAO PAULO, CP 9638, 01065 970 SAO PAULO SP, BRAZIL

Telephone: (55) $115778599 * 218$

Facsimile: (55) 11276,3848

Telex: 1156735 iagm br

Email: sylvio(a)vax.iagusp.usp).br

Commission(s): (07P,20

FERRER MARTINEZ SEBASTIAN, DPT FIS TIERRA \& COSMOS, UNIVERSIDAD DE ZARAGOZA

E 50009 ZARAGOZA, SPAIN

Telephone: (34) 76357011

Facsimile: (34)

Telex: $\mathbf{5 8 1 9 8}$

Email:

Commission(s): 07 
FERRER OSVALDO EDUARDO DR, OBSERVATORIO ASTRONOMICO, PASEO DEL BOSQUE S/N 1900 LA PLATA (BS AS), ARGENTINA

Telephone: (54) 21217308

Facsimile: (54) $21211761 \quad$ Telex:

Email: Commission(s): $26,+2$

FERRERI WALTER, OSS ASTRONOMICO DI TORINO, ST OSSERVATORIO 20, I 10025 PINO TORINESE, ITALY

Telephone: (39) $11842040 \quad$ Facsimile: (39)

Email:

Commission(s): 20

Telex: 213236 astr i

FERRIN IGNACIO, UNIVERSIDAD DE LOS ANDES, FACULTAD DE CIENCIAS, DPT DE FISICA MERIDA 5101 A, VENEZUELA

Telephone: (58) $7+639930$

Facsimile: (58)

Telex:

Email:

Commission(s): 15

FERRINI FEDERICO, IST DI ASTRONOMIA, UNIVERSITA DI PISA, PIAZZA TORRICELLI 2, I 56100 PISA, ITALY Telephone: (39) $50+33+3$ Facsimile: (39) 5048227 Telex:

Email: Commission(s): 28,34

FESTOU MICHEL C DR, OBS MIDI PYRENEES, 14 AVE E BELIN, F 31400 TOULOUSE CDX, FRANCE

Telcphone: (33) 61252101

Facsimile: (33)

Commission(s): 15

Telex: 530776

Email:

FEYNMAN JOAN DR, JPL, MS $14+218,4800$ OAK GROVE DR, PASADENA CA 91109, USA

Telephonc: (1) $81835+345+\quad$ Facsimile: (1) Telex: $675+29$

Email: $\quad$ Commission(s): 49

FIALA ALAN D DR, US NAVAL OBSERVATORY, ASTRONOMICAL APPLICAT DPT, 3450 MASSACHUSETTS AVE NW WASHINGTON DC $203925+20$, USA

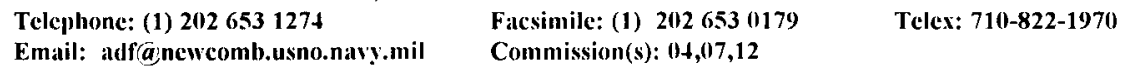

FICARRA ANTONINO DR, IST DI RADIOASTRONOMIA, CNR, VIA IRNERIO 46, I 40126 BOLOGNA, ITALY

Telephone: (39) $51232856 \quad$ Facsimile: (39) Telex: 211664 infn bo i

Email: $\quad$ Commission(s):

FICH MICHEL DR, DPT OF PHYSICS, UNIVERSITY OF WATERLOO, WATERLOO ON N2L, 3G1, CANADA

Telephone: (1) 5198851572

Email: bitnet:fichäwatsc1

Facsinile: (1) 5197468115

Telex: 06955259

FICHTEL CARL E DR, NASA GSFC, CODE 660, GREENBELT MD 20771, USA

Telephone: (1) 3012866281

Facsimilc: (1)

Telex: 89675

Email:

Commission(s): 4

FIEDLER RALPH L DR, NAVAL RESEARCH LABORATORY, CODE 4210,4555 OVERLOOK AVE SW WASHINGTON DC 20375 5000, USA
Telephone: (1) $20276706+4$
Facsimilc: (1)
Telex:
Email: fiedlerä rira.ntl.nary.mil
Commission(s):

FIEDLER RUSSELL, DPT OF MATHEMATICS, UNIVERSITY OF ST ANDREWS, ST ANDREWS, FIFE KY16 9SS, UK Telephone: (44) $133+76161$ Facsimilc: $(\$ 4) 1334637+8$ Commission(s):

Telex: $9312110846 \mathrm{sag}$

Email:

FIELD DAVID, SCHOOL OF CHEMISTRY, CANTOCKS CLOSE, BRISTOL BS\& 1TS, UK
Telephone: (4t) $11792+161^{*} 505$
Facsimile: $(+4)$

Email:

Commission(s): 34

FIELD GEORGE B PROF, CENTER FOR ASTROPHYSICS, HCO/SAO, 60 GARDEN ST, CAMBRIDGE MA 02138, USA

Telephone: (1) $617+95+721$

Email:

Facsimile: (1)

Telex: 921428 satellite cam

Commission(s): $28,34,40,44,47,49,51$

FIELDER GILBERT DR, WILLOW TREE, ELDROTH, AUSTWICK, LANCASTER LA2 8AH, UK

Telephone: (4)

Facsimile: $(4 t)$

Telex:

Email:

Commission(s): 16 
M 532

FIENBERG RICHARD T DR, SKY \& TELESCOPE, BOX 9111, BELMONT MA 01789111, USA

Telephone: (1) 6178647360

Facsimile: (1) $6178646117 \quad$ Telex:

Email: cfa::fienberg

Commission(s): 46

FIERRO JULIETA, INSTITUTO DE ASTRONOMIA, UNAM, APDO POSTAL 70-26 4 , 4510 MEXICO DF, MEXICO

Telephone: (52) $55484537 / 6223908$

Facsimile: (52) 56160653

Telex:

Email: fierro@affa.astroscu.unam.mx Commission(s): 34,46VP

FIGUERAS FRANCESCA DR, DPT ASTRONOMIA, UNIVERSIDAD DE BARCELONA, AVD DIAGONAL G47

E 08028 BARCELONA, SPAIN

Telephone: (34) 33307311

Facsimile: (34)

Telex:

Email: d3faffs0@eb0ub011

Commission(s): 33

FILIPOV LATCHEZAR, CTR LAB FOR SPACE RES, BULGARIAN ACAD SCIENCES, MOSKOVA ST 6

BG 1000 SOFIA, BULGARIA

Telephone: (359) 2870978

Facsimile: (359)

Telex: 23351 clsr bg

Email:

Commission(s):

FILIPPENKO ALEXEI V DR, ASTRONOMY DPT, UNIVERSITY OF CALIFORNIA, G01 CAMPBELL HALL BERKELEY CA 94720, USA

Telephone: (1) $\$ 156+21813$

Facsimile: (1)

Telex: 820181

Email; bitnet:alex(a)bkyast

Commission(s): $06,28,47$

FILLOY EMILIO MANUEL E E, IAR, CC 5, $189+$ VILLA ELISA (BS AS), ARGENTINA

Telephone: (54) 2143793

Facsimile: (54) 21211761 Telex:

Email:

Commission(s):

FINK UWE DR, LUNAR \& PLANETARY LAB, UNIVERSITY OF ARIZONA, TUCSON AZ 85721, USA

Telephone: (1) 5206212736

Facsimile: (1)

Telex: $91095211+3$

Email:

Commission(s): 14,16

FINKELSTEIN ANDREJ M DR, INST OF APPLIED ASTRONOMY, ACADEMY OF SCIENCES, ZDANOVSKAYA UL 8 $1970 \$ 2$ ST PETERSBURG, RUSSIA

Telephone: $(7)$

Email:

Facsimile: (7)

Telex:

Commission(s): +0

FINN G D DR, 22 NEIKAH CLOSE, KARANA DOWNS, QUEENS LAND 4305, AUSTRALIA

Telephone: (61)

Facsimile: (61)

Telex:

Email:

Commission(s): 36

FINN LEE SAMUEL, DPT PHYSICS \& ASTRONOMY, NORTHWESTERN UNIVERSITY

EVANSTON CA 60208 3112, USA

Telephone: (1) $708 \$ 914568$

Facsimile: (1) $708+919982$ Telex:

Email: Isf@holmes.astro.nwu.edu

Commission(s):

FINZI ARRIGO DR, DPT OF MATHEMATICS, IIT, TECHNION CITY, HAIFA 32000, ISRAEL

Telephone: (972)

Facsimile: (972)

Telex: $46+106$ tecon it

Email:

Commission(s):

FIRMANI CLAUDIO A PROF, INSTITUTO DE ASTRONOMIA, UNAM, APDO POSTAL 70-264,04510 MEXICO DF, MEXICO

Telephone: (52) $9055483712 \quad$ Facsimile: (52)

Commission(s): $\$ 2$

Telex: 1760155 cicme

Email:

FIRNEIS FRIEDRICH J DR, COMP CTR ACAD SCI, SONNENFELSGASSE 19/1, A 1010 WIEN, AUSTRIA

Telephone: (43)

Facsimile: (43)

Telex:

Email:

Commission(s): 24,51

FIRNEIS MARIA G DR, INSTITUT FUER ASTRONOMIE, UNIVERSITAET WIEN, TUERKENSCHANZSTR 17

A 1180 WIEN, AUSTRIA

Telephone: (43) 13453600

Facsimile: (43)

Telex:

Email:

Commission(s): $24,41,51$

FIROR JOHN W DR, HIGH ALTITUDE OBSERVATORY, NCAR, BOX 3000, BOULDER CO 80307 3000, USA

Telephone: (1) 3034971600

Facsimile: (1) $303+971568$

Telex: 45694

Email:

Commission(s): 
FISCHEL DAVID DR, EARTH OBSERVATION, SATELLITE COMPANY, 4300 FORBES BLVD, LANHAM MD 20706, USA Telephone: (1) 3015520623 Facsimile: (1) 3015523028 Telex: 277685

Email: Commission(s):

FISCHER JACQUELINE, NAVAL RESEARCH LABORATORY, CODE 4138F, 4555 OVERLOOK AVE SW WASHINGTON DC 203755000 , USA

Telephone: (1) 2027673058

Facsimile: (1)

Telex:

Email:

Commission(s): 3

FISCHER STANISLAV DR, ASTRONOMICAL INSTITUTE, CZECH ACADEMY SCIENCES, BOCNI II 1401 CZ 14131 PRAHA 4, CZECH R

Telephone: (42) $2766051 * 062$

Facsimile: (42) 2769023

Telex:

Email: fischer(âseis.ig.cas.cz

Commission(s):

FISHER GEORGE HEWITT DR, SPACE SCIENCES LABORATORY, UNIVERSITY OF CALIFORNIA

GRIZZLY PEAK BLVD, BERKELEY CA 94720, USA

Telephone: (1) $5106427297 \quad$ Facsimile: (1) 5106438302 Telex:

Email: finsher(asunspot.ssl.berkeley.edu Commission(s): 10,12

FISHER J RICHARD, NRAO, BOX 2, GREEN BANK WV 24944, USA

Telephone: (1) $3044562011 \quad$ Facsimile: (1)

Email: $\quad$ Commission(s):

Telex: 7109381530

FISHER PHILIP C, RUFFNER ASSOCIATES, BOX 1867, SANTA FE NM 8750+1867, USA

Telcphone: (1) Facsimile: (1) Telex:

Email: $\quad$ Commission(s): 44,51

FISHER RICHARD R DR, NASA GSFC, CODE 682, GREENBELT MD 20771, USA

Telephone: (1) Facsimile: (1) Telex:

Email: $\quad$ Commission(s):

FISHMAN GERALD J, NASA/MSFC, CODE ES 62, ASTROPHYSICS BRANCH, HUNTSVILLE AL 35812, USA

Telephone: (1) $2054530117 \quad$ Facsimile: (1)

Enail:

Commission(s): 44

Telex:

FITCH WALTER S DR, BOX 100, ORACLE AZ 85623, USA

Telephonc: (1) $60128962911 \quad$ Facsimile: (1)

Commission(s): 27

Telex: 467175

Email: Facsimile: (31)
Telex:

$\begin{array}{ll}\text { Telephone: (31) } 252+4635 & \text { Facsimile: (31) } \\ \text { Email: } & \text { Commission(s): } 44\end{array}$

Email:

FITZGERALD M PIM PROF, DPT OF PHYSICS, UNIVERSITY OF WATERLOO, WATERLOO ON N2L 3G1, CANADA

Telephone: (1) 5198851572

Facsimilc: (1) 5197468115

Telex: 06955259

Email:

Commission(s): 33,37

FITZPATRICK EDWARD L DR, PRINCETON UNIVERSITY OBS, PEYTON HALL, PRINCETON NJ 08544, USA

Telephone: (1) 6094523702 Facsimile: (1) $6092437333 \quad$ Telex:

Emajl: fitz(a)astrovax.princeton.edu Conmission(s): $29,36,45$

FIX JOHN D DR, DPT PHYSICS \& ASTRONOMY, UNIVERSITY OF IOWA, IOWA CITY IA 52240, USA

Telephone: (1) 319 $3537064 \quad$ Facsimile: (1)

Email:

Commission(s):

Telex: 910-525-1398

FLANAGAN CLAIRE SUSAN, HARTEBEESTHOEK RADIO, ASTRONOMY OBSERVATORY, BOX 443 KRUGERSDORP 1740, SOUTH AFRICA

Telephonc: (27) $116+24692$

Facsimile: (27) $116+22424 \quad$ Telex:

Email: claireabootes.hartrao.ac.zat

Commission(s):

FLANNERY BRIAN PAUL DR, EXXON RES \& ENGINEERING, ROUTE 22 EAST, ANNANDALE NJ 08801, USA

Telephonc: (1) 2017302540

Facsimile: (1)

Telex: 136140 exxonres

Email:

Commission(s): $34,35,42$ 
FLECK ROBERT CHARLES DR, DPT OF MATH \& PHYSICAL SC, EMBRY-RIDDLE AERON UNIVER DAYTONA FL 32114, USA

Telephone: (1) 9042266612

Email:

Facsimile: (1) $90+2266713 \quad$ Telex:

Commission(s): 34,46

FLEISCHER ROBERT DR, 108 OVERLOOK ST, ROUTE 2 BOX 515, MOOREFIELD WV 26836, USA

Telephone: (1)

Facsimile: (1)

Telex:

Email:

Commission(s): 40

FLEMING THOMAS ANTHONY, STEWARD OBSTERVATORY, UNIVERSITY OF ARIZONA, TUCSON AZ 85721, USA

Telephone: (1) 5206215049

Facsimile: (1) 5206211532

Telex:

Email: taf@as.arizona.edu

Commission(s):

FLETCHER J MURRAY, HERZBERG INST ASTROPHYS, DOMINION ASTROPHYS OBS, 5071 W SAANICH RD VICTORLA BC V8X 4M6, CANADA

Telephone: (1) 6043883905

Facsimile: (1) $60+36300+5$

Telex: 0497295

Email:

Commission(s): 09,26,30

FLETT ALISTAIR M, DPT OF PHYSICS, UNIVERSITY OF ABERDEEN, FRASER NOBLE BLDG ABERDEEN AB9 2UE, UK

Tclephone: (44) 122440241

Email:

Facsimile: (44)

Telex: $73+58$ uniabn g

Commission(s): 40

FLIEGEL HENRY F, 3730 EL MORENO AVE, BOX 8682, LA CRESCENTA CA 91214, USA

Tclephone: (1) 3103361710

Facsimile: (1) 3103365076 Telex:

Email: fliegcl@courier1.acro.org

Commission(s): 19,31P

FLIN PIOTR, PEDAGOGICAL UNIVERSITY, INST OF PHYSICS, UL PODCHORAZYCH 2

PL 30 084 KRAKOW, POLAND

Telephone: $(48)$

Facsimile: (48) 123722243

Telex: 0322297 uj pl

Email: sfllin@pllkey11

Commission(s): 28

FLOQUET MICHELE DR, OBSERVATOIRE DE PARIS, SECTION DE MEUDON, DEPEG F 92195 MEUDON PPL CDX, FRANCE

Telephone: (33) 145077851

Facsimile: (33)

Telex:

Email:

Commission(s): 20

FLORENTIN-NIELSEN RALPH, COPENHAGEN UNIVERSITY OBS, BRORFELDEVEJ 23, DK +34() TOLLOSE, DENMARK

Telephone: (45) 53488195

Facsimile: (45) 58488755

Telex: +4155 danast

Email:

Commission(s):

FLORIDES PETROS S PROF, SCHOOL OF MATHEMATICS, TRINITY COLLEGE, DUBLIN 2, IRELAND

Telephone: (353) 1772941

Facsimile: (353)

Telex: $25+42$ ted ei

Email:

Commission(s): 41,47

FLORKOWSKI DAVID R DR, US NAVAL OBSERVATORY, 3450 MASSACHUSETTS AVE NW

WASHINGTON DC 203925100 , USA

Telephone: (1) 2026531509

Email: drfämaia.usno.navy.mil

Facsimile: (1) $20126530587 \quad$ Telex

Commission(s): 40

FLORSCH ALPHONSE DR, OBS DE STRASBOURG, 11 RUE UNIVERSITE, F 67000 STRASBOURG, FRANCE

Telephone: (33) 88358200

Facsimile: (33) 88250160

Telex: 890506 starobs f

Email:

Commission(s): $28,30,38$

FLOWER DAVID R DR, DPT OF PHYSICS, UNIVERSITY OF DURHAM, SOUTH RD, DURHAM DH1 3LE, UK

Telephone: (44) 1913742145

Facsimile: $(4+) 19137+3749$ Telex:

Email: david.flower(a)durham.ac.uk

Commission(s): 14,34P

FLUDRA ANDRZEJ, ASTROPHYS DIV, RUTHERFORD APPLETON LAB, CHILTON DIDCOT OXON OX11 OQX, UK

Telephone: (44) 1235446497

Facsimile: (4t) $1235+46509 \quad$ Telex:

Email: af(a)ast.star.rl.ac.uk

Commission(s):

FOCARDI PAOLA DR, OSS ASTRONOMICO, UNIVERSITA DI BOLOGNA, CP 596, I 40100 BOLOGNA, ITALY

Telephone: (39) 51259301

Facsimile: (39)

Commission(s): 47

Telex: 520634 infnbo i 
FODERA SERIIO GIORGIA DR, OSS ASTRONOMICO, UNIVERSITA DI PALERNO, PALAZZO DEI NORMANNI I 90134 PALERMO, ITALY

Telephone: (39) 916570451

Email: astropa@ipacuc.bitnet
Facsimile: (39) 91488900

Commission(s): 41
Telex: 910402 astrop i

FOFI MASSIMO DR, OAR, VIA DEL PARCO MELLINI 84, I 00136 ROMA, ITALY

Telephonc: (39) 6347056

Email: 40061::fofi

Facsimile: (39)

Commission(s): 12
Telex: 626326 oaroma i

FOGARTY WILLIAM G DR, IBM, NCMD, 411 EAST WISCONSIN AVE, MILWAUKEE WI 53202, USA

Telephone: (1)

Facsimile: (1)

Commission(s):

Telex:

Email:
FOING BERNARD H DR, ESTEC, SPAC

Telephone: (31)

Email:

\section{Facsimile: (31)}

Commission(s): 10,29,4

FOING-EHRENFREUND PASCALE, LEIDEN OBSERVATORY, BOX 9513, NL 2300 RA LEIDEN, NETHERLANDS

Tclephone: (31) 71275872

Facsimile: (31) 71275819

Telex:

Email: pascale(a)rulhl1.lcidenuniv.nl

Commission(s):

FOLEY ANTHONY DR, NFRA, BOX 2, NL 7990 AA DWINGELOO, NETHERLANDS

Telephone: (31) $5219724 t$

Facsimile: (31) $52197332 \quad$ Telex:

Email: folrediànfra.nl

Commission(s): 40

FOLTZ CRAIG B, MULT MIRROR TELESCOPE OBS, UNIVERSITY OF ARIZONA, TUCSON AZ 85721, USA

Telephonc: (1) 520621 1269)

Facsimile: (1)

Commission(s): 28,30

Telex: 467175

Email:

BOX 0, SOCORRO NM 878010387 , USA

FOMALONT EDWARD B DR, NRAO, BOX 0, SOCORR

Telex: 910-988-1710

Email:

Commission(s): 40

FOMENKO ALEXANDR F DR, SPECIAL ASTROPHYSICAL OBS, ACADEMY OF SCIENCES, NIZHNIJ ARKHYZ 357147 STAVROPOLSKIJ, RUSSIA

Telephone: (7)

Facsimile: (7)

Telex:

Email:

Commission(s): (19)

FOMICHEV VALERI V DR, IZMIRAN, ACADEMY OF SCIENCES, 142092 TROITSK, RUSSIA

Telephone: (7)

Facsimile: (7)

Telex:

Email:

Commission(s): 12

FOMIN PIOTR IVANOVICH, METROLOGICHESKAYA ST 14B, 252143 KIEV 26, UKRAINE

Telephone: (7)

Facsimile: (7)

Telex:

Email:

Commission(s): 47

FOMIN VALERY A DR, PULKOVO OBSERVATORY, ACADEMY OF SCIENCES, 10 KUTUZOV QUAY 196140 ST PETERSBURG, RUSSIA

Telephone: (7)

Facsimile: (7)

Telex:

Email:

Commission(s): 08

FOMINOV ALEXANDR M DR, INST OF THEORET ASTRONOMY, ACADEMY OF SCIENCES, N KUTUZOVA 10 191187 ST PETERSBURG, RUSSIA

Telephone: (7) 2788898

Email:

Facsimile: (7)

Commission(s): (0)

Telex: 121578 ita su

FONG CHU-GANG, SHANGHAI OBSERVATORY, CAS, 80 NANDAN RD, SHANGHAI 200030, CHINA PR

Telephone: (86) 21386191

Facsimilc: (86)

Telex: 33164 shao cn

Email:

Commission(s): 07,19

FONG RICHARD, DPT OF PHYSICS, UNIVERSITY OF DURHAM, SOUTH RD, DURHAM DH1 3LE, UK

Telephone: (44) 19138564971

Facsimile: (4t)

Commission(s): 47

Telex: 537351

Email: 
FONTAINE GILLES DR, DPT DE PHYSIQUE, UNIVERSITE DE MONTREAL, CP 6128 SUCC A MONTREAL QC H3C 3J7, CANADA

Telephone: (1) 5143436680

Facsimile: (1) 5143432071

Telex: 05562425

Email:

Commission(s): 35,36

FONTENLA JUAN MANUEL DR, NASA/MSFC, CODE ES 52, HUNTSVILLE AL 35812, USA

Telephone: (1)

Facsimile: (1)

Telex:

Email:

Commission(s): 10,12,36

FORBES DOUGLAS DR, SIR WILFRED GRENFELL COLL, MEMORIAL UNIVERSITY, NEWFOUNDLAND CORNER BROOK NF A2H 6P9, CANADA

$\begin{array}{lll}\text { Telephone: (1) } 7096376295 & \text { Facsimile: (1) } 7096376390 & \text { Telex: } 01644190 \\ \text { Email: dforbes@kean.ucs.mun.ca } & \text { Commission(s): } 37 & \end{array}$

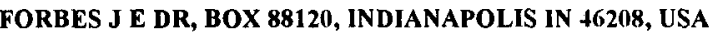

Telephone: (1)

Facsimile: (1)

Telex:

Email:

Commission(s): 35

FORBES TERRY G DR, SPACE SCIENCE CENTER/EOS, UNIV OF NEW HAMPSHIRE, SC ENGIN RES BLDG

DURHAM NH 03857, USA

Telephone: (1) 6038623872

Facsimile: (1)

Commission(s): 10

Telex: 950030 unh phys

Email: bitnet\%"t_forbesaunh"

FORD HOLLAND C RES PROF, STSCI, HOMEWOOD CAMPUS, 3700 SAN MARTIN DR, BALTIMORE MD 21218, USA

Telephone: (1) 3013384803

Facsimile: (1)

Telex:

Email:

Commission(s): $28,34,47$

FORD W KENT JR DR, DPT TERRESTR MAGNETISM, CARNEGIE INST WASHINGTON

5241 BROAD BRANCH RD NW, WASHINGTON DC 20015, USA

Tclephone: (1) $2029660863 \quad$ Facsimile: (1)

Telex: $\$ \$(0)+27$ magn ui

Email:

Commission(s): 09,28

FORMAN WILLIAM RICHARD DR, SMITHSONIAN ASTROPHYS OBS, 60 GARDEN ST, CAMBRIDGE MA 02138, USA

Telephone: (1) 6174957210

Facsimile: (1)

Telex: 921428

Email:

Commission(s): 44,47

FORREST WILLIAM JOHN, DPT PHYSICS \& ASTRONOMY, UNIVERSITY OF ROCHESTER ROCHESTER NY 14627, USA

Telephone: (1) 7162754343

Facsimile: (1)

Telex:

Email: forrest@boris.pas.rochester.ed Commission(s):

FORSTER JAMES RICHARD DR, HAT CREEK RADIO OBSERV, RT 2, BOX 500, CASSEL CA 96016, USA

Telephone: (1) 9163352364

Facsimile: (1) 9163353968

Telex:

Email:

Commission(s): $3+$

FORT BERNARD P DR, OBS MIDI PYRENEES, 14 AVE E BELIN, F 31400 TOULOUSE CDX, FRANCE

Telephone: (33) 61252101

Facsimile: (33)

Commission(s): 09

Telex: 530776 f

Email:

FORT DAVID NORMAN DR, JPL, 4800 OAK GROVE DR, PASADENA CA 91109, USA

Telephone: (1) $81835+9132$

Facsimile: (1)

Telex:

Email:

Commission(s): 40

FORTE JUAN CARLOS DR, OBSERVATORIO ASTRONOMICO, PASEO DEL BOSQUE S/N

1900 LA PLATA (BS AS), ARGENTINA

Telephone: (54) 21217308

Facsimile: (54) $21211761 \quad$ Telex:

Email:

Commission(s): 25,28,37

FORTI GIUSEPPE DR, OSS ASTROFISICO, DI ARCETRI, LARGO E FERMI 5, I 50125 FIRENZE, ITALY

Telephone: (39) 552752236

Facsimile: (39)

Email: fortiagarcetri.astro.it

Commission(s): $15,20,22$

Telex: 572268 arcetr

FORTINI TERESA DR, VIA F D GUERRAZZI 19, I 00152 ROMA, ITALY

Telephone: (39)

Facsimile: (39)

Commission(s): 10

Telex:

Email: 
FORVEILLE THIERRY DR, OBSERVATOIRE DE GRENOBLE, CERMO/ASTROPHYSIQUE BP 53X, F 38041 S MARTIN HERES CD, FRANCE
Telephone: (33) 76514567
Facsimile: (33) 76448821
Telex:

Email: forveill@frgag51.bitnet

Commission(s): 40

FOSBURY ROBERT A E DR, ESO, ST/ECF, KARL SCHWARZSCHILDSTR 2

D 85740 GARCHING MUENCHEN, GERMANY

$\begin{array}{ll}\text { Telephone: (49) } 8932006235 & \begin{array}{l}\text { Facsimile: (49) } \\ \text { Emais: }\end{array}\end{array}$

FOSSAT ERIC DR, OCA OBSERV DE NICE, BP 229, F 06304 NICE CDX 4, FRANCE

Tclephone: (33) 93529805 Facsimile: (33) 92003033 Telex: 460004 obsnice $f$

Email:

Commission(s): 10,12,35

FOSTER ROGER S, NAVAL RESEARCH LABORATORY, CODE 7210, WASHINGTON DC 20375, USA

Telephone: (1) 2027670669

Facsimile: (1) 2024048894 Telex:

Email: foster@irira.nrl.navy.mil

Commission(s):

FOUKAL PETER V DR, CAMBRIDGE RESEARCH \&, INSTRUMENTATIONM INC, 21 ERIE ST

CAMBRIDGE MA 02139, USA

Telephone: (1) 6174912627 Facsimile: (1) Telex:

Email:

Commission(s): 12VP

FOUQUE PASCAL DR, GRUPO ASTROFIS PONTIFICA, UNIVERSIDAD CATOLICA, CASILLA 104

SANTIAGO 22, CHILE

Telephone: (56)

Email: pfouque(a)astrouc.puc.cl

Facsimile: (56)

Telex:

Commission(s): $28,40,47$

FOWLER WILLIAM A PROF, CALTECH, MS 106 38, PASADENA CA 91125, USA

Telephone: (1) $8183564272 \quad$ Facsimile: (1) $81856+8708$ Telex:

Email:

Commission(s): 35,44

FOX KENNETH DR, DPT PHYSICS \& ASTRONOMY, UNIVERSITY OF TENNESSEE, 503 PHYSICS

KNOXVILLE TN 379961200 , USA

Telephone: (1) 6159742288 Facsimile: (1) Telex:

Email:

Commission(s): 16

FOX W E MR, BRITISH ASTRONOMICAL ASS, 40 WINDSOR RD, NEWARK NOTTINGHAMSHIRE, UK

Telephone: (44) 1636704932

Facsimile: (44)

Commission(s): 16

Telex:

Email:

FOY RENAUD DR, AIRI, OBSERVATOIRE DE LYON, 9 RUE CHARLES ANDRE, F 69561 ST GYENIS LAVAL, FRANCE

Telephone: (33) 78868546

Email: foy@image.univ-lyon 1.fr

Facsimilc: (33) 78868386

Telex:

Commission(s): 09,29,36

FRAIL DALE ANDREW, NRAO, VLA, BOX 0, SOCORRO NM 87801 0387, USA

Telephone: (1) $5058357338 \quad$ Facsimile: (1) 5058357027 Telex:

Email: d frail(a)nrao.edu

Commission(s): 40

FRAIX-BURNET DIDIER, OBS MIDI PYRENEES, 14 AVE E BELIN, F 31400 TOULOUSE CDX, FRANCE

Telephone: (33) 61332866

Email: fraix(a)obs-mip.fr

Facsimile: (33) 61536722 Telex:

Commission(s):

FRANCESCHINI ALBERTO, DPT DI FISICA G GALILAI, UNIVERSITA DI PADOVA, VIA MARZOLO 8

I 35131 PADOVA, ITALY

Telephone: (39) 49844111

Email:

Facsimile: (39) $498+4245 \quad$ Telex:

Commission(s): 44,47

FRANCHINI MARIAGRAZIA DR, OAT, BOX SUCC TRIESTE 5, VIA TIEPOLO 11, I 34131 TRIESTE, ITALY

Telephone: (39) 403199111

Facsimile: (39) 40309418

Telex: 461137 oat $i$

Email: 38439::franchini

Commission(s): 29

FRANCO JOSE DR, INSTITUTO DE ASTRONOMLA, UNAM, APDO POSTAL 70-264, 04510 MEXICO DF, MEXICO

Telephone: (52)

Facsimile: (52)

Telex:

Email: jjfranco(a)alfa.astroscu.unam.mx Commission(s): 
FRANCOIS PATRICK DR, OBSERVATOIRE DE PARIS, SECTION DE MEUDON, DASGAL F 92195 MEUDON PPL CDX, FRANCE

Tclephone: (33) $145977867 \quad$ Facsimile: (33) $145077472 \quad$ Telex: 270912

Email:

Commission(s): 29

FRANDSEN SOEREN PROF, INST OF PHYSICS \& ASTRON, UNIVERSITY OF AARHUS, NY MUNKEGADE DK 8000 AARHUS C, DENMARK

Telephone: (45) 86128899

Facsimile: (45) 86202711

Telex: 64767 aausci $d k$

Email:

Commission(s): 29,44

FRANK JUHAN, DPT PHYSICS \& ASTRONOMY, LOUISIANA STATE UNIV, BATON ROUGE LA 70803 4001, USA

Telephone: (1) 5043886845

Facsimile: (1) $5043885855 \quad$ Telex:

Email: phrank@Isuvm

Commission(s): 42,44

FRANKLIN FRED A DR, CENTER FOR ASTROPHYSICS, PLANETARY SCIENCE DIV, 60 GARDEN ST

CAMBRIDGE MA 02138, USA

Telephone: (1) 6174957230

Facsimile: (1)

Telex:

Email:

Commission(s): 20

FRANSSON CLAES, STOCKHOLM OBSERVATORY, $S 13336$ SALTSJOEBADEN, SWEDEN

Telephone: (46) 87170195

Facsimile: $(46) 87174719$

Telex: 12972 sobserv s

Email: claes@astro.su.se

Commission(s): 44

FRANTSMAN YU L DR, RADIOASTROPHYSICAL OBS, LATVIAN ACAD OF SCIENCES, TURGENEVA 19 226524 RIGA, LATVIA

Telephone: (371) 226006

Email:

Facsimile: (371)

Commission(s): 35,42

Telex:

FRANX MARIJN, KAPTEYN ASTRONOMICAL INST, BOX 800, NL 9700 AV GRONINGEN, NETHERLANDS

Telephone: (31) Facsimile: (31) Telex:

Email: $\quad$ Commission(s):

FRANZ OTTO G DR, LOWELL OBSERVATORY, 1400 W MARS HILL RD, BOX 1149, FLAGSTAFF AZ 86001, USA Telephone: (1) 6027743358

Facsimile: (1)

Telex:

Email:

Commission(s): 24,26

FRATER ROBERT H DR, CSIRO, DIV OF RADIOPHYSICS, BOX 76, EPPING NSW 2121, AUSTRALIA

Telephone: (61) 28680222

Facsimile: (61) 28680310

Telex: 26230 astro

Email:

Commission(s): 40

FRAZIER EDWARD N DR, TRW SPACE \& TECHNOLOGY, 1 SPACE PARK, REDONDO BEACH CA 90278, USA

Telephone: (1) 2135354723

Facsimile: (1)

Telex:

Email:

Commission(s): 12

FREDGA KERSTIN PROF, SWEDISH BOARD F SPACE ACT, BOX 4006, S 17154 SOLNA, SWEDEN

Telephone: (46) $87336486 \quad$ Facsimile: (46)

Email:

Conmission(s): 44

Telex: 17128 spaceco $s$

FREDRICK LAURENCE W PROF, UNIVERSITY STATION, UNIVERSITY OF VIRGINIA, BOX 3818 CHARLOTTESVILLE VA 22903, USA

Telephone: (1) 8049244905

Facsimile: (1)

Commission(s): $24,26,42,51$

Telex: $510-587-5453(t w x)$

Email: Jwf@virginia.edu

FREEDMAN WENDY L DR, CARNEGIE OBSERVATORIES, INSTITUTION OF WASHINGTON

813 SANTA BARBARA ST, PASADENA CA 91101 , USA

Telephone: (1) $8185771122 \quad$ Facsimile: (1)

Email: wendy@mwlco.caltech.edu Commission(s): 28

Telex:

FREEMAN KENNETH C PROF, MOUNT STROMLO OBSERVATORY, PRIVATE BAG, WESTERN CREEK PO CANBERRA ACT 2611, AUSTRALIA

Telephone: (61) 62490264

Facsimile: (61) 62490233

Telex:

Email: kcf(a)mso.anu.edu.au

Commission(s): 28,30C,33VP,37 
FREIRE FERRERO RUBENS G, OBS DE STRASBOURG, 11 RUE UNIVERSITE, F 67000 STRASBOURG, FRANCE

Telephone: (33) 88358200

Facsimile: (33) 88250160

Telex:

Email: freire@frias51.bitnet

Commission(s): 29,36,51

FREITAS MOURAO R R DR, AVE DE EXECITO 105, SAN CRISTOVAO, 20910 RIO DE JANEIRO RJ, BRAZIL

Telephone: (55) $215807154^{\star} 7204$

Facsimile: (55) $215800332 \quad$ Telex: 22653

Email:

Commission(s): 20,26,41

FRENCH RICHARD G, DPT OF EARTH \& PLANET SCI, MIT RM 54 422, BOX 165, CAMBRIDGE MA 02139, USA

Telephone: (1) 6172533392

Facsimile: (1)

Telex:

Email:

Commission(s):

FRENK CARLOS S, DPT OF PHYSICS, UNIVERSITY OF DURHAM, SOUTH RD, DURHAM DH1 3LE, UK

Telephone: (44) $19138564971 \quad$ Facsimile: (44)

Email:

Commission(s): 47

Tclex: $\mathbf{5 3 7 3 5 1}$ durlib g

FRESNEAU ALAIN DR, OBS DE STRASBOURG, 11 RUE UNIVERSITE, F 67000 STRASBOURG, FRANCE

Telephone: (33) 88358200

Facsimile: (33) 88250160

Telex: 890506 starobs $f$

Email:

Commission(s): 24

FRIBERG PER, JOINT ASTRONOMY CENTER, UKIRT, 665 KOMOHANA ST, HILO HI 96720, USA

Telephone: (1)

Facsimile: (1)

Telex:

Email: friberg(a)jach.hawaii.edu

Commission(s): 40

FRICKE KLAUS DR, UNIVERSITAETS STERNWARTE, GOETTINGEN, GEISMARLANDSTR 11 D 37083 GOETTINGEN, GERMANY

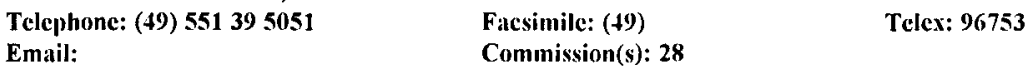

FRIDLUND MALCOLM DR, ESA/ESTEC, ASTROPHYSICS DIV, BOX 299, NL 2200 AG NOORDWIJK, NETHERLANDS

Telephone: (31) $171984768 \quad$ Facsimile: (31) $171984690 \quad$ Telex: 39098

Email: estes51::mfridlun Commission(s): 34

FRIDMAN ALEKSEY M DR, INST OF ASTRONOMY, ACADEMY OF SCIENCES, PYATNITSKAYA UL 48 109017 MOSCOW, RUSSIA

Telephone: (7) 952315461

Email: ialas $(\boldsymbol{a}$ node.iass.msk.su

Facsimile: (7)

Commission(s): 33C

Telex: $\mathbf{4 1 2 6 2 3}$ sestp su

FRIED JOSEF WILHELM DR, MPI FUER ASTRONOMIE, KOENIGSTUHL, D 69117 HEIDELBERG, GERMANY

Telephone: (49) $62215281 \quad$ Facsimile: (49)

Email:

Commission(s): 28

Telex: 461789 mpia d

FRIEDEMANN CHRISTIAN DR, UNIV STERNWARTE JENA, SCHILLERGAESSCHEN 2, D 07745 JENA, GERMANY

Telephone: (49) 78822 2637/27122 Facsimile: (49)

Email:

Commission(s): 34

Telex: 05886134

FRIEDJUNG MICHAEL DR, INSTITUT D'ASTROPHYSIQUE, 98BIS BD ARAGO, F 75014 PARIS, FRANCE

Telephone: (33) 144328111

Facsimile: (33) $14+328001$

Telex:

Email: fricdjung@friajs1

Commission(s): 27,29,42

FRIEDLANDER MICHAEL PROF, DPT OF PHYSICS, WASHINGTON UNIVERSITY, ST LOUIS MO 63130, USA

Telephone: (1) $3148896279 \quad$ Facsimile: (1)

Email:

Commission(s):

Telex:

FRIEDLI DANIEL, OBSERVATOIRE DE GENEVE, CHEMIN DES MAILLETTES 51

CH 1290 SAUVERNY, SWITZERLAND

Telephonc: (41) 227552611

Facsimile: (+1) 227553983

Telex: 45419209 obsg ch

Email: fri(a)scsun.unige.ch

Commission(s):

FRIEDMAN HERBERT DR, NAVAL RESEARCH LABORATORY, CODE 7100, 4555 OVERLOOK AVE SW WASHINGTON DC 20375 5000, USA

Telephone: (1)

Email:

Facsimile: (1)

Telex:

Commission(s): 10,12,40,44 
FRIEDMAN SCOTT DAVID DR, DPT PHYSICS \& ASTRONOMY, JOHNS HOPKINS UNIVERSITY CHARLES \& 34TH ST, BALTIMORE MD 21218, USA

Telephone: (1) $3013385317 \quad$ Facsimile: (1) $3013385494 \quad$ Telex:

Email: scivax::casa::scott Commission(s):

FRIEL EILEEN D DR, MARIA MICHEL OBSERVATORY, 3 VESTAL STREET, NANTUCKET MA 02554, USA

Telephone: (1) 5082289273

Facsimile: (1) $5082281031 \quad$ Telex:

Email: friel@hyades.bu.edu

Commission(s): 29,37

FRIEND DAVID B DR, DPT OF PHYS \& ASTRONOMY, WILLIAMS COLLEGE, WILLIAMSTOWN MA 01267, USA

Telephone: (1) $4135972817 \quad$ Facsimile: (1)

Telex:

Email: bitnet:dbfriend@williams Commission(s): 36

FRINGANT ANNE-MARIE DR, OBSERVATOIRE DE PARIS, 61 AVE OBSERVATOIRE, F 75014 PARIS, FRANCE

Telephone: (33) $140512248 \quad$ Facsimile: (33)

Telex: 270776 obs f

Email:

Commission(s):

FRISCH HELENE DR, OCA OBSERV DE NICE, BP 229, F 06304 NICE CDX 4, FRANCE

Telephone: (33) 93890420

Facsimile: (33) 92003033

Telex: 460004 obsnice $f$

Email:

Commission(s): 36

FRISCH PRISCILLA, ASTRONOMY \& ASTROPHYS CTR, UNIVERSITY OF CHICAGO, 5640 S ELLIS AVE CHICAGO IL 60637, USA

Telephone: (1) 3129628211

Email: frisch@oddjob.uchicago.edu

Facsimile: (1)

Telex: 9102215617

FRISCH URIEL DR, OCA OBSERV DE NICE, BP 229, F 0630 4 NICE CDX 4, FRANCE

Telephone: (33) 93890420

Facsimile: (33) 92003033

Telex: 460004 obsnice $f$

Email:

Commission(s): 36

FRISK URBAN DR, SPACE SCIENCE SECTION, SWEDISH SPACE CORPORATION, BOX 4207

S 17104 SOLNA, SWEDEN

Telephone: (46) 86276200

Facsimile: (46) 8987069

Telcx:

Email:

Commission(s): 40,44

FRITZE KLAUS, ASTROPHYSIKALISCHES INST, POTSDAM UNIVERSITY, AN DER STERNWARTE 16 D 14482 POTSDAM, GERMANY

Telephone: (49) $3317499202 \quad$ Facsimile: (49) $3317 \$ 99200 \quad$ Telex:

Email: kfritze@aip.de Commission(s): 28

FRITZE-VON ALVENSLEBEN U, UNIVERSITAETS STERNWARTE, GOETTINGEN, GEISMARLANDSTR 11 D 37083 GOETTINGEN, GERMANY

Telephonc: (49) 551395055

Email: ufritze@uni-sw.gwdg.de

Facsimile: (49) $551395043 \quad$ Telex:

Commission(s): 28

FRITZOVA-SVESTKA L DR, DOPPERSTRAAT 147, NL 3752 JC BUNSCHOTEN, NETHERLANDS

Telephone: (31) 349984403

Facsimile: (31)

Commission(s):

Telex:

Email:

FROEHLICH CLAUS, WORLD RADIATION CENTER, PHYSIKALISCH-METEOROL OBS, POSFACH 173

CH 7260 DAVOS-DORF, SWITZERLAND

Telephone: (41) $81462131 \quad$ Facsimile: (41)

Email:

Commission(s): 12

Telex: 853232 pmod ch

FROESCHLE CHRISTIANE D DR, OCA OBSERV DE NICE, BP 13\%, F 06003 NICE CDX, FRANCE

Telephone: (33) 93890420

Email:

Facsimile: (33) 92003033

Telex: 460004 obsnice $f$

Commission(s): 15,36

FROESCHLE CLAUDE DR, OCA OBSERV DE NICE, BP 139, F 06003 NICE CDX, FRANCE

Telephone: (33) 92003024

Email: claude@obs-nice.fr

Facsimile: (33) 92003033

Telex: 460004 obsnice $f$

FROESCHLE MICHEL DR, OCA CERGA, AVE COPERNIC, F 06130 GRASSE, FRANCE

Telephone: (33) 93355849

Facsimile: (33)

Telex:

Email:

Commission(s): 08 
FROGEL JAY ALBERT DR, DPT OF ASTRONOMY, OHIO STATE UNIVERSITY, 17 W $18 T H$ AVE COLUMBUS OH 432101106 , USA

Telephone: (1) 6142925651

Email: frogel(a)ohstpy.bitnet

Facsimile: (1)

Tclex:

Commission(s): 28

FROLOV M S DR, INST OF ASTRONOMY, ACADEMY OF SCIENCES, PYATNITSKAYA UL 48 109017 MOSCOW, RUSSIA

Telephone: (7) 952315461

Facsimile: (7)

Telex: 412623 scstp su

Email:

Commission(s): 27

FROST KENNETH J DR, NASA GSFC, CODE 600.2 BLDG 16, SPACE STATION OFFICE, GREENBELT MD 20771, USA

Telephone: (1) 3012868824

Facsimile: (1)

Telex:

Email:

Commission(s):

FRUCHTER ANDREW S, DPT OF ASTRONOMY, UNIVERSITY OF CALIFORNIA, BERKELEY CA 94720, USA

Telephone: (1) 5106438142

Facsimile: (1) $5106+23+11 \quad$ Telex:

Email: asf@orestes.berkeley.edu

Commission(s):

\section{FRUSCIONE ANTONELLA, CTR FOR EUV ASTROPHYSICS, UNIVERSITY OF CALIFORNIA, 2150 KITTREDGE ST BERKELEY CA 94720, USA \\ Telephone: (1) 5106436484 \\ Facsimile: (1) $5106435660 \quad$ Telex: \\ Email: antonella(a)cea.berkeley.edu \\ Commission(s):}

FRYE GLENN M PROF, PHYSICS DPT, CASE WESTERN RESERVE UNIV, ROCK BDG, CLEVELAND OH 44106, USA

Telephone: (1) 2163682997

\section{Facsimile: (1)}

Commission(s):

Telex:

Email:

FTACLAS CHRIST, PERKIN ELEMR CORP, MS 897, 100 WOOSTER HEIGHTS RD, DANBURY CT 06810 7859, USA

Telephone: (1) 2037976448

Facsimile: (1)

Telex:

Email:

Commission(s): 28

FU CHENG-QI, SHANGHAI OBSERVATORY, CAS, 80 NANDAN RD, SHANGHAI 200030, CHINA PR

Telephone: (86) $21386191 \quad$ Facsimile: $(86)$

Email:

Commission(s): +4

Telex: 33164 shao en

FU DELIAN, BEIJING ASTRONOMICAL OBS, CAS, W SUBURB, BEIJING 100080, CHINA PR

Telephone: (86) $1282070 \quad$ Facsimile: (86) $\quad$ Telex: 22040 baoas cn

Email:

Commission(s): 09

FU QI JUN, BEIJING ASTRONOMICAL OBS, CAS, W SUBURB, BEIJING 100080, CHINA PR

Telcphone: (86) $1275580 \quad$ Facsimile: (86)

Email:

Commission(s): 10

Telex: 22040 baons cn

FUCHS BURKHARD DR, ASTRON RECHEN INSTITUTE, MOENCHHOFSTR 12-14, D 69120 HEIDELBERG, GERMANY

Telephone: (\$9) 622149026

Facsimile: $(49)$

Commission(s): 28,33

Telex: 461336 arihd $d$

Email:

FUENMAYOR FRANCISCO J DR, UNIVERSIDAD DE LOS ANDES, FACULDAD DE CIENCIAS, DPT DE FISICA MERIDA 5101 A, VENEZUELA

Telephonc: (58) $74639930 \quad$ Facsimile: (58)

Email:

Commission(s):

Telex: 74173 cdch-ula

FUENSALIDA JIMENEZ J DR, INST DE ASTROFISICA, DE CANARIAS, OBS DEL TEIDE, E 38200 LA LAGUNA, SPAIN

Telephone: (34) 22262211

Facsimile: (3t)

Telex: 92640

Email:

Commission(s):

FUERST ERNST DR, MPI FUER RADIOASTRONOMIE, AUF DEM HUEGEL 69, D 53121 BONN, GERMANY

Telephone: (49)

Facsimile: (49)

Email:

Conmission(s): 40

Telex: $886+40$

FUHR JEFFREY ROBERT DR, DATA CTR ATOMIC TRANSIT, PROBABILITIES, NIST

GAITHERSBURG MD 20899, USA

Telephone: (1) 3019753204

Facsimile: (1) 3019753038

Commission(s): 14

Telex: $19767+$ nist ut

Email: fuhr@tiber.nist.gov 
FUJIMOTO MASAYUKI DR, FAC OF EDUCATION, NIIGATA UNIVERSITY, 8050 IKARASHI 2, NIIGATA 950 21, JAPAN Telephone: (81)

Facsimile: (81)

Commission(s): 28,35

Telex:

Email:

FUJIMOTO MASA-KATSU DR, TOKYO ASTRONOMICAL OBS, NAOJ, OSAWA MITAKA, TOKYO 181, JAPAN

Telephone: (81) 422325111

Facsinile: (81)

Email:

Commission(s): 31C,33,51

Telex: 2822307 taomtk j

FUJIMOTO MITSUAKI DR, DPT OF PHYSICS, NAGOYA UNIVERSITY, FUROCHO CHIKUSA KU

NAGOYA 464 01, JAPAN

Telephone: (81)

Facsimile: (81)

Telex:

Email:

Commission(s): $\mathbf{4 7 , 5 1}$

FUJISHITA MITSUMI DR, KYUSHU TOKAI UNIVERSITY, SCHOOL OF ENGINEERING, 9-1-1 TOROKU

KUMAMOTO 862, JAPAN

Telephone: (81) $963821141 * 1641$

Email:

Facsimile: (81) $963817956 \quad$ Telex:

Commission(s): 08,19

FUJITA YOSHIO PROF, DPT OF ASTRONOMY, UNIVERSITY OF TOKYO, BUNKYO KU, TOKYO 113, JAPAN

Telephone: (81) 423744186

Facsimile: (81)

Commission(s): 29

Telex:

Email:

FUJIWARA AKIRA DR, DPT OF PHYSICS, KYOTO UNIVERSITY, KITASHIRAKAWA SAKYO KU, KYOTO 606, JAPAN Telephone: (81) 757512111

Facsimile: (81)

Commission(s): $15,16,21$

Tclex: $5+22693$ libkyu

Email:

FUJIWARA TAKAO DR, KYOTO CITY UNIV OF ARTS, NISHIKYO KU, KYOTO 610 11, JAPAN
Telephoni: (81) 753320701
Facsimile: (81) 753320709

Telephone: (81) 753320701

Commission(s): 33

Telex:

Email: c53926@j.jnkudpc.bitnet

FUKUDA ICHIRO, KANAZAWA TECHNOLOGY INST, 7-1 OGIGAOKA, NONOICHIMACHI, ISHIKAWA 921, JAPAN

Telephone: (81) $762481100 \quad$ Facsimile: $(81)$

Email:

Commission(s):

Telex: 5122456 kit lc j

FUKUE JUN DR, ASTRONOMICAL INSTITUTE, OSAKA KYOIKU UNIVERSITY, 488 MINAMIKAWAHORICHO OSAKA 543, JAPAN

Telephone: (81)

Facsimile: (81)

Telex:

Email:

Commission(s):

FUKUGITA MASATAKA DR, RES INST FUNDAMENTAL PHYS, KYOTO UNIVERSITY

KITASHIRAKAWA SAKYO KU, KYOTO 606, JAPAN

Telephone: (81) $757111381 \quad$ Facsimile: (81)

Email:

Commission(s): 28,47

Telex: $5+23179$ rifpk

FUKUI TAKAO DR, DPT OF LIBERAL ARTS, DOKKYO UNIVERSITY, 1-1 GAKUENCHO, SOKA SAITAMA 340, JAPAN Telephone: (81) 489421111 Facsimile: (81) 489416621 Telex:

Email:

Commission(s): 47

FUKUI YASUO DR, DPT OF PHYSICS, NAGOYA UNIVERSITY, FUROCHO CHIKUSA KU, NAGOYA 464 01, JAPAN

Telephone: (81) 527815111

Facsimile: $(\mathbf{8 1})$

Commission(s): 34,40

Telex: $\$+77323$ scunag $j$

Email

FUKUNAGA MASATAKA DR, ASTRONOMICAL INSTITUTE, TOHOKU UNIVERSITY, SENDAI AOBA

MIYAGI 980, JAPAN

Telephone: (81) 222221800

Facsimile: (81)

Commission(s): 33

Telex:

Email:

FUKUSHIMA TOSHIO DR, ASTROMETRY CELE MECH DIV, NTL ASTRONOMICAL OBS, OSAWA MITAKA TOKYO 181, JAPAN

Telephone: (81) 422343613

Facsimile: $(81)+223+3793$

Telex: 2822307 taomtk j

Email: toshio@spacetime.mtk.nao.ac.jp

Commission(s): (4), $31 \mathrm{VP}$

FUKUSHIMA TOSHIO DR, JPL, MS 301 150, 4800 OAK GROVE DRIVE, PASADENA CA 91109 8099, USA

Telephone: (1) 8183541443

Facsimile: (1) 8183936388

Telex:

Email: toshio@grinch.jpl.nasa.gov

Commission(s): (0+C,31VP 
FULCHIGNONI MARCELLO PROF, OBSERVATOIRE DE PARIS, SECTION DE MEUDON, AEUROPA F 92195 MEUDON PPL CDX, FRANCE

Telephone: (33) 145077539

Email: 17736: :fulchignoni

Facsimile: (33) 145077469

Telex:

Commission(s): 15

FULLE MARCO, OSSERVATORIO ASTRONOMICO, VLA TIEPOLO 11, I 34131 TRIESTE, ITALY

Telephone: (39)

Facsimile: (39) $40309+18 \quad$ Telex: 461137 oat $i$

Email: fulle(a)ts.astro.it

Commission(s):

FURNISS IAN, DPT PHYSICS \& ASTRONOMY, UNIVERSITY COLLEGE LONDON, GOWER ST

LONDON WC1E 6BT, UK

Telephone: (44) 1713877050

Email:

Facsimile: (4t)

Commission(s): 34,44

Telex: 28722 ucphys

FURSENKO M A DR, INST OF THEORET ASTRONOMY, ACADEMY OF SCIENCES, N KUTUZOVA 10 191187 ST PETERSBURG, RUSSIA

Telephone: (7) 2788898

Facsimile: (7)

Commission(s): 04

Telex: 121578 ita su

Email:

FUSCO-FEMIANO ROBERTO, IAS, CNR, CP 67, 1 000 44 FRASCATI, ITALY

Telephone: (39) 69425655

Facsimile: (39) $69+168+7$

Telex: 610261

Email:

Commission(s):

FUSI PECCI FLAVIO, DPT DI ASTRONOMIA, UNIVERSITA DI BOLOGNA, CP 596, I 40100 BOLOGNA, ITALY

Telephone: (39) 51222956

Facsimile: (39)

Commission(s): 37

Telex: $21160+$ infnbo-i

Email:

FU-SHONG KUO, DPT OF PHYSICS, NTL CENTRAL UNIVERSITY, CHUNG LI TAIWAN, CHINA R

Telephone: (886)

Email:

Facsimile: $(\mathbf{8 8 6})$

Telex:

Commission(s):

GABRIEL ALAN H, LAS, BAT 121, UNIVERSITE PARIS XI, F 91405 ORSAY CEDEX, FRANCE

Telephone: (33) 169858500

Facsimile: (33) 169858675

Telex: 600252

Email:

Commission(s): 10,12,14,44

GABRIEL MAURICE R DR, INSTITUT D'ASTROPHYSIQUE, UNIVERSITE DE LIEGE, AVE COINTE 5

B 4000 COINTE-LIEGE, BELGIUM

Telephone: (32) 41529980

Email:

Facsimile: (32) $41527+74$

Telex: 41264 astrigg

Commission(s): 35

GADSDEN MICHAEL DR, DPT OF PHYSICS, UNIVERSITY OF ABERDEEN, FRASER NOBLE BLDG ABERDEEN AB9 2UE, UK

Telephone: (44) 1224574585

Email:

Facsimile: $(44) \quad 122+58+776$

Telex: $73+58$ uniabn $\mathrm{g}$

Commission(s): 21

GAETZ TERRANCE J DR, HARVARD SMITHSONIAN CFA, MS 81, 60 GARDEN ST, CAMBRIDGE MA 02138, USA

Tclephonc: (1) 6174967584

Facsimile: (1) $6174957356 \quad$ Telex:

Email: gactz(a)cfa.harvard.cdu

Commission(s):

GAHM GOESTA F DR, STOCKHOLM OBSERVATORY, S 133 36 SALTSJOEBADEN, SWEDEN

Telephone: (46) 87170637

Facsimile: $(46) 8717+719$ Telcx: 12972

Email: gahm@astro.su.se

Commission(s): 27

GAIGNEBET JEAN DR, OCA CERGA, AVE COPERNIC, F 06130 GRASSE, FRANCE

Telephone: (33) 93365849

Facsimile: (33)

Commission(s): 31

Telex: $\mathbf{4 7 0 8 6 5 f}$

Email:

GAIL HANS-PETER DR, INST F THEOR ASTROPHYSIK, DER UNIVERSITAET, IM NEUENHEIMER FELD 294 D 69120 HEIDELBERG, GERMANY

Telephone: (49)

Email:

Facsimile: (49)

Telex:

Commission(s): 36

GAISSER THOMAS K, BARTOL RESEARCH INSTITUTE, UNIVERSITY OF DELAWARE, NEWARK DE 19716, USA Telephonc: (1) $302+518111$

Facsimilc: (1)

Telex: 510-666-0805 bartol

Email:

Commission(s): 44 
GAIZAUSKAS VICTOR DR, HERZBERG INST ASTROPHYS, NTL RESEARCH COUNCIL, 100 SUSSEX DR, OTTAWA ON K1A 0R6, CANADA

Telephone: (1) 6139937395

Facsimile: (1) 6139520974

Commission(s): $10 \mathrm{C}, 12$
Telex: 0533715 nrcott

Telex:

Facsimile: (20)

Commission(s): 10

Telephone: (20) $780645 / 2683$

Email:

GALAN MAXIMINO J, M \& G ENGS, S MARTIN DE PORRES 45, E 28035 MADRID, SPAIN

Telephonc: (34) 12160995

Facsimilc: (34)

Telex:

Email:

Commission(s): 09,50

GALEEV ALBERT, SPACE RESEARCH INSTITUTE, PROFSOJUZNAYA UL 84/32, MOSCOW 117810, RUSSIA

Telephone: (7) 0953332588

Email: agaleev@esoc1.bitnet

Facsimile: (7) 0953107023

Telex: 411498 a star su

Commission(s):

GALEOTTI PIERO PROF, IST DI COSMO GEOFISICA, CNR, CORSO FIUME \, I 10133 TORINO, ITALY

Telephonc: (39) $11658979 \quad$ Facsimile: (39)

Email:

Commission(s): 4

Telex: 224379 cosmot $i$

GALIBINA I V DR, INST OF THEORET ASTRONOMY, ACADEMY OF SCIENCES, N KUTUZOVA 10, 191187 ST PETERSBURG, RUSSIA

Telephone: (7) 1861974

Email:
Facsimile: (7)

Commission(s): 07
Telex: 121578 ita su

GALINDO TREJO JESUS DR, INSTITUTO DE ASTRONOMIA, UNAM, APDO POSTAL 70-264, 04510 MEXICO DF, MEXICO

Telephone: (52) 9055485305

Email: bitnet:jgat(a)unamvm1

Facsimile: (52)

Commission(s):

GALLAGHER III JOHN S DR, LOWELL OBSERVATORY, 1400 W MARS HILL RD, BOX 1149, FLAGSTAFF AZ 86001, USA

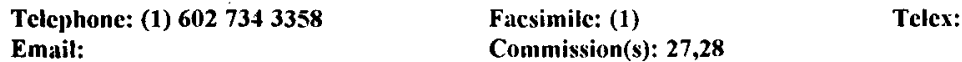

GALLAGHER JEAN W DR, NIST, PHYSICS BUILDIND 221, ROOM A 323, GAITHERSBURG MD 20899, USA

Telephone: (1) 3019752204

Facsimile: (1) $3019260416 \quad$ Telex: 197674 nistut

Email: jwg(a)nistes2.bitnet

Commission(s): 14

GALLARDO CASTRO C TABARE, FAC CIENCIAS, T NARVAJA 1674, MONTEVIDEO 11200, URUGUAY

Telephonc: (598) 2418004

Facsimilc: (598) 2409973

Telex:

Email: astronom@fisica.edu.uy

Commission(s):

GALLET ROGER M, 964 7TH ST, BOULDER CO 80302, USA

Telephone: (1)

Facsimilc: (1)

Commission(s):

Telex:

Email:

GALLETTA GIUSEPPE PROF, DPT DI ASTRONOMIA, UNIVERSITA DI PADOVA, VIC DELL OSSERVATORIO 5, I 35122 PADOVA, ITALY

Telephone: (39) 49661499

Facsimile: (39)

Commission(s): 28

Telex: $\mathbf{\$ 3 0 1 7 6}$ unpadu i

Email:

GALLETTO DIONIGI, IST DI FISICA MATEMATICA, UNIVERSITA DI TORINO, VIA C ALBERTO 10, I 10123 TORINO, ITALY

Telephonc: (39) 11539214

Facsimile: (39)

Telex:

Email:

Commission(s): $07,33,47$

GALLIANO PIER GIORGIO, IST ELETTRONICO NAZIONALE, ST DELLA CACCE 91, I 10135 TORINO, ITALY

Tclephone: (39) 3488933

Facsimile: (39)

Email:

Commission(s): 31

Telex: 211553 icngf $i$

GALLINO ROBERTO, IST DI FISICA GENERALE, CORSO D AZEGLIO 46, 110125 TORINO, ITALY

Tclephonc: (39) 11655103

Facsimile: (39)

Commission(s): $35,36,46$

Telex: $2110+$ infn to

Email: 
GALLOUET LOUIS DR, OBSERVATOIRE DE PARIS, 61 AVE OBSERVATOIRE, F 75014 PARIS, FRANCE

Telephone: (33) 140512207

Facsimile: (33)

Commission(s): 24,25

Telex: 270776 obs $f$

Email:

GALLOWAY DAVID DR, DPT OF APPLIED MATHS, UNIVERSITY OF SYDNEY, SYDNEY NSW 2006, AUSTRALIA

Telephone: (61) 26922222

Email:

Facsimile: (61) $26602903 \quad$ Telex: 26169 unisyd aa

Commission(s): 10

GALPERIN YU I PROF, SPACE RESEARCH INSTITUTE, ACADEMY OF SCIENCES, PROFSOJUZNAYA UL 84/32

117810 MOSCOW, RUSSIA

Telephone: (7) 953333122

Facsimile: (7)

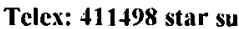

Email:

Commission(s): $21 \mathrm{C}$

GALT JOHN A DR, DOMINION RADIO ASTROPHYS, OBSERVATORY, BOX 248, PENTICTON BC V2A 6K3, CANADA Telephone: (1) 6044932277

Facsimile: (1) 6044937767

Tclex: 04888127

Email:

Commission(s): 40

GAMALELDIN ABDULLA I DR, HELWAN OBSERVATORY, HELWAN, EGYPT

Telephone: (20) $780645 / 2683$

Facsimile: (20)

Telex:

Email:

Commission(s): 28

GAMBIS DANIEL DR, OBSERVATOIRE DE PARIS, 61 AVE OBSERVATOIRE, F 75014 PARIS, FRANCE

Telephone: (33) 140512233

Facsimile: (33)

Telex: 270776 obs $f$

Email: gambis@friap51

Commission(s): 19,31

GAMMELGAARD PETER MAG SCI, INST OF PHYSICS \& ASTRON, UNIVERSITY OF AARHUS, NY MUNKEGADE DK 8000 AARHUS C, DENMARK

Telephone: (45) 86128899

Facsimile: (45) 86202711

Telex: 64767 aausci $d k$

Email:

Commission(s): 15

GAN WEI QUN, PURPLE MOUNTAIN OBS, NANJING 210008, CHINA PR

Telephone: (86) 253317874

Facsimile: (86) 253301459 Telex:

Email:

Commission(s): 10

GAO BILIE, NANJING ASTRONOMICAL, INSTRUMENT FACTORY, NANJING, CHINA PR

Telephone: (86) 2546191

Facsimile: (86)

Commission(s): (0)

Telex:

Email:

GAO BUXI, INSTITUTE OF GEODESY \&, GEOPHYSICS, XU DONG LU, WUCHANG HUBEI 430077, CHINA PR

Telephone: (86) 813805

Facsimile: (86)

Commission(s): 08,19

Telex:

Email:

GAPOSCHKIN EDWARD M DR, 55 FARMCREST AVE, LEXINGTON MA 02173, USA

Telcphone: (1) $6178622538 \quad$ Facsimile: (1) Telex:

Email: $\quad$ Commission(s): (17,19

GARAY GUIDO DR, DPT DE ASTRONOMIA, UNIVERSIDAD DE CHILE, CASILLA 36 D, SANTIAGO, CHILE

Telephonc: (56) 22294101

Facsimilc: (56)

Commission(s): 40

Telex:

Email:

GARCIA BEATRIZ ELENA, COMPLEJO ASTRON EL LEONCI, AV ESPANA 1512 SUR, CC 467

5400 SAN JUAN, ARGENTINA

Telephone: (54) $64213653 \quad$ Facsimile: (54) 64 213653 Telex: 59134 entop) ar

Email: bgarcia(a) castec.edu.ar

Commission(s):

GARCIA DE LA ROSA JOSE I, INST DE ASTROFISICA, DE CANARIAS, OBS DEL TEIDE, E 38071 LA LAGUNA, SPAIN

Telephonc: (34) 22262211

Facsimile: (3t)

Telex: 92640

Email:

Commission(s): 10

GARCIA DE MARIA J M DR, DPT FISICA APLICADA, RONDA DE VALENCIA 3, E 28012 MADRID, SPAIN

Tclephone: (34) 13367686

Facsimile: (34) 15309244

Commission(s): 42

Telex:

Email: 
M 546

GARCIA DOMINGO, DPT FISICA \& ENGINYERIA, UNIVERSIDAD POLITECNICA, C/ PAU GARGALLO 5 E 08028 BARCELONA, SPAIN

Telephone: (34) 34017421

Facsimile: (34)

Telex:

Email: domingo aff-720.upc.es

Commission(s): 35

GARCIA EDUARDO DEL POZO, INST GEOPHYS \& ASTRONOMY, CALLE 212 N 2906/29 Y 31, LISA

LA HABANA, CUBA

Telephone: (53)

Facsimile: (53)

Telex:

Email:

Commission(s):

GARCIA HOWARD A, SPACE ENVIRONMENT LAB, 325 BROADWAY R E SE, BOULDER CO 80303, USA

Telephone: (1) 3034973916

Facsimile: (1) 3034973645

Telex:

Email: hgarcia@solar.stanford.edu

Commission(s): $10,12,44$

GARCIA LAMBAS DIEGO DR, OBSERVATORIO ASTRONOMICO, DE CORDOBA, LAPRIDA 850

5000 CORDOBA, ARGENTINA

Telephone: (54) 51230491

Facsimile: (54) 51210613

Telex:

Email:

Commission(s): 28

GARCLA LOPEZ RAMON J, IAC, C/ VIA LACTEA S/N, E 38200 LA LAGUNA, SPAIN

Telephone: (34) 22605200

Facsimile: (34) 22605210

Telex: 92640 iac e

Email: rgl(a)iac.es

Commission(s): 29

GARCIA MICHAEL R DR, CENTER FOR ASTROPHYSICS, HCO/SAO, 60 GARDEN ST, CAMBRIDGE MA 02138, USA

Telephone: (1) 6174957169

Facsimile: (1) $617+957356$

Telex:

Email: garcia@cfa200.harvard.cdu

Commission(s):

GARCIA-BARRETO JOSE A, OBS ASTRONOMICO NACIONAL, UNAM, APDO POSTAL 877

22860 ENSENADA B CALIF, MEXICO

Telephone: (52) 66783093

Facsimile: (52)

Commission(s):

Telex:

Email:

GARCIA-BERRO ENRIQUE DR, DPT FISICA APLICADA, UNIV POLITEC DE CATALUNYA

E 08032 BARCELONA, SPAIN

Telephone: (34) 34016898

Facsimile: (34) 34016801

Telex: 52821 upe c

Email: garcia@etseccpb.upc.es

Commission(s): 12

GARCIA-PELAYO JOSE DR, INST ASTROFISICA, DE ANDALUCIA APD 3004, PROFESOR ALBAREDA 1

E 18080 GRANADA, SPAIN

Telephone: (34) 58256103

Facsimile: (34)

Email:

Commission(s):

Telex:

GARDNER FRANCIS F DR, 201B BEECROFT RD, CHELTENHAM NSW 2119, AUSTRALIA

Telephone: (61)

Facsimile: (61)

Telex:

Email:

Commission(s): 34,40

GARFINKEL BORIS DR, YALE UNIVERSITY OBS, YALE STATION, BOX 2023, NEW HAVEN CT 06520, USA

Telephone: (1) 2034363460

Facsimile: (1)

Telex:

Email:

Commission(s): 07,20

GARGAUD MURIEL DR, OBSERVATOIRE DE BORDEAUX, BP 89, F 33270 FLOIRAC, FRANCE

Telephonc: (33) 56864330

Facsimile: (33) $5640+251 \quad$ Telex:

Email: muriel@afrobor51

Commission(s): 14

GARILLI BIANCA, IFCTR CNR, VIA E BASSINI 15, I 20133 MILANO, ITALY

Telephonc: (39) 22665237

Facsimile: (39) 223629.46

Telex:

Email: bianca(âifetr.mi.cnr,it

Commission(s): $28,+7$

GARLICK GEORGE F DR, 267 SOUTH BELOIT AVE, LOS ANGELES CA 90049, USA

Telephonc: (1) $2134723512 \quad$ Facsimile: (1)

Email:

Commission(s):

Telex:

GARMANY CATHERINE D DR, JILA, UNIVERSITY OF COLORADO, BOX 440, BOULDER CO 80309 0440, USA

Tclephonc: (1) 3034927836

Facsimile: (1)

Telex:

Email:

Commission(s): $29,42,45 C$ 
GARMIRE GORDON P PROF, DPT OF ASTRONOMY, PENNSYLVANIA STATE UNIV, 525 DAVEY LAB UNIVERSITY PARK PA 16802, USA

Telephone: (1) 8148650418

Facsimile: (1)

Commission(s): 44

Telex: $8+2510$ pennstbstr sc

Email:

GARNETT DONALD ROY, ASTRONOMY DPT, UNIVERSITY OF MINNESOTA, 116 CHURCH ST SE MINNEAPOLIS MN 55455, USA

Telephone: (1) 6126241084

Facsimile: (1) $6126262029 \quad$ Telex:

Email: garnett(âoldstyle.spa.umn.edu

Commission(s): 34

GARNIER ROBERT ING, OBSERVATOIRE DE LYON, AVE CHARLES ANDRE, F 69561 S GENIS LAVAL CDX, FRANCE Telephone: (33) 78560705

Facsimile: (33) 72399791

Telex:

Email:

Commission(s):

GARRIDO RAFAEL, INST ASTROFISICA, DE ANDALUCIA APD 3004, PROFESOR ALBAREDA 1

E 18080 GRANADA, SPAIN

Telephone: (34) 58121311

Facsimile: (34)

Commission(s): 27

Telex: $\mathbf{7 8 7 5 3}$

Email:

GARRINGTON SIMON DR, NRAL, JODRELL BANK, MACCLESFIELD SK11 9DL, UK
Telephone: (44) 147771231

Telephone: (4) 147771231

Facsimile: (4+) 1+47771618

Telex: 36149

Email: janct stg@uk.ac.man.jb.star

Commission(s): 40

GARRISON ROBERT F PROF, STRONOMY DPR, UNIVERSITY OF TORONTO, 60 ST GEORGE ST TORONTO ON MS5 1A7, CANADA

Telephone: (1) 9058849562

Facsimile: (1) $9058842672 \quad$ Telex:

Email: garrison(a)astro.utoronto.ca

Commission(s): $29,45,47$

GARSTANG ROY H PROF, JILA, UNIVERSITY OF COLORADO, BOX 440, BOULDER CO 80309 0440, USA

$\begin{array}{lll}\text { Telephone: (1) } 3034927795 & \text { Facsimile: (1) } & \text { Telex: } 7558+2 \text { jila } \\ \text { Email: } & \text { Commission(s): } 05,14 & \end{array}$

GARTON W R S PROF, ASTROPHYSICS GROUP, IMPERIAL COLLEGE, BLACKETT LABORATORY

LONDON SW7 2BZ, UK

Telephonc: (44) 17123321657

Email:

Facsimile: $(44)$

Telex: 261503

Commission(s): 14

GARY DALE E, CALTECH, MS 26433, SA, PASADENA CA 91125, USA

$\begin{array}{ll}\text { Telephone: (1) } 8183563863 & \text { Facsimile: (1) } \\ \text { Email: } & \text { Commission(s): }\end{array}$

GARY GILMER ALLEN DR, NASA/MSFC, CODE ES 52, SPACE SCIENCE LAB, HUNTSVILLE AL 35812, USA

Telephone: (1) 2055447609

Facsimile: (1) 2055445862

Telex: 62026079

Email: span ssl::gary

Commission(s): 10

GARZON FRANCISCO DR, INST DE ASTROFISICA, DE CANARIAS, OBS DEL TEIDE, E 38200 LA LAGUNA, SPAIN

Telcphone: (34) 22262211

Email: span:iac::fgl

Facsimile: (34)

Commission(s): 33

Telex: 92640

GASCOIGNE S C B DR, MOUNT STROMLO \& SIDING, SPRING OBSERVATORIES, PRIVATE BAG WODEN PO ACT 2606, AUSTRALIA

Telcphone: (61) 62881111

Facsimilc: $(61) 62+90233$

Telex:

Email:

Commission(s): $27,28,37$

GASKA STANISLAW DR, INSTITUTE OF ASTRONOMY, N COPERNICUS UNIVERSITY, UL CHOPINA 12/18 PL 87100 TORUN, POLAND

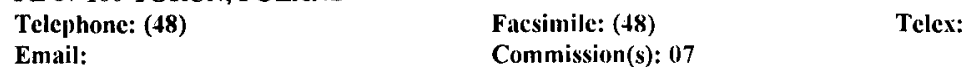

GATEWOOD GEORGE DIRECTOR, ALLEGHENY OBSERVATORY, OBSERVATORY STATION PITTSBURGH PA 15214, USA

Telephone: (1) 4123212400

Email:

Facsimile: (1)

Commission(s): $24,26,51$
Telex: 
GATLEY IAN, NOAO, BOX 26732, 950 N CHERRY AVE, TUCSON AZ 85726 6732, USA

Telephone: (1) 5203275511

Facsimile: (1)

Telex: $0666+8+$ aur

Email:

Commission(s):

GAUDENZI SILVIA DR, ISTITUTO ASTRONOMICO, UNIVERSITA DI ROMA, VIA G M LANCISI 29 I 00161 ROMA, ITALY

Telephone: (39) 64403734

Facsimile: (39) $64+03673$

Telex: 613255 infinro

Email: 40058::gaudenzi

Commission(s): 26

GAUME RALPH A DR, US NAVAL OBSERVATORY, 3450 MASS AVE NW, WASHINGTON DC 20392 5420, USA

Tclephonc: (1) 2026530947

Facsimile: (1) $2026530587 \quad$ Telex:

Email: gaume@nh3.usno.navy.mil

Commission(s):

GAUR V P, UTTAR PRADESH STATE, OBSERVATORY, PO MANORA PEAK 263 129, NAINITAL 263 129, INDLA

Telephone: (91) 59422136

Facsimile: (91)

Telex:

Email:

Commission(s): 12

GAUSS F STEPHEN, US NAVAL OBSERV, 3450 MASSACHUSETTS AVE NW, WASHINGTON DC 203925420 , USA

Telephone: (1) 2026531510 Facsimile: (1) 2026530944 Telex:

Email: fsg(asicon.usno.navy.mil

Commission(s): $08,09,24$

GAUSTAD JOHN E PROF, DPT OF ASTRONOMY, SWARTHMORE COLLEGE, SWARTHMORE PA 19081, USA

Tclephonc: (1) 2153288271

Facsimile: (1) 2153288673

Telex:

Email: jgausta1(a)ce.swarthmore.edu

Commission(s): 34

GAUTIER DANIEL, OBSERVATOIRE DE PARIS, SECTION DE MEUDON, F 92195 MEUDON PPL CDX, FRANCE

Telephone: (33) 145077707

Facsimilc: (33)

Telex: 201571

Email: gauticrd@17670.span.nasa.gov

Commission(s): 16C

GAUTSCHY ALFRED, ASTRONOMISCHES INSTITUT, UNIVERSITAET BASEL, VENUSSTRASSE 7

CH 4102 BINNINGEN, SWITZERLAND

Telephone: (\$1) 612717711

Facsimile: (41) $612717810 \quad$ Telex:

Email: gautschy a astro.unibas.ch

Commission(s): 35

GAVAZZI GIUSEPPE DR, OSS ASTRONOMICO DI BRERA, VIA BRERA 28, I 20121 MILANO, ITALY

Telephone: (39) 2874444

Facsimile: (39) $272001600 \quad$ Telex:

Email: 39216::gavazzi

Commission(s): 28

GAY JEAN DR, OCA CERGA, AVE COPERNIC, F 06130 GRASSE, FRANCE

Telephone: (33) 93365849

Facsimile: (33)

Commission(s): 09,34

Telex: $\mathbf{4 7 0 8 6 5}$

Email:

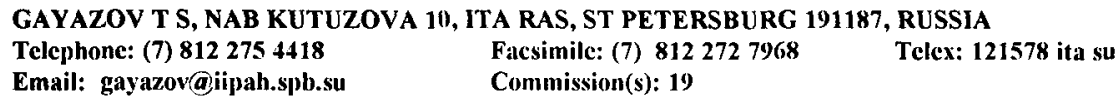

GAYLARD MICHAEL JOHN, HARTEBEESTHOEK RADIOASTRO, OBSERVATORY, BOX 443 KRUGERSDORP 1740, SOUTH AFRICA

Telephone: (27) $27116424692 \quad$ Facsimile: (27) 2711642 2446 $\quad$ Telex: 321006

Email:

Commission(s): 40

GEAKE JOHN E DR, DPT OF PHYSICS, UMIST, BOX 88, MANCHESTER M60 1QD, UK

Telephone: (44) $1612363311 \quad$ Facsimile: (44) Telex: 666094

Email:

Commission(s): 16

GEBALLE THOMAS R DR, JOINT ASTRONOMY CENTER, UKIRT, G65 KOMOHANA ST, HILO HI 96720, USA

Telephone: (1) 8089613756

Facsimile: (1)

Telex: 633135

Email: nssdca::psi\%ukth::tom

Commission(s): $3+$

GEBBIE KATHARINE B DR, JILA, UNIVERSITY OF COLORADO, BOX 440, BOULDER CO 80309 0440, USA

Telephone: (1) $303 \mathbf{4 9 2} 7825$

Facsimile: (1)

Telex: 755842 jila

Email:

Commission(s): 36 
GEBLER KARL-HEINZ DR, RADIOASTRONOMISCHES INST, UNIVERSITAET BONN, AUF DEM HUEGEL 71 D 53121 BONN, GERMANY

Telephone: (49) 228733662

Facsimile: (49)

Telex:

Email:

Commission(s): 40

GEFFERT MICHAEL DR, OBSERVATORIUM HOHER LIST, UNIV STERNWARTE BONN, D 5568 DAUN, GERMANY

Telephonc: (49)

Email: geffert@astro.uni-bonn.de

Facsimile: (49) 228733672 Telex:

Commission(s): 24,37

GEHRELS TOM PROF, LUNAR \& PLANETARY LAB, UNIVERSITY OF ARIZONA, TUCSON AZ 85721, USA

Telephone: (1) 5206216970

Facsimile: (1) 5206214933

Telex:

Email: gehreis@Ipl.arizona.cdu

Commission(s): $15,16,20,25,34,51$

GEHREN THOMAS PH D, INST ASTRON \& ASTROPHYSIK, UNIVERSITAETS STERNWARTE, SCHEINERSTR 1 D 81679 MUENCHEN, GERMANY

Telephone: (49) 89989021

Email:

Facsimilc: (49) 8992209427

Commission(s): 29

Telex: 529815 univers d

GEHRZ ROBERT DOUGLAS DR, DPT PHYSICS \& ASTRONOMY, UNIVERSITY OF WYOMING, BOX 3905

LARAMIE WY 82071, USA

Telephone: (1) 3077666176

Email:

Facsimile: (1)

Commission(s): 25

Telex:

GEISLER DOUGLAS P DR, CERRO TOLOLO, INTERAMERICAN OBSERVATORY, CASILLA GO3, LA SERENA, CHILE Telephone: (56) $51225415 * 208$

Email: Jgeisler(a)noa.edu Facsimile: (56) $51225+15^{\star} 3+2$ Telex: 620301 aural ct

Commission(s): 37

GEISS JOHANNES PROF, PHYSIKALISCHES INSTITUT, UNIVERSITAET BERN, SIDLERSTRASSE 5

CH 3012 BERN, SWITZERLAND

Telephone: (41) 31658645

Facsimile: (41)

Commission(s): 15,16

Telex: 32320

Email:

GELDZAHLER BERNARD J, NAVAL RESEARCH LABORATORY, CODE $4121.6,4555$ OVERLOOK AVE SW WASHINGTON DC 20375 5000, USA

Telephone: (1)

Facsimile: (1)

Telex:

Email:

Commission(s): $40,42 \mathrm{C}$

GELFREIKH GEORGIJ B DR, PULKOVO OBSERVATORY, ACADEMY OF SCIENCES, 10 KUTUZOV QUAY 196140 ST PETERSBURG, RUSSIA

Telephone: (7)

Email:

Facsimile: (7)

Telex:

Commission(s): 10,40

GELLER MARGARET JOAN, CENTER F ASTROPHYSICS, HCO/SAO, 60 GARDEN ST, CAMBRIDGE MA 02138, USA

Telephone: (1) 6174957409

Facsimile: (1)

Commission(s): $28,47 \mathrm{C}$

Telex: 921428 satellite cam

Email:

GEMMO ALESSANDRA DR, DPT DI ASTRONOMIA, UNIVERSITA DI PADOVA, VIC DELL OSSERVATORIO 5

I 35122 PADOVA, ITALY

Telephone: (39) 49661499

Email: 39003::gemmo

Facsimile: (39) 4938919

Telex: $\mathbf{4 3 2 0 7 1}$ astros $i$

Commission(s): 33

GENET R M DR, FAIRBORN OBSERVATORY, 3435 E EDGEWOOD ABE, MESA AZ 85204, USA

Telcphone: (1) $6029886561 * 223$

Facsimile: (1)

Telex:

Email: genet@aregasus.la.asu.cdu

Commission(s): 25,27

GENKIN IGOR L PROF DR, FACULTY OF PHYSICS, KAZAKH STATE UNIVERSITY, TOLE LI 96 480012 ALMA ATA, KAZAKHSTAN

Telcphonc: (7) 677018

Facsimile: (7)

Commission(s): 33

Telex:

Email:

GENOVA FRANCOISE DR, OBS DE STRASBOURG, 11 RUE UNIVERSITE, F 67000 STRASBOURG, FRANCE

Telephone: (33)

Facsimile: (33)

Telex:

Email:

Commission(s): 05 
GENT HUBERT MR, PROSPECT HOUSE, SHERWOOD LANE, WORCESTER WR2 4NX, UK Telephonc: (44) 1422186

Facsimile: (44)

Telex:

Email:

Commission(s): 40

GENZEL REINHARD DR, MPI F PHYSIK \& ASTROPHYS, KARL SCHWARZSCHILDSTR 1

D 85740 GARCHING MUENCHEN, GERMANY

Telephone: (49) 8932990

Facsimile: (49)

Telex: 524629

Email:

Commission(s): 34C,40

GEORGELIN YVON P DR, OBSERVATOIRE DE MARSEILLE, 2 PLACE LE VERRIER

F 13248 MARSEILLE CDX 04, FRANCE

Tclephone: (33) 91959088

Facsimile: (33)

Telex: $\mathbf{4 2 0 2 4 1 \mathrm { f }}$

Email:

Commission(s): $30,33,34$

GEORGELIN YVONNE M DR, OBSERVATOIRE DE MARSEILLE, 2 PLACE LE VERRIER

F 13248 MARSEILLE CDX 04, FRANCE

Tclephonc: (33) 91959088

Facsimile: (33)

Telex: $4202+1$ f

Email:

Commission(s): 33

GEORGIEV LEONID, DPT OF ASTRONOMY, SOFIA UNIVERSITY, 5 JAMES BOUCHIER AVE BG 1126 SOFIA, BULGARLA

Facsimile: (359)

Telex:

Email: physfac1@bgearn.bitnet

Commission(s):

GEORGIEV TSVETAN DR, DPT OF ASTRONOMY, BULGARIAN ACAD SCIENCES, 72 LENIN BLVD BG $178+$ SOFIA, BULGARIA

Telephone: (359) 2883503

Facsimilc: (359)

Telex: 23351 iki ban bg

Email:

Commission(s): 28

GERARD ERIC DR, OBSERVATOIRE DE PARIS, SECTION DE MEUDON, F 92195 MEUDON PPL CDX, FRANCE

Telephone: (33) 145077607

Facsimile: (33)

Telex: 270912

Email: gerard@frmeu51

Commission(s): $15,34,40 \mathrm{C}$

GERARD JEAN-CLAUDE M C DR, INSTITUT D'ASTROPHYSIQUE, UNIVERSITE DE LIEGE, AVE COINTE 5

B 4000 COINTE-LIEGE, BELGIUM

Telephone: (32) 41529980

Email: u2163jc at bliulg 11

Facsimile: (32) $\$ 1527474$

Telex: $\mathbf{4 1 2 6 4}$

GERASIMOV IGOR, STERNBERG ASTRONOMICAL, INSTITUTE, 13 UNIVERSITETSKIJ PROSP

MOSCOW 119899, RUSSIA

Telephone: (7) 0959391625

Email: snnasai.msk.su

Facsimile: (7)

Telex:

GERBAL DANIEL DR, OBSERVATOIRE DE PARIS, SECTION DE MEUDON, F 92195 MEUDON PPL CDX, FRANCE

Telephone: (33) 145077419

Facsimile: (33)

Telex: 201571

Email:

Commission(s):

GERBALDI MICHELE DR, INSTITUT D'ASTROPHYSIQUE, 98BIS BD ARAGO, F 75014 PARIS, FRANCE

Telephone: (33) 144328041

Facsimile: (33) 1 \$32 8001 Telex:

Email: gerbaldi@iap.fr

Commission(s): 25,29C,45VP, $46 \mathrm{C}$

GERGELY TOMAS ESTEBAN DR, NTL SCIENCE FOUNDATION, DIV ASTRONOMICAL SCIENCES, 1800 G ST NW WASHINGTON DC 20550, USA

Telephone: (1) 2023579696

Email:
Facsimile: (1)

Commission(s): 10,40,50C
Telex:

GERHARD ORTWIN, LANDESSTERNWARTE, KOENIGSTUHL, D 69117 HEIDELBERG, GERMANY

Telephone: (49) 6221509222

Email:

Facsimilc: (49)

Commission(s): 28

GERIN MARYVONNE DR, RADIOASTRONOMIE ENS, 24 RUE LHOMOND, F 75231 PARIS CDX 05, FRANCE

Telephone: (33) 143291225

Email: gerin@frulm11
Telex:
Commission(s): 34

Telex: 
GERLEI OTTO, HELIOPHYSICAL OBSERVATORY, BOC 30, H 4010 DEBRECEN, HUNGARY

Telephone: (36) 52311015

Facsimile: (36)

Telex:

Email:

Commission(s):

GEROLA HUMBERTO DR, 20390 KNOLLWOOD DRIVE, SARATOGA CA 95070, USA

Telephone: (1)

Facsimile: (1) Telex:

Email:

Commission(s): $3+$

GEROYANNIS VASSILIS S DR, DPT OF ASTRONOMY, UNIVERSITY OF PATRAS, GR 26110 RION, GREECE

Telephone: (30)

Facsimile: (30)

Telex:

Email:

Conmission(s): 35

GERSHBERG R E DR, CRIMEAN ASTROPHYS OBS, UKRAINIAN ACAD OF SCIENCE, NAUCHNY

33H413 CRIMEA, UKRAINE

Telephone: (7) 432945

Facsimile: (7) Telex:

Email:

Commission(s): 27,29

GESICKI KRZYSZTOF, INSTITUTE OF ASTRONOMY, N COPERNICUS UNIVERSITY, UL CHOPINA 12/18

PL 87100 TORUN, POLAND

Telephone: (48) 26018*50

Facsimile: $(48)$

Telex:

Email: gesicki@astri.uni.torun.pl

Commission(s): 29,36

GEYER EDWARD H PROF DR, OBSERVATORIUM HOHER LIST, UNIV STERNWARTE BONN

D 5568 DAUN, GERMANY

Telephone: (49) 65922150

Email:

Facsimile: (49)

Telex:

GEZARI DANIEL YSA DR, NASA GSFC, CODE 685, GREENBELT MD 20771, USA

Telephone: (1) 3012863432

Facsimilc: (1)

Telex:

Email:

Commission(s): 34,44

GHIGO FRANCIS D DR, NRAO, BOX 2, GREEN BANK WV 2494,, USA

Telephone: (1)

Facsimile: (1)

Telex:

Email:

Commission(s): $28,40,51$

GHOBROS ROSHDY AZER PROF, HELWAN OBSERVATORY, HELWAN, EGYPT

Telephone: (20) 780645

Facsimile: (20)

Telex:

Email:

Commission(s):

GHOSH KAJAL KUMAR DR, INDIAN INST OF ASTROPHYS, VAINU BAPPU OBSERVATORY, KAVALUR ALANGAYAM NAA TN 635701, INDIA

Telephone: (91) $530676 \quad$ Facsimile: (91)

Email: shakti!vigyan!iiap!gea Commission(s): 29

Telex: 8452763 iiab in

GHOSH P DR, TIFR, HOMI BHABHA RD, COLABA, BOMBAY 400005 , INDIA

Telephone: (91) $22219111 * 260 \quad$ Facsimile: (91) Telex: 011-3009

Email: $\quad$ Commission(s): 28

GHOSH S K DR, TIFR, HOMI BHABHA RD, COLABA, BOMBAY 400005 , INDIA

Telephone: (91) $222152311 \quad$ Facsimile: (91) $222152110 \quad$ Telex: 0113009 tifr in

Email: swarna $\underline{a}$ tifrvax.bitnet

Commission(s): 25

GIACAGLIA GIORgIO E PROF, CRAAE/ESCOLA POLITECNICA, UNIVERSIDADE DE SAO PAULO, CP 8174 05508 SAO PAULO SP, BRAZIL

Telephone: (55) 118159322

Email:

Facsimile: (55) $11815+272 \quad$ Telex:

Commission(s): 07

GIACANI ELSA BEATRIZ DR, IAR, CC 5, $189+$ VILLA ELISA (BS AS), ARGENTINA

Telephone: (54) 2143793

Facsimile: (54) 21211761

Telex: 18052 cicyt ar

Email:

Commission(s): 28,34

GIACCONI RICCARDO PROF, STSCI, HOMEWOOD CAMPUS, 3700 SAN MARTIN DR, BALTIMORE MD 21218, USA

Telephone: (1) 3013384711

Facsimile: (1)

Email:

Commission(s): 4

Telex: $68+9101$ st sci 
M 552

GIACHETTI RICCARDO PROF, DPT DI FISICA, UNIVERSITA DI FIRENZE, LARGO E FERMI 2

I SO100 FIRENZE, ITALY

Tclephonc: (39) 552298141

Email:

Facsimile: (39)

Telex: 572570

GIALLONGO EMANUELE DR, OAR, I 00040 MONTEPORZIO, ITALY

Telephone: (39) 69449019

Facsimile: (39)

Telex:

Email: decnet:17468::giallongo

Commission(s): 47

GIAMPAPA MARK S, NTL SOLAR OBSERVATORY, BOX 26732, 950 N CHERRY AVE, TUCSON AZ 85726 6732, USA

Telephone: (1) 5203275511

Facsimile: (1)

Telex: 0666484 aura noantuc

Email:

Commission(s): 29,36

GIANNONE PIETRO PROF, OAR, VIA DEL PARCO MELLINI 84, I 00136 ROMA, ITALY

Telephone: (39) 63452794

Facsimile: (39) Telex:

Email:

Commission(s): 35,42

GIANNUZZI MARIA A DR, DPT DI MATEMATICA, UNIV DI ROMA LA SAPIENZA, PIAZZA GRAMSCI 5 I 0004I ALBANO/LAZIALE, ITALY

Telephone: (39) 69321101

Facsimile: (39)

Telex:

Email:

Commission(s):

GIBSON DAVID MICHAEL DR, SITE MAN GEODSS FIELD ST, MIT LINCOLN LABORATORY, BOX 1707 SOCORRO NM 878011707 , USA

Telephone: (1) $505679+244$

Facsimile: (1)

Telex:

Email:

Commission(s): (99,10,50

GIBSON JAMES, 6838 GREELEY ST, TUJUNGA CA 910+2-2809, USA

Telephone: (1)

Facsimile: (1)

Telex:

Email:

Commission(s): 15,20

GICLAS HENRY L MR, 120 E ELM AVE, FLAGSTAFF AZ 8601, USA

Telcphonc: (1) $60277+4769$

Facsimile: (1)

Telex:

Email:

Commission(s): $16,20,24$

GIERASCH PETER J DR, DPT OF ASTRONOMY, CORNELL UNIVERSITY, SPACE SCIENCES BLDG

ITHACA NY 14853 , USA

Telephonc: (1) 6072563507

Facsimile: (1)

Telex:

Email:

Commission(s): 16

GIEREN WOLFGANG P DR, GRUPO ASTROFIS PONTIFICA, UNIVERSIDAD CATOLICA, CASILLA 104 SANTIAGO 22, CHILE

Telephone: (56)

Facsimile: (56)

Telex:

Email: wgicrenca astrouc.puc.cl

Commission(s): 27

GIES DOUGLAS R DR, DPT PHYSICS \& ASTRONOMY, GEORGIA ST UNIVERSITY, ATLANTA GA 30303 3083, USA

Telephone: (1) $40+6512932$

Facsimile: (1)

Commission(s): 27

Telex:

Email: bitnet:phyddy a gsuvm 1

GIESEKING FRANK DR, OBSERVATORIUM HOHER LIST, UNIV STERNWARTE BONN, D 5568 DAUN, GERMANY

Telephone: (49)

Facsimile: $(49)$

Telex:

Email:

Commission(s): 30

GIETZEN JOSEPH W, ROYAL GREENWICH OBS, HERSTMONCEUX CASTLE, HAILSHAM BN27 1RP, UK

Telephone: (4t) 1323813171

Facsimile: (4t)

Telex:

Email:

Commission(s):

GIGAS DETLEF DR, NASA GSFC, CODE 911 2, GREENBELT MD 20771, USA

Telephone: (1)

Facsimile: (1)

Telex:

Email:

Commission(s): 36

GIL JANUSZ A PROF, THE ASTRONOMICAL CENTER, LUBUSKA 2, PL 65 061 ZIELONA GORA, POLAND

Telephone: $(48) 486866385$

Email:

Facsimile: $(48)+86860385$

Commission(s): 40
Telex: 0433467 wsppl 
GILFANOV MARAT, SPACE RESEARCH INSTITUTE, PROFSOYUSNAYA UL 84/32, MOSCOW 117810, RUSSIA

Telephonc: (7) 0953333377

Email: mgilfano@hea.iki.rssi.ru
Facsimilc: (7) $0953335377 \quad$ Telex:

Commission(s):

GILLET D DR, OHP, F 04870 S MICHEL OBS, FRANCE

Telephonc: (33) 92766368

Email: bitnet:gillet@rronis1

Facsimile: (33)

Commission(s): 27

Telex: $\$ 10690$ ohp f

GILLILAND RONALD LYNN, STSCI, HOMEWOOD CAMPUS, 3700 SAN MARTIN DR, BALTIMORE MD 21218, USA

Telephone: (1)

Facsimile: (1)

Telex:

Email:

Commission(s): 10

GILLINGHAM PETER MR, WM HECK OBSERVATORY, BOX 220, KAMUELA HI 96743, USA

Telephone: (1) 8088857887

Facsimile: (1) $808885+464 \quad$ Telex:

Email: peter(a)keck.hawaii.edu

Commission(s): (09

GILMAN PETER A DR, HIGH ALTITUDE OBSERVATORY, NCAR, BOX 3000, BOULDER CO 80307 3000, USA

Telephone: (1) 3034971560

Facsimile: (1) $303+971568$

Telex:

Email:

Commission(s): 10

GILMORE ALAN C MR, MT JOHN OBSERVATORY, BOX 57, LAKE TEKAPO, NEW ZEALAND

Telephonc: (64) 645056813

Facsimile: (6t)

Telcx:

Email:

Commission(s): 06,20

GILMORE GERARD FRANCIS, INSTITUTE OF ASTRONOMY, THE OBSERVATORIES, MADINGLEY RD

CAMBRIDGE CB3 OHA, UK

Tclephone: (4t) 1223337548

Email:

Facsimile: (4t) $1223337523 \quad$ Tclex: 81797 astron g

Commission(s): 30,33C

GILMOZZI ROBERTO, ESA IUE OBSERVATORY, VILSPA, APD 50727, E 28080 MADRID, SPAIN

Telephone: (34) $18131100 \quad$ Facsimile: (34)

Telex: $\mathbf{2 5 5 5}$

Email:

Commission(s):

GILRA DAYA P DR, SM SYSTEMS \& RESEARCH CO, 8401 CORPORATE DR, SUITE 450, LANDOVER MD 20785, USA Telephone: (1) $301763+483$

Facsimile: (1)

Telex:

Email:

Commission(s): $29,34,44$

GIMENEZ ALVARO, LAB DE ASTROFISICA ESPACI, FISICA FUNDEMENTAL LAEFF, APD 50727

E 28080 MADRID, SPAIN

Telephone: (34)

Facsimile: (34)

Commission(s): 35,42

Telex:

Email:

GINGERICH OWEN PROF, CENTER FOR ASTROPHYSICS, HCO/SAO, 60 GARDEN ST, CAMBRIDGE MA 02138, USA

Telephone: (1) 6174957216

Email: ginger(â) cfa/cfa::ginger

Fackimile: (1) $6174967564 \quad$ Telex: 921428 satellite cam

Commission(s): $+1,+6$

GINGOLD ROBERT ARTHUR DR, AUSTRALIAN NTL UNIVERSITY, ANU SUPERCPTR FACILITY, BOX 4 CANBERRA ACT 2600, AUSTRALIA

Telephone: (61) $62+93437$

Facsimile: (61)

Commission(s): 35

Telex:

Email:

GINZBURG VITALY L PROF, LEBEDEV PHYSICAL INST, ACADEMY OF SCIENCES, LENINSKY PROSPEKT 53 $11792+$ MOSCOW, RUSSIA

Telephone: (7)

Facsimile: (7)

Telex:

Email:

Commission(s): $40,44,51$

GIOIA ISABELLA M DR, INSTITUTE FOR ASTRONOMY, UNIVERSITY OF HAWAII, 2680 WOODLAWN DR HONOLULU HI 96822, USA

Telephonc: (1)

Facsimile: (1)

Telex: 723 8459 whast hr

Email:

Commission(s): 40

GIOVANARDI CARLO, OSS ASTROFISICO, DI ARCETRI, LARGO E FERMI 5, I 50125 FIRENZE, ITALY

Telephone: (39) 552752239

Facsimile: (39)

Email:

Commission(s): 28

Telex: $\$ 72268$ arcetr $i$ 
GIOVANE FRANK, NASA HEADQUARTERS, CODE SZ, 600 INDEPENDENCE AVE SW, WASHINGTON DC 20546, USA Telephonc: (1) 2024531472

Facsimile: (1)

Telex:

Email: 6646::fgiovane

Commission(s): 15,21

GIOVANELLI RICCARDO DR, DPT OF ASTRONOMY, CORNELL UNIVERSITY, SPACE SCIENCES BLDG

ITHACA NY 14853, USA

Telephone: (1)

Facsimile: (1)

Telex:

Email:

Commission(s): $28,30,34$

GIOVANNELLI FRANCO DR, IAS, CNR, CP 67, I 00044 FRASCATI, ITALY

Telephone: (39) 69425655

Facsimile: (39) 69416847

Telex: 610261 enrfrai

Email:

Commission(s): $29,42,51$

GIOVANNINI GABRIELE, IST DI RADIOASTRONOMIA, VIA GOBETTI 101, I 40129 BOLOGNA, ITALY

Telephonc: (39)

Facsimile: (39)

Telex:

Email: ggiovannini@astb01.bo.cnt.it

Commission(s): 40

GIR BE YOUNG, DPT OF EARTH SCIENCES, NTL TAIWAN NORMAL UNIV, TAIWAN, CHINA R

Telephonc: (886)

Facsimile: (886)

Telex:

Email:

Commission(s):

GIRAUD EDMOND, CENTRE PHYSIQUE THEORIQUE, CNRS, LUMINY CASE 07, F 13288 MARSEILLE CDX, FRANCE

Telephone: (33) 91269519

Facsimile: (33)

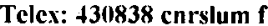

Email: carn::giraudaficptem51

Commission(s):

GIRIDHAR SUNETRA DR, INDIAN INSTITUTE OF, ASTROPHYSICS, KORAMANGALA, BANGALORE 560 034, INDIA

Telephone: (91) 803566585

Facsimile: (91)

Commission(s): 35

Telex: 845763 iiab in

Email:

GIURICIN GIULIANO, DPT DI ASTRONOMIA, UNIVERSITA DI TRIESTE, VIA TIEPOLO 11, I 34131 TRIESTE, ITALY

Telephone: (39) 40768005

Facsimile: (39)

Telex: 461137 oat $i$

Email:

Commission(s): $\mathbf{4 2 , 4 7}$

GLAGOLEVSKIJ JU V DR, SPECIAL ASTROPHYSICAL OBS, ACADEMY OF SCIENCES, NIZHNIJ ARKHYZ 357147 STAVROPOLSKIJ, RUSSIA

Telephone: (7) 93577

Facsimile: (7)

Telex:

Email:

Comimission(s): $14,27,29,45$

GLASER HAROLD DR, 1346 BONITA ST, BERKELEY CA 94709, USA

Telephone: (1) 4155271860 Facsimile: (1) Telex:

Email: $\quad$ Commission(s): 4

GLASNER SHIMON AMI, RACAH INST OF PHYSICS, HEBREW UNIV OF JERUSALEM, JERUSALEM 91904, ISRAEL

Telephone: (972) $258+521 \quad$ Facsimile: (972)

Email:

Commission(s):

Telex: 25391 huil

GLASPEY JOHN W DR, CANADA FRANCE HAWAII, TELESCOPE CORPORATION, BOX 1597

KAMUELA HI 96743, USA

Telephone: (1)

Facsimile: (1)

Telex:

Email: glaspey(a)uhefht.bitnet

Commission(s):

GLASS BILLY PRICE DR, DP'T OF GEOLOGY, UNIVERSITY OF DELAWARE, NEWARK DE 19716, USA

Telephone: (1) $3(1)+518458$

Facsimile: (1)

Telex:

Email:

Commission(s): 22

GLASS IAN STEWART DR, SAAO, BOX 9, OBSERVATORY 7935, SOUTH AFRICA

Telephone: (27) $21+70025$

Facsimile: (27)

Telex: 5720309 sa

Email: isgâasaao.ac.za

Commission(s): 09,25,28

GLASSGOLD ALFRED E PROF, DPT OF PHYSICS, NEW YORK UNIVERSITY, + WASHINGTON PLACE

NEW YORK NY 10001, USA

Telephone: (1) 2125982020

Facsimile: (1)

Email:

Commission(s):

Telex: 235128 nyu ur 
GLATZEL WOLFGANG DR, UNIVERSITAETS STERNWARTE, GOETTINGEN, GEISMARLANDSRE 11 D 37083 GOETTINGEN, GERMANY

Telephone: (49)

Facsimile: (49)

Commission(s):

Telex:

Email:

NATIONAL LAB, MS F665, ESS 5, LOS ALAMOS NM 87545, USA

GLATZMAIER GARY A, LOS ALAMOS NATIONAL LAB, MS F6rs, ESS S, LOS
Telephonc: (1) 505 6677647
Facsimile: (1)

Email:

Commission(s): $10,12,35$

GLEBOCKI ROBERT PROF, INST THEORETICAL PHYSICS, UNIVERSITY OF GDANSK, UL WITA STWOSZA 57 PL 80952 GDANSK, POLAND

Telephone: (48) 418700

Facsimile: (48)

Telex: 0512706 ifas

Email:

Commission(s): 36

GLEBOVA NINA I DR, INST OF THEORET ASTRONOMY, ACADEMY OF SCIENCES, N KUTUZOVA 10 191187 ST PETERSBURG, RUSSIA

Telephone: (7) 2788898

Facsimile: (7)

Telex: 121578 ita su

Email:

Commission(s): 04

GLEISSBERG WOLFGANG PROF, BUCHENWEG 12, D 6374 OBERURSEL, GERMANY

Telephone: (49)

Facsimile: (49)

Telex:

Email:

Commission(s): 10

GLENCROSS WILLIAM M DR, DPT PHYSICS \& ASTRONOMY, UNIVERSITY COLLEGE LONDON, GOWER ST L.ONDON WC1E 6BT, UK

Telephone: (4) 1713877050

Facsimile: (44)

Commission(s):

Telex: 28722 ucphys g

Email:

ATE ASTR INST, UNIVERSITETSKIJ PROSP 13, 119899 MOSCOW, RUSSIA

GLUSHNEVA I N DR, STERNBERG STATE ASTR INST, UNIVERSITETSKIJ PRO
Telephone: (7) 951392046
Felex:

Email:

Commission(s): 29

GNEDIN YURIJ N DR, IOFFE PHYSICAL TECH INST, ACADEMY OF SCIENCES, POLYTECHNICHESKAYA UL 26 194021 ST PETERSBURG, RUSSIA

$\begin{array}{ll}\text { Telephone: (7) } & \text { Facsimile: (7) } \\ \text { Email: } & \text { Commission(s): }\end{array}$

GODART ODON PROF, 9 AVE DES CERISERS, B 1470 BOUSVAL, BELGIUM

Telephone: (32) 10613817 Facsimile: (32) Telex:

Email:

Commission(s): $\$ 7$

GODFREY PETER DOUGLAS DR, DPT OF CHEMISTRY, MONASH UNIVERSITY, WELLINGTON RD

CLAYTON VIC 3168, AUSTRALIA

Telephone: (61) $35+10811$

Email:

Facsimile: (61)

Commission(s): 34

Telex: 32691 aa

GODOLI GIOVANNI PROF, DPT DI ASTRONOMIA, UNIVERSITA DI FIRENZE, LARGO E FERMI 5

I 50125 FIRENZE, ITALY

Telephone: (39) 5527521

Facsimile: (39) 55220039

Commission(s): $10,12,27,51$

Telex: 572268 arcetr $\mathrm{i}$

Email:

GODWIN JON GUNNAR DR, UNIVERSITY OF OXFORD, 85 CHERWELL DRIVE, OXFORD OX3 OND, UK

Telephone: (\$t) 1865722313 Facsimile: (4t) $1865721000 \quad$ Telex:

Email: uk.ac.rl.star::jg

Commission(s):

GOEBEL ERNST DR, INSTITUT FUER ASTRONOMIE, UNIVERSITAET WIEN, TUERKENSCHANZSTR 17

A 1180 WIEN, AUSTRIA

Telephone: (43) 1345360186

Facsimile: $(\$ 3)$

Commission(s): 50

Telex:

Email:

GOEBEL JOHN H DR, NASA AMES RESEARCH CTR, MS 244 7, SPACE SCIENCE DIV, MOFFETT FIELD CA 94035, US

Telephone: (1) $+1569+6525$

Facsimile: (1)

Telex:

Email:

Commission(s): 29,34 
GOEDBLOED JOHAN P PROF DR, INST VOOR PLASMAFYSICA, BOX 1207

NL $3+30$ BE NIEUWEGEIN, NETHERLANDS

Telephone: (31) 3+0231224 Facsimile: (31) 34 $0231204 \quad$ Telex:

Email: gocdblocd@sara.nl Commission(s): 10

GOELBASI ORHAN DR, FACULTY OF SCIENCE, INONU UNIVERSITY, 44069 MALATYA, TURKEY

Tetephone: (90) $82121871 \quad$ Facsimile: (90) $82118133 \quad$ Telex: 66140

Email:

Commission(s):

GOETZ WOLDEMAR DR, STERNWARTESTR 23A, POSTFACH 5527/28, D 96575 SONNEBERG, GERMANY

Telephone: (49) $96743793 \quad$ Facsimile: (49) Telex: 627180

Email:

Commission(s): 27

GOHARJI ADAN, SAUDI ARABIA

Telephone: (966)

Email:

Facsimile: (960) Telex:

Commission(s):

GOKDOGAN NUZHET PROF, UNIVERSITY OBSERVATORY, ISTANBUL UNIVERSITY, 34452 ISTANBUL, TURKEY

Telephone: (90) $15223597 \quad$ Facsimile: (90) Tclex:

Email:

Commission(s): 12,36

GOKHALE MORESHWAR HARI PR, INDIAN INSTITUTE OF, ASTROPHYSICS, KORAMANGALA

BANGALORE 560034 , INDIA

Telephone: (91) 803566585

Email:

Facsimile: (91)

Telex: 845763 iiab in

GOLAP KUMAR, FACULTY OF SCIENCE, UNIVERSITY OF MAURITIUS, REDUIT, MAURITIUS

Telephone: (230) $45+10+1$

Facsimile: (230) $+5+96+2 \quad$ Telex:

Email: kumarâf1.n726.z5.fidonct.org Commission(s):

GOLAY MARCEL PROF, OBSERVATOIRE DE GENEVE, CHEMIN DES MAILLETTES 51

CH 1290 SAUVERNY, SWITZERLAND

Telephone: (+1) 227552611

Facsimile: (+1) 227553983

Telex: $\$ 19209$ obs ch

Email: golayojobs.unige.ch

Commission(s): $25,37,45$

GOLD THOMAS PROF, CRSR, CORNELL UNIVERSITY, 306, SPACE SCIENCES BLDG, ITHACA NY 14853 6801, USA

Telephone: (1) 6072565284

Facsimile: (1)

Telex: $937+78$

Email:

Commission(s): $16,+40,44,47$

GOLDBACH CLAUDINE MME, INSTITUT D'ASTROPHYSIQUE, 98BIS BD ARAGO, F 75014 PARIS, FRANCE

Telephone: (33) $1+43281+1$

Facsimile: (33) 1 \& 328001

Telex:

Email:

Commission(s): 14

GOLDMAN ITZHAK DR, DPT OF PHYSICS \& ASTRON, TEL AVIV UNIVERSITY, RAMAT AVIV

TEL AVIV 69978, ISRAEL

Telephone: (972) 35450303

Email: goldman $\alpha$ uvm.tau.ac.il

Facsimile: (972)

Telex: 3+2171 rersy il

GOLDMAN MARTIN V, CASA, UNIVERSITY OF COLORADO, BOX 391, BOULDER CO 80309 (0391, USA

Telephone: (1) 3034928896

Facsimile: (1)

Telex:

Email:

Commission(s): 12,49

GOLDREICH P DR, CALTECH, PASADENA CA 91125, USA

Telephonc: (1) 2133566193

Facsimile: (1)

Telex:

Email: peteráciideimo

Commission(s): $07,16,33,34$

GOLDSMITH DONALD W DR, INTERSTELLAR MEDIA, 2153 RUSELL ST, BERKELEY CA 94705, USA

Telephonc: (1) +158481989

Facsimile: (1)

Telex:

Email:

Commission(s): $34,44,47,51$

GOLDSMITH PAUL F DR, LEADMINE HILL ROAD 5, AMHERST MA 01002, USA

Telephone: (1) $\$ 135450925 \quad$ Facsimile: (1)

Telex: 955491

Email:

Commission(s): 
GOLDSMITH S DR, DPT OF PHYSICS \& ASTRON, TEL AVIV UNIVERSITY, RAMAT AVIV, TEL AVIV 69978, ISRAEL Telephone: (972) 3420303 Facsimilc: (972)

Commission(s):

Telex: 342171 versy

Email:

ECH, MS 183 701, +800 OAK GROVE
Telex:

GOLDSTEIN RICHARD M DR, JPL CALTECH, MS 183 701,
Telephone: (1) $81835+6999$
$\begin{aligned} & \text { Facsimile: (1) } \\ & \text { Email: }\end{aligned}$

VE DR, PASADENA CA 91109, USA

GOLDSTEIN SAMUEL J PROF, UNIVERSITY STATION, UNIVERSITY OF VIRGINIA, BOX 3818

CHARLOTTESVILLE VA 22903, USA

Telephone: (1)

Facsimile: (1)

Telex:

Email: sjg(a)astsun.astro.virginia.edu

Commission(s): 34,40

GOLDSWORTHY FREDERICK A, DPT OF APPL MATHEMATICS, UNIVERSITY OF LEEDS, LEEDS LS2 9JT, UK

Telephone: (4t) 1132431751

Facsimile: $(4+)$

Telex:

Email:

Commission(s): 34

GOLDWIRE HENRY C JR, LAWRENCE LIVERMORE LAB, L \$51, BOX 808, LIVERMORE CA 94550, USA

Telephone: (1) $\$ 154230160$

Facsimile: (1)

Telex:

Email:

Commission(s): 40

GOLEV VALERY K DR, ASTRONOMICAL OBSERVATORY, BULGARIAN ACAD SCIENCES, BOX 36

BG 1504 SOFIA, BULGARIA

Telephone: (359) 27562324

Facsinile: (359)

Telex:

Email:

Commission(s):

GOLLNOW H DR, MOUNT STROMLO OBSERVATORY, PRIVATE BAG, WODEN PO, CANBERRA ACT 2606, AUSTRALIA

Telephone: (61) 6288111

Facsimile: (61)

Email:

Commission(s):

Telex:

GOLOVATYJ VOLODYMYR, ASTRONOMICAL OBSERVATORY, LVIV STATE UNIVERSITY

KJRIL \& MEFODIY STR 8, 290005 LVIV, UKRAINE

$\begin{array}{lll}\text { Telephone: (7) } 322729088 & \text { Facsimile: (7) } & \text { Telex: } \\ \text { Email: gol } \hat{a}_{\text {astro.lviv.ual }} & \text { Commission(s): } 34\end{array}$

GOLUB LEON DR, CENTER FOR ASTROPHYSICS, HCO/SAO, 60 GARDEN ST, CAMBRIDGE MA 02138, USA

Telephone: (1) $617+957177 \quad$ Facsimile: (1)

Telex:

Email:

Commission(s):

GOMBERT REGIS, LES OLIVETS, F 84400 APT, FRANCE

Telephonc: (33) $900+662+$

Facsimilc: (33)

Telex:

Email:

Commission(s):

GOMES ALERCIO M DR, R GAVIAO PEIXOTO 13, AP 1401, ICARAI 2400), NITEROJ ERJ, BRAZIL

Telephone: (55)

Facsimile: (55)

Telex:

Email:

Commission(s):

GOMES RODNEY D S DR, DPT DE ASTRONOMIAY, OBSERVATORIO NACIONAL, RUA GENERAL JOSE CRIS 77 20921 t010 RIO DE JANEIRO, BRAZIL

$\begin{array}{lll}\text { Telephone: (55) } 215892955 & \text { Facsimilc: (55) } & \text { Telex: } \\ \text { Email: rodncy(a)on.br } & \text { Commission(s): } 07 & \end{array}$

GOMEZ ANA E DR, OBSERVATOIRE DE PARIS, SECTION DE MEUDON, DAGAL F 92195 MEUDON PPL CDX, FRANCE

$\begin{array}{ll}\text { Telephonc: (33) } 1+50778+3 & \text { Facsimilc: (33) } \\ \text { Email: } & \text { Commission(s): } 33\end{array}$

Telex: 201571

GOMEZ GONZALEZ JESUS DR, PASEO IMPERIAL, 29 6H, E MADRID 5, SPAIN

Telephone: (34) Facsimile: (34) Telex:

Email:

Commission(s): 34,40C

GOMEZ MARIA THERESA DR, OSS ASTRONOMICO, 180131 NAPOLI, ITALY

Telephone: (39)

Facsimile: (39)

Telex:

Email:

Commission(s): 12 
M 558

GOMEZ MERCEDES DR, OBSERVATORIO ASTRONOMICO, LAPRIDA 854, 5000 CORDOBA, ARGENTINA

Telephone: (54) 51236876

Facsimile: (54) 51210613

Telex:

Email: mercedes $\boldsymbol{Q}$ uncbob.edu.ar

Commission(s):

GOMIDE FERNANDO DE MELLO, R ANGELICA LOPES DE, CASTRO 71, 25655430 PETROPOLIS RJ, BRAZIL

Telephone: (55)

Facsimilc: (55)

Telex:

Email:

Commission(s):

GONCZI GEORGES, OCA OBSERV DE NICE, BP 229, F 0630 4 NICE CDX 4 , FRANCE

Telephone: (33) $93890+20$

Facsimile: (33) 92003033

Telex: $46000+$ obsnice $f$

Email:

Commission(s):

GONDHALEKAR PRABHAKAR DR, RUTHERFORD APPLETON LAB, SPACE \& ASTROPHYSICS DIV, BLDG R25/R68 CHILTON DIDCOT OXI1 OQX, UK

Telephone: (4) $123521900 \quad$ Facsimile: (4)

Email:

Commission(s): $4+$

Telex: 83159

GONDOLATSCH FRIEDRICH PRF, ASTRON RECHEN INSTITUTE, MOENCHHOFSTR 12-14 D 69120 HEIDELBERG, GERMANY

Telephone: $(\$ 9) 622149026 \quad$ Facsimile: (\$9) Telex:

Email: $\quad$ Commission(s): 04

GONG SHOU-SHEN, SHANGHAI OBSERVATORY, CAS, 80 NANDAN RD, SHANGHAI 200030, CHINA PR

Telephone: (86) $21386191 \quad$ Facsimile: (86) Telex: 33164 shao cn

Email:

Commission(s): 09

GONG SHU-MO, PURPLE MOUNTAIN OBSERV, CAS, NANJING, CHINA PR

Telephone: (86) $25+6700 \quad$ Facsimile: (86) 25301459 Telex: $341+4$ pmonj cn

Email: $\quad$ Commission(s): 35,47

GONZALES-A WALTER D DR, INPE, CP 515,12200 S JOSE DOS CAMPOS, BRAZIL

Telephonc: (55) $123229977 \quad$ Facsimile: (55) $123218743 \quad$ Telex: 011-33530 inpe br

Email:

Commission(s): 44

GONZALEZ CAMACHO ANTONIO, INST DE ASTRON \& GEODESIA, FAC DE CIENCIAS MATEMAT UNIVERSIDAD COMPLUTENSE, E 28041) MADRID, SPAIN

Telephone: (34) $124+2501 \quad$ Facsimile: (34) Telex:

Email: $\quad$ Commission(s): 07

GONZALEZ G, INAOE, TONANTZINTLAZ, APDO POSTAL $216 \mathrm{y} 51,72000$ PUEBLA PUE, MEXICO

Telephone: (52) 9122470500 Facsimile: (52) Telex:

Email: $\quad$ Commission(s):

GONZALEZ J JESUS, INSTITUTO DE ASTRONOMIA, UNAM, AP 79 264, MEXICO DF 04510, MEXICO

Telephone: (52) $56223906 \quad$ Facsimile: (52) 56160653 Telex: 01760155 cicme

Email: jesus $\widehat{a}$ astroscu.unam.mx Commission(s):

GONZALEZ SERRANO J I DR, DPT DE FISICA MODERNA, UNIVERSIDAD DE CANTABRIA, AVD LOS CASTROS S/N E 39005 SANTANDER, SPAIN

Telephone: $(34)+2201450 \quad$ Facsimile: $(34)+2201402 \quad$ Telex: 35861 educi c

Email: gserranoaccuevx.unican.es Commission(s): 28

GONZALEZ-RIESTRA R DR, ESA IUE OBSERVATORY, VILSPA, APD 50727, E 28080 MADRID, SPAIN

Telephone: (34) $18131100 \quad$ Facsimile: (34) Telex: $\mathbf{2 5 5 5}$ vils e

Email: carn:iuchot(āddacsa 10 Commission(s):

GONZE ROGER F J IR, OBSERVATOIRE ROYAL DE, BELGIQUE, AVE CIRCULAIRE 3, B 1180 BRUSSELS, BELGIUM Telephone: (32) 23752484

Email: rogerg@astro.ome.be Facsimile: (32) 23749822

Telex: 21565

GOODE PHILIP R, DPT OF PHYSICS, NJ INST OF TECHNOLOGY, 323 HIGH ST, NEWARK NJ 07102, USA

Telephone: (1) 2015963562

Facsimile: (1)

Telex:

Email:

Commission(s): 
GOODRICH ROBERT W DR, CALTECH, MS 105 24, PASADENA CA 91125, USA

Telephone: (1) 8183563693 Facsimile: (1) Telex:

Email: rwg@deimos.caltech.cdu Commission(s): 28

GOODY R M, CEPP, PIERCE HALL, 29 OXFORD ST, CAMBRIDGE MA 02138, USA

Telephone: (1) $617495+517 \quad$ Facsimile: (1) Teicx:

Email:

Commission(s): 16

GOOSSENS MARCEL PROF, CTR PLASMA ASTROPHYSICS, KV LEUVEN, CELESTIJNENLAAN 200B

B 3001 HEVERLEE, BELGIUM

Telephonc: (32) 16200656

Facsimile: (32)

Telex:

Email:

Commission(s): 10

GOPALA RAO U V MR, SATELLITE METEOROLOGY, INDIAN METEOROLOGIC DPT, LODI RD/MAUSAM BHAVAN NEW DELHI 110 003, INDIA

Telephone: (91)

Facsimile: (91)

Telex:

Email:

Commission(s):

GOPALSWAMY N DR, ASTRONOMY PROGRAM, UNIVERSITY OF MARYLAND, COLLEGE PARK MD 20742, USA

Telephone: (1) $301+5+6649$

Facsimile: (1)

Telex: 62891478

Email:

Commission(s): 12,40

GOPASYUK S I DR, CRIMEAN ASTROPHYS OBS, UKRAINIAN ACAD OF SCIENCE, NAUCHNY

$33+413$ CRIMEA, UKRAINE

Telephone: (7) 432945

Facsimile: (7)

Telex:

Email:

Commission(s): 10,12

GORBATSKY VITALIJ G PROF, ASTRONOMICAL OBSERVATORY, ST PETERSBURG UNIVERSITY

BIBLIOTECHNAJA PL 2, 198904 ST PETERSBURG, RUSSIA

Telephone: (7) $812+287129$

Facsimile: (7)

Telex:

Email: vayak(a)astro.Jgu.spl).su

Commission(s): 27

GORDON CHARLOTTE PROF, 11 RUE TOURNEFORT, F 75015 PARIS, FRANCE

Telcphone: (33) Facsimile: (33) Telex:

Email: $\quad$ Commission(s): 12,36

GORDON COURTNEY P PROF, DPT OF ASTRONOMY, HAMPSHIRE COLLEGE, AMHERST MA 01002, USA

Telephone: (1)

Facsimile: (1)

Telex:

Email:

Commission(s): 34

GORDON ISAAC M DR, INST RADIOPHYS \& ELECTRON, UKRAINIAN ACAD OF SCIENCE

310085 KHARKOV, UKRAINE

Telephone: (7)

Facsimile: (7)

Telex:

Email:

Commission(s):

GORDON KURTISS J PROF, DPT OF ASTRONOMY, HAMPSHIRE COLLEGE, AMHERST MA 01002, USA

Telephonc: (1) $+13549+600$

Facsimile: (1)

Telex:

Email:

Commission(s):

GORDON MARK A DR, NRAO, CAMPUS BLDG 65, 949 N CHERRY AVE, TUCSON AZ 857210655 , USA

Telephonc: (1) 5208828250

Facsimile: (1)

Telex:

Email:

Commission(s): $33,34,4$ !

GORENSTEIN MARC V, CENTER FOR ASTROPHYSICS, MS 42, 60 GARDEN ST, CAMBRIDGE MA 02138, USA

Telephone: (1) $617+959296$

Facsimile: (1)

Telex:

Email:

Commission(s)

GORENSTEIN PAUL DR, CENTER FOR ASTROPHYSICS, HCO/SAO, 60 GARDEN ST, CAMBRIDGE MA 02138, USA

Telephone: (1) $617+957250$

Facsimile: (1)

Telex: $921+28$ satellite cam

Email:

Commission(s): 16

GORET PHILIPPE DR, CEA CEN, DAPNIA/SAP, BP 2, F 91191 GIF/YVETTE CDX, FRANCE

Telephone: (33) $16908+463$

Facsimile: (33)

Email:

Commission(s): 47

Telex: 690860 
GORGAS GARCIA JAVIER DR, DPT DE ASTROFISICA, FAC DE FISICA, UNIVERSIDAD COMPLUTENSE

E 28040 MADRID, SPAIN

Telephone: (34) 15495316

Email: carn/bitnet:w062aacemducm 11

Facsimile: (34)

Telex: $\mathbf{4 7 2 7 3}$

GORGOLEWSKI STANISLAW PR, INST OF RADIO ASTRONOMY, N COPERNICUS UNIVERSITY, UL CHOPINA 12/18 PL 87100 TORUN, POLAND

Telephone: (48) 20651 TORUN

Email:

Facsimile: $(48)$
Commission(s): 40

Telex: $155232+$ trano p)

GOSACHINSKIJ I V DR, PULKOVO OBSERVATORY, ACADEMY OF SCIENCES, IO KUTUZOV QUAY 196140 ST PETERSBURG, RUSSIA

Telephone: (7) 2979452

Email:

Facsimile: (7)

Telex: 321262

Commisxion(s): $34,40 \mathrm{C}$

GOSLING JOHN T DR, LOS ALAMOS NATIONAL LAB, MS D438, ESS 8, LOS ALAMOS NM 87545, USA

Telephone: (1) 51156675389

Facsimile: (1)

Telex: 660495

Email:

Commission(s): 49

GOSS W MILLER PROF, NRAO, VLA, BOX 0, SOCORRO NM 87801 0387, USA

Telephone: (1) 5057724011

Facsimile: (1)

Telex: 910-988-1710

Email:

Commission(s): $28,34,40$

GOSSET ERIC DR, INSTITUT D'ASTROPHYSIQUE, UNIVERSITE DE LIEGE, AVE COINTE 5 B 4000 COINTE-LIEGE, BELGIUM

Telephone: (32) +1529980

Email: u2t+legubliulg11

Facsimile: (32) 41527474

Telex: $+126+$ astrilg

Commission(s): 47

GOSWAMI J N DR, PHYSICAL RESEARCH LAB, NAVRANGPURA, AHMEDABAD 380 O09, INDIA

Telephone: (91) $272462129 \quad$ Facsimile: (91) $2724+5292$ Telex: 0121397 prl in

Eminil:

Commission(s): 22

GOTT III J RICHARD, DPT ASTROPIIYSICAL SCI, PRINCETON UNIVERSITY, PRINCETON NJ 085H 1001, USA Telephone: (1) 609 4523813 Facsimile: (1)

Telex:

Email:

Commission(s): 51

GOTTESMAN STEPHEN T DR, DPT OF ASTRONONIY, UNIVERSITY OF FLORIDA, 211 SSRB

GAINESVILLE FL 32611, USA

Telephonc: (1) $90+3922050 / 2052$

Email:

Ficsimile: (1)

Telex: 8108252308

GOTTLIEB CARL. A DR, INST FOR SPACE STUDIES, 2880 BROADWAY, NEW YORK NY 10025, USA

Telephone: (1) 2126785566,

Facsimile: $\{1)$

Telex:

Emati:

Commission(s):

GOTTLIEB KURT, $1+3$ STANLEY TERIRACE, TARINGA GLD +116, AUSTRALIA

Telephonc: (61) Facsimile: (61) Telex:

Em:til:

Commission(s):

GOTTLOEBER STEFAN, ASTROPIYSIKALISCHES INST, POTSBAM UNIVERSITY, AN DER STERNWARTE 16 D $1+482$ POTSDAM, GERMANY

Telephone: (49) 331749 91 $\quad$ Fatesimile: (49) 331749 9201 $\quad$ Telex: 361231 stggh d

Email: sgottlochera $a$ aip.de

Commission(s): 47

GOUDA NAOTERU DR, DPT EARTII SPACE SCIENCES, FAC OF SCIENCE, OSAKA UNIVERSITY

TOYONAKA OSAKA SG, JAPAN

Telephonc: (81) $684+1151 * 4117$

Email: gouda a oskatstro.kek.ate.jp

Facsimile: (81) $68531787 \quad$ Telex:

Commissiun(s): 47

GOUDAS CONSTANTINE L PROF, DPT OF MATHEMATICS, UNIVERSITY OF PATRAS, GR 261 10 RION, GREECE

Telephonc: (311) 6199 1889

Facsimile: (30)

Commission(s): 117,16

Telex: 312239 efap gr

Email:

GOUDIS CHRISTOS D PROF, DPT OF PHYSICS, UNIVERSITY OF PATRAS, GR 26110 PATRAS, GREECE

Telephone: (30)

Facsimile: (30)

Telex:

Email:

Commission(s): 51 
GOUGH DOUGLAS O DR, INSTITUTE OF ASTRONOMY, THE OBSERVATORIES, MADINGLEY RD

CAMBRIDGE CB3 OHA, UK

Telephonc: (4t) $12233375+8$

Email:

Facsimile: (4) 1223337523

Commission(s): 27,35,36

Telex: 817297

GOUGUENHEIM LUCIENNE, OBSERVATOIRE DE PARIS, SECTION DE MEUDON, RADIOASTRONOMIE F 92195 MEUDON PPL CDX, FRANCE

Telephone: (33) $1+5077604$

Email: gouguenhcinäfrmeu51

Facsimile: (33) $1+5077939$ Telex:

Commission(s): $28,30,46 \mathrm{C}$

GOULD ROBERT J PROF, DPT OF PHYSICS, UCSD, B 01, LA JOLLA CA 92093 (1216, USA

Telephone: (1) $619+523649$ Facsimile: (1) Telex:

Email:

Commission(s):

GOUPIL MARIE JOSE, OBSERVATOIRE DE PARIS, SECTION DE MEUDON, DASGAL

F 92195 MEUDON PPL CDX, FRANCE

Telephone: (33) $1+5077880$

Facsimile: (33)

Telex: 270912

Email: carn:goupilaf frmcusi

Commission(s): 27,35

GOUTTEBROZE PIERRE DR, IAS, BAT 121, UNIVERSITE PARIS XI, F $91+05$ ORSAY CEDEX, FRANCE

Teicphone: (33) 169858621

Facsinile: (33) 1 69 858675

Telex: 6001252

Email: iaslab::gouttebrome

Commission(s):

GOWER ANN C DR, DPT OF PHYSICS, UNIVERSITY OF VICTORIA, BOX 1700, VICTORIA BC V8W 2Y2, CANADA

Telephone: (1) 6047217700

Email:

Ficsimile: (1) 611 7217715

Telex:

Commission(s):

GOWER J F R DR, 1615 MCTAVISII ROAD RR 2, SIDNEY BC VRL 3SI, CANADA

Telephone: (1) $6046565+57$

Fatesimite: (1)

Telex:

Email:

Commission(s): 41

GOY GERALD PROF, OBSERVATOIRE DE GENEVE, CHEMIN DES MAILLETTES 51

CH 1290 SAUVERNY, SWITZERLAND

Telephone: (+1) 227552611

Email: goviagobs.unige.ch

Facsimile: (41) 227553983

Telex: 4192119 obs ch

Commission(s): 25

GOYAL A N DR, DPT OF MATHENATICS, UNIVERSITY OF RAJASTHAN, JAIPUR 3112 004, INDIA

Telephone: (91) $7+060$

Email:

Ficsimile: (1)

Commission(s): 24

Telex:

GRABOSKE HAROLD C JR, LAWRENCE LIVERNIORE LAB, L 23, BOX 808, LIVERMORE CA 94550, USA

Telephone: (1) $+15+227262$

Fatesimile: (1)

Telex:

Emiail:

Comminsion(s):

GRABOWSKI BOLESLAW DR, INSTITUTE OF PHYSICS, PEDAGOGICAL UNIVERSITY, UL OLESKA 48 PL 45 951 OPOLE, POLAND

Telephone: (48) $358+1 \quad$ Facsimile: $(48)$

Email:

Commission(s):

Telex: 10732230 wsp pl

GRACHEV STANISLAV, ASTRONONIICAL INSTITUTE, ST PETERSBURG UNIVERSITY, BYBLYOTECHNAYA PL 2 $198^{\circ} 0+$ ST PETERSBURG, RUSSIA

Telephone: (7) $812+28+163$

Email: vayalia astro.Igu.spl).su

Facsimile: (7) $812+2866+9 \quad$ Telex:

Commission(s):

GRADIE JONATHAN CAREY, INSTITUTE OF GEOPHYSICS, UNIVERSITY OF HAWAII

DIV PLANETARY GEOSCIENCES, HONOLULU HI 96822, USA

Telcplonc: (1) $8089566+88$

Facsimile: (1) 8089882790

Telex:

Email:

Commission(s): 15

GRADSZTAJN ELI DR, DPT OF PHYSICS \& ASTRON, TEL AVIV UNIVERSITY, RAMAT AVIV

TEL AVIV 69978, ISRAEL

Telephone: (972)

Facsimile: (972)

Email:

Commission(s):

Telex: 
GRADY CAROL ANNE DR, DPT OF PHYSICS, THE CATHOLIC UNIVERSITY, OF AMERICA, WASHINGTON DC 20064 USA

Telephone: (1) 2023195315

Email: iue::grady on span
Facsimile: (1)

Commission(s): 29
Telex:

GRAHAM DAVID A, MPI FUER RADIOASTRONOMIE, AUF DEM HUEGEL 69, D 53121 BONN, GERMANY

Telephone: (49) 228525282

Email:

Telex: $886+40$

Commission(s): 34,40

GRAHAM ERIC DR, DIRECT ALGORITHMS, BOX 2302, TUBAC AZ 856 66 , USA

Telephone: (1) Facsimile: (1) Telex:

Email:

Commission(s): 35

GRAHAM JOHN A DR, DPT TERRESTR MAGNETISM, CARNEGIE INST WASHINGTON, 5241 BROAD BRANCH RD NW, WASHINGTON DC 20015, USA

Telcphonc: (1) 2029660863

Facsimile: (1)

Email:

Commission(s): $25,27,28$

Telex: $\$+0+27$ magn ui

GRAHL BERND H DR, MPI FUER RADIOASTRONOMIE, AUF DEM HUEGEL 69, D 53121 BONN, GERMANY

Telephone: (49) $22573112 \quad$ Facsimile: (49)

Email:

Commission(s):

Telex: $8864+10$

GRAINGER JOHN F DR, DPT OF PHYSICS, UMIST, BOX 88, MANCHESTER MGO 1QD, UK

Telephone: (4) $1612363311 \quad$ Facsimile: (44)

Telex: 666094

Email:

Commission(s):

GRAMANN MIRT, TARTU ASTROPHYSICAL OBS, EE 24+4 TORAVERE, ESTONIA

Telephone: (372) $3410+50$

Facsimilc: (372) $3+10205$

Telex:

Email:

Commission(s):

GRANDI STEVEN ALDRIDGE DR, NOAO, BOX 26732,950 N CHERRY AVE, TUCSON AZ 85726 6732, USA

Telephone: (1) 5203275511

Facsimile: (1)

Telex:

Email:

Commission(s): 28

GRANDPIERRE ATTILA DR, KONKOLY OBSERVATORY, THEGE U 13/17, BOX 67, H 1525 BUDAPEST, HUNGARY

Telephone: (36) 1175 5866/175 $\$ 122$

Facsimile: (36) 11569640

Telex: 277 460 konob $h$

Email:

Commission(s): 10

GRANT IAN P DR, PEMBROKE COLLEGE, OXFORD OX1 1DW, UK

Telephone: (44) 1865242271

Facsimile: (4t)

Commission(s): 14,36

Telex:

Email:

GRANVEAUD MICHEL, OBSERVATOIRE DE PARIS, 61 AVE OBSERVATOIRE, LPTF, F 7501+ PARIS, FRANCE

Telephone: (33) 143201210

Facsimile: (33)

Commission(s): 31C

Telex: 270776 obs $f$

Email:

GRASDALEN GARY L DR, DPT PHYSICS \& ASTRONOMY, UNIVERSITY OF WYOMING, BOX 3905 UN STA, LARAMI WY 82071, USA

Telephone: (1) $307766+385$

Facsimilc: (1)

Telex:

Email:

Commission(s): $27,28,34$

GRATTON R G DR, OAR, VIA DELL'OSSERVATORIO, I (001040 MONTEPORZIO, ITALY

Telephonc: (39) $694+9019$

Facsimile: (39)

Commission(s): 29,37

Telex:

Email: span:17+68::raffacle

GRAUER ALBERT D, DPT PHYSICS \& ASTRONOMY, UALR, 33RD \& UNIVERSITY, LITTLE ROCK AR 72204, USA

Telephone: (1) 5015693275

Facsimile: (1)

Telex:

Email:

Conımission(s): 25

GRAY DAVID F PROF, DPT OF ASTRONOMY, UNIV OF WESTERN ONTARIO, LONDON ON N6A 3K7, CANADA

Telephone: (1) $5196792111 * 6715$

Facsimile: (1) 5196612009

Telex:

Email: Jfgray@uwo.ca

Conmmission(s): 29C,36

GRAY NORMAN, DPT PHYSICS \& ASTRONOMY, UNIVERSITY OF GLASGOW, GLASGOW G12 8QQ, UK

Telephone: (44) $1413398855 * \$ 153$

Facsimile: (4t) $1+133+9029$

Email: norman(a)astro.gla.ac.uk

Commission(s): 10

Telex: 777 (1770 unigla 
GRADY CAROL ANNE DR, DPT OF PHYSICS, THE CATHOLIC UNIVERSITY, OF AMERICA WASHINGTON DC 20064, USA

Telephone: (1) 2023195315

Email: iue::grady on span

Facsimile: (1)

Commission(s): 29

Telex:

GRAHAM DAVID A, MPI FUER RADIOASTRONOMIE, AUF DEM HUEGEL 69, D 53121 BONN, GERMANY

Telephone: (49) 228525282

Facsimile: (49)

Telex: $886+40$

Email:

Commission(s): $34,+10$

GRAHAM ERIC DR, DIRECT ALGORITHMS, BOX 2302, TUBAC AZ 85646, USA

Telephone: (1)

Facsimile: (1)

Commission(s): 35

Telex:

Email:

GRAHAM JOHN A DR, DPT TERRESTR MAGNETISM, CARNEGIE INST WASHINGTON 52+1 BROAD BRANCH RD NW, WASHINGTON DC 20015, USA

$\begin{array}{ll}\text { Telephone: (1) } 2029660863 & \text { Facsimile: (1) } \\ \text { Email: } & \text { Commission(s): 25,27,28 }\end{array}$

Telex: 440427 magn ui

GRAHL BERND H DR, MPI FUER RADIOASTRONOMIE, AUF DEM HUEGEL 69, D 53121 BONN, GERMANY

Telephone: (49) 22573112

Facsimile: (49)

Telex: $8864+0$

Email:

Commission(s):

GRAINGER JOHN F DR, DPT OF PHYSICS, UMIST, BOX 88, MANCHESTER MG0 1QD, UK

Telephone: (4t) $1612363311 \quad$ Facsimile: $(4 t) \quad$ Telex: 666094

Email:

Commission(s):

GRAMANN MIRT, TARTU ASTROPHYSICAL OBS, EE 2 $4+$ TORAVERE, ESTONIA

Telephonc: (372) $3+10+50$

Facsimile: (372) $3+10205 \quad$ Telex:

Email:

Commission(s):

GRANDI STEVEN ALDRIDGE DR, NOAO, BOX 26732, 950 N CHERRY AVE, TUCSON AZ 85726 6732, USA

Telephone: (1) 5203275511

Facsimile: (1)

Telex:

Email:

Commission(s): 28

GRANDPIERRE ATTILA DR, KONKOLY OBSERVATORY, THEGE U 13/17, BOX 67, H 1525 BUDAPEST, HUNGARY

Telephonc: (36) $11755866 / 175+122$

Facsimile: (36) 11569640

Telex: 277460 konob h

Email:

Commission(s): 10

GRANT IAN P DR, PEMBROKE COLLEGE, OXFORD OX1 1DW, UK

Telcphone: (\$4) $18652+2271$

Facsimile: $(4+)$

Commission(s): 14,36

Telcx:

Email:

GRANVEAUD MICHEL, OBSERVATOIRE DE PARIS, 61 AVE OBSERVATOIRE, LPTF, F 75014 PARIS, FRANCE
Telephonc: (33) 143201210
Facsimile: (33)

Email:

Commission(s): 31C

GRASDALEN GARY L DR, DPT PHYSICS \& ASTRONOMY, UNIVERSITY OF WYOMING, BOX 3905 UN STA

LARAMIE WY 82071, USA

Telephone: (1) $307766+385$

Email:

Facsimile: (1)

Tolex:

Commission(s): $27,28,34$

GRATTON R G DR, OAR, VIA DELL'OSSERVATORIO, I O0040 MONTEPORZIO, ITALY

Telephone: (39) 69449019

Email: span:17468::raffacle

Facsimile: (34)

Telex:

Commission(s): 29,37

GRAUER ALBERT D, DPT PHYSICS \& ASTRONOMY, UALR, 33RD \& UNIVERSITY, LITTLE ROCK AR 72204, USA

Telephone: (1) 5015693275

Facsimile: (1)

Commission(s): 25

Telex:

Email:

Commission(s): 25

GRAY DAVID F PROF, DPT OF ASTRONOMY, UNIV OF WESTERN ONTARIO, LONDON ON N6A 3K7, CANADA

Telephone: (1) 5196792111 *6715

Facsimile: (1) 5196612009

Telex:

Email: dfgrayiauwo.ca

Commission(s): 29C,36

GRAY NORMAN, DPT PHYSICS \& ASTRONOMY, UNIVERSITY OF GLASGOW, GLASGOW G12 8QQ, UK

Telephone: $(\downarrow+) 1+13398855 *+153$

Email: normancajastro.gla.ac.uk

Facsimile: (4t) $1+13349029$

Commission(s): 10

Telex: 7770770 unigla 
GRAY PETER MURRAY, AAO, BOX 296, EPPING NSW 2121, AUSTRALIA

Telephone: (61) 28681666

Facsimile: (61) 28768536

Telex: 23999 aaosyd aa

Email:

Commission(s): 09

GRAY RICHARD O, DPT PHYSICS \& ASTRONOMY, APPALACHIAN STATE UNIV, BOONE NC 28608, USA

Telephone: (1) 7042622430

Facsimile: (1) $70+2622049 \quad$ Telex:

Email: grayro@conrad.appstatc.cdu

Commission(s): $45 \mathrm{C}$

GRAYZECK EDWIN J DR, DPT OF PHYSICS, UNIVERSITY OF NEVADA, 4505 S MARYLAND PARKWAY

LAS VEGAS NV 8915 4 , USA

Telephone: (1) 7027393507

Facsimile: (1)

Telex:

Email:

Commission(s): 33

GREBENIKOV E A PROF DR, LOMONOSOV STATE UNIVER, 11723ł MOSCOW, RUSSIA

Telephone: $(7)$

Facsimile: (7) Telex:

Email:

Commission(s): (07

GREC GERARD, DPT ASTROPHYSIQUE, UNIVERSITE DE NICE, PARC VALROSE, F OGO3† NICE CDX, FRANCE

Telephone: (33) 93519100

Facsimile: (33)

Telex:

Email:

Commission(s):

GREEN DAVID DR, MULLARD RADIO ASTRON OBS, CAVENDISH LABORATORY, MADINGLEY RD

CAMBRIDGE CB3 OHE, UK

Telephone: (44) 1223337310

Facsimile: (\$4) $122335+599 \quad$ Telex:

Email: d.a.green(a)mrao.cam.ac.ul

Commission(s): 05,41

GREEN ELIZABETH M DR, MOUNT STROMLO \& SIDING, SPRING OBSERVATORIES, PRIVATE BAG

WODEN PO ACT 2606, AUSTRALIA

Telephone: (61) 62881111

Telex: 62270 ala

Email:

Commission(s): 37

GREEN JACK PROF, DPT OF GEOLOGY, CALIF STATE UNIVERSITY, LONG BEACH CA 90840, USA

Telephone: (1) $213+98+809$

Facsimilc: (1)

Telex:

Email:

Commission(s): 16

GREEN LOUIS C PROF, HAVERFORD COLLEGE, 7901 COLLEGE AVENUE, HAVERFORD PA 19041, USA

Telephone: (1) 2156490265

Facsimile: (1)

Telex:

Email:

Commission(s): 14

GREEN RICHARD F DR, KITT PEAK NTL OBS, BOX 26732, 950 N CHERRY AVE, TUCSON AZ 85726 6732, USA

Telephone: (1) $5203259299 \quad$ Facsimile: (1)

Email:

Commission(s):

Telex: $0666+84$ aura noan

GREEN ROBIN M DR, DPT OF ASTRONOMY, UNIVERSITY OF GLASGOW, GLASGOW G12 8QQ, UK

Telephone: (4) $1413398855 \quad$ Facsimile: (4t)

Telex: 778421

Email:

Commission(s):

GREEN SIMON F, PHYSICS LABORATORY, UNIVERSITY OF KENT, CANTERBURY CT2 7NR, UK

Telephone: (44) $1227764000 * 3780 \quad$ Facsimile: (4t) $1227762616 \quad$ Telex: $9654+4$

Email:

Commission(s): 15

GREENBERG J MAYO DR, STERREWACHT, HUYGENS LAB, BOX 9513, NL 2300 RA LEIDEN, NETHERLANDS

Telephone: (31) 71275700

Facsimile: (31)

Commission(s): $15,21,3+, 51$

Telex: 39058 astro $\mathrm{nl}$

Email:

GREENBERG RICHARD DR, LUNAR \& PLANETARY LAB, UNIVERSITY OF ARIZONA, TUCSON AZ 85721, USA

Telephone: (1) 5206216940

Facsimile: (1)

Telex:

Email:

Commission(s): $07,15,20$

GREENHILL JOHN DR, DPT OF PHYSICS, UNIVERSITY OF TASMANIA, GPO BOX 252C

HOBART TAS 7001, AUSTRALIA

Telephonc: (61) $2202+29$

Facsimile: (61) $2202+10$

Telex: at 58150

Email: grnhil@physvax.phys.utas.edu.a

Commission(s): 4 
GREENHILL LINCOLN J, CFA, MS 42, 60 GARDEN ST, CAMBRIDGE MA 02138, USA

Telephone: (1) 6174957194

Facsimile: (1) $6174957345 \quad$ Telex:

Email: greenhill@efa.harvard.edu

Commission(s):

GREENHOUSE MATTHEW A, NTL AIR \& SPACE MUSEUM, MRC 321, WASHINGTON DC 20560, USA

Telephone: (1) 2023571319

Facsimile: (1) 2026338174 Telex:

Email: matt@nasm.cdu

Commission(s):

GREENSTEIN GEORGE PROF, DPT OF ASTRONOMY, AMHERST COLLEGE, AMHERST MA 01002, USA

Telephone: (1) 4135422075

Facsimile: (1)

Telex:

Email:

Commission(s):

GREENSTEIN J L PROF, CALTECH, MS 105 24, PALOMAR OBS, PASADENA CA 91125, USA

Telephone: (1) 8183564006

Facsimile: (1)

Telex:

Email:

Commission(s): $29,36,51$

GREGG MICHAEL DAVID DR, LAWRENCE LIVERMORE NTL LB, BOX 808 L 413, LIVERMORE CA 94551 9900, USA

Telephone: (1)

Facsimile: (1)

Telex:

Email: gregg@fucgo.llni.gov

Commission(s): 28

GREGGIO LAURA DR, DPT DI ASTRONOMIA, UNIVERSITA DI BOLOGNO, CP 596, I 40125 BOLOGNA, ITALY

Telephone: (39) 51259413

Facsimile: (39)

Commission(s): 35

Telex: 520634 infnbo 1

Email: span/decnet:37928::laura

OASTRONOMIA, CNR, VIA IRNERIO 46 , I 40126 BOLOGNA, ITALY

$\begin{array}{lll}\text { GREGORINI LORETTA, IST DI RADIOASTRONOM, } \\ \text { Telephone: (39) } 51232856 & \text { Facsimile: (39) } & \text { Telex: } 21166+\text { infn boi }\end{array}$

Email:

Conımission(s): 40

GREGORIO-HETEM JANE, IAG, UNIVERSIDADE DE SAO PAULO, AV MIGUEL STEFANO 4200

$0430190+$ SAO PAULO, BRAZIL

Telephone: (55) $115778599 * 235$

Facsimile: (55) 112763848

Commission(s): 34,4 (1)

Telex: 1156735 iagm br

Email: janc(a)vax.iagusp.usp.br

GREGORY PHILIP C DR, DPT OF PHYSICS, UNIV OF BRITISH COLUMBIA, 224 AGRICULTURAL RD VANCOUVER BC VGT 1W5, CANADA

Telephone: (1) $60+2286+17$

Facsimile: (1) $60+228532 \downarrow$

Telex: 04508576

Email:

Commission(s): 40,51

GREGORY STEPHEN ALBERT DR, DPT PHYSICS \& ASTRONOMY, BOWLING GREEN STATE UNIV

BOWLING GREEN OH 43403 , USA

Telephonc: (1)

Email:

Facsimile: (1)

Commission(s): 47

Telex:

GREGUL A YA DR, ASTRONOMICAL OBSERVATORY, KIEV STATE UNIVERSITY, OBSERVATORNAYA UL 3 252053 KIEV, UKRAINE

Telcphonc: (7) $04+262391$

Facsimile: (7)

Telex:

Email:

Commission(s):

GREISEN KENNETH I PROF, 336 FOREST HOME DR, ITHACA NY 14850, USA

Telephone: (1) 6072571650

Email:

Facsimilc: (1)

Telex:

Commission(s): $4+$

GRENIER ISABELLE DR, CEA CEN, DAPNIA/SAP, BP 2, F 91191 GIF/YVETTE CDX, FRANCE

Telephone: (33) $16008+400$

Facsimile: (33) 169086577

Telex: 604860

Email: grenier@frsac11/32779::grenicr Commission(s): $4+$

GRENIER SUZANNE, OBSERVATOIRE DE PARIS, SECTION DE MEUDON, F 92195 MEUDON PPL CDX, FRANCE Telephone: (33) $1450778+1$

Facsinile: (33)

Email:

Commission(s):

Telex: 201571

GRENON MICHEL DR, OBSERVATOIRE DE GENEVE, CHEMIN DES MAILLETTES 51 CH 1290 SAUVERNY, SWITZERLAND

Telephone: (+1) 227552611

Email: grenon(a)obs.unige.ch

Facsimile: (41) 227553983

Telex: $\mathbf{4 1 9 2 0 9}$ obs ch

Commission(s): $25,33,+5 C$ 
M 566

GREVE ALBERT DR, IRAM, 300 RUE DE LA PISCINE, F 38406 S MARTIN HERES CD, FRANCE

Telephone: (33) 76824931

Facsimile: (33)

Telex:

Email:

Commission(s):

GREVESSE N DR, INSTITUT D'ASTROPHYSIQUE, UNIVERSITE DE LIEGE, AVE COINTE 5

B 4000 COINTE-LIEGE, BELGIUM

Telephone: (32) 41529980

Facsimile: (32) $41527+74$

Telex: 41264

Email:

Commission(s): 12,14C,36

GREWING MICHAEL PROF, ASTRONOMISCHES INSTITUT, UNIVERSITAET TUEBINGEN, WALDHAEUSERSTR 64 D 72076 TUEBINGEN, GERMANY

Telephone: (49) 7071292486

Facsimilc: (49)

Telex: 07262714 ait d

Email:

Commission(s): $25,34,40,44$

GREYBER HOWARD D DR, 10123 FALLS RD, POTOMAC MD 20854, USA

Telephone: (1)

Facsimile: (1)

Telex:

Email:

Commission(s): 44,47

GRIFFIN I P DR, ARMAGH PLANETARIUM, COLLEGE HILL, ARMAGH, N IRELAND, UK

Telephone: (44) 1861524725

Facsimile: (4) 1861526187 Telex:

Email: ipg@star.arm.ac.uk

Commission(s):

GRIFFIN MATTHEW J DR, DPT OF PHYSICS, QUEEN MARY/WESTFIELD COLL, MILE END RD LONDON E1 4NS, UK

Telephone: (44) 1719804811

Facsimile: $(4 t)$

Telex: 893750

Email: mjg@uk.ac.qme.star

Commission(s):

GRIFFIN RITA E M DR, INSTITUTE OF ASTRONOMY, THE OBSERVATORIES, MADINGLEY RD

CAMBRIDGE CB3 OHA, UK

Telephone: (44) $12233375+8$

Email:

Facsimile: (f4) 1223337523

Telex: 817297 astron g

Commission(s): 29

GRIFFIN ROGER F DR, INSTITUTE OF ASTRONOMY, THE OBSERVATORIES, MADINGLEY RD

CAMBRIDGE CB3 OHA, UK

Telephone: (44) 1223337548

Email: rff1@uk.ac.cam.phx

Facsimile: (4) $1223337523 \quad$ Telex: 817297 astion g

Commission(s): $05,29,30$

GRIFFITH JOHN S PROF, DPT MATH SCIENCE, LAKEHEAD UNIVERSITY, THUNDER BAY ON P7B 5E1, CANADA

Telephone: (1) 8073452121

Facsimile: (1)

Telex:

Email:

Commission(s):

GRIFFITHS RICHARD E DR, DPT PHYSICS \& ASTRONOMY, JOHNS HOPKINS UNIVERSITY, CHARLES \& 3+TH ST BALTIMORE MD 21218, USA

Telephone: (1) 4105164194

Facsimile: (1) 4105167239 Telex:

Email: griffith(a)stsci.edu

Commission(s): $09,28,+4$

GRIFFITHS WILLIAM K, DPT OF PHYSICS, UNIVERSITY OF LEEDS, LEEDS LS2 9JT, UK

Telephonc: (44)

Facsimilc: (4)

Telex:

Email:

Commission(s): 37

GRIGORJEV VICTOR M DR, SIBIZMIR, ACADEMY OF SCIENCES, 664697 IRKUTSK 33, RUSSIA

Telephone: (7) 629388

Facsimile: (7)

Telex: $1+2+07$

Email:

Commission(s): (0)

GRIJO DE OLIVEIRA A K DR, OBSERVATORIO NACIONAL, RUA GL BRUCE 586, SAN CRISTOVAO 20921 RIO DE JANEIRO RJ, BRAZIL

Telephone: (55) 215807181

Facsimile: (55) 215800332

Telex:

Email:

Commission(s):

GRINDLAY JONATHAN E DR, CENTER FOR ASTROPHYSICS, HCO/SAO, 60 GARDEN ST CAMBRIDGE MA 02138, USA

Telephone: (1) 6174957204

Facsimile: (1) $617+957356$

Telex: $921+28$ satellite cam

Email: cfa::"josh@cfa255"/6699::josh

Commission(s): $06, \mathrm{C}, 37,44$ 
GRININ VLADIMIR P DR, CRIMEAN ASTROPHYS OBS, UKRAINIAN ACAD OF SCIENCE, NAUCHNY $33+413$ CRIMEA, UKRAINE

Telephone: (7) $4329+5$

Facsimile: (7)

Telex:

Email:

Commission(s): 27,36

GRINSPOON DAVID HARRY, LASP, UNIVERSITY OF COLORADO, CAMPUS BOX 392, BOULDER CO 80309, USA

Telephone: (1) 3034926230

Facsimile: (1) 3034926946

Telex:

Email: david@sunra.colorado.edu

Commission(s):

GRISHCHUK L P DR, STERNBERG STATE ASTR INST, UNIVERSITETSKIJ PROSP 13, 119899 MOSCOW, RUSSIA

Telephone: (7) 951395006

Facsimile: (7)

Telex:

Email:

Commission(s): 47

GROOTE DETLEF, HAMBURGER STERNWARTE, GOJENSBERGSWEG 112, D 21029 HAMBURG, GERMANY

Telephone: (49) 4072524112

Facsimile: (49)

Telex:

Email:

Commission(s):

GROSBOL PREBEN JOHNSON DR, ESO, KARL SCHWARZSCHILDSTR 2

D 85740 GARCHING MUENCHEN, GERMANY

Telephone: (49) $8932006237 \quad$ Facsimile: (49) $893202362 \quad$ Telex: 52828222 cod

Email:

Commission(s): 05,09

GROSS PETER G PROF, 714 OXFORD RD, BALA CYNWYD PA 19004, USA

Telephone: (1)

Facsimile: (1)

Telex:

Email:

Commission(s):

GROSS RICHARD SEWART, JPL, MS 238 332, 4800 OAK GROVE DR, PASADENA CA 91109 8099, USA

Telephone: (1) $81835+4010$

Facsimile: (1) $8183936890 \quad$ Telex:

Email: rsg(a)logos.jpl.nasa.gov

Commission(s): 19

GROSSMAN ALLEN S PROF, ERWIN W FICK OBSERVATORY, IOWA STATE UNIVERSITY, AMES IA 50011, USA

Telephone: (1) $51529+3666$

Facsimile: (1)

Telex:

Email:

Commission(s):

GROSSMAN LAWRENCE PROF, DPT GEOPHYSICAL SCIENCES, UNIVERSITY OF CHICAGO, 5734 S ELLIS AVE, CHICAGO IL GO637, USA

Telephone: (1) 3129628153

Facsimile: (1)

Telex:

Email:

Commission(s): 15,16

GROSSMANN-DOERTH U DR, KIEPENHEUER INSTITUT, FUER SONNENPHYSIK, SCHOENECKSTR 6

D 7910+ FREIBURG BREISGAU, GERMANY

Telephone: (49) $76132864 \quad$ Facsimile: (49)

Email: $\quad$ Commission(s):

Telex: 7721552 kis d

GROTEN ERWIN PROF, INST/PHYSIKALISCHE GEOD, PETERSENSTR 13, D 6100 DARMSTADT, GERMANY

Telephone: (49) $6151163109 \quad$ Facsimile: $(49)$

Telex: 419579

Email:

Commission(s): 19

GROUSHINSKY N P PROF DR, STERNBERG STATE ASTR INST, UNIVERSITETSKIJ PROSP 13

119899 MOSCOW, RUSSIA

Telephone: (7)

Facsimile: (7)

Telex:

Email:

Commission(s): 07

GRUBISSICH C PROF DR, VI AOSTA 34/5, I 351+2 PADOVA, ITALY

Telephone: (39) +9 38301

Facsimile: (39)

Telex:

Email:

Commission(s): 37

GRUDLER PIERRE, OCA CERGA, AVE COPERNIC, F OG130 GRASSE, FRANCE

Telephone: (33) $93+05+24$

Facsimile: (33) $93+05+33 \quad$ Telex:

Email:

Commission(s): 08,31

GRUDZINSKA STEFANIA DR, INSTITUTE OF ASTRONOMY, N COPERNICUS UNIVERSITY, UL CHOPINA 12/18 PL 87100 TORUN, POLAND

Telephone: (48) 20655 Facsimile: $(48)$

Email:

Commission(s): 15

Telex: 0552234 astr pl 
M 568

GRUEFF GAVRIL DR, IST DI RADIOASTRONOMIA, CNR, VIA IRNERIO 46, I 40126 BOLOGNA, ITALY

Telephone: (39) 51232856

Facsimile: (39)

Tclex:

Email:

Commission(s):

GRUEN EBERHARD DR, MPI FUER KERNPHYSIK, POSTFACH 103 980, D 69029 HEIDELBERG, GERMANY

Telephone: (49) $6221516+78 \quad$ Facsimile: (49)

Telex: 461666 mpihd o

Email: gruen@dusty.mpi-hp.mpg.de Commission(s): 15,21,22C

GRUENWALD RUTH DR, IAG, UNIVERSIDADE DE SAO PAULO, CP 9638, 01065 SAO PAULO SP, BRAZIL

Telephone: (55) 115778599

Facsimile: (55) 112763848

Telex: 1156735 iagm br

Email:

Commission(s):

GRY CECILE DR, LAS, TRAVERSE DU SIPHON, LES TROIS LUCS, F 13012 MARSEILLE, FRANCE

Telephone: (33) 91055900

Facsimile: (33) $91661855 \quad$ Telex: 420584

Email: cecile@frlasm51

Commission(s):

GRYGAR JIRI DR, INSTITUTE OF PHYSICS, CZECH ACADEMY OF SCIENCES, NA SLOVANCE 2

CZ 18040 PRAHA 8, CZECH R

Telephone: (42) 266052660

Facsimile: (42) 2821227

Telex:

Email: grygar@fzu.cz

Commission(s): 27,42

GRZEDZIELSKI STANISLAW PR, SPACE RESEARCH CENTER, POLISH ACAD OF SCIENCES, UL ORDONA 21 PL 01237 WARSAW, POLAND

Telephone: (48)

Facsimile: (48)

Telex:

Email:

Commission(s): 49

GU XIAO-MA, YUNNAN OBSERVATORY, CAS, BOX 110, KUNMING 72946, YUNNAN, CHINA PR

Telephone: (86) $8712035 \quad$ Facsimile: (86) Telex: 64040 yuobs cn

Email:

Commission(s): 10,12

GUARNIERI ADRIANO DR, OSS ASTRONOMICO, UNIVERSITA DI BOLOGNA, VIA ZAMBONI 33 I 40126 BOLOGNA, ITALY

Telephone: (39) 51519593

Facsimile: (39)

Telex:

Email:

Commission(s):

GUBANOV VADIM S DR, PULKOVO OBSERVATORY, ACADEMY OF SCIENCES, 10 KUTUZOV QUAY 196140 ST PETERSBURG, RUSSIA

Telephone: (7) 2979481

Facsimile: (7)

Telex:

Email:

Commission(s): 08

GUDEHUS DONALD HENRY, CHARA, GEORGIA STATE UNIVERSITY, ATLANTA GA 30303, USA

Telcphone: (1) $40+6582932$

Facsimile: (1) 4046511389

Telex:

Email: gudehus(a)chara.gsu.edu

Commission(s):

GUDMUNDSSON EINAR H, RAUNVISINDASTOFNUN, HASKOLANS, DUNHAGA 3, IS 107 REYKJAVIK, ICELAND

Telephone: (354) 1694811

Facsimile: (354) 128911

Telex: 2307 isinfo

Email: cinar(a)raunvis.hi.is

Commission(s): 47

GUDUR N DR, OBSERVATORY, EGE UNIVERSITY, BOX 21, 35100 BORNOVA IZMIR, TURKEY

$\begin{array}{ll}\text { Telephone: (90) } 51180110 \star 2326 & \text { Facsimile: (90) } \\ \text { Email: } & \text { Commission(s): }\end{array}$

Telex:

Email:

GUEDEL MANUEL, PAUL SCHERRER INSTITUTE, WUERENLINGEN \& VILLIGEN

CH 5232 VILLIGEN PSI, SWITZERLAND

Telephone: (41) $56994265 \quad$ Facsimile: (41) 56993294 Telex:

Email: guedel(a)astro.phys.ethy.ch Commission(s):

GUELIN MICHEL DR, IRAM, 300 RUE DE LA PISCINE, F 38406 S MARTIN HERES CD, FRANCE

Telephone: (33) $76423383 \quad$ Facsimile: (33) Telex: $980753 \mathrm{iram}$

Email:

Commission(s): $34,+1)$

GUERIN PIERRE DR, INSTITUT D'ASTROPHYSIQUE, 98BIS BD ARAGO, F 75014 PARIS, FRANCE

Telephone: (33) 144328007

Facsimile: (33) $1+4328001 \quad$ Telex:

Email:

Commission(s): 16 
GUERRERO GIANANTONIO DR, OSS ASTRONOMICO DI MILANO, VIA E BIANCHI 46, I 22055 MERATE, ITALY

Telephone: (39) 592035

Facsimile: (39)

Telex:

Email:

Commission(s): 27

GUERTLER JOACHIN DR, UNIV STERNWARTE JENA, SCHILLERGAESSCHEN 2, D 07745 JENA, GERMANY

Telephone: (49) 3641630323

Facsimile: (49) $36+155594 \quad$ Telex: 588634 uni dd

Email:

Commission(s): 34

GUEST JOHN E DR, UNIVERSITY OF LONDON OBS, MILL HILL PARK, LONDON NW7 2QS, UK

Telephone: (44) $1819597367 \quad$ Facsimile: (44) Telex: 28722 ucphys

Email:

Commission(s): 16

GUESTEN ROLF, MPI FUER RADIOASTRONOMIE, AUF DEM HUEGEL 69, D 53121 BONN, GERMANY

Telephone: (49) 228525379

Facsimile: (49)

Telex:

Email:

Commission(s): 34,40

GUETTER HARRY HENDRIK, US NAVAL OBSERVATORY, FLAGSTAFF STATION, BOX 1149

FLAGSTAFF AZ 86002, USA

Telephone: (1) 6027795132

Facsimile: (1)

Telex:

Email:

Commission(s): $25,37,+5$

GUHATHAKURTA MADHULIKA, NASA GSFC, CODE 682, GREENBELT MD 20771, USA

Telephone: (1) 3012860722

Facsimile: (1) $3012861617 \quad$ Telex:

Email: likac@madhu.gsfc.nasa.gov

Commission(s): 10,12

GUIBERT JEAN DR, OBSERVATOIRE DE PARIS, 61 AVE OBSERVATOIRE, F 75014 PARIS, FRANCE

Telephone: (33) 140512098

Facsimile: (33)

Telex: 270776 obs $f$

Email:

Commission(s): $05,09,2+$

GUICHARD JOSE, INAOE, AP 51/216, PUEBLA PUE 72000, MEXICO

Telephone: (52)

Facsimile: (52)

Telex:

Email:

Commission(s):

GUIDERDONI BRUNO, INSTITUT D'ASTROPHYSIQUE, 98BIS BD ARAGO, F 75014 PARIS, FRANCE

Telcphone: (33) 144328098

Facsimile: (33) 1 +4328001 Telex:

Email: guider(a)iap.fr

Commission(s):

GUIDICE DONALD A DR, A F GEOPHYSICS LABORATORY, SPACE PHYSICS DIV, HANSCOM AFB

BEDFORD MA 01731, USA

Telephone: (1) 6178613989

Email:

Facsimile: (1)

Telex:

Commission(s): 40

GUINAN EDWARD FRANCIS DR, DPT OF ASTRONOMY, VILLANOVA UNIVERSITY, VILLANOVA PA 19085, USA

Telcphonc: (1) 2156454823

Email: guinan(a) ucis.vill.edu

Facsimile: (1) $2156457465 \quad$ Telex:

Commission(s): 27,42VP

GUINOT BERNARD R PROF, 2 RUE DES SOUPIRS, F 77590 CHARTRETTES, FRANCE

Telephone: (33)

Facsimilc: (33)

Telex:

Email:

Commission(s): 19,31C

GULKIS SAMUEL DR, JPL, MS 169 506, 4800 OAK GROVE DR, PASADENA CA 91109, USA

Telephone: (1) $81835+5708$

Facsimile: (1) $81835+2946$

Telex:

Email:

Commission(s): 16,40,51C

GULL STEPHEN F DR, MULLARD RADIO ASTRON OBS, CAVENDISH LABORATORY, MADINGLEY RD

CAMBRIDGE CB3 OHE, UK

Telephone: (44) 1223337294

Facsimile: (44) 1223354599

Telex: 81292

Email:

Commission(s): 40

GULL THEODORE R DR, NASA GSFC, CODE 680, LASP, GREENBELT MD 20771, USA

Telephone: (1) 3012868060

Facsimilc: (1)

Telex: 710-8289716

Email:

Commission(s): 34,44 
GULLIVER AUSTIN FRASER DR, DPT PHYSICS \& ASTRONOMY, UNIVERSITY OF BRANDON, 270 18TH STREET, BRANDON MB R7A 6A9, CANADA

Telephone: (1) 2047277441

Facsimile: (1) $20472873+6 \quad$ Telex:

Email: gulliver@brandonu.ca

Commission(s): 42

GULMEN OMUR DR, OBSERVATORY, EGE UNIVERSITY, BOX 21, 35100 BORNOVA IZMIR, TURKEY

Telephone: (90) 51180110

Facsimilc: (90)

Telex:

Email:

Commission(s):

GULSECEN HULUSI, ISTANBUL UNIVERSITY OBS, UNIVERSITY, ISTANBUL 34452, TURKEY

Telephone: (90) 2125223597

Facsimile: (90) 2125190834 Telex:

Email: ik001@triuvm11

Commission(s):

GULYAEV A P DR, STERNBERG STATE ASTR INST, UNIVERSITETSKIJ PROSP 13, 119899 MOSCOW, RUSSIA

Telephone: (7) 951391970

Facsimile: (7)

Telex:

Email:

Commission(s): 08,12

GULYAEV RUDOLF A DR, IZMIRAN, ACADEMY OF SCIENCES, 142092 TROITSK, RUSSIA

Telephone: (7)

Facsimilc: (7)

Telex:

Email:

Commission(s):

GULYAEV SERGEI, DPT OF ASTRONOMY, URAL STATE UNIVERSITY, LENIN PROSP 51, EKATERINBURG 620083, RUSSIA

Telephone: (7) 3432220729

Facsimile: (7) 3432555964

Telex:

Email: gulyaev@astro.urgu.e-burg.su

Commission(s):

GUNN JAMES E PROF, DPT ASTROPHYSICAL SCI, PRINCETON UNIVERSITY, PEYTON HALL, PRINCETON NJ 0854 1001, USA

Tclephone: (1) 6094523802

Email:

Facsimile: (1)

Telex:

Commission(s): 28,44,47,51

GUO NEI-SHU DR, NANJING ASTRONOMICAL, INSTRUMENT FACTORY, BOX 846, NANJING, CHINA PR

Telephonc: (86) 25646191

Facsimile: (86)

Telex: $3+136$

Email:

Commission(s):

GUO QUAN SHI, PURPLE MOUNTAIN OBSERV, CAS, NANJING, CHINA PR

Telephone: (86) 2546700

Facsimile: (86) 25301459

Telex: $3+144$ pmonj en

Email:

Commission(s):

GUPTA SUNIL K DR, TIFR, HOMI BHABHA RD, COLABA, BOMBAY 400 005, INDIA

Telephone: (91) $222152971 * 545$

Facsimile: (91) $222152110 \quad$ Telex:

Email: guptask@tifrvax.bitnet

Commission(s): 33

GURM HARDEV S PROF, DPT ASTRONOMY \& SPACE SCI, UNIVERSITY OF PANJABI, PATIALA 147 002, INDIA

Telcphone: (91) 73262*96

Facsimile: (91)

Telex:

Email:

Commission(s): $27,35,46,51$

GURMAN JOSEPH B DR, NASA GSFC, CODE 602.6, GREENBELT MD 20771, USA

$\begin{array}{lll}\text { Telephone: (1) } 3012867599 & \text { Facsimile: (1) } & \text { Telex: } 89675\end{array}$

Email: span:solmax::gurman

Commission(s): 10

GURSHTEIN ALEXANDER A DR, INST HIST OF SCI \& TECHN, ACADEMY OF SCIENCES, STAROPANSKY 1/5, 103012 MOSCOW, RUSSIA

Telephone: (7) 952015972

Facsimile: (7) 959259911

Telex:

Email: aag@ihst.msk.su

Commission(s): 16,41C

GURSKY HERBERT DR, NAVAL RESEARCH LABORATORY, CODE 4100, 4555 OVERLOOK AVE SW, WASHINGTON DC 20375 5000, USA

Telephone: (1) 2027676343

Facsimile: (1)

Telex:

Email:

Commission(s): $27,42,44$

GURZADIAN G A PROF DR, GARNY SPACE ASTRONOMY, INSTITUTE, BOX 370/15, 375002 YEREVAN, ARMENIA

Telephone: (7) 8852649001

Facsimile: (7)

Email:

Commission(s): $28,34,45$

Telex: $2+3344$ orrion su 
GUSEINOV O H PROF, INSTITUTE OF PHYSICS, AKADEMGORODOC, NARIMANOV UL 33, 370143 BAKU AZERBAIDZHAN

Telephone: (994) 393951

Facsimile: (994)

Commission(s): $34,42,44$

Telex:

Email:

GUSEJNOV RAGIM EH DR, SHEMAKHA ASTROPHYSICAL, OBSERVATORY, AZER ACADEMY OF SCIENCES 373243 SHEMAKHA, AZERBAIDZHAN

Telephone: (994)

Facsimile: (994)

Telex:

Email:

Commission(s):

GUSSMANN E A DR, ZNTRLINST F ASTROPHYSIK, STERNWARTE BABALSBERG, ROSA LUXEMBURG STR 17A D 1473 POTSDAM, GERMANY

Telephone: (49)

Facsimile: (49)

Telex:

Email:

Commission(s): 36

GUSTAFSON BO A S, DPT OF ASTRONOMY, UNIVERSITY OF FLORIDA, 211 SSRB, GAINESVILLE FL 32611, USA

Telephone: (1) 9043927677

Facsimile: (1) $90+3925089 \quad$ Telex:

Email: gustaf@astro.ufl.edu

Commission(s): $15,21 \mathrm{C}, 22$

GUSTAFSSON BENGT DR, ASTRONOMICAL OBSERVATORY, BOX 515, S 75120 UPPSALA, SWEDEN

Telephone: (46)

Facsimile: (46)

Telex:

Email:

Commission(s): 29,36

GUTCKE DIETRICH, CARL ZEISS STR 1, D (07740 JENA, GERMANY

Telephone: (49)

Facsimile: (49)

Commission(s): 09

Telex:

Email:

GUTHRIE BRUCE N G DR, 5 ARDEN STREET, EDINBURGH EH9 1BR, UK

\begin{tabular}{|c|c|}
\hline e: (44) 1312294957 & $\begin{array}{l}\text { Facsimile: }(4+) \\
\text { Compission(s): 20 }\end{array}$ \\
\hline
\end{tabular}

GUTIERREZ-MORENO A DR MRS, DPT DE ASTRONOMIA, UNIVERSIDAD DE CHILE, CASILLA 36 D SANTIAGO, CHILE

Telephone: (56) $2229+101 / 40122 \quad$ Facsimile: (56) Telex: $4+0001$

Email:

Commission(s): 25

GUZIK JOYCE ANN, LOS ALAMOS NATIONAL LAB, X 2 MS B220, LOS ALAMOS NM 87545 2345, USA

Telephone: (1) 5056678927

Facsimile: (1) 5056654080 Telex:

Email: joy a beta.lanl.gov

Commission(s): 27,35

GWINN CARL R DR, DPT OF PHYSICS, UNIVERSITY OF CALIFORNIA, SANTA BARBARA CA 93106, USA

Telephone: (1) 8059612814

Facsimile: (1) 8059614170

Telex:

Email: cgwinn(a)voodoo.ucsb.edu

Commission(s): 40

GYLDENKERNE KJELD DR, COPENHAGEN UNIVERSITY OBS, BRORFELDEVEJ 23, DK 4340 TOLLOSE, DENMARK

Telephone: (45) 53488195

Facsimile: (45) 58488755

Telex: $4+155$

Email:

Commission(s): 33,42

GYORI LAJOS, HELIOPHYSICAL OBSERVATORY, BOX 93, H 5701 GYULA, HUNGARY

Telephone: (36) 361553

Facsimilc: (36)

Commission(s): 10

Telex:

Email:

GYULBUDAGHIAN ARMEN, BYURAKAN OBSERVATORY, AIGESTAN 9TH ST $67+0,375009$ YEREVAN, ARMENIA

Telephone: (7) Facsimile: (7) $8852523640 \quad$ Telex:

Email: byurakan(ä)adonis.iass.msk.su Commission(s): 28

HABBAL SHADIA RIFAI, CENTER FOR ASTROPHYSICS, HCO/SAO, 60 GARDEN ST, CAMBRIDGE MA 02138, USA Telephone: (1) 6174957348

Facsimile: (1)

Telex: 921428

Email:

Commission(s): 49

HABE ASAO, DPT OF PHYSICS, HOKKAIDO UNIVERSITY, KITA 10 NISHI 8, SAPPORO 060, JAPAN

Telephone: (81) $117112111 \quad$ Facsimile: (81)

Email:

Commission(s): 33

Telex: 
HABIBULLIN SH T PROF DR, DPT OF ASTRONOMY, KAZAN STATE UNIVERSITY, LENIN UL 18 420008 KAZAN, RUSSIA

Tclephone: (7) 323641

Facsimile: (7)

Telex:

Email:

Commission(s): 16

HABING H J DR, STERREWACHT, BOX 9513, NL 2300 RA LEIDEN, NETHERLANDS

Telephone: (31) 71272727

Facsimile: (31) 71275819

Telex: 39058

Email:

Commission(s): 33,34C

HACHENBERG OTTO PROF DR, RADIOASTRONOMISCHES INST, UNIVERSITAET BONN, AUF DEM HUEGEL 71 D 53121 BONN, GERMANY

Tclephone: (49)

Facsimile: (49)

Telex:

Email:

Commission(s):

HACHISU IZUMI DR, DPT OF EARTH SCIENCES, UNIVERSITY OF TOKYO, MEGURO KU, TOKYO 153, JAPAN

Telephone: (81) 334653925

Facsimile: (81) 334653925 Telex:

Email: hachisu@kyohou.c.u-tokyo.ac.jp Commission(s): 35

HACK MARGHERITA PROF, OAT, BOX SUCC TRIESTE 5, VIA TIEPOLO 11, I $3 \$ 131$ TRIESTE, ITALY

Telephone: (39) $40793921 \quad$ Facsimile: (39)

Email:

Commission(s): $29,36,44,45$

Telex: 461137 oat i

HACKWELL JOHN A DR, AEROSPACE CORPORATION, BOX 92957, LOS ANGELES CA 90009, USA

Telephone: (1) 3077666296

Facsimile: (1)

Telex:

Email:

Commission(s): 27,34

HACYAN SHAHEN DR, INSTITUTO DE ASTRONOMIA, UNAM, APDO POSTAL 70-264, 0\$510 MEXICO DF, MEXICO Telcphone: (52) 9055485305

Facsimile: (52)

Email:

Commission(s): 47

Telex: 1760155 cicme

HADDOCK FRED T DR, DPT OF ASTRONOMY, UNIVERSITY OF MICHIGAN, DENNISON BLDG

ANN ARBOR MI 48109 1090, USA

Tclephone: (1) 3137643430

Facsimilc: (1)

Telex:

Email:

Commission(s): $40,44,51$

HADJIDEMETRIOU JOHN D, DPT THEORET MECHANICS, UNIVERSITY THESSALONIKI

GR 54006 THESSALONIKI, GREECE

Telephone: (30) 31991410

Facsimile: (30)

Telex:

Email:

Commission(s): 0?

HADLEY BRIAN W, ROYAL OBSERVATORY, BLACKFORD HILL, EDINBURGH EHO 3HJ, UK

Telephone: (44) 1316688296

Facsimile: (4t)

Commission(s): 09

Telex: 72383 roedin $g$

Email: bwh@uk.ac.roc.star.

HADRAVA PETR, ASTRONOMICAL INSTITUTE, CZECH ACADEMY OF SCIENCES, ONDREJOV OBSERVATORY CZ 251 65 ONDREJOV, CZECH R

Tclephonc: $(\$ 2) 20 \$ 8571+1 / 7111$

Email: aststclạcscarn
Facsimile: $(\$ 2) 20 \$ 851611$

Commission(s): 42
Telex: 121579 astr $\mathrm{c}$

HAEFNER REINHOLD DR, UNIVERSITAETS STERNWARTE, SCHEINERSTR 1, D 81679 MUENCHEN, GERMANY

Telephone: (49) 89989021

Facsimile: (49)

Commission(s): 27

Email:

HAEMEEN ANTTILA KAARLE A, DPT OF ASTRONOMY, UNIVERSITY OF OULU, SF 90570 OULU 57, FINLAND

Telephonc: (358)

Facsimile: (358) 81561278

Telex:

Email:

Commission(s):

HAENSEL PAWEL DR, COPERNICUS ASTRON CENTER, POLISH ACAD OF SCIENCES, UL BARTYCKA 18 PL 00716 WARSAW, POLAND

Telephone: (48) 410828

Facsimile: (48)

Email:

Commission(s):

Telex: 813978 zapjan pl

HAERENDEL G DR, MPI F PHYSIK \& ASTROPHYS, INST F EXTRATERR PHYSIK, KARL SCHWARZSCHILDSTR 1 D 85740 GARCHING MUENCHEN, GERMANY

Telephone: (49) 8932993516

Facsimile: (49) 8932993569

Telex: 05215845 xter d

Email:

Commission(s) 
HAGEN HANS-JUERGEN DR, HAMBURGER STERNWARTE, GOJENSBERGSWEG 112

D 21029 HAMBURG, GERMANY

Telephone: (49) $4072524136 \quad$ Facsimile: (49) $4072524198 \quad$ Telex: 217884

Email:

Commission(s): 47

HAGEN JOHN P, 4445 DENIA CIR, LAS VEGAS NV 89108, USA

Telephone: (1)

Facsimile: (1)

Telex:

Email:

Commission(s): 10,40

HAGEN-THORN VLADIMIR A DR, ASTRONOMICAL OBSERVATORY, ST PETERSBURG UNIVERSITY BIBLIOTECHNAJA PL 2, 198904 ST PETERSBURG, RUSSIA

$\begin{array}{lll}\text { Telephone: (7) } 2579491 & \text { Facsimile: (7) } & \text { Telex: } \\ \text { Email: } & \text { Commission(s): } 28 & \end{array}$

HAGFORS T DR, MPI FUER AERONOMIE, POSTFACH 20, D 37189 KALTENBURG, GERMANY

Telephone: (49) Facsimile: (49) Telex:

Email:

Commission(s): 16

HAGIO FUMIHIKO DR, KUMAMOTO INST TECHNOLOGY, IKEDA 4, KUMAMOTO, JAPAN

Telephone: (81) 963263111 Facsimile: (81) 963263000 Telex:

Email:

Commission(s): 28

HAGYARD MONA JUNE, NASA/MSFC, CODE ES 52, HUNTSVILLE AL 35812, USA

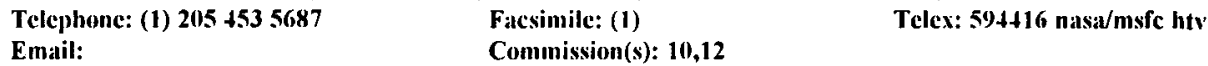

HAHN GERHARD J DR, INST F PLANETARY EXPLORAT, GERMAN AEOSPACE RES ESTAB

RUDOWER CHAUSSEE 5, D 12480 BERLIN, GERMANY

Telephone: (49) $3069545+17 \quad$ Facsimile: (49) $3069545386 \quad$ Telex:

Email: gerhard.hahn(a)dr.de Commission(s): 20

HAIKALA LAURI K, OBS \& ASTROPHYSICS LAB, UNIVERSITY OF HELSINKI, TAEHTITORNINMAKI SF 00130 HELSINKI 13, FINLAND

Telephone: (358) $1912948 \quad$ Facsimile: (358)

Telex: 124690 unih sf

Email:

Commission(s):

HAISCH BERNHARD MICHAEL, LOCKHEED PALO ALTO RES LB, DPT 91 30 BLDG 252, 3251 HANOVER ST PALO ALTO CA 94304 , USA

Telephonc: (1) 4158584073

Email: haisch(a)sag.dnet.nasa.gov

Facsimile: (1) $\$ 15+2+399+$

Telex: $3+6409$

Commission(s): 27,36,51

HAJDUK ANTON DR, ASTRONOMICAL INSTITUTE, SLOVAK ACADEMY SCIENCES SK $8+228$ BRATISLAVA, SLOVAK R

$\begin{array}{lll}\text { Telephonc: (42) } 7375157 & \text { Facsimile: }(42) & \text { Telex: } 93373 \text { scis } \\ \text { Email: astropor(â)savba.salvbi.sk } & \text { Commission(s): 15,22,51 } & \end{array}$

HAJDUKOVA MARIA, DPT OF ASTRONOMY, COMENIUS UNIVERSITY, MLYNSKA DOLINA

SK $8+215$ BRATISLAVA, SLOVAK R

Telephonc: (42) 7320003

Facsimile: $(+2)$

Telex:

Email:

Commission(s): 22

HAKKILA JON ERIC DR, DPT PHYSICS \& ASTRONOMY, MANKATO STATE UNIVERSITY, BOX 41

MANKATO MN 56002 8400, USA

Telephone: (1) 5073891840

Facsimile: (1)

Telex:

Email: jhakk@omsus1.msus.edu

Commission(s): 33

HALBWACHS JEAN LOUIS DR, OBS DE STRASBOURG, 11 RUE UNIVERSITE, F 67000 STRASBOURG, FRANCE

Telephonc: (33) 88358200

Facsimile: (33) 88250160

Telex: 890506

Email: earn:u01103(a)frecse21

Commission(s): 26,30

HALL ANDREW NORMAN, DPT OF ASTROPHYSICS, UNIVERSITY OF OXFORD, SOUTH PARKS RD, OXFORD OX1 3RQ, UK

Telephone: (44)

Facsimile: $(+4)$

Telex:

Email:

Commission(s): $4+$ 
HALL DONALD N DR, INSTITUTE FOR ASTRONOMY, UNIVERSITY OF HAWAII, 2680 WOODLAWN DR HONOLULU HI 96822, USA

Telephone: (1) 8089568312

Facsimile: (1) 8089882790

Telex: $723-8+59$

Email:

Commission(s):

HALL DOUGLAS S DR, DYER OBSERVATORY, VANDERBILT UNIVERSITY, NASHVILLE TN 37235, USA

Telephone: (1) 6153734897

Facsimile: (1)

Telex: $\mathbf{5 5 + 3 2 3}$

Email:

Commission(s): $25,27,36,42$

HALL PETER J DR, CSIRO AUSTRALIA TELESCOPE, NATIONAL FACULTY, BOX 94

NARRABRI NSW 2390, AUSTRALIA

Telephone: (61) 67959205

Facsimile: (61) 67959255

Telex:

Email: phall@atnf.csiro.au

Commission(s): 40

HALL R GLENN DR, 3612 SPRING ST, CHEVY CHASE MD 20815, USA

Telephone: (1) 3016527221

Facsimile: (1)

Telex:

Email:

Commission(s): 19,31

HALLAM KENNETH L DR, 701 E CAPITOL ST SE, WASHINGTON DC 20003, USA

Telephone: (1)

Facsimile: (1)

Telex:

Email:

Commission(s): $09,44,45$

HALLIDAY IAN DR, 825 KILLEEN AVENUE, OTTAWA ON K2A 2X8, CANADA

Telephone: (1) 6137281497

Facsimile: (1)

Telex:

Email:

Commission(s): $15,16,22$

HAMABE MASARU DR, INSTITUTE OF ASTRONOMY, UNIVERSITY OF TOKYO, OSAWA 2 CHOME

MITAKA 181, JAPAN

Telephone: $(81)+223+3802$

Facsimile: (81) $\$ 22343749$

Telex:

Email: hamabe(a)mtk.ioa.s.u-tokyo.ac.jp Commission(s): 28

HAMAJIMA KIYOTOSHI DR, 171 KAMI NO KURA 1 CHOME, MIDORI KU, NAGOYA 458, JAPAN

Telephone: (81)

Facsimile: (81)

Telex:

Email:

Commission(s): 33

HAMANN WOLF-RAINER, INST THEOR PHYS \& STERNW, UNIVERSITAET KIEL, OLSHAUSENSTR 40 D 24098 KIEL, GERMANY

Telephone: (49) $\$ 31880+110$

Facsimile: $(40)$

Telex: 292706

Email:

Commission(s): 36

.HAMBRAIAN VALERI, BYURAKAN OBSERVATORY, BLDG + APT 2,375(188 YEREVAN, ARMENIA

Telephonc: (7) $8852284+53$

Facsimile: (7)

Telex:

Email: byuzakan@adonis.ias.msk.su

Commission(s): 28

HAMDY M A M PROF, HELWAN OBSERVATORY, HELWAN, EGYPT

Telephone: (20) 78 0645/2683 Facsimile: (20)

Email:

Commission(s): 27

Telex: 93070 hiag un

'HAMID S EL DIN PROF, DPT OF ASTRONOMY, FACULTY OF SCIENCES, CAIRO UNIVERSITY, GEZA, EGYPT

Telephonc: (20)

Facsimile: (20)

Telex:

Email:

Commission(s): 07

HAMILTON ANDREW J S, JILA, UNIVERSITY OF COLORADO, CAMPUS BOX 440, BOULDER CO 80309, USA

Telephone: (1) 3034927833

Facsimile: (1) $303+925235$

Telex:

Email: ajsh(a)wild.colorado.cdu

Commission(s): 47

HAMILTON P A DR, DPT OF PHYSICS, UNIVERSITY OF TASMANIA, GPO BOX 252C, HOBART TAS 7001, AUSTRALI

Telephone: (61) 2202419

Facsimile: (61) $2202+10$

Telex: 58150

Email:

Commission(s): 40

HAMMER FRANCOIS DR, OBSERVATOIRE DE PARIS, SECTION DE MEUDON, DAEC

F 92195 MEUDON PPL CDX, FRANCE

Telephone: (33) 145077408

Email: hammer@frmeus1
Facsimile: (33) $1+5072806$

Commission(s): 28
Telex: 
HAMMER REINER, KIEPENHEUER INSTITUT, FUER SONNENPHYSIK, SCHOENECKSTR 6 D 79104 FREIBURG BREISGAU, GERMANY

Telephone: (49) $7613198216 \quad$ Facsimile: (49) $7613198111 \quad$ Telex:

Email: hammer@kis.uni-freiburg.de Commission(s): 10,12C

HAMMERSCHLAG ROBERT H DR, STERREKUNDIG INSTITUTE, BOX 80000, NL 3508 TA UTRECHT, NETHERLAND

Telephone: (31) $30535218 \quad$ Facsimile: (31)

Email: bitnet:wnmmailahutruul Commission(s): 09

Telex: 40048 fylut $n !$

HAMMERSCHLAG-HENSBERGE G, ASTRONOMICAL INSTITUTE, UNIVERSITY OF AMSTERDAM, KRUISLAAN 403 NL 1098 SJ AMSTERDAM, NETHERLANDS

$\begin{array}{lll}\text { Telephone: (31) } 205257491 / 7492 & \text { Facsimile: (31) } 205257484 & \text { Telex: } 10262 \mathrm{hef} \text { nl } \\ \text { Email: a } 410 \mathrm{inst}(\hat{a}) \text { hasara11.bitnet } & \text { Commission(s): } 42\end{array}$

HAMMOND GORDON L DR, DPT OF MATHEMATICS, UNIVERSITY OF S FLORIDA, ASTRONOMY PROGRAM TAMPA FL 33620, USA

Telephone: (1) 8137831226

Email: dkgawaa@efrvm.bitnet

Facsimile: (1) $\quad$ Telex:
Commission(s): 35

HAMZAOGLU ESAT E H DR, KING SAUD UNIVERSITY, COLLEGE OF SCIENCE, BOX 2455

RIYADH 11453, SAUDI ARABIA

Telephonc: (966)

Facsimilc: (966)

Telex:

Email:

Commission(s):

HAN FU, PURPLE MOUNTAIN OBSERV, CAS, NANJING, CHINA PR

Tclephonc: (86) $2533738 \quad$ Facsimilc: (86) 25301459

Email:

Commission(s): 40

Telex: $3 \$ 144$ pmonj cn

HAN TIANQI, INSTITUTE OF GEODESY \&, GEOPHYSICS, XU DONG LU, WUCHANG HUBEI 430077, CHINA PR

Telephone: (86) $813712 * 570$

Facsimile: $(86)$

Telex:

Email:

Commission(s): 19,31

HAN WENJUN, BEIJING ASTRONOMICAL OBS, CAS, W SUBURB, BEIJING 100080, CHINA PR

Telephonc: (86) 1281698

Email:

Facsimile: (86)

Telex:

HAN ZHENG-ZHONG, PURPLE MOUNTAIN OBSERV, CAS, NANJING, CHINA PR

Telephone: (86) 2533583

Email:

Facsimile: (86) 25301459

Telex: $341+4$ pmonj cn

HANAMI HITOSHI DR, COLL HUMANITIES SOCIAL SC, IWATE UNIVERSITY, UEDA 3, MORIOKA O20, JAPAN

Telephone: (81) $196235171 * 2284$

Facsimile: (81) $01965+2289$ Telex:

Email: d12697@jpnkudpc

Commission(s): $28,33,34$

HANAOKA YOICHIRO DR, NOBEYAMA RADIO OBS, NAOJ, MINAMIMAKI MURA, NAGANO 384 13, JAPAN

Telephone: (81) 267634381

Facsimilc: (81) 267982506

Commission(s): 10

Telex: 3329005 naonro $j$

Email: 52863@jpnkudpc.bitnet

HANASZ JAN DR, INSTITUTE OF ASTRONOMY, N COPERNICUS UNIVERSITY, UL CHOPINA 12/18

PL 87100 TORUN, POLAND

Telephone: (48) $26037 \quad$ Facsimile: (48)

Email:

Commission(s): 10,40

Telex: 055223+ astr pl

HANAWA TOMOYUKI DR, DPT OF ASTROPHYSICS, NAGOYA UNIVERSITY, FUROCHO CHIKUSA KU

NAGOYA 464 01, JAPAN

Telephone: (81) 527816769

Email: bitnet:b42287@jpnkudpc

Facsimile: (81)

Telex: 4477323

HANBURY BROWN ROBERT PROF, WHITE COTTAGE, PENTON MEWSEY, HANTS SP11 ORQ, UK

Telephone: (44) 1264772334

Facsimile: (4)

Telex:

Email:

Commission(s): 40

HANDA TOSHIHIRO DR, INST ASTRO FAC SCIENCE, UNIVERSITY OF TOKYO, OSAWA MITAKA TOKYO 181, JAPAN

Telephone: (81) 422343735

Facsimile: (81) 422343749

Telex: 2822307 taomtk j

Email: handa@gahz.mtk.iao.s.u-tokyo.ac.jp Commission(s): 28,40 
HANDLIROVA DAGMAR DR, N COPERNICUS OBSERVATORY, \& PLANETARIUM, KRAVI HORA

CZ 61600 BRNO, CZECH R

Telephone: (42) 5785759

Facsinile: (42)

Telex:

Email:

Commission(s):

HANES DAVID A DR, DPT OF PHYSICS, QUEEN'S UNIVERSITY, KINGSTON ON K7L 3N6, CANADA

Telephone: (1) 6135475750

Facsimile: (1) 6135456463

Telex:

Email:

Commission(s): 37

HANG HENG-RONG, PURPLE MOUNTAIN OBSERV, CAS, NANJING, CHINA PR

Telephonc: (86) 2533583

Facsimile: (86) $25301+59$

Telex: $3+1+4$ pmonj cn

Email:

Commission(s): 44

HANIFF CHRISTOPHER, MULLARD RADIO ASTRON OBS, CAVENDISH LABORATORY, MADINGLEY RD

CAMBRIDGE CB3 OHE, UK

Telephone: (44) 1223337363

Facsimile: (44) $1223354599 \quad$ Telex:

Email: cah@mrao.cam.ac.uk

Commission(s):

HANISCH ROBERT J DR, STSCI, HOMEWOOD CAMPUS, 3700 SAN MARTIN DR, BALTIMORE MD 21218, USA

Telephonc: (1) 3013384910

Facsimile: (1)

Telex: 6849101

Email: span:scivax::hanisch

Commission(s): $(15,(19,+11$

HANKINS TIMOTHY HAMILTON, DPT OF PHYSICS \& ASTRON, NEW MEXICO TECH, CAMPUS STATION SOCORRO NM 87801, USA

Telephone: (1) $505+768011$

Email:

Facsimile: (1)

Telex:

Commission(s): 40

HANNER MARTHA S DR, JPL, MS 183601,4800 OAK GROVE DR, PASADENA CA 91109, USA

Telephone: (1) $8183544321 \quad$ Facsimile: (1) 818393 4605 $\quad$ Telex: 675429

Email: msh@scn2.jpl.nasa.gov Commission(s): 15,21C,22

HANSEN CARL J PROF, JILA, UNIVERSITY OF COLORADO, BOX +40 , BOULDER CO 80309 (1440, USA

Telephone: (1) $3034927811 \quad$ Facsimile: (1) Telex: $7558+2$ jila

Email:

Commission(s): 27

HANSEN LEIF LECTURER, UNIVERSITY OBSERVATORY, OESTER VOLDGADE 3

DK 1350 COPENHAGEN K, DENMARK

Telephone: (45) 31141790

Email:

Facsimile: (45) 31389157

Telex: $\mathbf{H 1 5 5}$ danast $\mathbf{d k}$

HANSEN RICHARD T MR, ENGINEERING 138, VAMC, 150 S HUNTINGTON AVE, BOSTON MA 02130, USA

Telephone: (1) $61773+2534$

Facsimilc: (1)

Telex:

Email:

Commission(s): 10

HANSLMEIER ARNOLD, INSTITUT FUER ASTRONOMIE, KARL-FRANZENS-UNIVERSITAT

UNIVERSITAETSPLATZ 5, A 8010 GRAZ, AUSTRIA

Telephone: (43) $3163805275 \quad$ Facsimile: $(43)$

Email: $\quad$ Commission(s): 07,10

Telex:

HANSON ROBERT B DR, LICK OBSERVATORY, UNIVERSITY OF CALIFORNIA, SANTA CRUZ CA 95064, USA

Telephonc: (1) 4084292755

Facsimile: (1)

Telex:

Email:

Commission(s): 24

HANSSON NILS DR, LUND OBSERVATORY, BOX +3, S 22100 LUND, SWEDEN

$\begin{array}{lll}\text { Telephone: (46) } 46107000 & \text { Facsimile: }(46) & \text { Telex: } 33533 \text { luniver s } \\ \text { Email: } & \text { Commission(s): } & \end{array}$

HANSTEEN VIGGO, INST THEORET ASTROPHYSICS, UNIVERSITY OF OSLO, LANDINGSVEIEN 68, N 0767 OSLO, NORWAY

$\begin{array}{ll}\text { Telephonc: (47) } & \text { Facsimile: (47) } \\ \text { Email: } & \text { Commission(s): }\end{array}$ 
HANTZIOS PANAYIOTIS DR, ASTRONOMICAL INSTITUTE, NTL OBSERVATORY OF ATHENS, BOX 20048 GR 11810 ATHENS, GREECE

Telephone: (30) $13+61191$

Facsimile: (30)

Telex:

Email:

Commission(s): $\mathbf{4 2}$

HANUSCHIK REINHARD DR, ASTRONOMISCHES INSTITUT, RUHR UNIVERSITAET BOCHUM, POSTFACH 102148 D 44780 BOCHUM, GERMANY

Telephone: (49) 2347003450

Facsimilc: (49)

Commission(s): 29

Telex: 0825860

Email:

Facsimile: (86)
Telex:

$\begin{array}{ll}\text { Telephonc: (86) } 1656531 * 6285 & \text { Facsimile: (86) } \\ \text { Email: } & \text { Commission(s): } 09\end{array}$

HAPKE BRUCE W DR, DPT GEOL \& PLANETARY SCI, UNIVERSITY OF PITTSBURGH

321 OLD ENGINEERING HALL, PITTSBURGH PA 15235, USA

Telephone: (1) 4126244719 Facsimile: (1) Telex:

Email: $\quad$ Commission(s): 15

HARA TADAYOSHI DR, NTL ASTRONOMICAL OBS, DIV OF EARTH ROTATION, MIZUSAWA SHI IWATE 023, JAPAN

Telephone: (81) $1972+7111 \quad$ Facsimile: (81) Telex: 837628 ilsmiz j

Email: $\quad$ Commission(s):

HARA TETSUYA DR, DPT OF PHYSICS, KYOTO SANGYO UNIVERSITY, KAMIGAMO, KYOTO GO3, JAPAN

Telephone: (81) 757012151

Facsimile: (81)

Email: hara@jpnksuvx.bit

Commission(s): 28

Telex: $5 \$ 22661 \mathrm{ksu} \mathbf{j}$

HARDEBECK ELLEN G DR, 3106 TUMBLEWEED RD, BISHOP CA 93514, USA

Telephone: (1) Facsimile: (1) Telex:

Email: $\quad$ Commission(s): 34

HARDEE PHILIP, DPT OF PHYSICS \& ASTRON, UNIVERSITY OF ALABAMA, BOX 1921 UNIVERSITY AL 354870324 , USA

$\begin{array}{ll}\text { Telephone: (1) } 2053485050 & \text { Facsimile: (1) } \\ \text { Email: } & \text { Commission(s): } 40\end{array}$

HARDY EDUARDO, DPT DE PHYSIQUE, UNIVERSITE LAVAL, FAC SCIENCES \& GENIE

LAVAL QC G1K 7P4, CANADA

Telephone: (1) $4186562960 \quad$ Facsimile: (1) $4186562040 \quad$ Telex: 05131621

Email: hardy@phy.ujaval.ca Commission(s): 28,47

HARJU JORMA SAKARI, OBSERVATORY, BOX 14 , SF 00014 UNIV HELSINKI, FINLAND

Telephonc: (358)

Facsimile: (358)

Telex:

Email:

Commission(s):

HARLAFTIS EMILIOS, ROYAL GREENWICH OBS, APDO 321, SANTA CRUZ DE LA PALMA

E 38780 TENERIFE, SPAIN

Telephone: (34) 22405500

Facsimile: (34) $22405646 / 405501$ Telex: 92639 obrm e

Email: chh@/pve.ing.iac.es/29146::chh Commission(s):

HARMANEC PETR DR, ASTRONOMICAL INSTITUTE, CZECH ACADEMY OF SCIENCES ONDREJOV OBSERVATORY, CZ 25165 ONDREJOV, CZECH R

Telephone: (42) 204 8571+3/7111 Facsimile: (42) 20+851611

Email: aststel(a)scearn Commission(s): 27,42

Telex: 121579

HARMER CHARLES F W MR, NOAO, BOX 26732, 950 N CHERRY AVE, TUCSON AZ 85726 6732, USA

Telephone: (1) $5203275511 \quad$ Facsimile: (1) Telex: 0666-484 aura noao

Email: charmerânoa.edu

Commission(s): (09,29

HARMER DIANNE L MRS, NOAO, BOX 26732,950 N CHERRY AVE, TUCSON AZ 85726 6732, USA

Telephone: (1) 5203259218

Facsimile: (1)

Telex:

Email: diharmer(a)noao.edu

Commission(s): 09,29 
M 578

HARMS RICHARD JAMES DR, APPLIED RESEARCH CORP, 8201 CORPORATE DR, SUITE 920

LANDOVER MD 20785, USA

Telephone: (1) $30145984+2$

Facsimilc: (1)

Telex:

Email:

Commission(s): $28,44,47$

HARNDEN FRANK R Jr, CENTER FOR ASTROPHYSICS, HCO/SAO, 60 GARDEN ST, CAMBRIDGE MA 02138, USA

Telephone: (1) $6174957143 \quad$ Facsimile: (1)

Telex: 921428 sattellite cam

Email:

Commission(s):

HARNETT JULIEINE DR, SCHOOL OF PHYSICS, UNIVERSITY OF SYDNEY, SYDNEY NSW 2006, AUSTRALIA

Telephone: (61) 26922727

Facsimile: (61) 26602903

Tclex: aa 26169

Email: jhatnett(a) robin.rp.csiro.au

Commission(s): 28,40

HARPAZ AMOS DR, DPT OF PHYISICS/SPACE RES, IIT, TECHNION CITY, HAIFA 32000, ISRAEL

Tclephone: $(972)+293521$

Facsimile: (972)

Telex:

Email: phr89ah@technion

Commission(s):

HARPER DAVID DR, ASTRONOMY UNIT, QUEEN MARY/WESTFIELD COLL, MILE END RD, LONDON E1 4NS, UK Tclephone: (44) 1719755492 Facsimilc: (44) 1819819587 Telex: 893750

Email: adh@star.qmw.ac.uk Commission(s): 20

\author{
HARRINGTON J PATRICK DR, ASTRONOMY PROGRAM, UNIVERSITY OF MARYLAND \\ COLLEGE PARK MD 207 $\$ 2$, USA \\ Telephone: (1) 3014545944 \\ Facsimilc: (1) \\ Telex: 7108260352 \\ Email: \\ Commission(s): $3+$
}

HARRIS ALAN WILLIAM, DLR, INST F PLANETENERKUNDUNG, RUDOWER CHAUSSEE 5 D 12489 BERLIN ADLERSHOF, GERMANY

Telephone: (49) 30 69545324 Facsimile: (49) $30695+5313$ Telex:

Email: harris@saturn,pe.bat.dlr.de Commission(s): 16,34

HARRIS ALAN WILLIAM DR, JPL, MS 183 501, 4800 OAK GROVE DR, PASADENA CA 91109, USA

Facsimile: (1) $81835+0966$

Telex: $675429 / 9105883294 / 69$

Email: awharris@jpl.1354.nasa.gov

Commission(s): 15C,20

HARRIS DANIEL E DR, CENTER FOR ASTROPHYSICS, HCO/SAO, 60 GARDEN ST, CAMBRIDGE MA 02138, USA

Telephone: (1) 6174957148

Facsimile: (1)

Telex: 921428

Email:

Commission(s): 40

HARRIS GRETCHEN L H DR, DPT OF PHYSICS, UNIVERSITY OF WATERLOO, WATERLOO ON N2L 3G1, CANADA

Telephone: (1) 5198851211

Facsimile: (1) $5197468115 \quad$ Telex: 06955259

Email: glharris@astro.waterloo.edu

Commission(s): 37

HARRIS HUGH C, US NAVAL OBSERVATORY, FLAGSTAFF STATION, BOX 1149, FLAGSTAFF AZ 86002, USA

Telephone: (1) 6027795132

Facsimile: (1)

Telex:

Email:

Commission(s): 37

HARRIS STELLA, DPT OF PHYSICS, QUEEN MARY/WESTFIELD COLL, MILE END RD, LONDON E1 4NS, UK

Telephone: $(44) 1719804811^{*}+1150$

Facsimile: (44)

Telex: 893750

Email:

Commission(s): 34

HARRIS WILLIAM E DR, DPT OF PHYSICS, MCMASTER UNIVERSITY, HAMILTON ON L8S +M1, CANADA

Telephone: (1) 4165259140

Facsimile: (1) 4165461252

Telex: 0618347

Email:

Commission(s): 37

HARRISON EDWARD R PROF, DPT PHYSICS \& ASTRONOMY, UNIV OF MASSACHUSETTS, GRC

AMHERST MA 01003, USA

Telephone: (1) 4135452194

Email:

Facsimile: (1)

Telex:

Commission(s): $\$ 7,51$

HARRISON RICHARD A, RUTHERFORD APPLETON LAB, SPACE \& ASTROPHYSICS DIV, BLDG R25/R68 CHILTON DIDCOT OX11 0OX, UK

Telephone: (44) 1235446884

Facsimile: (44) $1235+46509$ Telex:

Email: rah@uk.ac.rl.astro

Commission(s): 10 
HARRISON RICHARD A DR, RUTHERFORD APPLETON LAB, SPACE \& ASTROPHYSICS DIV, BLDG R25/R68 CHILTON DIDCOT OX11 0QX, UK

Telephone: (44) 1235446497

Facsimile: (4)

Email: span:19457::rah

Commission(s): $10 \mathrm{C}$

Telex: 83159 ruthlb $g$

HARROWER GEORGE A DR, 2041033 BELMONT AVENUE, VICTORIA BC V8S 3T4, CANADA

Tclephone: (1) Facsimile: (1) Telex:

Email:

Commission(s):

HART MICHAEL H DR, 7301 MASONVILLE DR, ANNANDALE VA 22003, USA

Telephone: (1)

Facsimile: (1)

Telex:

Email:

Commission(s): 51

HARTEN RONALD H DR, RCA ASTRO ELECTRONIC, TB-1, BOX 800, PRINCETON NJ 08540, USA

Telephone: (1) 6094263551

Facsimile: (1)

Telex:

Email:

Commission(s): 34,40

HARTKOPF WILLIAM I DR, CHARA, GEORGIA STATE UNIVERSITY, ATLANTA GA 30303 3083, USA

Telephone: (1) 4046512932

Facsimile: (1)

Telex:

Email:

Commission(s): $24,26 \mathrm{C}, 33$

HARTL HERBERT DR, INSTITUT FUER ASTRONOMIE, TECHNIKERSTR 15, A 6020 INNSBRUCK, AUSTRIA

Telephonc: (43) 52227485263

Email: herbert@ast1.uibk.ac.at

Facsimile: (\$3) 52222185252

Telex: $\mathbf{5 3 3 8 0 8}$ unite a

Commission(s): 34

HARTMANN DIETER H, 202 TAMASSEE DR, CLEMSON SC 29633, USA

Telephone: (1) 8036565298

Facsimile: (1) 8036560805 Telex:

Email: hartmann(a)biophy,phys.clemson.edu

Commission(s):

HARTMANN LEE WILLIAM, CENTER FOR ASTROPHYSICS, HCO/SAO, 60 GARDEN ST, CAMBRIDGE MA 02138, US Telephone: (1) 6174957487

Facsimile: (1)

Commission(s): 29,36

Telex:

Email:

(s): 29,36

HARTMANN WILLIAM K, PLANETARY SCIENCE INST, 620 N SIXTH AVE, TUCSON AZ 85705 8331, USA

Telephone: (1) 5206626300

Facsimile: (1) 5206228060

Telex:

Email: psikey@psi.cdu

Commission(s): 15

HARTOOG MARK RICHARD DR, LICK OBSERVATORY, UNIV OF CALIFORNIA, SANTA CRUZ CA 95064, USA

Telephone: (1)

Facsimile: (1)

Commission(s):

Telex:

Email:

Commission(s):

HARTQUIST THOMAS WILBUR, MPI F PHYSIK \& ASTROPHYS, KARL SCHWARZSCHILDSTR 1

D 85740 GARCHING MUENCHEN, GERMANY

Telephone: (49) $893299838 \quad$ Facsimile: (49)

Email:

Commission(s): 34

Telex: $052158+5$ xterr d

HARTWICK F DAVID A DR, DPT OF PHYSICS, UNIVERSITY OF VICTORIA, BOX 1700

VICTORIA BC V8W 2Y2, CANADA

Telephone: (1) $6047217742 \quad$ Facsimile: (1) 6047217715 Telex:

Email:

Commission(s):

HARUTYUNIAN HAIK A DR, BYURAKAN ASTROPHYSICAL, OBSERVATORY, 378433 BYURAKAN, ARMENIA

Telephone: (7) $8852283453 / 4142$

Email:

Facsimile: (7) $885228+142$

Telex: 411576 as con su

Commission(s): 36

HARVEL CHRISTOPHER ALVIN, 6161 STEVEN'S FOREST RD, COLUMBIA MD 21045, USA

Telephone: (1) 3019640211

Facsimile: (1)

Telex:

Email:

Commission(s): 05,37

HARVEY CHRISTOPHER C DR, OBSERVATOIRE DE PARIS, SECTION DE MEUDON F 92195 MEUDON PPL CDX, FRANCE

Telephone: (33) 145077669

Facsimile: (33)

Commission(s): 44,49

Telex: 204464

Email: meudon::harvey 
M 580

HARVEY GALE A DR, NASA LANGLEY RESEARCH CTR, ATMOSPHERIC SCIENCE DIV, MS 401A HAMPTON VA 23665-5225, USA

Telephone: (1)

Facsimile: (1)

Telex:

Email:

Commission(s): 22

HARVEY JOHN W DR, NTL SOLAR OBSERVATORY, BOX 26732, 950 N CHERRY AVE, TUCSON AZ 85726 6732, USA

Telephone: (1) 5203275511

Facsimile: (1) 5203259278

Telex: 1561401 aura ut

Email: jharvey@noao.edu

Commission(s): 10C,12

HARVEY PAUL MICHAEL DR, ASTRONOMY DPT, UNIVERSITY OF TEXAS, RLM 15 308, AUSTIN TX 78712 1083, USA Telephone: (1) 5124714461

Facsimile: (1)

Telex: 910-87+-1351

Email:

Commission(s): $34,4+$

HARWIT MARTIN PROF, DPT OF ASTRONOMY, CORNELL UNIVERSITY, SPACE SCIENCES BLDG

ITHACA NY 14853, USA

Telephone: (1) 6072564805

Facsimile: (1)

Telex:

Email:

Commission(s): $15,21,44$

HARWOOD DENNIS MR, PERTH OBSERVATORY, BICKLEY WA 6076, AUSTRALIA

Telephone: (61) 92938255

Facsimile: (61) Telex:

Email:

Commission(s): 24,25

HASAN HASHIMA DR, STSCI, HOMEWOOD CAMPUS, 3700 SAN MARTIN DR, BALTIMORE MD 21218, USA

Telephone: (1) 3013384519

Facsimile: (1) $301338+767$

Telex: $30168+9101$ stsci

Email: hasan(a)stsci

Commission(s): 28

HASAN SAIYID STRAJUL, INDIAN INSTITUTE OF, ASTROPHYSICS, KORAMANGALA, BANGALORE 560 034, INDIA

Telephone: (91) $803566585 \quad$ Facsimile: (91)

Telex:

Email:

Commission(s): 10

HASCHICK AUBREY, HAYSTACK OBSERVATORY, WESTFORD MA 01886, USA

Telephone: (1) 6176924764

Facsimile: (1)

Telex:

Email:

Commission(s): 40

HASEGAWA ICHIRO DR, 4-18-5 FUJIWARADAI KITA, KIYA KU, KOBE 651-13, JAPAN

Telephone: (81) 789825255

Facsimile: (81)

Telex:

Email:

Commission(s): 15,20,22C

-HASEGAWA TATSUHIKO DR, DPT OF ASTRONOMY, ST MARY'S UNIVERSITY, 923 ROBIE ST

HALIFAX NS B3H 3C3, CANADA

Telcphone: (1) $902+205828$

Facsimile: (1) $902+205561 \quad$ Telex:

Email: hascgawa(a)stmarys.ca

Commission(s):

HASEGAWA TETSUO DR, INST OF ASTRONOMY, UNIVERSITY OF TOKYO, OSAWA MITAKA, TOKYO 181, JAPAN

Telephone: (81) 422413737

Facsimilc: (81)

Telex: 2822307

Email:

Commission(s): 40

. HASER LEO N K DR, MPI F EXTRATERRESTRISCHE, PHYSIK, KARL SCHWARZSCHILDSTR 1

D 85740 GARCHING MUENCHEN, GERMANY

Telephone: (49) 893299803

Facsimile: (49)

Commission(s): 15

Telex: $52158+5$ xter d

Email:

HASHIMOTO MASA-AKI DR, DPT OF PHYSICS, COLLEGE OF GENERAL EDU, KYUSHU UNIV ROPPONMATSU

FUKUOKA 810, JAPAN

Telephone: (81) $927714161 * 360$

Email: e76051ajpnccku

Facsimilc: (81) $0927318745 \quad$ Telex:

Commission(s): 35

HASLAM C GLYN T DR, MPI FUER RADIOASTRONOMIE, AUF DEM HUEGEL 69, D 53121 BONN, GERMANY

Telephone: (49)

Facsimile: (49)

Email:

Commission(s): 40

Telex: $886+40$

HASSALL BARBARA J M DR, CENTRE FOR ASTROPHYSICS, UNIVERSITY OF CENTRAL, LANCASHIRE

PRESTON PRI 2HE, UK

Telcphonc: (44) 1772893569

Facsimile: (4t) 1772892903

Commission(s): 42

Telex: 677409 uclan g

Email: b.j.m.hassall@uclan.ac.uk 
HASSAN S M PROF, HELWAN OBSERVATORY, HELWAN, EGYPT

Telephone: (20) 78 0645/2683

Email:
Facsimile: (20)

Commission(s): 37
Telex: 93070

HATHAWAY DAVID H DR, NASAMSFC, CODE ES 52, HUNTSVILLE AL 35812, USA

Telephone: (1) $2055 \$ 47610$

Facsimile: (1)

Telex:

Commission(s): 10

HATZES ARTIE P BR, ASTRONOMY DPT, UNIVERSITY OF TEXAS, RLM 15 308, AUSTIN TX 78712 1083, USA

Telephone: (1) 5124711473

Facsimile: (1)

Telex:

Email: artic(a)astro.as.utexas.edu

Commission(s):

HATZIDIMITRIOU DESPINA DR, AAO, EPPING LABORATORY, BOX 296, EPPING NSW 2121, AUSTRALIA

Telephone: (61) 28681666

Facsimile: (61) $28768536 / 5+2$

Telex: 123999 anosyd

Email: dh@aaocpp.oz.au

Commission(s): 37

HAUBOLD HANS JOACHIM PROF, OFFICE FOR OUTER SPACE, WIEN INTERNATIONAL CENTER

BLDG F 0839 BOX 500, A 1400 VIENNA, AUSTRIA

Telephone: (43) $1213455830 \quad$ Facsimile: (43) $1211314949 \quad$ Telex: hjh2(a)aip.org/at

Email: haubold@ekpvs2.dnet.tuwien.ac. Commission(s): 44

HAUCK BERNARD PROF, INSTITUT D'ASTRONOMIE, UNIVERSITE DE LAUSANNE

CH 1290 CHAVANNES-D-BOIS, SWITZERLAND

Telephonc: (41) 227552611

Email: hauck@scsun.unigech

Facsimile: (41) 227553983

Telex: 27720 obsg ch

HAUD URMAS, TARTU ASTROPHYSICAL OBS, EE 2 $44+$ TORAVERE, ESTONIA

Telephonc: (372) $7+10305 \quad$ Facsimile: (372) $7+10205$ Telex:

Email: urmas $(a)$ ati.ec Commission(s):

HAUG ULRICH PROF, HAMBURGER STERNWARTE, GOJENSBERGSWEG 112, D 21029 HAMBURG, GERMANY

Telephone: (49) 4072524131

Facsimile: (49)

Telex: 217884

Email:

Commission(s): 33

HAUGE OIVIND DR, INST THEORET ASTROPHYSICS, UNIVERSITY OF OSLO, BOX 1029

N 0315 BLINDERN OSLO 3, NORWAY

Telephone: (47) 245-65-06

Facsimile: $(47)$

Telex:

Email:

Commission(s):

HAUPT HERMANN F PROF, INSTITUT FUER ASTRONOMIE, KARL-FRANZENS-UNIVERSITAT

UNIVERSITAETSPLATZ 5, A 8010 GRAZ, AUSTRIA

Telephone: (43) 3163805271

Facsimile: (43) 316384091

Commission(s): 15,20,38,46

Telex: 31078a

Email:

HAUPT WOLFGANG DR, GIRONDELLE 105, D 4630 BOCHUM 1, GERMANY

Telephone: (49)

Facsimile: (49)

Commission(s):

Telex:

Email:

HAUSER MICHAEL G DR, NASA GSFC, CODE 680, LASP, GREENBELT MD 20771, USA

Telephone: (1) 3012868701

Facsimile: (1)

Telex:

Email: stars: hauser

Commission(s): $21 \mathrm{C},+$

HAVLEN ROBERT J DR, NRAO, BOX 0, SOCORRO NM 87801 0387, USA

Telephone: (1) 5058357330

Facsimile: (1) 5058357027

Telex: 9109881710

Email: bitnct: rhavien(a)nrao

Commission(s): 46

HAVNES OVE DR, AURORAL OBSERVATORY, UNIVERSITY OF TROMSO, BOX 953, N 9001 TROMSO, NORWAY Telephonc: (47) 83-86060

Facsimile: $(47)$

Commission(s):

Telex: $6+124$ aurob $n$

Emaii:

HAWARDEN TIMOTHY G DR, ROYAL OBSERVATORY, BLACKFORD HILL, EDINBURGH EH9 3HJ, UK

Tckphonc: (44) 1316673321

Facsimile: (4)

Email:

Commission(s): 37

Telex: 72383 roedin $g$ 
HAWKES ROBERT LEWIS DR, DPT OF PHYSICS, MOUNT ALLISON UNIVERSITY SACKVILLE NB EOA 3C0, CANADA

Telephone: (1) 5063642582

Facsimile: (1) $50636+2580 \quad$ Telex:

Email: rhawkes 0 mta.ca

Commission(s): $22 \mathrm{~S}$

HAWKING STEPHEN W PROF, DPT APPLIED MATHS, \& THEORETICAL PHYSICS, SILVER STREET

CAMBRIDGE CB3 9EW, UK

Telephone: (44) 1223337900

Email:

\author{
Facsimile: (44) $1223337918 \quad$ Telex: 81240 camspl g \\ Commission(s): 44,47
}

HAWKINS GERALD S DR, CONSUL 906, 2400 VIRGINIA AVE NW, WASHINGTON DC 20037, USA

Telephone: (1) $2024852050 \quad$ Facsimile: (1) Telex:

Email: $\quad$ Commission(s): 22,41

HAWKINS ISABEL DR, CTR FOR EUV ASTROPHYSICS, UNIVERSITY OF CALIFORNIA, 2150 KITTREDGE ST BERKELEY CA 94720, USA

Telephone: (1) 4156420816

Facsimile: (1) $4156+37629 \quad$ Telex:

Email:

Commission(s): 44

HAWKINS MICHAEL R S, ROYAL OBSERVATORY, BLACKFORD HILL, EDINBURGH EH9 3HJ, UK

Telephone: (4)

Facsimile: (44)

Telex:

Email:

Commission(s): 33

HAYASHI CHUSHIRO PROF, MOMOYAMA YOGORO-CHO 1, FUSHIMI KU, KYOTO 612, JAPAN

Telephone: (81) 756111062

Facsimile: (81)

Telex:

Email:

Commission(s): 35,47

HAYASHI MASAHIKO DR, DPT OF ASTRONOMY, UNIVERSITY OF TOKYO, BUNKYO KU, TOKYO 113, JAPAN

Telephone: (81) 38122111

Facsimile: (81)

Telex: 2722126 utgab $i$

Email:

Commission(s): 40

HAYASHI SAEKO S DR, TOKYO ASTRONOMICAL OBS, NAOJ, OSAWA MITAKA, TOKYO 181, JAPAN

Telephone: (81) $422 \$ 13609$

Facsimile: (81) $\$ 22+13608 \quad$ Telex:

Email: sacko@optik.mmtk.nao.ac.jp

Commission(s): 34

HAYES DONALD S DR, 6255 N PIMERIA ALTA, TUCSON AZ 85715, USA

Tclephone: (1) 5205777269

Facsimilc: (1)

Telex:

Email:

Commission(s): 25,45

HAYLI AVRAM PROF, OBSERVATOIRE DE LYON, AVE CHARLES ANDRE, F 69561 S GENIS LAVAL CDX, FRANCE Telephone: (33) 78560705

Facsimilc: (33) 72399791

Telex: 310916

Email:

Commission(s): 33,41

HAYMES ROBERT C PROF, DPT SPACE PHYS \& ASTRON, RICE UNIVERSITY, HOUSTON TX 77001, USA

Telephone: (1) 7135274045

Facsimilc: (1)

Telex: $556+57$

Email:

Commission(s): 44

- HAYNES MARTHA P, DPT OF ASTRONOMY, CORNELL UNIVERSITY, SPACE SCIENCES BLDG

ITHACA NY 14853, USA

Telephone: (1) 6072563734

Facsimile: (1)

Telex: 93245t

Email:

Commission(s): 40

HAYNES RAYMOND F PROF, CSIRO, DIV OF RADIOPHYSICS, BOX 76, EPPING NSW 2121, AUSTRALIA

Telephone: (61) 28680276

Facsimile: (61) 28680457

Telex: astro 26230

Email: rhaynes@rpepping.oz.au

Commission(s): $34,40,41$

HAYWARD JOHN, DPT MATHS \& COMPUTING, POLYTECHNIC OF WALES, PONTYPRIDD MID GLAMORGAN CF38 2PJ, UK

Telephone: (44)

Facsimile: (44)

Commission(s): 10

Telex:

Email:

Commission(s): 10

HAYWOOD J, DPT OF TEACHER EDUCATION, TRINITY COLLEGE, DUBLIN 2, IRELAND

Telephonc: (353) 1772941

Facsimile: (353)

Telex:

Email:

Commission(s): 46 
HAZARD CYRIL DR, INSTITUTE OF ASTRONOMY, THE OBSERVATORIES, MADINGLEY RD

CAMBRIDGE CB3 OHA, UK

Telephone: (44) 1223337548

Email:
Facsimile: (44) $1223337523 \quad$ Telex:

Commission(s): 40

HAZEN MARTHA L DR, CENTER FOR ASTROPHYSICS, HCO/SAO, 60 GARDEN ST, CAMBRIDGE MA 02138, USA

Telephone: (1) 6174953362

Facsimile: (1)

Telex:

Email:

Commission(s): 37

HAZER S DR, FACULTY OF SCIENCE, EGE UNIVERSITY, BOX 21, 35100 BORNOVA IZMIR, TURKEY

Telephone: (90)

Email:

Facsimile: (90)

Telex:

Commission(s):

HAZLEHURST JOHN DR, HAMBURGER STERNWARTE, GOJENSBERGSWEG 112, D 21029 HAMBURG, GERMANY

Telephonc: (49)

Facsimile: (49)

Telcx:

Email:

Commission(s): 42

HE MIAO-FU, SHANGHAI OBSERVATORY, CAS, 80 NANDAN RD, SHANGHAI 200030, CHINA PR

Telephone: (86) 21386191

Facsimile: (86) 214384618

Telex: $3316+$ shao cn

Email:

Commission(s): $0+C, 07,20$

HE XIANG-TAO, DPT OF ASTRONOMY, BEIJING NORMAL UNIVERSITY, BEIJING 100875, CHINA PR
$\begin{array}{ll}\text { Telephone: (86) } 1656531^{* 6285} & \text { Facsimile: }(86) \\ \text { Email: } & \text { Commission(s): } 28,47\end{array}$

HEAP SARA R DR, NASA GSFC, CODE 672, GREENBELT MD 20771, USA

Telephone: (1)

Email:

Facsimile: (1)

Telex:

Commission(s):

HEARN ANTHONY G DR, STERREKUNDIG INSTITUTE, BOX 80000, NL 3508 TA UTRECHT, NETHERLANDS

Telephone: (31) 30535202

Email: ahearn@afys.ruu.n]

Facsimile: (31) 30535201

Telex: 40048 fylut $n i$

Commission(s): $05 \mathrm{C}, 36,44$

HEARNSHAW JOHN B DR, DPT PHYSICS \& ASTRONOMY, UNIVERSITY OF CANTERBURY, PRIVATE BAG 4800, CHRISTCHURCH 1, NEW ZEALAND

Telephonc: $(64) 336+253371$

Email: j.hearnshaw@acsc.canterbury.ac.nz. Facsimile: $(64) 336+2469 \quad$ Telex:

Commission(s): 29,30VP

HEASLEY JAMES NORTON, INSTITUTE FOR ASTRONOMY, UNIVERSITY OF HAWAII, 2680 WOODLAWN DR HONOLULU HI 96822, USA

Telephonc: (1) 8089566826

Facsimile: (1) 8089882790

Telex: 7238459 uhast hr

Email:

Commission(s): 36

HEAVENS ALAN DR, ROYAL OBSERVATORY, BLACKFORD HILL, EDINBURGH EH9 3HJ, UK

Telephonc: (44) 1316688352

Facsimile: (\$4)

Commission(s): 47

Telex: 72383 roedin $g$

Email: afh@uk.ac.roc.star

HEBER ULRICH, DR REMEIS STERNWARTE, STERNWARTE STR 7, D 96049 BAMBERG, GERMANY

Telephone: (49) 9519522214

Facsimile: (49)

Telex:

Email:

Commission(s): 29,36

HECHT JAMES H DR, AEROSPACE CORPORATION, MS M2 255, BOX 92957, LOS ANGELES CA 90009, USA

Telephone: (1) 2133367017

Facsimile: (1)

Telex:

Email:

Commission(s): 21,34

HECK ANDRE DR, OBS DE STRASBOURG, 11 RUE UNIVERSITE, F 67000 STRASBOURG, FRANCE

Telephone: (33) 88358216

Facsimile: (33) 88250160

Telex: 890506 starobs $f$

Email: heck@frecse21.bitnet

Commission(s): $05,25,51$

HECKATHORN HARRY M, NAVAL RESEARCH LABORATORY, CODE $+1+32,+555$ OVERLOOK AVE SWWASHINGTON DC 20375 S000, USA

Telephone: (1) 2027672764

Email:

Facsimile: (1)

Telex:

Commission(s): 09,44 
HECKMAN TIMOTHY M, ASTRONOMY PROGRAM, UNIVERSITY OF MARYLAND, COLLEGE PARK MD 20742, USA

Telephenc: (1) 3014543001

Facsimilc: (1)

Telex: 7108260352 astr corp

Email:

Commission(s): 28

HECQUET JOSETTE DR, OBS MIDI PYRENEES, $1+$ AVE E BELIN, F 31400 TOULOUSE CDX, FRANCE

Telephone: (33) $61252101 \quad$ Facsimile: (33)

Telex: $\$ 30776$ f

Emaii:

Commission(s):

HEDDLE DOUGLAS W O PROF, DPT OF PHYSICS, ROYAL HOLLOWAY COLLEGE, UNIVERSITY OF LONDON EGHAM SURREY TW20 OEX, UK

Telephone: (44) $178+35351$

Facsimile: (4t)

Telcx: 935504

Email:

Commission(s): 14

HEDEMAN E RUTH MISS, 3440 ST JEFFERSON ST, FALLS CHURCH VA 220+1, USA

Telephone: (1)

Facsimile: (1)

Telex:

Email:

Commission(s):

HEESCHEN DAVID S DR, NRAO, 520 EDGEMONT RD, CHARLOTTESVILLE VA 22903, USA

Telephone: (1)

Facsimile: (1)

Telex:

Email:

Commission(s): 28,40,51

HEFELE HERBERT PH D, ASTRON RECHEN INSTITUTE, MOENCHHOFSTR 12-14

D 69120 HEIDELBERG, GERMANY

Telephone: (49)

Facsimile: (49)

Telex:

Email:

Commission(s): 05

HEFFERLIN RAY A PROF, DPT OF PHYSICS, SOUTHERN COLLEGE, DRAWER H COLLEGEDALE TN 37315 0370, USA

Telephonc: (1) 6152382869

Facsimile: (1)

Telex:

Email:

Commission(s): $1+$

HEFTY JAN DR, OBSERVATORY OF THE SLOVAK, TECHNICAL UNIVERSITY, RADLINSKEHO 11 SK 81368 BRATISLAVA, SLOVAK R

Telephone: (42) 7498047

Email: hefty@evt.stuba.cs

Facsimile: (\$2) $755+76$

Commission(s): 19

Telex: 92720 elfakc

HEGGIE DOUGLAS C DR, DPT OF MATHEMATICS, UNIVERSITY OF EDINBURGH, KING'S BUILDINGS, EDINBURGH EH9 3JZ, UK

Telephone: (44) 131667108

Email: d.c.heggie(a)ed.ac.uk

Facsimile: (4)

Commission(s): 07,37

Telex: 727442 univedg

HEGYI DENNIS J ASSOC PROF, RANDALL LABORATORY, UNIVERSITY OF MICHIGAN

ANN ARBOR MI 48109 1090, USA

Telephone: (1) 3137645448

Facsimile: (1)

Telex: $\mathbf{8 1 0 - 2 2 3 6 0 5 6}$

Email:

Commission(s):

HEIDMANN JEAN DR, OBSERVATOIRE DE PARIS, SECTION DE MEUDON, F 92195 MEUDON PPL CDX, FRANCE Telephone: (33) 145077598

Facsimile: (33)

Email:

Commission(s): $28,40,47,51 \mathrm{C}$

Telex: 270912

HEILES CARL PROF, ASTRONOMY DPT, UNIVERSITY OF CALIFORNIA, G01 CAMPBELL HALL BERKELEY CA 94720, USA

Telephone: (1) $\$ 156 \$ 24510$

Facsimile: (1)

Telex: 820181 ucb ast ral

Email:

Commission(s): $33,34,40$

HEIN RIGHINI GIOVANNA, 531 MAIN ST, ROOSEVELT ISLAND NY 10044, USA

Telcphonc: (1)

Email:

Facsimile: (1) Telex:

Commission(s): $12,34,44$

HEINRICH INGE, ASTRON RECHEN INSTITUTE, MOENCHHOFSTR 12-14, D 69120 HEIDELBERG, GERMANY

Telcphone: (49) 622149026

Facsinile: (49)

Telex:

Email:

Commission(s): 05

HEINTZ WULFF D DR, DPT OF ASTRONOMY, SWARTHMORE COLLEGE, SWARTHMORE PA 19081, USA

Telephone: (1) 2154477265

Facsimile: (1)

Telex:

Email:

Commission(s): 05,08,24,42 
HEINTZE J R W DR, STERREKUNDIG INSTITUTE, BOX 80000, NL 3508 TA UTRECHT, NETHERLANDS

Telephonc: (31) 30535235

Facsimile: (31)

Telex: 40048 fylut ni

Email: hitnet:wnmmail@ahutruu0

Commission(s): 29,3n

HEINZEL PETR DR, ASTRONOMICAL INSTITUTE, CZECH ACADEMY OF SCIENCES, ONDREJOV OBSERVATORY

CZ 251 65 ONDREJOV, CZECH R

Telephone: (42) 204 857233/7111

Facsimile: (42) 204851611

Telex: 121579 astr $\mathrm{c}$

Email: astsun(a)csearn

Commission(s): 10,12

HEISE JOHN DR, SPACE RESEARCH LABORATORY, SRON, SORBONNELAAN 2

NL 358+ CA UTRECHT, NETHERLANDS

Telephone: (31) 30535600

Email: j.heise(a)sron.ruu.nl

Facsimile: (31) 30540860

Telex: $\mathbf{4 7 2 2 4}$ astro $\mathrm{nI}$

Commission(s): 44

HEISER ARNOLD M DR, DYER OBSERVATORY, VANDERBILT UNIVERSITY, BOX 1803 STA B NASHVILLE TN 37235, USA

Tclephone: (1) 6153734897

Facsimile: (1)

Telex:

Email:

Commission(s): 27

HEJNA LADISLAV DR, FAC OF MATHS \& PHYSIC, CHARLES UNIVERSITY, MALOSTRANSKE NAM 2/25 CZ 11000 PRAHA 1, CZECH R

Telephonc: (+2) 2532132

Facsimile: (42) 2535222

Telex: $121673 \mathrm{mff} \mathrm{c}$

Email: Ihejna(a)spguk11

Commission(s): 12

HEKELA JAN DR, ASTRONOMICAL INSTITUTE, CZECH ACADEMY OF SCIENCES, ONDREJOV OBSERVATORY CZ 251 65 ONDREJOV, CZECH R

Telephone: (42) $20+857369$

Facsimile: $(+2) 20+851611 \quad$ Telex:

Email:

Commission(s): 36

HELALJ YHYA E DR, HELWAN OBSERVATORY, HELWAN, EGYPT

Telephone: (20) $7806+5 / 2683$

Facsimile: (20)

Commission(s): 07

Telex:

Email:

HELD ENRICO V, OSSERVATORIO ASTRONOMICO, VIA ZAMBONI 33, I 4126 BOLOGNA, ITALY
Telephone: (39) 51259301 Facsimile: (39) 51 259 +07 Telex:

Email: held(a)astbo3.bo.astro,it Commission(s): 5,28

HELFAND DAVID JOHN, COLUMBIA ASTROPHYSICS LAB, COLUMBIA UNIVERSITY, 538 W $120 T H$ ST NEW YORK NY 10027, USA

Telephone: (1) $21285+2150$

Email: bitnet:djht Qucuphyd

Facsimile: (1) 2123169504

Telex: 22009+ colu ur

HELFER H LAWRENCE PROF, DPT PIIYSICS \& ASTRONOMY, UNIVERSITY OF ROCHESTER ROCHESTER NY 1 4627 , USA

Telephone: (1) $716275+377$

Email:

Facsimile: (1)
Commission(s): 34

HELIN ELEANOR FRANCIS, JPL, MS 183,501, 4800 OAK GROVE DR, PASADENA CA 91109, USA

Telephone: (1) 8183544606

Facsimile: (1)

Telex: $675+29$

Email: eth051(a)jh.jpl.nasa.gov

Commission(s): $15,2(), 22$

HELlER MICHAEL PROF, POWSTANCOW WARSAWY 13/94, PL 33110 TARNOW, POLAND

Telephone: $(48)$

Facsimile: (48)

Telex:

Email:

Commission (s): 47

HELLWIG HELMUT WILHELM DR, FREQUENCY \& TIME SYSTEMS, 3 4 TOZER RD, BEVERLY MA 01915, USA

Telephone: (1) 6179278220

Facsimile: (1)

Telex: 940518

Email:

Commission(s): 31

HELMER LEIF, COPENHAGEN UNIVERSITY OBS, BRORFELDEVEJ 23, DK 4340 TOLLOSE, DENMARK

Telephone: $(45) 53+88195$

Facsimile: (45) $53+88755$

Telex: +4155

Email:

Commission(s): (88C,50)

HELMKEN HENRY F DR, CENTER FOR ASTROPIIYSICS, HCO/SAO, G0 GARDEN ST, CAMBRIDGE MA 02138, USA

Email:

Commission(s): 44 
M 586

HELOU GEORGE DR, CALTECH, MS 100 22, IPAC, PASADENA CA 91125, USA

Telephone: (1) 8183979555

Facsimile: (1) 8183979600

Telex: $58+9945$

Email: helou(a)ipac.caltech.edu

Commission(s): $05,28,34,40,44$

HELT BODIL E, UNIVERSITY OBSERVATORY, OESTER VOLDGADE 3, DK 1350 COPENHAGEN K, DENMARK

Telephone: (45) 31141790

Facsimile: (45) 31389157

Telex: $4+155$ danast $\mathbf{d k}$

Email:

Commission(s): 42

HEMENWAY MARY KAY M DR, ASTRONOMY DPT, UNIVERSITY OF TEXAS, RLM 15308

AUSTIN TX 78712 1083, USA

Telephone: (1) 5124711309

Facsimile: (1) $512+716016$

Telcx: $91087+1351$

Email: marykay@astro.as.utcxas.cdu

Commission(s): $41,+6$

HEMENWAY PAUL D DR, ASTRONOMY DPT, UNIVERSITY OF TEXAS, RLM 15 308, AUSTIN TX 78712 1083, USA

Telephone: (1) $512+71+461$

Facsimile: (1)

Tclex:

Email:

Commission(s): $08,20,2+C$

HEMMLEB GERHARD DR, ZNTRLINST F PHYSIK ERDE, TELEGRAFENBERG A 17, D 14473 POTSDAM, GERMANY

Telephonc: (49) $\$ 551$

Email:

Facsimile: (49)

Telex: 15305

HEMPE KLAUS, GRENZWEG $2+$ B, D 2057 REINBECK, GERMANY

Telcphone: (49) 407105628

Facsimile: (49)

Telex:

Email:

Commission(s):

HENDECOURT D' LOUIS DR, IAS, UNIVERSITE PARIS XI, BT 121, F 91405 ORSAY CEDEX, FRANCE

Telephonc: (33) 169858640

Facsimile: (33) 169858675

Telex: 6001252

Email:

Commission(s): $14,21,34$

HENDEN ARNE ANTHON, US NAVAL OBSERVATORY, BOX 1149, FLAGSTAFF AZ 86002 1149, USA

Telephone: (1) 6027795132

Facsimile: (1) $60277+3626 \quad$ Telex:

Email: aahanofs.navy.mil

Commission(s): 09,27

HENKEL CHRISTIAN, MPI FUER RADIOASTRONOMIE, AUF DEM HUEGEL 6\%, D 53121 BONN, GERMANY

Telephone: (49)

Facsimile: $\left(4^{\circ}\right)$

Telex: $886+40$

Email:

Commission(s): 34,40

HENNING THOMAS DR, UNIV STERNWARTE JENA, SCHILLERGAESSCHEN 2, D 07745 JENA, GERMANY

Telephòne: (49) 7827122

Facsimile: (49) $78+25(039)$

Telex: 05886134

Email: physfali@aphysik.uni-jena.dbp.de Commission(s): 34

HENON MICHEL C DR, OCA OBSERV DE NICE, BP 229, F 06304 NICE CDX 4 , FRANCE

Telephone: (33) $93890+20$

Facsimile: (33) 92 (1) 3033

Telex: $460000+$ obsnice $f$

Email:

Commission(s): $07,33,37$

HENOUX JEAN-CLAUDE DR, OBSERVATOIRE DE PARIS, SECTION DE MEUDON F 92195 MEUDON PPL CDX, FRANCE

Telephone: (33) 145077803

Facsimile: (33)

Telex:

Email:

Commission(s): 10,4t

HENRARD JACQUES PROF, DPT DE PHSIQUE, FACULTES UNIVERSITAIRES, RUE DE BRUXELLES 61 B 5000 NAMUR, BELGIUM

Telcphone: (32) 81229061

Email: jhenrardabnandps1

Facsimile: (32) 81230391

Telex: 59222

Commission(s): (14,07,20

HENRICHS HUBERTUS F DR, ASTRONOMICAL INSTITUTE, UNIVERSITY OF AMSTERDAM, KRUISLANN 403 NL 1098 SJ AMSTERDAM, NETHERLANDS

Telephone: (31) 205257491

Facsimile: (31) $31205257484 \quad$ Telex: 111262 hef $\mathrm{nl}$

Email:

Commission(s): 29)

HENRIKSEN RICHARD N DR, DPT OF PHYSICS, QUEEN'S UNIVERSITY, KINGSTON ON K7L 3N6, CANADA

Telephone: (1) 6135452719

Facsimile: (1) $6135456463 \quad$ Telex:

Email: henriksen $($ ) quednast.bitnet

Commission(s): $4+$ 
HENRY RICHARD B C DR, DPT PHYSICS \& ASTRONOMY, UNIVERSITY OF OKLAHOMA, NORMAN OK 73019, USA Telephone: (1) 4053253961

Facsimilc: (1)

Telex:

Email:

Commission(s): 28,35

HENRY RICHARD C PROF, DPT PHYSICS \& ASTRONOMY, JOHNS HOPKINS UNIVERSITY, CHARLES \& 3HTH ST BALTIMORE MD 21218, USA

Telephonc: (1) 3013387350

Facsimile: (1) Telex:

Email:

Commission(s): $21,+4$

HENSBERGE HERMAN, OBSERVATOIRE ROYAL BELGIQUE, AVE CIRCULAIRE 3, B 1180 BRUSSELS, BELGIUM

Telephonc: (32) 223730284

Facsimile: (32) $2237+9822$ Telex: 21565 obsbel

Email: hermanaastro.omo.be

Commission(s): 25,44

HENSLER GERHARD PROF, INST THEOR PHYS \& STERNW, UNIVERSITAET KIEL, OLSHAUSENSTR 40 D 24098 KIEL, GERMANY

Telephone: (49) 4318804125

Email: pas30@rani-kiel.dbp.de

Facsimile: (49) $\$ 318804+32 \quad$ Telex: 292706

Commission(s): 28,42

HERAS ANA M DR, ESAESTEC, SSD, BOX 299, NL 2200 AG NOORDWIJK, NETHERLANDS

Telejhone: (31) 171985016

Facsimile: (31) 171984698 Telex:

Email: estes1::aheras/aheras a estec

Commission(s): 49

HERBIG GEORGE H DR, INSTITUTE FOR ASTRONOMY, UNIVERSITY OF HAWAII, 2680 WOODLAWN DR HONOLULU HI 96822 , USA

Telephone: (1) 8089568312

Email:

Facsinile: (1) 8089882790

Telex: $723-8459$

Commission(s): 27,29

HERBST ERIC DR, DPT OF PHYSICS, OHIO STATE UNIVERSITY, 17+ W $18 T H$ AVE, COLUMBUS OH +3210, USA
Telephone: (1) $61+2922653$
$\begin{aligned} & \text { Email: } \\ & \text { Facsimile: (1) }\end{aligned}$

HERBST WILLIAM DR, DPT OF ASTRONOMY, VAN VLECK OBSERVATORY, WESLEYAN UNIVERSITY MIDDLETOWN CT 06457, USA

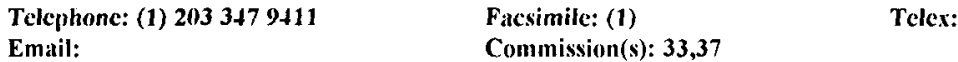

HERBSTMEIER UWE DR, MPI FUER ASTRONOMIE, KOENIGSTUHL, D 69117 HEIDELBERG, GERMANY

Telephone: (49) 6221528 355 Facsimile: (49) 6221528 246 Telex:

Email: herbsta astro Commission(s): 34

HERCZEG TIBOR J PROF DR, DPT PHYSICS \& ASTRONOMY, UNIVERSITY OF OKLAHOMA NORMAN OK 73019, USA

Telephone: (1) 4053253961

Email:

Facsimile: (1)

Telex:

Commission(s): $+2,51$

HERING ROLAND, ASTRON RECHEN INSTITUTE, MOENCHHOFSTR 12-14, D 69120 HEIDELBERG, GERMANY

Telephone: (49) 6221405157

Facsimile: (49) $6221405297 \quad$ Telex:

Email: s11ams.urz.uni-heidelberg.de Commission(s): 08

HERMAN JACOBUS DR, SOS, GUTENBERGSTR \%, POSTFACH 144\%, D 82199 GILCHING, GERMANY

Telephone: (49) 8153281167

Facsimile: (49)

Telex:

Email: jalap.hermanäudlr.de

Commission(s): 33

HERMANS DIRK DR, SCHOOL MATHS/STATISTICS, UNIVERSITY OF BIRMINGHAM, EDGBASTON BIRMINGHAM B15 2TT, UK

Telephone: (4) $121+1+3961$

Email: d.f.h.hermans(a)uk.ac.bham

Facsimile: (44) $121+1+3907$

Telex: 337262 uobham g

Commission(s): 10

HERMSEN WILLEM DR, SRL, HUYGENS LAB, BOX 95(4, NL 2300 RA LEIDEN, NETHERLANDS

Telephone: (31) $71275810 \quad$ Facsimile: (31)

Email: $\quad$ Commission(s):

Telex: 390158 astro $\mathrm{nl}$

HERNANDEZ CARLOS ALBERTO, OBSERVATORIO ASTRONOMICO, PASEO DEL BOSQUE S/N 1900 LA PLATA (BS AS), ARGENTINA

Telephone: (5t) 21217308

Facsimile: (54) 21211761

Telex:

Email:

Commission(s): 
HERNANDEZ-PAJARES MANUEL, DPT MATEMATICA APLI I TEL, UNIV POLITECNICA CATALUNA CAMPUS NORD ED C3, E 08034 BARCELONA, SPAIN

Telephone: (34) $34016029 \quad$ Facsimile: (34)

Email: matmhp@mat.upc.es

Facsimile: (34)

Telex:

HERNQUIST LARS ERIC DR, LICK OBSERVATORY, UNIVERSITY OF CALIFORNIA, NTL SCIENCES II SANTA CRUZ CA 95064, USA

Telephone: (1) $408 \$ 25 \$ 733$

Email: lars@ghelios.ucsu.edu

Facsimile: (1) $408 \$ 263115 \quad$ Telex: 7607936

Commission(s): 28

HEROLD HEINZ, LEHRSTUHL F THEORET ASTRO, PHYSIK DER UNIV TUEBINGEN

AUF DER MORGENSTELLE 12 C, D 72076 TUEBINGEN, GERMANY

Telephone: (49) 7071292043 Facsimile: (49) Telex:

Email:

Commission(s): 14,36

HERR RICHARD B DR, DPT OF PHYSICS, UNIVERSITY OF DELAWARE, NEWARK DE 19716, USA

Telephonc: (1) $302+512673$

Email:

Facsimile: (1)

Telex:

HERRERA MIGUEL ANGEL DR, INSTITUTO DE ASTRONOMIA, UNAM, APDO POSTAL 70-264

04510 MEXICO DF, MEXICO

Telephone: (52) $905 \$ 485305$

Email: bitnet:manher(a)unamvm 1

Facsimile: (52)

Telex: 1760155 cicme

HERRERO DAVO ARTEMIO DR, INST DE ASTROFISICA, DE CANARIAS, OBS DEL TEIDE, E 38200 LA LAGUNA, SPAIN

Telephone: (34) 22262211

Facsimile: (34)

Emait:

Comnission(s):

Telex: 92640

HERRMANN DIETER PROF DR, ARCHENHOLD STERNWARTE, ALT TREPTOW 1, D II93 BERLIN, GERMANY

Telephone: (49) $2728871 * 494$

Facsimile: (49)

Telex:

Email:

Conmission(s): 41

HERS JAN MR, BOX 48, SEDGEFIELD 6573, SOUTH AFRICA

Telephone: $(27)+455736$

Facsimile: $(27)$

Telex:

Email:

Commission(s): $06,20,27,31$

HERSHEY JOHN L DR, US NAVAL OBSERVATORY, 3450 MASSACHUSETTS AVE NW WASHINGTON DC 203925100 , USA

Telephone: (1) $2026531554 \quad$ Facsimile: (1)

Telex: $710-822-1970$

Enail:

Commission(s): $24,26,51$

HERTZ PAUL L DR, NAVAL RESEARCH LABORATORY, CODE $+1215,+555$ OVERLOOK AVE SW WASHINGTON DC 203755000, USA

Telephone: (1) 2027672438

Facsimile: (1)

Telex:

Email:

Commission(s):

HERZBERG GERHARD DR, HERZBERG INST ASTROPHYS, NTL RESEARCH COUNCIL, 100 SUSSEX DR OTTAWA ON K1A OR6, CANADA

Telephone: (1) 6139900917

Facsimile: (1) 61395266012

Telex: 0533715

Email:

Commission(s): 14,15,16,34

HESKE ASTRID DR, ESA/ESTEC, ASTROPHYSICS DIV, BOX 299, NL 2200 AG NOORDWIJK, NETHERLANDS Telephone: (31) 171983173

Facsimile: (31) 171984690

Telex: 39098

Email: estes1::aheske/aheskéajestec

Commission(s): $29,40,44$

HESSER JAMES E DR, HERZBERG INST ASTROPHYS, DOMINION ASTROPHYS OBS, 5071 W SAANICH RD VICTORIA BC V8X 4 M6, CANADA

Telephone: (1) $60+3883974$

Email:

Facsimile: (1) 6043630045

Telex: 0497295

Commission(s): $14,27,37$

HESSMAN FREDERIC VICTOR, UNIVERSITAETS STERNWARTE, GOETTINGEN, GEISMARLANDSTR 11 D 37083 GOETTINGEN, GERMANY

Telephone: (49) 551395052

Email: hessmanajuni-sw.gwdg.de

Facsimile: (49) $5513450+3$

Commistion(s): 29
Telex: 
HEUDIER JEAN-LOUIS DR, OCA OBSERV DE NICE, BP 22\%, F 06304 NICE CDX 4, FRANCE

Telephone: (33) 92003011

Facsimile: (33) 92003033

Telex: 460004 obsnice $f$

Email:

Commission(s): $09,20,24,37,46,51$

HEWETT PAUL, ASTRONOMY INSTITUTE, THE OBSERVATORIES, MADINGLEY RD, CAMBRIDGE CB3 OHA, UK Telcphone: (4t) $12233375+8$

Email: Facsimile: (44) $1223337523 \quad$ Telex: 817297 astron g

Commission(s): 30,47

HEWISH ANTONY PROF, MULLARD RADIO ASTRON OBS, CAVENDISH LABORATORY, MADINGLEY RD

CAMBRIDGE CB3 OHE, UK

Tclephone: (44) 1223337294

Email:

Facsimile: (44) $1223354599 \quad$ Telex: 81292

Commission(s): 40

HEWITT ADELAIDE, CASS, UCSD, C 011 , LA JOLLA CA 92093 (1216, USA

Telephonc: (1) $61953+66,27$

Facsimile: (1)

Telex:

Email: int:hewitt\%cass.span(a)ucsoledu Commission(s): 28,47

HEWITT ANTHONY V DR, GE MEDICAL SYSTEMS, PET ENGINEERING, BOX 206, PEWAUKEE WI 53072, USA

Telephone: (1) $+1+3205170$

Facsimile: (1)

Telex:

Email:

Commission(s): $(09,28$

HEY JAMES STANLEY DR, \& SHORTLANDS CLOSE, EASTBOURNE BN22 0JE, UK

Telephone: (4)

Email:

Facsimile: (4) Telex:

Commission(s): $22,+10$

HEYDARI-MALAYERI M, OBSERVATOIRE DE MEUDON, DEMIRM, F 92195 MEUDON PPL CDX, FRANCE

Telephone: (33)

Facsimile: (33)

Telex:

Email:

Commission(s): 34

HEYVAERTS JEAN DR, OBSERVATOIRE DE PARIS, SECTION DE MEUDON, F 92195 MEUDON PPL CDX, FRANCE

Telephone: (33) $1+5077+105$

Facsimile: (33)

Telex: 201571

Email:

Commission(s): 49

HibBS ALBERT R MGR PLANS, 781 PROSPECT BL, PASADENA CA 91103, USA

Telephonc: (1) Facsimile: (1) Telex:

Email:

Commission(s):

HICKSON PAUL DR, DPT GEOPHYS \& ASTRONOMY, UNIV OF BRITISH COLUMBIA, 2219 MAIN MALL

VANCOUVER BC VGT IW5, CANADA

Telephonc: (1) $60+2282267$

Facsimile: (1) $601+2286047 \quad$ Telex:

Email:

Commission(s): 28

HIDALGO MIGUEL A DR, FAC DE CIENCIAS FISICAS, UNIVERSIDAD DE ZARAGOZA, E 50009 ZARAGOZA, SPAIN Telephonc: (3ł) Facsimile: (34)

Commission(s):

Telex:

Email:

HIDAYAT BAMBANG PROF DR, BOSSCHA OBSERVATORY, LEMBANG 40391, INDONESIA

Telephone: (62) 2228 6001/60127 Facsimile: (62) $22287289 \quad$ Telex: 28234 bd itb

Email:

Commission(s): $26,34,46,50), E C$

HIDE RAYMOND PROF, GEOPHYSICAL FLUID, DYNAMICS LABORATORY, METEOROLOGICAL OFFICE BRACKNELL BERKS RG12 2SZ, UK

Telephone: $(4+) 13+4+22+2$

Facsinile: (4t)

Telex: 849801

Email:

Commission(s): 16,19

HIEI EIJIRO DR, TOKYO ASTRONOMICAL OBS, NAOJ, OSAWA MITAKA, TOKYO 18I, JAPAN

Telejhone: (81) +22325111

Facsimile: (81)

Telex: 2822307

Email:

Commission(s): 10,12

HIGGS LLOYD A DR, DOMINION RADIO ASTROPHYS, OBSERVATORY, BOX 248 PENTICTON BC V2A 6K3, CANADA

Telephone: (1) $60+4932277$

Facsimile: (1) $60+4937767$

Telex: 04888127

Email: lahodrao.nrc.ca

Commission(s): 34,40 
HILDEBRAND ROGER H, ENRICO FERMI INSTITUTE, UNIVERSITY OF CHICAGO, 5640 S ELLIS AVE CHICAGO IL 60637, USA

Telcphonc: (1) 3129627581

Email:

\author{
Facsimile: (1) \\ Commission(s): 34
}

Telex:

HILDEBRANDT JOACHIM, ASTROPHYSIKALISCHES INST, POTSDAM UNIVERSITY, AN DER STERNWARTE 1GD $1+482$ POTSDAM, GERMANY

Telephonc: (49) 33177138

Email: jhildebrandt(a)aip.de

Facsimile: (49) $33175105 \quad$ Telex: 361231 stbbg d

Commission(s): 10

HILDITCH RONALD W DR, DPT PHYSICS \& ASTRONOMY, UNIVERSITY OF ST ANDREWS, NORTH HAUGH ST ANDREWS FIFE KY16 9SS, UK

Telephonc: (44) 133+76161

Facsimile: $(44)$ 133 $7+487$

Telex: 93121108+6 sa g

Email: assrhauk.ac.st-and.star

Commission(s): $25,30,42$

r HILDNER ERNEST DR, DIRECTOR R/S/SE, SPACE ENVIRONMENT LAB, 325 BROADWAY

BOULDER CO 80303 3328, USA

Telephone: (1)

Facsimilc: (1)

Telex:

Email:

Commission(s): 10,12

HILF EBERHARD R H PH D, PESTRUPSWEG 30, D 2900 OLDENBURG, GERMANY

Telephone: (49)

Facsinile: $(49)$ Telex:

Email:

Commission(s): 35,40

HILL FRANK DR, NTL SOLAR OBSERVATORY, BOX 26732, 950 N CHERRY AVE, TUCSON AZ 85726 6732, USA

Telephone: (1) $520323+138$

Email: fhillanoao.edu

Facsimile: (1) 5203259278

Commission(s): 12C

Telex: $1561+111$ aura ut

HILL GRAHAM DR, HERZBERG INST ASTROPHYS, DOMINION ASTROPHYS OBS, 5071 W SAANICH RD VICTORIA BC V8X $4 M 6$, CANADA

Telephone: (1) 6023883935

Email:

Facsimile: (1) 6043630045

Commission(s): $24,26,31,+2 \mathrm{C}$

Telex: 0497295

-HILL HENRY ALLEN DR, DPT OF PHYSICS, UNIVERSITY OF ARIZONA, BLDG 81, TUCSON AZ 85721, USA

Telephone: (1) $5206216784 \quad$ Ficsimile: (1)

Telex: $910-95211+3$

Email:

Commission(s): 27

HILL PHILIP W DR, DPT PHYSICS \& ASTRONOMY, UNIVERSITY OF ST ANDREWS, NORTH HAUGH ST ANDREWS FIFE KY16 9SS, UK

Telephonc: (+4) $133+76161$

Facsimile: $(44) 133+7+487$

Telex: 76213 saulib g

Email: p.w.hilläuk.ac.st-andews

Commission(s): 25,27

: HILLEBRANDT WOLFGANG PH D, MPI F PHYSIK \& ASTROPHYS, KARL SCHWARZSCHILDSTR 1

D 85740 GARCHING MUENCHEN, GERMANY

$\begin{array}{ll}\text { Telephone: (49) } 8932999409 & \text { Facsimile: (49) } \\ \text { Ematil: } & \text { Commission(s): }\end{array}$

Telex:

HILLIARD R DR, OPTOMECHANICS RES INC, BOX 87, X9 RANCH, VAIL AZ 85641, USA

Telephonc: (1) 60126473332

Email:

Facsimile: (1) $6026+73312$ Telex:

Commission(s): (09)

HILLS JACK G DR, LOS ALAMOS NATIONAL LAB, MS B228, THEORETICAL DIV T6, LOS ALAMOS NM 87545, USA Telephonc: (1) 5056679152 Facsimile: (1)

Commission(s): 37,42

Telex:

Email:

HILLS RICHARD E DR, MULLARD RADIO ASTRON OBS, CAVENDISH LABORATORY, MADINGLEY RD CAMBRIDGE CB3 OHE, UK

Telephone: (44) 1223337294

Facsimile: (4) $1223354599 \quad$ Telex: 81282

Email:

Commission(s): 4l

HILTON JAMES LINDSAY, US NAVAL OBSERVATORY, 3450 MASSACHUSETTS NW, WASHINGTON DC 20392, USA Telephone: (1) 2026531568

Email: hiläham.usno.navy.mil

Facsimile: (1) 2026.531744

Commission(s): (14 
HILTON JOHN DR, DPT OF MATH SCIENCES, GOLDSMITHS' COLLEGE, NEW CROSS, LONDON SE14 GNW, UK

Telephonc: (4)

Email:
Facsimile: (4t)

Commission(s):
Telex:

HINDSLEY ROBERT BRUCE, † B SOUTHNAY RD, GREENBELT MD 20770, USA

Telephone: (1) 2026530951

Facsimile: (1) $2026531497 \quad$ Telex:

Commission(s): 26

HINKLE KENNETH H, KITT PEAK NTL OBS, BOX 26732, 950 N CHERRY AVE, TUCSON AZ 85726 6732, USA

Telephone: (1) 5203275511

Facsimile: (1)

Commission(s): 29

Telex: $0666-48+$ aura noan $t$

Email:

HINNERS NOEL W DR, LOCKHEED MARTIN ASTRONAUT, MS S8000, BOX 179, DENVER CO 80201, USA

Telephonc: (0) 3039711581

Facsimile: (0) $3039712390 \quad$ Telex:

Email: noel.w.hinners(a)den.mmn.com

Commission(s): 51

HINTZEN PAUL MICHAEL, N DR, NASA GSFC, CODE 681, GREENBELT MD 20771, USA

Telephone: (1) 3012865101

Facsimile: (1)

Telex:

Enail:

Commission(s): 28

HIOTELIS NICOLAOS DR, DPT OF ASTRONOMY, NTL UNIVERSITY OF ATHENS, PANEPISTIMIOPOLIS

GR 15783 ZOGRAFOS, GREECE

Telephone: (30) $172+3+14$

Email: nhiot@grathun1

Facsimile: (30)

Telex:

Commission(s): 28

HIPPELEIN HANS H DR, MPI FUER ASTRONOMIE, KOENIGSTUHL, D 69117 HEIDELBERG, GERMANY

Telephone: (49)

Facsimile: (49)

Telex:

Email:

Commission(s): 34

HIRABAYASHI HISASHI DR, NOBEYAMA RADIO OBS, NAOJ, MINAMIMAKI MURA, NAGANO 384 13, JAPAN

Telephonc: (81) 267982831

Facsimile: (81)

Commission(s): 40,51

Telex: 3329005

Email:

HIRAI MASANORI DR, DPT EARTH SCI \& ASTRONOMY, FUKUOKA UNIVERSITY OF EDU, 729 MUNAKATA

FUKUOKA 811-41, JAPAN

Telephone: (81) 940322381

Facsimile: (81)

Telex:

Email:

Commission(s): 29

HIRATA RYUKO, DPT OF ASTRONOMY, KYOTO UNIVERSITY, KITASHIRAKAWA SAKYO KU, KYOTO GOG, JAPAN

Telephone: $(81)$

Facsimile: (81)

Telex:

Email: hirata(äkuastro.kyoto-u.ac.jp Commission(s): 29

HIRAYAMA TADASHI PROF, TOKYO ASTRONOMICAL OBS, NAOJ, OSAWA MITAKA, TOKYO 181, JAPAN

Telephone: (81) $\$ 22325111$

Email:

Facsimile: (81)

Commission(s): 10,12

Telex: 2822307 taomtk-j

HIROMOTO NORIHISA DR, COMMUNICATIONS RES LAB, 4-2-1 NUKUIKITAMACHI, KOGANEI, TOKYO 184, JAPA

Telephone: $(81)+23277548$

Email: hiromoto a be.crl.go.jp

Facsimile: (81) $\$ 23276667$

Telex: 2832611 dempa j

Commission(s): $3 \downarrow$

HIRST WILLIAM P, 1 CLIFFORD CRESENT, BERGVLIET 7945, SOUTH AFRICA

Telephone: (27) Facsimile: (27) Telex:

Email:

Commission(s):

HIRTH WOLFGANG ERNST PH D, THEODOR-HEUS STR 18, D 535 $\downarrow$ WEILERSWIST, GERMANY

Telephone: (49)

Facsimile: (49)

Telex:

Email:

Commission(s):

HITOTSUYANAGI JUICHI PROF, KATAHIRA 1-4-(6-401, SENDAI 980, JAPAN

Telephonc: (81) 222279351

Facsimile: (81)

Commission(s): 35,36

Telex:

Email:

$(\mathrm{s}) .35,36$

HJALMARSON AKE G DR, ONSALA SPACE OBSERVATORY, CHALMERS UNIV OF TECH $S+3992$ ONSALA, SWEDEN

Telephone: (46) 317725500

Email: 
HJELLMING ROBERT M DR, NRAO, BOX 0, SOCORRO NM 87801 0387, USA

Telephone: (1) 5058357273

Facsimile: (1) 5058537027

Telex: 910-988-1710

Email: rhjellmi@inrao.edu

Commission(s): $34,40,42$

HNATYK BOHDAN, INST APPLIED PROBLEMS IN, MECHANICS \& MATHEMATICS, NAUKOV STR 3-6 290053 LVIV 53, UKRAINE

Telephone: (7) $03227+1114$

Facsimile: (7)

Telex:

Email:

Commission(s): 47

HO PAUL T P, SMITHSONIAN ASTROPHYS OBS, 60 GARDEN ST, CAMBRIDGE MA 02138, USA

Telejhone: (1) 6174953627

Facsimile: (1)

Telex: 921428

Email:

Commission(s): 40

HOAG ARTHUR A DR, \$110 E 14TH ST, TUCSON AZ 85711, USA

Telephone: (1) $520795864+$

Facsimile: (1)

Telex:

Email:

Commission(s): 50

HOANG BINH DY DR, OBSERVATOIRE DE PARIS, SECTION DE MEUDON, LAM F 92195 MEUDON PPL CDX, FRANCE

Telephone: (33) 145077445

Facsimile: (33)

Telex: 201571

Email:

Commission(s): $12,14,40,51$

HOARE MELVIN DR, MPI FUER ASTRONOMIE, KOENIGSTUHL, D 69117 HEIDELBERG, GERMANY

Telephone: (49)

Facsimile: (49)

Telex:

Email:

Commission(s): 36

HOBBS LEWIS M DR, YERKES OBSERVATORY, UNIVERSITY OF CHICAGO, BOX 258

WILLIAMS BAY WI 53191, USA

Telephone: (1) $41+2455555$

Facsimile: (1)

Telex:

Email:

Commission(s): 34

HOBBS ROBERT W DR, CTA INCORPORATED, 6116 EXECUTIVE BLVD, SUITE 800, ROCKVILLE MD 20852, USA

Telephone: (1)

Facsimile: (1)

Tclex:

Email:

Commission(s): 33,40

HODAPP KLAUS-WERNER, INSTITUTE FOR ASTRONOMY, UNIVERSITY OF HAWAII, 2680 WOODLAWN DR HONOLULU HI 96822 , USA

Telephonc: (1) 8089568968

Email: hodapp@afa.hawaii.edu

Facsimile: (1) $8089882790 \quad$ Telex:

Commission(s): 09,37

HODGE PAUL W PROF, DPT OF ASTRONOMY, UNIVERSITY OF WASHINGTON, FM 20, SEATTLE WA 98195, USA

Telephone: (1) 2065432888

Facsimile: (1)

Commission(s): 22,28

Telex: $910+740096$

Email:

HOEFLICH PETER DR, MPI F PHYSIK \& ASTROPHYS, KARL SCHWARZSCHILDSTR 1

D 857 40 GARCHING MUENCHEN, GERMANY

Telephone: (49) $8932993249 \quad$ Facsimile: (49) $8932993235 \quad$ Telex: 524629 astro d

Email: pah(â)dgaipp1s Commission(s): 29,36

HOEG ERIK DR, UNIVERSITY OBSERVATORY, OESTER VOLDGADE 3, DK 1350 COPENHAGEN K, DENMARK

Telephone: (45) 31141790

Facsimilc: (45) 31389157

Telex: +4155 danast

Email:

Commission(s): 08

HOEGBOM JAN A DR, STOCKHOLM OBSERVATORY, S 13336 SALTSJOEBADEN, SWEDEN

Telephone: (46) 87170195

Facsimile: (46) 87174719

Telex: 12972 sobserv s

Email: hogbomajastro.su.se

Commission(s): 40,51

HOEGLUND BERTIL PROF, ONSALA SPACE OBSERVATORY, CHALMERS UNIV OF TECH S $\$ 3992$ ONSALA, SWEDEN

Telephone: (46) 300606,52

Facsimile: $(46)$

Telex: 2400

Email:

Commission(s): 34,40

HOEKSTRA ROEL DR, TPD/TNO/TH, BOX 155, NL 2600 AD DELFT, NETHERLANDS

Telephone: (31)

Facsimile: (31)

Telex:

Email:

Commission(s): 
HOESSEL JOHN GREG, WASHBURN OBSERVATORY, UNIVERSITY OF WISCONSIN, 475 N CHARTER ST MADISON WI 53706, USA

Telephone: (1) 6082621752

Facsimile: (1)

Telex:

Email:

Commission(s):

HOEY MICHAEL J DR, DPT OF PHYSICS, UNIVERSITY COLLEGE, BELFIELD, DUBLIN 4, IRELAND

Telephone: (353) 1693244

Facsimile: (353)

Telex:

Email:

Commission(s):

HOFF DARREL BARTON, BOX 313, CALMAR IA 52132, USA

Telephone: (1)

Facsimile: (1)

Telex:

Email:

Commission(s): 46

HOFFLEIT E DORRIT DR, DPT OF ASTRONOMY, YALE UNIVERSITY, BOX 6666, NEW HAVEN CT 06520, USA

Telephone: (1)

Facsimile: (1)

Telex:

Email:

Commission(s): 24,27

HOFFMAN JEFFREY ALAN DR, NASAJOHNSON SPACE CENTER, CODE CB 4, HOUSTON TX 77058, USA

Telcphone: (1) $713+832+11 \quad$ Facsimile: (1)

Commission(s): 44

Telex:

Email:

HOFFMANN MARTIN DR, OBSERVATORIUM HOHER LIST, UNIV STERNWARTE BONN, D 5568 DAUN, GERMANY Tclephone: (49)

Facsimile: (49)

Commission(s): 42

Telex:

Email:

HOFMANN WILFRIED DR, ASTRON RECHEN INSTITUTE, MOENCHHOFSTR 12-14, D 69120 HEIDELBERG, GERMANY

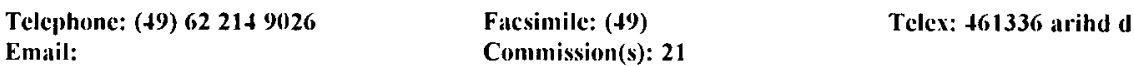

HOGAN CRAIG J DR, DPT OF ASTRONOMY, UNIVERSITY OF WASHINGTON, FM 20, SEATTLE WA 98195, USA

Telephone: (1)

Facsimile: (1)

Telex:

Email:

Commission(s):

HOGG DAVID E DR, NRAO, 520 EDGEMONT RD, CHARLOTTESVILLE VA 22903, USA

Telephone: (1) 80+2960220

Facsimile: (1)

Telex:

Email:

Commission(s): 40

HOHENKERK CATHERINE, HMNAUTICAL ALMANAC OFFICE, ROYAL GREENWICH OBS, MADINGLEY RD

CAMBRIDGE CB3 OEZ, UK

Telephone: (44) $122337+738$

Email: cyhâamail.ast.canı.ac.uk

Facsimile: (4) $122337+700$ Telex:

Commission(s): (0廿S

HOLBERG JAY B, LUNAR \& PLANETARY LAB, UNIVERSITY OF ARIZONA, 901 GOULD SIMPSON BLDG TUCSON AZ 85721, USA

Telephone: (1) $520621+301$

Facsimile: (1)

Telex: 9109521143

Email:

Commission(s): 16,4t

HOLLENBACH DAVID JOHN DR, NASA AMES RESEARCH CTR, MS 2456, MOFFETT FIELD CA 94035, USA

Telephone: (1) $\$ 159976+26$

Facsimile: (1)

Telex:

Email:

Commission(s): 34

HOLLIS JAN MICHAEL DR, NASA GSFC, CODE 930, GREENBELT MD 20771, USA

Telephone: (1)

Facsimile: (1)

Telex:

Email:

Commission(s): $34,40,51$

HOLLOWAY NIGEL J DR, SAFETY \& RELIABILITY DIR, WIGSHAW LANE, CULCHETH WARRINGTON WA3 +NE, UK

Telcphone: (4t) 19531244

Facsimile: (4t)

Commission(s): $4+$

Telex: 629301

Email:

ALAMOS NATIONAL LAB, MS B220 X2, LOS ALAMOS NM 87545, USA

HOLLOWELL DAVID EARL DR, LOS ALAMOS NATIO

Telex:

Email: u095874(a)lanl.gov

Commission(s): 35 
HOLLWEG JOSEPH V, DPT OF PHYSICS, UNIV OF NEW HAMPSHIRE, DEMERITT HALL, DURHAM NH O3824, USA Telephone: (1) 6038623869 Facsimile: (1) Telex:

Email:

Commission(s): 10,49C

HOLMAN GORDON D, NASA GSFC, CODE 682, GREENBELT MD 20771, USA

Telephone: (1) $3012864636 \quad$ Facsimile: (1) Telex:

Email: holman@stars.gsfc.nasa.gov Commission(s): 10

HOLMBERG ERIK B PROF, ENELIDEN 2, S 433 00 PARTILLE, SWEDEN

Telephone: (46) 31265842 Facsimile: (46) Telex:

Email:

Commission(s): 25,28

HOLT STEPHEN S, NASA GSFC, CODE 660, GREENBELT MD 20771, USA

Teleptione: (1) 3012868801

Facsimile: (1)

Telex:

Email:

Commission(s): 42,44

HOLWEGER HARTMUT PROF, INST THEOR PHYS \& STERNW, UNIVERSITAET KIEL, OLSHAUSENSTR 40

D 24098 KIEL, GERMANY

Telephone: (49) 4318804107

Facsimilc: (49)

Commission(s): 12,36

Telex: 292706

Email:

HQLZER THOMAS EDWARD DR, HIGH ALTITUDE OBSERVATORY, NCAR, BOX 3000, BOULDER CO 80307 3000, US Telephone: (1) $303+971536$

Facsimile: (1) $303+971568$

Telex: 45694

Email:

Commission(s): $10,36,49$

HONEYCUTT R KENT PROF, ASTRONOMY DPT, INDIANA UNIVERSITY, SWAIN WEST 319

BLOOMINGTON IN 47405, USA

Telephonc: (1) 8123356916

Facsimile: (1)

Telex:

Email:

Commission(s): 09,42

HONG HYON IK, DPT OF PHYSICS, KIM IL SUNG UNIVERSITY, TAESONG DISTRICT, PYONGYANG, KOREA DPR Telephone: (850)

Facsimile: (850)

Telex:

Email:

Commission(s): 10

HONG SEUNG SOO DR, DPT OF ASTRONOMY, SEOUL NTL UNIVERSITY, KWANAK KU, SEOUL 151, KOREA R

Telephone: (82) 8772131

Facsimile: (82)

Commission(s): $21 \mathrm{C}, 22,3+$

Telex: 29664

Email:

Facsimile: (86)

Telex:

HONG XIAO YU, CHINA PR

Telephone: (86)

Commission(s): 40

Email:

S, UNIVERSITY OF ST ANDREWS, NORTH HAUGH

HOOD ALAN, DPT OD APPLIED
ST ANDREWS FIFE KY16 9SS, UK

Telephone: (44) 1334761 61

Facsimile: (4t)

Telex: 76213

Email:

Commission(s): 10

HOOD ALAN DR, DPT OF MATHEMATICAL, UNIVERSITY OF ST ANDREWS, ST ANDREWS, FIFE KY16 9SS, UK

Telephone: (44) $133+76213 \quad$ Facsimile: (4)

Email: amsah(asavb.st-and.ac.uk. Commission(s):

Telex: 932110846 sa g

HOOGHOUDT B G IR, WATERTUIN 46, NL 2362 XD WARMOND, NETHERLANDS

$\begin{array}{ll}\text { Telephone: (31) } & \text { Facsimile: (31) } \\ \text { Email: } & \text { Commission(s): 09,40 }\end{array}$

HOPP ULRICH DR, MPI FUER ASTRONOMIE, KOENIGSTUHL, D 69117 HEIDELBERG, GERMANY

Telephonc: (49) 6221528224

Email: hopp@dhdmpisv.bitnct

Facsimile: (49) $6221528246 \quad$ Telex: $\$ 61789$ mpia d

Commission(s): 28

HOREDT GEORG PAUL DR, DFVLR, D 8031 WESSLING, GERMANY

Telephone: (49)

Facsimile: (49)

Commission(s): 16

Telex:

Email:

HORI GENICHIRO PROF, DPT OF ASTRONOMY, UNIVERSITY OF TOKYO, BUNKYO KU, TOKYO 113, JAPAN

Telephone: $(81) 38122111^{*}+251$

Facsimile: (81)

Telex: 33659 utyosci $j$

Email:

Commission(s): 07,33 
HORIUCHI RITOKU DR, NTL INSTITUTE FOR, FUSION SCIENCE, NAGOYA 464 01, JAPAN

Telephone: (81) 52781511

Facsimile: (81) 527827106

Telex: 0447-3691 nifs

Email:

Commission(s): 42

HORNE KEITH, DPT PHYSICS \& ASTRONOMY, UNIVERSITY OF ST ANDREWS, NORTH HAUGH

ST ANDREWS FIFE KY16 9SS, UK

Telephone: (44) 1334463322

Facsimile: (44) $133+463104$

Telex:

Email: keith.horne $a$ st-and.ac.uli

Commission(s):

HOROWITZ PAUL PROF, DPT OF PHYSICS, HARVARD UNIVERSITY, 60 GARDEN ST, CAMBRIDGE MA 02138, USA Telephone: (1) $617+953265$

Facsimile: (1)

Telex: 4992111

Email:

Commission(s): 51

HORSKY JAN PROF, DPT THEORET PHYS \& ASTRO, PURKYNE UNIVERSITY, KOTLARSKA 2

CZ 61137 BRNO, CZECH R

Telephone: (+2) 57129111

Email: horsky(a)clanor.sci.muni.cs

Facsimile: (42)

Telex:

Commission(s):

HORTON BRIAN H DR, JACABRI ENT, BOX 309, GOOLWA SA 5214, AUSTRALIA

Telephone: (61) $85553376 \quad$ Facsimile: (61) Telex:

Email:

Commission(s): 12

HORVATH ANDRAS DR, TIT PLANETARIUM \&, URANIA OBSERVATORY, BOX $\$ 6$, H $1+76$ BUDAPEST, HUNGARY

Telephone: (36) $133+25$

Facsimile: (36)

Commission(s):

Telex:

Email:

Facsimile: (972)

Commission(s):
TERTSALF, JERUSALEM 91904, ISRAEL

Telephone: (972) $2584 \$ 92$

Email:

Telex:
HOSHI REIUN DR, DPT OF PHYSICS, RIKKYO UNIVERSITY, NISHI-IKEBUKURO, TOSHIMA KU TOKYO 171, JAPAN Telcphone: (81) $39852+14$ Facsimile: (81)

Commission(s): 35

Telex:

Email:
HOSKIN MICHAEL A DR, CHURCHILL COLLEGE, CAMBRIDGE CB3 ODS, UK

Telephone: (t) $1223358381 \quad$ Facsimile: (tt)

Commission(s): +1

Teicx:

Email:

HOSKING ROGER J PROF, JAMES COOK UNIVERSITY, OF NORTH QUEENSLAND TOWNSVILLE QLD 4811, AUSTRALIA

$\begin{array}{ll}\text { Telephone: (61) } 778141113 & \text { Facsimile: }(61) \\ \begin{array}{ll}\text { Email: } & \text { Commission(s): }\end{array}\end{array}$

HOSOKAWA YOSHIMASA H PROF, SAKIGAOKA 3-4-9, FUNABASHI CITY, CHIBA PREFECTURE 274, JAPAN

Telephone: $(81)+74+86679$

Facsimile: (81)

Telex:

Email:

Commission(s):

HOTINLI METIN DR, UNIVERSITY RASATHANESI, ISTANBUL, TURKEY

Telephone: (90)

Facsimile: (90)

Commission(s): 12,36

Email:

HOUCK JAMES R, DPT OF ASTRONOMY, CORNELL UNIVERSITY, 220 SPACE SCIENCE BLDG, ITHACA NY 14853, USA

$\begin{array}{ll}\text { Telephone: (1) } 6072564806 & \text { Facsimile: (1) } 6072552365 \\ \text { Email: } & \text { Commission(s): }\end{array}$

HOUDEBINE ERIC, SPACE SCIENCE DPT, SOLAR SYSTEM DIV, ESTEC ESA POSTBUS 299

NL 2201 AG NOORDWIJK, NETHERLANDS

Telephone: (31) $17198+8208$

Facsimile: (31) $17198+697 \quad$ Telex:

Email: crhässo.estec.esa.nl

Commission(s): 10

HOUGH JAMES DR, DPT PHYSICS \& ASTRONOMY, UNIVERSITY OF GLASGOW, GLASGOW G12 8QQ, UK

Telephone: $(+4) 1+1330+706$

Facsimile: (4t) $14133+9029$

Telex: 777070 unigla

Email: gwot\%gla.ph.ila ak,ac,rl.bitnct

Commission(s): 09 
HOUK NANCY DR, DPT OF ASTRONOMY, UNIVERSITY OF MICHIGAN, 1045 PHYS-ASTRO BLDG ANN ARBOR MI 48109 1090, USA

Telephone: (1) 3137643436

Facsimile: (1)

Telex:

Email:

Commission(s): $27,29,45$

HOUSE FRANKLIN C DR, HEIDENREICHSTR +2, D 6100 DARMSTADT, GERMANY

Telephone: (49) $6151422+12$ Facsimile: (49) Telex:

Email: $\quad$ Commission(s):

HOUSE LEWIS L DR, US WEST ADVANCED TECHNOLO, 6200 S QUEBEC SUITE 320, ENGLEWOOD CO 80111, USA

Telephone: (1)

Facsimile: (1)

Telex:

Email:

Commission(s): $12,14,36$

HOUZIAUX L PROF, INSTITUT DE MATHEMATIQUES, 15 AV TILLEULS, B 4000 LIEGE, BELGIUM

Telejhone: (32) 41669494

Facsimile: (32) +1669493 Telex:

Email: u2141lh@bliulg11

Commission(s): $29,34,44,46 \mathrm{C}$

HOVENIER J W DR, DPT PHYSICS \& ASTRONOMY, FREE UNIVERSITY, DE BOELELAAN 1081

NL 1081 HV AMSTERDAM, NETHERLANDS

Telephone: (31) $205402414 \quad$ Facsimile: (31) Telex:

Email:

Commission(s): 16

HOWARD ROBERT F DR, NTL SOLAR OBSERVATORY, BOX 26732,950 N CHERRY AVE, TUCSON AZ 85726 6732, US

Telephone: (1) 5203275511

Facsimile: (1)

Telex: 0666484 aura noatuc

Email:

Commission(s): 10,12

HOWARD W MICHAEL DR, LAWRENCE LIVERMORE LAB, L 297, BOX 808, LIVERMORE CA 94550, USA

Telephonc: (1) $415+22+138$

Facsimilc: (1)

Telex:

Email: howvin (âphysics.llnl.gov

Commission(s):

HOWARD WILLIAM E III DR, 1653 QUAIL HOLLOW CT, MCLEAN VA 22101 3234, USA

Telepbone: (1) $70369514+7$

Facsimile: (1) $7036976956 \quad$ Telex:

Email: whoward@atecsun1.tec.army.mil Commission(s): 40

HOWARTH IAN DONALD, DPT PHYSICS \& ASTRONOMY, UNIVERSITY COLLEGE LONDON, GOWER ST

LONDON WC1E GBT, UK

Telephone: (4) $1713877050 \quad$ Facsimile: (4) $\quad$ Telex: 28722

Email:

Commission(s): $4 t$

HOWELL STEVE BRUCE DR, PLANETARY SCIENCE INST, 620 N SIXTH AVE, ASTROPHYSICS GP TUCSON AZ 85705, USA

Tclephone: (1) 5206226300

Facsimile: (1) $5206228060 \quad$ Telex:

Email: howellapsi.edu

Commission(s): 27

HOWSE H DEREK, 12 BARNFIELD RD, RIVERHEAD, SEVENOAKS TN13 2AY, UK

Telephonc: (4) $1732+5+366$

Facsimile: (4t)

Telex:

Email:

Commission(s): 41

HOYLE FRED SIR, 102 ADMIRALS WALK, WEST CLIFF RD, BOUREMOUTH, DORSET BH2 SHF, UK

Telephone: $(+4)$

Facsimile: (4t)

Telex:

Email:

Commission(s): $28,35,44,47$

HOYNG PETER DR, SPACE RESEARCH LABORATORY, SRON, SORBONNELAAN 2

NL $358+$ CA UTRECHT, NETHERLANDS

Telephonc: (31) 30535600

Facsimile: (31) 30540860

Telex: $\$ 722+$ sron ni

Email: phoyng@sron.ruu.nI

Commission(s): 10,12,4t

HRIC LADISLAV DR, ASTRONOMICAL INSTITUTE, SLOVAK ACADEMY SCIENCES SK 05960 TATRANSKA LOMNI, SLOVAK $R$

$\begin{array}{llll}\text { Telephone: (42) } 969967866 / 7 / 8 & \text { Facsimile: (42) } 969967656 & \text { Telex: } 78277\end{array}$

Email:

Commission(s): +2

HRIVNAK BRUCE J, DPT OF PHYSICS, VALPARAISO UNIVERSITY, VALPARAISO IN 46383, USA

Telephone: (1) 2194645379

Facsimile: (1)

Telex:

Email:

Commission(s): $30,+2$ 
HRON JOSEF DR, INSTITUT FUER ASTRONOMIE, UNIVERSITAET WIEN, TUERKENSCHANZSTR 17 A 1180 WIEN, AUSTRIA

Telephonc: (13) $13+53600$

Email: carn:awiuni11(a)a8201dah

Facsimile: (43)

Commission(s): 29,33

Telex: 133099 viast a

HSIANG YAN-YU, BEIJING ASTRONOMICAL OBS, CAS, W SUBURB, BEIJING 100080, CHINA PR

Telephonc: (86) $1281698 \quad$ Facsimile: (86)

Telex: 22040 baoas cn

Email:

Commission(s):

HSIANG-KUANG TSENG, DPT OF PHYSICS, NTL CENTRAL UNIVERSITY, CHUNG LI TAIWAN, CHINA R

Telephone: (886)

Facsimile: (886)

Telex:

Email:

Commission(s):

HU ESTHER M DR, INSTITUTE FOR ASTRONOMY, UNIVERSITY OF HAWAII, 2680 WOODLAWN DR, HONOLULU H 96822, USA

Telephone: (1) 8089567190

Facsimile: (1) 8089882790

Telex: 7238459 uhast hr

Email: hu(â)ifa.hawaii.edu

Commission(s): 47

HU FU-XING, PURPLE MOUNTAIN OBSERV, CAS, NANJING, CHINA PR

Telephonc: (86) $25 \$ 6700$

Facsimile: (86) 25301459

Telex: $3+1+4$ pmonj en

Email:

Commission(s): 28

HU JING-YAO, BEIJING ASTRONOMICAL OBS, CAS, W SUBURB, BEIJING 100080, CHINA PR

Telephone: (86) 1281698

Facsimile: (86)

Commission(s): 09,25

Telex: 22040 baoas en

Email:

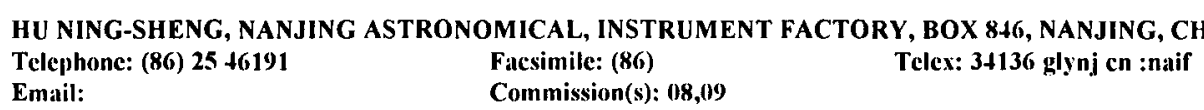

Email:

Commission(s): 08,09

HU WEN-RUI, INSTITUTE OF MECHANICS, CAS, W SUBURB, BEIJING 100080, CHINA PR

Telephone: (86) 1284185

Email:

Facsimile: (86)

Commission(s): 4

Telex: 22474 aschi cn

HU ZHONG-WEI, DPT OF ASTRONOMY, NANJING UNIVERSITY, NANJING 210008, CHINA PR

Telephone: (86) 2537651

Email:

Facsimile: (86)

Telex: 0909

Commission(s): 15,16

HUA CHON TRUNG DR, LAS, TRAVERSE DU SIPHON, LES TROIS LUCS, F 13012 MARSEILLE, FRANCE

Telephone: (33) 91055932

Facsimile: (33) 91661855

Telex: $\mathbf{4 2 0 5 8 4 \mathrm { f }}$

Email: hua@ofrlasm51

Commission(s): 28,34

HUA YING-MIN, SHANGHAI OBSERVATORY, CAS, 80 NANDAN RD, SHANGHAI 200030, CHINA PR

Telephone: (86) 21386191

Facsimilc: (86) 21384618

Telex: 33164 shao cn

Email:

Commission(s): 08,19

HUAN NGEYEN DUSH DR, UNIVERSITY OF VINH, PROVINCE NGH^E AN, VIETNAM

Telephone: (84)

Facsimile: (84)

Commission(s);

Telex:

Email:

HUANG BI-KUN, PURPLE MOUNTAIN OBSERV, CAS, LIBRARY, NANJING, CHINA PR

Telephonc: (86) 25307521

Facsimile: (86) 25301459

Telex: $3+14+$ pmonj en

Email:

Commission(s): 05

HUANG CHANG-CHUN, PURPLE MOUNTAIN OBSERV, CAS, NANJING, CHINA PR

Telephone: $(86) 25 \$ 6700 / \$ 2817$

Facsimile: (86) 25301459

Telex: $3+144$ pmonj en

Email:

Commission(s): 29,30

HUANG CHENG DR, SHANGHAI OBSERVATORY, CAS, 80 NANDAN RD, SHANGHAI 200030, CHINA PR

Telephone: (86) 21386191

Facsimile: (86)

Telex:

Email:

Commission(s): 07

hUANG GUANG LI, PURPLE MOUNTAIN OBS, NANJING 210008, CHINA PR

Telephone: (86) $25+432817$

Facsimile: (86) 253301459

Commission(s): 12

Telex: $3+14+$ pomtj en

Email: guangli.huangacetp.ipsl.fr 
HUANG JIE-HAO, DPT OF ASTRONOMY, NANJING UNIVERSITY, NANJING 210008, CHINA PR

Telephone: (86)

Facsimile: (86)

Commission(s): 28

Telex: $3+151$ prenu en

Email:

HUANG KE-LIANG, DPT OF ASTRONOMY, NANJING UNIVERSITY, NANJING 210008, CHINA PR
Telephone: (86) $2534651 * 2882$
Facsimile: (86)

Email: $\quad$ Commission(s): 28,44

HUANG KUN-YI, PURPLE MOUNTAIN OBSERV, CAS, NANJING, CHINA PR

Telephone: (86) $2532893 \quad$ Facsimile: (86) $25301459 \quad$ Telex: 34144 pmonj cn

Email:

Commission(s):

HUANG LIN, BEIJING ASTRONOMICAL OBS, CAS, W SUBURB, BEIJING 100080, CHINA PR Telephone: (86) 1281698 Facsimile: (86)

Commission(s): 25,45

Telex: 22040 batoas en

Email:

HUANG RUN-QIAN, YUNNAN OBSERVATORY, CAS, BOX 110, KUNMING 72946 YUNNAN, CHINA PR
Telephone: (86) 8712035
Facsimile: (86)

Email:

Commission(s): $35, \downarrow 2$

HUANG SONG-NIAN DR, ICTP CP 586, ST COSTIERA 11, MIRAMARE, I 3+100 TRIESTE, ITALY

Telephone: (39)

Facsimile: (39)

Telex:

Email:

Commission(s): 28,33

HUANG TIANYI, DPT OF ASTRONOMY, NANJING UNIVERSITY, NANJING 210008, CHINA PR

Telephonc: (86)

Facsimile: (86)

Commission(s): 07,46

Telex: $3+151$ prenu en

Email:

HUANG TIE-QIN, NANJING ASTRONOMICAL, INSTRUMENT FACTORY, NANJING, CHINA PR

Telephone: (86) 2546191

Facsimile: (86)

Commission(s): 09

Telex: 34136 glynj en

Email:

HUANG YINN-NIEN DR, DIR GEN OF TELECOMMUNICAT, MNSTR TRANSPORT \& COMMUNI, 31 AL KUO EAST RD TAIPEI 106, CHINA R

Telephone: $(886) 23+43604$

Email:

Facsimile: (886) $235611259 \quad$ Telex: 21733 gentel

Commission(s):

HUANG YIN-LIANG, TIANJIN INST OF TECH, HONG QI NAN RD, TIANJIN 300191, CHINA PR

Telephone: (86) 368787256

Facsimilc: (86)

Commission(s): 50

Telex:

Email:

ORY, NTL TSING HUA UNIVERSITY, HSIN CHU 3001043, CHINA R

HUANG YI-LONG DR, INST OF HISTORY, NTL TSING HUA UNIVERSITY, HSIN
Telephone: (886) 35716780
Facsimile: (886)

Commission(s):

Commission(s):

HUANG YONGWEI, BEIJING ASTRONOMICAL OBS, CAS, W SUBURB, BEIJING 100080, CHINA PR

Telephone: (86) $1281698 \quad$ Facsimile: (86)

Email: $\quad$ Commission(s): 28

Telex: 9053

HUANG YOU-RAN, DPT OF ASTRONOMY, NANJING UNIVERSITY, NANJING 210008, CHINA PR

Telephone: (86)

Facsimile: (86)

Telex: 34151

Email:

Commission(s): 10

HUBBARD WILLIAM B PROF, LUNAR \& PLANETARY LAB, UNIVERSITY OF ARIZONA, TUCSON AZ 85721, USA

Telephone: (1) 5206216942

Facsimile: (1)

Telex: $91095211+3$

Email: hubbard(a)lpl,arizona.edu

Commission(s): 16

HUBE DOUGLAS P DR, DPT OF PHYSICS, UNIVERSITY OF ALBERTA, EDMONTON AB TGG 2J1, CANADA

Telephone: (1) 4034325410

Facsimile: (1) $403+32+256$

Telex: 037 2979

Email:

Commission(s): $30,+2$

HUBENET HENRI DR, STERREKUNDIG INSTITUTE, BOX 80000, NL 3508 TA UTRECHT, NETHERLANDS

Telephone: (31) 30535200

Facsimile: (31)

Email:

Commission(s):

Telex: 40048 fylut $\mathrm{nl}$ 
HUBENY IVAN, NASA, GSFC, CODE 681, GREENBELT MD 20771, USA

Telephone: (1) 3012866072

Facsimile: (1) $3012861753 \quad$ Telex:

Email: hubeny@stars.gsfc.nasa.gov

Commission(s): $29,36 \mathrm{C}$

HUBER MARTIN C E DR, ESA/ESTEC, SSD, BOX 299, NL 2200 AG NOORDWIJK, NETHERLANDS

Telephone: (31) $171983552 \quad$ Facsimile: (31) 171984699 Telex: 39098

Email:

Commission(s): 14,44

HUBERT HENRI DR, OBSERVATOIRE DE PARIS, SECTION DE MEUDON, DASGA F 92195 MEUDON PPL CDX, FRANCE

$\begin{array}{lll}\text { Telephone: (33) } 145077850 & \text { Facsimile: (33) } & \text { Telex: } 270912 \\ \text { Email: hubert@frmeu51 } & \text { Commission(s): } 29 & \end{array}$

HUBERT-DELPLACE A-M DR, OBSERVATOIRE DE PARIS, SECTION DE MEUDON F 92195 MEUDON PPL CDX, FRANCE

$\begin{array}{lll}\text { Telephone: (33) } 145347856 & \text { Facsimile: (33) } & \text { Telex: } 270912 \\ \text { Email: } & \text { Commission(s): 29 } & \end{array}$

HUBRIG SWETLANA, ASTROPHYSIKALISCHES INST, POTSDAM UNIVERSITY, AN DER STERNWARTE 16 D 14482 POTSDAM, GERMANY

Telephone: (49) $3317499224 \quad$ Facsimile: (49) $3317499309 \quad$ Telex: 361231 stbg d

Email: shubrig(a)aip.de Commission(s): 29

HUCHRA JOHN PETER DR, CENTER FOR ASTROPHYSICS, HCO/SAO, 60 GARDEN ST, CAMBRIDGE MA 02138, USA

Telephone: (1) $6174957375 \quad$ Facsimile: (1)

Email: $\quad$ Commission(s): 28C,30, 47

Telex: $921+28$ satellite cam

HUCHTMEIER WALTER K DR, MPI FUER RADIOASTRONOMIE, AUF DEM HUEGEL 69, D 53121 BONN, GERMANY

Telephone: (49) 228525215

Facsimile: (49)

Telex: $8864+40$

Email:

Commission(s): 28,40

HUDEC RENE DR, ASTRONOMICAL INSTITUTE, CZECH ACADEMY OF SCIENCES, ONDREJOV OBSERVATORY

CZ 251 65 ONDREJOV, CZECH R

Telcphonc: (\$2) 204 857128/7111

Email: astmph(a)cscarn

Facsimile: (42) $20+851611$

Telex: 121579

HUDSON HUGH S DR, INSTITUTE FOR ASTRONOMY, UNIVERSITY OF HAWAII, 2680 WOODLAWN DR HONOLULU HI 96822 , USA

Tolephonc: (1) $8132769+531$

Email: hudson(āisass6.solar.isass.ac.jp)

Facsimile: (1) $81327694532 \quad$ Telex:

Commission(s): 10

HUEBNER WALTER F DR, SOUTHWEST RESEARCH INST, 6220 CULEBRA RD, BOX 28510

SAN ANTONIO TX 78284, USA

Telephone: (1) 5125222730

Facsimile: (1)

Telex: $\mathbf{2 4 4 8 4 6}$

Email:

Commission(s): $14,15 \mathrm{C}$

HUENEMOERDER DAVID P DR, DPT OF ASTRONOMY, PENNSYLVANIA STATE UNIV, 525 DAVEY LAB UNIVERSITY PARK PA 16812, USA

Telephone: (1) $81+8656601$

Facsimile: (1)

Telex:

Email: internet:dph(a) astro.psu.edu

Commission(s): 27,29

HUGGINS PATRICK J, DPT OF PHYSICS, NEW YORK UNIVERSITY, 4 WASHINGTON PLACE

NEW YORK NY 10003, USA

Telephone: (1) 2129987717

Facsimile: (1) $2129954016 \quad$ Telex:

Email: huggins(äacfeluster.nyu.edu

Commission(s): 34,35

HUGHES ARTHUR R W, UNIVERSITY OF NATAL, DPT OF PHYSICS, KING GEORGE V AVE

DURBAN 4001, SOUTH AFRICA

Telephone: (27) 312602775

Facsimile: (27) 312616550

Telex:

Email: hughes(aph.und.ac:za

Commission(s):

HUGHES DAVID W DR, DPT OF PHYSICS, THE UNIVERSITY, SHEFFIELD S3 7RH, UK

Telephone: (\$4) $17+278555 \quad$ Facsimile: $(+4)$

Email:

Commission(s): 15,22

Telex: $\mathbf{5 + 3 + 8}$ ulshef $\mathrm{g}$ 
M 600

HUGHES JOHN P DR, CENTER FOR ASTROPHYSICS, HCO/SAO, 60 GARDEN ST, CAMBRIDGE MA 02138, USA Telephone: (1) 6174957142

Facsimile: (1)

Telex: $921+28$ satellite cam

Email: bitnct:hughes(a)cfa.

Commission(s):

HUGHES PHILIP, DPT OF ASTRONOMY, UNIVERSITY OF MICHIGAN, DENNISON BLDG

ANN ARBOR MI 481091090 , USA

Telephone: (1) 3137643430

Facsimile: (1)

Telex:

Email:

Commission(s): 40

HUGHES SHAUN DR, ROYAL GREENWICH OBSERV, MADINGLEY RD, CAMBRIDGE CB3 OE, UK

Telephone: (44) 1223374000

Facsimile: (4) $1223374000 \quad$ Telex:

Email: hugnes@mail.ast.cam.ac.uk

Commission(s):

HUGHES VICTOR A PROF, DPT OF PHYSICS, QUEEN'S UNIVERSITY, KINGSTON ON K7L 3NG, CANADA

Telephone: (1) $6135+56000^{*}(\& S$

Facsimile: (1) 6135456463

Telex:

Email: hughesv(a)qucdn.bitnet

Commission(s): $33,34,40$

HUGUENIN G RICHARD, MULTITECH CORPORATION, BOX 109, SOUTH DEERFIELD RES PARK

SOUTH DEERFIELD MA 01373, USA

Telephone: (1) 4136658551

Facsimile: (1)

Telex: 3719862 trub

Email:

Commission(s):

HULSBOSCH A N M DR, STERRENKUNDIG INSTITUTE, KATHOLIEKE UNIVERSITEIT, TOERNOOIVELD NL 6525 ED NIJMEGEN, NETHERLANDS

Telephone: (31) 80558833

Facsimile: (31)

Commission(s): $33,34,40$

Telex: 48228

Email:

HUMBLE JOHN EDMUND DR, DPT OF PHYSICS, UNIVERSITY OF TASMANIA, GPO BOX 252C HOBART TAS 7001, AUSTRALIA

Facsimile: (61) $2202+10$

Telex: aa 58150

Email: john.humble $a_{\text {phys.utas.edu.au }}$

Commission(s): 49

HUMMEL EDSKO, KAPTEYN ASTRONOMICAL INST, BOX 800, NL 9700 AV GRONINGEN, NETHERLANDS

Telephone: (31) 50634073

Facsimile: (31) 50636100

Telex: $\mathbf{5 3 5 7 2}$ stars n!

Email: secra astro.rug.nl

Commission(s): 28

HUMMER DAVID G DR, 313 ALDER LANE, BOULDER CO 80304, USA

Telephone: (1)

Facsimile: (1)

Telex:

Email:

Commission(s): 34,36

HUMPHREYS ROBERTA M PROF, DPT OF ASTRONOMY, UNIVERSITY OF MINNESOTA, 116 CHURCH ST SE MINNEAPOLIS MN 55455, USA

Telephone: (1) 6123739747

Facsimile: (1)

Telex:

Email:

Commission(s): $28,33,35,45$

HUMPHRIES COLIN M DR, ROYAL OBSERVATORY, BLACKFORD HILL, EDINBURGH EH9 3HJ, UK

Telephone: (44) $1316673321 \quad$ Facsimile: (4)

Email:

Commission(s): 09

Telex: 72383

HUMUY MARIO, CTIO, CASILLA 603, LA SERENA, CHILE

Telephonc: (56) $51225+15$

Email: mhumuy(a)noao.edu

Facsimile: (56) $512053+2 \quad$ Telex:

Commission(s):

HUNDHAUSEN ARTHUR DR, HIGH ALTITUDE OBSERVATORY, NCAR, BOX 300, BOULDER CO 80307 3000, USA Telephone: (1) 3034971000 Facsimile: (1) $303+971568$ Telex:

Email:

Commission(s):

HUNGER KURT PROF, INST THEOR PHYS \& STERNW, NEUE UNIV PHYSIK ZENTRUM, OLSHAUSENSTR 40 N61C D $2+098$ KIEL, GERMANY

Telephone: (49) $\$ 318804110$

Facsimile: (49)

Telex: 292706

Email:

Commission(s): 29,36

HUNSTEAD RICHARD W DR, SCHOOL OF PHYSICS, UNIVERSITY OF SYDNEY, SYDNEY NSW 2006, AUSTRALIA

Telephone: (61) 26923871

Facsimile: (61)

Commission(s): 28,40

Telex: 26169 unisyd ai

Email: 
HUNT G E DR, ELBURY, 37 BLENHEIM ROAD, RAYNES PARK, LONDON SW20 9BA, UK

Telephone: (44) 1815422374

Facsimilc: (44)

Telex:

Email:

Commission(s): 16

HUNT LESLIE DR, OSS ASTROFISICO, DI ARCETRI CAISMI CNR, LARGO E FERMI 5, I 50125 FIRENZE, ITALY

Telephone: (39) 552752296

Facsimile: (39) 55220039

Telex: 572268 arcetr

Email: hunt@sisifo.arcetri.astro.it Commission(s): 44

HUNTEN DONALD M PROF, LUNAR \& PLANETARY LAB, UNIVERSITY OF ARIZONA, TUCSON AZ 85721, USA

Telephone: (1) 5206214002

Facsimile: (1)

Telex:

Email:

Commission(s): 16,51

HUNTER CHRISTOPHER PROF, DPT OF MATHEMATICS, FLORIDA STATE UNIVERSITY, TALLAHASSEE FL 32306, USA

Telephone: (1) 9046442488

Facsimile: (1)

Telex:

Email:

Commission(s): 28,33

HUNTER DEIDRE ANN, DPT TERRESTR MAGNETISM, CARNEGIE INST WASHINGTON S241 BROAD BRANCH RD NW, WASHINGTON DC 20015, USA

Telephone: (1) 2029660863

Facsimile: (1)

Commission(s):

Telex: $\mathbf{4 \$ 0 4 2 7}$ magn ui

Email:

Facsimile: (1)

Commission(s): 28,51

Telephone: (1) 9043921078

Email:

HUNTRESS WESLEY T DR, NASA HEADQUARTERS, CODE SL, 600 INDEPENDENCE AVE SW

WASHINGTON DC 20546, USA

Telephone: (1) 2024531588

Facsimile: (1) $202+261023$

Telex:

Email:

Commission(s): 15

HUOVELIN JUHANI DR, OBS \& ASTROPHYSICS LAB, UNIVERSITY OF HELSINKI, TAEHTITORNINMAKI

SF 00130 HELSINKI 13, FINLAND

Telephone: (358) 1912948

Facsimile: (358) 1912952

Commission(s): 25

Telex: 124690 unih sf

Email: huovelin at cchelsinki.fi

HURFORD GORDON JAMES, CALTECH, MS 26433, PASADENA CA 91125, USA

Telephone: (1) 8183563866

Facsimile: (1)

Telex: $675+25$

Email:

Commission(s): 10

\section{HURLEY KEVIN C, SPACE SCIENCES LABORATORY, UNIVERSITY OF CALIFORNIA}

BERKELEY CA 94720 7950, USA

Telephone: (1) 5106439173

Facsimilc: (1) 5106438602

Telex:

Email: khurley $(9$ sunspot.ssi.berkerly.edu Commission(s): $4 \downarrow$

HURNIK HIERONIM PROF, ASTRONOMICAL OBSERVATORY, A MICKIEWICZ UNIVERSITY, UL SLONECZNA 36 PL 60286 POZNAN, POLAND

Telephone: (48) 679670

Facsimile: (48)

Telex:

Email:

Commission(s): 20

HURUKAWA KIITIRO DR, 1329 OSAWA, MITAKA, TOKYO 181, JAPAN

Telephone: (81) 422323834

Facsimile: (81) 422338514

Telex:

Email:

Commission(s): 20

HURWITZ MARK V, CTR FOR EUV ASTROPHYSICS, UNIVERSITY OF CALIFORNIA, 2150 KITTREDGE ST BERKELEY CA 94720, USA

Telephone: (1) 5106435665

Email: markh@ssi.berkeley.edu

Facsimile: (1) $5106+35660 \quad$ Telex:

Commission(s): 21

HUSFELD DIRK DR, INST ASTRON \& ASTROPHYSIK, UNIVERSITAETS STERNWARTE, SCHEINERSTR 1

D 81679 MUENCHEN, GERMANY

Tclephone: (49) 8992209440

Facsimile: (49) $8992209427 \quad$ Telex:

Email: uh101bs@dm0irz01.earn

Commission(s): 36 
M 602

HUT PIET, INST FOR ADVANCED STUDY, SCHOOL OF NATURAL SCIENCE, PRINCETON NJ 08540, USA Telephone: (1) 6097348075

Facsimile: (1) $60992+8399$

Telex: 229734 ias ur

Email: piet@guinness.ias.edu

Commission(s): 07,37

HUTCHEON RICHARD J DR, DPT OF PHYSICS, UNIVERSITY OF LEICESTER, X-RAY ASTRONOMY GROUP LEICESTER LE1 7RH, UK

Telephone: (44)

Facsimile: (44)

Telex:

Email:

Commission(s):

HUTCHINGS JOHN B DR, HERZBERG INST ASTROPHYS, DOMINION ASTROPHYS OBS, 5071 W SAANICH RD VICTORIA BC V8X 4M6, CANADA

Telephone: (1) 6043883909

Facsimile: (1) $60+3630045$

Telex: 0497295

Email:

Commission(s): $27,34,36,+2,44$

HUTSEMEKERS DAMIEN DR, INSTITUT D'ASTROPHYSIQUE, UNIVERSITE DE LIEGE, AVE COINTE 5 B 4000 COINTE-LIEGE, BELGIUM

Telephone: (32) 41529980

Facsimilc: (32) 41527474

Telex: $4126+$ astrlg b

Email: u2141mg(a,bliulg11

Commission(s): 34

HWANG WOEI-YANN P PROF, DPT OF PHYSICS, NTL TAIWAN UNIVERSITY, TAIPEI TAIWAN 10764, CHINA R Telephone: (886) $23630231 * 3159$ Facsimile: (886) $23637204 / 9984$ Telex:

Email:

Commission(s):

HYDER C L DR, C/O ROZAMME SMITH, 2837 ALVARADO NE, ALBUQUERQUE NM 87110, USA

Telephone: (1)

Facsimile: (1)

Telex:

Email:

Commission(s): 10

HYLAND A R HARRY DR, FACULTY OF SCIENCE, UNIV OF NEW SOUTH WALES, SYDNEY NSW 2052, AUSTRALIA Telephone: (61) 23855750 Facsimile: (61) 26633028 Telex:

Email: h.hyland(a)unsw.edu.au

Commission(s): 25,29

HYSOM EDMUND J, 8 EAST DRIVE, CALDECOTE, CAMBRIDGE CB3 7NZ, UK

Telcphone: (44) 1954211137

Facsimile: (44)

Telex:

Email:

Commission(s): 09,41,51

HYUN JONG-JUNE PROF, DPT OF ASTRONOMY, SEOUL NTL UNIVERSITY, KWANAK KU, SEOUL 151, KOREA R Telephone: $(82) 8773010 * 25+2$ Facsimile: (82) Telex:

Email:

Commission(s):

IANNA PHILIP A, UNIVERSITY STATION, UNIVERSITY VIRGINIA, BOX 3818, CHARLOTTESVILLE VA 22903, USA

Telcphone: (1) $80+9244898$

Facsimilc: (1)

Telcx:

Email: pai@fermi.clas.virginia.edu

Commission(s): 20,24VP,26

IANNINI GUALBERTO DR, OBSERVATORIO ASTRONOMICO, DE CORDOBA, LAPRIDA 854 5000 CORDOBA, ARGENTINA

Telephone: (54) 51230491

Facsimile: (54) 51210613

Telex: 51822 bucor ar

Email:

Commission(s):

IBADINOV KHURSANDKUL DR, ASTROPHYSICAL INSTITUTE, TADJIK ACAD OF SCIENCES 734670 DUSHANBE, TAJIKISTAN

Telephone: (7)

Facsimile: (7)

Telex:

Email:

Commission(s): 15

IBANEZ S MIGUEL H DR, UNIVERSIDAD DE LOS ANDES, FACULDAD DE CIENCIAS, DPT DE FISICA MERIDA 5101 A, VENEZUELA

Telcphone: (58) $74639930 / 7477$

Facsimile: (58)

Commission(s):

Telex: $7 \mathbf{4 1 7 4}$ cida

Email:

IBANOGLU C DR, FACULTY OF SCIENCE, EGE UNIVERSITY, BOX 21, 35100 BORNOVA IZMIR, TURKEY

Telephone: (90) $51180110^{\star} 2332$

Facsimile: (9))

Telex:

Email:

Commission(s): 42

IBBETSON PETER AARON DR, WISE OBSERVATORY, TEL AVIV UNIVERSITY, RAMAT AVIV

TEL AVIV 69978, ISRAEL

Telephone: (972) 3413788

Email:

Facsimile: (972)

Telex: $3+2171$ versy il

Commission(s): 
IBEN ICKO JR PROF, DPT OF ASTRONOMY, UNIVERSITY OF ILLINOIS, 1011 W SPRINGFIELD AVE URBANA IL 61801, USA

Telephone: (1) 2173333090

Facsimile: (1)

Commission(s): $27,35 \mathrm{C}, 37$

Telex: $9102+52+34$ ast

Email:

IBRAHIM JORGA, DPT OF ASTRONOMY, BANDUNG INSTITUTE OF TECH, JL TAMANSARI 64

BANDUNG, INDONESIA

Telephone: (62)

Facsimile: (62)

Email:

Commission(s):

Telex:

ICHIKAWA SHIN-ICHI DR, TOKYO ASTRONOMICAL OBS, NAOJ, OSAWA MITAKA, TOKYO 181, JAPAN

Telephonc: (81) 422413604

Facsimile: (81) 422413608

Tclex: 02822307 taomtk j

Email: ichikawaac1.mtk.nao.ac.jp

Commission(s): 28

ICHIKAWA TAKASHI, DPT OF ASTRONOMY, KYOTO UNIVERSITY, KITASHIRAKAWA SAKYO KU

KYOTO 606, JAPAN

Telephone: (81) 757513890

Facsimile: (81)

Telex:

Email:

Commission(s): 28

ICHIMARU SETSUO DR, DPT OF PHYSICS, UNIVERSITY OF TOKYO, BUNKYO KU, TOKYO 113, JAPAN

Telephone: (81) 38122111

Facsimile: (81)

Telex: utphysic j23472

Email:

Commission(s): 44

ICKE VINCENT DR, STERREWACHT, BOX 9513, NL 2300 RA LEIDEN, NETHERLANDS

Telephone: (31) $71272727 \quad$ Facsimile: (31)

Email:

Commission(s): $\$ 7$

Telex: 39058 astro $\mathrm{nl}$

IDLIS G M DR, INST HIST OF SCI \& TECHN, ACADEMY OF SCIENCES, STAROPANSKY 1/5 103012 MOSCOW, RUSSIA

Telephone: (7) 952281969

Faesimile: (7)

Telex:

Email:

Commission(s): $\$ 1,51$

IIJIMA SHIGETAKA PROF, 423 6 OSAWA, MITAKA, TOKYO 181, JAPAN

Telephone: $\mathbf{8 1}$

Facsimile: $(81)$

Telex:

Email:

Commission(s): 19,31

IIJIMA TAKASHI DR, OSSERVATORIO ASTROFISICO, VIA DELL OSSERVATORIO 8, I 36012 ASIAGO, ITALY

Telephone: (39) 42462505

Facsimile: (39) $424+6288$

Telex:

Email: iijima(a)astras.cineca.it

Commission(s): 27

IKEUCHI SATORU DR, DPT EARTH SPACE SCIENCES, FAC OF SCIENCE, OSAKA UNIVERSITY TOYONAKA ASAKA 560, JAPAN

Telephone: (81)

Email:

Facsimile: (81)

Commission(s): 33,47

Telex:

IKHSANOV ROBERT N DR, PULKOVO OBSERVATORY, ACADEMY OF SCIENCES, 10 KUTUZOV QUAY 196140 ST PETERSBURG, RUSSIA

Telephone: ( 7 )

Facsimile: (7)

Telex:

Email:

Commission(s): 40

IKHSANOVA VERA N DR, PULKOVO OBSERVATORY, ACADEMY OF SCIENCES, 10 KUTUZOV OUAY 106140 ST PETERSBURG, RUSSIA

Telephone: (7)

Facsimile: (7)

Telex:

Email:

Commission(s): 40

ILIEV ILIAN, NTL ASTRONOMICAL OBS, BULGARIAN ACAD SCIENCES, BOX 136, BG 4700 SMOLJAN, BULGARIA

Telephone: (359) 7341559

Facsimile: (359)

Commission(s): 14,35

Telex: 23561

Email:

ILL MARTON J DR, KONKOLY OBSERVATORY, TOTH KALMAN U 19, H 6501 BAJA, HUNGARY

Telephone: (36) 7912110

Facsimile: (36)

Commission(s):

Telex: 281303

Email:

OLY OBSERVATORY, THEGE U 13/17, BOX 67, H 1525 BUDAPEST, HUNGARY

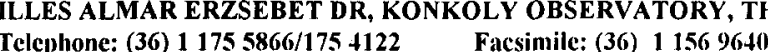

Telex: 227460

Email:

Commission(s): 
ILLING RAINER M E, BALL AEROSPACE SYSTEMS, DIVISION, BOX 1062, BOULDER CO 80306, USA

Telephone: (1) 3039395888

Facsimile: (1)

Telex:

Email:

Commission(s): 12

ILLINGWORTH GARTH D DR, LICK OBSERVATORY, UNIVERSITY OF CALIFORNIA, SANTA CRUZ CA 95064, USA

Telephone: (1)

Facsimilc: (1)

Telex:

Email:

Commission(s): 28,37

ILYAS MOHAMMAD DR, HEAD ASTRONOMY \&, ATMOSPHERIC RESEARCH UNIT, UNIVERSITY SAINS MALAYSI 11800 PENANG, MALAYSIA

Telephone: (60) 883822

Facsimilc: (60)

Telex: $4025+\mathrm{ma}$

Email:

Commission(s): 04,09,46

IMAMURA JAMES DR, DPT OF PHYSICS, UNIVERSITY OF OREGON, EUGENE OR 97403, USA

Telcphone: (1) 5036865212

Facsimile: (1)

Telex:

Email: imamura@astro.uoregon.edu

Commission(s): 42

IMBERT MAURICE DR, OBSERVATOIRE DE MARSEILLE, 2 PLACE LE VERRIER

F 13248 MARSEILLE CDX 04, FRANCE

Telephone: (33) 91959088

Facsimile: (33)

Telex: 420241

Email:

Commission(s): 30,42

IMHOFF CATHERINE L DR, NASA GSFC, CODE 684.9, IUE OBS, GREENBELT MD 20771, USA

Telephone: (1) 3012865749

Facsimile: $(1)$

Telex: 89675

Email:

Commission(s): 44

IMPEY CHRISTOPHER D DR, STEWARD OBSERVATORY, UNIVERSITY OF ARIZONA, TUCSON AZ 85721, USA

Telephone: (1) 5206216522

Facsimile: (1)

Telex: 467175

Email: impey@solpl.as.arizona.edu

Commission(s): $28,46,47$

IMSHENNIK V S DR, INST THEOR \& EXPER PHYS, CHEREMUSHKINSKAYA UL 25, 117259 MOSCOW, RUSSIA

Telephone: (7) 951230292

Facsimile: (7)

Telex: $\$ 11059$ cerii su

Email:

Commission(s): 35

INAGAKI SHOGO DR, DPT OF ASTRONOMY, KYOTO UNIVERSITY, KITASHIRAKAWA SAKYO KU

KYOTO 606, JAPAN

Telephone: (81) 757512111

Facsimile: $(\mathbf{8 1})$

Email: inagaki@a)kuastro.kyoto-u.ac.jp

Commission(s): $33,37,49$

Telex: $5+22693$ libkyu j

INATANI JUNJI, NOBEYAMA RADIO OBS, NAOJ, MINAMIMAKI MURA, NAGANO 38 $\downarrow 13$, JAPAN

Telephone: (81) $267982831 \quad$ Facsimile: (81) Telex: 3329005 tao nroj

Email:

Commission(s): 40

INFANTE LEOPOLDO, CASILlA 104, SAN JAOQUiN, SANTIAGO 22, CHILE

Telephone: (56)

Facsimile: (56)

Telex:

Email:

Commission(s):

INGERSON THOMAS DR, CERRO TOLOLO, INTERAMERICAN OBSERVATORY, CASILLA GO3, LA SERENA, CHILE

Telephone: (56) 51213352

Email: tingerson@noao,cdu Facsimile: (56) $51212466 * 342 \quad$ Telex: 620301 aura ct

Commission(s):

INNANEN KIMMO A PROF, DPT OF PHYSICS, YORK UNIVERSITY, 4700 KEELE ST

NORTH YORK ON M3J 1P3, CANADA

Telephone: (1) 4166673837

Facsimile: (1) $\mathbf{4 1 6} 736 \mathbf{5 3 8 6}$

Telex: $0652+736$

Email:

Commission(s): 33

INOUE HAJIME DR, INST SPACE \& ASTRON SCI, UNIVERSITY OF TOKYO, MEGURO KU, TOKYO 153, JAPAN

Telephone: (81) 34671111

Facsimile: (81)

Email:

Commission(s): $4+\mathrm{C}$

Telex: 24550

INOUE MAKOTO DR, VSOP PROJECT, NTL ASTRONOMICAL OBS, OSAWA MITAKA, TOKYO 181, JAPAN

Telephone: (81) 422343807

Facsimile: $(81)+223+3869$ Telex:

Email: inoue@nao.ac.jp

Commission(s): 40 
INOUE TAKESHI PROF, DPT OF PHYSICS, KYOTO SANGYO UNIVERSITY, KAMIGAMO, KYOTO G03, JAPAN

$\begin{array}{lll}\text { Telephone: (81) } 757012151 & \text { Facsimile: (81) } & \text { Telex: } 5 \$ 22661 \mathrm{ksu} \text { j } \\ \text { Email: } & \text { Commission(s): }\end{array}$

Email:

IOSHPA B A DR, IZMIRAN, ACADEMY OF SCIENCES, $1+2092$ TROITSK, RUSSIA

Telephone: (7) 2321921

Facsimile: (7)

Telex: $\$ 12623 \mathrm{scp}$

Email:

Commission(s): 10

IP WING-HUEN, MPI FUER ASTRONOMIE, D 37189 KATLENBURG LINDAU, GERMANY

Telephone: (49) 0049-555-6416 Facsimile: (49) Telex: 0965527

Email:

Commission(s): 15

IPATOV ALEXANDER, INST OF APPLIED ASTRONOMY, ZHDANOVSKAYA ST 8, ST PETERSBURG 197042, RUSSLA

Telephone: (7) 812235 3497/8122307\$14 Facsimile: (7) $8122307+13 \quad$ Telex: 122520 baste $\mathrm{ru}$

Email: iparan(a)sovam.com Commission(s): 40

IPSER JAMES R PROF, DPT OF PHYSICS, UNIVERSITY OF FLORIDA, WILLIAMSON HALL

GAINESVILLE FL 32611, USA

Telephone: (1) $90+3920521$

Facsinile: (1)

Telex:

Email:

Commission(s): 4

IRELAND JOHN G DR, C/O 13 GORDEN ROAD, BELVEDERE KENT DA17 GEA, UK

Telephone: (4) Facsimile: (4) Telex:

Email: $\quad$ Commission(s):

IRIGOYEN MAYLIS, UNIVERSITE DE PARIS II, 12 PLACE DU PANTHEON, F 75005 PARIS, FRANCE

Telephone: (33)

Email: $\quad$ Commission(s):

Telex:

IRIYAMA JUN DR, FAC OF ENGINEERING, CHUBU UNIVERSITY, 1200 MATSUMOTO, KASUGAI-SHI

AICHI 487, JAPAN

Telephone: (81) 568511111

Facsimile: (81)

Telex:

Email:

Commission(s): 17

IRVINE WILLIAM M PROF, FIVE COLLEGE, RADIO ASTRONOMY OBSERV, B619 LEDERLE GRAD RES TWR AMHERST MA 01003, USA

Telephone: (1) 4135450733

Facsimile: (1)

Commission(s): $15,16,34,51$

Telex: 955491 univ mass ams

Email:

IRWIN ALAN W DR, DPT OF PHYSICS, UNIVERSITY OF VICTORIA, BOX 1700, VICTORIA BC V8W 2Y2, CANADA

Telephonc: (1) $60+7217700$

Email: irwin(a)otto.phys.unic.ca

Facsimile: (1) $60+7217715$ Telex:

Commission(s): $14,25,30$

IRWIN JOHN B PROF, 2744 N TYNDALL AVE, TUCSON AZ 85719, USA

Telephone: (1) 5206237423

Facsimile: (1)

Commission(s): 33,42

Telex:

Email:

IRWIN JUDITH DR, DPT OF PHYSICS, QUEEN'S UNIVERSITY, STIRLING HALL RM 308E

KINGSTON ON K7L 3N6, CANADA

Telephonc: (1)

Enail:

Facsimile: (1)

Telex:

Commission(s): 28

IRWIN MICHAEL JOHN DR, INSTITUTE OF ASTRONOMY, THE OBSERVATORIES, MADINGLEY RD

CAMBRIDGE CB3 OHA, UK

Telephone: (44) 1223337548

Email: mike@uk.ac.cam.ast-star

Facsimile: (44) $1223337523 \quad$ Telex:

Commission(s): 24

ISAAK GEORGE R PROF, DPT OF PHYSICS, UNIVERSITY OF BIRMINGHAM, BOX 363, BIRMINGHAM B15 2TT, UK Telephone: (44) $1214721301 \quad$ Facsimile: (4)

Email:

Commission(s): 35

Telex: 338938 spaphy g

ISERN JORGE DR, C/SEPULVEDA 83-(6-3A, E 08015 BARCELONA, SPAIN

Telephone: (34)

Email:

Facsimile: (34)

Commission(s): 35
Telex: 
ISHIDA KEIICHI PROF, TOKYO ASTRONOMICAL OBS, NAOJ, OSAWA MITAKA, TOKYO 181, JAPAN

Telephone: (81) $422325211 \quad$ Facsimile: (81)

Email:

Commission(s): 37

Telex: 2822307 taomtk j

ISHIDA TOSHIHITO, NISHI HARIMA ASTRONOMICAL, OBSERVATORY, OHNADESAN SAYO CHO

HYOGO 679 53, JAPAN

Telephone: (81) $790823886^{\star} 1+2 \quad$ Facsimile: (81) $790823514 \quad$ Telex:

Email: ishida(a)nhao.go.jp Commission(s): 27

ISHIGURO MASATO PROF, NOBEYAMA RADIO OBS, NAOJ, MINAMIMAKI MURA, NAGANO 384 13, JAPAN

Telephone: (81) $267982831 \quad$ Facsimile: (81)

Email: ishiguro@nro.nao.ac.jp Commission(s): 40

Telex: 3329005 naonro $\mathrm{j}$

ISHHARA HIDEKI DR, DPT OF PHYSICS, KYOTO UNIVERSITY, KITASHIRAKAWA SAKYO KU, KYOTO 606, JAPAN

Telephone: (81) $757533850 \quad$ Facsimile: (81)

Email: ishihara (a)ws8 $+1 . s c$ phys.kyotoua Commission(s): 47

Telex:

ISHIZAWA TOSHIAKI A PROF, DPT OF ASTRONOMY, KYOTO UNIVERSITY, KITASHIRAKAWA SAKYO KU KYOTO 606, JAPAN

Telephone: (81) $757512111 \quad$ Facsimile: (81)

Email: ishizawa@kusastro.kyoto-u.ac.j Commission(s):

Telex: $5+22693$ libkyu j

ISHIZUKA TOSHIHISA DR, DPT OF PHYSICS, IBARAKI UNIVERSITY, BUNKYO, MITO 310, JAPAN

Telephone: (81) 292261621

Facsimile: (81)

Telex:

Email:

Commission(s): 35

ISOBE SYUZO DR, TOKYO ASTRONOMICAL OBS, NAOJ, OSAWA MITAKA, TOKYO 181, JAPAN

Telephone: (81) 422343645

Facsimile: $(81)+223+36+1 \quad$ Telex:

Email: oisobex(a)c1.mtk.nao.ac.jp

Commission(s): $06,15,20,33,34,46 \mathrm{C}, 50 \mathrm{P}$

ISRAEL FRANK P DR, STERREWACHT, BOX 9513, NL 2300 RA LEIDEN, NETHERLANDS

Telephone: (31)

Facsimile: (31)

Telex:

Email:

Commission(s): 28C,33,34,51

ISRAEL GUY MARCEL DR, SERVICE D'AERONOMIE, BP 3, F 91371 VERRIERES BUISSON, FRANCE

Telephone: (33) $16+47+289$

Facsimile: (33)

Telex:

Email:

Commission(s):

ISRAEL WERNER PROF, DPT OF PHYSICS, UNIVERSITY OF ALBERTA, EDMONTON AB T6G 2J1, CANADA

Telephone: (1) 4034323552

Facsimile: (1) $403+32+256$

Telex: 0372979

Email:

Commission(s): 4

ISSA ALI DR, ASTRONOMY DPT, KING SAUD UNIVERSITY, BOX 2455, RIYADH 11451, SAUDI ARABIA

Telephone: (966)

Facsimile: (966)

Telex:

Email:

Commission(s): 28,34

ISSERSTEDT JOERG DR, INST F ASTRONOMIE \&, ASTROPHYSIK, AM HUBLAND, D 9707\& WUERZBURG, GERMANY

Telephone: (49)

Facsimilc: $(49)$

Telex:

Email:

Commission(s):

ITO KENSAI A PROF, DPT OF PHYSICS, RIKKYO UNIVERSITY, NISHI-IKEBUKURO, TOKYO 171, JAPAN

Telephone: (81) 39852384

Facsimile: (81)

Telex:

Email:

Commission(s): 4

ITOH HIROSHI DR, MINAMIDA-CHO 27-160, JODOJI, SAKYO-KU, KYOTO 606, JAPAN

Telephone: (81) $757512111 \quad$ Facsimile: (81)

Telex:

Email:

Conmission(s): 34

ITOH MASAYUKI DR, ISAS, 3-1-1 YOSHINODAI, SAGAMIHARA, KANAGAWA 229, JAPAN

Telephone: (81) 427513911

Facsimile: $(81)+2759+253$

Telex: ¡27758 isas ero

Email:

Conmission(s): +4

ITOH NAOKI DR, DPT OF PHYSICS, SOPHIA UNIVERSITY, 7-1 KIOI-CHO CHIYODA KU, TOKYO 102, JAPAN

Telcphone: (81) 32383431

Facsimile: (81)

Telex:

Email:

Commission(s): 35 
IVANCHUK VICTOR I DR, ASTRONOMICAL OBSERVATORY, KIEV STATE UNIVERSITY, OBSERVATORNAYA UL 3 252053 KIEV, UKRAINE
Telephone: (7)
Facsimile: (7)
Telex:
Email:
Commission(s): 10

IVANOV EVGENY, IZMIRAN, TROITSK, MOSCOW 142092, RUSSIA

Telephone: (7) $09533 \downarrow 0282 \quad$ Facsimile: (7) $09533+012 \downarrow$

Telcx: $\$ 12623$ scstp

Email: ivanov@lars.izmiran.troitsk.su Commission(s): 10,12,4)

IVANOV GEORGI R DR, DPT OF ASTRONOMY, UNIVERSITY OF SOFIA, ANTON IVANOV ST 5

BG 1126 SOFIA, BULGARIA

Telephone: (359) 2544852

Facsimile: (359)

Telex:

Email:

Conmission(s):

IVANOV VSEVOLOD V DR PROF, ASTRONOMICAL OBSERVATORY, ST PETERSBURG UNIVERSITY BIBLIOTECHNAJA PL 2, 198904 ST PETERSBURG, RUSSIA

Telephone: (7) 2579491

Facsimile: (7)

Telex:

Email:

Commission(s): 36

IVANOVA VIOLETA DR, ASTRONOMICAL OBSERVATORY, BULGARIAN ACAD SCIENCES, 72 LENIN BLVD BG 1784 SOFIA, BULGARIA

Telephone: (359) 2758927

Facsimile: (359)

Telex: 23561 ecf ban bg

Email:

Commission(s): $07,15,20$

IVANOV-KHOLODNY G S DR, IZMIRAN, ACADEMY OF SCIENCES, $1+2092$ MOSCOW, RUSSIA

Telephone: (7)

Facsimile: (7)

Telex:

Email:

Commission(s): 21

IVCHENKO VASILY, DPT OF ASTRONOMY, UNIVERSITY OF KIEV, ACAD GLUSHKOV STR 6

252022 KIEV, UKRAINE

Telephone: (7) $04+2664457$

Facsimile: (7) $0+\$ 266+507 \quad$ Telex:

Email: ivchenko@astron.univ.kiev.ua

Commission(s): 09,10

IVES JOHN CHRISTOPHER MR, ESA/ESTEC, SSD, BOX 29\%, NL 2200 AG NOORDWIJK, NETHERLANDS

Telephone: (31) $171983629 \quad$ Facsimile: (31)

Email:

Commission(s)

Telex: 39098

IWANISZEWSKA CECYLIA DR, INSTITUTE OF ASTRONOMY, N COPERNICUS UNIVERSITY, UL CHOPINA 12/18 PL 87100 TORUN, POLAND

Telephone: (48) 5626018

Facsimile: $(48) 562+602$

Email:

Commission(s): $33,46 \mathrm{C}$

Telex: 0552234 astr pl

IWANOWSKA WILHELMINA PROF, INSTITUTE OF ASTRONOMY, N COPERNICUS UNIVERSITY

UL CHOPINA 12/18, PL 87 100 TORUN, POLAND

Telephone: (48) $26018 \quad$ Facsimile: $(48) \quad$ Telex: $86+12$ pl

Email:

Commission(s): 33

IWASAKI KYOSUKE DR, KYOTO GAKUEN UNIVERSITY, NANJO, SOGABECHO KAMEOKA, KYOTO 621, JAPAN

Tclephone: (81) 771222001

Email:

Facsimile: (81) $7712+8150$ Telex:

Commission(s): 16

IWATA TAKAHIRO, KASHIMA SPACE RESEARCH C, COMMUNICATIONS RES LAB, 8931 HIRAI, KASHIMA

IBARAKI 314, JAPAN

Tclephonc: (81) 299847140

Facsimilc: (81) $2998+7159$

Telex: $7604038 \mathrm{crl}$ uc

Email: iwat@crl.go.jp

Commission(s): 40

IYE MASANORI DR, TOKYO ASTRONOMICAL OBS, NAOJ, OSAWA MITAKA, TOKYO 181, JAPAN

Telcphone: (81) 422343703

Email: iye@optik.mtk.nao.ac.jp

Facsimile: (81) $4223+3608 \quad$ Telex:

Commission(s): 28,33

IYENGAR K V K PROF, INDIAN INSTITUTE OF, ASTROPHYSICS, KORAMANGALA, BANGALORE 560 034, INDIA Telephonc: (91) 80353 0672/0676

Facsimile: (91)

Telex: 8452763 iiab in

Email:

Commission(s): $25,34,4 \downarrow$ 
IYENGAR SRINIVASAN RAMA, INDIAN INST OF ASTROPHYS, SARJAPUR RD, KORAMANGALA BANGALORE 560034, INDIA

Telephone: (91) $80553(0672$

Facsimile: (91) $80553+043$

Telex: $8108+52763$

Email:

Commission(s): 9

IYER B R DR, RAMAN RESEARCH INSTITUTE, SADASHIVANAGAR, CV RAMAN AVE, BANGALORE 560 080, INDIA Telephone: (91) 803360122 Facsimile: (91) 803340492 Telex: $8+52671$ rri in

Email:

Commission(s): 47

IZOTOV YURI DR, MAIN ASTRONOMICAL OBS, UKRAINIAN ACAD OF SCIENCE, GOLOSEEVO 252127 KIEV, UKRAINE

Telephone: (7) 044663110

Facsimile: (7)

Telex: 131406 slị su

Email:

Commission(s): 28

IZUMIURA HIDEYUKI, DPT OF ASTRONOMY, TOKYO GAKUGEI UNIVERSITY, NUKUIKITA 411 KOGANEI TOKYO 184, JAPAN

Telephone: $(81)+23252111 * 2680$

Email: isumiurarayamabuki.u-gakugei.ac.jp

Facsimile: (81) $\$ 23249832$ Telex:

Commission(s): 29

IZVEKOV V A DR, INST OF THEORET ASTRONOMY, ACADEMY OF SCIENCES, N KUTUZOVA 10 191187 ST PETERSBURG, RUSSIA

Telephone: (7) 2724 (1) 23

Enrail:

Facsimile: (7)

Telex: 121578 ita su

Commission(s): 07,20

JAAKKOLA TOIVO S, OBS \& ASTROPHYSICS LAB, UNIVERSITY OF HELSINKI, TAEHTITORNINMAKI SF (00130 HELSINKI 13, FINLAND

Telephone: (358) 1912907

Facsimile: $(358)$

Telex: 124690 unih sf

Email:

Commission(s):

JABBAR SABEH RHAMAN, SARC, SCIENTIFIC RES COUNCIL, BOX 24+1, JADIRIYAH BAGHDAD, IRAQ

Telcphonc: (964) 17765127

Facsimile: $(964)$

Telex: 213976 sre ilk

Email:

Commission(s): 12,42

JABIR NIAMA LAFTA, SARC, SCIENTIFIC RES COUNCIL, BOX 2+41, JADIRIYAH BAGHDAD, IRAQ

Telcphone: (O64) $1776.5127 \quad$ Facsimile: (964)

Telex: 213976 sre ik

Email:

Commission(s): 34

JABLONSKI FRANCISCO DR, INPE, CP 515,12201 S JOSE DOS CAMPOS, BRAZIL

Telephone: (55) $123+18977$

Email:

Facsimile: (55) $1232187+3 \quad$ Telex:

Commission(s): 27

JACCHIA LUIGI G DR, CENTER FOR ASTROPHYSICS, HCO/SAO, G0 GARDEN ST, CAMBRIDGE MA (12138, USA

Telephone: (1) $617+957213$

Facsimile: (1)

Telex:

Email:

Commission(s): 22

JACKISCH GERHARD DR, ZNTRLINST FUER ASTROPHYS, STERNWARTE BABELSBERG

ROSA LUXEMBURG STR 17A, D 96575 SONNEBERG, GERMANY

Telephone: (49)

Facsimile: $(+9)$

Telex:

Enail:

Commission(s): +1

JACKSON BERNARD V DR, CASS, UCSD, C (111, LA JOLLA CA 92093 (1216, USA

Telephone: (1) $61953+3358 \quad$ Facsimile: (1) Telex:

Email: solar::bjackson/cassu1::bricme Commission(s): 12,21

JACKSON JOHN CHARLES DR, 16 THE PARK, NEWARK NG24 IS0, UK

Telephone: (4)

Facsimile: $(4+)$

Telex:

Email:

Commission(s): +4

JACKSON PAUL DR, INSTITUT FUER ASTRONOMIE, UNIVERSITAET WIEN, TUERKENSCHANZSTR 17 A 1180 WIEN, AUSTRIA

Tekphone: $(43) 13453601$

Facsimik: $(43)$

Telex:

Email:

Commission(s): $(18$ 
JACKSON PETER DOUGLAS DR, HUGHES STX CORP, 7601 ORA GLEN DRIVE, SUITE 100

GREENBELT MD 20770, USA

Telephonc: (1) 3015137735

Facsimile: (1) $3015137726 \quad$ Telex:

Email: jackson(atonga.gsfe.nasiı.gov Commission(s): 33

JACKSON WILLIAM M DR, DPT OF CHEMISTRY, UNIVERSITY OF CALIFORNIA, ROOM 214, DAVIS CA 95616, USA Telephone: (1) 9167520503

Facsimile: (1)

Telex:

Email:

Commission(s): 15

JACOBS KENNETH C DR, DPT OF PHYSICS, HOLLINS COLLEGE, BOX 9661, ROANOKE VA 24020, USA

Telephone: (1) 7033626478

Facsimile: (1)

Telex:

Email:

Commission(s):

JACOBSEN THEODOR S PROF, 6205 17TH PARK RD, SEATTLE WA 98115, USA

Telephone: (1) $20652352+5$ Facsimile: (1) Telex:

Email: $\quad$ Commission(s):

JACOBY GEORGE H, KITT PEAK NTL OBS, BOX 26732, 950 N CHERRY AVE, TUCSON AZ 85726 6732, USA

Telephone: (1) 5203259292

Facsimile: (1)

Telex:

Email:

Commission(s): 34

JACQ THIERRY, OBSERVATOIRE DE BORDEAUX, BP 89, F 33270 FLOIRAC, FRANCE

Telephone: (33) 56864330

Facsimile: (33) $56+0+251 \quad$ Telex:

Email: jacq@frobor51

Commission(s): 34,40

JACQUINOT PIERRE DR, LABORATOIRE AIME COTTON, UNIVERSITE PARIS XI, BT 505

F 91405 ORSAY CEDEX, FRANCE

Telephone: (33)

Email:

Facsimile: (33)

Commission(s): 14

Telex:

JAEGER FRIEDRICH W PROF, ZNTRLINST F ASTROPHYSIK, TELEGRAFENBERG A 33

D 14473 POTSDAM, GERMANY

Telephone: (49) 4551

Facsimile: (49)

Telex:

Email:

Commission(s):

JAFFE DANIEL T, SPACE SCIENCES LABORATORY, UNIVERSITY OF CALIFORNIA, BERKELEY CA 94720, USA

Telephone: (1) $\$ 156+21930$

Facsimile: (1)

Telex:

Email: dtj(a)astro.as.utexas.edu

Commission(s): 34

JAFPE WALTER JOSEPH DR, STSCI, HOMEWOOD CAMPUS, 3700 SAN MARTIN DR, BALTIMORE MD 21218, USA

Telephone: (1) 3013384762

Facsinuilc: (1)

Telex: 6849101 stsci

Email:

Commission(s): $28,40,44$

JAHREISS HARTMUT DR, ASTRON RECHEN INSTITUTE, MOENCHHOFSTR 12-14

D 69120 HEIDELBERG, GERMANY

Telephone: (49) 622149026

Email:

Facsimile: (49)

Commission(s): 24,33

Telex: 461336 arihd d

JAIN RAJMAL DR, UDAIPUR SOLAR OBSERVATORY, 11 VIDYA MARG, UDAIPUR 313 001, INDIA

Telephone: (91) $25626 / 27457$

Facsimile: (91)

Commission(s): 10

Telex:

Email:

JAKIMIEC JERZY PROF, ASTRONOMICAL INSTITUTE, WROCLAW UNIVERSITY, UL KOPERNIKA 11

PL 51622 WROCLAW, POLAND

Telephone: (48) 71482434

Facsimile: (48)

Commission(s): 10

Telex: 0712791 uwr pl

Email:

JAKOBSEN PETER, ESA/ESTEC, SSD, BOX 299, NL 2200 AG NOORDWIJK, NETHERLANDS

Telephone: (31) 1719833614

Facsimile: (31)

Telex: 39098

Email:

Commission(s):

JAKS WALDEMAR DR, ASTRONOMICAL LATITUDE OBS, BOROWIEC, BOX 62 035, PL 62 035 KORNIK, POLAND

Telephone: (48) 170187

Facsimile: $(48)$

Commission(s): 19

Telex: 0412623 ans pl

Email: 
M 610

JAMAR CLAUDE A J DR, IAL SPACE, UNIVERSITE DE LIEGE, AVE DU PRE AILY

B 4900 ANGLEUR-LIEGE, BELGIUM

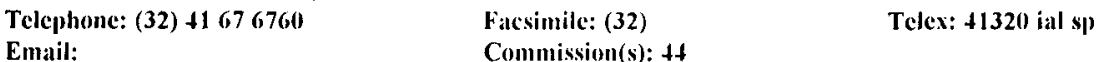

JAMES JOHN F MR, SCHUSTER LABORATORY, UNIVERSITY OF MANCHESTER, MANCHESTER M13 OPL, UK

Telephone: (H) $1612737121 \quad$ Facsimile: (4)

Email: $\quad$ Commission(s): 21

Telex:

JAMES RICHARD A DR, DPT OF ASTRONOMY, UNIVERSITY OF MANCHESTER, MANCHESTER M13 9PL, UK Telephone: (4)

Facsimile: (4t)

Telex:

Email:

Commission(s): 35

JAMESON RICHARD F DR, DPT OF ASTRONOMY, UNIVERSITY OF LEICESTER, UNIVERSITY RD LEICESTER LE1 7RH, UK

Telephone: (44) $1533554+55$

Facsimile: (4)

Telex: $3+1198$

Email:

Commission(s):

JANES KENNETH A DR, DPT OF ASTRONOMY, BOSTON UNIVERSITY, 725 COMMONWEALTH AVE BOSTON MA (2215, USA

$\begin{array}{lll}\text { Telephone: (1) } 6173532627 & \text { Facsimile: (1) } & \text { Telex: 95-1289 bos univ bsn }\end{array}$

Email:

Commission(s): 37

JANICZEK PAUL M DR, US NAVAL OBSERVATORY, 3450 MASSACHUSETTS AVE NW

WASHINGTON DC 203925100 , USA

Telephone: (1) 2026531569

Facsimile: (1)

Telex: $710-822-1970$

Email:

Commission(s): 04,07

JANKA HANS THOMAS, ASTRONOMY \& ASTROPHYS CTR, UNIVERSITY OF CHICAGO, $5640 \mathrm{~S}$ ELLIS AVE

CHICAGO IL 60637, USA

Telephone: (1) 3127027853

Facsimile: (1) $3127026645 \quad$ Telex:

Email: thomasa granta.uchicago.eda

Commission(s): +4

JANKOVICS ISTVAN DR, KONKOLY OBSERVATORY, THEGE U 13/17, BOX 67, H 1525 BUDAPEST, HUNGARY

Telephone: (36) $11755866 / 175+122 \quad$ Facsimile: (36) $11569640 \quad$ Telex: $227+60$

Email:

Commission(s):

JANOT-PACHECO EDUARDO DR, IAG, UNIVERSIDADE DE SAO PAULO, CP 9638,01065 SAO PAULO SP, BRAZIL Telephone: (55) 115778599 Facsimile: (55) 112763848 Telex: $1156735 \mathrm{iagm} \mathrm{br}$

Email:

Commission(s):

JANSSEN MICHAEL ALLEN, JPL, MS 183301,48011 OAK GROVE DR, PASADENA CA 91109, USA

Telephonc: (1) $21335+7247$ Facsimile: (1) Teic:

Email: $\quad$ Commission(s): 40

JARDINE MOIRA MARY DR, ASTRONOMY CENTRE, UNIVERSITY OF SUSSEX, FALMER, BRIGHTON BN1 9QH, UK Telephone: (4t) $1273606755 * 3085$ Facsimile: (4t) 1273678335

Telex: 877159 bhy txs g

Email: mmijajuk.ac.sussex.starlink

Commission(s): 10

JAROSZYNSKI MICHAL, ASTRONOMICAL OBSERVATORY, WARSAW UNIVERSITY, AL UJAZDOWSKIE \& PL 10478 WARSAW, POLAND

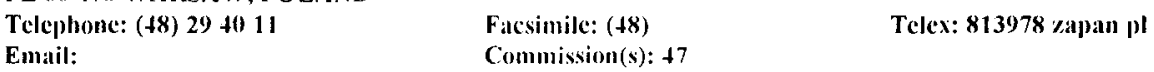

JARRETT ALAN H PROF, BOYDEN OBSERVATORY, BOX 334, BLOEMFONTEIN 9300, SOUTH AFRICA

Telephone: (27) 051-37605 Facsimile: (27) Telex: 267666 sal

Email:

Commission(s): 46

JARZEBOWSKI TADEUSZ DR, ASTRONOMICAL INSTITUTE, WROCLAW UNIVERSITY, UL KOPERNIKA 11 PL 51 (22 WROCLAW, POLAND

Telephone: (48)

Facsimile: $(48)$

Telex:

Email:

Commission(s): 27

JASCHEK CARLOS O R PROF, TESO DE LA FERIA 39, E 37018 SALAMANCA, SPAIN

Telephone: (34)

Facsimile: (34) 23216884

Telex:

Email:

Commission(s): $(15,26,33,42,45$ 
JASCHEK MERCEDES DR, TESO DE LA FERIA 39, E 37008 SALAMANCA, SPAIN

Telephone: (34) Facsimile: (34) 23216884 Telex:

Email:

Commission(s): 45

JASNIEWICZ GERARD DR, OBSERVATOIRE ASTRONOMIQUE, 11 RUE UNIVERSITE F 67000 STRASBOURG, FRANCE

Telephonc: (33) 88358200

Facsimile: (33) 88250160

Commission(s): 33,+2

Telex: 890506 starobs

Email: earn:u01109(a)frcesc21

JASTROW ROBERT, INST FOR SPACE STUDIES, 2880 BROADWAY, NEW YORK NY 10025, USA

$\begin{array}{lll}\text { Telephone: (1) } & \text { Facsimile: (1) } & \text { Telex: } \\ \text { Email: } & \text { Commission(s): } 51\end{array}$

JATENCO-PEREIRA VERA, IAG, UNIVERSIDADE DE SAO PAULO, AV MIGUEL STEFANO 4200 04301904 SAO PAULO, BRAZIL

$\begin{array}{lll}\text { Telephone: (55) } 115778599 & \text { Facsimile: (55) } 112763848 & \text { Telex: } 56735 \text { iagm br } \\ \text { Email: jatcneo@ax.iagusp.usp.br } & \text { Commission(s): } 36 & \end{array}$

JAUNCEY DAVID L DR, CSIRO, DIV OF RADIOPHYSICS, BOX 76, EPPING NSW 2121, AUSTRALIA

Telephone: (61) $28680222 \quad$ Facsimile: (61) $28680310 \quad$ Telex: 26230 astro

Email: djauncey@atnf.csiro.au Commission(s): $4(0,47 \mathrm{C}$

JAYANTHI UDAYA B DR, INPE, CP 515, 12201 S JOSE DOS CAMPOS, BRAZIL

Telephone: (55) $123418977^{\star} 392 \quad$ Facsimile: (55) $1232187+3 \quad$ Telex: 1233530 inpe br
Ematil:

JAYARAJAN A P MR, INDIAN INSTITUTE OF, ASTROPHYSICS, KORAMANGALA, BANGALORE 560 034, INDIA

Telephone: (91) 803566585

Facsimile: (91)

Commission(s): 09

Telex: 845763 iiab in

Email:

JEFFERIES JOHN T DR, NOAO, BOX 26732, 950 N CHERRY AVE, TUCSON AZ 85726 6732, USA

$\begin{array}{ll}\text { Telephone: (1) } 5208811960 & \text { Facsimile: (1) } \\ \text { Email: } & \text { Commission(s): 12,36 }\end{array}$

Telex: 0666484 auranoaotuc

JEFFERIES STUART DR, NTL SOLAR OBSERVATORY, BARTOL RESEARCH INST, 950 N CHERRY AVE TUCSON AZ 85726 6732, USA

Telephone: (1) $5203234182 \quad$ Facsimile: (1) Telex: 0666484 auranoatuc

Email: stuartj(ânoaocedu/5355::stuartị Commission(s): 12

JEFFERS STANLEY DR, CRESS PHYSICS DPT, YORK UNIVERSITY, ,700 KEELE ST

DOWNSVIEW ON M3J 1P3, CANADA

$\begin{array}{ll}\text { Telephone: (1) } 4166673851 & \text { Facsimile: (1) } \\ \text { Email: } & \text { Commission(s): } 09,51\end{array}$

JEFFERY CHRISTOPHER S DR, DPT PHYSICS \& ASTRONOMY, UNIVERSITY OF ST ANDREWS, NORTH HAUGH ST ANDREWS FIFE KY16 9SS, UK
Telephone: (44)
Facsimile: $(44)$
Telex:

Commission(s): 27

Email:

OMY DPT, UNIVERSITY OF TEXAS, RLM 15308 , AUSTIN TX 78712 1083, USA

JEFFERYS WILLIAM H DR, ASTRONOMY DPT, UNIVERSITY OF TEXAS, RLM
Telephone: (1) 5124714461
$\begin{array}{ll}\text { Email: } & \text { Facsimile: (1) } \\ \end{array}$

JENKINS CHARLES R, ROYAL GREENWICH OBS, MADINGLEY RD, CAMBRIDGE CB3 OEZ, UK

Telephone: (44)

Facsimile: (44)

Commission(s): 40

Telex:

Email:

Commission(s): 41

JENKINS EDWARD B DR, PRINCETON UNIVERSITY OBS, PEYTON HALL, PRINCETON NJ 08544, USA

Telephone: (1) $609+523826$

Facsimile: (1) $6092581020 \quad$ Telex: 322409 astro prin

Email: cbj(a) astro.princeton.edu

Commission(s): 34,44

JENKINS L F MS, YALE UNIVERSITY OBS, YALE STATION, BOX 2023, NEW HAVEN CT 06520, USA

Telephonc: (1)

Facsimilc: (1)

Commission(s): 34

Telex:

Email: 
JENKNER HELMUT DR, STSCI, HOMEWOOD CAMPUS, 3700 SAN MARTIN DR, BALTIMORE MD 21218, USA Telephonc: (1) 3013384842

Facsimile: (1)

Telex: 6849101 stsci

Email:

Commission(s): 05C,09

JENNER DAVID C DR, ASTROSFT CORPORATION, 3153 NE 84TH STREET, SEATTLE WA 98115 4717, USA

Telephone: (1) 2065272018

Facsimile: (1) 2065272019

Telex:

Email: davcj@astrosoft.com

Commission(s):

JENNINGS R E PROF, DPT PHYSICS \& ASTRONOMY, UNIVERSITY COLLEGE LONDON, GOWER ST

LONDON WC1E 6BT, UK

Telephone: (44) 171387 7050)

Facsimile: (4t)

Telex: 28722

Email:

Commission(s): 34

JENNISON ROGER C PROF, ELECTRONICS LABORATORY, UNIVERSITY OF KENT, CANTERBURY CT2 7NT, UK

Telephone: (4)

Facsimile: (4t)

Email:

Commission(s): $22,40,51$

Telex: 965449

JENSCH A, PESTALOZZISTR 9, D 07700 JENA, GERMANY

Telephone: (49)

Facsimile: (49)

Telex:

Email:

Commission(s):

JENSEN EBERHART PROF, INST THEORET ASTROPHYSICS, UNIVERSITY OF OSLO, BOX 1029 N 0315 BLINDERN OSLO 3, NORWAY

Telephone: (47) 2456502

Facsimile: (47)

Telex: $72+25 n$ unios

Email:

Commission(s): 10

JEONG JANG HAE, DPT ASTRONOMY \& SPACE SCI, CHUNGBUK NTL UNIVERSITY, SAN 48 GAESHIN DONG CHEINGJU 360763 , KOREA R

Telephone: (82) 431612313

Facsimile: (82) $\$ 3167 \$ 232$

Telex:

Email: jcongih(a)astro.chungbuk.ac.kr Commission(s):

JERZYKIEWICZ MIKOLAJ DR, ASTRONOMICAL INSTITUTE, WROCLAW UNIVERSITY, UL KOPERNIKA 11, PL 51622 WROCLAW, POLAND

Telephone: (48) 71482434

Facsimile: (48) 71482434

Telex: 0712791 uwr pl

Email: mjerzăii.uni.wroc.pl

Commission(s): 25,27P

JEWELL PHILIP R DR, NRAO, CAMPUS BLDG 65, 949 N CHERRY AVE, TUCSON AZ 857210655 , USA

Telephone: (1) 5208828250

Facsimile: (1)

Telex: 910240952+ nralo tuc

Email: bitnet:pjewellanrao

Commission(s): 27,40

JI HONG-QING, INTL LATITUTDE STATION, TIANJIN, CHINA PR

Telephonc: (86)

Facsimile: (86)

Commission(s):

Telex:

Email:

JI SHUCHEN DR, BOX 110 KUNMING, YUNNAN PROVINCE, YUNNAN, CHINA PR

Telephonc: (86) 72946

Email:

Facsimile: (86)

Telex: 64040 yuobs cn

JIANG CHONG-GUO, YUNNAN OBSERVATORY, CAS, BOX 110, KUNMING 72946 YUNNAN, CHINA PR

Telephone: (86) 8712035

Facsimile: (86)

Telex:

Email:

Commission(s): 08

JIANG DONG-RONG, SHANGHAI OBSERVATORY, CAS, 80 NANDAN RD, SHANGHAI 200030, CHINA PR

Telephone: (86) 21386191

Facsimile: (86)

Telex: 33164 shao $\mathrm{cn}$

Email:

Commission(s): 33

JIANG SHENGTAO, HARVARD SMITHSONIAN CFA, MS 42, 60 GARDEN ST, CAMBRIDGE MA 02138, USA

Telephone: (1) 6174957418

Facsimile: (1) $617+957345$ Telex:

Email: jiang@cfa.harvard.edu

Commission(s): 9

JIANG SHI-YANG, BEIJING ASTRONOMICAL OBS, CAS, W SUBURB, BEIJING 100080, CHINA PR

Telephone: (86) 1281698

Facsimile: (86)

Telex: 22040 badas cn

Email:

Commission(s): $09,29,27 \mathrm{C}, 50$ 
JIANG SHUDING, GRADUATE SCHOOL, UNIV SCIENCE \& TECHNOLOGY, BOX 3908, BEIJING 100039, CHINA PR Telephone: (86) $1817031 * 253$ Facsimile: (86)

Commission(s): 47 Telex:

Email:

OBSERVATORY, CAS, 80 NANDAN RD, SHANGHAI 200030, CHINA PR

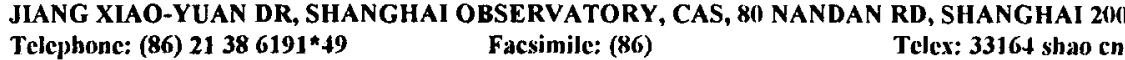

Email:

Commission(s): 41

JIANG YAO-TIAO, DPT OF ASTRONOMY, NANJING UNIVERSITY, NANJING 210008, CHINA PR

Telephone: (86) $2534151 \quad$ Facsimile: (86) Telex:

Email: $\quad$ Commission(s): 10

JIANG ZHAOJI, BEIJING ASTRONOMICAL OBS, CAS, W SUBURB, BEIJING 100080, CHINA PR

Telephone: (86)

Facsimile: (86)

Email:

Commission(s):

Telex: 22040 baoas en

JIMENEZ MANCEBO A J, IAC, C/ VIA LACTEA S/N, E 38200 LA LAGUNA, SPAIN

Telephone: (34) $22605200 \quad$ Facsimile: (34) $22605210 \quad$ Telex: 92640 iace

Email: ajm (a).iac.es Commission(s): 10

JIN BIAOREN DR, DPT OF GEOPHYSICS, WUHAN TECHNICAL UNIVER, 39 LO-YU RD, WUHAN 430070, CHINA PR

Telephonc: (86) $875571 \quad$ Facsimile: (86)

Email:

Commission(s):

Telex: 40210 wtusm cn

JIN SHEN-ZENG, BEIJING ASTRONOMICAL OBS, CAS, W SUBURB, BEIJING 100080, CHINA PR

Telephone: (86) $1281698 \quad$ Facsimile: (86) Telex: 22040 baobs cn

Email: $\quad$ Commission(s): 40

JIN WEN-JING, SHANGHAI OBSERVATORY, CAS, 80 NANDAN RD, SHANGHAI 200030, CHINA PR

Telephone: (86) $21386191 \quad$ Facsimile: (86) Telex: 33164 shao cn

Email:

Commission(s): $08,19,31$

JOCKERS KLAUS DR, MPI FUER AERONOMIE, POSTFACH 20, D 37189 KATLENBURG LINDAU, GERMANY

Telephone: (49) 5556411

Facsimile: (49)

Commission(s): 10,15

Telex: 965527

Email:

JOERSAETER STEVEN DR, STOCKHOLM OBSERVATORY, S 13336 SALTSJOEBADEN, SWEDEN

Telephone: (46) 8164463

Email: steven@astro.su.se

Facsimile: $(46) 87174719 \quad$ Telex:

Commission(s):

JOEVEER MIHKEL, TARTU ASTROPHYSICAL OBS, EE 244 TORAVERE, ESTONIA

Telephone: (372) $7410469 \quad$ Facsinile: (372) $7+10205 \quad$ Telex:

Email: mihkel@aai.ec Commission(s):

JOG CHANDA J DR, DPT OF PHYSICS, INDIAN INSTITUTE SCIENCES, BANGALORE 560 012, INDIA

Telephone: (91) 803344411

Facsimile: (91)

Commission(s): 28,33

Telex: 8458349 iisc in

Email:

JOHANSEN KAREN $T$ LEKTOR, COPENHAGEN UNIVERSITY OBS, BRORFELDEVEJ 23

DK 4340 TOLLOSE, DENMARK

Telephone: (45) 53488195

Email:

Facsimile: (45) $58+88758$

Telex:

Commission(s):

JOHANSSON LARS ERIK B DR, LADAEMNESGATAN 7, S 41679 GOETEBORG, SWEDEN

Telephone: (46)

Facsimilc: (46)

Telex:

Email:

Commission(s): 40

JOHANSSON LENNART DR, ASTRONOMICAL OBSERVATORY, BOX 515, S 75120 UPPSALA, SWEDEN

Telephone: (46) 18511274

Facsimile: (46) 18527583

Telex:

Email: laban::lj

Commission(s):

JOHANSSON SVENERIC DR, DPT OF PHYSICS, UNIVERSITY OF LUND, SOELVEGATAN 14, S 223 62 LUND, SWEDEN

Telcphone: (46) 46126097

Facsimile: $(46)+610+709$

Telex: 33533 luniver $s$

Email: atomsej@scidc52

Commission(s): 14C 
JOHNSON DONALD R DR, NTL BUREAU OF STANDARDS, BLDG 221 RM A363, GAITHERSBURG MD 20899, USA Telephonc: (1) 3019212828

Facsimile: (1)

Commission(s): $1+, 40$

Telex:

Email:

JOHNSON FRED M PROF DR, PHYSICS \& ASTRONOMY DPT, CALIFORNIA STATE UNIV, FULLERTON CA 92634, USA

Telephone: (1) 7147733366

Facsimile: (1)

Telex:

Email:

Commission(s): 14,34

JOHNSON HOLLIS R PROF, ASTRONOMY DPT, INDIANA UNIVERSITY, SWAIN WEST 319, BLOOMINGTON IN 47405 USA

Telephone: (1) 8123354172

Facsimile: (1)

Telex: 272279

Email:

Commission(s): 29,36

JOHNSON HUGH M DR, 1017 NEWELL RD, PALO ALTO CA 94303, USA

Telephone: (1) 4153267223

Facsimile: (1)

Telex:

Email:

Commission(s): 33,34

JOHNSON TORRENCE V DR, JPL, MS 183601,4800 OAK GROVE DR, PASADENA CA 91109, USA

Telephone: (1) 8183542761

Facsimile: (1)

Telex: $67-5+29$

Email:

Commission(s): 15,16

"JOHNSTON KENNETH J, NAVAL RESEARCH LABORATORY, CODE 7134, 4555 OVERLOOK AVE SW, WASHINGTON DC 20375 5000, USA

$\begin{array}{ll}\text { Telephone: (1) } 2027672351 & \text { Facsimile: (1) } \\ \text { Email: } & \text { Commission(s): 04,08,24C,34,40C }\end{array}$

JOHNSTONE RODERICK, INSTITUTE OF ASTRONOMY, THE OBSERVATORIES, MADINGLEY RD, CAMBRIDGE

CB3 OHA, UK

Telephone: (44) 1223337510

Email: rmj@mail.ast.cam.ac.uk

Facsimile: (44) $1223337523 \quad$ Telex:

Commission(s):

- JOKIPII J R PROF, LUNAR \& PLANETARY LAB, UNIVERSITY OF ARIZONA, TUCSON AZ 85721, USA

Telephone: (1) 5206214256

Facsimile: (1)

Telex:

Email:

Commission(s): 44,49

JOLY FRANCOIS DR, UNIVERSITE DE BORDEAUX, 123 RUE LAMARTINE, F 33405 TALENCE, FRANCE

Telephone: (33) 56864330

Facsimile: (33) $5640+251$

Telcx:

Email:

Commission(s): 14,40

-JOLY MONIQUE, OBSERVATOIRE DE PARIS, SECTION DE MEUDON, F 92195 MEUDON PPL CDX, FRANCE

Telephone: (33) 145077441

Facsimile: (33)

Commission(s):

Telex: 201571

JONAS JUSTIN LEONARD, DPT PHYSICS/ELECTRONICS, RHODES UNIVERSITY, BOX 94, GRAHAMSTOWN 6140, SOUTH AFRICA

Telephonc: (27) (461)22023 Facsimile: (27)

Email:

Commission(s): 33,40

Telex: $\mathbf{2 4 + 2 2 6}$ ruant sa

JONCAS GILLES DR, DPT DE PHYSIQUE, UNIVERSITE DE LAVAL, STE FOY QC G1K 7P4, CANADA

Telephone: (1) 4186562652

Email: joncas@phy.ulaval.ca

Facsimile: (1) $4186562040 \quad$ Telex: 05131621

Commission(s):

JONES ALBERT F MR, 31 RANUI RD, STOKE, NELSON, NEW ZEALAND

Telephonc: (64) 054-73-905

Facsimile: (6t)

Commission(s): 27

Telex:

Email:

JONES BARBARA, CASS, UCSD, C 011, LA JOLLA CA 92093 0216, USA

Telephone: (1) 714452447

Facsimile: (1)

Telex:

Email:

Commission(s): 09

JONES BARRIE W DR, DPT OF PHYSICS, THE OPEN UNIVERSITY, WALTON HALL, MILTON KEYNES MK7 GAA, U

Telephone: (44) 1908653378

Facsimile: (44) 1908653744

Telex: 825061

Email: bw jones 9 uk.ac.open.acs.vax

Commission(s): 40 
JONES BERNARD J T DR, NORDITA, BLEGDAMSVEJ 17, DK 2100 COPENHAGEN 0, DENMARK Telephone: (45) 31421616 Facsimile: (45) 31389157

Telex: $15216 \mathrm{nbi} \mathrm{dk}$

Email:

Commission(s): 47

JONES BURTON DR, LICK OBSERVATORY, UNIVERSITY OF CALIFORNIA, SANTA CRUZ CA 95064, USA Telephone: (1) 4084292384

Facsimilc: (1)

Telex:

Email:

Commission(s): 24

JONES DAYTON L, JPL, MS 238332,4800 OAK GROVE DR, PASADENA CA 91109, USA

Telephone: (1) $81835 \downarrow 7774$

Email: dj@bllac.jpl.nasa.gov

Facsimile: (1)

Telex: $675+29$

JONES DEREK H P DR, ROYAL GREENWICH OBS, MADINGLEY RD, CAMBRIDGE CB3 OEZ, UK

Telephone: (44) 1223374000

Facsimile: (4) $1223374700 \quad$ Telex:

Email: dhpj(amail.ast.cam.ac.uk

Commission(s): $24,33,37$

JONES ERIC M, LOS ALAMOS NATIONAL LAB, MS F665, BOX 1663, LOS ALAMOS NM 87545, USA

Telephone: (1) 5056676386

Facsimile: (1)

Telex:

Email:

Commission(s): 51

JONES FRANK CULVER DR, NASA GSFC, CODE 665, GREENBELT MD 20771, USA

Tclephone: (1) 3012865506

Facsimile: (1)

Telex: 710-828-9716

Email:

Commission(s): 34,44

JONES HARRISON PRICE DR, KITT PEAK NTL OBS, SOLAR STATION, 950 N CHERRY AVE

TUCSON AZ 85726 6732, USA

Telephone: (1) 5203259354

Facsimile: (1)

Telex:

Email:

Commission(s): 10,12

JONES JAMES DR, DPT OF PHYSICS, UNIV OF WESTERN ONTARIO, PHYS \& ASTRONOMY BLDG LONDON ON N6A 3K7, CANADA

Telephone: (1) $5196613283 * 6452$

Email: jimeteor@uwovas.uwo.ca
Facsinilc: (1) 5196612033

Commission(s): 22C
Telex:

JONES JANET E DR, NORDITA, BLEGDAMSVEJ 17, DK 2100 COPENHAGEN 0, DENMARK

Telephonc: (45) 31421616

Facsimile: (45) 31389157

Telex: 15216 nbi dk

Email:

Commission(s):

JONES MICHAEL, MULLARD RADIO ASTRON OBS, CAVENDISH LABORATORY, MADINGLEY RD CAMBRIDGE CB3 OHE, UK

Telcphone: (44) 1223337363

Email: mike@amrao.cam.ac.uk

Facsimile: (44) $1223354599 \quad$ Telex:

Commission(s):

JONES PAUL, SCHOOL OF PHYSICS, UWS NEPEAN, BOX 10, KINGSWOOD NSW 2747, AUSTRALIA

Telephone: (61) $47360+37$

Facsimile: (61) 47360779

Telex:

Email: p.jones(a)st-nepcan.uws.edu.au

Commission(s): 28,40

JONES THOMAS WALTER DR, DPT OF ASTRONOMY, UNIVERSITY OF MINNESOTA, 116 CHURCH ST SE MINNEAPOLIS MN 55455, USA

Telephone: (1) 6123733307

Email:

Facsimile: (1)

Tclex:

Commission(s): 28,4-

JORDAN CAROLE DR, DPT THEORETICAL PHYSICS, UNIVERSITY OF OXFORD, 1 KEBLE RD OXFORD OX1 3NP, UK

Telephone: (44) 18652733980

Facsimile: (44)

Commission(s): $12,14,29,44$

Telex: 83295 nuclox

Email:

JORDAN H L DR DIREKTOR, INST FUER PLASMAPHYSIK, KERNFORSCHUNGSANLAGE, JUELICH GMBH PF 365 D 5170 JUELICH 1, GERMANY

Telephone: (49)

Facsimile: (49)

Telex:

Email:

Commission(s): 14

JORDAN STEFAN, INST THEOR PHYS \& STERNW, UNIVERSITAET KIEL, OLSHAUSENSTR 40 D 24098 KIEL, GERMANY

Telephone: (49) 4318804105

Facsimile: $(49) 431880+100 \quad$ Telex:

Email: pas58@rz-uni-kicl.d400.de

Commission(s): 36 
JORDAN STUART D DR, NASA GSFC, CODE 682, LASP, GREENBELT MD 20771, USA

Telephone: (1) 3012868811

Facsimile: (1)

Telex: 89675

Email:

Commission(s): 10,12,4t

JORDEN PAUL RICHARD, ROYAL GREENWICH OBS, MADINGLEY RD, CAMBRIDGE CB3 OEZ, UK

Telephone: (44) 1223374000

Email: prj@mail.ast.cam.ac.uk

Facsimile: (4) $122337+700$

Telex: 265451/265871monrefg

JORDI NEBOT CARME DR, DPT ASTRONOMIA, UNIVERSIDAD DE BARCELONA, AVD DIAGONAL 647

E 08028 BARCELONA, SPAIN

Telcphonc: (34) 33307311

Facsimile: (34)

Telex:

Email: d3facjno@eb0ub011

Commission(s): 25

JORGENSEN HENNING E PROF, UNIVERSITY OBSERVATORY, OESTER VOLDGADE 3

DK 1350 COPENHAGEN K, DENMARK

Telephone: (45) 35323995

Email: henning@astro.ku.dk

Facsimile: (45) $35323989 \quad$ Telex:

Commission(s): 14,38P

JORGENSEN UFFE GRAE DR, NIELS BOHR INSTITUTE, BLEGDAMSVEJ 17, DK 2100 COPENHAGEN 0, DENMARK

Telephone: (45) 31421616

Facsimile: (45) 31389157

Tclex: 15216

Email: uffegi@nbivax.nbi.dk

Commission(s): 14C

'JORISSEN ALAIN, INST D'ASTRONOMIE, UNIV LIBRE DE BRUXELLES, CAMPUS PLAINE CP 226

B 1050 BRUSSELS, BELGIUM

Telephone: (32) 26502834

Facsimile: (32) $2650+226$

Telex:

Email: ajorisse (a) astro.ulb.ac.be

Commission(s):

JOSELYN JO ANN C DR, NOAA ERL R/E/SE2, SPACE ENVIRONMENT LAB, 325 BROADWAY BOULDER CO 80303, USA

Telephone: (1) 3034975147

Email: span:selvax::jjoselyn

Facsimile: (1)

Telex: 888776 noaa bldr

Commission(s): 10,49

\section{JOSEPH CHARLES LYNN DR, DPT ASTROPHYSICAL SCI, PRINCETON UNIVERSITY}

PRINCETON NJ 08544 1001, USA

Telephone: (1) 6092583808

Facsimile: (1) $6092581020 \quad$ Telex:

Email: clj@astro.princeton.edu

Commission(s): 09

JOSEPH J H DR, DPT GEOPHYS \& PLANET SCI, TEL AVIV UNIVERSITY, RAMAT AVIV, TEL AVIV, ISRAEL

Telephone: (972) 3420633

Facsimile: (972)

Telex: $3+2171$ versy il

Email:

Commission(s):

JOSEPH ROBERT D DR, ASTROPHYSICS GROUP, IMPERIAL COLLEGE, BLACKETT LABORATORY

LONDON SW7 2BZ, UK

Telephone: (44) $1715895111^{\star} 6660 \quad$ Facsimile: (4t)

Telex: 261503

Email:

Commission(s):

JOSHI G C DR, UTTAR PRADESH STATE, OBSERVATORY, PO MANORA PEAK 263 129, NAINITAL 263 129, INDIA

Telephone: (91) 594212136

Facsimile: (91)

Telex:

Email:

Commission(s): 12

JOSHI MOHAN N PROF, TIFR/RADIO ASTRONOMY CTR, BOX 8, UDHAGAMANDALAM GH3 OOI, INDIA

Telephone: (91) 4232032

Facsimile: (91)

Commission(s): 28,40,47

Telex: 8458488 tifr in

Email:

JOSHI SURESH CHANDRA DR, UTTAR PRADESH STATE, OBSERVATORY, PO MANORA PEAK 263 129, NAINITAL 263 129, INDIA

Telephone: (91) 59422136

Facsimile: (91)

Telex:

Email:

Commission(s): 25,29

JOSHI U C DR, PHYSICAL RESEARCH LAB, NAVRANGPURA, AHMEDABAD 380 009, INDIA

Telephone: (91) 272462129

Facsimile: (91) 272445292

Telex: 121397

Email:

Commission(s): $25,28,37$ 
JOSS PAUL CHRISTOPHER DR, DPT OF PHYSICS, MIT RM 6 203, BOX 165, CAMBRIDGE MA 02139, USA Telephone: (1) 6172434845

Facsimile: (1)

Telex:

Email:

Commission(s): 42,44

JOUBERT MARTINE, LAS, TRAVERSE DU SIPHON, LES TROIS LUCS, F 13012 MARSEILLE, FRANCE

Telephonc: (33) 91055900

Facsimile: (33) $91661855 \quad$ Telex: 420584

Email: joubert@frlasm51

Commission(s): 21

JOURDAIN DE MUIZON M DR, STERREWACHT, BOX 9513, NL, 2300 RA LEIDEN, NETHERLANDS

Telephone: (31) $71275840 \quad$ Facsimile: (31) $71275819 \quad$ Telex: 39058 astro nl

Email: muizon@hlerul51 Commission(s): 34

JOURNET ALAIN, OCA CERGA, AVE COPERNIC, F 06130 GRASSE, FRANCE

$\begin{array}{lll}\text { Telephonc: (33) } 93365849 & \text { Facsimile: }(33) & \text { Telex: } \$ 70865 \\ \text { Email: } & \text { Commission(s): } 07,08 & \end{array}$

JOVANOVIC BOZIDAR, FAC OF AGRICULTURE, INST WATERRANGING, VELIKA VLAHOVICA 2 YU 21000 NOVI SAD, YUGOSLAVIA-SERBIA \& MONTENEGRO

Telephone: (38) $215366 \quad$ Facsimile: (38) Telex:

Email:

Commission(s): 07,10

JOY MARSHALL J DR, NASA/MSFC, CODE ES 65, SPACE SCIENCE LAB, HUNTSVILLE AL 35812, USA

Telephone: (1) $2055+43+23$

Facsimile: (1) 205547754 Telex:

Email: ssl::joy/joy(a)ssl.msfc.nasa.gov Commission(s): 28

JUDGE PHILIP DR, HIGH ALTITUDE OBSERVATORY, NCAR, BOX 3000, BOULDER CO 80307 3000, USA

Telephone: (1) 3034971000

Facsimile: (1) $3034971568 \quad$ Telex:

Email:

Commission(s): 36

JUGAKU JUN DR, TOKYO ASTRONOMICAL OBS, NAOJ, OSAWA MITAKA, TOKYO 181, JAPAN

Telephone: (81)

Facsimile: (81)

Commission(s): 28,29,51

Telex: 2822307 taomtkj

Email:

JULIUSSON EINAR DR, MENNTASKOLINN, IS 840 LAUGARVATNI, ICELAND

$\begin{array}{lll}\text { Telephone: (354) } 861133 & \text { Facsimile: }(354) & \text { Telex: 0501 } 2307 \text { isinfo } \\ \text { Emalil: } & \text { Commission(s): } 4 \downarrow & \end{array}$

JUNG JEAN DR, 22 RUE BRIANT, F 92260 FONTENAY AUX ROSE, FRANCE

Telephone: (33) Facsimilc: (33) Telex:

Email:

Commission (s):

JUNKES NORBERT, INST FUER ASTRONOMIE UND, ASTROPHYSIK, UNIVERSITAET KIEL

D 24098 KIEL, GERMANY

Telephone: (49) 4318801575

Enail: junkesajastrophysik.uni-kicl,d $400 .(\mathrm{de}$

Facsimile: (49) $\$ 31880+100 \quad$ Telex:

Commission(s): 28

JUNKKARINEN VESA T DR, CASS, UCSD, C 011, LA JOLLA CA 92093 0216, USA

Telephone: (1) 6195340735 Facsimile: (1) Telex:

Email: span:27783::vesa Commission(s): 28,47

JUPP ALAN H DR, DPT APPL MATHS THEOR PHYS, UNIVERSITY OF LIVERPOOL, BOX 147

LIVERPOOL L69 3BX, UK

Telephone: (44) $1517096022 \quad$ Facsimile: (44) Telex: 627095

Email:

Commission(s): 07

JURA MICHAEL DR, DPT OF ASTRONOMY, UNIVERSITY OF CALIFORNIA, LOS ANGELES CA 90024, USA

Telcphone: (1) 3108254302

Facsimile: (1) 3102062096 Telex:

Email: jura@bonnic

Commission(s): $3+$

JURGENS RAYMOND F, JPL, MS 238 420, 4800 OAK GROVE DR, PASADENA CA 91119, USA

Telephone: (1) $81835+497+$

Facsimile: (1)

Telex: 675429

Email:

Commission(s): 16

JURKEVICH IGOR DR, 3130 PORT WAY, ANNAPOLIS MD 21403, USA

Telephone: (1) 2027672003

Facsimile: (1)

Telex:

Email:

Commission(s): $\$ 2$ 
JUST ANDREAS DR, ASTRON RECHEN INSTITUTE, MOENCHHOFSTR 12-14, D 69120 HEIDELBERG, GERMANY

Telephone: (49) 6221405129

Facsimile: (49) $6221405297 \quad$ Telex:

Email: s38@a)mvs.urz.uni-heidelberg.de Commission(s): 34

JUSZKIEWICZ ROMAN, COPERNICUS ASTRON CENTER, POLISH ACAD OF SCIENCES, UL BARTYCKA 18

PL 00716 WARSAW, POLAND

Telephone: (48)

Facsimile: (48)

Telex:

Email:

Commission(s): 47

KAASTRA JELLE S DR, SRL, HUYGENS LAB, BOX 9504, NL 2300 RA LEIDEN, NETHERLANDS

Telephone: (31) $71275818 \quad$ Facsimile: (31) Telex: 39058 astro nl

Email:

Commission(s):

KABURAKI MASAKI PROF, 4249 KICHIJYOJI, MINAMI MUSASHINO, TOKYO 180, JAPAN

Telephone: (81)

Facsimile: (81)

Telex:

Email:

Commission(s): 33

KABURAKI OSAMU DR, ASTRONOMICAL INSTITUTE, TOHOKU UNIVERSITY, ARAMAKI AOBA SENDAI 980, JAPAN

Telephonc: (81)

Facsimile: (81) 222612860

Telex:

Email: okabu(a)astroa.astr.tohodu.ac.jp) Commission(s):

KABURAKI OSAMU PROF, ASTRONOMICAL INSTITUTE, TOHOKU UNIVERSITY, ARAMAKI AOBA SENDAI 980, JAPAN

Telephone: (81) $222221800 \quad$ Facsimile: (81) $222612806 \quad$ Telex:

Email: okabu@astroa.astr.tohoku.ac.jp Commission(s): 10

KADLA ZDENKA I DR, PULKOVO OBSERVATORY, ACADEMY OF SCIENCES, 10 KUTUZOV QUAY 196140 ST PETERSBURG, RUSSIA

Telephone: (7)

Facsimile: (7)

Telex:

Email:

Commission(s): 05,37

KADOURI TALIB HADI, SARC, SCIENTIFIC RES COUNCIL, BOX 24+1, JADIRIYAH BAGHDAD, IRAQ

Telephone: (964) 17765127

Facsimile: $(\boldsymbol{9 6 4})$

Telex: 213976 src ik

Email:

Commission(s): 27,30,36,42

KAEHLER HELMUTH DR, HAMBURGER STERNWARTE, GOJENSBERGSWEG 112, D 21029 HAMBURG, GERMANY

Telephone: (49)

Facsimile: (49)

Telex:

Email:

Commission(s): 35

KAFATOS MINAS DR, DPT OF PHYSICS, GEORGE MASON UNIVERSITY, FAIRFAX VA 22030, USA

Tclephone: (1) 7033232303

Facsimile: (1)

Telex:

Email:

Commission(s): $34,4+, 51$

KAFKA PETER, MPI F PHYSIK \& ASTROPHYS, KARL SCHWARZSCHILDSTR 1

D 857\$0 GARCHING MUENCHEN, GERMANY

Telcphone: (49) 8932990

Facsimile: (49)

Commission(s): 44,51

Telex: 524629 astro d

Email:

GREEN BANK WV 24944 , USA

KAFTAN MAY A DR, NRAO, BOX 2, GREEN BANK
Telephone: (1)
Facsimile: (1)

Telex: $71(1-938-1530$

Email:

Commission(s): 34,40

KAHANE CLAUDINE DR, OBSERVATOIRE DE GRENOBLE, CERMO/ASTROPHYSIQUE, BP 53X F $380+1$ S MARTIN HERES CD, FRANCE

Telephone: (33) 76514600

Email: liahane/a frgag51

Facsimile: (33) $76+48821$

Commission(s):

Telex: $98013+$ usmg

KAHLER STEPHEN W DR, A F GEOPHYSICS LABORATORY, SPACE PHYSICS DIV PHP, HANSCOM AFB, BEDFORD MA 01731, USA

Telephone: (1) 6178613975

Email:

Facsimile: (1)

Telex:

Commission(s): $10 \mathrm{C}$ 
KAHLMANN HANS CORNELIS DR, RADIO OBS WESTERBORK, SCHATTENBERG 1

NL 9433 TA ZWIGGELTE, NETHERLANDS

Telephone: (31) $59392+21$

Facsimile: (31) 59392486

Telex:

Email: kahlmann_hansanfra.nl

Commission(s): $\$ 0,50$

KAHN FRANZ D PROF, DPT OF ASTRONOMY, UNIVERSITY OF MANCHESTER, MANCHESTER M13 9PL, UK

Telephone: (44) 1612737121

Email:

Facsimile: (44)

Commission(s): $3+, 40,44$

Telex: 668932 mchrul g

KAIFU NORIO DR, NOBEYAMA RADIO OBS, NAOJ, MINAMIMAKI MURA, NAGANO 38413, JAPAN

Telephone: (81)

Facsimile: (81)

Telex:

Email:

Commission(s): $34 \mathrm{C}, 40$

KAISER THOMAS R PROF, DPT OF PHYSICS, THE UNIVERSITY, SHEFFIELD S3 7RH, UK

Telephone: (4) $114278555^{\star}+277 \quad$ Facsimile: (4)

Email:

Commission(s): 22

Telex: 547216 ugshef $\mathrm{g}$

KAITCHUCK RONALD H, DPT OF PHYSICS \& ASTRON, BALL STATE UNIVERSITY, MUNCIE IN 47306, USA

Telephone: (1)

Facsimile: (1)

Telex:

Email:

Commission(s): 42

KAJINO TOSHITAKA, NTL ASTRONOMICAL OBS, MITAKA, TOKYO 188, JAPAN

Telephone: (81) $\$ 223+37+0$

Email: kajino(a) nao.ac.jp

Facsimile: $(81)+223+37+6$

Telex: 02822307 taomtk

KAKINUMA TAKAKIYO T PROF, INST FOR ATMOSPHERIC RES, NAGOYA UNIVERSITY, 3-13 HONOHARA TOYOKAWA AICHI $4+2$, JAPAN

$\begin{array}{ll}\text { Telephone: (81) } 533863154 & \text { Facsimile: }(81) \\ \text { Email: } & \text { Commission(s): } 40,49\end{array}$

KAKUTA CHUICHI DR, ASTROGEODYNAMICS OBS, HOSHIGAOKA 2-12, MIZUSAWA SHI, IWATE 023, JAPAN

Telephone: (81) 197247111

Facsimile: $(81)$

Commission(s): 19,31

Telex: 837628 ilsmiz j

Email:

KALAFI MANOUCHER, CTR FOR ASTRON RESEARCH, UNIVERISTY OF TABRIZ, TABRIZ 5IG64, IRAN

Telephone: (98) +132564

Facsimile: (98)

Commission(s): 28

Telex:

Email:

KALANDADZE N B DR, ABASTUMANI ASTROPHYSICAL, OBSERVATORY, GEORGIAN ACAD OF SCIENCES 383762 ABASTUMANI, GEORGIA

Telephone: (7) 227

Email:

Facsimile: (7)

Commission(s): 33

Telex: $327+109$

KALBERLA PETER, RADIOASTRONOMISCHES INST, UNIVERSITAET BONN, AUF DEM HUEGEL 7I

D 53121 BONN, GERMANY

Telephone: (49) 228733645

Email:

Facsimile: (49)

Commission(s): 05,40

Telex: $886+410$

KALER JAMES B PROF, DPT OF ASTRONOMY, UNIVERSITY OF ILLINOIS, 1002 W GREEN ST

URBANA IL 61801, USA

Telephone: (1) 2173339382

Facsimile: (1)

Commission(s): 34

Telex: $910-2+52+34$ ast

Email:

KALINKOV MARIN P DR, DPT OF ASTRONOMY, BULGARIAN ACAD SCIENCES, 72 LENIN BLVD BG 1784 SOFIA, BULGARIA

Telephone: (359) 2758927

Facsimile: (359)

Commission(s): 28

Telex: $2277+$ clanp bg

Email:

KALKOFEN WOLFGANG DR, CENTER FOR ASTROPHYSICS, HCO/SAO, G0 GARDEN ST

CAMBRIDGE MA 02138, USA

Telephone: (1) $617+957285$

Email: wolfacfa.harvard.cdu
Facsimile: (1) 6174957049

Commission(s): $12,36 \mathrm{C}$
Telex: 921428 satellite cam

KALLOGLIAN ARSEN T DR, BYURAKAN ASTROPHYSICAL, OBSERVATORY, 378433 BYURAKAN, ARMENIA Telephonc: (7) 8852283453

Email:
Facsimile: (7) $885228+1+2 \quad$ Telex:

Commission(s): 28 
KALMAN BELA DR, HELIOPHYSICAL OBSERVATORY, BOX 30, H 4010 DEBRECEN, HUNGARY Telephone: (36) $52311015 \quad$ Facsimile: (36) Telex: 72517 deobs $h$ Email:

Commission(s): 10,12

KALMYKOV A M DR, ASTRONOMICAL INSTITUTE, UZBEK ACADEMY OF SCIENCES 700000 TASHKENT, UZBEKISTAN

Telephone: (7)

Facsimile: (7)

Telex:

Email:

Commission(s):

KALNAJS AGRIS J DR, MOUNT STROMLO \& SIDING, SPRING OBSERVATORIES, PRIVATE BAG WODEN PO ACT 2606, AUSTRALIA

Telephone: (61) $62881111 * 248$

Facsimile: (61) $62+90233$

Telex: 62270 aa

Email: agris@mso.anu.oz

Commission(s): 33C

KALTCHEVA NADIA, DPT OF ASTRONOMY, SOFIA UNIVERSITY, 5 JAMES BOUCHIER AVE BG 1126 SOFLA, BULGARIA

Telephone: (359)

Facsimile: (359)

Telex:

Email: physfac1@bgearn.bitnet

Commission(s):

KALUZNY JANUSZ DR, ASTRONOMICAL OBSERVATORY, WARSAW UNIVERSITY, AL UJAZDOWSKIE 4 PL 00478 WARSAW, POLAND

Telephone: (48) 294011

Facsimile: (48)

Commission(s): $\$ 2$

Telex: 8171063

Email:

, NTL DEFENCE ACADEMY, YOKOSUKA, KANAGAWA 239, JAPAN

KAMBE EIJI, DPT OF GEOSCIENCE, NTL DEFENCE ACADEMY, YOKOSUKA,
Telephone: (81) $468413810 \quad$ Facsimile: (81) $468+45902$

Email: kambe(a)apsgw.aps.scikci.ac.jp Commission(s): 27

KAMEL OSMAN M PROF, DPT OF ASTRONOMY, FACULTY OF SCIENCES, CAIRO UNIVERSITY, GEZA, EGYPT Telephone: (20)

Facsimile: (20)

Email:

Commission(s):

Telex:

KAMEYA OSAMU DR, NOBEYAMA RADIO OBS, NAOJ, MINAMIMAKI MURA, NAGANO 38 4 13, JAPAN

Telephone: (81) 267982831

Facsimile: (81)

Commission(s): 19,40

Telex: 3329005 naionro $j$

Email:

KAMIJO FUMIO PROF DR, DPT OF ASTRONOMY, UNIVERSITY OF TOKYO, BUNKYO KU, TOKYO 113, JAPAN

Telephonc: (81)

Email:

Facsimile: (81)

Commission(s): 34

Telex:

KAMINISHI KEISUKE PROF, DPT OF PHYSICS, KUMAMOTO UNIVERSITY, 2-39-1 KUROKAMI KUMAMOTO 860, JAPAN

Telephone: $(81) 963+42111$

Email:

Facsimile: (81)

Telex:

Commission(s): 35

KAMMEYER PETER C DR, US NAVAL OBSERVATORY, 3450 MASSACHUSETTS AVE NW WASHINGTON DC 20392 5100, USA

Telephone: (1) 2026531563

Email:

Facsimile: (1)

Telex:

Commission(s): 07

KAMP LUCAS WILLEM DR, DPT OF ASTRONOMY, BOSTON UNIVERSITY, 725 COMMONWEALTH AVE BOSTON MA 02215, USA

Telephone: (1)

Facsimile: (1)

Telex:

Email:

Commission(s): 36,37

KAMPER KARL W DR, DAVID DUNLAP OBSERVATORY, UNIVERSITY OF TORONTO, BOX 360 RICHMOND HILL ON L+C +YG, CANADA

Telephone: (1) $41688+9562$

Facsimile: (1) 4169783921

Telex: 106986766

Email:

Commission(s):

KANAEV IVAN I DR, PULKOVO OBSERVATORY, ACADEMY OF SCIENCES, 10 KUTUZOV QUAY 196140 ST PETERSBURG, RUSSIA

Telephone: (7)

Facsimile: (7)

Telex:

Email:

Commission(s): 24 
KANBACH GOTTFRIED DR, MPI F EXTRATERRESTRISCHE, PHYSIK, KARL SCHWARZSCHILDSTR 1 D 85740 GARCHING MUENCHEN, GERMANY

$\begin{array}{lll}\text { Telephone: }(49) 893299544 & \text { Facsimile: }(49) & \text { Telex: } 5215845 \text { xter d } \\ \text { Email: bitnet:gok(a)dgaipp1s } & \text { Commission(s): } & \end{array}$

KANBUR SHASHI, DPT PHYSICS \& ASTRONOMY, UNIVERSITY OF GLASGOW, GLASGOW G12 8QQ, UK

Telephone: (4t) $1413398855 * 268 \quad$ Facsimile: (\$4) $14133+9(20 \quad$ Telex: 777070 unigla

Email: shash(a) astro.gla.ac.uk Commission(s): 27

KANDALIAN RAFIK, ASTROPHYSICAL OBSERVATORY, $378+33$ BYURAKAN, ARMENIA

Telephone: (7) $8852283453 \quad$ Facsimile: (7) $88525236+0 \quad$ Telex: $24334+$ orion su

Email: byurakan(a)adonis.ias.msk.su Commission(s): 28

KANDEL ROBERT S DR, LMD, ECOLE POLYTECHNIQUE, F 91128 PALAISEAU CDX, FRANCE

Telephone: (33) $169 \$ 18200 \quad$ Facsimile: (33) Telex: 691596

Email: $\quad$ Commission(s): 36

KANDEMIR GUELCIN, ISTANBUL TECHNICAL UNIV, FEN FAKULTESI FIZIK B, MASLAK $34+52$ ISTANBUL, TURKEY

$\begin{array}{ll}\text { Telephone: (90) } 1609109 & \text { Facsimile: (90) } \\ \text { Enrail: } & \text { Commission(s): }\end{array}$

KANDPAL CHANDRA D, UTTAR PRADESH STATE, OBSERVATORY, PO MANORA PEAK 263129

NAINITAL 263 129, INDIA

Telephone: (91) $59+22136,2325 \quad$ Facsimile: (91) Telex:

Email:

Commission(s): $\$ 2$

KANDRUP HENRY EMIL DR, DPT OF ASTRONOMY, UNIVERSITY OF FLORIDA, 211 SSRB

GAINESVILLE FL 32611, USA

Telcphone: (1) 90\$392 2681 Facsimile: (1) 90+3929741 Telex:

Email: kandrupáastro.ufl.edu Commission(s): $28,37,47$

KANE SHARAD R DR, SPACE SCIENCES LABORATORY, UNIVERSITY OF CALIFORNIA, BERKELEY CA 94720, USA

Telephone: (1) $\$ 156+21719$ Facsimile: (1)

Email: $\quad$ Commission(s): 10

Telex: 910-366-7945

KANEKO NOBORU DR, DPT OF PHYSICS, HOKKAIDO UNIVERSITY, KITA 10 NISHI 8, SAPPORO O60, JAPAN

Telephone: (81) $117162111 \quad$ Facsimile: (81)

Email:

Commission(s): 28

Telex: 932510 hokuse j

KANG GON IK, PYONGYANG ASTRON OBS, ACADEMY OF SCIENCES DPRK, TAESONG DISTRICT PYONGYANG, KOREA DPR

Telcphonc: (850)

Facsimile: $(850)$

Commission(s): $₫ 0$

Telex:

Email:

KANG JIN SOK, PYONGYANG ASTRON OBS, ACADEMY OF SCIENCES DPRK, TAESONG DISTRICT PYONGYANG, KOREA DPR

Telephonc: (850) Facsimile: $(\mathbf{8 5 0})$ Telex:

Email:

Commission(s): 10

KANG YOUNG WOON DR, DPT OF EARTH SCIENCE, KING SEJONG UNIVERSITY, 89 KOONJA-DONG SEOUL SUNGDONG $1337+7$, KOREA R

Tclephone: (82) 4675121 Facsimile: (82) Telex:

Email:

Commission(s): 42

KANYO SANDOR DR, KONKOLY OBSERVATORY, THEGE U 13/17, BOX 67, H 1525 BUDAPEST, HUNGARY

Telephone: (36) $11755866 / 175+122$ Facsimile: (36) 11569640 Telex:

Email:

Commission(s): 27

KAPAHI VIJAY K DR, TATA INST OF FUNDAMENTAL, RESEARCH, POONA UNIVERSITY CAMPUS

PUNE +11 007, INDIA

Telephone: (91) 212336105

Email: uunct!shalkti!gmrt!vija!

Facsimilc: (91) 212335760

Commission(s): 28,40
Telex: $01+5658 \mathrm{gmrt}$ in 
KAPISINSKY IGOR, ASTRONOMICAL INSTITUTE, SLOVAK ACADEMY SCIENCES, DUBRAVSKA 9 SK 84228 BRATISLAVA, SLOVAK R Telephone: (42) 7375157

Email:
Facsimile: (42)

Commission(s): 22
Telex: $(093355$

KAPLAN GEORGE H DR, US NAVAL OBSERVATORY, 3+50 MASSACHUSETTS AVE NW WASHINGTON DC $203925+20$, USA

Telephonc: (1) 2026530722

Facsimile: (1) 2026.530179

Telcx:

Email: ghk@eces.usno.navy.mil

Commission(s): $0+\mathrm{C}, 05,08$

KAPLAN J DR, DPT OF PHYSICS, UNIVERSITY OE CALIFORNIA, LOS ANGELES CA 90024, USA

Telephone: (1)

Facsimile: (1)

Tclex:

Email:

Commission(s):

- KAPLAN LEWIS D DR, ATMOSPH \& ENVIRONMENTAL, RESEARCH INC, 840 MEMORIAL DR CAMBRIDGE MA 02139, USA

Telephone: (1) 6175476207

Facsimile: (1)

Email:

Commission(s):

Telex: 951417 aerc

KAPOOR RAMESH CHANDER, INDIAN INSTITUTE OF, ASTROPHYSICS, KORAMANGALA BANGALORE 560034 , INDIA

Telephone: (91) 803566585

Email:

Facsimile: (91)

Commission(s): $4+, 47$

Telex: $845763 \mathrm{ijal}$ in

、 KARAALI SALIH DR, FACULTY OF SCIENCE, UNIVERSITY OF ISTANBUL, 3452 UNIVERSITY, TURKEY

Telephone: (90) $15224200 * 610 \quad$ Facsimile: (90)

Email:

Commission(s):

KARACHENTSEV I D DR, SPECIAL ASTROPHYSICAL OBS, ACADEMY OF SCIENCES, ZELENCHUKSKAJA 357147 STAVROPOLSKIJ, RUSSIA

Telephone: (7)

Email:

Facsimile: (7)

Telex:

Commission(s): $09,28,30,47$

KARACHENTSEVA VALENTINA, ASTRONOMICAL OBSERVATORY, KIEV UNIVERSITY, OBSERVATORNA STR 3 254053 KIEV 53, UKRAINE

Telephone: (7) $04+2161994$

Email: aolu(a)gluk.apc.org

Facsimile: (7)

Telex:

Commission(s): 28

KARANDIKAR R V PROF, DPT OF ASTRONOMY, UNIVERSITY OF OSMANIA, HYDERABAD 500 (007, INDIA

Telephone: (91) 71951

Facsimile: (\%1)

Telex:

Email:

Commission(s): 16,21

KARAS VLADIMIR, INSTITUTE OF ASTRONOMY, CHARLES UNIVERSITY, SVEDSKA 8 , CZ 15000 PRAHA S, CZECH R

Telephone: (42) Facsimile: (42)

Email: jana@aci.cvut.cz.

Commission(s):

Telex:

KARDASHEV N S DR, SPACE RESEARCH INSTITUTE, ACADEMY OF SCIENCES, PROFSOJUZNAYA UL 84/32 117810 MOSCOW, RUSSIA

$\begin{array}{ll}\text { Telephonc: (7) Facsimile: (7) } & \text { Telex: } \\ & \text { Comail: }\end{array}$

Email:

Commission(s): 40,51

- KARETNIKOV VALENTIN G R, ASTRONOMICAL OBSERVATORY, ODESSA STATE UNIVERSITY SHEVCHENKO PARK, 27001+ ODESSA, UKRAINE

Telejhone: (7) $048250356 \quad$ Facsimile: (7)

Email:

Commission(s): 42

Telex:

KARLICKY MARIAN, ASTRONOMICAL INSTITUTE, CZECH ACADEMY OF SCIENCES, ONDREJOV OBSERVATORY CZ 251 65 ONDREJOV, CZECH R

Telephone: (42) 20+857356/7111

Facsimilc: $(42) 20+851611$

Telex: 121579 astr c

Email: astsun(a)cscarn

Commission(s): 10,12

KAROJI HIROSHI DR, TOKYO ASTRONOMICAL OBS, NAOJ, OSAWA MITAKA, TOKYO 181, JAPAN

Tclephone: (81) $+22+136+3$

Facsimile: $(81)+22+13776$

Telex: 12822307 tanmtk j

Email: karojiáasxt1.mtk.nao.ac.jp

Commission(s): 28 
KAROVSKA MARGARITA DR, CENTER FOR ASTROPHYSICS, HCO/SAO, 60 GARDEN ST CAMBRIDGE MA 02138, USA

Telephone: (1) $617 \$ 957347$

Facsimile: (1)

Telex:

Email: karovska@efa

Commission(s): 27

KARP ALAN HERSH DR, HEWLETT PACKARD COMPANY, HP LABS 3U 7, 1501 PAGE MILL RD

PALO ALTO CA $9+304$, USA

Telephone: (1) 4158576766

Facsimile: (1) $\mathbf{4 1 5 8 5 7 5 1 7 2}$

Telex:

Email: karp@ahpl.hp.com

Commission(s): 27,36

KARPEN JUDITH T, NAVAL RESEARCH LABORATORY, CODE $\$ 175 \mathrm{~K}, 4555$ OVERLOOK AVE SW WASHINGTON DC 20375 5000, USA

Telephone: (1) 2027673441

Facsimile: (1)

Telex:

Email:

Commission(s): 10,12

KARPINSKIJ VADIM N DR, PULKOVO OBSERVATORY, ACADEMY OF SCIENCES, 10 KUTUZOV QUAY $1961+0$ ST PETERSBURG, RUSSIA

Telcphone: (7)

Facsimile: (7)

Telex:

Email:

Commission(s): $09,12,+4$

KARTTUNEN HANNU DR, CENTRE FOR SCIENTIFIC, COMPUTING, BOX 41, SF 02101 ESPOO, FINLAND

Telephone: (358) 4572709

Facsimile: $(358)+572302$

Telex:

Email: hkarttunen at csc.fi

Commission(s): 15

KARYGINA ZOYA V DR, ASTROPHYSICAL INSTITUTE, KAZAKH ACAD OF SCIENCES 480068 ALMA ATA, KAZAKHSTAN

Telephone: (7)

Facsimile: (7)

Telex:

Email:

Commission(s): 21

KASHSCHEEV B L PROF DR, KHARKOV INSTITUTE FOR, RADIOELECTRONICS, 310059 KHARKOV, UKRAINE

Telephone: (7)

Facsimile: (7)

Telex:

Email:

Commission(s): 22

KASPER U DR, ZNTRLINST F ASTROPHYSIK, STERNWARTE BABELSBERG, ROSA LUXEMBURG STR 17A

D 1473 POTSDAM, GERMANY

Telephone: (49)

Facsimile: (49)

Commission(s): 47

Telex:

Email:

KASTURIRANGAN K DR, ISRO SATELLITE CTR, AIRPORT RD, VIMANAPURA POST, BANGALORE 560 017, INDIA

Telephone: (91) 80 35+ 779

Facsimile: (91)

Commission(s): 4

Telex: $8+5325 \& 769$

Email:

KASUGA TAKASHI, COLLEGE OF ENGINEERING, HOSEI UNIVERSITY, KAJINOCHO KOGANEI

TOKYO 184, JAPAN

Telephone: (81) 423876244

Email:

Facsimile: (81) $423876123 \quad$ Telex: 3329005 tao nro j

Commission(s): 41

KASUMOV FIKRET K O DR, INSTITUTE OF PHYSICS, AKADEMGORODOC, NARIMANOV UL 33 370122 BAKU, AZERBAIDZHAN

Telephonc: (994) 396784

Facsimile: (994)

Telex:

Email:

Commission(s): 33

KATGERT PETER DR, STERREWACHT, BOX 9513, NL 2300 RA LEIDEN, NETHERLANDS

Telephone: (31) 71272727

Facsimile: (31)

Telex: 39058

Email:

Commission(s): 28

KATGERT-MERKELIJN J K DR, STERREWACHT, HUYGENS LAB, BOX 9513, NL 2300 RA LEIDEN, NETHERLANDS

Telephone: (31)

Facsimilc: (31)

Tclex:

Email:

Commission(s):

KATO MARIKO, DPT OF ASTRONOMY, KEIO UNIVERSITY, HIYOSHI KOULOKU-KU, YOKOHAMA SHI 223, JAPAN

Telephone: $(81)+4631111$

Facsimile: (81)

Email:

Commission(s): 35

Telex: 
KATO SHOJI PROF, DPT OF ASTRONOMY, KYOTO UNIVERSITY, KITASHIRAKAWA SAKYO KU KYOTO 606, JAPAN

Telephone: (81) 757512111

Facsimile: (81)

Commission(s): $12,33,47$

Telex: $5+22693$ libkyu j

Email: kato@kusastro.kyoto-u.ac.jp

KATO TAKAKO DR, INST OF PLASMA PHYSICS, NAGOYA UNIVERSITY, FUROCHO CHIKUSA KU NAGOYA 46401, JAPAN

Telephone: (81) 52781511

Email:

Facsimile: (81)

Commission(s): 14
Telex: 0\$47-3691 ippjinu j

KATSIS DEMETRIUS DR, 12 RUE VARNIS, GR 17124 NEA SMYRNE, GREECE

Telephone: (30) 9336014 Facsimile: (30) Telex:

Email:

Commission(s): 07

KATZ JONATHAN I, DPT OF PHYSICS, WASHINGTON UNIVERSITY, ST LOUIS MO 63130, USA

Telephone: (1) $31+8896202$

Facsimile: (1)

Telex:

Email:

Commission(s): 4

KATZ JOSEPH DR, RACAH INST OF PHYSICS, HEBREW UNIV OF JERUSALEM, JERUSALEM 91904, ISRAEL

Telephonc: (972) $258+604$

Facsimile: (972)

Telex: 25391 huil

Email:

Commission(s):

KAUFMAN MICHELE DR, DPT OF PHYSICS, OHIO STATE UNIVERSITY, 174 W 18TH AVE

COLUMBUS OH 43210, USA

Telephone: (1) $61+4225713$

Email:

Facsimilc: (1)

Commission(s): 28

Telex:

KAUFMANN JENS PETER DR, INST ASTRONOMIE/ASTROPHYS, TECHNISCHE UNIVERSITAT

HARDENBERGSTR 36, D 10623 BERLIN, GERMANY

Telephone: (49) $303145462 \quad$ Facsimile: (49)

Email: $\quad$ Commission(s):

Telex: 184262

KAUFMANN PIERRE PROF, CRAAE/ESCOLA POLITECNICA, UNIVERSIDADE DE SAO PAULO, CP 8174 05508 SAO PAULO SP, BRAZIL

Telephone: (55) 118159322

Facsimile: (55) $118154272 \quad$ Telex:

Email:

Commission(s): 10,12,40,51

KAULA WILLIAM M PROF, DPT OF EARTH \& SPACE SCI, UNIVERSITY OF CALIFORNIA

LOS ANGELES CA 90024, USA

Tclephone: (1)

Facsimile: (1)

Telex:

Email:

Commission(s): 07,16

KAWABATA KINAKI PROF, DPT OF PHYSICS, NAGOYA UNIVERSITY, FUROCHO CHIKUSA KU

NAGOYA 46401, JAPAN

Telephone: (81)

Facsimile: (81)

Commission(s): 40,47

Telex:

Email:

KAWABATA KIYOSHI, DPT OF PHYSICS COLL SCI, SCIENCE UNIVERSITY TOKYO, 1-3 KAGURAZAKA SHINJUKU, TOKYO, JAPAN

Telephone: (81) $3260+271 \quad$ Facsimile: (81) Telex:

Email:

Commission(s):

KAWABATA SHUSAKU PROF, KYOTO GAKUEN UNIVERSITY, NANJO, SOGABECHO KAMEOKA KYOTO 621, JAPAN

Telephone: (81) 771222001

Facsimile: (81)

Telex:

Email:

Commission(s): 42

KAWABE RYOHEI, NOBEYAMA RADIO OBS, NAOJ, MINAMISAKI MURA, NAGANO 384 13, JAPAN

Telephone: (81) $26763+385$

Email: kawabe (änro.nao.ac.j])

Facsimile: (81) $26763+339$ Telex:

Commission(s): 4 (1)

KAWAGUCHI ICHIRO PROF, DPT OF ASTRONOMY, KYOTO UNIVERSITY, KITASHIRAKAWA SAKYO KU

KYOTO 606, JAPAN

Telephone: (81)

Facsimile: (81)

Telex:

Email:

Commission(s): 12 
KAWAI NOBUYUKI, COSMIC RADLATION LAB, RIKEN, 2 HIROSAWA, WAKO SAITAMA 351 01, JAPAN

Telephone: (81) $484621111^{\star} 3226$

Facsimile: (81) $48+624640 \quad$ Telex:

Email: nkawai@postman.riken.go.jp

Commission(s): 44

KAWALER STEVEN D DR, DPT OF PHYSICS, IOWA STATE UNIVERSITY, AMES IA S0011, USA

Telephone: (1) 5152949728

Facsimile: (1) $51529+6027$ Telex:

Email: sdk@a)iastate.edu

Commission(s): 27

KAWARA KIMLAKI, TOKYO ASTRONOMICAL OBS, NAOJ, OSAWA MITAKA, TOKYO 181, JAPAN

Telephone: (81)

Facsimile: (81)

Telex:

Email:

Commission(s): 25

KAWASAKI MASAHIRO, INST COSMIC RAY RESEARCH, UNIVERSITY OF TOKYO, MIDORI CHO

TANASHI 188, JAPAN

Facsimile: (81) $42+623096 \quad$ Telex:

Email: kawasaki@etsun1.icrr.u-tokyo.a Commission(s): $\$ 7$

KAWATA YOSHIYUKI DR, KANAZAWA TECHNOLOGY INST, 7-1 OGIGAOKA, NONOICHOMACHI

ISHIKAWA 921, JAPAN

Telephone: (81)

Facsimile: (81)

Telex:

Email:

Commission(s):

KAYSER RAINER DR, HAMBURGER STERNWARTE, GOJENSBERGSWEG 112, D 21029 HAMBURG, GERMANY

Telephone: (49) 4072524126

Facsimilc: (49) $407252 \$ 198 \quad$ Telex: 217884

Email: st40010@dhhunit.bitnct

Commission(s): 47

KAZANTZIS PANAYOTIS DR, DPT OF MATHEMATICS, UNIVERSITY OF PATRAS, GR 26510 RION, GREECE

Telephone: (30) $61993456 \quad$ Facsimile: (30)

Telex:

Email:

Commission(s):

KAZES ILYA DR, OBSERVATOIRE DE PARIS, SECTION DE MEUDON, SECTION ASTROPHYSIQUE

F 92195 MEUDON PPL CDX, FRANCE

Telephone: (33) 145077606

Facsimilc: (33)

Telex:

Email:

Commission(s): $3+, 40$

KEAY COLIN S L PROF, DPT OF PHYSICS, NEWCASTLE UNIVERSITY, NEWCASTLE NSW 2308, AUSTRALIA

Telcphone: (61) $49215451 / 5440$

Facsimile: (61) 49216907

Telex: 28194 newun aa

Email: phcslk@ec.newcastle.cdu.au

Commission(s): 15,22,51

KEEL WILLIAM C, DPT OF PHYSICS \& ASTRON, UNIVERSITY OF ALABAMA, BOX 87032

TUSCALOOSA AL 35487 (324, USA

Telephone: (1) $2053+85050$

Facsimile: (1)

Telex:

Email: kecl@bildad.astr.ua.cdu

Commission(s): 28

KEENAN PHILIP C PROF EMER, PERKINS OBSERVATORY, OHIO STATE UNIVERSITY, BOX 449

DELAWARE OH 43015, USA

Telephone: (1) $61 \downarrow 3631257$

Facsimile: (1)

Telex: 810-482-1715

Email:

Commission(s): 29,45

KEGEL WILHELM H PROF, INST THEORETISCHE PHYSIK, UNIVERSITAET FRANKFURT

ROBERT MAYER STR 8-10, D GOOS 4 FRANKFURT A M, GERMANY

Telephone: (49) $697982357 \quad$ Facsimile: (49)

Telcx: $\mathbf{4 1 3 9 3 2}$

Email:

Commission(s): 34

KEIL KLAUS DR, DPT OF GEOLOGY, UNIVERSITY OF NEW MEXICO, 800 YALE BLVD NE ALBUQUERQUE NM 87131, USA

Telephone: (1) 5052774204

Facsimile: (1)

Telex:

Email:

Commission(s): 15

KEIL STEPHEN L, AIR FORCE GEOPHYSICS LAB, NTL SOLAR OBSERVATORY, SACRAMENTO PEAK OBS SUNSPOT NM 883 $\$ 9$, USA

Telephonc: (1) $505+341390$

Email:

Facsimile: (1)

Telex:

Commission(s): 12 
KELEMEN JANOS, KONKOLY OBSERVATORY, THEGE U 13/17, BOX 67, H 1525 BUDAPEST, HUNGARY

Telephone: (36) $11755866 / 175 \$ 122$

Facsimile: (36) 11569640

Telex: 227460 konob $h$

Email: kelemen@ogyalla.konkoly.hu

Commission(s):

KELLER CHARLES F, LOS ALAMOS NATIONAL LAB, MS F665, BOX 1663, LOS ALAMOS NM 87545, USA

Telephone: (1) 5056675648

Facsimile: (1)

Telex:

Lmail:

Commission(s):

KELLER CHRISTOPH U, NOAO NTL SOLAR OBS, BOX 26732, 950 N CHERRY AVE, TUCSON AZ 85726 6732, USA

Telephone: (1) 5203213445

Facsimile: (1) 5203259278

Telex:

Email: ckeller@noao.edu

Commission(s):

KELLER GEOFFREY, DPT OF ASTRONOMY, OHIO STATE UNIVERSITY, $17+$ W $18 T H$ AVE

COLUMBUS OH 432101106 , USA

Telephone: (1) 6144226279

Facsimile: (1)

Telex:

Email:

Commission(s):

KELLER HANS ULRICH DR, OBSERVATORY \& PLANETARIUM, NECKARSTR 47, D 70173 STUTTGART, GERMANY

Telephone: (49) 711162920

Facsimile: (49) 7112163912

Commission(s): $\$ 6,51$

Tclex: $711121+$ planstg

Email:

KELLER HORST UWE DR, MPI FUER AERONOMIE, POSTFACH 20, D 37189 KATLENBURG LINDAU, GERMANY

Telephone: (49) 555641419

Facsimilc: (49)

Commission(s): 15,49

Telex: 965527

KELLERMANN KENNETH I DR, NRAO, 520 EDGEMONT RD, CHARLOTTESVILLE VA 22903, USA

Telephone: (1) 8042960240

Facsimile: (1) $80+2960278$

Telcx:

Email: kkcllerm@nrao.edu

Commission(s): $28,40,44,47,51$

KELLOGG EDWIN M DR, CENTER FOR ASTROPHYSICS, HCO/SAO MS 3, 60 GARDEN ST

CAMBRIDGE MA 02138, USA

Telephone: (1)

Email:

Facsimile: (1)

Telex:

Commission(s): +4

KEMBALL ATHOL, NRAO, BOX 0, SOCORRO NM 87801 0387, USA

Telephone: (27) 5058357330

Facsimile: (27) 50583571127

Telex:

Email: akemball@nrao.edu

Commission(s):

KEMBHAVI AJIT K, TIFR, HOMI BHABHA RD, COLABA, BOMBAY 400 005, INDIA

Telephone: (91)

Facsimile: (91)

Telex:

Email:

Commission(s): 44,47

KENDERDINE SIDNEY DR, MULLARD RADIO ASTRON OBS, CAVENDISH LABORATORY, MADINGLEY RD CAMBRIDGE CB3 OHE, UK

Telephone: (44) 1223337294

Facsimile: (4t) $122335+599$

Tclex: 81292

Email:

Commission(s): 40

KENDZIORRA ECKHARD DR, ASTRONOMISCHES INSTITUT, UNIVERSITAET TUEBINGEN, WALDHAEUSERSTR 64 D 72076 TUEBINGEN, GERMANY

Telephone: (49) 7071296127

Email: 29382::kendziorra

Facsimile: (49) $7071293458 \quad$ Telex: 7262714 ait d

Commission(s):

KENNEDY EUGENE T, SCHOOL OF PHYSICAL SCI, NTL INSTITUTE HIGHER EDU, GLASNEVIN

DUBLIN 9, IRELAND

Telephone: (353) 1370071

Facsimile: (353)

Commission(s): 14

Telex: 30690 nihfd

Email:

KENNEDY HANS DANIEL, BOX 7243, TOOWOOOMBA MAIL CTR, QUEENSLAND \352, AUSTRALIA

Telephone: (61)

Facsimile: (61)

Email:

Commission(s):

Telex:

KENNEDY JOHN E PROF, 1003730 SPADINA, CRESCENT EAST, SASKATOON SK S7H 4H7, CANADA

Telephone: (1)

Facsimile: (1)

Telex:

Email:

Commission(s): $\$ 1,+6$ 
KENNICUTT ROBERT C JR, DPT OF ASTRONOMY, UNIVERSITY OF MINNESOTA, IIG CHURCH ST SE MINNEAPOLIS MN $55+55$, USA
Telephone: (1) 6123765224
Facsimile: (1)
Telex:
Entilil:
Commission(s): 28,34

KENT STEPHEN M, FERMILAB, MS 127, BOX 5U1, BA'TAVIA IL 60510, USA

Telephone: (1)

Facsimile: (1)

Telex:

Email:

Commission(s):

KENYON SCOTT J DR, CENTER FOR ASTROPHYSICS, HCO/SAO, 6I GARDEN ST, CAMBRIDGE MA (12138, USA

Telephone: (1) $617+957235$

Facsimile: (1)

Telex: 921428

Email: bitnet:kenyon ayefa

Commission(s): $+2 \mathrm{C}$

KEPLER S O, INSTITUTO DE FISICA, UFRGS, CP 15051, 90049 PORTO ALEGRE RS, BRAZIL

Telephone: (55) 512364677

Facsimile: $(55)$

Commission(s): 25,27

Telex: 051-1055 ufirs br

KERR FRANK J DR, ASTRONOMY PROGRAM, UNIVERSITY OF MARYLAND, COLLEGE PA
$\begin{array}{ll}\text { Telephone: (1) } 3014546302 & \text { Facsimile: (1) } \\ \text { Email: } & \text { Commission(s): } 28,33,34,40\end{array}$

KERR ROY P PROF, DPT PHYSICS \& ASTRONOMY, UNIVERSITY OF CANTERBURY, PRIVATE BAG

CHRISTCHURCH 1, NEW ZEALAND

Telephome: (64) 3482069 Facsimile: $(6+)$

Email: rpla a,math.canterburyac.me Commission(s):

Telex: $n z+1+4$

KERSCHBAUM FRANZ, INSTITUT HUER ASTRONOMIE, TUERKENSCHANZSTR 17, A 1180 WIEN, AUSTRIA

Telephone: $(\$ 3)+70600$

Ficsimile: $(43)+716688315$

Telex:

Email: kerschbaumaatsto.ast.univic.atc.at

Commission(s):

KESKIN VAROL, DPT ASTRONONI S SPACE SCI, EGE UNIVERSITY, SCIENCE FACULTY

BORNOVA IZMIR 35IIII), TURKEY

Telephone: (90) $232388(1110 / 1738$

Email: cfeastosarm3090.ege.edu.1\%

Facsimile: (90)

Telex:

KESSLER KARL G DR, NTL BUREAU OF STANDARDS, A 5015 ADMIN, GAITHERSBURG MD 211899, USA

Telephone: (1) $310192136+3$

Fatsimile: (1)

Telex: $19767+$ tit

Email:

Commission(s): 14,31

KESSLER MARTIN F DR, ESA/ESTE(, ASTROPHYSICS DIV, BOX 299, NL 2200 AG NOORDWIJK, NETHERLANDS

Telephone: (31) 171983623

Email: estes1::mkessler

Facsimile: (31) $171985+3-$

Telex: 39098

KESTEVEN MICHAEL J L DR, CSIRO, DIV OF RADIOPHYSICS, BOX 76, EPPING NSW 2121, AUSTRALIA

Telephone: (61) 28680222

Ficsimile: (61) 28680310

Telex: 26230 astro

Email: mlicvstereatatiosiro.au

Commission(s): 40

KHACHIKIAN E YE PROF, BYURAKAN ASTROPHYSICAL, OBSERVATORY, 378433 BYURAKAN, ARMENIA

Teleplsone: (7) 8852283453

Fasimile: (7) $88525236+1)$

Telex: $2+33+4$ orion su

Email: byumbancandonis.ias.mslisu Commission(s): $28 \mathrm{C}$

KHALESSEH BAHRAM DR, DPT OF PIIYSICS 91735654 , UNIVERSITY OF FERDOWSI, SCHOOL OF SCIENCES MASIIIIAD, IRAN

Telephone: (98) $51320121^{*} 64 \quad$ Filesimile: (98) 51871079

Telex: 512271 fuon ir

Emiıil:

Commixsion(s): 42

KHARADZE E K PROF, ABASTUMANI ASTROPHYŚICAL, OBSERVATORY, GEORGIAN ACAD OF SCIENCES 383762 ABASTUMANI, GEORGIA

$\begin{array}{ll}\text { Telephone: (7) } 998891 / 225460 & \text { Facsimile: (7) } \\ \text { Email: } & \text { Commisvion(s): } 33,34,45\end{array}$

Telex: $327+100$

KHARCIHENKO NINA, MAIN ASTRONOMICAL OBS, UKRAINIAN ACAD OF SCIENCE, GOLOSEEVO

252127 KIEV, IIKRAINE

Telephone: (7)

Facsimile: (7)

Email:

Commission(s):

Telex: 
KHARE BISHUN N DR, CRSR, CORNELL UNIVERSITY, 306 SPACE SCIENCES BLDG, ITHACA NY 14853 6801, USA Telephone: (I) 6072563934 Facsimile: (1) Telex:

Email:

Commission(s):

KHARE PUSHPA, PHYSICS DPT, UTKAL UNIVERSITY, BHUBANESWAR 751 004, INDIA

Telephone: (91) 674481079

Facsimile: (91) 67\$481 1+2 Telex:

Email: khare@iopb.ernet.in

Commission(s): 28,47

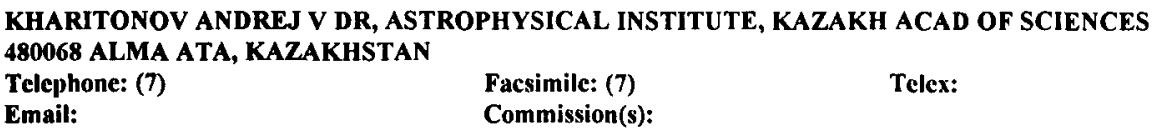

KHATISASHVILI ALFEZ SH DR, ABASTUMANI ASTROPHYSICAL, OBSERVATORY GEORGIAN ACAD OF SCIENCES, 383762 ABASTUMANI, GEORGIA

Telephone: (7)

Facsimile: (7)

Telex:

Email:

Commission(s): 20

KHETSURIANI TSIALA S DR, ABASTUMANI ASTROPHYSICAL, OBSERVATORY GEORGIAN ACAD OF SCIENCES, 383762 ABASTUMANI, GEORGIA

Telephone: (7)

Facsimile: (7)

Telex:

Email:

Commission(s): 12

KHOKHLOVA V L DR, INST OF ASTRONOMY, ACADEMY OF SCIENCES, PYATNITSKAYA UL 48 109017 MOSCOW, RUSSIA

Telephone: (7) 952315461

Facsimile: (7)

Commission(s): 29,36

Telex: $\$ 12623$ sestp su

Email:

KHOLSHEVNIKOV K V DR, ASTRONOMICAL OBSERVATORY, ST PETERSBURG UNIVERSITY BIBLIOTECHNAJA PL 2, $19890+$ ST PETERSBURG, RUSSIA

Telephone: (7) 2579488

Facsimile: (7)

Telex:

Email:

Commission(s): 07C

KHOLTYGIN ALEXANDER, ASTRONOMICAL INSTITUTE, ST PETERSBURG UNIVERSITY

BYBLYOTECHNAYA PL 2, ST PETERSBURG 198904, RUSSIA

Telephone: (7) 8124284163

Facsimile: (7) $812 \$ 286649$

Telex:

Email: afk@aispb4.spb.su

Commission(s):

KHOZOV GENNADIJ V, ASTRONOMICAL OBSERVATORY, ST PETERSBURG UNIVERSITY, BIBLIOTECHNAJA PL 2 198904 ST PETERSBURG, RUSSIA

Telephone: ( 7 ) 2579484

Facsimile: (7)

Email:

Commission(s): 35

Telex: 12168 phobos

KHROMOV G S DR, ASTRON GEOD SOCIETY OF, RUSSIA, 24 SADOVAJA KUDRINSKAJA S 103001 MOSCOW', RUSSIA

Telephone: (7) 952915896

Facsimile: (7)

Telex:

Email:

Commission(s): 34,41

KIANG TAO PROF, DUNSINK OBSERVATORY, DIAS, DUBLIN 15, IRELAND

Telephone: (353) 1387911

Facsimile: (353)

Commission(s): 20,41

Telex: 31687 dias ei

Email:

KIASATPOOR AHMAD PROF, DPT OF PHYSICS, UNIVERSITY OF ESFAHAN, DANESHGAH E, ESFAHAN, IRAN

Telephone: (98) 3144321

Facsimile: (98)

Email:

Commission(s):

Telex: 31-2295 ire u

KIBBLEWHITE EDWARD J DR, INSTITUTE OF ASTRONOMY, THE OBSERVATORIES, MADINGLEY RD, CAMBRIDGE CB3 OHA, UK

Telephone: (44) 1223337548

Email:

Facsimile: (44) $1223337523 \quad$ Telex:

Commission(s):

KIELKOPF JOHN F DR, DPT OF PHYSICS, UNIVERSITY OF LOUISVILLE, LOUISVILLE KY $4(1292$, USA

Telephone: (1) 5025886787

Email: bitnet:jfkiel01@ulkyvx

Facsimile: (1)

Commission(s): 14

Telex: 
KIGUCHI MASAYOSHI DR, RES INST SCIENCE \& TECH, KINKI UNIVERSITY, HIGASHI, OSAKA 577, JAPAN

Telephone: (81) 67212332

Facsimile: (81)

Commission(s): 35

Tclex:

Email:

KII TSUNEO DR, ISAS, 3-1-1 YOSHINODAI, SAGAMIHARA, KANAGAWA 229, JAPAN

Telephone: (81) 42751 3911*2624 Facsimile: (81) $427594253 \quad$ Telex: j27758iasa cro

Email:

Commission(s): 44

KIKUCHI SADAEMON PROF, ASTRONOMICAL INSTITUTE, TOHOKU UNIVERSITY, SENDAI AOBA

MIYAGI 980, JAPAN

Telephone: $(\mathbf{8 1})$

Facsimile: (81)

Email:

Commission(s):

Telex:

KILADZE R I DR, ABASTUMANI ASTROPHYSICAL, OBSERVATORY, GEORGIAN ACAD OF SCIENCES 383762 ABASTUMANI, GEORGIA

Telephone: (7)

Facsimile: (7) Telex:

Email: roki@abao.kheta.gcorgia.su

Commission(s): 16

KILAMBI G C DR, DPT OF ASTRONOMY, UNIVERSITY OF OSMANIA, HYDERABAD 500 007, INDIA

Telephone: (91) 71 951*247 Facsimile: (91)

Telex:

Email:

Commission(s): 37

KILAR BOGDAN DR, FAC OF GEODESY, UNIVERSITY OF LJUBLJANA, JAMOVA 2, LJUBLJANA, SLOVENIA

Telephone: (38)

Facsimile: (38)

Telex:

Email:

Commission(s):

KILIAN-MONTENBRUCK JUDITH, INST FUER ASTRONOMIE \&, ASTROPHYSIK, SCHEINERSTR 1

D 81679 MUENCHEN, GERMANY

Telephone: (49) 8992209429

Email: kilian@usm.uni-mucnchen.de

Facsimile: (49) $8992209+27 \quad$ Telex:

Commission(s):

KILKENNY DAVID DR, SAAO, BOX 9, OBSERVATORY 7935, SOUTH AFRICA

$\begin{array}{lll}\text { Telephone: (27) 021-47-0025 } & \text { Facsimile: (27) } & \text { Telex: 57-20309 sa } \\ \text { Email: } & \text { Commission(s): } 25 & \end{array}$

KILLEEN NEIL DR, AAO, ATNF, BOX 76, EPPING NSW 2121, AUSTRALIA

Telephone: (61) $28680222 \quad$ Facsimile: (61) $28680+00 \quad$ Telex:

Email: nikillcen(a)atnf.csiro.au Commission(s): $40,4+$

KILMARTIN PAMELA, MOUNT JOHN UNIVERSITY OBS, BOX 56, LAKE TEKAPO 8770, NEW ZEALAND

Telephone: (6t) $36806817 \quad$ Facsimile: (6t)

Telex:

Email: pokilmartin(a)csc.canterbury.ac. Commission(s): 20

KIM CHULHEE DR, DPT OF EARTH SCI EDU, CHONBUK NTL UNIVERSITY, CHONJU 560 756, KOREA R

Telephone: (82)

Facsimile: (82)

Telex:

Email:

Commission(s): 27

KIM CHUN HWEY, DPT ASTRONOMY \& SPACE SCI, CHUNGBUK NTL UNIVERSITY, CHEONGJU 360 763, KOREA R

Telephone: (82) 431613139

Facsimile: $(82)+3161+232$

Telex:

Email: kimch@astro.chungbuk.ac.kr

Commission(s):

KIM HO IL, KOREA ASTRONOMY OBS, 361 WHAAMDONG, YUSEONGGU, TAEJON 305 348, KOREA R

Telephone: (82)

Facsimile: (82)

Telex:

Email: hikim@hanul.issa.re.kr

Conmission(s):

KIM IRAIDA S, STERNBERG ASTRONOMICAL, INSTITUTE, 13 UNIVERSITETSKIJ PROSP MOSCOW 119899 RUSSIA

Telephone: (7) 0959392245

Facsimile: (7) 0951311357

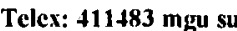

Email: kim@sai.msk.su

Commission(s): 10C,12

KIM JIK SU, PYONGYANG ASTRON OBS, ACADEMY OF SCIENCES DPRK, TAESONG DISTRICT, PYONGYANG KOREA DPR

Telephonc: (850)

Facsimile: $(\mathbf{8 5 0})$

Telex:

Email:

Commission(s): 47 
KIM KAP-SUNG DR, DPT ASTRONOMY SPACE SCI, KYUNG HEE UNIVERSITY, YONG IN KUN, KYUNG KIE DO 449 900, KOREA R

$\begin{array}{lll}\text { Telephone: (82) } 3312802443 & \text { Facsimile: (82) } 3312814964 & \text { Telex: } \\ \text { Email: } & \text { Commission(s): } 10\end{array}$

KIM KWANG-TAE DR, DPT ASTRON \& SPACE RES, CHUNGNAM NTL UNIVERSITY, DAEJOEN 304 764, KOREA R Telephone: (82) 428215463

Facsimile: (82)

Telex:

Email:

Commission(s): 40

KIM TU HWAN, KOREA ASTR/SPACE SCI INST, 36-1 WHAAM-DONG JUNG-GU, TAEJEON CHUNGCHUNGNAM-DO TAEJEON 30031 , KOREA R

Telephone: (82) 428231497

Email:

Facsimile: $(\mathbf{8 2})$

Telex: $45532 \mathrm{k}$

Commission(s): 27

KIM YONG HYOK DR, PYONGYANG ASTRON OBS, ACADEMY OF SCIENCES DPRK, TAESONG DISTRICT PYONGYANG, KOREA DPR

Telephone: (850) $53134 / 5$ \& 53239

Email:

Facsimile: (850)

Telex:

Commission(s):

KIM YONG UK, PYONGYANG ASTRON OBS, ACADEMY OF SCIENCES DPRK, TAESONG DISTRICT PYONGYANG, KOREA DPR

Telephone: $(850)$

Facsimile: $(\mathbf{8 5 0})$

Telex:

Email:

Commission(s):

KIM YUL, PYONGYANG ASTRON OBS, ACADEMY OF SCIENCES DPRK, TAESONG DISTRICT PYONGYANG, KOREA DPR

Telephone: (850)

Facsimile: (850)

Telex:

Email:

Commission(s):

KIM ZONG DOK, PYONGYANG ASTRON OBS, ACADEMY OF SCIENCES DPRK, TAESONG DISTRICT, PYONGYANG KOREA DPR

Telephone: $(\mathbf{8 5 0})$

Facsimile: $(\mathbf{8 5 0})$

Telex:

Email:

Commission(s): 14

KIMBLE RANDY A DR, DPT PHYSICS \& ASTRONOMY, JOHNS HOPKINS UNIVERSITY, CHARLES \& 3 4 TH ST BALTIMORE MD 21218, USA

Telejhone: (1) 3013388738

Email: span:scivax::casa::rak

Facsimile: (1)

Commission(s): 44

Telex: 9102400225 jhu casmd

KIMURA HIROSHI DR, PURPLE MOUNTAIN OBSERV, CAS, NANJING, CHINA PR

Telephone: (86) 2533921

Email:

Facsimile: (86) 25301459

Telex: $3+14+$ pmonj cn

Commission(s): 34

KIMURA TOSHIYA, COMPUTATIONAL SCIENCES DV, NTL AEROSPACE LABORATORY

JINDAIJI HIGASHIMACHI 744, CHOFU TOKYO 182, JAPAN

Telephone: $(81) 422475911 * 2550 \quad$ Facsimile: $(81)+22+38872 \quad$ Telex:

Email: kimura@nal.go.jp)

Commission(s): 34

KING ANDREW R DR, DPT OF ASTRONOMY, UNIVERSITY OF LEICESTER, UNIVERSITY RD

LEICESTER LE1 7RH, UK

Telephone: (44) 1162554455

Email:

Facsimile: (4)

Telex: 341198

KING DAVID LEONARD, ROYAL GREENWICH OBS, MADINGLEY RD, CAMBRIDGE CB3 OEZ, UK

Telephone: (44) 1223374749

Facsimile: (44) 1223374700 Telex:

Email: king@uk.ac.cam.ast-star

Commission(s): 34

KING DAVID S PROF, DPT PHYSICS \& ASTRONOMY, UNIVERSITY OF NEW MEXICO, 800 YALE BLVD NE ALBUQUERQUE NM 87131, USA

Telephone: (1)

Facsimile: (1)

Telex:

Email:

Commission(s): $35, \$ 1 \mathrm{C}$

KING HENRY C DR, TRILLIUM, 206 WHITE LION ROAD, LITTLE CHALFONT, BUCKS HP7 9NU, UK

Telephone: (44)

Facsimile: (4)

Email:

Commission(s): 41 
KING IVAN R PROF, ASTRONOMY DPT, UNIVERSITY OF CALIFORNIA, 601 CAMPBELL HALL

BERKELEY CA 94720, USA

Telephone: (1) 4156422206

Email: king@bkyast.berkelcy.edu

Facsimile: (1)

Commission(s): 25,28,33,37
Telex: 820181 ucb astral

Telex:

KING R B DR, BOX 725, MEDOCINO CA 95460, USA

Telephone: (1)

Facsimile: (1)

Commission(s): 14,29

KING ROBERT WILSON JR DR, DPT OF EARTH \& PLANET SCI, MIT RM 54620 , BOX 165 CAMBRIDGE MA 02139, USA

Telephone: (1) 6172537064

Facsimile: (1)

Telex: 921473 mit cam

Email:

Commission(s): 0-

KINGSTON ARTHUR E PROF, DPT OF APPLIED MATHS, \& THEORETICAL PHYSICS, QUEEN'S UNIVERSITY BELFAST BT7 1NN, UK

Telephone: (44) 1232245133

Email:

Facsimile: (4t)

Commission(s): 14

Telex: $\mathbf{7 4 8 7}$ qub amd $\mathrm{g}$

KING-HELE DESMOND G DR, ROYAL AIRCRAFT ESTABL, FARNBOROUGH HANTS, UK

Telephonc: (44) 125224461

Email:

Facsimile: (4)

Telex:

Commisxion(s): 07

KINMAN THOMAS D DR, KITT PEAK NTL OBS, BOX 26732, 950 N CHERRY AVE, TUCSON AZ 85726 6732, USA

Tclephonc: (1) 5203275511

Email:

Facsimile: (1)

Commission(s): 28,33

Telex: $0666-484$ au ra noao

KINNEY ANNE L DR, STSCI, HOMEWOOD CAMPUS, 3710 SAN MARTIN DR, BALTIMORE MD 21218, USA

Telephone: (1) $3013384831 \quad$ Facsimile: (1)

Email: 6549::kinney

Commission(s):

Telex: 6849101 stsci

KINOSHITA HIROSHI DR, TOKYO ASTRONOMICAL OBS, NAOJ, OSAWA MITAKA, TOKYO 181, JAPAN

Telephone: (81) 422413615

Facsimile: (81) $\$ 22+13793$

Telex: 02822307 taomtk

Email: kinoshita@c1.mtk.nao.ac.jp

Commission(s): 04P,07C,20

KIPLINGER ALAN L DR, APAS DPR, UNIVERSITY OF COLORADO, BOX 391, BOULDER CO 80309 0391, USA

Telephone: (1) 3034975892

Facsimile: (1)

Telex:

Email: span 9555::akiplinger

Commission(s): 10,27

KIPPENHAHN RUDOLF PROF, RAUTENBREITE 2, D 37083 GOETTINGEN, GERMANY

Telephonc: (49) 55124714

Facsimile: (49) 55122902

Telex:

Emait:

Commission(s): 27,35

KIPPER TONU DR, TARTU ASTROPHYSICAL OBS, ESTONIAN ACAD OF SCIENCES, EE 2024H TARTU, ESTONIA

Telephone: (372)

Facsimite: (372)

Telex:

Email:

Commission(s): $09,14,29$

KIRAL ADNAN PROF, UNIVERSITY OBSERVATORY, UNIVERSITY OF ISTANBUL, 3\$452 ISTANBUL, TURKEY

Telephone: (90)

Facsimile: (90)

Commission(s):

Telex:

Email:

KIRBIYIK HALIL DR, DPT OF PHYSICS, MIDDLE EAST TECH UNIV, 06531 ANKARA, TURKEY

Telephone: (90) $412237100 / 3528 \quad$ Facsimile: (90)

Telex: 42761 odtk tr

Email:

Commission(s):

KIRBY KATE P DR, CENTER FOR ASTROPHYSICS, HCO/SAO, 60 GARDEN ST, CAMBRIDGE MA 02138, USA

Telephone: (1) 6174957237

Facsimile: (1)

Commission(s): 14

Telex:

Email:

KIRIAN TATIANA, PULKOVO OBSERVATORY, GAO RAN, ST PETERSBURG 196140, RUSSIA

Telephone: (7) 8121234252

Facsimile: (7) 8123151701

Telex: 121351 pulob su

Email: anna@gaoranspb.su

Commission(s):

KIRK JOHN DR, MPI FUER KERNPHYSIK, POSTFACH 103980, D 69029 HEIDELBERG, GERMANY

Telephone: (49) 6221516248

Facsimile: (49) $6221516+82$

Telex:

Email:

Commission(s): tt 
KIRKPATRICK RONALD C DR, LOS ALAMOS NATIONAL LAB, MS 220, BOX 1663, LOS ALAMOS NM 87545, USA Telephone: (1) 5056674812

Facsimile: (1)

Telex:

Email:

Commission(s): 34

KIRSHNER ROBERT PAUL DR, DPT OF ASTRONOMY, HARVARD UNIVERSITY, 60 GARDEN ST CAMBRIDGE MA 02138, USA

Telephone: (1) 6174957390

Facsimile: (1)

Telex:

Email:

Commission(s): 28,34

KISELEV NIKOLAI N DR, ASTROPHYSICAL INSTITUTE, TADJIK ACAD OF SCIENCES, SVIRIDENKO UL 22 734042 DUSHANBE, TAJIKISTAN

Telephone: $(7)$

Email:

Facsimile: (7)

Telex:

Commission(s): 15C

KISELYOV ALEXEJ A DR, PULKOVO OBSERVATORY, ACADEMY OF SCIENCES, 10 KUTUZOV QUAY 196140 ST PETERSBURG, RUSSLA

Telephone: (7)

Facsimile: (7)

Telex:

Email:

Commission(s): 26

KISLYAKOV ALBERT G DR, INST OF APPLIED PHYSICS, ACADEMY OF SCIENCES, ULYANOV UL 46, 603600 N NOVGOROD, RUSSLA

Telephone: (7)

Facsimile: (7)

Telex:

Email:

Commission(s): 40

KISLYUK VITALIJ S DR, MAIN ASTRONOMICAL OBS, UKRAINIAN ACAD OF SCIENCE, GOLOSEEVO 252127 KIEV, UKRAINE

Telephone: (7)

Email:

Facsimile: (7)

Telex: 131406 sky us

KISSELEVA TAMARA P, PULKOVO OBSERVATORY, ACADEMY OF SCIENCES, 10 KUTUZOV QUAY 196140 ST PETERSBURG, RUSSLA

Telephone: (7)

Facsimile: (7)

Telex:

Email:

Commission(s): 20

KISSELL KENNETH E DR, ASTROMETROLOGY GROUP, UNIVERSITY OF MARYLAND, DPT OF PHYSICS COLLEGE PARK MD 207\$2, USA

Telephone: (1) 3013149531

Facsimile: (1) $3013149531 \quad$ Telex:

Email: kkissell@img.umd.edu

Commission(s): 09

KITAMOTO SHUNJI DR, FAC OF SCIENCES, OSAKA UNIVERSITY, MACHIKANEYAMA

TOYONAKA OSAKA 560, JAPAN

Telephone: (81) 68441151

Facsimile: (81)

Telex:

Email: bitnct:kitamoto@jjpnoskfm

Commission(s):

KITAMURA M PROF, TOKYO ASTRONOMICAL OBS, NAOJ, OSAWA MITAKA, TOKYO 181, JAPAN

Telephone: (81) $422325111 \quad$ Facsimile: (81)

Email:

Commission(s): 42

Telex: 2822307 taomtk j

KITCHIN CHRISTOPHER R DR, HATFIELD POLYTECHNIC, OBSERVATORY, BAYFORDBURY

HERTFORD HERTS SG13 8LD, UK

Telephone: (44) 1992558451

Facsimile: (4t)

Telex: $262+13$

Email:

Commission(s): 29,46

KIZILOGLU NILGUEN DR, DPT OF PHYSICS, MIDDLE EAST TECH UNIV, 06531 ANKARA, TURKEY

Telephone: (90) $412237100 * 3268$

Facsimilc: (O) $\$ 12868638$

Telex: 42761 odtu tr

Email: nlk@trmetu.bitnet

Commission(s): 35

KIZILOGLU UEMIT DR, DPT OF PHYSICS, MIDDLE EAST TECH UNIV, 06531 ANKARA, TURKEY

Telephone: (90) $412237100 * 3275$

Facsimile: (90) $\$ 12868638$

Telex: $\$ 2761$ odtu tr

Email: umk@trmetu.bitnet

Commission(s):

KJAERGAARD PER DR, UNIVERSITY OBSERVATORY, OESTER VOLDGADE 3

DK 1350 COPENHAGEN $K$, DENMARK

Telephone: (45) 31141790

Email:

Facsimile: (45) 31389157

Tclex: $\$+155$ danast $\mathbf{d k}$

Commission(s): 
KJELDSETH-MOE OLAV DR, INST THEORET ASTROPHYSICS, UNIVERSITY OF OSLO, BOX 1029 N 0315 BLINDERN OSLO 3, NORWAY

$\begin{array}{lll}\text { Telephone: (47) 47-2-456510 } & \text { Facsimile: (47) } & \text { Telex: } 72425 \text { unios n } \\ \text { Email: } & \text { Commission(s): } 10 & \end{array}$

KJURKCHIEVA DIANA DR, DPT OF PHYSICS, HIGHER PEDAGOGICAL INST, BG 9700 SHOUMEN, BULGARIA Telephone: (359) 63151216 Facsimile: (359) Telex:

Email:

Commission(s): 27,42

KLAPP JAIME DR, DPT DE FISICA, UNAM UNIDAD, APDO POSTAL 55-534, 09340 IZTAPALAPA0 DF, MEXICO Tclcphonc: (52) 5156442 Facsimile: (52)

Tclex: 1764186 kebeme

Email:

Commission(s):

KLARE GERHARD DR, LANDESSTERNWARTE, KOENIGSTUHL, D 69117 HEIDELBERG, GERMANY

Telephone: (49) 622110036

Facsimile: (49)

Telex:

Email:

Commission(s): 33

KLARMANN JOSEPH PROF, DPT OF PHYSICS, WASHINGTON UNIVERSITY, ST LOUIS MO 63130, USA

Telephone: (1) 3148896299

Facsimile: (1)

Telex: 650-2557719 mci

Email:

Commission(s):

KLECZEK JOSIP DR, ASTRONOMICAL INSTITUTE, CZECH ACADEMY OF SCIENCES, ONDREJOV OBSERVATORY CZ 25165 ONDREJOV, CZECH R

Telephone: (42) $204857157 / 7111$

Facsimile: $(+2) 204851611$

Telex: 121579 astr $\mathrm{c}$

Email: astsun@escarn

Commission(s): 05,10

KLEIN KARL LUDWIG DR, OBSERVATOIRE DE PARIS, SECTION DE MEUDON, DASOP

F 92195 MEUDON PPL CDX, FRANCE

Telephone: (33) 145347761

Facsimile: (33)

Telex:

Email: span:meudon::klein

Commission(s): $10,12,40$

KLEIN MICHAEL, J DR, JPL SSD, MS 303 401, 4800 OAK GROVE DR, PASADENA CA 91109, USA

Telephone: (1) 8183547132

Facsimile: (1)

Telex:

Email:

Commission(s): 51

KLEIN RICHARD I DR, LAWRENCE LIVERMORE LAB, L 23, BOX 808, LIVERMORE CA 94550, USA

Telephone: (1) 4154223548

Facsimile: (1)

Telex:

Email:

Commission(s): 36

KLEIN ULRICH, RADIOASTRONOMISCHES INST, UNIVERSITAET BONN, AUF DEM HUEGEL 71

D 53121 BONN, GERMANY

Telephone: (49) 228733644

Facsimile: (49)

Telex:

Email:

Commission(s): $\mathbf{2 8 , 4 0}$

KLEINMANN DOUGLAS E DR, HONEYWELL ELECTRO OPTICS, OPERATION, 2 FORBES RD LEXINGTON MA 02173, USA

Telephone: (1) 6178633841

Email:

Facsimile: (1)

Telex: $92-3+77$

Commission(s):

KLEMOLA ARNOLD R DR, LICK OBSERVATORY, UNIVERSITY OF CALIFORNIA, UCSC

SANTA CRUZ CA 95064, USA

Telephone: (1) 4084292907

Facsimilc: (1)

Telex:

Email:

Commission(s): 20,24

KLEMPERER W K DR, NBS, ELECTROMAGNETIC FIELDS D, 325 BROADWAY, BOULDER CO 80303, USA

Telephone: (1) $3034973757 \quad$ Facsimile: (1)

Email:

Commission(s)

Tclex: 592811 noaa masc bdr

KLEPCZYNSKI WILLIAM J DR, US NAVAL OBSERVATORY, 3450 MASSACHUSETTS AVE NW, WASHINGTON DC 203925100 , USA

Telephone: (1) 2026531521

Facsimile: (1)

Tclex: $710-822-1970$

Email:

Commission(s): 04,19,31C 
KLIEM BERNHARD DR, ASTROPHYSIKALISCHES INST, POTSDAM UNIVERSITY, AN DER STERNWARTE 16 D 14482 POTSDAM, GERMANY

Telephone: (49) 33174990

Facsimile: (49) 3317499200

Telex: 361231 stbbg d

Email: bkleim@aip.de Commission(s): 10

KLIMCHUK JAMES A DR, CTR FOR SPACE SCIENCES \&, ASTROPHYSICS, STANFORD UNIV ERL STANFORD CA 94305 4055, USA

Telephone: (1) 4157231765

Facsimile: (1) $4157252333 \quad$ Telex:

Email: klimchuk@flare.stanford.edu

Commission(s): 10

KLINGLESMITH DANIEL A DR, NASA GSFC, CODE 684, GREENBELT MD 20771, USA

Telephone: (1) $3012866541 \quad$ Facsimile: (1)

Telex:

Email:

Commission(s):

KLINKHAMER FRANS DR, INST F THEORETISCHE PHYSI, UNIVERSITAET KARLSRUHE

D 76128 KARLSRUHE, GERMANY

Telephone: (49) $7216082083 \quad$ Facsimile: (49) $7216083582 \quad$ Telex:

Email: frans.klinkhamer@physik.uni-karlsruhe.de Commission(s): 44

KLIORE ARVYDAS JOSEPH DR, JPL, 4800 OAK GROVE DR, PASADENA CA 91109, USA

Telephone: (1) 8183546164 Facsimile: (1) Telex: 675+29

Email: $\quad$ Commission(s):

KLOCK B L DR, 4509 BAYSIDE DRIVE, MILTON FL 32570, USA

Telephone: (1) 9049941728

Facsimilc: (1)

Telex:

Email:

Commission(s): $08,09,24$

\section{KLOCOK LUBOMIR DR, ASTRONOMICAL INSTITUTE, SLOVAK ACADEMY SCIENCES SK 05960 TATRANSKA LOMNI, SLOVAK R \\ Telcphone: (42) 96996 7866/7/8 $\quad$ Facsimile: (42) $969967656 \quad$ Telex: 78277 \\ Email: \\ Commission(s): 09}

KLOKOCNIK JAROSLAV DR, ASTRONOMICAL INSTITUTE, CZECH ACADEMY OF SCIENCES

ONDREJOV OBSERVATORY, CZ 25165 ONDREJOV, CZECH R

Telephone: (42) $204857158 / 7111$

Email: astfoz@csearn

Commission(s): 07

Telex: 121579 astr $\mathrm{c}$

KLVANA MIROSLAV, ASTRONOMICAL INSTITUTE, CZECH ACADEMY OF SCIENCES, ONDREJOV OBSERVATOR CZ 25165 ONDREJOV, CZECH R

Teicphone: (42) 204857221/7111 Facsimile: (42) 20\$851611 Telex: 121579 astr $\mathrm{c}$

Email: astsun@csearn

Commission(s): 10

KLYMYSHYN I A PROF, PUSHKIN UL 96 APT 66, 284000 IVANOFRANKOVSK, UKRAINE

$\begin{array}{ll}\text { Telephone: (7) } & \text { Facsimilc: (7) } \\ \text { Email: } & \text { Commission(s): }\end{array}$

Telex:

KNACKE ROGER F DR, PENN STATE ERIE, THE BEHREND COLLEGE, STATION RD, ERIE PA 16563-0203, USA Telephone: (1) 8148986105 Facsimilc: (1) 8148986213 Telcx:

Email: Commission(s): 15,34

KNAPENJOHAN HENDRIK, DPT DE PHYSIQUE, UNIVERSITE DE MONTREAL, CP 6128 SUCC CENTRE VILLE MONTREAL OC H3C 3J7, CANADA

Telephone: (1) $5143436111 * 3204$

Facsimile: (1) $5143432071 \quad$ Telex:

Email: knapen(a)astro.umontreal.ca

Commission(s): 28

KNAPP GILLIAN R DR, DPT ASTROPHYSICAL SCI, PRINCETON UNIVERSITY, PRINCETON NJ 08544 1001, USA Telephone: (1) 6094523824

Facsimile: (1)

Telex:

Email:

Commission(s): 28,33,34

KNEE LEWIS, ESO, CASILLA 19001, SEST PROJECT, SANTIAGO 19, CHILE

Telephone: (56) 26988757

Facsimile: (56) 26954263

Telex: 240881 esogo el

Email: Iknee@eso.org

Commission(s): 
KNEER FRANZ DR, UNIVERSITAETS STERNWARTE, GOETTINGEN, GEISMARLANDSTR 11 D 37083 GOETTINGEN, GERMANY

Telephone: (49) 551395042

Facsimile: (49)

Telex: 96753

Email:

Commission(s): 12

KNEZEVIC ZORAN, ASTRONOMICAL OBSERVATORY, VOLGINA 7

YU 11050 BEOGRAD, YUGOSLAVIA-SERBIA \& MONTENEGRO

Telephone: (38) $11419357 / 421875 \quad$ Facsimile: (38)

Email: zoran@aob.aob.bg.ac.yu

Commission(s): $07,15 C, 20$

Telex: 72610 aob yu

KNIFFEN DONALD A DR, DPT OF PHYSICS, BOX 862, HAMPDEN SYDNEY COLLEGE, VA 23943, USA

Telephonc: (1)

Facsimile: (1)

Telex:

Email:

Commission(s):

KNOLKER MICHAEL DR, KIEPENHEUER INSTITUT, FUER SONNENPHYSIK, SCHOENECKSTR 6 D 79104 FREIBURG BREISGAU, GERMANY

Telephone: (49)

Facsimile: (49)

Commission(s): 12,35

Telex:

Email:

INSTITUTE, SLOVAK ACADEMY SCIENCES

KNOSKA STEFAN, ASTRONOMICAL INSTI

Telephone: (42) $969967866 / 7 / 8 \quad$ Facsimile: $(\$ 2) 969967656 \quad$ Telex:

Email:

Commission(s): 10

KNOWLES STEPHEN H DR, CODE 02, NAVSPASUR, DAHLGREN VA $22+48$ 5180, USA

Telephone: (1)

Facsimile: (1)

Telex:

Email:

Commission(s): 19,51

KNUDE JENS KIRKESKOV DR, UNIVERSITY OBSERVATORY, OESTER VOLDGADE 3

DK 1350 COPENHAGEN K, DENMARK

Telephone: (45) 31141790

Email: indus@astro.ku.dk

Facsimile: (45) 31389157

Commission(s): 25C,3t

Telex: $\mathbf{4 1 5 5}$ danast $\mathbf{d k}$

KO HSIEN C PROF, DPT OF ELECT ENGINEERING, OHIO STATE UNIVERSITY, 1958 NEIL AVE

COLUMBUS OH 43210, USA

Telephone: (1) 6144222571

Facsimile: (1)

Telex: $24-5334$

Email:

Commission(s): 40

KOBAYASHI EISUKE DR, SCIENCE INST OF OSAKA, 1323 KARITA + CHOME, SUMIYOSHI KU, OSAKA 558, JAPAN Telephone: (81) 66921882

Facsimile: (81)

Telex:

Email:

Commission(s):

KOBAYASHI YUKISAYU, TOKYO ASTRONOMICAL OBS, NAOJ, OSAWA MITAKA, TOKYO 181, JAPAN

Telephone: (81) 422325111 Facsimile: (81)

Emait:

Commission(s): 31

Telex: 2822307 taomtk

KOCER DURCUN DR, DPT OF ASTRONOMY, UNIVERSIYT OF ISTANBUL, 34452 ISTANBUL, TURKEY

Telephone: (90) 15223597

Facsimile: (90) 15226123

Telex:

Email:

Commission(s): 51

KOCH DAVID G, NASA AMES RESEARCH CTR, MS 245 6, MOFFETT FIELD CA 94035, USA

Telephone: (1)

Facsimile: (1)

Telex:

Email:

Commission(s):

KOCH ROBERT H DR, DPT ASTRON \& ASTROPHYS, UNIV OF PENNSYLVANIA, 209 S 33RD ST, PHILADELPHIA PA 19104, USA

Telephone: (1) 2158987882

Email: rkoch@upenn.sas.edu

Facsimile: (1) 2158989336

Telex: $83+621$

Commission(s): $25,42,51$

KOCHAROV GRANT E PROF, IOFFE PHYSICAL TECH INST, ACADEMY OF SCIENCES

POLYTECHNICHESKAYA UL 26, 194021 ST PETERSBURG, RUSSIA

Telephone: (7) 2479167

Facsimile: (7)

Telex:

Email:

Commission(s): 4 
KOCHHAR R K DR, INDIAN INSTITUTE OF, ASTROPHYSICS, KORAMANGALA, BANGALORE 560 034, INDIA

Telephone: (91) 803566585

Facsimile: (91)

Email:

Commission(s): $28,35,41$

Tclex: $8+5763$ iiab in

KOCH-MIRAMOND LYDIE DR, CEA CEN, IRF/DPHG/SAP, BP 2, F 91191 GIF/YVETTE CDX, FRANCE

Telephone: (33) 169084329

Facsimile: (33)

Telex: 690860

Email:

Commission(s); 44

KODAIRA KEIICHI PROF, TOKYO ASTRONOMICAL OBS, NAOJ, OSAWA MITAKA, TOKYO 181, JAPAN

Telephone: (81) $422325111 \quad$ Facsimile: (81)

Email:

Commission(s): 28,29,36

Tclex: 2822307 taomtk j

KODAMA HIDEO, UJI RESEARCH CENTER, YUKAWA INSTITUTE, KYOTO UNIV GOKASHO, UJI 611, JAPAN

Telephone: (81) 774317423

Facsimile: (81) 774336226

Telex: kodama/ayisuni.

Email: .yukawa.kyoto.kyoto-u.ac.jp Commission(s): 47

KOEBERL CHRISTLAN DR, INSTITUTE OF GEOCHEMISTRY, UNIVERSITY OF VIENNA, DR KARL LUEGER RING 1 A 1010 WIEN, AUSTRIA

Telephone: (43) 122243002360

Facsimile: (43)

Telex:

Email: a8631dab@vm.univie.ac.at

Commission(s): 15,22C,51

KOEHLER H PROF DR, SAUERBRUCHSTR 6, D 7920 HEIDENHEIM, GERMANY

Telephone: (49) $732144560 \quad$ Facsimilc: (49) Telcx:

Email:

Commission(s): 09

KOEHLER JAMES A PROF, DPT OF PHYSICS, UNIV OF SASKATCHEWAN, SASKATOON SK STN OW0, CANADA Telephone: (1) 3069666442 Facsimile: (1) Telex:

Email: Commission(s):

KOEHLER PETER, GARL ZEISS JENA GMBH, ASTRON INSTRUMENTS DIV, BOX 125, D 07740 JENA, GERMANY Telephone: (49) 785882575

Email: Facsimile: (49) 785882083 Telex: $587452 \mathrm{czg}$ dd

KOEMPE CARSTEN, UNIVERSITAETS STERNWARTE, SCHILLERGAESSCHEN 2, D 07745 JENA, GERMANY

Telephone: (49) 3641630313

Facsimile: (49) $36+155594$

Telex: 0331506 uni d

Email: koempe@betty.astro.uni-jena.de Commission(s): 34

KOEN MARTHINUS DR, SAAO, BOX 9, OBSERVATORY 7935, SOUTH AFRICA

Telephone: (27) 2721470025

Facsimile: (27) 2721473639

Telex: 520309 saao sa

Email: ck@saao.ac.za

Commission(s): 27

KOENIGSBERGER GLORIA, INSTITUTO DE ASTRONOMIA, UNAM, APDO POSTAL 70-264 04510 MEXICO DF, MEXICO

Telephone: (52) $9055485305 / 06$

Email:

Facsimile: (52)

Telex:

Commission(s):

KOEPPEN JOACHIM DR, INST THEOR PHYS \& STERNW, UNIVERSITAET KIEL, OLSHAUSENSTR 40 D 24098 KIEL, GERMANY

Telephone: (49) 4318804103

Email: pas86@rz.uni,kicl.d400.de
Facsimile: $(49)+31880+\$ 32$

Commission(s): 34
Telex: 292706 iapki d

KOESTER DETLEV DR, INST THEOR PHYS \& STERNW, UNIVERSITAET KIEL, OLSHAUSENSTR 40 D 24098 KIEL, GERMANY

Telephone: (49)

Email:

Facsimile: $(49)$

Telex:

Commission(s): 35,36

KOFMAN LEV, TARTU ASTROPHYSICAL OBS, EE 2444 TORAVERE, ESTONIA

Telephone: (372) $3410450 \quad$ Facsimile: (372) 3410205 Telex:

Email: $\quad$ Commission(s):

KOGOSHVILI NATELA G, ABATSUMANI ASTROPHYSICAL, OBSERVATORY, MOUNT KANBILI 383762 ABASTUMANI, GEORGIA

Telephone: (7) 283

Facsimile: (7)

Telex:

Email:

Commission(s): 28 
KOGURE TOMOKAZU DR, TOGANO-O 1-10, HASHIMOTO, YAWATA, KYOTO 614, JAPAN

Telephone: (81) 759832984

Facsimile: (81)

Telex:

Email:

Commission(s): 29

KOHL JOHN L DR, CENTER FOR ASTROPHYSICS, HCO/SAO, 60 GARDEN ST, CAMBRIDGE MA 02138, USA

Telephone: (1) 6174957377

Facsimile: (1)

Telex: 921428

Email:

Commission(s): 14

KOHOUTEK LUBOS DR, HAMBURGER STERNWARTE, GOJENSBERGSWEG 112, D 21029 HAMBURG, GERMANY

Telephone: (49) 4072524112

Facsimile: (49)

Commission(s): $15,20,34$

Telex: 217884

Email:

KOJIMA MASAYOSHI DR, SOLAR TERRESTRIAL LAB, NAGOYA UNIVERSITY, 3-13 HONOHARA TOYOKAWA

AICHI 442, JAPAN

Telephone: (81) 53383154

Facsimile: (81) 533860811

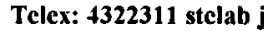

Email: kojima(a)stclab.nagoya-u.ac.jp)

Commission(s): 40

KOJIMA YASUFUMI, DPT OF PHYSICS, HIROSHIMA UNIVERSITY, HIGASHI, HIROSHIMA 739, JAPAN

Telephone: (81) 824247365

Facsimile: (81) $82+2+1) 717$

Telex:

Email: kojima@theo.phys.sci.hiroshima-u.ac.jp

Commission(s): 44

KOJOIAN GABRIEL DR, DPT OF PHYSICS, UNIVERSITY OF WISCONSIN, EAU CLAIRE WI 54701, USA

Telephone: (1) 7158363148

Facsimile: (1)

Telex:

Email:

Commission(s): 28,40

KOKKOTAS KONSTANTINOS DR, DPT ASTROPHY ASTRON MECH, UNIVERSITY THESSALONIKI

GR 54006 THESSALONIKI, GREECE

Telephone: (30) 31991357

Facsimile: (30) 31992777

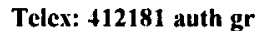

Email: caaz0104@grtheun1.carn

Commission(s): 47

KOKURIN YURIJ L DR, LEBEDEV PHYSICAL INST, ACADEMY OF SCIENCES, LENINSKY PROSPEKT 53

117924 MOSCOW, RUSSIA

Telephone: (7) 951350360

Facsimile: (7)

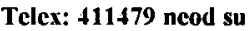

Email:

Commission(s): 08

KOLACZEK BARBARA DR, PLANETARY GEODESY DEPT, POLISH ACAD OF SCIENCES, UL BARTYCKA 18 PL 00716 WARSAW, POLAND

Telephone: (48) 22403766

Facsimile: (\$8) 39121273

Telex: 815670 cbk pl

Email: cbk@camk.edu.pl

Commission(s): 04,19C,31

KOLB EDWARD W DR, FERMILAB, MS 209, THEORETICAL ASTROPHYSICS, BATAVIA IL 60510, USA

Telephone: (1) 3128404695

Facsimile: (1)

Telex: $720+81$

Email:

Commission(s): $4+, 47$

KOLCHINSKIJ I G DR, MAIN ASTRONOMICAL OBS, UKRAINIAN ACAD OF SCIENCE, GOLOSEEVO 252127 KIEV, UKRAINE

Telephone: (7)

Facsimile: (7)

Telex:

Email:

Commission(s): 24

KOLESNIK IGOR G DR, MAIN ASTRONOMICAL OBS, UKRAINIAN ACAD OF SCIENCE, GOLOSEEVO

252127 KIEV, UKRAINE

Telephone: (7) 044663110

Facsimile: (7)

Commission(s): 33,3ł

Telex: 131406 sky su

Email:

KOLESNIK L N DR, MAIN ASTRONOMICAL OBS, UKRAINIAN ACAD OF SCIENCE, GOLOSEEVO 252127 KIEV, UKRAINE

Telephone: (7) 044660869

Facsimile: (7)

Telex: 131406

Email:

Commission(s): 33

KOLESOV A K DR, ASTRONOMICAL OBSERVATORY, ST PETERSBURG UNIVERSITY, BIBLIOTECHNAJA PL 2 199178 ST PETERSBURG, RUSSIA

Tclephone: (7)

Email:
Facsimile: (7)

Commission(s): 36
Telex: 
M 638

KOLEV DIMITAR ZDRAVKOV, NTL ASTRONOMICAL OBS, BULGARIAN ACAD SCIENCES, BOX 136

BG 4700 SMOLJAN, BULGARIA

Telephone: (359) 7341559

Facsimile: (359)

Telex:

Email:

Commission(s):

KOLKA INDREK, TARTU ASTROPHYSICAL OBS, EE 244 TORAVERE, ESTONIA

Telephone: (372) $7410438 \quad$ Facsimile: (372) $7+10205$ Telex:

Email: indrek@aai.ee Commission(s): 29,46

KOLLATH ZOLTAN DR, KONKOLY OBSERVATORY, THEGE U 13/17, BOX 67, H 1525 BUDAPEST, HUNGARY Telephone: (36) 1175 5866/175 $\$ 122 \quad$ Facsimile: (36)

Email:

Commission(s): 27

Telex: $227+60$ konob h

KOLLATSCHNY WOLFRAM DR, UNIVERSITAETS STERNWARTE, GOETTINGEN, GEISMARLANDSTR 11 D 37083 GOETTINGEN, GERMANY

Telephone: (49) $551395067 \quad$ Facsimile: (49)

Telex: 96753

Email: bitnet:wkollat@dgogwdg1 Commission(s): 28

KOLLBERG ERIK L PROF, DPT OF PHYSICS, CHALMERS TECHNICAL UNIV, S 41296 GOETEBORG, SWEDEN

Telephone: (46) $31810100 \quad$ Facsimilc: (46)

Email: $\quad$ Commission(s):

Telex: 2400 onspace

KOMAROV N S DR, ASTRONOMICAL OBSERVATORY, ODESSA STATE UNIVERSITY, SHEVCHENKO PARK 270014 ODESSA, UKRAINE

Telephone: (7) 048220396

Facsimile: (7)

Commission(s):

Telex:

Email:

KOMBERG BORIS, SPACE RESEARCH INSTITUTE, ACADEMY OF SCIENCES, PROFSOJUZNAYA UL 84/32 117810 MOSCOW, RUSSIA

Telephone: (7) 0953333366

Email: bkomberg@esoc1.bitnet

Facsinile: (7) 0953332378

Telex: $\$ 11+79$ neod su

KOMITOV BORIS, DPT OF ASTRONOMY, BLVD TSARIGRADSKO SH 72, BG 1784 SOFIA, BULGARIA

Telephone: (359) 2758827

Email: planet@bgearn.bitnet

Facsimile: (359) 2758927 Telex:

Commission(s):

KONDO MASAAKI DR, SENSHU UNIVERSITY, HIGASHI-MITA TAMA-KU, KAWASAKI SHI, KANAGAWA 214, JAPAN

Telephone: (81) 449117131

Email: 86123@nacsis.ac.jp

Facsimile: (81)

Telex:

Commission(s): 44

KONDO MASAYUKI DR, 125 OSAWA, MITAKA, TOKYO 181, JAPAN

Telephone: (81)

Facsimile: (81)

Commission(s):

Telex:

Email:

KONDO YOJI DR, NASA GSFC, CODE 684, GREENBELT MD 20771, USA
Telephone: (1) 3012866247
Facsimile: (1) 3012861752

Email: kondo@stars.gsfc.nasa.gov $\quad$ Commission(s): $34,36,+2 \mathrm{C}, 44$

KONIGL ARIEH DR, ASTRONOMY \& ASTROPHYS CTR, UNIVERSITY OF CHICAGO, 5640 S ELLIS AVE

CHICAGO IL 60637, USA

Telephone: (1) 3127027968

Email: lasr::oddjob::arieh

Facsimile: (1)

Telex: 282131

KONIN V V DR, NIKOLAEV OBSERVATORY, UKRAINIAN ACAD OF SCIENCE, 327000 NIKOLAEV, UKRAINE

Telephone: (7)

Facsimile: (7)

Telex:

Email: root@mao.nikolacv.ua

Commission(s): 08

KONONOVICH EDWARD V DR, STERNBERG STATE ASTR INST, UNIVERSITETSKIJ PROSP 13 119899 MOSCOW, RUSSIA

Telephone: (7)

Facsimile: (7)

Commission(s): 12,46

Telex:

Email:

KONOPLEVA VARVARA P DR, MAIN ASTRONOMICAL OBS, UKRAINIAN ACAD OF SCIENCE, GOLOSEEVO 252127 KIEV, UKRAINE

Telephone: (7) 044663110

Email: 
KONTIZAS EVANGELOS DR, ASTRONOMICAL INSTITUTE, NTL OBSERVATORY OF ATHENS, BOX 20048 GR 11810 ATHENS, GREECE

Telephone: (30) 13461191

Facsimile: (30)

Commission(s): $28,36,37,50$

Telex: 215530 obsa gr

Email:

KONTIZAS MARY DR, DPT OF ASTRONOMY, NTL UNIVERSITY OF ATHENS, PANEPISTIMIOPOLIS

GR 15771 ZOGRAFOS, GREECE

Telephone: (30) 17235122

Facsimile: (30)

Telex:

Email:

Commission(s): $\mathbf{3 7 , 5 0}$

KONTOROVICH VICTOR, INST OF RADIO ASTRONOMY, 4 CHERVONOPRAPORNA ST, KHARKIV 310002, UKRAIN

Telephone: (7) 572451014

Facsimile: (7) 572476506

Telex: 311039 space su

Email: rai@ira.kharkov.ua

Commission(s):

KOO BON CHUL, DPT OF ASTRONOMY, SEOUL NTL UNIVERSITY, SEOUL 151 742, KOREA R

Telcphone: (82) 28806623

Email: koo@astrohi.snu.ac.kr

Facsimile: (82) 28871435

Telex:

KOO DAVID C-Y DR, LICK OBSERVATORY, UNIVERSITY OF CALIFORNIA, NTL SCIENCES II SANTA CRUZ CA 95064, USA

Telephone: (1) 4084292130

Facsimile: (1) $408 \$ 263115$

Telex: $91099717+1$ unical

Email: koo@ahelios.ucsc.edu

Commission(s): $28,+7$

KOORNNEEF JAN DR, SPACE RESEARCH DPT, BOX 800, NL 9700 AV GRONINGEN, NETHERLANDS

Telephone: (31)

Facsimile: (31)

Telex:

Email:

Commission(s): 34

KOPECKY MILOSLAV DR, ASTRONOMICAL INSTITUTE, CZECH ACADEMY OF SCIENCES ONDREJOV OBSERVATORY, CZ 25165 ONDREJOV, CZECH R

Tclephone: (42) 204 857126/7111 Facsimile: (42) 204851611

Email: astsun(a)cscarn

Commission(s): 10,12

Telex: 121579

KOPP GREG, MEADOWLARK OPTICS, 7460 WELD COUNTY RD 1, LONGMONT CO 80504 9470, USA

Telephone: (1) 3037764068

Facsimile: (1) 3037765856

Telex:

Email: gkopp@meadowlark.com

Commission(s):

KOPP ROGER A DR, LOS ALAMOS NATIONAL LAB, MS F645, BOX 1663, LOS ALAMOS NM 87545, USA

Telephone: (1) 5056674398

Facsimile: (1)

Telex: 660495 los alamos

Email: rak@lanl.gov

Commission(s):

KOPYLOV I M DR, PULKOVO OBSERVATORY, ACADEMY OF SCIENCES, 10 KUTUZOV QUAY

196140 ST PETERSBURG, RUSSIA

Telephone: (7) 9282242

Facsimile: (7)

Telex:

Email:

Commission(s): 09,27

KORCHAK A A DR, IZMIRAN, ACADEMY OF SCIENCES, 1\$2092 TROITSK, RUSSIA

Telephone: (7)

Facsimile: (7)

Telex:

Email:

Commission(s):

KORMENDY JOHN DR, INSTITUTE FOR ASTRONOMY, UNIVERSITY OF HAWAII, 2680 WOODLAWN DR HONOLULU HI 96822, USA

Telephone: (1) 8089566680

Email: korment+dy@ifa.hawaii.edu

Facsimile: (1) 8089882790

Telex: 7238459

KOROVYAKOVSKIJ YURIJ P DR, SPECIAL ASTROPHYSICAL OBS, ACADEMY OF SCIENCES, NIZHNIJ ARKHYZ 357147 STAVROPOLSKIJ, RUSSIA

Telcphone: (7)

Facsimile: (7)

Telex:

Email:

Conmission(s): 09

KOSAI HIROKI, 488302 OSAWA, MITAKA, TOKYO 181, JAPAN

Telephone: (81) 422310874

Facsimile: (81)

Commission(s): 20

Email: 
KOSHIBA MASA-TOSHI DR, TOKAI UNIVERSITY, 2-28 TOMIGAYA, SHIBUYA, TOKYO 151, JAPAN

Telephone: (81) $334672211^{*} 483$

Facsimilc: (81) 34854958

Telex:

Email:

Commission(s): 44

KOSIN GENNADIJ S DR, PULKOVO OBSERVATORY, ACADEMY OF SCIENCES, 10 KUTUZOV QUAY 196140 ST PETERSBURG, RUSSIA

Telephone: (7)

Facsimile: (7)

Telex:

Email:

Commission(s): 08

KOSOVICHEV ALEXANDER, CTR FOR SPACE SCI \& ASTRO, ERL 328 MC 4055 , STANFORD UNIVERSITY STANFORD CA 94305 4055, USA

Telephone: (1) 4157237667

Facsimile: (1) 4157252333

Telex:

Email: akosovichev@solar.stanford.cdu

Commission(s): 35
KOSTIK ROMAN I, MAIN ASTRONOMICAL OBS, UKRAINIAN ACAD OF SCIENCE, GOLOSEEVO 252127 KIEV, UKRAINE
Telephone: (7) 044664762
Facsimilc: (7)
Commission(s): 10,12
Telex: 131406 sky su
Email:

KOSTINA LIDIJA D DR, PULKOVO OBSERVATORY, ACADEMY OF SCIENCES, 10 KUTUZOV QUAY 196140 ST PETERSBURG, RUSSIA

Telephone: (7)

Facsimile: (7)

Telex:

Email:

Commission(s):

KOSTYAKOVA ELENA B DR, STERNBERG STATE ASTR INST, 11723+ MOSCOW, RUSSIA

Telephone: (7)

Facsimile: (7)

Telex:

Email:

Commission(s): 34

KOSUGI TAKEO, TOKYO ASTRONOMICAL OBS, NAOJ, OSAWA MITAKA, TOKYO 181, JAPAN

Telephone: (81)

Facsimile: (81)

Telex:

Email:

Commission(s): 10C

KOTANYI CHRISTOPHE DR, NEPAE, UFSM, CIDADO UNIVERSITARIA, 97100 SANTA MARIA RS, BRAZIL

Telephone: (55) $552261616 \quad$ Facsimilc: (55)

Email:

Commission(s):

Telex: 0552230 ufsm br

KOTELNIKOV V A ACAD, INST OF RADIO \& ELECTRON, ACADEMY OF SCIENCES, 103907 MOSCOW, RUSSIA

Telephone: (7) 952036078

Facsimile: (7)

Telex:

Email:

Commission(s): 40

KOTHARI D S DR, DPT OF PHYSICS, UNIVERSITY OF DELHI, NEW DELHI 110 007, INDIA

Telephone: (91) 112918993

Facsimilc: (91)

Telex:

Email:

Commission(s): 35

KOTOV VALERY DR, CRIMEAN ASTROPHYS OBS, UKRAINIAN ACAD OF SCIENCE, NAUCHNY, 334413 CRIMEA, UKRAINE

Telephone: (7) $432945 \quad$ Facsimile: (7) Telex:

Email:

Commission(s): 12

KOTRC PAVEL, ASTRONOMICAL INSTITUTE, CZECH ACADEMY OF SCIENCES, ONDREJOV OBSERVATORY

CZ 251 65 ONDREJOV, CZECH R

Telephone: (42) $204857222 / 7111$

Facsimilc: (42) $20+851611$

Telex: 121579

Email: astsun@csearn

Commission(s): 10,12

KOUBSKY PAVEL, ASTRONOMICAL INSTITUTE, CZECH ACADEMY OF SCIENCES, ONDREJOV OBSERVATORY CZ 251 65 ONDREJOV, CZECH R

Telephone: (42) $204857127 / 7111$

Facsimile: (42) 204851611

Telex: 121579

Email: aststel@csearn

Commission(s): 29,42

KOUPELIS THEODOROS DR, DPT PHYSICS \& ASTRONOMY, UNIVERSITY OF ROCHESTER ROCHESTER NY 14627, USA

Telephone: (1) 7162754389

Email: tkou@uordbv.bitnet

Facsimile: (1) 7162758527

Commission(s); 4
Telex: 
KOURGANOFF VLADIMIR PROF, 20 AVE PAUL APELL, F 75014 PARIS, FRANCE

Telephone: (33) 145405053

Facsimile: (33)

Telex:

Email:

Commission(s): 46

KOUTCHMY SERGE DR, INSTITUT D'ASTROPHYSIQUE, 98BIS BD ARAGO, F 75014 PARIS, FRANCE

Telephonc: (33) 144328056

Facsimile: (33) $1+4328001 \quad$ Telex:

Email:

Commission(s): $10,12,21$

KOUVELIOTOU CHRYSSA DR, NASAMSFC, CODE ES 62, HUNTSVILLE AL 35812, USA

Telephonc: (1) 2055447711

Facsimile: (1) $2055+45800$

Telex:

Email: batse::kouveliotou ssl::kouvel

Commission(s):

KOVACHEV B J DR, DPT OF ASTRONOMY, BULGARIAN ACAD SCIENCES, 72 LENIN BLVD

BG 1784 SOFIA, BULGARIA

Telephone: (359) 2758827

Facsimile: (359)

Commission(s): 09,29

Telex: 23561 ecf ban bg

Email:

KOVACS AGNES DR, HELIOPHYSICAL OBSERVATORY, BOX 30, H 4010 DEBRECEN, HUNGARY

Telcphone: (36) $52311015 \quad$ Facsimilc: (36)

Telex: 72517 deobs $h$

Email:

Commission(s): 10

KOVACS GEZA DR, KONKOLY OBSERVATORY, THEGE U 13/17, BOX 67, H 1525 BUDAPEST, HUNGARY

Telephone: (36) 1175 5866/175 4122

Facsimile: (36) $115696+0$

Telex: 227460

Email:

Commission(s):

KOVAL I K DR, MAIN ASTRONOMICAL OBS, UKRAINIAN ACAD OF SCIENCE, GOLOSEEVO

252127 KIEV, UKRAINE

Telephone: (7) 044660869

Facsimile: (7)

Telex:

Email:

Commission(s):

KOVALEVSKY JEAN DR, OCA CERGA, AVE COPERNIC, F 0613) GRASSE, FRANCE

Telephone: (33) 93405353

Facsimile: (33) $93+05333$

Telex: 470865 cerga f

Email: kovalevsky@mrg.cnes.fr

Commission(s): $07,08 \mathrm{C}, 24,31 \mathrm{C}, 50 \mathrm{C}$

KOVAR N S DR, DPT OF PHYSICS, UNIVERSITY OF HOUSTON, HOUSTON TX 77004, USA

Telephone: (1)

Facsimile: (1)

Telex:

Email:

Commission(s):

KOVAR ROBERT P DR, 9666 E ORCHARD DR, ENGLEWOOD CO 80111, USA

Tclephone: (1) $3033944494 \quad$ Facsimile: (1) Telex:

Email:

Commission(s):

KOVETZ ATTAY PROF, DPT OF PHYSICS \& ASTRON, TEL AVIV UNIVERSITY, RAMAT AVIV

TEL AVIV 69978, ISRAEL

Telephone: (972) 3420234

Facsimile: (972)

Telex: 342-171 versy il

Email:

Commission(s): 35,47

KOWAL CHARLES THOMAS, STSCI, HOMEWOOD CAMPUS, 3700 SAN MARTIN DR, BALTIMORE MD 21218, USA

Telephone: (1)

Facsimilc: (1)

Telex:

Email:

Commission(s): $15,16,20$

KOYAMA KATSUJI, INST SPACE \& ASTRON SCI, UNIVERSITY OF TOKYO, MEGURO KU, TOKYO 153, JAPAN

Telephone: (81) $3+671111 \quad$ Facsimilc: (81)

Email:

Commission(s): +4

Telex: $3+757$ isastro

KOYAMA KO-U-ICHI DR, RES INST SCIENCE \& TECH, KINKI UNIVERSITY, HIGASHI, OSAKA 577, JAPAN

Telephone: (81) $67212332^{*} 4711$

Facsimile: (81) 67212353

Telex:

Email:

Commission(s): 35

KOYAMA SHIN PROF DR, KAGAWA VIDEO STUDY CENTER, UNIVERSITY OF THE AIR, SAIWAI CHO 21

TAKAMATSU 760, JAPAN

Telephone: (81)

Facsimile: (81)

Telex:

Email:

Commission(s): 12 
KOZAI YOSHIHIDE PROF, TOKYO ASTRONOMICAL OBS, NAOJ, OSAWA MITAKA, TOKYO 181, JAPAN

Telephone: (81) 422343650

Facsimile: (81) 422343690

Telex: 2822307 taomtk

Email: kozai@c1.mtk.nao.ac.jp

Commission(s): 07,20,38,50

KOZASA TAKASHI, DPT OF EARTH \& PLANATARY, SCIENCES, KOBE UNIVERSITY, KOBE 657, JAPAN

Telephone: (81) 788030978

Facsimile: (81) 788030490

Telex:

Email: kozasa(amambou.earth.s.kobe-u.ac.jp)

Commission(s): 15,34

KOZIEL KAROL PROF DR, ASTRON OBSERVATORY KRAKOW, UL 22 LIPCA 16, PL 43 460 WISLA, POLAND

Telephone: (48) 3242

Facsimile: (48)

Telex:

Email:

Commission(s):

KOZLOVSKY B Z DR, DPT OF PHYSICS \& ASTRON, TEL AVIV UNIVERSITY, RAMAT AVIV TEL AVIV 69978, ISRAEL

Telephone: (972)

Facsimile: (972)

Telex:

Email:

Commission(s): 47

KOZLOWSKI MACIEJ DR, COPERNICUS ASTRON CENTER, POLISH ACAD OF SCIENCES, UL BARTYCKA 18 PL 00716 WARSAW, POLAND

Telephone: (48) 411086

Facsimile: (48)

Commission(s): 35,44

Telex: 813978 zapan pl

Email:

KRAAN-KORTEWEG RENEE C DR, OBSERVATOIRE DE PARIS, DAEC, 5 PL JULES JANSSEN F 92195 MEUDON PPL CDX, FRANCE

Telephone: (33) 145077529

Facsimile: (33) 145077469 Telex:

Email: kraan@gin.obspm.fr

Commission(s): 28

KRAEMER GERHARD DR, ASTRONOMISCHES INSTITUT, UNIVERSITAET TUEBINGEN, WALDHAEUSERSTR 64 D 72076 TUEBINGEN, GERMANY

Telephone: (49) 7071292486

Email:

Facsimile: (49) 7071293458

Telex: 7262714 ait d

KRAFT ROBERT P PROF, LICK OBSERVATORY, UNIVERSITY OF CALIFORNIA, SANTA CRUZ CA 95064, USA

Telephone: (1) 4084593281

Facsimilc: (1) $408+263115$

Commission(s): $27,29,30,37,+2, \mathrm{EC}$

Telex: 9109971741

Email: kraft@helios.ucsc.cdu

KRAICHEVA ZDRAVSKA DR, DPT OF ASTRONOMY, BULGARIAN ACAD SCIENCES, 7TH NOVEMBER ST I

BG 1000 SOFIA, BULGARIA

Telephonc: (359) 27341

Facsimile: (359) 23561 ECFBAN BG Telex:

Email:

Commission(s): 42

KRAMER KH N DR, ASTRONOMICAL OBSERVATORY, ODESSA STATE UNIVERSITY, SHEVCHENKO PARK 270014 ODESSA, UKRAINE

Telephone: (7) 048220396

Facsimile: (7)

Telex:

Email:

Commission(s): 22

KRASINSKI ANDRZEJ PROF, COPERNICUS ASTRON CENTER, POLISH ACAD OF SCIENCES, UL BARTYCKA 18 PL 00716 WARSAW, POLAND

$\begin{array}{lll}\text { Telephone: }(\mathbf{4 8}) \mathbf{4 1} 0828 & \text { Facsimile: }(48) & \text { Telex: } 813978 \\ \text { Email: } & \text { Commission(s): } 47 & \end{array}$

KRASINSKY GEORGE A DR, INST OF APPLIED ASTRONOMY, ACADEMY OF SCIENCES, ZDANOVSKAYA UL 8 197042 ST PETERSBURG, RUSSIA

Telephone: $(7)$

Facsimile: (7)

Telex:

Email:

Commission(s): $04 \mathrm{C}, 07$

KRASSOVSKY V I DR, INST PHYSICS OF ATMOSPH, ACADEMY OF SCIENCES, PYSHEVSKY 3 109017 MOSCOW, RUSSIA

Telephone: (7) 952318862

Facsimile: (7)

Telex:

Email:

Commission(s):

KRAUS JOHN D PROF, RADIO OBSERVATORY, OHIO STATE UNIVERSITY, 2015 NEIL AVE

COLUMBUS OH 43210, USA

Telephone: (1) 6145487895

Facsimile: (1)

Telex:

Email:

Commission(s): 40,51 
KRAUSE F DR, ZNTRLINST F ASTROPHYSIK, ASTROPHYSIKALISCHES OBS, TELEGRAFENBERGD 14473 POTSDAM, GERMANY

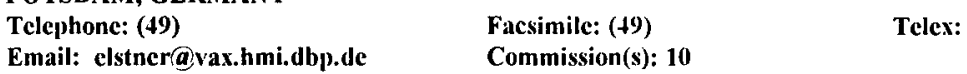

KRAUSHAAR WILLIAM L PROF, DPT OF PHYSICS, UNIVERSITY OF WISCONSIN, 1150 UNIVERSITY AVE MADISON WI 53706, USA

Telephone: (1) 6082625916

Email:

Facsimile: (1)

Commission(s): 44

Telex: $265+52$

KRAUTTER JOACHIM DR, LANDESSTERNWARTE, KOENIGSTUHL, D 69117 HEIDELBERG, GERMANY

Telephone: (49) 6221509209

Facsimile: (49) 6221509202

Telex:

Email: jkrautte(âhp2.Isw.uni-heidelberg.de

Commission(s): 27,34,42

KREIDL TOBIAS J N, LOWELL OBSERVATORY, 1400 W MARS HILL RD, BOX 1149, FLAGSTAFF AZ 86001, USA

Telephone: (1) $6027743358 \quad$ Facsimile: (1) Telex:

Email:

Commission(s): 09

KREINER JERZY MAREK DR, INSTITUTE OF PHYSICS, PEDAGOGICAL UNIVERSITY, UL PODCHORAZYCH 2 PL 30084 KRAKOW, POLAND

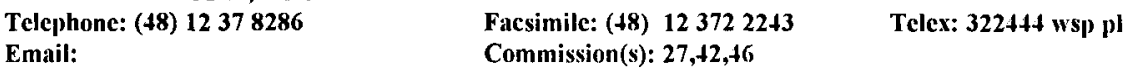

KREISEL E PROF, EINSTEIN LABORATORIUM, ROSA LUXEMBURG STR 17A, D 14473 POTSDAM, GERMANY

Telephone: (49) $762225 \quad$ Facsimile: (49)

Email: $\quad$ Commission(s): 44

Telex: 15471

KRELOWSKI JACEK DR, INSTITUTE OF ASTRONOMY, N COPERNICUS UNIVERSITY, UL CHOPINA 12/18PL 87 100 TORUN, POLAND

Telephone: (48) $85620655 \quad$ Facsimile: (48)

Email: $\quad$ Commission(s):

Telex: 055-2234 astr pl

KREMPEC-KRYGIER JANINA DR, INSTITUTE OF ASTRONOMY, N COPERNICUS UNIVERSITY, UL CHOPINA 12/18 PL 87100 TORUN, POLAND

$\begin{array}{lll}\text { Telephone: (48) } 26018 & \text { Facsimile: (48) } & \text { Telex: 0552234 astr pl } \\ \text { Email: } & \text { Commission(s): 29 } & \end{array}$

KRESAKOVA MARGITA DR, ASTRONOMICAL INSTITUTE, SLOVAK ACADEMY SCIENCES, DUBRAVSKA 9 SK 84228 BRATISLAVA, SLOVAK R

Telephone: (42) 7375157

Email:

Facsimile: (42)

Telex: 93373 seis

Commission(s): 15,22

KREYSA ERNST, MPI FUER RADIOASTRONOMIE, AUF DEM HUEGEL 69, D 53121 BONN, GERMANY

Telcphone: (49) 228525269

Facsimile: (49)

Telex: $886+40$

Email:

Commission(s): 34,40

KRIEGER ALLEN S DR, RADIATION SCIENCE INC, BOX 293, BELMONT MA 02178, USA

Telephone: (1) $6174940335 \quad$ Facsimile: (1) Telex:

Email:

Commission(s):

KRIKORIAN RALPH DR, INSTITUT D'ASTROPHYSIQUE, 98BIS BD ARAGO, F 75014 PARIS, FRANCE

Telcphone: (33) 144328145

Facsimile: (33) $144328001 \quad$ Telex:

Email:

Commission(s): 36

KRISCIUNAS KEVIN DR, JOINT ASTRONOMY CENTER, 665 KOMOHANA ST, HILO HI 96720, USA

Telephone: (1) 8089354332

Facsimile: (1)

Telex:

Email: kevin(a)jach.hawaii.edu

Commission(s): 27,41

KRISHNA GOPAL, TATA INST OF FUNDAMENTAL, RESEARCH, POONA UNIVERSITY CAMPUS, PUNE 411 007, INDI

Telephone: (91) 212337107

Email; uunet!shakti!gmrt!gk

Facsimile: (91) 212335760

Telex: $0145658 \mathrm{gmrt}$ in

KRISHNA SWAMY K S DR, TIFR/ASTROPHYSICS GROUP, HOMI BHABHA RD, COLABA, BOMBAY 400 005, INDIA

Telephone: (91) 22219111

Facsimile: (91)

Telex: 113009 tifr in

Email:

Commission(s): 15,34,36 
KRISHNAMOHAN S DR, TIFR/RADIO ASTRONOMY CTR, BANGALORE 560 012, INDIA

Telephone: (91) 803364062

Facsimile: (91)

Telex: $8+58488$ tifr in

Email:

Commission(s): 40

KRISHNAN THIRUVENKATA MR, HELIOS ANTENNAS/ELECTRON, 234 AVVAI SHANMUGHAM RD

GOPALAPURAM, MADRAS 600 086, INDIA

Telephone: (91) 44472680

Facsimile: (91)

Commission(s): 40

Telex:

Email:

KRISS GERARD A DR, DPT PHYSICS \& ASTRONOMY, JOHNS HOPKINS UNIVERSITY, CHARLES \& 34TH ST BALTIMORE MD 21218, USA

Telephone: (1) 3013387679

Facsimile: (1)

Commission(s): 47

Telex: 9101300225 jhu casmd

Email:

KRISTENSEN LEIF KAHL, INST OF PHYSICS \& ASTRON, UNIVERSITY OF AARHUS, NY MUNKEGADE DK 8000 AARHUS C, DENMARK

Telephone: (45) 86128899

Facsimile: (45) 86202711

Telex:

Email:

Commission(s): $05,15,20$

KRISTENSON HENRIK DR, DORTTININGGATAN 20, S 432410 VARBERG, SWEDEN

Telephone: (46)

Facsimile: (46)

Telex:

Email:

Commission(s):

KRISTIAN JEROME DR, CARNEGIE OBSERVATORIES, 813 SANTA BARBARA ST, PASADENA CA 91101, USA

Telcphone: (1) 8185771122

Facsimilc: (1) 8187958136

Telex: 1561318 ociw ut

Email:

Commission(s):

KRISTIANSSON KRISTER PROF, DPT OF PHYSICS, UNIVERSITY OF LUND, SOELVEGATAN 14, S 223 62 LUND, SWEDEN

Telephone: (46) 46107726

Facsimile: (46)

Telex:

Email:

Commission(s): 44

KRIVSKY LADISLAV DR, ASTRONOMICAL INSTITUTE, CZECH ACADEMY OF SCIENCES, ONDREJOV OBSERVATORY, CZ 25165 ONDREJOV, CZECH R

Telephone: (42) $204857225 / 7111 \quad$ Facsimile: (42) $204851611 \quad$ Telex: 121579 astr c

Email: astsun(a)cscarn Commission(s): 10

KRIZ SVATOPLUK DR, ASTRONOMICAL INSTITUTE, CZECH ACADEMY OF SCIENCES

ONDREJOV OBSERVATORY, CZ 25165 ONDREJOV, CZECH R

Telephone: (42) 2042486

Facsimile: (42) $20+851611$

Telex:

Email:

Commission(s): 42

KROGDAHL W S DR, DPT PHYSICS \& ASTRONOMY, UNIVERSITY OF KENTUCKY, LEXINGTON KY 40506, USA

Telephonc: (1) 6062722659

Facsimile: (1)

Telex:

Email:

Commission(s):

KROLIK JULIAN H, DPT PHYSICS \& ASTRONOMY, JOHNS HOPKINS UNIVERSITY, CHARLES \& 34TH ST

BALTIMORE MD 21218, USA

Telephone: (1) 3013387926

Facsimile: (1)

Telex:

Email:

Commission(s):

KRON RICHARD G, YERKES OBSERVATORY, UNIVERSITY OF CHICAGO, BOX 258, WILLIAMS BAY WI 53191, USA

Telephone: (1) 3122365468

Facsimile: (1)

Telex:

Email:

Commission(s): 28

KRONBERG PHILIPP DR, DPT OF ASTRONOMY, UNIVERSITY OF TORONTO, 60 ST GEORGE ST

TORONTO ON M5S 1A1, CANADA

Telephone: (1) 4169784971

Email:
Facsimilc: (1) 4169783921

Commission(s): 40
Telcx: 06986766

KROTO HAROLD PROF, SCHOOL OF CHEMISTRY, UNIVERSITY OF SUSSEX, FALMER, BRIGHTON BNI 9QJ, UK

Telcphone: (44) 1273678329

Facsimile: (4)

Commission(s): 14
Telex: 
KRUCHINENKO VITALIY G, ASTRONOMICAL OBSERVATORY, KIEV STATE UNIVERSITY OBSERVATORNAYA UL 3, 252053 KIEV, UKRAINE

Telephone: (7)

Facsimile: (7)

Email:

Commission(s): 22,42

Telex:

KRUEGEL ENDRIK DR, MPI FUER RADIOASTRONOMIE, AUF DEM HUEGEL 69, D 53121 BONN, GERMANY

Telephone: (49)

Facsimile: (49)

Commission(s): 40

Telex: $886+40$

Email:

KRUEGER ALBRECHT DR, ZNTRLINST F ASTROPHYSIK, TELEGRAFENBERG, D 14473 POTSDAM, GERMANY Telephone: (49) 4551

Email:

Telex: 15239

Commission(s): $10,12,40$

KRUMM NATHAN ALLYN, DPT OF PHYSICS, UNIVERSITY OF CINCINNATI, 210 BRAUNSTEIN ML 11

CINCINNATI OH 45221 0111, USA

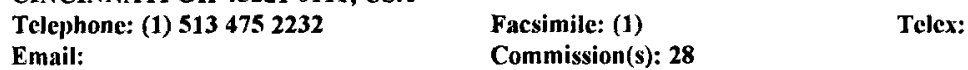

KRUPP EDWIN C DR, GRIFFITH OBSERVATORY, 2800 EAST OBS ROAD, LOS ANGELES CA 90027, USA

Telephone: (1) 2136641181

Facsimile: (1)

Telex:

Email:

Commission(s): 41,46

KRUSZEWSKI ANDRZEJ PROF, ASTRONOMICAL OBSERVATORY, WARSAW UNIVERSITY, AL UJAZDOWSKIE 4 PL 00478 WARSAW, POLAND

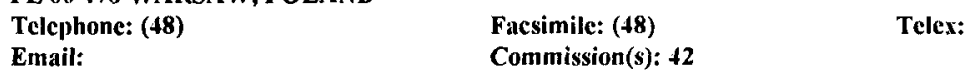

KRYGIER BERNARD DR, INST OF RADIO ASTRONOMY, N COPERNICUS UNIVERSITY, UL GAGARINA 11 PL 87100 TORUN, POLAND

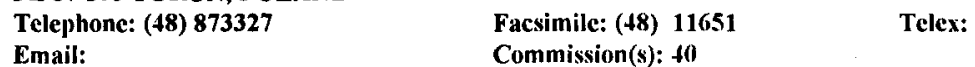

KRYVODUBSKYJ VALERY, ASTRONOMICAL OBSERVATORY, KIEV UNIVERSITY, OBSERVATORNA STR 3

254053 KIEV 53, UKRAINE

Telephone: (7) 0442162630

Email: aoku@gluk.apc.org

Facsimile: $(7)$
Commission(s): 10,12

KRZEMINSKI WOJCIECH DR, CARNEGIE INST WASHINGTON, LAS CAMPANAS OBSERVATORY, CASILLA 601 LA SERENA, CHILE

Telephone: (56) 51213032

Facsimile: (56)

Commission(s): 27,42

Telex: 645227 aura ct

Email:

KSANFOMALITI L V DR, SPACE RESEARCH INSTITUTE, ACADEMY OF SCIENCES, PROFSOJUZNAYA UL 84/32 117810 MOSCOW, RUSSIA
Telephone: (7) $953332322 / 3122$
Facsimile: (7)

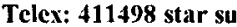

Email:

Commission(s): 16,51

KUBIAK MARCIN A DR, ASTRONOMICAL OBSERVATORY, WARSAW UNIVERSITY, AL UJAZDOWSKIE 4

PL 00478 WARSAW, POLAND

Telephone: (48) 295346/294011

Facsimile: $(48)$

Commission(s): 27

Telex: 813978 zapan pl

Email:

KUBICELA ALEKSANDAR DR, ASTRONOMICAL OBSERVATORY, VOLGINA 7

YU 11050 BEOGRAD, YUGOSLAVIA-SERBIA \& MONTENEGRO

Telephone: (38) 11419357

Facsimile: (38)

Telex:

Email:

Commission(s): 12

KUBO YOSHIO, HYDROGRAPHIC DPT, GEODESY \& GEOPHYSICS DIV, TSUKIJI 5 CHUO KU, TOKYO 104, JAPAN Telephone: (81) $35+13811$

Facsimile: $(\mathbf{8 1})$

Email:

Commission(s): 04 Telex: 02522222 jahyd $j$

KUBOTA JUN DR, KWASAN OBSERVATORY, KYOTO UNIVERSITY, YAMASHINA, KYOTO G07, JAPAN

Telephonc: (81) 755811235

Facsimile: (81)

Commission(s): 10

Telex: $5+22693$ libkyu j

Email: 
KUCERA ALES, ASTRONOMICAL INSTITUTE, SLOVAK ACADEMY SCIENCES SK 05960 TATRANSKA LOMNI, SLOVAK R

Telephone: (42)969967866 Facsimile: (42) $969967656 \quad$ Telex:

Email: akucera(gauriga.ta3.sk Commission(s): 10,12

KUDRITZKI ROLF-PETER PH D, INST ASTRON \& ASTROPHYSIK, UNIVERSITAETS STERNWARTE SCHEINERSTR 1, D 81679 MUENCHEN, GERMANY

Telephone: (49) $89989021 \quad$ Facsimile: (49) $8992209+27 \quad$ Telex: 529815 univm d

Email:

Commission(s): 36

KUEHNE CHRISTOPH F, KOEFLACHER STR 36, D 7928 GIENGEN/BRENZ, GERMANY

Telephone: (49) 732 24448 Facsimile: (49) Telex:

Email: $\quad$ Commission(s): 09

KUEHR HELMUT, MPI FUER ASTRONOMIE, KOENIGSTUHL, D 69117 HEIDELBERG, GERMANY

Telephone: (49) $62215281 \quad$ Facsimile: (49)

Email:

Commission(s):

Telex: 461789 mpia d

KUENZEL HORST, DIESELSTR 13, D 14482 POTSDAM, GERMANY

Telephone: (49) $33177318 \quad$ Facsimile: (49)

Commission(s): 10

Telex:

Email:

KUHI LEONARD V PROF, DPT OF ASTRONOMY, UNIVERSITY OF MINNESOTA, 116 CHURCH ST SE MINNEAPOLIS MN 55455, USA

Telcphone: (1)

Facsimile: (1)

Telex:

Email:

Commission(s): 27,36

KUHN JEFFERY RICHARD DR, PHYSICS \& ASTRONOMY DPT, MICHIGAN STATE UNIVERSITY EAST LANSING MI 48824, USA

$\begin{array}{lll}\text { Telcphone: (1) } 5173532986 & \text { Facsimile: (1) } & \text { Telex: } \\ \text { Email: kuhn(a)msupa.bitnet } & \text { Commission(s): }\end{array}$

KUIJPERS H JAN M E DR, STERREKUNDIG INSTITUTE, BOX 80000, NL 3508 TA UTRECHT, NETHERLANDS

Telephone: (31) 30535209

Email: kuijpers@hutruu51

Facsimile: (31) 30531601

Telex: 40048 fylut $n l$

Commission(s): 40

KUIN NICOLAAS PAULUS M, ASTRONOMICAL DATA CTR, NASA GSFC NSSDC, CODE 6332

GREENBELT MD 20771, USA

Telephone: (1) 3012860677

Facsimile: (1) 3012861771

Telex:

Email: kuin@nssdc.gsfc.nasa.gov

Commission(s): 05

KUIPER THOMAS B H DR, JPL, MS 169 5065, 4800 OAK GROVE DR, PASADENA CA 91109, USA

Telephone: (1) 8183545479

Facsimile: (1)

Telex: $675+29$

Email:

Commission(s): $34,40,51$

KUKLIN G V DR, SIBIZMIR, ACADEMY OF SCIENCES, 664697 IRKUTSK 33, RUSSIA

Telephone: (7) 629388

Facsimile: (7)

Telex: $1+2+07$

Email:

Commission(s): 10,12

KULCAR LADISLAV DR, PEDAGOGICKA FAKULTA; KATEDRA FYZIKY, TAJOVSKEHO 40 SK 97549 BANSKA BYSTRICE, SLOVAK R

Telephone: (42) 8834553

Facsimilc: (42) 8833132

Telex:

Email:

Commission(s): 12

KULKARNI PRABHAKAR V PROF, PHYSICAL RESEARCH LAB, NAVRANGPURA, AHMEDABAD 380 009, INDIA

Telephone: (91) $272462129 \quad$ Facsimile: (91) 272445292

Email:

Telex: 8458488 tifr in

KULKARNI SHRINIVAS R DR, CALTECH, MS 105 24, PASADENA CA 91125, USA

Telephone: (1) $8183564010 \quad$ Facsimile: (1)

Telex: 188192675425

Email:

Commission(s): 40

KULKARNI VASANT K DR, TATA INST OF FUNDAMENTAL, RESEARCH, POONA UNIVERSITY CAMPUS PUNE 411 007, INDIA

Telephone: (91) 212337107

Email: uunct!shakti!gmrt!vasant

Facsimile: (91) 212335760

Commission(s): 40
Telex: $01+5658$ gmrt in 
KULSRUD RUSSELL M DR, DPT ASTROPHYSICAL SCI, PRINCETON UNIVERSITY, PRINCETON NJ 08544 1001, USA Telephone: (1) 6096832613

Facsimile: (1)

Telex:

Email:

Commission(s): 33,44

KULTIMA JOHANNES, GEOPHYSICAL OBSERVATORY, EISCAT, SF 99600 SOKANKYLAE, FINLAND

Telephone: (358)

Facsimile: (358)

Telex:

Email:

Commission(s):

KUMAI YASUKI, DPT OF PHYSICS, NAGOYA UNIVERSITY, CHIKUSA KU, NAGOYA 464 01, JAPAN

Telephone: (81) 527892842

Facsimile: (81) $527892845 \quad$ Telex:

Email: e43211a@nucc.cc.nagoya-u.ac.jp Commission(s): 28

KUMAJGORODSKAYA RAISA DR, SPECIAL ASTROPHYSICAL OBS, ACADEMY OF SCIENCES, NIZHNIJ ARKHYZ 357147 STAVROPOLSKIJ, RUSSIA

Telephone: (7) 93515

Facsimile: (7)

Telex:

Email:

Commission(s):

KUMAR C KRISHNA DR, DPT PHYSICS \& ASTRONOMY, HOWARD UNIVERSITY, WASHINGTON DC 20059, USA

Telephone: (1) 2026366245

Facsimile: (1)

Telex:

Email:

Commission(s): 34

KUMAR SHAILENDRA, DPT OF ASTRONOMY, UNIVERSITY OF VIRGINIA, BOX 3818 UNIVERSITY STN, CHORLOTTESVILLE VA 22903, USA

Telephone: (1) 8049247494

Facsimile: (1) $8049243104 \quad$ Telex:

Email: ssk9u@virginia.edu

Commission(s):

KUMAR SHIV S PROF, UNIVERSITY STATION, UNIVERSITY OF VIRGINIA, BOX 3818

CHARLOTTESVILLE VA 22903, USA

Telephone: (1) 8049244896

Facsimile: (1)

Telex:

Email: ssk9u@virginia.cdu

Commission(s): $16,35,36$

KUMKOVA IRINA I DR, INST OF APPLIED ASTRONOMY, ACADEMY,OF SCIENCES, ZDANOVSKAYA UL 8 197042 ST PETERSBURG, RUSSLA

Telephone: (7) 8122353216

Facsimile: (7) $8122925704 \quad$ Telex:

Email: iparan@sovam.com

Commission(s): 24C

KUMSIASHVILY MZIA I DR, ABASTUMANI ASTROPHYSICAL, OBSERVATORY, GEORGIAN ACAD OF SCIENCES 383762 ABASTUMANI, GEORGIA

Telephone: (7) 252

Facsimile: (7)

Telex: 327409

Email:

Commission(s): 42

KUMSISHVILI J I DR, ABASTUMANI ASTROPHYSICAL, OBSERVATORY, GEORGIAN ACAD OF SCIENCES 383762 ABASTUMANI, GEORGIA

Telephone: (7) 279

Facsimile: (7)

Telex: 327409

Email:

Commission(s): 26,27

KUN MARIA DR, KONKOLY OBSERVATORY, THEGE U 13/17, BOX 67, H 1525 BUDAPEST, HUNGARY

Telephone: (36) $11755866 / 1754122$

Facsimile: (36) 11569640

Telex: 227460 konob h

Email:

Commission(s): 37

KUNCHEV PETER DR, DPT OF ASTRONOMY, UNIVERSITY OF SOFIA, ANTON IVANOV ST 5

BG 1126 SOFIA, BULGARIA

Telephone: (359) 2544852

Facsimile: (359)

Commission(s): 28

Telex:

Email:

KUNDT WOLFGANG PROF DR, INSTITUT FUER ASTROPHYSIK, EXTRATERR FORSCHUNG, AUF DEM HUEGEL 71 D 53121 BONN, GERMANY

Telephone: (49) 228267400

Facsimile: (49)

Telex: 886440

Email:

Commission(s): 40,44

KUNDU MUKUL R DR, ASTRONOMY PROGRAM, UNIVERSITY OF MARYLAND, COLLEGE PARK MD 20742, USA

Telcphone: (1) 3014543005

Facsimile: (1)

Commission(s): $10,12,34,40$

Telex: 710-826-0352

Email: 
M 648

KUNIEDA HIDEYO DR, DPT OF ASTROPHYSICS, NAGOYA UNIVERSITY, FUROCHO CHIKUSA KU

NAGOYA $\$ 6401$, JAPAN

Tclephone: (81) 527815111

Facsimile: (81) 527813541

Telex: $4+77323$ scunag $j$

Email:

Commission(s):

KUNITZSCH PAUL PROF, DAVIDSTR 17, D 81679 MUENCHEN, GERMANY

Telephonc: (49)

Facsimile: (49)

Telex:

Email:

Commission(s): +1

KUNKEL WILLIAM E DR, CARNEGIE INST WASHINGTON, LAS CAMPANAS OBSERVATORY, CASILLA 601 LA SERENA, CHILE

Telephone: (56) 51213032

Facsimile: (56)

Commission(s): 25,27

Telex: 645227 aura ct

Email:

PPYSIQUE, 98BIS BD ARAGO, F 75014 PARIS, FRANCE

KUNTH DANIEL, INSTITUT D'ASTROPHYSIQUE,
Telephonc: (33) 144328085

Email:

Commission(s): $28,34,47$

KUNZE RUEDIGER DR, INST THEOR PHYS \& STERNW, UNIVERSITAET KIEL, OLSHAUSENSTR 40

D 24098 KIEL, GERMANY

Telcphone: (49) $\$ 318801575$

Email: pas29(ä)rz.uni-kicl.dbp).dc
Facsimile: (\$9) $\$ 31880+432$

Commission(s): 34
Telex: 292706

KUPERUS MAX PROF DR, STERREKUNDIG INSTITUTE, BOX \$O00, NL 3508 TA UTRECHT, NETHERLANDS

Telephone: (31) 30535212
Facsimile: (31)

Commission(s): 10,12

Telex: 40048 fylut $n$ l

Email: bitnet:wnmmailähutruut

KUPLIAUSKIENE ALICIJA, INST THEORETICAL PHYSICS, \& ASTRONOMY, GOSTAUTO 12 VILNIUS 2600, LITHUANIA

Telephone: (370) 2612723

Facsimile: (370)

Telex:

Email: kupl(äitpa.fi.It

Commission(s):

KURFESS JAMES D, NAVAL RESEARCH LABORATORY, CODE +150, 4555 OVERLOOK AVE SW WASHINGTON DC 20375 5000, USA

Telephone: (1) 2027673182

Facsimilc: (1)

Emial:

Commission(s):

Telex:

KURIL-CHIK V N DR, STERNBERG STATE ASTR INST, UNIVERSITETSKIJ PROSP 13, 119899 MOSCOW, RUSSLA Telephone: (7) 951391030 Facsimile: (7)

Commission(s): 40

Telex:

Email:

KUROCHKA L N DR, ASTRONOMICAL OBSERVATORY, KIEV STATE'UNIVERSITY, OBSERVATORNAYA UL 3 252053 KIEV, UKRAINE

Telephone: (7) $04+262691$

Facsimile: (7)

Telex:

Email:

Commission(s): 10,12

KUROKAWA HIROKI DR, HIDA OBSERVATORY, UNIVERSITY OF KYOTO, KAMITAKARA, GIFU 506 13, JAPAN Telephone: (81) 57862628

Facsimile: (81)

Telex:

Email:

Commission(s): 10

KURPINSKA-WINIARSKA M DR, ASTRONOMICAL OBSERVATORY, JAGIELLONIAN UNIVERSITY, UL ORLA 171 PL 30 244 KRAKOW, POLAND

Telephone: (48)

Facsimile: (48) $12378(153$

Commission(s): 12

Tclex: 32 6203 ujoa pl

Email:

KURT V G DR, SPACE RESEARCH INSTITUTE, ACADEMY OF SCIENCES, PROFSOJUZNAYA UL 84/32 117810 MOSCOW, RUSSIA

Telephone: (7) 953333122

Facsimile: (7)

Commission(s): 16,4t

Telex: $\$ 11498$ star su

Email:

KURTANIDZE OMAR, KAZBEGI 2A, TBILISI, GEORGIA

Telephone: (7) 8832995367

Fatcsimile: (7) 8832998823

Telex:

Email: okur@abao.kheta.georgia.su

Commission(s): 45 
KURTZ DONALD WAYNE DR, DPT OF ASTRONOMY, UNIVERSITY OF CAPE TOWN, RONDEBOSCH 7700 SOUTH AFRICA

Telephone: (27) 21650 2394/2391

Facsimilc: (27) 216503726

Telex:

Email: dkurtz@uctvax.uct.ac.za

Commission(s): 25C,27VP,45C

KURTZ MICHAEL JULIAN, HARVARD SMITHSONIAN CFA, 60 GARDEN ST, CAMBRIDGE MA 02138, USA

Telephone: (1) 6174957434

Email: kurty@efa.harvard. cdu

Facsimile: (1) $6174957+76 \quad$ Telex:

Commission(s):

KURUCZ ROBERT L DR, CENTER FOR ASTROPHYSICS, HCO/SAO, 60 GARDEN ST, CAMBRIDGE MA 02138, USA

Telephone: (1) 6174957429

Facsimilc: (1)

Telex: $921+28$

Email: kurucz@cfa.harvard.edu

Commission(s): $36 \mathrm{C}$

KURZYNSKA KRYSTYNA DR, ASTRONOMICAL OBSERVATORY, A MICKIEWICZ UNIVERSITY, UL SLONECZNA 3 PL 60286 POZNAN, POLAND

Telephone: (48) 61679670

Email: kurzastr@plpuamm
Facsimile: (48) 61535535
Commission(s): 08

Telex: $\mathbf{4 1 3 2 6 0}$ uampl

KUS ANDRZEJ JAN DR, INST OF RADIOASTRONOMY, N COPERNICUS UNIVERSITY, UL CHOPINA 12/18

PL 87100 TORUN, POLAND

Telephone: (48) 485620651

Email:

Facsimile: (48)

Commission(s): 40

Telex: 055232+ trao pl

KUSHWAHA R S PROF, DPT OF MATHEMATICS, UNIVERSITY OF JODHPUR, JODHPUR, INDIA

Telephone: (91)

Facsimile: (91)

Commission(s): 35,36

Telex:

Email:

KUSTAANHEIMO PAUL E PROF, DANMARKS TEKN HOJSKOLE, LUNDTOFTEVEJ 7, DK 2800 LYNGBY, DENMARK

Telephone: (45) 42883022

Email:

Facsimile: (45)

Commission(s): $07,28,47$

Telex:

KUSUNOSE MASAAKI DR, DPT OF ASTRONOMY, UNIVERSITY OF TEXAS, AUSTIN TX 78712, USA

Telephonc: (1) $512+717426$

Facsimile: (1) $512+716016 \quad$ Telex:

Email: kusunose(a) astro.as.utexas.cdu

Commission(s): 44

KUTNER MARC LESLIE DR, DPT OF PHYSICS, RENSSELAER POLYTECHN INST, TROY NY 12180 3590, USA

Tclephone: (1) 5182666417

Facsimile: (1)

Telex:

Email:

Commission(s): 34,40

KUTTER G SIEGFRIED DR, NASA GSFC, CODE 681, GREENBELT MD 20771, USA

Telephone: (1)

Email:

Facsimile: (1)

Telex:

Commission(s):

KUTUZOV S A DR, ASTRONOMICAL OBSERVATORY, ST PETERSBURG UNIVERSITY, BIBLIOTECHNAJA PL 2 199164 ST PETERSBURG, RUSSIA

Telephone: (7) $812+2866+4 / 3151701$

Email: kvk@ast.lgu.spb.su

Facsinile: (7)

Telex:

Commission(s): 33

KUYKEN KOENRAAD H, KAPTEYN ASTRONOMICAL INST, BOX 800, NL 9700 AV GRONINGEN, NETHERLANDS

Telephone: (31)

Email:

Facsimile: (31)

Telex:

Commission(s):

KUZMANOSKI MIKE, INSTITUTE OF ASTRONOMY, UNIVERSITY OF BELGRADE, STUDENTSKI TRG 16

YU 11000 BEOGRAD, YUGOSLAVIA-SERBIA \& MONTENEGRO

Tclephonc: (38) 11638715

Facsimile: (38)

Telcx:

Email:

Commission(s):

KUZMIN ARKADII D PROF DR, LEBEDEV PHYSICAL INST, ACADEMY OF SCIENCES, LENINSKY PROSPEKT 53 $11792+$ MOSCOW, RUSSIA

Tclephonc: (7)

Facsimile: (7)

Email:

Commission(s): $16,40,51$

Telex: $\$ 11+79$ neod su

KWEE K K DR, STERREWACHT, BOX 9513, NL 23010 RA LEIDEN, NETHERLANDS

Telephonc: (31) 71272727

Facsímile: (31)

Commission(s): 42

Telex: 39058 astro $\mathrm{ni}$ 
KWITTER KAREN BETH DR, DPT OF PHYS \& ASTRONOMY, THOMPSON PHYSICS LAB, WILLIAMS COLLEGE WILLIAMSTOWN MA 01267, USA

Telephone: (1) 4135972272

Facsimile: (1)

Telex:

Email:

Commission(s): 34

KWOK SUN DR, DPT OF PHYSICS, UNIVERSITY OF CALGARY, 2500 UNIVERSITY DR NW

CALGARY AB T2N 1N4, CANADA

Telephonc: (1) $40328+5414 \quad$ Facsimile: (1) 403289333

Email:

Commission(s): $29,34,35,40$

Telcx: 03821545

KYLAFIS NIKOLAOS D DR, DPT OF PHYSICS, UNIVERSITY OF CRETE, BOX 1527, GR 71111 IRAKLION, GREECE

Telcphone: (30) 81239757

Facsimile: (30)

Telex:

Email:

Commission(s): 34

LA BONTE BARRY JAMES, INSTITUTE FOR ASTRONOMY, UNIVERSITY OF HAWAII, 2680 WOODLAWN DR HONOLULU HI 96822, USA

Telephone: (1) 8089566531

Email:

Facsimile: (1) 8089882790

Telex: 723-8+59 uhast hr

Commission(s): 12

LA DOUS CONSTANZE A DR, ESA IUE OBSERVATORY, VILLAFRANCA DEL CASTILLO, APD 50727

E 28080 MADRID, SPAIN

Telephone: (34) 18131100

Email: vilspa::cld/288+3::cld

Facsimile: (3†) 18131139

Commission(s): $\$ 2$

LA PADULA CESARE, IAS, CNR, CP 67,100044 FRASCATI, ITALY

Telephone: (39) 69425655

Email:

Facsimile: (39) $694168+7$

Commission(s):

Telex:

Telex: $\$ 2555$ vilse

LABAY JAVIER, DPT FISICA DE ATMOSFERA, UNIVERSIDAD DE BARCELONA, AVD DIAGONAL 645 E 08028 BARCELONA, SPAIN

Telcphonc: (34) 33307311

Facsimile: (34)

Commission(s): 35

Telex:

Email:

LABEYRIE ANTOINE DR, OCA CERGA, AVE COPERNIC, F 06130 GRASSE, FRANCE

Telephone: (33) $933658+9$

Facsimile: (33)

Commission(s): (09)

Telex: $\$ 61+(1) 2$

Email:

LABEYRIE JACQUES DR, CEA CEN, CFR, BP 2, F 91191 GIF/YVETTE CDX, FRANCE

Telephone: (33) 169677828

Facsimile: (33)

Commission(s):

Telex: 691137

Email:

LABHARDT LUKAS, ASTRONOMISCHES INSTITUT, UNIVERSITAET BASEL, VENUSSTRASSE 7

CH 4102 BINNINGEN, SWITZERLAND

Telephone: (41) $612717711 / 12$

Facsimile: $(41)$

Telex:

Email:

Commission(s): 25,45

LABS DIETRICH PROF, LANDESSTERNWARTE, KOENIGSTUHL, D 69117 HEIDELBERG, GERMANY

Telephone: (49) 622110036

Facsimile: (\$9)

Commission(s): 12

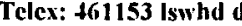

Email:

LACEY CEDRIC DR, DPT OF ASTROPHYSICS, UNIVERSITY OF OXFORD, KEBLE RD, OXFORD OX1 3RH, UK

Telephone: (44) 1865273352

Email: 19464::cgl (span)

Facsimile: (44) $1865273+18$

Telex: 83295 nuclox $g$

Commission(s): 47

LACHIEZE-REY MARC, CEA CEN, DAPNIA/SAP, BP 2, F 91191 GIF/YVETTE CDX, FRANCE

Telcphonc: (33) 169086292

Facsimile: (33)

Telex: 690860

Email:

Commission(s): 47

LACLARE F MR, OCA CERGA, AVE COPERNIC, F 06130 GRASSE, FRANCE

Telcphone: (33) 93365849

Email:

Facsimile: (33)

Commission(s): 08

Telex:

LACY CLAUD H DR, DPT OF PHYSICS, UNIVERSITY OF ARKANSAS, 104 PHYSICS BDG

FAYETTEVILLE AR 72701, USA

Telephone: (1) 5015752506

Facsimile: (1)

Telex:

Email:

Commission(s): $\$ 2$ 
LACY JOHN H DR, ASTRONOMY DPT, UNIVERSITY OF TEXAS, RLM 15 308, AUSTIN TX 78712 1083, USA

Telephone: (1) 5124711469

Facsimile: (1)

Telex:

Email:

Commission(s):

LADA CHARLES JOSEPH DR, STEWARD OBSERVATORY, UNIVERSITY OF ARIZONA, TUCSON AZ 85721, USA

Telephone: (1) 5206214878

Facsimile: (1)

Telex:

Email:

Commission(s): $34,37 \mathrm{C}, 40$

LAFFINEUR MARIUS MR, 21 BD BRUNE, F 7501† PARIS, FRANCE

Telephone: (33)

Facsimile: (33)

Telex:

Email:

Commission(s):

LAFON JEAN-PIERRE J DR, OBSERVATOIRE DE PARIS, SECTION DE MEUDON

F 92195 MEUDON PPL CDX, FRANCE

Tclephonc: (33) 145077858

Facsimile: (33)

Telex: 204464

Email:

Commission(s): $28,33,34,49,51$

LAGERKVIST CLAES-INGVAR, ASTRONOMICAL OBSERVATORY, BOX 515, S 75120 UPPSALA, SWEDEN

Telephonc: (46) 18113522

Facsimile: (46)

Telex: $7602+$ univ ups s

Email:

Commission(s): $15 \mathrm{C}, 20$

LAGERQVIST ALBIN PROF, INST OF THEORETICAL PHYS, VANADISVAEGEN 9, S 113 46 STOCKHOLM, SWEDEN

Telephone: $(46) 8164500$

Facsimile: $(46)$

Commission(s): 14

Telex: $15+33$ fystos

Email:

LAGO MARIA TERESA V T PR, CENTRO DE ASTROFISICA, UNIVERSIDADE DO PORTO, RUA CAMPO ALEGRE 823, P 4100 PORTO, PORTUGAL

Telephone: (351) 26001672

Email: mtlago@ciup1.ncc.up.pt

Facsimile: (351) 26003654

Telex:

Commission(s): $27,29,46$

LAHAV OFER DR, INSTITUTE OF ASTRONOMY, THE OBSERVATORIES, MADINGLEY RD

CAMBRIDGE CB3 (OHA, UK

Telephone: (4) 1223337548

Email: ol1(a)ast-star,cam.ac.uli

Facsimile: (4t) 1223337523

Telex: 817297 astron $g$

Commission(s): 47

LAHIRI N C, INDIAN METEOROLOGIC DPT, P 546 BLOCK N, NEW ALIPORE, CALCUTTA 700 053, INDIA

Telephone: (91)

Facsimile: (91)

Telex:

Email:

Commission(s): 04

LAHULLA J FORNIES DR, OBS ASTRONOMICO NCL, ALFONSO XII-3, E 2801+ MADRID, SPAIN

Telephone: (34) $12270107 \quad$ Facsimile: (34)

Telex: 22465 igc c

Email:

Commission(s):

LAI SEBASTIANA, ISTITUTO DI ASTRONOMIA, VIA OSPEDALE 72, I (19100 CAGLIARI, ITALY

Telephone: (39) 70663544

Facsimile: (39)

Telex:

Email:

Commission(s): 46,49

LAING ROBERT, ROYAL GREENWICH OBS, HERSTMONCEUX CASTLE, HAILSHAM BN27 I RP, UK

Telcphone: (4) $1323833171 \quad$ Facsimile: (4)

Email:

Commission(s): 40

Telex: $87+51$ rgobs $g$

LAIRD JOHN B DR, DPT PHYSICS \& ASTRONOMY, BOWLING GREEN STATE UNIV BOWLING GREEN OH $+3+103$, USA

Telephone: (1) 4193727244

Facsimile: (1)

Telex:

Email: lairda andy.bgsu.edu

Commission(s): 29

LAKE KAYLL WILLIAM DR, DPT OF PHYSICS, QUEEN'S UNIVERSITY, KINGSTON ON K7L 3N6, CANADA

Telephonc: (1) 6135473020

Facsimile: (1) 6135456463

Telex:

Email:

Commission(s): 47

LAL DEVENDRA, PHYSICAL RESEARCH LAB, NAVRANGPURA, AHMEDABAD 380 (010), INDIA

Telephone: (91) $272+62129$

Facsimile: (91) $272+45292$

Telex:

Email:

Commission(s): 
LALA PETR DR, OFFICE FOR OUTER SPACE, UN OFFICE IN VIENNA, BLDG F 0818 BOX 500 A 1400 VIENNA, AUSTRIA Telephone: (43) 1211314952 Facsimile: (43) $1213+55830 \quad$ Telex: 135612 Email: plala@unov.un.or.at Commission(s): 07

LALLEMENT ROSINE DR, SERVICE D'AERONOMIE, BP 3, F 91371 VERRIERES BUISSON, FRANCE Telephone: (33) $16447 \$ 235$

Email: bitnet:rosine(a)friap51

Telex: 602400

LAMB DONALD QUINCY JR DR, UNIV OF CHICAGO PRESS, UNIVERSITY OF CHICAGO, 5801 S ELLIS AVE CHICAGO IL 60637, USA

Telephone: (1) 3129628203

Facsimile: (1)

Telex: 269266

Email:

Commission(s): $35,42,44$

LAMB FREDERICK K PROF, DPT OF PHYSICS, UNIVERSITY OF ILLINOIS, 1110 W GREEN ST URBANA IL 61801, USA

Telcphone: (1) 2173336363

Facsimile: (1)

Telex: $6 \$ 02272050 \mathrm{mci}$

Email:

Commission(s): 4

LAMB RICHARD C DR, DPT OF PHYSICS, IOWA STATE UNIVERSITY, AMES IA 50011, USA

Telephone: (1) $51529+3873$

Email: bitnet:lamb(a) alisuvax

Facsimile: (1)

Telex:

Commission(s):

LAMB SUSAN ANN DR, DPT OF PHYSICS, UNIVERSITY OF MISSOURI, 8001 NATURAL BRIDGE RD ST LOUIS MO 63121, USA

Telephone: (1)

Facsimile: (1)

Telex:

Email:

Commission(s): 35,4-4

LAMBECK KURT PROF, AUSTRALIAN NTL UNIVERSITY, RES SCHOOL EARTH SCIENCE, BOX 4

CANBERRA ACT 2600, AUSTRALIA

Telephone: (61) 62492487

Facsimile: (61)

Telex: 62693

Email:

Commission(s):

LAMBERT DAVID L PROF, ASTRONOMY DPT, UNIVERSITY OF TEXAS, RLM 15 308, AUSTIN TX 78712 1083, USA

Tclephonc: (1) $5124717+38$

Email: dll@astro.as.utcxas.cdu

Facsimile: (1) $512+716016$

Telex: $91087+1351$

Commission(s): 14,29C,36

LAMERS HENNY J G L M DR, SPACE RESEARCH LABORATORY, SRON, SORBONNELAAN 2

NL 358+ CA UTRECHT, NETHERLANDS

Telephone: (31) 30535720

Facsimile: (31) 30540860

Telex: $\$ 7224$ sron $\mathrm{nl}$

Email: hennyle( $(\mathfrak{a})$ sron. Iruu.nl

Commission(s): 29,36,4t

LAMLA ERICH E DR, BRUESSELERSTR 9, D 5300 BONN 1, GERMANY

Telephone: (\$9)

Facsimile: (49)

Commission(s):

Telex:

Email:

LAMONTAGNE ROBERT DR, DPT DE PHYSIQUE, UNIVERSITE DE MONTREAL, CP 6128 SUCC A, MONTREAL QC H3C 3J7, CANADA

Telephonc: (1) $51+3+27273$

Facsimile: (1) $51+3+32017$

Telex: $05562+25$

Email: 5007(a)cc.umontreal.ca

Commission(s): 29

LAMPENS PATRICIA DR, OBSERVATOIRE ROYAL DE, BELGIQUE, AVE CIRCULAIRE 3 B 1180 BRUSSELS, BELGIUM

Telephone: (32) 23730263

Facsimile: (32) 23749822

Telex: 21565 obsbel

Email: patricia(a)astro.oma.be

Commission(s): 26

LAMPTON MICHAEL, SPACE SCIENCES LABORATORY, UNIVERSITY OF CALIFORNIA, BERKELEY CA 94720, USA

Telephone: (1) $+156+23576$

Facsimilc: (1)

Commission(s): +4

Telex: 9103667945

Email:

LAMY PHILIPPE DR, LAS, TRAVERSE DU SIPHON, LES TROIS LUCS, F 13012 MARSEILLE, FRANCE

Telephone: (33) 91055932

Email: lamy@afrlasm51
Facsimile: (33) 91661855

Commission(s): 15,21C,22
Telex: $+2058+f$ 
LANCASTER BROWN PETER, 10A ST PETER'S ROAD, ALDEBURGH SUFFOLK, UK

Telcphone: (44)

Email:

Facsimile: $(+4)$

Telex:

Commission(s): 15

LANDE KENNETH PROF, DPT OF PHYSICS, UNIV OF PENNSYLVANIA, PHILADELPHIA PA 19104, USA

Telephone: (1) 2158988177

Facsimile: (1)

Telex:

Email:

Commission(s):

LANDECKER PETER BRUCE DR, HUGHES AIRCRAFT CO, SPACE \& COMM GR BLDG S50, MS X371 BOX 92919

LOS ANGELES CA 90009 , USA

Telephone: (1) 2136480815

Facsimile: (1)

Commission(s):

Telex: 664480

Email:

LANDECKER THOMAS L DR, DOMINION RADIO ASTROPHYS, OBSERVATORY, BOX 248

PENTICTON BC V2A 6K3, CANADA

Telephone: (1) 6044932277

Facsimile: (1) $6044937767 \quad$ Telex: 04888127

Email:

Commission(s):

LANDI DEGL'INNOCENTI E PR, DPT DI ASTRONOMIA, UNIVERSITA DI FIRENZE, LARGO E FERMI 5 I 50125 FIRENZE, ITALY

Telephone: (39) 5527521

Facsimile: (39) 55220039

Telex: $\mathbf{5 7 2 2 6 8}$ arcetr $\mathrm{i}$

Email:

Commission(s): 12

LANDINI MASSIMO PROF, OSS ASTROFISICO, DI ARCETRI, LARGO E FERMI 5, I 50125 FIRENZE, ITALY
Telephone: (39) 55 2752247
$\begin{aligned} & \text { Email: } \\ & \text { Facsimile: (39) }\end{aligned}$

LANDMAN DONALD ALAN, 525 CAMINO LAGUNA VISTA, GOLETA CA 93117, USA

Tclephone: (1)

Facsimile: (1)

Telex:

Email:

Commission(s): 10,12,14

LANDOLFI MARCO, OSS ASTROFISICO, DI ARCETRI, LARGO E FERMI 5 , I 50125 FIRENZE, ITALY

Telephone: (39) $552752256 \quad$ Facsimile: (39) Telex: 572268 arcetr

Email: $\quad$ Commission(s): 12

LANDOLT ARLO U PROF, DPT PHYSICS \& ASTRONOMY, LOUISIANA STATE UNIV

BATON ROUGE LA 70803 4001, USA

Telephone: (1) $50+3888276 \quad$ Facsimile: (1) Telex: 559184

Email: phland(a)sn01.sncc.Isu.cdu Commission(s): 25C,27,37,42

LANDSTREET JOHN D PROF, DPT OF ASTRONOMY, UNIV OF WESTERN ONTARIO, LONDON ON N6A 3K7, CANAD

Telephone: (1) $5196792111 * 6707$

Facsimile: (1) 5196612001

Telex:

Email: jlandstraphobos.astro.uwo.ca

Commission(s): 25P,29,36

LANE ADAIR P, CFA, 60 GARDEN ST, CAMBRIDGE MA 02138, USA

Telephone: (1) 6174967654

Email: adain@ecfa.harvard.cdu

Facsimile: (1)

Commission(s):

Telex:

LANE ARTHUR LONNE DR, JPL, 4800 OAK GROVE DR, PASADENA CA 91109 , USA

Telephone: (1) 8183452725 Facsimile: (1) Telex:

Email:

Commission(s): 15,16

LANEY CLIFTON D DR, SAAO, BOX 9, OBSERVATORY 7935, SOUTH AFRICA

Telephone: (27) 470-(125 Facsimile: (27) Telex:

Email:

Commission(s): 27

LANG JAMES DR, RUTHERFORD APPLETON LAB, SPACE \& ASTROPHYSICS DIV, BLDG R25/R68

CHILTON DIDCOT OX11 OQX, UK

Telephone: (44) 123521900

Facsimile: (4t)

Telex: 83159

Email:

Commission(s): 14

LANG KENNETH R ASST PROF, DPT OF PHYSICS, TUFTS UNIVERSITY, ROBINSON HALL

MEDFORD MA 02155, USA

Tclephone: (1) 6173813390

Facsimile: (1)

Telex:

Email:

Commission(s): $10,+10,+1$ 
LANGER GEORGE EDWARD DR, PHYSICS DPT, COLORADO COLLEGE, COLORADO SPRINGS CO 8093, USA

Telephone: (1) $3034732233 * 578$

Facsimile: (1)

Telex:

Email:

Commission(s): 29

LANGER NORBERT DR, MPI FUER ASTROPHYSIK, KARL SCHWARZSCHILDSTR 1 D 85740 GARCHING MUENCHEN, GERMANY

Telephone: (49)

Email:

Facsimile: (49)

Telex:

LANGER WILLIAM DAVID DR, JPL, MS 169506,4800 OAK GROVE DR, PASADENA CA 91109, USA

Telephone: (1) 8183545823

Facsimile: (1)

Telex:

Email:

Commission(s): 34,40

LANGHOFF STEPHEN ROBERT, NASA AMES RESEARCH CTR, MS 230 3, MOFFETT FIELD CA 94035, USA

Telephone: (1) 4156046213

Facsimile: (1) $\$ 1660 \$ 0350$

Telex:

Email: langhoff(apegasms.arc.nasa.gov

Commission(s): 14,19

LANNES ANDRE DR, OBS MIDI PYRENEES, $1+$ AVE E BELIN, F 31400 TOULOUSE CDX, FRANCE

Telephone: (33) 61252101

Facsimile: (33)

Telex: $530776 \mathrm{f}$

Email:

Commission(s):

LANNING HOWARD HUGH, COMPUTER SCIENCES CORP, STSCI, 3700 SAN MARTIN DR

BALTIMORE MD 21218, USA

Telephone: (1) 4103384486

Facsimile: (1) $\$ 10338+767$ Telex:

Email: lanning(astsci

Commission(s):

LANTOS PIERRE DR, OBSERVATOIRE DE PARIS, SECTION DE MEUDON, DASOP

F 92195 MEUDON PPL CDX, FRANCE

Telephone: (33) 145077767

Email:

Facsimile: (33)

Telex:

LANZ THIERRY DR, NASA GSFC, CODE 681, LASP, GREENBELT MD 20771, USA

Telephone: (1)

Facsimile: (I)

Telex:

Email: lanzâatars.gsfc.nasa.gov

Commission(s): 29

LAPASSET EMILIO DR, OBSERVATORIO ASTRONOMICO, DE CORDOBA, LAPRIDA 854 5000 CORDOBA, ARGENTINA

Telephone: (5t) 51230491

Email: lapasset $(\vec{a})$ astro.edu.ar
Facsimile: (54) 51210613

Commission(s): $37,+2$
Telex: 51822 bucor ar

LAPUSHKA K K DR, ASTRONOMICAL OBSERVATORY, LATVIAN STATE UNIVERSITY, RAINIS BUL 19

226098 RIGA, LATVIA

Telephone: (371) $132223149 / 611984$

Email:

Facsimile: (371)

Commission(s): 24

Telex:

LAQUES PIERRE DR, OBS MIDI PYRENEES, 9 R PONT DE LA MOUETTE, F 65200 BAGNERES BIGORRE, FRANCE
Telephone: (33) 62951969

Facsimile: (33)

Commission(s): 09,51

Email:

Telex: 531625

LARGE MICHAEL I DR, SCHOOL OF PHYSICS, UNIVERSITY OF SYDNEY, SYDNEY NSW 2OOG, AUSTRALIA
Telephone: (61) 26922222

Email:

Facsimile: (61)

Commission(s): 40

Telex: 26169 unisyd aa

LARI CARLO DR, IST DI RADIOASTRONOMIA, CNR, VIA IRNERIO +6, I 40126 BOLOGNA, ITALY
Telephonc: (39) 51232856

Facsimile: (39)

Commission(s):

Telex:

Email:

LARSON HAROLD P DR, LUNAR \& PLANETARY LAB, UNIVERSITY OF ARIZONA, TUCSON AZ 85721, USA

Telephonc: (1) 5206216943

Email:

Facsimile: (1)

Telex:

LARSON RICHARD B PROF, DPT OF ASTRONOMY, YALE UNIVERSITY, BOX 6666, NEW HAVEN CT O6520, USA

Telephone: (1) $203+363015$

Commission(s): 15,16

Email:

Facsimile: (1)

Commission(s): $28,33,35$ 
LARSON STEPHEN M, LUNAR \& PLANETARY LAB, UNIVERSITY OF ARIZONA, TUCSON AZ 85721, USA

Telephone: (1) 5206214973

Facsimile: (1)

Telex: 910-952-11+3

Email:

Commission(s): 15,16

LARSSON STEFAN DR, STOCKHOLM OBSERVATORY, S 133 36 SALTSJOEBAIEN, SWEDEN

Telephone: (46) 8164464

Facsimile: (46) $8717+719$ Telex:

Email: larsson(a)astro.su.se

Commission(s): 42

LARSSON-LEANDER G PROF, LUND OBSERVATORY, BOX \&3, S 22100 LUND, SWEDEN

Telephone: (46) 46107000

Facsimile: (46)

Commission(s): 37,42

Telex: 33199obsnot s

Email:

LASALA GERALD J DR, DPT OF PHYSICS, UNIV OF SOUTHERN MAINE, 96 FALMOUTH ST PORTLAND ME 0\$103, USA

Telephonc: (1) 2077804557

Facsimile: (1) $207780+933 \quad$ Telex:

Email: lasala(aportland

Commission(s): 45

LASENBY ANTHONY, MULLARD RADIO ASTRON OBS, CAVENDISH LABORATORY, MADINGLEY RD CAMBRIDGE CB3 OHE, UK

Telephone: (44) 1223337294

Email:

Facsimile: (4t) $122335+599$

Telex: $\mathbf{8 1 2 9 2}$ cavlab g

Commission(s): 40

LASHER GORDON JEWETT DR, IBM, THOMAS J WATSON RES CTR, BOX 218 YORKTOWN HEIGHTS NY 10SO8, USA

Telephone: (1)

Facsimile: (1)

Commission(s): 44

Telex:

Email:

LASKAR JACQUES DR, BUREAU DES LONGITUDES, 77 AVE DENFERT ROCHEREAU, F 7501 4 PARIS, FRANCE Telephone: (33) 140512274

Facsimile: (33)

Telex:

Email:

Commission(s): 04,07

LASKARIDES PAUL G ASSPROF, DPT OF ASTRONOMY, NTL UNIVERSITY OF ATHENS, PANEPISTIMIOPOLIS GR 15771 ZOGRAFOS, GREECE

Telephone: (30) $172+3211$

Facsimile: (30)

Telex:

Email:

Commission(s): $25,27,35$

LASKER BARRY M DR, STSCI, HOMEWOOD CAMPUS, 3700 SAN MARTIN DR, BALTIMORE MD 21218, USA

Telephone: (1) $30133848+0$

Facsimile: (1)

Telex: 6849191 stsi

Email:

Commission(s): 09,25,28,34

LASOTA JEAN-PIERRE DR, OBSERVATOIRE DE PARIS, SECTION DE MEUDON F 92195 MEUDON PPL CDX, FRANCE
Telephone: (33) $1+5077+16$
Facsimile: (33)
Telex: 201571
Commission(s): 35,47

Email:

LATHAM DAVID W DR, CENTER FOR ASTROPHYSICS, HCO/SAO, 60 GARDEN ST, CAMBRIDGE MA 02138, USA

Telephone: (1) $617+957215$

Facsimile: (1)

Commission(s): 26,30,33,37

Telex: $921+28$ satellite cam

Email: latham(a)cfa3

LATOUR JEAN J, OBS MIDI PYRENEES, 14 AVE E BELIN, F 31400 TOULOUSE CDX, FRANCE

Telephone: (33) 61252101

Facsimile: (33)

Telex:

Email:

Commission(s): 35

LATTANZI MARIO G, OSS ASTRONOMICO DI TORINO, ST OSSERVATORIO 20, 1 10025 PINO TORINESE, ITALY Telephone: (39) 11841067 Facsimile: (39)

Commission(s): 08,26

Telex: 213236 to astr 1

Email: span:39181::lattanzi

LATTANZIO JOHN, DPT OF MATHEMATICS, MONASH UNIVERSITY, WELLINGTON RD CLAYTON VIC 3168, AUSTRALIA

Facsimile: (61) 39053867

Telex:

Email: johnl(anash.maths.monash.edu.a Commission(s): 35

LATTIMER JAMES M DR, DPT OF EARTH \& SPACE SCI, ASTRONOMY PROGRAM, SUNY AT STONY BROOK STONY BROOK NY $1179+2100$, USA

Telephone: (1) 5162468223

Facsimile: (1)

Telex:

Email:

Commission(s): 44 
M 656

LATYPOV A A DR, ASTRONOMICAL INSTITUTE, UZBEK ACADEMY OF SCIENCES 706052 TASHKENT, UZBEKISTAN

Telephone: (7) 358102

Facsimile: (7)

Telex: 116012 vremja

Email:

Commission(s): 24

LAUBERTS ANDRIS DR, FOA 3, BOX 1165, S 58111 LINKOPING, SWEDEN

Telcphonc: (46) 13118235

Facsimilc: (46) 13131665

Tclex: 50073 foatre s

Email:

Commission(s): 28

LAUNAY JEAN-MICHEL DR, OBSERVATOIRE DE PARIS, SECTION DE MEUDON F 92195 MEUDON PPL CDX, FRANCE

Telcphone: (33) $14507755 t$

Facsimilc: (33)

Telex: 201571

Email:

Commission(s): 14

LAURENT BERTEL E PROF, INST OF THEORETICAL PHYS, VANADISVAEGEN 9, S 11346 STOCKHOLM, SWEDEN

Telephone: (46) $816+500$

Facsimile: (46)

Commission(s):

Telex: $15+33$ fystos

Email:

LAURENT CLAUDINE DR, OBSERVATOIRE DE PARIS, 61 AVE OBSERVATOIRE, F 7501 4 PARIS, FRANCE

Telephone: (33)

Facsimile: (33)

Telex:

Email:

Commission(s): 34

LAUSBERG ANDRE DR, INSTITUT D'ASTROPHYSIQUE, UNIVERSITE DE LIEGE, AVE COINTE 5

B 4000 COINTE-LIEGE, BELGIUM

Telephone: (32) 41529980

Facsimile: (32) $\$ 1527+7 \downarrow \quad$ Telex:

Email:

Commission(s): 28,47

LAUTMAN D A DR, CENTER FOR ASTROPHYSICS, HCO/SAO, 60 GARDEN ST, CAMBRIDGE MA 02138, USA

Telephone: (1)

Facsimile: (1)

Telex:

Email:

Commission(s):

LAVAL ANNIE DR, OBSERVATOIRE DE MARSEILLE, 2 PLACE LE VERRIER, F 13248 MARSEILLE CDX 04, FRANCE

Telcphone: (33) 91959088

Facsimile: (33)

Commission(s): 37

Telex: $4202+1$ f

Email:

LAVROV M I PROF, ENGELHARDT ASTRONOMICAL, OBSERVATORY, OBSERVATORIA STATION \$22526 KAZAN, RUSSIA

Telephone: (7)

Facsimile: (7)

Telex:

Email:

Commission(s): $\$ 2$

LAVRUKHINA A K PROF DR, VERNADSKY INST GEOCHEM \&, ANALYTICAL CHEMISTRY

ACADEMY OF SCIENCES, $11733+$ MOSCOW, RUSSIA

Telephone: (7) $951377538 \quad$ Facsimile: (7)

Email:

Commission(s):

Telex:

LAWRENCE ANDREW DR, ROYAL OBSERVATORY, BLACKFORD HILL, EDINBURGH EH9 3HJ, UK

Telephone: (44) 1316688346

Facsimile: (44) 1316688356 Telex:

Email: al@roc.ac.uk

Commission(s): 28

LAWRENCE CHARLES R DR, CALTECH, MS 105 24, PASADENA CA 91125, USA

Telcphone: (1) 8183564976

Facsimile: (1)

Telex: $675+29$

Email: bitnet:crlacitdcimo

Commission(s): 40

LAWRENCE G M DR, LASP, UNIVERSITY OF COLORADO, BOX 392, BOULDER CO 80309 0392, USA

Telephone: (1)

Facsimilc: (1)

Telex:

Email:

Commission(s): $1+$

LAWRIE DAVID G, AEROSPACE CORPORATION, MS M4 041, BOX 92957, LOS ANGELES CA 90009, USA

Telephone: (1) $2136+861+2$

Facsimilc: (1)

Telex:

Email:

Commission(s):

LAYDEN CHOISY ANDREW, CERRO TOLOLO, INTERAMERICAN OBSERVATORY, CASILLA 6O3

LA SERENA, CHILE

Telephone: (56) 51205204

Email: alayden $(\hat{a}$ noa.cdu

Facsimile: (56) $512053+2 \quad$ Telex:

Commission(s): 
LAYZER DAVID PROF, CENTER FOR ASTROPHYSICS, HCO/SAO MS 31, 60 GARDEN ST CAMBRIDGE MA 02138, USA

Telephone: (1)

Facsimile: (1)

Telex:

Email:

Commission(s): $14,28,47$

LAZAREFF BERNARD DR, OBSERVATOIRE DE GRENOBLE, CERMO/ASTROPHYSIQUE, BP 53X

F 38041 S MARTIN HERES CD, FRANCE

Telephonc: (33) 76514600

Facsimile: (33) $76+48821$

Telex: $98013+$ f

Email:

Commission(s):

LAZARO CARLOS DR, INST DE ASTROFISICA, DE CANARIAS, OBS DEL TEIDE, E 38200 LA LAGUNA, SPAIN

Telephone: (34) 22262211

Facsimile: (34)

Telex: $926+0$

Email:

Commission(s): 27

LAZOVIC JOVAN P PROF, DPT OF ASTRONOMY, FACULTY OF SCIENCES, STUDENTSKI TRG 16

YU 11000 BEOGRAD, YUGOSLAVIA-SERBIA \& MONTENEGRO

Telephone: (38) 11638715

Facsimile: (38)

Telex:

Email:

Commission(s): 07

LAZZARO DANIELA DR, OBSERVATORIO NACIONAL, RUA GL BRUCE 586, SAN CRISTOVAO 20921 RIO DE JANEIRO RJ, BRAZIL

Telephone: (55) 215807181

Facsimile: (55) 215800332

Telex: 2121288 obsn br

Email: daza@inccvm

Commission(s): 20

LE BORGNE JEAN FRANCOIS, OBS MIDI PYRENEES, 14 AVE E BELIN, F 31400 TOULOUSE CDX, FRANCE

Telephone: (33) 61332929

Facsimile: (33) 61536722

Telex:

Email: Icborgne@obs-mip.fr

Commission(s):

LE BOURLOT JACQUES DR, OBSERVATOIRE DE PARIS, SECTION DE MEUDON, DAEC F 92195 MEUDON PPL CDX, FRANCE

Telephone: (33) $145077566 \quad$ Facsimile: (33) $145077+69$ Telex:

Email: lebourlot(afrmeu51 Commission(s): 14

LE CONTEL JEAN-MICHEL, OCA OBSERV DE NICE, BP 229, F 06304 NICE CDX 4 , FRANCE

Telephone: (33) $93890420 \quad$ Facsimile: (33) $92003033 \quad$ Telex: 460004 obsnice f

Email:

Commission(s): 29

LE DOURNEUF MARYVONNE, OBSERVATOIRE DE PARIS, SECTION DE MEUDON

F 92195 MEUDON PPL CDX, FRANCE

Telephone: (33) $145077555 \quad$ Facsimile: (33)

Email:

Commission(s): 14

Telex: 201571

LE FEVRE OLIVIER DR, OBSERVATOIRE DE PARIS, SECTION DE MEUDON, DAECC

F 92195 MEUDON PPL CDX, FRANCE

Telephone: (33) 145077555

Facsimile: (33)

Telex:

Email:

Commission(s): 28

LE POOLE RUDOLF S DR, STERREWACHT, BOX 9513, NL 2300 RA LEIDEN, NETHERLANDS

Telephone: (31) 71272727

Email:

Facsimile: (31)

Tclex: 39058 astro $n$

LE SQUEREN ANNE-MARIE DR, OBSERVATOIRE DE PARIS, SECTION DE MEUDON

F 92195 MEUDON PPL CDX, FRANCE

Telephone: (33) 145077595

Facsimile: (33)

Telex:

Email:

Commission(s): 34,40

LEA SUSAN MAUREEN DR, DPT PHYSICS \& ASTRONOMY, SAN FRANSISCO STATE UNIV, 1600 HOLLOWAY AVE SAN FRANCISCO CA 94132 , USA

Tclephone: (1) 4054691880

Facsimile: (1)

Telex:

Email:

Commission(s): 4

LEACH SYDNEY DR, OBSERVATOIRE DE PARIS, SECTION DE MEUDON, DAMAP

F 92195 MEUDON PPL CDX, FRANCE

Telephone: (33) 145077561

Facsimile: (33) $\$ 7077469$

Telex: 201571

Email: Icach(afrmeu51

Commission(s): 14 
LEACOCK ROBERT JAY, DPT OF ASTRONOMY, UNIVERSITY OF FLORIDA, 211 SSRB, GAINESVILLE FL 32611, USA Telephone: (1) 9043922052

Facsimile: (1)

Telex:

Email:

Commission(s): 28

LEAHY J PATRICK DR, NRAL, JODRELL BANK, MACCLESFIELD SK11 9DL, UK

Telephone: (44) 147771321

Facsimile: (4H) 147771618

Telex: 36149

Email: jpl@uk.ac.man.jb.star

Commission(s): 40

LEAHY DENIS A DR, DPT OF PHYSICS, UNIVERSITY OF CALGARY, 2500 UNIVERSITY DR NW

CALGARY AB T2N 1N4, CANADA

Telephonc: (1) 4032207192

Facsimile: (1) 4032893331

Telex:

Email: bitnet:leahy@uncamult

Commission(s):

LEBLANC YOLANDE DR, OBSERVATOIRE DE PARIS, SECTION DE MEUDON, F 92195 MEUDON PPL CDX, FRANCE Telephone: (33) 145077759

Facsimile: (33)

Tclex:

Email:

Commission(s): 40

LEBOFSKY LARRY ALLEN, LUNAR \& PLANETARY LAB, UNIVERSITY OF ARIZONA, TUCSON AZ 85721, USA

Telephone: (1) 5206216947

Facsimile: (1)

Telex:

Email:

Commission(s): 15

LEBOVITZ NORMAN R PROF, DPT OF MATHEMATICS, UNIVERSITY OF CHICAGO, 573+ S UNIVERSITY AVE CHICAGO IL 60637, USA

Telephone: (1) 3127538074

Facsimile: (1)

Telex:

Email:

Commission(s): 35

LEBRETON YVELINE DR, OBSERVATOIRE DE PARIS, SECTION DE MEUDON, F 92195 MEUDON PPL CDX, FRANCE Telephone: (33) 145077859

Facsimile: (33)

Commission(s): 35

Telex: 201571

Email: lcbreton@frmcu51

LECAR MYRON DR, CENTER FOR ASTROPHYSICS, HCO/SAO, 60 GARDEN ST, CAMBRIDGE MA 02138, USA

Telephone: (1) 6174957251

Facsimile: (1)

Telex: 921428 satellite cam

Email:

Commission(s): 33

LECKRONE DAVID S DR, NASA GSFC, CODE 681, GREENBELT MD 20771, USA

Telephone: (1) $3012868904 \quad$ Facsimile: (1) Telex:

Email: $\quad$ Commission(s): 29,4t

LEDERLE TRUDPERT DR, ASTRON RECHEN INSTITUTE, MOENCHHOFSTR 12-14 D 69120 HEIDELBERG, GERMANY

Telephone: (49) 622149026

Facsimile: $(49)$

Telex: 461336 arihd d

Email:

Commission(s): (04,05, 18

LEE DONG HUN, DPT ASTRONOMY \& SPACE SCI, KYUNGHEE UNIVERSITY, YONGIN KYUNGGEE 449 701, KOREA R

Telephone: (82)

Facsimile: (82)

Commission(s):

Telex:

Email: dhlce@nms.kyunghce.ac.kr

-LEE HYUNG MOK DR, DPT EARTH SCIENCES, PUSAN NTL UNIVERSITY, KUM JONG KU, PUSAN 609 735, KOREA R Telephone: (82) 515102702 Facsimile: (82) 515137495 Telex:

Email: hmlec@hyowon.jpusan.ac.kr Commission(s): 33

LEE JONG TRUENLIANG, TAIWAN, CHINA R

Telephone: (886)

Facsimile: (886)

Telex:

Email:

Commission(s):

LEE MYUNG GYOON, DPT OF ASTRONOMY, SEOUL NTL UNIVERSITY, SEOUL 151 742, KOREA R

Telephone: (82)

Facsimile: (82)

Telex:

Email: mglce@astrog.snu.ac.kr

Commission(s):

LEE PAUL D DR, DPT PHYSICS \& ASTRONOMY, LOUISIANA STATE UNIV, BATON ROUGE LA 70803 4001, USA

Telephone: (1)

Facsimile: (1)

Telex:

Email:

Commission(s): 
LEE SANG GAK, DPT OF ASTRONOMY, SEOUL NTL UNIVERSITY, KWANAK KU, SEOUL 151, KOREA R

Telephonc: (82) 877 2131/2139

Facsimile: (82)

Telex:

Email:

Commission(s): $33,45,51$

LEE SEE-WOO DR, DPT OF ASTRONOMY, SEOUL NTL UNIVERSITY, SEOUL CITY, KOREA R

Telephone: (82) 877 2131/9*3308 Facsimile: $(82)$

Email:

Commission(s):

Telex:

LEE TERENCE J DR, ROYAL OBSERVATORY, HEAD OF TECHNOLOGY, BLACKFORD HILL EDINBURGH EH9 3HJ, UK

Telephone: (44) 1316673321

Email:

Facsimile: (4t)

Commission(s): 34

Telex: 72383 roeding uk

LEE THYPHOON, INST EARTH SCIENCES, ACADEMIA SINICA, BOX 23 59, TAIPEI 107, CHINA R

Telephone: (886) 23963211

Facsimile: (886)

Commission(s): 15,35

Telex:

Email:

M DONG, YUSEONG GU, DAEJEON 305 348, KOREA R

LEE WOO-BAIK DR, ISSA, 36-1 WHAAM DONG, YUSEONG GU, DAEJEON 30S
Telephone: (82) 428615611
Facsimile: (82) 428615610

$\begin{array}{ll}\text { Telephone: (82) } 428615611 & \text { Facsimile: }(82) \text { (28 } \\ \text { Email: } & \text { Commission(s): } 42\end{array}$

elex:

LEE YONG-SAM DR, DPT ASTRONOMY \& SPACE SCI, CHUNGBUK NTL UNIVERSITY

CHEONGJU 360763 , KOREA R

Telephone: (82) $431612314 \quad$ Facsimile: (82) $\$ 31674232 \quad$ Telex:

Email: yslec@ebucc.cbnu.ac.kr

Commission(s): $\$ 2$

LEE YOUNG WOOK, DPT OF ASTRONOMY, YONSEI UNIVERSITY, SHINCHON I34 SEODAEMOON K

SEOUL 120 7\$9, KOREA R

Telephone: (82)

Facsimile: (82)

Telex:

Email: ywlec(agalaxy.yonsci.ac.kr

Commission(s):

LEEDJAERV LAURITS, TARTU ASTROPHYSICAL OBS, EE 2 $\$+4$ TORAVERE, ESTONIA

Telephone: (372) 7410343

Facsimile: (372) $7+10205$

Telex:

Email: lecd(a)aai.ee

Commission(s): 29, 42

LEER EGIL PROF, INST THEORET ASTROPHYSICS, UNIVERSITY OF OSLO, BOX 1029, N 0315 BLINDERN OSLO 3 , NORWAY

Telephone: (47) 2-456503

Email:

Facsimile: $(47) 2-456505$

Telex: $6+12+$ aurob $n$

LEFEBVRE MICHEL DR, CNES/GRGS, 18 AVE E BELIN, F 31055 TOULOUSE CDX, FRANCE

Telephone: (33)

Facsimile: (33)

Telex:

Email:

Commission(s):

LEFEVRE JEAN DR, OCA OBSERV DE NICE, BP 229, F 0630 $\downarrow$ NICE CDX $\downarrow$, FRANCE

Tclephonc: (33) 93890420

Facsimile: (33) 92003033

Telex: 460004 obsnice f

Email:

Commission(s):

LEGER ALAIN DR, IAS, UNIVERSITE PARIS XI, F 91405 ORSAY CEDEX, FRANCE

Telephone: (33) 169858580

Facsimile: (33) 169858675 Telex:

Email: leger(a)iaslab.ias.fr

Commission(s): $14,21,34,51 \mathrm{C}$

LEGG THOMAS H DR, HERZBERG INST ASTROPHYS, NTL RESEARCH COUNCIL, 100 SUSSEX DR OTTAWA ON K1A ORG, CANADA

Telephone: (1) 6139936060

Facsimile: (1) 6139526602

Telex: 0533715

Email:

Commission(s): \$0

LEHMANN MAREK DR, ASTRONOMICAL LATITUDE OBS, BOROWIEC, BOX 62 035, PL 62 (035 KORNIK, POLAND Telephone: (48) 170187

Email:

Facsimile: $(48)$

Commission(s): $(04,08,19$

Telex: $\$ 12623$ aos p!

LEHNERT B P PROF, DPT OF PLASMA PHYSICS, ROYAL INST OF TECHNOLOGY

S 10044 STOCKHOLM 70, SWEDEN

Telephone: (46) 7877763

Facsimile: (46)

Commission(s):

Telex: 10389 kthb s

Email:

Commission(s): 
LEIBACHER JOHN DR, NTL SOLAR OBSERVATORY, BOX 26732, 950 N CHERRY AVE, TUCSON AZ 85726 6732, USA

Telephone: (1) 5203259302

Facsimile: (1) 5203259305

Telex: 066484 aura noao tu

Email: jleibacher@noao.arizona.edu

Commission(s): 10,12,36

LEIBOWITZ ELIA M DR, DPT OF PHYSICS \& ASTRON, TEL AVIV UNIVERSITY, RAMAT AVIV, TEL AVIV 69978, ISRAEL

Telephone: (972) 3413788

Facsimile: (972)

Commission(s): 50

Telex: $3+3171$ versy il

Email:

LEIKIN G A DR, INST OF ASTRONOMY, ACADEMY OF SCIENCES, PYATNITSKAYA UL 48, 109017 MOSCOW, RUSSI

Telephone: (7) 952315461

Facsimile: (7)

Telex: 412623 sestp su

Email:

Commission(s): 16

LEINERT CHRISTOPH PROF, MPI FUER ASTRONOMIE, KOENIGSTUHL, D 69117 HEIDELBERG, GERMANY

Telephone: (49) 6221528264

Email: leinert@mpia-hd.mpg.de

Facsimile: (49) 6221528246

Telex: 461789 mpiad

LEISAWITZ DAVID DR, NASA GSFC, CODE 685, GREENBELT MD 20771, USA

Telephone: (1) $3012862150 \quad$ Facsimile: (1) $3012868709 \quad$ Telex:

Email: 6168::leisawitz

Commission(s): $33,34,37$

L,EISTER NELSON VANI, IAG, UNIVERSIDADE DE SAO PAULO, CP 9638, 01065970 SAO PAULO, BRAZIL

Telephone: (55) 115778599

Email: leister@vax.lagusp.usp.br

Facsimile: (55) $112763848 \quad$ Telex:

Commission(s):

LEITE SCHEID PAULO DR, OBSERVATORIO NACIONAL, RUA GL BRUCE 586, SAO CRISTOVAO

20921 RIO DE JANEIRO RJ, BRAZIL

Telephone: (55) 215807313

Facsimile: (55) 215800332 Telex:

Email:

Commission(s): 27

GEITHERER CLAUS, STSCI, HOMEWOOD CAMPUS, 3700 SAN MARTIN DR, BALTIMORE MD 21218, USA

Telephone: (1) 4103384425

Facsimile: (1) $4103384767 \quad$ Telex:

Email: stsci::leitherer

Commission(s):

LEKHT EVUENI, INAOE, AP 51/216, PUEBLA PUE 72000, MEXICO

Telephone: (52)

Facsimile: (52)

Telex:

Email:

Commission(s):

LELIEVRE GERARD DR, INSU/CNRS, BP 287, 3 RUE MICHEL ANGE, F 75766 PARIS CDX 16, FRANCE

Telephone: (33) 144964377

Facsimile: (33) 1 +496 4965 Telex:

Email: Ielievre@iap.fr

Commission(s): 09P,28

LEMAIRE JEAN-LOUIS DR, OBSERVATOIRE DE PARIS, SECTION DE MEUDON

F 92195 MEUDON PPL CDX, FRANCE

Telephone: (33) $145077563 \quad$ Facsimile: (33) 145077469 Telex:

Email:

Commission(s): 14

LEMAIRE PHILIPPE DR, IAS, BAT 121, UNIVERSITE PARIS XI, F 91405 ORSAY CEDEX, FRANCE

Telephone: (33) 169858622

Facsimile: (33) 169858675 Telex:

Email: iaslab::lemaire

Commission(s): 44

LEMAITRE ANNE DR, DPT DE MATHEMATIQUES, FACULTES UNIVERSITAIRES, REMPART DE LA VIERGE 8 B S000 NAMUR, BELGIUM

Telephone: (32) 81229061

Email:

Facsimilc: (32) 81230391

Tclex: 59222 facnam b

LEMAITRE GERARD R DR, OBSERVATOIRE DE MARSEILLE, 2 PLACE LE VERRIER

F 13248 MARSEILLE CDX 04, FRANCE

Telephone: (33) 91959088

Facsimile: (33)

Telex:

Email:

Commission(s): 09

LEMKE DIETRICH DR, MPI FUER ASTRONOMIE, KOENIGSTUHL, D 69117 HEIDELBERG, GERMANY

Telephone: (49) 6221528259

Facsimile: (49)

Email:

Commission(s): 21

Telex: 461789 impia-d 
LENA PIERRE J PROF, OBSERVATOIRE DE PARIS, SECTION DE MEUDON, F 92195 MEUDON PPL CDX, FRANCE

Telephone: (33) 145077719

Facsimile: (33)

Telex: 201571

Email:

Commission(s):

LENHARDT HELMUT, ASTRON RECHEN INSTITUTE, MOENCHHOFSTR 12-14, D 69120 HEIDELBERG, GERMANY

Telephone: (49) 6221405251

Facsimile: (49) 6221405297 Telex:

Email: s29@mvs.urz.uni-heidelberg.de Commission(s): 08

LENZEN RAINER DR, MPI FUER ASTRONOMIE, KOENIGSTUHL, D 69117 HEIDELBERG, GERMANY

Telephone: (49)

Facsimile: (49)

Telex:

Email:

Commission(s): 25

LEONE FRANCESCO, OSS ASTROFISICO, CITTA UNIVERSITARIA, VIA A DORLA 6, I 95125 CATANIA, ITALY

Telephone: (39) 957332229

Facsimile: (39) 95330592

Tclex: 970359 astret $i$

Email: fleone@astrct.ct.astro.it

Commission(s):

LEORAT JACQUES DR, OBSERVATOIRE DE PARIS, SECTION DE MEUDON, F 92195 MEUDON PPL CDX, FRANCE

Telcphone: (33) 145077421

Facsimile: (33)

Telex: 201571

Email:

Commission(s):

LEPINE JACQUES R D DR, IAG, UNIVERSIDADE DE SAO PAULO, AV MIGUEL STEFANO 4200

04301 SAO PAULO SP, BRAZIL

Telcphone: (55) $112753720 \quad$ Facsimile: (55) $112763848 \quad$ Telex: 1156735 iagm br

Email:

Commission(s): $34,35,40$

LEPP STEPHEN H DR, PHYSICS DPT, UNIVERSITY OF NEVADA, 4505 MARYLAND PARKWAY

LAS VEGAS NV 89154, USA

Telcphone: (1)

Email: lepp@cfaitamp.harvard.cdu

Facsimile: (1)

Telex:

LEQUEUX JAMES DR, RADIOASTRONOMIE ENS, 24 RUE LHOMOND, F 75231 PARIS CDX 05, FRANCE

Telephone: (33) $143291215 \quad$ Facsimile: (33)

Telex: 202601

Email: aanda@frmcu51/mesioa::aanda Commission(s): 05,28,34,40,47

LEROY BERNARD DR, OBSERVATOIRE DE PARIS, SECTION DE MEUDON, DASOP

F 92195. MEUDON PPL CDX, FRANCE

Telephone: (33) 145077812

Facsimile: (33)

Telex:

Email:

Commission(s): 10

LEROY JEAN-LOUIS, OBS MIDI PYRENEES, 14 AVE E BELIN, F 31400 TOULOUSE CDX, FRANCE

Telephone: (33) 61332929

Facsimile: (33)

Telex: $530776 \mathrm{f}$

Email: carn:leroy@frmeus1

Commission(s): 10,12

LESAGE ALAIN DR, OBSERVATOIRE DE PARIS, SECTION DE MEUDON, DASGAL

F 92195 MEUDON PPL CDX, FRANCE

Telephone: (33) 145077829

Facsimile: (33) $145077878 \quad$ Telex:

Email: Jesage@frmeu51/mesioa::Iesage

Commission(s): 14

LESCH HAROLD DR, MPI FUER RADIOASTRONOMIE, AUF DEM HUEGEL 69, D S3121 BONN, GERMANY

Telephone: (49) 2285251

Facsimile: (49)

Telex:

Email:

Commission(s): 40

LESCHIUTTA S PROF, DPT ELECTRONICA, POLITECNICO, CORSO DUCA D ABRUZZI 24, I 10129 TURINO, ITALY

Telephonc: (39) 115567235

Facsimile: (39)

Commission(s): 31

Telex: 220646 polito

Email: bitnet:leschiutta@itopoli

DPT, UNIVERSITY OF TEXAS, RLM 15308 , AUSTIN TX 78712 1083, USA

LESTER DANIEL F DR, ASTRONOMY DPT, UNIVERSITY OF TEXAS, RLM 15 308, AUSTIN
Telephone: (1) 5124713442
Facsimile: (1)

Commission(s):

Email: arpa:df@@astro.as.utexas.edu

LESTER JOHN B DR, DPT OF ASTRONOMY, UNIVERSITY OF TORONTO, ERINDALE COLLEGE

MISSISSAUGA ON LSL 1C6, CANADA

Telephone: (1) 4168285356

Facsimilc: (1) $\$ 168285328$

Telex:

Email:

Commission(s): 29 
LESTRADE JEAN FRANCOIS DR, BUREAU DES LONGITUDES, 77 AVE DENFERT ROCHEREAU F 75014 PARIS, FRANCE

Telephone: (33) 140512265

Facsimile: (33)

Telex: 2701070

Email:

Commission(s): 40

\section{LETFUS VOJTECH DR, ASTRONOMICAL INSTITUTE, CZECH ACADEMY OF SCIENCES} ONDREJOV OBSERVATORY, CZ 25165 ONDREJOV, CZECH R

Telephone: (42) 204857225/7111 Fatesimile: (42) 204851611 Telex: 121579

Email: astsun(ä)escain Conmission(s):

LEUNG CHUN MING DR, DPT OF PHYSICS, RENSSELAER POLYTECHN INST, TROY NY 12180 3590, USA

Tclephonc: (1) 5182666318

Facsimile: (1)

Telex:

Email:

Commission(s): $34,40,46$

LEUNG KAM CHING PROF, DPT PHYSICS \& ASTRONOMY, UNIVERSITY OF NEBRASKA, BEHLEN OBSERVATORY LINCOLN NE 685880111 , USA

Telephone: (1) 4024722770

Facsimile: (1)

Telex: $\mathbf{4 8 + 3 4 0}$ unl

Email:

Commission(s): $27,38,42$

LEVASSEUR-REGOURD A C PR, SERVICE D'AERONOMIE, BP 3, F 91371 VERRIERES BUISSON, FRANCE

Telephone: (33) $164+7+293$

Facsimile: (33) $1+3298673$

Email: chantal.levassucr(a) acrov,jussicu.fr Commission(s): 15C,21C,22,51

LEVATO ORLANDO HUGO DR, COMPLEJO ASTRONOMICO, EL LEONCITO, CC 467,5400 SAN JUAN, ARGENTINA

Telephone: (54) $6+213693$

Email: levato(a)castec.edi.ar

Facsimile: (54) $6+211+75$

Telex: $5913+$ entop ar

LEVINE RANDOLPH H DR, 50 CARVER RD, NEWTON MA (2161, USA

Telephone: (1) 6179655953

Facsimile: (1)

Commission(s):

Telex:

Email:

OBSERVATORY, FLAGSTAFF STATION, BOX 11+9, FLAGSTAFF AZ 86002, USA

LEVISON HAROLD F DR, US NAVAL OBSERVATORY, FLACSTAFF STATION,
Telephonc: (1) 60127795132

Email: percy::levison

Commission(s):

LEVREAULT RUSSELL M DR, 35 PAYSON AVE, EASTHAMPTON MA 01027, USA

Telephone: (1) $\$ 135279442$ Facsimile: (1) Telex:

Email:

Commission(s): 40

LEVY EUGENE H DR, LUNAR \& PLANETARY LAB, UNIVERSITY OF ARIZONA, TUCSON AZ 85721, USA

Telephone: (1) 5206216962

Facsimile: (1)

Telex: 9109521143

Email:

Commission(s): $+1,+4$

LEVY JACQUES R DR, OBSERVATOIRE DE PARIS, 61 AVE OBSERVATOIRE, F 75014 PARIS, FRANCE

Telephone: (33) $143201210 \quad$ Facsimile: (33)

Email:

Commission(s)

Telex: 270776 obs $f$

TEWIN WALTER H G PROF, DPT OF PHYSICS, MIT RM 37 627, BOX 165, CAMBRIDGE MA 02139, USA

Telephonc: (1) $617253+282$

Facsimilc: (1)

Telex:

Email:

Commission(s): th

LEWIS BRIAN MURRAY DR, ARECIBO OBSERVATORY, BOX 9\%, ARECIBO PR 00612, USA

Telephone: (1) 80987826.12

Facsimile: (1)

Telex:

Email:

Commission(s): 30

LEWIS J S, LUNAR \& PLANETARY LAB, UNIVERSITY OF ARIZONA, TUCSON AZ 85721, USA

Telephone: (1) 520 6214972

Facsimile: (1)

Telex:

Email:

Commission(s): 16

LI CHUN-SHENG, DPT OF ASTRONOMY, NANJING UNIVERSITY, NANJING 2100I8, CHINA PR

Telephone: (86) $2534651 * 2882 \quad$ Facsimile: $(86)$

Telex: $3+151$ prenu en

Email:

Commission(s): 10,40

LI DEPEI, NANJING ASTRONOMICAL, INSTRUMENT FACTORY, NANJING, CHINA PR

Telephone: (86) 2546191

Facsimile: $(86)$

Commission(s): (19)

Telex: 1131

Email: 
LI DONG-Ming, PURPLE MOUNTAIN OBSERV, CAS, NANJING, CHINA PR

Telephone: (86) 2546700

Facsimile: (86) 25301459

Telex: 34144 pmonj cn

Email:

Commission(s): 08

LI GI MAN, PYONGYANG ASTRON OBS, ACADEMY OF SCIENCES DPRK, TAESONG DISTRICT PYONGYANG, KOREA DPR
Telephone: (850)
Facsimile: (850)
Telex:

Email:

Commission(s): 04

LI GYONG WON, PYONGYANG ASTRON OBS, ACADEMY OF SCIENCES DPRK, TAESONG DISTRICT PYONGYANG, KOREA DPR

Telephone: (850) Facsimile: (850) Telex:

Email:

Commission(s): 40

LI HONG-WEI, JILA, UNIVERSITY OF COLORADO, CAMPUS BOX 440, BOULDER CO 80309, USA

Telephone: (1) $3034927789 \quad$ Facsimile: (1) 3034925235 Telex: 755842

Email: $\quad$ Commission(s): 40

LI HYOK HO, PYONGYANG ASTRON OBS, ACADEMY OF SCIENCES DPRK, TAESONG DISTRICT

PYONGYANG, KOREA DPR

Telephone: $(\mathbf{8 5 0})$

Facsimile: (850)

Commission(s): 04

Telex:

Email:

LI J Y, PYONGYANG ASTRON OBS, ACADEMY OF SCIENCES DPRK, TAESONG DISTRICT

PYONGYANG, KOREA DPR

Telephone: (850)

Email:

Facsimile: $(\mathbf{8 5 0})$

Telex:

Commission(s):

LI JING, BEIJING ASTRONOMICAL OBS, CAS, W SUBURB, BEIJING 100080, CHINA PR

Telephone: (86) 122040

Facsimile: (86)

Telex:

Email:

Commission(s): 28,33

LI NED C DR, CALIFORNIA UNIVERSITY, 6531 WITHWORTH RD, LOS ANGELES CA 90035, USA

Telephone: (1)

Facsimile: (1)

Telex:

Email:

Commission(s):

LI NENG-YAO, PURPLE MOUNTAIN OBSERV, CAS, NANJING, CHINA PR

Telephone: (86) $2537609 \quad$ Facsimile: (86) $25301459 \quad$ Telex: 34144 pmonj cn

Email:

Commission(s): (04,08

LI PENG, SPACE SCIENCES LABORATORY, UNIVERSITY OF CALIFORNIA, BERKELEY CA 94720, USA

Telephone: (1) 5106437728

Facsimile: (1) $5106438302 \quad$ Telex:

Email: pli@sunspot.ssl.berkeley.edu Commission(s):

LI QI-BIN, BEIJING ASTRONOMICAL OBS, CAS, W SUBURB, BEIJING 100080, CHINA PR

Telephone: (86) 1281968 Facsimile: (86) Telex: 22040 badas cn

Email: $\quad$ Commission(s): $05 \mathrm{C}, 28,44,46$

LI SIN HYONG, PYONGYANG ASTRON OBS, ACADEMY OF SCIENCES DPRK, TAESONG DISTRICT

PYONGYANG, KOREA DPR

Telephone: (850)

Facsimile: (850)

Telex:

Email:

Commission(s): 25

LI SON JAE, PYONGYANG ASTRON OBS, ACADEMY OF SCIENCES DPRK, TAESONG DISTRICT, YYONGYANG KOREA DPR

Telephone: (850)

Facsimile: (850)

Telex:

Email:

Commission(s): 10

LI TING, NANJING ASTRONOMICAL, INSTRUMENT FACTORY, NANJING, CHINA PR

Telephone: (86) 2546191

Facsimile: (86)

Telex:

Email:

Commission(s): 09

LI TIPEI, INSTITUTE OF HIGH ENERGY, PHYSICS, BOX 918 3, BEIJING, CHINA PR

$\begin{array}{ll}\text { Telephone: (86) } 1812971 * 464 & \text { Facsimile: (86) } \\ \text { Email: } & \text { Commission(s): } 44\end{array}$ 
LI WEI BAO, YUNNAN OBSERVATORY, CAS, BOX 110, KUNMING 729 $\$ 6$ YUNNAN, CHINA PR

Telephone: (86) 8712035

Facsimile: (86)

Telex:

Email:

Commission(s): 10

LI XIAO-QING, PURPLE MOUNTAIN OBSERV, CAS, NANJING, CHINA PR

Telephone: (86) 2531096

Facsimile: (86) 25301459

Telex: 34144 pmonj en

Email:

Commission(s): 28,49

LI YUAN-JIE, DPT OF PHYSICS, HUAZHONG UNIVERSITY OF, SCIENCE AND TECHNOLOGY, WUHAN, CHINA PR Telephone: (86) 870541

Facsimile: (86)

Telex: 7122

Email:

Commission(s): 44

LI ZHENG-XIN DR, SHANGHAI OBSERVATORY, CAS, 80 NANDAN RD, SHANGHAI 200030, CHINA PR

Telephone: (86) 21386191

Facsimile: (86)

Telex:

Email:

Commission(s): 19

LI ZHIAN DR, DPT OF ASTRONOMY, BEIJING NORMAL UNIVERSITY, BEIJING 100875, CHINA PR

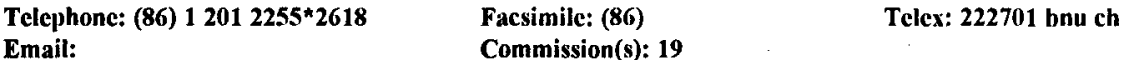

LI ZHIGANG, SHAANXI OBSERVATORY, CAS, LINTONG XIAN, SHAANXI, CHINA PR

Telephone: (86) 332255

Facsimilc: (86)

Telex: 70121 csalo cn

Email:

Commission(s): 08,09

LI ZHI-FANG, SHANGHAI OBSERVATORY, CAS, 80 NANDAN RD, SHANGHAI 200030, CHINA PR

Telephone: (86) 21386191

Facsimile: (86)

Commission(s): 08,47

Telex: $3316+$ shao cn

Email:

LI ZHI-SEN, BEIJING ASTRONOMICAL OBS, CAS, W SUBURB, BEIJING 100080, CHINA PR
Telephone: (86) 1281698
Facsimile: (86)

Email:

Commission(s): 41

LI ZHONGYUAN, DPT EARTH \& SPACE SCI, UNIV SCIENCE \& TECHNOLOGY, HEFEI 230026 ANHUI, CHINA PR

Telephone: (86) 551331134

Facsimile: (86)

Commission(s): $\mathbf{2}, \mathbf{4 4}$

Telex: 90028 uste en

Email:

BEIJING NORMAL UNIVERSITY, BEIJING 100875, CHINA PR

LI ZONG-WEI, DPT OF ASTRONOMY, BEIJING NORMAL UNIVERSITY, BEIJIN
Telephone: (86) $1656531^{* 683} \quad$ Facsimile: (86)

Email:

Commission(s): 35,4

LI ZONG-YUN, DPT OF ASTRONOMY, NANJING UNIVERSITY, NANJING, CHINA PR

$\begin{array}{ll}\text { Telephone: (86) } & \text { Facsimile: (86) } \\ \text { Email: } & \text { Commission(s): }\end{array}$

LIANG EDISON P DR, DPT SPACE PHYS \& ASTRON, RICE UNVIERSITY, BOX 1892, HOUSTON TX 77251 1892, USA

Telephone: (1) $7135278101 * 3524$

Facsimile: (1) $71328551+3$

Telex: $\$ 56457$

Email: liang@vega.rice.edu

Commission(s): $4+$

LIANG SHI-GUANG, SHANGHAI OBSERVATORY, CAS, 80 NANDAN RD, SHANGHAI 200030, CHINA PR

Telephone: (86) 21386191

Facsimile: (86)

Commission(s): 40

Telex: $3316+$ shao cn

Email:

LIANG ZHONG-HUAN, BOX 18, LINTONG, XIAN, CHINA PR
Telephone: (86) 332255
Facsimile: (86)

Commission(s): 31

Telex: 70121 csao cn

Email:

LIAO XINHAO DR, DPT OF ASTRONOMY, NANJING UNIVERSITY, NANJING 210008, CHINA PR

Telephone: (86) $25637651 * 2884$

Facsimile: (86)

Commission(s): 07

Telex: 34151 prenu en

Email: 210008

LIBBRECHT K G DR, CALTECH, MS 264 33, BIG BEAR SOLAR OBS, PASADENA CA 91125, USA

Telephone: (1) 8183563722

Email: kgl@asundog.caltech.edu
Facsimile: (1)

Commission(s):

Telex: 675425 caltech psd 
LIDDELL U MR, NASA HEADQUARTERS, SPACE SCI \& APPLICATIONS, 600 INDEPENDENCE AVE SW WASHINGTON DC 20546, USA
Telephone: (1)
Facsimile: (1)
Telex:
Email:
Commission(s):

LIDDLE ANDREW, ASTRONOMY CENTRE, UNIVERSITY OF SUSSEX, FALMER, BRIGHTON BN1 9QH, UK

Telephone: (44) $1273606755 * 2933$

Email: arl@starlink.sussex.ac.uk

Facsimile: (44) $1273678097 \quad$ Telex:

Commission(s): 47

LIEBERT JAMES W DR, STEWARD OBSERVATORY, UNIVERSITY OF ARIZONA, TUCSON AZ 85721, USA

Telephone: (1) 5206214513

Facsimile: (1)

Telex: 62141410

Email: liebert@arizrvax

Commission(s): 29,33,35,36

LIEBSCHER DIERCK-E DR, ZNTRLINST F ASTROPHYSIK, STERNWARTE BABELSBERG

ROSA LUXEMBURG STR 17A, D 1443 POTSDAM, GERMANY

Telephone: (49)

Facsimile: (49)

Telex:

Email:

Commission(s): 47

LIESKE JAY H DR, JPL CALTECH, MS 301 150, 4800 OAK GROVE DR, PASADENA CA 91109, USA

Telephone: (1) $81835436+2$

Email: jhl@naif.jpl.nasa.gov

Facsimile: (1) $81835+3437$

Telex: 675429

Commission(s): 0+C,07C,19,20

LFFMAN KURT, FLUID DYNAMICS LAB, CSIRO/DBCE, BOX 56, HIGHETT VIC 3190, AUSTRALIA

Telephone: (61) $\quad$ Facsimile: (61)

Email: $\quad$ Commission(s):

Telex:

LIKKEL LAUREN JONES, PROGRAM IN ASTRONOMY, WASHINGTON STATE UNIV, DPT OF MATHEMATICS PULLMAN WA 991653113 , USA

Telephone: (1) 5093353172

Facsimile: (1) $5093351188 \quad$ Telex:

Email: likkel@beta.math.wsu.edu

Commission(s): 34,40

LILJE PER VIDAR BARTH DR, INST THEORET ASTROPHYSICS, UNIVERSITY OF OSLO, BOX 1029 N 0315 BLINDERN OSLO 3, NORWAY

Telephone: (47) 2856501

Email: per.lilje@astro.uio.no

Facsimile: (47) 2856505

Commission(s): 47

Telex: 72705 astro n

LILLER WILLIAM DR, INSTITUTO ISAAC NEWTON, CASILLA 8-9, VINA DEL MAR, CHILE

Telephone: (56) 32970864

Email:

Facsimile: (\$6)

Telex:

Commission(s): $15,28,34$

LILLEY EDWARD A PROF, CENTER FOR ASTROPHYSICS, HCO/SAO, 60 GARDEN ST, CAMBRIDGE MA 02138, USA

Telephone: (1) 6174953971

Facsimile: (1)

Telex: 921428 satellite cam

Email:

Commission(s): 40,51

LILLIE CHARLES F DR, TRW SPACE \& TECHNOLOGY, 1 SPACE PARK, REDONDO BEACH CA 90278, USA

Telcphone: (1) 2138122248

Facsimile: (1)

Commission(s): 15,21

Telex: $910-325-6611$

Email:

LILLY SIMON J DR, DPT OF ASTRONOMY, UNIVERSITY OF TORONTO, 60 ST GEORGE ST

TORONTO ON MSS 1A1, CANADA

Telephone: (1) 4169783150

Facsimile: (1) 4169783921

Telex: 06286766

Email:

Commission(s): 28,47

LIMA BOTTI LUIZ CLAUDIO, CRAAE INPE, EPUSP/PTR/CRAAE, CP $61548,05+2+970$ SAO PAULO, BRAZIL

Telephone: (55) 118155936

Email: lclbotti@bruspvm.bitnet

Facsimile: (55) 118156289

Telex:

Commission(s):

LIN CHIA C PROF, DPT OF MATHEMATICS, MIT, 77 MASSACHUSETTS AVE, CAMBRIDGE MA 02139, USA

Telephone: (1) 6172531796

Facsimile: (1)

Commission(s): $28,33,34$

Telex: 921473 mit cam

Email:

LIN DOUGLAS N C DR, LICK OBSERVATORY, UNIVERSITY OF CALIFORNIA, SANTA CRUZ CA 95064, USA

Telephone: (1) 4084292732

Facsimile: (1)

Commission(s): 07

Telex:

Email: 
LIN YUANZHANG, BEIJING ASTRONOMICAL OBS, CAS, W SUBURB, BEIJING 100080, CHINA PR Telephone: (86) 1281698

Email:

Commission(s): 10,12

LINCOLN J VIRGINIA MISS, 2005 ALPINE DR, BOULDER CO 80304, USA

Telephone: (1) 3034426757

Facsimile: (1)

Telex:

Email:

Commission(s):

LINDBLAD BERTIL A DR, LUND OBSERVATORY, BOX $\$ 3$, S 221 OO LUND, SWEDEN

Telephone: (46) 46107000

Facsimile: (46)

Telex: 33199 obsnot s

Email: linasu@gemini.ldc.lu.se

Commission(s): $20,22,44$

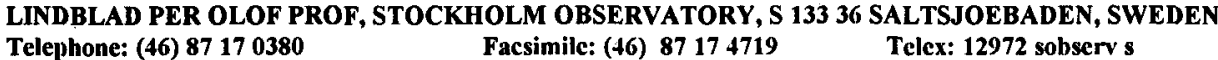

Email: lindblad@astro.su.se

Commission(s): 28,33

LINDE PETER DR, LUND OBSERVATORY, BOX 43, S 22100 LUND, SWEDEN

Telephone: (46) 46104701

Facsimile: (46)

Commission(s): 05,25

Telex: 33199 obsnot

Email: peter@astro.lu.se

LINDEGREN LENNART DR, LUND OBSERVATORY, BOX 43, S 22100 LUND, SWEDEN

Telephone: (46) $46107309 \quad$ Facsimile: (46) $46104614 \quad$ Telex: 33199 obsnot s

Email: $\quad$ Commission(s): 08C

LINDGREN HARRI, LUND OBSERVATORY, BOX 43, S 22100 LUND, SWEDEN

Telephone: (46)

Facsimile: (46)

Telex:

Email: harris@astro.lu.se

Commission(s): 30

LINDSEY CHARLES ALLAN, NTL SOLAR OBSERVATORY, BOX 26732, TUCSON AZ 85726 6732, USA

Telephone: (1)

Facsimile: (1)

Telex:

Email:

Commission(s): 15

LING CHIH-BING DR, INST OF MATHEMATICS, ACADEMIA SINICA, BOX NO 143, TAIPEI, CHINA R Telephone: (886)

Facsimile: (886)

Telex:

Email:

Commission(s):

LING J DR, OBSERVATORIA ASTRONOMICO, RAMON MARIA ALLER, AVD DE LAS CIENCIAS S/N E SANTIAGO DE COMPOSTELA, SPAIN

Telephone: (34)

Facsimile: (34)

Telex:

Email:

Commission(s): 26

LINGENFELTER RICHARD E, CASS, UCSD, C 011, LA JOLLA CA 92093 0216, USA

$\begin{array}{lll}\text { Telephone: (1) } 6194522464 & \text { Facsimile: (1) } & \text { Telex: } 9103371271 \text { sioccan } \\ \text { Email: } & \text { Commission(s): }\end{array}$

LINKE RICHARD ALAN DR, 8 ANDERSON LANE, PRINCETON NJ 08540, USA

$\begin{array}{ll}\text { Telephone: (1) } & \text { Facsimile: (1) } \\ \text { Email: } & \text { Commission(s): } 34,40\end{array}$

LINNELL ALBERT P PROF, 5323 NE 2ND ST, SEATTLE WA 98105, USA

Telephone: (1) Tacsimile: (1) 2065228319 Telex:

Email: linnell@msupa.pa.msu.edu Commission(s): 35,36,\$2

LINSKY JEFFREY L DR, JILA, UNIVERSITY OF COLORADO, BOX $4+0$, BOULDER CO 80309 0440, USA Telephone: (1) $3034927838 \quad$ Facsimile: (1) Telex: $7558+2$ jila

Email:

Commission(s): $12,36 \mathrm{C}, 44$

LINSLEY JOHN, DPT PHYSICS \& ASTRONOMY, UNIVERSITY OF NEW MEXICO, 800 YALE BLVD NE ALBUQUERQUE NM 87131, USA

Telephone: (1) 5052431924

Facsimile: (1)

Telex: 910989

Email:

Commission(s): 44

LIPOVETSKY V A, SPECIAL ASTROPHYSICAL OBS, ACADEMY OF SCIENCES, NIZHNIH ARKHYZ 357147 STAVROPOLSKIJ, RUSSIA

Telephone: (7) 93242

Facsimile: (7)

Telex:

Email:

Commission(s): 28 
LIPPINCOTT SARAH LEE DR, DPT OF ASTRONOMY, SWARTHMORE COLLEGE, SPOUL OBSERVATORY SWARTHMORE PA 19081, USA

Telephone: (1) 2155439058

Facsimile: (1)

Telex:

Email:

Commission(s): $24,26,51$

LIPSCHUTZ MICHAEL E DR, WETHERILL CHEMISTRY BLDG, PURDUE UNIVERSITY

W LAFA YETTE IN 47907, USA

Telephone: (1) 3174945326

Facsimile: (1)

Commission(s): 15

Telex: 272396

Email: bitnet:rnaajuml@purcevm

LIPUNOV V M, STERNBERG STATE ASTR INST, UNIVERSITETSKIJ PROSP 13, 119899 MOSCOW, RUSSIA

Telephone: (7) 0959395006

Facsimile: (7) 0959390126

Telex:

Email: lipunov@sai.msu.su

Commission(s):

LIRITZIS IOANNIS DR, RES CENTER FOR ASTRONOMY, ACADEMY OF ATHENS, 14 ANAGNOSTOPOULOU ST GR 10673 ATHENS, GREECE

Telephone: (30) 13613589

Facsimile: (30) 13631606

Telex:

Email:

Commission(s): 10

LIS DARIUSZ C, CALTECH, MS 320 47, DOWNS LABORATORY PHYSICS, PASADENA CA 91125, USA

Telephone: (1) 818.3956617

Email: dcl@atacos.caltech.cdu Facsimile: (1) $8187968806 \quad$ Telex:

Commission(s): 34,40

LISEAU RENE DR, IAS, CNR, VIA G GALILEI CP 27, I 00044 FRASCATI, ITALY

Telephone: (39) 69423801

Facsimile: (39) $69+168+7$

Telex: $610261 \mathrm{cnr}$ fra $\mathrm{i}$

Email:

Commission(s):

LISI FRANCO DR, OSS ASTROFISICO, DI ARCETRI, LARGO E FERMI 5, I 50125 FIRENZE, ITALY

Telephone: (39) 552752289

Facsimile: (39) 55220039

Telcx: 572268 i

Email: span:38954::lisi

Commission(s):

LISSAUER JACK J DR, DPT OF EARTH \& SPACE SCI, ASTRONOMY PROGRAM, SUNY AT STONY BROOK STONY BROOK NY 117942100 , USA

Telephone: (1) 5166328225

Facsimile: (1)

Telex:

Email:

Commission(s): 07,15,16

LISZT HARVEY STEVEN, NRAO, 520 EDGEMOMT RD, CHARLOTTESVILLE VA 22903, USA

Telephone: (1) 8042960344

Facsimilc: (1)

Telex:

Email:

Commission(s): 34

LTTLE LESLIE T DR, ELECTRONICS LABORATORY, UNIVERSITY OF KENT, CANTERBURY CT2 7NT, UK

Telephone: (44) 122766822

Facsimile: (44)

Telex: 965449 ukclib

Email:

Commission(s): 40

LITTLETON JOHN E, DPT OF PHYSICS, WEST VIRGINIA UNIVERSITY, BOX 6023

MORGANTOWN WV 26506 6023, USA

Telephone: (1) $3042933498 \quad$ Facsimile: (1)

Email:

Commission(s): 35

Telex: 710-921-0309

LITTLE-MARENIN IRENE R DR, WHITIN OBSERVATORY, WELLESLEY COLLEGE, WELLESLEY MA 02181, USA

Telephone: (1) 6172355303

Facsimile: (1)

Telex:

Email: ilittle@lucy.wellesley.edu

Commission(s): 27,29,46

LITVAK MARVIN M DR, TECHNOLOGY RESEARCH ASS, 1525 ESPINOSA CIRCLE

PALOS VERDES EST CA 90274 , USA

Telephone: (1)

Facsimile: (1)

Commission(s):

Telex:

Email:

Commission(s):

LITVINENKO LEONID N DR, INSTITUTE OF RADIO ASTRON, UKRAINIAN ACAD OF SCIENCE KRASNOZNAMENNAYA UL 4, 310002 KHARKOV, UKRAINE

Telephone: (7) 057451009

Facsimile: (7) 057320273

Email:

Commission(s): 40

Telex: 115144 metil su 
LIU BAO-LIN, PURPLE MOUNTAIN OBSERV, CAS, NANJING, CHINA PR

Telephone: (86) 25 42817/46700

Facsimile: (86) 25301459

Telex: $3+1+4$ pmonj cn

Email:

Commission(s): 04

LIU CAIPIN, PURPLE MOUNTAIN OBSERV, CAS, NANJING, CHINA PR

Telephone: (86) 2542817

Facsimile: (86) 25301459

Telex: $341 \$ 4$ pmonj cn

Email:

Commission(s): 36

LIU CI-YUAN DR, SHAANXI OBSERVATORY, CAS, LINTONG XIAN, SHAANXI, CHINA PR

Telephone: (86) 332255

Facsimile: (86) 92373496

Telex: 70121 csao

Email:

Commission(s): 19,41

LIU JINMING, SHANGHAI OBSERVATORY, CAS, 80 NANDAN RD, SHANGHAI 200030, CHINA PR

Telephone: (86) 21386191

Facsimile: (86)

Telex: $3316+$ shao $\mathrm{cn}$

Email:

Commission(s): 05,06,31

LIU LIAO, DPT OF PHYSICS, BEIJING NORMAL UNIVERSITY, BEIJING 100071, CHINA PR

Telephone: (86)

Facsimile: (86)

Telex:

Email:

Commission(s): 47

LIU LIN, DPT OF ASTRONOMY, NANJING UNIVERSITY, NANJING, CHINA PR

Telephone: (86) 25 34651*2882 Facsimile: (86)

Telex: $3+151$ prenu en

Email:

Commission(s):

LIU LIN-ZHONG, PURPLE MOUNTAIN OBSERV, CAS, NANJING, CHINA PR

Telephone: (86) 2546700

Facsimile: (86) 25301459

Telex: $3 \$ 1 \$ 4$ pmonj en

Email:

Commission(s): 15

LIU QINGYAO DR, YUNNAN OBSERVATORY, CAS, BOX 110, KUNMING 72946 YUNNAN, CHINA PR

Telephone: (86) 8712035

Facsimile: (86)

Telex: $6+040$ yuobs $\mathrm{cn}$

Email:

Commission(s): 42

LIU RU-LIANG, PURPLE MOUNTAIN OBSERV, CAS, NANJING, CHINA PR

Telephone: (86) 25 42817/46700

Facsimile: (86) 25301459

Tclex: 34144 pmonj cn

Email:

Commission(s): 28,44

- LIU SOU-YANG DR, COMPUTER SCIENCES CORP, SYSTEM SCIENCES DIV, 8728 COLESVILLE RD

SILVER SPRING MD 20910, USA

Telephone: (1) 3015891545

Facsimile: (1)

Telex:

Email:

Commission(s):

LIU XINPING PROF, INSTITUTE OF MECHANICS, CAS, BEIJING 100080, CHINA PR

Telephone: (86) 1284185

Facsimilc: (86) 12561284

Telex: 222554 mehas cn

Email:

Commission(s): 10

LIU XUEFU, DPT OF ASTRONOMY, BEIJING NORMAL UNIVERSITY, BEIJING 100875, CHINA PR

Telephone: (86) $1656531 * 6285$

Facsimile: (86)

Telex: 8511

Email:

Commission(s): 42

LIU YONG-ZHEN, GRADUATE SCHOOL, UNIV SCIENCE \& TECHNOLOGY, BOX 3908, BEIJING 100039, CHINA PR

Telephone: (86) 1817031

Facsimile: (86)

Commission(s): 28,47

Telex:

Email:

LIU ZONGLI, BEIJING ASTRONOMICAL OBS, CAS, W SUBURB, BEIJING 100080, CHINA PR

Telephone: (86)

Facsimile: (86)

Telex: 22040

Email:

Commission(s): 15,27

LIVINGSTON WILLIAM C, NOAO/NSO, BOX 26732, 950 N CHERRY AVE, TUCSON AZ 85726 6732, USA

Telephone: (1) 5203275511

Facsimile: (1)

Telex: $0666+8+$ aura noan tu

Email:

Conmission(s): 09,12

LIVIO MARIO PROF, DPT OF PHYSICS, IIT, TECHNION CITY, HAIFA 32000, ISRAEL

Telephone: (972) 4293549

Facsimile: (972)

Telex: 46650 tecli i)

Email: phr81ml@technion

Commission(s): 35 
LIVSHITS M A DR, IZMIRAN, ACADEMY OF SCIENCES, $1+2092$ TROITSK, RUSSIA

$\begin{array}{lll}\text { Telephone: (7) } & \text { Facsimile: }(7) & \text { Telex: } \$ 12623 \text { scstp su } \\ \text { Email: } & \text { Commission(s): } 10 & \end{array}$

LIZANO-SOBERON SUSANA DR, INSTITUTO DE ASTRONOMIA, UNAM, APDO POSTAL 70-264 04510 MEXICO DF, MEXICO

Telephone: (52) $5255485305 \quad$ Facsimile: (52) 5255483712 Telex: 01760155 cicme

Email: lizano\%alfa.astroscu.unam.mx Commission(s): 34

LLOYD EVANS THOMAS DR, SAAO, BOX 9, OBSERVATORY 7935, SOUTH AFRICA

Telephone: (27) 021-47-0026 Facsimile: (27) Telex: 5720309 sa

Email:

Commission(s): 37,45

LLOYD HUW, CHEMICAL \& PHYSICAL SCI, LIVERPOOL J M U, BYROM ST, LIVERPOOL L3 3AF, UK Telephone: (44) 1512314103 Facsimile: (4) 1512312337 Telex:

Email: hml@ustaru1.livjm.ac.uk Commission(s): 42

LO KWOK-YUNG DR, DPT OF ASTRONOMY, UNIVERSITY OF ILLINOIS, 1002 WEST GREEN STREET URBANA IL 61801, USA

Telephone: (1) 2173339381

Email: kyl@sgr.astro.uiuc.edu

Facsimile: (1)

Telex:

Commission(s): $28,34,40$

LOCANTHI DOROTHY DAVIS DR, 2180 PINECREST DR, ALTADENA CA 91001, USA

Telephone: (1) 2137970629

Facsimile: (1)

Telex:

Email:

Commission(s): 29

LOCHMAN JAN, ASTRONOMICAL INSTITUTE, CZECH ACADEMY OF SCIENCES, DVORAKOVA 298 CZ 51101 TURNOV, CZECH R

Telephone: (42) 43622913

Facsimile: (42) $\$ 3622913 \quad$ Telex:

Email:

Commission(s): 19

LOCKE JACK L DR, 2150 BRAESIDE AVENUE, OTTAWA ON K1H 7J5, CANADA

Telephone: (1) 6135230812 Facsimile: (1) Telex:

Email: $\quad$ Commission(s): 12,40

LOCKMAN FELIX J, NRAO, BOX 2, GREEN BANK WV 249+4, USA

Telephone: (1)

Facsinilc: (1)

Telex:

Email:

Commission(s): $33,34,40$

LOCKWOOD G WESLEY DR, LOWELL OBSERVATORY, 1400 W MARS HILL RD, BOX 1149

FLAGSTAFF AZ 86001, USA

Telephone: (1) 6077743358

Facsimile: (1)

Telex:

Email:

Commission(s): 16,25,27

LODEN KERSTIN R DR, STOCKHOLM OBSERVATORY, 13336 SALTSJOEBADEN, SWEDEN

Telephone: (46) 87170195

Facsimile: (46) $8717+719 \quad$ Telex: 12972 sobserv s

Email: loden_k@astro.su.se

Commission(s): $26,33,45$

LODEN LARS OLOF PROF, ASTRONOMICAL OBSERVATORY, BOX 515, S 75120 UPPSALA, SWEDEN

Telephone: (46) 18114490

Facsimile: (46)

Commission(s): $26,33,37,51$

Telex: 76024

Email:

LOISEAU NORA DR, INST DE ASTROFISICA, DE CANARIAS, OBS DEL TEIDE, E 38200 LA LAGUNA, SPAIN
Telephone: (34) 22605237
Facsimile: (34) 22605210

Email: nll@iac.dnet.nasa.gov

Commission(s): 40

LOMB NICHOLAS RALPH DR, SYDNEY OBSERVATORY, MUSEUM APPLIED ARTS \& SCI, BOX K3\$6 HAYMARKET NSW 2000, AUSTRALIA

Telephone: (61)

Facsimilc: (61)

Telex:

Email:

Commission(s): 20,46

LONG KNOX S DR, STSCI, HOMEWOOD CAMPUS, 3700 SAN MARTIN DR, BALTIMORE MD 21218, USA

Telcphonc: (1) 3013384862

Facsimile: (1)

Email:

Commission(s): 44

Telex: 9102400225 jhucasmd 
LONGAIR M S PROF, MULLARD RADIO ASTRON OBS, CAVENDISH LABORATORY, MADINGLEY RD CAMBRIDGE CB3 OHE, UK

Telephone: (4) 1223337083

Email: msl(a)uk.ac.cam.phy.ras

Facsimile: $(+4) 122335+599 \quad$ Telex: 81292 cavlab

Commission(s): $40,44,47$

LONGMORE ANDREW J, JOINT ASTRONOMY CENTER, UKIRT, 665 KOMOHANA ST, HILO HI 96720, USA

Telephone: (1) 8089613756

Facsimile: (1)

Telex: 633135

Email:

Commission(s): 27

LONSDALE CAROL J DR, CALTECH, MS 100 22, IPAC, PASADENA CA 91125, USA

Telephone: (1) 8183979529

Facsimile: (1) $818397 \% 600$

Telex: 675429

Email: cjlầipac.caltech.edu

Commission(s): $05,28,47$

LOOSE HANS-HERMANN DR, UNIVERSITAETS STERNWARTE, GOETTINGEN, GEISMARLANDSTR 11

D 37083 GOETTINGEN, GERMANY

Telephone: (49) $551395056 * 953$

Facsimile: (49)

Telex:

Email:

Commission(s): 28

LOPES DALTON DE FARIA DR, OBSERVATORIO NACIONAL, RUA GL J CRISTINO 77, SAO CRISTOVAO 20921 RIO DE JANEIRO TJ, BRAZIL

Telephone: (55) 215807181

Email: dflo(âlncevb.bitnet

Facsimile: (55) 215800332

Telex: 021288

LOPES-GAUTIER ROSALY DR, JPL, MS 183 601, 4800 OAK GROVE DR, PASADENA CA 91109, USA

Telephone: (1) 8183934584

Commission(s): 29

Telex: $7+018 \dashv 3$ jplr

Email:

Facsimile: (1) 8183540966

Commission(s): 15,16,41

LOPEZ CARLOS LIC, OBSERVATORIO ASTRONOMICO, FELIX AGUILAR, AV BENAVIDEZ 8175 OESTE 5407 MARQUESADO (S.J.), ARGENTINA

Telephone: (54) 64231494 Facsimile: (54)

Email:

Commission(s): $24 \mathrm{C}$

Telex:

LOPEZ DE COCA M D P DR, INST ASTROFISICA, DE ANDALUCIA APD 3004, PROFESOR ALBAREDA E 18080 GRANADA, SPAIN

Telephonc: (34) 58121311

Enail: pilarâjiaa.es

Facsimile: (3t) $5811+530$

Telex: 78573 ialage

Commission(s): 27

LOPEZ GARCIA ZULEMA L DR, OBSERVATORIO ASTRONOMICO, FELIX AGUILAR, AV BENAVIDEZ 8175 OESTE, S\$07 MARQUESADO (S.J.), ARGENTINA

Telephone: (54)

Facsimile: (5+)

Email:

Commission(s):

Tclex:

"LOPEZ JOSE A ING, OBSERVATORIO ASTRONOMICO, FELIX AGUILAR, AV BENAVIDEZ 8175 OESTE 5407 MARQUESADO (S.J.), ARGENTINA

Telephone: (54) $64231494 \quad$ Facsimile: (54)

Email:

Commission(s): $18 \mathrm{C}$

Telex: 59100 unsja ar

LOPEZ JOSE ALBERTO DR, INSTITUTO DE ASTRONOMIA, UNAM, APDO POSTAL 877, 22800 ENSENADA B CALIF, MEXICO

Telephonc: (52) 66744580

Facsimile: (52)

Commission(s):

Telex: 56539 ciceme

Email:

- LOPEZ ROSARIO DR, DPT FISICA, UNIVERSIDAD DE BARCELONA, AVD DIAGONAL 647

E O8028 BARCELONA, SPAIN

Telephone: (34) 33307311

Facsimile: $(3+)$

Telex:

Email: bitnct:d3farlho(âcboubo11

Commission(s): 28

LOPEZ-ARROYO M, OBS ASTRONOMICO NCL, ALFONSO XII-5, E 28014 MADRID, SPAIN

Telephone: (34)

Facsimile: (34)

Telex:

Email:

Commission(s): 12

LOPEZ-GARCIA FRANCISCO DR, OBSERVATORIO ASTRONOMICO, FELIX AGUILAR, AV BENAVIDEZ 8175 OESTE5 407 MARQUESADO (S.J.), ARGENTINA

Telephone: (54) 64231494

Facsimile: (54)

Email:

Commission(s):

Telex: 
LOPEZ-GONZALEZ MARIA J DR, INST ASTROFISICA, DE ANDALUCIA APD 3004, PROFESOR ALBAREDA 1 E 18080 GRANADA, SPAIN

Telephone: (34) 58121311

Email: 16488 ::mariajose

Facsimile: (34) 58814530

Commission(s): 21

Telex: 78573 iaag e

LOPEZ-MORENO JOSE JUAN, INST ASTROFISICA, DE ANDALUCLA APD 3004, PROFESOR ALBAREDA 1 E 18080 GRANADA, SPAIN

Telephone: (34) 58121300

Email:

Facsimile: (34)

Telex: 78573 iaag e

Commission(s): 16,21

LOPEZ-PUERTAS MANUEL, INST ASTROFISICA, DE ANDALUCIA APD 3004, PROFESOR ALBAREDA 1 E 18080 GRANADA, SPAIN

Telephone: (34) 58121300

Email:

Facsimile: (34)

Commission(s): 16,21

Telex: 78573 iaag $\mathrm{e}$

LOPEZ-VALVERDE M A DR, INST ASTROFISICA, DE ANDALUCIA APD 3004, PROFESOR ALBAREDA 1 E 18080 GRANADA, SPAIN

Telephone: (34) 58121311

Facsimile: (34) 58814530

Telex: 78573 iaag e

Email: 16488::valverde

Commission(s): 16

LORD STEVEN DONALD DR, MS 100 22, IPAC/CALTECH, PADADENA CA 91125, USA

Telephone: (1)

Facsimile: (1)

Telex:

Email: lord@gal.arc.nasa.gov

Commission(s): 28

LOREN ROBERT BRUCE DR, BOX 2915, SILVER CITY NM 88062, USA

Telephone: (1)

Facsimile: (1)

Commission(s): 34,40

Telex:

Email:

LORENZ HILMAR, ZNTRLINST F ASTROPHYSIK, AKAD WISSENSCHAFTEN DDR, ROSA LUXEMBURG STR 17A D 14473 PO'TSDAM, GERMANY

Telephone: (49)

Email:

Facsimile: (49)

Telex:

Commission(s): 28,40

LORTET MARIE CLAIRE, OBSERVATOIRE DE PARIS, SECTION DE MEUDON, DAPHE

F 92195 MEUDON PPL CDX, FRANCE

Telephone: (33) 145077426

Facsimile: (33)

Telex: 201571

Email:

Commission(s): $05,28,34$

LOSCO LUCETTE DR, FACULTE DES SCIENCES, F 25030 BESANCON CDX, FRANCE

Telephone: (33)

Facsimile: (33)

Telex:

Email:

Commission(s):

LOTOVA N A DR, IZMIRAN, ACADEMY OF SCIENCES, 1+2092 TROITSK, RUSSIA

Telephone: (7)

Facsimile: (7)

Commission(s): 49

Telex:

Email:

LOUCIF MOHAMMED LAKHDAR, INSTITUT D'ASTROPHYSIQUE, 98BIS BD ARAGO, F 75014 PARIS, FRANCE

Telephone: (33) 144328049

Email: mohal@iap.fr

Facsimile: (33) $14+328001$

Telex:

Commission(s):

LOUISE RAYMOND PROF, FACULTE DES SCIENCES, DEPT DE PHYSIOUE, 33 RUE ST-LEU

F 80039 AMIENS, FRANCE

$\begin{array}{ll}\text { Telephonc: (33) } & \text { Facsimile: (33) } \\ \text { Email: } & \text { Commission(s): } 34\end{array}$

LOULERGUE MICHELLE DR, OBSERVATOIRE DE PARIS, SECTION DE MEUDON

F 92195 MEUDON PPL CDX, FRANCE

Telephone: (33) $145077455 \quad$ Facsimile: (33)

Email:

Commission(s): 14

Telex: 270912

LOVAS FRANCIS JOHN DR, NTL BUREAU OF STANDARDS, DIV 545, MOLECULAR SPECTROSCOPIC

WASHINGTON DC 20234, USA

Telephone: (1) 3019212023

Facsimile: (1)

Telex: 898993

Email:

Commission(s): 1t,3t 
LOVAS MIKLOS, KONKOLY OBSERVATORY, THEGE U 13/17, BOX 67, H 1525 BUDAPEST, HUNGARY

Telephone: (36) 1175 5866/175 4122

Facsimile: (36) 11569640

Tclex: 227460 konob

Email:

Commission(s): 20

LOVELACE RICHARD V E DR, CORNELL UNIVERSITY, SPACE SCIENCES BLDG, ITHACA NY 14853, USA

Telephone: (1) 6072563968

Facsimile: (1)

Telex:

Email:

Commission(s): 44

LOVELL SIR BERNARD PROF, NRAL, JODRELL BANK, MACCLESFIELD SK11 9DL, UK

Telephone: (44) 147771321

Email:

Facsimile: ( +4$)$

Telex: 36149

Commission(s): $22,40,4+, 51$

LOW BOON CHYE, HIGH ALTITUDE OBSERVATORY, NCAR, BOX 3000, BOULDER CO 80307 3000, USA

Telephone: (1) 3034971553

Facsimile: (1) 3034971568

Telex: 45694

Email:

Commission(s): 10

LOW FRANK J DR, 4940 CALLE BARRIL, TUCSON AZ 85718, USA

Telephone: (1) 5206212779

Facsimile: (1)

Telex:

Email:

Commission(s): $28,34,45$

LOWE ROBERT P DR, DPT OF PHYSICS, UNIV OF WESTERN ONTARIO, LONDON ON N6A 3K7, CANADA

Telephone: (1) 5196613929

Facsimile: (1) 5196613129

Telex:

Email: lowe@canlon.physics.uwo.ca Commission(s):

LOYOLA PATRICIO DR, DPT DE ASTRONOMIA, UNIVERSIDAD DE CHILE, CASILLA 36 D, SANTIAGO, CHILE Telephone: (56) 22294101 Facsimile: (56)

Telex: $4+0001$

Email:

Commission(s): 08

LOZINSKAYA TAT'YANA A DR, STERNBERG STATE ASTR INST, UNIVERSITETSKIJ PROSP 13 119899 MOSCOW, RUSSIA

Telephone: (7) 951391030

Facsimilc: (7)

Telex:

Email:

Commission(s): $3+\mathrm{C}, 40$

LOZINSKIJ A M DR, INST OF ASTRONOMY, ACADEMY OF SCIENCES, PYATNITSKAYA UL 48 109017 MOSCOW, RUSSIA

Telephone: (7) 952315461

Facsimile: (7)

Telex: $\$ 12623$ sest]) su

Email:

Commission(s): 24

LOZITSKIJ VSEVOLOD, ASTRONOMICAL OBSERVATORY, KIEV UNIVERSITY, OBSERVATORNA STR 3

254053 KIEV 53, UKRAINE

Telephone: (7) 0442163910

Email: aoku@gluk.apc.org
Facsimile: (7)

Commission(s): 10
Telex:

LU BEN-KUI, PURPLE MOUNTAIN OBSERV, CAS, NANJING, CHINA PR

Telephone: (86) 2532893

Email:

Facsimile: (86) 25301459

Commission(s): 07,31
Telex: 34144 pmonj en

LU CHUN-LIN, PURPLE MOUNTAIN OBSERV, CAS, NANJING, CHINA PR

Telephone: (86) 2542700

Email:
Commission(s): 08
Telex: $3+144$ pmonj cn

LU JU FU DR, ASTROPHYSICS DIVISION, UNIV SCIENCE \& TECHNOLOGY, HEIFI 230026 ANHUI, CHINA PR

Telephone: (86) 551331134 *527

Facsimile: (86) 551331760

Telex: 90028 ustc cn

Email:

Commission(s): 4

LU PHILLIP K DR, DPT PHYSICS \& ASTRONOMY, W CONNECTICUT STATE UNIV, 181 WHITE ST, DANBURY CT 068107859 , USA

Telephone: (1) 2037974218

Facsimile: (1)

Telex:

Email:

Commission(s): $24,33,37$

LU RUWEI DR, YUNNAN OBERVATORY, CAS, BOX 110, KUNMING 72946 YUNNAN, CHINA PR

Telephone: (86) 8712035

Facsimile: (86) 87171845

Telex: $6+040$ yuobs

Email:

Commission(s): 09 
LUKS THOMAS, ASTRONOMISCHES INSTITUTE, RUHR UNIVERSITAET BOCHUM, D H780 BOCHUM, GERMANY

Telephone: (49) $2347005802 / 6660$

Facsimile: (49) $23+709+169$

Telex: $1723+356$

Email: astrorub@ruba.rz.ruhr-uni-boch Commission(s): 40

LUMINET JEAN-PIERRE, OBSERVATOIRE DE PARIS, SECTION DE MEUDON, F 92195 MEUDON PPL CDX, FRANCE

Telephone: (33) 145077423

Facsimile: (33)

Telex: 201571

Email:

Commission(s): $28,44,47$

LUMME KARI A DR, OBS \& ASTROPHYSICS LAB, UNIVERSITY OF HELSINKI, TAEHTITORNINMAKI SF 00130 HELSINKI 13, FINLAND

Telephone: (358) 1912910

Facsimile: (358) 1912952 Telex:

Email:

Commission(s): $15,16,21$

LUNA HOMERO G DR, IAR, CC 5, 1894 VILLA ELISA (BS AS), ARGENTINA

Telephone: (54) 2143793

Facsimilc: (54)

Tclex:

Email:

Commission(s): 25

LUND NIELS, DANISH SPACE RESEARCH INS, LUNDTOFTEVEJ 7, DK 2800 LYNGBY, DENMARK

Telcphone: (45) 42882277

Facsimilc: (45)

Tclex: 37198

Email:

Commission(s):

LUNDQUIST CHARLES A DR, RESEARCH INSTITUTE, UNIVERSITY OF ALABAMA, BOX 200

HUNTSVILLE AL 35899, USA

Telephone: (1) 2058956100

Facsimile: (1)

Telex:

Email:

Commission(s): 07

LUNDQVIST PETER, STOCKHOLM OBSERVATORY, S 13336 SALTSJOEBADEN, SWEDEN

Telephone: (46) 8164489

Facsimile: $(46) 8717 \$ 719 \quad$ Telex:

Email: peterasastro.su.se

Commission(s):

LUNDSTEDT HENRIK DR, LUND OBSERVATORY, BOX 43, S 22100 LUND, SWEDEN

Telephone: (46) 46107294

Facsimile: $(46)+610+614$

Telex: 33199 obsnot s

Email: henrik@astro.lu.se

Commission(s): 10,49

LUNDSTROM INGEMAR DR, LUND OBSERVATORY, BOX $\$ 3, S 22100$ LUND, SWEDEN

Telephone: (46) 46107300

Facsimile: $(46)$

Commission(s): 29

Telex: 33199

Email:

LUNEL MADELEINE DR, OBSERVATOIRE DE LYON, AVE CHARLES ANDRE, F 69561 S GENIS LAVAL CDX, FRANC

Telephone: (33) 78560705

Facsimile: (33) 72399791

Telex: $310-926$

Email:

Commission(s): 33

LUNGU NICOLAIE DR, INSTITUTE POLITEHNIC, CATEDRA DE MATEMATICA, STR EMIL ISAC 15

R 3400 CLUJ NAPOCA, RUMANIA

Telephonc: (40) 64117229

Facsimile: (f(0)

Telex:

Email:

Commission(s):

LUO BAO-RONG, YUNNAN OBSERVATORY, CAS, BOX 110, KUNMING 729+6 YUNNAN, CHINA PR

Telephone: (86) 8712035

Facsimile: (86)

Telex:

Email:

Commission(s): 10

LUO DINGCHANG, BEIJING ASTRONOMICAL OBS, CAS, W SUBURB, BEIJING 100080, CHINA PR

Telephone: (86) 1275580

Facsimile: (86)

Telex: 22040

Email:

Commission(s): 31

LUO DING-JIANG, BEIJING ASTRONOMICAL OBS, CAS, W SUBURB, BEIJING 100080, CHINA PR

Telephone: (86) 1281698

Facsimile: $(86)$

Telex: 22040 bao ascn

Email:

Commission(s): 08,19

LUO SHI-FANG, SHANGHAI OBSERVATORY, CAS, 80 NANDAN RD, SHANGHAI 200030, CHINA PR

Telephone: (86) 21386191

Facsimile: (86)

Telex: $3316+$ shao cn

Email:

Commission(s): 19,31 
LUO XIANHAN, DPT OF GEOPHYSICS, BEIJING UNIVERSITY, BEIJING 100071, CHINA PR

Telephone: (86) 122239

Facsimile: (86)

Telex:

Email:

Commission(s): 10,40

LUPISHKO DMITRIJ F, ASTRONOMICAL OBSERVATORY, KHARKOV UNIVERSITY, SUMSKAYA UL 35

310022 KHARKOV, UKRAINE

Telephone: (7) $057432428 \quad$ Facsimile: (7)

Email:

Commission(s): 15

Telex: 125531 icar

LUSTIG GUENTER DR, INSTITUT FUER ASTRONOMIE, KARL-FRANZENS-UNIVERSITAT, UNIVERSITAETSPLATZ 5 A 8010 GRAZ, AUSTRIA

Telephone: (43) 3163805272

Facsimile: (43)

Commission(s): 10,12

Telex: 0311662 ubgrz

Email:

LUTTERMOSER DONALD DR, IUE OBSERVATORY, CODE 684.9 UNIVERSITY, NASA GSFC GREENBELT MD 20771, USA

Telephone: (1)

Facsimile: (1)

Telex:

Email:

Commission(s): 36

LUTZ BARRY L DR, DPT PHYSICS \& ASTRONOMY, NORTHERN ARIZONA UNIV, BOX 6010

FLAGSTAFF AZ 86011 6010, USA

Telephone: (1)

Facsimile: (1)

Telex:

Email:

Commission(s): $14,15,16$

LUTZ JULIE H DR, PROGRAM IN ASTRONOMY, WASHINGTON STATE UNIV, PULLMAN WA 99164 2930, USA

Telephone: (1) 5093353136

Facsimile: (1)

Commission(s): 45

Telex: \$107741091 wsuoippma

Email:

LUTZ THOMAS E DR, PROGRAM IN ASTRONOMY, WASHINGTON STATE UNIV, PULLMAN WA 99164 2930, USA

Telephone: (1) 5093353141

Facsimile: (1)

Telex: 5107741091 wsuoippma

Email:

Commission(s): 24

LUYTEN WILLEM J PROF, SPACE SCIENCE CENTER, UNIVERSITY OF MINNESOTA, 100 UNION ST SE MINNEAPOLIS MN 55455, USA

Telephonc: (1) 6123733366

Facsimilc: (1)

Telex:

Email:

Commission(s): $24,26,33$

LYNAS-GRAY ANTHONY E, DPT PHYSICS \& ASTRONOMY, UNIVERSITY COLLEGE LONDON, GOWER ST LONDON WC1E GBT, UK

Telcphone: (44)

Facsimile: (4)

Telex:

Email:

Commission(s):

LYNCH DAVID K, AEROSPACE CORPORATION, MS M2 226, BOX 92957, LOS ANGELES CA 90009, USA

Telephone: (1) 2136486686

Facsimile: (1)

Commission(s): 09

Telex: 664460

Email:

LYNDEN-BELL DONALD PROF, INSTITUTE OF ASTRONOMY, THE OBSERVATORIES, MADINGLEY RD CAMBRIDGE CB3 OHA, UK

Telephone: (44) 1223337548

Email:

Facsimile: (4t) 1223337523

Telex: $\mathbf{8 1 7 2 9 7}$ astron $\mathbf{g}$

LYNDS BEVERLY T DR, KITT PEAK NTL OBS, BOX 26732, 950 N CHERRY AVE, TUCSON AZ 85726 6732, USA

Telephone: (1) 5203259396

Email:

Facsimile: (1)

Telex: 0666-484 aura noao

Commission(s): 28,3+

LYNDS ROGER C DR, KITT PEAK NTL OBS, BOX 26732,950 N CHERRY AVE, TUCSON AZ 85726 6732, USA

Telephone: (1) 5203275511

Facsimile: (1)

Telex:

Email:

Commission(s): 28

LYNE ANDREW G DR, NRAL, JODRELL BANK, MACCLESFIELD SK11 9DL, UK

Telephone: (44) 147771321

Facsimile: (4)

Telex: 36149

Email:

Commission(s): 40 
LYTTLETON RAYMOND A PROF, INSTITUTE OF ASTRONOMY, THE OBSERVATORIES, MADINGLEY RD CAMBRIDGE CB3 OHA, UK

Telephone: (44) 1223337548

Email:

Facsimile: (44) $1223337523 \quad$ Telex: 817297 astron g

Commission(s): 15

LYUBIMKOV LEONID S DR, CRIMEAN ASTROPHYS OBS, UKRAINIAN ACAD OF SCIENCE, NAUCHNY 334413 CRIMEA, UKRAINE

Telephone: (7) 432945

Facsimile: (7)

Telex:

Email:

Commission(s): 36

LYUTY VICTOR M DR, CRIMEAN STATION OF, STERNBERG INSTITUTE, NAUCHNY, 33 413 CRIMEA, UKRAINE

Telephone: (7) $432945 \quad$ Facsimile: (7)

Email: $\quad$ Commission(s): 42

Telex:

MA ER, BEIJING ASTRONOMICAL OBS, CAS, W SUBURB, BEIJING 100080, CHINA PR

Telephone: (86) 1281698

Facsimile: (86)

Telex: 22040 baoas en

Email:

Commission(s): 28

MA XING-YUAN, DPT OF GEOGRAPHY, BEIJING TEACHERS COLLEGE, BALIZHUANG, BEIJING, CHINA PR

Telephone: (86)

Facsimile: (86)

Telex:

Email:

Commission(s): 46

MA YU-QIAN, INSTITUTE OF HIGH ENERGY, PHYSICS, BOX 918 3, BEIJING, CHINA PR

Telcphone: (86) $1812971 * 464$

Facsimile: (86)

Commission(s): 44

Telex: 22082 ihep en

Email:

MACALPINE GORDON M, DPT OF ASTRONOMY, UNIVERSITY OF MICHIGAN, DENNISON BLDG

ANN ARBOR MI 481091090 , USA

Telephone: (1) 3137643433

Facsimile: (1)

Telex: 810-223-6056

Email:

Commission(s): 28

MACCACARO TOMMASO DR, CENTER FOR ASTROPHYSICS, HCO/SAO, GH GARDEN ST CAMBRIDGE MA 02138, USA

Telcphone: (1) 6174957253

Facsimile: (1)

Telex: 921428 satellite cam

Email:

Commission(s): 44

MACCAGNI DARIO, IST DI FISICA COSMICA, CNR, VIA BASSINI 15, I 20133 MILANO, ITALY

Telephone: (39) $2298237 \quad$ Facsimile: (39)

Email:

Commission(s): 4

Telex: 313839 muacnr i

MACCALLUM MALCOLM A H, SCHOOL OF MATHEMATICAL SC, QUEEN MARY/WESTFIELD COLL MILE END RD, LONDON E1 4NS, UK

Telephone: (44) $1719804811 \quad$ Facsimile: $(4+)$

Email:

Commission(s): 47

Telex: 893750 qmcuol

MACCHETTO FERDINANDO DR, STSCI, HOMEWOOD CAMPUS, 3700 SAN MARTIN DR, BALTIMORE MD 21218, USA

Telephone: (1) $3013384790 \quad$ Facsimilc: (1)

Email: $\quad$ Commission(s): 28,40,44

Telex: 6849101

MACCONNELL DARRELL J DR, STSCI/CSC, HOMEWOOD CAMPUS, 3700 SAN MARTIN DR, BALTIMORE MD 21218, USA

Telephone: (1) 4103384800

Facsimile: (1) $\$ 10338+767$

Telex:

Email: stscic::macconnell

Commission(s): $24,33,45 C$

MACDONALD GEOFFREY H DR, ELECTRONICS LABORATORY, UNIVERSITY OF KENT CANTERBURY CT2 7NT, UK

Telephone: (44) $122766822 * 258$

Email:

Facsimile: (4-4)

Telex: $965+49$ ukclib

Commission(s): 40

MACDONALD JAMES, DPT OF PHYSICS, UNIVERSITY OF DELAWARE, NEWARK DE 19716, USA

Telephone: (1) 3024512661

Facsimile: (1)

Telex:

Email:

Commission(s): 40,42

MACERONI CARLA, OAR, VIA DELL'OSSERVATORIO 2, I 00040 MONTEPORZIO C, ITALY

Telephone: (39) 9448028

Facsimile: (39) $9+472+3$

Telex:

Email: maceroni(a)astrmp.astro.it

Commission(s): 42 
MACGILLIVRAY HARVEY T DR, ROYAL OBSERVATORY, BLACKFORD HILL, EDINBURGH EH9 3HJ, UK Telephone: (44) 1316673321 Facsimilc: (4) Email: Commission(s): Tclex: 72383 rocdin $\mathrm{g}$

MACHADO LUIZ E DA SILVA, UNIV FED RIO DE JANEIRO, AV SERNAMBETIBA 3300 BL7 22630 BARRA DA TUUCA, 20080 RIO DE JANEIRO RJ, BRAZIL

Telephone: (55) 213992589

Facsimilc: (55)

Telex:

Email:

Commission(s): 20,24

MACHADO MARCOS, CNIE, AVENIDA MITRE 3100, 1663 SAN MIGUEL (BS AS), ARGENTINA

Telephone: (54) 16648371

Facsimile: (5t)

Telex: 17511 lanba ar

Email:

Commission(s): 10

MACHALSKI JERZY DR, ASTRONOMICAL OBSERVATORY, JAGIELLONIAN UNIVERSITY

UL MAZOWIECKA 36/33, PL 30019 KRAKOW, POLAND

Telephone: (48) Facsimile: (48)

Email:

Commission(s): 40

Telex: 0322297 uj pl

MACIEJEWSKI ANDRZEJ J DR, INSTITUTE OF ASTRONOMY, N COPERNICUS UNIVERSITY, UL CHOPINA $12 / 18$ PL 87100 TORUN, POLAND

Telephone: (48) $26018 * 53$

Email: macicjka@pltumk11

\author{
Facsimilc: (48) \\ Telex: $55223+$ astr $p$ I
}

Commission(s): 07

MACIEL WALTER J DR, IAG, UNIVERSIDADE DE SAO PAULO, CP 9638, 01065 SAO PAULO SP, BRAZIL

Telephone: (55) 115778599

Email:

Facsimile: (55) 112763848 Telex: 1156735 iagm br

Commission(s): 34,46

MACK PETER DR, ARIZONA GUEST OBSERVATORY, $4+1+$ W PLANTATION STR, TUCSON AZ 85741 4034, USA Telephone: (1) 5205790698

Facsimile: (1)

Telex:

Email:

Commission(s): 09

MACKAY CRAIG D DR, INSTITUTE OF ASTRONOMY, THE OBSERVATORIES, MADINGLEY RD

CAMBRIDGE CB3 OHA, UK

Telephone: (44) 1223337548

Email:

Facsimile: (4t) $1223337523 \quad$ Telex: 817297 astron $g$

Commission(s):

MACKINNON ALEXANDER L, DPT OF ASTRONOMY, UNIVERSITY OF GLASGOW, GLASGOW G12 80W, UK

Telephone: (44) 1413398855

Facsimile: (44)

Commission(s): 10

Telex: 777070 unigla

Email:

MACLEOD JOHN M DR, HERZBERG INST ASTROPHYS, NTL RESEARCH COUNCIL, 100 SUSSEX DR OTTAWA ON K1A OR6, CANADA

Telephone: (1) 6139936060

Facsimile: (1) 6139526602

Telex: 0533715

Email;

Commission(s): $3 \downarrow,+0$

MACQUEEN ROBERT M DR, DPT OF PHYSICS, RHODES COLLEGE, 2000 N PARKWAY, MEMPHIS TN 38112, USA Telephone: (1) 9017263000

Facsimile: (1)

Tclex:

Email:

Commission(s): 10,49

MACRAE DONALD A PROF, DAVID DUNLAP OBSERVATORY, UNIVERSITY OF TORONTO, BOX 360 RICHMOND HILL ON LAC \&YG, CANADA

Telephone: (1) 4168849562

Facsimile: (1) $\$ 169783921$

Telex: 06986766

Email: macrae@vela.astro.utoronto.ca

Commission(s): $33,38,40$

MACRIS CONSTANTIN J PROF, RES CENTER FOR ASTRONOMY, ACADEMY OF ATHENS

14 ANAGNOSTOPOULOU ST, GR 10673 ATHENS, GREECE

Telephone: (30) $13613589 \quad$ Facsimile: (30)

Telex:

Email:

Commission(s): 10

MACY WILLIAM WRAY DR, 151 MELVILLE AVE, PALO ALTO CA 94304, USA

Telephone: (1)

Facsimile: (1)

Commission(s):

Telex:

Email: 
MADDISON RONALD CH DR, UNIVERSITY OF KEELE, 2 CHURCH PLANTATION, KEELE PARK, KEELE STAFFS, UK

Telephone: (44) 1782621111

Email:

\author{
Facsimile: (44) \\ Commission(s): 46
}

Telex:

MADDOX STEPHEN DR, ROYAL GREENWICH OBS, MADINGLEY RD, CAMBRIDGE CB3 OEZ, UK

Telephone: (44)

Facsimile: (44)

Telex:

Commission(s): 47

MADEJ JERZY, ASTRONOMICAL OBSERVATORY, WARSAW UNIVERSITY, AL UJAZDOWSKIE 4 PL 00478 WARSAW, POLAND

Telephone: (48) 4822415648

Email: jm@alkor.astrouw.edu.pl

Facsimile: (48) $4822294967 \quad$ Telex:

Commission(s): 36

MADORE BARRY FRANCIS DR, CALTECH, NASA/IPAC, MS 100-22, PASADENA CA 91125, USA

Telephone: (1) 8183979512

Facsimile: (1) 8183979600

Telex:

Email: barry@ipac.caltech.edu

Commission(s): 27,28

MADSEN JES, INST OF PHYSICS \& ASTRON, UNIVERSITY OF AARHUS, NY MUNKEGADE DK 8000 AARHUS C, DENMARK

Telephone: (45) 86128899

Facsimile: (45) 86202711

Telex: 64767 aausci dk

Email:

Commission(s):

MAEDA KEI-ICHI DR, DPT OF PHYSICS, WASEDA UNIVERSITY, OKUBO 3-4-1 SHINJUKU-KU, TOKYO 160, JAPAN

Telephone: (81) 32034141

Facsimile: (81)

Commission(s): 47

Telex: 2323280 waseda $j$

Email: bitnet:macda@jpnwas00

MAEDA KOITIRO, DPT OF PHYSICS, HYOGO COLLEGE OF MEDICINE, NISHINOMIYA, HYOGO 663, JAPAN

Telephone: (81) 798456111

Facsimile: (81)

Telex:

Email:

Commission(s):

MAEDER ANDRE PROF, OBSERVATOIRE DE GENEVE, CHEMIN DES MAILLETTES 51

CH 1290 SAUVERNY, SWITZERLAND

Telephone: (41) 227552611

Facsimile: (\$1) 227553983

Telex: 419209 obs ch

Email: macder(a)cgeuge11

Commission(s): $27,35 \mathrm{C}, 37$

MAEHARA HIDEO DR, OKAYAMA ASTROPHYSICAL OBS, NAOJ, KAMOGATA ASAKUCHI

OKAYAMA 719 02, JAPAN

Telephone: (81) 865442155

Facsimile: (81) $865442360 \quad$ Telex:

Email: machara@kibi.oao.nao.ac.jp

Commission(s): $45 \mathrm{C}$

MAETZLER CHRISTIAN DR, PHYSIKALISCHES INSTITUT, UNIVERSITAET BERN, SIDLERSTRASSE 5, CH 3012 BERN, SWITZERLAND

Telephone: (41) $31654589 \quad$ Facsimile: (41)

Email:

Commission(s):

Telex: 32320 phybe ch

MAFFEI PAOLO PROF, CATTEDRA DI ASTROFISCA, UNIVERSITA DI PERUGIA, VLA DELL'ELCE DI SOTTO I 06100 PERUGIA, ITALY

Telephone: (39) 7545647

Facsimile: (39)

Telex:

Email:

Commission(s): 27,51

MAGAIN PIERRE DR, INSTITUT D'ASTROPHYSIQUE, UNIVERSITE DE LIEGE, AVE COINTE 5 B 4000 COINTE-LIEGE, BELGIUM

Telephone: (32) 41529980

Facsimile: (32) 41527474

Telex:

Email:

Commission(s): 29

MAGAKIAN TIGRAN Y DR, BYURAKAN ASTROPHYSICAL, OBSERVATORY, 378433 BYURAKAN, ARMENIA

Telephone: (7) 8852284142

Facsimile: (7) 8852523640

Telex: 411576 ascon su

Email:

Commission(s):

MAGALHAES ANTONIO A S ENG, OBSERVATORIO ASTRONOMICO, UNIVERSIDADE DO PORTO MONTE DA VIRGEM, 4400 VILA NOVA GAIA, PORTUGAL

Telephone: (351) 115778599

Facsimile: (351) 112763848

Telex:

Email:

Commission(s): 
MAGALHAES ANTONIO MARIO, IAG, UNIVERSIDADE DE SAO PAULO, CP 9638, 01065970 SAO PAULO SP, BRAZIL Telephone: (55) 115778599 Facsimile: (55) 115763848 Telex:

Email: magalhacs@vax.iagusp.usp.br Commission(s):

MAGAZZU ANTONIO DR, OSS ASTROFISICO, CITTA UNIVERSITARIA, VIA A DORIA 6, I 9S125 CATANIA, ITALY Telephone: (39) 95330533 Facsimile: (39) 95330592 Telex: 970359 astret $i$

Email: astrct::antonio Commission(s): 29

MAGGIO ANTONIO DR, OSS ASTRONOMICO, UNIVERSITA DI PALERNO, PALAZZO DEI NORMANNI
$\begin{array}{lll}\text { I } 90134 \text { PALERMO, ITALY } & & \\ \text { Telephone: (39) } 916570451 & \text { Facsimile: (39) } 9148890 & \text { Telex: } 910402 \text { astroj i } \\ \text { Email: astropa(9)ipacuc.bitnet } & \text { Commission(s): } 44 & \end{array}$

MAGNAN CHRISTIAN DR, GRAAL CC 72, USTL II, PLACE EUGENE BATAILLON F 34095 MONTPELLIER CDX 5, FRANCE
Telephone: (33) 67143902
Facsimile: (33)
Telex:
Email:
Commission(s):

MAGNANI LORIS ALBERTO DR, DPT PHYSICS \& ASTRONOMY, UNIVERSITY OF GEORGIA, ATHENS GA 30602, US Telephone: (1) 4045422876 Facsimile: (1) $4045+22492$ Telex: 414190

Email: loris@jove.physast.uga.edu

Commission(s):

MAGNARADZE NINA G DR, DPT OF ASTRONOMY, TBILISI UNIVERSITY, 3800 43 TBILISI, GEORGIA

Telephone: (7)

Facsimile: (7)

Telex:

Email:

Commission(s): 07

MAGNI GIANFRANCO, IAS, VIA DELL'UNIVERSITA 11, I 00185 ROMA, ITALY

Telephone: (39) Facsimile: (39) Telex:

Email:

Commission(s):

MAGUN ANDREAS DR, PHYSIKALISCHES INSTITUT, UNIVERSITAET BERN, SIDLERSTRASSE 5

CH 3012 BERN, SWITZERLAND

Telephone: (41) 31658914

Facsimile: ( $\$ 1)$

Telex:

Email:

Commission(s):

MAHAT ROSLI H DR, DPT OF ASTRONOMY, UNIVERSITY OF MALAYA, 59100 KUALA LUMPUR, MALAYSIA

Telephone: (60)

Facsimile: (60)

Telex:

Email:

Commission(s):

MAHESWARAN MURUGESAPILLAI, INST OF FUNDAMENT STUDIES, 380/72 BAUDDHALOKA, MAWATHA COLOMBO 7, SRI LANKA

Telephone: (94) 1-597538

Facsimile: (94)

Commission(s): 35

Telex: 21700 ifs ce

Email:

MAHMOUD FAROUK M A B DR, HELWAN OBSERVATORY, HELWAN, EGYPT

Telephone: (20) 78 0645/2683 Facsimile: (20) Telex:

Email:

Commission(s): 27

MAHRA H S DR, UTTAR PRADESH STATE, OBSERVATORY, PO MANORA PEAK 263 129, NAINITAL 263 129, INDIA Telephone: (91) 5942 2136/2583

Facsimile: (91)

Telex:

Email:

Commission(s): $09,16,20,27,50$

MAHTESSIAN ABRAHAM, BYURAKAN OBSERVATORY, 378433 BYURAKAN, ARMENIA

Telephone: (7) $8852283453 \quad$ Facsimile: (7) 8852523640 Telex: 243344 orion su

Email:

Commission(s): 28

MAIA MARCIO A G, OBSERVATORIO NACIONAL, RUA GL J CRISTINO 77, 20921400 RIO DE JANEIRO, BRAZIL Telephone: (55) 215896504 Facsimile: (55) $215800332 \quad$ Telex:

Email: maia@on.br

Commission(s):

MAIHARA TOSHINORI DR, DPT OF PHYSICS, KYOTO UNIVERSITY, KITASHIRAKAWA SAKYO KU

KYOTO 606, JAPAN

Telephone: (81) 757512111

Facsimile: (81)

Commission(s): 21,34

Telex: $5+22693$ libkyu j

Email: 
M 680

MAILLARD JEAN-PIERRE DR, INSTITUT D'ASTROPHYSIQUE, 98BIS BD ARAGO, F 75014 PARIS, FRANCE

Telephone: (33) 144328139

Facsimile: (33) 144328001

Telex:

Email:

Commission(s): $09,14,29$

MAITZEN HANS M DR, INSTITUT FUER ASTRONOMIE, UNIVERSITAET WIEN, TUERKENSCHANZSTR 17 A 1180 WIEN, AUSTRIA

Telephone: (43) 134536094

Facsimile: (43)

Commission(s): $\mathbf{2 5 , 2 9}$

Telex: 116222 physi a

Email:

MAJID ABDUL BIN A H DR, JABATAN UKUR DAN PEMETAAN, JALAN SEMARAK 50578 KUALA LUMPUR, MALAYSIA

Telephone: (60) 32926327

Facsimile: (60)

Telex:

Email:

Commission(s): 04

MAJOR JOHN DR, DPT OF PHYSICS, UNIVERSITY OF DURHAM, SOUTH RD, DURHAM DH1 3LE, UK

Telephone: (44) 1913742111

Facsimile: (4)

Telex:

Email:

Commission(s): 09

MAKARENKO EKATERINA N DR, ASTRONOMICAL OBSERVATORY, ODESSA STATE UNIVERSITY

SHEVCHENKO PARK, 270014 ODESSA, UKRAINE

Telephone: (7)

Facsimile: (7)

Telex:

Email:

Commission(s): 27

MAKAROV VALENTINE I, KISLOVODSK STATION OF THE, PULKOVO OBSERVATORY

357741 KISLOVODSK, RUSSIA

Telephone: (7)

Facsimile: (7)

Telex:

Email:

Commission(s): 10,12C

MAKAROV VALERI, UNIVERSITY OBSERVATORY, OESTER VOLDGADE 3, DK 1350 COPENHAGEN K, DENMARK

Telephone: (45) 35323966

Facsimile: (45) 35323989

Telex:

Email: makarov@astro.ku.dk

Commission(s): 4

MAKAROVA ELENA A DR, STERNBERG STATE ASTR INST, $11723+$ MOSCOW, RUSSIA

Telephone: (7) 951391973

Facsimile: (7)

Commission(s): 12

Telex: 113037 japet

Email:

MAKINO FUMIYOSHI DR, ISAS, 3-1-1 YOSHINODAI, SAGAMIHARA, KANAGAWA 229, JAPAN
Telephone: (81) 81427513911
Facsimile: (81) 81427594253

Email:

Commission(s):

MAKINO JUNICHIRO, DPT OF INFO SCI \& GRA, UNIVERSITY OF TOKYO, MEGURO KU, TOKYO 153, JAPAN

Telephone: (81) 334653925

Facsimile: (81) $334653925 \quad$ Telex:

Email: makino@kyohou.c.u-tokyo.ac.jp) Commission(s): 37

MAKISHIMA KAZUO, INST SPACE \& ASTRON SCI, UNIVERSITY OF TOKYO, MEGURO KU, TOKYO 153, JAPAN

Telephone: (81) $34671111 * 303 \quad$ Facsimile: $(81)$

Email:

Commission(s):

Telex: $3+757$ isastro

MAKITA MITSUGU DR, KWASAN \& HIDA OBS, KYOTO UNIVERSITY, YAMASHINA, KYOTO 607, JAPAN

Telephone: (81) 755811235

Facsimile: (81)

Commission(s): 10,12

Telex:

Email:

MALACARA DANIEL, CENTRO DE INVESTIGACIONES, EN OPTICA, APDO POSTAL 948, 37000 LEON GTD, MEXICO

Telephone: (52) 75823

Facsimile: (52)

Tclex:

Email:

Commission(s):

MALAGNINI MARIA LUCIA, OAT, BOX SUCC TRIESTE 5, VIA TIEPOLO 11, I 34131 TRIESTE, ITALY

Telephone: (39) $40793921 \quad$ Facsimile: (39)

Telex: 461137 oat $i$

Email:

Commission(s):

MALAISE DANIEL J DR, INSTITUT D'ASTROPHYSIQUE, UNIVERSITE DE LIEGE, AVE COINTE 5 B 4000 COINTE-LIEGE, BELGIUM

Telephone: (32) 41529980

Facsimile: (32) $41527+74$

Telex:

Email:

Commission(s): 15,44 
MALAKPUR IRADJ DR, INSTITUTE OF GEOPHYSICS, UNIVERSITY OF TEHRAN, KARGAR SHOMALI TEHRAN 1 4394 , IRAN

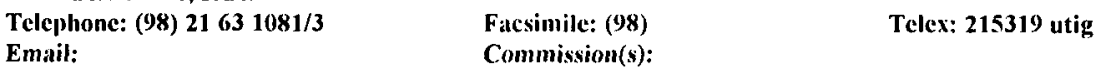

MALARODA STELLA M DR, COMPLEJO ASTRONOMICO, EL LEONCITO, CC 467,5400 SAN JUAN, ARGENTINA

Telephone: $(54) 6+225718$

Facsimile: (54)

Telex: $5913+$ entop ar

Email:

Commission(s): 29,45

MALASAN HAKIM LUTHFI, BOSSCHA OBSERVATORY, ITB, LEMBANG, BANDUNG 40391, INDONESIA

Telephone: (62) 22286001

Facsimile: (62) 22287289 Telex:

Email:

Commission(s): 42

MALAWI ABDULRAHMAN, ASTRONOMY DPT, BOX 9128, KAAU, JEDDAH 21+13, SAUDI ARABIA

Telephone: (966) 26870571

Facsimile: (966) $26870571 \quad$ Telex:

Email: scf3010/äsakatau(33

Commission(s):

MALHERBE JEAN MARIE DR, OBSERVATOIRE DE PARIS, SECTION DE MEUDON, DASOP F 92195 MEUDON PPL CDX, FRANCE

Telephone: (33) 145077629

Email: malherrbe(äobspm.fi

Facsimile: (33) 1 t5 $077634 \quad$ Telex:

Commission(s): 11

MALIN DAVID F DR, AAO, BOX 296, EPPING NSW 2121, AUSTRALIA

Telephonc: (61) $23724800 \quad$ Facsimile: (61) $23724860 \quad$ Telex:

Email: Jfm (a) aaocpp.aao.gov:au Commission(s): 09VP

MALIN STUART, NATIONAL MARITIME MUSEUM, GREENWICH, LONDON SE11 9NF, UK

Telephone: (+H) 1818581167 Facsimile: (+4) Telex:

Email: $\quad$ Commission(s): +1

MALINA ROGER FRANK DR, CTR FOR EUV ASTROPHYSICS, UNIVERSITY OF CALIFORNIA, 2150 KITTREDGE ST BERKELEY CA 94720, USA

Telephone: (1) 5106+35636 Facsimile: (1) $5116435660 \quad$ Telex:

Email: rmalina ässl.berkeley.edı Commission(s):

MALITSON HARRIET H MS, 13315 MAGELLAN AVE, ROCKVILLE MD 20853, USA

Telephone: (1) $3019+60 \$ 96 \quad$ Facsimile: (1) Telex:

Email:

Commission(s): 10,44

MALKAMAEKI LAURI J DR, ELSON RESEARCH INC, BOX 6356, INGWOOD TX 77325 6356, USA

Telephone: (1)

Facsimile: (1)

Telex:

Email:

Commission(s): (19)

MALKAN MATTIEW ARNOLD DR, DPT OF ASTRONOMY, UNIVERSITY OF CALIFORNIA

LOS ANGELES CA 910124, USA

Telephone: (1) 2138253404

Facsimile: (1)

Telex:

Email: malkancastro.ucla.edu

Commision(s); tt

MALKOV OLEG YU, INSTITUTE OF ASTRONOMY, ACADEMY OF SCIENCES, 48 PYATNITSKAYA ST MOSCOW 109017, RUSSIA

Telephone: (7) 1952331702

Email: omalkova inasan.rssi.ru

Fatsimile: (7) 10952302081

Telex: +11576 iscon su

MALLIA EDWARD A DR, DPT OF ASTROPIYSICS, UNIVERSITY OF OXFORD, SOUTH PARKS RD OXFORD OX1 3RQ, UK
Telephone: $(+4)$
Facsimile: (4t)
Telex:
Email:
Commixsion(s):

MALLIK D C V DR, INDIAN INSTITUTE OF, ASTROPHYSICS, KORAMANGALA, BANGALORE 56O O34, INDIA

Telephone: (91) $8013566585 / 6+497$

Facsimile: (91)

Telex: $8+5763 \mathrm{jiab}$ in

Email:

Commission(s): 34,35

MALTBY PER PROF, INST THEORET ASTROPIIYSICS, UNIVERSITY OF OSLO, BOX 1029

N (1315 BLINDERN OSLO 3, NORWAY

Telephone: (47) 2-4565(19)

Facsimilc: (47)

Commission(s): 10

Telex:

Email: 
MALVILLE J MCKIM PROF, CASA, UNIVERSITY OF COLORADO, BOX 391, BOULDER CO 80309 0391, USA Telephone: (1) 3034928788

Facsimilc: (1) Telex:

Email:

Commission(s): 10

MALYUTO VALERI, TARTU ASTROPHYSICAL OBS, EE 2H+4 TORAVERE, ESTONIA

Telephonc: (372) 7410305

Facsimile: (372) $7+10205 \quad$ Telex:

Email: valcri@aai.ec

Commission(s):

MAMMANO AUGUSTO DR, OSSERVATORIO ASTROFISICO, VIA DELL OSSERVATORIO 8, I 36012 ASIAGO, ITALY

Telephone: (39) 42462665

Facsimile: (39)

Telex:

Email:

Commission(s): $\$ 2$

MAMPASO ANTONIO, IAC, C/ VIA LACTEA S/N, E 38200 LA LAGUNA, SPAIN

Telephone: (34) 22605200

Facsimile: (34) 22605210

Telex: $926+10$ iac e

Email: amr@iac.es

Commission(s): 34

MANABE SEIJI DR, INTL LATITUDE OBSERVATORY, NAOJ, HOSHIGAOKA MIZUSAWA SHI, IWATE 023, JAPAN

Telcphonc: (81)

Facsimile: (81)

Telex:

Email:

Commission(s): 19C

MANARA ALESSANDRO A DR, OSS ASTRONOMICO DI BRERA, VIA BRERA 28, 120121 MILANO, ITALY

Telephone: (39) 2874444

Facsimile: (39) 272001600

Telex:

Email:

Commission(s): 20,4t

MANCHADO ARTURO DR, INST DE ASTROFISICA, DE CANARIAS, OBS DEL TEIDE, E 38200 LA LAGUNA, SPAIN

Telephone: (34) 22262211

Facsimile: (34) 22263005

Telex: $926+0$ jac e

Email:

Commission(s): 3

MANYCHANDA R K DR, TIFR, HOMI BHABHA RD, COLABA, BOMBAY 400005 , INDIA

Telephone: (91) $22219111 * 336 \quad$ Facsimile: (91)

Telex: 113009 tifr in

Email:

Commission(s):

MANCHESTER RICHARD N DR, CSIRO, DIV OF RADIOPHYSICS, BOX 76, EPPING NSW 2121, AUSTRALIA

Telephone: (61) $28680225 \quad$ Facsimile: (61)

Telex: 26320 astro

Email: rmanches(a) atnf.csiro.au

Commission(s): $33,34,40$

MANCUSO SANTI PROF, DPT DI SCIENZE FISICHE, UNIVERSITA DI NAPOLI, MOSTRA D OLTREMARE PAD 19 I 80125 NAPOLI, ITALY

Telephone: (39)

Facsimile: (39)

Telex:

Email:

Commission(s):

MANDEL HOLGER, LANDESSTERNWARTE, KOENIGSHUHL, D 69117 HEIDELBERG, GERMANY

Telcphone: (49) 622150923

Facsimile: (49) 6221509202

Telex:

Email: hmandel@hp2.Isw.uni-heudelberg.de

Commission(s):

MANDOLESI NAZZARENO, IST TESRE, CNR, VIA DE CASTAGNOLI 1, I 40126 BOLOGNA, ITALY

Telephone: (39) 51238022

Facsimile: (39)

Telex: 511350

Email:

Commission(s): $40,44,47$

MANDRINI CRISTINA HEMILSE, IAFE, CC 67 SUC 28, 1428 BUENOS AIRES, ARGENTINA

Telcphone: (54) 17816755

Facsinile: (54) 17868114

Telex:

Email: mandriniabiafe.uba.ar

Commission(s): 10,12

MANDZHOS ANDREJ V DR, SPECIAL ASTROPHYSICAL OBS, ACADEMY OF SCIENCES, NIZHNIJ ARKHYZ 3571 +7 STAVROPOLSKIJ, RUSSIA

Telephone: (7) 8657892501

Facsimile: (7)

Telex: $1232+4$ zenit

Email: amand(a)sio.stavropol.su

Commission(s): 47

MANFROID JEAN DR, INSTITUT D'ASTROPHYSIQUE, UNIVERSITE DE LIEGE, AVE COINTE 5

B 4000 COINTE-LIEGE, BELGIUM

Telcphone: (32) 41529980

Facsimile: (32) 41527474

Email:

Commission(s): 25,34

Telex: $\$ 1264$ astrlg 
MANGENEY ANDRE DR, OBSERVATOIRE DE PARIS, SECTION DE MEUDON, F 92195 MEUDON PPL CDX, FRANCE Telephone: (33) 145077661

Facsimile: (33)

Telex:

Email:

Commission(s): 49

MANGUM JEFFREY GARY, STEWARD OBSERVATORY, SUBMILLIMETER TELESCP OBS

UNIVERSITY OF ARIZONA, TUCSON AZ 85721, USA

Telephone: (1) 5206215685

Facsimile: (1) 5206215554

Telex:

Email: jmangum(a) as.arizona.edu

Commission(s):

MANN GOTTFRIED DR, ZNTRLINST F ASTROPHYSIK, OBS FUER SOLARE, RADIO ASTRONOMIE

D 14473 TREMSDORF, GERMANY

Telephone: (49)

Email:

Facsimile: (49)

Telex: $15+20$

Commission(s): 10

MANN INGRID, MPI FUER AERONOMIE, POSTFACH 20, D 37189 KALTENBURG LINDAU, GERMANY

Telephone: (49) 5556979291

Facsimile: (49) $5556979240 \quad$ Telex:

Email: mann@linax1.dnet.gwdg.de

Commission(s): 21,22

MANN PATRICK J DR, DPT OF ASTRONOMY, UNIV OF WESTERN ONTARIO, LONDON ON N6A 3K7, CANADA

Telephone: (1) 5196613183

Facsimile: (1) 5196613486

Telex: $06+713+$

Email: bitnet:2014-562 (a)uwovax

Commission(s):

MANNHEIM KARL DR, UNIVERSITAETS STERNWARTE, GOETTINGEN, GEISMARLANDSTR 11

D 37083 GOETTINGEN, GERMANY

Telephone: (49) 551395050

Facsimile: (49) $551395043 \quad$ Telex: 96753

Email: kmannhe@unj-sw.gwdg.de

Commission(s):

MANNINO GIUSEPPE PROF, IST MATEMATICO, VIA CAMPI 181, I 11100 MODENA, ITALY

Telephone: (39)

Facsimile: (39)

Telex:

Email:

Commission(s): 27

MANRIQUE WALTER T PROF, OBSERVATORIO ASTRONOMICO, FELIX AGUILAR, AV BENAVIDEZ 8175 OESTE 5407 MARQUESADO (S.J.), ARGENTINA

Telephone: (54) 6423149

Facsimile: (54)

Telex:

Email:

Commission(s): 08

MANSFIELD VICTOR N PROF, DPT OF PHYSICS \& ASTRON, COLGATE UNIVERSITY, HAMILTON NY 13346, USA
Telephone: (1) 3158241000

Telephone: (1) 3158241000

Email:

Commission(s):

MANTEGAZZA LUCLANO, OSS ASTRONOMICO DI MILANO, VIA E BIANCHI \$6, I 22055 MERATE, ITALY

Telephone: (39) 592035

Facsimilc: (39)

Telex:

Email:

Commission(s): 27

MANTOVANI FRANCO, IST DI RADIOASTRONOMIA, CNR, VIA IRNERIO 46, I 40126 BOLOGNA, ITALY

Telephone: (39) 51232856

Facsimile: (39)

Telex: $21166+$ infnbo i

Email:

Commission(s):

MAO WEI, YUNNAN OBSERVATORY, CAS, BOX 110, KUNMING 729 46 YUNNAN, CHINA PR

Telephone: (86) $8712(135$

Facsimile: (86)

Telex:

Email:

Commission(s): 08

MARABINI RODOLFO JOSE ING, OBSERVATORIO ASTRONOMICO, PASEO DEL BOSQUE S/N

1900 LA PLATA (BS AS), ARGENTINA

Telephone: (54) 21217308

Facsimile: (54) 21211761

Telex:

Email:

Commission(s):

MARAN STEPHEN P DR, NASA GSFC, CODE 600, GREENBELT MD 20771, USA

Telcphonc: (1) 3012865154

Facsimile: (1) 3012861772

Telex: 89675

Email: hrsmaran(a)stars.gsfc.nasa.gov

Commission(s): $15,40,+4$

MARANO BRUNO, DPT DI ASTRONOMIA, UNIVERSITA DI BOLOGNA, CP \$96, I 40100 BOLOGNA, ITALY

Telephone: (39) 51222956

Facsimile: (39)

Telex: $21166+$ infnbo i

Email:

Commission(s): 47 
MARAR T M K, ISRO SATELLITE CTR, AIRPORT RD, VIMANAPURA POST, BANGALORE 560 017, INDIA Telephone: (91) 803566251

Facsimile: (91)

Telex:

Email:

Commission(s): 4

MARASCHI LAURA DR, IST DI FISICA, UNIVERSITA DI MILANO, VIA CELORLA 16, I 20133 MILANO, ITALY

Telephone: (39) 22392275

Facsimile: (39) 22366583

Telex:

Email:

Commission(s):

MARCAIDE JUAN-MARIA DR, DPT DE ASTRONOMIA, UNIVERSIDAD DE VALENCIA, C/DR MOLINER S/N E 46100 BURRJASSOT, SPAIN

Telephone: (34) 63864549

Facsimile: (34) $63864364 \quad$ Telex:

Email: jmm(a)vibi.matapl.uv.cs

Commission(s): 40

MARCELIN MICHEL, OBSERVATOIRE DE MARSEILLE, 2 PLACE LE VERRIER

F 13248 MARSEILLE CDX 04, FRANCE

Telephone: (33) 91959088

Fucsimile: (33)

Telex: $\$ 20241 \mathrm{f}$

Email:

Commission(s): 28

MARCHAL CHRISTIAN DR, DPT ETUDES DE SYNTHESE, ONERA, F 92320 CHATILLON, FRANCE

Tełephone: (33) 146571160

Facsimile: (33)

Telex: 260907

Email:

Commission(s): 07

MARCIALIS ROBERT, LUNAR \& PLANETARY LAB, UNIVERSITY OF ARIZONA, TUCSON AZ 85721, USA

Telephonc: (1) $520327+827$

Facsimile: (1) 5206214933 Telex:

Email: umpire(a)lpl.arizona.edu

Commission(s): 15,16

MARDIROSSIAN FABIO, DPT ASTRONOMIA, UNIVERSITA DI TRIESTE, VIA TIEPOLO 11,134131 TRIESTE, ITALY

Telephone: (39) $40793921 * 221$

Facsimile: (39)

Commission(s): $\$ 2,47$

Telex: 461137 oat i

Email:

MAREK JOHN, \& PERCY RD, WREXHAM CLWYD, UK

Telephonc: (4)

Conmission(s): 47

Telex:

Email:

MARGON BRUCE H PROF, DPT OF ASTRONOMY, UNIVERSITY OF WASHINGTON, FM 20, SEATTLE WA 98195, USA

Telephone: (1) $2065+30089 \quad$ Facsimile: (1)

Email: margon(äjanusastro.washington. Commission(s):

Telex: $47+0096$

MARGONI RINO, OSSERVATORIO ASTROFISICO, VIA DELL OSSERVATORIO 8, I 36012 ASIAGO, ITALY

Telephone: (39) $42462665 \quad$ Facsimile: (39)

Telex: $\$ 30110$ setour

Email:

Commission(s)

MARGRAVE THOMAS EWING JR, 400 JOHNSON ST, VIENNA VA 22180, USA

Telephone: (1) Facsimile: (1) Telex:

Email: $\quad$ Commission(s): 27,51

MARIE M A DR, DPT OF ASTRONOMY, FACULTY OF SCIENCES, CAIRO UNIVERSITY, GEZA, EGYPT

Telephone: (20)

Facsimile: (20)

Telex:

Email:

Commission(s):

MARIK MIKLOS DR, ASTRONOMY DPT, EOTVOS UNIVERSITY, KUN BELA TER 2, H 1083 BUDAPEST, HUNGARY

Telephone: (36) $111+1019$

Facsimile: (36) 1210 1089)

Telex:

Email: marik(äinnin.eltc.hu

Commission(s): 12,38

MARILLI ETTORE DR, OSS ASTROFISICO, CITTA UNIVERSITARIA, VIA A DORIA 6, I 95125 CATANIA, ITALY

Telephone: (39) 95330533

Facsimile: (39)

Telex: 970359 astret

Email:

Commission(s): $12,29,42$

MARINO BRIAN F ENG, 156 QUEEN ST, NORTHCOTE, AUCKLAND 9, NEW ZEALAND

Telephone: (64)

Facsimile: $(6 \downarrow)$

Telex:

Email:

Commission(s): $\$ 2$

MARIOTTI JEAN MARIE DR, OBSERVATOIRE DE PARIS, SECTION DE MEUDON F 92195 MEUDON PPL CDX, FRANCE

Telephone: (33) 145077570

Email: bitnet:mariottiafímeusi

Facsimile: (33)

Commission(s): (1)

Telex: 204464 
MARIS GEORGETA DR, ASTRONOMICAL INSTITUTE, CUTITUL DE ARGINT 5, BOX 28

R 75212 BUCHAREST, RUMANIA

Telephone: (40) 16413686

Facsimile: (40) 13123391

Telex: 11882 astro $r$

Email: gmaris@imar.ro

Commission(s): 10

MARISKA JOHN THOMAS, NAVAL RESEARCH LABORATORY, CODE 7673, 4555 OVERLOOK AVE SW

WASHINGTON DC 20375 5000, USA

Telephone: (1) 2027672605

Email: jmariska@solar.stanford.cdu

Facsimile: (1) 2024047997

Telex:

Commission(s): 10,12

MARK JAMES WAI-KEE DR, LAWRENCE LIVERMORE LAB, L 477, BOX 808, LIVERMORE CA 94550, USA

Telephone: (1) 4154225931

Facsimile: (1)

Telex: 910-386-8339 ucclll

Email:

Commission(s): 33

MARKELLOS VASSILIS V DR, DPT ENFINEERING SCIENCE, UNIVERSITY OF PATRAS, GR 26000 RION, GREECE

Telephone: (30) 61991465

Facsimile: (30)

Telex:

Email:

Commission(s): 07

MARKERT THOMAS H DR, CENTER FOR SPACE RESEARCH, MIT RM 37 621, BOX 165, CAMBRIDGE MA 02139, USA

Telephone: (1) 6172535169

Facsimilc: (1)

Telex:

Email: thm(a)space.mit.erlu

Commission(s):

MARKKANEN TAPIO DR, OBS \& ASTROPHYSICS LAB, UNIVERSITY OF HELSINKI, TAEHTITORNINMAKI

SF 00130 HELSINKI 13, FINLAND

Telephone: (358) 0908391

Facsimile: (358)

Telex:

Email:

Commission(s): $25,37,50$

MARKOVA NEVJANA, BOX 136, BG 4700 SMOLJAN, BULGARIA

Telephonc: (359) 30128901

Facsimile: (359) 302 1356

Telex:

Email:

Commission(s):

MARKOWITZ WILLIAM DR, 651 SW 6TH ST, CT 1012, POMPANO BEACH FL 33060, USA

Telephone: (1) 3059410083

Facsimile: (1)

Telex:

Email:

Commission(s): 19,31

MARKWORTH NORMAN LEE, DPT PIYSICS \& ASTRONOMY, STEPHEN F AUSTIN STATE UN, BOX 13044

NACOGDOCHES TX 75962, USA

Telephone: (1) $409 \$ 683001$

Facsimile: (1) $4094681226 \quad$ Telex:

Email: f_markwort(âtitan.sfasu.edu

Commission(s): $\$ 2$

MARLBOROUGH J M PROF, DPT OF ASTRONOMY, UNIV OF WESTERN ONTARIO, LONDON ON NGA 3K7, CANADA

Telephone: (1) 5196793184

Facsimile: (1) 5196613486

Telex: $064713-$

Email:

Commission(s): 36

MARMOLINO CIRO, DPT DI FISICA, UNIVERSTIA DI NAPOLI, MOSTRA D OLTREMARE PAD 19

I 80125 NAPOLI, ITALY

Telephone: (39) 817253428

Facsimile: (39)

Telex: 720320 infnna i

Email:

Commission(s): 12C

MAROCHNIK L S PROF DR, COMPUTOR SCIENCES CORP, SYSTEM SCIENCES DIV, 10000 A AREOSPACE ROAD

LAUHAM SEABROOK MA 201706, USA

Telephone: (1) $30179 \$ 1483$

Facsimile: (1) 301 $459+482$

Telex:

Email: Iconid@ecexsels.gsfe.nasa.gov

Commission(s): 33

MAROV MIKHAIL YA PROF, INST OF APPLIED MATHS, ACADEMY OF SCIENCES, MIUSSKAJA SQ 4

$1250+7$ MOSCOW, RUSSIA

Telephone: (7) 095250 (1485

Email: marov(a)applmat.msk.su

Facsimile: (7) 0959720737 Telex:

Commission(s): $16 \mathrm{P}, 4+, 51$

MARQUES DOS SANTOS P PROF, IAG, UNIVERSIDADE DE SAO PAULO, CP 9638, 01065 SAO PAULO SP, BRAZIL

Telephone: (55) 112763941

Facsimile: (55) 11276,3848

Telex: $1156735 \mathrm{iagm}$ br

Email:

Commission(s): 28,40 
MARQUES MANUEL N DR, OBSERVATORIO ASTRONOMICO, TAPADA DA AJUDA, P 1300 LISBOA 3, PORTUGAL Telephone: (351)

Facsimile: (351)

Telex:

Email:

Commission(s):

MARRACO HUGO G DR, OBSERVATORIO ASTRONOMICO, PASEO DEL BOSQUE S/N

1900 LA PLATA (BS AS), ARGENTINA

Telephone: (54) 21217308

Facsimile: (54) 21211761

Telex: 31151 bulap ar

Email:

Commission(s): 25,37

MARSCHALL LAURENCE A, DPT OF PHYSICS, GETTYSBURG COLLEGE, GETTYSBURG PA 17325, USA

Telephone: (1) 7173371865

Facsimile: (1)

Telex:

Email:

Commission(s): 24,30

MARSCHER ALAN PATRICK, DPT OF ASTRONOMY, BOSTON UNIVERSITY, 725 COMMONWEALTH AVE BOSTON MA 02215, USA

Telephone: (1) 6173535029

Facsimile: (1)

Telex: 951289 bos univ bsn

Email:

Commission(s): 40

MARSDEN BRIAN G DR, CENTER FOR ASTROPHYSICS, HCO/SAO, 60 GARDEN ST, CAMBRIDGE MA 02138, USA

Telephone: (1) 6174957244

Facsimile: (1) $6174977345 \quad$ Telex: $71032068+2$ astrogram

Email: marsden@efa.harvard.edu

Commission(s): (66VP,07,15,20C

MARSDEN PHILIP L PROF, DPT OF PHYSICS, UNIVERSITY OF LEEDS, LEEDS LS2 9JT, UK

Telephone: (44) 1135431751

Facsimile: (4)

Email:

Commission(s):

Telex: $556+73$ unids

MARSH JULIAN C D, HATFIELD POLYTECHNIC, OBSERVATORY, BAYFORDBURY

HERTFORD HERTS SG13 8LD, UK

Telephone: (44) $1992558451 \quad$ Facsimile: (44)

Email:

Facsimile: $(+4)$

Telex: $262+13$

MARSH THOMAS DR, DPT OF ASTROPHYSICS, UNIVERSITY OF OXFORD, KEBLE RD, OXFORD OX1 3RH, UK

Tclephonc: (44) 1865273303

Facsimile: (4t) $1865273+18$

Telex: 83295 nuclox g

Email: 19464::trm

Commission(s): 42

MARSHALL HERMAN LEE DR, CTR FOR EUV ASTROPHYSICS, UNIVERSITY OF CALIFORNIA 2150 KITTREDGE ST, BERKELEY CA 94720 , USA

Telephone: (1) $4156+35671 \quad$ Facsimile: (1) $\$ 156+35660 \quad$ Telex: $910+14061$ ucbsag

Email: hermanm(a)ssl.bereley.edu Commission(s):

MARSHALL KEVIN P, INSTITUTE OF ASTRONOMY, THE OBSERVATORIES, MADINGLEY RD

CAMBRIDGE CB3 OHA, UK

Telephone: (44) 1223337548

Email: kpm@uk.ac.cam.ast-star

Facsimile: (44) 1223337523

Telex: 817297 astron g

Commission(s): 37

MARSOGLU A DR, UNIVERSITY OBSERVATORY, UNIVERSITY OF ISTANBUL, 34+52 ISTANBUL, TURKEY

Telephone: (90) 15223597

Facsimile: (90)

Telex:

Email:

Commission(s):

MARSTON ANTHONY PHILIP, DPT PHYSICS \& ASTRONOMY, DRAKE UNIVERSITY, DES MOINES IA 50311, USA

Tclephone: (1) 5152713034

Facsimile: (1) 5152713977 Telex:

Email: in\%tm9991 roacad.drake.edu

Commission(s): 28,34

MARTENS PETRUS C DR, ESA/ESTEC, SPACE SCIENCE DIVISION, PO BOX 299

NL 2200 AG NOORDWIJK, NETHERLANDS

Telephone: (31) 171985295

Facsimile: (31) $171984698 \quad$ Telex:

Email: pmartens(a) soho.esil.estec.nl

Commission(s): 10

MARTIN ANTHONY R DR, UK CULHAM LABORATORY, RM F $4 / 135$, ABINGDON OX1+ 3DB, UK

Telephonc: (44) 123521840

Facsimilc: (4)

Telex: 83189

Email:

Commission(s): 51

MARTIN DEREK H PROF, DPT OF PHYSICS, QUEEN MARY/WESTFIELD COLL, MILE END RD LONDON E1 4NS, UK

Telephonc: (4)

Facsimile: (4t)

Email:

Commission(s): 
MARTIN FRANCOIS DR, DPT ASTROPHYSIQUE, UNIVERSITE DE NICE, PARC VALROSE F 06034 NICE CDX, FRANCE

Telephone: (33) 93519100

Facsimile: (33)

Telex: 970281

Emanil:

Commission(s):

Telephonc: (55)

Facsimile: (55)

Telex:

Email: martinaifi.unicamp.br

Commission(s): +4

MARTIN JEAN-MICHEL P, OBSERVATOIRE DE PARIS, ARPEGES, 5 PL JULES JANSSEN

F 92195 MEUDON PPL CDX, FRANCE

Telephone: (33) 145077608

Facsimile: (33) 145077939

Telex: 270912 obsastr

Email: jmmartin(a)obspm.fr

Commission(s):

MARTIN MARIA CRISTINA DR, IAR, CC 5, 189 4 VILLA ELISA (BS AS), ARGENTINA

Telephone: (54) $21+3793$

Facsimile: (54)

Telex: 31216 cesla ar

Email:

Commission(s): 28,51

MARTIN NICOLE DR, OBSERVATOIRE DE MARSEILLE, 2 PLACE LE VERRIER

F 13248 MARSEILLE CDX (4, FRANCE

Telephone: (33) $91959088 \quad$ Facsinile: (33)

Commission(s): 30

Telex: $\$ 202+1$

Email:

MARTIN PETER G PROF, CITA MCLENNAN LABS, UNIVERSITY OF TORONTO, 60 ST GEORGE ST

TORONTO ON MSS 1A1, CANADA

Telephone: (1) $\$ 1697868+10$

Facsimile: (1) $\$ 169783921$

Telex:

Email: pgmartin(acita.utoronto.ca

Commission(s): $3+\mathrm{C}$

MARTIN ROBERT N DR, STEWARD OBSERVATORY, UNIVERSITY OF ARIZONA, TUCSONAZ 85721, USA

$\begin{array}{ll}\text { Telephone: (1) } 5206211539 & \text { Facsimile: (1) } \\ \text { Email: } & \text { Commission(s): 34,40 }\end{array}$

Telex: 467175

MARTIN WILLIAM C DR, NTL BUREAU OF STANDARDS, PHYSICS BLDG A167, GAITHERSBURG MD 20899, USA

Telephone: (1) 3019212011

Facsimile: (1)

Telex:

Email:

Commission(s): 14

MARTIN WILLIAM L DR, ROYAL GREENWICH OBS, MADINGLEY RD, CAMBRIDGE CB3 03Z, UK

Telephone: (4t) $1323833171 \quad$ Facsimile: $(4 t)$

Telex:

Email:

Commission(s): 27

MARTINET LOUIS PROF, OBSERVATOIRE DE GENEVE, CHEMIN DES MAILLETTES 51

CH 1290 SAUVERNY, SWITZERLAND

Telephone: (41) 227552611

Email: martinet(a)obs.unige.ch

Facsimile: (41) 227553983

Telex: 419209 obs ch

MARTINEZ MARIO DR, DPT DE GEOFISICA, CIESE, APDO POSTAL 2732, 22860 ENSENADA B CALIF, MEXICO

Telephone: (52)

Commission(s): $07,28,33,46$

Telex:

Email:

Facsimile: (52)

Commission(s):

MARTINEZ PILLET VALENTIN, IAC, C/ VIA LACTEA S/N, E 38200 LA LAGUNA, SPAIN

Telephone: (34) 22605200

Email: imprajiac.es

Facsimilc: (34) 22605210

Telex: $92+60$ iac e

MARTINEZ ROGER CARLOS DR, INST DE ASTROFISICA, DE CANARIAS, OBS DEL TEIDE

E 38200 LA LAGUNA, SPAIN

$\begin{array}{ll}\text { Telephone: (34) } 22262211 & \text { Facsimile: (34) } \\ \text { Email: } & \text { Commission(s): }\end{array}$

Email:

MARTINEZ-GONZALEZ E DR, DPT DE FISICA MODERNA, UNIVERSIDAD DE CANTABRIA, AVD LOS CASTROS S/N E 39005 SANTANDER, SPAIN

Telephone: $(34)+2201+68$

Facsimile: (34) $\$ 2201402$

Telex: 35861 educi e

Email: 16+38::ccucve::c_martines

Commission(s): $\$ 7$

MARTINI ALDO DR, IAS, CNR, CP 67, I 0004 4 FRASCATI, ITALY

Telephone: (39) 69425655

Facsimile: (39) 69416847

Email:

Commission(s):

Telex: 
MARTINS DONALD HENRY DR, DPT PHYSICS \& ASTRONOMY, UNIVERSITY OF ALASKA, 3221 UAA DRIVE ANCHORAGE AK 99508, USA

Telephone: (1) 9077861238

Facsimile: (1)

Telex:

Email:

Commission(s): 09,37

MARTIN-DIAZ CARLOS DR, INST DE ASTROFISICA, DE CANARIAS, OBS DEL TEIDE, E 38200 LA LAGUNA, SPAIN Telephone: (34) 22262211

Facsimile: (34) 22263005

Telex: 92640 iace

Email: span:iac::cmd

Commission(s):

MARTIN-LORON M DR, HERMANOS MIRALLES 14, E MADRID 1, SPAIN

Telephone: (34)

Facsimile: (34)

Email:

Commission(s):

Telex:

MARTIN-PINTADO JESUS, CTR ASTRON DE YEBES, OAN, APD 148, E 19080 GUADALAJARA, SPAIN

Telephone: (34) 11223358

Facsimile: (34)

Telex:

Email:

Commission(s): 34,40

MARTRES MARIE-JOSEPHE, OBSERVATOIRE DE PARIS, SECTION DE MEUDON F 92195 MEUDON PPL CDX, FRANCE

Telephone: (33) 145347530

Facsimile: (33)

Telex:

Email:

Commission(s): 10

MARVIN URSULA B DR, CENTER FOR ASTROPHYSICS, HCO/SAO, 60 GARDEN ST, CAMBRIDGE MA 02138, USA Telephone: (1) 6174957270

Facsimile: (1)

Telex: $921+28$ satcllite cam

Email:

Commission(s): 22

MARX GYORGY PROF, DPT ATOMIC PHYSICS, EOTVOS UNIVERSITY, PUSHKIN U 5-7

H 1088 BUDAPEST, HUNGARY

Telephone: (36) 1187902

Email: kuerti $(a)$ awiraf

Facsimile: (36) 1180206

Telex: 225459 atomf h

MARX SIEGFRIED DR, ZNTRLINST F ASTROPHYSIK, KARL-SCHWARZSCHILD OBS D G901 TAUTENBURG, GERMANY

Telephone: (49) 7823530

Email:

Facsimile: (49)

Commission(s): 50

Telex: $588628+$ ksot dd

MASANI A PROF, OSS ASTRONOMICO DI BRERA; VIA BRERA 28, I 20100 MILANO, ITALY

Telephone: (39)

Facsimile: (39)

Telex:

Email:

Commission(s): $25,27,35$

MASEGOSA GALLEGO J DR, INST ASTROFISICA, DE ANDALUCIA APD 3004, C/SANCHO PANZA S/N

E 18080 GRANADA, SPAIN

Telephone: (34) $\$ 8121311$

Facsimile: $(34) 5881+530$

Telex: 78573 ialag c

Email:

Commission(s): 28

MASLOWSKI JOZEF DR, ASTRONOMICAL OBSERVATORY, JAGIELLONIAN UNIVERSITY, UL ORLA 171

PL 30 $2+4$ KRAKOW, POLAND

Telephonc: $(48) 3+10+1$

Facsimile: (48) 12378053

Telex: 32 6203 ujoa pl

Email:

Commission(s): 40

MASNOU FRANCOISE DR, 28 ALLEE GAMBAUBERIE, F 91190 GIF/YVETTE, FRANCE

Telephonc: (33)

Facsimile: (33)

Telex:

Email:

Commission(s):

MASNOU J L DR, OBSERVATOIRE DE PARIS, SECTION DE MEUDON, ER 176 DARC F 92195 MEUDON PPL CDX, FRANCF

Telephone: (33) $1+53+7570$

Facsimile: (33)

Telex: 201571

Email:

Commission(s):

MASON GLENN M, ASTRONOMY PROGRAM, UNIVERSITY OF MARYLAND, COLLEGE PARK MD 20742, USA

Telephonc: (1) $301+5+2616$

Facsimile: (1)

Telex: $71-8261125$

Email:

Commission(s): $10,4+, 4)$ 
MASON HELEN E DR, DPT APPLIED MATHS, \& THEORETICAL PHYSICS, SILVER STREET

CAMBRIDGE CB3 9EW, UK

Telephone: (44) 1223337898

Facsimile: (44) 1223337918

Telex: $\mathbf{8 1 2 4 0}$

Email:

Commission(s): $14 \mathrm{C}$

MASON JOHN WILLIAM DR, S1 ORCHARD WAY, WEST BARNHAM, BOGNOR REGIS, WEST SUSSEX PO22 OHX, UK Telephone: (44) $12435532+4$ Facsimile: (4t) $12+355+272$ Telex:

Email:

Commission(s): 22

MASON KEITH OWEN, MULLARD SPACE SCIENCE LAB, UNIVERSITY COLLEGE LONDON, HOLMBURY ST MARY DORKING SURREY RH5 6NT, UK

Telephone: (44) 130670292

Facsimile: (\$t)

Telex: $\mathbf{8 5 9 1 8 5}$

Email:

Commission(s): 4

MASSA DERCK LOUIS, APPLIED RESEARCH CORP, 8201 CORPORATE DR, LANDOVER MD 20785, USA

Telephone: (1) $301+598+42$

Facsimile: (1) 3017310765 Telex:

Email: massa(g)godot.arcich.com

Commission(s):

MASSAGLIA SILVANO, IST DI FISICA GENERALE, CORSO D AZEGLIO 46, I 10125 TORINO, ITALY

Telephone: (39) $11657694 \quad$ Facsimile: (39)

Telex: $2110+1$

Email:

Commission(s): 36

MASSAGUER JOSEP PROF, DPT FISICA APLICADA, UNIV POLITEC DE CATALUNYA, E 0803+ BARCELONA, SPAIN Telephone: (34) 34016827

Email: massaguer fa.upe.es Facsimile: (3†) 34016090

Telex:

MASSEVICH ALLA G DR, INST OF ASTRONOMY, ACADEMY OF SCIENCES, PYATNITSKAYA UL 48 109017 MOSCOW, RUSSIA

Telephone: (7) 952315461

Facsimile: (7)

Commission(s): 35

Telex: $\mathbf{\$ 1 2 6 2 3}$ scstp su

Email:

MASSEY PHILIP L, KITT PEAK NTL OBS, BOX 26732, 950 N CHERRY AVE, TUCSON AZ 85726 6732, USA

Telephone: (1) 5203275511

Facsimile: (1)

Telex:

Email:

Commission(s): 29

MASSON COLIN R, CENTER FOR ASTROPHYSICS, HCO/SAO, (0) GARDEN ST, CAMBRIDGE MA 02138, USA

Telephone: (1) $617+957000$

Facsimile: (1)

Telcx:

Email:

Commission(s): 34,40

MATAS VLADIMIR R DR, ASTRON RECHEN INSTITUTE, MOENCHHOFSTR 12-14, D 69120 HEIDELBERG, GERMANY

Telephone: (\$) $6221+9(126$

Facsimile: (\$9)

Telex:

Email:

Commission(s): 07

MATERNE JUERGEN DR, ARETINSTR 27, D 81545 MUENCHEN, GERMANY

Telephone: (49)

Facsimile: (49)

Telex:

Email:

Commission(s): $\$ 7$

MATHER JOHN CROMWELL, NASA GSFC, CODE 685, LASP, GREENBELT MD 20771, USA

Telephone: (1) 3012868720

Facsimile: (1)

Telex: 89675

Email: matherastars.gsfc.nasa.gov

Commission(s): $21,34,44,47$

MATHESON DAVID NICHOLAS, RUTHERFORD APPLETON LAB, SPACE \& ASTROPHYSICS DIV, BLDG R25/R68 CHILTON DIDCOT OX11 0QX, UK

Telephone: (\$4) 1235219 (10

Facsimile: (tt)

Telex: 83159

Email:

Conmission(s): 40

MATHEWS WILLIAM G PROF, LICK OBSERVATORY, UNIVERSITY OF CALIFORNIA, SANTA CRUZ CA 95064, USA Telephone: (1) 408 \$29 2074

Facsimile: (1)

Telex:

Email:

Commission(s): 34

MATHEWSON DONALD S PROF, MOUNT STROMLO \& SIDING, SPRING OBSERVATORIES, PRIVATE BAG

WODEN PO ACT 2611, AUSTRALIA

Telephonc: (61) 62881111

Email: Jsmamso.anu.edu.au

Facsimile: (61) $62+91233$

Commission(s): $28,33,34, \mathrm{EC}$
Telex: 62270 aa 
MATHEZ GUY, OBS MIDI PYRENEES, 14 AVE E BELIN, F 31400 TOULOUSE CDX, FRANCE Telephone: (33) 61252101 Facsimile: (33)

Email:

Commission(s): 47

Telex: 530776 obstlse f

MATHIEU ROBERT D DR, DPT OF ASTRONOMY, UNIVERSITY OF WISCONSIN, 475 N CHARTER ST MADISON WI 53706, USA

Telephone: (1) 6082625679

Facsimile: (1)

Telex: 265452

Email: bitnet:mathieu@wiscmac3

Commission(s): 30,42

MATHIS JOHN S PROF, DPT OF ASTRONOMY, UNIVERSITY OF WISCONSIN, 475 N CHARTER ST MADISON WI 53706, USA

Telephone: (1) 6082625994

Facsimile: (1) 6082630361

Telex: $265+52$ uofwisc mds

Email: 7309::mathis/mathis(âwisemacc

Commission(s): $3+\mathrm{C}$

MATHUR B S DR, NTL PHYSICAL LABORATORY, TIME \& FREQUENCY SECTION, HILLSIDE RD NEW DELHI 110 012, INDIA

Telephone: (91) 11586168

Facsimile: (91)

Email:

Commission(s): 31

Telex: 31-6245+ rsd in

MATHYS GAUTIER DR, ESO, CASILLA 19001, SANTIAGO 19, CHILE

Telephone: (56) 26988757

Facsimile: (56) $2695+263$

Telex: $2+0881$ esogo cl

Email:

Commission(s): 29C,36

MATSAKIS DEMETRIOS N, US NAVAL OBSERVATORY, 3450 MASSACHUSETTS AVE NW WASHINGTON DC 20392 5100, USA

Telephone: (1) 2026531823

Facsimile: (1)

Telex:

Email:

Commission(s): 19,31,40,51

MATSON DENNIS L DR, JPL, MS 183 501, 4800 OAK GROVE DR, PASADENA CA 91109, USA

Telephone: (1) 2133542984

Facsimile: (1)

Telex:

Email:

Commission(s): 15,16

MATSUDA TAKUYA PROF, DPT OF EARTH SCIENNCES, KOBE UNIVERSITY, ROKKOUDAI-MACHI NADA KU KOBE 657, JAPAN

Telephone: $(81) 788811212^{*}+421 \quad$ Facsimile: $(81) 7888215+9 \quad$ Telex:

Email: tmatsuda(a)icluna.kobe-u.ac.jp) Commission(s): 51

MATSUHARA HIDEO, DPT OF ASTROPHYSICS, NAGOYA UNIVERSITY, CHIKUSA KU, NAGOYA 46401, JAPAN

Telephonc: (81) 527892560

Facsimile: (81) 527892919

Telex:

Email: maruma $a$ toyo.phys.nagoya-u.ac.jp

Commission(s): 34

MATSUI TAKAFUMI DR, DPT EARTH \& PLANETARY PHY, UNIVERSITY OF TOKYO, BUNKYO KU

TOKYO 113, JAPAN

Telephonc: (81) $338122111 * 4305$

Facsimile: (81) 338183247

Telex: 2722126 utgah

Email:

Commission(s): 16

MATSUMOTO MASAMICHI PROF, FACULTY OF ENGINEERING, GIFU UNIVERSITY, GIFU 501 11, JAPAN

Telephonc: (81) 878361663

Facsimile: (81) $878361652 / 1962$ Telex:

Email: matsu(ged.kagawa-u.ac.jp)

Commission(s): 36

MATSUMQTO RYOJI DR, DPT PHYSICS/FAC OF SCIENC, CHIBA UNIVERSITY, 1-33 YAYOICHO INAGE KU CHIBA 263, JAPAN

Telephone: (81) 432903724

Facsimile: $(81)+32903720$

Telex:

Email: matumoto(a)c.chiba-u.ac.jp)

Commission(s): +4

MATSUMOTO TOSHIO DR, DPT OF PHYSICS, NAGOYA UNIVERSITY, FUROCHO CHIKUSA KU

NAGOYA $\$ 6401$, JAPAN

Telephone: (81) 527815111

Email:

Fatcsimile: (81)

Commission(s): $21 \mathrm{C},+7$
Telex: $\$+77323$ scunag.

MATSUMURA MASAFUMI DR, FAC OF EDUCATION, KAGAWA UNIVERSITY, SAIWAI CHO TAKAMATSUSHI KAGAWA 760, JAPAN

Telephone: (81) $878614141 * 400$

Facsimilc: (81) 878347144

Telex:

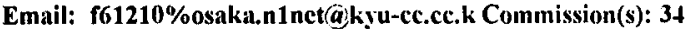


MATSUOKA MASARU DR, INST PHYS \& CHEMICAL RES, KENKYUSHITSU, 2-1 HIROSAWA, WAKO 351 01, JAPAN

Telephone: (81) $48+621111 \quad$ Facsimile: (81)

Email:

Commission(s): 44

Telex: 02962818 riken $j$

MATSUURA OSCAR T DR, IAG, UNIVERSIDADE DE SAO PAULO, CP 9638,01065 SAO PAULO SP, BRAZIL

Telephone: (55) $112753720 \quad$ Facsimile: (55) $112763848 \quad$ Telex: 1156735 iagm br

Email:

Commission(s): $10,15,49$

MATTEI JANET AKYUZ DR, AAVSO, 25 BIRCH ST, CAMBRIDGE MA 02138, USA

Telephone: (1) $6173540484 \quad$ Facsimile: (1) $61735+0665$ Telex:

Email:

Commission(s): $27 \mathrm{C}, 42$

MATTEUCCI FRANCESCA DR, IAS, CNR, CP 67, I O0044 FRASCATI, ITALY

Telephone: (39) $69425655 \quad$ Facsimile: (39) $694168+7 \quad$ Telex:

Email:

Commission(s): 33C,35,37

MATTHEWS CLIFFORD PROF, DPT OF CHEMISTRY M/C111, UNIVERSITY OF ILLINOIS, BOX 4338

CHICAGO IL G0680, USA

Telephone: (1) 3129963161

Facsimile: (1) $3129960431 \quad$ Telex:

Email:

Commission(s): 51

MATTHEWS HENRY E DR, JOINT ASTRONOMY CENTER, JCMT, G65 KOMOHANA ST, HILO HI 96720, USA

Telcphonc: (1) 8089613756

Facsimile: (1)

Telex: 633135

Email:

Commission(s): 40

MATTHEWS JAYMIE, DPT GEOPHYS \& ASTRONOMY, UNIV OF BRITISH COLUMBIA, 2075 WESBROOK PL VANCOUVER BC VGT 1ZH, CANADA

Telephone: (1) $60+8222696 / 2267$

Facsimile: (1) 6048226147

Telex:

Email: matthews $(\hat{a})$ astro.ubc.ci

Commission(s): 27

MATTHEWS THOMAS A DR, ASTRONOMY PROGRAM, UNIVERSITY OF MARYLAND

COLLEGE PARK MD 20742, USA

Telephone: (1)

Facsimile: (1)

Telex:

Email:

Commission(s):

MATTIG W PROF DR, KIEPENHEUER INSTITUT, FUER SONNENPHYSIK, SCHOENECKSTR G

D 79104 FREIBURG BREISGAU, GERMANY

Telephone: (49) $76132864 \quad$ Facsimile: (49) Telex: 7721552 kis d

Emıtil:

Commission(s): $10,12,50$

MATTILA KALEVI DR, OBS \& ASTROPHYSICS LAB, UNIVERSITY OF HELSINKI, TAEHTITORNINMAKI

SF 00130 HELSINKI 13, FINLAND

Telephone: (358) 1912947

Facsimile: (358) 1912952

Telex: $12+690$ unih sf

Email: kalevi.mattilaa helsinki.fi

Commission(s): $21,34,40$

MATTOX JOHN, NASA GSFC, CODE 660, GREENBELT MD 20771, USA

Telephone: (1) 3014056856

Facsimile: (1) $3012861611 \quad$ Telex:

Email: john_mattoxägsfc.nasa.gov

Commission(s):

MATYEYENKO L I DR, SPACE RESEARCH INSTITUTE, ACADEMY OF SCIENCES, PROFSOJUZNAYA UL 84/32, 117810 MOSCOW, RUSSIA

Telephone: (7) 953333122

Facsimile: (7)

Telex: $\mathbf{4 1 1}+98$ star su

Email:

Commission(s): 40

MATZ STEVEN MICHEAL DR, DPT PHYSICS \& ASTRONOMY, NORTHWESTERN UNIVERSITY

DEARBORN OBSERVATORY, EVANSTON IL 601208, USA

Telephone: (1) $708+9186+3 \quad$ Facsimile: (1) Telex:

Email: matzajossenu.astro.nwu.edu Commission(s): $05,09,4+$

MATZNER RICHARD A PROF, ASTRONOMY DPT, UNIVERSITY OF TEXAS, RLM 15 308, AUSTIN TX 78712 1083, USA Telephone: (1) $512+715062$

Facsimile: (1)

Telex:

Email:

Commission(s): +7

MAUAS PABLO, IAFE, CC 67 SUC $28,1+28$ BUENOS AIRES, ARGENTINA

Telephone: (5t) 17816755

Email: pablo áiafe.uba.ar

Facsimile: (5t) $17868114 \quad$ Telex:

Commission(s): 
M 692

MAUCHERAT J DR, LAS, TRAVERSE DU SIPHON, LES TROIS LUCS, F 13012 MARSEILLE, FRANCE

Telephone: (33) 91055900

Facsimile: (33) 91661855

Telex:

Email:

Commission(s): 21

MAUDER HORST PROF DR, ASTRONOMISCHES INSTITUT, UNIVERSITAET TUEBINGEN, WALDHAEUSERSTR 64 D 72076 TUEBINGEN, GERMANY

Telephone: (49)

Email:

Facsimile: (49)

Telex:

Commission(s): $\$ 2$

MAUERSBERGER RAINER DR, STEWARD OBSERVATORY, UNIVERSITY OF ARIZONA, TUCSON AZ 85721, USA

Telephone: (1) 5206211532

Facsimile: (1) $5206215751 \quad$ Telex:

Email: mauersa as.arizona.edu

Commission(s): 40

MAURICE ERIC N, OBSERVATOIRE DE MARSEILLE, 2 PLACE LE VERRIER, F 13248 MARSEILLE CDX 04, FRANCE

Telephone: (33) 91959088

Facsimile: (33)

Commission(s): 28,30

Telex: $4202+1 \mathrm{f}$

Email:

MAUROGORDATO SOPHIE, LABORATOIRE D'ASTRONOMIE, OBSERVATOIRE DE MEUDON, CNRS URA 173 F 92195 MEUDON PPL CDX, FRANCE

Telephone: (33) $145077407 \quad$ Facsimile: $(33) 1+5077469 \quad$ Telex:

Email: maurogordato(a)messiob.obspm.f. Commission(s):

MAURON NICOLAS DR, OBS MIDI PYRENEES, 14 AVE E BELIN, F 31400 TOULOUSE CDX, FRANCE

Telephone: (33) $61252101 \quad$ Facsimile: (33)

Telex: $530776 \mathrm{f}$

Email:

Commission(s):

MAVRAGANIS A G PROF, DPT OF ENG SECT OF MECH, NTL TECHN UNIV/5 HEROES, POLYTECH AVE

GR 15773 ZOGRAFOS, GREECE

Telejhone: (30) 16433170

Facsimile: (30)

Telex:

Email:

Commission(s): 07

MAVRIDES STAMATIA DR, OBSERVATOIRE DE PARIS, SECTION DE MEUDON, DPT RADIOASTRONOMIE F 92195 MEUDON PPL CDX, FRANCE

Telephone: (33) $1+5077597$

Facsimile: (33)

Telex:

Email:

Commission(s): 28,47

MAVRIDIS L N PROF, DPT GEODETIC ASTRONOMY, UNIVERSITY THESSALONIKI, UNIV BOX 503 GR $5+006$ THESSALONIKI, GREECE

Telephonc: (30) 31992693

Facsimile: (30)

Telex:

Email:

Commission(s): $08,27,33,46,51$

MAVROMICHALAKI HELEN DR, DPT PHYSICS/UNIVERSITY, NUCLEAR PHYSICS SECTION, 104 SOLONOS ST GR 10680 ATHENS, GREECE

Telephone: (30) 13639439

Email:

Facsimile: (30)

Commission(s): 49

Telex:

MAX CLAIRE E DR, LAWRENCE LIVERMORE LAB, L 413, BOX 808, LIVERMORE CA 94550, USA

Tclephone: (1) $4154225+42$

Email:

Facsimilc: (1)

Commission(s):

Telex: 9103868339 ucllllvmr

MAXWELL ALAN DR, CENTER FOR ASTROPHYSICS, HCO/SAO, 60 GARDEN ST, CAMBRIDGE MA 02138, USA

Telephone: (1) 6174959059

Facsimile: (1)

Telex:

Email:

Commission(s): 10,+0

MAY J, OBS RADIOASTR DE MAIPU, UNIVERSIDAD DE CHILE, CASILlA 68, SANTIAGO, CHILE

Telephone: (56) $2229+101$

Facsimilc: (56)

Telex: +40001

Email:

Commission(s): 40

MAYALL MARGARET W, 5 SPARKS ST, CAMBRIDGE MA 02138, USA

Telephone: (1) 6178761563

Facsimile: (1)

Telex:

Email:

Commission(s): 27

MAYER CORNELL H, 1209 VILLAMAY BLVD, ALEXANDRIA VA 22307, USA

Telephone: (1)

Facsimile: (1)

Telex:

Email:

Commission(s): 16,40 
MAYER PAVEL DR, DPT OF ASTRONOMY, CHARLES UNIVERSITY, SVEDSKA 8, CZ 15O OO PRAHA 5, CZECH R Telephonc: (32) 2540395

Facsimile: (32) 2299272

Telex: $121673 \mathrm{mff}$

Email: mayer(a)csearn

Conmission(s): 25,42

\section{MAYFIELD EARLE B DR, CALIFORNIAN POLYTECHNIC, STATE UNIVERSITY, 1427 BAYVIEW HEIGHTS DR LOS OSOS CA 93403, USA \\ Telephone: (1) 8055285231 \\ Email:

\author{
Facsimile: (1) \\ Telex:
} \\ Commission(s):}

MAYOR MICHEL PROF, OBSERVATOIRE DE GENEVE, CHEMIN DES MAILLETTES 51

CH 1290 SAUVERNY, SWITZERLAND

Telephone: (41) 227552611

Email: mayor(a)obs.unige.ch

Facsimile: (41) $227553983 \quad$ Telex: 419209 obs ch

Commission(s): $30 \mathrm{C}, 33 \mathrm{C}, 37$

MAZA JOSE, DPT DE ASTRONOMIA, UNIVERSIDAD DE CHILE, CASILlA 36 D, SANTIAGO, CHILE

Telephonc: (56) 22294101

Facsimile: (56)

Commission(s): 46 Telex: 440001

Email: masa(a)uchcecvm

Commission(s): 46

MAZEH TSEVI DR, WISE OBSERVATORY, TEL AVIV UNIVERSITY, RAMAT AVIV, TEL AVIV 69978, ISRAEL Telephone: (972) 35450729

Email: bitnet:k23@taunos

Facsimile: (972)

Commission(s): 30,42

Telex: 342171 versy il

MAZURE ALAIN DR, LAB ASTRONOMIE, USTL II, PLACE EUGENE BATAILLON

F 34095 MONTPELLIER CDX 5, FRANCE

$\begin{array}{lll}\text { Telephone: (33) } 671+3468 & \text { Facsimile: (33) } 671+\$ 535 & \text { Telex: } 490944 \\ \text { Email: mazare(g)frmops3 } & \text { Commission(s): } & \end{array}$

MAZUREK THADDEUS JOHN DR, 6920 AVE ROTELLA, SAN JOSE CA 95139, USA

$\begin{array}{ll}\text { Telephone: (1) } & \text { Facsimile: (1) } \\ \text { Email: } & \text { Commission(s): 35,44 }\end{array}$

MAZZITELLI ITALO DR, IAS, CNR, CP 67, I 00044 FRASCATI, ITALY

Telephone: (39) 69421483

Facsimile: (39) 69416847

Telex: 610261 cnrfra

Email:

Commission(s): 35

MAZZONI MASSIMO DR, DPT DI ASTRONOMIA, UNIVERSTIA DI FIRENZE, LARGO E FERMI 5

I 50125 FIRENZE, ITALY

Telephone: (39) 5527521

Facsimile: (39) 55220039

Telex: 572268 arcetr i

Email:

Commission(s):

MAZZUCCONI FABRIZIO DR, OSS ASTROFISICO, DI ARCETRI, LARGO E FERMI S, I 50125 FIRENZE, ITALY
Telephonc: (39) 552752250
Facsimile: (39)

Email:

Commission(s):

MCADAM W BRUCE DR, SCHOOL OF PHYSICS, UNIVERSITY OF SYDNEY, SYDNEY NSW 2006, AUSTRALIA

Telephonc: (61) 26922222

Email:

Facsimile: (61)

Commission(s): 40

Telex: 26169 unisyd

MCALISTER HAROLD A DR, DPT PHYSICS \& ASTRONOMY, GEORGIA STATE UNIVERSITY

ATLANTA GA 303033083 , USA

Telephone: (1) 4046582932

Email: hal@gchara.gsu.edu

Facsimile: (1) $4046512013 \quad$ Telex:

Commission(s): $24,26,51$

MCBREEN BRIAN PHILIP DR, DPT OF PHYSICS, UNIVERSITY COLLEGE, BELFIELD, DUBLIN 4, IRELAND

Telephone: (353) $1693244 \quad$ Facsimile: (353)

Email:

Commission(s): 28,44

Telex: 36293

MCCABE MARIE K MS, 1617 S BERETANIA ST \#801, HONOLULU HI 96826, USA

Telephone: (1) $8089560923 \quad$ Facsimile: (1) 8089882790 Telex:

Email:

Commission(s): 10

MCCALL MARSHALL LESTER DR, DPT OF PHYSICS, YORK UNIVERSITY, 4700 KEELE ST NORTH YORK ON M3J 1P3, CANADA

Telephone: (1) 4167362100

Facsimile: (1) $\$ 167365386$

Telex: 06524736

Email: bitnet:fs300050(a)yusol

Commission(s): 34 
MCCAMMON DAN, DPT OF PHYSICS, UNIVERSITY OF WISCONSIN, 1150 UNIVERSITY AVE

MADISON WI 53706, USA

Telephone: (1) 6082625916

Email:

Facsimile: (1)

Commission(s):

Telex: $265+52$ uofwisc mds

MCCARROLL RONALD PROF, UNIVERSITE DE BORDEAUX, LAB ASTROPHYSIQUE, 123 RUE LAMARTINE

F 33405 TALENCE, FRANCE

Telephone: (33) 56864330

Facsimile: (33) $56404251 \quad$ Telex:

Email:

Commission(s):

MCCARTHY DENNIS D DR, US NAVAL OBSERVATORY, 3450 MASSACHUSETTS AVE NW

WASHINGTON DC 20392 5100, USA

Telephone: (1) 2026530066

Facsimile: (1) 2026520587

Telex: 7108221970

Email: dme(a)maia.usno.navy.mil

Commission(s): 19VP,31

MCCARTHY MARTIN F DR, SPECOLA VATICANA, I 00120 VATICAN CITY, VATICAN CITY STATE

Telephone: (39) 669885266

Email:

Facsimile: (39) 669884671

Telex: $50+2020$ vat obs va

MCCLAIN EDWARD F, $\$ 133$ MAPLE RD, MORNINGSIDE MD 207 $\$ 6$, USA

Telephone: (1) 3017368933

Facsimile: (1)

Tclex:

Email:

Commission(s):

MCCLINTOCK JEFFREY E DR, CENTER FOR ASTROPHYSICS, HCO/SAO, 60 GARDEN ST

CAMBRIDGE MA 02138, USA

Telephone: (1) 6174957136

Facsimilc: (1)

Telex:

Email:

Commission(s):

MCCLURE ROBERT D PROF, HERZBERG INST ASTROPHYS, DOMINION ASTROPHYS OBS, 5071 W SAANICH RD VICTORIA BC V8X 4M6, CANADA

Telephone: (1) 6043880230

Email:

Facsimile: (1) $60+3630045 \quad$ Telex: 0497295

Commission(s): 30,45

MCCLUSKEY GEORGE E JR DR, ASTRONOMY DIV/MATHS DPT, LEIGH UNIVERSITY, BETHLEHEM PA 18015, USA Telephone: (1) 2158613721

Facsimilc: (1)

Telex:

Email:

Commission(s): $\$ 2,+4$

MCCONNELL DAVID DR, CSIRO AUSTRALIA TELESCOPE, NATIONAL FACULTY, BOX 94

NARRABRI NSW 2390, AUSTRALIA

Telephone: (61) 67959205

Facsimile: (61) $67959255 \quad$ Telex:

Email: Imcconne(a)rpepping.0\%.,au

Commission(s): 40

MCCORD THOMAS B DR, PLANETARY GEOSCIENCES DIV, HAWAII INST OF GEOPHYSICS, 2525 CORREA RD HONOLULU HI 96822, USA

Telephonc: (1) 8089566488

Facsimile: (1) 8089882790

Telex:

Email:

Commission(s): 15,16

MCCRAY RICHARD DR, JILA, UNIVERSITY OF COLORADO, BOX $4+10$, BOULDER CO 80309 0440, USA

Telephone: (1) 3034927835

Facsimile: (1)

Telex:

Email: dick@jila.colorado.cdu

Commission(s): $34,4 \mathrm{C}$

MCCREA J DERMOTT, DPT OF MATHS/PHYSICS, UNIVERSITY COLLEGE, BELFIELD, DUBLIN 4, IRELAND

Telephone: (353) 1693244

Facsimile: (353)

Telex:

Email:

Commission(s): $34,35,47$

MCCROSKY RICHARD E DR, CENTER FOR ASTROPHYSICS, HCO/SAO, 60 GARDEN ST, CAMBRIDGE MA 02138, US Telephone: (1) 6174957212

Facsimile: (1)

Telex:

Email:

Commission(s): $15,20,22$

MCCULLOCH PETER M DR, DPT OF PHYSICS, UNIVERSITY OF TASMANIA, GPO BOX 252C

HOBART TAS 7001, AUSTRALIA

Telephone: (61) $2202+20$

Facsimile: (61) $22102+10$

Telex: $\mathbf{5 8 1 5 0}$

Email:

Commission(s): 40 
MCCUTCHEON WILLIAM H PIROF, DPT OF PHYSICS, UNIV OF BRITISH COLUMBIA, 2075 WESBROOK PL VANCOUVER BC VGT 2AG, CANADA

Telephone: (1) $60+2283853$

Facsimile: (1) 6042285324

Telex: 04508576 ubcphysics

Email:

Commission(s):

MCDONALD FRANK B DR, INST PHYSICAL SCI \& TECH, UNIVERSITY OF MARYLAND, RM 3245 CSS BLDG COLLEGE PARK MD 20742, USA

Telephone: (1) 3014054874

Facsimile: (1) 301 $31+9363$

Telex:

Email:

Commission(s):

MCDONALD J K PETRIE DR, 768 RICHMOND AVE, VICTORIA BC V8S 3Z1, CANADA

Telephone: (1) 6015592 6880

Facsimile: (1)

Telex:

Email:

Comminsion(s):

MCDONNELL J A M PROF, UNIT FOR SPACE SCIENCES, UNIVERSITY OF KENT, CANTERBURY CT2 7NR, UK

Telephone: $(+4) 1227+59616$

Facsimile: (4t)

Telex: 965449 ukclib

Email:

Commission(s): $15,21,22$

MCDONOUGH THOMAS R DR, CALTECH, 500 S OAK KNOLL NO 46, PASADENA CA 91101, USA

Telephone: (1) 8187950147

Facsimile: (1)

Telex:

Email:

Commission(s): 51

MCELROY M B DR, DPT OF EARTH \& PLANET SCI, HARVARD UNIVERSITY, G0 GARDEN ST

CAMBRIDGE MA 02138, USA

Telephone: (1)

Facsimile: (1)

Telex:

Email:

Commission(s): 16

MCFADDEN LUCY ANN DR, ASTRONOMY PROGRAM, UNIVERSITY OF MARYLAND, COLLEGE PARK MD 20742, USA

Telephone: (1)

Facsimile: (1)

Commission(s): 15

Telex:

Email:

MCGEE RICHARD X DR, CSIRO, DIV OF RADIOPHYSICS, BOX 76, EPPING NSW 2121, AUSTRALIA

Telephone: (61) 28680222

Facsimile: (61) 28680310

Telex: 26230 astro

Email:

Commission(s): 34

MCGIMSEY BEN Q JR DR, DPT PIIYSICS \& ASTRONOMY, GEORGIA STATE UNIVERSITY

ATLANTA GA 303033083 , USA

Telephone: (1) $40+6582279$ Facsimile: (1) Telex:

Email:

Commission(s):

MCGRAW JOHN T DR, STEWARD OBSERVATORY, UNIVERSITY OF ARIZONA, TUCSON AZ 85721, USA

Telephone: (1) 5206215381

Ficsimilc: (1)

Telex: 467175

Email:

Commission(s): 27

MCGREGOR PETER JOHN DR, MOUNT STROMLO \& SIDING, SPRING OBSERVATORIES, PRIVATE BAG WODEN PO ACT 2611, AUSTRALIA

Telephone: (61) 62881111

Facsimile: (61) $62+90233$

Email:

Commission(s): (09,29,33,34

Telex: 62270 canopus ala

MCHARDY IAN MICHAEL DR, DPT OF PHYSICS, SOUTHAMPTON UNIVERSITY, ASTRO \& SPACE PHYSICS GP SOUTHAMPTON SO' 5 NH, UK

Telephonc: $(4+)$

Facsimile: $(t+)$

Email:

Commission(s):

Telex:

MCINTOSH BRUCE A DR, 25 SEGUIN STR, GLOUCESTER, ONTARIO K1J 6P $\downarrow$, CANADA

Telephone: (1)

Facsimile: (1)

Telex:

Email:

Commission(s): 22

MCINTOSH PATRICK S, NOAA ERL, R/E/SE3, SPACE ENVIRONMENT LAB, 325 BROADWAY

BOULDER CO 80303, USA

Telephonc: (1) $303+973795$

Email: span:0555::pmcintosh

Facsimile: (1)

Telex:

Commission(s): 10 
M 696

MCKEE CHRISTOPHER F PROF, PHYSICS DPT, UNIVERSITY OF CALIFORNIA, BERKELEY CA 94720, USA Telephonc: (1) $\$ 156420805$

Facsimile: (1)

Commission(s): 34

Telex: 820181 uch ast ralud

Email:

MCKEITH CONAL D DR, DPT OF PURE \& APPL PHYS, QUEEN'S UNIVERSITY, BELFAST BT7 1NN, UK Telephone: (44) $1232245133 \quad$ Facsimile: (44)

Email:

Commission(s): 34

Telex: 74487 qub adm

MCKEITH NIALL ENDA DR, DPT OF PHYSICS, ST PATRICK'S COLLEGE, MAYNOOTH, CO KILDARE, IRELAND Telephone: (353) 1285222 Facsimile: (353) Telex:

Email:

Commission(s):

MCKENNA LAWLOR SUSAN, DPT OF EXPERIMENTAL PHYS, ST PATRICK'S COLLEGE, MAYNOOTH CO KILDARE, IRELAND

Telephone: (353) 1285222

Facsimile: (353)

Commission(s): $10,12,15,40,41$

Telex: 31493 spem ei

Email:

MCLAREN ROBERT A DR, INSTITUTE FOR ASTRONOMY, UNIVERSITY OF HAWAII, 2680 WOODLAWN DR HONOLULU HI 96822, USA

Telephone: (1) 8089568768

Email: mclaren@ifa.hawaii.cdu
Facsimile: (1) 8089463467

Conmission(s):
Telex: $8+59$ uhast hr

MCLEAN BRIAN JOHN, STSCI, HOMEWOOD CAMPUS, 3700 SAN MARTIN DR, BALTIMORE MD 21218, USA

Telephone: (1) 3013339101

Email:

Facsimile: (1)

Telex: 6849101 stsci

Commission(s): 05,2t

MCLEAN DONALD J DR, CSIRO, DIV OF RADIOPHYSICS, BOX 76, EPPING NSW 2121, AUSTRALIA

Telephone: (61) 28680222

Facsimile: (61) 28680310

Telex: 26230 astro a2

Email: dmclean@rp.csiro.au

Commission(s): 10,40

MCLEAN IAN S DR, DPT OF ASTRONOMY, UNIVERSITY OF CALIFORNIA, 405 HILGARD AVENUE LOS ANGELES CA 90024, USA

Telephonc: (1) 2138251140

Email: mclean@abonnic.astro.ucla.edu

Facsimile: (1) 2132062096

Telex:

Commission(s): (09C,25C

MCMAHAN ROBERT KENNETH DR, DPT PHYSICS \& ASTRONOMY, UNIVERSITY NORTH CAROLINA, PHILLIPS HALL CB 3255, CHAPEL HILL NC 27599 3255, USA

Telephone: (1) $9199627168 \quad$ Facsimile: (1) 9199620480 Telex:

Email:

Commission(s):

MCMAHON RICHARD, INSTITUTE OF ASTRONOMY, THE OBSERVATORIES, MADINGLEY RD

CAMBRIDGE CB3 OHA, UK

Telephonc: (44) 1223337548

Email: rgm(a)mail.ast.cam.ac.uk

Facsimilc: (4) $1223337523 \quad$ Telex:

Commission(s):

MCMILLAN ROBERT S DR, LUNAR \& PLANETARY LAB, UNIVERSITY OF ARIZONA, SPACE SCIENCES BLDG TUCSON AZ 85721, USA

Telephone: (1) 5206216968

Facsimilc: (1)

Telex:

Email: bob@krzysztof.lpl.arizona.edu

Commission(s): 30

MCMULLAN DENNIS DR, MULLARD RADIO ASTRON OBS, CAVENDISH LABORATORY, MADINGLEY RD CAMBRIDGE CB3 OHE, UK

Telephone: (44) 1223337294

Email:

Facsimile: (4) $1223354599 \quad$ Telex:

Commission(s): 09

MCNALLY DEREK DR, UNIVERSITY OF LONDON OBS, MILL HILL PARK, LONDON NW7 2QS, UK

Telephone: (44) 1819590421

Facsimile: (44) $181819+151$

Telex: 28722 ucphys g

Email: dmn(a)starlink.ucl.ac.uk

Commission(s): 05,34,46C, ,50C

MCNAMARA DELBERT H DR, DPT PHYSICS \& ASTRONOMY, BRIGHAM YOUNG UNIVERSITY

PROVO UT 84602, USA

Tclephone: (1) 8013782298

Facsimile: (1)

Telex:

Email:

Commission(s): $05,27,29,45$ 
MCNAUGHT ROBERT H, SIDING SPRING OBSERVATORY, PRIVATE BAG COONABARABRAN NSW 2357, AUSTRALIA
Telephone: (61) 68426269
Facsimile: (61) $688+2298$
Telex: canopus aa 163945
Email: nssdca::psi\%aaocbn::ukesru
Commission(s): 20

MCWHIRTER R W PETER DR, RUTHERFORD APPLETON LAB, SPACE \& ASTROPHYSICS DIV, BLDG R25/R68 CHILTON DIDCOT OX11 0QX, UK

Telephone: (44) 1235446424

Facsimile: (44)

Commission(s): 14,44

Telex: 83159 ruthlb g

Email:

Facsimile: (44) Telex:

MEABURN J DR, DPT OF ASTRONOMY, UNIVERSITY OF MANCHESTER, MAN
$\begin{array}{ll}\text { Telephone: (44) } & \text { Facsimile: (44) } \\ \text { Email: } & \text { Commission(s): } 34\end{array}$

MEAD JAYLEE MONTAGUE DR, 2700 VIRGINIA AVE NW, APT 701, WASHINGTON DC 20037, USA

Telcphone: (1) 2023380208

Facsimile: (1) $202338+407$ Telex:

Enuil: jmead@ablackholc.aas.org

Commission(s): $05,44,45$

MEADOWS A JACK PROF, DPT ASTRONOMY \& HISTORY, UNIVERSITY OF LEICESTER, UNIVERSITY RD LEICESTER LE1 7RH, UK

Telephone: (44)

Facsimile: (44)

Telex:

Email:

Commission(s): 05,16

MEATHERINGHAM STEPHEN DR, MOUNT STROMLO \& SIDING, SPRING OBSERVATORIES, PRIVATE BAG, WESTON CREEK PO ACT 2611, AUSTRALIA

Telephone: (61) 62490293

Email: sjm(a)mso.anu.edu.au

Facsimile: (61) 62490233

Commission(s): 33

Telex: 62270 canopous aa

MEBOLD ULRICH DR PROF, RADIOASTRONOMISCHES INST, UNIVERSITAET BONN, AUF DEM HUEGEL 71 D 53121 BONN, GERMANY

Telephone: (49)

Facsimile: (49)

Telex:

Email:

Commission(s): 34,40

MEDIAVILLA EVENCIO DR, INST DE ASTROFISICA, DE CANARIAS, OBS DEL TEIDE, E 38200 LA LAGUNA, SPAIN

Telephone: (34) 22262211

Facsimile: (34)

Telex: 92640

Email:

Commission(s): 28

MEDINA JOSE DR, DPT FISICA, UNIVERSIDAD DE ALCALA, APD 20, E 28871 ALCALA DE HENARES, SPAIN

Telephone: (34) 18854940

Facsimile: (34) $1885+953$

Telex:

Email:

Commission(s): $4 t$

MEDVEDEV YURI A DR, ASTRONOMICAL OBSERVATORY, ODESSA STATE UNIVERSITY, SHEVCHENKO PARK

$27001+$ ODESSA, UKRAINE

Telephone: (7) $048228+42$

Facsimile: (7)

Telex:

Email:

Commission(s):

MEECH KAREN DR, INSTITUTE FOR ASTRONOMY, UNIVERSITY OF HAWAII, 2680 WOODLAWN DR HONOLULU HI 96822, USA

Telcphone: (1) 8089566828

Facsimile: (1) 8089882790

Telex: $8+59$ uhast hr

Email: meech@ifa.hawaii.edu

Commission(s): 15

MEEKS M LITTLETON DR, MEEKS ASSOCIATES INC, BOX 643, LINCOLN MA 01773, USA

Telephone: (1) 6172590093

Facsimile: (1)

Telex:

Email:

Commission(s): 40

MEERSON BARUCH DR, RACAH INST OF PHYSICS, HEBREW UNIV OF JERUSALEM, BOX 4040 JERUSALEM 91904, ISRAEL

Telephonc: (972) 2584470

Facsimile: (972) 2584437

Telex: 25391 huil

Email: meerson(a)hujivms

Commission(s): 10

MEGESSIER CLAUDE DR, OBSERVATOIRE DE PARIS, SECTION DE MEUDON, LAM

F 92195 MEUDON PPL CDX, FRANCE

Tclephone: (33) 145077862

Facsimilc: (33)

Telex: 201571

Email:

Commission(s): 29 
MEGEVAND DENIS DR, OBSERVATOIRE DE GENEVE, CHEMIN DES MAILLETTES 51 CH 1290 SAUVERNY, SWITZERLAND

Tclephone: (41) 227552611

Facsimile: (41) 227553983

Commission(s): 09

Telex: 45419209 obsq ch

MEIDAV MEIR DR, SCHOOL OF EDUCATION, TEL AVIV UNIVERSITY, TEL AVIV 69978, ISRAEL

Telephone: (972) 35450840

Facsimilc: (972) 36413944

Telex:

Email:

Commission(s): 46

MEIER DAVID L, JPL, MS 264 700, 4800 OAK GROVE DR, PASADENA CA 91109, USA

Telephone: (1) 2133545062

Facsimile: (1)

Telex: 675429

Email: dlm@cena.jpl.nasa.gov/cena::dl Commission(s): 28,44,40

MEIER ROBERT R, NAVAL RESEARCH LABORATORY, CODE 4140, 4555 OVERLOOK AVE SW

WASHINGTON DC 20375 5000, USA

Telephone: (1) 2027672773

Facsimile: (1)

Telex:

Email:

Commission(s): 34

MEIKLE WILLIAM P S, ASTROPHYSICS GROUP, IMPERIAL COLLEGE, BLACKETT LABORATORY

LONDON SW7 2BZ, UK

Telephone: (44) 171589511

Facsimile: (4t)

Telex: 261503

Email:

Commission(s): 28

MEIKSIN AVERY ABRAHAM DR, CITA MCLENNAN LABS, UNIVERSITY OF TORONTO, 60 ST GEORGE ST TORONTO ON M5S 1A1, CANADA

Telephone: (1) 416978849

Facsimile: (1) $4169783921 \quad$ Telex:

Email: meiksin(a)mouse.cita.utoronto.ca Commission(s): 44

MEIN NICOLE DR, OBSERVATOIRE DE PARIS, SECTION DE MEUDON, DASOP, F 92195 MEUDON PPL CDX, FRANC

Telephone: (33) 145077801

Facsimile: (33)

Telex:

Email:

Commission(s):

MEIN PIERRE, OBSERVATOIRE DE PARIS, SECTION DE MEUDON, F 92195 MEUDON PPL CDX, FRANCE

Telephone: (33) 145077801

Facsimile: (33)

Telex: 270912

Email:

Commission(s): $05,10,12$

MEINEL ADEN B PROF, JPL, MS $186,134,4800$ OAK GROVE DR, PASADENA CA 91109, USA

Telephone: (1) 8183546827

Facsimilc: (1)

Telex:

Email:

Commission(s): 09,24

MEINIG MANFRED DR, INST F ANGEW GEODAESIE, AUSSENSTELLE POTSDAM, MICHENDORFER CHAUSSEE 23 D 14473 POTSDAM, GERMANY

Tclephonc: (49) 331316615

Email: mg@potsdam.ifag.de

Facsimile: (49) $331316602 \quad$ Telex:

Commission(s): 19,31

MEIRE RAPHAEL, ASTRON STERRENWACHE, RIJKSUNIVERSITEIT GENT, WEIDESTR 11 B 9050 EVERGEM, BELGIUM

Telephone: (32) 9153875

Email:

\author{
Facsimilc: (32) \\ Telex:
}

Commission(s): 07

MEISEL DAVID D DR, DPT PHYSICS \& ASTRONOMY, STATE UNIVERSITY COLLEGE, SUNY

GENESEO NY 14454, USA

Telephone: (1) $716245528-$

Telex:

Email: meisel(ä)uno.cc.geneseo.edu

Commission(s): 15,22

MEISENHEIMER KLAUS DR, MPI FUER ASTRONOMIE, KOENIGSTUHL, D 69117 HEIDELBERG, GERMANY

Telephone: (49) 6221528206

Facsimile: (49) 6221528246

Telex: $461789 \mathrm{mpi} \mathrm{d}$

Email: meise@dhdmpisv

Commission(s): 28

MEISTER CLAUDIA VERONIKA, WIP PROJ SPACE PLASMA PHY, INST THEOR PHYS \& ASTROPH AM NEUEN PALAIS 10, D 14469 POTSDAM, GERMANY

Telephone: (49) 3317499327

Facsimile: (49) 3317499309

Telex:

Email:

Commission(s): 49 
MEKARNIA DJAMEL DR, OCA OBSERV DE NICE, BP 229, F 06304 NICE CDX 4, FRANCE

Telephone: (33) 92003011

Facsimile: (33) 92003033

Telex: 460004

Email: mekarnia(a)fronis1

Commission(s):

MEKLER YURI PROF, DPT GEOPHYS \& PLANET SCI, TEL AVIV UNIVERSITY, TEL AVIV, ISRAEL

Telephone: (972) $3413505 \quad$ Facsimile: (972)

Telex:

Email:

Commission(s):

MELBOURNE WILLIAM G DR, JPL, MS 238 540, 4800 OAK GROVE DR, PASADENA CA 91109, USA

Telephone: (1) 8183545071

Facsimile: (1)

Telex:

Email:

Commission(s): 07,19C,31

MELCHIOR PAUL J PROF DIR, OBSERVATOIRE ROYAL DE, BELGIQUE, AVE CIRCULAIRE 3

B 1180 BRUSSELS, BELGIUM

Telejhone: (32) 23752484

Facsimile: (32) 23748822

Telex: 21565 obsbel b

Email:

Commission(s): 08,19,31

MELIA FULVIO DR, DPT PHYSICS \& ASTRONOMY, NORTHWESTERN UNIVERSITY, DEARBORN OBSERVATORY

EVANSTON IL 60208, USA

Telephone: (1) 3124914568

Facsimile: (1)

Telex:

Email: span:nssdca::11340::melia

Commission(s): $+2,+4$

MELIKIAN NORAIR, BYURAKAN OBSERVATORY, $378+33$ BYURAKAN, ARMENIA

Telephonc: (7) $8852283+53 \quad$ Facsimile: (7)

Telex: $2+33+4$ orion su

Email: byurakan $(\mathfrak{a}$ )adonis.ias.msk.su

Commission(s): 28

MELIK-ALAVERDLAN YU DR, BYURAKAN ASTROPHYSICAL, OBSERVATORY, 378433 BYURAKAN, ARMENIA

Telephone: (7) 8852283453

Facsimile: (7) $8852284142 \quad$ Telex:

Email:

Commission(s): 35

MELLIER YANNICK DR, OBS MIDI PYRENEES, 14 AVE E BELIN, F 31400 TOULOUSE CDX, FRANCE

Telephone: (33) 61252101

Facsimile: (33)

Tclex: 530776

Email:

Commission(s): 47

MELNICK GARY J, CENTER FOR ASTROPHYSICS, HCO/SAO, 60 GARDEN ST, CAMBRIDGE MA 02138, USA

Telephone: (1) 6174957388

Facsimile: (1)

Telex:

Email:

Commission(s): $30,34,44$

MELNICK JORGE, DPT DE ASTRONOMIA, UNIVERSIDAD DE CHILE, CASILLA 36 D, SANTIAGO, CHILE

Telephone: (56) $2229+101 \quad$ Facsimile: (56)

Email:

Commission(s):

Telex: 440001

MELOTT ADRIAN L PROF, DPT PHYSICS \& ASTRONOMY, UNIVERSITY OF KANSAS, LAWRENCE KS 66045, USA

Telephone: (1) 9138644626

Facsimile: (1)

Commission(s): 47

Telex:

Email: bitnet:melottajukanvax

MELROSE DONALD B PROF, DPT THEORETICAL PHYSICS, UNIVERSITY OF SYDNEY

SYDNEY NSW 2006, AUSTRALIA

Telephone: (61) 26922222

Facsimile: (61)

Telex:

Email:

Commission(s): 10,12,44,49

MENDES DA COSTA ARACY DR, CRAAE/PTR ESCOLA POLI USP, CP 8174 CEP 05508, 01051 SAO PAULO SP, BRAZIL

Telephone: (55) 118155936

Email: amdcosta(a)brusp.bitnet

Facsimile: (55) 118156289

Telex: 1180127 inpe br

Commission(s): 10

MENDEZ MANUEL DR, INSTITUTO DE ASTRONOMIA, UNAM, APDO POSTAL 70-264, 04510 MEXICO DF, MEXICO

Telephone: (52)

Facsimile: (52)

Telex:

Email:

Commission(s):

MENDEZ MARIANO DR, OBSERVATORIO ASTRONOMICO, PASEO DEL BOSQUE S/N 1900 LA PLATA (BS AS), ARGENTINA

Telephone: (54) 21216357

Email: mmendez@fcaglp.edu.ar

Facsimile: (54) 21211761

Commission(s): 37
Telex: 31151 bulap ar 
M 700

MENDEZ ROBERTO H DR, IAFE, CC 67 SUC 28, 1428 BUENOS AIRES, ARGENTINA

Telephone: (54) $17816755 \quad$ Facsimile: (54) Telex: 22414 cedoc ar

Email: $\quad$ Commission(s): 34

MENDIS DEVAMITTA ASOKA DR, EECS, UCSD, LA JOLLA CA 92093 0216, USA

Telephone: (1) $6194522719 \quad$ Facsimile: (1) Telex:

Email:

Commission(s): 15,49

MENDOZA CLAUDIO, IBM VENEZUELA SCIENT CTR, BOX 388, CARACAS 1010 A, VENEZUELA

Telephone: (58) $29088697 \quad$ Facsimile: (58) Telex: 23283 ibmve ve

Email: $\quad$ Commission(s):

MENDOZA V EUGENIO E DR, INSTITUTO DE ASTRONOMIA, UNAM, APDO POSTAL 20-158 01000 MEXICO DF, MEXICO

Telephone: (52) 6833094

Facsimile: (52)

Email:

Commission(s): $25,45,51$

MENDOZA-TORRES JOSE-ED, INAOE, AP 51/216, PUEBLA PUE 72000, MEXICO

$\begin{array}{lll}\text { Telephone: (52) } & \text { Facsimile: }(52) & \text { Telex: } \\ \text { Email: } & \text { Commission(s): }\end{array}$

MENEGUZZI MAURICE M DR, CEA CEN, DAPNIA/SAP, BP 2, F 91191 GIF/YVETTE CDX, FRANCE

Telephone: (33) 169084438

Facsimile: (33)

Telex: 690860

Email:

Commission(s):

MENG XINMIN, YUNNAN OBSERVATORY, CAS, BOX 110, KUNMING 72946 YUNNAN, CHINA PR

Telephone: (86) 8712035

Facsimile: (86)

Telex: 64040 yuobs en

Email:

Commission(s): 09

MENNESSIER MARIE-ODILE DR, GR DE RECHERCHE EN ASTRON, USTL II GRAAL CCO72

PLACE EUGENE BATALLON, F 34095 MONTPELLIER CDX 5, FRANCE

Telephone: (33) 67143469

Facsimile: (33) 67143031

Telex: 490944 ustmont $f$

Email:

Commission(s): $24,27,33$

MENON T K PROF, DPT GEOPHYS \& ASTRONOMY, UNIV OF BRITISH COLUMBIA, 2219 MAIN MALL

VANCOUVER BC VGT 1ZA, CANADA

Telephone: (1)

Facsimile: (1) Telex:

Email:

Commission(s): $28,34,37,40$

MENTESE HUSEYIN DR, UNIVERSITY OBSERVATORY, UNIVERSITY OF ISTANBUL, 34452 ISTANBUL, TURKEY

Telephone: (90) $15223597 \quad$ Facsimile: (90)

Telex:

Email:

Commission(s):

MENZIES JOHN W DR, SAAO, BOX 9, OBSERVATORY 7935, SOUTH AFRICA

Telephone: (27) $470025 \quad$ Facsimile: $(27) 473639$ Telex:

Email: jwm@saao.ac.za

Commission(s): $25 \mathrm{C}, 34,37,50$

MEN' A V DR, INST RADIOPHYS \& ELECTRON, UKRAINIAN ACAD OF SCIENCE, 310085 KHARKOV, UKRAINE

Telephone: (7)

Facsimile: (7)

Telex:

Email:

Commission(s):

MERAT PARVIZ, INSTITUT D'ASTROPHYSIQUE, 98BIS BD ARAGO, F 75014 PARIS, FRANCE

Telephone: (33) 144328108

Email:

Facsimile: (33) $144328001 \quad$ Telex:

Commission(s): 47

MERAT PARVIZ DR, INSTITUT D'ASTROPHYSIQUE, 98BIS BD ARAGO, F 75014 PARIS, FRANCE

Telephone: (33) 144328108

Facsimile: (33) $14+328001$ Telex:

Email:

Commission(s):

MERCIER CLAUDE DR, OBSERVATOIRE DE PARIS, SECTION DE MEUDON, DASOP F 92195 MEUDON PPL CDX, FRANCE

Telephone: (33) 14507781

Facsimile: (33)

Telex: 201571

Email:

Commission(s): 
MERGENTALER JAN PROF, ASTRONOMICAL INSTITUTE, UL KOPERNIKA 19, PL 51 617 WROCLAW, POLAND

Telephone: (48) 482329

Facsimile: (48)

Telex:

Email:

Commission(s): 10,12

MERIGHI ROBERTO DR, OSS ASTRONOMICO, UNIVERSITA DI BOLOGNA, CP 596, I 40100 BOLOGNA, ITALY

Telephone: (39) 51259401

Facsimile: (39)

Telex: $52063+$ infnbo

Email: span:37929::merighi

Commission(s): $\$ 7$

MERKLE FRITZ DR, CARL ZEISS JENA GMBH, TATZENDPROMENADE 1A, D 07740 JENA, GERMANY

Telephonc: (49) 3614588 2454/5880 Facsimilc: (49) 3614588 2023/5881 Telex: 331545

Email:

Commission(s): 09C

MERLEAU-PONTY J PROF, 5 RUE GL DE CASTELNAU, F 75015 PARIS, FRANCE
Telcphonc: (33)
$\begin{array}{ll}\text { Email: } & \text { Facsimile: (33) } \\ \end{array}$

MERMAN G A DR, INST OF THEORET ASTRONOMY, ACADEMY OF SCIENCES, N KUTUZOVA 10 192187 ST PETERSBURG, RUSSIA

Telcphone: (7)

Facsimilc: $(7)$

Telex:

Email:

Commission(s): 07

MERMAN NATALIA V DR, PULKOVO OBSERVATORY, ACADEMY OF SCIENCES, 10 KUTUZOV QUAY 196140 ST PETERSBURG, RUSSIA

Telephone: (7)

Facsimile: (7)

Telex:

Email:

Commission(s):

MERMILLIOD JEAN-CLAUDE DR, INSTITUT D'ASTRONOMIE, UNIVERSITE DE LAUSANNE

CH 1290 CHAVANNES-D-BOIS, SWITZERLAND

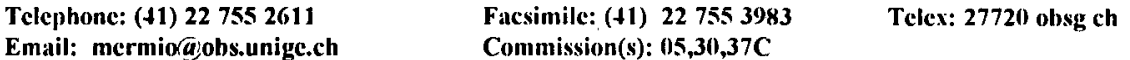

MERRIAM JAMES B, DPT GEOLOGICAL SCIENCES, UNIV OF SASKATCHEWAN

SASKATOON SK S7N OWO, CANADA

Telephone: (1) 3069665716

Facsimile: (1) $3069668593 \quad$ Telex:

Email: merriam(a)geoid.usask.ca

Commission(s): 19

MERTŻ LAWRENCE N DR, 287 FAIRFIELD CT, PALO ALTO CA 94306, USA

Telephone: (1)

Facsimile: (1)

Telex:

Email:

Commission(s): 09

MERZANIDES CONSTANTINOS, ZALOGOU 15, GR 65403 KAVALA, GREECE

Telephone: (30) $5122840 \quad$ Facsimile: (30)

Email:

Commission(s):

MESSAGE PHILIP J DR, DPT APPL MATHS THEOR PHYS, UNIVERSITY OF LIVERPOOL, BOX 147, LIVERPOOL L69 3BX, UK

Telephone: (4t) 1517096022

Facsimile: (44)

Telex: 627095

Email:

Commission(s): 07,20

MESSEROTTI MAURO DR, SOLAR ASTROPHYSICS GROUP, ASTRONOMICAL OBSERVATORY, BASOVIZZA 302 I 34012 TRIESTE, ITALY

Telephone: (39) 40226176

Facsimile: (39) $40226630 \quad$ Telex:

Email: messerotti@oat.ts.astro.it

Commission(s): 10

MESSINA ANTONIO, DPT DI ASTRONOMIA, UNIVERSTIA DI BOLOGNA, CP 596, I 40100 BOLOGNA, ITALY

Telephone: (39) 51222956

Facsimile: (39)

Commission(s):

Telex:

Email:

MESTEL LEON PROF, ASTRONOMY CENTRE, UNIVERSITY OF SUSSEX, FALMER, BRIGHTON BN1 9QH, UK
Telephone: (44) 1273606755
Facsimile: (44)

Email:

Commission(s): $35,44,49$

877159 unisex $\mathrm{g}$

MESZAROS ATTILA DR, DPT ASTRONOMY/ASTROPHYS, CHARLES UNIVERSITY, SVEDSKA 8

CZ 150 00 PRAHA S, CZECH R

Telephone: (42) $25+0395$

Email:

Facsimile: (42) 2299272

Telcx:

Commission(s): 47 
M 702

MESZAROS PETER DR, DPT OF ASTRONOMY, PENNSYLVANIA STATE UNIV, 525 DAVEY LAB UNIVERSITY PARK PA 16802, USA

Telephone: (1) 8148650418

Facsimile: (1)

Telex: $8+2510$

Email:

Commission(s): $34,4+, 47$

METCALFE LEO DR, ESAESTEC, SSD, BOX 299, NL 2200 AG NOORDWIJK, NETHERLANDS

Telephone: (31) $171983616 \quad$ Facsimile: (31)

Telex: 39098

Email: carn:Imetcalf@estec

Commission(s):

METZ KLAUS DR, "NOVALIS-HAUS" ZIMMER 217, GHERSBURGSTR 19, D 83043 BAD AIBLING, GERMANY

Telephone: (49)

Facsimile: (49)

Telex:

Email:

Commission(s): 27

MEURS EVERT DR, MPE, GIESSENBACHSTR, D 85740 GARCHING MUENCHEN, GERMANY

Telephone: (49)

Facsimile: (49)

Telex:

Email:

Commission(s):

MEWE R DR, SPACE RESEARCH LABORATORY, SRON, SORBONNELAAN 2, NL 358+ CA UTRECHT, NETHERLAND

Telephone: (31) 30535600

Facsimile: (31) 30540860

Telex: $\$ 7224$

Email:

Commission(s): $12,14,4+$

MEYER CLAUDE DR, OCA CERGA, AVE COPERNIC, F 06130 GRASSE, FRANCE

Telephone: (33) 93405379

Facsimile: (33) $93405333 \quad$ Telex:

Email: meyer@ocar01.obs-azur.fr Commission(s): 26

MEYER DAVID M DR, DPT PHYSICS \& ASTRONOMY, NORTHWESTERN UNIVERSITY, DEARBORN OBSERVATOR EVANSTON IL 60208, USA

Telephone: (1) 3124914516

Email:

Facsimile: (1)

Telex:

Commission(s): 47

MEYER FRIEDRICH DR, MPI F PHYSIK \& ASTROPHYS, KARL SCHWARZSCHILDSTR 1

D 85740 GARCHING MUENCHEN, GERMANY

$\begin{array}{lll}\text { Telephone: (49) } 8932990 & \text { Facsimile: }(49) & \text { Telex: } 524629 \text { astro d } \\ \text { Email: } & \text { Commission(s): } 12,44 & \end{array}$

MEYER JEAN-PAUL DR, CEA CEN, DAPNIA/SAP, BP 2, F 91191 GIF/YVETTE CDX, FRANCE

Telephone: (33) $169085025 \quad$ Facsimile: (33) Telex: 690860

Email:

Commission(s): 44

MEYERS KARIE ANN, NOAO, BOX 26732, 950 N CHERRY AVE, TUCSON AZ 85726 6732, USA

Telcphone: (1) 5203259202

Facsimile: (1) $5203259360 \quad$ Telex:

Email: kmeyersanoao.edu

Commission(s):

MEYER-HOFMEISTER E DR, MPI F PHYSIK \& ASTROPHYS, KARL SCHWARZSCHILDSTR 1

D 857 $\$ 0$ GARCHING MUENCHEN, GERMANY

$\begin{array}{lll}\text { Telephone: (49) } 8932990 & \text { Facsimile: (49) Telex: } 524629 \text { astro d }\end{array}$

Email:

Commission(s): $35,+2$

MEYLAN GEORGES DR, ESO, KARL SCHWARZSCHILDSTR 2, D 85740 GARCHING MUENCHEN, GERMANY

Telephone: (49) 8932006293

Facsimile: (49) 893202362

Telex:

Email: gmeylan(a)eso.org

Commission(s): $30,37 \mathrm{C}, 47$

MEYNET GEORGES DR, OBSERVATOIRE DE GENEVE, CHEMIN DES MAILLETTES 51

CH 1290 SAUVERNY, SWITZERLAND

Telephone: (41) 227552611

Email: meynet@scsun.unigc.ch

Facsimile: (41) 227553983

Tclex: 419209 obsg ch

MEZGER PETER G PROF, MPI FUER RADIOASTRONOMIE, AUF DEM HUEGEL 69, D 53121 BONN, GERMANY

Telephone: (49) 228525297

Facsimile: (49) 228525229

Tclex: 886440

Email:

Commission(s): $33,34,40$

MEZZETTI MARINO, OAT, BOX SUCC TRIESTE 5, VIA TIEPOLO 11, I 34131 TRIESTE, ITALY

Telephone: (39) $\$ 0793221$

Facsimile: (39)

Telex: 461137 oat $i$

Email:

Commission(s): $\$ 2,47$ 
MIANES PIERRE DR, OBS MIDI PYRENEES, 14 AVE E BELIN, F 31400 TOULOUSE CDX, FRANCE Telephone: (33) 61252101 Facsimile: (33)

Commission(s): 25

Email:

MIAO YONG-KUAN, DPT OF ASTRONOMY, NANJING UNIVERSITY, NANJING 210008, CHINA PR

Telephone: (86)

Facsimile: (86)

Telex:

Email:

Commission(s):

MIAO YONG-RUI, SHAANXI OBSERVATORY, CAS, LINTONG XIAN, SHAANXI, CHINA PR

Telephone: (86) 332255

Facsimile: (86)

Telex: 70121 csao cn

Email:

Commission(s): 31

MICELA GIUSEPPINA DR, OSS ASTRONOMICO, UNIVERSITA DI PALERNO, PALAZZO DEI NORMANNI I 90134 PALERMO, ITALY

Telephone: (39) 916570451

Facsimile: (39) 91488900

Telex: 910402 astrop $i$

Email: astropa(a)ipacuc

Commission(s): 44

MICHALEC ADAM, ASTRONOMICAL OBSERVATORY, JAGIELLONIAN UNIVERSITY, UL ORLA 171

PL 30 244 KRAKOW, POLAND

Telephone: (48) 221817/3856

Facsimile: (48) 12378053

Telex: 32 6203 ujoa pl

Email:

Commission(s): 40

MICHALITSIANOS ANDREW, NASA GSFC, CODE 684.1, GREENBELT MD 20771, USA

Telephonc: (1) 3012866177

Facsimilc: (1)

Telex:

Email:

Commission(s): $10,34,4+S$

MICHALOWSKI TADEUSZ DR, ASTRONOMICAL OBSERVATORY, A MICKIEWICZ UNIVERSITY

UL SLONECZNA 36, PL 60286 POZNAN, POLAND

Telephone: (48) 61679670

Facsimile: (48) 61535535

Telex: $\$ 13260$ uam pl

Email: michastr@aplpuam 11

Commission(s): 15

MICHARD RAYMOND DR, OCA OBSERV DE NICE, BP 229, F 06304 NICE CDX 4, FRANCE

Telephonc: (33) 93890420

Facsimile: (33) 92003033

Telex: 460004 obsnice f

Email:

Commission(s): 12

MICHAUD GEORGES J DR, 250 DU FINISTERE, ST LAMBERT JłS 1P5, CANADA

Telephone: (1) 5143436672

Facsimile: (1)

Telex: 05562425 udemphysas

Email:

Commission(s): 35C,36

MICHEL F CURTIS PROF, DPT PHYSICS \& ASTRONOMY, RICE UNIVERSITY, BOX 1892

HOUSTON TX 77251 1892, USA

Telephone: (1) 7135274925

Facsimile: (1)

Telex: 556457

Email:

Commission(s): 44,49

MICKELSON MICHAEL E DR, DPT OF PHYSICS \& ASTRON, DENISON UNIVERSITY, GRANVILLE OH 43023, USA

Telephone: (1) 6145876467

Facsimile: (1)

Telex:

Email: mickelson(a)denison

Commission(s): 14,16,41

MIDDLEHURST BARBARA M MS, 16567 EL CAMINO REAL, HOUSTON TX 77062, USA

Telephone: (1)

Facsimile: (1)

Telex:

Email:

Commission(s): 16

MIETELSKI JAN S DR, ASTRONOMICAL OBSERVATORY, JAGIELLONIAN UNIVERSITY, UL ORLA 171

PL 30244 KRAKOW, POLAND

Telephone: (48) 48122238

Email:

Facsimile: (48) 12378053

Telex: 326203 ujoa pl

Commission(s):

MIGENES VICTOR, CSIRO ATNF, GPO BOX 3(123, ANU CAMPUS, CANBERRA ACT 2601, AUSTRALIA

Telephone: (61) 62790811

Facsimile: (61) 62790812

Telex:

Email: vmigenes@atnf.csiro.au

Commission(s):

MIGHELL KENNETH JOHN, DPT OF ASTRONOMY, COLUMBIA UNIVERSITY, 538 W 120TH ST

NEW YORK NY 10027, USA

Telephone: (1) 2128546899

Facsimile: (1) 2123169504

Telex:

Email: mighell@figaro.phys.columbia.cdu Commission(s): 
MIGNARD FRANCOIS DR, OCA CERGA, AVE COPERNIC, F 06130 GRASSE, FRANCE

Telephone: (33) $93365849 \quad$ Facsimile: (33) Telex: 470865

Email:

Commission(s): 07

MIHAILA IERONIM PROF, BUCHAREST UNIVERSITY, STR ACADEMIEI 14, R 70109 BUCAREST, RUMANIA

Telephone: (40) $16230819 \quad$ Facsimile: (40)

Imail:

Commission(s):

Telex:

MIHALAS BARBARA R WEIBEL, NCSA, BECKMAN INSTITUTE DRAW 25, 405 MATHEWS AVE

URBANA IL 61801, USA

Telephone: (1)

Facsimile: (1)

Telex:

Email:

Commission(s):

MIHALAS DIMITRI DR, DPT OF ASTRONOMY, UNIVERSITY OF ILLINOIS, 1011 W SPRINGFIELD AVE URBANA IL 61801, USA

Telephone: (1) 2173333090

Facsimile: (1)

Telex:

Email:

Commission(s): 12,36

MIKAMI TAKAO DR, OSAKA GAKUIN UNIVERSITY, 2-36-1 KISHIBE MINAMI, SUITA SHI, OSAKA 564, JAPAN

Telephone: (81) 63818434

Facsimile: (81)

Telex:

Email:

Commission(s):

MIKESELL ALFRED H MR, 8316 WALDNUT RD NE, OLYMPIA WA 9850669550, USA

Telephone: (1) 2064931457

Facsimile: (1)

Telex:

Email:

Commission(s):

MIKHAIL FAHMY I PROF DR, AIN SHAMS UNIVERSITY, FACULTY OF SCIENCES CAIRO UNIVERSITY CAIRO, EGYPT

Telephone: (20) 575887

Facsimile: $(20)$

Telex: 94070 ushms un

Email:

Commission(s): 21

MIKHAL JOSEPH SIDKY PROF, HELWAN OBSERVATORY, HELWAN, EGYPT

$\begin{array}{lll}\text { Telephone: }(20) 780645 / 2683 & \text { Facsimile: }(20) & \text { Telex: } 93070 \text { hiag un } \\ \text { Email: } & \text { Commission(s): } 16 & \end{array}$

MIKHELSON NIKOLAJ N DR, PULKOVO OBSERVATORY, ACADEMY OF SCIENCES, 10 KUTUZOV QUAY 196140 ST PETERSBURG, RUSSIA

Telephone: (7) 2979465

Email:

Facsimile: (7)

Telex:

Commission(s): 09

MIKKOLA SEPPO DR, TURKU UNIVERSITY OBS, TUORLA, SF 21500 PIIKKIO, FINLAND

Telephone: (358) $21435822 \quad$ Facsimile: (358) 2143376

Email:

Commission(s): $07,26,33,37$

Telex: 62638 tyf sf

MIKOLAJEWSKA JOANNA DR, INSTITUTE OF ASTRONOMY, N COPERNICUS UNIVERSITY, UL BARTYCKA 18 PL 00716 WARSAW, POLAND

Telephone: (48) 410041- $\$ 6$

Facsimile: $(48)+10046$

Commission(s): $\$ 2$

Telex: 552234 astr pl

Email: mikolaj(âcamk.edu.pl

MIKULASEK ZDENEK DR, N COPERNICUS OBSERVATORY, \& PLANETARIUM, KRAVI HORA

CZ 61600 BRNO 16, CZECH R

Telephonc: $(42) 5744347$

Facsimile: $(42)$

Telex:

Email:

Commission(s): 29,42

MILANI ANDREA, IST DI MATEMATICA, UNIVERSITA DI PISA, VIA BUONARROTI 2, I 56127 PISA, ITALY

Telephone: (39)

Facsimile: (39)

Telex:

Email:

Commission(s): 07C,15,20

MILANO LEOPOLDO DR, DPT DI SCIENZE FISICHE, UNIVERSITA DI NAPOLI, MOSTRA D OLTREMARE PAD 19 I 80125 NAPOLI, ITALY

Telephone: (39) $817253+47$

Facsimile: (39)

Telex: 720320

Email:

Commission(s): 42

MILES HOWARD G MR, LANE PARK, PTIYNE, ST MINVER, WADEBRIDGE PL27 6PN, UK

Telephone: (44) 1208863153

Facsimile: (4)

Telex:

Emait:

Commission(s): 22 
MILET BERNARD L DR, OCA OBSERV DE NICE, BP 229, F 06304 NICE CDX 4, FRANCE

Telephone: (33) $93890 \$ 20$

Facsimile: (33) 92003033

Telex: 460004 obsnice $f$

Email:

Commission(s): $15,20,28,51$

MILEY G K DR, STERREWACHT, NIELS BOHRWEG 2, BOX 9513, NL 2333 RA LEIDEN, NETHERLANDS

Telephone: (31) 71275849

Facsimile: (31) 71275819

Telex: 39058 astro nl

Email:

Commission(s): 28,40

MILKEY ROBERT W DR, AMERICAN ASTRON SOCIETY, 2000 FLORIDA AVE NW, SUITE 400

WASHINGTON DC 20009, USA

Telephone: (1) 2023282010

Facsimile: (1) $20223+2560 \quad$ Telex:

Email: i:milkey@aas.org

Commission $(s): 12$

MILLAR THOMAS J DR, DPT OF MATHEMATICS, UMIST, BOX 88, MANCHESTER MG0 1QD, UK

Telephone: (14) 1612363311

Facsimile: (4)

Telex: 666094

Email:

Commission(s): 34

MILLER FREEMAN D PROF, DPT OF ASTRONOMY, UNIVERSITY OF MICHIGAN, DENNISON BLDG ANN ARBOR MI 48109 1090, USA
Telephone: (1) 3137643447
Facsimile: (1)
Telex:
Email:
Commission(s): 15

MILLER GUY SCOTT, DPT OF PHYSICS, NORTHWESTERN UNIVERSITY, 2145 SHERIDAN RD

EVANSTON IL 60208, USA

Telephone: (1) 7084918647

Emait: gsmiller(a)casbah.acns.nwu.edu

Facsimile: (1) $7084913135 \quad$ Telex:

Commission(s): 44

MILLER HUGH R PROF, DPT PHYSICS \& ASTRONOMY, GEORGIA STATE UNIVERSITY

ATLANTA GA 303033083 , USA

Telephone: (1) 4046582279

Facsimile: (1)

Telex:

Email:

Commission(s): 28

MILLER JOHN C DR, DPT OF ASTROPHYSICS, UNIVERSITY OF OXFORD, SOUTH PARKS RD OXFORD OX1 3RQ, UK

Telephone: (44) 1865511336

Facsimile: (4)

Telex: 83295 nuclox $\mathbf{g}$

Email:

Commission(s): 44

MILLER JOSEPH S PROF, LICK OBSERVATORY, UNIVERSITY OF CALIFORNIA, SANTA CRUZ CA 95064, USA

Telephone: (1) 4084292135

Facsimile: (1)

Telex:

Email:

Commission(s): $25,28,34$

MILLER RICHARD H DR, ASTRONOMY \& ASTROPHYS CTR, UNIVERSITY OF CHICAGO, 5640 S ELLIS AVE CHICAGO IL 60637, USA

Telephone: (1) 3129628201

Email:

Facsimile: (1)

Telex: 6871133

Commission(s): 28,33

MILLET JEAN DR, LAS, TRAVERSE DU SIPHON, LES TROIS LUCS, F 13012 MARSEILLE, FRANCE

Telephone: (33) 91055900

Facsimile: (33) 91661855

Telex: $\mathbf{4 2 0 5 8 4}$ astrosp $f$

Email:

Commission(s):

MILLIARD BRUNO, LAS, TRAVERSE DU SIPHON, LES TROIS LUCS, F 13012 MARSEILLE, FRANCE

Telephone: (33) 91055900

Facsimile: (33) 91661855

Telex: $\mathbf{4 2 0 5 8}+$ astrosp f

Email: milliard@frlasm51

Commission(s):

MILLIGAN J E, NASA GSFC, INFRARED ASTROPHYSICS BR, GREENBELT MD 20771, USA

Telephone: (1)

Facsimile: (1)

Telex:

Email:

Commission(s):

MILLIKAN ALLAN G MR, 7061 BOUGHTON HILL RD, VICTOR NY 14564, USA

Telephone: (1) 7169249802

Facsimile: (1)

Telex:

Email:

Commission(s): 09

MILLIS ROBERT L DR, LOWELL OBSERVATORY, 1400 W MARS HILL RD, BOX 1149, FLAGSTAFF AZ 86001, USA Telephone: (1) 6027743358

Facsimile: (1)

Telex:

Email:

Commission(s): 15,16,20 
MILLS ALLAN A DR, DPT OF ASTRONOMY, UNIVERSITY OF LEICESTER, UNIVERSITY RD LEICESTER LE1 7RH, UK

Telephone: (44) 1533554455

Email:

Facsimile: (44) Telex:

Commission(s):

MILLS BERNARD Y PROF, SCHOOL OF PHYSICS, UNIVERSITY OF SYDNEY, SYDNEY NSW 2006, AUSTRALIA

Telephone: (61) $26922544 \quad$ Facsimile: (61)

Email:

Commission(s): 28,40

Telex: 26169 unisyd

MILNE DOUGLAS K DR, CSIRO, DIV OF RADIOPHYSICS, BOX 76, EPPING NSW 2121, AUSTRALIA

Tclephone: (61) 28680222

Email: dmilne(a)atnf.csiro.au

Facsimile: (61) 28680310

Telex: 26230 astro

MILOGRADOV-TURIN JELENA, INSTITUTE OF ASTRONOMY, UNIVERSITY OF BELGRADE, STUDENTSKI TRG 16 YU 11000 BEOGRAD, YUGOSLAVIA-SERBIA \& MONTENEGRO

Telepbone: (38) 11638715

Facsimile: (38)

Telex:

Email:

Commission(s): 40

MILONE EUGENE F PROF, DPT OF PHYSICS \& ASTRON, UNIVERSITY OF CALGARY, 2500 UNIVERSITY DR NW CALGARY AB T2N 1N4, CANADA

Telephone: (1) 4032205412

Facsimile: (1) 4032893331

Telex:

Email: milone(a)acs.ucalgary.ca

Commission(s): $25 \mathrm{C}, 27,37,42$

MILONE LUIS A DR, OBSERVATORIO ASTRONOMICO, DE CORDOBA, LAPRIDA 854, 5000 CORDOBA, ARGENTINA

Telephone: (54) 51230491

Facsimile: (54) 51210613

Telex: 51822 bucor ar

Email: milone@astro.edu.ar

Commission(s): 27

MILOVANOVIC VLADETA DR, INSTITUT ZA GEODEZIJU, BULEVAR REVOLUCIJE 73

YU 11000 BEOGRAD, YUGOSLAVIA-SERBIA \& MONTENEGRO

Telephone: (38) Facsimile: (38) Telex:

Email:

Commission(s):

MINAROVJECH MILAN, ASTRONOMICAL INSTITUTE, SLOVAK ACADEMY SCIENCES

SK 059 60 TATRANSKA LOMNI, SLOVAK R

Telephone: (42) $969967866 / 7 / 8 \quad$ Facsimile: (42) $969967656 \quad$ Telex: 78277

Email:

Commission(s): 09

MINESHIGE SHIN DR, DPT OF ASTRONOMY, KYOTO UNIVERSITY, KITASHIRAKAWA SAKYO KU

KYOTO 606, JAPAN

Telephone: (81)

Facsimile: (81)

Telex:

Email: minesige@kusastro.kyoto-u.ac.j Commission(s): 42

MINEVA VENETA DR, DPT OF ASTRONOMY, BULGARIAN ACAD SCIENCES, 72 LENIN BLVD

BG 1784 SOFIA, BULGARIA

Telephone: (359) 2758827

Facsimile: (359)

Telex: 23561

Email:

Commission(s): 28

MINGALIEV MARAT, SPECIAL ASTROPHYSICAL OBS, NIZJNIJ ARKHYZ, KARACHAI KARACHAI

CHERKESSIAN 357147, RUSSIA

Telephone: (7)

Email: marat(a)sao.stavropol.su

Facsimile: (7) $8711406337 \quad$ Telex: 123060 sao ru

Commission(s):

MINH YOUNG CHOL, KOREA ASTRONOMY OBS, 36 WHAAMDOG, YUSEONGGU, TAEJON 305 348, KOREA R

Telephone: (82)

Facsimile: (82)

Commission(s):

Telex:

Email: minh@hanul.issa.re.kr

ASTROPHYSICS, BUKHORO STR 22, 734042 DUSHANBE, TAJIKISTAN

MINIKULOV NASRIDIN K, INST OF ASTROPHYSICS, BUKHORO STR 22, 734047
Telephone: (7) $3772274351 \quad$ Facsimile: (7)

Email: $\quad$ Commission(s): 27

MININ I N PROF, ASTRONOMICAL OBSERVATORY, ST PETERSBURG UNIVERSITY, BIBLIOTECHNAJA PL 2 198904 ST PETERSBURG, RUSSIA

Telephone: (7) 2579489

Facsimile: (7)

Telex:

Email:

Commission(s): 34 
MINN YOUNG KEY DR, DPT ASTRONOMY SPACE SCI, KYUNG HEE UNIVERSITY, YONG IN KUN KYUNGGI DO 170) 73, KOREA R

Telephone: $(82) 276+6131$

Facsimile: (82)

Telex:

Email:

Commission(s): 34,51

MINNET HARRY C MR, CSIRO, DIV OF RADIOPHYSICS, BOX 76, EPPING NSW 2121, AUSTRALIA

Telephone: (61) 28680222

Facsimile: (61) 28680310

Telex:

Email:

Commission(s):

MINNITI DANTE, ESO, KARL SCHWARZSCHILDSTR 2, D 85740 GARCHING MUENCHEN, GERMANY

Telephone: (49) $8932006532 \quad$ Facsimile: (49) 893202362 Telex:

Email: dante(ajeso.org Commission(s):

MINTZ BLANCO BETTY MRS, CERRO TOLOLO, INTERAMERICAN OBSERVATORY, CASILLA G03

LA SERENA, CHILE

Telephone: (56) $51213352 \quad$ Facsimile: (56) $51212466 * 3+2 \quad$ Telex: 620 301 aura ct

Email:

Commission(s): 20,25

MIOC VASILE DR, ASTRONOMICAL OBSERVATORY, STR CIRESILOR 19, R 3400 CLUJ NAPOCA, RUMANIA Telephone: $(+0) 6+19+592$

Facsimile: (40)

Telex:

Email:

Commission(s): (07

MIRABEL IGOR FELIX DR, CEA CEN, DAPNIA/SAP, BP 2, F 9119 I GIF/YVETTE CDX, FRANCE

Telephonc: (33) $169083492 \quad$ Facsimile: (33) $169086577 \quad$ Telex: 604860

Email: 32773 ::mirabcl

Commission(s): $28,33,41,51$

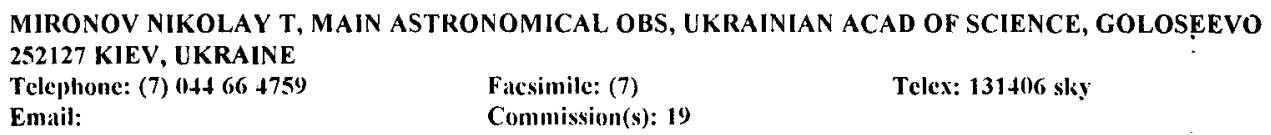

Telex: 131406 sky

MIRZOYAN L V DR PROF, BYURAKAN ASTROPHYSICAL, OBSERVATORY, 378433 BYURAKAN, ARMENIA Telephonc: (7) 8852283453 Facsimile: (7) $885228+142 \quad$ Telex:

Email:

Commission(s): 27,33

MISCONI NEBIL YOUSIF DR, FIT, 328 W HIBISCUS, MELBOURNE 32901, USA

Telephone: (1) 40776880011 Facsimile: (1) Telex:

Email:

Commission(s): 21,22

MISNER CHARLES W PROF, ASTRONOMY PROGRAM, UNIVERSITY OF MARYLAND

COLLEGE PARK MD 207+2, USA

Telephone: (1)

Facsimile: (1)

Telex:

Email:

Commission(s): 47

MISSANA MARCO DR, OSS ASTRONOMICO DI BRERA, VIA CREMAGNANI 13/11, I 20159 VIMERCATE, ITALY
Telephonc: (39)
$\begin{array}{ll}\text { Email: } & \text { Facsimile: (39) } \\ \text { Commission(s): } 30\end{array}$

MISSANA NATALE PROF, VIA PUCCINI 2, I 10025 PINO TORINESE, ITALY

Telephone: (39) Facsimile: (39) Telex:

Email:

MITALAS ROMAS ASSOC PROF, DPT OF ASTRONOMY, UNIV OF WESTERN ONTARIO

LONDON ON NGA 3K7, CANADA

Telephone: (1) 519679318

Fatcsimile: (1) 5196613486

Telex: $06+7134$

Email:

Commission(s): 35

MITCHELL GEORGE F DR, DPT OF ASTRONOMY, ST MARY'S UNIVERSITY, HALIFAX NS B3H 3C3, CANADA Telephonc: (1) $902+299780$

Email:

Facsimile: (1) $902+205561 \quad$ Telex:

Commission(s): 34

MITCHELL KENNETH J DR, GENERAL SCIENCES CORP, G100 CHEVY CHASE DR, LAUREL MD 20707, USA

Telephone: (1) 3015137815

Facsimile: (1)

Telex:

Email: mitchelläaruha.gsfc.nasa.gov

Commission(s): t0 
MITCHELL PETER, SCHOOL OF PHYSICS, UNIV OF NEW SOUTH WALES, SYDNEY NSW 2052, AUSTRALIA Telephone: (61) 23855168 Facsimile: (61) Telex:

Email: Commission(s): 9

MITCHELL WALTER E JR, DPT OF ASTRONOMY, OHIO STATE UNIVERSITY, 174 W 18TH AVE COLUMBUS OH 432101106 , USA

Telephone: (1) 6144225554

Facsimile: (1)

Telex:

Email:

Commission(s):

MITIC LJUBISA A DR, ASTRONOMICAL OBSERVATORY, VOLGINA 7

YU 11050 BEOGRAD, YUGOSLAVIA-SERBIA \& MONTENEGRO

Telephone: (38) $11419357 / 421875$

Facsimile: (38)

Telex:

Email:

Commission(s): 08

MITRA A P DR, NTL PHYSICAL LABORATORY, HILLSIDE RD, NEW DELHI 110 012, INDIA

Telephone: (91) 11585 298/440 Facsimile: (91)

Email:

Commission(s)

Telex: $3162+54$ rsd in

MITROFANOVA LYUDMILA A DR, PULKOVO OBSERVATORY, ACADEMY OF SCIENCES, 10 KUTUZOV QUAY 196140 ST PETERSBURG, RUSSIA

Telephone: (7)

Facsimile: (7)

Telex:

Email:

Commission(s):

MITSUDA KAZUHISA DR, ISAS, 3-1-1 YOSHINODAI, SAGAMIHARA, KANAGAWA 229, JAPAN

Telephone: (81) 427513911

Facsimile: (81) $427594253 \quad$ Telex: j34757 isastro

Email:

Commission(s):

MITTON JACQUELINE, 8A CANTERBURY ClOSE, CAMBRIDGE CB $43 Q Q$, UK

Telephone: (44) $1223355924 \quad$ Facsimile: (44) Telex:

Email:

Commission(s):

MITTON SIMON DR, CAMBRIDGE UNIV PRESS, SHAFTSBURY RD, CAMBRIDGE CB2 2RU, UK

Telephone: (44) 1223312393

Facsimilc: (4)

Email: sam11@phx.cam.ac.uk

Commission(s): 05

Telex: 817256 cupcam uk

MIYAJI SHIGEKI DR, COLLEGE OF ARTS \& SCIENCE, CHIBA UNIVERSITY, 1-33 YAYOICHO, CHIBA 260, JAPAN

Telephone: (81) 472511111

Facsimile: (81)

Telex:

Email:

Commission(s): $\mathbf{3 5 , 4 2 , 4 4}$

MIYAMA SYOKEN, DPT OF PHYSICS, KYOTO UNIVERSITY, KITASHIRAKAWA SAKYO KU, KYOTO 606, JAPAN

Telephone: (81) 757512111

Facsimile: $(\mathbf{8 1})$

Telex:

Email:

Commission(s): 34

MIYAMOTO MASANORI DR, TOKYO ASTRONOMICAL OBS, NAOJ, OSAWA MITAKA, TOKYO 181, JAPAN

Telephone: (81) 422325111

Facsimile: (81) 422321924

Telex: 2822307 taomtk j

Email:

Commission(s): $08,24,33$

MIYAMOTO SIGENORI PROF, DPT OF PHYSICS/FAC SCI, OSAKA UNIVERSITY, MACHIKANEYAMA

TOYONAKA OSAKA 560, JAPAN

Telephone: (81) 68441151

Email:

Facsimile: (81)

Telex:

Commission(s): $16,36,44$

MIZUNO AKIRA, DPT OF ASTROPHYSICS, NAGOYA UNIVERSITY, FUROCHO CHIKUSA KU

NAGOYA 46401, JAPAN

Telephone: (81) 527816769

Facsimile: (81) $5278206+7 \quad$ Telex:

Email: mizuno@a.phys.nagoya-u.ac.jp Commission(s): 40

MIZUNO SHUN, KANAZAWA TECHNOLOGY INST, 7-1 OGIGAOKA, NONOICHIMACHI, ISHIKAWA 921, JAPAN

Telephone: (81) 762481100

Facsimile: (81)

Telex: 5122456 kitlc j

Email:

Commission(s): 34

MIZUNO TAKAO, TOKYO GAKUGEI UNIVERSITY, KOGANEI CITY, TOKYO 184, JAPAN

Telephone: (81) 423252111

Facsimile: (81) $\$ 23249832$

Telex:

Email: mizuno@yamabuki.u-gakugci.ac.jp

Commission(s): 28 
MNATSAKANIAN MAMIKON A DR, BYURAKAN ASTROPHYSICAL, OBSERVATORY, 378433 BYURAKAN, ARMENIA Telephone: (7) 8852283453 Facsimilc: (7) $885228+1+2$ Telex:

Email:

Commission(s): 36

MO JING-ER, PURPLE MOUNTAIN OBSERV, CAS, NANJING, CHINA PR

Telephone: (86) 2536967

Facrimile: (86) 25301459

Telex: 3\$144 pmonj en

Email:

Commission(s): 34

MOCHKOVITCH ROBERT DR, INSTITUT D'ASTROPHYSIQUE, 98BIS BD ARAGO, F 75014 PARIS, FRANCE

Telephone: (33) 144328187

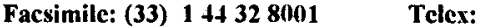

Email: bitnet:mochko@rriap 51

Commission(s):

MOCHNACKI STEPHAN W DR, DAVID DUNLAP OBSERVATORY, UNIVERSITY OF TORONTO, GO ST GEORGE ST TORONTO ON M5S 1A1, CANADA

Telephone: (1) 4169782016

Facsimile: (1) 4169783921

Telex: 06986766

Email:

Commission(s): 42

MODALI SARMA B DR, SM SYSTEMS \& RESEARCH C0,8401 CORPORATE DR, LANDOVER MD 20785, USA

Telephone: (1) 3014593322

Facsimile: (1)

Telex:

Email:

Commission(s):

MODISETTE JERRY L PROF, 18323 HEREFORD LN, HOUSTON TX 77058, USA

Telephone: (1)

Facsimile: (1)

Telex:

Email:

Commission(s): 4

MOEHLER SABINE, LANDESSTERNWARTE, KOENIGSTUHL, D 69117 HEIDELBERG, GERMANY

Telephonc: (49) 6221509255

Facsimile: (49) 6221509202

Telex:

Email: smochler@mail.lsw.uni-heidelberg.de

Commission(s):

MOEHLMANN DIEDRICH PROF, INST F RAUMSIMULATION, POSTFACH 9060 58, D 51140 KOELN, GERMANY

Telephone: (49) 22036013205

Facsimile: (\$9) $2203601209+/ 2352$ Telex: 88100 der d

Email: dirk.mochlmann(@)europa.rs.kp.dl Commission(s): 15,16

MOELLENHOFF CLAUS DR, LANDESSTERNWARTE, KOENIGSTUHL, D 69117 HEIDELBERG, GERMANY

Telephone: (49) 6221509210

Facsimile: (49) 6221509202

Telex:

Email: cmocllen@hp2.Isw.uni-heidelberg.de

Commission(s): 35

MOELLER PALLE, STSCI, HOMEWOOD CAMPUS, 3700 SAN MARTIN DR, BALTIMORE MD 21218, USA

Telephone: (1)

Facsimile: (1)

Telex:

Email:

Commission(s):

MOERDIJK WILLY G DR, STERREKUNDIG OBSERV, ST PIETERSAALSTSTRAAT 171, B 9000 GENT, BELGIUM

Telephone: (32) 91221233

Facsimile: (32)

Telex:

Email:

Commission(s):

MOESGAARD KRISTIAN P, HISTORY OF SCIENCE INST, UNIVERSITY OF AARHUS, BYGADAN 1/ TORRILD

DK 8300 ODDER, DENMARK

Telephone: (45) 86531004

Facsimile: (45)

Telex:

Email:

Commission(s): 41

MOFFAT ANTHONY F J DR, DPT DE PHYSIQUE, UNIVERSITE DE MONTREAL, CP 6128 SUCC A MONTREAL QC H3C 3J7, CANADA

Telephone: (1) 5143436682

Email:

Facsimile: (1) $51+3+32071$

Telex: 05562425 udemphysas

Commission(s): $29,33,37$

MOFFAT JOHN W DR, DPT OF PHYSICS, UNIVERSITY OF TORONTO, 60 ST GEORGE ST

TORONTO ON M5S 1A1, CANADA

Telephone: (1) 4169782949

Facsimile: (1) 4169783921

Telex: 06986766

Email:

Commission(s):

MOFFATT HENRY KEITH PROF, DPT APPLIED MATHS, \& THEORETICAL PHYSICS, SILVER STREET

CAMBRIDGE CB3 9EW, UK

Telephone: (44) 1223337900

Facsimilc: (44) 1223337918

Commission(s):

Telex: 81249 camspl g

Email: 
MOFFETT THOMAS J PROF, DPT OF PHYSICS, PURDUE UNIVERSITY, WEST LAFAYETTE IN 47907, USA

Telephonc: (1) 3174945508

Facsimile: (1)

Telex:

Email: moffett(a)physics.purdue.edu

Commission(s): 25C,27

MOGILEVSKIJ EH I DR, IZMIRAN, ACADEMY OF SCIENCES, 142092 TROITSK, RUSSIA

Telephone: (7) 2321931

Facsimile: (7)

Telex: 412623 scstp su

Email:

Commission(s): 10

MOHAN CHANDER DR, DPT OF MATHEMATICS, UNIVERSITY OF ROORKEE, ROORKEE 247 667, INDIA

Telephone: (91)

Facsimile: (91)

Telex:

Email:

Commission(s): $26,27,35$

MOHAN RAO D, INDIAN INSTITUTE OF, ASTROPHYSICS, KORAMANGALA, BANGALORE 560 034, INDIA

Telephone: (91) 805530672

Email: mohan@iap.ernet.in

Facsimile: (91) $80553+043 \quad$ Telex:

Commission(s): 36

MOHAN VIJAY DR, UTTAR PRADESH STATE, OBSERVATORY, PO MANORA PEAK 263129

NAINITAL 263 129, INDIA

Telephone: (91) 59422136

Facsimile: (91)

Email:

Commission(s): 37

Telex:

MOHD ZAMBRI ZAINUDDIN DR, CENTRE FOR FOUNDATION, UNIVERSITY OF MALAYA 59100 KUALA LUMPUR, MALAYSIA

Telephone: (60) 37552744

Facsimile: (60) 37573661

Telex: ma39845

Email:

Commission(s):

MOISEEV I G DR, CRIMEAN ASTROPHYS OBS, UKRAINIAN ACAD OF SCIENCE, NAUCHNY 334413 CRIMEA, UKRAINE

Telephone: (7) 432945

Facsimilc: (7)

Telex:

Email:

Commission(s): 10,40

MOLARO PAOLO DR, OAT, BOX SUCC TRIESTE 5, VIA TIEPOLO 11, I 34131 TRIESTE, ITALY

Telephone: (39) 40309342

Facsimilc: (39)

Commission(s): 29

Telex: 461137 oat i

Email:

CAL OBSERVATORY, ST PETERSBURG UNIVERSITY

MOLCHANOV A P PROF, ASTRONOMICAL OBSERVATORY,
BIBLIOTECHNAJA PL 2, 199178 ST PETERSBURG, RUSSIA

Telephone: (7)

Facsinile: (7)

Telex:

Email:

Commission(s): 40

MOLES MARIANO J DR, INST ASTROFISICA, DE ANDALUCIA APD 3004, PROFESOR ALBAREDA 1 E 18080 GRANADA, SPAIN

Telephone: (34) 58121311

Email:

Facsimilc: (34)

Commission(s): 28

Telex: 78573 iaag e

MOLINA ANTONIO, INST ASTROFISICA, DE ANDALUCIA APD 3004, PROFESOR ALBAREDA 1

E 18080 GRANADA, SPAIN

Telephone: (34) 58121300

Facsimile: (34)

Commission(s): 16

Telex: 78573 iaag e

Email:

MOLNAR MICHAEL R PROF, MOLNAR TECHNOLOGIES, 3 STONINGHAM DR, WARREN NJ 07060, USA

Telephone: (1) 2015801404

Facsimile: (1)

Telex:

Email:

Commission(s):

MOMCHEV GOSPODIN, ASTRONOMICAL OBSERVATORY, BOX 7, BG 8800 SLIVEN, BULGARIA

Telephone: (359) $4 \$ 2720+$

Facsimilc: (359)

Telex:

Email:

Commission(s): 46

MONAGHAN JOSEPH J DR, DPT OF MATHEMATICS, MONASH UNIVERSITY, WELLINGTON RD CLAYTON VIC 3168, AUSTRALIA

Telephone: (61) 35412563

Facsimile: (61)

Telex: monash aa 32691

Email:

Commission(s): 35

MONET ALICE K B DR, US NAVAL OBSERVATORY, FLAGSTAFF STATION, BOX 1149, FLAGSTAFF AZ 86002, USA

Telephone: (1) 6027795132

Facsimile: (1) $60277+3626$

Telex:

Email: alice@nofs.navy.mil

Commission(s): 19,20 
MONET DAVID G, US NAVAL OBSERVATORY, FLAGSTAFF STATION, BOX 1149, FLAGSTAFF AZ 86002, USA

Telephone: (1) 6927795132

Facsimile: (1)

Telex:

Email: percy::dmonet

Commission(s): $24,33,44$

MONFILS ANDRE G PROF, IAL SPACE, UNIVERSITE DE LIEGE, AVE DU PRE AILY

B 4900 ANGLEUR-LIEGE, BELGIUM

Telephone: (32) 41676668

Facsimile: (32)

Commission(s): 44

Telex: $\mathbf{4 1 3 2 0}$ ial sp b

Email:

MONIN JEAN-LOUIS DR, OBSERVATOIRE DE GRENOBLE, CERMO/ASTROPHYSIQUE, BP 53X F 38041 S MARTIN HERES CD, FRANCE

Telephone: (33) $76514786 \quad$ Facsimile: (33) $76448821 \quad$ Telex: $980134 \mathrm{f}$

Email: monin@frgag51/gagvx3::monin Commission(s): 34

MONNET GUY J DR, OBSERVATOIRE DE LYON, AVE CHARLES ANDRE, F 69561 S GENIS LAVAL CDX, FRANCE

Telephone: (33) 78560705

Facsimile: (33) 72399791

Telex: 310926

Email:

Commission(s): 33

MONSIGNORI FOSSI BRUNA DR, OSS ASTROFISICO, DI ARCETRI, LARGO E FERMI 5, I 50125 FIRENZE, ITALY

Telephone: (39) 552752239

Facsimile: (39)

Telex:

Email:

Commission(s): 10,12

MONTEIRO TANIA S DR, DPT OF MATHEMATICS, ROYAL HOLLOWAY COLLEGE, UNIVERSITY OF LONDON EGHAM SURREY TW20 0EX, UK

Telephone: (44) $178434455 * 3106$

Email: as10703@uicc

Facsimile: (44)

Telex:

MONTES CARLOS DR, OCA OBSERV DE NICE, BP 229, F 0630 4 NICE CDX 4, FRANCE

Telephone: (33) 93890420

Facsimile: (33) 92003033

Telex: $\mathbf{4 6 0 0 0 4}$ obsnice $f$

Email:

Commission(s):

MONTMERLE THIERRY DR, CEA/DAPNIA/SAP, CTR D'ETUDES DE SACLAY, F 91191 GIF/YVETTE CDX, FRANCE

Telephone: (33) 169085722

Facsimile: (33) 169089266

Telex:

Email: montmerle@sapvig.saclay.cea.fr Commission(s): $3+$

MOOK DELO E PROF, DPT OF PHYSICS \& ASTRON, DARTMOUTH COLLEGE, HANOVER NH 03755, USA

Telephone: (1) 6036462972

Facsimile: (1)

Telex:

Email;

Commission(s):

MOON SHIN HAENG DR, ISSA, 36-1 WHAAM DONG, YUSEONG GU, DAEJEON 305 348, KOREA R

Telephone: (82) 428611497

Facsimile: (82) $\$ 28815610 \quad$ Telex:

Email:

Commission(s): 44

MOONS MICHELE B M M, DPT DE MATHEMATIQUE, FACULTES UNIVERSITAIRES, REMPART DE LA VIERGE 8 B 5000 NAMUR, BELGIUM

Telephone: (32) $81229061^{\star 2} 2+38 \quad$ Facsimile: (32) $81230391 \quad$ Telex: 59222 facnam b

Email:

Commission(s): 07,20

MOORE DANIEL R DR, DPT OF MATHEMATICS, IMPERIAL COLLEGE, HUXLEY QUEEN'S GATE

LONDON SW7 2BZ, UK

Telephone: (44)

Facsimile: (44)

Commission(s): 35

Telex:

Email:

MOORE ELLIOTT P PROF, JOINT OBSERVATORY, NEW MEXICO TECH, CAMPUS STATION

SOCORRO NM 87801, USA

Telephone: (1) 5058355431

Facsimile: (1)

Telex:

Email:

Commission(s): 15

MOORE PATRICK DR, FARTHINGS, 39 WEST ST, SELSEY SUSSEX, UK

Telephonc: (44) 1243603668

Facsimile: (44)

Commission(s): 16

Tclex:

Email:$$
\text { Commission(s): } 16
$$

MOORE RONALD L DR, NASA/MSFC, CODE ES 52, SPACE SCIENCE LAB, HUNTSVILLE AL 35812, USA

Telephone: (1) 2054530118

Facsimile: (1)

Telex: 59-4416 nasa msfc ht

Email:

Commission(s): 12 
MOORHEAD JAMES M DR, DPT OF ASTRONOMY, UNIV OF WESTERN ONTARIO, LONDON ON N6A 3K7, CANADA

Telephone: (1) $5196792111 * 6712$

Facsimile: (1) 5196612009

Telex:

Email: moorhead@phobos.astro.uwo.ca Commission(s):

MOORWOOD ALAN F M, ESO, KARL SCHWARZSCHILDSTR 2, D 85740 GARCHING MUENCHEN, GERMANY

Telephone: (49) 8932006294

Facsimilc: (49) 893202362

Telex: 052828224 co d

Email:

Commission(s): 28

MOOS HENRY WARREN DR, DPT PHYSICS \& ASTRONOMY, JOHNS HOPKINS UNIVERSITY, CHARLES \& 34TH ST BALTIMORE MD 21218, USA

Telephone: (1) 3013387337

Facsimilc: (1)

Email:

Commission(s): 29,44

Telex: 7102341090

MORALES-DURAN CARMEN, LAB DE ASTROFISICA ESPACI, FISICA FUNDAMENTAL LAEFF, APD 50727

E 28080 MADRID, SPAIN

Telcphone: (34) 16750700

Facsimile: (34)

Email:

Commission(s):

Telex: 22026 inta e

MORAN JAMES M DR, CENTER FOR ASTROPHYSICS, HCO/SAO, 60 GARDEN ST, CAMBRIDGE MA 02138, USA

Telephone: (1) 6174957477

Facsimile: (1) $617 \$ 977345$

Telex: 7103206842 astr

Email: moran(a)cfa.harvard.edu

Commission(s): 40VP

MORANDO BRUNO L DR, BUREAU DES LONGITUDES, 77 AVE DENFERT ROCHEREAU, F 75014 PARIS, FRANCE

Telephone: (33) 140512276

Facsimile: (33) 14633283

Telex:

Email: morando@bdl.fr

Commission(s): 04,07,20,41

MORBEY CHRISTOPHER L, HERZBERG INST ASTROPHYS, DOMINION ASTROPHYS OBS, 5071 W SAANICH RD VICTORIA BC V8X 4M6, CANADA

Telephone: (1) 6043880220

Email:

Facsimile: (1) $60 \$ 3630045 \quad$ Telex: 0497295

Commission(s): 26,30

MORBIDELLI LORENZO, CAISMI CNR, LARGO E FERMI 5, I 50125 FIRENZE, ITALY

Telephone: (39) Facsimile: (39)

Telex:

Email: lorenzo@arcetri.astro.it

Commission(s):

MORBIDELLI ROBERTO, OSSERVATORIO ASTRONOMICO, DI TORINO, STR OSSERVATORIO 20

I 10025 PINO TORINESE, ITALY

Telephone: (39) 114619034

Facsimile: (39) 114619030

Telex: 213236 toastr i

Email: morbidelti@astto2.astro.it

Commission(s): 5,26

MORCOS ABD EL FADY B, HALWAN OBSERVATORY, PO BOX +71, CAIRO MAADI 11728, EGYPT

Telephone: (20) 202789645

Facsimile: (20) 202782683

Telex: 93070 hiag un

Email: astro@frcu.eun.eg

Commission(s):

MORDECAI-MARK MAC LOW, ASTRONOMY \& ASTROPHYS CTR, UNIVERSITY OF CHICAGO, 5640 S ELLIS AVE CHICAGO IL 60615, USA

Telephone: (1) 3127020598

Email: mordecai $\left(a_{\text {jets.uchicago.edu }}\right.$

Facsimile: (1) 3127028212

Telex:

MOREELS GUY DR, OBSERVATOIRE DE BESANCON, 41 BIS AVE OBSERVATOIRE

F 25000 BESANCON CDX, FRANCE

Telephone: (33) 81502266

Telex: 361144 f

Email:

Commission(s): 46

MOREL PIERRE JACQUES DR, OCA OBSERV DE NICE, BP 229, F 06304 NICE CDX 4, FRANCE

Telephone: (33) 93890420

Facsimile: (33) 92003033

Telex: 460004 obsnice f

Email:

Commission(s): 26

MORENO CORRAL MARCO A DR, OBS ASTRONOMICO NACIONAL, UNAM, APDO POSTAL 877 22830 ENSENADA B CALIF, MEXICO

Teicphonc: (52) 66744580

Facsimile: (52) 66744607

Telex: 56539 ciceme

Email:

Commission(s): 34 
MORENO EDMUNDO DR, INSTITUTO DE ASTRONOMIA, UNAM, APDO POSTAL 70-264, 04510 MEXICO DF, MEXICO Telephone: (52) 5255485306 Facsimile: (52) $5255483712 \quad$ Telex:

Email:

Commission(s): 28

MORENO FERNANDO DR, INST ASTROFISICA, DE ANDALUCIA APD 3004, PROFESOR ALBAREDA 1

E 18080 GRANADA, SPAIN

Tclephone: (34) 58121311

Facsimile: (34) 58814530

Telex: 78573 iaag c

Email: 16488::fernando

Commission(s): 16

MORENO HUGO PROF, DPT DE ASTRONOMIA, UNIVERSIDAD DE CHILE, CASILLA 36 D, SANTIAGO, CHILE

Telephone: (56) $22294101 / 4002$

Facsimile: (56)

Telcx: 440001

Email:

Commission(s): 25

MORENO-INSERTIS FERNANDO, INST DE ASTROFISICA, DE CANARIAS, OBS DEL TEIDE

E 38200 LA LAGUNA, SPAIN

Telephone: (34) 22605200

Facsimile: (34) $22605210 \quad$ Telex:

Email: fmi@iac.es

Commission(s): 10C,12

MORETON G E, 155 THE ESPLANADE, BALMORAL BEACH NSW 2088, AUSTRALIA

Telephone: (61)

Facsimile: (61)

Telex:

Email:

Commission(s): 10

MORGAN BRIAN LEALAN, ASTROPHYSICS GROUP, IMPERIAL COLLEGE, BLACKETT LABORATORY

LONDON SW7 2BZ, UK

Telephone: (44) $1715895111 \quad$ Facsimile: (44)

Email:

Commission(s): 09

Telex: 261503

MORGAN DAVID H DR, ROYAL OBSERVATORY, BLACKFORD HILL, EDINBURGH EH9 3HJ, UK

Telephone: (\$4) 1316673321

Facsimile: (4t)

Telex: 72383 roedin $\mathrm{g}$

Email:

Commission(s): $21,3 \uparrow$

MORGAN JOHN ADRIAN, AEROSPACE CORPORATION, MS M $4+1$, BOX.92957, LOS ANGELES CA 90009, USA

Tclejphonc: (1)

Facsimile: (1)

Commission(s): 35

Telex:

Email:

MORGAN PETER DR, CANBERRA COLL ADV EDUC, SCHOOL OF APPLIED SCIENCE, BOX 1 BELCONNEN ACT 2616, AUSTRALIA

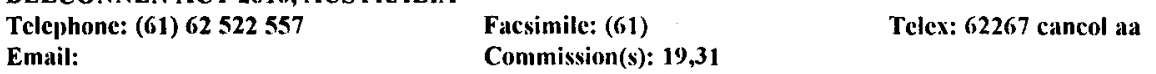

MORGAN THOMAS H DR, SOUTHWEST RESEARCH INST, PO DRAWER 28510, SAN ANTONIO TX 78228 0510, USA Telephone: (1) 2105223985 Facsimile: (1) $2106+7+325 \quad$ Telex:

Email: swri::tmorgan

Commission(s): 42,44

MORIARTY-SCHIEVEN G H DR, DOMINION RADIO ASTROPHYS, OBSERVATORY, BOX 248

PENTICTON BC V2A 6K3, CANADA

Telephone: (1) $60+4932277$

Facsimile: (1) $604+937767 \quad$ Telex:

Email: schieven@drao.nrc.ca

Commission(s):

MORIMOTO MASAKI DR, TOKYO ASTRONOMICAL OBS, NAOJ, OSAWA MITAKA, TOKYO 181, JAPAN

Telcphonc: (81) 992858960

Facsimile: (81) $99258+866$

Tclex:

Email:

Commission(s): $34,38 \mathrm{C}, 40 \mathrm{C}, 51$

MORISON IAN MR, NRAL, JODRELL BANK, MACCLESFIELD SK11 9DL, UK

Telephone: (4) $147771321 \quad$ Facsimile: (4)

Email:

Commission(s): 40

MORITA KAZUHIKO, DPT OF PHYSICS, HOKKAIDO UNIVERSITY, KITA 10 NISHI 8, SAPPORO 060, JAPAN

Telephone: (81) 11711211

Facsimile: (81)

Telex:

Email:

Commission(s): 40

MORITA KOH-ICHIRO, NOBEYAMA RADIO OBS, NAOJ, MINAMISAKI MURA, NAGANO 38 13 , JAPAN

Telephone: (81) $26763+331$

Email: moritarajnro.nao.ac.jp

Facsimile: (81) 267634339 Telex:

Commission(s): 40 
MORIYAMA FUMIO PROF, OSAKA GAKUIN UNIVERSITY, 2-36-1 KISHIBE MINAMI, SUITA SHI, OSAKA 564, JAPAN

Telephone: (81) 63818434

Facsimile: (81)

Telex:

Email:

Commission(s): 10,12,40

MOROSSI CARLO, OAT, BOX SUCC TRIESTE 5, VIA TIEPOLO 11, I $3+131$ TRIESTE, ITALY

Telephone: (39) 40768506

Facsimile: (39)

Telex: 461137 oat

Email:

Commission(s): 29,45

MOROZ V I PROF DR, SPACE RESEARCH INSTITUTE, ACADEMY OF SCIENCES, PROFSOJUZNAYA UL 84/32 117810 MOSCOW, RUSSIA

Telephone: (7) 953333122

Facsimile: (7)

Email:

Commission(s): 15,16,51

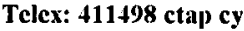

MOROZHENKO A V DR, MAIN ASTRONOMICAL OBS, UKRAINIAN ACAD OF SCIENCE, GOLOSEEVO 252127 KIEV, UKRAINE

Telephone: (7) 044663110

Facsimile: (7)

Commission(s): 16

Telex: 131406 sky

Email:

MOROZHENKO N N DR, MAIN ASTRONOMICAL OBS, UKRAINIAN ACAD OF SCIENCE, GOLOSEEVO

252127 KIEV, UKRAINE

Telephone: (7) 044663110

Email:

\author{
Facsimile: (7) \\ Commission(s): 10
}

Telex: 131406 sky

MORRAS RICARDO DR, IAR, CC 5, 189+ VILLA ELISA (BS AS), ARGENTINA

Telejhone: (54) 21254909

Email: r morras@irma.edu.ar

Facsimile: (54) $2125+909$

Telex: 18052 cicyr ar

Commission(s): 40)

MORRELL NIDIA DR, OBSERVATORIO ASTRONOMICO, PASEO DEL BOSQUE S/N

1900 LA PLATA (BS AS), ARGENTINA

Telephone: (54) 21217308

Email: nidia(a)fcaglp.edu.ar

Facsimile: (54) 21211761

Telex: 31151 bulap ar

MORRIS CHARLES S, JPL, MS 300 319, 4800 OAK GROVE DR, PASADENA CA 91109, USA

Telephonc: (1) $81835+8074 \quad$ Facsimilc: (1)

Email: span:stans::csm

Commission(s):

Telex: $675+29 \mathrm{jpl} \mathrm{usa}$

MORRIS DAVID DR, IRAM, 300 RUE DE LA PISCINE, F 38406 S MARTIN HERES CD, FRANCE

Telephone: (33) $76423383 \quad$ Facsimile: (33)

Telex: 950753

Email:

Commission(s): 40

MORRIS MARK ROOT DR, DPT OF ASTRONOMY, UNIVERSITY OF CALIFORNIA, MATH SCIENCES BLDG LOS ANGELES CA 90024, USA

Telephone: (1) 2138253320

Facsimile: (1)

Telex:

Email:

Commission(s): 33C,34,40,51

MORRIS MICHAEL C, ROYAL GREENWICH OBS, MADINGLEY RD, CAMBRIDGE CB3 OEZ, UK

Telephone: (44) 1323833171

Facsimile: (t4)

Telex: 87451

Email: janet:uk.ac.rgo bowen

Commission(s): 09

MORRIS SIMON, HERZBERG INST ASTROPHYS, DOMINION ASTROPHYS OBS, 5071 W SAANICH RD

VICTORIA BC V8X 4MG, CANADA

Telephonc: (1) $60+3630062$

Email: simon $a$ dao.nrc.ca

Facsimile: (1) $6043630045 \quad$ Telex:

Commission(s):

MORRIS STEPHEN C DR, HERZBERG INST ASTROPHYS, DOMINION ASTROPHYS OBS, 5071 W SAANICH RD VICTORIA BC V8X 4M6, CANADA

Telephone: (1) 6043883976

Email:

Facsimile: (1) 6043630045

Telex: 0497295

MORRIS STEVEN DR, APT 2, 2860 W 235TH ST, TORRANCE CA 90505, USA

Telephone: (1) 2135308708

Facsimile: (1)

Telex:

Email:

Commission(s): 
MORRISON DAVID PROF, NASA AMES RESEARCH CTR, MS 245 1, SPACE SCIENCE DIV

MOFFETT FIELD CA 94035 , USA

Telephone: (1) 4156045028

Facsimile: (1) 4156046779

Telex:

Email: david_morrison(a)qmgate.arc.nasa.gov

Commission(s): 15,16C,51

MORRISON LESLIE V DR, ROYAL GREENWICH OBS, MADINGLEY RD, CAMBRIDGE CB3 OEZ, UK

Telephone: (44) 1223374000

Facsimile: (44) $122337 \$ 700 \quad$ Telex: $265451 / 265871$

Email: merrlp(a)uk.ac.ro-greenwich.sıf Commission(s): 04,08C,19C,24

MORRISON NANCY DUNLAP DR, DPT PHYSICS \& ASTRONOMY, UNIVERSITY OF TOLEDO, 2801 W BANCROFT ST TOLEDO OH 43606, USA

Telcphone: (1) 4195372659

Facsimile: (1)

Telex:

Email:

Commission(s): 27,29

MORRISON PHILIP PROF, DPT OF PHYSICS, MIT RM 6 205, BOX 165, CAMBRIDGE MA 02139, USA

Telephone: (1) 6172535086

Facsimile: (1)

Telex:

Email:

Commission(s): $44,47,51$

MORTON DONALD C DR, HERZBERG INST ASTROPHYS, NTL RESEARCH COUNCIL, 5071 W SAANICH RD VICTORIA BC V8X 4M6, CANADA

Telephone: (1)

Facsimilc: (1)

Commission(s): $09,34,44$

Telex:

Email: $\operatorname{dcm}(\hat{a})$ hiaras.hia.nrc.ca

MORTON G A DR, 1122 SKYCREST DR, APT 6, WALNUT CREEK CA 94595 , USA

Telephone: (1) 4159333802

Facsimile: (1)

Telex:

Email:

Commission(s):

MOSKALIK PAWEL DR, COPERNICUS ASTRON CENTER, POLISH ACAD OF SCIENCES, UL BARTYCKA 18 PL 00716 WARSAW, POLAND

Telephone: (48)

Facsimile: (48)

Telex:

Email:

Commission(s): 35

MOSS CHRISTOPHER DR, INSTITUTE OF ASTRONOMY, THE OBSERVATORIES, MADINGLEY RD

CAMBRIDGE CB3 OHA, UK

Telephone: (44) 1223337548

Email:

Facsimile: (4) 1223337523

Commission(s): 28

Telex: 817297 astron g

MOSS DAVID L DR, DPT OF MATHEMATICS, UNIVERSITY OF MANCHESTER, MANCHESTER M13 9PL, UK Telephone: $(4+)$

Facsimile: (4t)

Telex:

Email:

Commission(s): 35

MOTTA SANTO DR, DPT DI MATIMATICA, CITTA UNIVERSITARIA, VIA A DORIA 6, I 95125 CATANIA, ITALY

Telephone: (39) 9533 (0533*668

Facsimile: (39)

Commission(s): 10

Telex: 970359 astret-i

MOTZ LLOYD PROF, DPT OF ASTRONOMY, COLUMBIA UNIVERSITY, PUPIN HALL 538 W 120TH ST

NEW YORK NY 10027, USA

Telcphonc: (1) 2122803279

Facsimile: (1)

Telex:

Email:

Commission(s):

MOUCHET MARTINE DR, OBSERVATOIRE DE PARIS, SECTION DE MEUDON, DAEC

F 92195 MEUDON PPL CDX, FRANCE

Telephone: (33) 145077522

Facsimile: (33) $145077469 \quad$ Telex: 201571

Email: melama::mouchet/mouchet afimens

Commission(s): 42

MOULD JEREMY R, MOUNT STROMLO \& SIDING, SPRING OBSERVATORIES, PRIVATE BAG WESTON CREEK PO ACT 2611, AUSTRALIA

Telephone: (61) 62490266

Facsimile: (61) 62490233

Telex:

Email: jrma mso.anu.edu.au

Commission(s): 28,37

MOURADIAN ZADIG M DR, OBSERVATOIRE DE PARIS, SECTION DE MEUDON, DASOP F 92195 MEUDON PPL CDX, FRANCE

Telephone: (33) 145077800

Facsimile: (33)

Telex:

Email:

Commission(s): 12 
MOURARD DENIS, OCA OBSERV DU CALERN, CAUSSOLS, F $06 \$ 60$ S VALLIER THIEY, FRANCE

Telephone: (33) 93405492

Email: mourand@obs-nice.fr Facsimile: (33) $93404431 \quad$ Telex:

MOUSCHOVIAS TELEMACHOS CH, DPT OF ASTRONOMY, UNIVERSITY OF ILLINOIS, 1011 W SPRINGFIELD AVE URBANA IL 61801, USA

Telephone: (1) 2173333090

Facsimile: (1) 2172447638

Telex: 9102409464 astodept

Email:

Commission(s): 34

MOUSSAS XENOPHON PH D, DPT OF ASTROPHYSICS, NTL UNIVERSITY OF ATHENS, PANEPISTIMIOPOLIS GR 15783 ATHENS, GREECE

Telephone: (30) 17235122

Facsimile: (30)

Telex:

Email: moussas@grathon1.earn

Commission(s): $\$ 9$

MOUTSOULAS MICHAEL PROF, DPT OF EARTH SCIENCES, NTL UNIVERSITY OF ATHENS, PANEPISTIMIOPOLIS GR 15784 ATHENS, GREECE

Telephonc: (30) 17247569

Facsimile: (30)

Commission(s): 16,51

Telex: $215255 \mathrm{gr}$

Email:

MOZURKEWICH DAVID DR, US NAVAL OBSERVATORY, AD S BLDG 22 RM 220, 3450 MASSACHUSETTS AVE NW WASHINGTON DC 203925100 , USA

Telephone: (1) 2026530948

Facsimile: (1)

Telex:

Email:

Commission(s):

MRKOS ANTONIN DR, DPT OF ASTRONOMY, CHARLES UNIVERSITY, SVEDSKA 8, CZ 150 O0 PRAHA 5, CZECH R Telephonc: (42) $25 \$ 0395$

Email:

Facsimile: (42)

Telex: 144307 klet $c z$

Commission(s): 06,15,20

MUECKET JAN P, ASTROPHYSIKALISCHES INST, POTSDAM UNIVERSITY, AN DER STERNWARTE 16 D 14482 POTSDAM, GERMANY

Telephone: (49) 33174990

Email: jpmuecket(a)ajp.de

Facsimile: (49) $33174992(m) \quad$ Telex:

Commission(s): 47

MUELLER EDITH A PROF, RENNWEG 15, CH +052 BASEL, SWITZERLAND

Telephone: (41) 613123168 Facsimile: (41) Telex:

Email:

Commission(s): $12,38,+4$

MUELLER EWALD, MPI F PHYSIK \& ASTROPHYS, KARL SCHWARZSCHILDSTR 1 D 85740 GARCHING MUENCHEN, GERMANY

$\begin{array}{lll}\text { Telephone: (49) } 8932990 & \text { Facsimile: (49) } & \text { Telex: 524629 astro d } \\ \text { Email: } & \text { Commission(s): } 35 & \end{array}$

MUELLER IVAN I PROF, GEODETIC SCI \& SURVEYING, OHIO STATE UNIVERSITY, 1958 NEIL AVE COLUMBUS OH 432101247 , USA

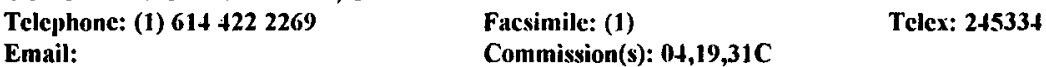

MUELLER VOLKER, ASTROPHYSIKALISCHES INST, POTSDAM UNIVERSITY, AN DER STERNWARTE 16 D 14482 POTSDAM, GERMANY

Telephone: (49) 33174990

Email: vmueller(âaip.de

Facsimile: (49) $3317499200 \quad$ Telex: 361231 stbbg d

Commission(s): 28

MUENCH GUIDO PROF, INST DE ASTROFISICA, DE CANARIAS, VIA LACTEA S/N, E 38200 LA LAGUNA, SPAIN Telephone: (34)

Facsimile: (34) 22605210

Telex:

Email:

Commission(s): 33,34,36

MUFSON STUART LEE DR, ASTRONOMY DPT, INDIANA UNIVERSITY, SWAIN WEST 319 BLOOMINGTON IN $\$ 7+105$, USA

Telephone: (1) 8123356927

Facsimile: (1)

Telex:

Email:

Commission(s): $3-$

MUINONEN KARRI DR, LOWELL OBSERVATORY, 1400 W MARS HILL RD, BOX 1149, FLAGSTAFF AZ 86001, USA

Telephone: (1) $60277 \$ 3358$

Facsimilc: (1) $601277+6296$

Telex:

Email: s:percy::kom

Commission(s): 21 
MUINOS JOSE L DR, REAL INST Y OBSERVATORIO, DE LA ARMADA, CECILIO PUJAZON S/N

E 11110 SAN FERNANDO, SPAIN

$\begin{array}{ll}\text { Telephone: }(34) 56883548 & \text { Fansimile: }(34) 56881732 \quad \text { Telex: } 76108 \text { iom e } \\ \text { Email: } & \text { Commission(s): } 08\end{array}$

MUKAI SONOYO DR, KANAZAWA TECHNOLOGY INST, 7-1 OGIGAOKA, NONOICHIMACHI, ISHIKAWA 921, JAPA

Telephone: (81) $762+81100 \quad$ Facsimile: (81) Telex: $5122+56$ kitlcj

Email: $\quad$ Commission(s): 21,36

MUKAI TADASHI DR, DPT OF EARTH SCIENCES, FACULTY OF SCIENCES, KOBE UNIVERSITY NADA

KOBE 6.57, JAPAN

$\begin{array}{ll}\text { Telephone: (81) } 788030574 & \text { Facsimile: (81) } 788030490 \quad \text { Telex: } \\ \text { Email: mukaíajasmin.kobe-u. ac.jp) } & \text { Commission(s): 15,21 }\end{array}$

, MUKHERJEE KRISHNA, DPT OF PHYSICS \& ASTRON, SLIPPERY ROCK UNIV OF PEN, 327 VINCENT SCIENCE

SLIPPERY ROCK PA 16057, USA

$\begin{array}{ll}\text { Telephone: (1) }+1+7382858 & \text { Facsimile: (1) } \\ \text { Email: } & \text { Commission(s): }\end{array}$

MULHOLLAND J DERRAL DR, OCA CERGA, AVE COPERNIC, F 06130 GRASSE, FRANCE

Telephone: (33) 9336584 ) Facsimile: (33) Telex:

Email:

Commission(s): $(17,16,20$

MULLALY RICHARD F DR, 31 EDEN AVE, TURRAMURRA NSW 2017, AUSTRALIA

Telephone: (61) Ficsimile: (61) Telex:

Email:

Commission(s):

MULLAN DERMOTT J DR, BARTOL RESEARCH INSTITUTE, UNIVERSITY OF DELAWARE, NEWARK DE 19716, US

Telephonc: (1) 3113983368

Facsimile: (1)

Telex: 51106660805

Email:

Commission(s):

MULLER A B DR, THOPMASLAAN 40, NL 5631 GNI EINDHOVEN, NETHERLANDS

Telephone: (31) $+11+3$ (1322 Facsimile: (31) Telex:

Email: $\quad$ Commission(s): 25

MULLER C A PROF JR, ODINKSVELD 8, NL 7491 HD DELDEN, NETHERLANDS

Telephone: (31) $5+072+28 \quad$ Facsimile: (31) Telex:

Email:

Commission(s):

MULLER PAUL, 3 RUE CHAUVAIN, F UGHOU NICE, FRANCE

Telephone: (33) Facsimile: (33) Telex:

Email: Commission(s): 26

MULLER RICHARD A, LAWRENCE BERKELEY LAB, BLDG SU RM 238, BERKELEY CA 94720, USA

Telephone: (1) $+15+865235$

Facsimile: (1)

Telex:

Email:

Commission(s): 47,51

MULLER RICHARD DR, OBS MIDI PYRENEES, I AVE E BEL.IN, F $31+100$ TOULOUSE CDX, FRANCE

Telephone: (33) 62950069

Facimile: (33)

Telex:

Emial:

Commission(s): 10,12

MUMFORD GEORGE S PROF, DPT OF EDUCATION, TUFTS UNIVERSITY, FILENE CENTER

MEDFORD MA 02155, USA

Telephonc: (1) 6176.538923

Email:

Facsimile: (1)

Telex:

Commission(s): $25,27,42$

MUMMA MICHAEL JON, NASA GSFC, CODE 693, GREENBELT MD 20771, USA

Telephonc: (1) $3012866994 \quad$ Facsimile: (1) Telex:

Enail: mmummaajlepwax.dnct.nasal.gov Commission(s): 14,15,16

MUNDT REINHARD DR, MPI FUER ASTRONOMIE, KOENIGSTUHL, D G9117 HEIDELBERG, GERMANY

Telephone: (49) $6221528227 \quad$ Facsinile: (49)

Telex: 461789

Email:

Commission(s): 
M 718

MUNDY LEE G DR, ASTRONOMY PROGRAM, UNIVERSITY OF MARYLAND, COLLEGE PARK MD 20742, USA

Telephone: (1)

Facsimile: (1)

Commission(s): 40

Telex:

Email:

Commission(s): +1

MUNOZ-TUNON CASIANA, INST DE ASTROFISICA, DE CANARIAS, OBS DEL TEIDE, E 38200 LA LAGUNA, SPAIN

Telephone: (34) 22262211

Facsimile: (34)

Telex: 92640

Email:

Commission(s): 28

MUNRO RICHARD H DR, 2378 DENNISON LANE, BOULDER CO 80302, USA

$\begin{array}{lll}\text { Telephone: (1) } & \text { Facsimile: (1) } & \text { Telex: } \\ \text { Email: } & \text { Commission(s): } 12 & \end{array}$

MURAKAMI HIROSHI, ISAS, 3-1-1 YOSHINODAI, SAGAMIHARA, KANAGAWA 229, JAPAN

Telephone: $(81) 427513911 * 2603 \quad$ Facsimilc: $(81)+2759+253 \quad$ Telex:

Email: hmurakam(a)astro.isas.ac.jp) Commission(s): 44

MURAKAMI TOSHIO, INST SPACE \& ASTRON SCI, UNIVERSITY OF TOKYO, MEGURO KU, TOKYO IS3, JAPAN

Telephone: (81) $34671111 * 303 \quad$ Facsimile: (81)

Email:

Commission(s): 44

Telex: $3+757$ isastro $\mathrm{j}$

MURDIN PAUL G DR, PART PHYS \& ASTRON RES CO, POLARIS HOUSE, NORTH STAR AVE, SWINDON SN2 ISZ, UK

Telephone: (44) 1793442075

Facsimile: (4+) $1793+42003 \quad$ Telex:

Email: murdin(a)mail.ast.cam.ac.uk Commission(s): 27,50C

MURDOCH HUGH S DR, DPT OF ASTROPHYSICS, UNIVERSITY OF SYDNEY, SYDNEY NSW 20OG, AUSTRALIA

Telephone: (61) 26922222

Email:

Facsimile: (61)

Commission(s): to

Telex: 26169 unisyd

MURDOCK THOMAS LEE, GENERAL RESEARCH CORP, DPT OF TECHNOLOGY, 5 CHERRY HILL, DANVERS MA 01923, USA

Telephone: (1) $6177776323 \quad$ Facsimile: (1) Telex:

Email:

Commission(s): 44

MUREDDU LEONARDO DR, STAZIONE ASTRONOMICA, VIA OSPEDALE 72, I 09100 CAGLIARI, ITALY

Telephone: (39) $70663544 \quad$ Facsimile: (39) 70657657 Telex:

Email:

Commission(s):

"MURIEL HERNAN, OBSERVATORIO ASTRONOMICO, LAPRIDA 85 4,5000 CORDOBA, ARGENTINA

Telephone: (54) 51214059

Email: muriclauncbob.cdu

Facsimile: (54) 51210613 Telex:

Commission(s):

MURPHY BRIAN WILLIAM DR, DPT PHYSICS \& ASTRONOMY, BUTLER UNIVERSITY, 4600 SUNSET AVE INDIANAPOLIS IN $\$ 6208$, USA

Telephonc: (1) 3172839282

Facsimile: (1) $3172839950 \quad$ Telex:

Email: murphy(a)astrosun.tu.cornell.cdu Commission(s):

MURPHY ROBERT E DR, NASA HEADQUARTERS, CODE Y, WASHINGTON DC 20546, USA

Telephone: (1) $202+531720 \quad$ Facsimile: (1) Telex: 89530 nasa wsh

Email:

Commission(s): 16

MURRAY C ANDREW, DERWENT COTTAGE, 12 DERWENT RD, MEADS, EASTBOURNE DN20 7PH, UK

Telephone: (\$t)

Facsimile: $(4)$

Telex:

Email:

Commission(s): $(18,22,24,33,37$

MURRAY CARL D DR, SCHOOL OF MATHEMATICAL SC, QUEEN MARY/WESTFIELD COLL, MILE END RD LONDON E1 $+\mathrm{NS}$, UK

$\begin{array}{lll}\text { Telephone: (4f) } 171980+811 & \text { Facsimile: }(4) & \text { Telex: } 893750 \\ \text { Email: } & \text { Commission(s): } 20,22 & \end{array}$

MURRAY JOHN B DR, DPT OF EARTH SCIENCES, THE OPEN UNIVERSITY, WALTON HALL

MILTON KEYNES MK7 GAA, UK

Telephone: (4t) 1908652118

Email: j.b.murray âopen.ac.uk

Facsimile: (4) $1908655151 \quad$ Telex:

Commission(s): 
MURRAY STEPHEN DAVID, LAWRENCE LIVERMORE LAB, L 58, BOX 808, LIVERMORE CA 94550, USA

Telephone: (1) 5104239382

Facsimile: (1) $510+225102$ Telex:

Email: murray@astron.berkeley.edu

Commission(s):

MURRAY STEPHEN S DR, CENTER FOR ASTROPHYSICS, HCO/SAO, 60 GARDEN ST, CAMBRIDGE MA 02138, USA

Telephone: (1) 6174957205

Facsimile: (1)

Telex: $921+28$ satellite cam

Email:

Commission(s): 09,28

MURTHY JAYANT, DPT PHYSICS \& ASTRONOMY, JOHNS HOPKINS UNIVERSITY, CHARLES \& 34TH ST

BALTIMORE MD 21218, USA

Telephonc: (1) 4105167027

Email: murthy@pha.jhu.edu

Facsimile: (1) $410516+109$

Telex:

Commission(s): 34,44

MUSEN PETER DR, 880 4 ORBIT LANE, LANHAM MD 20801, USA

Telephonc: (1) 3015523848

Facsimile: (1)

Commission(s): 07

Telex:

Email:

MUSIELAK ZDZISLAW E DR, NASA/MSFC, CODE ES 52, SPACE SCIENCE LAB, HUNTSVILLE AL 35812, USA

Telephone: (1) $2055+47619$

Facsimile: (1)

Commission(s): 10,36 Telex: 59+416

Email; span:ssl::musiclak

MUSMAN STEVEN DR, NGS, N/CG 112, ROCKVILLE MD 20852, USA

Telephone: (1)

Facsimile: (1)

Commission(s):

Telex:

Email:

ICS \& ASTRONOMY, UNIVERSITY OF IOWA, IOWA CITY IA 52242, USA

MUTEL ROBERT LUCIEN, DPT PHYSICS \& ASTRONOMY, UNIVERSITY OF IO
Telephone: (1) 3193537205
Facsimile: (1)

Email:

Commission(s): $\$ 1,+2$

MUTSCHLECNER J PAUL DR, ASTRONOMY DPT, INDIANA UNIVERSITY, SWAIN WEST 319

BLOOMINGTON IN $47+05$, USA

Telephonc: (1)

Facsimile: (1)

Telex:

Email:

Commission(s): 36

MUXLOW THOMAS, NRAL, JODRELL BANK, MACCLESFIELD SK11 9DL, UK

Telephone: (4) $1 \$ 7771321$

Facsimile: (4)

Telex: 36149

Email:

Commission(s): 40

MUZzIO JUAN C PROF, OBSERVATORIO ASTRONOMICO, PASEO DEL BOSQUE S/N

1900 LA PLATA (BS AS), ARGENTINA

Telephonc: (45) 21217308

Facsimile: (45) 2121 1761/25 5004 Telex: 31216 cesla ar

Email: jcmuzzio\%fcaglp.cdu.arâuucpnct Commission(s): 07C,28,33,37,46

MYACHIN VLADIMIR F DR, INST OF THEORET ASTRONOMY, ACADEMY OF SCIENCES, N KUTUZOVA 10 192187 ST PETERSBURG, RUSSIA

Tclephonc: (7)

Email:

Facsimilc: (7)

Commission(s): 07

Telex:

MYERS PHILIP C, CENTER FOR ASTROPHYSICS, HCO/SAO MS $\$ 2,60$ GARDEN ST, CAMBRIDGE MA 02138, USA Telephone: (1)

Facsimile: (1)

Telex:

Email:

Commission(s): $3+\mathrm{C}, 40$

NACOZY PAUL E DR, FEDEREAL SPACE SYSTEMS, BOX 26712, AUSTIN TX 78755, USA

Telephone: (1) $512+676659$

Facsimile: (1)

Telex:

Email:

Commission(s): 07,20

NADAL ROBERT, OBS MIDI PYRENEES, $1+$ AVE E BELIN, F 31400 TOULOUSE CDX, FRANCE

Telephonc: (33) 61252101

Facsimile: (33)

Telex: $5301776 \mathrm{f}$

Email:

Commission(s): $\$ 1$

NADEAU DANIEL DR, DPT DE PHYSIQUE, UNIVERSITE DE MONTREAL, CP G128 SUCC A

MONTREAL QC H3C 3.J7, CANADA

Telephone: (1) 5143436676

Facsimile: (1) $51+3+32071$

Telex: $05562+25$

Email:

Commission(s): 40 
NADOLSCHI V PROF DR, COM ARDEOANI OF TESCANI, JUD, R BACAU, RUMANIA

Tclephone: $(40)$

Facsimile: (40)

Telex:

Email:

Commission(s):

NADYOZHIN D K DR, INST THEOR \& EXPER PHYS, CHEREMUSHKINSKAJA UL 25, 117259 MOSCOW, RUSSIA

Telephone: (7) $951230292 \quad$ Facsimile: (7)

Email:

Commission(s): 35

Telex: $\mathbf{4 1 0 5 9}$ cerii su

NAGASAWA SHINGO PROF, 3209 HIGASHI MACHI, KICHIJOJI, MUSASHINO SHI, TOKYO 180, JAPAN

Telephone: (81)

Facsimile: (81)

Telex:

Email:

Commission(s): 10

NAGASE FUMIAKI DR, DPT OF ASTROPHYSICS, NAGOYA UNIVERSITY, FUROCHO CHIKUSA KU

NAGOYA 464 01, JAPAN

Telephone: (81) 527815111

Facsimile: (81)

Commission(s):

Telex: $\$ \$ 77323$ scunag j

Email:

OF, ASTROPHYSICS, KORAMANGALA, BANGALORE 560 034, INDIA

NAGENDRA K N, INDIAN INSTITUTE OF, ASTROPHYSICS, KORA
Telephone: (91) 805530672

Telex: $8+52763$ iab in

Email: knn@iiap.ernet.in

Commission(s): 36

NAGIRNER DMITRIJ I DR, ASTRONOMICAL OBSERVATORY, ST PETERSBURG UNIVERSITY, BIBLIOTECHNAJA PL 2, 198904 ST PETERSBURG, RUSSIA

Tclephone: (7) 2579489

Facsimile: (7)

Telex:

Email:

Commission(s): 36

NAGNIBEDA VALERY G DR, ASTRONOMICAL OBSERVATORY, ST PETERSBURG UNIVERSITY, BIBLIOTECHNAJ PL 2, 198904 ST PETERSBURG, RUSSIA

Telephone: (7) 2579491

Facsimile: (7)

Telex:

Email:

Commission(s): 40

NAGOVITSYN YURI, PULKOVO OBSERVATORY, ST PETERSBURG 196140, RUSSIA

Telephone: (7) 8121231997

Facsimile: (7) 8123151701

Telex: 121351 pulob

Email: sol@gaoran.spb.su

Commission(s):

NAHON FERNAND PROF, 25 AVE DE L'EUROPE, F 92310 SEVRES, FRANCE

Telephone: (33) $1453+1805$

Facsimile: (33)

Telex:

Email:

Commission(s): 07,33

NAIDENOV VICTOR O, IOFFE PHYSICAL TECH INST, ACADEMY OF SCIENCES, POLYTECHNICHESKAYA UL 26 194021 ST PETERSBURG, RUSSIA

Telephonc: (7)

Email:

Facsimile: (7)

Telex:

NAKADA YOSHIKAZU DR, KISO OBSERVATORY, MITAKE MURA, KISO GUN, NAGANO 397 01, JAPAN

Telephone: (81) $26+523360$

Facsimile: (81) 264523361

Telex:

Email: Inakada(âc1.mtk.nao.ac.jp

Commission(s): 34

NAKAGAWA NAOYA DR, UNIV ELECTRO-COMMUNICATIO, CHOFU-SHI, TOKYO 182, JAPAN

Telephonc: (81) $42+832161 \quad$ Facsimile: (81)

Email:

Commission(s):

Telex: $2822+46$ uec j

NAKAGAWA TAKAO DR, ISAS, 3-1-1 YOSHINODAI, SAGAMIHARA, KANAGAWA 229, JAPAN

Telephone: (81) +2751 3911

Facsimile: (81) $42759+253 \quad$ Telex: j 34757 isast ro

Email: nakagawa(ajastro.isas.ac.jp)

Commission(s): 34

NAKAGAWA YOSHINARI DR, CHIBA INST OF TECHNOLOGY, NARASHINO 275, JAPAN

Telephone: (81) $\$ 7+752111$

Facsinile: (81)

Telex:

Email:

Commission(s): 10,49,51

NAKAGAWA YOSHITSUGU DR, EARTH \& PLANETARY SCIENCE, FACULTY OF SCIENCE, KOBE UNIVERSITY KOBE 657, JAPAN

Telephone: (81) 788030563

Facsimile: (81) 788821549

Telex:

Email: yoshi(a)saturn.jphys.kobe-u.ac.jp

Commission(s): 16 
NAKAI NAOMASA DR, NOBEYAMA RADIO OBS, NAOJ, MINAMIMAKI MURA, NAGANO 38 4 13, JAPAN Telephone: (81) 267634367 Facsimilc: (81) $26763+387$

Telex: 3329005 naonro $j$

Email: nronaka@jpnnro Commission(s): 28

NAKAI YOSHIHIRO, KWASAN \& HIDA OBS, KYOTO UNIVERSITY, YAMASHINA, KYOTO 607, JAPAN

Telephone: (81) 755811235

Facsimile: (81)

Telex:

Email:

Commission(s): 09

NAKAJIMA HIROSHI, NOBEYAMA RADIO OBS, NAOJ, MINAMIMAKI MURA, NAGANO 384 13, JAPAN

Telephone: (81) 267982034

Facsimile: (81)

Email:

Commission(s): 10

Telex: 3329005 tionro $j$

NAKAJIMA KOICHI DR, FAC OF ECONOMICS, HITOTSUBASHI UNIVERSITY, NAKA 2-1 KUNITACHI TOKYO 186, JAPAN

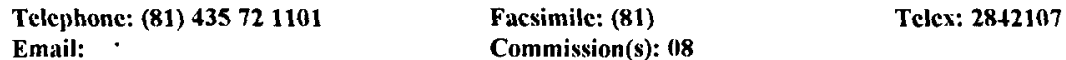

NAKAMURA TAKASHI DR, DPT OF PHYSICS, KYOTO UNIVERSITY, KITASHIRAKAWA SAKYO KU KYOTO 606, JAPAN

Telephone: (81)

Facsimile: (81)

Telex:

Email:

Commission(s): 35

NAKAMURA TSUKO DR, TOKYO ASTRONOMICAL OBS, NAOJ, OSAWA MITAKA, TOKYO 181, JAPAN

Telephone: (81) 38122111

Facsimile: (81)

Telex:

Email:

Commission(s): 15,20

NAKAMURA YASUHISA, DPT OF EARTH SCINECE, FUKUSHIMA UNIVERSITY, MATSUKAWA MACHI FUKUSHIMA 960-12, JAPAN

Telephone: (81) $245+85151^{*}+21$

Email:

Facsimile: (81)
Commission(s): +2

Telex:

NAKANO MAKOTO, FACULTY OF EDUCATION, OITA UNIVERSITY, 700 DANNOHARU, OITA 870 11, JAPAN

Telephone: (81) $975693311^{* 360}$

Email: mnakano(a)cc.oita-u.ac.jp

Facsimile: (81) 975688319 Telex:

Commission(s): $06 \mathrm{C}, 34$

NAKANO SABURO DR, KOBINATO 121 7, BUNKYO KU, TOKYO 112, JAPAN

Telephonc: (81)

Facsimile: (81)

Telex:

Email:

Commission(s):

NAKANO SYUICHI, 3-19 1 CHOMO, TAKENOKUCHI, SUMOTO, HYOGO KEN 656, JAPAN

Telephone: (81)

Facsimile: (81)

Telex:

Email:

Commission(s): 06,20

NAKANO TAKENORI DR, DPT OF PHYSICS, KYOTO UNIVERSITY, KITASHIRAKAWA SAKYO KU KYOTO 606, JAPAN

Telephone: (81) 757512111

Facsimile: (81)

Telex:

Email:

Commission(s): 35

NAKAYAMA SHIGERU DR, 3711 CHUOU, NAKANO KU, TOKYO 164, JAPAN

Telephone: (81)

Facsimile: (81)

Telex:

Email:

Commission(s): $41 \mathrm{C}$

NAKAZAWA KIYOSHI DR, TOKYO INST OF TECH, OHOKAYAMA 2-12-1, MEGUROKU TOKYO 152, JAPAN

Telephone: (81)

Facsimile: (81)

Commission(s): 22,35

Telex:

Email:

NAMBA OSAMU DR, MARCO POLOLAAN 319, NL 3526 GE UTRECHT, NETHERLANDS

Telephonc: (31)

Facsimile: (31)

Commission(s): 10,12

Telex:

Email:

NAMBOODIRI P M S, INDIAN INSTITUTE OF, ASTROPHYSICS, KORAMANGALA, BANGALORE 560 034, INDIA

Telephonc: (91)

Email: pmsn@aiiap.ernet.in
Facsimile: (91)

Commission(s): 28,33

Telex: 
NAMBU YASUSADA DR, DPT OF PHYSICS, KYOTO UNIVERSITY, KITASHIRAKAWA SAKYO KU KYOTO 606, JAPAN

Telephone: (81) 757533844

Facsimile: (81) 757533866

Telex:

Email: nambu@jpnrifp

Commission(s): 47

NAN REN-DONG, BEIJING ASTRONOMICAL OBS, CAS, W SUBURB, BEIJING 100080, CHINA PR

Telephone: (86) 1281698

Facsimile: (86)

Telex: 22040 baobs cn

Email:

Commission(s): $\$ 0$

NANDY KASHINATH DR, ROYAL OBSERVATORY, BLACKFORD HILL, EDINBURGH EH9 3HJ, UK

Telephone: (44) 1316673321

Facsimile: (44)

Telex: 72383 rocding

Email:

Commission(s): 34,45

NAPIER WILLIAM M DR, ROYAL OBSERVATORY, BLACKFORD HILL, EDINBURGH EH9 3HJ, UK

Telephone: (44) 1316673321

Email:

Facsimile: $(4+)$

Commission(s): 15,22

Telex: $\mathbf{7 2 3 8 3}$ roedin $\mathrm{g}$

NAQVI S I H PROF, DPT PHYSICS, UNIVERSITY OF REGINA, REGINA, SASKATCHEWAN S4S OA2, CANADA

Telephone: (1) 3065854258

Email: naqvi@max.cc.uregina.ca

Facsimilc: (1) $306585+894$

Telex:

Commission(s):

NARAIN UDIT, ASTROPHYSICS RESEARCH GP, MEEUT COLLEGE, MEERUT 250 001, INDIA

Telephone: (91)

Facsimile: (91)

Telex:

Email:

Commission(s): $10,+3$

NARANAN S PROF, TIFR, HOMI BHABHA RD, COLABA, BOMBAY 400 005, INDIA

Telephone: (91) 22219111

Facsimile: (91)

Telex: 0113009

Email:

Commission(s):

NARASIMHA DELAMPADY DR, TIFR, HOMI BHABHA RD, COLABA, BOMBAY 400 005, INDIA

Telephone: (91) 224952311

Facsimile: (91)

Telex: 3009 in

Email:

Commission(s): $35,36,47$

NARAYAN RAMESH DR, CFA, MS 51, 60 GARDEN ST, CAMBRIDGE MA 02138, USA

Telephone: (1) 6174957000

Facsimile: (1)

Tclex: 921428

Email:

Commission(s):

NARAYANA J V, REGIONAL METEOROLOGICAL, OFFICE, + COLLEGE RD, MADRAS G00 006, INDIA

Telephone: (91)

Facsimile: (91)

Commission(s):

Telex:

Email:

NARLAI KYOJI PROF, NTL ASTRONOMICAL OBS, 136 KINOOLE ST, HILO HI 96720, USA

Telephone: (1) 8089354067

Facsimilc: (1) $80893+7198$

Telex:

Email: nariai@subaru.naoj.hawaii.edu

Commission(s): $36, \$ 2$

NARITA SHINJI DR, DOSHISHA UNIVERSITY, KYOTO 602, JAPAN

Telephone: (81)

Facsimile: (81)

Telex:

Email:

Commission(s): 35

NARLIKAR JAYANT V PROF, IUCAA, PO BOX 4 , GANESHKHIND, PUNE $\$ 11007$, INDIA

Telephone: (91) $212336415 \quad$ Facsimile: (91) 212335760 Telex: 01 $\$ 57658$ gmrt in

Email: jvn@iucaa.ernct.in

Commission(s): $28,+6 \mathrm{C}, 47 \mathrm{P}$

NASIRI GHEIDARI S DR, DPT OF PHYSICS, UNIVERSITY OF ZANJAN, KMG TABRIZ ROAD 45195 313, ZANJAN, IRAN

Telephone: (98) 2821270014

Facsimile: (98)

Telex:

Email:

Commission(s): 35

NATALI GIULIANO DR, IAS, CNR, CP 67, I 0004 FRASCATI, ITALY

Telephone: (39) 69425655

Facsimile: (39) 69416847

Telex:

Email:

Commission(s):

NATHER R EDWARD, ASTRONOMY DPT, UNIVERSITY OF TEXAS, RLM 15 308, AUSTIN TX 78712 1083, USA

Telephone: (1)

Facsimile: (1)

Telex:

Email:

Commission(s): $27,+2$ 
NATTA ANTONELLA DR, CENTRO PER ASTRONOMIA IR, LARGO E FERMI 5, I 50125 FIRENZE, ITALY

Telephone: (39) 552752239

Facsimile: (39)

Telex: 572268 arcetri

Email:

Commission(s):

NAUMOV VITALIJ A DR, PULKOVO OBSERVATORY, ACADEMY OF SCIENCES, 10 KUTUZOV QUAY 196140 ST PETERSBURG, RUSSIA

Telephone: (7) 2982242

Email:

Facsimile: (7)

Telex:

Commission(s): 19,31

NAVARRO JULIO FERNANDO DR, DPT OF PHYSICS, UNIVERSITY OF DURHAM, SOUTH RD, DURHAM DH1 3LE, U Telephone: (44) $19137 \$ 2194$

Facsimile: (4+) $19137+3749$

Telex: 537351 durlib $g$

Email: jfn(a)star.dur.ac.uk

Commission(s): 28

NAWAR SAMIR DR, HELWAN OBSERVATORY, HELWAN, EGYPT

Telephone: (20) 78 06+5/2683

Facsimile: (20)

Commission(s): 21

Telex:

Email:

TERNWARTE, GOJENSBERGSWEG 112, D 21029 HAMBURG, GERMANY

NECKEL HEINZ DR, HAMBURGER STERNWARTE, GOJENSBERGSWEG 112, D 21029
Telephone: $(49)+07252+130$

Email: $\quad$ Commission(s): 12,29

NECKEL TH DR, MPI FUER ASTRONOMIE, KOENIGSTUHL, D 69117 HEIDELBERG, GERMANY

$\begin{array}{lll}\text { Telephone: (\$9) } 6221528288 & \text { Facsimile: (49) } & \text { Telex: } 461789 \text { mpia d } \\ \text { Email: } & \text { Commission(s):33 }\end{array}$

Email: $\quad$ Commission(s): 33

NEE TSU-WEI DR, DPT OF PHYSICS, NTL CENTRAL UNIVERSITY, CHUNG LI TAIWAN, CHINA R

Telephone: (886) $\quad$ Facsimile: (886)

Telex:

Email:

Commission(s):

NEEMAN YUVAL PROF, DPT OF PHYSICS \& ASTRON, TEL AVIV UNIVERSITY, RAMAT AVIV

TEL AVIV 69978, ISRAEL

Telephone: $(972) 3+25+11$

Facsimile: (972)

Commission(s): 44,47

Telex: $3+2171$ versy il

Email:

NEFEDEVA ANTONINA I PROF, ENGELHARDT ASTRONOMICAL, OBSERVATORY, OBSERVATORIA STATION 422526 KAZAN, RUSSIA

Telephone: (7) 324827

Facsimils: (7)

Telex:

Email:

Commission(s): 08

NEFF JAMES EDWARD DR, DPT OF ASTRONOMY, PENNSYLVANIA STATE UNIV, 525 DAVEY LAB

UNIVERSITY PARK PA 16802, USA

Telephone: (1) 8148639551

Email: jneffäastro.psu.edu

Facsimile: (1) $81+8633399 \quad$ Telex:

Commission(s):

NEFF JOHN S, DPT PHYSICS \& ASTRONOMY, UNIVERSITY OF IOWA, 605 BROOKLAND PARK DR

IOWA CITY IA 52240, USA

Telephone: (1) 3193534340

Facsimile: (1)

Telex:

Email:

Commission(s): $15,27,36,40$

NEFF SUSAN GALE DR, NASA GSFC, CODE 684.1, GREENBELT MD 20771, USA

Telephone: (1) 3012865137

Facsimile: (1)

Telex: 89675

Email: neff(astars.gsfc.nasa.gov

Commission(s): +4

NEIDIG DONALD F DR, AIR FORCE GEOPHYSICS LAB, NTL SOLAR OBSERVATORY, SUNSPOT NM 88349, USA

Telephone: (1) $5054347000 \quad$ Facsimile: (1)

Email: bitnet:dneidig(äsunspot.noas.ed Commission(s): 10

Telex: 0666484 noao tuc

NELSON ALISTAIR H DR, DPT OF PHYSICS, UNIV WALES COLLEGE, BOX 913, CARDIFF CF1 3TH, UK

Telephone: (4) $122287+785$

Email: nelsonarăuk.ac.cardiff

Facsimile: (4) $1222371921 \quad$ Telex:

Commission(s): 33

NELSON BURT DR, DPT OF ASTRONOMY, SAN DIEGO STATE UNIV, SAN DIEGO CA 92182, USA

Telephone: (1) 6192656175

Facsimile: (1)

Telex:

Email: nclson $a$ minaka.sdsu.edu

Commission(s): $\mathbf{4 2 , 5 0}$ 
NELSON GEORGE DRIVER DR, DPT OF ASTRONOMY, UNIVERSITY OF WASHINGTON, FM 20

SEATTLE WA 98195, USA

Telephone: (1) 2065436616

Facsimile: (1) $2066853218 \quad$ Telex:

Email: pnclson@cosmos.astro.washinton Commission(s):

NELSON GRAHAM JOHN DR, CSIRO, DIV OF RADIOPHYSICS, BOX 76, EPPING NSW 2121, AUSTRALIA

Telephone: (61) 28680222

Facsimile: (61) 28680310

Telex: 26230

Email: gnelson@atnf.csiro.au

Commission(s): 10

NELSON JERRY E DR, LICK OBSERVATORY, UNIVERSITY OF CALIFORNIA, 181 NATURAL SCIENCES II

SANTA CRUZ CA 95064, USA

Telephone: (1)

Facsimile: (1)

Telex:

Email:

Commission(s): 09

NELSON ROBERT M, JPL, MS 183 501, 4800 OAK GROVE DR, PASADENA CA 91109, USA

Telephone: (1) 2133546893

Facsimile: (1)

Telex:

Email:

Commission(s): 51

NEMEC JAMES, DPT GEOPHYS \& ASTRONOMY, UNIV OF BRITISH COLUMBIA, 2075 WESBROOK PL VANCOUVER BC V6T 1W5, CANADA

Telephone: (1) 6046524517

Facsimile: (1) $6042286047 \quad$ Telex:

Email:

Commission(s): 37

NEMIRO ANDREJ A DR PROF, PULKOVO OBSERVATORY, ACADEMY OF SCIENCES, 10 KUTUZOV OUAY 196140 ST PETERSBURG, RUSSIA

Tclephone: (7) 2982242

Facsimile: (7)

Telex:

Email:

Commission(s): 08

NEMIROFF ROBERT DR, RACAH INST OF PHYSICS, HEBREW UNIV OF JERUSALEM, GIVAT RAM JERUSALEM 91904, ISRAEL

Telephone: (972) 2584928 Facsimile: (972) Telex

Email:

Commission(s):

NESCI ROBERTO, ISTITUTO ASTRONOMICO, UNIVERSITA DI ROMA, VIA G M LANCISI 29, I 00161 ROMA, ITALY Telephone: (39) $6867525 \quad$ Facsimile: (39)

Email:

Commission(s): 37

Telex: 613255 infnro

NESIS ANASTASIOS DR, KEIPENHEUER INSTITUT, FUER SONNENPHYSIK, SCHOENECKSTR 6

D 79104 FREIBURG BREISGAU, GERMANY

Telephone: (49) $761382067 \quad$ Facsimile: (49) $76132280 \quad$ Telex: 7721552 kisd

Email:

Commission(s): 12

NESS NORMAN F DR, BARTOL RESEARCH INSTITUTE, UNIVERSITY OF DELAWARE, NEWARK DE 19716, USA

Telephone: (1) $3024518116 \quad$ Facsimile: (1)

Email: $\quad$ Commission(s): 16,44

Telex: 510-6665

NESTEROV NIKOLAI S, RT 22, KATSIVELI, 33+247 CRIMEA, UKRAINE

Telephone: (7) 654727952

Facsimile: (7) $655727961 / 655+4070+$ Telex:

Email: nesterov@rt22.crimea.ua

Commission(s):

- NETZER HAGAI PROF, DPT OF PHYSICS \& ASTRON, TEL AVIV UNIVERSITY, RAMAT AVIV

TEL AVIV 69978, ISRAEL

Telephonc: (972) 35450208

Email: bitnet:h31@taunos

Facsimile: (972)

Tefex: $3+2171$ versy it

NEUGEBAUER GERRY DR, CALTECH, MS 320 47, DOWNES LAB OF PHYSICS, PASADENA CA 91125, USA

Telephonc: (1) 8183564284

Commission(s):

Telex: $675+25$

Email:

Facsimilc: (1)

Commission(s): 34

NEUKIRCH THOMAS, DPT OF MATHEMATICS, UNIVERSITY OF ST ANDREWS, ST ANDREWS, FIFE KY16 9SS, UK Tclephone: (44) 1334463713 Facsimilc: (4t) $1334+63748$

Telex:

Email: thomas@dcs.st-andrews.ac.uk

Commission(s): 10

NEUKUM G DR, D L R, NE PE, RUDOWER CHAUSSEE 5, D 12+8+ BERLIN, GERMANY

Telephonc: (49) 3069545300

Facsimile: (49) $30695+5303$

Telex: $0526+19$ dvlop d

Email:

Commission(s): 15,16 
NEUPERT WERNER M DR, NASA GSFC, CODE 680, GREENBELT MD 20771, USA

Telephone: (1) $3012868169 \quad$ Facsimile: (1) Telex:

Email: Commission(s): 10,44

NEUZIL LUDEK DR, ASTRONOMICAL INSTITUTE, CZECH ACADEMY OF SCIENCES, ONDREJOV OBSERVATORY CZ 251 65 ONDREJOV, CZECH R

Telephone: (42) $204857328 / 7111 \quad$ Facsimile: (42) 204851611 Telex: 121579

Email: astsun(a)csearn Commission(s):

NEWBURN RAY L JR, JPL, 169-237, 4800 OAK GROVE DR, PASADENA CA 91109, USA

Telephone: (1) $8183542319 \quad$ Facsimile: (1) $8183934619 \quad$ Telex: 675429

Email: ray@sch5.jpl.nasa.gov Commission(s): 15,22

NEWELL EDWARD B DR, MOUNT STROMLO \& SIDING, SPRING OBSERVATORIES, PRIVATE BAG WODEN PO ACT 2606, AUSTRALIA

Telephone: (61) $62881111 \quad$ Facsimile: (61) $62490233 \quad$ Telex: aa 62270 canopus

Email:

Commission(s): 37

NEWHALL X X DR, JPL, MS 238 332, 4800 OAK GROVE DR, PASADENA CA 91109, USA

Telephone: (1) $8183540000 \quad$ Facsimile: (1) Telex: 192961003

Email: span:logos:xxn Commission(s): 04,19,31

NEWMAN MICHAEL JOHN DR, LOS ALAMOS NATIONAL LAB, MS B220 X 2, BOX 1663, LOS ALAMOS NM 87545, US Telephone: (1) 5056677698

Facsimile: (1)

Telex:

Email:

Commission(s): 35

NEWSOM GERALD H PROF, DPT OF ASTRONOMY, OHIO STATE UNIVERSITY, 17† W 18TH AVE

COLUMBUS OH 432101106 , USA

Telephone: (1) $6144227082 \quad$ Facsimile: (1) Telex:

Email:

Commission(s): $1+,+2$

NEWTON GAVIN DR, DPT PHYSICS \& ASTRONOMY, UNIVERSITY OF GLASGOW, GLASGOW G12 8QQ, UK

Telephone: (44) $1413398855^{\star 4196}$ Facsimile: (44) 1413349029 Telex: 777070 unigla

Email:

Commission(s): 09

NEWTON ROBERT R DR, APPLIED PHYSICS LAB, JOHNS HOPKINS UNIVERSITY, JOHNS HOPKINS RD

LAUREL MD 20707, USA

Telephone: (1) 3019537100

Email:

Facsimile: (1)

Telex:

Commission(s):

NEY EDWARD P PROF, DPT OF ASTRONOMY, UNIVERSITY OF MINNESOTA, 116 CHURCH ST SE

MINNEAPOLIS MN 55455, USA

Telephone: (1) 6123734687

Facsimile: (1)

Commission(s): 21

Telex:

Email:

NEZLIN MIKHAIL, RRC KURCHATOV INSTITUTE, KURCHATOV SQ 1, MOSCOW 123182, RUSSIA

Telephone: (7) 0951967976

Email: nezlin(a)wowa.net.kiae.su

Facsimile: (7) $0959430073 \quad$ Telex: 411594 shuga su

Commission(s):

NG KIN-WANG, INSTITUTE OF PHYSICS, ACADEMIA SINICA, TAIPEI TAIWAN 11529, CHINA R

Telephone: (886) 27823075

Facsimilc: (886) $2783+187 \quad$ Telex:

Email: phkwng@twnas886

Commission(s):

NGUYEN MAU TUNG DR, COMMITTEE FOR SPACE RESEARCH, 201 K16 BACH KHOA, BOX 429 BO HO

HANOI 10000 , VIETNAM

Telephone: (84) 42258333

Facsimilc: (84) 4252483

Telex: 411525 ncsr vt

Email:

Commission(s): 04

NGUYEN-QUANG RIEU DR, OBSERVATOIRE DE PARIS, SECTION DE MEUDON

F 92195 MEUDON PPL CDX, FRANCE

Telephone: (33) 145347530

Facsimilc: (33)

Telex: 270912

Email:

Commission(s): 34,40 
NHA IL-SEONG DR, YONSEI UNIVERSITY OBS, 134 SINCHON-DONG, SUDAEMUN, SEOUL 120, KOREA R Telephone: (82) 3920131 Facsimile: (82) Telex:

Email:

Commission(s): $38,41,42,46$

NIARCHOS PANAYIOTIS PH D, DPT OF ASTRONOMY, NTL UNIVERSITY OF ATHENS, PANEPISTIMIOPOLIS GR 15771 ZOGRAFOS, GREECE

Telephone: (30) 17243414

Facsimile: (30)

Telex:

Email:

Commission(s): $27,42,51$

NLAZY ADNAN MOHAMMAD DR, KACST, BOX 6086, RIYADH 11 $\$ 42$, SAUDI ARABIA

Telephone: (966) 14883751

Facsimile: (966) 14883756

Telex: 404017

Email:

Commission(s):

NICHOLLS JENNIFER, RCFTA, SCHOOL OF PHYSICS, UNIVERSITY OF SYDNEY, SYDNEY NSW 2006, AUSTRALIA

Telcphone: (61) 23512621

Facsimile: (61) 26602903

Telex:

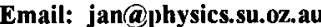

Commission(s): 40

NICHOLLS RALPH W PROF, DPT PHYSICS \& ASTRONOMY, YORK UNIVERSITY, 4700 KEELE ST NORTH YORK ON M3J 1P3, CANADA

Telephone: (1) 4167365247

Facsimile: (1) 4167365626

Telex: $0652+736$

Email: fs300003ayusol

Commission(s): 14,29

NICHOLS-BOHLIN JOY DR, COMPUTOR SCIENCES CORP, 10000A AEROSPACE RD

LANHAM SEABROOK MD 20706, USA

Telephonc: (1) 3017941410

Facsimile: (1)

Telex:

Email: 6890: nichols

Commission(s): 44

NICOLAIDIS EFTHYMIOS DR, NTL RESEARCH FOUNDATION, 48 VAS CONSTANTINOU AVE GR 11635 ATHENS, GREECE

Telephone: (30) 17210554

Facsimilc: (30) 17212729

Telex: 224064

Email:

Commission(s): 41

NICOLAS KENNETH ROBERT, NAVAL RESEARCH LABORATORY, CODE 4163,4555 OVERLOOK AVE SW WASHINGTON DC 203755000 , USA

Telephone: (1) 2027672517

Facsimile: (1)

Telex:

Email:

Commission(s): 12

NICOLET BERNARD, OBSERVATOIRE DE GENEVE, CHEMIN DES MAILLETTES 51 CH 1290 SAUVERNY, SWITZERLAND

Telephone: (41) 227552611

Email: nicolct@obs.unige.ch

Facsimile: (41) 227553983

Telex: 419209 obs ch

NICOLET MARCEL PROF, IASB, AVE DEN DOORN 30, B 1180 BRUSSELS, BELGIUM

Tclephone: (32) 23742948

Facsimile: (32)

Commission(s): 12,21

Telex: 21563 espace b

NICOLL JEFFREY FANCHER, INST FOR DEFENCE ANALYSES, 1801 N BEAUREGARD ST ALEXANDRIA VA 22311, USA

Telephone: (1) 7035782987

Facsimilc: (1) 7035782877

Telex:

Email: jnicoll@ida.org

Commission(s): $\$ 7$

NICOLOV NIKOLAI S DR, DPT OF ASTRONOMY, UNIVERSITY OF SOFIA, ANTON IVANOV ST 5 BG 1126 SOFIA, BULGARIA

Telephone: (359) 2544852

Facsimile: (359)

Commission(s): 27,46

Telex:

Email:

NICOLSON GEORGE D DR, HARTEBEESTHOEK RADIOASTRO, OBSERVATORY, BOX 443 KRUGERSDORP 1740, SOUTH AFRICA

Telephone: (27) $27116424692 \quad$ Facsimile: (27) 2711642 2446 Telex: 321006 hart sa

Email:

Commission(s): 40

NICOLSON IAIN, UNIV OF HERTFORDSHIRE, BAYFORDBURY, LOWER HATFIELD RD, HERTFORD SG13 8 LD, UK Telephone: (44) 1707285560

Facsimile: (44) 1707285562

Telex:

Email:

Commission(s): 46 
NIEDNER MALCOLM B DR, NASA GSFC, LASP, GREENBELT MD 20771, USA

Telejhone: (1)

Facsimile: (1)

Telex:

Email:

Commission(s): 15

NIEDNER MALCOLM DR, NASA GSFC, GREENBELT MD 20771, USA

$\begin{array}{ll}\text { Telephone: (1) } 3012862000 & \text { Facsimile: (1) } \\ \text { Email: } & \text { Commission(s): }\end{array}$

NIELL ARTHUR E DR, HAYSTACK OBSERVATORY, WESTFORD MA 01886, USA

Telephone: (1) 617692476

Facsimile: (1)

Telex: 948149

Email:

Commission(s):

NIEMELA VIRPI S DR, CALLE 51 ESQ 11, 189+ VILLA ELISA (BS AS), ARGENTINA

Telephone: (54) 2143793

Facsimile: (5t)

Email: ncf::psi\%telenet.iafe::virpi

Commission(s): 29.42

NIEMI AIMO, TURKU UNIVERSITY OBS, TUORLA, SF 21500 PIIKKIO, FINLAND

Telephone: (358) 21435822

Facsimile: (358) 21433767

Telex: 62638 tyf sf

Email:

Commission(s): 09,19

NIEUWENHUIJZEN HANS DR, STERREKUNDIG INSTITUTE, BOX 80000, NL 3508 TA UTRECHT, NETHERLANDS

Telephone: (31) $30535237 \quad$ Facsimile: (31)

Email: bitnet:xmhansen(âhutruu0 Commission(s):

Telex: 40048 fylut $n l$

NIIMI YUKIO, TOKYO ASTRONOMICAL OBS, NAOJ, OSAWA MITAKA, TOKYO 181, JAPAN

Telephone: (81) $422325111 \quad$ Facsimile: (81)

Email:

Commission(s): 3

Telex: 2822307 taomtk j

NIKITIN A A DR, ASTRONOMICAL OBSERVATORY, ST PETERSBURG UNIVERSITY, BIBLIOTECHNAJA PL 2 198904 ST PETERSBURG, RUSSIA

Telephone: (7) 2932262

Facsimile: (7)

Telex:

Email:

Commission(s):

NIKOGHOSSIAN ARTHUR G DR, BYURAKAN ASTROPHYSICAL, OBSERVATORY, 378433 BYURAKAN, ARMENIA

Telephone: (7) 8852283453

Facsimilc: (7) $885228+142$

Telex: 411576 ascon su

Email:

Commission(s): 36

NIKOLOFF IVAN DR, PERTH OBSERVATORY, BICKLEY WA 6076, AUSTRALIA

Telephone: (61) 92931865

Facsimilc: (61)

Telex:

Email:

Commission(s): 08

NIKOLOV ANDREJ DR, DPT OF ASTRONOMY, UNIVERSITY OF SOFIA, ANTON IVANOV ST 5

BG 1126 SOFIA, BULGARIA

Telephonc: (359) 2544852

Facsimile: (359)

Commission(s): 27

Telex: 23296 sukor bg

Email:

NILSON PETER DR, ASTRONOMICAL OBSERVATORY, BOX 515, S 75120 UPPSALA, SWEDEN

Telephone: (46)

Facsimilc: (46)

Telex:

Email:

Commission(s):

NILSSON CARL DR, CENTER FOR ASTROPHYSICS, HCO/SAO, 60 GARDEN ST, CAMBRIDGE MA 02138, USA

Telephone: (1)

Facsimilc: (1)

Tclex:

Email:

Commission(s):

NINKOV ZORAN, CTR FOR IMAGING SCIENCE, ROCHESTER INSTITUTE OF, TECHNOLOGY

ROCHESTER NY 14623 5604, USA

Telephone: (1) 7164757195

Email: zxnpcißaborg.cis.rit.edu

Facsimile: (1) $716+755988 \quad$ Telex:

Commission(s): 37,15

NINKOVIC SLOBODAN, ASTRONOMICAL OBSERVATORY, VOLGINA 7

YU 11050 BEOGRAD, YUGOSLAVIA-SERBIA \& MONTENEGRO

Telcphonc: (38) 11419 357/421 $875 \quad$ Facsimile: (38)

Telex:

Email:

Commission(s): $28,33,38$ 
NISHI KEIZO DR, TOKYO ASTRONOMICAL OBS, NAOJ, OSAWA MITAKA, TOKYO 181, JAPAN Telephone: (81) $422325111 \quad$ Facsimile: (81) Telex: (02822307 taomtk j

Email:

Commission(s): 10,12

NISHIDA MINORU PROF, DPT OF PHYSICS, KYOTO UNIVERSITY, KITASHIRAKAWA SAKYO KU

KYOTO 606, JAPAN

Telephone: (81)

Facsimile: (81)

Commission(s): $33,35,47$

Telex: $5+22693$ libkyu $j$

Email:

NISHIDA MITSUGU, DPT OF LITERATURE, KOBE WOMEN'S UNIVERSITY, SUMA KU, KOBE 654, JAPAN

Telephone: (81) 787314416

Facsimile: (81)

Telex:

Email:

Commission(s): 33

NISHIKAWA JUN, HIRAISO SOLAR TERRESTRIAL, ISOZAKI 3601, HITACHINAKA, IBARAKI 311 12, JAPAN

Telephone: (81) 292659723

Facsimile: (81) $292659726 \quad$ Telex:

Email: nisikawa(a)planet.crl.go.jl) Commission(s): 09

NISHIMURA JUN DR, INST SPACE \& AERON SCI, UNIVERSITY OF TOKYO, MEGURO KU, TOKYO 153, JAPAN

Telephone: (81) 34671111 *388 Facsimile: $(81)$

Email:

Commission(s):

Telex: j 24550 spacetky

NISHIMURA MASAKI, DPT OF PHYSICS, HOKKAIDO UNIVERSITY, KITA 10 NISHI 8, SAPPORO 060, JAPAN

Telephone: (81) $117112111 \quad$ Facsimile: (81)

Telex:

Email:

Commission(s):

NISHIMURA SHIRO PROF EMER, NTL ASTRONOMICAL OBS, NAOJ, OSAWA MITAKA, TOKYO 181, JAPAN

Telephone: (81) 422343761

Facsimile: $(81)$

Telex:

Email: bnishim@c1.mtk.nao.ac.jp

Commission(s): $(05,09,29$

NISHIMURA TETSUO DR, STEWARD OBSERVATORY, UNIVERSITY OF ARIZONA, TUCSON AZ 85721, USA

Telephone: (1) 5206212054

Facsimile: (1)

Telex: 467175

Email:

Commission(s): 21

NISHIO MASANORI DR, NOBEYAMA RADIO OBS, NAOJ, MINAMIMAKI MURA, NAGANO 384 13, JAPAN

Telephone: (81) 267634381

Facsimile: (81) 267982506

Telex: 3329005

Email:

Commission(s): 40

NISSEN POUL E PROF, INST OF PHYSICS \& ASTRON, UNIVERSITY OF AARHUS, NY MUNKEGADE

DK 8000 AARHUS C, DENMARK

Telephone: (45) 86128899

Email:

Facsimile: (45) $86202711 \quad$ Telex: $6+767$ aausci $d k$

Commission(s): 29,37

NITTMANN JOHANN, DIGITAL EOUIPMENT CBT, FAVORITENSTR 7, A 1040 WIEN, AUSTRIA

Telephone: (43) $1505487012 \quad$ Facsimile: (43) $1505+87022$ Telex:

Email: johannnittmann(a)cbt.mts.dec.com Commission(s):

NITYANANDA R DR, RAMAN RESEARCH INSTITUTE, SADASHIVANAGAR, CV RAMAN AVE

BANGALORE 560 080, INDIA

Telephone: (91) 803360122

Facsimile: (91) $8033+0492$

Telex: 8452671 rri in

Email: najaram@rri.ernet.in

Commission(s): 28,44

NOBILI ANNA M, IST DI MATEMATICA, UNIVERSITA DI PISA, VIA BUONARROTI 2, I 56127 PISA, ITALY

Telephone: (39)

Facsimile: (39)

Telex:

Email:

Commission(s): 07,20

NOBILI L DR, DPT DI FISICA G GALILEI, UNIVERSITA DI PADOVA, VIA MARZOLO 8, I 35131 PADOVA, ITALY

Telephone: (39) 4984 4205*111 Facsimile: (39)

Email: $\quad$ Commission(s):

Tclex: 430308 dfggpdi

NOCERA LUIGI DR, IST DI FISICA, ATOMICA E MOLECOLARE, VIA GIARDINO 7, I 56127 PISA, ITALY

Telephonc: (39) 50501384

Facsimilc: (39) 5025175

Telex:

Email: bistab@icnuccvm

Commission(s): 10 
NOCI GLANCARLO PROF, DPT DI ASTRONOMIA, UNIVERSITA DI FIRENZE, LARGO E FERMI 5

I 50125 FIRENZE, ITALY

Telephone: (39) 5527521

Facsimile: (39) 55220039

Telex: 572268 arcetr $i$

Email:

Commission(s):

NOEL FERNANDO, DPT DE ASTRONOMIA, UNIVERSIDAD DE CHILE, CASILLA 36 D, SANTIAGO, CHILE

Telephone: (56) 22294101

Facsimile: (56)

Telex: 40853

Email:

Commission(s): 08C,31

NOELS ARLETTE DR, 50 AVE DE LA PAIX, BOX 063, B 4030 GRIVEGNEE, BELGIUM

Telephone: (32) $41529980^{* 7517}$

Facsimile: (32)

Telex:

Email:

Commission(s): 35,46

NOENS JACQUES-CLAIR DR, OBS MIDI PYRENEES, 9 R PONT DE LA MOUETTE

F 65200 BAGNERES BIGORRE, FRANCE

Telephone: (33) 62951969

Facsimile: (33) 62951070

Commission(s): 10

Telex: $\mathbf{5 3 1 6 2 5}$

Email:

NOERDLINGER PETER D PROF, APPLIED RESEARCH CORP, 8201 CORPORATE DR, SUITE 1120

LANDOVER MD 20785, USA

Telephone: (1) 3014598442

Facsimile: (1)

Telex:

Email:

Commission(s): 47

NOGUCHI KUNIO, DPT OF ASTROPHYSICS, NAGOYO UNIVERSITY, FURO CHO CHIKUSA KU

NAGOYO 46401, JAPAN

Telephonc: (81) 527892926

Facsimilc: (81) 527892922

Telex: $\mathbf{4 7 7 3 2 3}$ scunag j

Email:

Commission(s): 25

NOGUCHI MASAFUMI DR, ASTRONOMICAL INSTITUTE, TOHOKU UNIVERSITY, AOBA KU, SENDAI 980, JAPAN

Telephone: (81)

Facsimile: (81)

Telex:

Email:

Commission(s): 28

NOLL KEITH STEPHEN DR, STSCI, HOMEWOOD CAMPUS, 3700 SAN MARTIN DR, BALTIMORE MD 21218, USA

Telephone: (1) 4103385080

Facsimile: (1)

Telex:

Email: noli@snoqualm.stsci.cdu

Commission(s): $15,16 \mathrm{C}$

NOLLEZ GERARD DR, INSTITUT D'ASTROPHYSIQUE, 98BIS BD ARAGO, F 75014 PARIS, FRANCE

Telephone: (33) 144328142

Facsimile: (33) 1 i\$ $328001 \quad$ Telex:

Email:

Commission(s): 14

NOMOTO KEN'ICHI DR, DPT OF ASTRONOMY, UNIVERSITY OF TOKYO, BUNKYO KU, TOKYO 113, JAPAN

Telephone: (81) $338122111 * 4255 \quad$ Facsimile: $(81) 338139439$

Telex: 2722126 utgabj

Email: nomoto@apsun1.astron.s.u.-toky Commission(s): 35C,44

NOONAN THOMAS W PROF, SUNY AT BROCKPORT, DPT OF PHYSICS, BROCKPORT NY 14420, USA

Telejphone: (1) 7163955581

Facsimile: (1)

Telex:

Email:

Commission(s): 28,47

NORDH H LENNART DR, STOCKHOLM OBSERVATORY, S 133 36 SALTSJOEBADEN, SWEDEN

Telephone: (46) $87170195 \quad$ Facsimile: (46) $8717+719 \quad$ Telex: 12972

Email: nordh@astro.su.se Commission(s): 34,44

NORDLUND AKE DR, UNIVERSITY OBSERVATORY, OESTER VOLDGADE 3, DK 1350 COPENHAGEN K, DENMARK

Telephone: (45) 31141790

Facsimilc: (45) 31389157

Email:

Commission(s): 12,36

Telex: $\mathbf{4 4 1 5 5}$ danast

NORDSTROEM BIRGITTA DR, COPENHAGEN UNIVERSITY OBS, BRORFELDEVEJ 23

DK 4340 TOLLOSE, DENMARK

Telephone: (45) 53488195

Facsimilc: (45) 53488755

Commission(s): $30 \mathrm{C}, 33,42$

Telex: $\mathbf{4 4 1 5 5}$ danast dk

Email: birgitta@astro.ku.dk

NORGAARD-NIELSEN HANS U, UNIVERSITY OBSERVATORY, OESTER VOLDGADE 3

DK 1350 COPENHAGEN K, DENMARK

Telejhone: (45) 31141790

Facsimile: (45) 31389157

Telex:

Email:

Commission(s): 
M 730

NORIEGA-CRESPO ALBERTO DR, DPT OF ASTRONOMY, UNIVERSITY OF WASHINGTON, FM 20

SEATTLE WA 98195, USA

Telephone: (1) $2066852155 \quad$ Facsimile: (1) Telex:

Email: aipsac@phast.phys.washinton.ed Commission(s):

NORMAN COLIN A PROF, STSCI, HOMEWOOD CAMPUS, 3700 SAN MARTIN DR, BALTIMORE MD 21218, USA

Telephone: (1) 3013384895

Facsimile: (1)

Telex: 6849101

Email: stscic::norman

Commission(s): $28,33,34,44,47$

NORRIS JOHN DR, MOUNT STROMLO \& SIDING, SPRING OBSERVATORIES, PRIVATE BAG WODEN PO ACT 2606, AUSTRALIA

Telephone: (61) 62881111

Facsimile: (61) $62+90233$

Telex: aa 62270 canopus

Email:

Commission(s): 29

NORRIS RAYMOND PAUL, CSIRO, DIV OF RADIOPHYSICS, BOX 76, EPPING NSW 2121, AUSTRALIA

Telephone: (61) $23724416 \quad$ Facsimile: (61) $23724400 \quad$ Telex: 26230 astro

Email: rnorris@rp.csiro.au

Commission(s): 51

NORTH JOHN DAVID PROF, FILOSOFISCH INST, RIJKSUNIVERSITEIT, A WEG 30

NL 9718 CW GRONINGEN, NETHERLANDS

Telephone: (31) $59071846 \quad$ Facsimile: (31) $50636160 \quad$ Telex:

Email:

Commission(s): $\$ 1 \mathrm{C}$

NORTH PIERRE, INSTITUT D'ASTRONOMIE, UNIVERSITE DE LAUSANNE

CH 1290 CHAVANNES-D-BOIS, SWITZERLAND

Telephone: (41) $227552611 \quad$ Facsimile: (41) $227553983 \quad$ Telex: 27720 obsg ch

Email: north@obs.unige.ch Commission(s): 45C

NOSKOV BORIS N DR, STERNBERG STATE ASTR INST, UNIVERSITETSKIJ PROSP 13, 119899 MOSCOW, RUSSIA

Telephone: (7)

Facsimile: (7)

Telex:

Email:

Commission(s): 07

NOTNI P DR, ZNTRLINST F ASTROPHYSIK, STERNWARTE BABELSBERG, ROSA LUXEMBURG STR 17A

D 14473 POTSDAM, GERMANY

Telephone: (49)

Email:

Facsimile: (49) $\quad$ Telex:
Commission(s): 25,45

NOTTALE LAURENT, OBSERVATOIRE DE PARIS, SECTION DE MEUDON, DAF, F 92195 MEUDON PPL CDX, FRANC

Telephone: (33) $145077403 \quad$ Facsimile: (33)

Email:

Commission(s): 47

Telex: 201571

NOVELLO MARIO DR, CTR BRAS PESQUISAS FISIC, RUA DR XAVIER SIGAUD, 150 URCA 22290 RIO DE JANEIRO RJ, BRAZIL

$\begin{array}{lll}\text { Telephone: (55) } 215410337 & \text { Facsimile: (55) } & \text { Telex: } 2122563 \\ \text { Email: } & \text { Commission(s): } 47 & \end{array}$

NOVICK ROBERT, DPT OF PHYSICS, COLUMBIA UNIVERSITY, 538 W 120TH ST, NEW YORK NY 10027, USA

Telephone: (1) 2122803293

Facsimile: (1)

Telex: 22094 colu ur

Email:

Commission(s): 44

NOVIKOV I D DR, SPACE RESEARCH INSTITUTE, ACADEMY OF SCIENCES, PROFSOJUZNAYA UL 84/32

117810 MOSCOW, RUSSIA

Telephone: (7)

Email:

Facsimile: (7)

Telex:

Commission(s): 47

NOVIKOV SERGEJ B DR, STERNBERG STATE ASTR INST, 117234 MOSCOW, RUSSIA

Telephone: (7)

Facsimile: (7)

Telex:

Email:

Commission(s):

NOVOSELOV V S PROF DR, ASTRONOMICAL OBSERVATORY, ST PETERSBURG UNIVERSITY BIBLIOTECHNAJA PL 2, 19894 ST PETERSBURG, RUSSIA

Telephone: (7) 2579491

Facsimile: (7)

Telex:

Email:

Commission(s): 07 
NOVOTNY JAN DR, FAC OF SCIENCE, MSARYK UNIVERSITY, KOTLARSKA 2, CZ 61137 BRNO, CZECH R

Telephone: (42) 57129466

Facsimile: (42) $57+0108$

Telex:

Email: novotny@elanor.sci.muni.cs

Commission(s): 47

NOYES ROBERT W PROF, CENTER FOR ASTROPHYSICS, HCO/SAO, 60 GARDEN ST, CAMBRIDGE MA 02138, USA Telephone: (1) 6174957424

Facsimile: (1)

Telex: 921428 satellite cam

Email:

Commission(s): 10,12,44

NUGIS TIIT, TARTU ASTROPHYSICAL OBS, ESTONIAN ACAD OF SCIENCES, EE 202444 TARTU, ESTONIA

Telephone: (372)

Facsimile: (372)

Telex:

Email:

Commission(s): 27,29

NULSEN PAUL DR, DPT OF PHYSICS, UNIVERSITY OF WOLLONGONG, WOLLONGONG NSW 2522, AUSTRALIA

Telephone: (61)

Facsimile: (61)

Telcx:

Email: p.nulsen(a)now.edu.au

Commission(s): $28,34,44$

NUNES ROGERIO S DE SOUSA, GRUPO DE MATEM APLICADA, UNIVERSIDADE DO PORTO, RUA DAS TAIPAS 135 P 4000 PORTO, PORTUGAL

Telephonc: (351) 380313/769

Email:
Facsimile: (351)

Commission(s): 09
Telex:

NUNEZ JORGE DR, OBSERVATORIO FABRA, TIBIDADO, E 08022 BARCELONA, SPAIN

Telephone: (34) $32475736 \quad$ Facsimile: (34)

Email: . Commission(s): 24

Telex:
NUNEZ JOSUE ARTURO, OBSERVATORIO ASTRONOMICO, PASEO DEL BOSQUE S/N 1900 LA PLATA (BS AS), ARGENTINA

Telephone: (54) 2138810

Email: jan@fcaglp.edu.ar

Facsimile: (54) $21258985 \quad$ Telex:

Commission(s):
NUSSBAUMER HARRY PROF, INSTITUT FUER ASTRONOMIE, ETH ZENTRUM, CH 8092 ZUERICH, SWITZERLAND Telephone: (41) 12563631

Email:

Facsimile: (41)

Commission(s): 10,14,34

Telex: 53178 ethbi ch

NUTH JOSEPH A III, NASA GSFC, CODE 691, LEP, GREENBELT MD 20771, USA

Telephone: (1) 3012866364

Facsimile: (1)

Telex:

Email:

Commission(s): $22,3+$

NYMAN LARS-AKE DR, ONSALA SPACE OBSERVATORY, CHALMERS UNIV OF TECH, $\mathrm{S} 439$ 92 ONSALA, SWEDEN Telejhone: (46) 317725500

Facsimile: (\$6) 317725550

Tclex: 2400

Email:

Commission(s):

\section{OBASHEV SAKEN O DR, ASTROPHYSICAL INSTITUTE, KAZAKH ACAD OF SCIENCES}

480068 ALMA ATA, KAZAKHSTAN

Telephone: (7)

Facsimile: (7)

Telex: 275

Email:

Commission(s):

OBI SHINYA PROF, UNIVERSITY OF THE AIR, 2-11 WAKABA, MIHAMA KU, CHIBA BITY 261, JAPAN

Telephone: (81) 432765111

Email:

Facsimile: (81) $\$ 32766130$

Telex:

Commission(s): 14

OBLAK EDOUARD, OBSERVATOIRE DE BESANCON, 41 BIS AVE OBSERVATOIRE

F 25000 BESANCON CDX, FRANCE

Tclephone: (33) 81503088

Facsimile: (33)

Telcx: 361144

Email:

Commission(s): $25,26,33$

OBREGON DIAZ OCTAVIO J DR, DPT DE FISICA, UNAM UNIDAD, APDO POSTAL 55-534, 09340 MEXICO DF, MEXIC

Telephone: (52) 6860322

Facsimile: (52)

Commission(s):

Telex: $176+296$ uam me

Email:

OBRIDKO VLADIMIR N DR, IZMIRAN, ACADEMY OF SCIENCES, $1+2092$ TROITSK, RUSSIA

Telephone: (7) 2321921

Facsimilc: (7)

Email: obridko@lars.izmiran.troitsk.s

Commission(s): 10
Telex: $\mathbf{4 1 2 6 2 3}$ scstp 
OCCHIONERO FRANCO PROF, OAR, VIA DEL PARCO MELLINI 84, I OO136 ROMA, ITALY

Telephone: (39) 63452656 Facsimile: (39) Tek:

Email:

Commission(s):

OCHSENBEIN FRANCOIS DR, OBSERVATOIRE ASTRONOMIQUE, 11 RUE UNIVERSITE F 67000 STRASBOURG, FRANCE

Telephone: (33) 88358218

Facsimile: (33) 88250160

Telex: 890506 starobsf

Email:

Commission(s): 05

ODA MINORU PROF, WAKO SHI, SAITAMA 351 01, JAPAN

Telephone: (81) 484621111

Facsimile: (81) $4846759+2$

Commission(s): 44,51

Telex: 02962818 riken $j$

Email:

ODA NAOKI, MICRO-ELECTRONICS RES LAB, NIPPON ELECTRIC COMPANY, 4-1-1 MIYAZAKI MIYAMAE KU KAWASAKI KANAGAWA 213, JAPAN

Telephone: (81)

Facsimile: (81)

Telex:

Email:

Commission(s): 44

ODELL ANDREW P, ASTROPHYSICAL OBSERVATORY, N ARIZONA UNIVERSITY, FLAGSTAFF AZ 86011, USA

Telephone: (1)

Facsimile: (1)

Telex:

Email:

Commission(s): 35

ODENWALD STEN F DR, NAVAL RESEARCH LABORATORY, CODE 4138 0, 4555 OVERLOOK AVE SW

WASHINGTON DC 20375 5000, USA

Telephone: (1) 2027673010

Facsimile: (1)

Telex:

Email:

Commission(s):

ODGERS GRAHAM J DR, HERZBERG INST ASTROPHYS, DOMINION ASTROPHYS OBS, 5071 W SAANICH RD VICTORIA BC V8X 4M6, CANADA

Telephone: (1) 6043883977

Facsimile: (1) 6043630045

Telex: 0497295

Email:

Commission(s): 09,27

ODSTRCIL DUSAN, CZECH ACADEMY OF SCIENCES, CZ 25165 ONDREJOV, CZECH R

Telephone: (42) 204857126

Facsimile: (42) $2881611 \quad$ Telex

Email: adstrcila)asu.cas.cz

Commission(s):

OEGELMAN HAKKI B DR, MPI F EXTRATERRESTRISCHE, PHYSIK, KARL SCHWARZSCHILDSTR 1

D 85740 GARCHING MUENCHEN, GERMANY

Telephone: (49) $8932993833 \quad$ Facsimile: (49) 8932993569 Telex:

Email: hbo at dgaipp1s Commission(s):

OEGERLE WILLIAM R, DPT PHYSICS \& ASTRONOMY, JOHNS HOPKINS UNIVERSITY, HOMEWOOD CAMPUS BALTIMORE MD 21218, USA

Telephone: (1) 4105166732

Email: oegerle(a)pha.jhu.edu

Facsimile: (1) $4105165494 \quad$ Telex:

Commission(s):

OEHMAN YNGVE PROF, THULELEM 53, S 22367 LUND, SWEDEN

Tclephone: (46) 46143362

Facsimile: (46)

Commission(s):

Telex:

Email:

BSERVATORY, UNIVERSITY OF ISTANBUL, 34452 ISTANBUL, TURKEY

Telephone: (90) 15223597 Facsimile: (90) 15226123 Telex:

Email: jk017@triuvm11

Commission(s): 10

OEMLER AUGUSTUS JR DR, YALE UNIVERSITY OBS, BOX 6666, NEW HAVEN CT 06511, USA

Telephonc: (1) 2034363460

Facsimile: (1)

Telex:

Email:

Commission(s): 28,47

OERTEL GOETZ K DR, AURA INC, 1625 MASSACHUSETTS AVE NW, SUITE 701, WASHINGTON DC 20036, USA

Telephone: (1) 2024832101

Facsimile: (1)

Telex:

Email: telemail: g.ocrtel

Commission(s): 4

OESTERWINTER CLAUS, BOX 1270, DAHLGREN VA 22448 5180, USA

Telephone: (1) 7036632555

Facsimile: (1)

Telex:

Email:

Commission(s): 04,07 
OESTGAARD ERLEND, DPT OF PHYSICS, UNIVERSITY OF TRONDHEIM, AVH, N 70S5 DRAGVOLLM, NORWAY

Telephone: (47) 07-920411 $\times 117$

Facsimile: (47)

Telex:

Email:

Commission(s):

OESTREICHER ROLAND, LANDESSTERNWARTE, KOENIGSTUHL, D 69117 HEIDELBERG, GERMANY

Telephone: (49) $622110036 \quad$ Facsimile: (49)

Telex:

Email:

Commission(s): 25

OETKEN L DR, ZNTRLINST F ASTROPHYSIK, ASTROPHYSIKALISCHES OBS, GRENZSTR 4

D 14552 WILDENBRUCH, GERMANY

Telephone: (49)

Facsimile: (49)

Telex:

Email:

Commission(s): 14,30

OEZEL MEHMET EMIN DR, SPACE SCIENCES DPR, MARMARA RESEARCH CENTRE, PK 21

41470 GEBZE KOCAELI, TURKEY

Telephone: (90) 2626412300

Facsimile: (90) $2626412309 \quad$ Telex:

Email: ozel@trmbeam

Commission(s): $40,44,50$

OEZKAN MUSTAFA TUERKER DR, UNIVERSITY OBSERVATORY, UNIVERSITY OF ISTANBUL 34452 ISTANBUL, TURKEY

Telephone: (90) 15283847

Facsimile: (90) 15226123

Telex:

Email: jk017@triuvm11

Commission(s): 42

OGAWARA YOSHIAKI, INST SPACE \& ASTRON SCI, UNIVERSITY OF TOKYO, MEGURO KU, TOKYO 153, JAPAN

Telephone: (81) 34671111

Facsimile: (81)

Commission(s): 44

Telex: $3 \$ 757$ isastro $\mathrm{j}$

Email:

OGELMAN HAKKI B DR, DPT OF PHYSICS, UNIVERSITY OF WISCONSIN, 1150 UNIVERSITY AVE MADISON WI 53706, USA

Telephone: (1) 6082652052

Facsimile: (1) 6082630800

Telex: 265452 uofwisc mds

Email: 47452::ogelman

Commission(s): +4

OGIR MAYA DR, CRIMEAN ASTROPHYS OBS, UKRAINIAN ACAD OF SCIENCE, NAUCHNY

334413 CRIMEA, UKRAINE

Telephone: (7) 432945

Facsimile: (7)

Telex:

Email:

Commission(s): 10

OGURA KATSUO DR, COL OF LITERATURE, KOKUGAKUIN UNIVERSITY, HIGASHI 4-10-2

IBUYAKU TOKYO 150, JAPAN

Telephone: (81) $298426913 \quad$ Facsimile: $(81)$

Email:

Commission(s): 37

Telex: 28899 sibinbth j

OH KYU DONG DR, DPT EARTH SCIENCE, CHUNGNAM NTL UNIVERSITY, KWANGJU CHONNAN, KOREA R

Telephonc: (82)

Facsimilc: (82)

Telex:

Email:

Commission(s): 42

OHASHI TAKAYA DR, DPT OF PHYSICS, TOKYO METROPOLITAN UNIV, 1-1 MINAMI OHSAWA

HACHIOJI TOKYO 192-03, JAPAN

Telephone: (81) 42677111 *3245

Email: ohashi(a)phys.metro-u.ac.jp

Facsimilc: (81) 426772483

Telex:

OHISHI MASATOSHI DR, NOBEYAMA RADIO OBS, NAOJ, MINAMIMAKI MURA, NAGANO 38+ 13, JAPAN

Telephone: (81) 267634373

Email: nrooisi@jipnnro.bitnct

Facsimile: (81) 267982884

Telex: 3329005 naonro $j$

OHKI KENICHIRO DR, TOKYO ASTRONOMICAL OBS, NAOJ, OSAWA MITAKA, TOKYO 181, JAPAN

Telephone: (81) $422325111 \quad$ Facsimilc: (81)

Telex: 2822307

Email:

Commission(s): 10

OHRING GEORGE PROF, DPT GEOPHYS \& PLANET SCI, TEL AVIV UNIVERSITY, TEL AVIV, ISRAEL

Telephone: (972)

Facsimile: (972)

Telex:

Email:

Commission(s):

OHTA KOUJI, DPT OF ASTRONOMY, FACULTY OF SCIENCE, KYOTO UNIVERSITY, KYOTO 606, JAPAN

Telephonc: (81) 757533896

Facsimilc: (81) 757533897

Email: ohta@kusastro.kyoto-u.ac.jp

Commission(s): 28

Telex: 
OHTANI HIROSHI DR, DPT OF ASTRONOMY, KYOTO UNIVERSITY, KITASHIRAKAWA SAKYO KU KYOTO 606, JAPAN

Email: ohtani@kusastro.kyoto-u.ac.jp Commission(s): 34

Telex: $5+22693$ libkyu j

OHTSUBO JUNJI DR, FACULTY OF ENGINEERING, SHIZUOKA UNIVERSITY, 3 CHOME JYOHOKU

HAMAMATSU 432, JAPAN

Telephone: (81) 534711171 *585

Facsimile: (81) 53\$75 $1764 \quad$ Telex:

Email:

Commission(s): 09

OHYAMA NOBORU PROF, FACULTY OF ENGINEERING, SHIZUOKA UNIVERSITY, 3 CHOME JYOHOKU

HAMAMATSU 432, JAPAN

Telephone: (81)

Facsimile: (81)

Telex:

Email:

Commission(s): 35

OJA HEIKKI DR, OBS \& ASTROPHYSICS LAB, UNIVERSITY OF HELSINKI, TAEHTITORNINMAKI

SF 00130 HELSINKI 13, FINLAND

Telephone: (358) 1912942

Facsimile: (358)

Telex:

Email:

Commission(s): 46

OJA TARMO PROF, KVISTABERG OBSERVATORY, S 197 00 BRO, SWEDEN

Telephone: (46) 75840157

Facsimile: $(40)$

Telex:

Email:

Commission(s): $24,33,45$

OKA TAKESHI DR, DPT OF CHEMISTRY, UNIVERSITY OF CHICAGO, 5735 S ELLIS AVE, CHICAGO IL 60637, USA

Telephone: (1) 3129627070

Facsimile: (1)

Telex:

Email:

Commission(s): 14

OKAMOTO ISAO DR, INTL LATITUDE OBSERVATORY, NAOJ, HOSHIGAOKA MIZUSAWA SHI, IWATE 023, JAPAN

Telephone: (81) $197247111 \quad$ Facsimile: (81)

Telex: 8376-28 ilsmiz j

Email:

Commission(s): 35

OKAMURA SADANORI DR, DPT OF ASTRONOMY, UNIVERSITY OF TOKYO, BUNKYO KU, TOKYO 113, JAPAN

Telephone: (81) 358006880

Facsimile: (81) 338139439

Telex:

Email: okamura(a)apsunl.astron.s.u-toky Commission(s): 28

OKAZAKI AKIRA DR, DPT SCIENCE EDUCATION, GUNMA UNIVERSITY, MAEBASHI, GUNMA 371, JAPAN

Telephone: (81) 272321611

Facsimilc: (81) 27233923

Telex:

Email:

Commission(s): 42

OKAZAKI ATSUO T DR, COL OF GEN EDUCATION, HOKKAI GAKUEN UNIVERSITY, TOYOHIRA-KU

SAPPORO 062, JAPAN

Telephone: (81) 118411161 *84

Facsimile: (81)

Telex:

Email: a10935@jpnkudpc.bitnet

Commission(s): 29

OKAZAKI SEICHI DR, 244 OSAWA, MITAKA, TOKYO 181, JAPAN

Telephone: (81) 422316770

Facsimile: (81)

Telex:

Email:

Commission(s):

OKE J BEVERLEY PROF, CALTECH, MS 105 24, PASADENA CA 91125, USA

Telephone: (1) 8183564007

Facsimile: (1)

Telex:

Email:

Commission(s): 28

OKEKE PIUS N DR, DPT OF PHYSICS \& ASTRON, UNIVERSITY OF NIGERIA, NSUKKA ANAMBRA STATE, NIGERIA

Telephone: (234) $42771911 \quad$ Facsimile: (234)

Email: $\quad$ Commission(s): 44

Telex:

OKOYE SAMUEL E PROF, DPT OF PHYSICS \& ASTRON, UNIVERSITY OF NIGERIA NSUKKA ANAMBRA STATE, NIGERIA

Telephone: (234) 042-770752

Facsimile: (234)

Telcx:

Email:

Commission(s): $38,40,44,46,47$ 
OKUDA HARUYUKI DR PROF, INST SPACE \& ASTRON SCI, UNIVERSITY OF TOKYO, MEGURO KU TOKYO 153, JAPAN

Telephone: (81) 34671111

Email:
Facsimile: (81)

Commission(s): 33,34
Telex: 24550 spacetky j

OKUDA TORU, INST OF EARTH SCIENCE, HOKKAIDO UNIV OF EDUCAT, 1-2 HACHIMAN CHO

HAKODATE 040, JAPAN

Tclephonc: (81) 138411121

Email:

Facsimilc: (81)

Telex:

Commission(s): 44

OKUMURA SACHIKO DR, NOBEYAMA RADIO OBS, NAOJ, MINAMISAKI MURA, NAGANO 38 4 13, JAPAN

Telephone: (81) 267634366

Email: sokumura@nro.nao.ac.jp

Facsimile: (81) 267634339

Commission(s): 40

Tclex: 3329005 naonro j

OLAH KATALIN DR, KONKOLY OBSERVATORY, THEGE U 13/17, BOX 67, H 1525 BUDAPEST, HUNGARY

Telephone: (36) $11755866 / 175 \$ 122 \quad$ Facsimile: (36) 11569640

Telex: $227+60$ konob $h$

Email: olah@ogyalla.konkoly.hu

Commission(s): 27,42

OLANO CARLOS ALBERTO DR, IAR, CC 5, 189 \& VILLA ELISA (BS AS), ARGENTINA

Telephone: (54) 2143793

Facsimile: (54)

Commission(s): 33

Telex:

Email:

OLBERG MICHAEL DR, ONSALA SPACE OBSERVATORY, CHALMERS UNIV OF TECH, 5 439 92 ONSALA, SWEDEN

Telephone: (46) 317725500

Facsimile: (46) 317725550

Telex: 2400 onspace $s$

Email: olberg@oso.chalmers.se

Commission(s): 40

OLEAK H DR, ZNTRLINST F ASTROPHYSIK, STERNWARTE BABELSBERG, ROSA LUXEMBURG STR 17A . D 14473 POTSDAM, GERMANY

Telephone: (49)

Email:

Facsimile: (49)

Telex:

Commission(s): 28

OLIVA ERNESTO DR, OSS ASTROFISICO, DI ARCETRI, LARGO E FERMI 5, I 50125 FIRENZE, ITALY

Telephone: (39) 552752310

Facsimile: (39) (5 220039

Telex: 572268

Email: span:38954::oliva

Commission(s):

OLIVER BERNARD M DR, NASA AMES RESEARCH CTR, MS 229 8, MOFFETT FIELD CA 94035, USA

Telephonc: (1) 4156945166

Facsimile: (1)

Telex: $348+108$ nasa ames

Email:

Commission(s): 51

OLIVER BERNARD M PROF, NASA AMES RESEARCH CTR, CHEIF SETI PROGRAM OFFICE

MOFFETT FIELD CA 94035, USA

Telephone: (1) 4156945166

Facsimile: (1)

Telex:

Email:

Commission(s): 51

OLIVER JOHN PARKER DR, DPT OF ASTRONOMY, UNIVERSITY OF FLORIDA, 211 SSRB

GAINESVILLE FL 32611, USA

Telephone: (1)

Email:

Facsimile: (1)

Telex:

Commission(s): 42

OLIVIER SCOT STEWART, IGPP LLNL L 413, BOX 808, LIVERMORE CA 94551 9900, USA

Telephonc: (1) 5104238129

Email: olivier@sunlight.IInl.gov

Facsimile: (1) $510+230238 \quad$ Telex:

Commission(s):

OLLONGREN A PROF DR, STERREWACHT, DPT MATHS \& COMPUTER SCI, BOX 9512

NL 2300 RA LEIDEN, NETHERLANDS

Telephone: (31) $71272727{ }^{\star 5006}$

Facsimile: (31)

Telex: 39058 astro $\mathrm{nl}$

Email:

Commission(s): 07,33,51

OLMI LUCA, CORNELL UNIVERSITY, NAIC ARECIBO OBSERVATORY, BOX 995, ARECIBO PR 00613, USA

Telephone: (1) 8098782612

Email: olmi@naic.edu

Facsimile: (1) 8098781861 Telex:

Commission(s):

OLNON FRISO, NFRA, BOX 2, NL 7990 AA DWINGELOO, NETHERLANDS

Telephone: (31) 52197244

Facsimile: (31)

Commission(s): 
M736

OLOFSSON HANS, STOCKHOLM OBSERVATORY, S 13336 SALTSJOEBADEN, SWEDEN

Telephone: (46) 8164448

Facsimile: (46) 87174719 Telex:

Email: hans@astro.su.se

Commission(s): 34

OLOFSSON S GOERAN DR, STOCKHOLM OBSERVATORY, S 133 36 SALTSJOEBADEN, SWEDEN

Telephone: (46) 87172639

Facsimile: (46) $8717 \$ 719$

Telex: 12972

Email: olofsson(a)astro.su.se

Commission(s):

OLOWIN RONALD PAUL DR, DPT OF PHYS \& ASTRONOMY, SAINT MARY'S COLLEGE, 207 D GALILEO HALL MORAGA CA 94575, USA

Telephone: (1) 5106314428

Facsimile: (1)

Telex:

Email: rpolowin@galileo.stmarys-ca.ed Commission(s): 47

OLSEN ERIK H, COPENHAGEN UNIVERSITY OBS, BRORFELDEVEJ 23, DK 4340 TOLLOSE, DENMARK

Telephone: (45) 53488195

Facsimile: (45) $58+88758$

Telex: $4 \$ 155$ danast dk

Imail:

Commission(s): 45

OLSEN FOGH H J, COPENHAGEN UNIVERSITY OBS, BRORFELDEVEJ 23, DK 4340 TOLLOSE, DENMARK

Telephone: (45) 53488195

Facsimile: (45) 58488758

Telex: $4 \$ 155$ dqnqst

Email:

Commission(s): 08,46

OLSEN KENNETH H DR, GCS, BOX 1273, LYNNWOOD WA 98046 1273, USA

Telephone: (1)

Facsimile: (1)

Telex:

Email:

Commission(s):

OLSON EDWARD C PROF, DPT OF ASTRONOMY, UNIVERSITY OF ILLINOIS, 1002 W GREEN ST

URBANA IL 61801, USA

Telephone: (1) 217333553

Facsimile: (1)

Telex:

Email: olsomed(a)uxh.cso.uiuc.edu

Commission(s): $\$ 2$

OLTHOF HINDERICUS DR, ESA/ESTEC, SSD, BOX 299, NL 2200 AG NOORDWIJK, NETHERLANDS

Telephone: (31) 171986555

Facsimile: (31)

Telex: 39098 estc nl

Email: span:estec 1::holthof

Commission(s): 44

OMAROV TUKEN B PROF, ASTROPHYSICAL INSTITUTE, KAZAKH ACAD OF SCIENCES

480068 ALMA ATA, KAZAKHSTAN

Telephone: (7) 644040

Facsimile: (7)

Telex:

Email:

Commission(s): 07

OMNES ROLAND PROF, LPTHE, UNIVERSITE PARIS XI, BT 211, F 91405 ORSAY CEDEX, FRANCE

Telephone: (33) 169417744

Facsimile: (33)

Telex: 692166

Email:

Commission(s): 47

OMONT ALAIN PROF, INSTITUT D'ASTROPHYSIQUE, 98BIS BD ARAGO, F 7501 + PARIS, FRANCE

Telephone: (33) 144328071

Facsimile: (33) 1 +\$328001 Telex:

Email:

Commission(s): 14,34

ONAKA TAKASHI, DPT OF ASTRONOMY, UNIVERSITY OF TOKYO, BUNKYO KU, TOKYO 113, JAPAN

Telephone: (81) 38122111

Facsimile: (81)

Commission(s): 34

Telex: 33659 utyosci j

Email:

ONDERLICKA BEDRICH DR, DPT OF ASTROPHYSICS, PURKYNE UNIVERSITY, KOTLARSKA 2 CZ 61137 BRNO, CZECH R

Telephone: (42) 57129482

Facsimile: (42)

Telex:

Email:

Commission(s):

ONEGINA A B DR, MAIN ASTRONOMICAL OBS, UKRAINIAN ACAD OF SCIENCE, GOLOSEEVO 252127 KIEV, UKRAINE

Telephone: (7) 044663744

Facsimile: (7)

Telex:

Email:

Commission(s): 24

ONO YORO PROF, DPT OF PHYSICS, HOKKAIDO UNIVERSITY, KITA 10 NISHI 8, SAPPORO 063, JAPAN

Telephone: (81)

Facsimile: (81)

Telex:

Email:

Commission(s): 
ONUORA LESLEY IRENE DR, DPT OF PHYSICS \& ASTRON, UNIVERSITY OF NIGERIA NSUKKA ANAMBRA STATE, NIGERIA

Telephone: (23t)

Facsimile: (234) Telex: 51496 ulions

Email:

Commission(s): $40,46,+7$

OOE MASATSUGU DR, INTL LATITUDE OBSERVATORY, NAOJ, HOSHIGAOKA MIZUSAWA SHI, IWATE 023, JAPA

Telephone: (81) $1972+7111 \quad$ Facsimile: (81)

Email:

Commission(s): 19

Telex: 837628 miz. i

OPENDAK MICHAEL, DPT ASTRON \& ASTROPHYS, UNIV OF PENNSYLVANIA, 209 SOUTH 33 ST PHILADELPHIA PA 19104, USA

Telephone: (1) 2158985066

Facsimile: (1) $2158989336 \quad$ Telex:

Email: mopendak(a)mait.sas.upenn.edu Commission(s): 34

OPHER REUVEN PROF, IAG, UNIVERSJDADE DE SAO PAULO, CP 9638, 01065 SAO PAULO SP, BRAZIL

Telephone: (55) 112753720

Facsimile: (55) 112763848

Telex: 1156735 iagm br

Ematil:

Commission(s):

OPOLSKI ANTONI PROF, ASTRONOMICAL INSTITUTE, WROCLAW UNIVERSITY, UL KOPERNIKA 11 PL 51622 WROCLAW, POLAND

Telephone: (48)

Facsimile: (48)

Telex:

Email:

Commission(s): 27

OPRESCU GABRIELA, ASTRONOMICAL INSTITUTE, CUTITUL DE ARGINT 5, BOX 28

R 75212 BUCHAREST, RUMANIA

Telephone: (40) 16236892

Email: goprescu(a) imar.ro

Facsimile: (41) $13123391 \quad$ Telex:

Commission(s):

OPROIU TIBERIU DR, ASTRONOMICAL OBSERVATORY, BLOC R-5 SC I \& II AP 11, STR BUCIUM 25

R 3H00 CLUJ NAPOCA, RUMANIA

Telejhonc: $(40) 6+162616$

Email:

Facsimile: (t(1) $6+194592$

Telex:

Commission(s):

ORCHISTON WAYNE DR, CARTER OBSERVATORY, NTL OBS OF NEW ZEALAND, BOX 2909

WELLINGTON, NEW ZEALAND

Telephonc: $(6+)+\$ 728167$

Facsimilc: $(6 t)+4728320$

Telex:

Enail: cartdir $(\bar{a}$ matai.vuw.ac.n\%

Commission(s): +1

ORELLANA ROSA BEATRIZ DR, OBSERVATORIO ASTRONOMICO, PASEO DEL BOSQUE S/N 1900 LA PLATA (BS AS), ARGENTINA

Telephone: (54) 21217308

Facsimile: (54) 21211761

Telex:

Email: rorellana\%faglp.edu.atr.

Commission(s): 177

ORLIN HYMAN DR, NTL ACADEMY OF SCIENCES, NTL RESEARCH COUNCIL, 2101 CONSTITUTION AVE NW WASHINGTON DC $20+18$, USA

Telephone: (1)

Facsimile: (1)

Commission(s):

Telex:

Email:

MICAL OBS, UKRAINIAN ACAD OF SCIENCE, GOLOSEEVO

ORLOV MIKHAIL DR,
252127 KIEV, UKRAINE

Telephone: (7) $04+663110$

Email:

Facsimile: (7)
Commission(s): 2y

Telex: 131406 sky

ORMES JONATHAN F DR, NASA GSFC, CODE 660, GREENBELT MD 20771, USA

Telephone: (1) 3012868801

Email: span lheavx: :ormes

Facsimile: (1)

Commission(s)

Telex: 89675

ORRALL FRANK Q PROF, INSTITUTE FOR ASTRONOMY, UNIVERSITY OF HAWAII, 2680 WOODLAWN DR HONOLULU HI 96822 , USA

Telephone: (1) 8089568667

Facsimile: (1) 8089882790

Telex: 723-8459

Email:

Commission(s): 10,12,36

ORSATTI ANA M DR, OBSERVATORIO ASTRONOMICO, PASEO DEL BOSQUE S/N 1900 LA PLATA (BS AS), ARGENTINA

Telephone: (54) 21217308

Email: amoūircagtp.edu.ar

Facsimile: (54) 21258985

Comnission(s): 25
Telex: 31151 bulap 
M 738

ORTE AlbERTO, CECILIO PUJAZON 22-3 A, E 11100 SAN FERNANDO, SPAIN

Telephone: (34) 56895441 Facsimile: (34) Telex:

Email:

Commission(s):

ORTOLANI SERGIO, OSS ASTRONOMICO DI PADOVA, VIC DELL OSSERVATORIO 5, I 35122 PADOVA, ITALY

Telephone: (39) 49661499

Facsimile: (39)

Telex: $\mathbf{4 3 2 0 7 1}$ astros

Email:

Commission(s): 37

ORTON GLENN S DR, JPL, MS 183 301, 4800 OAK GROVE DR, PASADENA CA 91109, USA

Telephone: (1) 8183544321

Facsimile: (1)

Telex:

Email:

Commission(s): 14

ORUS JUAN J PROF, DPT FISICA DE ATMOSFERA, UNIVERSIDAD DE BARCELONA, AVD DIAGONAL 645 E 08028 BARCELONA, SPAIN

Telephone: (34)

Facsimile: (34)

Telex:

Email:

Commission(s): 07

OSAKI TORU DR, RYUKOKU UNIVERSITY, FUKAKUSA TSUKAMOTO, FUSHIMI KU, KYOTO G12, JAPAN

Telephone: (81) 75642111

Facsimile: (81)

Telex:

Email:

Commission(s): 34

OSAKI YOJI DR, DPT OF ASTRONOMY, UNIVERSITY OF TOKYO, BUNKYO KU, TOKYO 113, JAPAN

Telephone: (81) 38122111

Facsimile: (81)

Commission(s): $35,+2$

Telex: 33659 utyosei j

Email:

OSAWA KIYOTERU DR, TOKYO ASTRONOMICAL OBS, NAOJ, OSAWA MITAKA, TOKYO 181, JAPAN

Telephone: (81) 422325111

Facsimile: (81)

Telex:

Email:

Commission(s):

OSBORN WAYNE DR, DPT OF PHYSICS, CENTRAL MICHIGAN UNIV, MT PLEASANT MI 48859, USA

Telephone: (1) 5177743321

Facsimile: (1) $51777+7106$

Telex:

Email: 3y2lw5g@cmuvm.bitnet

Conmission(s): $24,45,46$

OSBORNE JOHN L DR, DPT OF PHYSICS, UNIVERSITY OF DURHAM, SOUTH RD, DURHAM DH1 3LE, UK Telephone: (44) 19138564971

Facsimile: $(4+)$

Telex: $\mathbf{5 3 7 3 5 1}$ durlib

Email:

Commission(s): 34

OSMAN ANAS MOHAMED PROF, HELWAN OBSERVATORY, HELWAN, EGYPT

Telephone: (20) 78 0645/2683

Facsimile: (20)

Telex:

Email:

Commission(s): 28,37

OSMER PATRICK S DR, NOAO, BOX 26732, 950 N CHERRY AVE, TUCSON AZ 85726 6732, USA

$\begin{array}{ll}\text { Telephonc: (1) } 5203275511 & \text { Facsimile: (1) } \\ \text { Email: } & \text { Commission(s): }\end{array}$

Telex: $666+8+$

OSORIO ISABEL MARIA T V P, OBSERVATORIO ASTRONOMICO, UNIVERSIDADE DO PORTO MONTE DA VIRGEM, P 4400 VILA NOVA DE GAIA, PORTUGAL

Telephone: (351) $27820404 \quad$ Facsimile: (351) $27827253 \quad$ Telex:

Email:

Commission(s): 7

OSORIO JOSE J S P PROF, OBSERVATORIO ASTRONOMICO, UNIVERSIDADE DO PORTO, MONTE DA VIRGEM

P 400 VILA NOVA DE GAIA, PORTUGAL

Telephone: (351) 7820404

Facsimile: (351)

Telex: 22367

Email:

Commission(s): $07,08,46,50$

OSTER LUDWIG F PROF DR, NTL SCIENCE FOUNDATION, DIV ASTRONOMICAL SCIENCES, 1800 G ST NW WASHINGTON DC 20550, USA

Telephone: (1) 2023579857

Facsimile: (1)

Commission(s): 12

Telex:

Email:

OSTERBROCK DONALD E PROF, LICK OBSERVATORY, UNIVERSITY OF CALIFORNIA SANTA CRUZ CA 95064, USA

Telephone: (1) 4084292605

Facsimile: (1) $408+263115$

Telex:

Email:

Commission(s): $28,34.40,41$ 
OSTRIKER JEREMIAH P PROF, PRINCETON UNIVERSITY OBS, PEYTON HALL, PRINCETON NJ 08544, USA

Telephone: (1) 6092583800

Email: jpo@astro.princeton.edu
Facsimile: (1)

Commission(s): $33,35,44 C, 51$
Telcx: 322409

Telex:

OSTRO STEVEN J, JPL, MS 300 233, PASADENA CA 91109 8099, USA

Telephone: (1) 8183543173

Facsimile: (1) 8183549476

Email: ostro@echo.jpl.nasa.gov

Commission(s):

OSWALT TERRY D DR, DPT PHYSICS \& SPACE SCI, FLORIDA INST TECHNOLOGY, 150 W UNIVERSITY BLVD MELBOURNE FL 32901, USA

Telephone: (1) $4077688000 \star 7325$

Facsimile: (1) 4079848461

Telex:

Email: oswawlt@tycho.pss.fit.edu

Commission(s): 26,27,35,46

OTERMA LIISI PROF, SIRKKALANKATU 31, SF 20700 TURKU, FINLAND

Telephone: (358) 21332081

Facsimile: (358)

Commission(s): 20

Telex:

Email:

OTHMAN MAZLAN DR, PLANETARIUM DIVISION, PRIME MINISTER'S DPT, 2731/5 JALANJOHOR SELATAN 50480 KUALA LUMPUR, MALAYSIA

Telephone: (60) 32824463

Facsimile: (60) 32824507

Telex:

Email:

Commission(s): 46

OTT HEINZ-ALBERT, ASTRONOMISCHES INSTITUTE, UNIVERSITAET MUENSTER, WILHELM KLEMM STR 10 D 48149 MUENSTER, GERMANY

Telephone: (49) 251833561

Facsimilc: (49) 251833669

Telex:

Email: ott@cygnus.uni-muenster.de

Commission(s): 47

OTTELET I J DR, INSTITUT D'ASTROPHYSIQUE, UNIVERSITE DE LIEGE, AVE COINTE 5

B 4000 COINTE-LIEGE, BELGIUM

Telephone: (32) 41529980

Facsimile: (32) $\$ 1527474$

Telex: 41264

Email:

Commission(s): 16

OUHRABKA MIROSLAV DR, COLLEGE OF EDUCATION IN, HRADEC KRALOVE, NAM SVOBODY 301

CZ 50191 HRADEC KRALOVE, CZECH R

Telephone: (42) $4925226 \quad$ Facsimile: $(42) 4925785 \quad$ Telex:

Email:

Commission(s):

OVERBEEK MICHIEL DANIEL, BOX 212, EDENVALE 1610, SOUTH AFRICA

Telephone: (27) $11535447 \quad$ Facsimile: (27) Telex:

Email:

Commission(s): 20

OWAKI NAOAKI DR, DPT ASTRON \& EARTH SCI, TOKYO GAKUGEI UNIVERSITY, KOGANEI, TOKYO 184, JAPAN

Telephone: $(\mathbf{8 1})$

Facsimile: $(\mathbf{8 1})$

Telex:

Email:

Commission(s): 46

OWEN FRAZER NELSON DR, NRAO, VLA, BOX 0, SOCORRO NM 87801 0387, USA

Telephone: (1) 505772401

Facsimile: (1)

Telex: 9109881710

Email:

Commission(s): $28,40,50$

OWEN TOBIAS C PROF, INSTITUTE FOR ASTRONOMY, UNIVERSITY OF HAWAII, 2680 WOODLAWN DR HONOLULU HI 96822, USA

Telephone: (1) 8089568007

Email: owen@hubble.ifa.hawaii.edu

Facsimile: (1) 8089882790

Telex:

OWOCKI STANLEY PETER DR, BARTOL RESEARCH INSTITUTE, UNIVERSITY OF DELAWARE

NEWARK DE 19716, USA

Telephone: (1) 3024518357

Email: bartol::owocki

Facsimile: (1) 3024511843

Telex: 5106660805

OXENIUS JOACHIM DR, UNIV LJBRE DE BRUXELLES, CP 231, B 1050 BRUSSELS, BELGIUM

Telephone: (32) 26400015

Facsimile: (32) 26505824

Telex: 23069 unilib b

Email:

Commission(s): 7236

OZERNOY LEONID M PROF, NASA GSFC, CODE 665, LAB HIGH ENERGY ASTROPHYS, GREENBELT MD 20771, US

Telephone: (1) 3012868801

Facsimile: (1)

Telex:

Email: ozernoy@lheavx.gstc.nasa.gov

Commission(s): $34,44,47$ 
OZGUC ATILA, KANDILLI OBSERVATORY, BUGAZICI UNIVERSITY, CENGELKOY, 81220 ISTANBUL, TURKEY Telephone: (90) 1332 0240/41 Facsimile: (90)

Telex:

Email:

Commission(s): 10

OZSVATH I PROF, UNIVERSITY OF TEXAS, PROGRAMS IN MATHEMAT SCI, BOX 830688 RICHARDSON TX 750830688 , USA

Telephone: (1) 2146902174

Facsimile: (1)

Telex:

Email:

Commission(s): 47

O'BRIEN PAUL THOMAS DR, DPT OF ASTROPHYSICS, UNIVERSITY OF OXFORD, KEBLE RD

OXFORD OX1 3RH, UK

Telephone: (44)

Facsimilc: (4)

Telex:

Email: rlesis::0xvad::pto

Commission(s): 44

O'BRIEN TIM DR, LIVERPOOL JMU, ASTROPHYSICS GROUP, BYROM ST, LIVERPOOL L3 3AF, UK

Teicphonc: (44) 1512312337

Facsimile: (44) 1512312337

Telex:

Email: tob@staru1.livjm.ac.uk

Commission(s):

O'CONNELL ROBERT F PROF, DPT PHYSICS \& ASTRONOMY, LOUISIANA STATE UNIV

BATON ROUGE LA 708034001 , USA

Telephone: (1) 5043886848

Facsimilc: (1)

Telex:

Email:

Commission(s): 44

O'CONNELL ROBERT WEST DR, UNIVERSITY STATION, UNIVERSITY OF VIRGINIA, BOX 3818

CHARLOTTESVILLE VA 22903, USA

Telephone: (1) 8049247494

Facsimile: (1)

Telex: $510-587-5453$

Email: rwo@virginia.edu

Commission(s): 28,47

O'CONNOR SEAMUS L DR, DPT OF PHYSICS, UNIVERSITY COLLEGE, BELFIELD, DUBLIN 4, IRELAND

Telephone: (353) 1693244

Facsimile: (353)

Telex: 32693 ued ei

Email:

Commission(s):

O'DEA CHRISTOPHER P DR, STSCI, HOMEWOOD CAMPUS, 3700 SAN MARTIN DR, BALTIMORE MD 21218, USA

Telephone: (1) 3013382590

Facsimile: (1) 3013385085

Telex: $68+9101$

Email:

Commission(s): $28,+0$

O'DELL CHARLES R DR, DPT SPACE PHYS \& ASTRON, RICE UNIVERSITY, BOX 1892, HOUSTON TX 77251 1892, US

Telephone: (1) 7135278101

Facsimile: (1)

Telex: 556457

Email:

Commission(s): 09,15,34

O'DELL STEPHEN L, NASA/MSFC, CODE ES 65, SPACE SCIENCE LAB, HUNTSVILLE AL 35812, USA

Telephone: (1) $20554+7708$

Facsimile: (1)

Telex:

Email:

Commission(s): 3t

O'DONOGHUE DARRAGH DR, DPT OF ASTRONOMY, UNIVERSITY OF CAPE TOWN RONDEBOSCH 7700, SOUTH AFRICA

Telephone: (27)

Facsimile: (27)

Telex:

Email:

Commission(s): 27

O'HANDLEY DOUGLAS A DR, AMES RESEARCH CENTER, CENTER FOR MARS EXPL MOFFETT FIELD CA 94035, USA

Email: doug_ohandley@qmgate.arc.nasa.gov

Telex:

Commission(s): 04,07

O'KEEFE JOHN A DR, NASA GSFC, CODE 681, GREENBELT MD 20771, USA

Telephone: (1) 3012868445

Facsimile: (1)

Telex: 89675

Email:

Commission(s): 15,16,22

O'LEARY BRIAN T, FUTURE FOCAS, 5136 E KAREN DR, SCOTTSDALE AZ 8525,, USA

Telephone: (1)

Facsimile: (1)

Telex:

Email:

Commission(s): 
O'MARA BERNARD J PROF, DPT OF PHYSICS, UNIVERSITY OF QUEENSLAND, ST LUCIA BRISBANE QLD 4067, AUSTRALIA

Telephone: (61) 73773429

Facsimile: (61)

Telex:

Email:

Commission(s): 36

O'MONGAIN EON, DPT OF PHYSICS, UNIVERSITY COLLEGE, BELFIELD, DUBLIN 4, IRELAND

Telephone: (353) 1693244

Facsimile: (353)

Telex: 32693 ucd

Email:

Commission(s): 4

O'SULLIVAN DENIS F, DIAS, SCHOOL OF COSMIC PHYSICS, 5 MERRION SQ, DUBLIN 2, IRELAND

Telephone: (353) 1774321

Facsimile: (353)

Telex: 31687 dias ei

Email:

Commission(s): 44

O'SULLIVAN JOHN DAVID DR, CSIRO, DIV OF RADIOPHYSICS, BOX 76, EPPING NSW 2121, AUSTRALIA

Telephone: (61) 28680222

Facsimile: (61) 28680310

Telex: 26230 astro

Email:

Commission(s): 40

PACHARIN-TANAKUN P DR, DPT OF PHYSICS, CHULALONGKORN UNIVERSITY, 10330 BANGKOK, THAILAND Telephone: (66) 22514902

Facsimile: (66) 22531150

Telex: 20217 unichul th

Email:

Commission(s): 36

PACHNER JAROSLAV PROF, 60655 WYNFORD HTS CR, TORONTO ON M3C 1LA, CANADA

Telephone: (1) $4164471015 \quad$ Facsimile: (1)

Telex:

Email:

Compission(s): 47

PACHOLCZYK ANDRZEJ G PROF, STEWARD OBSERVATORY, UNIVERSITY OF ARIZONA, TUCSON AZ 85721, USA

Telephone: (1) 5206216928

Facsimilc: (1)

Telex: 467175

Email:

Commission(s): $28,40,44$

PACIESAS WILLIAM S DR, DPT OF PHYSICS, UNIVERSITY OF ALABAMA, HUNTSVILLE AL 35899, USA

Telephone: (1) 2055447712

Facsimile: (1)

Telex: 594416 es62

Email:

Commission(s): 44

PACINI FRANCO PROF, DPT DI ASTRONOMIA, UNIVERSITA DEGLI STUDI, LARGO E FERMI 5

I S0125 FIRENZE, ITALY

Telephone: (39) 55275 21/2232

Facsimile: (39) 55220039

Telex: 0572268 arcetr $\mathrm{i}$

Email: pacini@arcetri.astro.it

Commission(s): $\$ 4,51, \mathrm{EC}$

PACZYNSKI BOHDAN PROF, COPERNICUS ASTRON CENTER, POLISH ACAD OF SCIENCES, UL BARTYCKA 18 PL 00716 WARSAW, POLAND

Telephone: (48)

Facsimile: (48)

Commission(s): 35,42

Telex:

Email:

PADALIA T D DR, UTTAR PRADESH STATE, OBSERVATORY, PO MANORA PEAK 263 129, NAINITAL 263 129, INDLA Telephone: (91) 59422136

Facsimile: (91)

Commission(s): 42

Telex:

Email:

PADMAN RACHAEL, MULLARD RADIO ASTRON OBS, CAVENDISH LABORATORY, MADINGLEY RD

CAMBRIDGE CB3 OHE, UK

Telephone: (44) 1223337294

Facsimilc: (44) 1223354599

Telex: $\mathbf{8 1 2 9 2}$

Email:

Commission(s): 40

PADMANABHAN JANARDHAN, RADIO ASTRONOMY DIV, PHYSICAL RESEARCH LAB, NAVRANGPURA

AHMEDABAD 380 009, INDIA

Telephone: (91)

Facsimile: (91)

Telex:

Email:

Commission(s): 10,12

PADMANABhAN T DR, IUCAA, PO BOX 4, GANESHKHIND, PUNE 411 007, INDIA

Telephone: (91) 212336415

Email: paddy@iucaa.ernet.in

Facsimile: (91) 212335760

Telex: 0145658 gmrt in

PADOVANI PAOLO, DPT DI FISICA, II UNIVERSITA DI ROMA, VIA DELLA RICERCA SCI 1, I 00133 ROMA, ITALY

Telephone: (39)

Facsimile: (39)

Telex:

Email: padovani@roma2.infn.it

Commission(s): 
PADRIELLI LUCLA, IST DI RADIOASTRONOMIA, CNR, VIA IRNERIO $\downarrow 6$, I 40126 BOLOGNA, ITALY

Telephone: (39) 51232856

Email:
Facsimile: (39)

Commission(s): $40 \mathrm{C}, 47$
Telex: 211664 inf bo i

PAERELS FREDERIK B S, DPT OF PHYSICS, UNIVERSITY OF CALIFORNIA, BERKELEY CA 94720 7300, USA
Facsimilc: (1) $5106+35204$

Commission(s):
Telephone: (1) 5106428137

Email: frits@physics.berkeley.cdu

Telex:

PAGE ARTHUR MR, DPT OF PHYSICS, UNIVERSITY OF QUEENSLAND, BRISBANE QLD 4072, AUSTRALIA

Telephone: (61) 73652422

Email:

Telex:

Facsimile: (61) 7365119

Commission(s): 25

PAGE CLIVE G DR, DPT OF PHYSICS, UNIVERSITY OF LEICESTER, UNIVERSITY RD, LEICESTER LE1 7RH, UK Telephone: (44) $1162554455 * 23 \quad$ Facsimile: (t4)

Email:

Commission(s): 4

Telex: $3 \$ 1664$ luxray $\mathrm{g}$

PAGE DON NELSON, 412 PHYSICS LAB, UNIVERSITY OF ALBERTA, EDMONTON, ALBERTA TGG 2J1, CANADA

Telephone: (1) 4034924129

Facsimile: (1) $403 \$ 920714$

Telex: 0372979

Email: don(aphys.ualberta.ca

Commission(s): 47

PAGE THORNTON L DR, NASA/JOHNSON SPACE CENTER, 18639 POINT LOOKOUT DR, HOUSTON TX 77058, USA

Telephone: (1) 7134833728

Facsimile: (1)

Telex:

Email:

Commission(s): 28,51

PAGEL BERNARD E J PROF, NORDITA, BLEGDAMSVEJ 17, DK 2100 COPENHAGEN 0, DENMARK

Telephone: (45) 31421616

Facsimile: (45) 31389157

Telex: 15216 nbj dk

Email: nordita(a)nbivax.nbi.dk

Commission(s): $29,34,36$

PAKVOR IVAN, ASTRONOMICAL OBSERVATORY, VOLGINA 7

YU 11050 BEOGRAD, YUGOSLAVIA-SERBIA \& MONTENEGRO

Telephone: (38) $11419357 / 421875 \quad$ Facsimile: (38)

Email:

Commission(s): 08

Telex: 72610 aob yu

PAL ARPAD PROF DR, FAC OF MATHEMATICS, UNIVERSITY OF CLUJ NAPOCA, STR RAKOCZI 72

R 3400 CLUJ NAPOCA, RUMANIA

Telephone: (40) 64116101

Facsimile: $(40) 64194592$

Telex:

Email:

Commission(s): 07

PALAGI FRANCESCO, OSS ASTROFISICO, DI ARCETRI, LARGO E FERMI 5, I 50125 FIRENZE, ITALY

Telephonc: (39) $5527521 \quad$ Facsimile: (39)

Email:

Commission(s):

Telex: 572268 arcetr $\mathrm{i}$

PALLA FRANCESCO, OSS ASTROFISICO, DI ARCETRI, LARGO E FERMI 5, I 50125 FIRENZE, ITALY

Telephonc: (39) $552752242 \quad$ Facsimile: (39)

Telex: 572268 arcetr i

Email:

Commission(s): 34

PALLAVICINI ROBERTO DR, OSS ASTROFISICO, DI ARCETRI, LARGO E FERMI 5, I 50125 FIRENZE, ITALY

Telephone: (39) 552752252

Facsimile: (39) 55220039

Telex: 572268 arcetr i

Email: pallavic(a)arcetri.astro.it

Commission(s): 10,36VP

PALLE PERE-LLUIS DR, INST DE ASTROFISICA, DE CANARIAS, OBS DEL TEIDE, E 38200 LA LAGUNA, SPAIN

Telephone: (34) 22262211

Facsimile: (34) 22263005

Telex: $926+0$

Email: span:iac::plp

Commission(s): 10,12

PALMEIRA RICARDO A R DR, INPE, CP 515, 12200 S JOSE DOS CAMPOS, BRAZIL

Telephone: (55) 123229977

Facsimile: (55) $1232187+3$

Telex:

Email:

Commission(s):

PALMER PATRICK E PROF, ASTRONOMY \& ASTROPHYS CTR, UNIVERSITY OF CHICAGO, 5640 S ELLS AVE CHICAGO IL 60637, USA

Telephone: (1) 3129627972

Facsimilc: (1)

Telex: 6871133

Email: ppalmer(a)oskar.uchicago.edu

Commission(s): $33,34,40$

PALMER PHILIP DR, ASTRONOMY UNIT, QUEEN MARY/WESTFIELD COLL, MILE END RD, LONDON E1 4NS, UK

Telephonc: (44) 1719755462

Facsimilc: $(4)$

Telex: 893750

Email: philip (a) qme.maths.

Commission(s): 28 
PALOUS JAN DR, ASTRONOMICAL INSTITUTE, CZECH ACADEMY SCIENCES, BOCNI II 1401 CZ 14131 PRAHA 4, CZECH R

Telephone: (42) 2766051

Facsimile: (42) 2769023

Telex:

Email: palousj@cscarn

Commission(s): 33

PALUMBO GIORGIO G C DR, DPT DI ASTRONOMIA, UNIVERSITA DI BOLOGNA, VIA ZAMBONI 33 I 40126 BOLOGNA, ITALY

Telcphone: (39) 51259424

Facsimilc: (39) 51259407

Telex: $52063+$ infn bo i

Email: ggcpalumbo(a)alma02.cineca.it Commission(s): 28,44

PALUS PAVEL DR, FAC OF MATH \& PHYSICS, DPT ASTRONOMY \& ASTROPHYS, MLYNSKA DOLINA SK 842 15 BRATISLAVA, SLOVAK R

Telcphone: (42) 7723611

Facsimile: (42) $7325882 \quad$ Telex:

Email:

Commission(s): 10,12

PAMYATNIKH A A DR, INST OF ASTRONOMY, ACADEMY OF SCIENCES, PYATNITSKAYA UL 48 109017 MOSCOW, RUSSIA

Telephone: (7) 952315461

Facsimile: (7)

Commission(s): 05,35

Telex: $\mathbf{4 1 2 6 2 3}$ scstp su

Email:

PAN JUN-HUA, NANJING ASTRONOMICAL, INSTRUMENT FACTORY, BOX 846, NANJING, CHINA PR

Telcphone: (86) $2546191 \quad$ Facsimile: (86)

Telex: $3 \$ 136$ glynj cn

Email:

Commission(s):

PAN LIANDE, SHAANXI OBSERVATORY, CAS, LINTONG XIAN, SHAANXI, CHINA PR

Telephone: (86) 332255

Facsimilc: (86)

Telex: $70121 \mathrm{csao} \mathrm{cn}$

Email:

Commission(s): 10

PAN NING-BAO, BEIJING ASTRONOMICAL OBS, CAS, W SUBURB, BEIJING 100080, CHINA PR

Telephone: (86) 1281698

Facsimile: (86)

Telex:

Email:

Commission(s):

PAN RONG-SHI, SHANGHAI OBSERVATORY, CAS, 80 NANDAN RD, SHANGHAI 200030, CHINA PR

Telephone: (86) 21386191

Facsimile: $(86)$

Telex: 33164 shao en

Email:

Commission(s): $24,28,47$

PAN XIAO-PEI, CALTECH, MS 105 24, PASADENA CA 91125, USA

Telephone: (1) $8183564015 \quad$ Facsimile: (1)

Email: x]p(a)deimos.caltech.edu Commission(s):

Telex: $67+25$ caltech psd

PANAGIA NINO DR, STSCI, HOMEWOOD CAMPUS, 3700 SAN MARTIN DR, BALTIMORE MD 21218, USA

Telephone: (1) 3013384916

Facsimile: (1)

Commission(s): 34

Telex: $68+9101$ st sci

Email:

PANCHUK VLADIMIR, SPECIAL ASTROPHYSICAL OBS, SAO N ARKHYZ, N ARKHYZ 357147, RUSSIA

Telephone: (7) 8657893527

Facsimile: (7)

Commission(s):

Telex: 123060 salo su

Email: panchuk(asao.stavropol.su

PANDE GIRISH CHANDRA PROF, 126 ARYANAGAR, LUCKNOW 226004 , INDIA
Telephone: (91)
Facsimile: (91)

Email:

Commission(s): 35

PANDE MAHESH CHANDRA DR, UTTAR PRADESH STATE, OBSERVATORY, PO MANORA PEAK 263129

NAINITAL 263 129, INDIA

Telephone: (91) 59422136

Email:

Facsimile: (91)

Commission(s): 12

Telex:

PANDEY A K, UTTAR PRADESH STATE, OBSERVATORY, PO MANORA PEAK 263 129, NAINITAL 263 129, INDIA

Telephonc: (91) 59422136

Facsimile: (91)

Telex:

Email:

Commission(s): 20,33,37

PANDEY S K, DPT OF PHYSICS, UNIVERSITY OF RAVISHANKAR, RAIPUR \$92 010, INDIA

Telephone: (91) 27064

Facsimile: (91)

Telex:

Email:

Commission(s): 
PANDEY UMA SHANKAR DR, DPT OF PHYSICS, UNIVERSITY OF GORAKHPUR, GORAKHPUR 273 O09, INDIA Telephone: (91) 336601

Facsimile: (91)

Telex:

Email:

Commission(s): $42,44,46$

PANEK ROBERT J DR, DPT OF ASTRONOMY, PENNSYLVANIA STATE UNIV, 525 DAVEY LAB

UNIVERSITY PARK PA 16802, USA

Telephone: (1)

Facsimile: (1)

Telex:

Email:

Commission(s): 36

PANG KEVIN, JPL, MS T11 823, 4800 OAK GROVE DR, PASADENA CA 91109, USA

Telephone: (1) $81835+5392$

Facsimilc: (1)

Telex: $675+29$

Email:

Commission(s): 16

PANKONIN VERNON LEE DR, NTL SCIENCE FOUNDATION, DIV ASTRONOMICAL SCIENCES, 1800 G ST NW WASHINGTON DC 20550, USA

Telcphone: (1) 2023579696

Facsimile: (1)

Telex:

Email: vpankoni@nsf.gov

Commission(s): $34,40,50$

PANNUNZIO RENATO, OSS ASTRONOMICO DI TORINO, ST OSSERVATORIO 20, I 10025 PINO TORINESE, ITALY

Telephone: (39) 114619034

Facsimile: (39) $11+619030$

Telex: 213236 toastr $\mathrm{i}$

Email: pannunzio@to.astro.it

Commission(s): 26

PANOV KIRIL DR, DPT OF ASTRONOMY, BULGARIAN ACAD SCIENCES, 7TH NOVEMBER ST 1 BG 1000 SOFIA, BULGARIA

Telephone: (359) 27341

Email:

Facsimile: (359) 23561 ECFBAN BG Telex: 23561 ecf ban bg

Commission(s):

PAOLICCHI PAOLO DR, IST DI ASTRONOMIA, UNIVERSITA DI PISA, PIAZZA TORRICELLI 2, I 56100 PISA, ITALY

Telephone: (39) 5043343

Facsimile: (39)

Telex:

Email:

Commission(s): 15,16

PAP JUDIT, JPL CALTECH, MS 171 400, 4800 OAK GROVE DR, PASADENA CA 91109, USA

Telephone: (1) 8183542662

Facsimile: (1) $81835+4707 \quad$ Telex

Email: jpap(a)solar.stanford.edu

Commission(s): 10

PAPAELIAS PHILIP DR, DPT OF PHYSICS, NTL UNIVERSITY OF ATHENS, PANEPISTIMIOPOLIC

GR 15771 ZOGRAFOS, GREECE

Telephone: (30) $17235122 \quad$ Facsimile: (30)

Email:

Commission(s):

Telex: 223815 univgr

PAPAGIANNIS MICHAEL D PR, DPT OF ASTRONOMY, BOSTON UNIVERSITY, 725 COMMONWEALTH AVE BOSTON MA 02215, USA

Telephone: (1) 6173532626

Email:

Facsimile: (1)

Telex:

Commission(s): $40,44,51$

PAPALIOLIOS COSTAS DR, CENTER FOR ASTROPHYSICS, HCO/SAO, , G GARDEN ST, CAMBRIDGE MA 02138, USA

Telephone: (1)

Facsimile: (1)

Telex:

Email:

Commission(s):

PAPALOIZOU JOHN C B DR, SCHOOL OF MATHEMATICAL SC, QUEEN MARY/WESTFIELD COLL, MILE END RD LONDON E1 4NS, UK

Telephone: (44)

Email:

Facsimile: $(+4)$

Telex:

Commission(s): 27,35

PAPARO MARGIT DR, KONKOLY OBSERVATORY, THEGE U 13/17, BOX 67, H 1525 BUDAPEST, HUNGARY

Telephone: (36) $11755866 / 1754122$

Facsimile: (36) 11569640

Telex: $227+60$

Email: paparo a)ogyalla.konkoly.hu

Commission(s): 27

PAPATHANASOGLOU D DR, DPT OF ASTRONOMY, NTL UNIVERSITY OF ATHENS, PANEPISTIMIOPOLIS

GR 15771 ZOGRAFOS, GREECE

Telephone: (30) 17243414

Facsimile: (30)

Telex:

Email:

Commission(s): 12 
PAPAYANNOPOULOS TH DR, DPT OF ASTRONOMY, NTL UNIVERSITY OF ATHENS, PANEPISTIMIOPOLIS GR 15783 ZOGRAFOS, GREECE

Telephone: (30) 17243414

Email: spm25@grathun1

\author{
Facsimilc: (30) \\ Tclex: 223815 univ gr
}

Commission(s): $\mathbf{2 8 , 3 3}$

PAPOUSEK JIRI, DPT OF ASTROPHYSICS, PURKYNE UNIVERSITY, KOTLARSKA 2, CZ 61137 BRNO, CZECH R

Telephone: (42) 57129483

Email: papousck(a)csbrmu 11

Facsimile: (+2)

Telex:

Commission(s): 27

PAQUET PAUL EG DR, OBSERVATOIRE ROYAL DE, BELGIQUE, AVE CIRCULAIRE 3, B 1180 BRUSSELS, BELGIUM

Telephone: (32) 23730211

Facsimile: (32) 23749822

Telex: 21565 obsbel

Email: paulpaq@astro.ome.be

Commission(s): $19,31 \mathrm{C}$

PARCELIER PIERRE DR, OBSERVATOIRE DE PARIS, 61 AVE OBSERVATOIRE, F 75014 PARIS, FRANCE

Telephone: (33) $143201210 \quad$ Facsimile: (33)

Email:

Commission(s):

Telex: 270776 obs $\mathrm{f}$

PAREDES JOSE MARIA DR, DPT ASTRONOMIA, UNIVERSIDAD DE BARCELONA, AVD DIAGONAL 647

E 08028 BARCELONA, SPAIN

Telephone: (34) 33307311

Facsimile: (34)

Telex:

Email: d3fajpp0 (a) eb0ub011

Commission(s): 40

PARESCE FRANCESCO DR, ESO, KARL SCHWARZSCHILDSTR 2, D 85740 GARCHING MUENCHEN, GERMANY

Telephone: (49)

Facsimile: (4)

Telex:

Email:

Commission(s): 21,49

PARIJSKIJ N N PROF, INST PHYSICS OF THE EARTH, ACADEMY OF SCIENCES, GRUZINSKAYA 10

123342 MOSCOW, RUSSIA

$\begin{array}{ll}\text { Telephone: (7) } 2520721 & \text { Facsimile: (7) } \\ \text { Email: } & \text { Commission(s): }\end{array}$

PARIJSKIJ YU N DR, PULKOVO OBSERVATORY, ACADEMY OF SCIENCES, 10 KUTUZOV QUAY

196140 ST PETERSBURG, RUSSIA

Telephone: (7) 2979452

Facsimile: (7)

Telex:

Email:

Commission(s): 40,51

PARISE RONALD A DR, NASA GSFC, CODE 684.9, GREENBELT MD 20771, USA

Telephone: (1) 3012863896

Facsimile: (1)

Telex:

Email: span:uit::parise

Commission(s):

PARISOT JEAN-PAUL, OBSERVATOIRE DE BORDEAUX, BP 89, F 33270 FLOIRAC, FRANCE

Telephonc: (33) 56864330

Facsimile: (33) $56+0+251$

Telex:

Email: carn:<userid $>$ affrobors1

Commission(s): 15,46

PARK CHANGBOM, DPT OF ASTRONOMY, SEOUL NTL UNIVERSITY, SEOUL 151 742, KOREA R

Telephone: (82) 28806769

Facsimile: (82) $28871+35 \quad$ Telex:

Email: cbprasastrogate.snu.ac.kr

Commission(s):

PARK HONG SUH DR, KOREA ASTRONOMY OBS, SAN 36 1, WHAAM DONG, TAEJEON 305 348, KOREA R

Telephonc: (82) 428653300

Facsimile: $(82)+28615610 \quad$ Telex:

Email: hspark(a)apissa.issa.yc.kr

Commission(s): $\$ 2$

PARK MYEONG-GU DR, DPT ASTRON \& METEOROLOGY, COLLEGE OF NTL SCIENCES

KYUNGPOOK NTL UNIVERSITY, TAEGU 7112 701, KOREA R

Telephone: (82) 539506364

Facsimile: (82) 539 $570431 \quad$ Telex:

Email:

Commission(s): +4

PARK SEOK JAE DR, KOREA ASTRONOMY OBS, 36-1 WHAAM DONG, YUSEONG GU, TAE JON 305 348, KOREA R

Telcphone: (82) +28653266

Facsimilc: (82) $+28615610 \quad$ Telex:

Email: sjpark(a)apissa.issa.rc.kr

Commission(s):

PARKER EDWARD A DR, ELECTRONICS LABORATORY, UNIVERSITY OF KENT, CANTERBURY CT2 7NT, UK

Telephone: (44) 122766822

Facsimile: $(+4)$

Email:

Commission(s): 41)

Telcx: $965+49$ 
PARKER EUGENE N, ASTROPHYS \& SPACE RES LAB, UNIVERSITY OF CHICAGO, 933 E 56TH ST CHICAGO IL 60637, USA

Telephone: (1) 3129627847

Facsimile: (1)

Telex: 910-221-5617

Email:

Commission(s): $34,44,4$ )

PARKER QUENTIN DR, ROYAL OBSERVATORY, BLACKFORD HILL, EDINBURGH EHO 3HJ, UK

Telephone: (4) 1316 688 379 Facsimile: (H)

Email:

Commission(s): 28

Telex: 72383 roedin $g$

PARKER ROBERT A R, NASA/JOHNSON SPACE CENTER, CODE CB, HOUSTON TX 77158, USA

Telephone: (1) 7134832221

Facsimile: (1)

Telex:

Email:

Commission(s):

PARKINSON JOHN H DR, MULLARD SPACE SCIENCE LAB, UNIVERSITY COLLEGE LONDON HOLMBURY ST MARY, DORKING SURREY RHS GNT, UK

Telephone: (4) $148327+111 \quad$ Facsimile: (4t) $1483287312 \quad$ Telex: 859185 ucmssl g

Email:

Commission(s): 10,4t

PARKINSON TRUMAN DR, KITT PEAK NTL OBS, BOX 26732, 950 N CHERRY AVE, TUCSON AZ 85726 6732, USA

Telephone: (1)

Facsimile: (1)

Telex:

Email:

Commission(s):

PARKINSON WILLIAM H DR, CENTER FOR ASTROPHYSICS, HCO/SAO MS 51), 6) GARDEN ST CAMBRIDGE MA 02138. USA

Telephone: (1) $617+95+865$

Facsimile: (1) $617+957105$

Telex: $921+28$

Email: parkinsonacfatharvard.edu

Commission(s): 111,12,1+P,+4

PARMA PAOLA, IST DI RADIOASTRONOMIA, CNR, VIA IRNERIO +6, I +0126 BOLOGNA, ITALY

Telephonc: (39) 51232850

Facsimilc: (3)

Telex:

Email:

Commission(s): 410

PARMAR ARVIND NICHOLAS, SPACE SCIENCE DPT, ESTEC, KEPLERLAAN 1

NL 2200 AG NOORDWIJK, NETHERLANDS

Telephone: (31) $71654532 \quad$ Facsimile: (31) $716.54960 \quad$ Telex:

Email: aparmaräastro.estec.esa.nd Commission(s):

PARRAVANO ANTONIO, UNIVERSII)AD DE LOS ANDES, APARTADO 26 IPOSTEL, LA HECHICERA MERIDA 5101 A, VENEZUELA

Telephone: (58) $74+1013311 / 401331$

Email: parravanaciens.ulave

Facsimile: (58) $7+401286 \quad$ Telex:

Commission(s):

PARRISH ALLAN DR, STATE UNIV OF NEW YORK, UNIV OF MASSACHUSETTS, GRC GI9I

AMHERST MA (110)13, USA

Telephone: (1)

Facsimile: (1)

Telex:

Email:

Commission(s): 40

PARSAMYAN ELMA S DR, BYURAKAN ASTROPHYSICAL, OBSERVATORY, 378433 BYURAKAN, ARMENIA

Telephone: (7) 8852283453

Facsimile: (7) $885228+1+2$

Telex:

Email:

Commistion(s): 27,37

PARSÓNS SIDNEY B DR, STSCI, HOMEWOOD CAMPUS, 37!I SAN MARTIN DR, BALTIMORE MD 21218, USA

Telephone: (1) $311338+807$

Facsimile: (1)

Telex:

Email:

Commission(s): 29,45

PARTHASARATHY M DR, INDIAN INSTITUTE OF, ASTROPHYSICS, KORAMANGALA, BANGALORE 560 034, INDIA

Telephone: (91) $803566585 / 6+97 \quad$ Fitesimile: (91)

Telex: $8+5763$ iiab in

Emiil:

Commission(s): $27,29,42$

PARTRIDGE ROBERT B PROF, IIAVERFORD COLLEGE, IAVERFORD PA 19041, USA

Telephone: (1) 2158961145 Facsimile: (1) 2158961224 Telex:

Email: b_partridge a ance.harverford.edu Commission(s): $+7 \mathrm{C}$

PASACHOFF JAY M PROF, IIOPKINS OBSERVATORY, WILLIAMIS COLLEGE, WILLIAMSTOWN MA 01267, USA

Telelolone: (1) +13 507 21105

Facsimile: (1) +135973200

Email: jay.m.pasachoftawilliams.edu

Telex: 
PASCOAL ANTONIO J B SCI, OBSERVATORIO ASTRONOMICO, UNIVERSIDADE DO PORTO, MONTE DA VIRGEM P 4H00 VILA NOVA DE GAIA, PORTUGAL

$\begin{array}{ll}\text { Telephone: (351) } 7820404 & \text { Facsimile: }(351) \\ \text { Email: } & \text { Commission(s): }\end{array}$

PASCU DAN DR, US NAVAL OBSERVATORY, 34.50 MASSACHUSETTS AVE NW, WASHINGTON DC 20392 5100, USA

Telephone: (1) 2026531178

Facsimile: (1)

Telex:

Email:

Commission(s): 20,24

PASIAN FABIO, OAT, BOX SUCC TRIESTE 5, VIA TIEPOLO 11, I 34131 TRIESTE, ITALY

Telephone: (39) $\$(1) 768005 \quad$ Facsimile: (39) Telex: 461137 oat

Email:

Commission(s): (19)

PASINETTI LAURA E PROF, DPT DI FISICA, UNIVERSITA DI MILANO, VIA CELORIA 16, I 20133 MILANO, ITALY

Telephone: (39) $22392275^{\star} 272$

Facsimile: (39) 22366583

Telex: 334687 infn mi

Email: pasinettiamilano.infn.it

Commission(s): $05,29,36,45,51$

PASTORI LIVIO, OSS ASTRONOMICO DI MILANO, VIA E BIANCHI +6, I 22055 MERATE, ITALY

Telephone: (39) 5921035

Facsimile: (39)

Commission(s):

Telex:

Email:

PASTORIZA MIRIANI G DR, INSTITUTO DE FISICA, UFRGS, CP 15051, 90900 PORTO ALEGRE RS, BRAZIL
Telephone: (55) 512364677
Facsimile: (55)

Email:

Commission(s): 28

PATERNO LUCIO PROF, OSS ASTROFISICO, CITTA UNIVERSITARIA, VIA A DORIA 6, 195125 CATANIA, ITALY

Telephone: (39) 95330533

Facsimile: (39)

Telex: 970359 astret $i$

Emait:

Commission(s): 10,27

PATERSON-BEECKMANS F, VINCEN'T VAN GOGHLAAN 19, NL $23+3$ RH OEGSTGEEST, NETHERLANDS

Telephone: (31) 7117 (182) Facsimile: (31)

Telex:

Email:

Commission(s): 29

PATHRIA RAJ K PROF, DPT OF PIIYSICS, UNIVERSITY OF WATERLOO, WATERLOO ON N2L 3G1, CANADA

Telephone: (1) 5198851211

Facsimilc: (1) 519 746,8115

Telex: 06955259

Email:

Comminsion(s):

PATI A K, INDIAN INSTITU'TE OF, ASTIROPIIYSICS, KORAMANGALA, BANGALORE \$60 (134, INDIA

Telephone: (91) $803569702 \quad$ Facsimile: (91)

Entail:

Commission(s): (1)

Telex: 08452763

PATKOS LASZLO DR, KONKOLY OBSERVATORY, THEGE U 13/17, BOX 67, H 1525 BUDAPEST, HUNGARY

Telephonc: (36) $11755866 / 175+122$

Email:

Facsimile: $(36) 11569640$

Telex: $227+60$

PATNAIK ALOK DR, NRAL, JODRELL BANK, MACCLESFIELD SK11 9DL, UK

Telephone: (\$t) 1+7771321 Facsimile: (+4) 147771618 Telex:

Email: alok'auk,ac.man.jb.stat $\quad$ Commission(s):

PATRIARCHI PATRIZIO DR, OSS ASTROFISICO, DI ARCETRI, LARGO E FERMI S, I 50125 FIRENZE, ITALY

Telephone: (39) $552752282 \quad$ Facsimite: (39)

Email:

Commission(s):

Telex: 572268 arcetr $\mathrm{i}$

PATUREL GEORGES, OBSERVATOIRE DE LYON, AVE CHARLES ANDRE, F 6956I S GENIS LAVAL CDX, FRANCE Telephone: (33) 78560705

Facsimile: (33) 72399791

Telex: $310 \% 26$

Email:

Commission(s): 05,28

PAUL JACQUES DR, CEA CEN, DAPNIA/SAP, BP 2, F 91191 GIF/YVETTE CDX, FRANCE

Telephone: (33) $16908+462$

Facsimile: (33)

Telex:

Emiail:

Commission(s):

PAULDRACH ADALBERT W A DR, UNIVERSITAETS STERNWARTE, SCHEINERSTR I

D 81679 MUENCHEN, GERMANY

Telephone: (49) 89 $92209+36$

Ficsimile: (40)

Telex:

Enail:

Commission(s): 
PAULINY TOTH IVAN K K DR, MPI FUER RADIOASTRONOMIE, AUF DEM HUEGEL 69, D 53121 BONN, GERMANY Telephone: (49) 228525243

Facsimile: (\$9)

Telex: 886440

Email:

Commission(s): 40,44

PAULS THOMAS ALBERT DR, NAVAL RESEARCH LABORATORY, CODE 4130,4555 OVERLOOK AVE SW, WASHINGTON DC 20375 5000, USA

Telephone: (1)

Facsimile: (1)

Telex:

Email:

Cemmission(s): $33,34,4$ (1)

PAUWELS T DR, OBSERVATOIRE ROYAL DE, BELGIQUE, AVE CIRCULAIRE 3, B 1180 BRUSSELS, BELGIUM

TeJephone: (32) 2373 0225/0211

Facsimile: (32) $237+9822$

Telex: 21565 obs bel

Email: thicrry@astro.oma.be

Commission(s): 07,20

PAVLENKO ELENA, CRIMEAN ASTROPHYS OBS, UKRAINIAN ACAD OF SCIENCE, NAUCHNY, 334413 CRIMEA, UKRAINE

Telephone: (7) 655471124

Facsimile: (7) 655440704

Telex:

Email: pavienko(a)crao.crimea.ua

Commission(s): $\$ 2$

PAVLENKO YAKOV V, MAIN ASTRONOMICAL OBS, DPT PHYSICS STARS \& GALAX, GOLOSIIV, 252127 KIEV 650 GSP, UKRAINE

Telephone: (7) 0442664771

Email: maouas(a)gluk.apc.org
Facsimile: (7) $04426621+7$

Commission(s): 29
Telex: 131406 sky

PAVLINSKY MIKHAIL, SPACE RESEARCH INSTITUTE, PROFSOYUSNAYA UL 84/32, MOSCOW 117810, RUSSIA

Telephone: (7) 0953332366

Email: mykleärea.iki.rssi.ru
Telex:

Commission(s):

PAVLOVSKI KRESIMIR, HVAR OBSERVATORY, FACULTY OF GEODESY, KACICEVA 26, ZAGREB, CROATIA

Telephone: (38) 41442600

Facsimilc: (38)

Telex:

Email:

Commission(s): $27,+2$

PAXTON HAROLD J B R, ROYAL GREENWICH OBS, HERSTMONCEUX CASTLE, HAILSHAM BN27 1RP, UK

Tclephonc: (4t)

Facsimilc: (4)

Telex:

Email:

Commission(s):

PAYNE DAVID G, JPL, MS $26+748,4800$ OAK GROVE DR, PASADENA CA 91109 , USA

Telephone: (1)

Facsimile: (1)

Telex:

Email:

Commission(s): 40

PEACH GILLIAN DR, DPT PHYSICS \& ASTRONOMY, UNIVERSITY COLLEGE LONDON, GOWER ST, LONDON WC1 GBT, UK

Telephone: (4t) $1713877050 \quad$ Facsimile: (4t)

Conmission(s): 14

Telex: 28722

Email:

PEACH JOHN V DR, DPT OF ASTROPHYSICS, UNIVERSITY OF OXFORD, SOUTH PARKS RD, OXFORD OX1 3RQ, U

Telephone: (t4) 1865511336

Facsimile: $(+4)$

Email:

Commission(s):

Telex:

'PEACOCK ANTHONY DR, ESA/ESTEC, SSD, BOX 299, NL 2200 AG NOORDWIJK, NETHERLANDS

Telephone: (31) 171983563

Email:

Facsimile: (31) $171917+00$

Telex: 39098

PEACOCK JOHN ANDREW, ROYAL OBSERVATORY, BLACKFORD HILL, EDINBURGH EH9 3HJ, UK

Telephone: (44) 1316673321

Facsimile: (4)

Commission(s): 47 Telex: 72383 roedin $\mathrm{g}$

Email:

PEALE STANTON J PROF, DPT OF PHYSICS, UNIVERSITY OF CALIFORNIA, SANTA BARBARA CA 93106, USA

Telephone: (1) 8059612977

Facsimile: (1)

Telex:

Email:

Commission(s): (07C

PEARCE GILLIAN DR, DPT OF ASTROPHYSICS, UNIVERSITY OF OXFORD, KEBLE RD, OXFORD OX1 3RH, UK

Telephonc: (44) 1865273297

Ematil:
Facsimile: (4t)

Commission(s): 35
Telex: 83295 nuclox g 
PAULINY TOTH IVAN K K DR, MPI FUER RADIOASTRONOMIE, AUF DEM HUEGEL 69, D 53121 BONN, GERMANY Telephone: (49) 228525243

Facsimile: (49)

Commission(s): $\$ 0,+4$

Telex: 8864+0

Email:

PAULS THOMAS ALBERT DR, NAVAL RESEARCH LABORATORY, CODE 4130,4555 OVERLOOK AVE SW WASHINGTON DC 20375 5000, USA

Telejhonc: (1)

Facsimile: (1)

Telex:

Email:

Commission(s): $33,34,40$

PAUWELS T DR, OBSERVATOIRE ROYAL DE, BELGIQUE, AVE CIRCULAIRE 3, B 1180 BRUSSELS, BELGIUM

Telephone: (32) 2373 0225/10211

Facsimile: (32) $237+9822$

Telex: 21565 obs bel

Email: thicrry@astro.oma.be

Commission(s): (07,20

PAVLENKO ELENA, CRIMEAN ASTROPHYS OBS, UKRAINIAN ACAD OF SCIENCE, NAUCHNY

334413 CRIMEA, UKRAINE

Telephone: (7) 655471124

Facsimile: (7) $655+4070 \downarrow$

Telex:

Email: pavlenko@erao.crimea.ua

Commission(s): $\$ 2$

PAVLENKO YAKOV V, MAIN ASTRONOMICAL OBS, DPT PHYSICS STARS \& GALAX, GOLOSIIV

252127 KIEV 650 GSP, UKRAINE

Telephone: (7) 0442664771

Email: maouasagluk.apc.org

Facsimile: (7) 0442662147

Telex: 131406 sky

Commission(s): 29

PAVLINSKY MIKHAIL, SPACE RESEARCH INSTITUTE, PROFSOYUSNAYA UL 84/32, MOSCOW 117810, RUSSIA

Telephone: (7) 0953332366

Facsimile: (7) 0953335377 Telex:

Email: mykle(a)rea.iki.rssi.ru

Commission(s):

PAVLOVSKI KRESIMIR, HVAR OBSERVATORY, FACULTY OF GEODESY, KACICEVA 26, ZAGREB, CROATIA

Telephone: (38) $414+2600$

Facsimile: (38)

Commission(s): 27,42

Telex:

Email:

PAXTON HAROLD J B R, ROYAL GREENWICH OBS, HERSTMONCEUX CASTLE, HAILSHAM BN27 1RP, UK

Telephone: $(+t)$

Facsimile: (+4)

Telex:

Email:

Commission(s):

PAYNE DAVID G, JPL, MS $26+7+8,4800$ OAK GROVE DR, PASADENA CA 91109 , USA

Telephone: (1)

Facsimile: (1)

Commission(s): 40

Telex:

Email:

PEACH GILLIAN DR, DPT PHYSICS \& ASTRONOMY, UNIVERSITY COLLEGE LONDON, GOWER ST

LONDON WC1E GBT, UK

Telephone: (\$t) 1713877050

Email:

Facsimile: (4)

Commission(s): 14

Telex: 28722

PEACH JOHN V DR, DPT OF ASTROPHYSICS, UNIVERSITY OF OXFORD, SOUTH PARKS RD, OXFORD OX1 3RQ, U Telcphone: (4) 1865511336 Facsimile: (4)

Commission(s): Telex:

Email:

PEACOCK ANTHONY DR, ESA/ESTEC, SSD, BOX 29\%, NL 2200 AG NOORDWIJK, NETHERLANDS

Telephone: (31) 171983563

Email: Facsinile: (31) 171917400

Telex: 39098

Commission(s): $\$ 4$

PEACOCK JOHN ANDREW, ROYAL OBSERVATORY, BLACKFORD HILL, EDINBURGH EH9 3HJ, UK

Telephone: (4) 1316673321

Facsimile: (44)

Email:

Commission(s): 47

Telex: 72383 roedin $\mathrm{g}$

PEALE STANTON J PROF, DPT OF PHYSICS, UNIVERSITY OF CALIFORNIA, SANTA BARBARA CA 93106, USA

Telephone: (1) 8059612977

Facsimile: (1)

Commission(s): 07C

Telex:

Email:

PEARCE GILLIAN DR, DPT OF ASTROPHYSICS, UNIVERSITY OF OXFORD, KEBLE RD, OXFORD OX1 3RH, UK Telephone: (4) 1865273297 
PEARSON TIMOTHY J, CALTECH, MS 105 24, OWENS VALlEY RADIO OBS, PASADENA CA 91125 , USA

Telephone: (1) $8183564980 \quad$ Facsimile: (1)

Email:

Commission(s): 40

Telcx: $675+25$

PECINA PETR, ASTRONOMICAL INSTITUTE, CZECH ACADEMY OF SCIENCES, ONDREJOV OBSERVATORY CZ 251 65 ONDREJOV, CZECH R

Telephonc: (42) $204857129 / 7111$

Facsimilc: (42) $20+851611$

Telex: 122486

Email: astmph@cscarn

Commission(s): 22

PECKER JEAN-CLAUDE PROF, COLLEGE DE FRANCE, 3 RUE D'ULM, F 75331 PARIS CDX 05, FRANCE

Telephone: (33) $14+271695$

Facsimile: (33)

Telex:

Email:

Commission(s): $05,12,34,36,47$

PEDERSEN BENT M DR, OBSERVATOIRE DE PARIS, SECTION DE MEUDON, F 92195 MEUDON PPL CDX, FRANCE Telephone: (33) 145077809

Facsimile: (33)

Telex:

Email: mesopd::pedersen

Commission(s): 10

PEDERSEN HOLGER DR, UNIVERSITY OBSERVATORY, OESTER VOLDGADE 3

DK 1350 COPENHAGEN K, DENMARK

Telephone: (45) $311+1790$

Facsimile: (45) 31389157

Telex:

Email:

Commission(s):

PEDERSEN OLAF PROF, HISTORY OF SCIENCE INST, UNIVERSITY OF AARHUS, NY MUNKEGADE

DK 8000 AARHUS C, DENMARK

Telephone: (45) $86127188 \quad$ Facsimile: (45) $86202711 \quad$ Telex:

Email:

Commission(s): 41

PEDLAR ALAN DR, NRAL, JODRELL BANK, MACCLESFIELD SK11 9DL, UK

Telephone: (4) Facsimile: (4) Telex:

Email:

Commission(s): 40

PEDOUSSAUT ANDRE, OBS MIDI PYRENEES, 14 AVE E BELIN, F 31400 TOULOUSE CDX, FRANCE

Telephone: (33) 61252101

Facsimile: (33)

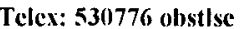

Em:ail:

Commission(s): 29,30

PEDREROS MARIO DR, DPT DE FISICA, UNIV DE TARAPACA, CASILLA 7 D, ARICA, CHILE

Tclephone: (1)

Facsimile: (1)

Telex:

Email: mpedrerora vitor.faci.uta.cl

Commission(s): 25,37

PEEBLES P JAMES E, DPT OF PHYSICS, PRINCETON UNIVERSITY, JADWIN HALL, PRINCETON NJ (85544, USA

Telephonc: (1) $609+52+386$

Facsimile: (1)

Telex: 499-3512

Email:

Commission(s): 47

PEERY BENJAMIN F PROF, DPT PHYSICS \& ASTRONOMY, HOWARD UNIVERSITY, WASHINGTON DC 20059, USA

Telephone: (1) 2026366267

Facsimile: (1)

Telex:

Email:

Commission(s): 29

PEIMBERT MANUEL DR, INSTITUTO DE ASTRONOMIA, UNAM, APDO POSTAL 70-264, 04510 MEXICO DF, MEXICO Tclephonc: (52) 9055485306

Facsimile: (52)

Telex: 01760155 cimce

Email:

Commission(s): 28,33,34

PEKERIS CHAIM LEIB PROF, DPT OF APPLIED MATHS, WEIZMANN INSTITUTE OF SCI, BOX 26

REHOVOT 76100, ISRAEL

Telephone: (972) 8483292

Facsimile: (972)

Commission(s):

Telex: 361900

Email:

OF SCIENCE, EGE UNIVERSITY, BOX 21, 35100 BORNOVA IZMIR, TURKEY

PEKUENLUE E RENNAN DR, FACULTY OF SCIENCE, EGE UNIVERSITY, BOX
Telephone: (90) 222295

Email:

Commission(s):

PEL JAN WILLEM DR, KAPTEYN ASTRONOMICAL INST, BOX 800, NL 9700 AV GRONINGEN, NETHERLANDS Telephone: (31) 50634082

Email: pelóanstro.rug.n!

Facsimile: $(31)$ 50 $63+033$

Telex:

Commission(s): 25 
PENSTON MARGARET, ROYAL GREENWICH OBS, MADINGLEY RD, CAMBRIDGE CB3 OEZ, UK

Telcphone: (44) $1223374000 \quad$ Facsimilc: (4) $122337+700 \quad$ Telex:

Email: $\quad$ Commission(s): 46

PENZIAS ARNO A DR, AT\&T BELL LABORATORIES, RM 6A- $4(19,600$ MOUNTAIN AVENUE, MURRAY HILL NJ 07974, USA

Telephone: (1) $2015823361 \quad$ Facsimile: (1) Telex: 13-8650 or 219348

Email:

Commission(s): $34,40,47$

PEQUIGNOT DANIEL, OBSERVATOIRE DE PARIS, SECTION DE MEUDON, DAF, F 92195 MEUDON PPL CDX,

FRANCE

Telephone: (33) $145077438 \quad$ Facsimile: (33)

Email:

Commission(s): 34

Telex: 201571

PERAIAH ANNAMANENI DR, INDIAN INSTITUTE OF, ASTROPHYSICS, KORAMANGALA, BANGALORE 560 034, INDIA

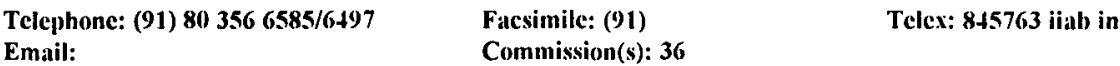

PERAULT MICHEL, RADIOASTRONOMIE ENS, 24 RUE LHOMOND, F 75231 PARIS CDX 05, FRANCE

Telcphone: (33) 143291225

Facsimile: (33) $1+5873489$

Telex: 2026,1

Email: decnet:iapobs::perault

Commission(s): 34

PERCY JOHN R PROF, DIV OF SCIENCE, UNIVERSITY OF TORONTO, ERINDALE COLLEGE, MISSISSAUGA ON LSL 1C6, CANADA

Telephone: (1) 9058285351

Facsimile: (1) 9058285328

Telex:

Email: jpercy(a)credit.erin.utoronto.ca Commission(s): 27C,46P

PERDANG JEAN M DR, INSTITUT D'ASTROPHYSIQUE, UNIVERSITE DE LIEGE, AVE COINTE 5, B 4000 COINTELIEGE, BELGIUM

Telephone: (32) $41529980 \quad$ Facsimile: (32) $+1527+74 \quad$ Telex: +1264 astrlg b

Email:

Commission(s):

PERDOMO RAUL LIC, OBSERVATORIO ASTRONOMICO, PASEO DEL BOSQUE S/N, 1900 LA PLATA (BS AS), ARGENTINA

Telephone: (54) 21217308

Email: perdomo(a)feagcp.edu.ar

Facsimile: (54) 21211761

Telex: 31151 bulap

PEREA-DUARTE JAIME D DR, INST ASTROFISICA, DE ANDALUCIA APD 300 $\nrightarrow$, PROFESOR ALBAREDA 1, E 18080 GRANADA, SPAIN

Telephone: (34) $58121311 \quad$ Facsimile: (34)

Email: jaime $(\bar{a})$ iata:es

Commission(s): 28

Telex: 78573 ialag c

PEREK LUBOS DR, ASTRONOMICAL INSTITUTE, CZECH ACADEMY SCIENCES, BOCNI II 1401, CZ 141 31 PRAHA 4, CZECH R

Telephone: (\$2) $2766051^{* 068}$

Facsimile: (42) 2769023

Telex:

Email:

Commission(s): 33,51

PERES GIOVANNI DR, INST OSSERVATORIO ASTRON, PALAZZO DEI NORMANNI, I 9013+ PALERMO, ITALY

Telephone: (39) 916517998

Facsimile: (39) 916517292

Telex:

Email: peres@oapa.astrpa.unipa.it

Commission(s): 10

PEREZ ENRIQUE DR, INST DE ASTROFISICA, DE CANARIAS, OBS DEL TEIDE, E 38200 LA LAGUNA, SPAIN

Telephone: (34) 22262211

Email: iac::eps/epjajiac.es

Facsimile: (34) 222630115

Telex: $926+0$ iac e

PEREZ FOURNON ISMAEL DR, INST DE ASTROFISICA, DE CANARIAS, OBS DEL TEIDE, E 38200 LA LAGUNA, SPAI

Telephone: (34) 22262211

Commission(s): 28

Email:

Facsimilc: (34) 22263005

Telex: 92640 iac e

Commission(s): 28,40

PEREZ-DE-TEJADA H A DR, INSTITUTO DE GEOFISICA, UNAM, APDO POSTAL 877, 22860 ENSENADA B CALIF, MEXICO

Telephone: (52) 7066740601

Facsimile: (52)

Telex:

Email:

Commission(s): 15 
PENSTON MARGARET, ROYAL GREENWICH OBS, MADINGLEY RD, CAMBRIDGE CB3 OEZ, UK Telephone: (44) 1223374000 Facsimile: $(4) 122337+700 \quad$ Telex: Email: Commission(s): 46

PENZIAS ARNO A DR, AT\&T BELL LABORATORIES, RM 6A- 409,600 MOUNTAIN AVENUE MURRAY HILL NJ 07974, USA Telephone: (1) 2015823361

Facsimile: (1) Emait: Commission(s): $34,40,47$

Telex: $13-8650$ or $2193+8$

PEQUIGNOT DANIEL, OBSERVATOIRE DE PARIS, SECTION DE MEUDON, DAF F 92195 MEUDON PPL CDX, FRANCE

Telephone: (33) $145077+38$

Facsimile: (33)

Telex: 201571

Email:

Commission(s): 34

PERAIAH ANNAMANENI DR, INDIAN INSTITUTE OF, ASTROPHYSICS, KORAMANGALA BANGALORE 560034 , INDIA

Telephone: (91) $803566585 / 6497$

Facsimile: (91)

Telex: 845763 iiab in

Email:

Commission(s): 36

PERAULT MICHEL, RADIOASTRONOMIE ENS, 24 RUE LHOMOND, F 75231 PARIS CDX OS, FRANCE

Telephone: (33) 143291225

Facsimile: (33) 145873489 Telex: 202601

Email: decnet:iapobs::perault

Commission(s): 34

PERCY JOHN R PROF, DIV OF SCIENCE, UNIVERSITY OF TORONTO, ERINDALE COLLEGE MISSISSAUGA ON LSL 1C6, CANADA

Telephone: (1) 9058285351

Email: jpercy(a)credit.erin.utoronto.ca

Facsimile: (1) 9058285328

Telex:

PERDANG JEAN M DR, INSTITUT D'ASTROPHYSIQUE, UNIVERSITE DE LIEGE, AVE COINTE 5 B 4000 COINTE-LIEGE, BELGIUM

Telephone: (32) 41529980

Conmission(s): $27 \mathrm{C}, 46 \mathrm{P}$

Email:

Facsimile: (32) +1527474

Telex: $\$ 126+$ astrig b

PERDOMO RAUL LIC, OBSERVATORIO ASTRONOMICO, PASEO DEL BOSQUE S/N 1900 LA PLATA (BS AS), ARGENTINA

Telephone: (54) 21217308

Email: perdomorafcagcp.edu.ar

Facsimile: (54) 21211761

Telex: 31151 bulap

PEREA-DUARTE JAIME D DR, INST ASTROFISICA, DE ANDALUCIA APD 3004, PROFESOR ALBAREDA 1 E 18080 GRANADA, SPAIN

Telephone: (34) 58121311

Facsimile: (34)

Telex: 78573 iang e

Email: jaimea

Commission(s): 28

PEREK LUBOS DR, ASTRONOMICAL, INSTITUTE, CZECH ACADEMY SCIENCES, BOCNI II 1401

CZ 14131 PRAHA 4, CZECH R

Telephone: (42) 2766051 *068

Facsimile: (42) 276,9023

Telex:

Email:

Commission(s): 33,51

PERES GIOVANNI DR, INST OSSERVATORIO ASTRON, PALAZZO DEI NORMANNI, I 90134 PALERMO, ITALY

Telephone: (39) 916517998

Facsimile: (39) 916517292

Telex:

Email: peres(a)oapa.astrpa.unipa.it

Commission(s): 10

PEREZ ENRIQUE DR, INST DE ASTROFISICA, DE CANARIAS, OBS DEL TEIDE, E 38200 LA LAGUNA, SPAIN

Telephone: (34) 22262211

Facsimile: (34) 22263005

Telex: 92640 iac e

Email: iac::eps/cpialac.es

Commission(s): 28

PEREZ FOURNON ISMAEL DR, INST DE ASTROFISICA, DE CANARIAS, OBS DEL TEIDE, E 38200 LA LAGUNA, SPAI

Telephone: (34) 22262211

Facsimile: (34) 22263005

Telex: 92640 iac e

Email:

Commission(s): $28,+11$

PEREZ-DE-TEJADA H A DR, INSTITUTO DE GEOFISICA, UNAM, APDO POSTAL 877

22860 ENSENADA B CALIF, MEXICO

Telephone: (52) $70667+0601$

Facsimile: (52)

Telex:

Email:

Commission(s): 15 
PEREZ-PERAZA JORGE A PROF, INSTITUTO DE GEOFISICO, UNAM, CIUDAD UNIVERSITAIRE 04510 MEXICO DF, MEXICO

Telephone: (52) 9055481375

Facsimile: (52) $9055505215 \quad$ Telex: 1760155 cicme

Email: japercz

Commission(s):

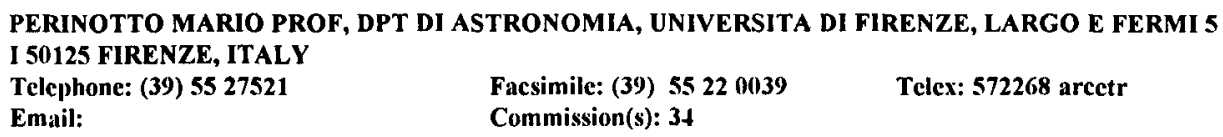

Commission(s): 34

PERKINS FRANCIS W DR, PLASMA PHYSICS LAB, PRINCETON UNIVERSITY, BOX 451, PRINCETON NJ 08544, USA
Telephone: (1) 6096832603
Facsimile: (1)

Email:

Commission(s): 49

PERLEY RICHARD ALAN, NRAO, BOX 0, SOCORRO NM 87801 0387, USA

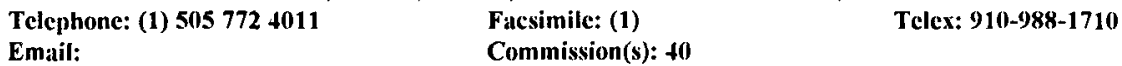
PEROLA GIUSEPPE C DR, ISTITUTO ASTRONOMICO, UNIVERSITA DI ROMA, VIA G M LANCISI 29
I 00161 ROMA, ITALY
Telephone: (39) $6867525 \quad$ Facsimile: (39) Telex: 613255 infnro
Email: $\quad$ Commission(s): $4+$

PERRIER CHRISTIAN DR, OBSERVATOIRE DE GRENOBLE, LABORATOIRE D'ASTROPHYS, BP S3X/UJF F 38041 GRENOBLE CEDEX, FRANCE

Telephone: (33)

Email:

Facsimile: (33)

Commission(s):

Telex:

PERRIN JEAN MARIE DR, LAS, TRAVERSE DU SIPHON, LES TROIS LUCS, F 13012 MARSEILLE, FRANCE

Telephone: (33) $91055900 \quad$ Facsimile: (33) $91661855 \quad$ Telex: $\$ 20$ 584 c astrosp

Email: perrinâfrlasm51

Commission(s): 21

PERRIN MARIE-NOEL DR, OBSERVATOIRE DE PARIS, 61 AVE OBSERVATOIRE, F $7501+$ PARIS, FRANCE

Telephone: (33) 140512245

Facsimile: (33)

Telex: 270776 obs $f$

Email:

Commission(s): 29

PERRY CHARLES L DR, DPT PHYSICS \& ASTRONOMY, LOUISIANA STATE UNIV, BATON ROUGE LA 708034001, USA

Telephone: (1) 5043888287

Facsimilc: (1)

Commission(s): $25,30,33,45$

Telex: 559184

Email:

PERRY JUDITH J DR, INSTITUTE OF ASTRONOMY, THE OBSERVATORIES, MADINGLEY RD

CAMBRIDGE CB3 OHA, UK

Telephone: (4t) $1223337548 \quad$ Facsimile: (4t) $1223337523 \quad$ Telex: 817297

Email:

Commission(s): 28

PERRY PETER M DR, COMPUTER SCIENCES CORP, 10000A AEROSPACE RD, LANHAM SEABROOK MD 20706, USA

Telephone: (1)

Facsimile: (1)

Commission(s): +4

Telex:

Email: pperry(a)explorer.csc.com

PERRYMAN MICHAEL A C DR, ESA/ESTEC, SSD, BOX 299, NL 2200) AG NOORDWIJK, NETHERLANDS

Telephone: (31) 171983615

Facsimile: (31) 171984690

Telex: 39098

Email: estes1::mperrrma

Commission(s): $08,(09,24,47$

PERSI PAOLO, IAS, CNR, CP 67, I0004+ FRASCATI, ITALY

$\begin{array}{ll}\text { Telephone: (39) } 69425655 & \text { Facsimilc: (39) } 69416847 \quad \text { Telex: } 610261 \mathrm{cnr} \text { fra } \\ \text { Email: } & \text { Commission(s): } 34\end{array}$

PERSIDES SOTIRIOS C, DPT OF ASTRONOMY, UNIVERSITY THESSALONIKI, GR 54006 THESSALONIKI, GREECE

Telephone: (30) 31991357

Facsimile: (30)

Telex:

Email:

Commission(s): 47

PESCH PETER DR, NTL SCIENCE FOUNDATION, DIV ASTRONOMICAL SCIENCES, 1800 G ST NW

WASHINGTON DC 20550, USA

Telephone: (1) 2023577622

Facsimile: (1)

Telex:

Email:

Commission(s): 33 


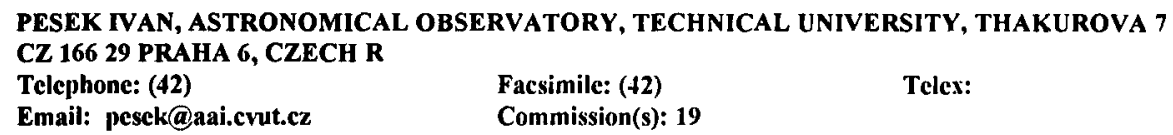

Commission(s): 19

Telex:

Email: pesek@aai.crut.cz

PETERS GERALDINE JOAN DR, SPACE SCIENCES CENTER, UNIV SOUTHERN CALIFORNIA, UNIVERSITY PARK LOS ANGELES CA 900891341 , USA

Telephone: (1) 2137436962

Facsimile: (1)

Commission(s): 29,36,42,44

Telex: $\$ 720+90$ usc Isa

Email:

PETERS WILLIAM L III DR, STEWARD OBSERVATORY, UNIVERSITY OF ARIZONA, TUCSON AZ 85721, USA

Telephonc: (1) 5206215380

Facsimile: (1)

Telex:

Email: peters@as.arizona.edu

Commission(s): $28,34,40$

PETERSEN $J$ O DR, UNIVERSITY OBSERVATORY, OESTER VOLDGADE 3, DK 1350 COPENHAGEN K, DENMARK Telephone: (45) 31141790

Facsimile: (45) 31389157

Telex:

Email:

Commission(s): 27
PETERSON BRADLEY MICHAEL, DPT OF ASTRONOMY, OHIO STATE UNIVERSITY, 17 4 W 18TH AVE COLUMBUS OH 432101106 , USA
Telephonc: (1) $6142927886 \quad$ Facsimilc: (1) Telex:
Email: peterson@payne.mps.ohio-state.edu Commission(s): 28

PETERSON BRUCE A DR, MOUNT STROMLO \& SIDING, SPRING OBSERVATORIES, PRIVATE BAG WODEN PO ACT 2606, AUSTRALIA

Telephone: (61) $62881111 \quad$ Facsimile: (61) $62490233 \quad$ Telex: 62270

Email:

Commission(s): $\$ 4,+7$

PETERSON CHARLES JOHN DR, DPT PHYSICS \& ASTRONOMY, UNIVERSITY OF MISSOURI, 223 PHYSICS BLDG COLUMBIA MO 65211, USA

Telephone: (1) 3148823217

Facsimile: (1)

Commission(s): $28,37,41$

Telex:

Email:

PETERSON DEANE M DR, DPT OF EARTH \& SPACE SCI, ASTRONOMY PROGRAM, SUNY AT STONY BROOK STONY BROOK NY 1179+ 2100, USA

Telephone: (1) 5166328223

Facsimile: (1) $5166326240 \quad$ Telex:

Email: Upeterson@astro.sunysb.edu

Commission(s): 26

PETERSON LAURENCE E PROF, CASS, UCSD, C 011, LA JOLLA CA 92093 0216, USA

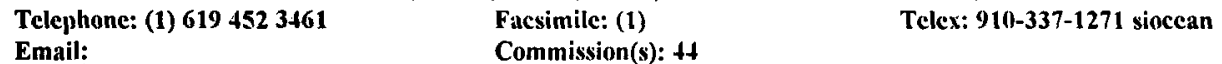

PETERSON RUTH CAROL DR, 607 MARION PL, PALO ALTO CA 9\$301, USA

Telephone: (1) $4153211281 \quad$ Facsimile: (1) Telex:

Email: $\quad$ Commission(s): 29C,30

PETFORD A DAVID DR, DPT OF ASTROPHYSICS, UNIVERSITY OF OXFORD, SOUTH PARKS RD, OXFORD OX1 3RQ, UK

$\begin{array}{ll}\text { Telephone: (44) } 1865511336 & \text { Facsimile: (44) } \\ \text { Email: } & \text { Commission(s): (19) }\end{array}$

PETHICK CHRISTOPHER J DR, NORDITA, BLEGDAMSVEJ 17, DK 2100 COPENHAGEN 0, DENMARK

Telephone: (45) 31421616

Facsimile: (45) 31389157

Telex: 15216 nbidk

Email: pethick@nbivax.nbi.dk

Commission(s): 44

PETIT GERARD DR, BIPM, PAVILLON DE BRETEUIL, F 92312 SEVRES CDX, FRANCE

Telephone: (33) 145077067

Facsimile: (33) $1+53+2021$

Telex: 631351

Email: gpetit@bipm.fr

Commission(s): 31

PETIT JEAN-MARC DR, OCA OBSERV DE NICE, BP 229, F 06304 NICE CDX $\downarrow$, FRANCE

Telephone: (33) 92003089

Facsimile: (33) 92003033

Telex:

Email: petit@fronis1

Commission(s): 07,16 
PETON ALAIN DR, OBSERVATOIRE DE MARSEILLE, 2 PLACE LE VERRIER, F 13248 MARSEILLE CDX 04, FRANCE Telephone: (33) 91959088

Facsimile: (33)

Telex:

Email:

Commission(s):

PETRE ROBERT, GSFC, CODE 666, GREENBELT MD 20771, USA

Telephone: (1) $3012863844 \quad$ Facsimile: (1) 3012861684

Email: petre(alheavx.gsfc.nasa.gov Commission(s);

Telex:

PETRI WINFRIED PROF DR, UNTERLEITEN 2, POSTFACH 106, D 83722 SCHLIERSEE, GERMANY

Telephone: (49) 80266428

Facsimile: (49)

Telex:

Email:

Commission(s): +1

PETRINI DANIEL DR, OCA OBSERV DE NICE, BP 229, F 0630 4 NICE CDX 4 , FRANCE

Telephone: (33) 93890420

Facsimile: (33) 92003033

Telex: $\$ 60004$ obsnice f

Email:

Commission(s): 14

PETRO LARRY DAVID, STSCI, HOMEWOOD CAMPUS, 3700 SAN MARTIN DR, BALTIMORE MD 21218, USA

Telephone: (1) 4103384501

Facsimile: (1) +103381592

Telex: $68+9101$

Email: petroßastsci.edu

Commission(s):

PETROPOULOS BASIL CH DR, RES CENTER FOR ASTRONOMY, ACADEMY OF ATHENS

14 ANAGNOSTOPOULOU ST, GR 10673 ATHENS, GREECE

Telephone: (30) $13613589 \quad$ Facsimile: (30)

Email:

Commission(s): 14,16

Telex:

PETROSIAN ARTASHES R DR, BYURAKAN ASTROPHYSICAL, OBSERVATORY, 378433 BYURAKAN, ARMENIA

Telephone: (7) 8852283453

Facsimile: (7) $885228+142$

Telex: $2433+4$ orion su

Email:

Commission(s): 28

PETROSIAN VAHE PROF, CTR FOR SPACE SCIENCES \&, ASTROPHYSICS, STANFORD UNIV ERL 304

STANFORD CA 943054055 , USA

Telephone: (1) 4154971435

Facsimile: (1) Telex:

Email:

Commission(s): $10,34, \downarrow 4,47$

PETROV G M DR, NIKOLAEV OBSERVATORY, UKRAINIAN ACAD OF SCIENCE, 327000 NIKOLAEV, UKRAINE

Telephone: (0) 361824

Facsimile: (0)

Telex:

Email: $\operatorname{root}(\mathfrak{a})$ ma.nikolacv.ua

Commission(s): (18

PETROV GENNADIJ M, INST OF RADIO \& ELECTRON, ACADEMY OF SCIENCES, MARKS AVENJU 18 103907 MOSCOW, RUSSIA

Telephone: (7)

Facsimile: (7)

Telex:

Email:

Commission(s):

PETROV GEORGIJ I PROF DR, SPACE RESEARCH INSTITUTE, ACADEMY OF SCIENCES

PROFSOJUZNAYA UL 84/32, 117810 MOSCOW, RUSSIA

Telephone: (7)

Facsimile: (7)

Commission(s):

Telex:

Email:

DPT OF ASTRONOMY, BULGARIAN ACAD SCIENCES, 72 LENIN BLVD

PETROV GEORGY TRENDA
BG $178+$ SOFIA, BULGARIA

Telephone: (359) 2758927

Facsimile: (359)

Commission(s): 28

Telex: 23561 eef ban bg

Email:

PETROV PETER P DR, CRIMEAN ASTROPHYS OBS, UKRAINIAN ACAD OF SCIENCE, NAUCHNY

$33+413$ CRIMEA, UKRAINE

Telephone: (7) $4329+5$

Facsimile: (7)

Telex:

Email:

Commission(s): (09,27

PETROVAY KRISTOF, ASTRONOMY DPT, EOTVOS UNIVERSITY, LUDOVIKA TER 2, H 1083 BUDAPEST, HUNGARY

Telephone: (36) $111+1019$

Facsimilc: (36) 12101089

Telex:

Email: krisâinnin.clte.hu

Commission(s): 10,12

PETROVSKAYA M S DR, INST OF THEORET ASTRONOMY, ACADEMY OF SCIENCES, N KUTUZOVA 10 191187 ST PETERSBURG, RUSSIA

Telephone: (7) 121578 ITA SU

Facsimile: (7)

Telex:

Email:

Commission(s): 07,37 
PETTENGILL GORDON H PROF, CENTER FOR SPACE RESEARCH, MIT $372+1$, BOX 165 CAMBRIDGE MA 02139, USA

Telephone: (1) 6172537501

Facsimile: (1)

Commission(s): 16,40

Telex: 92-1473

Email:

PETTERSEN BJOERN RAGNVALD, GEODETIC INSTITUTE, NORWEGIAN MAPPING AUTH N 3500 HONEFOSS, NORWAY

Telephone: (47) 6718100

Facsimile: (47) 6718101

Telex:

Email: bjornr|@gdiv.statkart.no

Commission(s): 27

PETTINI MARCO, OSS ASTROFISICO, DI ARCETRI, LARGO E FERMI 5, 1 50125 FIRENZE, ITALY

Telephone: (39) $552752282 \quad$ Facsimilc: (39)

Telex: 572268 arcetr

Email:

Commission(s): 14

PETTINI MAX, ROYAL GREENWICH OBS, HERSTMONCEUX CASTLE, HAILSHAM BN27 IRP, UK

Telephonc: (44) $1323833171 \quad$ Facsimile: (4)

Email:

Commission(s)

Telex: 87451 rgobsy g

PETUCHOWSKI SAMUEL J, NASA GSFC, CODE 685, GREENBELT MD 20771, USA

Telephone: (1) 3012863901

Facsimile: (1) 3012861617 Telex:

Email: spet@stars.gsfc.nasa.gov

Commission(s): 34.28

PEYTURAUX ROGER H PROF, INSTITUT D'ASTROPHYSIQUE, 98BIS BD ARAGO, F 7501 4 PARIS, FRANCE Telejphone: (33) 144328007

Facsimile: (33) $1+4328001$

Telex:

Email:

Commission(s): 12

PFAU WERNER, UNIV STERNWARTE JENA, SCHILLERGAESSCHEN 2, D 077+5 JENA, GERMANY

Telephone: (49) 7858861347

Facsimile: (49)

Telex:

Email:

Commission(s): 25

PFEIFFER RAYMOND J, 8 BARBARA LANE, TITUSVILLE NJ 08560, USA

Telephone: (1) 6098834612

Facsimile: (1)

Commission(s): 25

Telex:

Email:

\& ASTROPHYS, KARL SCHWARZSCHILDSTR 1

PFENNIG HANS H DR, MPI F PHYSIK \& ASTRO

Telephone: (49) $8932999435 \quad$ Facsimile: (49)

Telex: $52+629$ astro d

Email:

Commission(s): 14

PFENNIGER DANIEL DR, OBSERVATOIRE DE GENEVE, CHEMIN DES MAILLETTES 51

CH 1290 SAUVERNY, SWITZERLAND

Telephone: (\$1) 227552611

Facsimile: (41) 227553983

Telex: 419209 obs ch

Email: bitnet:pfennige(a)egeugest

Commission(s): 28

PFLEIDERER JORG PROF, INSTITUT FUER ASTRONOMIE, TECHNIKERSTR 15, A 6020 INNSBRUCK, AUSTRIA

Telephone: (43) 5125076030

Facsimile: (43) 5125072923

Telex:

Email: chef@ast1.uibk.ac.at

Commission(s): 21

PFLUG KLAUS DR, ZNTRLINST F ASTROPHYSIK, SONNENOBSERVATORIUM, EINSTEINTURM D 14473 POTSDAM, GERMANY

Telephone: (49)

Facsimile: $(49)$

Telex:

Email:

Commission(s): 10,12,49

PHAM-VAN JACQUELINE MME, OCA CERGA, AVE COPERNIC, F 06130 GRASSE, FRANCE

Telephone: (33) 93365849

Facsimile: (33)

Commission(s): 08

Telex: 470865

Email:

PHILIP A G DAVIS, 1125 OXFORD PL, SCHENECTADY NY 12308, USA

Telephone: (1) 5183745636

Facsimilc: (1) 5183465781

Telex:

Email: agdp@gar.union.edu

Commission(s): $05,25,30,33,37,45$

PHILLIPS JOHN G PROF, ASTRONOMY DPT, UNIVERSITY OF CALIFORNIA, 601 CAMPBELL HALL BERKELEY CA 94720, USA

Telephone: (1) $4156+25275$

Facsimile: (1)

Telex:

Email:

Commission(s): 14,36 
PHILLIPS JOHN PETER, DPT OF PHYSICS, QUEEN MARY/WESTFIELD COLL, MILE END RD, LONDON E1 4NS, UK Telephone: (44) 1719804811

Facsimile: (4)

Commission(s): 34

Telex: 893750 qmeuol g

Email:

PHILLIPS KENNETH J H, RUTHERFORD APPLETON LAB, SPACE \& ASTROPHYSICS DIV, BLDG R25/R68

CHILTON DIDCOT OX11 0QX, UK

Telephone: (44) 123521900

Facsimile: (4)

Telex:

Email:

Commission(s): 10,12,44

PHILLIPS MARK M DR, CERRO TOLOLO, INTERAMERICAN OBSERVATORY, CASILLA 603, LA SERENA, CHILE

Telephone: (56) 51213352

Email:

Facsimile: (56) $51212+66 * 3+2 \quad$ Telex: 620301 aura ct

Commission(s): $06,28,35$

PHILLIPS THOMAS GOULD DR, CALTECH, MS 320 47, PASADENA CA 91125, USA

Telephone: (1) 8183564278

Facsimile: (1)

Telex:

Email:

Commission(s): $34,+0$

PIACENTINI RUBEN DR, OBSERVATORIO ASTRONOMICO, DE ROSARIO, CC 606, 2000 ROSARIO, ARGENTINA

Telephone: (54) $4163084 \quad$ Facsimile: (54)

Telex: $\$ 1817$ ciros ar

Email: ruben\%ifir.edu.ar(ä)unet.uu.net Commission(s): 14

PIAZZA LILIANA RIZZO, CRAAE/EPUSP, RUA S VICENTE DE PAULA, 705/82 VINCENTE DE PAULA

01229010 SAO PAULO SP, BRAZIL

Telephone: (55)

Facsimile: (55)

Email:

Commission(s):

Telex:

PICAT JEAN-PIERRE DR, OBS MIDI PYRENEES, 14 AVE E BELIN, F 31400 TOULOUSE CDX, FRANCE

Telephone: (33) 61252101

Facsimile: (33)

Telex:

Email:

Commission(s): 09

PICCA DOMENICO DR, DPT DI FISICA, UNIVERSITA DI BARI, VIA G AMENDOLA 173, I 70123 BARI, ITALY

Telephone: (39) 80243215

Facsimile: (39) $802+2+3+$

Telex:

Email:

Commission(s): 19

PICCIONI ADALBERTO, OSS ASTRONOMICO, UNIVERSITA DI BOLOGNA, CP 596, I 40100 BOLOGNA, ITALY

Telephone: (39) 51222956

Facsimile: (39)

Telex: 211664 infnbo

Email:

Commission(s): 42

PICK MONIQUE DR, OBSERVATOIRE DE PARIS, SECTION DE MEUDON, DASOP, F 92195 MEUDON PPL CDX, FRANCE

$\begin{array}{lll}\text { Telcphone: (33) } 145077811 & \text { Facsimile: (33) } & \text { Telex: 200590 } \\ \text { Email: } & \text { Commission(s): 10,40 } & \end{array}$

PICKLES ANDREW JOHN DR, INSTITUTE FOR ASTRONOMY, UNIVERSITY OF HAWAII, 2680 WOODLAWN DR HONOLULU HI 96822, USA

Telephone: (1) 8089566756

Email: pickles(a)ifa.hawaii.edu

Facsimile: (1) 8089882790

Telex: 7238459 uhast hr

PIDDINGTON JACK H RES FEL, CSIRO NML, LINDFIELD, SYDNEY NSW 2070, AUSTRALIA

Telephone: (61) 24676211

Email:

Facsimilc: (61)

Telex: 26296 a

Commission(s): 10,4t

PIER JEFFREY R DR, US NAVAL OBSERVATORY, FLAGSTAFF STATION, BOX 1149, FLAGSTAFF AZ 86002, USA

Telcphonc: (1) 6027795132

Facsimile: (1)

Telex:

Email:

Commission(s): 33

PIERCE A KEITH DR, NTL SOLAR OBSERVATORY, BOX 26732, 950 N CHERRY AVE, TUCSON AZ 857266732 , USA

Telephone: (1) 5203275511

Facsimile: (1)

Telex:

Email:

Commission(s): 07,12

PIERCE DAVID ALLEN DR, 7706 WASTLAWN AVE, LOS ANGELES CA 900\$5, USA

Telephonc: (1)

Facsimile: (1)

Telex:

Email:

Commission(s): 20 
M 758

PIERRE MARGUERITE DR, ESO, KARL SCHWARZSCHILDSTR 2, D 85740 GARCHING MUENCHEN, GERMANY

Telephone: (49) 8932006293

Facsimile: (49) 893202362

Telex: $\$ 282820$ co d

Email: bitnct:pierre(a)esomc1

Commission(s):

PIGATTO LUISA DR, OSS ASTRONOMICO DI PADOVA, VIC DELL OSSERVATORIO 5, I 35122 PADOVA, ITALY

Telephone: (39) 49661499

Facsimile: (39)

Telex: 432071

Email:

Commission(s): 41

PIIROLA VILPPU E DR, OBS \& ASTROPHYSICS LAB, UNIVERSITY OF HELSINKI, TAEHTITORNINMAKI SF 00130 HELSINKI 13, FINLAND

Telephone: (358) 1912801

Facsimile: (358)

Commission(s): $25,27,42$

Telex: $12+690$ unih sf

Email:

PIKE CHRISTOPHER DAVID, RUTHERFORD APPLETON LAB, SPACE \& ASTROPHYSICS DIV, BLDG R25/R68 CHILTON DIDCOT OX11 OQX, UK

Telephone: (44) 123521900

Facsimile: ( $4+4)$

Email:

Commission(s):

Telex:

PIKICHIAN HOVHANNES, BYURAKAN OBSERVATORY, ASHTARAK DISTZ, 378+33 BYURAKAN, ARMENIA

Telephone: (7) 8852283453

Facsimile: (7) 8852523640

Telex: $2 \$ 3121$ nauka

Email:

Commission(s): 28

PILACHOWSKI CATHERINE DR, KITT PEAK NTL OBS, BOX 26732, 950 N CHERRY AVE, TUCSON AZ 85726 6732, US

Telephone: (1) 5203275511

Facsimile: (1)

Commission(s): 29,37

Telex: 0666484 aura noaotuc

Email: catyp@noao.edu

PILCHER CARL BERNARD DR, $\$ 316$ ELLICOTT STREET NW, WASHINGTON DC 20016, USA

Telephone: (1)

Facsimile: (1)

Telex:

Email:

Commission(s): 15

PHLKINGTON JOHN D H DR, ROYAL GREENWICH OBS, MADINGLEY RD, CAMBRIDGE CB3 OEZ, UK

Telephonc: (44) 1223374758

Facsimile: (4) $122337+700 \quad$ Telex:

Email: jdhp@mail.ast.cam.ac.uk

Commission(s): 19,31

PILLINGER COLIN DR, DPT OF EARTH SCIENCES, THE OPEN UNIVERSITY, WALTON HALL

MILTON KEYNES MK7 6AA, UK

Telephone: (44) 1908652119

Facsimilc: (44) $1908655910 \quad$ Telex:

Email:

Commission(s): $15,16,22$

PILOWSKI K PROF DR, GEODAETISCHES INST, TECHNISCHE UNIVERSITAET, NIENBURGER STR 1 D 3000 HANNOVER, GERMANY

Telephonc: (49)

Facsimile: (49)

Telex:

Email:

Commission(s): 08,33

PILYUGIN LEONID, MAIN ASTRONOMICAL OBS, UKRAINIAN ACAD OF SCIENCE, GOLOSEEVO

252127 KIEV, UKRAINE

Telephone: (7)

Facsimile: (7)

Telex:

Email:

Commission(s):

PINEAU DES FORETS G DR, OBSERVATOIRE DE PARIS, SECTION DE MEUDON, DAEC

F 92195 MEUDON PPL CDX, FRANCE

Telephone: (33) 145077454

Email: mesioa::forets/forets/afrmeus1

Facsimile: (33) $145077469 \quad$ Telex: 631987

Commission(s): 34

PINEAULT SERGE DR, DPT DE PHYSIQUE, UNIVERSITE DE LAVAL, SAINTE-FOY QC G1K 7P4, CANADA

Telephone: (1) 4166563901

Facsimile: (1) 4186562040

Telex:

Email: pincault@phy.ulaval.ca

Commission(s):

PINEDA DE CARIAS MARIA C, SECCION DE ASTRONOMIA, DPT DE FISICA UNAH, AP 4332

TEGUCIGALPA, HONDURAS

Telephone: (504) 3221 10*230

Facsimile: (504) 310678

Telex:

Email:

Commission(s):

PINES DAVID PROF, DPT OF PHYSICS, UNIVERSITY OF ILLINOIS, 1110 W GREEN ST, URBANA IL 61801, USA

Telephone: (1) 2173330115

Facsimile: (1)

Email:

Commission(s): 35

Telex: 9103806599 physics s 
PINGREE DAVID PROF, BROWN UNIVERSITY, PO BOX 1900, PROVIDENCE RI 02912, USA

Telephone: (1) 4018632101

Facsimile: (1)

Telex:

Email:

Commission(s): 41

PINIGIN GENNADIJ I DR, NIKOLAEV OBSERVATORY, UKRAINIAN ACAD OF SCIENCE

327001 NIKOLAEV, UKRAINE

$\begin{array}{lll}\text { Telcphone: (7) } & \text { Facsimile: (7) } & \text { Telex: } \\ \text { Email: pinigin@mao.nikolaev.ua } & \text { Commission(s): } 08 & \end{array}$

PINKAU K PROF, MPI FUER PLASMAPHYSIK, KARL SCHWARZSCHILDSTR 1

D 85740 GARCHING MUENCHEN, GERMANY

Telephone: (49) $893299342 \quad$ Facsimile: (49)

Email:

Commission(s): 44

Telex: 05-215-808

PINOTSIS ANTONIS D DR, DPT OF ASTRONOMY, NTL UNIVERSITY OF ATHENS, PANEPISTIMIOPOLIS

GR 15771 ZOGRAFOS, GREECE

Telephone: (30) 17243414

Facsimile: (30)

Commission(s): 35

Telex:

Email:

$$
\text { Commission(s): } 35
$$

PINSONNEAULT MARC HOWARD, DPT OF PHYSICS, YALE UNIVERSITY, 260 WHITNEY AVE

NEW HAVEN CT 06511, USA

Telepphone: (1) 2034323012

Facsimile: (1) $203+325048 \quad$ Telcx:

Email: pinsono(a)ctoile.astro.yale.edu

Commission(s):

PINTO GIROLAMO PROF, OSS ASTRONOMICO DI PADOVA, VIC DELL OSSERVATORIO 5, I 35122 PADOVA, ITALY

Telephone: (39) 49661499

Facsimile: (39)

Email:

Commission(s):

Telex:

PINTO PHILIP ALFRED DR, CENTER FOR ASTROPHYSICS, HCO/SAO, 60 GARDEN ST, CAMBRIDGE MA 02138, USA

Telephone: (1) 6174957174

Email: pinto (a) cfa.harvard.edu

Facsimile: (1) Telex:

Commission(s): 36,44

PIOTTO GIAMPAOLLO, DPT DI ASTRONOMIA, UNIVERSITA DI PADOVA, VIC DELL OSSERVATORIO 5

I 35122 PADOVA, ITALY

Telephone: (39) 49661499

Facsimile: (39) 4938919

Telex: $\mathbf{4 3 2 0 7 1}$ astros $i$

Email:

Commission(s):

PIPHER JUDITH L, DPT PHYSICS \& ASTRONOMY, UNIVERSITY OF ROCHESTER, ROCHESTER NY 14627, USA

Telephone: (1) 7162754402

Facsimile: (1)

Telex:

Email: jlpipher@boris.pas. rochester.e

Commission(s): +4

PIRO LUIGI DR, IAS, CNR, CP 67, I 0004 FRASCATI, ITALY

Telephonc: (39) 69424589

Facsimilc: (39) 69416847

Telex: $610261 \mathrm{cnr}$ fra

Email: 40607::piro

Commission(s): 4

PIRRONELLO VALERIO, OSS ASTROFISICO, CITTA UNIVERSITARIA, VIA A DORIA 6, I 95125 CATANIA, ITALY

Telephone: (39) 95330533

Facsimile: (39)

Commission(s):

Telex: 970359 astret $i$

Email:

PISKUNOV ANATOLY E, INST OF ASTRONOMY, ACADEMY OF SCIENCES, PYATNITSKAYA UL 48 109017 MOSCOW, RUSSIA

Telephone: (7) $952315461 \quad$ Facsimile: (7) Telex: 412623 scstp su

Email:

Commission(s): 05C,37

PISKUNOV NIKOLAI E DR, INST OF ASTRONOMY, ACADEMY OF SCIENCES, PYATNITSKAYA UL 48 109017 MOSCOW, RUSSIA

Telephone: (7) 952315461

Email:

Facsimilc: (7)

Telex:

Commission(s): 36

PISMIS DE RECILLAS PARIS, INSTITUTO DE ASTRONOMIA, UNAM, APDO POSTAL 70-264

04510 MEXICO DF, MEXICO

Telephone: (52) 9055485306

Facsimile: (52) 9055483712

Telex: 1760155 cicme

Email:

Commission(s): $28,33,34$ 
PITTICH EDUARD M DR, ASTRONOMICAL INSTITUTE, SLOVAK ACADEMY SCIENCES, DUBRAVSKA 9 SK 84228 BRATISLAVA, SLOVAK R

Telephone: (42) 7375157

Facsimile: (42)

Telex: 93373 seis

Email:

Commission(s): 15,20

PITZ ECKHART DR, MPI FUER ASTRONOMIE, KOENIGSTUHL, D 69117 HEIDELBERG, GERMANY

Telephone: (49) 62215281

Facsimile: (49)

Telex: 461789 mpia d

Email:

Commission(s):

PIZZELLA G DR, DPT DI FISICA, UNIVERSITA DI ROMA, PA MORO 2, I 00185 ROMA, ITALY

Telephone: (39) $64940156 \quad$ Facsimile: (39)

Telex: 613255 infnro

Email:

Commission(s):

PIZZICHINI GRAZIELLA, IST TESRE, CNR, VIA DE CASTAGNOLI 1, I 40126 BOLOGNA, ITALY

Telephone: (39) 51519593

Facsimile: (39)

Telex: 511350 cnr bo

Email:

Commission(s): $05,24,45$

PLANESAS PERE, CTR ASTRON DE YEBES, OAN, APD 148, E 19080 GUADALAJARA, SPAIN

Telephone: (34) 11223358

Facsimile: (34)

Telex:

Email:

Commission(s): 40

PLASSARD J DR, KSARA OBSERVATORY, KSARA, LEBANON

Telephone: (961)

Facsimile: (961)

Telcx:

Email:

Commission(s):

PLATAIS IMANTS K DR, YALE UNIVERSITY OBS, BOX 208101, NEW HAVEN CT 06520, USA

Telephone: (1) 2034323021

Facsimile: (1) $203 \mathbf{4 3 2} 5048 \quad$ Telex:

Email: imants $(a)$ astro.yale.edu

Commission(s): $24,25,37$

PLAVEC MIREK J PROF, DPT OF ASTRONOMY, UNIVERSITY OF CALIFORNIA, MS 8979 LOS ANGELES CA 90024 , USA

Telephone: (1) 2138251672

Facsimile: (1)

Telex:

Email:

Commission(s): $29,35,42$

PLAVEC ZDENKA DR, DPT OF ASTRONOMY, UNIVERSITY OF CALIFORNIA, 405 HILGARD AVE LOS ANGELES CA 90024, USA

Telephone: (1) 2132068596

Facsimile: (1)

Telex:

Email:

Commission(s): 22

PLEZ BERTRAND, NIELS BOHR INSTITUTE, BLEGDAMSVEJ 17, DK 2100 COPENHAGEN 0, DENMARK

Telephone: (45)

Email: plezanbi.dk

Facsimile: ( $\mathbf{4 5})$

Telex:

Commission(s): 29

PNEUMAN GERALD W, 550 ROE RD, PARADISE CA 95969, USA

Telephone: (1)

Facsimile: (1)

Telex:

Email:

Commission(s): 10,49

PODSIADLOWSKI PHILIPP, INSTITUTE OF ASTRONOMY, THE OBSERVATORIES, MADINGLEY RD, CAMBRIDGE CB3 0HA, UK

Telephone: (44) 1223337502

Email: ppodsi@mail.ast.cam.ac.uk

Facsimile: (4) $1223337523 \quad$ Telex:

Commission(s):

POECKERT ROLAND H DR, DEFENCE RESEARCH, ESTABLISHMENT PACIFIC, FMO CFB ESQUIMALT VICTORIA BC VOS 1BO, CANADA

Telephone: (1)

Facsimile: (1)

Telex:

Email:

Commission(s):

POEPPEL WOLFGANG G L DR, IAR, CC 5, $189+$ VILLA ELISA (BS AS), ARGENTINA

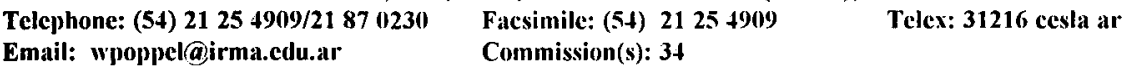

POHL ECKHARD DR, STERNWARTE NUERNBERG, REGIOMONTANUSWEG 1, D 8500 NUERNBERG 20, GERMANY Telephone: (49) 911593540

Facsimile: $(49)$

Telex:

Email:

Commission(s): 
POKORNY ZDENEK DR, N COPERNICUS OBSERVATORY, \& PLANETARIUM, KRAVI HORA CZ 616 00 BRNO 16, CZECH R

Telephone: $(\$ 2) 57+437+$

Facsimile: (+2)

Telex:

Email:

Commission(s): 16,46

POLAND ARTHUR I DR, NASA GSFC, CODE 682, GREENBELT MD 20771, USA

Telephone: (1) 3012867334

Facsimile: (1)

Telex: 89675

Email:

Commission(s): 10

POLCARO V F, IAS, CNR, CP 67,100014 FRASCATI, ITALY

Telephone: (39) 69425651

Facsimile: (39) 69416847

Commission(s): 29

Telex: 610261

Email:

PLANE

Telephonc: (42) 25353513

Email:

Facsimile: (42)

Commission(s): 05

Telex:

POLETTO GIANNINA PROF, OSS ASTROFISICO, DI ARCETRI, LARGO E FERMI 5, I 50125 FIRENZE, ITALY

Tclephonc: (39) $552752252 \quad$ Facsimile: (39)

Email:

Commission(s): 10C

Telex: 572268 arcetr $\mathrm{i}$

POLIAKOV EUGENE, PULKOVO OBSERVATORY, PULKOVO SHOSSE 65, M TOREZ AVE 106 36 196140 ST PETERSBURG, RUSSIA

Telephonc: (7) $812123 \mathbf{4 5 5 5}$

Email: poliakov(a)gao.spl).su

Facsimile: (7) 8123151701

Telex: 121351 pulob su

POLIDAN RONALD S, NASA GSFC, CODE 681.1, LASP, GREENBELT MD 20771, USA

Telephonc: (1) 301 286 5013)

Email: (span)stars::polidan

Facsimile: (1) 301 286 8709

Telex: 89675

Commission(s): $29,+2,+4$

POLLACCO DON, SCHOOL OF CHEMICAL \&, PHYSICAL SCIENCES, LIVERPOOL JMU, LIVERPOOL L3 3AF, UK

Telephone: (44) 1512312337

Email: Ilp(a)staru1.livjm.ac.uli

Facsimile: (4+) 1512312337

Telex:

Commission(s): 34

POLLAS CHRISTIAN, OBSERVATOIRE COTE D'AZUR, TELESCOPE DE SCHMIDT

2130 ROUTE DE L'OBSERVATOIRE, F 16460 CAUSSOLS, FRANCE

Telephone: (33) 93+0 5437 Facsimile: (33) 9340 $5+33$

Email: pollasgocaro1.obs.azur.fr Commission(s):

Telex:

POLNITZKY GERHARD DR, INSTITUT FUER ASTRONOMIE, UNIVERSITAET WIEN, TUERKENSCHANZSTR 17 A 1180 WIEN, AUSTRIA

Telephone: $(\$ 3) 134536090$

Email:

Facsimilc: (43)

Commission(s): 08,22

Telex; 116222 physi a

POLOSUKHINA-CHUVAEVA N DR, CRIMEAN ASTROPHYS OBS, UKRAINIAN ACAD OF SCIENCE, NAUCHNY $33+413$ CRIMEA, UKRAINE

Telephone: (7) $4329+5$

Facsimile: (7)

Commission(s):

Telex:

Email:

POLOZHENTSEV DIMITRIJ DR, PULKOVO OBSERVATORY, ACADEMY OF SCIENCES PULKOVSKOE SHOSSE 65-8-11, 1961 40 ST PETERSBURG, RUSSIA

Telephone: (7) $812123+453$

Facsimile: (7)

Email:

Commission(s): $08 \mathrm{C}, 2+, 41$

Telex: 121351 pulob su

POLUPAN P N DR, ASTRONOMICAL OBSERVATORY, KIEV STATE UNIVERSITY, OBSERVATORNAYA UL 3 252053 KIEV, UKRAINE

Telephone: (7) 044260908

Email:

Facsimile: (7)

Telex: 132201

Commission(s): 10

POLYACHENKO VALERIJ L DR, INST OF ASTRONOMY, ACADEMY OF SCIENCES, PYATNITSKAYA UL 48 109017 MOSCOW, RUSSIA

Telephone: (7) 952313980

Email: iaasânodc.ias.msk.su

Facsimile: (7) 952302081

Commission(s): 33
Telex: $\$ 11576$ ascon su 
POLYMILIS CHRONIS DR, DPT OF ASTRONOMY, NTL UNIVERSITY OF ATHENS, PANEPISTIMIOPOLIS GR 15783 ZOGRAFIG, GREECE

Telephone: (30) 17243414

Facsimile: (30)

Telex:

Email: spmso@grathun1

Commission(s): 33

POMA ANGELO DR, ISTITUTO DI ASTRONOMIA, VIA OSPEDALE 72, 109100 CAGLIARI, ITALY

Telephone: (39) 70663544

Facsimile: (39)

Commission(s): 08,19

Telex: 790326 ossast

Email:

PONCE G A, APARTADO POSTAL 3023, TEGUCIGALPA MDC, HONDURAS
Telephone: (504)
Facsimile: (504) 327196

Email:

Commission(s): 46

PONGRACIC HELEN, RCFTA, SCHOOL OF PHYSICS, UNIVERSITY OF SYDNEY, SYDNEY NSW 2006, AUSTRALIA

Telephone: (61) 23512546

Facsimile: (61) 26602903

Telex:

Email: helenp@physics.su.oz.au

Commission(s): 34,35

PONMAN TREVOR DR, DPT OF SPACE RESEARCH, UNIVERSITY OF BIRMINGHAM, BOX 363

BIRMINGHAM B15 2TT, UK

Telephone: (44)

Facsimile: (44)

Telex:

Email:

Commission(s):

RONNAMPERUMA CYRIL PROF, DPT OF CHEMISTRY, UNIVERSITY OF MARYLAND

COLLEGE PARK MD 20472, USA

Telephone: (1)

Facsimile: (1)

Telex:

Email:

Commission(s): 51

PONSONBY JOHN E B DR, NRAL, JODRELL BANK, MACCLESFIELD SK11 9DL, UK

Telephone: (44) 147771321

Facsimile: (44)

Commission(s): 40,51

Telex: 36149

Email:

POOLE GRAHAM, DPT PHYSICS \& ELECTRONICS, RHODES UNIVERSITY, BOX 94, GRAHAMSTOWN 6140 SOUTH AFRICA

Telephone: (27) $461318454 / 318450 \quad$ Facsimile: (27) $46125049 \quad$ Telex:

Email: phg)(aruchem.ru.ac.za Commission(s): 22

POOLEY GUY DR, MULLARD RADIO ASTRON OBS, CAVENDISH LABORATORY, MADINGLEY RD

CAMBRIDGE CB3 OHE, UK

Telephone: (44) 1223337294

Facsimile: (44) $1223354599 \quad$ Telex: 81292

Email:

Commission(s): 40

POP VASILE DR, FAC OF MATHEMATICS, UNIVERSITY OF CLUJ-NAPOCA, STR KOGALNICEANU 1

R 3400 CLUJ-NAPOCA, RUMANIA

Telephone: (40) 64116101

Facsimile: $(40) 64194592$

Telex:

Email:

Commission(s): 27

POPELAR JOSEF DR, GEODETIC SURVEY DIVISION, CANADA CENTRE FOR SURVEY, 615 BOOTH ST OTTAWA ON K1A 0E9, CANADA

Telephone: (1)

Facsimile: (1)

Telex:

Email:

Commission(s): 19,31

'POPESCU PETRE, ASTRONUMICAL INSTITUTE, CUTITUL DE ARGINT 5, BOX 28, R 75212 BUCHAREST, RUMANIA Telephone: (40) 16236892 Facsimilc: (40) 1312339 Telex:

Email: ppopescu@imar.ro

Commission(s):

POPOV MICHKAIL, SPACE RESEARCH INSTITUTE, ACADEMY OF SCIENCES, PROFSOJUZNAYA UL 84/32 117810 MOSCOW, RUSSIA

Telephone: (7) 0953332512

Email: pian(a)sovam.com

Facsimile: (7) $0953332378 \quad$ Telex:

Commission(s):

POPOV VASIL NIKOLOV, DPT OF ASTRONOMY, BULGARIAN ACAD SCIENCES, 72 LENIN BLVD

BG 1784 SOFIA, BULGARIA

Telephone: (359) 2758927

Facsimile: (359)

Commission(s): 28

Telex:

Email: 
POPOV VICTOR S DR, PULKOVO OBSERVATORY, ACADEMY OF SCIENCES, 10 KUTUZOV QUAY 196140 ST PETERSBURG, RUSSIA

Telephone: (7)

Email:
Facsimile: (7)

Commission(s): 30
Telex:

POPOVIC GEORGIJE DR, ASTRONOMICAL OBSERVATORY, VOLGINA 7

YU 11050 BEOGRAD, YUGOSLAVIA-SERBIA \& MONTENEGRO

Telephone: (38) $11419357 \quad$ Facsimile: (38) Telex:

Email:
Commission(s): 26

POPPER DANIEL M PROF, DPT OF ASTRONOMY, UNIVERSITY OF CALIFORNIA, LOS ANGELES CA 90024, USA

Telephone: (1) $2138253622 \quad$ Facsimile: (1)

Telex: 9103427597

Email:

Commission(s): 42

POQUERUSSE MICHEL, OBSERVATOIRE DE PARIS, SECTION DE MEUDON, DESPA F 92195 MEUDON PPL CDX, FRANCE

Telephone: (33) 145077530

Facsimile: (33)

Telex: 204464

Email:

Commission(s): 10,12

PORCAS RICHARD DR, MPI FUER RADIOASTRONOMIE, AUF DEM HUEGEL 69, D 53121 BONN, GERMANY

Telephone: (49) 228525282

Facsimile: (49)

Telex: 886440

Email: porcas(ampifr-bonn.mpg.de

Commission(s): 40

PORCEDDU IGNAZIO E P DR, ISTITUTO DI ASTRONOMIA, VIA OSPEDALE 72, I 09124 CAGLIARI, ITALY

Telephonc: (39) 70663544

Facsimile: (39) 70657657 Telex:

Email: 40588::porceddu

Commission(s): 34

PORETTI ENNIO, OSS ASTRONOMICO DI MILANO, VIA E BIANCHI 46, I 22055 MERATE, ITALY

Telephone: (39) 596412

Facsimile: (39)

Telex:

Email: porctti(a)astmib.infn.it

Commission(s):

PORFIR'EV V V DR, PEDAGOGOC INSTITUTE, MINISTRY OF EDUCATION, 107846 MOSCOW, RUSSIA

Telephone: (7)

Facsimile: (7)

Commission(s): 35

Telex:

Email:

PORTER JASON G DR, NASA/MSFC, CODE E5 52, SPACE SCIENCE LAB, HUNTSVILLE AL 35812, USA

Telephonc: (1) $2055+47607$

Facsimile: (1) $2055+45862$

Telex: $62026079 \mathrm{esl}$

Email: ssl::porterj

Commission(s): 10

PORTER NEIL A PROF, DPT OF PHYSICS, UNIVERSITY COLLEGE, BELFIELD, DUBLIN 4, IRELAND

Telephonc: (353) $1693244 \star 211$

Facsimile: (353)

Telex: 32693 ucdei

Email:

Commission(s): 41,44

PORUBCAN VLADIMIR DR, ASTRONOMICAL INSTITUTE, SLOVAK ACADEMY SCIENCES, DUBRAVSKA 9 SK 84228 BRATISLAVA, SLOVAK R

Telephone: (42) 7375157

Email: astropor@asaba.sk

Facsimile: (42)

Commission(s): $22 \mathrm{C}$

Telex: 93373 seis

POTTASCH STUART R PROF, KAPTEYN ASTRONOMICAL INST, BOX 800, NL 9700 AV GRONINGEN, NETHERLAND

Telephone: (31) $501166+1$

Facsimile: (31)

Telex: 53572 stars $\mathrm{nl}$

Email:

Commission(s): $3+C, 36$

POTTER ANDREW E DR, NASA/JOHNSON SPACE CENTER, CODE SN3, HOUSTON TX 77058, USA

Telephone: (1) 7134835276

Facsimile: (1) $7134835347 \quad$ Telex:

Email: sn:;potter

Commission(s): 16

POTTER HEINO I DR, PULKOVO OBSERVATORY, ACADEMY OF SCIENCES, 10 KUTUZOV QUAY 196140 ST PETERSBURG, RUSSIA

Telephone: (7) 2982242

Email:

Facsimile: (7)

Telex:

Commission(s): 24

POULAKOS CONSTANTINE DR, RES CENTER FOR ASTRONOMY, ACADEMY OF ATHENS

14 ANAGNOSTOPOULOU ST, GR 10673 ATHENS, GREECE

Telephone: (30) 13613589

Facsimile: (30)

Email:

Commission(s):

Telex: 
POULLE EMMANUEL PROF, ECOLE NTLE DES CHARTES, 19 RUE DE LA SORBONNE, F 75005 PARIS, FRANCE Telephone: (33) 145894857

Facsimile: (33)

Telex:

Email:

Commission(s): 41

POUNDS KENNETH A PROF, DPT OF PHYSICS, UNIVERSITY OF LEICESTER, UNIVERSITY RD

LEICESTER LE1 7RH, UK

Telephonc: (44) $1533554455^{\star} 151$

Facsimile: (44)

Commission(s): 06,44

Telex: 341664 luxrayg

Email:

POUQUET ANNICK DR, OCA OBSERV DE NICE, BP 229, F 06304 NICE CDX 4, FRANCE
Tclephonc: (33) 92003057

Email: pouquet@rameau.obs-nicc.fr Commission(s): 34

POVEDA ARCADIO DR, INSTITUTO DE ASTRONOMIA, UNAM, APDO POSTAL 70-264, 04510 MEXICO DF, MEXICO

Telephone: (52) 5255505805

Facsimile: (52)

Telex: 1760155 eicme

Email:

Commission(s): $26,28,35,37$

POVEL HANSPETER, INSTITUT FUER ASTRONOMIE, ETH ZENTRUM, CH 8092 ZUERICH, SWITZERLAND

Telephone: (41) 16324222

Facsimile: (41) $12631205 \quad$ Telex:

Email: povel@astro.phys.ethz.ch

Commission(s): 09,12

POYET JEAN-PIERRE DR, OBS MIDI PYRENEES, 14 AVE E BELIN, F 31400 TOULOUSE CDX, FRANCE

Telephone: (33) 61252101

Facsimile: (33)

Telex:

Email:

Commission(s):

PRABHAKARAN NAYAR S R DR, DPT OF PHYSICS, UNIVERSITY OF KERALA, KARIYAVATTOM TRIVANDRUM 695 581, INDIA

Telcphone: (91) 471418920

Facsimile: (91)

Commission(s): 46

Telex:

Email:

PRABHU TUSHAR P, INDIAN INSTITUTE OF, ASTROPHYSICS, KORAMANGALA, BANGALORE 560 034, INDIA

Tclephone: (91) 803566585

Facsinilc: (91)

Commission(s): 28

Telex: 845763 iiah in

Email:

PRADERIE FRANCOISE DR, OBSERVATOIRE DE PARIS, SECTION DE MEUDON, DPT RECHERCHE SPATIALE F 92195 MEUDON PPL CDX, FRANCE

Telephone: (33) 145077651

Facsimile: (33)

Commission(s): 29,36

Telex: 204464

Email:

PRADHAN ANIL DR, DPT OF ASTRONOMY, OHIO STATE UNIVERSITY, 174 W 18TH AVE COLUMBUS OH 43210 1106, USA

Telephone: (1) 6142925850

Facsimile: (1) 6142922928

Telex: $7558+2$

Email: pradhan@ohstpy,bitnet

Commission(s): 14

PRANTZOS NIKOS DR, INSTITUT D'ASTROPHYSIQUE, 98BIS BD ARAGO, F 7501+ PARIS, FRANCE

Telephone: (33) 144328188

Facsimile: (33) 1 \$ 328001 Telex:

Email: nikos@friap51

Commission(s):

PRASAD SHEO S, LOCKHEED PALO ALTO RES LB, DPT 9120 BLDG 255, 3251 HANOVER ST PALO ALTO CA 94304, USA

Telephonc: (1) 4154242659

Facsimile: (1) 4154243333

Telex:

Email: prasad\%lparl1

Commission(s): 34

PRASANNA A R DR, PHYSICAL RESEARCH LAB, NAVRANGPURA, AHMEDABAD 380 009, INDIA

Telephone: (91) 272462129

Facsimile: (91) 272445292

Telex: 021-397 prl in

Email:

Commission(s): 44

PRATAP R DR, INST OF APPLIED SCIENCES, COCHIN 682 317, INDIA

Telephone: (91)

Facsimilc: (91)

Email:

Commission(s):

Telex:

PRAVDO STEVEN H, JPL, MS 168 222, 4800 OAK GROVE DR, PASADENA CA 91109, USA

Telephonc: (1) $8183544134 \quad$ Facsimile: (1)

Email:

Commission(s):

Telex: 910-588-3294 
PREDEANU IRINA DR, ASTRONOMICAL INSTITUTE, CUTITUL DE ARGINT 5, BOX 28

R 75212 BUCHAREST, RUMANIA

Telephone: (40) 16413686

Facsimile: (40) 13123391

Telex: 11882 astro $r$

Email: ipredeanu@imar.ro

Commission(s): 16

PREITE-MARTINEZ ANDREA DR, IAS, CNR, CP 67, I 00044 FRASCATI, ITALY

Telephone: (39) $69425655 \quad$ Facsimile: (39) $69416847 \quad$ Telex: 610261

Email: $\quad$ Commission(s): 34

PREKA-PAPADEMA P DR, DPT OF ASTROPHYSICS, NTL UNIVERSITY OF ATHENS, PANEPISTIMIOPOLIS GR 15783 ATHENS, GREECE

Telephone: (30) 17235122

Facsimile: (30)

Telex:

Email: spm75ograthun 1

Commission(s): 10

PRENDERGAST KEVIN H PROF, DPT OF ASTRONOMY, COLUMBIA UNIVERSITY, PUPIN HALL 538 W 120 TH ST NEW YORK NY 10027, USA

Telephone: (1) 2122803280

Facsimile: (1)

Telex:

Email:

Commission(s): 28

PRENTICE ANDREW J R DR, DPT OF MATHEMATICS, MONASH UNIVERSITY, WELLINGTON RD

CLAYTON VIC 3168, AUSTRALIA

Telephone: (61) $35+10811$

Facsimile: (61)

Telex:

Email:

Commission(s): 35

PRESS WILLIAM H DR, CENTER FOR ASTROPHYSICS, HCO/SAO, 60 GARDEN ST, CAMBRIDGE MA 02138, USA

Telcphonc: (1) $617+954908$

Facsimile: (1)

Commission(s): 28,47

Telex: $921+28$ satellite cam

Email:

PRESTON GEORGE W DR, CARNEGIE OBSERVATORIES, 813 SANTA BARBARA ST, PASADENA CA 91101, USA

Telephone: (1) 8185771122

Facsimile: (1) 8187958136

Telex: 1561318 ociw ut

Email:

Commission(s): 30,45

PRESTON ROBERT ARTHUR, JPL, MS 138307,4800 OAK GROVE DR, PASADENA CA 91 109, USA

Telephone: (1) $21335+6895 \quad$ Facsimile: (1)

Email:

Commission(s): 40

Telex: 675429

PREUSS EUGEN DR, MPI FUER RADIOASTRONOMIE, AUF DEM HUEGEL 69, D 53121 BONN, GERMANY

Telephone: (49) 2285251

Facsimile: (49)

Telex: 886440

Email:

Commission(s): 40,44

PREVOT LOUIS DR, OBSERVATOIRE DE MARSEILLE, 2 PLACE LE VERRIER, F 13248 MARSEILLE CDX 04, FRANC

Telephone: (33) 91959088

Facsimile: (33)

Telex: $\mathbf{4 2 0 2 4 1}$

Email:

Commission(s): 30

PREVOT-BURNICHON M L DR, OBSERVATOIRE DE MARSEILLE, 2 PLACE LE VERRIER, F 13248 MARSEILLE CDX O4, FRANCE

Telephone: (33) 91959088

Facsimile: (33)

Telex: $\mathbf{\$ 2 0 2}+1$

Email:

Commission(s): 28

PRIALNIK-KOVETZ DINA DR, DPT GEOPHYS \& PLANET SCI, TEL AVIV UNIVERSITY, TEL AVIV, ISRAEL

Telephone: (972) 35450633

Facsimile: (972)

Commission(s): 15,35

Telex: $\mathbf{3 4 2 1 7 1}$ versy il

Email: bitnet:b13@ataunos

PRICE MICHAEL J DR, SCIENCE APPLICATIONS, 5151 E BROADWAY, SUITE 1100, TUCSON AZ 85711, USA

Telephone: (1) 5207487400

Facsimile: (1)

Telex:

Email:

Commission(s):

PRICE R MARCUS DR, DPT PHYSICS \& ASTRONOMY, UNIVERSITY OF NEW MEXICO, 800 YALE BLVD NE ALBUQUERQUE NM 87131, USA

Telephonc: (1) 5052772616

Facsimile: (1)

Telex:

Email:

Commission(s): $33,34,40$

PRICE STEPHAN DONALD, 2 POLLEY RD, WESTFORD MA 01886, USA

Telephone: (1) 6178614552

Email:

Facsimile: (1)

Commission(s): th 
PRIEST ERIC R PROF, DPT OF APPLIED MATHS, UNIVERSITY OF ST ANDREWS, NORTH HAUGH ST ANDREWS FIFE KY16 9SS, UK

Telephone: (44) $133476161 * 8156$
Facsimile: (44) $133+7487$

Commission(s): 10,12
Telex: 76213 saulib

Email: eric(a)cs.st-andrews.ac.uk
PRIESTER WOLFGANG PRO
D S3121 BONN, GERMANY

Telephone: (49) 228733671

Email:

\author{
Facsimile: (49) Telex: 886440 \\ Commission(s): 33,40
}

PRIETO MERCEDES, INST DE ASTROFISICA, DE CANARIAS, OBS DEL TEIDE, E 38071 LA LAGUNA, SPAIN

Telephone: (34) $22262211 \quad$ Facsimile: (34)

Email:

Commission(s):

Telex: 92640

PRIEUR JEAN-LOUIS DR, OBS MIDI PYRENEES, 14 AVE E BELIN, F 31400 TOULOUSE CDX, FRANCE

Telephone: (33) 61332929

Facsimile: (33) 61536722

Telex: $\mathbf{5 3 0 7 7 6}$

Email: pricur at fromp51 bitnct

Commission(s): 28

PRINCE HELEN DODSON PROF, 4800 FILLMORE AVE, ALEXANDRIA VA 22311, USA

Telephone: (1) 7035781000

Facsimile: (1)

Telcx:

Email:

Commission(s):

PRINGLE JAMES E DR, INSTITUTE OF ASTRONOMY, THE OBSERVATORIES, MADINGLEY RD

CAMBRIDGE CB3 OHA, UK

Telephonc: (44) 1223337548

Email:

Facsimile: (4) 1223337523

Commission(s): 27,42

Telex: 817297 astron $g$

PRINJA RAMAN DR, DPT PHYSICS \& ASTRONOMY, UNIVERSITY COLLEGE LONDON, GOWER ST

LONDON WC1E 6BT, UK

Telephone: (44) 1713877050

Facsimile: (4t)

Telex: 28722

Email: bitnet:rkp(a)uk.ac.ucl.starlink

Commission(s): 29

PRITCHET CHRISTOPHER J DR, DPT OF PHYSICS, UNIVERSITY OF VICTORIA, BOX 1700 VICTORIA BC V8W 2Y2, CANADA

Telcphone: (1) 6047217704

Facsimile: (1) $6047217715 \quad$ Telex:

Email:

Commission(s): $09,28,37$

PROBSTEIN R F DR, DPT MECHANICAL ENGINEERG, MIT, BOX 165, CAMBRIDGE MA 02139, USA

Telephone: (1) $6172532240 \quad$ Facsimile: (1)

Telex: 921473 mit cam

Email:

Commission(s):

PROCHAZKA FRANZ V DR, INST F INTERDISZIPLINARE, FORSCHUNG \& FORTBILDUNG, STERNECKSTR 15 A 9010 KLAGENFURT, AUSTRIA

Telephone: (43) $4632700754 \quad$ Facsimile: $(43) 4632700759$ Telex:

Email:

Commission(s): 24,51

PRODAN Y I DR, STERNBERG STATE ASTR INST, UNIVERSITETSKIJ PROSP 13, 119899 MOSCOW, RUSSIA

Telephone: (7) 951395543

Facsimile: (7)

Telex:

Email:

Commission(s):

PROFFITT CHARLES R DR, GSFC, CODE 684.9, IUE OBSERVATORY, GREENBELT MD 20771, USA

Telephone: (1) 3012863608

Facsimile: (1) $30128676+2 \quad$ Telex:

Email: proffitt@iuesoc.gsfc.nasa.gov

Commission(s): 35

PROISY PAUL E DR, OBSERVATOIRE DE LYON, AVE CHARLES ANDRE, F 69561 S GENIS LAVAL CDX, FRANCE Telephone: (33) 78560705 Facsimile: (33) 72399791 Telex:

Email:

Commission(s): 15

PROKAKIS THEODORE J DR, ASTRONOMICAL INSTITUTE, NTL OBSERVATORY OF ATHENS, BOX 20048 GR 11810 ATHENS, GREECE

Telephone: (30) $13+6$ 1191/1 80+ 0619

Facsimile: (30)

Email:

Commission(s): 10,12,41

Telex: 215530 
PROKOF'EV VLADIMIR K PROF, CRIMEAN ASTROPHYS OBS, UKRAINIAN ACAD OF SCIENCE, NAUCHNY 334413 CRIMEA, UKRAINE

Telephone: (7) 432945

Facsimile: (7)

Telex:

Email:

Commission(s): 14,44

PROKOF'EVA IRINA A DR, PULKOVO OBSERVATORY, ACADEMY OF SCIENCES, 10 KUTUZOV QUAY 196140 ST PETERSBURG, RUSSIA

Telephone: (7)

Facsimile: (7)

Telex:

Email:

Commission(s):

PROKOF'EVA VALENTINA V DR, CRIMEAN ASTROPHYS OBS, UKRAINIAN ACAD OF SCIENCE, NAUCHNY 334413 CRIMEA, UKRAINE

Telephone: (7) 432945

Facsimile: (7)

Telex:

Email:

Commission(s): 09

PRONIK I I DR, CRIMEAN ASTROPHYS OBS, UKRAINIAN ACAD OF SCIENCE, NAUCHNY

334413 CRIMEA, UKRAINE

Telephone: (7) 432945

Facsimile: (7)

Telex:

Email:

Commission(s): 28,34

PRONIK V I DR, CRIMEAN ASTROPHYS OBS, UKRAINIAN ACAD OF SCIENCE, NAUCHNY

33\$413 CRIMEA, UKRAINE

Telephone: (7) $432945 \quad$ Facsimile: (7) Telex:

Email:

Commission(s): 28

PROSZYNSKI MIECZYSLAW, COPERNICUS ASTRON CENTER, POLISH ACAD OF SCIENCES, UL BARTYCKA 18 PL 00716 WARSAW, POLAND

Telephone: (48)

Facsimile: (48)

Commission(s): 44

Telex:

Email:

PROTHEROE RAYMOND J DR, DPT OF PHYSICS, UNIVERSITY OF ADELAIDE, BOX 498

ADELAIDE SA 5001, AUSTRALIA

Telephone: (61) 82285996

Facsimile: (61)

Commission(s): 44

Telex: $891+1$ univad aa

Email:

PROTHEROE WILLIAM M PROF, DPT OF ASTRONOMY, OHIO STATE UNIVERSITY, 174 W 18TH AVE, COLUMBUS OH 43210 1106, USA

Telephone: (1) 6144227891

Email:

Facsimile: (1)

Telex:

Commission(s):

PROTICH MILORAD B, ASTRONOMICAL OBSERVATORY, VOLGINA 7

YU 11050 BEOGRAD, YUGOSLAVIA-SERBIA \& MONTENEGRO

$\begin{array}{lll}\text { Telephone: (38) } 11+02365 & \text { Facsimile: (38) } & \text { Telex: } \\ \text { Email: } & \text { Commission(s): } 20\end{array}$

PROUST DOMINIQUE, OBSERVATOIRE DE PARIS, SECTION DE MEUDON, DAEC

F 92195 MEUDON PPL CDX, FRANCE

Telephone: (33) 145077411

Facsimilc: (33) 145077469

Telex: $631987 \mathrm{lam}$

Email: proust/afrmeu51/mesioa::proust

Commission(s): 28

PROVERBIO EDOARDO PROF, ISTITUTO DI ASTRONOMIA, VIA OSPEDALE 72, 1 09100 CAGLIARI, ITALY

Telephone: (39) 70663544

Facsimile: (39) 70657657 Telex:

Email: proverbio@astrca.astro.it

Commission(s): $08,19,31 \mathrm{C}, 46,41$

PROVOST JANINE DR, OCA OBSERV DE NICE, BP 229, F 0630 4 NICE CDX $\downarrow$, FRANCE

Telephone: (33) $93890+20$

Facsimile: (33) 92003033

Telex: 460004 obsnice f

Email:

Commission(s): 27,35

PRUSTI TIMO, ASTROPHYSICS DIV, ESTEC ESA, POSTBUS 299, NL 2200 AG NOORDWIJK, NETHERLANDS

Telephone: (31) 171985669

Facsimile: (31) 171985434

Telex: 39098

Email: tprusti@iso.estec.esa.n!

Commission(s):

PRYCE MAURICE H L DR, DPT OF PHYSICS, UNIV OF BRITISH COLUMBIA, 2075 WESBROOK PL

VANCOUVER BC VGT 1W5, CANADA

Tclephonc: (1) $60+2286+17$

Facsimile: (1) $60+2285324$

Telex: $045(88576$

Email:

Commission(s): 
PRYOR CARLTON PHILIP DR, DPT PHYSICS \& ASTRONOMY, RUTGERS UNIVERSITY, BOX 849 PISCATAWAY NJ 08854 0849, USA

Telephone: (1) 9089325462

Facsimile: (1) $9089324343 \quad$ Telex:

Email: pryor@pryor.rutgers.edu

Commission(s):

PSKOVSKIJ JU P DR, STERNBERG STATE ASTR INST, UNIVERSITETSKIJ PROSP 13, 119899 MOSCOW, RUSSIA Telephone: (7) 951393721

Facsimile: (7)

Telex:

Email:

Commission(s): 27,3†

PUCHE DANIEL, CENTRE FOR ASTROPHYSICS, 60 GARDEN ST, CAMBRIDGE MA 02138, USA

Telephone: (1) 6174957344

Facsimile: (1) $617+957014 \quad$ Telex:

Email: dpuche@gefa.harvard.cdu

Commission(s):

PUCILLO MAURO DR, OAT, BOX SUCC TRIESTE 5, VIA TIEPOLO 11, I 3+131 TRIESTE, ITALY

Telephone: (39) 40793921

Facsimile: (39)

Telex: 461137 oat $i$

Email:

Commission(s): 05,09

PUETTER RICHARD C DR, CASS, UCSD, C 011, LA JOLLA CA 92093 0216, USA

Telephone: (1) 6195344995 Facsimile: (1) Telex:

Email:

Commission(s):

PUGACH ALEXANDER F DR, MAIN ASTRONOMICAL OBS, UKRAINIAN ACAD OF SCIENCE, GOLOSEEVO 252127 KIEV, UKRAINE

Telephone: (7) 044664771

Email:

Facsimile: (7)

Telex: 131406 sky su

PUGET JEAN-LOUP DR, RADIOASTRONOMIE ENS, 24 RUE LHOMOND, F 75231 PARIS CDX 05, FRANCE

Telephone: (33) 143291225

Facsimile: (33)

Commission(s): $3 \downarrow, 47$

Telex: 270912

Email:

PUGLIANO ANTONIO PROF, IST UNIVERSITARIO NAVALE, VIA ACTON 38, I 80133 NAPOLI, ITALY

Telephone: (39) 815512330

Facsimile: (39) 815521485

Telex: $710+17$

Email:

Commission(s): 08

PULS JOAHIM DR, INST ASTRON \& ASTROPHYSIK, UNIVERSITAETS STERNWARTE, SCHEINERSTR 1, D 81679 MUENCHEN, GERMANY

Telephone: (49) $8992209+36$

Facsimile: (49) $8992209427 \quad$ Telex:

Email: uh101aw@dm01rz01/carn

Commission(s): 36

· PUNETHA LALIT MOHAN DR, UTTAR PRADESH STATE, OBSERVATORY, PO MANORA PEAK 263129

NAINITAL 263 129, INDIA

Telephone: (91) 59422136

Facsimile: (91)

Telex:

Email:

Commission(s):

PURCELL EDWARD M PROF, DPT OF PHYSICS, HARVARD UNIVERSITY, 60 GARDEN ST

CAMBRIDGE MA 02138, USA

Telephone: (1) 6174952860

Facsimile: (1)

Telex:

Email:

Commission(s): $\mathbf{5 1}$

PURTON CHRISTOPHER R DR, DOMINION RADIO ASTROPHYS, OBSERVATORY, BOX 248

PENTICTON BC V2A 6K3, CANADA

Telephone: (1)

Facsimile: (1)

Telex:

Email:

Commission(s):

PUSCHELL JEFFERY JOHN, MARTIN MARIETTA, 103 CHESAPEAKE PARK PLAZA, E460

BALTIMORE MD 21220, USA

Telcphone: (1) 3016820885

Facsimile: (1)

Telex: 908225

Email:

Commission(s): 40

PUSHKIN SERGEY B DR, TIME \& FREQUENCY SERVICE, GOSSTANDARD USSR, 117049 MOSCOW, RUSSIA

Telephone: (7)

Facsimile: (7)

Telex:

Email:

Commission(s): 31 
PUSTYL'NIK IZOLD B DR, TARTU ASTROPHYSICAL OBS, ESTONIAN ACAD OF SCIENCES

EE 202444 TARTU, ESTONIA

Telephone: (372) $33 \mathbf{4 3 9}$

Facsimile: (372)

Commission(s): $\mathbf{4 1 , 4 2}$

Telex:

Email:

PYATUNINA TAMARA, INST OF APPLIED ASTRONOMY, 8 ZHDANOVSKAYA ST, ST PETERSBURG 197042, RUSSIA

Telephone: (7) 8122307414

Facsimile: (7) $8122307+13$

Telex: 121391 ipa su

Email: iparan(a)sovam.com

Commission(s):

PYE JOHN P DR, DPT OF PHYSICS, UNIVERSITY OF LEICESTER, UNIVERSITY RD, LEICESTER LE1 7RH, UK

Telephone: (44) $1162554455{ }^{\star} 23 \quad$ Facsimile: (4)

Email:

Commission(s):

Telex: 341664 luxray $g$

PYPER SMITH DIANE M DR, DPT OF PHYSICS, UNIVERSITY OF NEVADA, 4505 S MARYLAND PARKWAY

LAS VEGAS NV 89154, USA

Telephone: (1)

Facsimile: (1)

Telex:

Email:

Commission(s):

QI GUAN RONG, SHANNKI OBSERVATORY, CAS, LINTONG XIAN, SHAANXI, CHINA PR

Telephone: (86) 332255 Facsimile: (86) Telex: 70121 csao cn

Email:

Commission(s): 08,31C

QLAN BO-CHEN, SHANGHAI OBSERVATORY, CAS, 80 NANDAN RD, SHANGHAI 200030, CHINA PR

Telephone: (86) $21386191 \quad$ Facsimile: (86)

Telex: 33164 shao cn

Email:

Commission(s): 37

QIAN SHAN-JIE, BEIJING ASTRONOMICAL OBS, CAS, W SUBURB, BEIJING 100080, CHINA PR

Telephone: (86) $1282194 \quad$ Facsimilc: (86)

Email:

Commission(s): 40

Telex: 22040 baoas en

QIAN ZHI-HAN DR, SHANGHAI OBSERVATORY, CAS, 80 NANDAN RD, SHANGHAI 200030, CHINA PR

Telephone: (86) $21386191 \quad$ Facsimile: (86)

Telex: $3316+$ shao $\mathrm{cn}$

Email:

Commission(s): 08

QIAO GUOJUN, DPT OF GEOPHYSICS, BEIJING UNIVERSITY, BEIJING 100871, CHINA PR

Telephene: (86)

Facsimile: (86) 12564095

Telex: 22239 pkuni cn

Email:

Commission(s): $\$ 2$

QIN DAO, PURPLE MOUNTAIN OBSERV, CAS, NANJING, CHINA PR

Telephone: (86) 2546700

Email:

Facsimile: (86) 25301459

Telex: 34144 pmonj cn

QIN SONG-NIAN, YUNNAN OBSERVATORY, CAS, BOX 110, KUNMING 72946 YUNNAN, CHINA PR

Telephonc: (86) 8712035

Facsimile: (86)

Telex:

Email:

Commission(s):

QIN ZHI-HAI, DPT OF ASTRONOMY, NANJING UNIVERSITY, NANJING, CHINA PR

Telephone: (86) $2534651 * 2882 \quad$ Facsimile: (86) Telex: 0909

Email:

Commission(s): 34

QIU PUZHANG ASS PROF, YUNNAN OBSERVATORY, CAS, BOX 110, KUNMING 72946 YUNNAN, CHINA PR

Telephone: (86) 8712035

Facsimile: (86)

Telex: $6+1040$ yuobs cn

Email:

Commission(s): 09,51

QIU Y.U-HAI, BEIJING ASTRONOMICAL OBS, CAS, W SUBURB, BEIJING 100080, CHINA PR

Telephone: (86) 1281698

Facsimilc: (86)

Telex: 22040 baoas cn

Email:

Commission(s): 40

QU QIN-YUE, DPT OF ASTRONOMY, NANJING UNIVERSITY, NANJING, CHINA PR

Telephone: (86) $25637551 * 3186 \quad$ Facsimile: (86) $25302728 \quad$ Telex: 34151 prenu cn

Email:

Commission(s): $35,47,44$

QUAMAR JAWAID, C 6 STAFF TOWN, UNIVERSITY OF KARACHI, KARACHI 75270, PAKISTAN

Telephone: $(92)+65+91$

Facsimile: (92)

Telex:

Email: bartel@s.sgl.ists.ca

Commission(s): 46 
M 770

QUAN HEJUN, SHANGHAI OBSERVATORY, CAS, 80 NANDAN RD, SHANGHAI 200030, CHINA PR

Telephone: (86) $21386191 \quad$ Facsimile: (86)

Commission(s): 41

Telex: $3316+$ shao en

Email:

QUARTA MARIA LUCIA, IAG, UNIVERSIDADE DE SAO PAULO, CP 9638, 01065 SAO PAULO SP, BRAZIL
Telephone: (55) 115778599

Email:

Commission(s):

QUAST GERMANO RODRIGO, OBSERVATORIO NACIONAL, RUA COLONEL RENNO O7, CP 21 37500 ITAJUBA MG, BRAZIL

Telephone: (55) 356220788

Facsimile: (55)

Email:

Commission(s):

Telex: 0312603

QUENBY JOHN J DR, ASTROPHYSICS GROUP, IMPERIAL COLLEGE, BLACKETT LABORATORY

LONDON SW' 2BZ, UK

Telephone: (44) $1715895111 * 6661 \quad$ Facsimile: $(44) \quad$ Telex: 261503

Email:

Commission(s):

QUERCI FRANCOIS R DR, OBS MIDI PYRENEES, 14 AVE E BELIN, F 31400 TOULOUSE CDX, FRANCE

Telephone: (33) 61332929

Facsimile: (33) 61536722

Telex: $\mathbf{5 3 0 7 7 6}$ f obstlse

Email: frompsi

Commission(s): $14,29,36$

QUERCI MONIQUE DR, OBS MIDI PYRENEES, 14 AVE E BELIN, F $31+10$ TOULOUSE CDX, FRANCE

Telephone: (33) 61332929

Facsimilc: (33) 61536722

Telex: 530776 f obstlse

Email: fromp51

Commission(s): 29,36

QUESADA VINICIO DR, STAZIONE ASTRONOMICIA, VIA OSPEDALE 72, I 09124 CAGLIARI, ITALY

Telephone: (39) 70663544

Facsimile: (39) 70657657 Telex:

Email: vaxca2::quesada

Commission(s): 22

QUINN PETER DR, MOUNT STROMLO \& SIDING, SPRING OBSERVATORIES, PRIVATE BAG WESTON CREEK PO ACT 2611, AUSTRALIA

Telephonc: (61) 62490272

Email: pjq@ominuet.anu.0z.au

Telex:

Commission(s): 28

QUINTANA HERNAN DR, GRUPO ASTROFIS PONTIFICA, UNIVERSIDAD CATOLICA, CASILLA IO4 SANTIAGO 22, CHILE

Telephone: (56) 2775474

Email; hquintana(a)astrouc.puc.cl

Facsimile: (56) 25525692

Telex: $2+0395$

Commission(s): $05,28,31), 4+, 51$

QUINTANA JOSE M DR, INST ASTROFISICA, DE ANDALUCIA APD 300 4 , PROFESOR ALBAREDA 1

E 18080 GRANADA, SPAIN

Telephone: (34) 58121300

Email:

Facsimile: (34)

Commission(s): 51

Telex: 78573 ialag e

QUIRK WILLIAM J DR, LAWRENCE LIVERMORE LAB, L 35, BOX 808, LIVERMORE CA 94550, USA

Telephone: (1) 4154221852

Facsimile: (1)

Telex:

Email:

Commission(s):

RAADU MICHAEL A DR, DPT OF PLASMA PHYSICS, ROYAL INST OF TECHNOLOGY S 10044 STOCKHOLM 70, SWEDEN

Telephone: (46) 87877000

Facsimile: $(46)$

Telex: 10389 kthb stockholm

Email:

Commission(s): 10,49

RABBIA YVES DR, OCA CERGA, AVE COPERNIC, F 06130 GRASSE, FRANCE

Telephone: (33) $93365849 \quad$ Facsimile: (33)

Email: carn::yvrabb(afroni51

Commission(s):

Telex: 470865 cerga

RABIN DOUGLAS MARK, NTL SOLAR OBSERVATORY, BOX 26732, 950 N CHERRY AVE, TUCSON AZ 85726 6732, US

Telephone: (1) 5203259331

Facsimile: (1) $5203259360 \quad$ Telex: 0666484 aura noatuc

Email: rabin(a)noao.edu

Commission(s): 10,12

RABOLLI MONICA DR, OBSERVATORIO ASTRONOMICO, PASEO DEL BOSQUE S/N 1900 LA PLATA (BS AS), ARGENTINA

Telephone: (54) 21217308

Facsimile: (54) 17868114

Telex:

Email: mrabolli\%psi\#iafe\%ssl.span

Commission(s): 33 
RACHKOVSKY D N DR, CRIMEAN ASTROPHYS OBS, UKRAINIAN ACAD OF SCIENCE, NAUCHNY 33+413 CRIMEA, UKRAINE

Telephone: (7) 432945

Facsimile: (7)

Telex: 192

Email:

Commission(s): 36

RACINE RENE DR, DPT DE PHYSIQUE, UNIVERSITE DE MONTREAL, CP 6128 SUCC A MONTREAL QC H3C 3J7, CANADA

Telcphone: (1) 5143436718

Email:
Facsimile: (1) $51+3432071$

Commission(s): 09
Telex: 05561359 rzlpnum ml

RADHAKRISHNAN V PROF, RAMAN RESEARCH INSTITUTE, SADASHIVANAGAR, CV RAMAN AVE BANGALORE 560 080, INDIA

Tclephone: (91) 803340522

Facsimile: (91) $8033+0492$

Telex: 8452671 rri in

Email: rad(ärri.ernet.in

Commission(s): $34,40,44$

RADICK RICHARD R DR, AIR FORCE GEOPHYSICS LAB, NTL SOLAR OBSERVATORY, SUNSPOT NM 88349, USA Telephone: (1) 505 43+ 1390

Facsimile: (1)

Telex:

Email:

Commission(s): 12

RADIMAN IRATIUS, BOSSCHA OBSERVATORY, LEMBANG +(0391, INDONESIA

Telephone: (62) $2296001 \quad$ Facsimile: (62) Telex:

Email:

Commission(s):

RADOSKI HENRY R DR, AFOSR/NP, BUILDING 410, BOLLING AIR FORCE BASE, WASHINGTON DC 20332, USA

Telephone: (1) $202767+906$

Facsimile: (1)

Telex:

Email:

Commission(s): 21

RADU EUGENIA, ASTRONOMICAL OBSERVATORY, STR CIRESILOR 19, R 3400 CLUJ NAPOCA, RUMANIA

Telephone: (40) 64194592

Facsimile: (40) $6419+592$

Telex:

Email:

Commission(s):

RAEDLER K H DR, ZNTRLINST F ASTROPHYSIK, STERNWARTE BABELSBERG, ROSA LUXEMBURG STR 17A

D 14473 POTSDAM, GERMANY

Telephone: (49)

Facsimile: (49)

Telex:

Email:

Commission(s): 35

RAFANELLI PIERO DR, OSS ASTRONOMICO DI PADOVA, VIC DELL OSSERVATORIO 5, I 35122 PADOVA, ITALY

Telephone: (39) 49661499

Facsimile: (39)

Telex:

Email:

Commission(s): 28

RAFERT JAMES BRUCE, DPT PHYSICS \& SPACE SCI, FLORIDA INST TECHNOLOGY, 150 W UNIVERSITY BLVD MELBOURNE FL 32901, USA

Telephone: (1)

Facsimile: (1)

Telex:

Email:

Commission(s): 42

RAFFERTY THEODORE J, US NAVAL OBSERVATORY, ASTROMETRY DPT, 3450 MASSACHUSETTS AVE NW WASHINGTON DC 203925400 , USA

Telephone: (1) 2026531511

Email: tjrasicon.usno.navy.mil

Facsimile: (1) 2026531497

Telex:

Commission(s): $08,2+$

RAGHAVAN NIRUPAMA DR, IIT, 6 WEST AVE, NEW DELHI 110016 , INDIA

Telephone: (91)

Facsimile: (91)

Email:

Commission(s):

Telex:

RAHARTO MOEDJI, DPT OF ASTRONOMY, BANDUNG INSTITUTE OF TECH, JL GANESHA 10

BANDUNG 40132, INDONESIA

Telephone: (62) $6222+40252$

Email:

Facsimile: (62) $6222+38388$

Telex: $2832+$ bd

RAHE JURGEN PROF, NASA HEADQUARTERS, CODE EL, 600 INDEPENDENCE AVE SW

WASHINGTON DC 20546, USA

Telephone: (1) 2024531590

Facsimile: (1) $202+261023$

Telex: $\mathbf{4 9 7 4 8 4 3}$

Email:

Commission(s): $15,16,42,44$ 
RAHUNEN TIMO, TAMPERE SAERKAENNIEMI OY, SAERKAENNIEMI, SF $33+10$ TAMPERE, FINLAND Telephone: (358) 3131333 Facsimile: (358) Telex:

Email:

Commission(s): 42

RAIKOVA DONKA DR, DPT OF ASTRONOMY, BULGARIAN ACAD SCIENCES, 7TH NOVEMBER ST 1 BG 1000 SOFIA, BULGARIA

Telephonc: (359) 27341

Email:

Facsimile: (359) 23561 ECFBAN BG Telex: 23561 ecf ban bg Commission(s):

RAIMOND ERNST DR, NFRA, BOX 2, NL 7990 AA DWINGELOO, NETHERLANDS

Telephonc: (31) 52197244

Facsimile: (31) 52197332

Telex:

Email: exranfra.nl

Commission(s): $05 C, 08,34,40$

RAINE DEREK J DR, DPT OF ASTRONOMY, UNIVERSITY OF LEICESTER, UNIVERSITY RD LEICESTER LE1 7RH, UK

Telephone: (44) 1162554455

Email:

Facsimile: $(4 t)$

Telex: $3 \$ 1198$ Ieicul

RAITALA JOUKO T, DPT OF ASTRONOMY, UNIVERSITY OF OULU, SF 90570 OULU 57, FINLAND

Telephone: (358) 81352106

Facsimile: (358) 81561278

Tclex: 32375

Email:

Commission(s):

RAJAMOHAN R DR, INDLAN INSTITUTE OF, ASTROPHYSICS, KORAMANGALA, BANGALORE 560 034, INDIA

Telephone: (91) 80356 6497/6585 Facsimile: (91)

Telex: 845763 iiab in

Email:

Commission(s): 20,51

RAJCHL JAROSLAV DR, ASTRONOMICAL INSTITUTE, CZECH ACADEMY OF SCIENCES ONDREJOV OBSERVATORY, CZ 25165 ONDREJOV, CZECH R

Telephone: (42) 204 857245/7111 Facsimile: (42) 204851611 Telex: 121579

Emait: astmph(a)csearn Commission(s): 22

RAJU P K DR, INDIAN INSTITUTE OF, ASTROPHYSICS, KORAMANGALA, BANGALORE 560 034, INDIA

Telephonc: (91) $803566585 \quad$ Facsimile: (91)

Telex: $8+5763$ iiab in

Email:

Commission(s):

RAKAVY GIDEON PROF, EINSTEIN INST OF PHYSICS, HEBREW UNIV OF JERUSALEM, JERUSALEM 91904, ISRAE

Telephone: (972)

Facsimile: (972)

Telex:

Email:

Comımission(s):

RAKOS KARL D PROF, INSTITUT FUER ASTRONOMIE, UNIVERSITAET WIEN, TUERKENSCHANZSTR 17

A 1180 WIEN, AUSTRIA

Telephone: (43) 134536095

Email:

Facsimile: $(+3)$

Telex: 133099 viast a

RAKSHIT H PROF, BENGAL ENGINEERG COLLEGE, SIBPORE, HEWRAH, INDIA

Telephone: $(91)$

Commission(s): $(09,26,27,42$

Email:

Facsimile: (91)

Telex:

RAM SAGAR DR, INDIAN INSTITUTE OF, ASTROPHYSICS, KORAMANGALA, BANGALORE 560 034, INDIA

Telephone: (91) 80356 6585/6497 Facsimile: (91)

Email: sagar(a)iap.ernct.in

Telex: $8+5763$ iiab in

RAMADURAI SOURIRAJA DR, TIFR, HOMI BHABHA RD, COLABA, BOMBAY 400005 , INDIA

Telephone: (91) 224952311

Facsimile: (91)

Telex: 0113009 tifr in

Email: shakti!tifr!tap15 a uunet.uu.net

Commission(s): $35,44,46$

RAMAMURTHY SWAMINATHAN, CASA, UNIVERSITY OF OSMANIA, HYDERABAD 500 007, INDIA

Telephone: (91) 851672

Facsimile: (91)

Telex:

Email:

Commission(s):

RAMANA MURTHY P V DR, TIFR, HOMI BHABHA RD, COLABA, BOMBAY 400 005, INDIA

Telephone: (91) 224952979

Facsimile: (91)

Telex: 0113009 tifr in

Email: ra1mana(a)tifrvax.bitnet

Commission(s): 
RAMATY REUVEN DR, NASA GSFC, CODE 665, LAB HIGH ENERGY ASTROPHYS, GREENBELT MD 20771, USA

Telephone: (1) 3012868715

Facsimile: (1)

Telex:

Email:

Commission(s): 40

RAMELLA MASSIMO, OAT, BOX SUCC TRIESTE 5, VIA TIEPOLO 11, I 34131 TRIESTE, ITALY

Telcphone: (39) 40768506

Facsinile: (39)

Commission(s): 47

Telex: 461137 oat $i$

Email:

RAMOS ISABEL FERRO DR, INST GEOPHYS \& ASTRONOMY, CALLE 212 N 2906/29 Y 31, LISA, LA HABANA, CUBA

Telephone: (53) 21 8416/0644

Facsimilc: (53)

Commission(s):

Telex: 511240 geoas cu

Email:

RAMPAZZO ROBERTO DR, OSS ASTRONOMICO DI BRERA, VIA BRERA 28, I 2012I MILANO, ITALY

Telephone: (39) 2874444

Facsimile: (39) 272001600

Telex:

Email: rampazzo@astmib.astro.it

Commission(s): 28

RAMSEY LAWRENCE W DR, DPT OF ASTRONOMY, PENNSYLVANIA STATE UNIV, 525 DAVEY LAB UNIVERSITY PARK PA 16802, USA

Telephone: (1) 8148650418

Facsimile: (1)

Telex:

Email:

Commission(s): 09,36

RANA NARAYAN CHANDRA DR, IUCAA, POST BAG 4, GANESHKHIND PUNE 411 007, INDIA

Telephone: (91) 2012336415

Facsimile: (91) 2012335760

Telex:

Emaik: ncrana(a)iucaa.ernet.in

Commission(s): 28,33

RANDIC LEO PROF DR, GEODETICAL FACULTY, GUNDULICEVA 54, ZAGREB, CROATIA

Telephone: (38) 41446675

Facsimile: (38)

Telex:

Email:

Commission(s): 19,31

RANGARAJAN K E, INDIAN INSTITUTE OF, ASTROPHYSICS, KORAMANGALA, BANGALORE 560 034, INDLA

Telephone: (91) 805530672

Facsimile: (91) 805534043

Telex:

Email: rangaraj@iiap.ernet.in

Commission(s): 36

RANIERI MARCELLO, IAS, CNR, CP 67, I 00044 FRASCATI, ITALY

Telephonc: (39) 69425655

Facsimile: (39) $694168+7$

Telex:

Email:

Commission(s):

RANK DAVID M PROF, LICK OBSERVATORY, UNIVERSITY OF CALIFORNIA, SANTA CRUZ CA 95064, USA

Telephone: (1) 4084292277

Facsimile: (1)

Telex:

Email:

Commission(s):

RANKIN JOANNA M DR, PHYSICS DPT, UNIVERSITY OF VERMONT, A 405 COOK BUILDING BURLINGTON VT 05405, USA

Telephone: (1) $8026562644 \quad$ Facsimile: (1)

Telex: 5102990021

Email: rankin\%merlin.uvm-gen.uvm.edu Commission(s):

RAO A PRAMESH DR, TATA INST OF FUNDAMENTAL, RESEARCH, POONA UNIVERSITY CAMPUS

PUNE 411 007, INDIA

Telephone: (91) 212337107

Facsimile: (91) 212335760

Commission(s): 10,40

Telex: 0145658 gmrt in

Email: uunet!shakti!gmrt!pramesh

RAO ARIKKALA RAGHURAMA, SPACE PHYSICS GROUP, TIFR, BOMBAY 400 005, INDIA

Telephonc: (91) 222152971

Facsimile: (91) 222152110

Telex: 01183009 tifr in

Email: arrao@tifrvax.tifr.res.in

Commission(s): 4

RAO K NARAHARI, DPT OF PHYSICS, OHIO STATE UNIVERSITY, 174 W 18TH AVE, COLUMBUS OH 43210, USA

Telephone: (1) 6144226505

Facsimile: (1)

Telex:

Email:

Commission(s): 14

RAO K RAMANUJA DR, C/O DR K SURENDRA, RUA CEL JOAO CURSINO 210, APT 92 VILA ADYANA 12200 S JOSE DOS CAMPOS, BRAZIL

Telephone: (55)

Facsimile: (55)

Telex:

Email:

Commission(s): 
RAO M N DR, PHYSICAL RESEARCH LAB, NAVRANGPURA, AHMEDABAD 380 O09, INDIA

Telephone: (91) 272462129

Facsimile: (91) $272+\downarrow 5292$

Telex: 121397

Email:

Commission(s): 16

RAO N KAMESWARA, INDIAN INSTITUTE OF, ASTROPHYSICS, KORAMANGALA, BANGALORE 560 034, INDIA Telephone: (91) 803566585 Facsimile: (91)

Telex:

Email:

Commission(s): $27,29 \mathrm{C}$

RAO P VIVEKANANDA DR, DPT OF ASTRONOMY, UNIVERSITY OF OSMANIA, HYDERABAD 500 007, INDIA

Telephone: (91) 71951

Facsimile: (91)

Telex:

Email:

Commission(s): 25

RAO RAMACHANDRA V PROF, ISRO SATELLITE CENTER, PEENYA, BANGALORE 560 058, INDIA

Telephone: (91)

Facsimile: (91)

Telex:

Email:

Commission(s): 44

RAOULT ANTOINETTE DR, OBSERVATOIRE DE PARIS, SECTION DE MEUDON, DASOP, F 92195 MEUDON PPL CDX FRANCE

Telephone: (33) 145077766

Facsimile: (33)

Telex: 200590

Email:

Commission(s): 10,12,40

RAPAPORT MICHEL DR, OBSERVATOIRE DE BORDEAUX, BP 89, F 33270 FLOIRAC, FRANCE

Telephone: (33) 56864330

Facsimile: (33) $56+0+251$

Telex:

Email:

Commission(s): 20

RAPLEY CHRISTOPHER G DR, MULLARD SPACE SCIENCE LAB, UNIVERSITY COLLEGE LONDON

HOLMBURY ST MARY, DORKING SURREY RH5 GNT, UK

Telephone: (44) $130670292 \quad$ Facsimile: (44)

Telex: 859185

Email:

Commission(s):

¿RATAG MEZAK ARNOLD, LAPAN, NTL INST AERONAUTICS \& SP, UL JUNJUNAN 133, BANDUNG 40173, INDONESI

Telephone: (62)

Facsimile: (62)

Telex:

Email:

Commission(s):

RATNATUNGA KAVAN U, DPT PHYSICS \& ASTRONOMY, JOHNS HOPKINS UNIVERSITY, BLOOMBERG CT SAN MART DR, BALTIMORE MD 21218 2695, USA

Telephone: (1) $4105164553 \quad$ Facsimile: (1) 4105168260

Email: kavan(a)Jmds.pha.jhu.cdu

Commission(s): $05,30,33$

Telex: 089675 nascom ghlt

RAUBENHEIMER BAREND C PR, COSMIC RAY RESEARCH UNIT, POTCHEFSTROOM UNIVERSITY

POTCHEFSTROOM 2520, SOUTH AFRICA

$\begin{array}{lll}\text { Telephone: (27) } 148127511 & \text { Facsimile: (27) } & \text { Telex: } \mathbf{2 1 3 6 3} \\ \text { Email: } & \text { Commission(s): } 44 & \end{array}$

RAUTELA B S DR, UTTAR PRADESH STATE, OBSERVATORY, PO MANORA PEAK 263 129, NAINITAL 263 129, INDIA

Telephone: (91) 59422136

Facsimile: (91)

Telex:

Email:

Commission(s): 29,45

- RAVEENDRAN A V, INDIAN INSTITUTE OF, ASTROPHYSICS, KORAMANGALA, BANGALORE 560 034, INDIA Telcphone: (91) 805530672

Email: avn a iiap.ernet.in Facsimile: (91) $80553+043$ Telex: 8452763 iiab in

RAWLINGS JONATHAN DR, DPT OF ASTROPHYSICS, UNIVERSITY OF OXFORD, KEBLE RD, OXFORD OXI 3RH, U Telephone: (44) 133865273292

Email: jr@uk.ac.ox.astro Facsimile: (4) $1865273+18$

Telex: 83295 nuclox g

RAWLINGS STEVEN DR, DPT OF ASTROPHYSICS, UNIVERSITY OF OXFORD, KEBLE RD, OXFORD OX1 3RH, UK Telephone: (44) 1865273303

Email: sr(a)uk.ac.ox.astro Facsimile: (44) $1865273+18$

Commission(s): 47

Telex: 83295 nuclox $g$

RAY ALAK DR, TIFR, HOMI BHABHA RD, COLABA, BOMBAY 400 005, INDIA

Telephone: (91) 222152971

Facsimile: (91) 222152110

Telex: 1183009 tifr in

Email: akr(a)tifrvax

Commission(s): 35 
RAY CHOUDHURI ARNAB DR, DPT OF PHYSICS, INDIAN INSTITUTE SCIENCES, BANGALORE 560 012, INDIA

Telephone: (91) 803344411

Facsimile: (91)

Telex:

Email:

Commission(s): 10

RAY JAMES R, NOAA N/OES13 SSMC4, 1305 EAST WEST HWY, SILVER SPRING MD 20910, USA

Telephone: (1) 3017132850

Facsimile: (1) $3017134475 \quad$ Telex:

Email: jimr@ray.grdl.noaa.gov

Commission(s): 19,31

RAY THOMAS P, DIAS, SCHOOL OF COSMIC PHYSICS, 5 MERRION SQ, DUBLIN 2, IRELAND

Telephone: (353) 1774321

Facsimile: (353)

Telex: 31687 dias ei

Email:

Commission(s): 40

RAYCHAUDHURI AMALKUMAR DR, PRESIDENCY COLlEGE, COLlEgE ST, CALCUTTA 73, INDIA

Telephone: (91)

Facsimile: (91)

Telex:

Email:

Commission(s): 47

RAYET MARC, ASTROPHYSICS INSTITUTE, UNIV LIBRE DE BRUXELLES, CAMPUS PLAINE CP 226

B 1050 BRUSSELS, BELGIUM

Telephone: (32) 26503572

Facsimile: (32) $26504226 \quad$ Telex:

Email: mrayet $\hat{a}$ astro.ulb.ac.be

Commission(s): 35

RAYMOND JOHN CHARLES, CFA, HCO/SAO, 60 GARDEN ST, CAMBRIDGE MA 02138, USA

Telephone: (1)

Facsimile: (1)

Telex:

Email:

Commission(s): 34

RAYROLE JEAN R DR, OBSERVATOIRE DE PARIS, SECTION DE MEUDON, F 92195 MEUDON PPL CDX, FRANCE Telephone: (33) 145077789

Facsimile: (33)

Telex:

Email:

Commission(s): 10

RAZDAN HIRALAL, TECHNICAL COORDINATION GP, BHABHA ATOMIC RESEARCH CT

TF CNTRL COMPLEX TROMBAY, BOMBAY 400 085, INDIA

Telephone: (91) 225511859

Facsimile: (91) 225560750

Telex: 01171017 in

Email:

Commission(s): 44

RAZIN V A DR, RADIOPHYSICAL RESEARCH, INSTITUTE, LYADOV UL 25/14, 603600 N NOVGOROD, RUSSIA

Telephone: (7) 367294

Facsimile: (7)

Commission(s): 40

Telex:

Email:

READHEAD ANTHONY C S DR, CALTECH, ROBINSON BLDG, PASADENA CA 91125, USA

Telephone: (1) 2133564972

Facsimile: (1)

Telex: 675425 caltech psd

Email:

Commission(s): 40,49

REAMES DONALD V, NASA GSFC, CODE 661, GREENBELT MD 20771, USA

Telephone: (1) 3012866454

Facsimile: (1) $3012861682 \quad$ Telex:

Email: reames(a)lheavx.gsfc.nasa.gov

Commission(s):

REASENBERG ROBERT D DR, CENTER FOR ASTROPHYSICS, HCO/SAO RM B 217, 60 GARDEN ST CAMBRIDGE MA 02138, USA

Telephone: (1) 6174957108

Facsimile: (1)

Telex: 921428 satelite cam

Email:

Commission(s): 04

REAVES GIBSON PROF, DPT OF ASTRONOMY, UNIV SOUTHERN CALIFORNIA, LOS ANGELES CA 90089 1342, USA

Telephonc: (1) 2137432039

Facsimile: (1)

Telex:

Email:

Commission(s): 28,41

REAY NEWRICK K DR, ASTROPHYSICS GROUP, IMPERIAL COLLEGE, BLACKETT LABORATROY

LONDON SW7 2BZ, UK

Telephone: (H) $1715895111 * 6669 \quad$ Facsimile: (44)

Email:

Commission(s): $09,+9,51$

Telex: 261503 impcol

REBEIROT EDITH DR, OBSERVATOIRE DE MARSEILLE, 2 PLACE LE VERRIER

F 13248 MARSEILLE CDX 04, FRANCE

Telephone: (33) 91959088

Facsimile: (33)

Telex: $\mathbf{4 2 0 2 4 1}$

Email:

Commission(s): $28,30,33$ 
REBER GROTE DR, C/O POST OFFICE, BOTHWELL TAS 7030, AUSTRALIA

Telephone: (61) $2237371 \quad$ Facsimile: (61) Telex

Email:

Commission(s): 40

REBOLO RAFAEL DR, INST DE ASTROFISICA, DE CANARIAS, OBS DEL TEIDE, E 38201 LA LAGUNA, SPAIN

Telephone: (34) 22262211

Facsimile: (34)

Telex: 92640

Email: span:iac::rr|

Commission(s): 29

RECILLAS-CRUZ ELSA DR, INSTITUTO DE ASTRONOMIA, UNAM, APDO POSTAL 70-264

04510 MEXICO DF, MEXICO

Telephone: (52)

Facsimile: (52) Telex:

Email:

Commission(s):

REDFERN MICHAEL R DR, DPT OF PHYSICS, UNIVERSITY COLLEGE, GALWAY, IRELAND

Telephone: (353) 4124411

Facsimile: (353)

Commission(s): 09

Telex: $\mathbf{5 0 0 2 3}$

Email:

REED B CAMERON DR, DPT OF PHYSICS, ALMA COLLEGE, ALMA MI 48801 1599, USA

Telephone: (1) $5174637111 \quad$ Facsimile: (1) $517+637277$ Telex:

Email:

Commission(s):

REES DAVID ELWYN DR, CSIRO, DIV OF RADIOPHYSICS, BOX 76, EPPING NSW 2121, AUSTRALIA

Telephone: (61) 28680493

Facsimile: (61) 28680411

Telex:

Email: drees(a)rp.csiro.au

Commission(s): 10,12

REES MARTIN J PROF, INSTITUTE OF ASTRONOMY, THE OBSERVATORIES, MADINGLEY RD

CAMBRIDGE CB3 OHA, UK

Telephone: (44) 1223337548

Facsimile: (t4) 1223337523

Telex: 817297 astron $\mathrm{g}$

Email:

Commission(s): $44,44,47 \mathrm{C}, 51$

REEVES EDMOND M DR, NASA HEADQUARTERS, CODE EM, 600 INDEPENDENCE AVE SW

WASHINGTON DC 20546, USA

Telephone: (1) 2024531571

Facsimile: (1)

Telex: 89530

Email:

Commission(s): 10,12,44

REEVES HUBERT PROF, CEA CEN, SEP-SES BAT 28, BP 2, F 91191 GIF/YVETTE CDX, FRANCE

Telephone: (33) 169085159

Facsimile: (33)

Telex:

Email:

Commission(s): 10,35,44,47C

REFSDAL S PROF DR, HAMBURGER STERNWARTE, GOJENSBERGSWEG 112, D 21029 HAMBURG, GERMANY

Telephone: (49) 4072524124

Facsimile: (49)

Telex: 21788

Email:

Commission(s): 42,47

REGEV ODED DR, DPT OF PHYSICS, IIT, TECHNION CITY, HAIFA 32000, ISRAEL

Telephone: (972) 4293992

Facsimilc: (972) $4221514 \quad$ Telex:

Email: phre1or@technion

Commission(s):

REGLERO-VELASCO VICTOR DR, DPT MATEMATICA Y ASTRON, UNIVERSIDAD DE VALENCIA, BURJASOT E 46100 VALENCLA, SPAIN

Telephone: (34) 63864326

Facsimile: (34) 63864302

Telex: 61071

Email:

Commission(s): 25,42

REGO FERNANDEZ M DR, DPT DE ASTROFISICA, FAC DE FISICA, UNIVERSIDAD COMPLUTENSE

E 28040 MADRID, SPAIN

Telephone: (34) 14495316

Facsimilc: (34)

Commission(s): 29

Telex: $47273 \mathrm{ff}$ uc

Email:

REGOES ENIKOE, INSTITUTE OF ASTRONOMY, THE OBSERVATORIES, MADINGLEY RD

CAMBRIDGE CB3 OHA, UK

Telephone: (44) 1223330894

Email: eniko@mail.ast.cam.ac.uk

Facsimile: (44) 1223337523

Telex: 817297 astron $g$

REGULO CLARA DR, INST DE ASTROFISICA, DE CANARIAS, OBS DEL TEIDE, E 38200 LA LAGUNA, SPAIN

Telephone: (34) 22262211

Facsimile: (34)

Telex: 92640

Email: span:iac::crr

Commission(s): 10,12 
REICH WOLFGANG, MPI FUER RADIOASTRONOMIE, AUF DEM HUEGEL 69, D 53121 BONN, GERMANY

Telephone: (49)

Facsimile: (49)

Telex: 886440

Email:

Commission(s): 40

REICHERT GAIL ANNE DR, NASA GSFC, CODE 666, GREENBELT MD 20771, USA

Telephonc: (1) $3012865307 \quad$ Facsimilc: (1) 3012863391 Telex:

Email: iuc::rcichert/zhgar(a)sefvm Commission(s): 28,44

REID MARK JONATHAN DR, CENTER FOR ASTROPHYSICS, HCO/SAO, 60 GARDEN ST, CAMBRIDGE MA 02138, US

Telephone: (1) 6174957470

Facsimilc: (1)

Telex: 921428 satellite cam

Email:

Commission(s): 40

REID NEILL, CALTECH, PALOMAR OBS MS 105 24, PASADENA CA 91101, USA

Telephone: (1) 8183566586 Facsimile: (1) Telex:

Email: $\quad$ Commission(s); 33

REIF KLAUS DR, RADIOASTRONOMISCHES INST, UNIVERSITAET BONN, AUF DEM HUEGEL 71 D 53121 BONN, GERMANY

Telephone: (49) 228733657

Facsimile: (4))

Telex:

Email: u.1+5ref\%mpichu\%unido.uuc) Commission(s): 33,40

REIMERS DIETER PROF, HAMBURGER STERNWARTE, UNIVERSITAET HAMBURG, GOJENSBERGSWEG 112 D 21029 HAMBURG, GERMANY

Telephone: (49) $407252+112$

Ficsimile: (49)

Email:

Commission(s): 29,36

Telex: $\mathbf{2 1 7 8 8 4}$

REINISCH GILBERT DR, OCA OBSERV DE NICE, BP 229, F 0630屯 NICE CDX \&, FRANCE

Telephone: (33) $93890420 \quad$ Facsimile: (33) 92003033 Telex:

Email:

Commission(s):

Telex: 240881

REIPURTH BO, ESO, CASILLA 19001, SANTIAGO 19, CHILE

Telephone: (56) $26988757 \quad$ Facsimile: (56)

Commission(s): 34

REITSEMA HAROLD J, BALL AEROSPACE SYSTEMS, DIVISION, BOX 1062, BOULDER CO 80306, USA

Tclephone: (1) $303+415026$

Facsimilc: (1)

Telex:

Email:

Commission(s): 15,20

REIZ ANDERS PROF, LOVSPRINGSVEJ 3 B, DK 2920 CHARLOTTENLUND, DENMARK

Telephonc: (45) 31632536

Facsimile: $(45)$

Telex:

Email:

Commission(s): 08,35

REMY BATTIAU LILIANE G A, CONSEIL DE LA RECHERCHE, UNIVERSITE DE LIEGE, 7 PLACE DU XX AOUT B 4000 COINTE-LIEGE, BELGIUM

Tclephone: $(32)+1+20080$

Facsimile: (32)

Commission(s): 05,15

Telex: $\$ 1397$ univ ulg

Email:

RENGARAJAN T N DR, TIFRIR ASTRONOMY, HOMI BHABHA RD, COLABA, BOMBAY 400 005, INDIA

Telephone: (91) 22219111

Facsimile: (91)

Telex: 011-3009 tifr in

Email:

Commission(s): $34,+4$

RENSE WILLIAM A DR, CASA, UNIVERSITY OF COLORADO, BOX 391, BOULDER CO 80309 (039), USA

Telcphonc: (1) $303+920111$

Facsimile: (1)

Telex:

Email:

Commission(s): ft

RENSON P F M DR, INSTITUT D'ASTROPHYSIQUE, UNIVERSITE DE LIEGE, AVE COINTE 5

B 4000 COINTE-LIEGE, BELGIUM

Telephone: (32) 41529980

Email: u2148ap(a) bliulg11

Facsimile: (32) $+1527+74 \quad$ Telex:

Commission(s): 05,27

RENZINI ALVIO PROF, DPT DI ASTRONOMIA, UNIVERSITA DI BOLOGNA, VIA ZAMBONI 33

I 40126 BOLOGNA, ITALY

Telephone: (39) 51222956

Facsimile: (39)

Commission(s): $35 \mathrm{C}, 37$

Telex: $21166+$ infnbo

Enail: astb(3):alvio 
M 778

REPHAELI YOEL DR, DPT OF PHYSICS \& ASTRON, TEL AVIV UNIVERSITY, RAMAT AVIV, TEL AVIV 69978, ISRAE Telephone: (972)

Facsimile: (972)

Commission(s): 28

Telex: 342171 versy il

Email:

REQUIEME YVES DR, OBSERVATOIRE DE BORDEAUX, BP 89, F 33270 FLOIRAC, FRANCE

Telephone: (33) 56864330

Facsimile: (33) $56+0+251 \quad$ Telex:

Email:

Commission(s): $08 \mathrm{C}, 24$

REVELLE DOUGLAS ORSON DR, LOS ALAMOS NATIONAL LAB, BOX 1663, MS K490, LOS ALAMOS NM 87544, USA

Telephone: (1)

Facsimile: (1)

Telex:

Email: dorageddie.lanl.gov

Commission(s): 15,22

REYES FRANCISCO DR, DPT OF ASTRONOMY, UNIVERSITY OF FLORIDA, 211 SSRB, GAINESVILLE FL 32611, USA

Telephone: (1) 9043922049

Facsimile: (1)

Telex:

Email: bitnet freyes(a)ufpine

Commission(s): 40

REYNOLDS JOHN DR, AAO, ATNF, BOX 76, EPPING NSW 2121, AUSTRALIA

Telephone: (61) 28680222

Facsimilc: (61) 28680400

Telex: 26230 astro aa

Email: jreynold(a)atnf.csiro.au

Commission(s): $\mathbf{0 8 , 4 0}$

REYNOLDS JOHN H PROF, PHYSICS DPT, UNIVERSITY OF CALIFORNIA, BERKELEY CA 94720, USA

Telephone: (1) $\$ 156+24863 \quad$ Facsimile: (1)

Email:

Commission(s):

Telex: 9103667114

REYNOLDS RONALD J DR, DPT OF PHYSICS, UNIVERSITY OF WISCONSIN, 1150 UNIVERSITY AVE

MADISON WI 53706, USA

Telephonc: (1) 6082625916

Email:

Facsimile: (1)

Telex:

Commission(s): 34

REYNOLDS STEPHEN P, DPT OF PHYSICS, N CAROLINA STATE UNIV, BOX 8201, RALEIGH NC 27695 8202, USA

Telephone: (1) $9197377751 \quad$ Facsimile: (1)

Email:

Commission(s):

Telex:

RHODES EDWARD J JR, 11801 KILLIMORE AVE, NORTHRIDGE CA 91326, USA

Telephone: (1) Facsimile: (1) Telex:

Email:

Commission(s):

RIBES ELIZABETH DR, OBSERVATOIRE DE PARIS, SECTION DE MEUDON, F 92195 MEUDON PPL CDX, FRANCE

Telephone: (33) $1+5077786$

Facsimile: (33)

Telex:

Email:

Commission(s): 36

RIBES JEAN-CLAUDE DR, INSU, 77 AVE DENFERT ROCHEREAU, F 75014 PARIS, FRANCE

Telephonc: (33) $143201330 \quad$ Facsimile: (33)

Telex:

Email:

Commission(s): 40

RICE JOHN B DR, DPT PHYSICS \& ASTRONOMY, UNIVERSITY OF BRANDON, 270 18TH STREET

BRANDON MB R7A 6A9, CANADA

Telephone: (1) 2047279693

Facsimile: (1) $20+7287346$

Telex:

Email: riceabbrandonu.ca

Commission(s):

RICHARDS MERCEDES T DR, UNIVERSITY STATION, UNIVERSITY OF VIRGINIA, BOX 3818

CHARLOTTESVILLE VA 22\%03, USA

Telephone: (1) $804924+895$

Facsimile: (1) $80492+3104 \quad$ Telex:

Email: mtr8r(a)virginia.edu

Commission(s): $\$ 2$

RICHARDSON E HARVEY DR, HERZBERG INST ASTROPHYS, DOMINION ASTROPHYS OBS, 5071 W SAANICH RD VICTORIA BC V8X +MG, CANADA

Telephone: (1) 6043880001

Facsimile: (1) $601363(0145$

Telex: 0497295

Email:

Commission(s): (09

RICHARDSON KEVIN J, DPT OF PIIYSICS, QUEEN MARY/WESTFIELD COLL, MILE END RD, LONDON E1 4NS, UK Telephone: (4)

Facsimile: (4)

Email:

Commission(s):

Telex: 
RICHARDSON LORNA LOGAN, DPT PHYSICS \& ASTRONOMY, UNIVERSITY OF GLASGOW

GLASGOW G12 8QQ, UK

Telephone: (44) $1413398855^{*}+153$

Email: lorna(a)astro.gla.ac.uk

Facsimile: (44) $1413349029 \quad$ Telex: 777070 unigla

Commission(s): 25

RICHARDSON R S, GRIFFITH OBSERVATORY, BOX 27787, LOS FELIX STATION, LOS ANGELES CA 90027, USA

Telephone: (1)

Facsimile: (1)

Telex:

Email:

Commission(s):

RICHER HARVEY B DR, DPT GEOPHYS \& ASTRONOMY, UNIV OF BRITISH COLUMBIA, 2075 WESBROOK PL

VANCOUVER BC VGT 1W5, CANADA

Telephone: (1) $60+228+134$

Facsimile: (1) $60+2286047$

Telex:

Email:

Commission(s): 28,37

RICHSTONE DOUGLAS O DR, DPT OF ASTRONOMY, UNIVERSITY OF MICHIGAN, DENNISON BLDG, ANN ARBOR MI 481091090 , USA

Telephone: (1) $31376+3441 \quad$ Facsinile: (1)

Email: d_richstone(a) $u b . c c . u m i c h . e d u \quad C o m m i s s i o n(s): 28$

Telex:

RICHTER G A DR, ZENTRLINST FUER ASTROPHYS, STERNWARTE BABELSBERG, ROSA LUXEMBURG STR 17A D 96575 SONNEBERG, GERMANY

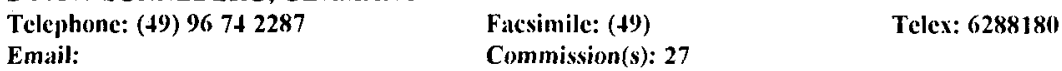

RICHTER JOHANNES PROF, INST F EXPERIMENT PHYSIK, PHYSIKZENTRUM, OLSHAUSENSTR

D 24098 KIEL, GERMANY

Telephone: (49) 4318803835

Email:

Facsimile: (49)

Telex: 292706

Commission(s): 14

RICHTLER TOM DR, UNIVERSITAETS STERNWARTE, AUF DEM HUEGEL 71, D 53121 BONN, GERMANY

Telephone: (49) $228733669 \quad$ Ficsimile: $(49)$

Email: $\$ 5228090071:$ richtler $\quad$ Commission(s): 37

Telex: $886+411$

RICKARD JAMES JOSEPH DR, BOX 777, BORREGO SPRINGS CO 92004, USA

Telephone: (1) $71+7675462$ Facsimile: (1) Telex:

Email:

Commission(s):

RICKARD LEE J DR, NAVAL RESEARCH LABORATORY, CODE 4138 RRD, 4555 OVERLOOK AVE SW

WASHINGTON DC 20375 5000, USA

Telephone: (1) 2027672495

Facsimile: (1)

Telex:

Email:

Commission(s): 34,40

RICKER GEORGE R DR, CENTER FOR SPACE RESEARCH, MIT RM 37 527, BOX 165, CAMBRIDGE MA (12139, USA

Telephone: (1) 6172537532

Facsimile: (1)

Commission(s):

Telex: 92-14-73

Email:

RICKETT BARNABY JAMES DR, DPT OF ELECTRICAL ENGIN, \& COMPUTER SCIENCE, UCSD

LA JOLLA CA 92093 0216, USA

Telephone: (1) 6194522731

Facsimile: (1)

Commission(s): 40,49

Telex:

Email:

AL OBSERVATORY, BOX 515, S 75120 UPPSALA, SWEDEN

RICKMAN HANS DR, ASTRONOMICAL OBSERVATORY, BOX 515, S 751 20 UPPSALA, SWEDE:
Telephone: (46) 18530265
Facsimile: (46) 18527583

Email: rickmanoban.uni.se

Commission(s): $15,20 \mathrm{VP}$

RICORT GILBERT DR, DPT ASTROPHYSIQUE, UNIVERSITE DE NICE, PARC VALROSE, F (G034 NICE CDX, FRANCE

Telephone: (33) 93519100

Facsimile: (33)

Telex:

Email:

Commission(s):

RIDDLE ANTHONY C DR, 700 GRANT PL, BOULDER CO 80302, USA

Telephone: (1) 3034478127

Facsimile: (1)

Commission(s): 49

Telex:

Email:

RIEGEL KURT W DR, NTL SCIENCE FOUNDATION, 3019 N OAKLAND ST, ARLINGTON VA 22207, USA

Telephone: (1) 202 3579450

Facsimile: (1)

Telex:

Email: kriegelänote.nsf.gov

Commission(s): 33 
RIEGER ERICH DR, MPI F EXTRATERRESTRISCHE, PHYSIK, KARL SCHWARZSCHILDSTR 1 D 85740 GARCHING MUENCHEN, GERMANY

Telephone: (49) $8932993511 \quad$ Facsimile: (49) 8932993539 Telex:

Email: cir@dgaipp1s

Commission(s): 10

RIEGLER GUENTER R DR, NASA HEADQUARTERS, CODE SZE, G00 INDEPENDENCE AVE SW WASHINGTON DC 20546, USA

Telephone: (1) 2024531435

Facsimile: (1)

Telex: 89530

Email: ames::"gricgleranasamail"

Commission(s): 05C,4t

RIEUTORD MICHEL, OBS MIDI PYRENEES, 14 AVE E BELIN, F 31400 TOULOUSE CDX, FRANCE

Telephone: (33) 61332949

Facsimile: (33) 61536722 Telex:

Email: ricutord@obs-mip.fr

Commission(s):

RIGHINI ALBERTO PROF, DPT DI ASTRONOMIA, UNIVERSITA DI FIRENZE, LARGO E FERMI S I 50125 FIRENZE, ITALY

Telephone: (39) 5527521

Facsimile: (39) 55220039

Telex: $\mathbf{5 7 2 2 6 8}$ arcetri i

Email:

Commission(s):

RIIHIMAA JORMA J DR. DPT OF ASTRONOMY, UNIVERSITY OF OULU, SF 90570 OULU 57, FINLAND

Tclephonc: (358)

Facsimile: (358) 81561278

Telex:

Email:

Commission(s): 40,51

RIJNBEEK RICHARD DR, SPACE \& PLASMA PHYSICS GP, SCHOOL MATHES \& PHYS SCI UNIVERISITY OF SUSSEX, BRIGHTON BN1 9QH, UK

Telephonc: (4) Facsimile: (4) 1273678097 Telex:

Enail: richardrâcentral.sussex.ac.uk Commission(s): 10

RILEY JULIA M DR, MULLARD RADIO ASTRON OBS, CAVENDISH LABORATORY, MADINGLEY RD

CAMBRIDGE CB3 OHE, UK

Telephone: (44) 1223337294

Facsimile: (44) $1223354599 \quad$ Telex: 81292

Email:

Commission(s): 40

RINDLER WOLFGANG PROF, UNIVERSITY OF TEXAS, UTD, BOX 830688, RICHARDSON TX 75083 0688, USA

Telephone: (1) 2146902885

Facsimile: (1)

Telex: 791-880

Email:

Commission(s): $\$ 7$

RING JAMES PROF, ASTROPHYSICS GROUP, IMPERIAL COLLEGE, BLACKETT LABORATORY

LONDON SW7 2BZ, UK

Telephone: (44) 1715895111

Facsimile: $(+4)$

Telex: 261503

Email:

Commission(s): 09

RINGNES TRULS S DR, INST THEORET ASTROPHYSICS, UNIVERSITY OF OSLO, BOX 1029 N 0315 BLINDERN OSLO 3, NORWAY
Telephone: $(+7)+72-456-503$
Facsimile: (47)
Telex: $72+25$ unios $n$
Email:
Commission(s):

RINGUELET ADELA E DR, 49 3\$2, 1900 LA PLATA (BS AS), ARGENTINA

$\begin{array}{ll}\text { Telephone: (54) } 2131063 & \text { Facsimile: (54) } 21211761 \quad \text { Telex: } 31151 \text { bulap ar } \\ \text { Email: } & \text { Commission(s): } 29\end{array}$

Email:

Commission(s): 29

RIPKEN HARTMUT W DR, DARA GERMAN SPACE AGENCY, KOENIGWINTERERSTR 522-524 D 53227 BONN, GERMANY

Telephone: $(49) 228+47203$

Email:

Facsimile: (49) $228+47700$

Telex:

RITTER HANS DR, MPI F PHYSIK \& ASTROPHYS, KARL SCHWARZSCHILDSTR 1 D 85740 GARCHING MUENCHEN, GERMANY

Telephone: (49)

Facsimilc: (49)

Commission(s): $35,+2$

Telex:

Email:

RIVOLO ARTHUR REX, DPT ASTRON \& ASTROPHYS, UNIV OF PENNSYLVANIA, PHILADELPHIA PA 19104, USA

Telephone: (1) 2158986250

Facsimile: (1)

Telex: 358300

Email:

Commission(s): 47 
RIZVANOV NAUFAL G DR, ENGELHARDT ASTRONOMICAL, OBSERVATORY, OBSERVATORIA STATION 422526 KAZAN, RUSSIA

Telephone: (7) 324827

Facsimile: (7)

Telex:

Email:

Commission(s): 24

ROARK TERRY P PROF, UNIVERSITY OF WYOMING, BOX 3434E, LARAMIE, WYOMING 82071, USA

Telephone: (1) 3077664121

Facsimile: (1)

Telex:

Email:

Commission(s):

ROBB RUSSEL M, DPT PHYSICS \& ASTRONOMY, UNIVERSITY OF VICTORIA, BOX 3055

VICTORIA BC V8W 3P6, CANADA

Telephone: (1) 6047217750

Email: robbaupupys

Facsimile: (1) 604 $7217715 \quad$ Telex:

Commission(s): 25,42

ROBBINS R ROBERT PROF, ASTRONOMY DPT, UNIVERSITY OF TEXAS, RLM 15 308, AUSTIN TX 78712 1083, USA

Telephone: (1) $512+717312$

Facsimile: (1)

Telex:

Email:

Commission(s): $34,46 C$

ROBE H A G DR, INSTITUT D'ASTROPHYSIQUE, UNIVERSITE DE LIEGE, AVE COINTE 5

B 4000 COINTE-LIEGE, BELGIUM

Telephone: (32) 41529980

Email:

Facsimile: (32) $\$ 1527474$

Telex: $\$ 1264$ astrilg

Commission(s):

ROBERGE WAYNE G DR, DPT OF PHYSICS, RENSSELAER POLYTECHN INST, TROY NY 12180 3590, USA

Telephone: (1) $5182766+54$

Facsimilc: (1)

Telex:

Email: robergéąorion.phys.rpi.clu

Commission(s): 34

ROBERTI GIUSEPPE DR, IST DI FISICA, UNIVERSITA DI NAPOLI, MOSTRA D OLTREMARE PAD 19 I 80125 NAPOLI, ITALY

$\begin{array}{ll}\text { Telephone: (39) } & \text { Facsimile: (39) } \\ \text { Email: } & \text { Commission(s): } 12\end{array}$

ROBERTS DAVID HALL DR, DPT OF PHYSICS, BRANDEIS UNIVERSITY, WALTHAM MA 02254, USA

Telephone: (1) $6176+72846$

Facsimile: (1)

Telex: 703013

Email:

Commission(s): $4(1,+7$

ROBERTS MORTON S DR, NRAO, 520 EDGEMONT RD, CHARLOTTESVILLE VA 229(1), USA

Telephone: (1) $80+296(1233$

Facsimile: (1) $80+2960278 \quad$ Telex:

Email: mrobertsiajurao.edu

Commission(s): $28,33,38 V P, 40$

ROBERTS WILLIAM W JR PROF, DPT OF APPLIED MATHS, UNIVERSITY OF VIRGINIA, THORNTON HALL CHARLOTTESVILLE VA 22003, USA

$\begin{array}{ll}\text { Telephone: (1) } 8049241038 & \text { Facsimile: (1) } \\ \text { Email: } & \text { Commission(s): } 28,33,34\end{array}$

ROBERTSON DOUGLAS S, NGS, N/CG 114 NOAA, 11400 ROCKVILLE PIKE, ROCKVILLE MD 20852, USA

Telephone: (1) $301+438+23$

Facsimile: (1)

Telex:

Email:

Commission(s): 19C,31,40

ROBERTSON JAMES GORDON DR, DPT OF ASTROPHYSICS, UNIVERSITY OF SYDNEY

SYDNEY NSW 2006, AUSTRALIA

Telephone: (61) 26922222

Email:

Facsimile: (61)

Telex:

Commission(s): 28,4i)

ROBERTSON JOHN ALISTAIR, DPT OF APPLIED MATHS, UNIVERSITY OF ST ANDREWS, NORTH HAUGH, S ANDREWS FIFE KY16 9SS, UK

Telephone: (4t) $133+76161$

Facsimile: (4)

Telex: 76213

Email:

Commission(s): 42

ROBERTSON NORNA DR, DPT PHYSICS \& ASTRONOMY, UNIVERSITY OF GLASGOW, GLASGOW G12 8QQ, UK

Telephone: ( $\$ 4) 1+13398855$

Email: gw05áulk.ac.gla.ph.i1
Facsimile: (t+) $1+13349029$

Commission(s): (09)
Telex: 777070 unigla

ROBILLOT JEAN-MAURICE DR, OBSERVATOIRE DE BORDEAUX, BP 8\%, F 33270 FLOIRAC, FRANCE

Telephonc: (33) $5686+330$

Email:
Facsimile: (33) $5640+251$

Commission(s):
Telex: 
ROBIN ANNIE C DR, OBSERVATOIRE DE BESANCON, BP 1615, F 25010 BESANCON CDX, FRANCE

Telephone: (33) 81666941

Facsimile: (33) 81666944

Telex: 361144

Email: robin(a)frobes51/obsbea::annic

Commission(s): 33

ROBINSON ANDREW, INSTITUTE OF ASTRONOMY, THE OBSERVATORIES, MADINGLEY RD

CAMBRIDGE CB3 OHA, UK

Telephone: (44) 1223337530

Email: ar(a)mail.ast.cam.ac.uk

Facsimile: (4) $1223337523 \quad$ Telex:

Commission(s):

ROBINSON BRIAN J DR, BOX 256, MILSONS POINT NSW 2(161, AUSTRALIA

Telephone: (61)

Facsimile: $(61)$

Telex:

Email:

Commission(s): $33,34,4$ (1)

ROBINSON EDWARD LEWIS DR, ASTRONOMY DPT, UNIVERSITY OF TEXAS, RLM 15308 AUSTIN TX 78712 1083, USA

Telephone: (1) 5124713401

Facsimile: (1)

Telex:

Email:

Commission(s): $25,27,42$

ROBINSON GARRY DR, DPT PHYSICS UNIV COLLEGE, UNIVER OF NEW SOUTH WALES, NORTHCOTT DR CAMPBELL ACT 2600, AUSTRALIA

Telephonc: (61) 6268880

Facsimile: (61) $62688786 \quad$ Telex:

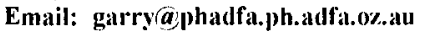

Commission(s): 34

ROBINSON I PROF, UNIVERSITY OF TEXAS, MS BE 32, BOX 688, RICHARDSON TX 75080, USA

Telephone: (1) $21+6902176$

Facsimile: (1)

Telex:

Email:

Commission(s): $\$ 7$

ROBINSON JR RICHARD D DR, GSFC, CODE 681/CSC, GREENBELT MD 20771, USA

Telephone: (1)

Facsimile: (1)

Telex:

Email:

Commission(s): 10,40

ROBINSON LEIF J, SKY \& TELESCOPE, t9 BAY STATE RD, CAMBRIDGE MA 02238, USA

Telephone: (1) $6178647360 \quad$ Filcsimile: (1) Telex:

Email:

Commisxion(s): 46,51

ROBINSON LLOYD B DR, LICK OBSERVATORY, UNIVERSITY OF CALIFORNIA, SANTA CRUZ CA 9506 4 , USA

Telephonc: (1) $408+292437$

Facsimile: (1)

Telex:

Email:

Commission(s): 09

ROBINSON WILLIAM J DR, 18 HOLLINGWOOD RISE, ILKLEY, W YORKSHIRE LS29 9PW, UK

Telephone: (4)

Facsimile: (4)

Telex:

Email:

Commission(s): 07

ROBLEY R DR, 9 ALLEE F VERDJER, F 31009) TOULOUSE, FRANCE

Telephonc: (33) 61522273

Facsimile: (33)

Telex:

Email:

Commission(s): 21

ROBSON IAN E DR, JOINT ASTRONOMY CENTRE, 660 N A'OHOKU PL, UNIVERSITY PARK, HILO HI 96720, USA

Telephone: (1)

Facsimile: (1)

Email:

Commission(s):

Telex:

ROCA CORTES TEODORO, INST DE ASTROFISICA, DE CANARIAS, OBS DEL TEIDE, E 38III LA LAGUNA, SPAIN

Telephone: (34) 22262211

Facsimile: (34)

Telex: 92640

Email:

Commission(s): 10,12

ROCCA-VOLMERANGE BRIGITTE, INSTITUT D'ASTROPHYSIQUE, 98BIS BD ARAGO, F 75014 PARIS, FRANCE

Telephone: (33) $14+328091$

Facsimile: (33) $1++328001$

Email:

Commission(s):

ROCHE PATRICK F DR, DPT OF ASTROPHYSICS, UNIVERSITY OF OXFORD, KEBLE RD, OXFORD OX1 3RH, UK

Telephone: (44) 1865273338

Facsimile: (44) $1865273+18$

Telex: 83295 nuclox g

Email: pfr(a)uk.ac.oxford.astrphysics

Commission(s): 34 
ROCHESTER MICHAEL G PROF, DPT OF EARTH SCIENCES, MEMORIAL UNIVERSITY OF, NEWFOUNDLAND ST JOHNS NF A1B 3X57, CANADA

Telephonc: (1) 7097377565

Facsimile: (1) 7097372589

Telex: $016+101$

Email: mrochest akean.ucs.mun.ca

Commission(s): 19

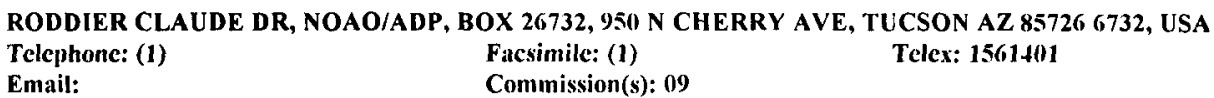

Email:

Commission(s): 09

RODDIER FRANCOIS PROF, NOAO/ADP, BOX 26732, 950 N CHERRY AVE, TUCSON AZ 85726 6732, USA

Telephone: (1) $5203259220 \quad$ Facsimile: (1) Telex: 0666484 aura noao tu

Email:

Commission(s): 09,12

RODGERS ALEX W DR, MOUNT STROMLO \& SIDING, SPRING OBSERVATORIES, PRIVATE BAG WODEN PO ACT 2606, AUSTRALIA

Telephone: (61) 62881111

Email:

Facsimile: (61) $62+90233$

Telex: 62270 canopus aa

Commission(s): $27,29,46$

RODMAN RICHARD B DR, 65 LOCUST AVE, LEXINGTON MA 02173 , USA

Telephone: (1) 6178618149 Facsimile: (1) Telex:

Email:

Commission(s):

RODONO MARCELLO DR, IST DI ASTRONOMIA, CITTA UNIVERSITARIA, VIA A DORIA 6, I 95125 CATANLA, ITALY

Telephone: (39) $95337308 \quad$ Facsimile: (39) 95330592

Email: marcellajastret.astro.it Commission(s): $27 \mathrm{C}, 36,42 \mathrm{P}$

Telex: 970359 astret $i$

RODRIGO RAFAEL, INST ASTROFISICA, DE ANDALUCIA APD 3004, PROFESOR ALBAREDA 1

E 18080 GRANADA, SPAIN

Telephone: (34) 58121300

Facsimilc: (34)

Telex: 78573 iaag e

Email:

Commission(s): 16,21

RODRIGUEZ ELOY DR, INST ASTROFISICA, DE ANDALUCIA APD 3004, PROFESOR ALBAREDA 1

E 18080 GRANADA, SPAIN

Telephone: (34) 58121311

Facsimilc: (34) $5881+530$

Telex: 78573 iaag e

Email: 16488::cloy

Commission(s): 27

RODRIGUEZ HILDAGO INES L, IAC, C/ VIA LACTEA S/N, E 38200 LA LAGUNA, SPAIN

$\begin{array}{lll}\text { Telephone: (34) } 22605200 & \text { Facsimile: (34) } 22605210 & \text { Telex: } 92640 \text { iac c }\end{array}$

Email: irhoulac.es Commission(s): 12

RODRIGUEZ LUIS F, INSTITUTO DE ASTRONOMIA, UNAM, APDO POSTAL 70-264, 04510 MEXICO DF, MEXICO

Telephone: (52) 9055485306

Email:

Facsimile: (52)

Telex: 1760155 cicme

Commission(s): $34 \mathrm{C}, 40$

RODRIGUEZ-ESPINOSA JOSE, INST DE ASTROFISICA, DE CANARIAS, OBS DEL TEIDE, E 38200 LA LAGUNA, SPAIN

Telephone: (34) $22262211^{\star}+4$ )

Facsinilc: (34) 222630105

Telex: 92640 iace

Email: span 288+4::ire

Commission(s): 28

RODRIGUEZ-VILLAMIL R DR, REAL INST Y OBSERVATORIO, DE LA ARMADA, CECILIO PUJAZON S/N E 11110 SAN FERNANDO, SPAIN

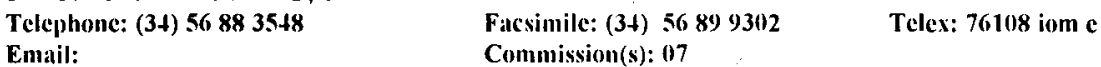

ROEDER ROBERT C PROF, DPT OF PHYSICS, SOUTHWESTERN UNIVERSITY, UNIVERSITY AVENUE

GEORGETOWN TX 78626, USA

Telephone: (1) $5128631633 \quad$ Facsimile: (1) Telex: 910-350-1677

Email:

Commission(s): 40,47

ROELFSEMA PETER DR, KAPTEYN ASTRONOMICAL INST, BOX 800, NL 9700 AV GRONINGEN, NETHERLANDS

Telephone: (31) $5063+043$

Facsimile: (31) 50636100

Telex:

Email: pjutrâahgrug

Commission(s): $3 \downarrow,+0$

ROEMER ELIZABETH PROF, LUNAR \& PLANETARY LAB, UNIVERSITY OF ARIZONA, TUCSON AZ 85721, USA

Telephone: (1) 5206212897

Facsimile: (1) $520621+933$

Email:

Commission(s): $06 \mathrm{C}, 15,20,24$

Telex: 467175 
ROEMER MAX PROF, INSTITUT FUER ASTROPHYSIK, EXTRATERR FORSCHUNG, AUF DEM HUEGEL 71 D 53121 BONN, GERMANY

Telephone: (49) 228733670

Facsimile: (49)

Telex:

Email:

Commission(s): 10

ROENNAENG BERNT O DR, ONSALA SPACE OBSERVATORY, CHALMERS UNIV OF TECH

S 43992 ONSALA, SWEDEN

Telephone: (46) 317725500

Facsimile: (46) 317725550

Telex: $2+00$

Email:

Commission(s): 40

ROESER HANS-PETER, DLR, INST F WELTRAUMSENSORIK, RUDOWER CHAUSSEE 5, D 12+89 BERLIN, GERMANY

Telephone: (49) $30695+5500$

Facsimile: (49) $30695+5502$ Telex:

Email: hans-peter.roeser(a)dir.de

Commission(s): 34,40

ROESER HERMANN-JOSEF DR, MPI FUER ASTRONOMIE, KOENIGSTUHL, D 69117 HEIDELBERG, GERMANY

Telephonc: (49) 6221528206

Facsimile: (49)

Telex: 461789 mpia d

Email:

Commission(s): 28

ROESER SIEGFRIED DR, ASTRON RECHEN INSTITUTE, MOENCHHOFSTR 12-14, D 69120 HEIDELBERG, GERMANY

Telcphone: (\$9) 622149026

Facsimile: (49)

Enail:

Commission(s): $08,20,24$

Telex: 461336 arihd d

ROESSIGER SIEGFRIED DR, ZNTRLINST FUER ASTROPHYS, STERNWARTE SONNEBERG ROSA LUXEMBURG STR 17A, D 96575 SONNEBERG, GERMANY

Telephone: (49) $967+2287 \quad$ Facsimile: (49) $967+2836$

Email: $\quad$ Commission(s): 05

Telex: 627180

ROGER ROBERT S DR, DOMINION RADIO ASTROPHYS, OBSERVATORY, BOX 248 PENTICTON BC V2A 6K3, CANADA

Telephone: (1) $60+\$ 932277$

Facsimile: (1) $604+937767$

Telex: 04888127

Email:

Commission(s): 34,40

ROGERS ALAN E E DR, HAYSTACK OBSERVATORY, WESTFORD MA 01886, USA

Telephone: (1) $617692+764$

Facsimile: (1)

Telex: 948149 haystack wfrd

Email:

Commission(s): 34,40

ROGERS CHRISTOPHER DR, DOMINION RADIO ASTROPHYS, OBSERVATORY, BOX 248

PENTICTON BC V2A 6K3, CANADA

Telephone: (1) $604+932277$

Facsimile: (1) $604+937767$

Telex: $0+888127$

Email: crogersadrao.nrc.ca

Commission(s):

ROGERSON JOHN B PROF, DPT ASTROPHYSICAL SCI, PRINCETON UNIVERSITY, PEYTON HALL PRINCETON NJ 085 441001 , USA

Telephonc: (1) 6094523806

Facsimile: (1)

Telex: 322409

Email:

Commission(s):

ROGSTAD DAVID H DR, JPL, MS 11/116, 4800 OAK GROVE DR, PASADENA CA 91109, USA

Telephone: (1)

Facsimile: (1)

Telex:

Email:

Commission(s): 40

ROHLFS K PROF DR, INST FUER THEORET PHYSIK, RUHR UNIVERSITAET BOCHUM, POSTFACH 102148 D $4+780$ BOCHUM, GERMANY
Telephone: (49) $23+70(1581) 2$
Facsimile: (49)
Telex: 0825860

Email:

Commission(s): $33,34,40$

ROLAND GINETTE DR, INSTITUT D'ASTROPHYSIQUE, UNIVERSITE DE LIEGE, AVE COINTE 5

B 4000 COINTE-LIEGE, BELGIUM

Telephone: (32) 41529980

Facsimile: (32) 41527474

Telex: 41254 astrlg b

Email:

Commission(s): 12

ROLLAND ANGEL DR, INST ASTROFISICA, DE ANDALUCIA APD 3004, PREFESOR ALBAREDA 1

E 18080 GRANADA, SPAIN

Telephone: (34) 58121300

Facsimile: (34)

Email:

Commission(s):

Telex: 78573 
ROMAN NANCY G DR, $\$ 260$ N PARK AVE, APT 306W, CHEVY CHASE MD 20815, USA

Telephone: (1) 3016566092

Facsimile: (1)

Telex:

Email:

Conmmission(s): $05,44,45$

ROMANCHUK PAVEL R DR, ASTRONOMICAL OBSERVATORY, KIEV STATE UNIVERSITY OBSERVATORNAYA UL 3, 252053 KIEV, UKRAINE

Telephone: (7)

Facsimile: (7)

Telex:

Email:

Commission(s): 10

ROMANI ROGER WILLIAM, DPT OF PHYSICS, STANFORD UNIVERSITY, STANFORD CA, USA

Telephone: (1) 4157257595

Facsimile: (1) +157239389 Telex:

Email: rwrāgeminga.stanford.edu

Commission(s):

ROMANISHIN WILLIAM DR, DPT PHYSICS \& ASTRONOMY, UNIVERSITY OF OKLAHOMA NORMAN OK 73019, USA

Telephone: (1) 4053253961

Facsimile: (1)

Telex:

Email:

Commission(s):

ROMANO GIULIANO PROF, V S ANTONIO DA PADOVA 7, I 31100 TREVISO, ITALY

Telephone: (39)

Facsimile: (39)

Tclex:

Email:

Commission(s): 27

ROMANOV YURI S DR, ASTRONOMICAL OBSERVATORY, ODESSA STATE UNIVERSITY, SHEVCHENKO PARK 27001 + ODESSA, UKRAINE

Telephone: (7) 048220396

Facsimile: (7)

Telex:

Email:

Commission(s): 27,30

ROMERO PEREZ M PILAR, INST DE ASTRON \& GEODESIA, FAC DE CIENCIAS MATEMAT

UNIVERSIDAD COMPLUTENSE, E 28040 MADRID, SPAIN

Telephone: (34) $12+42501 \quad$ Facsimile: (34)

Email: $\quad$ Commission(s): 04

Telex:

ROMNEY JONATHAN D DR, NRAO, BOX 0, SOCORRO NM 87801 0387, USA

Telephone: (1)

Facsimile: (1)

Telex:

Email:

Commission(s): $\$ 0$

ROMPOLT BOGDAN DR, ASTRONOMICAL INSTITUTE, WROCLAW UNIVERSITY, UL KOPERNIKA 11

PL 51 622 WROCLAW, POLAND

Tclephone: (48) $71+82+34 \quad$ Facsimile: (48)

Email:

Telex: 0712791 uwr pl

Commission(s): 10

RONAN COLIN A, FLAT 6, BOURNE CT, THE BOURNE, HASTINGS, EAST SUSSEX TN34 3UZ, UK

Telephone: $(4+) 1+2+\$ 46362$

Facsimile: $(+4)$

Telex:

Email:

Commission(s): +1

RONG JIAN-XIANG, DPT OF ASTRONOMY, NANJING UNIVERSITY, NANJING, CHINA PR

Telephone: (86) $2534651 * 2882$

Facsimile: (86)

Telex: $3+151$ prenu cn

Email:

Commission(s): 33

ROOD HERBERT J, INST FOR ADVANCED STUDY, SCHOOL OF NATURAL SCIENCE, PRINCETON NJ 08540, USA

Telephone: (1)

Facsimile: (1)

Telex:

Email:

Commission(s): 28

ROOD ROBERT T DR, UNIVERSITY STATION, UNIVERSITY OF VIRGINIA, BOX 3818

CHARLOTTESVILLE VA 22903, USA

Telephone: (1) 80+924+904

Facsimile: (1)

Telex:

Email: rtrâurginia.edu

Commission(s): 35,51

ROOS MATTS, HIGH ENERGY PHYSICS LAB, PB 9, SF 0001+ UNIV HELSINKI, FINLAND

Telephone: (358) $1918+40$

Facsimile: (358) 1918366

Telex:

Email: mroosâphcu.hclsinki.fi

Commission(s):

ROOS NICOLAAS DR, STERREWACHT, BOX 9513, NL 2300 RA LEIDEN, NETHERLANDS

Telephone: (31) 71275864

Facsimile: (31)

Telex:

Email: roosahierul151,bitnet

Commission(s): 28 
M 786

ROOSEN ROBERT G DR, 3760 TEXAS ST 24, SAN DIEGO CA 92014, USA

$\begin{array}{ll}\text { Telephone: (1) } 6192958724 & \text { Facsimilc: (1) } \\ \text { Email: } & \text { Commission(s): }\end{array}$

ROQUES FRANCOISE DR, OBSERVATOIRE DE PARIS, SECTION DE MEUDON, LAM

F 92195 MEUDON PPL CDX, FRANCE

Telephone: (33) $145077409 \quad$ Facsimile: (33) $145077469 \quad$ Telex: 201571

Email: mesioa::roques/roquesaffrmeus1 Commission(s): 16

ROQUES SYLVIE DR, OBS MIDI PYRENEES, 14 AVE E BELIN, F 31400 TOULOUSE CDX, FRANCE

Telephone: (33) $61252101 \quad$ Facsimile: (33) Telex: 530776 obstlse

Email:

Commission(s):

ROS ROSA M, DPT MATEMATICA APLICADA, EUPVG UPC, VICTOR BALAGUER SN

E 08800 VILANOVA I LA GEL, SPAIN

Telephone: (34) 38967720

Facsimilc: (34) $38967700 \quad$ Tclex:

Email: ros@mat.upc.cs

Commission(s): 46

ROSA DOROTHEA DR, EMIL-KURZ STR 4, D 8045 ISMANING, GERMANY

Telephone: (49) 89964299 Facsimile: (49) Telex:

Email:

Commission(s):

ROSA MICHAEL RICHARD DR, ESO, ST/ECF, KARL SCHWARZSCHILDSTR 2

D 85740 GARCHING MUENCHEN, GERMANY

Telephone: (49) $89320060 \quad$ Facsimile: (49) $893202362 \quad$ Telex: $528-282-22-c 0 \mathrm{~d}$

Email: Commission(s): $28,3+\mathrm{C}$

ROSADO MARGARITA DR, INSTITUTO DE ASTRONOMIA, UNAM, APDO POSTAL 70-26ł

04510 MEXICO DF, MEXICO

Telephone: (52) 9055485306

Email:

Facsimile: (52)

Telex:

Commission(s): 28,34

ROSCH JEAN PROF, OBS MIDI PYRENEES, 9 R PONT DE LA MOUETTE, F 6520 4 BAGNERES BIGORRE, FRANCE

Telephone: (33) 62951969

Facsimile: (33)

Telex:

Email:

Commission(s): 09,10,16

ROSE JAMES ANTHONY, DPT PHYSICS \& ASTRONOMY, UNIVERSITY NORTH CAROLINA

204 PHILLIPS HALL 039A, CHAPEL HILL NC 27514, USA

Telcphone: (1) 9199627170

Facsimile: (1)

Telex:

Email:

Commission(s): 28,29

ROSE WILLIAM K DR, ASTRONOMY PROGRAM, UNIVERSITY OF MARYLAND, COLLEGE PARK MD 20742, USA

Telephone: (1) 3012992777

Facsimile: (1)

Telex:

Email:

Commission(s): 34

ROSEN EDWARD DR, DPT OF HISTORY, CITY COLLEGE OF NY, NEW YORK NY 10031, USA

Telephone: (1)

Facsimile: (1)

Telex:

Email:

Commission(s):

ROSENBERG J DR, STATE UNIV OF UTRECHT, HEIDELBERLAAN 8, NL 358+ CA UTRECHT, NETHERLANDS

Telephone: (31) 30535124

Facsimile: (31)

Telex:

Email:

Commission(s):

ROSENDHAL JEFFREY D DR, NASA HEADQUARTERS, CODE $Z$, ASS DIRECTOR SPACE EXPLO

WASHINGTON DC 20546, USA

Telephone: (1) 2024539181

Facsimile: (1) $202+260408$

Telex:

Email:

Commission(s): 44

ROSER PELLO DESCAYRE, OBS MIDI PYRENEES, 14 AVE E BELIN, F $31+00$ TOULOUSE CDX, FRANCE

Telephone: (33) 61332812

Facsimile: (33) 61536722

Telex:

Email: roser(a)obs-mij.fr

Commission(s): 28,47

ROSINO LEONIDA PROF, OSS ASTRONOMICO DI PADOVA, VIC DELL OSSERVATORIO 5, I 35122 PADOVA, ITALY

Telephone: (39) 49661499

Facsimile: (39)

Email:

Commission(s): 06,27,34,37

Tclex: $\mathbf{4 3 0 1 7 6}$ unpadu 
ROSLUND CURT DR, DPT OF ASTRONOMY, CHALMERS TECHNICAL UNIV, S 41296 GOETEBORG, SWEDEN Telephone: (46) 31810100 Facsímile: (46) Telex:

Email:

Commission(s): 25,46

ROSNER ROBERT, ASTRONOMY \& ASTROPHYS CTR, UNIVERSITY OF CHICAGO, 5640 S ELLIS AVE

CHICAGO IL 60637, USA

Telephone: (1)

Facsimile: (1)

Telex:

Email:

Commission(s): $\mathbb{4 , 4 9}$

ROSQUIST KJELL, INST OF THEORETICAL PHYS, VANADISVAEGEN 9, S 11346 STOCKHOLM, SWEDEN

Telephone: (46) $8228160 * 225$

Facsimile: (46)

Telex: $15+33$ fysto s

Email:

Commission(s): 47

ROSS DENNIS K PROF, DPT OF PHYSICS, IOWA STATE UNIVERSITY, AMES IA 50011, USA

Telephone: (1) 5152946010

Facsimile: (1)

Telex:

Email:

Commission(s):

ROSS JOHN E R DR, DPT OF PHYSICS, UNIVERSITY OF QUEENSLAND, ST LUCIA

BRISBANE QLD 4067, AUSTRALIA

Telephone: (61) $73773+29$

Facsimile: (61)

Telex: $\mathbf{4 0 3 1 5}$ univqld aa

Email:

Commission(s): 14,36

ROSSELLO GASPAR, DPT FISICA DE ATMOSFERA, UNIVERSIDAD DE BARCELONA, AVD DIAGONAL 645 E 08028 BARCELONA, SPAIN

Telephone: (34)

Facsimile: (34)

Telex;

Email:

Commission(s): 04

ROSSI CORINNE DR, ISTITUTO ASTRONOMICO, UNIVERSITA DI ROMA, VIA G M LANCISI 29

I 00161 ROMA, ITALY

Telephonc: (39) $64+03734$

Facsimile: (39) 6 +4 03673

Telex: 613255 infuro

Email: 40058::rossi

Commission(s): 29

ROSSI LUCIO, IAS, CNR, CP 67, $1000+4$ FRASCATI, ITALY

Telephone: (39) $69425651 / 2 / 3$

Facsimile: (39) 69416847

Commission(s): 29

Telex: $610261 \mathrm{cnr}$ fra

Email:

FE DE PARIS, SECTION DE MEUDON, F 92195
Facsimile: (33) $1+5077469)$

ROSTAS FRANCOIS DR, OB
Telephonc: (33) $1+5077565$

Commission(s): 14VP

Email: rostasajobspm.fr

ROTH MIGUEL R DR, CARNEGIE INST WASHINGTON, LAS CAMPANAS OBSERVATORY, CASILLA 601

LA SERENA, CHILE

Telephone: (56) $51213032 \quad$ Facsimile: (56)

Email: mrothauchecrm

Commission(s):

Telex: 645227 aura ct

ROTHENFLUG ROBERT DR, CEA CEN, DAPNIA/SAP, BP 2, F 91191 GIF/YVETTE CDX, FRANCE

Telephone: (33) $16908+327 \quad$ Facsimile: (33)

Telex: 604806

Email: rothenflug@32779.decnet.cern Commission(s):

ROTH-HOPPNER MARIA LUISE, HAMBURGER STERNWARTE, GOJENSBERGSWEG 112

D 21029 HAMBURG, GERMANY

Telephone: (49) $407252+112$

Facsimile: (49)

Telex: 217884

Email:

Commission(s): 37

ROTS ARNOLD H DR, GSFC, CODE 668, GREENBELT MD 20771, USA

Telephone: (1) 3012861061

Facsimile: (1) 301286168

Telex:

Email: arotsaxebec.gsfc.nasa.gov

Commission(s): 28

ROUAN DANIEL DR, OBSERVATOIRE DE PARIS, SECTION DE MEUDON, F 92195 MEUDON PPL CDX, FRANCE

Telephone: (33) 145077715

Facsimile: (33) 145072806

Telex:

Email: megasy::rouan rouanafimeus 1 Commission(s): 34

ROUDIER THIERRY DR, OBS MIDI PYRENEES, 14 AVE E BELIN, F 31400 TOULOUSE CDX, FRANCE

Telephone: (33) 61252101

Facsinile: (33)

Telex:

Email:

Commission(s): 10,12 
ROUEFF EVELYNE M A DR, OBSERVATOIRE DE PARIS, SECTION DE MEUDON, DAF F 92195 MEUDON PPL CDX, FRANCE

Telephone: (33) 145077435

Facsimile: (33)

Telex: 201571

Email:

Commission(s): 14

ROUNTREE JANET DR, 6001 WYNNWOOD RD, BETHESDA MD 20816, USA

Telephone: (1)

Facsimilc: (1)

Telcx:

Email: rountrec(a)nssdca.gsfc.nasa.gov

Commission(s): $09,27,37,45$

ROUSE CARL A DR, 62715 TH STR, DEL MAR CA 92014, USA

Telephone: (1) $6194554015 \quad$ Facsimile: (1)

Telex: 695065

Email:

Commission(s): 35

ROUSSEAU JEANINE DR, OBSERVATOIRE DE LYON, AVE CHARLES ANDRE, F 69561 S GENIS LAVAL CDX, FRANC Telephone: (33) 78560705

Facsimile: (33) 72399791

Telex: 310926

Email:

Commission(s):

ROUSSEAU JEAN-MICHEL MR, OBSERVATOIRE DE BORDEAUX, BP 89, F 33270 FLOIRAC, FRANCE

Telephone: (33) 56864330

Facsimile: (33) $56+0+251$

Telex:

Email:

Commission(s): 08

ROUTLEDGE DAVID DR, DPT OF ELECTRICAL ENGEENR, UNIVERSITY OF ALBERTA

EDMONTON AB T6G 2J7, CANADA

Telephone: (1) 4034325668

Facsimile: (1)

Telex: 0372979

Email:

Commission(s):

ROUTLY PAUL M DR, US NAVAL OBSERVATORY, 3450 MASSACHUSETTS AVE NW

WASHINGTON DC 203925100, USA

Telephone: (1) 2026531532

Email:

Facsimile: (1) Telex:

Commission(s): 38

ROVIRA MARTA GRACIELA, IAFE, CC 67 SUC 28, 1+28 BUENOS AIRES, ARGENTINA

Telephone: (54) 17816755

Facsimile: (54) Telex:

Email:

Commission(s): 10C,12,36

ROVITHIS PETER DR, ASTRONOMICAL INSTITUTE, NTL OBSERVATORY OF ATHENS, BOX 20048 GR 11810 ATHENS 306, GREECE

$\begin{array}{lll}\text { Telephone: (30) } 13463803 & \text { Facsimile: }(30) & \text { Telex: 215530 obsa gr } \\ \text { Email: } & \text { Commission(s): } 42 & \end{array}$

ROVITHIS-LIVANIOU HELEN, DPT OF ASTROPHYSICS, NTL UNIVERSITY OF ATHENS, PANEPISTIMIOPOLIS GR 15771 ZOGRAFOS, GREECE

Telephonc: (30) 17243414

Email:

Facsimile: (30)

Telex:

Commission(s): $\$ 2$

ROWAN-ROBINSON MICHAEL DR, ASTROPHYSICS GROUP, IMPERIAL COLLEGE, BLACKETT LABORATORY LONDON SW7 2BZ, UK

Telephone: (44) 1712258825

Facsimile: (†) $1715899463 \quad$ Telex:

Email: uk.ac.ic.ph.star::mrr/icva::mr

Commission(s): 47,51

ROWSON BARRIE DR, NRAL, JODRELL BANK, MACCLESFIELD SK11 9DL, UK

Telephone: (44) $147771321 \quad$ Facsimile: (44) Telex: 36149

Email:

Commission(s): 40

ROXBURGH IAN W PROF, SCHOOL OF MATHEMATICAL SC, QUEEN MARY/WESTFIELD COLL, MILE END RD LONDON E1 4NS, UK

Telephone: (44) 1719804811

Facsimile: (\$)

Telex:

Email:

Commission(s): $10,3+, 35,+2,47,49$

ROY ARCHIE E PROF, DPT OF ASTRONOMY, UNIVERSITY OF GLASGOW, GLASGOW G12 8QQ, UK

Telephone: (44) $1413398855 * 502$

Facsimile: (44)

Commission(s): (77C, 46

Telex: $778+21$ glasul

Email:

ROY JEAN-RENE, DPT DE PHYSIQUE, UNIVERSITE LAVAL, FAC SCIENCES 2 GENIE, LAVAL QC G1K 7P4, CANAD

Telephone: (1) $\$ 186565816$

Facsimile: (1) 4186562040

Telex: 05131621 unilaval

Email: 1150005/a)saphir.ulaval.ca

Commission(s): 
ROZELOT JEAN P, OCA CERGA, AVE COPERNIC, F 06130 GRASSE, FRANCE

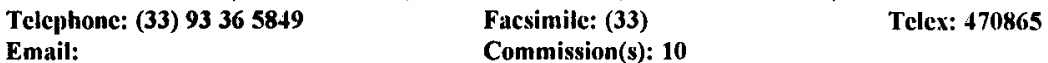

ROZHKOVSKIJ DIMITRIJ A, ASTROPHYSICAL INSTITUTE, KAZAKH ACAD OF SCIENCES

480068 ALMA ATA, KAZAKHSTAN

Telephone: (7) 624040

Facsimile: (7) Telex:

Email:

Commission(s): 21,34

ROZYCZKA MICHAL, ASTRONOMICAL OBSERVATORY, WARSAW UNIVERSITY, AL UJAZDOWSKIE 4

PL 00478 WARSAW, POLAND

Telephonc: (48)

Facsimile: (48)

Telex: 813978 zapan pl

Email:

Commission(s): 34

RUBASHEV BORIS M DR, PULKOVO OBSERVATORY, ACADEMY OF SCIENCES, 10 KUTUZOV QUAY 196140 ST PETERSBURG, RUSSIA

Telephone: (7)

Facsimile: (7)

Commission(s): 10

Telex:

Emait:

RUBEN G PROF DR, KANTSTR 6, STERNWARTE BABELSBERG, D 1471 POTSDAM, GERMANY

Telephone: (49)

Facsimilc: (4))

Telex:

Email:

Commission(s): 35,44

RUBIN ROBERT HOWARD, NASA AMES RESEARCH CTR, MS 2456 , MOFFETT FIELD CA 94035, USA

Telephone: (1) 4159655528

Facsimilc: (1)

Telex: 348408

Email:

Commission(s): 40,51

RUBIN VERA C DR, DPT TERRESTR MAGNETISM, CARNEGIE INST WASHINGTON, 5241 BROAD BRANCH RD NW WASHINGTON DC 20015, USA

Telephone: (1) 2029660863

Facsimile: (1)

Email:

Commission(s): $28,30,33,47$

Telex: 440427 magn ui

RUBIO MONICA DR, DPT DE ASTRONOMIA, UNIVERSIDAD DE CHILE, CASILLA 36 D, SANTIAGO, CHILE

Telephone: (56) $2229+101$

Facsimile: (56) 22712799

Telex: $\$+0001$

Email:

Commission(s): 40

RUCINSKI DANIEL, SPACE RESEARCH CENTER, POLISH ACAD OF SCIENCES, UL BARTYCKA 18

PL 00716 WARSAW, POLAND

Telephone: (48) 22403766

Email: rucinski âcbk.waw.pl

Facsimile: (48) 39121 273/22 403131 Telex: 825670 chk pl

Commission(s): 49

RUCINSKI SLAWOMIR M DR, SAL/ISTS, YORK UNIVERSITY, +700 KEELE ST, NORTH YORK ON M3J 1P3, CANADA

Telephone: (1) 4166653311

Facsimile: (1)

Telex: 06524736

Email: bitnet:fs300516(a) yusol

Commission(s): 36,+2

RUDAK BRONISLAW, INSTITUTE OF ASTRONOMY, N COPERNICUS UNIVERSITY, UL CHOPINA 12/18

PL 87100 TORUN, POLAND

Telephone: (48) $26037^{\star} 10 \quad$ Facsimile: $(48)$

Emait:

Commission(s):

Telex: 813978 zapan pl

RUDER HANNS, LEHRSTUHL F THEORET ASTRO, PHYSIK DER UNIV TUEBINGEN

AUF DER MORGENSTELLE 12 C, D 72076 TUEBINGEN, GERMANY

Telephone: (49) $7071292487 \quad$ Facsimile: (49)

Telex:

Email:

Commission(s): $09,14,19,24,4$

RUDERMAN MALVIN A, DPT OF PHYSICS, COLUMBIA UNIVERSITY, 538 W 120TH ST, NEW YORK NY 10027, USA

Telephone: (1) 2122803317

Facsimile: (1)

Telex:

Email:

Commission(s):

RUDKJOBING MOGENS PROF, INST OF PHYSICS \& ASTRON, UNIVERSITY OF AARHUS, NY MUNKEGADE DK 8000 AARHUS C, DENMARK

Telephone: (45) 86128899

Email:
Facsimile: (45) 86202711

Commission(s): 45
Telex: 64767 aausci dk 
RUDNICK LAWRENCE DR, DPT OF ASTRONOMY, UNIVERSITY OF MINNESOTA, 116 CHURCH ST SE MINNEAPOLIS MN 55455, USA

Telephone: (1) 6123735457

Facsimile: (1)

Telex:

Email:

Commission(s): 40,47

RUDNICKI KONRAD PROF, ASTRONOMICAL OBSERVATORY, JAGIELLONIAN UNIVERSITY, UL ORLA 171 PL 30 244 KRAKOW, POLAND

Telephone: (48) 226933

Email:

Facsimile: (48) 12378053

Commission(s): 28

Telex: 32 6203 ujoa pl

RUDNITSKIJ GEORGIJ, STERNBERG ASTRONOMICAL, INSTITUTE, 13 UNIVERSITETSKIJ PROSP 119899 MOSCOW, RUSSIA

Telephone: (7) 0959391030

Email: gmr(a)sai.msk.su

Facsimile: (7) 0959391661

Commission(s):

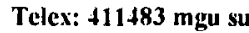

RUDZIKAS ZENONAS B, INST THEORETICAL PHYSICS, \& ASTRONOMY, GOSTAUTO 12, VILNIUS 2600, LITHUANI Telephone: (370) 2620939

Facsimile: (370) 2224694

Commission(s): 14

Telex: 261135 fisu

Email: astro@itpa.fi.It

RUEDIGER GUENTHER DR, ZNTRLINST F ASTROPHYSIK, STERNWARTE BABELSBERG ROSA LUXEMBURG STR 17A, D $1+473$ POTSDAM, GERMANY

Telephone: (49) $373377138 \quad$ Facsimile: (4) $(66915+71$

Email: $\quad$ Commission(s): 10

Telex:

RUELAS-MAYORGA R A DR, INSTITUTO DE ASTRONOMIA, UNAM, APDO POSTAL 70-264 04510 MEXICO DF, MEXICO

Telephone: (52) 5255485306

Email: rarm@alfa.astroscu.unam.mx

Facsimile: (52) $5255483712 \quad$ Telex:

Commission(s): 33

RUFENER FREDY G PROF, OBSERVATOIRE DE GENEVE, CHEMIN DES MAILLETTES $\$ 1$ CH 1290 SAUVERNY, SWITZERLAND

Tclephonc: (41) 227552611

Email:

Facsinile: (+1) $227553983 \quad$ Telex: +19209 obs ch

Commission(s): 25

RUFFINI REMO, DPT DI FISICA, UNIVERSITA DI ROMA, PA MORO 2, 100185 ROMA, ITALY

Telephone: (39) $64976304 \quad$ Facsimile: (39) Telex: 613255 infnro $i$

Email:

Commission(s): 44,47

RUGGE HUGO R DR, AEROSPACE CORPORATION, MS M2 226, BOX 92957, LOS ANGELES CA 90009, USA

Telephone: (1) 2136487086

Facsimile: (1)

Commission(s):

Telex:

Email:

RUIZ LAPUENTE PILAR, DPT D' ASTRONOMIA I METEO, UNIVERSIDAD DE BARCELONA E 08028 BARCELONA, SPAIN

Telephone: $(3+) 3+021121$

Email: pilar@ararcmo.ub.es

Facsimilc: (34) $340121133 \quad$ Telex:

Commission(s): 35

RUIZ MARIA TERESA DR, DPT DE ASTRONOMIA, UNIVERSIDAD DE CHILE, CASILLA 36 D, SANTIAGO, CHILE Telephone: (56) 22294101

Facsimile: (56)

Commission(s): 33

Telex: $4+0001$

Email:

RULE BRUCE H, HALE OBSERVATORIES, 2205 MONTE VISTA ST, PASADENA CA 91107, USA

Telephone: (1) 8187946593

Facsimilc: (1)

Commission(s):

Telex:

Email:

RUMSEY NORMAN J, 21 MALONE RD, LOWER HUTT, NEW ZEALAND

Telephone: $(64) 4696787 \quad$ Facsimile: $(6 t)$

Email:

Commission(s):

Telex:

RUNCORN S K PROF, DPT OF PHYSICS, IMPERIAL COLLEGE, PRINCE CONSORT RD, LONDON SW7 2BZ, UK

Telephone: (44)

Facsimile: (4)

Telex:

Email:

Commission(s): 16,19

RUPPRECHT GERO DR, ESO, KARL SCHWARZSCHILDSTR 2, D 85740 GARCHING MUENCHEN, GERMANY

Telephone: (49) 8932006355

Facsimile: (49) 893202362

Telex: 5282820 cod

Email: grupprecäaso.org

Commission(s): (19) 
RUPRECHT JAROSLAV DR, ASTRONOMICAL INSTITUTE, CZECH ACADEMY SCIENCES, BOCNI II 1401 CZ 14131 PRAHA 4, CZECH R

Telephone: (42) 2258757

Facsimile: (42) 2255010

Telex: 122486

Email: astdss@csearn

Commission(s): 37

RUSCONI LUIGLA DR, DPT DI ASTRONOMIA, UNIVERSITA DI TRIESTE, VIA TIEPOLO 11, I 34131 TRIESTE, ITALY

Telephone: (39) 40794863

Facsimile: (39)

Telex: 461137 oaoti

Email:

Commission(s): 09

RUSIN VOJTECH, ASTRONOMICAL INSTITUTE, SLOVAK ACADEMY SCIENCES

SK 05960 TATRANSKA LOMNI, SLOVAK R

Telephone: (42) $969967866 / 7 / 8 \quad$ Facsimile: (+2) $969967656 \quad$ Telex: 8078277 aussav c

Email:

Commission(s): 10,12

RUSKOL EUGENIA L DR, INST PHYSICS OF THE EARTH, ACADEMY OF SCIENCES, GRUZINSKAYA 10 123342 MOSCOW, RUSSIA

Telephone: (7) $2520726 \quad$ Facsimile: (7) Telex: 411196 ifzan su

Email:

Commission(s): 16

RUSSELL CHRISTOPHER T, INST OF GEOPHYS \& PLANET, UNIVERSITY OF CALIFORNIA

LOS ANGELES CA 90024, USA

Telephonc: (1) 3108253188

Facsimile: (1) $3102063051 \quad$ Telex:

Email: ctrussellajigpp.ucla.cdu

Commission(s): 49

RUSSELL JANE L DR, NAVAL RESEARCH LABORATORY, CODE 4130 R, 4555 OVERLOOK AVE SW

WASHINGTON DC 203755000 , USA

Telephone: (1) 2027670171

Facsimile: (1)

Telex:

Email:

Commission(s): 08,24,26,40,51

RUSSELL JOHN A PROF, DPT OF ASTRONOMY, UNIV SOUTHERN CALIFORNIA, UNIVERSITY PARK LOS ANGELES CA 90089, USA

Telephonc: (1) 2137430231

Facsimile: (1)

Telex:

Email:

Commission(s): 22

RUSSELL KENNETH S DR, UK SCHMIDT TELESCOPE, AAO, PRIVATE BAG COONABARABRAN NSW 2357, AUSTRALIA

Telephonc: (61) $68426311 \quad$ Facsimile: (61) $68846298 \quad$ Telex: canopus aa 163945

Email: nssdca::psi\%anocbn::ksr Commission(s): 20

RUSSELL STEPHEN DR, DIAS, SCHOOL OF COSMIC PHYSICS, 5 MERRION SQ, DUBLIN 2, IRELAND

Telephone: (353) 1774321

Email: 29536::sr/sr@dias.ic

Facsimilc: (353) 1682003

Commission(s): 34

Tclex: 31687 dias ei

RUSSEV RUSCHO DR, DPT OF ASTRONOMY, UNIVERSITY OF SOFIA, ANTON IVANOV ST 5

BG 1126 SOFIA; BULGARIA

Telephonc: (359) 2544852

Facsimile: (359)

Commission(s): 27

Telex:

Email:

RUSSEVA TATJANA, DPT OF ASTRONOMY, BULGARIAN ACAD SCIENCES, 72 LENIN BLVD BG 178 4 SOFIA, BULGARIA

Telephone: (359) 2758927

Facsimile: (359)

Telex: 23561 ecf ban bg

Email:

Commission(s): 37

RUSSO GUIDO DR, DPT DI SCIENZE FISICHE, UNIVERSITA DI NAPOLI, MOSTRA D OLTREMARE PAD 19 I 80125 NAPOLI, ITALY

Telephone: (39) $8172534+7$

Facsimile: (39)

Commission(s): $05,+2$

Telex: 720320

Email:

RUST DAVID M DR, APPLIED PHYSICS LAB, JOHNS HOPKINS UNIVERSITY, JOHNS HOPKINS RD

LAUREL MD 20707, USA

Telephone: (1) $3019535+14$

Facsimile: (1)

Email:

Commission(s): 10

Telex: 89-5+8 apl jhu laur 
RUSU I DR, ASTRONOMICAL INSTITUTE, CUTITUL DE ARGINT 5, BOX 28, R 75212 BUCHAREST, RUMANIA Telephone: (40) 16236301 Facsimile: (40) Telex:

Email: Commission(s): 08,19

RUSU L DR, STR MITROPOLITUL IOSIF 47, R 75217 BUCHAREST, RUMANIA

Telephone: (40) Facsimile: (40) Telex:

Email:

Commission(s):

RUTTEN RENEE G M, ROYAL GREENWICH OBS, APDO 321, SANTA CRUZ DE LA PALMA

E 38780 SANTA CRUZ, SPAIN

$\begin{array}{lll}\text { Telephone: (34) } & \text { Facsimile: (34) } & \text { Telex: } \\ \text { Email: } & \text { Commission(s): }\end{array}$

RUTTEN ROBERT J DR, STERREKUNDIG INSTITUTE, BOX 80000, NL 3508 TA UTRECHT, NETHERLANDS

Telephone: (31) 30535200

Facsimile: (31) 34572101

Telex: 40048 fylut $n$ l

Email: rutten(a)ruunsc.fys.ruu.nl

Commission(s): 12C,29,36

RUZDJAK VLADIMIR DR, INSTITUTE OF PHYSICS, UNIVERSITY OF ZAGREB, BOX 304, ZAGREB, CROATIA

Telephone: (38)

Facsimilc: (38)

Telex:

Email:

Commission(s): 10

RUZICKOVA-TOPOLOVA B DR, ASTRONOMICAL INSTITUTE, CZECH ACADEMY OF SCIENCES ONDREJOV OBSERVATORY, CZ 25165 ONDREJOV, CZECH R

Telephone: (42) $20485201 \quad$ Facsimile: (42) 204851611

Email:

Commission(s): 10

Telex: 121579

RYABCHIKOVA TANYA DR, INST OF ASTRONOMY, ACADEMY OF SCIENCES, PYATNITSKAYA UL 48 109017 MOSCOW, RUSSIA

Telephone: (7) 952315461

Facsimile: (7)

Telex:

Email:

Commission(s): 36

RYABOV YU A PROF DR, DPT OF MATHEMATICS, MADI, LENINGRADSKY PROSP 64, 125319 MOSCOW, RUSSIA Telephone: (7) 1550326

Email:

Facsimile: (7)

Telex:

Commission(s): 07

RYAN SEAN GERARD, ANGLO AUSTRALIAN OBS, BOX 296, EPPING NSW 2121, AUSTRALIA

Telephone: (61) 23724843

Facsimile: (61) 23724880

Telex:

Email: sgra aacepp.aao.gov.au

Commission(s): 29

RYBANSKY MILAN, ASTRONOMICAL INSTITUTE, SLOVAK ACADEMY SCIENCES

SK 059 60 TATRANSKA LOMNI, SLOVAK R

Telephone: (42) $969967866 / 7 / 8$

Facsimile: (42) 969967656

Telex: 8(1-78277 ausav c

Email:

Commission(s): 10,12

RYBICKI GEORGE B DR, CENTER FOR ASTROPHYSICS, HCO/SAO, 60 GARDEN ST, CAMBRIDGE MA 02138, USA

Telephone: (1) 6174957452

Facsimile: (1)

Commission(s): 33,36

Telex: $92-1428$

Email:

RYBKA PRZEMYSLAW DR, INST HISTORY OF SCIENCES, POLISH ACAD OF SCIENCES, NOWY SWIAT 72 PL 00330 WARSAW, POLAND

Telephone: (48)

Facsimile: $(48)$

Telex:

Email:

Commission(s):

RYDBECK GUSTAF H B DR, ONSALA SPACE OBSERVATORY, CHALMERS UNIV OF TECH S 43992 ONSALA, SWEDEN

Telephone: (46) 317725500

Facsimile: (46) $317725550 \quad$ Telex:

Email:

Commission(s): 28,40

RYDBECK OLOF E H PROF, ONSALA SPACE OBSERVATORY, CHALMERS UNIV OF TECH S 43992 ONSALA, SWEDEN

Telcphone: (46) 317725500

Facsimile: (46) 317725550

Telex: 85\$2400 onspace

Email:

Commission(s): 40

RYDGREN ALFRED ERIC JR DR, BOEING AEROSPACE CO, MS 87 08, BOX 3999, SEATTLE WA 9812+ 2499, USA

Telephone: (1) 2067732155

Facsimile: (1)

Telex:

Email:

Commission(s): 25 
RYKHLOVA LIDIJA V DR, INST OF ASTRONOMY, ACADEMY OF SCIENCES, PYATNITSKAYA UL 48 109017 MOSCOW, RUSSIA

Telephone: (7) 952315461

Facsimile: (7)

Email:

Commission(s): 19C

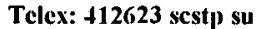

RYLOV VALERIJ S DR, SPECIAL ASTROPHYSICAL OBS, ACADEMY OF SCIENCES, NIZHNIJ ARKHYZ 357147 STAVROPOLSKIJ, RUSSIA

Telephone: (7)

Facsimile: (7)

Telex:

Email:

Commission(s): 09

RYS STANISLAW, ASTRONOMICAL OBSERVATORY, JAGIELLONIAN UNIVERSITY, UL ORLA 171

PL 30244 KRAKOW, POLAND

Telcphonc: (48) 12376455

Facsimile: (48) $12378053 \quad$ Telex:

Email: strys@oa.uj.edu.pl

Commission(s): 40

RYTER CHARLES E DR, CEA CEN, DAPNIA/SAP, BP 2, F 91191 GIF/YVETTE CDX, FRANCE

Telephonc: (33) 169083912

Facsimile: (33)

Telex:

Email:

Commission(s):

RYU DONGSU, DPT ASTRONOMY \& SPACE SCI, CHUNGNAM NTL UNIVERSITY, DAEJEON, KOREA R

Telephone: (82) 428215466 Facsimile: (82) $428228380^{\circ}$ Telex:

Email: ryu@sirius.chungnam.ac.kr Commission(s):

RYUTOVA MARGARITA P DR, INSTITUTE OF NUCLEAR PHYS, ACADEMY OF SCIENCES, SIBERIAN DIV 630090 NOVOSIBIRSK, RUSSIA

Telephone: (7) $3832359943 \quad$ Facsimile: (7) $3832352163 \quad$ Telex: 133116 atom su

Email: ryutova(a)vinpb.inp.nsk.su

Commission(s): 10

RZHIGA OLEG N DR, INST OF RADIO \& ELECTRON, ACADEMY OF SCIENCES, 103907 MOSCOW, RUSSIA

Telephone: (7)

Facsimile: (7)

Telex:

Email:

Commission(s):

SAAR ENN DR, TARTU ASTROPHYSICAL OBS, ESTONIAN ACAD OF SCIENCES, EE 202444 TARTU, ESTONIA

Tclephonc: (372)

Facsimilc: (372)

Telex:

Email: saar@aai.tartu.ew.su

Commission(s): 33,47

SABANO YUTAKA DR, ASTRONOMICAL INSTITUTE, TOHOKU UNIVERSITY, SENDAI AOBA, MIYAGI 980, JAPAN

Telephone: (81) $222221800 \quad$ Facsimile: (81)

Telex:

Email:

Commission(s): $3+$

SABBADIN FRANCO DR, OSSERVATORIO ASTROFISICO, VIA DELL OSSERVATORIO 8, I 36012 ASIAGO, ITALY

Telephone: (39) 42462665

Facsimile: (39)

Telex: setur $\mathbf{4 3 0 1 1 0}$

Email:

Commission(s): 34

SACK NOAM DR, DPT OF THEORETICAL PHYS, HEBREW UNIV OF JERUSALEM, JERUSALEM 91904, ISRAEL

Telephone: (972)

Facsimile: (972)

Telex:

Email:

Commission(s):

SACKMANN I JULIANA DR, CALTECH, KELlOGG RADIATION LAB, PASADENA CA 91125, USA

Telephone: (1) $818356+256$

Facsimile: (1)

Telex:

Email:

Commission(s): 35

SADAKANE KOZO DR, ASTRONOMICAL INSTITUTE, OSAKA KYOIKU UNIVERSITY, 4 88 MINAMIKAWAHORICHO OSAKA 543, JAPAN

Telephone: (81)

Email:

Facsimile: (81)

Telex:

Commission(s): 29C

SADAT RACHIDA DR, OBS DE STRASBOURG, 11 RUE UNIVERSITE, F 67000 STRASBOURG, FRANCE

Telephone: (33) 88358220

Facsimile: (33) 88250160

Telex:

Email:

Commission(s): 28,47

SADEH D DR, DPT OF PHYSICS \& ASTRON, TEL AVIV UNIVERSITY, RAMAT AVIV, TEL AVIV 69978, ISRAEL

Telephone: (972) 3420553

Facsimile: (972)

Telex: 34271 versy

Email:

Commission(s): 
$\mathbf{M} 794$

SADIK AZIZ R DR, SARC, SCIENTIFIC RES COUNCIL, BOX 2441, JADIRIYAH BAGHDAD, IRAQ
Tclephone: (964) 17765127
Facsimile: (964)

Email: $\quad$ Commission(s): 27,42

SADLER ELAINE MARGARET, AAO, BOX 296, EPPING NSW 2121, AUSTRALIA

Telephone: (61) $28681666 \quad$ Facsimile: (61) $28768536 \quad$ Telex: 123999 aaosyd aa

Email:

Commission(s): 28

SADUN ALBERTO CARLO DR, BRADLEY OBSERVATORY, AGNES SCOTT COLLEGE, DECATUR GA 30030, USA

Telephone: (1) 4043716265

Facsimile: (1)

Telex:

Email: phyaas@gsuvm1 bitnet

Commission(s): 28

SADZAKOV SOFIJA DR, ASTRONOMICAL OBSERVATORY, VOLGINA 7

YU 11050 BEOGRAD, YUGOSLAVIA-SERBIA \& MONTENEGRO

Telephone: (38) $11419357 / 421875 \quad$ Facsimile: (38) Telex:

Email:

Commission(s): 08,19

SAEMUNDSON THORSTEINN, RAUNVISINDASTOFNUN, HASKOLANS, DUNHAGA S, IS 107 REYKJAVIK, ICELAND Telephone: (354) 16222607 Facsimile: (354) 128911 Telex: 2307 isinfo is

Email: halo(a)ravnis.hi.is

Commission(s): 10

SAFKO JOHN L, DPT PHYSICS \& ASTRONOMY, UNIVERSITY S CAROLINA, COLUMBIA SC 29208, USA

Telephone: (1) $8037776466 \quad$ Facsimile: (1)

Email: $\quad$ Commission(s): 46

Telex: univscarol clb

SAFRONOV VICTOR S DR, INST PHYSICS OF THE EARTH, ACADEMY OF SCIENCES, GRUZINSKAYA 10, 123242 MOSCOW, RUSSIA

Telephone: (7) 2520726

Email:

Facsimile: (7)

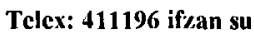

Commission(s): 16

SAGAN CARL DR, CORNELL UNIVERSITY, 302 SPACE SCIENCE BLDG, ITHACA NY 1†853, USA

Telephonc: (1) 6072564971

Facsimilc: (1)

Email: bland@astrosun.tn.cornell.edu

Commission(s): 16C,51

Telex: 937478

SAGDEEV ROALD Z DR, SPACE RESEARCH INSTITUTE, ACADEMY OF SCIENCES, PROFSOJUZNAYA UL 84/32 117810 MOSCOW, RUSSIA

Telephone: (7) 953331466

Facsimile: (7)

Commission(s): $15,44,49$

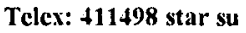

Email:

TL PRESS BLDG, WASHINGTON DC 20045, USA

SAGE LESLIE JOHN, NATURE, 1234 NTL PRESS BLDG, WASHINGTON DC 21045
Telephone: (1) $2027372355 \quad$ Facsimile: (1) 2026281609 Telex:

Email: nature@inaturedc.com Commission(s):

SAGGION ANTONIO PROF, DPT DI FISICA G GALILEI, UNIVERSITA DI PADOVA, VIA MARZOLO 8
I 35131 PADOVA, ITALY
$\begin{array}{lll}\text { Telephone: (39) } 49844254 & \text { Facsimile: (39) } & \text { Telex: } 430308 \text { dfggidi } \\ \text { Email: } & \text { Commission(s): } & \end{array}$

SAGLA ROBERTO PHILIP, INST FUER ASTRONOMIE \&, ASTROPHYSIK, SCHEINERSTR 1 D 81679 MUENCHEN, GERMANY

Telephone: (49) 8992209425 • Facsimile: (49) 8992209427 Telex:

Email: saglia@hal1.usm.uni-muenchen.de Commission(s):

SAGNIER JEAN-LOUIS DR, BUREAU DES LONGITUDES, 77 AVE DENFERT ROCHEREAU, F 75014 PARIS, FRANCE

$\begin{array}{ll}\text { Telephone: (33) } 140512261 & \text { Facsimile: (33) } \\ \text { Email: } & \text { Commission(s): 07,20 }\end{array}$

SAHA SWAPAN KUMAR DR, INDIAN INSTITUTE OF, ASTROPHYSICS, KORAMANGALA BANGALORE 560034 , INDIA

Telephone: (91) $803569902 \quad$ Facsimile: (91)

Email:

Commission(s): 09

Telex: $8+5-2763$ iiab in

SAHADE JORGE PROF, OBSERVATORIO ASTRONOMICO, PASEO DEL BOSQUE S/N, CC 677 1900 LA PLATA (BS AS), ARGENTINA

Telephone: (54) 21217308

Facsimile: (54) 2121 1761/25 8985 Telex: 31151 bulap ar

Email: sahade@gfcaglp.fcaglp.unlp.edu.ar Commission(s): 29,38C,42,44 
SAHAI RAGHVENDRA DR, JPL, MS 169 506, 4800 OAK GROVE DR, PASADENA CA 91109, USA

Telephone: (1) 8183540452

Facsimile: (1) $81835+8895 \quad$ Telex:

Email: sahai@jplsp.jpl.nasa.gov

Commission(s):

SAHAL-BRECHOT SYLVIE DR, OBSERVATOIRE DE PARIS, SECTION DE MEUDON, DAMAP F 92195 MEUDON PPL CDX, FRANCE

Facsimile: (33) $145077469 \quad$ Telex: 201571

Email: mesioa::sahal/sahal(a)frmeu51

Commission(s): 10,14

SAHIBOV FIRUZ H, INST OF ASTROPHYSICS, BUHORO (SVIRIDENKO)STR 22, DUSHANBE 734042, TAJIKISTAN

Telephonc: (7)

Facsimile: (7)

Telex:

Email:

Commission(s): 28

SAHU KAILASH C DR, KAPTEYN ASTRONOMICAL INST, BOX 800, NL 9700 AV GRONINGEN, NETHERLANDS

Telephonc: (31) 50634073

Email: kailash(a)hgrrug5

Facsimile: (31) 50636100

Telex: $\mathbf{5 3 5 7 2}$ stars $\mathbf{n l}$

SAIJO KEIICHI, DPT OF PHYSICAL SCIENCES, NATIONAL SCIENCE MUSEUM, 7-20 UENO PARK TAITO KU TOKYO 110, JAPAN

Telephone: (81) 38220111

Facsimile: (81)

Commission(s): 42

Telex:

Email:

SAIKIA DHRUBA JYOTI DR, TATA INST OF FUNDAMENTAL, RESEARCH, POONA UNIVERSITY CAMPUS

PUNE 411 007, INDIA

Telcphone: (91) 212337107

Email: uunet!shakti!gmrt!djs

Facsimile: (91) 212335760

Telex: $0145658 \mathrm{gmrt}$ in

Commission(s): 40

SAIO HIDEYUKI DR, ASTRONOMICAL INSTITUTE, TOHOKU UNIVERSITY, SENDAI AOBA, MIYAGI 980, JAPAN

Telephone: (81)

Facsimile: (81)

Telex:

Email:

Commission(s): 35

SAISSAC JOSEPH DR, 7 RUE AUGUSTE LIMOUZI, F 11100 NARBONNE, FRANCE

Telephone: (33)

Facsimile: (33)

Telex:

Email:

Commission(s): 16

SAITO KUNIJI PROF, TOKYO ASTRONOMICAL OBS, NAOJ, OSAWA MITAKA, TOKYO 181, JAPAN

Telephone: (81)

Facsimile: (81)

Telex:

Email:

Commission(s): 10,36

SAITO MAMORU DR, DPT OF ASTRONOMY, KYOTO UNIVERSITY, KITASHIRAKAWA SAKYO KU

KYOTO 606, JAPAN

Telephone: (81) 757512111 *3904 Facsimile: (81)

Email: saitom@kusastro.kyoto-u.ac.jp Commission(s):

Tclex: $5+22693$ libkyu j

SAITO SUMISABURO DR, KWASAN OBSERVATORY, KYOTO UNIVERSITY, YAMASHINA, KYOTO 607, JAPAN

Telephone: (81) $755811235 \quad$ Facsimile: (81)

Email:

Commission(s):

Telex: $5+22693$ libkyu j

SAITO TAKAO, TAIHAKU 3629 , SENDAI 980, JAPAN

Telephone: (81) 222458437

Email:

Facsimile: (81) $222458+37 \quad$ Telex:

Commission(s): 15

SAITO TAKAO PROF, GEOPHYS INST SCIENCE FAC, TOHOKU UNIVERSITY, AOBA AOBA-KU, SENDAI 980, JAPAN Telephone: (81) 222684508

Email:

Facsimile: (81) $22268+508$

Telex: mta1s5c(ājpntohok

Commission(s): 15

SAKAI JUNICHI, FAC OF ENGINEERING, TOYAMA UNIVERSITY, TOYAMA 930, JAPAN

Telephone: (81) 764411271

Facsimile: (81)

Telex:

Email:

Commission(s): 12

SAKASHITA SHIRO PROF, DPT OF PHYSICS, HOKKAIDO UNIVERSITY, KITA 10 NISHI 8, SAPPORO O60, JAPAN Telephone: (81) 117162111

Facsimile: (81)

Email:

Commission(s): 35

Telex: 
M 796

SAKHIBOV FIROUZ, INSTITUTE OF ASTRONOMY, ACADEMY OF SCIENCES, 48 PYATNITSKAYA ST 109017 MOSCOW, RUSSIA

Telephone: (7) 0952331624

Email: sakhibov@inasan.rssi.ru
Facsimile: (7) 0952302081

Commission(s): 28
Telex: 411576 ascon

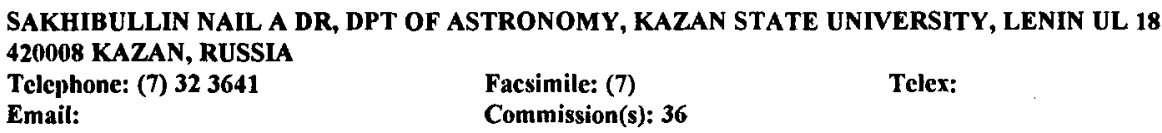

Telcx:

Commission(s): 10,51

SAKURAI TAKASHI DR, TOKYO ASTRONOMICAL OBS, NAOJ, OSAWA MITAKA, TOKYO 181, JAPAN

Telephonc: (81) 422325111

Facsimile: (81)

Email:

Commission(s): 10,12C

SAKURAI TAKEO T PROF, DPT AERONAITIC ENGINEER, KYOTO UNIVERSITY, KITASHIRAKAWA SAKYO KU KYOTO 606, JAPAN

Telephone: (81) 757512111 \$5792 Facsimile: (81) Telex: 05422693 libkyuj

Email:

Commission(s):

SALA FERRAN DR, DPT FISICA, UNIVERSIDAD DE BARCELONA, AVD DIAGONAL 647, E 08028 BARCELONA, SPAIN Telephone: (34) 33307311 Facsimile: (34) Telex:

Email:

Commission(s): 28,33

SALAS LUIS, OBSERVATORIO ASTRONOMICO, NACIONAL, BOX 439027, SAN DIEGO CA 92143 9027, USA Telephone: (1) 5261744580 Facsimilc: (1) 5261744607 Telex:

Email: salas@bufadora.astrosen.unam.mxCommission(s):

SALAZAR ANTONIO DR, REAL INST Y OBSERVATORIO, DE LA ARMADA, CECILIO PUJAZON S/N E 11110 SAN FERNANDO, SPAIN Telephone: (34) 56883548 Facsimilc: (34) 56899302 Telex: 76108 iom c

Email:

Commission(s): 04

SALETIC DUSAN, ASTRONOMICAL OBSERVATORY, VOLGINA 7 YU 11050 BEOGRAD, YUGOSLAVIA-SERBIA \& MONTENEGRO

Telcphone: (38) 11157022

Facsimile: (38)

Telex:

Email:

Commission(s): 08

SALINARI PIERO, OSS ASTROFISICO, DI ARCETRI, LARGO E FERMI 5, I 50125 FIRENZE, ITALY

Telephone: (39) $552752231 \quad$ Facsimile: (39)

Telex: 572268

Email:

Commission(s): $3+$

SALISBURY J W DR, US GEOLOGICAL SURVEY, 927 NATIONAL CENTER, RESTON VA 22092, USA

Telephone: (1) 7038606668

Facsimilc: (1)

Email:

Commission(s):

Telex: 92178

SALO HEIKKI, JPL, MS 183 501, 4800 OAK GROVE DR, PASADENA CA 91109, USA

Telephone: (1) $8183543833 \quad$ Facsimile: (1) 35881561278 Telex:

Email: salo@finfun.bitnet Commission(s):

SALPETER EDWIN E PROF, CORNELL UNIVERSITY, NEWMAN LAB OF NUCLEAR STU, ITHACA NY 14853, USA Telephone: (1) 6072563302 Facsimile: (1) Telex: 937478

Email:

Commission(s): $34,35,40,44$

SALTER CHRISTOPHER JOHN, ARECIBO OBSERVATORY, BOX 995, ARECIBO PR 00613, USA

Telephone: (1) 8098782612

Facsimile: (1) 8098781861 Telex:

Email: csalter@naic.edu

Commission(s): 34,40

SALUKVADZE G N DR, ABASTUMANY ASTROPHYSICAL, OBSERVATORY, GEORGIAN ACAD OF SCIENCES 383762 ABASTUMANI, GEORGIA

Telephone: (7)

Facsimile: (7)

Telex:

Email:

Commission(s): 26,37 
SALVADOR-SOLE EDUARDO, DPT FISICA DE ATMOSFERA, UNIVERSIDAD DE BARCELONA, AVD DIAGONAL 645 E 08028 BARCELONA, SPAIN

Telephone: (34) 33307311

Facsimile: (34)

Telex:

Email:

Commission(s): 28,47

SALVATI MARCO, OSS ASTROFISICO, DI ARCETRI, LARGO E FERMI 5, I S0125 FIRENZE, ITALY

Tclephonc: (39) $552752268 \quad$ Facsimilc: (39)

Telex: 572230 arcetr i

Email:

Commission(s): 44

SALZER JOHN JOSEPH DR, DPT OF ASTRONOMY, VAN VLECK OBSERVATORY, WESLEYAN UNIVERSITY

MIDDLETOWN CT 06457, USA

Telephone: (1) $2033479411 * 2827$

Facsimile: (1) $2033+47981$

Telex:

Email: slaz@parcha.astro.wesleyan.cdu

Commission(s): 47

SAMAIN DENYS DR, IAS, BAT 121, UNIVERSITE PARIS XI, F 91\$05 ORSAY CEDEX, FRANCE

Telephone: (33) 169858578

Facsimile: (33) 169858675

Telex: 600252

Email: iaslab::samain

Commission(s): 12

SAMEC RONALD G DR, DPT PHYSICS \& ASTRONOMY, MILLIKIN UNIVERSITY, 1184 WEST MAIN ST

DECAUTER IL 62522, USA

Telephone: (1)

Facsimile: (1)

Telex:

Email: rsamec(a)mail.millikin.cdu

Commission(s): $\$ 2$

SAMPSON DOUGLAS H PROF, DPT OF ASTRONOMY, PENNSYLVANIA STATE UNIV, 525 DAVEY LAB

UNIVERSITY PARK PA 16802, USA

Telephonc: (1) $8148650261 \quad$ Facsimile: (1)

Telex: $\mathbf{8 4 2 5 1 0}$

Email:

Commission(s):

SAMS BRUCE JONES III, MPI FUER EXTERRESTRISCHE, GIESSENBACH ST

D 85748 GARCHING MUENCHEN, GERMANY

Telephone: (49) $8932993587 \quad$ Facsimile: (49) $8932993569 \quad$ Telex:

Email: sams@mpa-garching.mpg.de Commission(s):

SAMUS NIKOLAI N DR, INST OF ASTRONOMY, ACADEMY OF SCIENCES, PYATNITSKAYA UL 48 109017 MOSCOW, RUSSIA

Telcphonc: (7) $952315461 \quad$ Facsimile: (7)

Email:

Commission(s): $27,30,37$

Telex: $\$ 12623$ sestp) su

SANAHUJA BLAS, DPT FISICA DE ATMOSFERA, UNIVERSIDAD DE BARCELONA, AVD DIAGONAL 645

E 08028 BARCELONA, SPAIN

Telephone: (34) $33307311 * 298$

Email:

Facsimilc: (34)

Telex:

Commission(s): 28,46

SANAMIAN V A DR, BYURAKAN ASTROPHYSICAL, OBSERVATORY, 378433 BYURAKAN, ARMENIA

Telephonc: (7) 8852283453

Facsimile: (7) 8852284142 Telex:

Email:

Commission(s): 40

SANCHEZ ALMEIDA JORGE DR, INST DE ASTROFISICA, DE CANARIAS, OBS DEL TEIDE

E 38200 LA LAGUNA, SPAIN

Telephone: (34) 22262211

Facsimile: (34) 222630105

Telex: 92640 iac e

Email: iac::jos/jos@ias.es

Commission(s): $10,12,29$

SANCHEZ FRANCISCO PROF, INST DE ASTROFISICA, DE CANARIAS, OBS DEL TEIDE, E 38071 LA LAGUNA, SPAIN

Telephonc: (34) 22262211

Facsimile: (34)

Telex: 92640 iac e

Email:

Commission(s): 21,50

SANCHEZ MANUEL, REAL INST Y OBSERVATORIO, DE LA ARMADA, CECILIO PUJAZON S/N

E 11110 SAN FERNANDO, SPAIN

Telephone: (34) 56883548

Facsimile: (34)

Telex: 76108

Email:

Commission(s): 08,19

SANCHEZLAVEGA AGUSTIN DR, DPT FISICA APLICADA, ETS INGENIEROS IND Y TEL, AVD URQUIJO S/N

E 18013 BILBAO, SPAIN

Telephone: (34) $44416400 \star 353$

Facsimile: $(34) \$+414041$

Commission(s): 16

Telex:

Email: 
SANCHEZ-SAAVEDRA M LUISA, FAC DE CIENCIAS, UNIVERSIDAD DE GRANADA, E 18080 GRANADA, SPAIN Telephone: (34) 58202212

Email:

Commission(s): $21,33,3+$

SANCISI RENZO DR, KAPTEYN ASTRONOMICAL INST, BOX 800, NL 9700 AV GRONINGEN, NETHERLANDS Telephonc: (31) 50116695

Facsimile: (31)

Telex: $\mathbf{5 3 5 7 2}$ stars $\mathbf{n l}$

Email:

Commission(s): 28,34,51

SANDAGE ALLAN, CARNEGIE OBSERVATORIES, 813 SANTA BARBARA ST, PASADENA CA 91101, USA

Telephonc: (1) 8185771122

Facsimilc: (1) 8187958136

Telex: 156131118 ociv ut

Email:

Commission(s):

SANDAKOVA E V DR, ASTRONOMICAL OBSERVATORY, KIEV STATE UNIVERSITY, OBSERVATORNAYA UL 3 252053 KIEV, UKRAINE

Telephone: (7)

Facsimile: (7)

Telex:

Email:

Commission(s):

SANDELL GORAN HANS L DR, 655 KOMOHAWA ST, HILO HI 96720, USA

Telephonc: (1)

Facsimile: (1)

Telex:

Email:

Commission(s): 34,40

SANDERS DAVID B DR, INSTITUTE FOR ASTRONOMY, UNIVERSITY OF HAWAII, 2680 WOODLAWN DR HONOLULU HI 96822, USA

Telephone: (1) 8089567399

Facsimile: (1) 8089882790

Telex: 7238459 uhast hr

Email: sanders@ifa.hawaii.edu

Commission(s): 28,40

SANDERS ROBERT DR, KAPTEYN ASTRONOMICAL INST, BOX 800, NL 9700 AV GRONINGEN, NETHERLANDS

Telephone: (31) 50116695

Facsimile: (31)

Commission(s): 28

Telex: $\mathbf{5 3 5 7 2}$ stars $\mathrm{nl}$

Email:

SANDERS WALT L PROF, 2395 DELAWARE AVE 68, SANTA CRUZ CA 95060, USA
Telephone: (1)

Email: wsanders(anmsu.edu $\quad$ Commission(s): 24,37

SANDERS WILTON TURNER III, DPT OF PHYSICS, UNIVERSITY OF WISCONSIN, 1150 UNIVERSITY AVE MADISON WI 53706, USA

Telephone: (1) 6082625916

Email:

Facsimile: (1)

Telex:

Commission(s): 44

SANDFORD MAXWELL T II, LOS ALAMOS SCIENTIFIC LAB, LOS ALAMOS NM 875+5, USA

Telephone: (1) 5056676384

Facsimile: (1)

Telex:

Email:

Commission(s):

SANDFORD SCOTT ALAN, NASA AMES RESEARCH CTR, MS 245 6, MOFFETT FIELD CA 94035, USA

Telephone: (1) 4156046849

Facsimile: (1) $4156046779 \quad$ Telex:

Email: sandford(a)ssa1.arc.nasa.gov Commission(s):

SANDMANN WILLIAM HENRY, DPT OF PHYSICS, HARVEY MUDD COLLEGE, CLAREMONT CA 91711, USA

Telephone: (1) $7146218024 \quad$ Facsimile: (1)

Telex:

Email:

Commission(s): 27

SANDQVIST AAGE DR, STOCKHOLM OBSERVATORY, S 133 36 SALTSJOEBADEN, SWEDEN

Telephone: (46) 87170380

Email: sandqvis@astro.su.se

Facsimile: $(46) 8717+719$

Telex: 12972 sobserv

SANFORD PETER WILLIAM MR, DPT PHYSICS \& ASTRONOMY, UNIVERSITY COLLEGE LONDON, GOWER ST LONDON WC1E 6BT, UK

Telephone: (44)

Facsimile: (44)

Telex:

Email:

Commission(s):

SANIGA METOD, ASTRONOMICAL INSTITUTE, SLOVAK ACADEMY SCIENCES

SK 05960 TATRANSKA LOMNI, SLOVAK R

Telephone: (42) 969967866

Facsimile: (42) 969967656

Telex:

Email: msaniga@auriga.ta3.sk

Commission(s): 10,12 
SANROMA MANUEL DR, DPT FISICA, UNIVERSIDAD DE BARCELONA, AVD DIAGONAL 647 E 08028 BARCELONA, SPAIN Telephonc: (34) 33307311

Facsimile: (34)

Telex:

Email:

Commission(s): 28

SANSATURIO MARIA E DR, ETS, INGENIEROS INDUSTRIALES, PASEO DEL CAUCE S/N

E 47011 VALLADOLID, SPAIN

Telephone: (34) 83304899

Facsimile: (34) 83392026

Telex:

Email:

Commission(s): 07

SANTAMARIA RAFFAELE DR, IST UNIVERSITARIO NAVALE, VIA ACTON 38, I 80133 NAPOLI, ITALY

Telephonc: (39) 815475135

Email:

Facsimilc: (39) 815521485

Telex: $710+17$

Commission(s):

SANTIN PAOLO DR, OAT, BOX SUCC TRIESTE 5, VIA TIEPOLO 11, I 34131 TRIESTE, ITALY

Telephone: (39) 40793921 Facsimile: (39) Telex: 461137 oat $i$

Email:

Commission(s):

SANTOS FILIPE D DR, CENTRO DE FISICA NUCLEAR, UNIVERSIDADE DE LISBOA, AV PROF GAMMA PINTO N2 P 1699 LISBOA CODEX, PORTUGAL

Telephone: (351) 35117950790

Facsimile: (351) $3511765622 \quad$ Telex: 62593 p

Email: santos $\sqrt[a]{p}$ tifm

Commission(s): 35

SANTOS NILTON OSCAR DR, OBSERVATORIO NACIONAL, RUA GL BRUCE 586, SAO CRISTOVAO 20921 RIO DE JANEIRO RJ, BRAZIL

Telephone: (55) 215800235

Facsimile: (55) 215800332

Telex:

Email:

Commission(s): 44

SANWAL BASANT BALLABH DR, UTTAR PRADESH STATE, OBSERVATORY, PO MANORA PEAK 263129

NAINITAL 263 129, INDIA

$\begin{array}{lll}\text { Telephone: (91) } 59422136 / 2583 & \text { Facsimile: (91) } & \text { Telex: } \\ \text { Email: sanwal(abison,obs-besancon.fr } & \text { Commission(s): } 29\end{array}$

SANWAL N B DR, DPT OF ASTRONOMY, UNIVERSITY OF OSMANIA, HYDERABAD 500 007, INDIA

Telephone: (91) $71951 * 247$

Facsimilc: (91)

Telex:

Email:

Commission(s): 30,42,45

SANYAL ASHIT DR, 7505 RIDGEWELL CT, BELTSVILLE MD 20705, USA

Telephone: (1)

Facsimile: (1)

Telex:

Email:

Commission(s): 27,42

SANZ I SUBIRANA JAUME DR, DPT MATEMATICA APLICADA, UNIV POLITEC DE CATALUNYA, BOX 30002 E 08080 BARCELONA, SPAIN

Telephone: (34) 34016799

Facsimile: (34) 34016801

Telex: 281 upc e

Email: matjss@mat.upc.cs

Commission(s): 33

SANZ JOSE L DR, DPT DE FISICA MODERNA, UNIVERSIDAD DE CANTABRIA, AVD LOS CASTROS S/N

E 39005 SANTANDER, SPAIN

Telephone: (34) 42201452

Facsimile: (34)

Commission(s): 47

Telex: 35681 cduci e

Email:

YSICAL OBS, ESTONIAN ACAD OF SCIENCES, EE 202444 TARTU, ESTONIA

Telephone: (372)

Facsimile: (372)

Commission(s): 36,47

Telex:

Email:

SAPRE A K DR, DPT OF PHYSICS, UNIVERISTY OF RAVISHANKAR, RAIPUR 429 010, INDIA

Telephone: (91) 27064

Facsimile: (91)

Telex:

Email:

Commission(s): 28

SARASSO MARIA DR, OSS ASTRONOMICO DI TORINO, ST OSSERVATORIO 20, I 10025 PINO TORINESE, ITALY

Telephonc: (39) 11841067

Email: 39181::sarasso
Facsimile: (39) 11841281

Commission(s): 05,08
Telex: 213236 toastr $i$ 
SARAZIN CRAIG L DR, UNIVERSITY STATION, UNIVERSITY OF VIRGINIA, BOX 3818 CHARLOTTESVILLE VA 22903, USA

Telephone: (1) 8049244903

Facsimile: (1) $80492+3104 \quad$ Telex:

Email: cls7i@virginia.edu

Commission(s): 28,34

SAREYAN JEAN-PIERRE DR, OCA OBSERV DE NICE, BP 229, F 0630 4 NICE CDX 4, FRANCE

Telephone: (33) 93890420

Facsimile: (33) 92003033

Telex: 460004 obsnice $f$

Email:

Commission(s): 27,29

SARGENT ANNEILA I, CALTECH, MS 320 47, DOWNES LAB OF PHYSICS, PASADENA CA 91125, USA

Telephone: (1) 8183566622

Facsimile: (1)

Telex: 675425

Email:

Commission(s): $33,34,40$

SARGENT WALlACE L W DR, CALTECH, MS 105 24, PASADENA CA 91125, USA

Telephone: (1) 8183564055

Facsimile: (1)

Telex: $675+25$ caltech psd

Email:

Commission(s): 28,47

SARMA M B K PROF, DPT OF ASTRONOMY, UNIVERSITY OF OSMANLA, HYDERABAD 500 007, INDIA Telcphone: (91) 65228

Facsimile: (91)

Telex:

Email:

Commission(s): 25,27

SARMA N V G PROF, RAMAN RESEARCH INSTITUTE, SADASHIVANAGAR, CV RAMAN AVE

BANGALORE 560080 , INDIA

Telcphone: (91) 803360122

Facsimilc: (91) 803340492

Telex: 8452671 rri in

Email: sarma@rri.crnet.in

Commission(s): 34,40

SARMIENTO-GALAN A F DR, INSTITUTO DE ASTRONOMIA, UNAM, APDO POSTAL 70-264 04510 MEXICO DF, MEXICO

Tclephone: (52) 5255485305

Facsimile: (52)

Commission(s):

Telex: 1760155 cicme

Email: ansar@unamvm1.bitnet

Facsimile: (48)

Telex:

Telephone: (48)

Email:

\author{
Commission(s):
}

SARRIS ELEFTHERIOS PH D, ASTRONOMICAL INSTITUTE, NTL OBSERVATORY OF ATHENS, BOX 20048 GR 11810 ATHENS, GREECE

$\begin{array}{ll}\text { Telephonc: (30) } 13461191 & \text { Facsimilc: (30) } \\ \text { Email: } & \text { Commission(s): }\end{array}$

Telex: 215530 obsa gr

SARRIS EMMANUEL T PH D, DPT OF ELECT ENGINEERING, DEMOCRITOS UNIV OF THRACE

GR 67100 XANTHI, GREECE

$\begin{array}{lll}\text { Tclephone: (30) } 54126948 & \text { Facsimile: (30) } & \text { Telex: } 452312 \text { polx gr } \\ \text { Email: } & \text { Commission(s): } 49 & \end{array}$

SARTORI LEO PROF, DPT PHYSICS \& ASTRONOMY, UNIVERSITY OF NEBRASKA, BEHLEN OBSERVATORY LINCOLN NE 68588 0111, USA

Telephone: (1)

Facsimile: (1)

Telex:

Email:

Commission(s): 44

SASAKI MISAO, PHYSICS DPT, KYOTO UNIVERSITY, KYOTO 606, JAPAN

Telephone: (81) 757513883

Email: misao@jpnyitp.bitnet

Facsimile: (81) $757533886 \quad$ Telex:

Commission(s): 47

SASAKI TOSHIYUKI DR, NTL ASTRONOMICAL OBS, 221 OSAWA, MITAKA, TOKYO 181, JAPAN

Telephone: (81)

Facsimile: (81)

Telex:

Email: sasaki@oan.nno.ac.jp

Commission(s): 28

SASAO TETSUO DR, INTL LATITUDE OBSERVATORY, NAOJ, HOSHIGAOKA MIZUSAWA SHI, IWATE 023, JAPAN

Telcphonc: (81) $197247111 \quad$ Facsimile: (81)

Email:

Commission(s): 19

Telex: 837628 ilsmirj

SASLAW WILLIAM C PROF, UNIVERSITY STATION, UNIVERSITY OF VIRGINIA, BOX 3818

CHARLOTTESVILLE VA 22903, USA

Telephone: (1) 8049244892

Facsimile: (1)

Telex:

Email:

Commission(s): 28,44 
SASSELOV DIMITAR D DR, CENTER FOR ASTROPHYSICS, HCO/SAO, 60 GARDEN ST, CAMBRIDGE MA 02138, USA Telephone: (1) 6174957451

Email: sassclov@cfa.harvard.cdu Facsimile: (1) $6174957049 \quad$ Telex:

Commission(s): $36 \mathrm{C}$

SASTRI HANUMATH J DR, INDIAN INSTITUTE OF, ASTROPHYSICS, KORAMANGALA, BANGALORE 560 034, INDIA Telephone: (91) 803566585

Facsimile: (91)

Telex: $8+5763$ iiab in

Email:

Commission(s): 49

SASTRY CH V, INDIAN INSTITUTE OF, ASTROPHYSICS, KORAMANGALA, BANGALORE 560 034, INDIA

Telephone: (91) 803566585

Facsimile: (91)

Telex:

Email:

Commission(s): 40

SASTRY SHANKARA K, DPT OF ASTRONOMY, UNIVERSITY OF OSMANIA, HYDERABAD SO0 O07, INDIA

Telephone: (91) 71951

Facsimile: (91)

Telex:

Email:

Commission(s): 28

SATO FUMIO DR, DPT ASTRON \& EARTH SCI, TOKYO GAKUGEI UNIVERSITY, KOGANEI, TOKYO 184, JAPAN

Telephone: (81) 423252111

Facsimile: (81)

Telex:

Email:

Commission(s): $\mathbf{3 4 , 4 0}$

SATO HUMITAKA PROF, DPT OF PHYSICS, KYOTO UNIVERSITY, KITASHIRAKAWA SAKYO KU

KYOTO 606, JAPAN

Telephone: (81)

Facsimile: (81)

Telex:

Email:

Commission(s): 47

SATO KATSUHIKO PROF, DPT OF PHYSICS, UNIVERSITY OF TOKYO, BUNKYO KU, TOKYO 113, JAPAN

Telephone: (81) $38122111^{*}+207$

Email: satơ(âphys.s.u-tokyo.ac.jp)

Facsimile: (81) 356890465

Telex: $23+72$ utphysic

Commission(s): $35,+4,47 \mathrm{C}$

SATO KOICHI DR, INTL LATITUDE OBSERVATORY, NAOJ, HOSHIGAOKA MIZUSAWA SHI, IWATE 023, JAPAN

Telephone: (81) 197247111

Facsimile: (81)

Telex: 837628 ilsmizj

Email:

Commission(s): 08,19

SATO MASSAE DR, IAG, UNIVERSIDADE DE SAO PAULO, AV MIGUEL STEFANO 4200 04301 SAO PAULO SP, BRAZIL

Telephone: (5S) $115778599 \quad$ Facsimile: (55) $112763848 \quad$ Telex: 1156735 iagm br

Email: 47556::massac Commission(s): 07,20

SATO NAONOBU PROF, AKITA UNIVERSITY, 1-1 TEGATA GAKUENCHO, AKITA 010, JAPAN

Telephone: (81) 188335261

Facsimile: (81)

Telex:

Email:

Commission(s): 27

SATO SHINJI, DPT OF ASTROPHYSICS, NAGOYA UNIVERSITY, FUROCHO CHIKUSA KU, NAGOYA 464 01, JAPAN

Facsimile: (81) 527892919

Telex:

Email: sato@toyo.phys.nagoya-u.ac.jp Commission(s): 47

SATO SHUJI DR, TOKYO ASTRONOMICAL OBS, NAOJ, OSAWA MITAKA, TOKYO 181, JAPAN

Telephone: (81) 422413643

Facsimile: (81) $422+13776$

Telex:

Email:

Commission(s): 34

SATO YUZO DR, 4819 OSAWA, MITAKA, TOKYO 181, JAPAN

Telephone: (81) Facsimile: (81)

Email:

Commission(s):

Telex:

SAULT ROBERT DR, AAO, ATNF, BOX 76, EPPING NSW 2121, AUSTRALIA

Telephone: (61) 28680222

Facsimile: (61) 28680310

Telex: 26230

Email: rsault@atnf.csiro.auu

Commission(s): 09

SAUNDERS RICHARD D E, MULLARD RADIO ASTRON OBS, CAVENDISH LABORATORY, MADINGLEY RD

CAMBRIDGE CB3 OHE, UK

Telephone: (44) 1223337294

Facsimile: (44) $122335+599$

Commission(s): 40

Telex: $\mathbf{8 1 2 9 2}$

Email: 
$\mathrm{M} 802$

SAUVAL A JACQUES DR, OBSERVATOIRE ROYAL DE, BELGIQUE, AVE CIRCULAIRE 3

B 1180 BRUSSELS, BELGIUM

Telephone: (32) 23752484

Facsimile: (32) 23749822

Telex: 21565 obsbel b

Email: jacques@astro.ome.be

Commission(s): 12

SAVAGE ANN DR, UK SCHMIDT TELESCOPE, AAO, PRIVATE BAG, COONABARABRAN NSW 2357, AUSTRALIA Telephone: (61) 68426311

Facsimile: (61) 68846298

Telex:

Email:

Commission(s): $28,40,47$

SAVAGE BLAIR D DR, DPT OF ASTRONOMY, UNIVERSITY OF WISCONSIN, 475 N CHARTER ST

MADISON WI 53706, USA

Telephone: (1) 6082623072

Facsimile: (1)

Commission(s): 34,44

Telex: $\mathbf{2 6 5 4 5 2}$ uofwisc-mds

Email:

SAVANOV IGOR S, CRIMEAN ASTROPHYS OBS, UKRAINIAN ACAD OF SCIENCE, NAUCHNY 6A 23 334413 CRIMEA, UKRAINE

Telephonc: (7) 0655471113

Facsimile: (7) 0655440704

Telex:

Email: savanov(acrao.crimea.ua

Commission(s): 36,14

SAVEDOFF MALCOLM P PROF, DPT PHYSICS \& ASTRONOMY, UNIVERSITY OF ROCHESTER BAUSCH AND LOMB BLDG, ROCHESTER NY 14627, USA

$\begin{array}{lll}\text { Telephone: (1) } 7162754357 & \text { Facsimile: (1) } & \text { Telex: } 978374 \text { unibook roc } \\ \text { Email: } & \text { Commission(s): } 34,35,44 & \end{array}$

SAVONIJE GERRIT JAN DR, ASTRONOMICAL INSTITUTE, UNIVERSITY OF AMSTERDAM, KRUISLAAN 403 NL 1098 SJ AMSTERDAM, NETHERLANDS

Telephone: (31) $205257491 \quad$ Facsimile: (31) $205257484 \quad$ Telex: 10262 hef $\mathrm{nl}$

Email:

Commission(s): 35,42

SAWA TAKEYASU, DPT PHYSICS \& ASTRONOMY, AICHI UNIVERSITY OF EDU, KARIYA, AICHI 448, JAPAN

Telephone: (81) 566363111

Facsimile: (81) $566364337 \quad$ Telex:

Email: sawa@aucphyas.aichi-cdu.ac.jp Commission(s): 28

SAWANT HANUMANT S DR, INPE, CP 515, 12200 S JOSE DOS CAMPOS, BRAZIL

Telephone: (55) 123229977

Facsimile: (55) 123218743

Telex: 1233530

Email:

Commission(s): 40

SAWYER CONSTANCE B DR, 850 20TH ST \# 705, 325 BROADWAY, BOULDER CO 80302, USA

Telephone: (1)

Facsimile: (1)

Telex:

Email:

Commission(s): 10,49

SAXENA A K DR, INDIAN INSTITUTE OF, ASTROPHYSICS, KORAMANGALA, BANGALORE 560 034, INDIA

Telephone: (91) $803566585 / 6497$

Facsimile: (91)

Telex: $8+5763$ iiab in

Email:

Commission(s): (0)

SAXENA P P DR, DPT OF MATHS \& ASTRONOMY, UNIVERSITY OF LUCKNOW, LUCKNOW, INDIA

Telephone: (91)

Facsimile: (91)

Telex:

Email:

Commission(s): 21,46

SAYGAC A TALAT, ISTANBUL UNIVERSITY OBS, UNIVERSITY 34452, ISTANBUL, TURKEY

Tclephone: (90) 2125223597

Facsimile: (90) $2125190834 \quad$ Telex:

Email: ik131@triuvm11

Commission(s):

SAZHIN MICHAIL DR, STERNBERG STATE ASTR INST, UNIVERSITETSKIJ PROSP 13, 119899 MOSCOW, RUSSIA

Telephone: (7) 959395006

Facsimile: (7)

Telex:

Email: snn@sai.msk.su

Commission(s): 47

SBIRKOVA-NATCHEVA T, PLANETARIUM AND PUBLIC, ASTRONOMICAL OBSERVATORY, BOX 136

BG 4700 SMOLJAN, BULGARIA

Telephone: (359) 3022953

Facsimile: (359)

Commission(s): 41,46

Telex:

Email:

SCALISE JR EUGENIO DR, INPE, CP 515, 12200 S JOSE DOS CAMPOS, BRAZIL

Telephone: (55) 123229977

Facsimile: (55) $1232187+3$

Commission(s): 40

Telex: 34061 inpe br

Email: 
SCALO JOHN MICHAEL, ASTRONOMY DPT, UNIVERSITY OF TEXAS, RLM 15308 , AUSTIN TX 78712 1083, USA

Telephone: (1) 5124714461

Facsimile: (1)

Commission(s): 34,35

Telex:

Email:

SCALTRITI FRANCO DR, OSS ASTRONOMICO DI TORINO, ST OSSERVATORIO 20, I 10025 PINO TORINESE, ITALY

Telephone: (39) 11841067

Facsimile: (39) $118+1281$

Telex: 213236 toastr $i$

Email:

Commission(s): 15,42

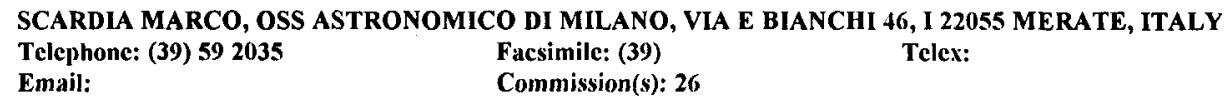

SCARFE COLIN D DR, DPT PHYSICS \& ASTRONOMY, UNIVERSITY OF VICTORIA, BOX 3055

VICTORIA BC V8W 3P6, CANADA

Telephone: (1) 6047217740

Email: scarfe@uvphys.uvic.ca

Facsimile: (1) $60+7217715$ Telex:

Commission(s): 26C,30P, $\$ 2$

SCARGLE JEFFREY D DR, NASA AMES RESEARCH CTR, MS 245 3, MOFFETT FIELD CA 94035, USA

Telephone: (1) 4156946330

Facsimile: (1)

Telex:

Email:

Commission(s): 44,51

SCARIA K K DR, INDIAN INSTITUTE OF, ASTROPHYSICS, KORAMANGALA, BANGALORE 560 034, INDIA

Telephone: (91) $803566585 \quad$ Facsimile: (91)

Commission(s): 37

Telex: 845763 iiab in

Email:

SCARROTT STANLEY M DR, DPT OF PHYSICS, UNIVERSITY OF DURHAM, SOUTH RD, DURHAM DH1 3LE, UK Telephone: (44)

Facsimilc: (4)

Commission(s): 34

Telex:

Email:

SCHAAL RICARDO E DR, CRAAE/ESCOLA POLITECNICA, UNIVERSIDADE DE SAO PAULO, CP 8174 05508 SAO PAULO SP, BRAZIL

Telephonc: (55) 118156289

Email: ccorrcia(a)brusp.bitnet
Facsimile: (55) $11815+272$

Commission(s): 40
Telex: 1180127 inpe br

SCHADEE AERT DR, STERREKUNDIG INSTITUTE, BOX 80000, NL 3508 TA UTRECHT, NETHERLANDS

Telephone: (31) $30535200 \quad$ Facsimile: (31)

Commission(s): 14

Email: bitnet/wnmaacrs(a)hutruu(0)

Telex: $400+8$ fylut $n$

SCHAEFER BRADLEY E DR, NASA GSFC, CODE 661 , GREENBELT MD 20771, USA

Telephone: (1) 3012866955

Facsimile: (1)

Telex:

Email: batse1::schaefer

Commission(s): 27,41

SCHAEFER GERHARD DR, MAX PLANCK GESELLSCAFT, AG GRAVITATIONSTHEORIE, MAX WIEN PLATZ 1 D O7745 JENA, GERMANY

Telephone: (49) 36418225263

Email:

Facsimile: (49) $36+18225186 \quad$ Telex:

Commission(s): 44

SCHAEFER MARTHA W, YALE UNIVERSITY, DPT GEOLOGY \& GEO PHYS, BOX 208109

NEW HAVEN CT 065208109 , USA

Facsimilc: (1) $203+323134$

Telex:

Email: schaefer@syrtis.geology.yaale.edu Commission(s):

SCHAEFFER RICHARD DR, CEA CEN, PHYSIQUE THEORIQUE, BP 2, F 91191 GIF/YVETTE CDX, FRANCE

Telephone: (33) 169087376

Facsimile: (33)

Email:

Commission(s):

Telex: 690641

SCHAIFERS KARL DR, STEINBACHWEG 37, D 69000 HEIDELBERG, GERMANY

Telephone: (49) $6221801511 \quad$ Facsimile: (49) Telex:

Email:

Commission(s):

SCHALLER GERARD DR, UNIVERSITE DE GENEVE, UNI DUFOUR, SERVICE INFORMATIQUES

CH 1211 GENEVE 4, SWITZERLAND

Telephonc: (41) 227057187

Facsimile: (41) 227057986

Telex:

Email: schaller@jdivsun.unige.ch

Commission(s): 
SCHANDA ERWIN PROF, PHYSIKALISCHES INSTITUT, UNIVERSITAET BERN, SIDLERSTRASSE 5 CH 3012 BERN, SWITZERLAND

Telephone: (41) 31658910

Facsimile: $(+1)$

Telex: 32320

Email:

Commission(s):

SCHARMER GOERAN BJARNE, STOCKHOLM OBSERVATORY, S 133 36 SALTSJOEBADEN, SWEDEN

Telephone: (46) 87170195

Facsimile: (46) $8717+719 \quad$ Telex: 12972

Email: scharmer ăastro.su.sc

Commission(s): 36

SCHATTEN KENNETH H DR, DPT OF PHYSICS, VICTORIA UNIVERSITY, PRIVATE BAG

WELLINGTON, NEW ZEALAND

Telephone: (64)

Facsimile: (64)

Telex:

Email:

Commission(s): $10,35,44$

SCHATZMAN EVRY PROF, OBSERVATOIRE DE PARIS, SECTION DE MEUDON, DASGAL

F 92195 MEUDON PPL CDX, FRANCE

Telephone: (33) 145077873

Facsimilc: (33)

Telex: 201571

Email:

Commission(s): $34,35,44,47,49,51$

SCHECHTER PAUL L DR, DPT OF PHYSICS, MIT RM 6 206, BOX 165, CAMBRIDGE MA 02139, USA

Telephone: (1) 617253069

Facsimile: (1)

Telex:

Email: schech(a)achernar.mit.edu

Commission(s): $28,33,47$

SCHEEPMAKER ANTON DR, COSMIC RAY WORKING GROUP, HUYGENS LAB, WASSENAARSEWEG 78 NL 2300 RA LEIDEN, NETHERLANDS

Telephone: (31)

Facsimile: $(31)$

Telex:

Email:

Commission(s):

SCHEFFLER HELMUT PROF, CARL-ORFF-WEG 16, D 6906 LEIMEN 3, GERMANY

Telephone: (49)

Facsimile: (49)

Telex:

Email:

Commission(s):

SCHEIDECKER JEAN-PAUL DR, OCA OBSERV DE NICE, BP 229, F 06304 NICE CDX 4, FRANCE

Telephone: (33) 93890420

Facsimile: (33) 92003033

Telex: 460004 obsnice $f$

Email:

Commission(s):

SCHERB FRANK PROF, DPT OF PHYSICS, UNIVERSITY OF WISCONSIN, 1150 UNIVERSITY AVE MADISON WI 53706, USA

Telephone: (1) 6082626879

Email:

Facsimile: (1)

Telex:

Commission(s): 34,4 (4)

SCHERRER PHILIP H DR, CTR FOR SPACE SCIENCES \&, ASTROPHYSICS, STANFORD UNIV ERL, STANFORD CA 94305 4055, USA

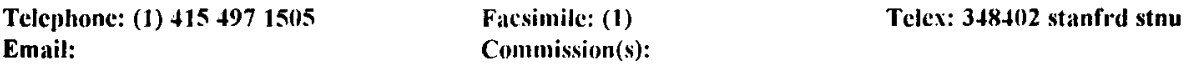

SCHEUER PETER A G DR, MULLARD RADIO ASTRON OBS, CAVENDISH LABORATORY, MADINGLEY RD

CAMBRIDGE CB3 OHE, UK

Telephone: (H) 1223337307

Facsimile: $(+4) 122335+509$

Telex: 81292

Email:

Commission(s): $34,40,44,47$

SCHILBACH ELENA DR, ZNTRLINST F ASTROPHYSIK, STERNWARTE BABELSBERG, ROSA LUXEMBURG STR 17 D $1+473$ POTSDAM, GERMANY

Telephone: (49)

Email:

Facsimile: $(49)$
Commission(s): $05,2+\mathrm{C}$

SCHILD HANSRUEDI, INST FUER ASTRONOMIE, ETH ZENTRUM, CH 8092 ZUERICH, SWITZERLAND

Telephone: (41) 12563633

Facsimile: (\$1) $12620003 \quad$ Telex:

Email: hschild(a)astro.phys.ctly..cl

Commission(s): 35,37

SCHILD RUDOLPH E DR, CENTER FOR ASTROPHYSICS, HCO/SAO, 60 GARDEN ST, CAMBRIDGE MA 02138, USA

Telephonc: (1) $617 \$ 957 \$ 26$

Facsimile: (1)

Telex: $921+28$ satellite cam

Email:

Commission(s): $29,45,51$ 
SCHILIZZI RICHARD T DR, NFRA, BOX 2, NL 7990 AA DWINGELOO, NETHERLANDS

Telephone: (31) 52197244

Facsimile: (31)

Telex:

Email:

Commission(s): $40,44,50$

SCHILLER KARL PROF DR, PIRSCHWEG 6, D 6072 DREIEICH BUCHSCHLA, GERMANY

Telephone: (49)

Facsimile: (49)

Telex:

Email:

Commission(s):

SCHILLER STEPHEN, PHYSICS DPT, SOUTH DAKOTA STATE UNIV, BOX 2219 ROOM $310 B$ BROOKINGS SD 57007, USA

Telephone: (1) 6056884293

Facsimile: (1)

Telex:

Email:

Commission(s): 42

SCHINDLER KARL PROF DR, INST FUER THEORET PHYSIK, RUHR UNIVERSITAET BOCHUM

D 44780 BOCHUM, GERMANY

Telephone: (49)

Email:

\author{
Facsimile: (49) \\ Telex: \\ Commission(s): 10,49
}

SCHLEGEL ERIC MATTHEW DR, NASA GSFC, CODE 668, LAB HIGH ENERGY ASTROPHYS

GREENBELT MD 20771, USA

Telephone: (1) 3012866636

Facsimile: (1) $30128633 \% 1 \quad$ Telex:

Email: cric@heasfs.gsfc.nasa.gov

Commission(s): 27

' SCHLEICHER DAVID G DR, LOWELL OBSERVATORY, 1400 W MARS HILL RD, BOX 1149

FLAGSTAFF AZ 86001, USA

Telephone: (1) 6027743358

Facsimile: (1)

Telex:

Email:

Commission(s): $15,16,46$

SCHLESINGER BARRY M DR, ST SYSTEMS CORP (STX), 4 H00 FORBES BLVD, LANHAM MD 20706, USA

Telephone: (1)

Facsimile: (1)

Telex:

Email:

Commission(s): 05

SCHLICKEISER REINHARD DR, MPI FUER RADIOASTRONOMIE, AUF DEM HUEGEL 69, D 53121 BONN, GERMANY Telephone: (49) 2285251

Facsimile: (49)

Commission(s): 40

Telex: 886440

Email:

ASTRONOMY, UNIV OF MASSACHUSETTS, GRC, AMHERST MA 01003, USA

SCHLOERB F PETER, DPT PHYSICS \& ASTRONOMY, UNIV OF MASSACHUSETTS,
Telcphone: (1) 4135454303
Facsimile: (1)

Email:

Commission(s): 15,16

SCHLOSSER WOLFHARD PROF, ASTRONOMISCHES INSTITUT, RUHR UNIVERSITAET BOCHUM POSTFACH 102148, D 4+780 BOCHUM, GERMANY

Tclephone: (49) $2347003454 \quad$ Facsimile: (49)

Email:

Commission(s): 46

Telex: 0825860

SCHLUETER A PROF DR, MPI FUER PLASMAPHYSIK, KARL SCHWARZSCHILDSTR 1 D 85740 GARCHING MUENCHEN, GERMANY

$\begin{array}{lll}\text { Telephone: (49) } 893299347 & \text { Facsimile: (49) } & \text { Telex: } 5215808 \text { ip|) d } \\ \text { Email: } & \text { Commission(s): 05,10 } & \end{array}$

SCHLUETER DIETER PROF, INST THEOR PHYS \& STERNW, NEUE UNIV PHYSIK ZENTRUM OLSHAUSENSTR GEB N 61C, D 24098 KIEL, GERMANY

Telephone: (49) $4318804109 \quad$ Facsimile: (49)

Email:

Commission(s):

Telex:

SCHMADEL LUTZ D DR, ASTRON RECHEN INSTITUTE, MOENCHHOFSTR 12-14, D 69120 HEIDELBERG, GERMANY Tclephone: (49) 622149026

Facsimile: (49)

Telex: 461336 arihd $d$

Email:

Commission(s): 05,20

SCHMAHL EDWARD J DR, ASTRONOMY PROGRAM, UNIVERSITY OF MARYLAND, COLLEGE PARK MD 20742, US Telephone: (1) 3014546074

Facsimile: (1)

Telex:

Email:

Commission(s): 10,12

SCHMALBERGER DONALD C DR, THE ALBANY ACADEMY, ACADEMY RD, ALBANY NY 12208, USA

Telephone: (1) 5184651461

Facsimile: (1)

Telex:

Email:

Commission(s): 36 
SCHMEIDLER F PROF DR, MAUERKIRCHERSTR 17, D 8000 MUENCHEN 80, GERMANY

Telephone: (49)

Facsimile: (49)

Telex:

Email:

Commission(s): 08

SCHMELZ JOAN T DR, NASA GSFC, CODE 602.6, SMM XRP, GREENBELT MD 20771, USA

Telephone: (1) $3012204164 \quad$ Facsimile: (1) $301220+171 \quad$ Telex: 248496

Email: solmax::jts

Commission(s): 10

SCHMID HANS MARTIN DR, MOUNT STROMLO OBSERVATORY, PRIVATE BAG, WESTERN CREEK PO CANBERRA ACT 2611, AUSTRALIA

Telephone: (61)

Facsimile: (61)

Telex:

Email:

Commission(s): 42

SCHMIDT EDWARD G, DPT PHYSICS \& ASTRONOMY, UNIVERSITY OF NEBRASKA, BEHLEN OBSERVATORY LINCOLN NE 68588 0111, USA

Telephone: (1) 4024722788

Facsimile: (1)

Telex:

Email:

Commission(s): 25,27

SCHMIDT H U DR, MPI F PHYSIK \& ASTROPHYS, KARL SCHWARZSCHILDSTR 1

D 85740 GARCHING MUENCHEN, GERMANY

Telephone: (49) 89329 99413/4 Facsimile: (49) Telex: 524629 astro d

Email:

Commission(s): $10,15,49$

SCHMIDT HANS PROF, UNIVERSITAETS STERNWARTE, AUF DEM HUEGEL 71, D 53121 BONN, GERMANY

Telephonc: (49)

Facsimile: (49)

Tclex:

Email:

Commission(s): 33,42

SCHMIDT K H DR, ZNTRLINST F ASTROPHYSIK, STERNWARTE BABELSBERG, ROSA LUXEMBURG STR 17A D 14473 POTSDAM, GERMANY

Telephone: (49)

Email:

Facsimile: (49)

Telex:

Commission(s): $05,28,33,44$

SCHMIDT MAARTEN PROF, CALTECH, MS 105 24, ASTRONOMY DPT, PASADENA CA 91125 , USA

Telephone: (1) 8183564204

Facsimile: (1)

Telex: 675425

Email:

Commission(s): $15,28,33,40,47$

SCHMIDT THOMAS DR, RUDOLF STEINER SCHULE, AN DER PROBSTEI 23, D 4800 BIELEFELD 1, GERMANY

Telephone: (49) 521880407

Facsimilc: $(49)$

Telex:

Email:

Commission(s): 3ł,46

SCHMIDT WOLFGANG DR, KIEPENHEUER INSTITUT, FUER SONNENPHYSIK, SCHOENECKSTR 6 D 79104 FREIBURG BREISGAU, GERMANY

Telephonc: (49) $761382067 \quad$ Facsimile: (49) $76132280 \quad$ Telex: 7721552 kis d

Email:

Commission(s): 12

SCHMIDTKE PAUL C DR, DPT OF PHYSICS, ARIZONA STATE UNIVERSITY, TEMPE AZ 85287, USA

Telephone: (1) 6029652918

Facsimile: (1)

Telex:

Email: bitnet:schmidtke(a) asucps

Commission(s): 26,42

SCHMIDT-KALER TH PROF, ASTRONISCHES INSTITUT, RUHR UNIVERSITAET BOCHUM, STEINHUEGEL 10S D 5810 WITTEN, GERMANY

Telephone: (49) 2347003454

Facsimile: (49)

Telex: 0825860

Email:

Commission(s): $33,34,45$

SCHMID-BURGK J DR PROF, MPI FUER RADIOASTRONOMIE, AUF DEM HUEGEL 69, D 53121 BONN, GERMANY

Telephone: (49) 228525271

Facsimile: $(49)$

Telex: $8864+10$

Email:

Commission(s): $3+, 36$

SCHMIEDER BRIGITTE DR, OBSERVATOIRE DE PARIS, SECTION DE MEUDON

F 92195 MEUDON PPL CDX, FRANCE

Telephone: (33) 145077817

Facsimile: (33)

Telex:

Email:

Commission(s): 10C 
SCHMITT DIETER DR, UNIVERSITAETS STERNWARTE, GOETTINGEN, GEISMARLANDSTR 11 D 37083 GOETTINGEN, GERMANY
Telcphone: (49) 551395046
Facsimile: (49)
Telex: 96753
Email: bitnet:dschmit@dgogwdg1
Commission(s): 12
SCHMITTER EDWARD F DR, DPT OF PHYSICS, UNIVERSITY OF LAGOS, AKOKA, LAGOS, NIGERIA
Telephone: (234) 1837864 Facsimile: (234) Telex:
Email:
Commission(s): 46

SCHMUTZ WERNER, INSTITUT FUER ASTRONOMIE, ETH ZENTRUM, CH 8092 ZUERICH, SWITZERLAND

Telephone: (41) 12563806

Facsimile: (41)

Telex:

Email:

Commission(s): 36

SCHNEIDER DONALD P, DPT OF ASTRONOMY, PENNSYLVANIA STATE UNIV, 525 DAVEY LAB

UNIVERSITY PARK PA 16802, USA

Telephone: (1) 8148639554

Email: dps(a)astro.psu.edu

Facsimile: (1) $8148633399 \quad$ Telex:

Commission(s): 47

SCHNEIDER GLENN H DR, STCTI, HOMEWOOD CAMPUS, 3700 SAN MARTIN DR, BALTIMORE MD 21218, USA

Telephone: (1) 3013384717

Facsimilc: (1)

Commission(s):

Telex: 6849101 stsci

Email: gschncider(a)scivax

SCHNEIDER HARTMUT DR, UNIVERSITAETS STERNWARTE, GOETTINGEN, GEISMARLANDSTR 11

D 37083 GOETTINGEN, GERMANY

$\begin{array}{lll}\text { Telephone: (49) } 551395042 & \text { Facsimile: (49) } 551395043 & \text { Telex: } 96753 \\ \text { Email: bitnet: hschnei(a)dgogwdg1 } & \text { Commission(s): } 27 & \end{array}$

SCHNEIDER JEAN, OBSERVATOIRE DE PARIS, SECTION DE MEUDON, F 92195 MEUDON PPL CDX, FRANCE

Telephonc: (33) 145077430

Email: schneider(a)frmeusi

Facsimile: (33)

Telex: 201517

SCHNEIDER NICHOLAS M, LASP, UNIVERSITY OF COLORADO, CAMPUS BOX 392, BOULDER CO 80309, USA

Telephone: (1) 3034927672

Facsimile: (1) $3034926946 \quad$ Telex:

Email: nick@pelc.colorado.cdu

Commission(s): 9,16

SCHNEIDER PETER DR, MPI F PHYSIK \& ASTROPHYS, KARL SCHWARZSCHILDSTR 1

D 85740 GARCHING MUENCHEN, GERMANY

$\begin{array}{lll}\text { Telephone: (49) } 8932990 & \text { Facsimile: }(49) & \text { Telex: } 524629 \\ \text { Email: } & \text { Commission(s): } 47 & \end{array}$

SCHNELL ANNELIESE DR, INSTITUT FUER ASTRONOMIE, UNIVERSITAET WIEN, TUERKENSCHANZSTR 17 A 1180 WIEN, AUSTRIA

Telephonc: (43) 134536093

Email:

Facsimile: (43)

Commission(s):

Telex:

SCHNEPS MATTHEW H, CENTER FOR ASTROPHYSICS, HCO/SAO, 60 GARDEN ST, CAMBRIDGE MA 02138, USA

Telephone: (1) $6174957472 \quad$ Facsimile: (1)

Telex: 921428 satellite cam

Email:

Commission(s):

SCHNOPPER HERBERT W DR, DANISH SPACE RESEARCH INS, LUNDTOFTEVEJ 7, DK 2800 LYNGBY, DENMARK

Telephone: (45) $\$ 2882277 \quad$ Facsimile: (45)

Email:

Commission(s): $4+$

Telex: 37198 danru

SCHNUR GERHARD F O, ASTRONOMISCHES INSTITUT, RUHR UNIVERSITAET BOCHUM, POSTFACH 102148 D 44780 BOCHUM, GERMANY

Telephone: (49)

Facsimile: (49)

Telex:

Email:

Commission(s):

SCHOBER HANS J DR, INSTITUT FUER ASTRONOMIE, UNIVERSITAETSPLATZ 5, A 8010 GRAZ, AUSTRIA

Telephone: (43) 3163805273

Facsimile: (43)

Email:

Commission(s): $10,12,15,20,42,51$

Telex: 31078 obsigz

SCHOEFFEL EBERHARD F DR, MERIANERSTR +2, D 8600 BAMBERG, GERMANY

Telephone: (49)

Facsimile: (49)

Telex:

Email:

Commission(s): $\$ 2$ 
M 808

SCHOEMBS ROLF DR, INST ASTRON \& ASTROPHYSIK, UNIVERSITAETS STERNWARTE, SCHEINERSTR 1 D 81679 MUENCHEN, GERMANY

Telephone: (49) 89989021

Facsimile: (49) 8992209427

Telex:

Email:

Commission(s): 27

SCHOENBERNER DETLEF PROF, ASTROPHYSIKALISCHES INST, POTSDAM, TELEGRAFENBERG A 27

D 14473 POTSDAM, GERMANY

Telephone: (49) 331310603

Facsimile: (49) 331310223

Telex:

Email: deschoenberner(a) aip.de

Commission(s): 35,36

SCHOENEICH W DR, ZNTRLINST F ASTROPHYSIK, STERNWARTE BABELSBERG, ROSA LUXEMBURG STR 17A D 14473 POTSDAM, GERMANY

Telephone: (49)

Email:

Facsimile: (49)

Telex:

Commission(s): 25,44

SCHOENFELDER VOLKER DR, MPI F EXTRATERRESTRISCHE, PHYSIK, KARL SCHWARZSCHILDSTR 1 D 85740 GARCHING MUENCHEN, GERMANY

Telephone: (49) 893299578 Facsimile: (49)

Telex: 5215845 xter d

Email:

Commission(s):

SCHOLL HANS DR, OCA OBSERV DE NICE, BP 229, F 06304 NICE CDX 4, FRANCE

Telephone: (33) $93890420 \quad$ Facsimile: (33) 92003033 Telex:

Email:

Commission(s): $07,15,20$

SCHOLZ GERHARD DR, ZNTRLINST F ASTROPHYSIK, AKAD WISSENSCHAFTEN DDR ROSA LUXEMBURG STR 17A, D 14473 POTSDAM, GERMANY

Telephone: (49)

Facsimile: (49)

Telex:

Email:

Commission(s): 24,29

SCHOLZ M PROF, INST F THEOR ASTROPHYSIK, DER UNIVERSITAET, IM NEUENHEIMER FELD 561 D 69120 HEIDELBERG, GERMANY

Telephone: (49)

Facsimile: (49)

Telex:

Email: b15@dhdurz2.bitnet

Commission(s): 36

SCHOLZ RALF DIETER, WIP ASTROMETRIE, POTSDAM UNIVERSITY, AN DER STERNWARTE 16

D 14482 POTSDAM, GERMANY

Telephone: (49) 3317499336

Facsimile: (49) $3317499309 \quad$ Telex:

Email: rdscholz@a),aip.de

Commission(s): 24

SCHOOLMAN STEPHEN A DR, LOCKHEED PALO ALTO RES LB, DPT 9120 BLDG 255, 3251 HANOVER ST PALO ALTO CA 94304, USA

Telephonc: (1)

Facsimile: (1)

Commission(s):

Telex:

Email:

SCHRAMM DAVID N PROF, ASTRONOMY \& ASTROPHYS CTR, UNIVERSITY OF CHICAGO, 5640 S ELLIS AVE CHICAGO $1 \mathrm{~L}$ 60637, USA

Telephone: (1) 3129628202

Facsimile: (1)

Commission(s): $35,47,44$

Telex: 6871133 uncgo uw

Email:

SCHRAMM K JOCHEN, MEGAPHOT EV, GOJAPHOT, GOJENBERGSWEG 112, D 21029 HAMBURG, GERMANY

Telephone: (49) 4072524121

Facsimile: (49) 4072524198

Telex:

Email: jschramm(ahs.uni-hamburg.de Commission(s): 47

SCHRAMM THOMAS, HAMBURGER STERNWARTE, GOJENBERGSWEG 112, D 21029 HAMBURG, GERMANY

Telephone: (49) 4072524126

Facsimile: (49) 4072524198

Telex: 217884 hamst d

Email: tschramm(ahs.uni-hamburg.de Commission(s): 47

SCHREIBER ROMAN, INSTITUTE OF ASTRONOMY, N COPERNICUS UNIVERSITY, UL CHOPINA 12/18

PL 87100 TORUN, POLAND

Telephone: (48) 485626017

Email: schreibe@pltumk11

Facsimile: (48)

Commission(s): 49

Telex: 0552234 astr pl

SCHREIER ETHAN J DR, STSCI, HOMEWOOD CAMPUS, 3700 SAN MARTIN DR, BALTIMORE MD 21218, USA

Telephone: (1) 4103384740

Facsimile: (1) 4103382519

Telex:

Email:

Commission(s): 44 
SCHRIJVER C J DR, STERREKUNDIG INSTITUTE, BOX 80000, NL 3508 TA UTRECHT, NETHERLANDS

Telephone: (31) 30535224

Facsimilc: (31) 30531601

Telex:

Email: kschrijver(a)solar

Commission(s): 10C,36C

SCHRIJVER JOHANNES DR, SPACE RESEARCH LABORATORY, SRON, SORBONNELAAN 2

NL 3584 CA UTRECHT, NETHERLANDS

Telephone: (31) $30535600 \quad$ Facsimile: (31) $30540860 \quad$ Telex: 47224 astro $\mathrm{nl}$

Email: $\quad$ Commission(s): 14

SCHROEDER DANIEL J PROF, DPT PHYSICS \& ASTRONOMY, BELOIT COLLEGE, BELOIT WI 53511, USA

Telephone: (1) 6083653391

Facsimile: (1)

Telex:

Email:

Commission(s): 09,46

SCHROEDER KLAUS PETER DR, HAMBURGER STERNWARTE, GOJENSBERGSWEG 112

D 21029 HAMBURG, GERMANY

Telephone: (49) 4072524141

Facsimile: (49) $407252+198$

Telex: 217884

Email:

Commission(s): 29

SCHROEDER ROLF DR, MOEOERKENWEG 37, D 21029 HAMBURG, GERMANY

Telephone: (49) Facsimile: (49) Telex:

Email; $\quad$ Commission(s):

SCHROETER EGON H PROF, KIEPENHEUER INSTITUT, FUER SONNENPHYSIK, SCHOENECKSTR 6 D 79104 FREIBURG BREISGAU, GERMANY

$\begin{array}{lll}\text { Telephone: (49) } 76132864 & \text { Facsimile: (49) } & \text { Telex: } 7721552 \text { kis d } \\ \text { Email; } & \text { Commission(s): } 10 & \end{array}$

SCHROLL ALFRED DR, SONNENOBSERVATORIUM, KANZELHOEHE, A 9521 TREFFEN, AUSTRIA

Telephone: $(43) \quad 42 \$ 82717 \quad$ Facsimile: $(\$ 3)$

Telex: $\$ 5699$ solobs a

Email:

Commission(s):

SCHRUEFER EBERHARD DR, INSTITUT FUER ASTROPHYSIK, UNIVERSITAET BONN, AUF DEM HUEGEL 71

D 53121 BONN, GERMANY

Telephone: (49) 228733390

Facsimile: (\$9)

Commission(s):

Telex: $8864+0$

Email:

SCHRUTKA-RECHTENSTAMM PR, WILLERGASSE 27/4/7, A 1238 WIEN, AUSTRIA
Telephone: (43) 18848132
Facsimile: $(43)$

Email:

Commission(s): 20

SCHUBART JOACHIM DR, ASTRON RECHEN INSTITUTE, MOENCHHOFSTR 12-14

D 69120 HEIDELBERG, GERMANY

Telephone: (49) $6221+9026$

Email:

Facsimile: (49)

Commission(s): $07,15,20$

Telex: 461336 arihd d

SCHUCH NELSON JORGE, OBSERVATORIO NACIONAL, UFSM/CTRO TECNOLOGIA, CIDADE UNIVERSITARIA 97100 SANTA MARIA RS, BRAZIL

Telephone: (55) 552261616

Email:

Facsimile: (55)

Telex: 0552230 ufsm

Commission(s): $40,47,51$

SCHUECKER PETER, ASTRONOMISCHES INSTITUT, UNIVERSITAET MUENSTER, WILHELM KLEMM STR 10 D 48149 MUENSTER, GERMANY

Telephone: (49) 251839128

Facsimile: (49) 251833669

Telex: 892529 unims d

Email: peterácygnus.uni-muenster.de

Commission(s): 47

SCHUECKING E L DR, DPT OF PHYSICS, NEW YORK UNIVERSITY, NEW YORK NY 10003, USA

Telephone: (1)

Facsimile: (1)

Telex:

Email:

Commission(s): 28,47

SCHUESSLER MANFRED DR, KIEPENHEUER INSTITUT, FUER SONNENPHYSIK, SCHOENECKSTR 6 D 7910 \& FREIBURG BREISGAU, GERMANY

Telephone: (49) $7613286+$

Facsimile: (49)

Telex: 7721552 kis d

Email:

Commission(s): 10,12 
M 810

SCHULER WALTER DR, SONNENRAIN 15, CH 4533 RIEDHOLZ, SWITZERLAND

Telephone: (41) 65232055 Facsimile: (41) Telex:

Email: $\quad$ Commission(s): 31

SCHULTE D H DR, ITEK CORPORATION, 10 MAGUIRE RD, LEXINGTON MA 02173, USA

Telephone: (1) Facsimile: (1) Telex:

Email:

Commission(s):

SCHULTE-LADBECK REGINA E, DPT PHYSICS \& ASTRONOMY, UNIVERSITY OF PITTSBURGH, 3941 O'HARA ST PITTSBURGH PA 15260, USA

Telephone: (1) 4126249013

Facsimile: (1) $4126241833 \quad$ Telex:

Email: rslavms.cis.pitt.edu

Commission(s):

SCHULTZ ALFRED BERNARD DR, STSCI/CSC, HOMEWOOD CAMPUS, 3700 SAN MARTIN DR BALTIMORE MD 21218, USA

Telephone: (1) 301338504

Email: scivax::schultz

Facsimile: (1)

Telex: 6849101 stsci

SCHULTZ G V DR, MPI FUER RADIOASTRONOMIE, AUF DEM HUEGEL 69, D 53121 BONN, GERMANY

Telephone: (49) 228525291

Facsimilc: (49)

Telex: $886+40$

Email:

Commission(s): $09,28,34,40,44,47$

SCHULZ HARTMUT DR, ASTRONOMISCHES INSTITUT, RUHR UNIVERSITAET BOCHUM, POSTFACH 102148 D 44780 BOCHUM, GERMANY

Telephone: (49) 2347003454

Facsimile: (49)

Telex: 0825860

Email:

Commission(s): 28

SCHULZ ROLF ANDREAS, MPI FUER RADIOASTRONOMIE, AUF DEM HUEGEL 69, D 53121 BONN, GERMANY

Telephone: (49) 228525232

Facsimilc: (49)

Telex: 886440

Email:

Commission(s): 34,40

SCHUMACHER GERARD DR, OCA OBSERV DU CALERN, CAUSSOLS, F 06460 S VALLIER THIEY, FRANCE

Telephone: (33) 93426270

Facsimile: (33)

Telex: 460004

Email:

Commission(s): 47

SCHUMANN JOERG DIETER DR, OBSERVATORIUM HOHER LIST, UNIV STERNWARTE BONN, D 5568 DAUN, GERMANY

Telephone: (49) 65922937

Facsimile: (49)

Commission(s): 09

Telex:

Email:

TITUTO DE ASTRONOMIA, UNAM, APDO POSTAL 877

SCHUSTER WILLIAM JOHN DR, INST
22860 ENSENADA B CALIF, MEXICO

Telephone: (52) 7066783093

Facsimilc: (52)

Commission(s): 20,25

Telex: 56539 cice me

Email:

SCHUTTE WILLEM ALBERT, STERREWACHT, BOX 9513, NL 2300 RA LEIDEN, NETHERLANDS

Facsimile: (31) 71275819

Telex: 39058 astro $\mathrm{nl}$

Email: schutte(a)strwehem.Ieidenuniv.nl Commission(s):

SCHUTZ BERNARD F PROF, DPT OF PHYSICS, UNIV WALES COLLEGE, BOX 913, CARDIFF CF1 3TH, UK

Telephone: (44) $122287+785$

Facsimile: (44) 1222371921

Telex: 498635

Email:

Commission(s): 35

SCHUTZ BOB EWALD, ASTRONOMY DPT, UNIVERSITY OF TEXAS, AUSTIN TX 78712 1083, USA

Telephone: (1) $512+711356 \quad$ Facsimile: (1)

Email:

Commission(s): 19

Telex: $70+265$ csrutx ud

SCHWAN HEINER DR, ASTRON RECHEN INSTITUTE, MOENCHHOFSTR 12-14, D 69120 HEIDELBERG, GERMANY

Telephone: (49) 62214050

Facsimile: (49) $6221+0,5297$

Telex: 461336 arihd $d$

Email: s25 (âm)s.urz.uni-heidelherg.de

Commission(s): 04C, $08 \mathrm{VP}$

SCHWARTZ DANIEL A DR, CENTER FOR ASTROPHYSICS, HCO/SAO, 60 GARDEN ST, CAMBRIDGE MA 02138, USA

Telephone: (1) $617+957232$

Facsimile: (1)

Telex:

Emajl:

Commission(s): +4 
SCHWARTZ PHILIP R DR, NAVAL RESEARCH LABORATORY, CODE 4138, 4555 OVERLOOK AVE SW WASHINGTON DC 20375 5000, USA

Telephone: (1) 2027673391

Facsimile: (1)

Telex:

Email:

Commission(s): $27,34,40$

SCHWARTZ RICHARD D, DPT OF PHYSICS, UNIVERSITY OF MISSOURI, 8001 NATURAL BRIDGE RD ST LOUIS MO 63121, USA

Telephone: (1) 3145535025

Email:

Facsimile: (1)

Telex: $\mathbf{4} 7658$ umsl bookstor

Commission(s): 34

SCHWARTZ ROLF PH D, MPI FUER RADIOASTRONOMIE, AUF DEM HUEGEL 69, D 53121 BONN, GERMANY

Telephone: (49) $228525303 \quad$ Facsimile: (49)

Telcx:

Email:

Commission(s):

SCHWARTZ STEVEN JAY, ASTRONOMY UNIT, QUEEN MARY/WESTFIELD COLL, MILE END RD

LONDON E1 4NS, UK

Telephone: (44) $1719804811 * 3849$

Facsimile: (44)

Telex:

Email:

Commission(s): $12,4+, 49$

SCHWARZ HUGO E, ESO, CASILLA 19001, SANTIAGO 19, CHILE

Telephone: (56) 21213249

Facsimile: (56)

Commission(s):

Telex: 240853

Email:

FTRONOMICAL INST, BOX 800, NL 9700 AV GRONIN
Telex: 53572 stars n]

$\begin{array}{ll}\text { Telephone: (31) } 50116695 & \text { Facsimile: (31) } \\ \text { Email: } & \text { Commission(s): 28,34,40 }\end{array}$

Telex: $\mathbf{5 3 5 7 2}$ stars $\mathrm{nl}$

SCHWARZENBERG-CZERNY A, ASTRONOMICAL OBSERVATORY, WARSAW UNIVERSITY, AL UJAZDOWSKIE 4 PL 00 478 WARSAW, POLAND

Telephone: (48) 294011

Email: czerny(a)camk.edu.pl

Facsimile: (48) 22410046

Commission(s): 27

Telcx: 813978 zapan pl

SCHWARZSCHILD MARTIN PROF, K102 PENNSWOOD VILLAGE, NEWTOWN PA 18940, USA

Telephone: (1) 2155797885

Facsimile: (1)

Telex:

Ematil:

Commission(s): 35

SCHWEHM GERHARD DR, ESA/ESTEC, SSD, BOX 299, NL 2200 AG NOORDWIJK, NETHERLANDS

Telephone: (31) $1719865555 \quad$ Facsimile: (31) $171917400 \quad$ Telex: 39098

Email: $\quad$ Commission(s): 21,44

SCHWEIZER FRANCOIS DR, DPT TERRESTR MAGNETISM, CARNEGIE INST WASHINGTON 5241 BROAD BRANCH RD NW, WASHINGTON DC 20015, USA

Telephone: (1) $2029660863 \quad$ Facsimile: (1) Telex: $4+0427$ magn ui

Email: schweizer(âbmit.ciw.edu Commission(s): 28

SCHWEKENDIEK PETER, ASTRON RECHEN INSTITUTE, MOENCHHOFSTR 12-14

D 69120 HEIDELBERG, GERMANY

Telephone: (49) 6221405128

Facsimile: (49) $6221405297 \quad$ Telex:

Email: st1(a)mvs.urz.uni-heidelberg.de Commission(s): 08

SCIAMA DENNIS W DR, SISSA, ST COSTIERA 11, MIRAMARE, I 3+0) $1+$ TRIESTE, ITALY

Telephone: (39) $4022+118$

Facsimile: (39)

Telex: 460392

Email:

Commission(s): $28,44,47$

SCIORTINO SALVATORE DR, OSS ASTRONOMICO, UNIVERSITA DI PALERNO, PALAZZO DEI NORMANNI I 9013+ PALERMO, ITALY

Telephone: (39) 91657 (0451

Email: astropa(a) ipacuc.

Facsimile: (39) 91488900

Telex: 910402 astrop $\mathrm{i}$

SCONZO PASQUALE DR, 29 OLD MYSTIC ST, ARLINGTON MA 02174, USA

Telephone: (1) 6176469315

Facsimile: (1)

Telex:

Email:

Commission(s): 07 
SCOTT ELIZABETH L PROF, STATISTICS DPT, UNIVERSITY OF CALIFORNIA, 367 EVANS HALL BERKELEY CA 94720, USA

Telephone: (1) 4156422777

Facsimile: (1)

Telex: 910-366-7114 uc berk

Email:

Commission(s): 47

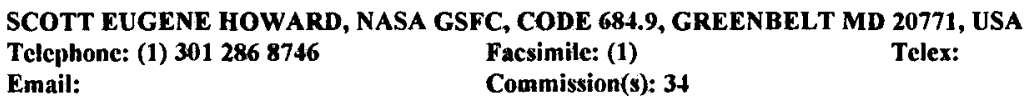

SCOTT JOHN S DR, STEWARD OBSERVATORY, UNIVERSITY OF ARIZONA, TUCSON AZ 85721, USA

Telephonc: (1)

Facsimile: (1)

Tclex:

Email:

Commission(s): 40,44

SCOTT PAUL F DR, MULLARD RADIO ASTRON OBS, CAVENDISH LABORATORY, MADINGLEY RD

CAMBRIDGE CB3 OHE, UK

Telephone: (44) 1223337294

Facsimile: (44) 1223354599

Telex: 81292

Email:

Commission(s): 40

SCOVILLE NICHOLAS Z, ASTRONOMY DPT, CALTECH, MS 105 24, PASADENA CA 91125, USA

Telephone: (1) 8183564979

Facsimile: (1)

Telex:

Email:

Commission(s): 28,34

SCRIMGER J NORMAN DR, DPT OF ASTRONOMY, ST MARY'S UNIVERSITY, HALIFAX NS B3H 3C3, CANADA

Telephone: (1) 9024205633

Facsimile: (1) $902 \$ 205561$

Telex:

Email:

Commission(s):

SCUFLAIRE RICHARD DR, INSTITUT D'ASTROPHYSIQUE, UNIVERSITE DE LIEGE, AVE COINTE S, B 4000 COINTELIEGE, BELGIUM

Telephonc: (32) 41529980

Email:

Facsimile: (32) $41527+74$

Telex: 41264 astrig b

Commission(s): 27,35

'SEAQUIST ERNEST R PROF, DPT OF ASTRONOMY, UNIVERSITY OF TORONTO, 60 ST GEORGE ST TORONTO ON MSS 1A1, CANADA

Telephone: (1) 4169783146

Facsimilc: (1) 4169783921

Telex: 06986766

Email:

Commission(s): 40

SEARLE LEONARD DR, CARNEGIE OBSERVATORIES, 813 SANTA BARBARA ST, PASADENA CA 91101, USA

Telephone: (1) 8185771122

Facsimile: (1) 8187958136

Telex: 1561318 ociw ut

Email:

Commission(s): 28

SEARS RICHARD LANGLEY DR, DPT OF ASTRONOMY, UNIVERSITY OF MICHIGAN, DENNISON BLDG

ANN ARBOR MI 48109 1090, USA

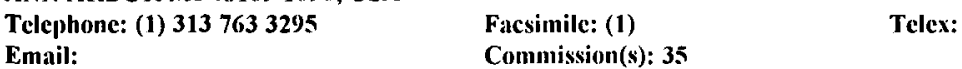

SEATON MICHAEL J PROF, DPT PHYSICS \& ASTRONOMY, UNIVERSITY COLLEGE LONDON, GOWER ST LONDON WC1E 6BT, UK

Telephone: (4) 1713877050

Email:

Facsimile: $(4 t)$

Commission(s): $12,14,34,36$

Telcx: $\mathbf{2 8 7 2 2}$

-SECCO LUIGI DR, DPT DI ASTRONOMIA, UNIVERSITA DI PADOVA, VIC DELL OSSERVATORIO 5

I 35122 PADOVA, ITALY

Telephone: (39) 49661499

Email:

Facsimilc: (39)

Conmission(s):

Telex: $\$ 30176$ unpadu $i$

SEDLMAYER ERWIN DR, INST ASTRONOMIE/ASTROPHYS, TECHNISCHE UNIVERSITAT, ERNST-REUTER-PL 7 D 10623 BERLIN, GERMANY

Telephone: (49)

Facsimile: (49)

Telex:

Email:

Commission(s): 36

SEDMAK GIORGIO PROF, DPT DI ASTRONOMIA, UNIVERSITA DI TRIESTE, VIA TIEPOLO 11

I 34131 TRIESTE, ITALY

Telephone: (39) 40794863

Facsimile: (39)

Telex: 461137

Email:

Commission(s): (09 
SEDRAKIAN DAVID, NTL ACADEMY OF SCIENCES, BAGRAMIAN 24, 375049 YEREVAN, ARMENIA

Telephonc: (7) 8852527051

Facsimile: (7)

Telex:

Email:

Commission(s): 28

SEEDS MICHAEL AUGUST DR, ASTRONOMY PROGRAM, FRANKLIN/MARSHALL COLLEGE

LANCASTER PA 17604 3003, USA

Telephonc: (1) 7172913800

Facsimile: (1) $717291+1+3 \quad$ Telex:

Email: bitnet\%"m_seeds(a)fandm"

Commission(s): 27,46

SEEGER CHARLES LOUIS III, SAN FRANCISCO STATE UNIV, 473 JAMES RD, PALO ALTO CA 9\$306, USA

Telephone: (1) 4154936005

Facsimile: (1)

Telex:

Email:

Commission(s): 51

SEEGER PHILIP A DR, LOS ALAMOS NATIONAL LAB, MS H805, BOX 1663, LOS ALAMOS NM 87545, USA

Telephone: (1) $50566788+3$

Facsimile: (1)

Telex:

Email:

Commission(s):

SEGAL IRVING E DR, DPT OF MATHEMATICS, MIT RM 224 , BOX 165, CAMBRIDGE MA 02139, USA

Telephone: (1) 6172534985

Facsimile: (1)

Telex:

Email:

Commission(s): 47

SEGALUVITZ ALEXANDER DR, BOX 659, KEFAR SAVA, ISRAEL

Telephone: (972)

Facsimile: (972)

Telex:

Email:

Commission(s):

SEGAN STEVO, INSTITUTE OF ASTRONOMY, UNIVERSITY OF BELGRADE, STUDENTSKI TRG 16, YU 11000 BEOGRAD, YUGOSLAVIA-SERBIA \& MONTENEGRO

Telephone: (38)

Email:

Commission(s): 07

Telex:

SEGGEWISS WILHELM PROF, OBSERVATORIUM HOHER LIST, UNIV STERNWARTE BONN

D 5568 DAUN, GERMANY

Telephone: (49) 65922150

Facsimile: (49)

Telex:

Email:

Commission(s): 29,33,42

SEHNAL LADISLAV DR, ASTRONOMICAL INSTITUTE, CZECH ACADEMY OF SCIENCES ONDREJOV OBSERVATORY, CZ 251 G5 ONDREJOV, CZECH R

Telephone: (\$2) 20\$857113/7111 Facsimile: (\$2) 204851611

Email: astdiracscarn Commission(s): 07

Telex: 121579

SEIDELMANN P KENNETH DR, US NAVAL OBSERVATORY, 3450 MASSACHUSETTS AVE NW WASHINGTON DC 203925100 , USA

Telephone: (1) $20265315+5$

Facsimile: (1) 2026.531744

Telex: 7108221970

Email: usnao ajphobos.usno.navy.mil

Commission(s): (0+C,07,20

SEIDEN PHILIP E, IBM, THOMAS J WATSON RES CTR, BOX 218, YORKTOWN HEIGHTS NY 10598, USA

Telephone: (1) 9149451424

Facsimile: (1)

Telex: $\mathbf{3 3 7 + 5 6}$

Email:

Commission(s): 28,47

SEIDOV ZAKIR F DR, SHEMAKHA ASTROPHYSICAL, OBSERVATORY, AZER ACADEMY OF SCIENCES 373243 SHEMAKHA, AZERBAIDZHAN

Telephone: (994)

Facsimile: (994)

Telex:

Email:

Commission(s): 35

SEIELSTAD GEORGE A, NRAO, BOX 2, GREEN BANK WV 24944, USA

Telephone: (1) $30+4562301$

Facsimile: (1)

Commission(s): $40,44, \downarrow 7,51$

Telex: 710-938-1530

Email:

SEIMENIS JOHN DR, DPT OF MATHEMATICS, UNIVERSITY OF THE AEGEAN, GR 83200 SAMOS, GREECE

Telephone: (30) 27333896

Facsimile: (30) 27333896

Telex: 294268 vasm gr

Email: jscim(ägrathun1

Commission(s): 33

SEIN-ECHALUCE M LUISA DR, DPT MATEMATICA APLICADA, UNIVERSIDAD DE ZARAGOZA

AVD MARIA ZAMBRANO 50, E 50009 ZARAGOZA, SPAIN

Telephonc: (34) 76518143

Facsimile: (34) 76565852

Telex: $\mathbf{5 8 1 9 8}$

Email:

Commission(s): 107 
SEIRADAKIS JOHN HUGH DR, DPT OF PHYSICS, UNIVERSITY THESSALONIKI, SEC ASTROPHYSICS \& ASTRON, GR 54006 THESSALONIKI, GREECE

Telephone: (30) 31998173

Facsimile: (30) 31995384

Telex:

Email: jhs@astro.auth.gr

Commission(s): 40,51

SEITTER WALTRAUT C PROF, ASTRONOMISCHES INSTITUT, UNIVERSITAET MUENSTER

WILHELM KLEMM STR 10, D 48149 MUENSTER, GERMANY

Telephone: (49) $251833561 \quad$ Facsimile: (49) 251833669

Telex: 892529

Email: waltraut@eygnus.uni-muenster.de Commission(s): 45

SEKANINA ZDENEK DR, JPL MS 169 237, EARTH \& SPACE SCI DIV, 4800 OAK GROVE DR, PASADENA CA 91109, US Telephone: (1) 8183547589 Facsimile: (1)

Email: zs@sek.jpl.nasa.gov

Commission(s): $15,20,22$

Telex:

SEKI MUNEZO DR, DPT OF EARTH SCIENCES, TOHOKU UNIVERSITY, KAWAUCHI, SENDAI 980, JAPAN

Telephone: (81) 222121800

Facsimile: (81)

Commission(s): 34

Telex:

Email:

SEKIGUCHI KAZUHIRO DR, DPT OF ASTRONOMY, NEW MEXICO STATE UNIV, BOX 4500

LAS CRUCES NM 88003, USA

Telephone: (1) 5056462613

Facsimile: (1)

Telex:

Email:

Commission(s):

SEKIGUCHI MAKI, NTL ASTRONOMICAL OBS, 211 OSAWA, MITAKA, TOKYO 181, JAPAN

Telephone: (81) $\$ 223+36+3$

Facsimile: $(81)+223+3776$

Telex:

Email: osckigu(acl.mtk.nato.ac.jp

Commission(s): 28

SEKIGUCHI NAOSUKE PROF, MUSASHIDAI 3-16-8, FUCHU, TOKYO 183, JAPAN

Telephone: (81)

Facsimile: (81)

Telex:

Enıil:

Commission(s): 19

SELLWOOD JERRY A, DPT PHYSICS \& ASTRONOMY, RUTGERS UNIVERSITY, BOX 849

PISCATAWAY NJ $0885+(0849$, USA

Telephone: (1)

Email:

Facsimile: (1) $908932+343$

Telex: 703528

Commission(s): 28,33

SELVELLI PIERLUIGI DR, OAT, BOX SUCC TRIESTE 5, VIA TIEPOLO 11, I 3+131 TRIESTE, ITALY

Telephone: (39) 40793221

Facsimile: (39)

Email:

Commission(s): $4+$

Telex: $\$ 61137$ ogt $i$

- SEMEL MEIR DR, OBSERVATOIRE DE PARIS, SECTION DE MEUDON, F 92195 MEUDON PPL CDX, FRANCE

Telephone: (33) $1+5077790$

Facsimile: (33)

Commission(s): 10,12

Telex:

Email:

SEMENIUK IRENA DR, ASTRONOMICAL OBSERVATORY, WARSAW UNIVERSITY, AL UJAZDOWSKIE \ PL 00 478 WARSAW, POLAND

$\begin{array}{lll}\text { Telephone: }(48) 29-40-11 / 12 & \text { Facsimile: }(48) & \text { Telex: } 815548 \text { oaluw } \\ \text { Email: } & \text { Commission(s): } 42 & \end{array}$

I SEMENZATO ROBERTO, DPT DI FISICA G GALILEI, UNIVERSITA DI PADOVA, VIA MARZOLO 8

I 35131 PADOVA, ITALY

Telephone: (39) $498+\$ 247$

Email:

Facsimile: (39)

Commission(s):

Telex: $\$ 30308$ off ggpd i

SEN S N DR, INDIAN ASSOCIATION FOR, THE CULTIVATION OF SCI, JADAVPUR, INDIA

Telephone: $(91)$

Facsimile: (91)

Telex:

Email:

Commission(s):

SENGBUSCH KURT V DR, MPI F PHYSIK \& ASTROPHYS, KARL SCHWARZSCHILDSTR 1 D 85740 GARCHING MUENCHEN, GERMANY

Telephone: (49)

Facsimile: (49)

Email:

Commission(s): 35

Telex: 
SHAHAM JACOB PROF, DPT OF PHYSICS, COLUMBIA UNIVERSITY, 538 W 120TH ST, NEW YORK NY 10027, USA Telephonc: (1) 2122803349

Facsimile: (1)

Telex: 220094 colu ur

Email:

Commission(s): 44

SHAKESHAFT JOHN R DR, MULLARD RADIO ASTRON OBS, CAVENDISH LABORATORY, MADINGLEY RD

CAMBRIDGE CB3 OHE, UK

Telephonc: (44) 1223337294

Email: jrs@phy-ravx.cam.ac.uk

Facsimile: (4) 1223354599

Telex: 81292 cavlab g

Commission(s): $05,28,40$

SHAKHBAZIAN ROMELIA K DR, BYURAKAN ASTROPHYSICAL, OBSERVATORY, 378 433 BYURAKAN, ARMENIA

Telephone: (7) 8852283453

Facsimile: (7) $88 \$ 228+142 \quad$ Telex:

Email:

Commission(s): 28

SHAKHBAZYAN YURIJ L DR, BYURAKAN ASTROPHYSICAL, OBSERVATORY, 378433 BYURAKAN, ARMENIA

Telephone: (7) 8852283435

Email:

Facsimile: (7) $885228+142$ Telex:

Commission(s): 09,

SHAKHOVSKAYA NADEJDA 1, CRIMEAN ASTROPHYS OBS, UKRAINIAN ACAD OF SCIENCE, NAUCHNY

33\$413 CRIMEA, UKRAINE

Telejhone: (7) 6554 71164

Email: nishôacrno.crimea.ua

Facsimile: (7) $6554+0704 \quad$ Telex:

Commission(s): 27

SHAKHOVSKOJ NIKOLAY M DR, CRIMEAN ASTROPHYS OBS, UKRAINIAN ACAD OF SCIENCE, NAUCHNY

$33 \$ 13$ CRIMEA, UKRAINE

Telephone: (7) 432945

Facsimile: (7)

Commission(s): 25

Telex:

Email:

SHAKURA NICHOLAJ I DR, STERNBERG STATE ASTR INST, $11723+$ MOSCOW, RUSSIA

Telephone: (7)

Facsimile: (7)

Commission(s): 42,44

Telex:

Email:

SHALLIS MICHAEL J DR, DPT OF ASTROPHYSICS, UNIVERSITY OF OXFORD, SOUTH PARKS RD

OXFORD OX1 3RQ, UK

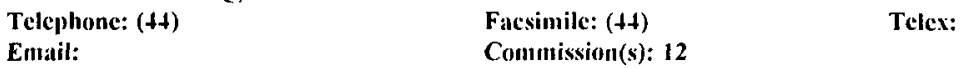

SHALTOUT MESALAM A M PROF, HELWAN OBSERVATORY, HELWAN, EGYPT

Telephone: (20) $7806+5 / 2683 \quad$ Facsimile: (20) Telex:

Email:

Commission(s):

SHANDARIN SERGEI F DR, INST FOR PHYSICS PROBLEMS, KOSYGIN 2, 117334 MOSCOW, RUSSIA

Telephone: (7) 951373248

Enail:

Facsimile: (7)

Commission(s): $47 \mathrm{C}$

Telex: $113+51$ magnit

SHANE WILLIAM W DR, 9095 COKER RD, PRUNEDALE CA 939017, USA

Telephone: (1)

Email:

Facsimile: (1)

Commission(s): 33,34

Telex:

SHANKS THOMAS, DPT OF PHYSICS, UNIVERSITY OF DURHAM, SOUTH RD, DURHAM DH1 3LE, UK

Telephone: ( $\$ 4) 19137+2171$

Facsimile: (44) $19137+3749$ Telex:

Email: tom.shanks(a)durhan.ac.uk Commission(s): 47

SHAO CHENG-YUAN, CENTER FOR ASTROPHYSICS, HCO/SAO, 60 GARDEN ST, CAMBRIDGE MA 02138, USA

Telephone: (1) $617+957212$

Facsimile: (1)

Commission(s): 22,34

Telex:

Email:

SHAPERO DONALD C DR, NTL ACADEMY OF SCIENCES, NTL RESEARCH COUNCIL, 2101 CONSTITUTION AVE N WASHINGTON DC 20418, USA

Telephone: (1) 2023343520

Facsimile: (1)

Email: bitnet:dshaperoünas

Commission(s):

Telex: 248664

SHAPIRO IRWIN I PROF, CENTER FOR ASTROPHYSICS, HCO/SAO RM P 2019, 60 GARDEN ST

CAMBRIDGE MA 02138, USA

Telephone: (1) $617+957100$

Facsimile: (1)

Email:

Commission(s): $04,07,16,19$

Telex: $921+28$ satellite cam 
SHAPIRO MAURICE M PROF, UNIVERSITY OF MARYLAND, SUITE ISI4, 205 YOAKUM PARKWAY ALEXANDRIA VA 22304, USA

Telephone: (1) 7033701985

Facsimile: (1)

Telex:

Email:

Commission(s): 44,51

SHAPIRO STUART L, CRSR, CORNELL UNIVERSITY, 306 SPACE SCIENCES BLDG, ITHACA NY 14853 6801, USA

Telephone: (1) 6072564936

Facsimile: (1)

Telex:

Email:

Commission(s): 34

SHAPLEY ALAN H, NOAA, 325 BROADWAY, BOULDER CO 80303, USA

Telephone: (1)

Facsimile: (1)

Telex:

Email:

Commission(s): 10

SHARA MICHAEL DR, STSCI, HOMEWOOD CAMPUS, 3700 SAN MARTIN DR, BALTIMORE MD 21218, USA

Telephone: (1) $301338+743$

Facsimile: (1)

Telex: 6849101 stsci uw

Email:

Commission(s): 27

SHARAF MOHAMED ADEL PROF, DPT OF ASTRONOMY, FACULTY OF SCIENCES, CAIRO UNIVERSITY GEZA, EGYPT

Telephone: (20)

Facsimile: (20)

Telex:

Email:

Commission(s):

SHARAF SH G DR, INST OF THEORET ASTRONOMY, ACADEMY OF SCIENCES, N KUTUZOVA 10 192187 ST PETERSBURG, RUSSIA

Telephone: (7)

Facsimile: (7)

Telex:

Email:

Commission(s): 07

SHARMA DHARMA PAL DR, DPT OF PHYSICS, UNIVERSITY OF TASMANIA, GPO BOX 252C HOBART TAS 7(101, AUSTRALIA

Telephone: (61) $2202+28 \quad$ Facsimile: (61) $2202+10 \quad$ Telex: an 58150

Email: sharma(âjphsvax,phys.utas.edu.a Commission(s): 27

SHAROV A S DR, STERNBERG STATE ASTR INST, UNIVERSITETSKIJ PROSP 13, 119899 MOSCOW, RUSSIA

Telephone: (7) 951392657

Facsimile: (7)

Telex:

Email:

Commission(s): 06,33,37

SHARP CHRISTOPHER DR, SERVICE D'ASTROPHYSIQUE, CEN SACLAY, F 91191 GIF SUR YVETTE, FRANCE

Telephone: (33)

Facsimile: (33)

Email:

Commission(s): 14,15

Telex:

SHARPLES RAY DR, DPT OF PHYSICS, UNIVERSITY OF DURHAM, SOUTH RD, DURHAM DH1 3LE, UK

Telephone: $(44)$

Facsimile: $(44)$

Telex:

Email:

Commission(s): 28

SHARPLESS STEWART PROF, DPT PHYSICS \& ASTRONOMY, UNIVERSITY OF ROCHESTER ROCHESTER NY $1+627$, USA

Telephone: (1) $716275 \mathbf{4 3 8 9}$

Facsimile: (1)

Telex:

Email:

Commission(s): 34,45

SHASTRI PRAJVAL, ASTRONOMY DPT, UNIVERSITY OF CALIFORNIA, 611 CAMPBELL HALL

BERKELEY CA $9+720$, USA

Telephone: (1) $5106+26708$

Facsimile: (1) $5106+23+11 \quad$ Telex:

Email: shastria ucdast.berkeley.edu

Commission(s):

SHAVER PETER A DR, ESO, KARL SCHWARZSCHILDSTR 2, D 85740 GARCHING MUENCHEN, GERMANY

Telephone: (49) 89 32006233

Facsimile: (49) 89 320 06480

Telex:

Email: pshaverácso.org

Commission(s): $28,34,40,44,47 \mathrm{VP}$

SHAVIV GIORA PROF, DPT OF PHYSICS, IIT, TECHNION CITY, HAIFA 32000, ISRAEL

Telephone: (972) 293020

Facsinile: (972) 221680

Commission(s): $35,+2,44,47$

Telex: 46406 tecon il

Email:

SHAW JAMES SCOTT DR, DPT PHYSICS \& ASTRONOMY, UNIVERSITY OF GEORGIA, ATHENS GA 30602, USA

Telephonc: (1) $40+5+22485$

Facsinile: (1)

Telex:

Email:

Commission(s): 
SHAW JOHN H PROF, DPT OF ASTRONOMY, OHIO STATE UNIVERSITY, $17+\mathrm{W}$ 18TH AVE

COLUMBUS OH 432101106 , USA

Telephone: (1) $614+227968$

Facsimile: (1)

Telex:

Email:

Commission(s):

SHAW R WILLIAM PROF, 105 HALCYON HILL, ITHACA NY 14850, USA

Telcphonc: (1) $60725719+8$

Facsimile: (1)

Comnission(s):

Telex:

Email:

\& ASTRONOMY, UNIVERSITY OF KA
Facsimile: (1)
Telex:

$\begin{array}{ll}\text { Telephone: (1) } & \text { Facsimile: (1) } \\ \text { Email: } & \text { Commission(s): 25,34,37 }\end{array}$

SHAYA EDWARD J DR, ASTRONOMY PROGRAM, UNIVERSITY OF MARYLAND, COLLEGE PARK MD 20742, USA

Telephone: (1)

Facsimile: (1)

Telex:

Email:

Commission(s): 28,47

SHCHEGLOV P V DR, STERNBERG STATE ASTR INST, UNIVERSITETSKIJ PROSP 13, 119899 MOSCOW, RUSSIA

Telephone: (7) 951391973

Facsimile: (7)

Telex:

Email:

Commission(s): $(09,3+, 50 \mathrm{C}$

SHCHEGOLEV DIMITRIJ E DR, PULKOVO OBSERVATORY, ACADEMY OF SCIENCES, 10 KUTUZOV QUAY 196140 ST PETERSBURG, RUSSIA

Telephone: (7)

Facsimile: (7)

Telex:

Email:

Commission(s):

SHCHERBINA-SAMOJLOVA I DR, INST OF SCIENCE \& TECH, 125219 MOSCOW, RUSSIA

Telephone: (7) $155+237$

Facsimile: (7)

Telex:

Email:

Commission(s): 05

SHEA MARGARET A DR, A F GEOPHYSICS LABORATORY, SPACE PHYSICS DIV PHC, HANSCOM AFB

BEDFORD MA 01732, USA

Telephone: (1)

Ficsimile: (1)

Telex:

Email:

Commission(s): 10, (1) $^{\prime}$

SHEARER ANDREW, DPT OF PHYSICS, UNIVERSITY COLLEGE GALWAY, GALWAY, IRELAND

Facsimile: (353) 91257700

Telex:

Email: shearerajeponit.physics.ncg.ic

Commission(s):

SHEELEY NEIL R DR, NAVAL RESEARCH LABORATORY, CODE 4172, 4555 OVERLOOK AVE SW

WASHINGTON DC 20375 5001), USA

Telephone: (1) 2027672777

Facsimile: (1)

Telex:

Email:

Commission(s): 10,12

SHEFFER EUGENE K DR, STERNBERG STATE ASTR INST, UNIVERSITETSKIJ PROSP 13, 119899 MOSCOW, RUSSIA

Telephone: (7) 951392046

Facsimile: (7)

Telex:

Email:

Commission(s):

SHEFFIELD CHARLES DR, EARTH SATELLITE CORP, ,011 EXECUTIVE BLVD, SUITE 400

ROCKVILLE MD 20852 3804, USA

Tclephone: (1) 3012310660

Facsimile: (1) 3012315020

Telex: 248618 esco ur

Email: cshefficarearthsat.com

Commission(s): 44

SHEFOV NICOLAI N, INST PHYSICS OF ATMOSPH, ACADEMY OF SCIENCES, PYZHEVSKY 3

109017 MOSCOW, RUSSIA

Telephone: (7)

Facsimile: (7)

Telex:

Email:

Commission(s): 21

SHELUS PETER J DR, ASTRONOMY DPT, UNIVERSITY OF TEXAS, RLM 15 316, AUSTIN TX 78712 1083, USA

Telephonc: (1) $512+713339$

Facsimile: (1)

Telex: 910-874-1351

Email:

Commission(s): 20,2t

SHEN BENJAMIN S P PROF, DPT OF ASTRONOMY E1, UNIV OF PENNSYLVANIA, PHILADELPHIA PA 19104, USA

Telephone: (1) 2158988176

Facsimile: (1)

Telex:

Email:

Commission(s): 
SHEN CHANGJUN, PURPLE MOUNTAIN OBSERV, CAS, NANJING, CHINA PR

$\begin{array}{ll}\text { Telephone: (86) } 2546700 & \text { Facsimile: (86) } 25301459 \quad \text { Telex: } 34144 \text { pmonj cn } \\ \text { Email: } & \text { Commission(s): } 09\end{array}$

SHEN CHUN-SHAN, ASTRONOMICAL STY OF CHINA, NTL TSING HUA UNIVERSITY, HSIN CHU 300043, CHINA R Telephone: (886) 35719039 Facsimile: (886) Telex:

Email:

Commission(s): 46,51

SHEN KAIXIAN, SHAANXI OBSERVATORY, CAS, LINTONG XIAN, SHAANXI, CHINA PR

Telephone: (86) 332255

Facsimile: (86)

Commission(s): 08,20

Telex: 70121 csao en

Email:

SHEN LIANG-ZHAO, BEIJING ASTRONOMICAL OBS, CAS, W SUBURB, BEIJING 100080, CHINA PR
Telephone: (86) 1281698
Facsimile: (86)

Email:

Commission(s): 42

SHEN LONG-XIANG, BEIJING ASTRONOMICAL OBS, CAS, W SUBURB, BEIJING 100080, CHINA PR

Telephone: (86) 1281698

Facsimile: $(86)$

Commission(s): 12

Telex: 22040 baoas en

Email:

SHEN PARN-AN, NANJING ASTRONOMICAL, INSTRUMENT FACTORY, BOX 846, NANJING, CHINA PR

Telephone: (86) 2546191

Facsimile: (86)

Telex: 34136 glynj c/o naif

Email:

Commission(s): 09

SHER DAVID DR, BOX 9624, CINCINNATI OH 452098, USA

Telephone: (1) $5138718850 \quad$ Facsimile: (1)

Telex:

mail:

Commission(s): 33,37

SHERIDAN K V DR, 17B/23 THORNTON ST, DARLING POINT NSW 2027, AUSTRALIA

Telephone: $(61)$

Facsimile: (61)

Telex:

Email:

Commission(s): 40

SHERWOOD WILLIAM A DR, MPI FUER RADIOASTRONOMIE, AUF DEM HUEGEL 69, D 53121 BONN, GERMANY

Telephone: (49) $228525362 \quad$ Facsimile: (49)

Email:

Commission(s): $27,28,34$

Telex: 886440

SHEVCHENKO VLADISLAV V DR, STERNBERG STATE ASTR INST, UNIVERSITETSKIJ PROSP 13 119899 MOSCOW, RUSSIA

Telephone: $(7)$

Facsimile: (7)

Telex:

Email:

Commission(s): 16

SHEVGAONKAR R K DR, DPT OF ELECTRICAL ENGG, IIT, POWAI, BOMBAY, INDIA

Telephone: (91) $225782545^{\star} 2440 \quad$ Facsimile: (91) $225783480 \quad$ Telex: 01172313

Email:

Commission(s): 40

SHI GUANG-CHEN, PURPLE MOUNTAIN OBSERV, CAS, NANJING, CHINA PR

Telephone: (86) $2533921 \quad$ Facsimile: (86) $25301459 \quad$ Telex: $341+4$ jmonj en

Email:

Commission(s): 08,24

SHI ZHONG-XIAN, BEIJING ASTRONOMICAL OBS, CAS, W SUBURB, BEIJING 100080, CHINA PR

Telephone: (86) 1281698

Facsimile: $(86)$

Telex: 9053

Email:

Commission(s): 10

SHIBAHASHI HIROMOTO DR, DPT OF ASTRONOMY, UNIVERSITY OF TOKYO, BUNKYO KU, TOKYO 113, JAPAN

Facsimile: (81) 338129439

Telex: 33659 utyosci $\mathrm{j}$

Email: shibahashi@astron.s.u-tokyo.ac.jp Commission(s): 35

SHIBAI HIROSHI, ISAS, 3-1-1 YOSHINODAI, SAGAMIHARA, KANAGAWA 229, JAPAN

Telephone: (81) $427513911 * 2602$

Facsimile: (81) 427594253

Telex:

Email: shibai@astro.isas.ac.jp

Commission(s): 4

SHIBASAKI KIYOTO, NOBEYAMA RADIO OBS, NAOJ, MINAMIMAKI MURA, NAGANO 384 13, JAPAN

Telephone: (81)

Facsimile: (81)

Telex:

Email:

Commission(s): 10 
SHIBATA KAZUNARI DR, NTL ASTRONOMICAL OBS, SOLAR PHYSICS DIV, MITAKA, TOKYO 181, JAPAN

Telephone: (81) $\$ 223+3712$

Facsimile: $(81)+223+3700 \quad$ Telex:

Email: shibata(a)spot.mtk.nao.ac.jp

Commission(s): 10

SHIBATA SHINPEI DR, DPT OF PHYSICS, YAMAGATA UNIVERSITY, KOJIRAKAWA, YAMAGATA 990, JAPAN

Tclephone: (81) 236311421

Facsimile: (81)

Telex:

Email: bitnct:b26416,ajpnkudpc

Commission(s):

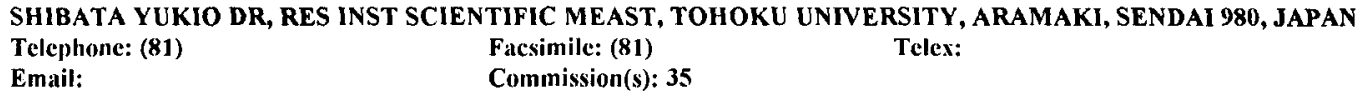

SHIELDS GREGORY A DR, ASTRONOMY DPT, UNIVERSITY OF TEXAS, RLM 15 212, AUSTIN TX 78712 1083, USA

Telephonc: (1) $512+71+461$

Facsimile: (1)

Telex: 910-874-1351

Email:

Commission(s): $28,34,44$

SHIGEYAMA TOSHIKAZU, DPT OF ASTRONOMY, UNIVERSITY OF TOKYO, BUNKYO KU, TOKYO 113, JAPAN
Telephone: (81) 338129224
Facsimile: (81) 338139439

Telephone: (81) 338129224

Email: shigeyama(a)astron.s.u-tokyo.ac.jp Commission(s): 44

\section{SHIMIZU MIKIO PROF, ISAS, 3-1-1 YOSHINODAI, SAGAMIHARA, KANAGAWA 229, JAPAN}

Telephone: $(81)+27513911$

Facsimile: (81)

Telex:

Email:

Comınission(s): $15,16,51$

SHIMIZU TSUTOMU PROF EMER, TERADA OOTANTI 26-16, JOYO SHI, KYOTO 61001 , JAPAN

Telephone: (81)

Facsimile: (81)

Telex:

Email:

Commission(s): 16,33

SHIMMINS ALBERT JOHN, 18 PAGE ST, ALBERT PARK VIC 3206, AUSTRALIA

Telephone: (61) 36903803

Facsimile: (61)

Telex:

Email:

Commission(s): 40

SHINE RICHARD A DR, LOCKHEED PALO ALTO RES LB, DPT 9130 BLDG 256, 3170 PORTER DR

PALO ALTO CA $9+30+1211$, USA

Telephone: (1) $415858+135$

Facsimile: (1)

Telex:

Email:

Commission(s): 10,12,36

SHIPMAN HENRY L DR, DPT OF PHYSICS, UNIVERSITY OF DELAWARE, NEWARK DE 19716, USA

Telephone: (1) $302+512986$

Facsimile: (1)

Telex:

Email:

Commission(s): 36,46

SHIRYAEV ALEXANDER A DR, ASRI TECHSAT PROJECT, IIT, TECHNION CITY, HAIFA 32000, ISRAEL

Telephone: (972) +292398

Facsimile: $(972)+230956$

Telex:

Email: astchsat $(\mathfrak{a}) \mathrm{msa}$.technion.ac.il

Conmission(s): 04

SHISHOV VLADIMIR I DR, LEBEDEV PHYSICAL INST, ACADEMY OF SCIENCES, LENINSKY PROSPEKT 53, 117924 MOSCOW, RUSSIA

Telephonc: (7)

Facsimile: (7)

Commission(s):

Telex:

Email:

SHIVANANDAN KANDIAH DR, NAVAL RESEARCH LABORATORY, CODE +138 S, 4555 OVERLOOK AVE SW WASHINGTON DC 203755000 , USA
Telephone: (1) 2027672749
Facsimile: (1)
Commission(s): $09,4+, 47$
Email:

Telex: 202-767-6473

SHKODROV V G DR, DPT OF ASTRONOMY, BULGARIAN ACAD SCIENCES, 72 LENIN BLVD

BG $178+$ SOFIA, BULGARIA

Telephone: (359) 2758927

Facsimile: (359)

Commission(s): 15,20

Telex: 23761 ecf ban bg

Email:

SHLOSMAN ISAAC, DPT PHYSICS \& ASTRONOMY, UNIVERSITY OF KENTUCKY, LEXINGTON KY 40506 0055, USA

Telephone: (1) 606 2573461

Facsimile: (1) $60632328+6$

Telex:

Email: shlosman(a)asta.pa.uky.edu

Commission(s): 
SHOBBROOK ROBERT R DR, DPT OF ASTRONOMY, UNIVERSITY OF SYDNEY, SYDNEY NSW 2OO6, AUSTRALIA Telephonc: (61) 26923604 Facsimile: (61) Telex: 26169 unisyd aa Email: Commission(s): 27,37

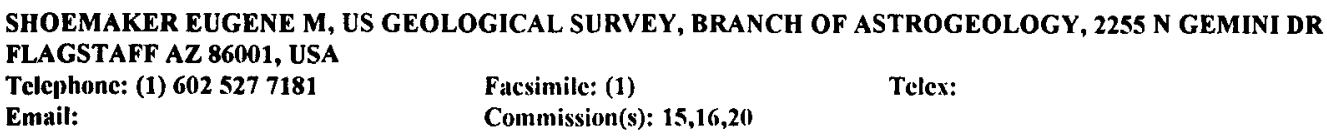

SHOLOMITSKY G B DR, SPACE RESEARCH INSTITUTE, ACADEMY OF SCIENCES, PROFSOJUZNAYA UL 84/32 117810 MOSCOW, RUSSIA

Telephone: (7) 95 333-31-22

Enual:
Facsimile: (7)

Commission(s): $40,+4$
Telex: $\$ 11498$ star su

SHOR VIKTOR A DR, INST OF THEORET ASTRONOMY, ACADEMY OF SCIENCES, N KUTUZOVA 10 191187 ST PETERSBURG, RUSSIA

Telephone: (7)

Email:

Facsimile: (7)

Telex: 121578

Commission(s): $15,20 \mathrm{C}$

SHORE BRUCE W, LAWRENCE LIVERMORE LAB, BOX 808, LIVERMORE CA 94550, USA

Telephone: (1) 4154471100

Ficsimile: (1)

Telex:

Email:

Commission(s): 14

SHORE STEVEN N, DPT PHYSICS \& ASTRONOMY, INDINIA UNIVERSITY, 1700 MISHAWAKA AVE SOUTH BEND IN $4663+7111$, USA

Telcphonc: (1) 2192374401

Facsimile: (1)

Telex:

Email:

Commission(s): $28,29,45$

SHORTRIDGE KEITH, ANGLO AUSTRALIAN OBS, BOX 296, EPPING NSW 2121, AUSTRALIA

Tclephone: (61) 23724822

Email: ks@aancpp.ano.gov.au

Facsinile: (61) 23724880

Telex:

Commission(s): 9

SHOSTAK G SETH DR, 1372 CUERNAVACA CIRC, MOUNTAIN VIEW CA 94(140, USA

Telephone: (1) $\$ 159678193$

Facsimile: (1)

Telex:

Email:

Commission(s): 28,51

SHU CHENGGANG, SHANGHAI OBSERVATORY, CAS, 80 NANDAN RD, SHANGHAI 200030, CHINA PR Telephone: (86) 214386191 Facsimile: (86) $21+38+618$ Telex:

Email:

SHU FRANK H PROF, ASTRONOMY DPT, UNIVERSITY OF CALIFORNIA, G01 CAMPBELL HALL BERKELEY CA 94720, USA

Telephone: (1) 4156422529

Email:

Facsimile: (1)

Telex:

Commission(s): $33,34,42$

SHUKLA K, DPT OF MATHS \& ASTRONOMY, UNIVERSITY OF LUCKNOW, LUCKNOW, INDIA

Telephone: (91)

Facsimile: (91)

Commission(s): 41

Telex:

Email:

SHUKRE C S DR, RAMAN RESEARCH INSTITUTE, SADASHIVANAGAR, CV RAMAN AVE BANGALORE 560080 , INDIA

Telephone: (91) 803360122

Facsimile: (91) $8033+0 \$ 92$

Telex: $8 \$ 25671$ rri in

Email: shukre(a)rri.ernet.in

Commission(s): 4

SHULL JOHN MICHAEL, JILA, UNIVERSITY OF COLORADO, BOX 440, BOULDER CO 80309 0440, USA

Telephone: (1) 3034927827

Facsimile: (1)

Telex:

Email:

Commission(s): $3+$

SHULL PETER OTTO DR, DPT OF PHYSICS, OKLAHOMA STATE UNIVERSITY, STILLWATER OK 74078 0444, USA Telephone: (1) 4057445785

Facsimile: (1) Telex:

Email: physpos(a)osucc.bitnet

Commission(s): $3+$ 
SHULOV OLEG S DR, ASTRONOMICAL OBSERVATORY, ST PETERSBURG UNIVERSITY, BIBLIOTECHNAJA PL 2 199178 ST PETERSBURG, RUSSIA

Telephone: (7)

Facsimile: (7)

Email:

Commission(s):

Telex:

\begin{tabular}{|c|c|c|}
\hline $\begin{array}{l}\text { Telephone: (7) } 048250356 \\
\text { Email: }\end{array}$ & $\begin{array}{l}\text { Facsimile: (7) } \\
\text { Commission(s): } 26,42\end{array}$ & Telex: \\
\hline
\end{tabular}

SHUL'MAN L M DR, MAIN ASTRONOMICAL OBS, UKRAINIAN ACAD OF SCIENCE, GOLOSEEVO 252127 KIEV, UKRAINE

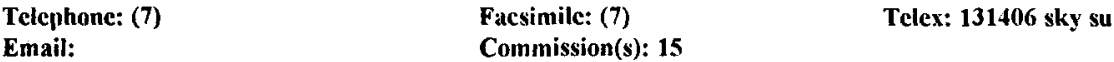

SHUSTOV. BORIS M DR, INST OF ASTRONOMY, ACADEMY OF SCIENCES, PYATNITSKAYA UL 48 109017 MOSCOW, RUSSIA

Telephone: (7) $952315+61$

Facsimile: (7)

Commission(s): 34,35

Telex: $\mathbf{4 1 2 6 2 3}$ scstp su

Email:

SHUTER WILLIAM L H DR, DPT OF PHYSICS, UNIV OF BRITISH COLUMBIA, 6224 AGRICULTURE RD VANCOUVER BC VGT 2A6, CANADA

Telephone: (1) $60+228+269$

Facsimile: (1) 6042285324

Telex: 04508576

Email:

Commission(s): $33,34,40,51$

SIBILLE FRANCOIS, OBSERVATOIRE DE LYON, AVE CHARLES ANDRE, F 69561 S GENIS LAVAL CDX, FRANCE Telephone: (33) 78560705 Facsimilc: (33) 72399791 Telex: 310926

Email:

Commission(s):

SICARDY BRUNO DR, OBSERVATOIRE DE PARIS, SECTION DE MEUDON, EUROPA F 92195 MEUDON PPL CDX, FRANCE

Telephone: (33) $145077409 \quad$ Facsimile: (33) $145077469 \quad$ Telex: 201571

Email: sicardy(a)frmeus1/mesioa::sicard Commission(s): 16

SIDLICHOVSKY MILOS DR, ASTRONOMICAL INSTITUTE, CZECH ACADEMY SCIENCES, BOCNI II 1401 CZ 14131 PRAHA 4, CZECH R

Telephone: (42) 2258757

Facsimile: (42) 2255010

Telex: 122486

Email: sidlich(a)csearn

Commission(s): 07

SIDORENKOV NIKOLAY S, HYDROMETEOROLOGICAL CTR, 123376 MOSCOW, RUSSIA

Telephone: (7) Facsimile: (7) Telex:

Email: $\quad$ Commission(s): 19

SIEBER WOLFGANG PH D, FACH HOCHSCHULE NIEDERRHEI, FACHBEREICH ELECTR, REINARZSTR 4969 D 4150 KREFELD 1, GERMANY

Telephone: (49) 21518220

Facsimile: (49)

Commission(s): 40

Telex:

Enail:

SIENKIEWICZ RYSZARD DR, COPERNICUS ASTRON CENTER, POLISH ACAD OF SCIENCES, UL BARTYCKA 18 PL 00716 WARSAW, POLAND

$\begin{array}{ll}\text { Telephone: (48) } 411086 & \text { Facsimile: (48) } \\ \text { Email: } & \text { Commission(s): } 35\end{array}$

Telex: 813878 zapan pl

SIGNORE MONIQUE DR, RADIOASTRONOMIE ENS, 24 RUE LHOMOND, F 75231 PARIS CDX 05, FRANCE

Telephone: (33) 145291225

Facsimile: (33)

Telex:

Email:

Commission(s): $35,41,44,47$

SIKORA MAREK, COPERNICUS ASTRON CENTER, POLISH ACAD OF SCIENCES, UL BARTYCKA 18

PL 00716 WARSAW, POLAND

Telephone: (48)

Email:

Facsimile: (48)

Commission(s): 44

Telex:

SIKORSKI JERZY DR, INST THEORETICAL PHYSICS, UNIVERSITY OF GDANSK, UL WITA STWOSZA 57 PL 80 952 GDANSK, POLAND

Telephone: (48) Facsimile: (48)

Email: fr.jks(ahalina.univ.gda.pl Commission(s):

Telex: 0512706 ifas pl 
SILANT'EV NIKOLAI, PULKOVO OBSERVATORY, ST PETERSBURG 196140, RUSSIA

Telephone: (7) $8121234090 \quad$ Facsimile: (7) $8123151701 \quad$ Telex:

Email: alexceva(aggoran.spb.su Commission(s):

SILBERBERG REIN DR, NAVAL RESEARCH LABORATORY, CODE 4154,4555 OVERLOOK AVE SW WASHINGTON DC 203755000 , USA

Telephone: (1) 2027672803

Facsimile: (1)

Telex:

Email:

Commission(s): $10,34,44$

SILICH SERGEY, MAIN ASTRONOMICAL OBS, UKRAINIAN ACAD OF SCIENCE, GOLOSEEVO 252127 KIEV, UKRAINE

Telephone: (7) 0442664771

Facsimile: (7) 0442242147

Telex: 131406 sky su

Email: maouas@gluk.apc.org

Commission(s): 34

SILK JOSEPH I PROF, ASTRONOMY DPT, UNIVERSITY OF CALIFORNIA, 601 CAMPBELL HALL BERKELEY CA 94720, USA

Telephone: (1) 4156422113

Email:

Facsimile: (1)

Telex: 820181 ucb ast

SILLANPAA AIMO KALEVI DR, TURKU UNIVERSITY OBS, TUORLA, SF 21500 PIIKKIO, FINLAND

Telephone: (358) 21435822

Email: aimosill@kontu.utu.fi

Facsimile: (358) $21433767 \quad$ Telex:

Commission(s): 28

SILVERBERG ERIC C DR, MCDONALD OBSERVATORY, UNIVERSITY OF TEXAS, BOX 1337

FT DAVIS TX 79734, USA

Telephone: (1)

Facsimile: (1)

Commission(s):

Telex:

Email:

SILVESTRO GIOVANNI, IST DI FISICA, UNIVERSITA DI TORINO, CORSO D AZEGLIO 46, I 10125 TORINO, ITALY

Telcphone: (39) 11658623

Facsimile: (39)

Commission(s): $34,35,44$

Telex: 211041 infinto

Email:

SIM MARY E MISS, ROYAL OBSERVATORY, BLACKFORD HILL, EDINBURGH EH9 3HJ, UK
Telephone: (44) 1316673321
Facsimile: (44)

Email:

Commission(s): (0)

SIMA ZDISLAV DR, ASTRONOMICAL INSTITUTE, CZECH ACADEMY SCIENCES, BOCNI II 1401

CZ 14131 PRAHA 4, CZECH R

Telephone: (42) 2258757

Facsimile: (42) 2255010

Telex: $122+86$

Email: sima(a)csearn

Commission(s): $07,+1,+2$

SIMEK MILOS DR, ASTRONOMICAL INSTITUTE, CZECH ACADEMY OF SCIENCES, ONDREJOV OBSERVATORY CZ 25165 ONDREJOV, CZECH R

Telephonc: (42) 204857252/7111

Facsimile: (42) 20+851611

Telex: 121579

Email: astmph@escarn

Commission(s): 22

SIMIEN FRANCOIS DR, OBSERVATOIRE DE LYON, AVE CHARLES ANDRE, F 69561 S GENIS LAVAL CDX, FRANCE

Telephone: (33) 78560705

Facsimile: (33) 72399791

Telex: 310926

Email:

Commission(s): 28

SIMKIN SUSAN M DR, PHYSICS \& ASTRONOMY DPT, MICHIGAN STATE UNIVERSITY

EAST LANSING MI 48824 , USA

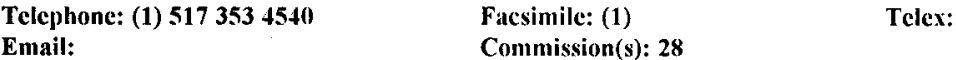

SIMMONS JOHN FRANCIS L, 31 HAVELOCK STREET, GLASGOW G11 5HA, UK

Telephone: (4) Facsimile: (4) Telex:

Email:

Commission(s): 42

SIMNETT GEORGE M, DPT OF SPACE RESEARCH, UNIVERSITY OF BIRMINGHAM, BOC 363 BIRMINGHAM B15 2TT, UK

Telephone: (44)

Email:
Facsimile: (44)

Commission(s): 10

Telex: 
SIMO CHARLES DR, FAC DE MATEMATICAS, UNIVERSIDAD DE BARCELONA, AV JOSE ANTONIO S85 E 08028 BARCELONA, SPAIN
Telephone: $(3+)$
Facsimile: (34)
Telex:
Email:
Commission(s):

SIMODA MAHIRO PROF, 1-362-6, SUZUKI, KODAIRA, TOKYO 187, JAPAN

Telephone: (81)

Facsimile: (81)

Telex:

Email:

Commission(s): 37

SIMON GEORGE W DR, AIR FORCE GEOPHYSICS LAB, NTL SOLAR OBSERVATORY, SUNSPOT NM 88349, USA

Telephone: (1) $505+3+1390$

Facsimile: (1)

Tclex:

Email:

Commission(s): 12

SIMON GUY, OBSERVATOIRE DE PARIS, SECTION DE MEUDON, F 92195 MEUDON PPL CDX, FRÁNCE

Telephone: (33) 145077787

Facsimile: (33)

Telex:

Enail:

Commission(s): 10,12

SIMON JEAN-LOUIS MR, BUREAU DES LONGITUDES, 77 AVE DENFERT ROCHEREAU, F 75014 PARIS, FRANCE

Telephone: (33) 143201210

Facsimile: (33)

Telex:

Email:

Commission(s): 04,07

SIMON KLAUS PETER, INST ASTRON \& ASTROPHYSIK, UNIVERSITAETS STERNWARTE, SCHEINERSTR 1 D 81679 MUENCHEN, GERMANY

Telephone: (49) 89989021

Facsimile: (49) 89922 09427

Telex: 529815 univm d

Email:

Commission(s): 36

SIMON MICHAL PROF, DPT OF EARTH \& SPACE SCI, ASTRONOMY PROGRAM, SUNY AT STONY BROOK STONY BROOK NY 1179+2100, USA

Telephone: (1) 5162467672

Facsimile: (1)

Telex: 510-228-7767

Email:

Commission(s):

SIMON NORMAN R PROF, DPT PHYSICS \& ASTRONOMY, UNIVERSITY OF NEBRASKA, BEHLEN OBSERVATORY

LINCOLN NE 685880111 , USA

$\begin{array}{ll}\text { Telephone: (1) } 4024722788 & \text { Facsimile: (1) } \\ \text { Email: } & \text { Commission(s): }\end{array}$

SIMON PAUL C DR, IASB, AVE CIRCULAIRE 3, B 1180 BRUSSELS, BELGIUM

Telcphonc: (32) 2375 1579) Facsimile: (32) Telex: 21563

Email:

Commission(s): 44

SIMON RENE L E PROF, INSTITUT D'ASTROPH YSIQUE, UNIVERSITE DE LIEGE, AVE COINTE 5

B 4000 COINTE-LIEGE, BELGIUM

Telcphone: (32) 41529980

Facsimile: (32) 41527474

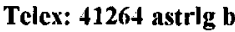

Email:

Commission(s): 47

SIMON THEODORE, INSTITUTE FOR ASTRONOMY, UNIVERSITY OF HAWAII, 2680 WOODLAWN DR

HONOLULU HI 96822 , USA

Telephone: (1) 8089566317

Facsimile: (1) 8089882790

Telex: 723-8459 uhast hr

Email:

Commission(s): 29,36

SIMONNEAU EDUARDO DR, INSTITUT D'ASTROPHYSIQUE, 98BIS BD ARAGO, F 75014 PARIS, FRANCE

Telephone: (33) 144328143

Facsimile: (33) 1 \$4 $328001 \quad$ Telex:

Email:

Commission(s): 36C

SIMONS STUART DR, SCHOOL OF MATHEMATICAL SC, QUEEN MARY/WESTFIELD COLL, MILE END RD LONDON E1 4NS, UK

Telephone: (4t) $171980+811$

Ficsimile: $(44)$

Telex:

Email:

Commission(s): 34

SIMONSON S CHRISTIAN DR, 1061 RUSSELL AVE, LOS ALTOS CA 94022, USA

Telephone: (1) $\$ 159680473$

Facsimile: (1)

Telex:

Email:

Commission(s): 33 
SIMOVLJEVITCH JOVAN L DR, DPT OF ASTRONOMY, FACULTY OF SCIENCES, STUDENTSKI TRG 16 YU 11000 BEOGRAD, YUGOSLAVIA-SERBIA \& MONTENEGRO

Telephone: (38) 11638715

Facsimile: (38)

Telex:

Email:

Commission(s):

SIMS KENNETH P DR, SYDNEY OBSERVATORY, OBSERVATORY PARK, SYDNEY NSW 2000, AUSTRALIA

Telephone: (61)

Facsimile: (61)

Telex:

Email:

Commission(s): 08,24

SINACHOPOULOS D DR, OBSERVATOIRE ROYAL DE, BELGIQUE, AVE CIRCULAIRE 3

B 1180 BRUSSELS, BELGIUM

Telephone: (32) 23730291

Email: dimitris@astro.oma.be

Facsimile: (32) 23749822

Telex: 21565 obsbel

Commission(s): 26

SINCLAIR ANDREW T DR, ROYAL GREENWICH OBS, MADINGLEY RD, CAMBRIDGE CB3 0EZ, UK

Telephone: (44) 1323833171

Facsimile: (44)

Telex: 87451 rgobsy g

Email:

Commission(s): 07,20

SINGH H P, ASTRONOMY UNIT, QUEEN MARY/WESTFIELD COLL, MILE END RD, LONDON E1 4AS, UK

Telephone: (44) 1719755440

Facsimile: (4) $1819819587 \quad$ Telex: 893750

Email: h.p.singh(a)qmw.ac.uk

Commission(s): 5

- SINGH JAGDEV DR, INDIAN INSTITUTE OF, ASTROPHYSICS, KORAMANGALA, BANGALORE 560 034, INDIA

Telephone: (91) $803566585 / 6497$

Facsimile: (91)

Commission(s): 12

Telex: 845763 iiab in

Email:

SINGH KULINDER PAL DR, TIFR, HOMI BHABHA RD, COLABA, BOMBAY 400 005, INDIA
Telephone: (91) 224952971

Email: bitnet:uunct!shakti!tifr!root Commission(s):

SINGH PATAN DEEN DR, IAG, UNIVERSIDADE DE SAO PAULO, CP 9638, 01065 SAO PAULO SP, BRAZIL

Telephone: (55) 112753720

Email: iagusp@brfapesp

Facsimile: (55) $1127638+8$

Telex: 1156735 iagm br

SINHA K DR, UTTAR PRADESH STATE, OBSERVATORY, PO MANORA PEAK 263 129, NAINITAL 263 129, INDIA

Telephone: (91) 59422136

Commission(s): 34

Email:

Facsimile: (91)

Commission(s): 10,12,14

Telex: cable : astronomy

SINHA RAMESHWAR P, TATA INST OF FUNDAMENTAL, RESEARCH, POONA UNIVERSITY CAMPUS PUNE 411 007, INDIA

Telephone: (91) 212337107

Facsimile: (91) 212335760

Telex: $0145658 \mathrm{gmrt}$ in

Email: sinha(a)gmrt.ernet.in

Commission(s): 40

SINNERSTAD ULF E PROF, STOCKHOLM OBSERVATORY, S 133 36 SALTSJOEBADEN, SWEDEN

Telephone: (46) 87170195

Facsimile: (46) $8717+719$

Telex:

Email:

Commission(s): 29,45

SINTON WILLIAM M, 850 E DAVID DR, FLAGSTAFF AZ 86001, USA

Tclephonc: (1) 6027748308

Facsimile: (1)

Telex:

Email:

Commission(s): 16

SINVHAL SHAMBHU DAYAL DR, 4/3 SNEHALATAGANG, INDORE 452 003, INDIA

Telephone: (91)

Facsimile: (91)

Telex:

Email:

Commission(s): 27,42

SINZI AKIRA M DR, HYDROGRAPHIC DPT, GEODESY \& GEOPHYSICS DIV, TSUKIJI 5 CHUO KU TOKYO 104, JAPAN

Telephone: (81)

Email:

Facsimile: (81)

Telex:

Commission(s): 0 -

SION EDWARD MICHAEL, DPT OF ASTRONOMY, VILLANOVA UNIVERSITY, VILLANOVA PA 19085, USA

Telephone: (1) $215645+822$

Email: $\operatorname{scion}\left(a_{3}\right.$ scivax.stsci.edu
Facsimile: (1)

Telex: 
SIREGAR SURYADI DR, DPT OF ASTRONOMY, BANDUNG INSTITUTE OF TECH, JL GANESHA 10 BANDUNG 40132, INDONESIA

$\begin{array}{ll}\text { Telephone: (62) } 84254^{*}+76 & \text { Facsimile: (62) } \\ \text { Email: } & \text { Commission(s): }\end{array}$

SIROKY JAROMIR DR, PALACKY UNIVERSITY, DPT PHYSICS \& ASTRONOMY, LENIN ST 26 CZ 771 46 OLOMOUC, CZECH R

Telephone: (42) 6822451

Facsimile: (42)

Commission(s): 46

Telex:

Email:

SIROUSSE ZIA HAYDEH, GRAVITATION \& COSMOLOGIE, RELATIVISTES UPMC TOUR 22, 4 PL JUSSIEU F 75252 PARIS CEDEX 15, FRANCE

Telephone: (33) 144277292

Email: strousse(a)cer.jussicu.fr

Facsimile: (33) $144277287 \quad$ Telex:

Commission(s):

SIRY JOSEPH W, 4438 42ND ST NW, WASHINGTON DC 20016, USA

Telephone: (1)

Facsimile: (1)

Telex:

Ematil:

Commission(s): 07

SiSSON GEORge M MR, PLANETREeS, WALL, HEXHAM NE46 4EQ, UK

Telephone: (44) 143481434

Facsimilc: (4)

Commission(s):

Telex:

Email:

SISTERO ROBERTO F DR, OBSERVATORIO ASTRONOMICO, DE CORDOBA, LAPRIDA 854 5000 CORDOBA, ARGENTINA

Telephonc: (54) 5123 (1491

Email: sistcro(a)astro.edu.ar

Facsimile: (54) 51210613

Telex: $\mathbf{5 1 8 2 2}$ bucor ar

SITARSKI GRZEGORZ PROF, SPACE RESEARCH CENTER, POLISH ACAD OF SCIENCES, UL ORDONA 21 PL 01237 WARSAW, POLAND

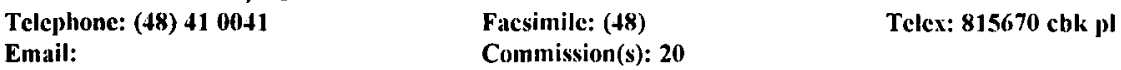

SITKO MICHAEL L, DPT OF PHYSICS, UNIVERSITY OF CINCINNATI, 210 BRAUNSTEIN ML 11 CINCINNATI OH 45221 0111, USA

Telephone: (1)

Email:

Facsimile: (1)

Telex:

Conmission(s): 28,34

SITNIK G F PROF, STERNBERG STA'TE ASTR INST, UNIVERSITETSKIJ PROSP 13, 119899 MOSCOW, RUSSIA Telephone: (7) 951391973

Facsimile: (7)

Telex:

Email:

Commission(s): $10,12,36$

SIVAN JEAN-PIERRE DR, LAS, TRAVERSE DU SIPHON, LES TROIS LUCS, F 13012 MARSEILLE, FRANCE

Telephone: (33) 91055900

Facsimile: (33) 91661855

Telex: $\mathbf{4 2 0 5 8 4}$ astrosp

Email: sivan@frlasm51

Commission(s): 34

SIVARAM C DR, INDIAN INSTITUTE OF, ASTROPHYSICS, KORAMANGALA, BANGALORE 560 034, INDIA

Telephonc: (91) 80 356 6585/6497

Facsimile: (91)

Telex: 845763 iiab in

Email:

Commission(s): 51

SIVARAMAN K R DR, INDIAN INSTITUTE OF, ASTROPHYSICS, KORAMANGALA, BANGALORE 560 034, INDIA

Telephone: (91) 803566585

Facsimile: (91)

Commission(s): 12,15

Telex: 845763 iiab in

Email:

ECH, MS 264664, 4800 OAK GROVE DR, PASADENA CA 91109, USA

SJOGREN WILLLAM L MR, JPL CALTECH, MS 264 664, 4800 OAK GROVE DR, PASADE
Telephone: (1) 8183544868
Facsimile: (1)

Email: $\quad$ Commission(s): 16

SKALAFURIS ANGELO J, NAVAL RESEARCH LABORATORY, CODE 5307, 4555 OVERLOOK AVE SW

WASHINGTON DC 20375 5000, USA

Telephone: (1) 3027673227

Facsimilc: (1)

Telex:

Email:

Commission(s): 
SKILLEN IAN DR, INSTITUTE OF ASTRONOMY, THE OBSERVATORIES, MADINGLEY RD

CAMBRIDGE CB3 OHA, UK

Telephone: (44) 1223337548

Facsimile: (4t) $1223337523 \quad$ Telex:

Email:

Commission(s):

SKILLING JOHN DR, DPT APPLIED MATHS, \& THEORETICAL PHYSICS, SILVER STREET

CAMBRIDGE CB3 9EW, UK

Telephone: (44) 1223337887

Email:

Facsimile: (44) 1223337918 Telex:

Commission(s): 34,44

SKILLMAN EVAN D DR, DPT OF ASTRONOMY, UNIVERSITY OF MINNESOTA, 116 CHURCH ST SE MINNEAPOLIS MN 55455, USA

Telephone: (1) 6126244523

Facsimile: (1) 6126262029

Telex:

Email: skillman@ast1.spa.umn.edu

Commission(s): 28,40

SKINNER GERALD DR, SCHOOL PHYSICS/RESEARCH, UNIVERSITY OF BIRMINGHAM, BOX 363 BIRMINGHAM B15 2TT, UK

Telephone: (44) 1214146450

Email: span 19457::bhvad::gks

Facsimile: $(44)$

Telcx: 338938

SKOPAL AUGUSTIN, ASTRONOMICAL INSTITUTE, SLOVAK ACADEMY SCIENCES

SK 05960 TATRANSKA LOMNI, SLOVAK R

Telephone: (42) 969967866

Facsimile: (42) 969967656

Telex:

Email: astrskop@a ta3.sk

Commission(s): 42

SKRIPYICHENKO VLADIMIR DR, INST OF APPLIED ASTRONOMY, ACADEMY OF SCIENCES, ZDANOVSKAYA UL 197042 ST PETERSBURG, RUSSIA

Telephone: (7)

Facsimile: (7)

Telex:

Email:

Commission(s): 07

SKULACHOV DMITRY, SPACE RESEARCH INSTITUTE, PROFSOJUZNAYA UL 84/32, MOSCOW 117810, RUSSIA

Telephone: (7) 0953332588

Email:

Facsimile: (7) 0953107023

Telex: 411498 a star su

SKULSKYJ MYCHAJLO Y, DPT OF PHYSICS, STATE UNIV LVIVSKA POLITE, S BANDERA STR 290646 LVIV, UKRAINE

Telephone: (7) 322744300

Email: msky(a)astro.lviv.ua

Facsimile: (7) $3227+4300 \quad$ Telex:

Commission(s): 34

SKUMANICH ANDRE PROF, HIGH ALTITUDE OBSERVATORY, NCAR, BOX 3000, BOULDER CO 80307 3000, USA

Telephone: (1) 3034971528

Facsimile: (1) $3034971568 \quad$ Telex: 45694

Email:

Commission(s): 12,36

SLADE MARTIN A III DR, JPL, MS 238 420, 4800 OAK GROVE DR, PASADENA CA 91109, USA

Telephone: (1) 8183546538

Facsimile: (1)

Telex:

Email:

Commission(s): 40

SLEE O B DR, CSIRO, DIV OF RADIOPHYSICS, BOX 76, EPPING NSW 2121, AUSTRALIA

Telephone: (61) 28680222

Email:

Facsimile: (61) 28680310

Telex: 26230 astro

Commission(s): 40

SLETTEBAK ARNE PROF, DPT OF ASTRONOMY, OHIO STATE UNIVERSITY, 174 W 18TH AVE

COLUMBUS OH 432101106, USA

Telephone: (1) 614292786

Facsimile: (1) $61+2922928 \quad$ Telex:

Email: slettebk(a)ohstpy.mps.ohio-state

Commission(s): $29,33,45$

SLEZAK ERIC DR, OCA OBSERV DE NICE, BP 229, F $1630+$ NICE CDX 4 , FRANCE

Facsimile: (33) 92003033

Telex: 470865

Email: slezak@obs-nice.fr/17499::slezak Commission(s): 28

SLONIM E M DR, ASTRONOMICAL INSTITUTE, UZBEK ACADEMY OF SCIENCES, 700000 TASHKENT, UZBEKISTA

Telephone: (7)

Facsimile: (7)

Telex:

Email:

Commission(s): 10 
SLOVAK MARK HAINES DR, PO BOX 751044, MEMPHIS TN 38175, USA

Telephone: (1)

Facsimile: (1) Telex:

Email: madraf::slovak

Commission(s): 09,42

SLYSH VJACHOSLAV I DR, SPACE RESEARCH INSTITUTE, ACADEMY OF SCIENCES, PROFSOJUZNAYA UL 84/32 117810 MOSCOW, RUSSIA

Telephone: (7)

Facsimile: (7)

Telex:

Email:

Commission(s): 40,51

SMAK JOSEPH I PROF, COPERNICUS ASTRON CENTER, POLISH ACAD OF SCIENCES, UL BARTYCKA 18 PL 00716 WARSAW, POLAND

Telephone: (48) 22410041

Email: jis@alfa.camk.edu.pl

Facsimile: (48) 22410828

Commission(s): 26,27,42,EC

Telex: 813978 zapan pl

SMALDONE LUIGI ANTONIO, DPT DI FISICA, UNIVERSITA DI NAPOLI, MOSTRA D OLTREMARE PAD 19

I 80125 NAPOLI, ITALY

Telephone: (39) 817253428

Facsimile: (39)

Telex: 720320 infinna i

Email:

Commission(s): 10

SMALE ALAN PETER, NASA GSFC, LAB HIGH ENERGY ASTROPHYS, CODE 668, GREENBELT MD 20771, USA

Telephone: (1) 3012867063

Facsimile: (1) 3012863391

Telex:

Email: smale@/heaux.gsfc.nasa.gov Commission(s): 44

SMALLEY BARRY, DPT OF PHYSICS, UNIVERSITY OF KEELE, KEELE ST5 SBG, UK

Telephone: (44) 1782621111

Facsimile: (44) 1782711093

Telex: 36113 unklib $g$

Email: bs@astro.keele.ac.uk

Commission(s): 29

SME YERS PAUL PROF, INST VOOR STERRENKUNDE, KU LEUVEN, CELESTIJNENLAAN 200B

B 3001 HEVERLEE, BELGIUM

Telephone: (32) 16200656

Facsimile: (32) 16201241

Telex:

Email:

Commission(s): 27,35

SMIRNOV MICHAEL, INSTITUTE OF ASTRONOMY, ACADEMY OF SCIENCES, 48 PYATNITSKAYA ST, MOSCO 109017, RUSSIA

Telephone: (7) 0952331624

Facsimile: (7) 0952302081

Telex: 411576 ascon

Email: ·rykhlova@inasan.rssi.ru

Commission(s):

SMIT J A PROF, STERREKUNDIG INSTITUTE, BOX 80000, NL 3508 TA UTRECHT, NETHERLANDS

Telephone: (31) 30535200

Facsimile: (31)

Telex:

Email:

Commission(s):

SMITH ALEX G PROF, DPT OF ASTRONOMY, UNIVERSITY OF FLORIDA, 211 SSRB, GAINESVILLE FL 32611, USA

Telephone: (1) 9043926135

Facsimile: (1)

Telex:

Email:

Commission(s): 40

SMITH ANDREW M DR, NASA GSFC, CODE 681, GREENBELT MD 20771, USA

Telephone: (1) $30128686+8$

Facsimile: (1)

Telex:

Email:

Commission(s):

SMITH BARHAM W DR, LOS ALAMOS NATIONAL LAB, MS D436, BOX 1663, LOS ALAMOS NM 87545, USA

Telephone: (1) 5056671585

Facsimilc: (1)

Telex:

Email:

Commission(s): 34,44

SMITH BRADFORD A PROF, 826012 PU'UHONUA RD, NAPO'OPO'O HI 96740 8226, USA

Telephone: (1)

Facsimile: (1)

Telex:

Email: basmith@galico.ifa.hawaii.edu

Commission(s): $15,16,44$

SMITH BRUCE F DR, NASA AMES RESREACH CTR, MS 245 3, THEORETICAL STUDIES BR

MOFFETT FIELD CA 94035 , USA

Telephone: (1) 4156945515

Facsimile: (1)

Telex:

Email:

Commission(s): 28

SMITH CHARLES DITTO, N PALMA BEACH FL 33410, USA

Telephone: (1) 4076224044

Facsimile: (1)

Telex:

Email:

Commission(s): 09,25 
SMITH CRAIG H DR, DPT PHYSICS UNIV COLLEGE, UNIVER OF NEW SOUTH WALES, NORTHCOTT DR CAMPBELL ACT 2600, AUSTRALIA

Telephone: (61) 62688790

Facsimile: (61) 62688786

Telex: 62030 adfadm an

Email: craig@phadfa.ph.adfa.oz.au

Commission(s): 34

SMITH DEAN F DR, BERKELEY RESEARCH ASS, 290 GREEN ROCK DRIVE, BOULDER CO 80302, USA
$\begin{aligned} & \text { Telex: } \\ & \text { Telphonc: (1) } 3034441922\end{aligned} \quad \begin{aligned} & \text { Facsimile: (1) } \\ & \text { Email: }\end{aligned}$

SMITH ELSKE V P DR, COLL HUMANITIES/SCIENCES, VIRGINIA COMMON UNIV, 900 PARK AVENUE

RICHMOND VA 23284, USA

Telephonc: (1) 8042571674

Facsimile: (1)

Telex:

Email:

Commission(s):

SMITH ERIC PHILIP DR, NASA GSFC, CODE 681, LASP, GREENBELT MD 20771, USA

Telephone: (1) 3012868549

Facsimile: (1) $3012868709 \quad$ Telex:

Email: esmith@hubble.gsfc.nasa.gov

Commission(s): 28

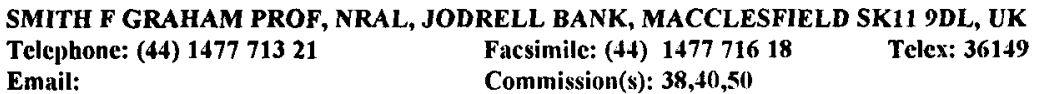

SMITH GEOFFREY DR, DPT OF ASTROPHYSICS, UNIVERSITY OF OXFORD, SOUTH PARKS RD

OXFORD OX1 3RQ, UK

Telephonc: (44) 1865511336

Facsimile: (44)

Telex:

Email:

Commission(s): 14

SMITH GRAEME H DR, LICK OBSERVATORY, UNIVERSITY OF CALIFORNIA, SANTA CRUZ CA 95064, USA

Telephone: (1)

Facsimilc: (1)

Telcx:

Email:

Commission(s): 29,37

SMITH HARDING E JR DR, CASS, UCSD, C 011, LA JOLLA CA 92093 0216, USA

Telephone: (1) $4195344558 \quad$ Facsimile: (1) $41953+2294$ Telex:

Email: 27778: harding (span) Commission(s): 28,47

SMITH HAYWOOD C DR, DPT OF ASTRONOMY, UNIVERSITY OF FLORIDA, 211 SSRB, GAINESVILLE FL 32611, US

Telephone: (1) 9043921079

Facsimile: (1)

Telex:

Email:

Commission(s): 28

SMITH HORACE A, PHYSICS \& ASTRONOMY DPT, MICHIGAN STATE UNIVERSITY, EAST LANSING MI 48824, USA

Telephone: (1) $5173536784 \quad$ Facsimile: (1)

Telex:

Email:

Conmmission(s):

SMITH HOWARD ALAN, NTL AIR \& SPACE MUSEUM, SMITHSONIAN INSTITUTION, WASHINGTON DC 20560, USA

Telephone: (1)

Facsimilc: (1) 2027862262

Telex: 264729

Email:

Commission(s): $34,44,51$

SMITH HUMPHRY M, 23 NORMANDALE, BEXHILL ON SEA TN39 3LU, UK

Telephone: (44) $1424214288 \quad$ Facsimile: (44) Telex:

Email:

Commission(s): 19,31

SMITH KEITH COLIN, DPT PHYSICS \& ASTRONOMY, UNIVERSITY COLLEGE LONDON, GOWER ST LONDON WC1E 6BT, UK

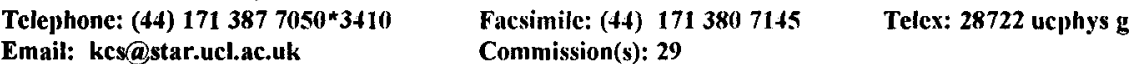

SMITH LINDA J, DPT PAYSICS \& ASTRONOMY, UNIVERSITY COLLEGE LONDON, GOWER ST LONDON WC1E 6BT, UK

Telephone: (44) $1713877050 * 788 \quad$ Facsimile: $(44) \quad$ Telex: 28722 ucphys g

Email:

Commission(s): 44

SMITH LINDSEY F DR, 6B/26 ETHAM AVE, DARLING POINT NSW 2027, AUSTRALIA

Telephone: (61) 23262536

Facsimile: (61) $23262536 \quad$ Telex:

Email: Ismith $a$ physics.su.oz.au

Commission(s): 
SMITH MALCOLM G DR, CTIO SUPPORT OFFICE, BOX 26732, TUCSON AZ 85726-6732, USA

Telephone: (1)

Facsimile: (1)

Telex:

Email:

Commission(s): 28

SMITH MYRON A ASST PROF, NTL SCIENCE FOUNDATION, DIV ASTRONOMICAL SCIENCES, 1800 G ST NW WASHINGTON DC 20550, USA

Telephone: (1)

Facsimile: (1)

Telex:

Email:

Commission(s): 27C,29,30

SMITH PETER L DR, CENTER FOR ASTROPHYSICS, HCO/SAO MS 50, 60 GARDEN ST, CAMBRIDGE MA 02138, USA

Telephone: (1) $6174954984 \quad$ Facsimile: (1)

Telex: 921428 satellite cam

Email: plsmith(a)cfa.harvard.edu

Commission(s): $12,1+C, 3+,+4$

SMITH ROBERT CONNON DR, ASTRONOMY CENTRE, UNIVERSITY OF SUSSEX, FALMER

BRIGHTON BN1 9QH, UK

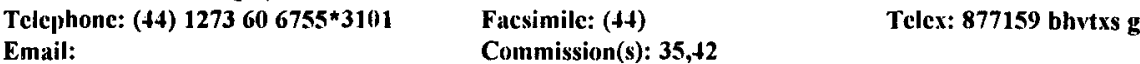

SMITH ROBERT G DR, DPT PHYSICS UNIV COLLEGE, UNIVER OF NEW SOUTH WALES, NORTHCOTT DR CAMPBELL ACT 2600, AUSTRALIA

Telephone: (61) 62688746

Facsimile: (61) 62688786

Tclex: adfadm aa 62030

Email: rgs(a)phadfa.ph.adfa.oz..au

Commission(s): 34

SMITH RODNEY M DR, DPT OF PHYSICS, UNIVERSITY OF DURHAM, SOUTH RD, DURHAM DH1 3LE, UK

Telephone: (4)

Facsimile: (4t)

Telex:

Email:

Commission(s): 47

SMITH VERNE V DR, ASTRONOMY DPT, UNIVERSITY OF TEXAS, RLM 15 308, AUSTIN TX 78712 1083, USA

Telephonc: (1) $512+713351$

Facsimile: (1)

Telex:

Email:

Commission(s): 29

SMITH WM HAYDEN PROF, DPT OF PHYSICS, WASHINGTON UNIVERSITY, MCDONNEL CTR SPACE SCI

ST LOUIS MO 63130, USA

Tclephonc: (1) 3148896574

Facsimile: (1)

Telex:

Email:

Commission(s): 14

SMITS DERCK P, HARTEBEESTHOEK RADIO, ASTRONOMY OBSERVATORY, BOX $\$ 43$

KRUGERSDORP 1740, SOUTH AFRICA

Telephone: (27) $11642+692$

Facsimile: (27) 116422424

Telex:

Email: derck@bootes.hartrao.ac.za Commission(s):

SMOLINSKI JAN DR, INSTITUTE OF ASTRONOMY, N COPERNICUS UNIVERSITY, UL CHOPINA 12/18

PL 87100 TORUN, POLAND

Tclephonc: (48)

Facsimile: (48)

Telex:

Email:

Commission(s): 29

SMOLUCHOWSKI ROMAN PROF, ASTRONOMY DPT, UNIVERSITY OF TEXAS, RLM 15314

AUSTIN TX 78712 1083, USA

Telephone: (1) 5124711305

Facsimile: (1)

Telex: 910-874-1351

Email:

Commission(s): 15,16

SMOL'KOV GENNADIJ YA DR, SIBIZMIR, ACADEMY OF SCIENCES, $66+697$ IRKUTSK 33, RUSSIA

Telephone: (7) 629388

Facsimile: (7)

Telex: $1+2+07$

Email:

Commission(s): 10,40

SMOOT III GEORGE F, LAWRENCE BERKELEY LAB, 1 CYCLOTRON RD, BLDG 50 230, BERKELEY CA 94720, USA

Telcphone: (1) $\$ 154865237$

Facsimilc: (1)

Telex:

Email:

Commission(s): 47

SMRIGLIO FILIPPO PROF, ISTITUTO ASTRONOMICO, UNIVERSITA DI ROMA, VIA G M LANCISI 29

I 00161 ROMA, ITALY

Telephone: (39) 68442977

Facsimile: (39)

Telex:

Email:

Commission(s): 
SMYLIE DOUGLAS E DR, DPT EARTH \& ATMOSPH SCI, YORK UNIVERSITY, 4700 KEELE ST DOWNSVIEW ON M3J 1P3, CANADA
Telephone: (1) 4167365245
Facsimile: (1)
Telex:

Email:

Commission(s): 31

SMYTH MICHAEL J DR, ROYAL OBSERVATORY, BLACKFORD HILL, EDINBURGH EH9 3HJ, UK

Telephone: (44) 1316673321

Facsimile: (4t)

Telex: 72383

Email:

Commission(s): 09,25

SNEDEN CHRISTOPHER A, ASTRONOMY DPT, UNIVERSITY OF TEXAS, RLM 15 308, AUSTIN TX 78712 1083, USA

Telephone: (1) 5124714461

Facsimile: (1)

Telex:

Email:

Commission(s): 29

SNELL RONALD L, FIVE COLLEGE, RADIO ASTRONOMY OBSERV, B619 LEDERLE DRAD RES TWR

AMHERST MA 01003, USA

Telephone: (1) 4135451949

Facsimile: (1)

Telex: $955+91$

Email:

Commission(s): 34

SNEZHKO LEONID I, SPECIAL ASTROPHYSICAL OBS, ACADEMY OF SCIENCES, NIZHNIJ ARKHYZ 357147 STAVROPOLSKIJ, RUSSIA

Telephone: (7) 93513

Facsimile: (7)

Telex: 297140 zenit

Email:

Commission(s): 09,36

SNIJDERS MATTHEUS A J DR, IRAM, 300 RUE DE LA PISCINE, F 38406 ST MARTIN D'HERES, FRANCE

Telephone: (33)

Facsimile: (33)

Telex:

Email:

Commission(s): 36

SNOW THEODORE P PROF, CASA CB 391, UNIVERSITY OF COLORADO, BOX 391, BOULDER CO 80309 0391, USA

Telephone: (1) 3034926857

Facsimile: (1)

Telex:

Email:

Commission(s): $29,34,4$

SNYDER LEWIS E, DPT OF ASTRONOMY, UNIVERSITY OF ILLINOIS, 1011 W SPRINGFIELS AVE URBANA IL 61801, USA

Telephone: (1) 2173335531

Facsimile: (1)

Telex: 910-245-2+34

Email:

Commission(s): 15,51

SOBERMAN ROBERT K DR, DPT ASTRON \& ASTROPHYS, UNIV OF PENNSYLVANIA, DAVID RITTENHOUSE LAB PHILADELPHIA PA 19104, USA

Telephone: (1) 2158988176

Facsimile: (1)

Telex:

Email:

Commission(s): 21,22

SOBIESKI STANLEY DR, NASA GSFC, CODE 673, GREENBELT MD 20771, USA

Telephone: (1)

Facsimile: (1)

Telex:

Email:

Commission(s): 42

SOBOLEV V V DR, ASTRONOMICAL OBSERVATORY, ST PETERSBURG UNIVERSITY, BIBLIOTECHNAJA PL 2 199178 ST PETERSBURG, RUSSIA

Telephone: (0)

Email:

Facsimile: (0)

Telex:

Commission(s): 34,36

SOBOLEV VLADISLAV M DR, PULKOVO OBSERVATORY, ACADEMY OF SCIENCES, 10 KUTUZOV QUAY 196140 ST PETERSBURG, RUSSIA

Telcphone: (7) $29822+2$

Facsimile: (7)

Telex:

Email:

Commission(s): 12

SOBOLEVA N S DR, PULKOVO OBSERVATORY, ACADEMY OF SCIENCES, 10 KUTUZOV QUAY 196140 ST PETERSBURG, RUSSIA

Telephone: (7)

Email:

Facsimile: (7)

Telex:

Commission(s): 40

SOBOTKA MICHAL DR, ASTRONOMICAL INSTITUTE, CZECH ACADEMY OF SCIENCES ONDREJOV OBSERVATORY, CZ 25165 ONDREJOV, CZECH R

Telephone: (42) 20+857249/7111 Facsimilc: (42) 20+851611

Email: astsun(a)csearn

Commission(s): 10

Telex: 121579 
SOBOUTI YOUSEF PROF, DPT OF PHYSICS, UNIVERSITY OF SHIRAZ, BIRUNI OBSERVATORY, SHIRAZ 71459, IRA

Telephone: (98) 7157339

Facsimile: (98)

Commission(s): $28,33,35$

Telex:

Email:

SOCHILINA ALLA S DR, INST TO THEORET ASTRONOMY, ACADEMY OF SCIENCES, N KUTUZOVA 10 191187 ST PETERSBURH, RUSSIA

Telephone: (7) 2788898

Email:

Facsimile: (7)

Telex: 121578 ita su

Commission(s): 04

SODEMANN M DR, INSTITUTE OF PHYSICS, \& ASTRONOMY, AARHUS UNIVERSITY

DK 8000 AARHUS C, DENMARK

Telephone: (0)

Facsimile: (0)

Telex:

Email:

Commission(s):

SODEMANN M DR, INSTITUTE OF PHYSICS, \& ASTRONOMY, AARHUS UNIVERSITY

DK 8000 AARHUS C, DENMARK

Telephone: (0)

Facsimile: (0)

Telex:

Email:

Commission(s):

SODERBLOM DAVID R, STSCI, HOMEWOOD CAMPUS, 3700 SAN MARTIN DR, BALTIMORE MD 21218, USA

Telephone: (1) 3013384830

Email:

Facsimilc: (1)

Telex: 6849101 stsci

Commission(s): 29

SODERBLOM LARRY DR, US GEOLOGICAL SURVEY, BRANHC OF ASTROGEOLOGY, 2255 N GEMINI DR

FLAGSTAFF AZ 80001, USA

Telephone: (1)

Ficsimile: (1)

Telex:

Email:

Commission(s): 16

SODRE LAERTE, IAG, UNIVERSIDADE DE SAO PAULO, AV MIGUEL STEFANO \$200, 04301 904 SAO PAULO, BRAZIL

Telephone: (55) 115778599

Facsimile: (55) 112763848

Telex:

Email: lacrte(a)astro1.iagusp.usp.br

Commission(s):

SOEDERHJELM STAFFAN DR, LUND OBSERVATORY, BOX 43, S 22100 LUND, SWEDEN

Telephone: (46) 46107303

Facsimile: (46)

Telex: 33199 obsnot s

Email:

Commission(s): 08,42

SOFFEL MICHAEL DR, LEHRSTUHL F THEORET ASTRO, PHYSIK DER UNIV TUEBINGEN AUF DER MORGENSTELLE $10 \mathrm{C}$, D 72076 TUEBINGEN, GERMANY

Telcphone: (49) $707+12920+3$

Email: soffel(a)tat.physik.uni-tucbinge Commission(s): 07C,19

Telex: 7262867 utna d

SOFIA SABATINO PROF, YALE UNIVERSITY OBS, BOX 6666, NEW HAVEN CT 06511, USA

Telephonc: (1) 2034363460

Facsimile: (1) $203+323048$

Telex: 7104653041

Email:

Commission(s): $34,35,44$

SOFUE YOSHIAKI PROF, INST OF ASTRONOMY, UNIVERSITY OF TOKYO, OSAWA MITAKA, TOKYO 181, JAPAN

Telephone: (81) $422+13734$

Facsimile: $(81)+22+137+9$

Telex: 2822307

Email: y.sifyi\%tansci.cc.u-tokyo.ac.

Commission(s): $34,40,51$

SOIFER BARUCH T DR, CALTECH, MS 320 47, DOWNES LAB OF PHYSICS, PASADENA CA 91125 , USA

Telephone: (1) 8183566626

Facsimile: (1)

Telex: 675425

Email:

Commission(s):

SOKOLOWSKI LECH, ASTRONOMICAL OBSERVATORY, JAGIELLONIAN UNIVERSITY, UL ORLA 171

PL 30244 KRAKOW, POLAND

Telephone: (48) 12223856

Email:

Facsimile: (48) 12378053

Commission(s): 47

Telex: 326203 ujoa pl

SOKOLSKY ANDREJ G DR, INST OF THEORET ASTRONOMY, ACADEMY OF SCIENCES, N KUTUZOVA 10 191187 ST PETERSBURG, RUSSIA

Telephonc: (7) 8122790667

Email: sokolsky(a)iiii.spb.su

Facsimile: (7) 8122727968

Telex: 121578 ita su

Commission(s): 05,07C,20

SOL HELENE DR, OBSERVATOIRE DE PARIS, SECTION DE MEUDON, DAEC, F 92195 MEUDON PPL CDX, FRANCE

Telephone: (33) 145077428

Facsimile: (33)

Telex: 20157

Email: bitnet:solaffrmeus1

Commission(s): 
M 832

SOLANES MAJUA JOSE M, DPT OF ASTRONOMY, CORNELL UNIVERSITY, 410 SPACE SCIENCES BLDG ITHACA NY 14853, USA

Telephone: (1) 6072556915

Facsimile: (1) 6077255 8803

Telex:

Email: solanes $(\hat{a}$ astrosun.tn.cornell.edu Commission(s):

SOLANKI SAMI K DR, INSTITUT FUER ASTRONOMIE, ETH ZENTRUM, CH 8092 ZUERICH, SWITZERLAND

Telephone: (41) 12563810

Facsimile: (\$1) 12512172 Telex:

Email: solanki(a)ifa.ethz.ch

Commission(s): 10,12

SOLARIC NIKOLA, HVAR OBSERVATORY, FACULTY OF GEODESY, KACICEVA 26, ZAGREB, CROATIA

Telephone: (38) $\$ 1521548$

Facsimile: (38)

Telex:

Email:

Comnission(s): 08

SOLC MARTIN, DPT ASTRONOMY/ASTROPHYS, CHARLES UNIVERSITY, SVEDSKA 8, CZ 150 00 PRAHA 5, CZECH

Telephone: (42) 2540395

Facsimile: (42) 2299272

Telex: $121673 \mathrm{mff}$

Emai): solc(a)csearn

Commission(s): 15,34,41

SOLF JOSEF DR, THUERINGER LANDESSTERN, WARTE, STERNWARTE 5, D 07778 TAUTENBURG, GERMANY

Telephone: (49) $36+278630$

Facsimile: (49) 3642786329

Telex:

Email:

Commission(s):

SOLHEIM JAN ERIK, INST MATHS \& PHYSICAL SCI, UNIVERSITY OF TROMSO, BOX 953 N 901 TROMSO, NORWAY

Telephone: (47) 083-86060

Facsimile: $(47)$

Telex: 64124

Email:

Commission(s): $+2,46 \mathrm{NR}$

SOLIMAN MOHAMED AHMED DR, AL TAIF CO LTD, KFCC PROJECT, BOX 3181, RIYADH 11471, SAUDI ARABIA

Telephone: $(966) 4824987$ *238

Facsimile: $(966) \$ 822933$

Telex: 405581 al taif sj

Email:

Commission(s): 10,27

SOLIVELLA GLADYS R LIC, OBSERVATORIO ASTRONOMICO, PASEO DEL BOSQUE S/N 1900 LA PLATA (BS AS), ARGENTINA

Telephone: (54) 21217308

Facsimile: $(54) 2138810$

Telex: 31151 bulap ar

Email: gladys(äfeaglp.cdu.ar

Commission(s): 30

SOLLAZZO CLAUDIO, EUROPEAN SPACE OPERATIONS, CENTER, ROBERT BOSCH STR 5

D 64293 DARMSTADT, GERMANY

Telephone: (49) 61518861

Facsimile: $(49)$

Email:

Commission(s):

Telex: 419453

SOLOMON PHILIP M DR, DPT OF EARTH \& SPACE SCI, ASTRONOMY PROGRAM, SUNY AT STONY BROOK STONY BROOK NY $1179+2100$, USA

Telephone: (1) 5162468383

Facsimile: (1)

Telex: 510-228-7767

Email:

Commission(s): 33,34

SOLOVAYA NINA A, STERNBERG STATE ASTR INST, UNIVERSITETSKIJ PROSP 13, 119899 MOSCOW V 234, RUSSIA

Telephone: (7) 0959393764

Facsimile: (7) 0959390126

Telex: 411483 mgu su

Email: ursa@sai.msk.su

Commission(s): 20

SOLTAN ANDRZEJ MARIA DR, COPERNICUS ASTRON CENTER, POLISH ACAD OF SCIENCES, UL BARTYCKA 18 PL 00 716 WARSAW, POLAND

$\begin{array}{lll}\text { Telephone: (48) } & \text { Facsimile: (48) } & \text { Telex: } \\ \text { Email: } & \text { Commission(s): } 28 & \end{array}$

SOMA MITSURU DR, TOKYO ASTRONOMICAL OBS, NAOJ, OSAWA MITAKA, TOKYO I81, JAPAN

Telephone: $(81)+22+13788$

Facsimile: $(81)$

Telex: 02822307

Email:

Commission(s): $04,08,20$

SOMERVILLE WILLIAM B DR, DPT PHYSICS \& ASTRONOMY, UNIVERSITY COLLEGE LONDON, GOWER ST

LONDON WC1E 6BT, UK

Telephonc: (44) 1713827050

Facsimile: $(4+)$

Telex: $\mathbf{2 8 7 2 2}$

Email:

Commission(s): 14,34 
SOMMER-LARSEN JESPER DR, NIELS BOHR INSTITUTE, BLEGDAMSVEJ 17, DK 2100 COPENHAGEN 0, DENMARK Telephone: (45) 31421616 Facsimilc: (45) 31389157 Telex:

Email: Commission(s):

SOMOV BORIS V DR, STERNBERG STATE ASTR INST, UNIVERSITETSKIJ PROSP 13, 119899 MOSCOW, RUSSIA Telephone: (7) $9593916+4$ Facsimile: (7) 7959390126 Telex: $\mathbf{4 1 1 4 8 3} \mathbf{m g u}$ su

Email: snna sai.msk.su Commission(s): 10

SONETT CHARLES P PROF, LUNAR \& PLANETARY LAB, UNIVERSITY OF ARIZONA, TUCSON AZ 85721, USA

Telephone: (1) 5206216935

Facsimile: (1)

Telex: 9109521143

Email:

Commission(s): 16,49

SONG DOO JONG DR, ISSA, 36-1 WHAAM DONG, YUSEONG GU, DAEJEON 305 348, KOREA R

Telephone: $(82)+28611502$

Facsimile: (82) 428615610

Telex: $\mathbf{4 5 5 3 2}$ tdtdrok $k$

Email: djsong\%apiss .issa. re.krâgaram Commission(s): 47

SONG GUO-XUAN, SHANGHAI OBSERVATORY, CAS, 80 NANDAN RD, SHANGHAI 200030, CHINA PR

Telephone: (86) 21386191

Facsimile: (86)

Telex: 33164 shao en

Email:

Commission(s): 28,33

SONG JIN-AN, SHAANXI OBSERVATORY, CAS, LINTONG XIAN, SHAANXI, CHINA PR

Telephone: (86) 332255

Facsimile: (86)

Telex: 70121 csao cn

Email:

Commission(s): 31

SONG MU-TAO, PURPLE MOUNTAIN OBSERV, CAS, NANJING, CHINA PR

Telephone: (86) $2546700 \quad$ Facsimile: $(86) 25301459 \quad$ Telex: 34144 pmonj cn

Email: Commission(s): 12

SONGSATHAPORN RUANGSAK DR, DPT OF PHYSICS, CHIANG MAI UNIVERSITY, FACULTY OF SCIENCES CHIANG MAI 50002, THAILAND

Telephone: (66) $53221934^{*} 135$

Facsimile: (66)

Telex: $\mathbf{4 3 5 5 3}$ unichim th

Email:

Commission(s):

SONNEBORN GEORGE DR, NASA GSFC, CODE 681, LASP, GREENBELT MD 20771, USA

Telephonc: (1) $3012863665 \quad$ Facsimile: (1) Telex: 89675

Email: span:6471::sonncborn

Commission(s): 29,44

SONTI SREEDHAR RAO, DPT OF ASTRONOMY, UNIVERSITY OF OSMANIA, HYDERABAD 500 007, INDIA

Telephone: (91)

Facsimile: (91)

Telex:

Email:

Commission(s): 29,42,45

SOOD RAVI, DPT OF PHYSICS, UNIV COLLEGE ADFA, UNSW, CANBERRA ACT 2600, AUSTRALIA

Telephone: (61) 62688765

Facsimile: (61) 62688786

Telex: aa 62030

Email: r-sood(a)udfa.edu.au

Commission(s): 44

SOON WILLIE H, CFA, MS 16, 60 GARDEN ST, CAMBRIDGE MA 02138, USA

Telephone: (1) 6174957488

Facsimile: (1) $6174957049 \quad$ Telex:

Email: wsoon(a)cfassp3t.harvard.edu Commission(s):

SOONTHORNRHUM BOONRUCKSAR, DPT OF PHYSICS, FACULTY OF SCIENCES, CHIANG MAI UNIVERSITY CHIANG MAI 50200, THAILAND

Telephone: (66) $53221699 * 3367$

Facsimile: (66) 53222268

Telex: unichim th $\mathbf{4 3 5 5 3}$

Email: boonraksâacmu.chianmati.ac.th

Commission(s):

SORENSEN GUNNAR DR, INST OF PHYSICS \& ASTRON, UNIVERSITY OF AARHUS, NY MUNKEGADE

DK 8000 AARHUS C, DENMARK

Telephone: (45) 86128899

Facsimile: $(45) 86202711$

Telex:

Email:

Commission(s): 14

SORENSEN SOREN-AKSEL DR, DPT COMPUTER SCIENCE, UNIVERSITY COLLEGE LONDON

LONDON WC1E 6BT, UK

Telephone: (44)

Facsimile: (44)

Email:

Commission(s):

Telex: 
SOROCHENKO R L DR, LEBEDEV PHYSICAL INST, ACADEMY OF SCIENCES, LENINSKY PROSPEKT 53 117924 MOSCOW, RUSSLA

Telephone: (7) 951350171

Facsimile: (7)

Telex: $+11+79$ neod su

Email:

Commission(s): 40

SORU-ESCAUT IRINA MRS, OBSERVATOIRE DE PARIS, SECTION DE MEUDON F 92195 MEUDON PPL CDX, FRANCE

Telephone: (33) 145347530

Facsimile: (33)

Telex:

Email:

Commission(s):

SOTIROVSKI PASCAL DR, OBSERVATOIRE DE PARIS, SECTION DE MEUDON, F 92195 MEUDON PPL CDX, FRANCE

Telephone: (33) 145077802

Facsimile: (33)

Telex: 270912

Email:

Commission(s): 10,12

SOUCAIL GENEVIEVE, OBS MIDI PYRENEES, 14 AVE E BELIN, F 31400 TOULOUSE CDX, FRANCE

Telephone: (33) 61332819

Email: soucail(a)obs-mip.fr

Facsimile: (33) $61536722 \quad$ Telex:

Commission(s):

SOUCHAY JEAN, OBSERVATOIRE DE PARIS, 61 AVE OBSERVATOIRE, F 75014 PARIS, FRANCE

Telephone: (33) 140512322

Facsimile: (33) $140512291 \quad$ Telex:

Email: souchay@obspm.fr

Commission(s): 07,19

SOUFFRIN PIERRE B DR, OCA OBSERV DE NICE, BP 229, F 0630 + NICE CDX 4, FRANCE

Telephone: (33) 93890420

Facsimile: (33) 92003033

Telcx: $\$ 6000+$ obsnice $f$

Email:

Commission(s): 12,35,36

SOULIE GUY, OBSERVATOIRE DE BORDEAUX, BP 89, F 33270 FLOIRAC, FRANCE

Telephone: (33) 56864330

Facsimile: (33) $56+04251 \quad$ Telex:

Email:

Commission(s):

SOWELL JAMES ROBERT DR, GEORGIA INSTITUTE, OF TECHNOLOGY, SCHOOL OF PHYSICS ATLANTA GA 30332, USA

Telcphonc: (1) 4048943628

Email: js58@hydra.gatech.edu

Facsimile: (1)

Commission(s): 26

Telex:

SPADA GIANFRANCO DR, IST TESRE, CNR, VIA DE CASTAGNOLI 1, I 40126 BOLOGNA, ITALY

Telephonc: (39) 51519593

Facsimile: (39)

Telex: $511350 \mathrm{cnr}$ bo

Email:

Commission(s): 44

SPADARO DANIELE DR, OSS ASTROFISICO, CITTA UNIVERSITARIA, VIA A DORIA 6, I 95125 CATANIA, ITALY

Telephone: (39) 95330533

Facsimile: (39) 95330592

Telex: 970359 astrct $i$

Email: 40297::daniele

Commission(s): 10

SPAENHAUER ANDREAS MARTIN, ASTRONOMISCHES INSTITUT, UNIVERSITAET BASEL, VENUSSTRASSE 7 CH 4102 BINNINGEN, SWITZERLAND

$\begin{array}{ll}\text { Telephone: (41) } 612717711 / 12 & \text { Facsimile: (41) } \\ \text { Email: } & \text { Commission(s): }\end{array}$

Telex:

SPAGNA ALESSANDRO, OSSERVATORIO ASTRONOMICO, DI TORINO, STR OSSERVATORIO 20 I 10025 PINO TORINESE, ITALY

Telephone: (39) $114619034 \quad$ Facsimile: (39) $114619030 \quad$ Telex:

Email: 32056::spagna/spagna(ato.astro.it Commission(s):

SPARKE LINDA, WASHBURN OBSERVATORY, UNIVERSITY OF WISCONSIN, 475 N CHARTER ST

MADISON WI 53706, USA

Telephone: (1) 6082623071

Email: sparke(a)wiscmac3 bitnet

Facsimile: (1)

Telex:

Commission(s): 33

SPARKS WARREN M DR, LOS ALAMOS NATIONAL LAB, MS F669, BOX 1663, LOS ALAMOS NM 87545, USA

Telephone: (1) 5056674922

Facsimile: (1)

Telex:

Email:

Commission(s): $35,+2$

SPARKS WILLIAM BRIAN, STSCI, HOMEWOOD CAMPUS, 3700 SAN MARTIN DR, BALTIMORE MD 21218, USA Telephone: (1) 4103384843

Facsimile: (1)

Telex:

Email: scivax::sparks

Commission(s): 28 
SPARROW JAMES G DR, AERONAUTICAL RESEARCH, LABORATORIES, 4331, MELBOURNE VIC 3001, AUSTRALIA Telephone: (61) $36 \$ 77623$ Facsimile: (61)

Email:

Commission(s): 21

Telex: 39391 arl aa

SPASOVA NEDKA MARINOVA, DPT OF ASTRONOMY, BULGARIAN ACAD SCIENCES, 72 LENIN BLVD BG $178+$ SOFIA, BULGARIA

Telephone: (359) 2758927

Facsimile: (359)

Telex:

Email:

Commission(s):

SPEER R J DR, DPT OF PHYSICS, IMPERIAL COLLEGE, PRINCE CONSORT RD, LONDON SW7 2BZ, UK

Telephone: (4) $1715895111 \quad$ Facsimile: (4)

Telex: 261503 impcol

Email:

Commission(s): +4

SPENCER JOHN HOWARD, NAVAL RESEARCH LABORATORY, CODE 4134, 4555 OVERLOOK AVE SW WASHINGTON DC 20375 5000, USA

Telephone: (1) 2027673050

Facsimile: (1)

Telex:

Email:

Commission(s): 40

SPENCER RALPH E DR, NRAL, JODRELL BANK, MACCLESFIELD SK11 9DL, UK

$\begin{array}{ll}\text { Telephone: (44) } 147771321 & \text { Facsimile: (4) } \\ \text { Email: } & \text { Commission(s): 40 }\end{array}$

SPERAUSKAS JULIUS, ASTRONOMICAL OBSERVATORY, CIURLIONIO 29, VILNIUS 2009, LITHUANIA

Telephonc: (370) 2633343

Facsimilc: (370) 2223563

Telex: 261212 vusu

Email:

Commission(s):

SPERGEL DAVID N DR, PRINCFTON UNIVERSITY OBS, PEYTON HALL, PRINCETON NJ 08544, USA

Telephone: (1) 6092583589

Facsimile: (1)

Commission(s): 33

Telex:

Email: Ins(a)astro.princeton.cdu

SPICCER DANIEL SHIELDS DR, NASA GSFC, CODE 682, GREENBELT MD 20771, USA

Telephone: (1) 3012867334

Facsimile: (1)

Telex:

Email:

Commission(s): 10,12

SPIEGEL E DR, DPT OF ASTRONOMY, COLUMBIA UNIVERSITY, PUPIN HALL 538 W $120 T H$ ST

NEW YORK NY 10027, USA

Telephone: (1)

Facsimile: (1)

Telex:

Email:

Commission(s): $33,35,36$

SPIELFIEDEL ANNIE DR, OBSERVATOIRE PARIS, SECTION DE MEUDON, DAMAP F 92195 MEUDON PPL CDX, FRANCE

Telephone: (33) 145077453

Email: spilfildâfrmeusi

Facsimile: (33) $145077469 \quad$ Telex: 201571

Commission(s): 14

SPINRAD HYRON PROF, ASTRONOMY DPT, UNIVERSITY OF CALIFORNIA, 601 CAMPBELL HALL

BERKELEY CA $9 \$ 720$, USA

Telephone: (1) $4156+22078$

Facsimile: (1)

Telex:

Email:

Commission(s): 15,28

SPITE FRANCOIS M DR, OBSERVATOIRE DE PARIS, SECTION DE MEUDON, F 92195 MEUDON PPL CDX, FRANCE

Telephonc: (33) $1450778+0$

Facsimile: (33) $1+5077878$

Telcx: 270912

Email: francois.spite(a)obspm.fr

Commission(s): $05,29,36$

SPITE MONIQUE DR, OBSERVATOIRE DE PARIS, SECTION DE MEUDON, F 92195 MEUDON PPL CDX, FRANCE

Telephonc: (33) 145077839

Facsimile: (33)

Telex: 270912

Email: monique.spitc(äobspm.fr

Commission(s): 29,36

SPITHAS ELEFTERIOS N DR, DPT OF ASTRONOMY, NTL UNIVERSITY OF ATHENS, PANEPISTIMIOPOLIS

GR 15771 ZOGRAFOS, GREECE

Telephone: (30) $172+3414$

Facsimile: (30)

Telex:

Email:

Commission(s):

SPITZER LYMAN JR DR, PRINCETON UNIVERSITY OBS, PEYTON HALL, PRINCETON NJ 08544, USA

Telephone: (1) 6094523809

Facsimile: (1)

Telex: $322+09$

Email:

Commission(s): 34,44 
SPOELSTRA T A TH DR, NFRA, BOX 2, NL 7991 PD DWINGELOO, NETHERLANDS

Telephone: (31) $52197244 \quad$ Facsimile: (31) Telex:

Email: $\quad$ Commission(s): $08,40,50$

SPRAGUE ANN LOUISE, LUNAR \& PLANETARY LAB, ROOM 325 BLDG 92, UNIVERSITY OF AZRIZONA TUCSON AZ 85721, USA

Telephone: (1) 5206212282

Facsimile: (1) $5206214933 \quad$ Telex:

Email: sprague $(\bar{a})$ titan.Jpl.arizona.colu

Commission(s): 16

SPRUIT HENK C DR, MPI F PHYSIK \& ASTROPHYS, KARL SCHWARZSCHILDSTR 1 D 85740 GARCHING MUENCHEN, GERMANY

Telephonc: (49) 8932990

Facsimile: (49)

Email:

Commission(s): 10,36

Telex: 524629 astro d

SPURNY PAVEL, ASTRONOMICAL INSTITUTE, CZ 25165 ONDREJOV, CZECH R

Telephone: (42) 204857153

Facsimile: (\$2) 2881611 Telex:

Email: spurny(a)asu.cas.cz

Conmission(s): 22

SPURZEM RAINER DR, INST THEOR PHYS \& STERNW, UNIVERSITAET KIEL, OLSHAUSENSTR 40 D 24098 KIEL, GERMANY

Telephone: (49) $43188(1574$

Email: pas28(a) rz.uni-kicl.db)p.de

Facsimile: $(49) \mathbf{4 3 1} 8804432 \quad$ Telex: 292706

Commission(s): 37

SPYROU NICOLAOS PROF, DPT OF ASTRONOMY, UNIVERSITY THESSALONIKI

GR 54006 THESSALONIKI, GREECE

Telephone: (30) 31992658

Facsimile: (30)

Commission(s): 47

Telex: $\mathbf{4 1 2 1 8 1}$

Email:

SRAMEK RICHARD A DR, NRAO, BOX 0, SOCORRO NM 87801 0387, USA
Telephonc: (1) $5057724011 \quad$ Facsinile: (1)

Email: Commission(s): 40

Telex: 9109881710

SREEKANTAN B V DR, TIFR, HOMI BHABHA RD, COLABA, BOMBAY 400005 , INDIA

Telephone: (91) $22219111 \quad$ Facsimile: (91) Telex: 011-3009

Email:

Commission(s):

SREENIVASAN S RANGA PROF, DPT OF PHYSICS, UNIVERSITY OF CALGARY, 2500 UNIVERSITY DR NW CALGARY AB T2N IN4, CANADA

Telephone: (1) 4032845385

Email:

Facsimile: (1) $\$ 032893331 \quad$ Telex:

Commission(s): 35

SRINIVASAN G, RAMAN RESEARCH INSTITUTE, SADASHIVANAGAR, CV RAMAN AVE, BANGALORE 560 080, INDI Telephone: (91) 803360122

Email: srini(a)rri.ernet.in

Facsimile: (91) $8033+0492$

Commission(s): +4VP

Telex: 8452671 rri in

SRIVASTAVA J B DR, UTTAR PRADESH STATE, OBSERVATORY, PO MANORA PEAK 263129

NAINITAL 263 129, INDLA

Telephone: (91) 59422136

Email: srini(a)rri.crnet.in

Facsimile: (91)

Commission(s): 12

Telex:

SRIVASTAVA RAM KUMAR DR, UTTAR PRADESH STATE, OBSERVATORY, PO MANORA PEAK 263 129

NAINITAL 263 129, INDIA

Telephone: (91) 59422136

Facsimile: (01)

Telex:

Email:

Commission(s): 27,42

STABELL ROLF DR, INST THEORET ASTROPHYSICS, UNIVERSITY OF OSLO, BOX 1029

N O315 BLINDERN OSLO 3, NORWAY

Telephone: (47) 2-456-530

Facsimile: (47)

Commission(s):

Telex: 72705 astro $n$

STACEY GORDON J DR, DPT OF ASTRONOMY, CORNELL UNIVERSITY, SPACE SCIENCES BLDG

ITHACA NY 14853, USA

Telephone: (1) 6072555900

Emai): stacey(a)astrosun.tn.edu

Facsimile: (1) 6072555875

Telex: 
STACHNIK ROBERT V, NASA HEADQUARTERS, ASTROPHYSICS DIV, CODE SZ, WASHINGTON DC 20546, USA

Telephone: (1) $202+531469$

Facsinile: (1)

Telcx:

Email: champ::stachnik

Commission(s): 4

STAGG CHRISTOPHER DR, DPT OF PHYSICS \& ASTRON, UNIVERSITY OF CALGARY, 2500 UNIVERSITY DR NW CALGARY AB T2N 1 N4, CANADA

Telephone: (1) 4032207423

Facsimile: (1) $\$ 1032893331$

Telex: 03821545

Email: crstagg(a)acs.ucalgary.ca

Commission(s): $\$ 2$

STAGNI RUGGERO, OSSERVATORIO ASTROFISICO, VIA DELL OSSERVATORIO 8, 1 36112 ASIAGO, ITALY

Telephone: (39) $42 \$ 62665 \quad$ Facsimile: (39)

Email:

Commission(s):

Telex: 430110 setour $i$

STAHL OTMAR RICHARD DR, LANDESSTERNWARTE, KOENIGSTUHL, D 69117 HEIDELBERG, GERMANY Tetephone: (49) $6221509232 \quad$ Facsimile: (49) 6221509202 Telex:

Email: ostahl(a)mail.lsw.uni-heidetberg.de Commission(s):

STAHLER SETVEN W DR, DPT OF PHYSICS, MIT, BOX 165, CAMBRIDGE MA 02139, USA

Telephone: (1) $6172530905 \quad$ Facsimile: (1) 6172539798 Telex:

Email:

Commission(s): 34

STAHR-CARPENTER M DR, 1101 HILL TOP RD, CHARLOTTESVILLE VA 22903, USA

Telephone: (1) $80+2937063$

Facsimile: (1)

Telex:

Email:

Commission(s): 40

STALIO ROBERTO DR, DPT DI ASTRONOMIA, UNIVERSITA DI TRIESTE, VIA TIEPOLO 11, I 34131 TRIESTE, ITALY

Telephonc: (39) $40793921 * 221 \quad$ Facsimile: (39)

Telex: 461137 oat $i$

Email:

Commission(s): 29,36,51

STANDISH E MYLES DR, JPL, MS 301 150, 4800 OAK GROVE DR, PASADENA CA 91109, USA

Telephone: (1) $81835+3959$

Facsimile: (1) 8183936388

Telex:

Email: cms(a)smyles.jpl.nasa.gov

Commission(s): 0+VP,07,20

STANFORd SPENCER A, CALTECH, MS 100 22 IPAC, PASADENA CA 91125, USA

Telephone: (1)

Facsimile: (1)

Telex:

Email:

Commission(s):

STANGA RUGGERO, DPT DI ASTRONOMIA, UNIVERSITA DI FIRENZE, LARGO E FERMI 5, I 50125 FIRENZE, ITALY

Telephone: (39) 5527521

Facsimile: (39) 55220039

Telex: $\mathbf{5 7 2 2 6 9}$ arcetr i

Email:

Commission(s): 34

STANGE LOTHAR, TECHNICAL UNIVERSITY, DRESDEN, MOMMSENSTR 13, D 8027 DRESDEN, GERMANY

Telephone: (49) $514634652 \quad$ Facsimile: $(49)$

Telex: 02278

Email:

Commission(s): 08

STANILA GEORGE DR, MITROPOLIT GRIGORE 28, BOX $28, \mathrm{R} 75215$ BUCHAREST, RUMANIA

Telephone: (40) $16233+26$

Facsimile: (40)

Telex:

Email:

Commission(s): 19,31

STANKEVICH KAZIMIR S DR, RADIOPHYSICAL RESEARCH, INSTITUTE, LYADOV UL 25/14

603600 N NOVGOROD, RUSSIA

Telephone: (7) 389091

Facsimile: (7)

Telex:

Email:

Commission(s):

Telex:

STANLEY G J, BOX 1348, CARMEL VALLEY CA 93924, USA

Telephone: (1) 4086592940

Facsimile: (1)

Email:

Commission(s): 40

STANNARD DAVID DR, NRAL, JODRELL BANK, MACCLESFIELD SK11 9DL, UK

$\begin{array}{lll}\text { Telephone: (44) } 147771321 & \text { Facsimile: (44) } & \text { Telex: } 36149 \text { jodrcl g } \\ \text { Email: } & \text { Commission(s): } 40 & \end{array}$

STARK ANTONY A, SMITHSONIAN ASTROPHYS LAB, MA 78, 60 GARDEN ST, CAMBRIDGE MA 02138, USA

Telephone: (1) 6174967648

Email: aas(a)cfa.harvard.cdu

Faesimile: (1) 6174967554

Telex:

Commission(s): 
STARK GLEN DR, WHITIN OBSERVATORY, WELLESLEY COLLEGE, DPT OF PHYSICS, WELLESLEY MA 02181, US

Telephone: (1) 6172350320 .

Facsimile: (1)

Telex:

Email: gstark@alucy.wcllesley.edu

Commission(s): 14

STARRFIELD SUMNER PROF, DPT OF PHYSICS, ARIZONA STATE UNIVERSITY, TEMPE AZ 85287, USA

Telephone: (1) 6029653561

Facsimile: (1)

Email:

Commission(s): $27,35,42$

Telex: 667391 ariz st u tmp

STASINSKA GRAZYNA DR, OBSERVATOIRE DE PARIS, SECTION DE MEUDON, F 92195 MEUDON PPL CDX, FRANC Telephone: (33) $145077+22$

Facsimile: (33)

Telex: 201571

Email:

Commission(s):

STATHOPOULOU MARIA DR, DPT OF ASTROPHYSICS, NTL UNIVERSITY OF ATHENS, PANEPISTIMIOPOLIS GR 15783 ZOGRAFOS, GREECE

Telephone: (30) 17243414

Facsimile: (30) 17228981

Telex:

Email: marstath(a)grathun1

Commission(s): 12

STAUBERT RUDIGER PROF DR, ASTRONOMISCHES INSTITUT, UNIVERSITAET TUEBINGEN

WALDHAEUSERSTR 64, D 72076 TUEBINGEN, GERMANY

Telephone: (49) $7071294980 \quad$ Facsimile: (49)

Email:

Commission(s): 4

Telex: 7262714 ait d

STAUDE HANS JAKOB PH D, MPI FUER ASTRONOMIE, KOENIGSTUHL, D 69117 HEIDELBERG, GERMANY

Telephone: (49) $6221528229 \quad$ Facsimile: (49)

Email:

Commission(s): 21

Telex: 461789 mpia d

STAUDE JUERGEN DR, ASTROPHYS INST POTSDAM, SONNEN OBS EINSTEINTURM

D 14473 POTSDAM, GERMANY

Telephone: (49) 3312882300

Email: jstaude a aip.de

Facsimile: (49) $3312882310 \quad$ Telex:

Commission(s): 12

STAUFFER JOHN RICHARD, 117 SYLVESTER AVE, WINCHESTER MA 01890, USA

Telephone: (1) 6174957024

Facsimile: (1) $617+957490 \quad$ Telex:

Email: stauffera efa.harvard.edu

Commission(s): 37

STAVELEY-SMITH LISTER DR, CSIRO, DIV OF RADIOPHYSICS, BOX 76, EPPING NSW 2121, AUSTRALIA

Telephone: (61) 28680222

Facsimile: (61) 28680310

Telex: 26230 astro aa

Email: lstavele(a)atnf.csiro.au

Commission(s): 28

STAVINSCHI MAGDALENA, ASTRONOMICAL INSTITUTE, CUTITUL DE ARGINT 5, BOX 28 R 75212 BUCHAREST, RUMANIA

Telephone: (40) 16236892

Facsimile: (40) 13123391

Telex: 11882 astro $\mathrm{r}$

Email: mstavins(a)roearn.bitnet

Commission(s): $08,19,41$

STAWIKOWSKI ANTONI DR, INSTITUTE OF ASTRONOMY, N COPERNICUS UNIVERSITY, UL CHOPINA 12/18

PL 87100 TORUN, POLAND

Telephone: (48)

Facsimilc: (48)

Commission(s): 29

Telex:

Email:

.STEBBINS ROBIN, JILA, UNIVERSITY OF COLORADO, BOX 440, BOULDER CO 80309 0440, USA

Telephone: (1) 3034926073

Facsimile: (1)

Telex: 755842

Email:

Commission(s): 12

STECHER THEODORE P, NASA GSFC, CODE 680, GREENBELT MD 20771, USA

Telephone: (1) $3012868718 \quad$ Facsimile: (1) Telex:

Email:

Commission(s): $29,44,34$

STECKER FLOYD W DR, NASA GSFC, CODE 660, LAB HIGH ENERGY ASTROPHYS, GREENBELT MD 20771, USA

Telephone: (1) 3012866057

Facsimile: (1)

Telcx:

Email:

Commission(s): $33,44,47$

STEEL DUNCAN I DR, UK SCHMIDT TELESCOPE, AAO, PRIVATE BAG, COONABARABRAN NSW 2357, AUSTRALIA

Telephone: (61) 68842298

Facsimile: (61) 68426282

Telex:

Email: dis(a)anocbn3.aao.gov:alu

Commission(s): 15,20,22C 
STEELE COLIN D C, DPT OF MATHEMATICS, UMIST, BOX 88, MANCHESTER MGO 1QD, UK

Telephone: (4) 1612003632

Email: cds(aast.ma.umist.ac.uk

Facsimile: (4) 1612003669

Telex:

Commission(s):

STEENMAN-CLARK LOIS DR, OCA OBSERV DE NICE, BP 229, F 06304 NICE CDX 4, FRANCE

Telephone: (33) 93890420

Facsimile: (33) 92003033

Telex: 460004 obsnice $f$

Email:

Commission(s): 14

STEFANIK ROBERT DR, OAK RIDGE OBSERVATORY, HARVARD SMITHSONIAN CTR, PINNACLE ROAD HARVARD MA 01451, USA

Telephone: (1) 6174957070

Email: span:cfaz::stefanik
Facsimile: (1)

Commission(s): $30 \mathrm{C}$
Telex:

STEFFEN MATTHIAS DR, INST THEOR PHYS \& STERNW, UNIVERSITAET KIEL, OLSHAUSENSTR 40 D 24098 KIEL, GERMANY

Telephone: (49) 4318804101

Email:

Facsimile: (49)

Telex: 292706

Commission(s): $12,29,36,40$

STEFL STANISLAV DR, ASTRONOMICAL INSTITUTE, CZECH ACADEMY OF SCIENCES

ONDREJOV OBSERVATORY, CZ 2516.5 ONDREJOV, CZECH R

Tclephone: (42) $20485314 \times 143$

Email: aststel(â)csearn Commission(s): 29

Telex:

STEFL VLADIMIR, DPT THEORET PHYS \& ASTRO, PURKYNE UNIVERSITY, KOTLARSKA 2

CZ 61137 BRNO, CZECH R

Telephone: (42) 57129482

Facsimile: $(+2)$

Telex:

Email: stchla)csbrmul1

Commission(s): 46

STEHLE CHANTAL DR, OBSERVATOIRE DE PARIS, SECTION DE MEUDON, DPT ATOMES \& MOLECULES F 92195 MEUDON PPL CDX, FRANCE

Telephone: (33) 145077453

Facsimile: (33)

Telex: 201571

Email:

Commission(s): 14

STEIGER W R PROF, 30 KJELE PLACE, HILO HI 96720, USA

Telephonc: (1) 8089614980

Facsimile: (1)

Telex:

Email:

Commission(s):

STEIGMAN GARY PROF, DPT OF PHYSICS, OHIO STATE UNIVERSITY, $17+\mathrm{W}$ 18TH AVE, COLUMBUS OH 43210, US

Telephonc: (1) $61+2921999$

Facsimile: (1)

Telex: 8104821715

Email:

Commission(s): 44,47

STEIMAN-CAMERON THOMAS DR, NASA AMES RESEARCH CTR, MS 245 3, THEORETICAL STUDIES BR MOFFETT FIELD CA 94035, USA

Telephone: (1) $41569+3120$

Facsimilc: (1)

Telex:

Email: span:gal::tomsc

Commission(s): 42

STEIN JOHN WILLIAM, 555 HILL ST, SEWICKLEY PA 15143, USA

Telephone: (1) $4127+1+182$

Facsimile: (1)

Telex:

Email:

Commission(s): $24,26,51$

STEIN ROBERT F ASSOC PROF, PHYSICS \& ASTRONOMY DPT, MICHIGAN STATE UNIVERSITY

EAST LANSING MI 48824 , USA

Telephone: (1) 5173538661

Email:

Facsimile: (1)

Telex:

Commission(s): 36

STEIN WAYNE A PROF, SCHOOL OF PHYS \& ASTRON, UNIVERSITY OF MINNESOTA, 116 CHURCH ST SE MINNEAPOLIS MN 55+55, USA

Telephone: (1) 6123739963

Facsimile: (1)

Telex:

Email:

Commission(s):

STEINER JOAO E DR, INPE, CP 515, AV DOS ASTRONAUTAS 1758, 12200 S JOSE DOS CAMPOS, BRAZIL

Telephone: (55) 123229977

Facsimile: (55)

Telex:

Email:

Commission(s): $\mathbf{4 2 , 4 4}$ 
STEINER OSKAR, KIEPENHEUER INSTITUT, FUER SONNENPHYSIK, SCHOENECKSTR 6 D 79104 FREIBURG BREISGAU, GERMANY

Telephone: (49) $76131980 \quad$ Facsimile: (49) 7613198111 Telex:

Email: steiner(akis.uni.freiburg.de Commission(s): 10,12

STEINERT KLAUS GUENTER DR, LORHMANN OBSERVATORY, DRESDEN UNIVERSITAET, MOMMSENSTR 13 D 8027 DRESDEN, GERMANY

Telephone: (49) 514634097

Facsimile: (49) 514637106

Telex: 2278 teuni dd

Email:

Commission(s): 24

STEINITZ RAPHAEL PROF, DPT OF PHYSICS, BEN GURION UNIVERSITY, BOX 653, BEERSHEVA 84105, ISRAEL Telephone: (972) 5770985

Facsimile: (972)

Telex:

Email:

Commission(s):

STEINLE HELMUT DR, MPI F EXTRATERRESTRISCHE, PHYSIK, POSTFACH 1603 D 85740 GARCHING MUENCHEN, GERMANY

Telephone: (49) $8932993374 \quad$ Facsimile: (49) $8932993569 \quad$ Telex: 215845 exter d

Email: hes(a)mpe-garching.mpg.de Commission(s):

STEINLIN ULI PROF, SCHULGASSE 7, CH +105 BIEL-BENKEN, SWITZERLAND

Telephone: (41)61721 4811 Facsimile: (41) Telex:

Email:

Commission(s): $25,33,45$

STEINOLFSON RICHARD S DR, SOUTHWEST RESEARCH INST, 6220 CULEBRA RD

SAN ANTONIO TX 78228 2510, USA

Telephone: (1) 5125222822

Facsimile: (1) 5126474325

Telex: 244046

Email: swri:arich/rich(a) 129.162.150.30 Commission(s):

STELLINGWERF ROBERT F DR, LOS ALAMOS NATIONAL LAB, MS F645, X DIV, LOS ALAMOS NM 87545, USA

Telephone: (1) $505667+370$

Facsimilc: (1)

Telex:

Email:

Commission(s): 27,35

STELLMACHER GOETZ, INSTITUT D'ASTROPHYSIQUE, 98BIS BD ARAGO, F 7501 4 PARIS, FRANCE

Telephone: (33) $14+328057$

Facsimilc: (33) $1+4328001$

Telex:

Email:

Commission(s): 10

STELLMACHER IRENE DR, BUREAU DES LONGITUDES, 77 AVE DENFERT ROCHEREAU, F 75014 PARIS, FRANCE

Telephone: (33) 143201210

Facsimilc: (33)

Commission(s): 07,20

Telex:

Email:

STENCEL ROBERT EDWARD, DPT OF PHYSICS, UNIVERSITY OF DENVER, DENVER CO 80208, USA

Telephone: (1)

Facsimile: (1)

Telex:

Email:

Commission(s): $29,+2,44$

STENFLO JAN O DR, INSTITUT FUER ASTRONOMIE, ETH ZENTRUM, CH 8092 ZUERICH, SWITZERLAND

Telephone: (41) 12563804

Facsimile: (41) 126200013

Telex: 817379 chhg ch

Email: stenflo(a)ifa.ethz.ch

Commission(s): 10,12C

STENHOLM BJOERN DR, LUND OBSERVATORY, BOX 43, S 221 00 LUND, SWEDEN

Telephone: (46) $46107306 \quad$ Facsimile: (46) $46104614 \quad$ Telcx: 33199

Email: bjorn(a)astro.lu.se

Commission(s): 34,46

STENHOLM LARS, ASTRONOMICAL OBSERVATORY, BOX 515, S 75120 UPPSALA, SWEDEN

Telephone: (46) 18112488

Facsimile: (46) 18111853

Telex: 76024 univups s

Email: stenholmâataban.uv.se

Conmission(s): 34

STEPANIAN A A DR, CRIMEAN ASTROPHYS OBS, UKRAINIAN ACAD OF SCIENCE, NAUCHNY 33+413 CRIMEA, UKRAINE

Tclephone: (7) 432945

Facsimile: (7)

Telex:

Email:

Commission(s): 28,4

STEPANIAN JIVAN A DR, BYURAKAN ASTROPHYSICAL, OBSERVATORY, $378+33$ BYURAKAN, ARMENIA

Telephone: (7) 8852283435

Facsimile: (7) 8852284142

Telex:

Email:

Commission(s): 28 
STEPANIAN N N DR, CRIMEAN ASTROPHYS OBS, UKRAINIAN ACAD OF SCIENCE, NAUCHNY 33+413 CRIMEA, UKRAINE

$\begin{array}{ll}\text { Telephone: (7) } 432945 & \text { Facsimile: (7) } \\ \text { Email: } & \text { Commission(s): } 10\end{array}$

STEPANOV ALEXANDER V DR, CRIMEAN ASTROPHYS OBS, UKRAINIAN ACAD OF SCIENCE, NAUCHNY $33+247$ KATZIVELY RT 22, UKRAINE
Telephone: (7) 727906
Facsimile: (7)
Telex: 222192 voshod
Email:
Commission(s): 10

STEPHENS S A DR, TIFR, HOMI BHABHA RD, COLABA, BOMBAY +100 005 , INDIA
Telephone: (91) 22219111
Facsimile: (91)
Commission(s): $4+$
Telex: 011-3009 tifr in
Email:

STEPHENSON C BRUCE PROF, $1+205$ WASHINGTON BVD, UNIVER HEIGHTS OH 44181 , USA
Telephone: (1)

Telephone: (1)

Commission(s): $33,+5$
STEPHENSON F RICHARD DR, DPT OF PHYSICS, UNIVERSITY OF DURHAM, SOUTH RD, DURHAM DH1 3LE, UK Telephone: (44) $19138564971 * 208$
Fincsimile: (44)
Commission(s): 19,41

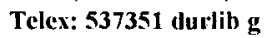
Email:

STEPIEN KAZIMIERZ DR, ASTRONOMICAL OBSERVATORY, WARSAW UNIVERSITY, AL UJAZDOWSKIE 4 PL 00478 WARSAW, POLAND

Telephone: (48) 29 4011

Facsimile: (48) 294967

Telex:

Email: kstiátaurus.astrouw.edu.pl

Commission(s): 27,36

STEPINSKI TOMASZ DR, LUNAR \& PLANETARY INST, 3313 NASA RD, HOUSTON TX 77058, USA

Telephone: (1) 7134862170

Ficsinile: (1)

Telex:

Email: lpi::stepinski

Commission(s):

STEPPE HANS DR, IRAM, AVD DIVINA PASTORA 7, BLOQUE 6/2B, E 18012 GRANADA, SPAIN

Telephonc: (34)

Facsimile: (34)

Telex:

Email:

Commission(s):

STERKEN CHRISTIAAN LEO DR, VRIJE UNIV BRUSSELS, FAC SCIENCES, PLEINLAAN 2

B 1050 BRUSSELS, BELGIUM

Telephone: (32) $26293+69$

Facsimile: (32) 293623976

Telex:

Email: csterken(a)vub.ac.be

Commission(s): 25VP,27

STERN ROBERT ALLAN, LOCKHEED PALO ALTO RES LB, DPT 9130 BLDG 252, 3251 HANOVER ST

PALO ALTO CA 94304 , USA

Telephone: (1) $+15+2+3272 \quad$ Facsimile: $(1)+15+2+399+$ Telex:

Email: sternassag.space. lockhed.com Commission(s): 4

STERN S ALAN, SOUTHWEST RESEARCH INST, 6220 CULEBRA RD, SAN ANTONIO TX 78238 5166, USA

Telephone: (1) 3035469670

Facsimile: (1) 31135469687

Telex:

Email: alan(âswri.space.swri.colu

Commission(s): 15,16

STESHENKO N V DR, CRIMEAN ASTROPHYS OBS, UKRAINIAN ACAD OF SCIENCE, NAUCHNY

$33+\$ 13$ CRIMEA, UKRAINE

Telephone: (7) $\$ 329+5$

Facsimile: (7)

Telex:

Email:

Commission(s): (19,10

STETSON PETER B. DR, HERZBERG INST ASTROPHYS, DOMINION ASTROPHYS OBS, 5071 W SAANICH RD VICTORIA BC V8X $\ M G$, CANADA

Telephone: (1) $60+3390001$

Email:

Fatcimile: (1) $60+36300+5$

Telex: 0497295

STEVENS GERARD A DR, SPACE RESEARCH LABORATORY, SRON, SORBONNELAAN 2

NL $358+$ CA UTRECHT, NETHERLANDS

Telephonc: (31) 30535600$)$

Email:

Facsimile: (31) $305+0860$

Telex:

Commission(s): 
STEWART JOHN MALCOLM DR, DPT APPLIED MATHS, $\&$ THEORETICAL PHYSICS, SILVER STREET CAMBRIDGE CB3 9EW, UK

Telephone: (4) 1223337900

Facsimile: (44) $1223337918 \quad$ Telex:

Email:

Commission(s): 47

STEWART PAUL DR, DPT OF MATHEMATICS, UNIVERSITY OF MANCHESTER, MANCHESTER MI3 9PL, UK

Telephone: (4)

Facsimile: (4)

Telex:

Email:

Commission(s): 40

STEWART RONALD T MR, CSIRO, DIV OF RADIOPHYSICS, BOX 76, EPPING NSW 2121, AUSTRALIA

Telephone: (61) 28680222

Facsimile: (61) $28680310 \quad$ Telex: 26230

Email: rstewart(a)atnf.csiro.au

Commission(s): 10,40

STEYAERT HERMAN PROF DR, STERIREKUNDIG OBSERV, RIJKSUNIVERSITEIT GENT, KRIJGSLAAN 28I

B 9000 GENT, BELGIUM

Telephone: (32) $91225715 * 2572 \quad$ Facsimile: (32) $91240634 \quad$ Telex:

Email:

Commission(s):

STIAVELLI MASSIMO DR, VIA F CORRIDONI 25, I 56100 PISA, ITALY

Telephone: (39) 50 48806 Facsimile: (39)

Telex:

Email: stiaveliádgacs(151/39196::stiav

Commission(s): 28

STIBBS DOUGLAS W N PROF, MOUNT STROMLO \& SIDING, SPRING OBSERVATORIES, PRIVATE BAG

WODEN PO ACT 2606, AUSTRALIA

Telephone: (61) 62881111

Email: dworämso.anu.colu.au

Facsimile: (61) $62+90) 233$

Telex: 62270 canopus ala

STICKLAND DAVID J DR, RUTHERFORD APPLETON LAB, SPACE \& ASTROPHYSICS DIV, BLDG R25/R68 CHILTON DIDCOT OX $110 \mathrm{QX}, \mathrm{UK}$

Telephone: (4t) 1235446523

Fatcimile: (44) $12354+5848 \quad$ Telex: 83159

Email: uk.ac.rl.star.ast::ds

Commission(s):

STIER MARK T, HUGHES DANBURY OP'TICAL SY, MS 813, 100 WOOSTER HEIGHTS RD, DANBURY CT 06810, USA Telephone: (1) 20137975708

Facsimilc: (1) 2037976259

Telex:

Email: nasamailamsticr

Commission(s): 44

STIFT MARTIN JOHANNES DR, INSTITUT FUER ASTRONOMIE, UNIVERSITAET WIEN, TUERKENSCHANZSTR I7 A 1180 WIEN, AUSTRIA

Telephonc: $(43) 134536096$

Einail:

Facsimile: $(\$ 3)$

Telex:

STINEBRING DANIEL R, DPT OF PHYSICS, PRINCETON UNIVERSITY, JADWIN HALL, PRINCETON NJ OS544, USA

Telephone: (1) $609+525578$

Email:

Facsimile: (1)

Telex:

Commission(s):

STIRPE GIOVANNA M DR, OSS ASTRONOMICO, UNIVERSITA DI BOLOGNA, VIA ZAMBONI 33

I 40126 BOLOGNA, ITALY

Telephone: (39) 51259301

Email: stirpeqastbo3.cineca.it

Facsimile: (39) 51259407

Telex:

Commission(s): 28

STIX MICHAEL DR, KIEPENHEUER INSTITUT, FUER SONNENPHYSIK, SCHOENECKSTR 6

D 79104 FREIBURG BREISGAU, GERMANY

Telephone: (49)

Fassimile: (49)

Commission(s): 10.12

Telex: 7721552 kis d

Email:

STOBIE ROBERT S DR, SAAO, BOX 9, OBSERVATORY 7935, SOUTH AFRICA

Teleplone: (27) $21+70025$

Facsimile: (27) $21+73639$

Telex:

Email: rssóasalo.ac.za

Commission(s): 27

STOCK JURGEN D, CIDA, BOX 264, MERIDA 5101 A, VENEZUELA

Teleplone: (58) $7+639930$

Facsimile: $(58)$

Commission(s): $24 \mathrm{C}, 25,31), 45$

Telex: 74174

Email:

STOCKE JOHN T, CASA, UNIVERSITY OF COLORADO, CAMPUS BOX 389, BOULDER CO 80309, USA

Telephone: (1) $303+921521$

Facsimile: (1) $303+927178$

Telex:

Email: stocke::hyades

Commission(s): 
STOCKMAN HERVEY S JR DR, STSCI, HOMEWOOD CAMPUS, 3700 SAN MARTIN DR, BALTIMORE MD 21218, USA Telephone: (1) $301338 \mathbf{4 2 0}$ Facsimite: (1)

Telex: 6849101 stsci uw

Email:

Commission(s): 25,44

STOCKTON ALAN N DR, INSTITUTE FOR ASTRONOMY, UNIVERSITY OF HAWAII, 2680 WOODLAWN DR HONOLULU HI 96822 , USA

Telephone: (1)

Facsimile: (1) 8089882790

Telex:

Email:

Commission(s):

STOEGER WILLIAM R DR, SPECOLA VATICANA, I 00120 VATICAN CITY, VATICAN CITY STATE
Telephone: (39) $66983+11$
Facsimile: (39)

Telephone: (39) 66983411

Telex: 504-2020 vatobs val

Email:

Commission(s): +7

STOEV ALEXEI, PEOPLE'S ASTRONOMICAL OBS, \& PLANETARIA IN BULGARIA, YURI GAGARIN BG 6000 STARA ZAGORA, BULGARIA

Telephone: (359) $\$ 2+3183$

Email:

Facsimile: (359)

Telex:

STOKER PIETER H, COSMIC RAY RESEARCH UNIT, POTCHEFSTROOM UNIVERSITY POTCHEFSTROOM 2520, SOUTH AFRICA

$\begin{array}{lll}\text { Telephone: (27) } 27148125360 & \text { Facsimile: (27) } & \text { Telex: } \mathbf{2 1 3 6 3} \\ \text { Email: } & \text { Commission(s): } 10 & \end{array}$

STONE EDWARD C DR, JPL, MS 180904,4800 OAK GROVE DR, PASADENA CA 91100, USA

Telcphonc: (1) $81835+3+115$

Facsimile: (1) $818393+218 \quad$ Telex:

Email:

Commission(s): 16

STONE R G DR, NASA GSFC, CODE (190, LEP, GREENBELT MD 20771, USA

Telcphonc: (1) 3012868631

Facsimilc: (1)

Telex: $710-82089716$

Email:

Commission(s): $4(0,44,49$

STONE REMINGTON P S DR, LICK OBSERVATORY, MOUNT HAMILTON CA 9514(), USA

Telephone: (1) $40827+1809$

Facsimile: (1)

Telex:

Email: renoalick.ucsc.edu

Commission(s): 25,28

STONE RONALD CECIL, US NAVAL OBSERVATORY, FLAGSTAFF STATION, BOX 1149, FLAGSTAFF AZ 86002, USA Telcphone: (1) 60127795132

Facsimile: (1)

Telex:

Email:

Commission(s): $08 \mathrm{C}, 24$

STORCHI-BERGMAN THAISA DR, INSTITUTO DE FISICA, UFRGS, CP 150151, 91500) PORTO ALEGRE RS, BRAZIL

Telephone: (55) 512364677

Facsimile: (55)

Commission(s): 28

Telex: $\$ 15730$ ccuf br

Email:

STOREY JOHN WV DR, SCHOOL OF PHYSICS, UNIV NEW SOUTH WALES, BOX 1

KENSINGTON NSW 21133, AUSTRALIA

Telephone: (61) 26974591

Facsimile: (61)

Commission(s): (09)

Telex: $2605+$ ata

Email:

STOREY MICHELLE DR, DPT THEORETICAL, PHYSICS, UNIVERSITY OF SYDNEY, SYDNEY NSW 2006, AUSTRALIA

Telephone: (61) 26922538

Facsimile: (61) 2660129013

Telex:

Email:

Commission(s): 40

STORM JESPER, ESO, CASILLA 19001, SANTIAGO 19, CHILE

Telcphone: (45) Facsimile: (45) Telex:

Emaiil:

Commission(s):

STRAFELLA FRANCESCO, DPT DI FISICA, UNIVERSITA DI LECCE, CP 193, I 73100 LECCE, ITALY

Telephone: (39) 83 2627/247 Facsimile: (39)

Telex: 86,6830 unstle $i$

Email:

Commission(s):

STRAIZYS V PROF DR, INST THEORETICAL PHYSICS, \& ASTRONOMY, GOSTAUTO 12, VILNIUS 2600, LITHUANIA

Telephonc: (370) $2613+40$ Facsimile: (371) 2818464

Email: straizysäitpa.fi.It/straizysaitpa.chet.

Telex: $2611+1 \mathrm{lma}$ su

Commission(s): $25 \mathrm{C}, 45,51$ 
STRAND KAJ AA DR, 3200 ROWLAND PL NW, WASHINGTON DC 2000, USA

Telephone: (1) $2029660495 \quad$ Facsimile: (1) Telex:

Email: Commission(s): 24,26

STRASSL HANS L PROF, ASTRONOMISCHES INSTITUT, UNIVERSITAET MUENSTER, WILHELM KLEMM STR I0 D 48149 MUENSTER, GERMANY

Telephone: (49) $251862463 \quad$ Facsimile: (49) Telex:

Email:

Commission(s):

STRASSMEIER KLAUS G DR, INSTITUT FUER ASTRONOMIE, UNIVERSITAET WIEN, TUERKENSCHANZSTR 17 A 1180) WIEN, AUSTRIA

Telephone: (43) 134031695

Facsimile: (43) 134031615

Telex: 133099

Email: strassmeicr(âavia.una.ac.at

Commission(s): 27

STRAUMANN NORBERT, INST THEORETISCHE PHYSIK, UNIVERSITAET ZUERICH, WINTERTHURERSTR 190 CH 8057 ZUERICH, SWITZERLAND

Telephone: (41) 12575815

Facsimile: (+1) 12575704

Telex:

Email:

Commission(s): $4+47$

STRAZZULLA GIOVANNI, OSS ASTROFISICO, CITTA UNIVERSITARIA, VIA A DORIA G, I 95125 CATANIA, ITALY

Telephone: (39) 9533 (1533 Facsimile: (39)

Email:

Commission(s):

Telex: 970359 astret $i$

STREL'NITSKIJ VLADIMIR DR, INST OF ASTRONOMY, ACADEMY OF SCIENCES, PYATNITSKAYA UL 48 109017 MOSCOW, RUSSIA

Telcphone: (7) 952315461

Facsimile: (7)

Commission(s): 14

Telex: $\$ 12623$ scstp su

Email:

STRINGFELLOW GUY SCOTT, DPT OF ASTRONOMY, PENNSYLVANIA STATE UNIV, 525 DAVEY LAB

UNIVERSITY PARK PA 168012, USA

Telephone: (1) $81+8639553$

Email: guyrasastro.psu.edu

Facsimile: (1) $81+8633399 \quad$ Telex:

Commission(s):

STRITTMATTER PETER A PROF, STEWARD OBSERVATORY, UNIVERSITY OF ARIZONA, TUCSON AZ 85721, USA

Telephonc: (1) 5206216532

Facsimile: (1)

Telex:

Email:

Commission(s): 35

STROBEL ANDRZEJ DR, INSTITUTE OF ASTRONOMY, N COPERNICUS UNIVERSITY, UL CHOPINA 12/18

PL 87 100 TORUN, POLAND

Tclephonc: (48) 26018

Facsinite: (48)

Commission(s): 33,45

Telex: 0552234 astr pl

Email:

STROBEL DARRELL F, DPT EARTH \& PLANETARY SCI, JOHNS HOPKINS UNIVERSITY, CHARLES \& 34TH ST BALTIMORE MD 21218, USA

Telephone: (1)

Facsimile: (1)

Telex:

Email:

Commission(s): 16

STROHMEIER WOLFGANG PROF, VOLKFELDSTR 5, D 8600 BAMBERG, GERMANY

Telephonc: (49) 95155394

Facsimile: (49)

Telex:

Emait:

Commision(s): $25,27,42$

STROM KAREN M, DPT OF ASTRONOMY, UNIV OF MASSACHUSETTS, GRC 518 B 6732, AMHERST MA 01002, USA

Telephonc: (1) 4135452290

Facsimile: (1)

Telex:

Email:

Commission(s): 27

STROM RICHARD G DR, NFRA, BOX 2, NL 7990 AA DWINGELOO, NETHERLANDS

Telephone: (31) 52197244

Facsimile: (31)

Telex:

Email:

Commission(s): $28,34,40$

STROM ROBERT G PROF, LUNAR \& PLANETARY LAB, UNIVERSITY OF ARIZONA, TUCSON AZ 85721, USA

Telephone: (1) 5206212720

Facsimile: (1)

Telex: 9110521143

Email:

Commission(s): 16,28 
STROM STEPHEN E, DPT PHYSICS \& ASTRONOMY, UNIV OF MASSACHUSETTS, GRC 518 B

AMHERST MA 01003, USA

Telephone: (1) 418545229 )

Facsimile: (1)

Telex:

Email:

Commission(s): 27,36

STRONG IAN B DR, LOS ALAMOS NATIONAL LAB, MS 436, BOX 1663, LOS ALAMOS NM 87545, USA

Telephone: (1) $505667+823$

Facsimile: (1)

Telcx:

Email:

Commission(s): 44

STRONG JOHN D PROF, ASTRON RESEARCH FACILITY, UNIV OF MASSACHUSETTS, AMHERST MA 01003, USA Telephone: (1)

Facsimile: (1)

Telex:

Email:

Commission(s): 16

STRONG KEITH T DR, LOCKHEED PALO ALTO RES LB, DPT 9130 BLDG 252, 3251 HANOVER ST

PALO ALTO CA 94304, USA

Tclephone: (1) $+1535+5136$

Facsimile: (1) $+15+2+399+$

Telex: $3+6409$ Imsc

Email: strong(äsag.space. Ioclihecd.com

Commission(s): 10

STRUBLE MITCHELL F, DPT ASTRON \& ASTROPHYS, UNIV OF PENNSYLVANIA, DAVID RITTENHOUSE LAB PHILADELPHIA PA 19104, USA

Tclephone: (1) 2152438176

Fancsimile: (1)

Telex:

Email:

Commission(s): 47

STRUCK-MARCELL CURTIS J, DPT OF PHYSICS, IOWA STATE UNIVERSITY, AMES IA 50011, USA

Telephone: (1) $5152945440 \quad$ Facsimile: (1)

Telex:

Email:

Commission(s):

STRUKOV IGOR A DR, SPACE RESEARCH INSTITUTE, ACADEMY OF SCIENCES, PROFSOJUZNAYA UL $84 / 32$ 117811 MOSCOW, RUSSIA

Telephone: (7) 953331466

Email:

Ficsimile: (7)

Commission(s): 40,47

Telex: $+11+98$ star su

STRYKER LINDA L, ARIZONA STATE UNIV WEST, BOX 37100, PHOENIX AZ 85069-7100, USA

Telephone: (1) $60125+360000$

Facsimile: (1) 602543600

Telex:

Email: strykeraphyast.la.asu.edu

Commission(s):

STUMPFF PETER PROF DR, MPI FUER RADIOASTRONOMIE, AUF DEM HUEGEL 69, D 53121 BONN, GERMANY

Telcphonc: (49) 228525360 Facsinile: (49)

Telex: $886+40$

Email:

Commission(s):

STURCH CONRAD R DR, STSCI/CSC, HOMEWOOD CAMPUS, 3700 SAN MARTIN DR, BALTIMORE MD 21218, USA

Tclephonc: (1) $301338+856$

Facsimile: (1)

Telex:

Email:

Commission(s): 33

STURROCK PETER A PROF, CTR FOR SPACE SCIENCES \&, ASTROPHYSICS, STANFORD UNIV ERL

STANFORD CA $9+3054(1) 55$, USA

Telephone: (1) $+157231+38$

Email:

Facsimile: (1)

Commission(s): $11,44,49,51$

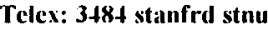

STUTZI JUERGEN DR, I PHYSIKALISCHE INSTITUT, UNIVERSITAET KOELN, ZUELPICHERSTR 77

D 5000 KOELN 11 , GERMANY

Telephone: (49) $221+703494 / 3567$

Email: stutrki@aphl.uni-kocln.de

Facsimile: (49) $221+705162 \quad$ Telex:

Commission(s):

ST-LOUIS NICOLE, DPT DE PIIYSIQUE, UNIVERSITE DE MONTREAL, CP 6128 SUCC CENTRE VILLE MONTREAL QC H3C 3J7, CANADA

Telephone: (1) $51+3+36932$

Facsimilc: (1) $51+3+32071$

Telex:

Email: stlouis/äastro.umontreal.cal

Commission(s): 29

SU BUMEI, YUNNAN OBSERVATORY, CAS, BOX 110, KUNMING 72946 YUNNAN, CHINA PR

Telephonc: (86) 8712035

Facsimile: (86)

Telex: 64040 yuobs cn

Email:

Commission(s): 34

SU DING-OIANG, NANJING ASTRONOMICAL, INSTRUMENT RESEARCH CTR, NANJING 2100+2, CHINA PR

Telephone: (86) $25 \$+11776$

Emait:

Facsimile: (86) $255+11872$

Commission(s): (19C

Telex: 
SU HONG-JUN, PURPLE MOUNTAIN OBSERV, CAS, NANJING, CHINA PR

$\begin{array}{lll}\text { Telephone: }(86) 25306967 & \text { Ficsimile: (86) } 253114 \text { 459) } & \text { Telex: } 34144 \text { pmonj cn } \\ \text { Email: } & \text { Commission(s): } 28,38 \mathrm{C}\end{array}$

SU QING-RUI DR, PURPLE MOUNTAIN OBSERV, CAS, NANJING, CHINA PR

$\begin{array}{lll}\text { Telephone: (86) } 256+2817 & \text { Facsimile: (86) } 25301459 & \text { Telex: } 34144 \text { pmonj cn } \\ \text { Email: } & \text { Commission(s): } 10 & \end{array}$

SU WAN-ZHEN, PURPLE MOUNTAIN OBSERV, CAS, NANJING, CHINA PR

Telephone: (86) $2533583 \quad$ Facsimile: (86) $25301459 \quad$ Telex: $3+14+$ pmonj cn

Email: Commission(s): 44

SUBRAHMANYA C R, TATA INST OF FUNDAMENTAL, RESEARCH, POONA UNIVERSITY CAMPUS

PUNE 411 007, INDIA

Telephone: (91) $212337107 \quad$ Facsimile: (91) $212335760 \quad$ Telex: $0145658 \mathrm{gmrt}$ in

Email: uunet!shaliti!gmrt!crs Commission(s): 47

SUBRAHMANYAM P V DR, DPT OF ASTRONOMY, UNIVERSITY OF OSMANIA, HYDERABAD 500 007, INDIA

Telephone: $(91) 71951 * 2+7 \quad$ Facsimile: $(91) \quad$ Telex:

Email: $\quad$ Commission(s): 28

SUBRAMANIAN KANDASWAMY DR, TATA INST OF FUNDAMENTAL, RESEARCH, POONA UNIVERSITY CAMPUS PUNE $\$ 11007$, INDIA

Telephone: (91) $212337107 \quad$ Facsimile: (91) $212335760 \quad$ Telex: 0145658 gmit in

Email: unnet!shakti!gust!lsandu Commission(s):

SUDA KAZUO PROF, ASTRONOMICAL INSTITUTE, TOHOKU UNIVERSITY, SENDAI AOBA, MIYAGI 980, JAPAN

Telcphonc: (81) $222221800 \quad$ Facsimile: (81)

Email: $\quad$ Commission(s): 35

Telex:

SUDZIUS JOKUBAS, ASTRONOMICAL OBSERVATORY, CIURLIONIO 29, VILNIUS 2009, LITHUANIA

Telephone: (370) 2633343

Email: jokubas.sudriusaffivu.It

Facsimilc: (370) $26356+8 / 223563$ Telex: 261212 vusu

Commission(s):

SUEMATSU YOSHINORI DR, TOKYO ASTRONOMICAL OBS, NAOJ, OSAWA MITAKA, TOKYO 181, JAPAN

Telephone: $(81)+22+13705$

Facsimile: $(81)+22+13700 \quad$ Telex: 02822307 taomtk j

Email: sucmatsu(ajspot.mtk,nao.ac.jp) Commission(s): 12

SUESS STEVEN T DR, NASA/MSFC, CODE ES 52, SPACE SCIENCE LAB, HUNTSVILLE AL 35812, USA

Telephone: (1) $205+53282$

Facsimile: (1)

Telex:

Email:

Commission(s): 49

SUGAWA CHIKARA DR, HANANOI 1586-25, KASHIWA SHI, CHIBA KEN 277, JAPAN

Telephone: (81) 471333825

Facsimile: (81)

Commission(s): 19

Telex:

Email:

SUGIMOTO DAIICHIRO PROF, DPT EARTH SCI \& ASTRON, UNIVERSITY OF TOKVO, KOMABA, MEGUROK TOKYO 153, JAPAN

Telephone: (81) 33+671171* $452 \quad$ Facsimile: (81) $33+653925 / 3485 / 2904$ Telex: $012+26728$ todaik j

Email: sugimoto äkyolou.c.u-tolṣo.ac.j Commission(s): $35,37,42$

SUGITANI KOJI DR, COLL OF GENERAL. EIUCAT, NAGOYA CITY UNIVERSITY, MIZUHO KU, NAGOYA 467, JAPA

Telephone: (81) 528725846

Email: d+30(0)äjombudpe.bitnet

Facsimile: (81) $528 \times 23075 \quad$ Telex:

Commixsion(s): 40

SUGIYAMA NAOSHI DR, DPT OF PIIYSICS, UNIVERSITY OF TOKYO, HONGO 7-3-1 BUNKYO-KU

KYOTO 113, JAPAN

Telephone: (81) $338122111 * 4191 \quad$ Facsimile: (81) $356890465 \quad$ Telex: 23472 utphysic j

Email: +1610::nsugiyama

Commission(s): 47

SUH KYUNG-WON DR, DPT ASTRONOMY \& SPACE SCI, CHUNGBUK NTL UNIVERSITY

CHEONGJU 360) 763, KOREA R

Telephone: $(82)+31612315$

Email: kwsuhácbucc.cbnu.ac.kı

Facsimile: $(82)+3167+232 \quad$ Telex:

Commission(s): 34 
SUKARTADIREDJA DARSA, PLANETARIUM DKI, JL CIKINI RAYA 73, JAKARTA 10330, INDONESIA

Telephonc: (62) 21377531

Facsimile: (62)

Telex:

Email:

Commission(s): 10,46

SUKUMAR SUNDARAJAN DR, DOMINION RADIO ASTROPHYS, OBSERVATORY, BOX 248

PENTICTON BC V2A 6K3, CANADA

Telephone: (1) $60+4932277$

Facsimile: (1) 6044937767

Telex: 04888127

Email:

Commission(s): 40

SULENTIC JACK W DR, DPT OF PHYSICS \& ASTRON, UNIVERSITY OF ALABAMA, BOX 1921

TUSCALOOSA AL 35487 (0324, USA

Telephone: (1) 2053485050

Facsimile: (1)

Telex: 81107295845

Email: jsulentic $(\bar{a} u a 1 v m$

Commission(s): 28

SULLIVAN DENIS JOHN DR, DPT OF PHYSICS, VICTORIA UNIVERSITY, PRIVATE BAG, WELLINGTON NEW ZEALAND

Telephone: (6t) 721000

Facsimile: (64)

Telex:

Email:

Commission(s): 25

SULLIVAN WOODRUFF T 11, DPT OF ASTRONOMY, UNIVERSITY OF WASHINGTON, FM 20

SEATTLE WA 98195, USA

Telephone: (1) $2025+37773$

Facsimile: (1) $2026850403 \quad$ Telex:

Email: woody (a)astro.washington.edu

Commission(s): $28,40,41,510 \mathrm{VP}, 51$

SULTANOV G F ACAD, SHEMAKHA ASTROPHYSICAL, OBSERVATORY, AZER ACADEMY OF SCIENCES 3732+3 SHEMAKHA, AZERBAIDZHAN

Telejhone: (994)

Facsimile: (994)

Telex:

Email:

Commission(s): 017,211

SUMMERS HUGH P DR, JET JOINT UNDERTAKING, CULHAM LABORATORY, ABINGDON OX14 3EA, UK

Telephone: (4) $123528822 \quad$ Facsimile: (4)

Email:

Commission(s): 14

Telex: 837505 jetcur g

SUN JIN, DPT OF ASTRONOMY, BEIJING NORMAL UNIVERSITY, BEIJING 100875, CHINA PR

Telephone: $(86) 1656531 * 6285 \quad$ Facsimile: $(86)$

Telex:

Email:

Commission(s): 34

SUN KAI, DPT OF GEOPHYSICS, BEIJING UNIVERSITY, BEIJING 100071, CHINA PR

Telephonc: $(86)$

Facsimile: (86)

Commission(s): 10

Telex: 22239 pkuni cn

Email:

SUN WEI-HSIN DR, INST PHYSICS \& ASTRONOMY, NTL CENTRAL UNIVERSITY, CHUNG LI TAIWAN, CHINA R

Telephone: (886) $3+227151^{* 5302}$

Facsimile: $(886) 3+251175 \quad$ Telex:

Email: suncaphỵast.dnct.ncu.edu.t”

Commission(s): $4+$

SUN YI-SUI, DPT OF ASTRONOMY, NANJING UNIVERSITY, NANJING, CHINA PR

Telephone: (86) 253755

Facsimile: (86)

Commission(s): 07C

Telex: $3+1.51$ prenu en

Email:

SUN YONGXIANG, INSTITUTE OF GEODESY \&, GEOPHYSICS, XU DONG LU, WUCHANG HUBEI 430077, CHINA PR Telephone: (86)

Facsimile: (86)

Telex:

Email:

Commisxion(s): 19

SUNDELIUS BJOERN DR, DPT OF ASTRONOMY, CHALMERS TECHNICAL UNIV, S +1296 GOETEBORG, SWEDEN

Telephone: (46) 31723141

Facsimile: $(46)$

Telcx:

Email: bjorncajoso.chalmers.se

Commission(s): 28

SUNDMAN ANITA DR, STOCKHOLM OBSERVATORY, S 13336 SALTSJOEBADEN, SWEDEN

Telcphonc: (46) 87170634

Facximile: (46) $8717+71$ )

Telex: 12972 sweden

Email:

Commission(s): $41,+2$

SUNTZEFF NICHOLAS B, CERRO TOLOLO, INTERAMERICAN OBSERVATORY, CASILLA 603, LA SERENA, CHILE Telcphone: (56) 51213352

Facsimile: (56) $51212466 * 3+2$

Commission(s): 29,317,37,50

Telex: 620 301 alura ct

Email: 
SUNYAEV RASHID A DR, SPACE RESEARCH INSTITUTE, ACADEMY OF SCIENCES, PROFSOJUZNAYA UL 84/32 117810 MOSCOW, RUSSIA

Telephonc: (7) 952302585

Facsimilc: (7) 953107023

Telex: 411498 star su

Email:

Commission(s): $4+, 47$

SURAN DORU MARIAN, ASTRONOMICAL INSTITUTE, CUTITUL DE ARGINT 5, BOX 28

R 75212 BUCHAREST, RUMANIA

Telephone: (40) 16236892

Email: masuranatimar.ıo

Facsimile: (40) $13123391 \quad$ Telex:

Commission(s):

SURDEJ JEAN M G, STSCI, HOMEWOOD CAMPUS, 3700 SAN MARTIN DR, BALTIMORE MD 21218, USA

Telephone: (1) 4103384861

Facsinile: (1) $\$ 103382617 \quad$ Telex:

Email: surdejástsci.edu

Commission(s): $15,+7$

SUTANTYO WINARDI, BOSSCHA OBSERVATORY, LEMBANG 40391, INDONESIA

Telejhone: (62) 229 6001 Facsimile: (62) Telex:

Email:

Commission(s):

SUTHERLAND PETER G DR, DPT OF PHYSICS, MCMASTER UNIVERSITY, HAMILTON ON L8S +M1, CANADA

Telephone: (1) 4165259140

Facsimilc: (1) $+165+61252$

Telex: 0618347

Email:

Commission(s):

SUTHERLAND WILLIAM DR, DPT OF ASTROPHYSICS, UNIVERSITY OF OXFORD, KEBLE RD

OXFORD OX1 3RH, UK

Telephone: (44) 1865273310

Facsimile: (44) $1865273+18$

Telex: 83295 nuclox $g$

Email: wjsäuk.ac.ox.astro/1946t::wis

Commission(s): 47

SUTO YASUSHI DR, DPT OF PHYSICS, UNIVERSITY OF TOKYO, BUNKYO KU, TOKYO 113, JAPAN

Telephone: (81) $338123111^{*}+195 \quad$ Facsimile: (81) $3568+96+2 \quad$ Telex:

Email:

Commission(s): 47

SUTTON EDMUND CHARLES, DPT OF ASTRONOMY, UNIVERSITY OF ILLINOIS, 1002 W GREEN ST

URBANA IL 61801, USA

Telephone: (1) 2173339339

Email: suttoncáastro.uiuc.edu

Facsimile: (1) $21724+76,38 \quad$ Telex:

Commission(s):

SUZUKI HIDEYUKI, KEK NTL LABORATORY FOR, HIGH ENERGY PHYSICS, 11 OHO

TSUKUBA IBARAKI 305, JAPAN

Telephone: (81) 298645400

Facsimile: (81) $2986+2580 \quad$ Telex:

Email: suzakiaglieth1.kek.jo

Commission(s): 4

SUZUKI YOSHIMASA PROF, 23-I NAKAJIMA, HIRONOMACHI, UJI 611, JAPAN

Telephonc: (81) Facsimile: (81) Telex:

Email:

Commission(s): 34

SVALGAARD LEIF DR, HERTOGENLAAN 31, B 3202 LUBBEEK, BELGIUM

Telephone: (32) Facsimile: (32) Telex:

Enail: $\quad$ Commission(s):

SVECHNIKOVA MARIA A DR, DPT OF ASTRONOMY, URALSKIJ STATE UNIVERSITY, 620083 SVERDLOVSK, RUSSI

Telephone: (7)

Facsimile: (7)

Telex:

Email:

Commission(s): 42

SVENSSON ROLAND, STOCKHOLM OBSERVATORY, S 13336 SALTSJOEBADEN, SWEDEN

Telephone: $(46) 816+472$

Facsimile: (46) $8717+719$ Telex:

Email: svensson'a astro.su.se

Commission(s): $4+$

SVESHNIKOV MIKHAIL, ITA RAS, 10 NAB KUTUZOVA, ST PETERSBURG 191187, RUSSIA

Telcphone: (7) 8122750360

Facsimile: (7) 8122727968

Telex: 121578 ital su

Email: $1088 / a ;$ iipalit.spb.su

Commission(s):

SVESTKA JIRI DR, OBS AND PLANETARIUM, PETRIN 215, CZ 118 46 PRAIIA 1, CZECH R

Telephone: (42) 25353513

Facsimile: (42)

Commission(s): 22,66

Telex:

Email: 
SVESTKA ZDENEK DR, SPACE RESEARCH LABORATORY, SRON, SORBONNELAAN 2

NL 358+ CA UTRECHT, NETHERLANDS

Telephonc: (31) 30535600$)$

Facsimile: (31) $305+10860$

Telex: $\mathbf{4 2 2 4}$ astro $\mathrm{nl}$

Email:

Commission(s): 10,12

SVOLOPOULOS SOTIRIOS PROF, DPT OF ASTROPHYSICS, NTL UNIVERSITY OF ATHENS, PANEPISTIMIOPOLIS GR 15771 ZOGRAFOS, GREECE

Telephone: (30) $172+3414$

Facsimile: (30)

Telex:

Email:

Commission(s): 29,33,41

SVOREN JAN, ASTRONOMICAL INSTITUTE, SLOVAK ACADEMY SCIENCES SK 059 60 TATRANSKA LOMNI, SLOVAK R

Telephone: (42) 969 96 7866/7/8 $\quad$ Facsimile: (+2) $969967656 \quad$ Telex: 78277 au sav cs

Email:

Commission(s): 15,20

SWADE DARYL ALLEN, STSCI, HOMEWOOD CAMPUS, 3700 SAN MARTIN DR, BALTIMORE MD 21218, USA

Telephone: (1) $410338+480$

Facsimile: (1) $+10338+767$

Telex:

Email: swade $\mathbf{a}$ stsci.edu

Commission(s): 15,34

SWANENBURG B N DR, SRL, IUYGENS LAB, BOX 9504, NL 2300 RA LEIDEN, NETHERLANDS

Telephone: (31) $71272727 \quad$ Facsimile: (31)

Email:

Commission(s):

Telex: $3 \% 158$ astro $\mathrm{nl}$

SWANK JEAN HEBB, NASA GSFC, CODE 661, GREENBELT MD 20771, USA

Telephone: (1) 301 2866188

Facsimile: (1)

Telex: 89675 nascom gblt

Email:

Commission(s): H

SWARUP GOVIND PROF, TATA INST OF FUNDAMENTAL, RESEARCH, POONA UNIVERSITY CAMPUS

PUNE +11 007, INDIA

Telephone: (91) 212336111

Email: uunet!shakti!gmrt!gswarup

Facsimile: (91) 212335760

Telex: 0145658 gmrt in

SWEET PETER A PROF, DPT OF ASTRONOMY, UNIVERSITY OF GLASGOW, GLASGOW G12 8QQ, UK

Telephonc: (t+) $1+13398855$

Facsimile: $(4+)$

Telex:

Email:

Commission(s): 35

SWEIGART ALLEN V DR, NASA GSFC, CODE 681, GREENBELT MD 20771, USA

$\begin{array}{lll}\text { Telephonc: (1) 301 } 2866274 & \text { Facsimile: (1) } & \text { Telex: } 89675 \\ \text { Email: } & \text { Commission(s): } 35\end{array}$

SWEITZER JAMES STUART DR, ASTRONOMY \& ASTROPHYS CTR, UNIVERSITY OF CHICAGO, 5640 S ELLIS AVE CHICAGO IL 60637, USA

Telephonc: (1) $3127(127783$

Email: jssäuodujob.uchicago.cdu

Facsimile: (1) $3127028212 \quad$ Telex:

Commission(s):

SWENSON GEORGE W JR PROF, DPT ELECT \& COMPUTER ENG, UNIVERSITY OF ILLINOIS, 1406 W GREEN ST URBANA IL 61801, USA

Telephone: (1) $217333+498$

Facsimile: (1)

Telex:

Enail:

Commission(s): 40

SWENSSON JOHN W DR, DPT OF THEORETICAL PHYS, UNIVERSITY OF LUND, SOELVEGATAN 14 A

S 22362 LUND, SWEDEN

Telephone: (46) 10969686

Email:

Facsimile: (46)

Telex:

Commission(s): 12,29

SWERDLOW NOEL PROF, ASTRONOMY \& ASTROPHYS CTR, UNIVERSITY OF CHICAGO, 5640 S ELLIS AVE

CHICAGO IL 60637 , USA

Telephone: (1) 3129627969

Email:
Facsimilc: (1)

Commission(s): +1
Telex:

SWIHART THOMAS L DR, 448 W HARDY RD, TUCSON AZ 85737, USA

Telephone: (1)

Facsimile: (1)

Commission(s): 36
Telex: 
SWINGS JEAN-PIERRE DR, INSTITUT D'ASTROPHYSIQUE, UNIVERSITE DE LIEGE, AVE COINTE 5 B $\$ 000$ COINTE-LIEGE, BELGIUM

Tclephone: (32) +1529980

Facsimile: (32) $+1527+74$

Telex: $+126+4$ astrolg b

Email: jpswingsāam $1 . u l g$. ac.be

Commission(s): $119,14,29$

SYGNET JEAN FRANCOIS DR, INSTITUT D'ASTROPHYSIQUE, 98BIS BD ARAGO, F 7501+ PARIS, FRANCE

Telephone: (33) $14 \$ 328094$

Facsimile: (33) $1+4328001$

Telex:

Email: syget(afriap51

Commission(s): 33

SYKES MARK VINCENT DR, STEWARD OBSERVATORY, UNIVERSITY OF ARIZONA, TUCSON AZ 85721, USA

Telephone: (1) 5206212054

Facsinile: (1)

Telex: 467175

Email: msykesias.arizona.edu

Commission(s): $15,21 \mathrm{C}$

SYKES-HART AVRIL B DR, DPT OF ASTROPHYSICS, UNIVERSITY OF OXFORD, SOUTH PARKS RD

OXFORD OX1 3RQ, UK

Telephone: (44)

Facsimile: (4t)

Telex:

Email:

Commission(s):

SYKORA JULIUS DR, ASTRONOMICAL INSTITUTE, SLOVAK ACADEMY SCIENCES

SK 059 60 TATRANSKA LOMNI, SLOVAK R

Telephone: (42) $969967866 / 7 / 8 \quad$ Facsimile: (42) $969967656 \quad$ Telex: 78277 ausav cz

Email:

Commission(s): 10

SYLWESTER BARBARA DR, ASTRONOMICAL INSTITUTE, WROCLAW UNIVERSITY, UL KOPERNIKA 11

PL 51 622 WROCLAW, POLAND

Telephone: $(48)+83238$

Email:

Facsinile: (48)

Commission(s): 10

Telex: 0712791 uwrpl

SYLWESTER JANUSZ, ASTRONOMICAL INSTITUTE, WROCLAW UNIVERSITY, UL KOPERNIKA II

PL 51 622 WROCLAW, POLAND

Telephone: (48) 481801

Facsimile: (48)

Commission(s): 10C

Telex:

Email:

SYNNOTT STEPHEN P, JPL, MS 264686,4800 OAK GROVE DR, PASADENA CA 91109, USA

Telephone: (1) 8183546933

Facsimile: (1)

Telex:

Email:

Commission(s): 16,20

SZABADOS LASZLO PH D, KONKOLY OBSERVATORY, THEGE U 13/17, BOX 67, H 1525 BUDAPEST, HUNGARY

Telephone: (36) $11755866 / 175+122$

Facsimile: (36) 11569640

Telex: $227 \$ 60$ konobh

Email:

Commission(s): $26,27,42$

SZAFRANIEC ROZALIA DR, UL KOPERNIKA 27, PL 31 501 KRAKOW, POLAND

Telephone: (48)

Facsimile: (48)

Telex:

Email:

Commission(s): 42

SZALAY ALEX DR, DPT ATOMIC PHYSICS, EOTVOS UNIVERSITY, PUSHKIN U 5-7, H IOB8 BUDAPEST, HUNGARY

Telephone: (36) $111+1019$

Email: szalai(âludens.elte.hu Fatesimile: (36) $12101689 \quad$ Telex:

Commission(s): 47VP

SZATMARY KAROLY DR, JATE UNIVERSITY, DPT OF PHYSICS, DOM TER 9, H 6720 SZEGED, HUNGARY

Telephone: (36) 366211622 Facsimile: (36) 366212921

Telex:

Email: h267+sza(a) cllı.hu

Commission(s): 27

SZEBEHELY VICTOR G PROF, AEROSPACE ENGINEERING DPT, UNIVERSITY OF TEXAS, WRW 414, AUSTIN TX 78712, USA

Telephone: (1) $512+71+239$

Facsimile: (1) $512+713788$

Telex: 9108741305

Email:

Commission(s): 07,33

SZECSENYI-NAGY GABOR DR, ASTRONOMY DPT, EOTVOS UNIVERSITY, KUN BELA TER 2

H 1083 BUDAPEST, HUNGARY

Telephone: (36) $111+1019$

Email:

Facsimile: (36) Telex:

Commission(s): 27,36,37,46

SZEGO KAROLY DR, CENTRAL RESEARCH INST, FOR PHYSICS, BOX 49, H 1525 BUDAPEST, HUNGARY

Telephone: (36) 1551682

Facsimile: (36) 1696567

Telex: $22+722$

Email:

Commission(s): 15 


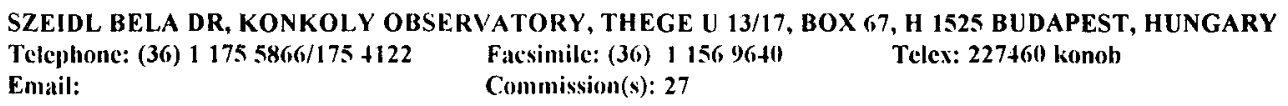

TABARA HIROTO DR, FAC OF EDUCATION, UTSUNOMIYA UNIVERSITY, MINEMACHI, UTSUNOMIYA 321, JAPAN Telephone: (81) $286361515 \quad$ Facsimile: (81)

Email:

Commission(s): 41 Telex:

TABOADA RAMON RODRIGUEZ, INST GEOPHYS \& ASTRONOMY, CALLE 212 N 2906/29 Y 31, LISA

LA HABANA, CUBA

Telephone: (53)

Facsimile: $(53)$

Email:

Commission(s):

Telex:

TABORDA JOSE ROSA DR, FACULTY OF SCIENCES, ASTRONOMICAL OBSERVATORY

R ESCOLA POLITECNICA 58, P 1200 LISBOA, PORTUGAL

Telephone: (351)

Facsimile: (351) Telex:

Email:

Commission(s): 07,46

TACCONI LINDA J DR, MPI F EXTRATERRESTRISCHE, PHYSIK, KARL SCHWARZSCHILDSTR 1

D 857+1 GARCHING MUENCHEN, GERMANY

Telephone: (49) 89 329 93289) Facsimile: (49) 89 32993569 Telex: 5215845 xter d

Enali: lindaladgampc.bitnat Commission(s): 28

TACCONI-GARMAN LOWELL E, MPI FUER EXTERRESTRISCHE, PHYSIK, GIESSENBACHSTR

D 85748 GARCHING MUENCHEN, GERMANY

Telephone: (\$9) $8932993288 \quad$ Fatesimile: (49) 8932993569 Telex:

Email: lowellajmpa-garching.mpg.de Commission(s): 15,28

TADEMARU EUGENE DR, DPT OF ASTRONOMY, UNIV OF MASSACHUSETTS, AMHERST MA 01002, USA

Telephonc: (1)

Facsimile: (1)

Telex:

Email:

Commission(s):

TAFF LAURENCE G DR, 5208 SPRINGLAKE WAY, BALTIMORE MD 212123240 , USA

Telephone: $(1)+10338+799 \quad$ Facsimile: $(1)+10338+796 \quad$ Telex:

Email: Commission(s): 08

TAFFARA SALVATORE PROF, VIA CALZA 5BIS, 135128 PADOVA, ITALY

Telephone: (39) $498071624 \quad$ Facsimile: (39)

Email:

Commission(s)

Telex:

TAGLIAFERRI GIUSEPPE PROF, OSS ASTROFISICO, DI ARCETRI, LARGO E FERMI 5, I 50125 FIRENZE, ITALY

Telephonc: (39)

Facsimile: (39)

Telex:

Ematil:

Commission(s):

TAGO ERIK, TARTU ASTROPHYSICAL OBS, EE 2H+4 TORAVERE, ESTONIA

Telephone: (372) $7+10+34 \quad$ Facsimile: (372) $7+10205 \quad$ Telex:

Email: crikajupiter.atai.ee Commission(s):

TAKABA HIROSHI DR, KASHIMA SPACE RES CENTER, CRL, 893-1 HIRAI, IBARAKI 314, JAPAN

Telephone: (81) 290 82 1211

Facsimile: (81) 299835728

Telex: $7604(138 \mathrm{crl}$ uc

Email: lashimaralatair.gstc.nasa.gov

Commission(s): 41,51 
TAKADA-HIDAI MASAHIDE DR, RES INST OF CIVILIZATION, TOKAI UNIVERSITY, 1117 KITAKANAME KANAGAWA 259-12, JAPAN

Telephone: (81) 463581211

Facsimile: $(81)$

Telex: $2+23+02$ unitok $j$

Email:

Commission(s): 29,51

TAKAGI KOJIRO PROF, DPT OF PHYSICS, TOYAMA UNIVERSITY, 3190 GOFUKU, TOYAMA 930, JAPAN

Telephone: $(81) 76423+716$

Facsimile: (81)

Telex:

Email:

Commission(s): 40

TAKAGI SHIGETSUGU DR, DPT DE FISICA-CCE-UFRN, CAMPUS UNIVERSITARIO, 59000 NATAL RN, BRAZIL

Telephone: (55)

Facsimile: (55)

Telex:

Email:

Commission(s):

TAKAHARA FUMIO DR, DPT OF PHYSICS, TOKYO METROPOLITAN UNIVER, MINAMIOHSAWA 1-1, HACHIOJI TOKYO 192-(1), JAPAN

Telephone: (81) +26771111

Facsimilc: (81) $\$ 26772483$

Telex:

Email:

Commission(s): 44,47

TAKAHARA MARIKO, DPT OF ASTRONOMY, UNIVERSITY OF TOKYO, BUNKYO KU, TOKYO I13, JAPAN

Telephonc: (81) 38122111

Email:

Facsimile: (81)

Telex: 33659 utyosci

Commission(s): 35

TAKAHASHI MASAAKI, DPT PHYSICS \& ASTRONOMY, AICHI UNIVERSITY OF EDU, KARIYA, AICHI 4H8, JAPAN

Telephone: (81) 566363111 *538

Facsimile: $(81) \quad 56636+337$

Telex:

Email: takahasiagaucphyas.aichi-e(u.ac.jp)

Commission(s): 4

TAKAKUBO KEIYA PROF, ASTRONOMICAL INSTITUTE, TOHOKU UNIVERSITY, SENDAI AOBA

MIYAGI 980, JAPAN

Telephone: (81) 222221800

Email:

Facsimile: (81)

Commission(s): 34,40

Telex: 852246 thucom $\mathbf{j}$

TAKAKURA TATSUO PROF EMER, DPT OF ASTRONOMY, UNIVERSITY OF TOKYO, BUNKYO KU TOKYO 113, JAPAN

Telephone: (81) 38122111

Facsimile: (81)

Commission(s): $10,+1),+4$

Telex: 336.59 utyosci j

Email:

TAKALO LEO O DR, TURKU UNIVERSITY OBS, TUORLA, SF 21500 PIIKKIO, FINLAND

Telephone: (358) $21+35822$

Facsimile: (358) $21+33767 \quad$ Telex:

Email: takalorakontu.utu.fi

Commission(s): 28

TAKAMI HIDEKI DR, NTL ASTRONOMICAL OBS, 211 OSAWA, MITAKA, TOKYO 181, JAPAN

Telephonc: (81) $+223+3865$

Facsimile: $(81)+223+3864$

Telex:

Email: takami@optik.mtk.nao.ac.jp

Commission(s): 28

TAKANO TOSHIAKI DR, NOBEYAMA RADIO OBS, NAOJ, MINAMIMAKI MURA, NAGANO 38+ 13, JAPAN

Telephone: (81) $26763+487$

Email: takano(a)nro.nao.ac.jp

Facsimile: (81) 26763444

Telex:

Commission(s): $10,34,40$

TAKARADA KATSUO DR, KYOTO INST OF TECHNOLOGY, MATSUGASAKI, KITASHIRAKAWA SAKYO KU KYOTO 606, JAPAN

Telephone: (81) 757913211

Facsimile: $(81)$

Commission(s):

Telex:

Email:

TRONOMICAL OBS, NAOJ, OSAWA MITAKA, TOKYO 181, JAPAN

Telephone: (81) +22325111 Facsimile: (81)

Email:

Commission(s): 28

Telex: 2822307 taomtk

TAKAYANAGI KAZUO PROF, SYSTEMS ENGINEERING, SHIBAURA INST TECHNOLOGY TAMEIHARA 307 FUKASAKU, OMIYA SAITAMA KEN 330, JAPAN

Telephone: (81) $486875153 \quad$ Facsimile: $(81) 486875198$

Email:

Commission(s): 14

Telex: 
TAKEDA HIDENORI DR, DPT AERONAUTIC ENGINEER, KYOTO UNIVERSITY, KITASHIRAKAWA SAKYO KU KYOTO 606, JAPAN

Telephone: (81)

Facsimile: (81)

Telex:

Email:

Commission(s): 15

TAKEDA YOICHI DR, INST OF ASTRONOMY, UNIVERSITY OF TOKYO, OSAWA MITAKA, TOKYO 181, JAPAN

Telephonc: (81) 42241373 )

Facsimile: (81) $+22+13749$

Telex: 2822307 taomtk j

Email:

Commission(s): 36

TAKENOUCHI TADAO DR, 12830 KICHIJYOJI, KITA MACHI, MUSASHINO SHI, TOKYO 180, JAPAN

Telephone: (81) Facsimile: (81)

Email: $\quad$ Commission(s):

Telex:

TAKENS ROELF JAN DR, ASTRONOMICAL INSTITUTE, UNIVERSITY OF AMSTERDAM, KRUISLAAN 403

NL 1098 SJ AMSTERDAM, NETHERLANDS

Telephone: (31) $205257491 \quad$ Facsimile: (31) $205257484 \quad$ Telex: $10262 \mathrm{hef} \mathrm{nl}$

Email:

Commission(s):

TAKEUTI MINE DR, ASTRONOMICAL, INSTITUTE, TOHOKU UNIVERSITY, SENDAI AOBA, MIYAGI 980, JAPAN

Telephonc: (81) 222221800

Facsimile: (81) 222612806

Telex: 852246 thucom $j$

Email: ifatosda (ajpntoholi

Commission(s): $27 \mathrm{C}$

TALAVERA A DR, ESA IUE OBSERVATORY, VILSPA, APD 50727, E 28080 MADRID, SPAIN

Telephone: (34) $18131100 \quad$ Facsimile: (34) $18131139 \quad$ Telex: 42555 vilse

Email:

Commission(s): 29

TALBOT RAYMOND J JR DR, THE AEROSPACE CORPORATION, 1927 CURTIS AVENUE

REDONDO BEACH CA 90278, USA

Telephonc: (1) 2133799927

Facsimile: (1)

Telex:

Email:

Commission(s): 28

TALON RAOUL DR, CESR, BP $\$ 3+6$, F 31129 TOULOUSE CDX, FRANCE

Telephone: (33) 61556666

Facsimile: (33) 61556701

Commission(s): 10

Telex: $\mathbf{5 3 1 3 2 9}$ unspat $f$

Email:

TALWAR SATYA P DR, DPT OF PHYSICS, UNIVERSITY OF DELHI, NEW DELHI 110 007, INDIA

Telephone: (91) $112918993 \quad$ Facsimile: (91)

Telex:

Email:

Commission(s)

TAMENAGA TATSUO DR, FAC OF EDUCATION, MIE UNIVERSITY, TSU-SHI, MIE 514, JAPAN

Telephonc: (81)

Facsimilc: (81)

Telex:

Email:

Commission(s): 10

TAMMANN G ANDREAS PROF DR, ASTRONOMISCHES INSTITUT, UNIVERSITAET BASEL, VENUSSTRASSE 7

CH +1112 BINNINGEN, SWITZERLAND

Telejhone: (\$1) $612717711 / 12$

Facsimule: (41) 612717810

Telex:

Email:

Commission(s): $27,28,33,47$

TAMURA MOTOHIDE, NTL ASTRONOMICAL OBS, 2211 OSAWA, MITAKA, TOKYO 181, JAPAN

Telephone: $(81)+223+3705$

Facsimile: $(81)+223+3608 \quad$ Telex:

Email: otamura(ajc1.mtk.nao.ac.jp)

Commission(s): 34

TAMURA SHINICHI DR, DPT OF ASTRONOMY, TOHOKU UNIVERSITY, ARAMAKI, SENDAI 980, JAPAN

Telcphone: (81) $222221800 \quad$ Facsimile: (81)

Commission(s): 34

Telex: 852246 thucom $j$

Email:

TAN DETONG, SHANGHAI OBSERVATORY, ACADEMIA SIMICA, 80 NANDAN RD, SHANGHAI 200030, CHINA PR

Telephone: (86) 214386191

Facsimile: (86) $21+38+618$

Telex: 33164 shao cn

Email:

Commixsion(s): 8

TAN HUISONG, YUNNAN OBSERYATORY, CAS, BOX 110, KUNMING 729 46 YUNNAN, CHINA PR

Telephone: (86) 8712035

Facsimile: $(86)$

Commission(s): 42

Telex: 64040 yuobs co

Email: 
TANABE HIROYOSHI DR, TOKYO ASTRONOMICAL OBS, NAOJ, OSAWA MITAKA, TOKYO 181, JAPAN

Telephome: (81) 422325111

Facsimile: (81)

Telex: (02822307 taomtk j

Email:

Commission(s): 15,21

TANABE KENJI DR, OKAYAMA UNIV OF SCIENCE, 1-1 RIDAI-CHO, OKAYAMA 700, JAPAN

Telephone: (81) 862523161

Facsimile: (81) 862553847 Telex:

Email:

Commission(s): 47

TANABE TOSHIHIKO DR, TOKYO ASTRONOMICAL OBS, NAOJ, OSAWA MITAKA, TOKYO 181, JAPAN

Telcphonc: (81) 422325111

Facsimile: (81)

Telex:

Email:

Commission(s):

TANAKA MASUO DR, INST OF ASTRONOMY, UNIVERSITY OF TOKYO, OSAWA MITAKA, TOKYO 181, JAPAN

Telephone: $(81)+22+137+3$

Facsimile: (81) $+22+13749$ Telex:

Email:

Commission(s): 34

TANAKA RIICHIRO PROF, FAC OF EDUCATION, NIIGATA UNIVERSITY, 8050 IKARASHI 2, NIIGATA 950 21, JAPAN

Telephone: $(81)$

Facsimile: (81)

Telex:

Email:

Commission(s): 40

TANAKA WATARU DR, OPT \& IR ASTRO DIV, NAOJ, OSAWA MITAKA, TOKYO 181, JAPAN

Telephone: (81) $4223+3603$

Facsimile: (81) $+223+3608$ Telex:

Email: otanak2@c1.mtk.nao.ac.jp) Commission(s):

TANAKA YASUO DR, DPT OF PHYSICS, IBARAKI UNIVERSITY, BUNKYO, MITO 310, JAPAN

$\begin{array}{ll}\text { Telephonc: (81) } 292261621^{\star} 372 & \text { Facsimile: (81) } \\ \text { Email: } & \text { Commission(s): } 4+\end{array}$

Telex:

TANAKA YASUO PROF, ISAS ASTROPHYSICS, 3-1-1 YOSHINODAI, SAGAMIHARA, KANAGAWA 229, JAPAN

Telephone: (81) 427513111 Facsimile: $(81)+2759+253$ Telex:

Email: $\quad$ Commission(s): th

TANAKA YUTAKA D DR, KOBE YAMATE WOMEN'S, JUNIOR COLLEGE, SUWAYAMA CHUO-KU, KOBE G50, JAPA

Telephone: (81) $783+161600 \quad$ Facsimile: $(81)$

Email:

Commission $(\mathrm{s}): 28$

Telex:

TANCREDI GONZALO, DPT DE ASTRONOMIA, FAC CIENCIAS, TRISTAN NARAJA 1674

MONTEVIDEO 11200, URUGUAY

Telephone: (598) $2418004 \quad$ Facsimile: (508) $2421957 \quad$ Telex:

Email: gonzalo(afisica.cdu.uy Commission(s): 20

TANDBERG-HANSSEN EINAR A, NASA/MSFC, CODE ES 01, HUNTSVILLE AL 35812, USA

Telephone: (1) $2055447578 \quad$ Facsimile: (1) Telex:

Email:

Commission(s): 10,12

TANDON JAGDISH NARAIN DR, DPT PHYSICS \& ASTROPHYS, UNIVERSITY OF DELHI, NEW DELHI 110 007, INDIA

Telephone: (91) 112521521

Facsimile: (91)

Telex:

Email:

Commission(s): 10

TANDON S N PROF, IUCAA, PO BOX 4 , GANESHKHIND, PUNE 411 (007, INDIA

Telephone: (91) 212336105 Facsimile: (91) 212335760 Telex:

Email:

Commission(s): 25

TANG YU-HUA, DPT OF ASTRONOMY, NANJING UNIVERSITY, NANJING, CHINA PR

Telephone: (86) $2537651 \quad$ Facsimile: (86)

Email:

Commission(s): 10

TANGO WILLIAM J DR, SCHOOL OF PHYSICS, UNIVERSITY OF SYDNEY, SYDNEY NSW 2006, AUSTRALIA

Telephone: (61) 26923953

Facsimile: (61)

Commission(s): (19)

Telex: 26169 unisyd ata

Email:

TANIGUCHI YOSHIAKI DR, ASTRONOMICAL INSTITUTE, TOHOKU UNIVERSITY, ARAMAKI AOBA

SENDAI 980, JAPAN

Telephone: $(81) 222221800 * 3319$

Facsimile: (81) 222612806

Telex:

Email: tani(a)astroa.astr.tohoku.ac.jp

Commission(s): 28 
TANIKAWA KIYOTAKA, NTL ASTRONOMICAL OBS, 211 OSAWA, MITAKA, TOKYO 181, JAPAN

Telephone: (81) $4+23+3634$

Facsimile: $(81)+\$ 23+37+6$

Telex:

Email: tanikawa@ferio.mtk.nao.ac.jp

Commission(s):

TANZELLA-NITTI GIUSEPPE, ROMAN ATHENAEUM OF THE, HOLY CROSS, S GIROLAMO DELA CARITA 64 I 00186 ROME, ITALY

Telephonc: (39)

Facsimile: (39)

Telex:

Email:

Commission(s):

TANZI ENRICO G, IST DI FISICA COSMICA, CNR, VIA BASSANI 15, I 20133 MILANO, ITALY

Telephone: (39) $2298237 \quad$ Facsimile: (39)

Telex:

Email:

Commission(s):

TAPDE SURESH CHANDRA, NCRA/TIFR, POONA UNIV CAMPUS PB 3, GANESHKHIND, PUNE 411 007, INDIA

Telephone: (91) 2123336108

Email: sct(a)gmrt.crnet.in

Facsimile: (91) 212345149

Telex: 8101457658 gmrt in

TAPIA MAURICIO DR, INSTITUTO DE ASTRONOMIA, UNAM, APDO POSTAL 877

22860 ENSENADA B CALIF, MEXICO

Telephone: (52) 4 (0880/8 3093

Facsimile: (52)

Telex: $\mathbf{5 6 7 3 9}$ ciceme

Email:

Commission(s):

TAPIA-PEREZ SANTIAGO, PHILLIPS LAB, LIMA, KIRTLAND AFB NM 87117, USA

Telephone: (1) $5058+65045$

Facsimilc: (1)

Telex:

Email: tapiaáplli.af.mil

Conmission(s): 25

TAPLEY BYRON D DR, AEROSPACE ENGINEERING DPT, UNIVERSITY OF TEXAS, WRW 402, AUSTIN TX 78712, US Telephonc: (1) $512+711356$

Facsimile: (1)

Telex:

Email:

Commission(s): 19

TAPPING KENNETH F, HERZBERG INST ASTROPHYS, NTL RESEARCH COUNCIL, 100 SUSSEX DR OTTAWA ON K1A ORG, CANADA

Telejphone: (1) $61399158+2$

Facsimile: (1) 6139526602

Telex: 0533715

Email:

Commission(s): 10

TARADY VLADIMIR K DR, MAIN ASTRONOMICAL OBS, UKRAINIAN ACAD OF SCIENCE, GOLOSEEVO 252127 KIEV, UKRAINE

Teleplione: (7) 044662286

Email:

Facsimile: (7)

Commission(s): 19C

Telex: 131406 sky su

TARAFDAR SHANKAR P DR, TIFR, HOMI BHABHA RD, COLABA, BOMBAY +00 005, INDIA

Telejphonc: (91) 22219111

Facsimile: (91)

Telex: 0113009 tifr in

Email: tarafdaratifrvax

Commisuion(s): 34,36

TARENGHI MASSIMO DR, ESO, KARL SCHWARZSCHILDSTR 2, D 85740 GARCHING MUENCHEN, GERMANY

Tele|phone: (49) 89 32006236

Facsimile: (49) 893202362

Telex: 52828223 co d

Email:

Conmission(s):

TARNSTROM GUY DR, MIT LINCOLN LABORATORY, BOX 73, LEXINGTON MA 02173, USA

Telephone: (1) 6178635500

Facsimile: (1)

Telex: 923355

Email:

Commission(s):

TARRAB IRENE, 221 RUE LA FAYETTE, F 75010 PARIS, FRANCE

Telephone: (33)

Facsimile: (33)

Telex:

Email:

Commission(s):

TARTER C BRUCE DR, LAWRENCE LIVERMORE LAB, L 295, BOX 808, LIVERMORE CA 94550, USA

Telephone: (1) $+15+22+169$

Facsimilc: (1)

Telex:

Email:

Commission(s):

TARTER JILL C DR, SETI INSTITUTE, PROJECT PHOENIX OFFICE, 2035 LANDINGS DRIVE MOUNTAIN VIEW CA $9+11+3$, USA

Telephone: (1) $+15960+555$

Facsimile: (1) 4159685830

Tclex:

Email: tarterablisyast.berliclev.edu

Commission(s): $411,+7,51 P$ 
TASSOUL JEAN-LOUIS PROF, 6000 CH DEACON APP J2, MONTREAL QC H3S 2T9, CANADA

Telephone: (1)

Facsimilc: (1)

Telex:

Email:

Commission(s):

TASSOUL MONIQUE DR, 6000 CH DEACON APP J2, MONTREAL QC H3S 2T\%, CANADA

Telephone: (1)

Facsimile: (1)

Telex:

Email:

Commission(s): 35

TATEMATSU KEN'ICHI DR, IBARAKI UNIVERSITY PHYS, 2-1-1 BUNKYO, MITO 310, JAPAN

Telephone: (81) 292261621

Facsimile: (81) $292278222 \quad$ Telex:

Email: ktäro.nao.ac.jp

Commission(s): 40

TATEVYAN S K DR, INST OF ASTRONOMY, ACADEMY OF SCIENCES, PYATNITSKAYA UL 48 109017 MOSCOW, RUSSIA

Telephone: (7) 952315461

Facsimile: (7)

Commission(s): (07

Telex: +12623 sestp su

Email:

TATEYAMA CLAUDIO EIICHI, CRAAE INPE, EPUSP/PTR/CRAAE, CP 61548, 05+24970 SAO PAULO, BRAZIL

Telephone: (55) 118155936

Facsimile: (55) 118156280

Telex:

Email: cetateya(a)bruspvm.bitnet

Commission(s):

TATON RENE PROF, CENTRE ALEXANDRE KOYRE, 12 RUE COLBERT, F 75002 PARIS, FRANCE

Telephone: (33) 142975245

Facsimile: (33)

Telex:

Email:

Commission(s): 41

TATUM JEREMY B DR, CLIMENHOGA OBSERVATORY, UNIVERSITY OF VICTORIA, BOX 1700

VICTORIA BC V8W 2Y2, CANADA

Telephone: (1) 6047217750

Facsimile: (1) $60+7217715 \quad$ Telex:

Email:

Commission(s): $14,15,20$

TAUBER JAN, ASTROPHYSICS DIV, ESA/ESTEC, KEPLERLAAN 1, NL 2201 AZ NOORDWIJK, NETHERLANDS

Telephone: (31) $1719853+2$

Facsimile: (31) 1719 84690 Telex:

Email: jtauber(a)astro.estec.esa.nl

Commission(s):

TAUTVAISIENE GRAZINA, INST THEORETICAL PHYSICS, \& ASTRONOMY, GOSTAUTO 12

VILNIUS 2600, LITHUANIA

Telephone: (370) 2621117 Facsimile: (370) $224694 \quad$ Telex:

Email: taut $(a$ itpal.fi.lt

Commission(s)

TAVAKOL REZA, SCHOOL OF MATHEMATICAL SC, OUEEN MARY/WESTFIELD COLL, MILE END RD LONDON E1 4NS, UK

$\begin{array}{ll}\text { Telephone: }(44) & \text { Facsimile: (44) } \\ \text { Email: } & \text { Commission(s): } 51\end{array}$

TAVARES J T L DR, AVE DIAS DA SILVA, 173 R/C ESQ, P 3000 COIMBRA, PORTUGAL

Telephone: (351)

Facsimile: (351)

Telex:

Email:

Commission(s):

TAWADROS MAHER JACOUB DR, HELWAN OBSERVATORY, HELWAN, EGYPT

Telephone: (20) $780645 / 2683$

Facsimile: (20)

Telex: 93070 hiag

Email:

Commission(s): 07

TAWARA YUZURU DR, DPT OF ASTROPHYSICS, NAGOYA UNIVERSITY, FUROCHO CHIKUSA KU

NAGOYA 46401, JAPAN

Telephonc: (81) 527815111

Facsimile: (81) $5278135+1$

Tclex: $\$ 477323$ scungy j

Email:

Commission(s):

TAYLER ROGER J PROF, ASTRONOMY CENTRE, UNIVERSITY OF SUSSEX, FALMER, BRIGHTON BN1 9QH, UK Telephone: (44) 1273606755

Facsimile: (4)

Commission(s): $35,44,47$

Telex: 877159 unisex g

Email:

TAYLOR A R DR, DPT OF PHYSICS, UNIVERSITY OF CALGARY, 2500) UNIVERSITY DR NW

CALGARY AB T2N 1N4, CANADA

Telephone: (1) 4032205385

Facsimile: (1) 4032893331

Telex: 0821545

Email: bitnet:artayloräuncamult

Commission(s): 41) 
TAYLOR ANDREW, DPT OF PHYSICS \& MATHEMAT, PHYSICS, UNIVERSITY OF ADELAIDE

ADELIADE SA 5005, AUSTRALIA
Telephonc: (64) 83035313
Facsimile: $(6+4) 83034380$
Telex:

Email: ataylor(appysics.adelaide.edu.au Commission(s): 22

TAYLOR DONALD BOGGIA DR, ROYAL GRENWICH OBS, MADINGLEY RD, CAMBRIDGE CB3 OEZ, UK

Telephonc: (4) 122337 4739 Facsimile: (4) 122337 4700 Telex: 87451 rgohsy g

Email: dbtäuk.ac.ro-greenwich.starlin Commission(s): 07,20

TAYLOR DONALD J DR, DPT PHYSICS \& ASTRONOMY, UNIVERSITY OF NEBRASKA, BEHLEN OBSERVATORY LINCOLN NE 685880111 , USA

$\begin{array}{ll}\text { Telephone: (1) } 4024723686 & \text { Facsimile: (1) } \\ \text { Email: } & \text { Commission(s): }\end{array}$

TAYLOR GREGORY BENJAMIN, CALTECH, RADIO ASTRONOMY, MS 105 24, PASADENA CA 91001, USA

Telephone: (1) $818395+024$

Email: ght(a)astro.caltech.cdu

Facsimile: (1) $8185689352 \quad$ Telex:

Conminssion(s):

TAYLOR JOSEPH H PROF, DPT OF PHYSICS, PRINCETON UNIVERSITY, JADWIN HALL, PRINCETON NJ 08544, USA

Telephone: (1) $6094524368 \quad$ Facsimile: (1)

Email: Commission(s): Telex: 4993512

TAYLOR KEITH DR, AAO, BOX 296, EPPING NSW 2121, AUSTRALIA

Telephone: (61) 28680222 Facsimile: (61) 28680310 Telex:

Email:

Comunission(s):

TAYLOR KENNETH N R PROF, 105A COPELAND RD, BEECROFT NSW 2119, AUSTRALIA

Telephone: (61) Facsimile: (61) Telex:

Email: $\quad$ Commission(s): 34

TCHANG-BRILLET LYDIA DR, OBSERVATOIRE UE PARIS, SECTION DE MEUDON, DAMAP F 92195 MEUDON PPL CDX, FRANCE

$\begin{array}{llll}\text { Telephonc: (33) } 1+5077576 & \text { Facsimile: (33) } 145077469 & \text { Telex: } 631987\end{array}$

Email: brilletafrmeu51 Commission(s): 14C

TEAYS TERRY J DR, NASA GSFC, CODE 684.9, IUE OBS, GREENBELT MD 20771, USA

Telephone: (1) $3012865749 \quad$ Facsimile: (1) 3012867642 Telex:

Email: iucsoc::tcays $\quad$ Commission(s): 42

TEDESCO EDWARD F, MISSION RESEARCH CORP, SUITE 302, ONE TARA BLVD, NASHUA NH 03062 2801, USA

Telephone: (1)

Facsimile: (1)

Telex:

Email:

Commission(s): $15,22,51$

TEERIKORPI VELI PEKKA DR, TURKU UNIVERSITY OBS, TUORLA, SF 21500 PIIKKIO, FINLAND

Telephone: (358) 21435822

Email:

Facsimile: (358) $21+33767$

Telex: 62638 tyf sf

Commission(s):

TEHERANY D, 83 AVENUE REY, TEHERAN, IRAN

Telephone: (98) Facsimile: (98)

Email:

Commission(s):

Telex:

TEIXEIRA RAMACHRISNA, IAG, UNIVERSIDADE DE SAO PAULO, AV MIGUEL STEFANO +200 04301904 SAO PAULO, BRAZIL

Telephone: (55) 115778599

Email: tcixcira(a)iag.usp.ansp.br

Facsimile: (55) $115763848 \quad$ Telex:

Commission(s): 08

TEJFEL VIKTOR G DR, ASTROPHYSICAL INSTITUTE, KAZAKH ACAD OF SCIENCES 480068 ALMA ATA, KAZAKHSTAN

Telephonc: (7) 683053

Facsimile: (7) Telex:

Email: tcjf@afi.academ.alma-atu.su

Commission(s): $16 \mathrm{C}, 51$

TEKTUNALI H GOKMEN DR, UNIVERSITY OBSERVATORY, UNIVERSITY OF ISTANBUL, 34452 ISTANBUL, TURKE

Telephone: (90) 15223597

Facsimile: (90)

Email:

Commission(s):

Telex: 
TELESCO CHARLES M DR, NASA/MSFC, CODE ES 63, SPACE SCIENCE LAB, HUNTSVILLE AL 35812, USA Telephone: (1) 2055447723 Facsimile: (1) Telex: 594416

Email:

Commission(s): 28

TEL'NYUK-ADAMCHUK V, ASTRONOMICAL OBSERVATORY, KIEV UNIVERSITY, OBSERVATORNA STR 3 254053 KIEV 53, UKRAINE

Telephone: (7) 0442162691

Email: aoku(g)gluk.apc.org

Facsimile: (7) $0442246387 \quad$ Telex:

Commission(s): 188

TEMPESTI PIERO PROF, ISTITUTO ASTRONOMICO, UNIVERSITA DI ROMA, VIA G M LANCISI 29 I 00161 ROMA, ITALY

Telephonc: (39) $684 \mathbf{4 2 9 7 7}$

Facsimile: (39)

Telex:

Email:

Commission(s): 27

TENORIO-TAGLE G DR, INST DE ASTROFISICA, DE CANARIAS, VIA LACTEA S/N, E 38200 LA LAGUNA, SPAIN Telephone: (34) 22605200

Email: gttaiac.es Facsimile: (34) 22605210

Telex: $926+0$ iac e

TEPLITSKAYA R B DR, SIBIZMIR, ACADEMY OF SCIENCES, 664697 IRKUTSK 33, RUSSIA

Telephone: (7) 629388

Commission(s): 34

Email:

Facsimile: (7)

Telex: $1+2+107$

TER HAAR DIRK, BOX 10, 349 MIDDLE ST, PETWORTH GU28 0RY, UK

Telephone: $(44)$

Facsimile: (44)

Telex:

Email:

Commission(s):

TERASHITA YOICHI PROF, KANAZAWA TECHNOLOGY INST, 7-1 OGIGAOKA, NONOICHIMACHI

ISHIKAWA 921, JAPAN

Telephone: (81)

Facsimile: (81)

Telex:

Email:

Commission(s): 05

TEREBIZH VALERY YU DR, CRIMEAN STATION OF, STERNBERG INSTITUTE, NAUCHNY 334413 CRIMEA, UKRAINE

Telephone: (7) $432945 \quad$ Facsimile: (7) Telex: 222192 voshod

Email:

Commission(s):

TERENTJEVA ALEXANDRA K DR, INST OF ASTRONOMY, ACADEMY OF SCIENCES, PYATNITSKAYA UL 48 109017 MOSCOW, RUSSIA

Telephone: (7) 952315461

Email: ater(a)airas.msk.su

Facsimile: (7)

Commission(s): 15,22

Telex: $\$ 12623$ scstp su

TERLEVICH ELENA DR, ROYAL GREENWICH OBS, HERSTMONCEUX CASTLE, HAILSHAM BN27 1RP, UK
Telephone: (44) $1323833171 \quad$ Facsimile: (44)

Email: et(astarlink.ro.greenwich.ac.uk Commission(s):

TERLEVICH ROBERTO JUAN, ROYAL GREENWICH OBS, HERSTMONCEUX CASTLE, HAILSHAM BN27 1RP, UK Telephonc: (44)

Facsimilc: (44)

Commission(s): 28

Telex:

Email:

TERNULLO MAURIZIO DR, OSS ASTROFISICO, CITTA UNIVERSITARIA, VIA A DORIA 6, I 95125 CATANIA, ITALY Telephone: (39) 95330533

Facsimile: (39) 95330592

Telex: 970359 astret $\mathrm{i}$

Email: astrct::maurizio

Commission(s): 10

TERRELL NELSON JAMES JR, LOS ALAMOS NATIONAL LAB, MS D436 ESS 9, BOX 1663

LOS ALAMOS NM 87545, USA

Telephone: (1) 5056672044

Facsimile: (1)

Telex:

Email:

Commission(s): 4

TERRILE RICHARD JOHN, JPL, MS 183 \$01, 4800 OAK GROVE DR, PASADENA CA 91109, USA

Telephone: (1)

Facsimile: (1)

Telex:

Email:

Commission(s): 16

TERZAN AGOP DR, OBSERVATOIRE DE LYON, AVE CHARLES ANDRE, F 69561 S GENIS LAVAL CDX, FRANCE

Telephone: (33) 78560705

Facsimile: (33) 72 39 9791

Telex: 310926

Email:

Commission(s): 27,37 
TERZIAN YERVANT PROF, CORNELL UNIVERSITY, SPACE SCIENCES BLDG, ITHACA NY 14853, USA

Telejhone: (1) 6072554935

Facsimile: (1) 6072559817

Telex: 932454

Enail: terøiana astrosun.tn.cornell.ed

Commission(s): $28,34,40,51$

TERZIDES CHARALAMBOS DR, DPT OF ASTRONOMY, UNIVERSITY THESSALONIKI GR 54006 THESSALONIKI, GREECE

Telephonc: (30) 31991357

Facsimilc: (30)

Telex:

Email:

Commission(s): 33

TESKE RICHARD G PROF, DPT OF ASTRONOMY, UNIVERSITY OF MICHIGAN, DENNISON BLDG ANN ARBOR MI 48109 1090, USA

Telephone: (1) 3137643398

Facsimilc: (1)

Telex:

Email:

Commission(s): 10

TEUBEN PETER J DR, ASTRONOMY DPT, UNIVERSITY OF MARYLAND, COLLEGE PARK MD 20742, USA

Telephone: (1) 3014051540

Facsimile: (1) 3013149067

Telex:

Email: teuben(a)astro.umd.edu

Commission(s): 05

TEXEREAU JEAN M, OCA CERGA, AVE COPERNIC, F 06130 GRASSE, FRANCE

Telephone: (33) 93365849 Facsimile: (33) Telex:

Email:

Commission(s):

THADDEUS PATRICK PROF, CENTER FOR ASTROPHYSICS, HCO/SAO, 60 GARDEN ST, CAMBRIDGE MA 02138, US Telephone: (1) $617+957340$

Email:

Facsimile: (1)

Commission(s): 34,51

Telex: 921428 satellite cam

THAKUR RATNA KUMAR DR, DPT OF PHYSICS, UNIVERSITY OF RAVISHANKAR, RAIPUR 492 010, INDIA

Telephone: (91) 27064

Facsimile: (91)

Telex:

Email:

Commission(s): 28

THE PIK-SIN PROF, ASTRONOMICAL INSTITUTE, UNIVERSITY OF AMSTERDAM, KRUISLAAN 403

NL 1098 SJ AMSTERDAM, NETHERLANDS

Telephone: (31) $202527491 \quad$ Facsimile: (31) $205257484 \quad$ Telex: 10262 hef $\mathrm{nl}$

Email:

Commission(s): $33,34,37^{\circ}$

THEJLL PETER ANDREAS DR, NIELS BOHR INSTITUTE, BLEGDAMSVEJ 17, DK 2100 COPENHAGEN 0, DENMARK Telephone: (45) 35325339

Email: thejllanordita.dk

Facsimile: (45) $31+21016$

Telex:

Commission(s): 36

THEODOSSIOU EFSTRATIOS DR, DPT OF PHYSICS, NTL UNIVERSITY OF ATHENS, PANEPISTIMIOPOLIS

GR 15783 ZOGRAFOS, GREECE

Telephone: (30) 17243414

Facsimile: (30)

Telex:

Email:

Commission(s):

THEVENIN FREDERIC DR, OCA OBSERV DE NICE, BP 229, F 06314 NICE CDX 4, FRANCE

Telephone: (33) 92003011

Facsimile: (33) 920113033

Telex:

Email: thevenin(a)fronis1

Commission(s): 29

THIELEMANN FRIEDRICH-KARL, CENTER FOR ASTROPHYSICS, HCO/SAO MS 10, 60 GARDEN ST CAMBRIDGE MA 02138, USA

Telephone: (1) 6174954979

Facsimile: (1) $617+955374$

Telex:

Email: fkt (a) cfa.3.bitnet

Commission(s): 35

THIELHEIM KLAUS O DR, ABT MATHEMATIPHYSIK, UNIVERSITAET KIEL, OLSHAUSENSTR 40/60

D 24098 KIEL, GERMANY

Telephone: $(49) \$ 318803216$

Email:

Facsimile: (49)

Telex: 292979

Commission(s): 33

THIRY YVES R PROF, UNIVERSITE PARIS VI, + PLACE JUSSIEU TOUR 66, F 75230 PARIS CDX 05, FRANCE

Telephone: (33) 143362525

Facsimile: (33)

Commission(s): 07

Telex:

Email:

THOBURN CHRISTINE, ROYAL GREENWICH OBS, MADINGLEY RD, CAMBRIDGE CB3 OEZ, UK

Telephone: (4t) 1323833171

Facsimile: (\$t) $122337 \$ 700$

Telex: $87+51$

Email:

Commission(s): 08 
THOLEN DAVID J DR, INSTITUTE FOR ASTRONOMY, UNIVERSITY OF HAWAII, 2680 WOODLAWN DR HONOLULU HI 96822, USA

Telephone: (1) 808956 6030

Email: tholen(a)hubble.ifa.hawaii.edu
Facsimile: (1) 8089882790

Commission(s): $06,15,16 \mathrm{C}, 20$
Telex: 8459 uhast $\mathrm{hr}$

THOMAS CLAUdiNe DR, BIPM, PAVILLON DE BRETEUIL, F 92312 SEVRES CDX, FRANCE

Telephone: (33) 145077073

Email: bij)m(âfrmeus1
Facsimile: (33) $1+53+2021$

Commission(s): $31 \mathrm{C}$
Telex: 631351
THOMAS DAVID V DR, DPT OF PHYSICS, UNIVERSITY OF EDINBURGH, MAYFIELD RD, EDINBURGH EH9 3JZ, UK Telephonc: (44) 1316505307 Email:
Telex: 727442 unived g

Commission(s): 08,24

THOMAS HANS-CHRISTOPH DR, MPI F PHYSIK \& ASTROPHYS, KARL SCHWARZSCHILDSTR 1

D 85740 GARCHING MUENCHEN, GERMANY

Telephone: (49)

Facsimile: (49)

Telex:

Email:

Commission(s): 35

THOMAS JOHN H PROF, DPT MECH \& AEROSPACE SCI, UNIVERSITY OF ROCHESTER

ROCHESTER NY 14627, USA

Telephone: (1) 7162754083

Facsimile: (1)

Telex:

Email:

Commission(s): 10,12

THOMAS PETER A DR, ASTRONOMY CENTRE, UNIVERSITY OF SUSSEX, FALMER, BRIGHTON BN1 9QH, UK

Telephonc: (44) $1273606755 * 3099$

Email: petertosyma.sussex.ac.uk

Facsimile: (4) 1273678097

Telex: 877159 bhvtxs.g

THOMAS RICHARD N DR, 1155 TIMBERLANE, PINEBROOK HILL, BOULDER CO 80302, USA

Telephone: (1) 3034439290

Facsimile: (1)

Telex:

Email:

Commission(s): 12,36

THOMAS ROGER J DR, NASA GSFC, CODE 682, GREENBELT MD 20771, USA

Telephone: (1) 3012867921 Facsimile: (1) Telex:

Email: pal::thomas Commission(s): 10,44

THOMASSON PETER DR, NRAL, JODRELL BANK, MACCLESFIELD SK11 9DL, UK

Telephone: (44) $147771321 \quad$ Facsimile: (4+) Telex: 36149

Email:

Commission(s): + (1)

THOMPSON A RICHARD DR, NRAO, VLBA PROJECT, 2015 IVY RD, CHARLOTTESVILLE VA 22903, USA

Telephone: (1) 8042960211

Facsimile: (1) 8042960278 Telex:

Email:

Commission(s): 34,40

THOMPSON G I DR, 7 MACDOWALL RD, EDINBURGH EH9 3HJ, UK

Telephone: (44)

Facsimilc: (4t)

Conmossion(s):

Telex:

Email:

THOMPSON KEITH DR, DPT OF PHYSICS, MONASH UNIVERSITY, WELLINGTON RD

CLAYTON VIC 3168, AUSTRALIA

Telephone: (61) 35654 (k00

Facsimile: (61)

Commission(s): 27,42

Telex: 32691 aa

Email:

THOMPSON LAIRD A DR, DPT OF ASTRONOMY, UNIVERSITY OF ILLINOIS, 1011 W SPRINGFIELD AVE URBANA IL 61801, USA

Telephone: (1) 2173333090

Email:

Facsimile: (1)

Telex:

Commission(s): $28,+7$

THOMPSON MICHAEL J DR, ASTRONOMY UNIT, QUEEN MARY WESTFIELD COLL, MILE END RD

LONDON E1 4NS, UK

Telephone: (44) 1719755462

Email: mthompso(a)solar.bitnet

Facsimile: (44) 1819819587

Commission(s): 10,35

THOMPSON RODGER I PROF, STEWARD OBSERVATORY, UNIVERSITY OF ARIZONA, TUCSON AZ 85721, USA

Telephonc: (1) 5206211891

Facsimile: (1)

Telex: 467175

Email: rthompson(a) as.arizona.celu

Commission(s): 
THOMSEN BJARNE B LECT, INST OF PHYSICS \& ASTRON, UNIVERSITY OF AARHUS, NY MUNKEGADE DK 8000 AARHUS C, DENMARK

Telephonc: (45) 86128899

Facsimile: (45) 86202711

Telex:

Email:

Commission(s):

THOMSON ROBERT, INSTITUTE OF ASTRONOMY, THE OBSERVATORIES, MADINGLEY RD

CAMBRIDGE CB3 UHA, UK

Telephonc: (44) $12233375+8$

Email: rețâmail.ast.cam.ac.uk

Facsimile: (44) $1223337523 \quad$ Telex:

Commission(s):

THONNARD NORBERT DR, ATOM SCIENCES, $11+$ RIDGEWAY CENTER, OAK RIDGE TN 37830, USA

Telephone: (1) 6154831113

Facsimile: (1)

Telex:

Email:

Commission(s): 28,34

THORNE KIP S PROF, CALTECH, MS 130 33, PASADENA CA 91125, USA

Telephone: (1) $213356+598$

Facsimile: (1)

Telex: $675+25$

Email:

Conmission(s): 4

THORSETT STEPHEN ERIK, DPT OF PHYSICS, PRINCETON UNIVERSITY, JADWIN HALL

PRINCETON NJ 08544, USA

Telephone: (1) 6092581245

Email: steve $(\hat{a}$ pulsar.princeton.colu

Facsimile: (1) 6092586853

Telex:

Commission(s):

THORSTENSEN JOHN R, DPT OF PHYSICS \& ASTRON, DARTMOUTH COLLEGE, HANOVER NH 03755, USA

Telephone: (1) $603646286^{\circ}$

Facsimile: (1)

Telex:

Email:

Commission(s):

THRONSON HARLEY ANDREW JR, DPT PHYSICS \& ASTRONOMY, UNIVERSITY OF WYOMING

LARAMIE WY 82071, USA

Telephone: (1) 3077666150

Facsimile: (1)

Telex:

Email:

Conmission(s): 34

THUAN TRINH XUAN DR, UNIVERSITY STATION, UNIVERSITY OF VIRGINIA, BOX 3818 CHARLOTTESVILLE VA 22913, USA

Telephonc: (1) $804924+894$

Facsimile: (1)

Telex:

Email: txt(a)virginia.edu

Commission(s): 28,47

THUILLOT WILLIAM, BUREAU DES LONGITUDES, 77 AVE DENFERT ROCHEREAU, F 75014 PARIS, FRANCE Tetephone: (33) 140512262

Email: thuillot $\bar{a}$ bdl.fi

Facsimilc: (33) $1+633283+$

Tclex:

Commission(s): 04,07

THUM CLEMENS DR, IRAM, AVD DIVINA PASTORA 7, BLOQUE 6/2B, E 18012 GRANADA, SPAIN

Telephonc: (34) 5848 (0413 Facsimile: (34)

Telex: 78521 iram c

Email:

Commission(s): 40

TIERSCH HEINZ, POTSDAM UNIVERSITY, STERNWARTE BABELSBERG, AN DER STERNWARTE 16

D $1+482$ POTSDAM, GERMANY

Telephone: (49) 3317499206

Email: hticrsch(a)aip.de

Facsimile: (49) 3317499309

Telex: 361231 stbbg a

TIFFT WILLIAM G PROF, STEWARD OBSERVATORY, UNIVERSITY OF ARIZONA, TUCSON AZ 85721, USA

Telephonc: (1) 5206216532

Facsimile: (1)

Telex: $\mathbf{4 6 7 1 7 5}$

Email:

Commission(s): 28,47

TIFREA EMILIA DR, ASTRONOMICAL INSTITUTE, CUTITUL DE ARGINT 5, BOX 28

R 75212 BUCHAREST, RUMANIA

Telephone: (40) 16413686

Facsimile: (4)

Telex:

Email:

Commission(s): 10

TIMOTHY J GETHYN DR, CTR FOR SPACE SCIENCES \&, ASTROPHYSICS, STANFORD UNIV ERL 314 STANFORD CA $9+305$ +1055, USA
Telephone: (1) $415+97005$ )
Facsimile: (1)
Telex: $\mathbf{3 4 8 + 0 2}$ stanfid stnu
Email:
Commission(s): 
TINBERGEN JAAP DR, KAPTEYN STERREWACHT, WERKGROEP, MENSINGHEWEG 20 NL 9301 KA RODEN, NETHERLANDS

Telephone: (31) 590819631

Facsimile: (31)

Telex: 53767 kswro nl

Email:

Commission(s): 25

TING YEOU-TSWEN, ASTRONOMY SECTION, CENTRAL WEATHER BUREAU, G† KUNG YUEN RD TAIPEI 100, CHINA R

Telephone: (886) $23713181 * 281$

Facsimile: $(886)$

Telex:

Email:

Commission(s): 04

TIPLER FRANK JENNINGS DR, DPT OF PHYSICS, TULANE UNIVERSITY, NEW ORLEANS LA 70118, USA Telephonc: (1)

Facsinile: (1)

Telex:

Email:

Commission(s): 47

TIURI MARTTI PROF, HELSINKI UNIV TECHNOLOGY, RADIO LABORATORY, OTAKAARI 5 A SF 02150 ESPOO 15, FINLAND

Telephone: (358) $45125+5$

Facsimile: (358)

Telex: 122771 rorta sf

Email:

Commission(s):

TJIN-A-DJIE HERMAN R E DR, KOEKOELAAN 106, NL 1403 EJ BUSSUM, NETHERLANDS

Telephone: (31) 215917076

Facsimile: (31)

Commission(s): 27,35

Telex: 10460 facwn nl

Email:

TLAMICHA ANTONIN DR, ASTRONOMICAL INSTITUTE, CZECH ACADEMY OF SCIENCES ONDREJOV OBSERVATORY, CZ 2516.5 ONDREJOV, CZECH R

Telephone: (42) 20+857324/7111 Ficsimile: (42) $20+851611$

Email: astsuñäcsearn Commission(s): 10,40

Telex: 121579 astr e\%

TOBIN WILLIAM, DPT PHYSICS \& ASTRONOMY, UNIVERSITY OF CANTERBURY, PRIVATE BAG 4800 CHRISTCHURCH 1, NEW ZEALAND

Telephone: $(64) 336+2531 \quad$ Facsimile: $(64) 336+2469$ Telex:

Email: w.tobin(a)esc.canterbury.ac.ny. Commission(s): 33

TODORAN IOAN DR, ASTRONOMICAL OBSERVATORY, STR CIRESILOR 19, R 3400 CLUJ NAPOCA, RUMANIA

Telephone: (40) 64194592

Facsimile: (40)

Telex:

Email:

Commission(s): $25,+2$

TOFANI GIANNI PROF, OSS ASTROFISICO, DI ARCETRI, LARGO E FERMI 5,150125 FIRENZE, ITALY

Telephone: (39) 552752217

Facsinile: (39)

Commission(s): 40

Telex: 572268 alcetri

Email:

TOHLINE JOEL EDWARD, DPT PHYSICS \& ASTRONOMY, LOUISIANA STATE UNIV

BATON ROUGE LA 70803 W(1)1, USA

Telephone: (1) 5043886851

Facsimile: (1)

Telex: 559184

Email:

Commission(s): 35

TOKAREV YURIJ V DR, RADIOPHYSICAL RESEARCH, INSTITUTE, LYADOV UL 25/14

603600 N NOVGOROD, RUSSIA

Telephone: (7) 978312360188

Facsimile: (7) $978312369902 \quad$ Telex:

Email:

Commission(s): 34

TOKOVININ ANDREJ A DR, STERNBERG STATE ASTR INST, UNIVERSITETSKIJ PROSP 13 119899 MOSCOW, RUSSIA

Telephone: (7) 959393318

Facsimile: (7)

Telex:

Enıail;

Commission(s): $26 \mathrm{C}, 30 \mathrm{C}$

TOKUNAGA ALAN TAKASHI DR, INSTITUTE FOR ASTRONOMY, UNIVERSITY OF HAWAII, 2680 WOODLAWN DR HONOLULU HI 96822, USA

Telephone: (1) 8089566691

Facsimile: (1) 8089882790

Telex: 7238459 uhast hr

Email: tokunaga(a)galileo.ifa.hawaii.ed

Commission(s): 25

TOLBERT CHARLES R DR, UNIVERSITY STATION, UNIVERSITY OF VIRGINIA, BOX 3818 CHARLOTTESVILLE VA 22903, USA

Telephone: (1) 80+9247494

Facsimile: (1)

Telex:

Email: crtävirginia.edu

Commission(s): $25,38,40,46,51$ 
TOLLER GARY N DR, NASA GSFC, CODE 685.3, GREENBELT MD 20771, USA

Telephone: (1) Facsimile: (1) Telex:

Email:

Commission(s): 21

TOMASI PAOLO DR, IST DI RADIOASTRONOMIA, CNR, VIA IRNERIO +6, I +1126 BOLOGNA, ITALY

Telephonc: (39) $51232856 \quad$ Ficsimile: (39) Telex:

Enail:

Commission(s): +1

TOMASKO MARTIN G DR, LUNAR \& PLANETARY LAB, UNIVERSITY OF ARIZONA, SPACE SCIENCES BLDG TUCSON AZ 85721, USA

Telephone: (1) 5206216969

Email:

Facsimile: (1)

Commission(s):

Telex:

TOMBAUGH CLYDE W PROF, DPT OF ASTRONOMY, NEW MEXICO STATE UNIV, BOX 4500

LAS CRUCES NM 88003, USA

Telephone: (1) 5056462107

Facsimile: (1)

Telex:

Email:

Commission(s): 16

TOMIMATSU AKIRA DR, DPT OF PHYSICS, NAGOYA UNIVERSITY, FUROCHO CHIKUSA KU

NAGOYA 46401, JAPAN

$\begin{array}{ll}\text { Telephone: (81) } & \text { Facsimile: }(81) \\ \text { Email: } & \text { Commission(s): } 44,47\end{array}$

Email:

TOMISAKA KOHJI DR, FAC OF EDUCATION, NIIGATA UNIVERSITY, 8050 IKARASHI 2, NIIGATA 950 21, JAPAN

Telephone: (81) $252627269 \quad$ Facsimile: (81)

Email: tomisalia(ajed.nitgata-u.ac.jp Commission(s): 33

Telex: 81252631277

TOMITA KENJI PROF, UJI RESEARCH CENTER, YUKAWA INSTITUTE, KYOTO UNIV GOKASHO, UJI 611, JAPAN

Telcphone: (81)

Facsinuile: (81)

Telex:

Email: tomita(âyisun 1.kyoto-u.ac.jp)

Commission(s): 47

TOMITA KOICHIRO MR, 4 -11-20 YOGA, SETAGAYAKU, TOKYO 158, JAPAN

Telephone: (81) 370 00066

Facsimile: (81)

Telex:

Email:

Commission(s): 15,20,22

TOMOV NIKOLAI, NTL ASTRONOMICAL OBS, BULGARIAN ACAD SCIENCES, BOX 136 BG 4700 SMOLJAN, BULGARIA

Tclephone: (359) 3021357283

Facsimile: (359) 3021356

Telex:

Email:

Commission(s):

TOMOV TOMA V DR, NTL ASTRONOMICAL OBS, BULGARIAN ACAD SCIENCES, BOX 136 BG 4700 SMOLJAN, BULGARIA

Telephonc: (359) $73+1599 \quad$ Facsimile: $(359) \quad$ Telex: $\mathbf{4 8 4 4 6}$

Email:

Commission(s): 29

TONER CLIFFORD GEORGE, NTL SOLAR OBSERVATORY, BOX 26732, 950 N CHERRY AVE TUCSON AZ 85726 6732, USA

Telephone: (1) $520323+111$

Facsimile: (1) $5203259278 \quad$ Telex:

Email: toncränoao.cdu

Commission(s):

TONG FU, PURPLE MOUNTAIN OBSERV, CAS, NANJING, CHINA PR

Telephonc: (86) 2533921

Facsimile: (86) 25301459

Commission(s): $(14,07$

Telex: $341+4$ pmonj $\mathrm{en}$

Email:

$$
\text { COMTING }
$$

TONG YI, DPT OF ASTRONOMY, BEIJING NORMAL UNIVERSITY, 19 XINJISKOW OUT ST BEIJING 100875, CHINA PR

Telephone: (86) $1656531 * 6285$

Email:
Facsimilc: (86)
Commission(s): 28,33
Telcx:

TONRY JOHN DR, DPT OF MATHEMATICS, MIT RM 6 204A, 77 MASSACHUSETTS AVE, CAMBRIDGE MA 02139, US

Telephone: (1) 6172537528

Email: jtiâalcor.mit.edu

Facsimile: (1)

Telex:

TONWAR SURESH C PROF, TIFR, HOMI BHABHA RD, COLABA, BOMBAY 400 005, INDIA

Telephonc: (91) $224952311 \quad$ Facsimile: (91)

Email:

Commission(s):

Telex: 0113009 tifi in 
TOOMRE ALAR DR, DPT OF MATHEMATICS, MIT RM 2372,77 MASSACHUSETTS AVE

CAMBRIDGE MA 02139, USA

Telephone: (1) $617253+326$

Facsimile: (1) $\quad$ Telex:

Email:

Commission(s): 28,33

TOOMRE JURI, JILA/DAG, UNIVERSITY OF COLORADO, BOX +41, BOULDER CO 80309 0440, USA

Telcphone: (1) 3034927854

Facsimile: (1)

Telex:

Email:

Commission(s): $33,35,36$

TOPAKTAS LATIF A DR, KING SAUD UNIVERSITY, COLLEGE OF SCIENCE, BOX 2455

RIYADH 11453, SAUDI ARABIA

Telephone: $(966)$

Facsimile: (966) $\quad$ Telex:

Email:

Commission(s):

TORAO MASAHISA, 41011 , SENPUKU GA OKA 2-11-9, SUSONO CITY, JAPAN

Telephone: (81)

Facsimile: (81)

Telex:

Cmail:

Commission(s): 19

TORELLI M DR, OAR, VIA DEL PARCO MELLINI 84, I 00136 ROMA, ITALY

Telcphone: (39) 6347056 Facsimile: (39) Telex:

Email:

Commission(s): 12

TORNAMBE AMEDEO, IAS, CNR, CP 67, I 00044 FRASCATI, ITALY

Telephone: (39) $69425655 \quad$ Facsimile: (39) $69+16847 \quad$ Telex:

Email:

Commission(s): 37

TORO TIBOR PROF, INSTITUTE OF THEORETICAL, PHYSICS, UNIVERSITY TIMISOARA, TIMISOARA, RUMANIA

Telephone: $(40) 56130823$

Facsimile: (f0)

Tclex:

Email:

Commission(s): 51

TOROSHLIDZE TEIMURAZ I DR, ABASTUMANI ASTROPHYSICAL, OBSERVATORY

GEORGIAN ACAD OF SCIENCES, 383762 ABASTUMANI, GEORGIA

Telephone: (7)

Facsimilc: (7)

Telex:

Email:

Commission(s): 21

TORRA JORDI DR, DPT FISICA, UNIVERSIDAD DE BARCELONA, AVD DIAGONAL 647, E OBO28 BARCELONA, SPAIN

Telephone: (34) 33307311

Ficsimile: (34)

Commission(s): 33

Telex:

Email:

TORRELLES JOSE M DR, INST ASTROFISICA, DE ANDALUCIA APD 3OH4, C/ SANCHO PANZA S/N

E 18080 GRANADA, SPAIN

Telephone: (34) 58121311

Facsimile: (34) 58814530

Telex:

Email: torrelles(a)ian.es

Commission(s): 34

TORRES CARLOS ALBERTO DR, OBSERVATORIO NACIONA, RUA CORONEL RENNO 07, CP 21 37500 ITAJUBA MG, BRAZIL

Telephone: (55) 356220788

Facsimile: (55)

Telex: (131-2603

Email:

Commission(s): 27,50

TORRES CARLOS DR, DPT DE ASTRONOMIA, UNIVERSIDAD DE CHILE, CASILLA 36 D, SANTIAGO, CHILE

Telephone: (56) $2229+101$

Email:

Facsimilc: (56)

Telex: $\$+0(0) 1$

TORRES DODGEN ANA V, 15735 FOREST HILL DR, BOULDER CREEK CA 950OG, USA

Telephone: (1) 4083380158

Email: 3303pannavpgs.bitnet

Facsimile: (1)

Telex:

TORRES-PEIMBERT SILVIA DR, INSTITUTO DE. ASTRONOMIA, UNAM, APDO POSTAL 70-264 04510 MEXICO DF, MEXICO

Telcphone: (52) 56223900

Facsimile: (52) 55483712

Telex: 1760155 cic me

Email:

Commission(s): 34,46

TORRICELLI GUIDETTA DR, OSS ASTROFISICO, DI ARCETRI, LARGO E FERMI 5, I 50125 FIRENZE, ITALY

Telephone: (39) 552752260

Facsimile: (39)

Email:

Commission(s): 
TORROJA J PROF, DPT DE ASTRONOMIA, FAC DE CIENCIAS, UNIVERSIDAD COMPLUTENSE

E 28040 MADRID, SPAIN

Telephone: (34)

Facsimile: (34)

Commission(s):

Tclex:

Email:

TOSA MAKOTO DR, ASTRONOMICAL INSTITUTE, TOHOKU UNIVERSITY, SENDAI AOBA, MIYAGI 980, JAPAN

Telephone: (81) 222221800

Email:

Facsimile: (81)

Commission(s): 33

Telex: 852246 thucom $\mathbf{j}$

TOSHIKI AIKAWA DR, ASTRONOMICAL INSTITUTE, TOHOKU GAKUIN UNIVERSITY, ICHINAZAKA IZUMI-KU SENDAI 981 31, JAPAN

Telephone: (81) $223751111 \star 318 \quad$ Facsimile: (81) $223754040 \quad$ Telex:

Email:

Commission(s):

TOSI MONICA, OSS ASTRONOMICO, UNIVERSITA DI BOLOGNA, CP 596, I 40100 BOLOGNA, ITALY

Telephone: (39) 51222956

Facsimile: (39)

Telex: 211664 infin bo $\mathrm{i}$

Email:

Commission(s): 34

TOTH IMRE DR, KONKOLY OBSERVATORY, THEGE U 13/17, BOX 67, H 1525 BUDAPEST, HUNGARY

Telephone: (36) $11755866 / 175+122$

Facsimile: (36) 115696410

Telex: $227+60$ konob h

Email: ho97kno(äjogyalla.konkoly.hu

Commission(s): 15

TOTSUKA YOJI DR, INST COSMIC RAY RESEARCH, UNIVERSITY OF TOKYO, MIDORICHO TANASHI TOKYO 188, JAPAN

Telephone: (81) $42461+131$

Email: totsuka $\left(a_{j}\right.$ jpnutins.bitnet

Facsimile: $(81)+24681438$

Telex: 02822371 iertu j

TOUSEY RICHARD DR, NAVAL RESEARCH LABORATORY, CODE 7140, 4555 OVERLOOK AVE SW WASHINGTON DC 20375 50(0), USA

$\begin{array}{ll}\text { Telephone: (1) } 2027673441 & \text { Facsimile: (1) } \\ \text { Email: } & \text { Commission(s): } 12,14\end{array}$

TOUT CHRISTOPHER DR, INSTITUTE OF ASTRONOMY, THE OBSERVATORIES, MADINGLEY RD

CAMBRIDGE CB3 OHA, UK

Telephone: (44) $1223337548 \quad$ Facsimile: (44) $1223337523 \quad$ Telex:

Email: ct23(aphoenix.cambridge.ac.uk Commission(s): 42

TOVMASSIAN GAGHIK, INSTITUTO DE ASTRONOMIA, OBSERVATORIO ASTRONOMICO, AP 22860 ENSENADA, MEXICO

Telephone: (52) $617+4580$ Facsimile: (52) $617+4607$ Telex:

Email: gagạ bufadora.astrosen.unam.mx Commission(s):

TOVMASSIAN H M DR, BYURAKAN ASTROPHYSICAL, OBSERVATORY

375433 BYURAKAN, ARMENIA

Telephone: (7) $8852283453 \quad$ Ficsimile: (7) $885228+142$ Telex:

Email:

Commission(s): $28,40,+4,51$

TOWNES CHARLES HARD DR, PHYSICS DPT, UNIVERSITY OF CALIFORNIA, RM 557 BIRGE HALL BERKELEY CA 94720, USA

Telcphone: (1) 4156421128

Facsimile: (1)

Commission(s): $34,40,51$

Telex:

Email:

TOYAMA`KIYOTAKA, HOKKAIDO INFORMATION UNIV, NISHINOPPORO 59-2, EBETSU (69), JAPAN

Telephone: (81) $11385+411$

Facsimile: (81) 113840134

Telex:

Email:

Commission(s): 28

TOZER DAVID C DR, SCHOOL OF PHYSICS, UNIVERSITY OF NEWCASTLE, NEWCASTLE/TYNE NE1 7RU, UK

Telephone: (44)

Facsimile: (4t)

Telex:

Email:

Commission(s):

TOZZI GIAN PAOLO, OSS ASTROFISICO, DI ARCETRI, LARGO E FERMI 5, I 50125 FIRENZE, ITALY

Telephone: (39) 552752250

Facsimile: (39)

Telex: 572268 arcetr $i$

Email:

Commission(s): 14 
TRAAT PEETER, TARTU ASTROPHYSICAL OBS, TARTU TAEHETORN, TOOMEMAEGI, EE 202400 TARTU, ESTONI Telephone: (372) $27+34932$ Facsimile: (372) $27+35+30 \quad$ Telex:

Email: traatia obs.ec Commission(s): 28

TRAFTON LAURENCE M DR, ASTRONOMIY DPT, UNIVERSITY OF TEXAS, RLM 15 308, AUSTIN TX 78712 1083, USA Telephonc: (1) $512+711+76$ Facsimile: (1) Telex:

Email: Commission(s): 16

TRAN MINH NGUYET DR, OBSERVATOIRE DE PARIS, SECTION DE MEUDON, F 92195 MEUDON PPL CDX, FRANCE Telephonc: (33) $1 \$ 5077+47$ Facsimile: (33)

Email:

Commission(s): 14

Telex: 270912

TRAN-MINH FRANCOISE DR, OBSERVATOIRE DE PARIS, SECTION DE MEUDON, DASGAL F 92195 MEUDON PPL CDX, FRANCE. Telephone: (33) 145077553

Facsimile: (33) 145077469

Telex: 201571

Email:

Commission(s): 16

TRAUB WESLEY ARTHUR, CENTER FOR ASTROPHYSICS, HCO/SAO, 60 GARDEN ST, CAMBRIDGE MA 02138, USA Telephone: (1) 6174957406

Facsimile: (1)

Telex: $921+28$ satellite cam

Email:

Commission(s): 09,44

TRAVING GERHARD PROF, INST F THEOR ASTROPHYSIK, DER UNIVERSITAET, IM NEUENHEIMER FELD S6I D 69120 HEIDELBERG, GERMANY

Telephone: (49) $6221562815 \quad$ Ficsimile: (49)

Commission(s): 36

Telex: $\$ 61515$

Email:

TREDER H J PROF DR, ZNTRLINST F ASTROPHYSIK, STERNWARTE BABELSBERG, ROSA LUXEMBURG STR 17A D 14473 POTSDAM, GERMANY

Telephone: (49) 762225

Facsimile: (49)

Commission(s): 28,47

Telex: $15+71$

Email:

TREFFERS RICHARD R, ASTRONOMY DPT, UNIVERSITY OF CALIFORNIA, G01 CAMPBELL HALL

BERKELEY CA 94720, USA

Telephone: (1) $4156+2+223$

Facsimile: (1)

Telex:

Email:

Commission(s): 34

TREFFTZ ELEONORE E DR, MPI F PHYSIK \& ASTROPHYS, KARL SCHWARZSCHILDSTR 1 D 85740 GARCHING MUENCHEN, GERMANY

$\begin{array}{lll}\text { Telephone: }(49) 8932990 & \text { Facsimile: }(49) & \text { Telex: } 524629 \text { astro d } \\ \text { Email: } & \text { Commission(s): } 14 & \end{array}$

TREFZGER CHARLES F DR, ASTRONOMISCHES INSTITUT, UNIVERSITAET BASEL, VENUSSTRASSE 7 CH $\$ 102$ BINNINGEN, SWITZERLAND

$\begin{array}{lll}\text { Telephone: }(+1) 612717711 / 12 & \text { Facsimile: }(+1) & \text { Telex: } \\ \text { Email: } & \text { Commission(s): } 33\end{array}$

TREHAN SURINDAR K PROF, DPT OF MATHEMATICS, UNIVERSITY OF PANJAB, CHANDIGARH IG0 014, INDIA

Telephone: (91) $29938 \quad$ Facsimile: (91)

Telex:

Email:

Commission(s):

TRELLIS MICHEL DR, OCA OBSERV DE NICE, BP 229, F 16304 NICE CDX 4, FRANCE

Telephone: (33) $93890420 \quad$ Facsimile: (33) 92003033 Telex: 460004 obsnice $f$

Email:

Commission(s): 10

TREMAINE SCOTT DUNCAN, CITA MCLENNAN LABS, UNIVERSITY OF TORONTO, GO ST GEORGE ST TORONTO ON M5S 1A1, CANADA

Telephone: (1) 4169786879

Facsimile: $(1)+169783921$

Telex:

Email: tremaine(a)cita.utoronto.ca

Commission(s): $07,28,47$

TREMKO JOZEF DR, ASTRONOMICAL INSTITUTE, SLOVAK ACADEMY SCIENCES SK 059 60 TATRANSKA LOMNI, SLOVAK R

Telephone: $(42) 969967866 / 7 / 8$

Facsimile: $(42) 969967656$

Telex: 78277 ausav cz

Email:

Commission(s): $27,42,50$ 
TREUMANN RUDOLF A DR, MPI F PHYSIK \& ASTROPHYS, INST F EXTRATERR PHYSIK KARL SCHWARZSCHILDSTR 1, D 857+0 GARCHING MUENCHEN, GERMANY
Telephone: (49) 893299831
Facsimile: (49)
Telex: $52158+5$ xter d
Email:
Commission(s): 10

TREVESE DARIO, ISTITUTO ASTRONOMICO, UNIVERSITA DI ROMA, VIA G M LANCISI 29, I 00161 ROMA, ITALY

Telephone: (39) $6+403734$

Facsimile: (39) $6+\$ 13673$

Telex: 613255 infinro

Email:

Commission(s): 47

TREXLER JAMES H MR, 1921 SO ABREGO DR, GREENVALLEY AZ 85614 1403, USA

Telephone: (1)

Facsimile: (1)

Telex:

Email:

Commission(s):

TRIMBLE VIRGINIA L DR, DPT OF PHYSICS, UNIVERSITY OF CALIFORNIA, IRVINE CA 92717, USA

Telephone: (1) $71+82+69+8 / 301+1055822$ Facsimile: (1) $71+82+217+30131+9067$ Telex:

Email: vtrimble(ā)uci.edu/astro.umd.edu Commission(s): 26,28P,35,42,44,47,51,EC

TRINCHIERI GINEVRA, OSS ASTROFISICO, DI ARCETRI, LARGO E FERMI 5, I 50125 FIRENZE, ITALY

Telephonc: (39) $552752230 \quad$ Facsimile: (39)

Telex: $\$ 72268$ arcetr i

Email:

Commission(s): 28

TRIPATHI B M DR, UTTAR PRADESH STATE, OBSERVATORY, PO MANORA PEAK 263 129, NAINITAL 263 129, INDI

Telephone: (91) $59+22136$

Facsimile: (91)

Commission(s): 12

Telex: cable : astronomy

Email:

TRIPICCO MICHAEL J DR, ASTRONOMY PROGRAM, UNIVERSITY OF MARYLAND

COLLEGE PARK MD 20742, USA

Telephone: (1) 3014051548

Facsimile: (1)

Commission(s): 37

Telex:

Email: miket(a) astro.umd.edu

TRITAKIS BASIL P DR, DPT ASTRONOMY APPL MATH, ACADEMY OF ATHENS, $1+$ ANAGNOSTOPOULOU ST GR 10673 ATHENS, GREECE

Telephone: (30) 13613589

Facsimile: (30)

Telex:

Email:

Commission(s): 10,49

TRITTON KEITH P DR, ROYAL GREENWICH OBS, MADINGLEY RD, CAMBRIDGE CB3 OEZ, UK

Telephone: (44) $122337+1000$

Facsimile: (4) 1223374700

Telex: 817235 rgostr g

Email:

Commission(s): 411

TRITTON SUSAN BARBARA, ROYAL OBSERVATORY, BLACKFORD HILL, EDINBURGH EH9 3HJ, UK

Telephone: (44) $1316673321 \quad$ Facsimile: (4t) Telex: 72383 roeding

Email: $\quad$ Commission(s): 05

TROCHE-BOGGINO A E DR, FAC CIENC EXACTAS/NATURAL, UNIV NACIONAL DE ASUNCION

SUC DE CORREOS 19, AGEN POS CAMPUS UNA KM10, PARAGUAY

Telephone: (92) $501517 \quad$ Facsimile: (92) Telex:

Email:

Commission(s): 46

TRODAHL HARRY JOSEPH DR, DPT OF PHYSICS, VICTORIA UNIVERSITY, PRIVATE BAG WELLINGTON, NEW ZEALAND

Telephone: (64) 721000

Facsimile: $(64)$

Commission(s): 25

Telex:

Email:

TROITSKY V S PROF DR, RADIOPHYSICAL RESEARCH, INSTITUTE, LYADOV UL 25/14

603600 N NOVGOROD, RUSSIA

Telephone: (7) $360+40$

Facsimile: (7) $83123609012 \quad$ Telex:

Email: vstrounirfi.sandy.nnov:su

Commission(s): $16,+11,51$

TROLAND THOMAS HUGH, DPT PHYSICS \& ASTRONOMY, UNIVERSITY OF KENTUCKY

LEXINGTON KY 40506 , USA

Telephone: (1) 6062578620

Facsimile: (1)

Telex:

Email:

Commission(s): 4 () 
TROTTET GERARD DR, OBSERVATOIRE DE PARIS, SECTION DE MEUDON, DASOP F 92195 MEUDON PPL CDX, FRANCE Telephone: (33) 145077808

Facsimile: (33)

Telex:

Email:

Commission(s): 10

TRUEMPER JOACHIM PROF, MPI F EXTRATERRESTRISCHE, PHYSIK, KARL SCHWARZSCHILDSTR 1 D 85740 GARCHING MUENCHEN, GERMANY

Telephone: (49) $8932993559 \quad$ Facsimile: (49) $8932993315 \quad$ Telex: 5215845 xter (J

Enail:

Commission(s): $4+\mathrm{C}$

TRUJILLO BUENO JAVIER DR, INST DE ASTROFISICA, DE CANARIAS, OBS DEL TEIDE, E 38200 LA LAGUNA, SPAI Telephone: (34) 22605266

Email: iac::jtb Facsimile: (34) 22 (10 5210

Telex: 92640 iac e

TRULLOLS I FARRENY ENRIC, MATEPATICA APLICADA, U P CATALUNYA, VICTOR BALAGUER S/N E 08800 VILANOVA, SPAIN

Telejhone: (34) 38967738

Facsimile: (34) $38967700 \quad$ Telex:

Email: enric(amat.upc.es

Commission(s): 37

TRULSEN JAN K PROF, INST THEORET ASTROPHYSICS, UNIVERSITY OF OSLO, BOX IO29

N (I315 BLINDERN OSLO 3, NORWAY

Telephone: $(+7)$

Facsimile: (47)

Telex:

Enail:

Commission(s):

TRUONG-BACH, OBSERVATOIRE DE PARIS, SECTION DE MEUDON, F 92195 MEUDON PPL CDX, FRANCE

Telejhone: (33) 145077897

Facsimile: (33) $1+5077893 \quad$ Telex: 270912

Email: tbach(âfrmcuฐ1

Commission(s): 40

TRURAN JAMES W JR, DPT OF ASTRONOMY, UNIVERSITY OF ILLINOIS, 1011 W SPRINGFIELD AVE

URBANA IL 61801, USA

Telephonc: (1) 2173333690

Facsimile: (1)

Telex:

Email:

Commission(s): 35,44

TRUSSONI EDOARDO, IST DI COSMO GEOFISICA, CNR, CORSO FIUME 4, I 10133 TORINO, ITALY

Telephone: (39) $11657694 / 8979 \quad$ Facsimile: (39)

Telex: $2110+1$ infinto

Email:

Commission(s): 44

TRUTSE YU L DR, INST PHYSICS OF ATMOSPH, ACADEMY OF SCIENCES, PYZHEVSKY 3 109017 MOSCOW, RUSSIA

Telephonc: (7)

Facsimile: (7)

Telex:

Email:

Commission(s):

TSAI CHANG-HSIEN DIRECTOR, TAIPEJ OBSERVATORY, YUAN SHAN, TAIPEI 104 TAIWAN, CHINA R Telephone: (886) $259+7+32$

Email: Facsimile: (886)

Telex:

TSAMPARLIS MICHAEL DR, DPT OF ASTROPHYSICS, NTL UNIVERSITY OF ATHENS, PANEPISTIMIOPOLIS, GR 15773 ZOGRAFOS, GREECE

Telephone: (30) $17243414 * 211$

Email: rich(agrathun1
Facsimile: (30) $1962+430$

Commission(s): 47
Telex: 223815 univ gr

TSAO MO PROF, NO 47 SEC 3, HSIN-I RD, TAIPEI 106, CHINA R

Telephone: $(886) 27047795$

Email:
Faesimile: (886)

Commission(s): 19
Telex:
TSAP T T DR, CRIMEAN ASTROPHYS OBS, UKRAINIAN ACAD OF SCIENCE, NAUCHNY, 334413 CRIMEA, UKRAINE Telephone: (7) 432945

Email:

Telex:

Facsinile: (7)

Commission(s): 12

TSAREVSKY GREGORY, SPACE RESEARCH INSTITUTE, ACADEMY OF SCIENCES, PROFSOJUZNAYA UL 84/32 117810 MOSCOW, RUSSIA

Telephone: (7) 0953332133

Email: gtsarvacsoc1. bitnet

Facsimile: (7) $0953332378 \quad$ Telex:

Commission(s): 
TSAY WEAN-SHUN DR, INST PHYSICS \& ASTRONOMY, NTL CENTRAL UNIVERSITY CHUNG LI TAIWAN 32054, CHINA R

Telephone: $(886) 34227151^{* 5335 / 5310}$

Email: tsay@aphyast.dnet.ncu.edu.tw

Facsimile: $(886) 34251175 \quad$ Telex:

Commisvion(s): 26

TSCHARNUTER WERNER M DR, INST F THEOR ASTROPHYSIK, DER UNIVERSITAET

IM NEUENHEIMER FELD 561, D 6912(। HEIDELBERG, GERMANY

Telephone: (49) $6221562815 \quad$ Facsimile: (49) Telex:

Email: $\quad$ Commiswion(s): 35

TSEYTLIN NAUM M, RADIOPHYSICAL RESEARCH, INSTITUTE, LYADOV UL 25/14, 603600 N NOVGOROD, RUSSIA

Telephonc: (7) 360129

Facsimile: (7)

Commission(s):

Telex: 1113 luna

Email:

TSIKOUDI VASSILIKI PH D, DPT OF PHYSICS, UNIVERSITY OF IOANNINA, GR 45332 IOANNINA, GREECE

Telephone: (30) $65191084 \quad$ Facsimile: (30)

Telex:

Email:

Commission(s):

TSINGANOS KANARIS DR, DPT OF PHYSICS, UNIVERSITY OF CRETE, BOX 1527, GR 71110 HERAKLION, GREECE

Telephonc: (30) 8123 9757*15t

Facsimile: (30) 81239735

Telex: 262860

Email: tsingan(a)iest.forth.gr

Commission(s): 10

TSIOUMIS ALEXANDROS DR, DPT GEODETIC ASTRONOMY, UNIVERSITY THESSALONIKI, UNIV BOX 503 GR 54006 THESSALONIKI, GREECE

Tclephonc: (30) 31992693

Facsimile: (30)

Telex:

Email:

Commission(s): 27,33

TSIROPOULA GEORGIA DR, ASTRONOMICAL INSTITUTE, NTL OBSERVATORY OF ATHENS, BOX 20048 GR 11810 ATHENS, GREECE

Telephone: (30) 13461191

Facsimile: (30)

Commission(s): 12

Telex: $\mathbf{2 1 5 5 3 0}$ obs.gr

Email:

TSUBAKI TOKIO PROF, DPT OF EARTH SCIENCE, SHIGA UNIVERSITY, 2-5-1 HIRATSU, OHTSU 520, JAPAN

Telephone: (81) 77537081

Facsimile: (81)

Telex:

Email:

Commission(s): 10,12

TSUBOI MASATO DR, NOBEYAMA RADIO OBS, NAOJ, MINAMIMAKI MURA, NAGANO 384 13, JAPAN

Telephone: (81) $26763+314$

Facsimile: (81) 267982927

Telex: 3329005 naonro i

Email: nrotsub@jpnnro.bitnet

Commission(s): 40

TSUBOKAWA IETSUNE DR, INTL LATITUDE OBSERVATORY, NAOJ, HOSHIGAOKA MIZUSAWA SHI IWATE 023, JAPAN

$\begin{array}{lll}\text { Telephotke: (81) } 197247111 & \text { Facsimile: (81) } & \text { Telex: } 837628\end{array}$

Email:

Commission(s): 19

TSUCHIDA MASAYOSHI DR, IAG, UNIVERSIDADE DE SAO PAULO, AV MIGUEL STEFANO 4200 04301 SAO PAULO SP, BRAZIL

Telephone: (55) $115778599 \quad$ Facsimile: (55) $112763848 \quad$ Telex: 1156735 iagm br

Email: masa\%iagusp(ährfapesp.bitnet Commission(s): 17,20

TSUCHIYA ATSUSHI DR PROF, OMACHI +-2-18, KAMAKURA 248, JAPAN

Telephone: (81)

Facsimile: (81)

Telex:

Email:

Commission(s): 31

TSUJI TAKASHI, INST OF ASTRONOMY, UNIVERSITY OF TOKYO, OSAWA MITAKA, TOKYO 181, JAPAN

Telephone: (81) $422325111 \quad$ Facsimile: (81)

Email:

Commission(s): 36

Telex: 02822307 taomtk $\mathrm{i}$

TSUNEMI HIROSHI DR, FAC OF SCIENCES, OSAKA UNIVERSITY, MACHIKANEYAMA

TOYONAKA OSAKA 560, JAPAN

Telephone: (81) $684+1151$

Facsimile: (81)

Telex:

Email: bitnet:tsunemiâjipnoskfm

Commission(s): H

TSUNETA SAKU DR, INST OF ASTRONOMY, UNIVERSITY OF TOKYO, OSAWA MITAKA, TOKYO 181, JAPAN

Telephone: $(81)+22324710$

Facsimile: (81)

Email: stsuncta:nasamail

Commission(s): 10

Telex: 2822307 taomik $\mathbf{j}$ 
TSURUTA SACHIKO DR, DPT OF PHYSICS, MONTANA STATE UNIVERSITY, BOZEMAN MT 59715, USA

Telephone: (1) $40699+3614$

Facsimile: (1)

Telex:

Email:

Commission(s): $4+$

TSVETANOV ZLATAN IVANOV, DPT PHYSICS \& ASTRONOMY, JOHNS HOPKINS UNIVERSITY CTR FOR ASTROPHYSICAL SCI, BALTIMORE MD 21218, USA

Telephone: (1) $4105168585 \quad$ Facsimile: (1) +105168260 Telex:

Email: ztsvetanov(âpha.jhu.edu Commission(s):

TSVETKOV MILCHO K DR, DPT OF ASTRONOMY, BULGARIAN ACAD SCIENCES, 72 LENIN BLVD

BG 178+ SOFIA, BULGARIA

Telephone: (359) 2758927

Email:

Facsimile: (359)

Commission(s): $015,27,37$

Telex: 23561 ecf ban bg

TSVETKOV TSVETAN DR, DPT OF ASTRONOMY, UNIVERSITY OF SOFIA, ANTON IVANOV ST 5

BG 1126 SOFIA, BULGARIA

Telephonc: (359) $254+852$

Facsimile: (359)

Telex:

Email:

Commission(s):

TSVETKOVA KATYA, ASTRONOMICAL OBSERVATORY, BULGARIAN ACAD SCIENCES, 72 LENIN BLVD BG $178+$ SOFIA, BULGARIA

Telephonc: (359) 2758927

Email: liatyal7(a)bgearn.bituct

Facsimile: (359) 2757053

Telex: 23561 ecf ban bg

Commission(s): 27,37

TSYGAN ANATOLII I PROF, IOFFE PHYSICAL TECH INST, ACADEMY OF SCIENCES POLYTECHNICHESKAYA UL 26, 19+(021 ST PETERSBURG, RUSSIA

Telephone: (7) 8122479326

Email: varsh(a)eo.pti.spb.su

Facsimile: (7) 8122471963

Telex: 121453 ftian su

TUCHMAN YTZHAK, RACAH INST OF PHYSICS, HEBREW UNIV OF JERUSALEM, JERUSALEM 91904, ISRAEL

Telephone: (972) $2584+17 \quad$ Facsimile: (972)

Telex: 25391 huil

Email:

Commiswion(s):

TUCHOLKE HANS-JOACHIM, UNIVERSITAETS STERNWARTE, AUF DEM HUEGAL 71, D 53121 BONN, GERMANY

Telephone: (49) 228733 649

Facsimile: (49) 228733672

Telex:

Email: tucholke(a)astro.uni-bonn.de

Commission(s): 37

TUCKER WALLACE H DR, BOX 266, BONSALL CA 92003, USA

Telephone: (1) 6197287103

Facsimile: (1)

Cammission(s):

Telex:

Email:

Camissime

TUEG HELMUT DR, ALFRED-WEGENER INSTITUT, FUR POLARFORSCHUNG, COLUMBUS CENTER D 2850 BREMERHAVEN, GERMANY

Telephone: (49)

Email:

Facsimile: (49)

Commission(s): 09

Telex:

TUFEKCIOGLU ZEKI DR, ASTRONOMY DPT, SCIENCE FACULTY, ANKARA UNIVERSITY, 06100 ANKARA, TURKE

Telephone: (90) 42126720

Facsimile: (90)

Commission(s):

Telex:

Email:

TULL ROBERT G, ASTRONOMY DPT, UNIVERSITY OF TEXAS, RLM 15 308, AUSTIN TX 78712 1083, USA

$\begin{array}{ll}\text { Telephone: (1) } 5124713337 & \text { Facsimile: (1) } \\ \text { Email: } & \text { Commission(s): 09 }\end{array}$

Telex: $91087+1351$

TULLY JOHN A DR, OCA OBSERV DE NICE, BP 229, F $0630+4$ NICE CDX 4 , FRANCE

Telephonc: (33) 93890420

Facsimile: (33) 92 (00) 3033

Telex: $46000+$ obsnice $f$

Email:

Commission(s):

TULLY RICHARD BRENT DR, INSTITUTE FOR ASTRONOMY, UNIVERSITY OF HAWAII, 2680 WOODLAWN DR HONOLULU HI 96822, USA

Telephone: (1) 8089568606

Email:

Facsimile: (1) 8089882790

Commission(s): 28,47
Telex: 723-8459 uhast hr 
TUNCA ZEYNEL DR, FACULTY OF SCIENCE, EGE UNIVERSITY, BOX 21, 35100 BORNOVA IZMIR, TURKEY

Telephone: (90) $51180110 * 2332$

Facsimile: (90)

Telex:

Email:

Commission(s):

TUOHY IAN R DR, BRITISH AEROSP AUSTRALIA, I PARK WAY, TECHNOLOGY PARK

SALISBURY 5095, AUSTRALIA

Telephone: (61) 834322111

Facsimile: (61) 83496629

Telex: $883+2$

Email;

Commission(s):

TUOMINEN ILKKA V DR, OBS \& ASTROPHYSICS LAB, UNIVERSITY OF HELSINKI, TAEHTITORNINMAKI SF 00130 HELSINKI 13, FINLAND

Telephone: (358) 1912946

Facsimile: (358)

Telex:

Email: tuominen $(a)$ finuh

Commission(s): $10,29,35,36$

TURATTO MASSIMO DR, OSS ASTRONOMICO DI PADOVA, VIC DELL OSSERVATORIO 5, I 35122 PADOVA, ITALY

Telephonc: (39) 49661499

Facsimile: (39)

Telex: 432071

Email: span:39003::turatto

Commission(s):

TURLO ZYGMUNT DR, INSTITUTE OF ASTRONOMY, N COPERNICUS UNIVERSITY, UL CHOPINA 12/18

PL 87 100 TORUN, POLAND

$\begin{array}{ll}\text { Telephone: }(48) & \text { Facsimile: }(48) \\ \text { Email: } & \text { Commission(s): } 40 .\end{array}$

Email:

TURNER BARRY E DR, NRAO, 520 EDGEMONT RD, CHARLOTTESVILLE VA 22903, USA

Telephone: (1) 8042960337

Facsimile: (1) Telex:

Email:

Commission(s): $34,+0$

TURNER DAVID G DR, DPT OF ASTRONOMY, ST MARY'S UNIVERSITY, HALIFAX NS B3H 3C3, CANADA Telephone: (1) $9024299780 \star 2254$ Facsimile: (1) $902+205561 \quad$ Telex:

Email:

Commission(s): 27,37

TURNER EDWIN L DR, PRINCETON UNIVERSITY OBS, PEYTON HALL, PRINCETON NJ 08544, USA

Telcphone: (1) 6092583577

Facsimile: (1) $6082581020 \quad$ Telex: 322409 astro prin

Email: zltâastrouvav.princeton.colu

Commission(s): $28,47,51$

TURNER KENNETH C DR, NTL SCIENCE FOUNDATION, DIV ASTRONOMICAL SCIENCES, 1800 G ST NW WASHINGTON DC 20550 , USA

Telephone: (1)

Facsinile: (1)

Telex:

Email: kturneränote.nsf.gov

Commission(s): $05,34,40,51$

TURNER MARTIN J L DR, DPT OF PHYSICS, UNIVERSITY OF LEICESTER, X-RAY ASTRONOMY GROUP LEICESTER LE1 7RH, UK

Telephone: (4) $1533554455 \quad$ Facsimile: (4t)

Email:

Commission(s):

Telex: $3+166+$ luxiay g

TURNER MICHAEL S, ASTRONOMY \& ASTROPHYS CTR, UNIVERSITY OF CHICAGO, 5640 S ELLIS AVE

CHICAGO IL 60637, USA

Telephone: (1) 3129627974

Email:

\author{
Facsimile: (1) \\ Commission(s): 47
}

Telex: 6871133 uncog vw

TURON C DR, OBSERVATOIRE DE PARIS, SECTION DE MEUDON, DASGAL, F 92195 MEUDON PPL CDX, FRANCE

Telephone: (33) 145077837

Email: turonabobspm.fr Ficsimile: (33) $1+5077878 \quad$ Telex:

Commission(s): $108,2+\mathrm{P}, 33$

TURTLE A J DR, DPT OF PHYSICS, UNIVERSITY OF SYDNEY, SYDNEY NSW 2006, AUSTRALIA

Telephone: (61) 26922222

Email:

Facsinile: $(61) 266029013$

Telex: 26169 unisyd

Commission(s): 40

TUTUKOV A V DR, INST OF ASTRONOMY, ACADEMY OF SCIENCES, PYATNITSKAYA UL 48 $109017^{\circ}$ MOSCOW, RUSSIA

Telephone: (7) 952315461

Facsimile: (7)

Commission(s): $27,35 \mathrm{C},+2 \mathrm{C}$

Telex: 412623 sestp su

Email:

TWAROG BRUCE A, DPT PHYSICS \& ASTRONOMY, UNIVERSITY OF KANSAS, LAWRENCE KS 66045, USA

Telephone: (1) 9138645163

Facsimile: (1)

Telex:

Email:

Commission(s): 37 
TWISS R Q DR, C/O A.R. BOSCHI, 96A HOLLAND RDOSCHI, LONDON WI 4 8BD, UK Telephone: (4)

Facsimile: (4t)

Telex:

Email:

Commission(s):

TWORKOWSKI ANDRZEJ S, SCHOOL OF MATHEMATICAL SC, QUEEN MARY/WESTFIELD COLL, MILE END RD LONDON E1 4NS, UK

Telephone: (4) $1719804822 \quad$ Facsimile: (t4)

Telex: 893750

Email:

Commission(s):

TYLENDA ROMUALD DR, INSTITUTE OF ASTRONOMY, N COPERNICUS UNIVERSITY, UL CHOPINA 12/18 PL 87100 TORUN, POLAND

Telephone: (48) $26018 * 10$

Facsimilc: (48)

Telcx: $55223+$ astr pl

Email:

Comnission(s): 27

TYLER JR G LEONARD DR, RADAR ASTRONOMY INST, STANFORD UNIVERSITY, STANFORD CA 94305 4035, USA

Telephone: (1) $415+973535$

Facsimile: (1)

Telex:

Email:

Commission(s): 16

TYLKA ALLAN J DR, NAVAL RESEARCH LABORATORY, CODE +154 GAMMA \& COSMIC

RAY ASTROPHYSICS BRANCH, WASHINGTON DC 20375 \$000, USA

Telephone: (1) 2027672200

Facsimile: (1) $2027676+73$

Telex:

Email: 11335::tylka

Commission(s): 44

TYSON JOHN A DR, BELL LABS, RM 1D-316, 600 MOUNTAIN AVE, MURRAY HILL NJ 07974, USA

Telephont: (1) 2015826028

Facsimile: (1)

Commission(s): $21,28,47$

Telex: 138650 hell labs muh

Email:

TYTLER DAVID DR, CASS, UCSD, C 011 , LA JOLLA CA 92093 1216, USA

$\begin{array}{ll}\text { Telephone: (1) } 61953+3460 & \text { Facsimile: (1) } \\ \text { Email: } & \text { Commission(s): } 47\end{array}$

Telex: 5106010681 cass uq

TZIOUMIS ANASTASIOS DR, AUSTRALIA TELESCOPE, NATIONAL FACILITY, BOX 76

EPPING NSW 2121, AUSTRALIA

Telephone: (61) 28680222

Facsinile: (61) $28680310 \quad$ Telex:

Email: atzioumiagatuf.csiro.au

Comunission(s): 40

UBERTINI PIETRO, IAS, CNR, CP 67. I 00044 FRASCATI, ITALY

Telephone: (39) $69425132 \quad$ Fatcsimile: (39) 69416847

Email:

Commission(s):

Telex: 610261 cnifra

UCHIDA JUICHI DR, TOHOKU GAKUEN UNIVERSITY, TAGAYO UNIVERSITY, MIYAGI 985, JAPAN

Telephone: (81)

Facsimile: (81)

Telex:

Email:

Commission(s): 35

UCHIDA YUTAKA PROF, DPT OF ASTRONOMY, UNIVERSITY OF TOKYO, BUNKYO KU, TOKYO 113, JAPAN

Tclephonc: (81) 757533890

Email:

Facsimile: (81) 757533897

Telcx:

UDALSKI ANDRZEJ DR, ASTRONOMICAL OBSERVATORY, WARSAW UNIVERSITY, AL UJAZDOWSKIE 4 PL 00 478 WARSAW, POLAND

Telephonc: (48) $294011 \quad$ Facsimile: (48)

Email: vdalski(aphwanw61.bitnet Commission(s):

Telex: 817063 oauw pl

UDAL'TSOV V A DR, LEBEDEV PHYSICAL INST, ACADEMY OF SCIENCES, LENINSKY PROSPEKT 53, 117924

MOSCOW, RUSSIA

Telephone: (7) $951358560 \quad$ Facsimile: (7)

Email:

Commission(s): 40

Telex: 411479 neod su

UDAYA SHANKAR N DR, RAMAN RESEARCH INSTITUTE, SADASHIVANAGAR, CV RAMAN AVE, BANGALORE 560 080, INDIA

Telephone: (91) 803360122

Facsimile: (91) 803340492

Telex: $08+52671$ rri in

Email: uday@arri.ernct,in

Commission(s): 40 
UDRY STEPHANE, DPT PHSYICS ¿ ASTRONOMY, RUTGERS UNIVERSITY, BOX 849, PISCATAWAY NJ 08855, USA

Telephone: (1) 9089322513

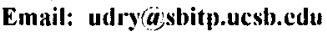

Facsimile: (1)

Commission(s): 28

Telex:

UENO SUEO PROF, KANAZAWA TECHNOLOGY INST, 7-1 OGIGAOKA, NONOICHIMACHI, ISHIKAWA 921, JAPAN

Telephone: (81) $762+81100$

Facsimile: (81)

Telex: $5122+56$ kiy lej

Email:

Commission(s): 36

UESUGI AKIRA DR, DPT OF ASTRONOMY, KYOTO UNIVERSITY, KITASHIRAKAWA SAKYO KU

KYOTO GO6, JAPAN

Telephone: (81)

Facsimile: (81)

Telex:

Email:

Commission(s): 05,36

UKITA NOBUHARU, NOBEYAMA RADIO OBS, NAOJ, MINAMIMAKI MURA, NAGANO 384 13, JAPAN

Telephone: (81) $267982831 \quad$ Facsimile: (81)

Enail:

Commission(s): 40

Telex: 3329005

ULFBECK OLE DR, NIELS BOHR INSTITUTE, BLEGDAMSVEJ 17, DK 2100 COPENHAGEN 0, DENMARK

Telcphone: (45) $31+21616$

Facsimile: (45) 31389157

Telex:

Email:

Commission(s):

ULICH BOBBY LEE, KAMAN AEROSPACE CORP, ELECTRO OPTICS DEVELOP CT, 3480 E BRITANNIA DR

TUCSON AZ 85711, USA

Telephone: (1) 5202952114

Facsimile: (1) 5208890211

Telex:

Email:

Commission(s): (19)

ULMER MELVILLE P PROF, DPT PIIYSICS \& ASTRONOMY, NORTHWESTERN UNIVERSITY

DEARBORN OBSERVATORY, EVANSTON IL $6(12018$, USA

Tclephonc: (1) $312+915633 \quad$ Fitcsimile: (1)

Telex: 9102310040

Email:

Commission(s):

ULMSCHNEIDER PETER PROF, INST F THEOR ASTROPHYSIK, DER UNIVERSITAET, IM NEUENHEIMER FELD 561 D 69120 HEIDELBERG, GERMANY
Telephone: (49) 6221562837
Facsimile: (49)
Telex: $\$ 61515$ unihd d

Email: i98/ädhdurzi

Commission(s): 36

ULRICH BRUCE T PROF, HILTENSPERGERSTR 93, D 8100 MUENCHEN 40, GERMANY

Telephone: (49)

Email:

Facsimile: (40)

Telex:

Commission(s): 25,41

ULRICH MARIE-HELENE D DR, ESO, KARL SCHWARZSCHILDSTR 2, D 85740 GARCHING MUENCHEN, GERMANY

Telephone: (49) 89320106229

Facsimile: (49) 893202362

Telex: $\$ 2828222$ cod

Email:

Commission(s): $28 \mathrm{C}, 3+, 40$

ULRICH ROGER K PROF, DPT OF ASTRONOMY, UNIVERSITY OF CALIFORNIA, 8931 MSB

LOS ANGELES CA 90124 , USA

Telephone: (1) $213825+270$

Facsimile: (1)

Telex:

Email:

Commission(s): 35

UMEMURA MASAYUKI DR, TOKYO ASTRONOMICAL OISS, NAOJ, OSAWA MITAKA, TOKYO 181, JAPAN

Telephone: $(81)+22+13731$

Facsimile: $(81)+22+137+6$

Telex: 028223117 taomtk j

Email: umemuräaume.m1k.nao.ac.jp

Commission(s): +7

UMLENSKI VASIL, DPT OF ASTRONOMY, BULGARIAN ACAD SCIENCES, 72 LENIN BLVD BG 1784 SOFIA, BULGARIA

Telephone: (359) 2758927

Facsimile: (350)

Telex: 23561 eef ban bg

Email:

Commission(s):

UNDERHILL ANNE B DR, 4696 WEST 10TH AVE \#301, VANCOUVER BC V6R 2J5, CANADA

Telephone: (1) $60+2243552$

Facsimile: (1)

Telex:

Email:

Commission(s): 29,36,44

UNDERWOOD JAMES H DR, LAWRENCE BERKELEY LAB, UNIVERSITY OF CALIFORNIA

$X$ RAY OPTICS LAB 80 101, BERKELEY CA 94720, USA

Telephone: (1) $+15+86$ 4958 Fasimile: (1)

Email:

Commission(s): $10,4 t$

Telex: 9103662037 
UNGER STEPHEN DR, ROYAL GREENWICH OBS, MADINGLEY RD, CAMBRIDGE CB3 OEZ, UK

Telephonc: (4) 1323833171

Facsimile: $(+4)$

Telex: $87 \$ 51$

Email: uk.ac.rgo.star

Commission(s): 28,40

UNNO WASABURO PROF, RES INST SCIENCE \& TECH, KINKI UNIVERSITY, HIGASHI, OSAKA 577, JAPAN

Telephonc: (81) 67212332

Facsimile: (81)

Telex:

Enaril:

Commission(s): $12,35,36$

UNSOELD ALBRECHT PROF, INST THEOR PHYS \& STERNW, NEUE UNIV PHYSIK ZENTRUM OLSHAUSENSTR GEB N 61C, D $24098 \mathrm{KJEL}$, GERMANY

Telephone: (49) $\$ 31880+205 \quad$ Facsimile: $(49) \quad$ Telex:

Email:

Commission(s):

UNWIN STEPHEN C, CALTECH, MS 105 24, PASADENA CA 91125, USA

Telephone: (1) $213356+973$

Facsimile: (1)

Telex: $675+25$

Email:

Commission(s): 40

UOMOTO ALAN K DR, DPT PHYSICS \& ASTRONOMY, JOHNS HOPKINS UNIVERSITY, CHARLES \& 34TH ST BALTIMORE MD 21218, USA

Telephone: (1) 3013388.594

Facsimile: (1)

Telex:

Email: auajpha.jhu.colu

Commission(s):

UPGREN ARTHUR R DR, DPT OF ASTRONOMY, VAN VLECK OBSERVATORY, WESLEYAN UNIVERSITY MIDDLETOWN CT $06+57$, USA

Telephone: (1) 2033479411

Facsimilc: (1)

Telex:

Email:

Commission(s): $24,26,33,37,45,50$

UPSON WALTER L II DR, PRINCETON UNIVERSITY OBS, PEYTON HALL, PRINCETON NJ O854, USA

Telephone: (1)

Facsimile: (1)

Telex:

Email:

Commission(s): $4+$

UPTON E K L DR, DPT OF ASTRONOMY, UNIVERSITY OF CALIFORNIA, LOS ANGELES CA 90124, USA

Telephone: (1)

Facsimile: (1)

Telex:

Email:

Commission(s):

URAS SILVANO DR, ISTITUTO DI ASTRONOMIA, VIA OSPEDALE 72, I 09100 CAGLIARI, ITALY

Telephone: (39) 70711246

Email:

Commission(s):

Telex: 7911326 ossast i

URASIN LIRIK A DR, ENGELHARDT ASTRONOMICAL, OBSERVATORY, OBSERVATORIA STATION $\$ 22526$ KAZAN, RUSSIA

Telephone: (7) $32+827$

Facsimile: (7)

Telex:

Email:

Commission(s): $3+$

URBANIK MAREK DR, ASTRONOMICAL OBSERVATORY, JAGIELLONIAN UNIVERSITY, UL ORLA 171 PL $302+4$ KRAKOW, POLAND

Telephone: (48) 12221877

Email:
Facsimile: (48) $12378(153$

Commission(s): 28
Telex: 326203 ujoa pl

URBARZ H DR, ASTRONOMISCHES INSTITUT, DER UNIVERSITAET, AUSSENSTELLE WEISSENAU D 7989 RAVENSBURG/RASTHAL, GERNIANY

Telephone: (49) $75161621 \quad$ Facsimile: (49) Telex:

Email:

Commission(s):
URECHE VASILE DR, FAC OF MATIIEMATICS, UNIVERSITY OF CLUJ-NAPOCA, STR M KOGALNICEANU 1 R 3400 CLUJ NAPOCA, RUMANIA

Telephone: (40) 64116111

Email:

Facsimile: $(411) 6+19+592 \quad$ Telex:

Commission(s): 25,42

URPO SEPPO I, HELSINKI UNIV TECIINOLOGY, RADIO LABORATORY, OTAKAARI 5 A

SF (215I ESPOO 15, FINLAND

Telephone: (358) $+5125+8 \quad$ Facsimile: (358)

Email:

Commission(s): $10,+10$

Telex: 122771 rorta sf 
URRY CLAUDIA MEGAN DR, STSCI, HOMEWOOD CAMPUS, 3700 SAN MARTIN DR, BALTIMORE MD 21218, USA

Telephone: (1) $301338+593$

Facsimile: (1)

Telex: 6849101 stsci

Email: scivax::cmu/cmu/astsci.bitnet

Commission(s):

USHER PETER D DR, DPT OF ASTRONOMY, PENNSYLVANIA STATE UNIV, 507 DAVEY LAB

UNIVERSITY PARK PA 168112 , USA
Telephone: (1) 8148653509
Facsimile: (1)
Telex: 842510 pennstbstreg

Email:

Commission(s): 27

USON JUAN M DR, NRAO, VLA, BOX 0, SOCORRO NM 878010387 , USA

Telephone: (1) 5058357237

Facsimile: (1)

Telcx: 9109881710

Email:

Commission(s): 40,47

USOWICS JERZY BOGDAN DR, INST OF RADIO ASTRONOMY, N COPERNICUS UNIVERSITY, UL CHOPINA 12/18 PL 87100 TORUN, POLAND

Telephone: $(48)$

Facsimile: (48)

Telex:

Email:

Commission(s):

UTSUMI KAZUHIKO DR, DPT OF ASTRONOMY, HIROSHIMA UNIVERSITY, HIGASHI SENDA MACHI

HIROSHIMA 730, JAPAN

Telephone: (81) $822+11221$

Email:

Facsimile: (81)

Telex:

Commission(s): 29

UUS UNDO DR, TARTU ASTROPHYSICAL OBS, ESTONIAN ACAD OF SCIENCES, EE 202 44 TARTU, ESTONIA

Telephone: (372)

Facsimile: (372)

Telex:

Email:

Commission(s): 12,35

VAGER ZEEV DR, DPT OF PIIYSICS, WEIZMANN INSTITUTE OF SCI, BOX 26, REHOVOT 76100, ISRAEL

Telephone: (972) $\quad$ Facsimile: (972)

Telex:

Email:

Commission(s):

VAGHI SERGIO DR, ESA/ESTEC, PHA, BOX 299, NL 22100 AG NOORDWIJK, NETHERLANDS

Telephonc: (31) 171983453

Facsimile: (31)

Telex: 391988

Email:

Commission(s): 20

VAGNETTI FAUSTO, DPT DI FISICA, UNIV DI ROMA TOR VERGATA, VIA DELLA RICERCA SCIENTI

I 00133 ROMA, ITALY

Tclephone: (39) $67259+426$

Facsimile: (39) 62023507

Telex: 626382 fiunty

Email: vagnettiatovf2.roma2.infu.it

Commission(s): 47

VAHIA MAYANK N, TIFR, HOMI BIIABHA RD, COLABA, BOMBAY 400005 , INDIA

Telephonc: (91) 222152971

Facsimile: (91) $222152110 \quad$ Telex:

Email: vahiaâ158.144.1.11

Commission(s): 4

VAIDYA P C PROF, 3† SHARDA NAGA, PALDI, AHMEDABAD 380007 , INDIA

Telephone: (91) $272+13322$ Facsimile: (91) Telex:

Email: $\quad$ Commission(s): 47

VAINSTEIN L A DR, LEBEDEV PHYSICAL INST, ACADEMY OF SCIENCES, LENINSKY PROSPEKT 53 11792 + MOSCOW, RUSSIA

Telephone: (7) 951352250

Email:

Facsimile: (7)

Telex:

Commission(s): +9

VAKILI FARROKH DR, OCA OBSERV DU CALERN, CAUSSOLS, F 16460 S VALLIER THIEY, FRANCE

Telephone: $(33) 93+26270$

Email: valkiliafronis1

Facsimile: (33) 93 (09 2613

Telex: $4(61+02 \mathrm{f}$

Commission(s): 09,36

VALBOUSQUET ARMAND DR, OBS DE STRASBOURG, 1 I RUE UNIVERSITE, F 67000 STRASBOURG, FRANCE

Telephone: (33) 88358200

Facsimile: (33) 88250160

Telex:

Email:

Commission(s): $24,26,51$

VALENTIJN EDWIN A DR, KAPTEYN ASTRONOMICAL INST, BOX 800, NL 9700 AV GRONINGEN, NETHERLANDS

Telephone: (31) 50116695

Facsimile: (31)

Telex:

Email:

Commission(s): 28 
VALIRON PIERRE DR, OBSERVATOIRE DE GRENOBLE, CERMOIASTROPHYSIQUE, BP 53X F 380 $\$ 1$ S MARTIN HERES CD, FRANCE

Telephone: (33) 76514787

Facsimile: (33) 76448821

Telex: 980753 iram f

Email: bitnct:valiron(äfrgag 51

Commission(s):

VALLEE JACQUES P DR, HERZBERG INST ASTROPHYS, NTL RESEARCH COUNCIL, IOI SUSSEX DR OTTAWA ON K1A IRG, CANADA

Telephone: (1) 613993 6060

Facvimilc: (1) 6139526602

Telex: 0533715

Email:

Commission(s): 40,51

VALNICEK BORIS DR, ASTRONOMICAL INSTITUTE, CZECH ACADEMY OF SCIENCES ONDREJOV OBSERVATORY, CZ 251 65 ONDREJOV, CZECH R

Telephenc: (42) 2048573247111

Email: astsun $(a) c s c a r n$

Facsimile: $(\$ 2) 20+851611$

Telex: 121579

Commission(s): $(9), 10,44$

VALSECCHI GIOVANNI B DR, IAS, REPARTO DI PLANETOLOGIA, VIA DELL'UNIVERSTA 11, I OOIS5 ROMA, ITAL

Telephone: (39) 64456951

Facsimile: (39) $6+45+969$

Telex: cnr fra 610261

Email: giovanni(a)m-ias.fra.cnr.it

Commission(s): $07,15,20$

VALTAOJA ESKO, TURKU UNIVERSITY OBS, TUORLA, SF 21500 PIIKKIO, FINLAND

Telephone: (358) $21 \$ 35822$

Email:

Facsionile: (3.58) $21+33767$

Telex: 62638 tyf

VALTAOJA LEENA DR, TURKU UNIVERSITY OBS, TUORLA, SF 21500 PIIKKIO, FINLAND

Telephone: (358) $21+35822$

Email: Ivaltaoja(âkontu.utu.fi

Facsinile: (358) $21+33767$

Telex:

VALTIER JEAN-CLAUDE DR, OCA OBSERV DE NICE, BP 229, F $0630 \downarrow$ NICE CDX 4, FRANCE

Telcphone: (33) 93890420

Emili:

Facsimile: (33) 920031133

Telex: $f(0)(0)+$ obsnice $f$

Commission(s): 27,29

VALTONEN MAURI J PROF, TURKU UNIVERSITY OBS, TUORLA, SF 2150I PIIKKIO, FINLAND

Telephone: (358) $21+35822 \quad$ Facsimile: (358) $21+33767 \quad$ Telex: 62638 tyf sf

Email:

Commission(s): $47,09,26,28,33,411,44$

VALYAEV VALERY, 10 NAB KUTUSOVA, ST PETERSBURG, RUSSIA

Telephone: (7) 8122751006

Email: solkolsky a iipah.spb.su

Facsimile: (7) $8122727 \% 68$

Telex: 121578 ifa su

VAN AGT S L TH J DR, STERRENKUNDIG INSTITUTE, KATHOLIEKE UNIVERSITEIT, TOERNOOIVELD NL 6525 ED NIJMEGEN, NETHERLANDS

Telephone: (31) $80558833 \quad$ Facsimile: (31)

Commission(s): 27

Telex: 48228 winat nl

Email:

VAN ALBADA TJEERD S DR, KAPTEYN ASTRONOMICAL INST, BOX 800, NL 9700 AV GRONINGEN, NETHERLANDS Telephone: (31) 50 $116695 \quad$ Facsimile: (31)

Email: $\quad$ Commission(s): 28 Telex: $\mathbf{5 3 5 7 2}$ stars nl

VAN ALLEN JAMES A PROF, DPT PHYSICS \& ASTRONOMY, UNIVERSITY OF IOWA, IOWA CITY IA 52242, USA Telephone: (1) $319353+531$ Fatesimile: (1) Telex:

Email: Commission(s): $10,16,49$

VAN ALTENA WILLIAM F PROF, YALE UNIVERSITY OBS, BOX 6666, NEW HAVEN CT (I65II, USA

Telephone: (1) $203+368318 \quad$ Filsimile: (1) $203+325048$ Telex: 9102508365

Email: vanalten(a) valastio

Commission(s): $08,24,26,37$

VAN BEEK FRANK PROF DR, DPT OF MECHAN ENGINEERING, TECHNICAL UNIV OF DELFT, MEKELWEG 2 NL 2628 CD DELFT, NETHERLANDS

Telephone: (31) 15785396

Ficsimile: (31)

Commission(s): +4

Telex:

Email:

OF ASTRONOMY, UNIV OF MASSACHUSETTS, AMHERST MA 01002, USA

VAN BLERKOM DAVID J PROF, DPT OF ASTRONOMY, UNIV OF MASSACHUS
Telephone: (1)

Email:

Commission(s): 
VAN BREDA IAN G DR, DUNSINK OBSERVATORY, SCHOOL OF COSMIC PHYSICS, DUBLIN 15, IRELAND

Telephone: (353)

Facsimile: (353)

Telex:

Email:

Commission(s):

VAN BREUGEL WIL, RADIO ASTRONOMY LAB, UNIVERSITY OF CALIFORNIA, G(II CAMPBELL HALL

BERKELEY CA 9\$720, USA

Telephone: (1) $+156+25275$

Email:

Facsimile: (1)

Commission(s):

Telex:

VAN BUEREN HENDRIK G PROF, MEIDOORNLAAN 13, NL 3461 ES LINSCHOTEN, NETHERLANDS

Telephone: (31) $348115406 \quad$ Facsimile: (31)

Telex:

Email:

Commission(s):

VAN CITTERS GORDON W DR, NTL SCIENCE FOUNDATION, DIV ASTRONOMICAL SCIENCES, 1800 G ST NW WASHINGTON DC 20550, USA

Telephone: (1)

Facsimile: (1)

Telex:

Email:

Commission(s): (0)

VAN DE HULST H C PROF DR, STERREWACHT, BOX 9513, NL 2300 RA LEIDEN, NETHERLANDS

Telephonc: (31) $711+8333$

Facsimile: (31)

Telex: 39058

Email:

Commission(s): $21,34,40,44$

VAN DE KAMP PETER, AMSTEL 244, NL 1017 AK AMSTERDAM, NETHERLANDS

Telephonc: (31) 20223377

Facsimile: (31)

Telex:

Email:

Commission(s): $24,26,51$

VAN DE STADT HERMAN DR, SPACE RESEARCH DPT, BOX 800, NL 9700 AV GRONINGEN, NETHERLANDS

Telephonc: (31) 50116695

Facsimilc: (31)

Telex: 53572

Email:

Commission(s):

VAN DEN BERGH SIDNEY PROF, HERZBERG INST ASTROPHYS, DOMINION ASTROPHYS OBS, 5071 W SAANICH

RD, VICTORIA BC V8X +MG, CANADA

Telephone: (1) 6043630006

Facsimile: (1) 6043630045

Commission(s): 28,37,50

Telex: 0497295

Enail: vandenberghäadao.nec.ca

VAN DEN HEUVEL EDWARD P J, ASTRONOMICAL INSTITUTE, UNIVERSITY OF AMSTERDAM, KRUISLAAN 403 NL 1098 SJ AMSTERDAM, NETHERLANDS

Telephone: (31) $205257491 \quad$ Facsimile: (31) 205257484

Telex: 10262 hef $\mathrm{nl}$

Email:

Commission(s): $35,38,+2,44$

VAN DEN OORD BERT H J DR, STERREKUNDIG INSTITUTE, BOX 8OOM, NL 3500 TA UTRECHT, NETHERLANDS

Telephone: (31) 30535200

Email: oordậ̣s.ruu.ul

Facsimile: (31) $30531601 \quad$ Telex: $\$ 0048$ fylut

Commission(s): 10

VAN DER BORGHT RENE PROF, 31 THE PROMENADE, ISLE OF CAPRI, SURFERS PARADISE \$217, AUSTRALIA

Telephone: (61) 385712

Facsimile: (61)

Telex:

Email:

Commission(s): 35

VAN DER HUCHT KAREL A DR, SPACE RESEARCH LABORATORY, SRON, SORBONNELAAN 2

NL $358+$ CA UTRECHT, NETHERLANIS

Telephone: (31) 30535600

Ficsimile: (31) 30540860

Telex: 47224

Email:

Commission(s): 26,29,4t

VAN DER HULST JAN M DR, KAPTEYN ASTRONOMICAL INST, BOX 800, NL 9700 AV GRONINGEN, NETHERLANDS

Telephonc: (31) $5063+1154$

Email: voluulstệhgrrugs

Facsimile: (31) $50634033 \quad$ Telex: 53572 starz nl

Commission(s): 28,40

VAN DER KLIS MICHIEL DR, ASTRONOMICAL INSTITUTE, UNIVERSITY OF AMSTERDAM, KRUISLAAN 403 NL 1098 SJ AMSTERDAM, NETHERLANDS

Telephone: (31) $205257498 / 7+91 / 7+92$ Facsimile: (31) $205257484 \quad$ Telex: 10262 hef nl

Email: carn/bitnet: af10mvdlk(ahasarall Commission(s):

VAN DER KRUIT PIETER C DR, KAPTEYN ASTRONOMICAL INST, BOX 800

NL 9700 AV GRONINGEN, NETHERLANDS

Telephone: (31) $5063+073$

Facsimile: (31)

Commission(s); $28,33,41$

Telex: 53572 stars nl

Email: 
VAN DER LAAN H PROF DR, STERREKUNDIG INSTITUTE, BOX 80000, NL 3508 TA UTRECHT, NETHERLANDS Telephone: (31) 30535200

Email: vdlaan(a)ys.ruu.n!

Facsimile: (31) 30535201

Telex:

VAN DER LINDEN RONALD, JET JOINT UNDERTAKING, ABINGDON, OXFORDSHIRE OX14 3EA, UK

Telephone: (44) $133+76161 \quad$ Facsimile: (4+) $133+637+8 \quad$ Telex: 9312110846 sag

Email: ramvdlajjet.uk

Commission(s): 10

VAN DER RAAY HERMAN B, DPT OF PHYSICS, UNIVERSITY OF BIRMINGHAM, BOX 363 BIRMINGHAM B15 2TT, UK

Telephonc: (4+) $121+721301$

Email:
Facsimile: (4t)

Commission(s): 35
Telex: 228938 spaphy g

VAN DER VEEN WILHELMUS EC, ASTRONOMY DPT, COLUMBIA UNIVERSITY, 538 W 120TH ST NEW YORK NY 10027, USA

Telephone: (1) 2128546831

Facsimile: (1) 2128549504

Telex:

Email: weci@acarmen.phys.columbia.celu Commission(s):

VAN DER WALT D J DR, DPT OF PHYSICS, POTCHEFSTROOM UNIVERSITY, PRIVATE BAG XG001 POTCHEFSTROOM, SOUTH AFRICA

$\begin{array}{lll}\text { Telephonc: (27) } 27148992408 & \text { Facsimile: (27) } 27148992421 & \text { Telex: } 346019 \text { sa } \\ \text { Email: fskdjudwapukvml.puk.ac.zal } & \text { Commission(s): } 44 & \end{array}$

VAN DER WERF PAUL P, LEIDEN OBSERVATORY, BOX 9513, NL 2300 RA LEIDEN, NETHERLANDS

Telephone: (31) 71275861

Facsimile: (31) 71275819 Telex:

Email: pwdwerfastrw.Icidemuniv.nt

Commission(s):

VAN DESSEL EDWIN LUDO DR, OBSERVATOIRE ROYAL DE, BELGIQUE, AVE CIRCULAIRE 3

B 1180 BRUSSELS, BELGIUM

Telephone: (32) 26735366

Facsimile: (32) 23749822

Telex: 21565 obsbel

Email: evdesaastro.ome,be

Commission(s): 26,30

VAN DIGGELEN J DR, OBSERVATORY UTRECHT, AETSVELDSELAAN 12, NL 1381 EA WEESP, NETHERLANDS

Telephone: (31)

Email:

Facsimile: (31)

Commission(s):

Telex:

VAN DISHOECK EWINE F DR, STERREWACHT, BOX 9513, NL 2300 RA LEIDEN, NETHERLANDS

Telephone: (31) $71275874 / 5835 \quad$ Fatesimile: (31) $71275819 \quad$ Telex: 39058 astro $\mathrm{nl}$

Email:

Commission(s): 14,34

VAN DORN BRADT HALE DR, CTR FOR SPACE RESEARCH, MIT RM 37 587, BOX 165, CAMBRIDGE MA 02139, USA

Telephonc: (1) 6172537550

Email: halceaspace.mit.edu

Facsimile: (1) 6172530861

Commission(s):

Telex: $921+73$ mitcam

VAN DRIEL WILLEM DR, OBSERVATOIRE DE PARIS, UNITE SCIENTIFIQUE NANCAY

F 92195 MEUDON PPL CDX, FRANCE

Telephone: (33)

Facsimile: (33)

Commission(s): 28

Telex:

Email: wim.van.driclagin.obspm.fy

VAN DRIEL-GESZTELYI L DR, OBSERVATOIRE DE PARIS, SECTION DE MEUDON, DASOP F 92195 MEUDON PPL CDX, FRANCE

Telephone: (33)

Facsimile: (33)

Commission(s): 10

Telex:

Email:

VAN DUINEN R J DR, FOKKER BV, SPACE DIVISION, BOX 7600, NL 1117 ZJ SCHIPHOL, NETHERLANDS

Telephone: (31) $205++2030$

Facsimile: (31)

Telex: 12227

Email:

Commission(s): 44

VAN DYK SCHUYLER, ASTRONONY DPT, UNIVERSITY OF CALIFORNIA, BERKELEY CA 9+720 3411, USA

Telephone: (1) $51064381+3$

Facsimile: (1) $5106+23+11$

Telex:

Email: vandykapopsicle.berlicley.edu

Commission(s):

VAN FLANDERN TOM DR, META RESEARCH, 6327 WESTERN AVE NW, WASHINGTON DC 20015, USA

Telephone: (1) 2023633860

Facsimile: (1)

Telex:

Email:

Commission(s): $04,15,16,20,51$ 
VAN GENDEREN A M DR, STERREWACHT, BOX 9513, NL 2300 RA LEIDEN, NETHERLANDS

Telephone: (31) 71272727

Facsimilc: (31)

Telex: 31476 astro nl

Enail:

Commission(s): 27,28

VAN GORKOM JACQUELINE H, NRAO, BOX 0, SOCORRO NM 87801 10387, USA

Telephone: (1) $5057724302 \quad$ Facsimile: (1) Telex: 910-997-0174

Email: $\quad$ Commission(s): $28,34,40$

VAN GRONINGEN ERNST DR, ASTRONOMICAL OBSERVATORY, BOX 515, S 75120 UPPSALA, SWEDEN

Telephone: (46) Facsimile: (46) Telex:

Email: $\quad$ Commission(s):

VAN HAMME WALTER, DPT PHYSICS \& ASTRONOMY, FLORIDA ITL UNIVERSITY, UNIVERSITY PARK MIAMI FL 33199, USA

Telephone: (1)

Facsimile: (1)

Commission(s): $\$ 2$

Telex:

Email:

VAN HERK G, STERREWACHT, BOX 9513, NL 2300 RA LEIDEN, NETHERLANDS

Telephonc: (31) $71272727 \quad$ Ficsimile: (31) Telex:

Email: $\quad$ Commission(s):
VAN HORN HUGH M PROF, DPT PHYSICS \& ASTRONOMY, UNIVERSITY OF ROCHESTER ROCHESTER NY $1+627$, USA
Telephone: (1) $716275+3+4 \quad$ Facsimile: (1) Telex:
Email: $\quad$ Commission(s): 35

VAN HOUTEN C J DR, STERREWACHT, BOX 9513, NL 2300 RA LEIDEN, NETHERLANDS

Telephone: (31) $71272727 \quad$ Falcsimile: (31) Telex: 39058 astro nl

Email: $\quad$ Commission(s): 20

VAN HOUTEN-GROENEVELD I, STERREWACHT, BOX 9513, NL 23\%0 RA LEIDEN, NETHERLANDS

$\begin{array}{ll}\text { Telephone: (31) } 71272727 & \text { Fancsimile: (31) } \\ \text { Email: } & \text { Commission(s): 20. Telex: } 39058 \text { astro nl }\end{array}$

Email:

Commission(s): 20

VAN HOVEN GERARD DR, DPT OF PHYSICS, UNIVERSITY OF CALIFORNIA, IRVINE CA 92717, USA

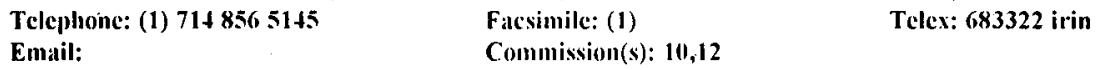

VAN LEEUWEN FLOOR DR, ROYAL GREENWICH OBS, MADINGLEY RD, CAMBRIDGE CB3 OEZ, UK

Telephone: (4) $122337+765 \quad$ Facsimile: (4) 1223374700 Telex:

Email: $\quad$ Commission(s): 108

VAN MOORSEL GUSTAAF DR, NRAO, BOX 0 , SOCORRO NM 87801 (0387, USA

Telephone: (1) 5058357396 Facsimile: (1) 511583571127 Telex:

Email: granmoorạnrao.edu Commission(s): 28

VAN NIEUWKOOP J DR IR, PRINSESSELAAN 12, NL 7316 CN APELDOORN, NETHERLANDS

$\begin{array}{ll}\text { Telephone: (31) } & \text { Facsimile: (31) } \\ \text { Email: } & \text { Commission(s): t0 }\end{array}$

VAN PARADIJS JOHANNES DR, ASTRONOMICAL INSTITUTE, UNIVERSITY OF AMSTERDAM, KRUISLAAN 403 NL 1098 SJ AMSTERDAM, NETHERLANDS

Telephone: (31) $20 \$ 257+91 \quad$ Facsinile: (31) $205257484 \quad$ Telex: 10262 hef $\mathrm{nl}$

Email:

Commission(s): $\$ 2$

VAN REGEMORTER HENRI DR, OBSERVATOIRE DE PARIS, SECTION DE MEUDON

F 92195 MEUDON PPL CDX, FRANCE

Telephone: (33) 1 +5 $077+4+\quad$ Facsimile: (33) Telex: 201571

Email: $\quad$ Commission(s): 14,36

VAN RENSBERGEN WALTER DR, IAAG, VRIJE UNIV BRUSSELS, CP 165, B 1050 BRUSSELS, BELGIUM

Telephone: (32) $26+13497$

Facsimile: (32)

Telex:

Email:

Commission(s): 14 
VAN RIPER KENNETH A DR, LOS A LAMOS NATIONAL LAB, MS B 226 X 6, BOX 1663, LOS ALAMOS NM 87545, USA Telephone: (1) 5056678104

Facsimile: (1)

Telex:

Email:

Commission(s): 35,44

VAN SPEYBROECK LEON P DR, CENTER FOR ASTROPHYSICS, HCO/SAO, 60 GARDEN ST CAMBRIDGE MA (12138, USA

Telephone: (1) 6174957233

Facsimile: (1)

Telex:

Email:

Commission(s): $4+$

VAN WOERDEN HUGO PROF DR, KAPTEYN ASTRONOMICAL INST, BOX 800 NL 97(0) AV GRONINGEN, NETHERLANDS

$\begin{array}{lll}\text { Telephonc: (31) } 50634073 / 4066 & \text { Facsimile: }(31) & \text { Telex: } 53572 \text { stars n\} } \\ \text { Email: secr(â)astro.rug.nl } & \text { Commission(s): } 28,33,34,40 & \end{array}$

VANDAS MAREK, ASTRONOMICAL INSTITUTE, CZECH ACADEMY SCIENCES, BOCNI II 1401

CZ 14131 PRAHA 4, CZECH R

Telephone: (42) 2267103061

Email: vandas(ajig.cas.cz

Ficsimile: (42) 2769012

Telex: 121579

Commission(s): 49

VANDEN BOUT PAUL A, NRAO, 520 EDGEMONT RD, CHARLOTTESVILLE VA 22003, USA

Telephonc: (1) 8042960241

Facsimile: (1) 8042960385

Telex:

Email: pwandenbrajnra.cdu

Commission(s): 34,40

VANDENBERG DON DR, DPT OF PHYSICS, UNIVERSITY OF VICTORIA, BOX 1700, VICTORIA BC V8W 2Y2, CANAD Telephonc: (1) $60+7217739$

Facsimile: (1) $6047217715 \quad$ Telex:

Email:

Commission(s): $35 \mathrm{C}, 37$

VANDERVOORT PETER O DR, ASTRONOMY \& ASTROPHYS CTR, UNIVERSITY OF CHICAGO, 5640 S ELLIS AVE CHICAGO IL 60637, USA

Telephone: (1) 3129628209

Email:

Facsimile: (1)

Telex:

Commission(s): 33

VANYSEK VLADIMIR PROF, DPT OF ASTRONOMY, CHARLES UNIVERSITY, SVEDSKA 8

CZ 150 00 PRAHA 5, CZECH R

Telephone: $(42) 25 \$ 10395$

Email: vanysckácscarn

Facsimile: (42) 2299272

Telex: $121673 \mathrm{mff}$

VAN'T VEER FRANS DR, OBSERVATOIRE DE PARIS, 61 AVE OBSERVATOIRE, F 7501+ PARIS, FRANCE

Telephone: (33) 140512221

Email:

Facsimile: (33)

Commission(s): $10,36,42$

Telex: $27(0776$ obs p

VAN'T VEER-MENNERET CL DR, OBSERVATOIRE DE PARIS, 1 AVE OBSERVATOIRE, F 75014 PARIS, FRANCE

Telephone: (33) 1 40 512249

Facsimile: (33)

Telex:

Email:

Commission(s): 29,36

VAPILLON LOIC J DR, OBSERVATOIRE DE PARIS, SECTION DE MEUDON, F 92195 MEUDON PPL CDX, FRANCE

Telephone: (33) 145077623

Facsimile: (33)

Telex: 211571

Email:

Commission(s):

VARDANIAN R A DR, BYURAKAN ASTROPHYSICAL, OBSERVATORY, 378\$33 BYURAKAN, ARMENIA

Telephone: (7) $885228+142$

Facsimile: (7) $885228+1+2 \quad$ Telex:

Email:

Commission(s): 25

VARDAVAS ILIAS MIHAIL, DPT OF PIIYSICS, UNIVERSITY OF CRETE, BOX 1527, GR 71111 IRAKLION, GREECE

Telephone: (30) 81236.589

Facsimile: (30) $02+167902$

Telex: 262728

Email:

Commission(s): 36

VARDYA M S DR, TIFR, HOMI BHABHA RD, COLABA, BOMBAY 400 (0)5, INDIA

Telephone: (91) $22219111 * 221$

Facsimile: (91)

Telex: 113009 tifr in

Email:

Commission(s): 35,36

VARMA RAM KUMAR PROF, PHYSICAL RESEARCH LAB, NAVRANGPURA, AHMEDABAD 380 009, INDIA

Telephone: (91) $272+6212$ )

Email:

Facsimile: (ソ1) $272+45292$

Commission(s): 28
Telex: 0121397 pril in 
VARSHALOVICH DIMITRIJ PR, IOFFE PHYSICAL TECH INST, ACADEMY OF SCIENCES POLYTECHNICHESKAYA UL 26, 194021 ST PETERSBURG, RUSSIA

Telephone: (7) 2472255

Facsimile: (7)

Telex:

Email:

Commission(s): $14,34,51$

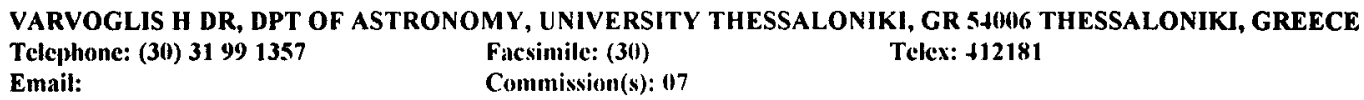

VASHKOV'YAK SOF'YA N DR, STERNBERG STATE ASTR INST, UNIVERSITETSKIJ PROSP 13 119899 MOSCOW, RUSSIA

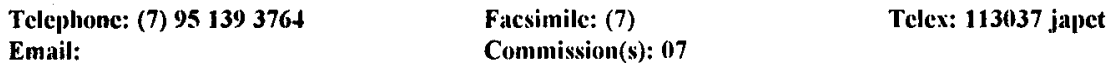

VASILEVA GALINA J DR, PULKOVO OBSERVATORY, ACADEMY OF SCIENCES, 10 KUTUZOV QUAY 196140 ST PETERSBURG, RUSSIA

Telephone: (7)

Facsimile: (7)

Telex:

Email:

Commission(s): 12

VASS GHEORGHE, ASTRONOMICAL INSTITUTE, CUTITUL DE ARGINT 5, BOX 28, R 75212 BUCHAREST, RUMANIA

Telephone: (40) 16236892

Email: ghevaswaroean.bitnet

Fatesimile: (\$1) 13123391

Telex: 11882 astro $r$

VASU-MALLJK SUSHMA DR, INDIAN INSTITUTE OF, ASTROPHYSICS, KORAMANGALA

BANGALORE 560 034, INDIA

Telephonc: (91) $803569179 / 9180$

Ficsimile: (91)

Telex: 845763 iiab in

Email:

Commission(s): 29,36

VATS HARI OM DR, PHYSICAL RESEARCH LAB, NAVRANGPUVA, AHMEDABAD 9, INDIA

Telephonc: (91) $272462129 \quad$ Facsimile: (91) $272+4 \$ 292 \quad$ Telex: 01216397

Email: ipsaprl.crnet.in

Commission(s): 4 (1)

VAUCLAIR GERARD P DR, OBS MIDI PYRENEES, 14 AVE E BELIN, F 31 H 1 TOULOUSE CDX, FRANCE

Telephone: (33) 61252101

Facsimile: (33)

Telex: $\$ 30776$

Email:

Commission(s): $35 \mathrm{C}$

VAUCLAIR SYLVIE D DR, OBS MIDI PYRENEES, 14 AVE E BELIN, F 31400 TOULOUSE CDX, FRANCE

Telephone: (33) 61252101

Facsimile: (33)

Telex:

Email:

Commission(s): 46

VAUGHAN ALAN DR, SCHOOL OF MATHS/PHYSICS, COMPUTING AND ELECTRONICS, MACQUARIE UNIVERSITY MACQUARIE 2109, AUSTRALIA

Facsimile: (61) 28058083

Email: alanv(a)macastro.mpes.mq.0\%..atu Commission(s): 41)

VAUGHAN ARTHUR H DR, PERKIN-ELMER CORP, 7421 ORANGEWOOD AVE, GARDEN GROVE CA 92641, USA

Telephone: (1) $7148951667 \quad$ Facsimile: (1) Telex:

Email:

Commission(s): $10,12,25,30$

VAUGLIN ISABELLE DR, OBSERVATOIRE DE LYON, AVE CHARLES ANDRE, F 69561 S GENIS LAVAL CDX, FRANC

Telephone: (33) 72399098

Facsimile: (33) 72399791

Telex: 31092

Email: vauglin(a)castor.univ-lyon 1.fr Commission(s): 28

VAVROVA ZDENKA DR, NA KOPECKU 346, CZ 379 01 TREBON, CZECH R

Telephone: (42) Facsimile: (\$2) Telex:

Email:

Commission(s): 20

VAZ LUIZ PAULO RIBEIRO, OBSERVATORIO ASTRONOMICO, DPT DE FISICA ICEX UFMG, CP 702 30161 BELO HORIZONTE MG, BRAZIL

Telephone: (55) 314412541

Facsimile: (55)

Email:

Commission(s): +2

Telex: 312308 ufmg br

VAZQUEZ MANUEL DR, INST DE ASTROFISICA, DE CANARIAS, OBS DEL TEIDE, E 38071 LA LAGUNA, SPAIN

Telephone: (34)

Facsimile: (34)

Telex: $926+0$ iace e

Email:

Commission(s): 51 
VAZQUEZ RUBEN ANGEL DR, OBSERVATORIO ASTRONOMICO, PASEO DEL BOSQUE S/N 1900 LA PLATA (BS AS), ARGENTINA

Tclephonc: (54) 21217308

Facsimile: (54) 21211761

Telex: 31216 cesla ar

Email: Ivazquez(âfcagl).edu.ar

Commission(s): 37

VAZQUEZ-SEMADENI ENRIQUE, INSTITUTO DE ASTRONOMIA, UNAM, APDO 70 264, MEXICO DF 04510, MEXICO Telephone: (52) 56223906 Facsimile: (52) 56160653 Telex:

Email: enro(a)astroscu.unam.mx

Commission(s):

VECK NICHOLAS, MARCONI RESEARCH CENTRE, WEST HANNINGFIELD RD, GT BADDOW CHELMSFORD ESSEX CM2 $8 \mathrm{HN}$, UK

Telephone: (44) 124573331

Email: ye08\%a.gec-nrc.co.uk aucl-es

Facsimile: (4t) $12+5752+4$

Telex: 995016 gecres g

VEEDER GLENN J DR, JPL, MS 183 \$01, 4800 OAK GROVE DR, PASADENA CA 91109, USA

Telephonc: (1) $21335+7388$

Facsimile: (1)

Telex:

Email:

Commission(s): 15

VEGA E IRENE DR, OBSERVATORIO ASTRONOMICO, PASEO DEL BOSQUE S/N 1900 LA PLATA (BS AS), ARGENTINA

$\begin{array}{ll}\text { Telephonc: (54) } 212173108 & \text { Facsimile: (54) } 21211761 \quad \text { Telex: } 31151 \text { bulap ar } \\ \text { Email: } & \text { Commission(s): } 33\end{array}$

VEILLET CHRISTIAN, OCA CERGA, AVE COPERNIC, F 06130 GRASSE, FRANCE

$\begin{array}{ll}\text { Telephone: (33) } 93365849 & \text { Facsimile: (33) } \\ \text { Email: } & \text { Commission(s): 07,19C,26,31C }\end{array}$

VEIS GEORGE PH D, GEODESY LABORATORY, NTL TECHNICAL UNIVERSITY, PANEPISTIMIOPOLIS GR 15773 ZOGRAFOS, GREECE

Telephone: (30) $17243414 \quad$ Facsimile: (30) Telex:

Email:

Commission(s):

VEISMANN UNO DR, TARTU ASTROPHYSICAL OBS, ESTONIAN ACAD OF SCIENCES, EE 202444 TARTU, ESTONIA Telephone: (372) Facsimile: (372) Telex:

Email:

Commission(s):

VEKSTEIN GREGORY DR, DPT OF PHYSICS, UMIST, BOX 88, MANCHESTER MGO 1QD, UK

Telephone: (4) 1612003913

Facsimile: (\$4) 1612003941 Telex:

Email: grigory.velstein(äumist.ac.uli

Commission(s): 11

\begin{tabular}{|c|c|c|}
\hline $\begin{array}{l}\text { Telephone: (359) } 2758927 \\
\text { Email: }\end{array}$ & $\begin{array}{l}\text { Facsimile: (359) } \\
\text { Commission(s): (09,10 }\end{array}$ & Telex: 23561 ecf bqn bg \\
\hline
\end{tabular}

VELLI MARCO DR, OBSERVATOIRE DE PARIS, SECTION DE MEUDON, DESPA F 92195 MEUDON PPL CDX, FRANCE

Telephonc: (33) $1+5077659$

Facsimile: (33) $145072806 \quad$ Telex:

Email: velli@frmeu51/17710::velli

Commission(s): 10

VELLI MARCO DR, INST DI ASTRONOMINA, LARGO FERMI 5 , I 50125 FIRENZE, ITALY

Telephone: (39)

Facsimile: (39)

Telex:

Email:

Commission(s): 10

VELUSAMY T DR, TIFR/RADIO ASTRONOMY CTR, BOX 8 , UDHAGAMANDALAM $6+3$ O01, INDIA

Telephone: (91) $\$ 232651 / 2032$

Facsimile: (91)

Commission(s): $410 \mathrm{C}$

Telex: $8+58488$ tifr in

Email:

SFC

VENKATAKRISHNAN P DR
Telephone: (1) 2055449404
Email:

Facsimile: (1)

Commission(s): 12C

L 35812, USA

Telex:

VENKATESAN DORASWAMY DR,
CALGARY AB T2N 1N4, CANADA
Telcphonc: (1) $4032205389 / 3689$

Email:
Facsimile: (1) $\$ 032893331$ Telex:

Commission(s): 10 
VENNIK JAAN, TARTU ASTROPHYSICAL OBS, EE 2444 TORAVERE, ESTONIA

Telephone: (372) $7+10274 \quad$ Ficsimile: (372) $7+10205$ Telex:

Email: vennikiäati.ce Commission(s):

VENTURA JOSEPH DR, DPT OF PHYSICS, UNIVERSITY OF CRETE, BOX 1527, GR 71111 IRAKLION, GREECE

Telephone: (30) $81239757 \quad$ Facsimile: (30)

Telex: 262728

Email: venturaä)grearn

Commission(s):

VENTURA RITA, OSS ASTROFISICO, CITTA UNIVERSITARIA, VIA A DORIA 6, I 95125 CATANIA, ITALY

Tclephone: (39) 957332258

Facsimile: (39) $95330592 \quad$ Telex:

Email: rventura(äastret.ct.astro.it Commission(s): 10,27

VENUGOPAL V R DR, TIFR/RADIO ASTRONOMY CTR, BOX 8, UDHAGAMANDALAM 643 001, INDIA

Telephone: (91) 423 2651/2032 Facsimile: (91) Telex: 0853-241 rac in

Email:

Commission(s): $33,+10,51$

VERBEEK PAUL DR, GEORGE MINNELAAN 50, B 9830 S MARTENS-LATEM, BELGIUM
Telephone: (32) 9826119

Email:

Commission(s):

VERBUNT FRANCISCUS DR, STERREKUNDIG INSTITUTE, BOX 80000, NL 3508 TA UTRECHT, NETHERLANDS

Telephone: (31) $893299833 \quad$ Fikesimile: (31)

Telex: $05218+5$ xter d

Email: bitnet:fivoädgaippls

Commission(s):

VERDET JEAN-PIERRE DR, OBSERVATOIRE DE PARIS, 61 AVE OBSERVATOIRE, F 75014 PARIS, FRANCE

Telephone: (33) 1 40 $512206 \quad$ Facsimile: (33) Telex:

Entail: $\quad$ Commission(s): 41

VERES FERENC, KONKOLY OBSERVATORY, THEGE U 13/17, BOX 67, H 1525 BUDAPEST, HUNGARY

Telephone: (36) $11755866 \quad$ Facsimile: (36) $11569640 \quad$ Telex: 227460

Email: $\quad$ Commission(s):

VERESHCHAGIN SERGEI V, INSTITUTE OF ASTRONOMY, ACADEMY OF SCIENCES, 48 PYATNITSKAYA ST 1001) 17 MOSCOW, RUSSIA

Telephone: (7) $0955921207 \quad$ Facsimile: (7) $0952302081 \quad$ Telex:

Email:

Commission(s):

VERGEZ MADELEINE DR, OBS MIDI PYRENEES, 9 R PONT DE LA MOUETTE

F 65200 BAGNERES BIGORRE, FRANCE

Telephone: (33) 6295 1969) Facsimile: (33)

Email: $\quad$ Commission(s): 10

Telex: $\$ 31625$ obspic $f$

VERGNANO A PROF, OSS ASTRONOMICO DI TORINO, ST OSSERVATORIO 20, 110025 PINO TORINESE, ITALY Telephone: (39) 11811061

Facsimile: (39) $118+1281$

Telex:

Email:

Commission(s):

VERGNE MARIA MARCELA DR, OBSERVATORIO ASTRONOMICO, PASEO DEL BOSQUE S/N 1900 LA PLATA (BS AS), ARGENTINA

Telephone: (54) $21217308 \quad$ Facsimile: (54) 21258985 Telex:

Email: mvergne(âfcaglp.edu.ar $\quad$ Commission(s): 33

VERHEEST FRANK PROF, STERRENKUNDIG OBSERV, UNIVERSITEIT GENT, KRIJGSLAAN 281

B 9000 GENT, BELGIUM

Telephone: (32) $926+4799$ Facsimile: (32) 9264 +989 Telex:

Email: frank.verheest/ä,rug.ac.be Commission(s): 10,27,49VP

VERMA R P DR, TIFR, HOMI BHABHA RD, COLABA, BOMBAY 400 005, INDIA

Telephone: (91) 22 219 $111 \quad$ Facsimile: (91) Telex: 0113009 tifr in

Email:

Commission(s): 25

VERMA SATYA DEV DR, DPT PHYSICS \& SPACE SCI, UNIVERSITY SCHOOL OF SCI, GUJARAT UNIVERSITY AHMEDABAD 380 (909, INDIA

Telephone: (91) $272+40920$

Facsimile: (91)

Email:

Commission(s):

Telex: 
VERMA V K DR, UTTAR PRADESII STATE, OBSERVATORY, PO MANORA PEAK 263 129, NAINITAL 263 129, INDIA Telephone: (91) $59+22136$ Facsimile: (91)

Telex: 59+2 2+01

Email:

Conmmission(s): I0

VERMEULEN RENE CORNELIS, CALTECH, MS 10524 , RADIO ASTRONOMY DPT, PASADENA CA 91125, USA

Telephone: (1) 8383958495

Fatsimile: (1) 8185689352

Telex:

Email: revianastro.caltech.cdu

Commission(s): $28,+(0$

VERNIANI FRANCO PROF, DPT DI FISICA, CNR, VIA IRNERIO \$6, I \$1126 BOLOGNA, ITALY

Telephone: (39) 5126 (1991 Fasimile: (39)

Telex: 211664

Email:

Commission(s): 22

VERON MARIE-PAULE DR, OHP, F O487( S MICHEL OBS, FRANCE

Telephone: (33) $92766368 \quad$ Facsimile: (33)

Email:

Commission(s): 28

Telex: $\$ 10690$ ohp f

VERON PHILIPPE DR, OHP, F (4870 S MICHEL OBS, FRANCE

Telephone: (33) 92766368 Facsimile: (33)

Email:

Commission(s): 28,40

Telex: $\$ 10690$ ohp f

VERSCHUEREN WERNER, ASTROPHYSICS RESEARCH GRP, UNIVERSITY OF ANTWERP GROENENBORGERLAAN 171, B 2020 ANTWERPEN, BELGIUM

Telephonc: (32) 32180356

Email: verschue(a) ruca.ual.ac.be Commission(s): 31,37

Telex:

VERSCHUUR GERRIT L PROF, DPT OF PHYSICS, RHODES COLLEGE, 200I N PARKWAY MEMPHIS TN 381121690 , USA

Telephone: (1) 9017263585

Facsimile: (1)

Telex:

Email: verschuur(a) rhodes.bitnct

Commission(s): $33,34,4(1,51$

VERTER FRANCES DR, NASA GSFC, CODE 685, GREENBELT MD 20771, USA

Telephone: (1) 3012867860

Facsimile: (1)

Telex:

Email:

Commission(s): 40

VESECKY J F DR, RADAR ASTRONOMY INST, STANFORD UNIVERSITY, 233 DURAND STANFORD CA $9+305+1135$, USA

Telephone: (1)

Email:

Facsimile: (1)

Telex:

Commission(s):

VETESNIK MIROSLAV DR, DPT OF ASTROPHYSICS, PURKYNE UNIVERSITY, KOTLARSKA 2 CZ 61137 BRNO, CZECH R

Telephone: (42) 57129481

Email: vetesnik(a)csbrmu11

Facsimile: $(+2) 57+0108 \quad$ Telex:

Commission(s): $33,+2,511$

VETTOLANI GIAMPAOLO, IST DI RADIOASTRONOMIA, CNR, VIA IRNERIO \$6, I 41126 BOLOGNA, ITALY

Telephone: (39) 51232856

Facsimile: (39)

Commission(s): $\$ 7$

Telex: 211664 infn bo i

Email:

VEVERKA JOSEPH DR, CORNELL UNIVERSITY, 312 SPACE SCI BLDG, ITHACA NY 14853 , USA

Telephone: (1) $6072563507 \quad$ Facsimile: (1)

Email:

Commission(s): 15,16

VIAL JEAN-CLAUDE, IAS, BAT 121, UNIVERSITE PARIS XI, F $91+15$ ORSAY CEDEX, FRANCE

Telephone: (33) $169858631 \quad$ Facsimile: (33) $169858675 \quad$ Telex: 6001252

Email; vialiátiaslab.ias.fr Commission(s): $10,12,+t$

VIALA YVES, OBSERVATOIRE DE PARIS, SECTION DE MEUDON, DENIRM, F 92195 MEUDON PPL CDX, FRANCE

Telephone: (33) $145077912 \quad$ Facsimile: (33)

Email:

Commission(s): 34

Telex: 270912

VIALLEFOND FRANCOIS, OBSERVATOIRE DE PARIS, SECTION DE MEUDON, DENIRM

F 92195 MEUDON PPL CDX, FRANCE

Telephone: (33) 145077905

Facsimile: (33) 145077893

Telex: 270912

Email: fviallef(a) frmeus1

Commission(s): 34 
VICENTE RAIMUNDO O PROF, FACULDADE CIENCIAS LISBOA, RUA MESTRE AVIZ 30 R/C

P 1495 LISBOA, PORTUGAL

Telephone: (351) $2112666 \quad$ Facsimile: (351) Telex:

Emait: $\quad$ Commission(s): 19,31

VIDAL JEAN-LOUIS DR, UNIVERSITE MONTPELLIER II, USTL II, PLACE EUGENE BATAILLON

F 34095 MONTPELLIER CDX 5, FRANCE

$\begin{array}{lll}\text { Telephonc: }(33) 67143901 & \text { Facsimile: (33) } & \text { Telex: } 490944 \text { ustmont } \\ \text { Email: } & \text { Commission(s): } 34 & \end{array}$

VIDAL NISSIM V DR, INST FOR SCIENCES \& TECHN, 92 BAYIT VEGAN ST, JERUSALEM 96427, ISRAEL

Telephone: (972) $2+13+11 \quad$ Ficsimile: (972) $2+30(1+1)$ Telex:

Email:

Commission(s): 44

VIDAL-MADJAR ALFRED DR, INSTITUT D'ASTROPHYSIQUE, 98BIS BD ARAGO, F 7501 4 PARIS, FRANCE

Telephone: (33) 1 \$\$328073

Facsimile: (33) $1+432$ 8001 Telex:

Email:

Commission(s): 34,44

VIEIRA MARTINS ROBERTO DR, OBSERVATORIO NACIONAL, RUA GL BRUCE 586, SAO CRISTOVAO 20921 RIO DE JANEIRO RJ, BRAZIL

Telephone: (55) 215807313

Facsimile: (55) 215800332

Telex: $021-21288$

Email:

Commission(s): $(17,20$

VIENNE ALAIN, LABORATOIRE D'ASTRONOMIE, UNIVERSITE DE LILLE, 1 IMPASSE DE L'OBSERVAT F 59000 LILLE, FRANCE

Telephone: (33) $2052+42+$

Email: vienne $a g a t . u n i-l i l l e 1 . f r$

Facsimile: (33)

Commission(s): 07,20

Telex:

VIETRI MARIO DR, OSS ASTRONOMICO, $1000+0$ MONTE CATONE, ITALY

$\begin{array}{ll}\text { Telephone: (39) } 69449019 & \text { Facsimile: (39) } \\ \text { Email: victri(ä)astrmp.astro.it } & \text { Commission(s): }\end{array}$

VIGIER JEAN-PIERRE DR, INSTITUT H POINCARE, 11 RUE P \& M CURIE, F 75005 PARIS, FRANCE

Telephone: (33) Facsimile: (33)

Email: $\quad$ Commission(s):

Telex:

VIGOTTI MARIO, IST DI RADIOASTRONOMIA, CNR, VIA IRNERIO \$6, I \$1126 BOLOGNA, ITALY

$\begin{array}{ll}\text { Telephone: (39) } 51232856 & \text { Facsimile: (39) } \\ \text { Ematil: } & \text { Commission(s): }\end{array}$

VIIK TONU DR, TORAVERE OBSERVATORY, EE 2112444 TARTU, ESTONIA

Telephone: (372) 3411265 Facsimile: (372) 34111205 Telex:

Email: viik(a)jupieter.aai.ec

Commission(s): 36

VILÁ SAMUEL C PROF, DR ULLES 184, E 03224 TARRASA (BRNA), SPAIN

$\begin{array}{ll}\text { Telephone: (34) } 37880310 & \text { Facsimile: (34) } \\ \text { Email: } & \text { Commission(s): } 35\end{array}$

VILAS FAITH DR, NASA/JOHNSON SPACE CENTER, CODE SN3, HOUSTON TX 77058, USA

Telephone: (1) $713+835056$

Facsimile: (1) $\quad$ Telex:

Email: sn::vilas

Commission(s): 15,40

VILAS-BOAS JOSE W DR, CRAAE/PTR ESCOLA POLI USP, CP $817+$ CEP 05508, 01051 SAO PAULO SP, BRAZIL

Telephone: (55) 118155936

Facsimile: (55) 118156289

Telex: 1180127 inpe br

Email: jwdsvboa(äbrusp.bitnet

Commission(s): 40

VILCHEZ MEDINA JOSE M DR, INST DE ASTROFISICA, DE CANARIAS, OBS DEL TEIDE, E 38200 LA LAGUNA, SPAI

Telephone: (34) 22262211

Facsimile: (34)

Telex: $926+0$

Email:

Commission(s):

VILHENA DE MORAES R DR, INPE, CP 515,12200 S JOSE DOS CAMPOS, BRAZIL

Telephone: (55) 123229088

Facsimile: (55)

Commission(s): 07

Telex: 01173+37 awo-24-73

Email: 
VILHU OSMI DR, OBS \& ASTROPHYSICS LAB, UNIVERSITY OF HELSINKI, TAEHTITORNINMAKI SF 00130 HELSINKI 13, FINLAND

\begin{tabular}{|c|c|}
\hline Telephone: (358) 1912941 & Facsimile: (358) 1912952 \\
\hline
\end{tabular}

VILKKI ERKKI U, + PARC DE LA LONDE, F 76130 MONT SAINT AIGNAN, FRANCE

Telephone: (33) Facsimile: (33) Telex:

Email:

Commission(s): 24

VILKOVISKIJ EMMANUIL Y DR, ASTROPHYSICAL INSTITUTE, KAZAKH ACAD OF SCIENCES 480068 ALMA ATA, KAZAKHSTAN

Telephone: (7)

Facsimile: (7)

Telex:

Email:

Commission(s): 35

VILLADA MONICA MARIA, OBSERVATORIO ASTRONOMICO, LAPRIDA 854, 5000 CORDOBA, ARGENTINA Telephone: (54) 51230491 Facsimile: (54) 51210613 Telex:

Email: villada(a)uncbob.colu.ar

Commission(s):

VILLELA THYRSO NETO DR, INPE, CP \$15, 12201 S JOSE DOS CAMPOS, BRAZIL

Telephone: (55) $123418977^{\star 278} \quad$ Facsimile: (55) Telex:

Email:

Commission(s):

VILMER NICOLE DR, OBSERVATOIRE DE PARIS, SECTION DE MEUDON, DASOP F 92195 MEUDON PPL CDX, FRANCE

Telephonc: (33) 145077806

Facsimile: (33)

Telex: 2010590

Email: span:meudon::vilmer

Commission(s): 111,12

VINCE ISTVAN, ASTRONOMICAL OBSERVATORY, VOLGINA 7

YU 11050 BEOGRAD, YUGOSLAVIA-SERBIA \& MONTENEGRO

$\begin{array}{lll}\text { Telephonc: (38) } 11419357 / 421875 & \text { Facsimile: (38) } & \text { Telex: } 72610 \text { aob yu } \\ \text { Email: caop001(a)yubgss21.bitnet } & \text { Commission(s): } & \end{array}$

VINKO JOZSEF, DPT OF OPTICS, JATE UNIVERSITY, H 6720 SZEGED, HUNGARY

Telephonc: (36) Falesimile: (36) Telex:

Email:

Commission(s):

VINLUAN RENATO, UNIVERSITY OF SOUTHERN, PHILIPPINES, OBRERO DAVAO CITY 9501, PHILIPPINES

Telephonc: (63)

Facsimile: (63)

Telex:

Email:

Commission(s): 11

VINOD S KRISHAN DR, INDIAN INSTITUTE OF, ASTROPHYSICS, KORAMANGALA, BANGALORE 560 (034, INDIA

Telephonc: (91) $803566585 / 6+97$

Facsimile: (91)

Telex: $8+5763$ iial) in

Email:

Commission(s): 10.49C

VIOTTI ROBERTO DR, IAS, CNR, CP 67, I $0004+$ FRASCATI, ITALY

Telephone: (39) $69+25655$

Email: viottioârirmiass,bitnet

Facsimile: (39) 6941 6847

Telex: 611261 cnitia

VIRGOPIA NICOLA PROF, DPT DI MATEMATICA, UNIV DI ROMA LA SAPIENZA, CITTA UNIVERSITARIA I 00185 ROMA, ITALY

Telephonc: (39) Facsimile: (39) Telex:

Email:

Commission(s):

VISHNIAC ETHAN T, ASTRONOMY DPT, UNIVERSITY OF TEXAS, RLM 15308 , AUSTIN TX 78712 1083, USA Telephone: (1) $5124711+29$

Facsimile: (1)

Telex:

Email:

Commission(s): 47

VISHVESHWARA C V PROF, RAMAN RESEARCH INSTITUTE, SADASHIVANAGAR, CV RAMAN AVE

BANGALORE 560080 , INDIA

Telephonc: (91) 803360122

Facsimile: (91) $8033+0492$

Telex: $8+52671$ rri in

Email:

Commission(s): 47

VISVANATHAN NATARAJAN DR, MOUNT STROMLO \& SIDING, SPRING OBSERVATORIES, PRIVATE BAG WODEN PO ACT 2606, AUSTRALIA

Telcphone: (61) 62881111

Facsimile: (61) $62+90233$

Telex: 62270 tlg canopus aa

Email:

Commission(s): $25,28,34$ 
VITINSKIJ YURIJ I DR, PULKOVO OBSERVATORY, ACADEMY OF SCIENCES, 10 KUTUZOV QUAY 196140 ST PETERSBURG, RUSSIA

$\begin{array}{ll}\text { Telephone: (7) } 2982242 & \text { Facsimile: (7) } \\ \text { Email: } & \text { Commission(s): 10,12 Telex: }\end{array}$

VITON MAURICE DR, LAS, TRAVERSE DU SIPHON, LES TROIS LUCS, F 13012 MARSEILLE, FRANCE

Telephone: (33) 9105 5900) Facsimile: (33) 91661855 Telex:

Email: viton(afrilasm51 Commission(s):

VITTONE ALBERTO ANGELO, OSS ASTRONOMICO, DI CAPODIMONTE, VIA MOIARIELLO 16 I 80131 NAPOLI, ITALY

Telephone: (39) $8140101 \quad$ Facsimile: (39) Telex:

Email: $\quad$ Commission(s):

VITTORIO NICOLA, ISTITUTO ASTRONOMICO, UNIVERSITA DI ROMA, VIA G M LANCISI 29, I 00161 ROMA, ITAL

Telephone: (39)

Facsimile: (39)

Telex:

Email:

Commission(s):

VITYAZEV ANDREI, GRUZINSKAYA 10, MOSCOW 123810, RUSSIA

Telephone: (7) $0952549006 \quad$ Facsimile: (7) 0952549088

Email: avit(a)orig.ipg.msk.su Commission(s):

Telex: $\mathbf{4 1 1 9 6}$ ifzan su

VITYAZEV VENEAMIN V DR, ASTRONOMICAL OBSERVATORY, ST PETERSBURG UNIVERSITY BIBLIOTECHNAJA PL 2, 198904 ST PETERSBURG, RUSSIA

$\begin{array}{ll}\text { Telephone: (7) } & \text { Facsimile: (7) } \\ \text { Email: } & \text { Commission(s): }\end{array}$

VIVEKANAND M DR, RAMAN RESEARCH INSTITUTE, SADASHIVANAGAR, CV RAMAN AVE

BANGALORE 560080 , INDIA

Telephene: (91) $803360122 \quad$ Facsimile: (91) $803340492 \quad$ Telex: 8452671 rit in

Email:

Coumunission(s): 40

VIVEKANANDA RAO, CASA, UNIVERSITY OF OSMANIA, HYDERABAD 500 007, INDIA

Telephone: (91) 851672

Facsimile: (91)

Telex:

Email:

Commission(s):

VIVES TEODORO JOSE DR, CTR ASTRON HISPANO ALEMAN, REINA 66 9B, CORREOS 511

E 04002 ALMERIA, SPAIN

Telephone: (34) 23 (0988 Facsimile: (34)

Email: $\quad$ Commission(s):

Telex: 78812 dsaze

VLACHOS DEMETRIUS G PROF, DPT GEODESY \& SURVEYING, UNIVERSITY THESSALONIKI

FACULTY OF ENGINEERING, GR 54066 THESSALONIKI, GREECE

Telephone: (30) $31991520 \quad$ Facsimile: (30)

Email: $\quad$ Commission(s):

Telex: $\$ 12181$ auth $\mathrm{gr}$

VLADILO GIOVANNI DR, OAT, BOX SUCC TRIESTE 5, VIA TIEPOLO 11, 134131 TRIESTE, ITALY

Telephone: (39) $40309342 \quad$ Facsimile: (39) Telex: 461137 oat $\mathrm{i}$

Email:

Commission(s): 29

VLADIMIROV SIMEON, ASTRONOMICAL OBSERVATORY, BULGARIAN ACAD SCIENCES, BOX 15 BG 1309 SOFIA, BULGARIA

Telephone: (359) $2758927 \quad$ Facsimile: (359) Telex: 23561 ecf ban bg

Email:

Commission(s): $09,+6$

VLAHOS LOUKAS DR, DPT OF ASTROPHYSICS, UNIVERSITY THESSALONIKI, GR 54006 THESSALONIKI, GREECE

Telephone: (30) $31991357 \quad$ Facsimile: (30)

Email: $\quad$ Commission(s):

Telex: 0412181 avth gr

VOELK HEINRICH J PROF, MPI FUER KERNPHYSIK, POSTFACH 103 980, D 69029 HEIDELBERG, GERMANY

Telephone: (49) 6221516295

Facsimile: (49)

Telex: 461666

Email:

Comnission(s): $14,4+$ 
VOGEL MANFRED DR, INSTITUT FUER ASTRONOMIE, ETH ZENTRUM, CH 8092 ZUERICH, SWITZERLAND

Telephone: (41) 12563806

Facsimile: (+1) 12620003

Telex: 817379 chhg ch

Email: vogel(a)chethsa

Commission(s): 34

VOGEL STUART NEWCOMBE DR, ASTRONOMY PROGRAM, UNIVERSITY OF MARYLAND, COLLEGE PARK MARYLAND 20742, USA

Telephonc: (1)

Facsimile: (1)

Telex:

Email:

Commission(s): 40

VOGLIS NIKOS DR, DPT OF ASTROPHYSICS, NTL UNIVERSITY OF ATHENS, PANEPISTIMIOPOLIS GR 15783 ZOGRAFOS, GREECE

Telephonc: (30) 17243414

Facsimilc: (30)

Telex: 223815 univ gr

Email: node:grathun 1, userid:spm 70

Commission(s): 28,47

VOGT NIKOLAUS DR, GRUPO ASTROFIS PONTIFICA, UNIVERSIDAD CATOLICA, CASILLA 104 SANTIAGO 22, CHILE

Telephone: $(56)$

Facsimilc: (56)

Telex:

Email: nvogt(âastrouc.puc.cl

Commission(s): 27,29,51

VOGT STEVEN SCOTT, LICK OBSERVATORY, UNIVERSITY OF CALIFORNIA, SANTA CRUZ CA 95064, USA

Telephone: (1) 4084292844

Facsimile: (1)

Telex: 910-598-4408

Email:

Commission(s): 29

VOIGT HANS.H PROF, CHARLOTTENBURGER STR 19, D 37070 GOETTINGEN, GERMANY

Telephone: (49)

Facsimile: (49)

Telex:

Email;

Commission(s):

VOKROUHLICKY DAVID, INSTITUTE OF ASTRONOMY, CHARLES UNIVERSITY, SVEDSKA 8

CZ 150 00 PRAHA 5, CZECH R

Telephonc: (42) 2540395

Facsimile: (42) $2299272 \quad$ Telex:

Email: davok@aci.cvut.cz

Commission(s):

VOLK KEVIN DR, DPT OF PHYSICS \& ASTRON, UNIVERSITY OF CALGARY, 2500 UNIVERSITY DR NW

CALGARY AB T2N 1N4, CANADA

Telephone: (1) 4039312366

Facsimile: (1) $41132893331 \quad$ Telex:

Email:

Commission(s): 34

VOLLAND H DR, ASTRONOMISCHES INSTITUT, UNIVERSITAET BONN, AUF DEM HUEGEL 71

D 53121 BONN, GERMANY

Telephone: (49) 228733674

Facsimile: (49)

Telex: $0886+40$

Email:

Commission(s):

VOLONTE SERGE DR, ESA, 8-10 RUE MARIO NIKIS, F 75738 PARIS CDX 15, FRANCE

Tclephonc: (33) 153697654

Facsimile: (33) 153697236 Telex:

Email:

Conmission(s): 12,14

VOLYANSKAYA MARGARITA YU, DPT OF ASTRONOMY, ODESSA STATE UNIVERSITY, T G SCHECHENKO PARK 270014 ODESSA, UKRAINE

Telephone: (7) 048228442

Email: $\operatorname{root}(\widehat{a})$ ast ${ }^{\prime} 0.0 d e s s a . u a$

Facsimile: (7) $048228+42 \quad$ Telex:

Commission(s): 08,41

VON BORZESZKOWSKI H H DR, EINSTEIN LABORATORIUM, AKAD WISSENSCHAFTEN DDR

ROSA LUXEMBURG STR 17A, D $1+473$ POTSDAM, GERMANY

Telephonc: (49) 762225

Facsimile: (49)

Telex:

Email:

Commission(s): 47

VON DER HEIDE JOHANN DR, ALARDUSSTR 12, D 2000 HAMBURG 20, GERMANY

Telephonc: (49) 404914016

Facsimile: (49)

Telex:

Email:

Commission(s): 08

VON HIPPEL THEODORE A, INSTITUTE OF ASTRONOMY, THE OBSERVATORIES, MADINGLEY RD CAMBRIDGE CB3 OHA, UK

Telephone: (44) 1223337516

Email: ted@mail.ast.cam.ac.uk

Facsimile: (44) 1223337523 Telex:

Commission(s): 
VON HOERNER SEBASTIAN DR, KRUMMENACKERSTR 186, D 7300 ESSLINGEN, GERMANY

Telephone: (49)

Facsimile: (49)

Telex:

Email:

Commission(s): 51

VON STEIGER RUDOLF, PHYSIKALISCHES INSTITUT, UNIVERSITAET BERN, SIDLERSTRASSE 5

CH 3012 BERN, SWITZERLAND

Telephone: (41) $31631+\$ 19$

Facsimile: (+1) $31631+405 \quad$ Telex:

Email: vsteigeraphim.unibe.ch

Commission(s): 49

VON WEIZSAECKER C F PROF, MAXIMILLIANSTR 15, D 8130 STARNBERG, GERMANY

Telephone: (\$9) Facsimile: (49)

Email: $\quad$ Commission(s):

Telex:

VONDRAK JAN DR, ASTRONOMICAL INSTITUTE, CZECH ACADEMY SCIENCES, BOCNI II 1401

CZ 14131 PRAHA 4, CZECH R

Telephone: (42) $267103043 \quad$ Facsimile: $(42) 2769023 \quad$ Telex:

Email: rondrak@aig.cas.ce

Commission(s): 19P

VOROSHILOV V I DR, MAIN ASTRONOMICAL OBS, UKRAINIAN ACAD OF SCIENCE, GOLOSEEVO

252127 KIEV, UKRAINE

Telephone: (7) 044663110

Facsimile: (7)

Telex: 131406 sky su

Email:

Commission(s): 33

VORPAHL JOAN A DR, 748 23RD ST, SANTA MONICA CA 904112, USA

Telephone: (1)

Facsimile: (1)

Commission(s):

Telex:

Email:

VOSHCHINNIKOV NICOLAI DR, ASTRONOMICAL OBSERVATORY, ST PETERSBURG UNIVERSITY BIBLIOTECHNAJA PL 2, 198\%)+ ST PETERSBURG, RUSSIA

Telephone: (7) $428+162 \quad$ Facsimile: (7) $121+81$ LSU SU Telex:

Email: $\quad$ Commission(s): 34

VRBA FREDERICK J DR, US NAVAL OBSERVATORY, FLAGSTAFF STATION, BOX 1149, FLAGSTAFF AZ 86002, USA

Telephone: (1) 6012 7795132

Facsimile: (1)

Telex:

Email: fjvanofs.navy,mi

Commission(s): $09,25 \mathrm{C}, 34$

VREUX JEAN MARIE DR, INSTITUT D'ASTROPHYSIQUE, UNIVERSITE DE LIEGE, AVE COINTE 5

B 4000 COINTE-LIEGE, BELGIUM

Telephone: (32) 41529980

Facsimile: (32) $\$ 1527+74$

Commission(s): 29

Telex: $\$ 1264$

Email:

VRSNAK BOJAN DR, HVAR OBSERVATORY, FACULTY OF GEODESY, KACICEVA 26, ZAGREB, CROATIA

Telephone: $(38)+1+426(10 \star 335$

Facsimile: $(38)+1+45+10$

Telex:

Email: bojan.vrsnakiäuni-fg.ac.mail.yu

Commission(s): 10

VRTILEK JAN M DR, NASA HEADQUARTERS, ASTROPHYS DIV CODE SZF, 300 E ST SW

WASHINGTON DC 20546, USA

Telephone: (1) 2023580351

Facsimile: (1) 2023583096

Telex:

Enatil: vrtilekrästars.gsfc.nasi. gov

Commission(s): 21,28

VRTILEK SAEQA DIL DR, CENTER FOR ASTROPHYSICS, HCO/SAO MS 4, 60 GARDEN ST

CAMBRIDGE MA 02138, USA

Telephone: (1) $6174957400 \quad$ Facsimile: (1) 617 \$95 $7356 \quad$ Telex: $921+28$ satellite cam

Email: svrtilekiâcfa

VU DUONG TUYEN DR, BUREAU DES LONGITUDES, 77 AVE DENFERT ROCHEREAU, F 75014 PARIS, FRANCE

Telephone: (33) 145072262

Commission(s): 4

Email:

Facsimile: (33)

Telex:

Commission(s): 20

VUCETICH HECTOR DR, UNIV NACIONAL DE LA PLATA, DPT FISICA, CCN 67, 1900 LA PLATA (BS AS), ARGENTIN

Telephone: (54) 21217308

Facsimile: (54) 21252006

Telex: 31151 bulap ar

Email: atina!fisilp!vucetich

Commission(s): 49

VUILLEMIN ANDRE DR, LAS, TRAVERSE DU SIPHON, LES TROIS LUCS, F 13012 MARSEILLE, FRANCE

Telephone: (33) 91055900

Facsimile: (33) $9166,1855 \quad$ Telex:

Email: andre(afriasm51

Commission(s): 
VUJNOVIC VLADIS DR, INSTITUTE OF PIIYSICS, UNIVERSITY OF ZAGREB, BOX 304, ZAGREB, CROATIA

Telephone: (38) 41271211

Facsimile: (38)

Email:

Commission(s): 14,46

Telex: 22203 ifs yu

VUKICEVIC K M PROF DR, DPT OF ASTRONOMY, FACULTY OF SCIENCES, STUDENTSKI TRG 16 YU $11(100)$ BEOGRAD, YUGOSLAVIA-SERBIA \& MONTENEGRO

Telephone: (38) $11638715 \quad$ Facsimile: (38) Telex:

Email:

Commission(s): 12

VYALSHIN GENNADIJ F DR, PULKOVO OBSERVATORY, ACADEMY OF SCIENCES, 10 KUTUZOV QUAY 196140 ST PETERSBURG, RUSSIA

Telephone: (7)

Email:

Facsimile: (7)

Telex:

Commission(s): 10

VYKUTILOVA MARIE, OBSERVATORY, VALASSKE MEZIRICI, VSETINSKA 7

CZ 75701 VALASSKE MEZIRIC, CZECH R

Telephonc: (\$2) $65121928 \quad$ Facsimile: $(\$ 2) \quad$ Telex:

Email:

Commission(s):

WACKERNAGEL H BEAT DR, 5I BROADMOOR HILLS DR, COLORADO SPRINGS CO 80OO, USA

Telephone: (1) 3035543801

Facsimile: (1)

Telex:

Email:

Commission(s): 04,31

WADDINGTON C JAKE PROF, DPT OF PHYSICS, UNIVERSITY OF MINNESOTA, 11G CHURCH ST SE MINNEAPOLIS MN 55+55, USA

Telephone: (1) $6126242566 \quad$ Facsimile: (1)

Telex: 910-576-2955

Email:

Commission(s):

WADE RICHARD ALAN DR, DPT OF ASTRONOMY, PENNSYLVANIA STATE UNIV, 525 DABEY LAB UNIVERSITY PARK PA 16802, USA

Telephone: (1)

Facsimile: (1)

Telex:

Email: wade(äastro.psu.edu

Commission(s): 42

WAELKENS CHRISTOFFEL, ASTRONOMISCH INSTITUUT, KATHOLIEKE UNIV LEUVEN, CELESTIJNENLAAN 200B B 3030 HEVERLEE, BELGIUM

$\begin{array}{lll}\text { Telephonc: (32) } 16200656 & \text { Facsimile: (32) } & \text { Telex: } 25715 \\ \text { Email: } & \text { Commission(s): } 27 & \end{array}$

WAGNER RAYMOND L DR, ROCKWELL INTERNATIONAL, BOX 3105, MS 031 BA06, ANAHEIM 928033105 , USA

Telephone: (1) $71+7621754$

Facsimilc: (1) $71+76220117$

Telex:

Ematil: ray_l._wagneräaccmail.anatcp).rockwell.con

Commission(s):

WAGNER ROBERT M DR, LOWELL OBSERVATORY, 14011 W MARS HILL RD, BOX 1149, FLAGSTAFF AZ 86001, USA Telephone: (1) $6027790106 \quad$ Facsimile: (1)

Email: $\quad$ Commission(s):

Telex:

WAGNER STEFAN, LANDESSTERNWARTE, KOENIGSTUHL, D 69117 HEIDELBERG, GERMANY

Telephone: (49) 6221509212

Facsimile: (\$9) 62215092612 Telex

Email: swagnerämail.Isw.uni-heidelberg.de

Commission(s): 28

WAGNER WILLIAM J DR, NASA HEADQUARTERS, CODE SS, SPACE PHYSICS DIV, WASHINGTON DC 20546, USA

Telephone: (1)

Ficsimile: (1)

Tclex:

Email:

Commission(s):

WAGONER ROBERT V PROF, VARIAN PHYSICS BLDG, STANFORD UNIVERSITY, STANFORD CA 94305, USA

Telephone: (1) $+15723+561 \quad$ Facsimile: (1)

Email:

Commission(s): 47

Telex: $348+112$

WAINWRIGHT JOHN DR, DPT OF APPLIED MATHS, UNIVERSITY OF WATERLOO

WATERLOO ON N2L 3G1, CANADA

Facsimile: (1) 5197466530

Email: jwainwright(a)math.uwatcrloo.ca Commission(s): 17

Telex: 06955259 
WAKAMATSU KEN-ICHI DR, FACULTY OF ENGINEERING, GIFU UNIVERSITY, GIFU 501 11, JAPAN

Telephonc: (81) 582301111

Facsimilc: (81)

Telex:

Enuail:

Commission(s): 28

WAKO KOJIRO DR, INTL LATITUDE OBSERVATORY, NAOJ, HOSHIGAOKA MIZUSAWA SHI, IWATE 023, JAPAN

Telephone: (81)

Facsimilc: (81)

Telex:

Email:

Commission(s):

WALBORN NOLAN R DR, STSCI, HOMEWOOD CAMPUS, 3716) SAN MARTIN DR, BALTIMORE MD 21218, USA

Telephone: (1) $301338+915$

Facsimile: (1)

Tclex: 6849101 stsci uw

Email: stsci::walborn

Commission(s): 45

WALCH JEAN-JACQUES, OCA CERGA, AVE COPERNIC, F 06130 GRASSE, FRANCE

Telephone: (33) 93365849

Facsimile: (33)

Telex: $\$ 70865$ cerga f

Email:

Commission(s): 177

WALDHAUSEN SILVIA, OBSERVATORIO ASTRONOMICO, PASEO DEL BOSQUE S/N

1900) LA PLATA (BS AS), ARGENTINA

Telephone: (54) 21217308

Email: silvia(a)feaglp.fcagl|p.unl|p.edu.ar

Facsimile: (54) 21258985

Telex: 31151 bulap)

WALDMEIER MAX PROF DR, SWISS FEDERAL OBSERVATORY, WIRZENWEID 15

CH 8053 ZUERICH, SWITZERLAND

Telephone: (41) $138162+2$

Facsimile: ( +1$)$

Telex:

Email:

Commission(s): 10,12

WALKER ALISTAIR ROBIN DR, CERRO TOLOLO, INTERAMERICAN OBSERVATORY, CASILLA 603

LA SERENA, CHILE

Telephone: (56) 51213352

Email:

Facsimile: (56) $51212466 * 342 \quad$ Telex: 621301 aura et

Commission(s): $09,15,25$

WALKER ALTA SHARON DR, US GEOLOGICAL SURVEY, 927 NATIONAL CENTER, RESTON VA 22092, USA

Telephone: (1) 7036486387

Facsimile: (1) 7036486684

Telex:

Email:

Commission(s): 16

WALKER ARTHUR B C JR PROF, CTR FOR SPACE SCIENCES \&, ASTROPHYSICS, STANFORD UNIV ERL 310 STANFORD CA 9\$305 4055, USA

Telephone: (1) +15 +97 1486

Facsimile: (1)

Commission(s):

Telex:

Email:

HYSICS \& ASTRONOMY, UNIVERSITY COLLEGE LONDON, GOWER ST

WALKER DAVID DOUG

Telephone: (44) 171387705()$^{*} 3510$

Facsimile: (44) 1713807145

Telex: 28722

Email: ddw(âak,ac.ulc.starlink

Commission(s): (1)

WALKER EDWARD N MR, DEUDREYS COTTAGE, OLD RD, HERSTMONCEUR, EAST SUSSEX BN27 1PU, UK

Telephone: (44)

Facsimile: (4)

Telex:

Email:

Commission(s): 27

WALKER GORDON A H PROF, DPT GEOPHYS \& ASTRONOMY, UNIV OF BRITISH COLUMBIA

2075 WESBROOK PL, VANCOUVER BC VGT IW5, CANADA

Telephone: (1) 604228 4133

Facsimile: (1) 6042286047

Telex:

Email:

Commission(s): $09,30,34,37,45$

WALKER HELEN J, RUTHERFORD APPLETON LAB, SPACE \& ASTROPHYSICS DIV, BLDG R25/R68, CHILTON DIDCOT OX11 0QX, UK

Telephone: (44) 123582190

Facsimile: (44) $1235+45848$

Telex: 83159 ruthlb g

Email:

Commission(s):

WALKER IAN WALTER, DPT OF ASTRONOMY, UNIVERSITY OF GLASGOW, GLASGOW G12 8QQ, UK

Telephone: (44)

Facsimile: $(+t)$

Telex:

Email:

Commission(s): 07

WALKER MERLE F PROF, LICK OBSERVATORY, UNIVERSITY OF CALIFORNIA, SANTA CRUZ CA 95064, USA

Telephone: (1) $408 \$ 292526$

Email:
Facsimile: (1)

Commission(s): 09,27,37,50
Telex: 
WALKER RICHARD L, US NAVAL OBSERVATORY, FLAGSTAFF STATION, BOX 1149, FLAGSTAFF AZ 86002, USA

Telephone: (1) 60127746623

Facsimile: (1)

Telicx:

Email:

Commission(s): 26,42

WALKER ROBERT C DR, NRAO, BOX 1, SOCORRO NM 87801 0387, USA

Telephonc: (1) $50583572+7$

Facsimile: (1)

Telex: 9109881710

Email: bitnet:cwallker(änrato

Commission(s): 40

WALKER ROBERT M A PROF, DPT OF PHYSICS, WASHINGTON UNIVERSITY, BOX 1105, ST LOUIS MO 63130, USA Telephone: (1) $31+8896225$ Facsimile: (1) Telex:

Email:

Commission(s): 16

WALKER WILLIAM S G, 14 APPLEYARD CRES, AUCKLAND 5, NEW ZEALAND

Telejhone: (64) 09-548-736 Facsimile: (64) Telex:

Email:

Commission(s): 27,42

WALL J W, ROYAL GREENWICH OBS, MADINGLEY RD, CAMBRIDGE CB3 OEZ, UK

Telephone: (4t)

Facsimile: (4)

Telex:

Email:

Commission(s):

WALL JASPER V DR, ROYAL GREENWICH OBS, MADINGLEY RD, CAMBRIDGE CB3 OEZ, UK

Telephonc: (4t) 1223374000

Facsimile: (4t) $122337+700 \quad$ Telex: $265+51 / 265871$

Email: 19463::cavad::jon

Commission(s): 40

WALLACE LLOYD V DR, KITT PEAK NTL OBS, BOX 26732, 950 N CHERRY AVE, TUCSON AZ 85726 6732, USA

Telephonc: (1) 520 3275511

Facsimile: (1)

Telex:

Email:

Commission(s): 16

WALLACE PATRICK T MR, RUTHERFORD APPLETON LAB, SPACE \& ASTROPHYSICS DIV, BLDG R25/R68 CHILTON DIDCOT OX110QX, UK

Telcphonc: (44) $1235+45+72$

Facsimile: (4)

Telex: 83159

Email:

Commission(s): $05,(18,09$

WALLACE RICHARD K, LOS ALAMOS NATIONAL LAB, MS B257, X 7, LOS ALAMOS NM 87545, USA

Telephone: (1) 5056675000

Facsimile: (1)

Telex:

Email:

Commission(s):

WALLENQUIST AAKE A E PROF, ASTRONOMICAL OBSERVATORY, NORRLANDSGATAN 34 D S 75220 UPPSALA, SWEDEN

Telephone: (46) 18135685

Facsimile: (46)

Telex:

Email:

Commission(s): 25,37

WALLER WILLIAM H, NASA GSFC, LAB ASTRONOMY SOLAR PHYS, CODE 681, GREENBELT MD 20771, USA

Telephone: (1) 3012865351

Facsimile: (1) $301286,1753 \quad$ Telex:

Email: waller(âstars.gsfc.nasa.gov

Commission(s):

WALLERSTEIN GEORGE PROF, DPT OF ASTRONOMY, UNIVERSITY OF WASHINGTON, FM 20 SEATTLE WA 98195, USA

Telephone: (1) 2065432888

Email:

Facsimile: (1)

Telex:

Commission(s): 27,29

WALLINDER FREDERICK, LUND OBSERVATORY, BOX 43, S 22100 LUND, SWEDEN

Telephone: $(46)+610+702$

Email: fredrikiâastro.lu.se

Facsimile: $(46)+6104614 \quad$ Telex

Commission(s):

WALLIS MAX K DR, DPT APPLIED MATHS/ASTRON, UNIVERSITY COLLEGE, BOX 78, CARDIFF CF1 1XL, UK

Telephone: (4t) 12224.4211

Facsimile: $(44)$

Telex: 488635

Email:

Commission(s): $15,21,51$

WALMSLEY C MALCOLM DR, MPI FUER RADIOASTRONOMIE, AUF DEM HUEGEL 69, D 53121 BONN, GERMANY

Telephone: (49) 228525305

Facsimile: (49)

Commission(s): $34,+0$

Telex: $886+40$ 
WALRAVEN TH DR, BOX 98, ORANGE FREESTATE 9850, SOUTH AFRICA

Telephone: (27)

Facsimile: (27)

Commission(s): 25,27

Telex:

Email:

WALSH DENNIS DR, NRAL, JODRELL BANK, MACCLESFIELD SK11 9DL, UK

Telephonc: $(+4) 1 \$ 7771321$

Facsimile: (4)

Telex: 36149

Email:

Commission(s): $41,+4$

WALTER FREDERICK M, DPT OF EARTH \& SPACE SCI, ASTRONOMY PROGRAM, SUNY AT STONY BROOK STONY BROOK NY $1179+2100$, USA

Telephone: (1) 5166328221

Facsimile: (1)

Commission(s): 36

Telex:

Email:

$\begin{array}{lll}\text { Felephone: }(49) 622149026 & \text { Facsimile: }(49) & \text { Telex: } 461336 \text { arihd d } \\ \text { Email: } & \text { Commission(s): } 08,2+\mathrm{C} & \end{array}$

Email:

Commission(s): $08,2+\mathrm{C}$

WALTER ROLAND, OBSERVATOIRE DE GENEVE, CHEMIN DES MAILLETTES 51

CH 1290 SAUVERNY, SWITZERLAND

Telephone: (41) 227552611

Fatcsimile: (\$1) 227553983

Telex: $45+19209$ obsg ch

Email: 'walterascsun.unige.ch

Commission(s):

WALTERBOS RENE A M DR, DPT OF ASTRONOMY, NEW MEXICO STATE UNIV, BOX 4500

LAS CRUCES NM 88003, USA

Telephone: (1) 5056466.522

Facsimile: (1)

Commission(s): 28

Telex:

Email:

WALTON NICHOLAS A DR, ROYAL GREENWICH OBS, APD 321, E 38780 SANTA CRUZ, SPAIN
Telephonc: (34) 22405500
Facsimile: (34) 22405501

Email: naw(älpve.ing.iac.es

Commission(s): 34

WAMBSGANSS JOACHIM, ASTROPHYSIKALISCHES INST, POTSDAM UNIVERSITY, AN DER STERNWARTE 16 D 14482 POTSDAM, GERMANY

Telephone: (49) 3317499316

Email: jwambsganss äaip.de

Facsimile: (49) $3317499267 \quad$ Telex:

Commission(s): 47

WAMPLER E JOSEPH PROF, ESO, KARL SCHWARZSCHILDSTR 2, D 85740 GARCHING MUENCHEN, GERMANY

Telephonc: (49) 8932006297

Facsimile: (49) 893202362

Telex: 52828222 eo d

Email:

Commission(s): 199

WAMSTEKER WILLEM DR, ESA IUE GROUND STATION, VILSPA, APD 50727, E 28080 MADRID, SPAIN

Tclephone: (3ł) 18131100

Facsimile: (34) 11831139

Telex: 42555

Email: ww(a)vilspa.csi.es

Commission(s): +tVP

WAN FOOK SUN, DPT OF MATHEMATICS, NTL UNIVERSITY SINGAPORE, KENT RIDGE

SINGAPORE 0511, SINGAPORE

Telephone: (65) $772-27+2$

Facsimilc: (65)

Email:

Commission(s):

Telex:

WAN LAI, SHANGHAI OBSERVATORY, CAS, 80 NANDAN RD, SHANGHAI, CHINA PR

Tclephone: (86) 21380696

Facsimilc: (86)

Commission(s): 24,37

Telex: $3316+$ shato $\mathrm{cn}$

Email:

WAN TONG-SHAN, SHANGHAI OBSERVATORY, CAS, 80 NANDAN RD, SHANGHAI 200030, CHINA PR

Telephonc: (86) 21386191

Facsimile: (86)

Telex: $3316+$ shato en

Email:

Commission(s): 40

WANAS M I PROF, DPT OF ASTRONOMY, FACULTY OF SCIENCES, CAIRO UNIVERSITY, GEZA ORMAN, EGYPT

Telephone: (20)

Facsimile: (20)

Telex:

Email:

Commission(s): 44

WANG CHUAN-JIN, PURPLE MOUNTAIN OBSERV, CAS, NANJING, CHINA PR

Telephone: (86) $25+6700$

Facsimile: (86) 25301459

Telex: $3 \$ 1+4$ pmonj en

Email:

Conmission(s): 09,25 
WANG DEYU, PURPLE MOUNTAIN OBSERV, CAS, NANJING, CHINA PR

Telephone: (86) $25+2817 / 46704$

Facsimile: (86) 25301459

Telex: $3 \$ 1 \$+$ pmonj en

Email:

Commission(s): 44

WANG DE-CHANG, PURPLE MOUNTAIN OBSERV, CAS, NANJING, CHINA PR

Telephone: (86) $256+6700 / 4205 \quad$ Faesimile: (86) 25301459

Telex: $3+14+$ pmonj en

Email:

Commission(s): 22,41

WANG GANG, BEIJING ASTRONOMICAL OBS, CAS, BEIJING 160080, CHINA PR

Telephone: (86) 12569840

Facsimile: (86) 12561085 Telex:

Email:

Commission(s):

WANG HAIMIN DR, CALTECH, MS $26+33$, PASADENA CA 91125 , USA

Telephonc: (1) 8183563858

Facsimile: (1)

Telex:

Email: haiminasunsog.caltech.edu

Commission(s): 10

WANG JIA-JI, SHANGHAI OBSERVATORY, CAS, 80 NANDAN RD, SHANGHAI 200030, CHINA PR

Telephone: (86) 21386191

Facsimile: $(86)$

Commission(s): $24 \mathrm{C}$

Telex: 33164 shao en

Email:

WANG JIA-LONG, BEIJING ASTRONOMICAL OBS, CAS, W SUBURB, BEIJING 100080, CHINA PR

Telephone: (86) 1281698

Facsimile: (86)

Telex: 22040 baobs en

Email:

Commission(s): 11)

WANG JING-SHENG, PURPLE MOUNTAIN OBS, IBID, NANJING 210018, CHINA PR

Telephonc: (86) 25313738

Facsimite: (86) 2530 1459) Telex:

Email:

Commission(s): +0

WANG JING-XIU, BEIJING ASTRONOMICAL OBS, CAS, W SUBURB, BEIJING 100080, CHINA PR

Telephone: (86)'1 1281698

Facsimile: (86)

Telex: 22(1) bat) en

Email:

Commission(s): 10,12

WANG LAN-JUAN, SHANGHAI OBSERVATORY, CAS, 80 NANDAN RD, SHANGHAI 200030, CHINA PR

Telephone: (86) 21386191

Facsimile: (86)

Commission(s): (09)

Telex: $3316+$ shao cn

WANG QINGDE DANIEL, CASA, UNIVERSITY OF COLORADO, CAMPUS BOX 389, BOULDER CO 80309 0389, USA

Telephonc: (1) $303+926784$

Facsimile: (1) $303+927178$

Telex:

Enual: wqdáavela.colorado.edu

Commission(s):

WANG RENCHUAN, CFA, UNIV SCIENCE TECHNOLOGY, HEFEI 230026 ANHUI, CHINA PR

Telephonc: (86) 551331134 Facsimile: (86)

Telex: 90028 uste cn

Email:

Commission(s): 47

WANG SHOU-GUAN, BEIJING ASTRONOMICAL OBS, CAS, W SUBURB, BEIJING 100080, CHINA PR

Telepbonc: (86) 1281261

Facsimile: (86)

Telex: $220+0$ badas en

Email:

Commission(s): $38,40,44$

WANG SHUI, DPT EARTH \& SPACE SCI, UNIV SCIENCE \& TECHNOLOGY, HEFEI 230026 ANHUI, CHINA PR

Telephonc: (86) $551331134 * 209$

Facsimile: (86)

Telex: $\mathbf{4 + 3 0}$

Email:

Commission(s): +4

WANG SHUNDE DR, BEIJING ASTRONOMICAL OBS, CAS, W SUBURB, BEIJING 1OOOB(I, CHINA PR

Telephonc: (86) 12561264

Facsimile: (86)

Telex: 220+(0 baoas en

Email:

Commission(s): 46,49

WANG SI-CHAO, PURPLE MOUNTAIN OBSERV, CAS, NANJING, CHINA PR

Telephone: (86) $25 \mathbf{4 4 2 0 5}$

Facsinile: (86) $25311+59$

Telex: $3+1+4$ pmonj en

Email:

Commission(s): 15

WANG TING-GUI, CFA, UNIVERSITY OF SCIENCE \&, TECHNOLOGY, HEFEI 2301026, CHINA PR

Telephonc: (86) 551301852

Facsimile: (86) 551331760

Telex: 90028 ustc en

Email:

Commission(s): 28 
WANG YANAN, NANJING ASTRONOMICAL, INSTRUMENT RESEARCH CTR, NANJING 210042, CHINA PR

Telephone: (86) $255+11776$

Facsimile: (86) $255+11872$

Telex:

Email:

Commission(s): (6)

WANG YIMING, YUNNAN OBSERVATORY, CAS, BOX 110, KUNMING 72946 YUNNAN, CHINA PR

Telephone: (86) 8712035

Facsimile: (86)

Telex:

Email:

Commission(s): (09)

WANG YI-MING DR, NAVAL RESEARCH LABORATORY, CODE $\$ 172$ W, 4555 OVERLOOK AVE SW

WASHINGTON DC 20375 5000, USA

Telephone: (1) 2027676202

Facsimile: (1)

Telex:

Email:

Commission(s): $10,44,49$

WANG YONG, BOX 846, NANJING $2106+2$, CHINA PR

Telephone: (86) 255507485

Facsimile: (86) 255507872 Telex:

Email: ylab(aba001.bao.ac.co

Commission(s): (19)

WANG ZHENG MING, SHAANXI OBSERVATORY, CAS, LINTONG XIAN, SHAANXI, CHINA PR

Telephone: (86) 332255

Facsimile: (86)

Telex: 70121 csio cn

Email:

Commission(s): (09,19

WANG ZHEN-RU, DPT OF ASTRONOMY, NANJING UNIVERSITY, NANJING, CHINA PR

Telephone: (86) 2537551 *2685 Facsimile: (86)

Telex: $3+151$ prenu en

Email:

Commission(s): +4

WANG ZHEN-YI, PURPLE MOUNTAIN OBSERV, CAS, NANJING, CHINA PR

$\begin{array}{ll}\text { Telephone: }(86) 2546700 & \text { Facsimile: }(86) 25301459 \\ \text { Email: } & \text { Commission(s): } 12\end{array}$

WANG ZHONG, CENTER FOR ASTROPHYSICS, MS 66, 60 GARDEN ST, CAMBRIDGE MA (12138, USA

Telephone: (1) $617+967632$

Facsimile: (1) 617 \$95 7\$90 Telex:

Email: mangéacfa.havard.celu

Commission(s):

WANNIER PETER GREGORY DR, JPL, MS 169506, 4800 OAK GROVE DR, PASADENA CA 91109, USA

Telephone: (1) 8183543347

Facsimile: (1)

Telex: $675+29$

Emait:

Commission(s): 34,411

WARD HENRY DR, DPT PHYSICS \& ASTRONOMY, UNIVERSITY OF GLASGOW, GLASGOW G12 8QQ, UK

Telephone: $(+4) 1+13398855^{\star}+705$

Facsimile: (4t) $1+13349$ (12)

Telex: 777070 uniglil

Email: gw 10äjuk.ac.glit.ph.il

Commission(s): (19)

WARD MARTIN JOHN, INSTITUTE OF ASTRONOMY, THE OBSERVATORIES, MADINGLEY RD

CAMBRIDGE CB3 OHA, UK

Telephone: (4t) 1223337548

Facsimile: (4) 1223337523 Telex:

Email:

Commission(s): $28,+2$

WARD RICHARD A DR, LAWRENCE LIVERMORE LAB, L 58, BOX 808, LIVERMORE CA 94551, USA

Telephone: (1) $510+232679$

Email: rawardällinl.gov Facsimile: (1) $510+2251112$ Telex: 9103868339

Commission(s): 35

WARD WILLIAM R DR, JPL, MS 183 501, \$800 OAK GROVE DR, PASADENA CA 91109, USA

Telephone: (1)

Facsimile: (1)

Telex:

Email:

Commission(s):

WARDLE JOHN F C PROF, DPT OF PIIYSICS, BRANDEIS UNIVERSITY, WALTHAM MA (02154, USA

Telephone: (1) 617 647 2889

Facsimile: (1)

Telex:

Email:

Commission(s): fo

WARES GORDON W DR, 73 PERKINS ST, WEST NEWTON MA 12165, USA

Telephone: (1)

Facsimile: (1)

Telex:

Email:

Commission(s): 14

WARGAU WALTER F DR, DPT OF MATHS NASTRONOMY, UNIVERSITY OF S AFRICA, BOX 392 PRETORIA (OMOI, SOUTH AFRICA

Telephonc: (27) $2712+402133$

Facsimile: (27)

Telex: 350068 ta unisa ttx

Email:

Commission(s): +2 
WARMAN JOSEF DR, INSTITUTO DE ASTRONOMIA, UNAM, APDO POSTAL 70-26 4,04510 MEXICO DF, MEXICO

Telephone: (52)

Facsimile: $(52)$

Telex:

Email:

Commission(s):

WARMELS REIN HERM DR, ESO, KARL SCHWARZSCHILDSTR 2, D 857†1 GARCHING MUENCHEN, GERMANY

Telephone: (49) 8932006292

Facsimile: (4) $893202362 \quad$ Telex:

Email: rwarmels âjeso.ore

Commission(s): 40

WARNER BRIAN PROF, DPT OF ASTRONOMY, INST OF THEOR PHYSICS, RONDEBOSCH 7700, SOUTH AFRICA

Telephone: (27) 6502391

Facsimile: (27)

Commission(s): 27,42

Telex: $521+39$

Emili:

ER CORP, MS
Facsimile: (1)

Telex:

Telephone: (1) 2037967919

Commission(s): $28,+4$

WARNER PETER J DR, MULLARD RADIO ASTRON OBS, CAVENDISH LABORATORY, MADINGLEY RD

CAMBRIDGE CB3 OHE, UK

Telephone: (44) 1223337294

Facsimile: (4+) $1223354599 \quad$ Telex: 81292 cavlab g

Email:

Coumission(s): 40

WARREN WAYNE H JR DR, NASA GSFC, CODE 681, LAB ASTRON \& SOLAR PHYS, GREENBELT MD 20771, USA

Telephone: (1) $3012868701{ }^{*} 5+19$

Facsimile: (1)

Telex:

Email: w3whwásefmrs.gsfe.nasa.gov

Commission(s): $05 \mathrm{C}, 25,37,45$

WARWICK JAMES W DR, RADIOPIIYSICS CORP, 5475 WESTERN AVE, BOULDER CO 80301, USA

Telephone: (1) $303+47952 t$

Facsimile: (1)

Telex:

Email:

Commixsion(s): 12,40

WARWICK ROBERT S DR, DPT OF PHYSICS, UNIVERSITY OF LEICESTER, UNIVERSITY RD

LEICESTER LE 1 7RH, UK

Telephone: $(44) 1533554+55$

Facsimile: (ft)

Telex: $34166+$ luxiay g

Email:

Commission(s):

WASHIMI HARUICHI DR, INST FOR ATMOSPHERIC RES, NAGOYA UNIVERSITY, 3-13 HONOHARA

TOYOKAWA AICHI +12, JAPAN

Telephone: (81) 533863154 Facsimile: (81)

Telex: 4322311

Email:

Commission(s):

WASSERMAN LAWRENCE H DR, LOWELL OBSERVATORY, 1400 W MARS HILL RD, BOX 1149

FLAGSTAFF AZ 86001, USA

Telephone: (1) $601277+3358$

Facsimile: (1)

Telex:

Email:

Commission(s): $16,211 \mathrm{C}, 2 t$

WASSON JOHN T, INST OF GEOPHYS \& PLANET, UNIVERSITY OF CALIFORNIA, LOS ANGELES CA 90024, USA

Telephone: (1) 2138251986

Facsimile: (1)

Telex:

Email:

Commission(s): 15,16

WATANABE JUN-ICHI DR, TOKYO ASTRONOMIICAL OBS, NAOJ, OSAWA MITAKA, TOKYO 181, JAPAN

Telephone: (81) $+22+1361$

Facsimile: $(81)+22+13608$

Commission(s): 15,22

Telex: 028223117 taomtk j

Email: owatana/acl.mtk.nato.ac.jp

WATANABE TAKASHI DR, INST FOR ATMOSPHERIC RES, NAGOYA UNIVERSITY, 3-13 HONOHARA

TOYOKAWA +42, JAPAN

Telephonc: (81) $53386315+$

Facsimile: (81) 533860811

Telex: +322311 rianag $j$

Emitil:

Commission(s): 40

WATANABE TETSUYA, NTL ASTRONOMICAL OBS, 211 OSAWA, MITAKA, TOKYO 181, JAPAN

Telephonc: $(81)+223+371+$

Facsimile: $(81)+223+3700$

Telex:

Email: watamaberaubab.mtk.nao.ac.jp

Commission(s): 36

WATERS LAURENS B F M DR, SRON, BOX 800 , NL9700 AV GRONINGEN, NETHERLANDS

Telephone: (31) 50634090

Facsimile: (31) $5063+1133$

Telex:

Email: renswarug.nl

Commission(s): 36 
WATERWORTH MICHAEL DR, SCHOOL OF PHYSICS, QUEENSLAND UNIV OF TECHN, GPO BOX $2+3$ BRISBANE QLD 4001, AUSTRALIA

Telephone: (61)

Facsimile: (61)

Telex:

Email:

Commmission(s): 29

WATSON FREDERICK GARNETT, ROYAL GREENWICH OBS, MADINGLEY RD, CAMBRIDGE CB3 OEZ, UK

Telephone: (\$4) 122337 \$(1010

Facsimile: (4t) $122337+700 \quad$ Telex:

Email: uk.ac.rgo.starafgw

Commission(s): 09,51

WATSON MICHEAL G DR, DPT OF PIIYSICS, UNIVERSITY OF LEICESTER, UNIVERSITY RD

LEICESTER LEI 7RH, UK

Telephone: (4t) $1162523553 \quad$ Ficsimile: (4t) 1162550182 Telex:

Email: ngwäuk,ac.le.star Commission(s):

WATSON ROBERT DR, DPT OF PIIYSICS, UNIVERSITY OF TASMANIA, GPO BOX 252C HOBART TAS 7001, AUSTRALIA

Telephone: (61) $2202+15 \quad$ Ficsimile: (61) $22112+10 \quad$ Telex: aa 58150

Email: $\quad$ Commission(s): 27

WATSON WILLIAM D PROF, DPT OF PHYSICS, UNIVERSITY OF ILLINOIS, 1110 W GREEN ST

URBANA IL 61811, USA

Telephone: (1) $2173337240 \quad$ Facsimile: (1) Telex:

Email:

Commission(s):

WATT GRAEME DAVID, ROYAL OBSERVATORY, BLACKFORD HILL, EDINBURGH EHO 3HJ, UK

Telephone: (4)

Facsimile: (4)

Telex:

Email:

Commission(s): 34

WATTENBERG D PROF, LINDERIIOFSTR 57, D I $1+7$ BERLIN, GERMANY

Telephone: (49) 5277772

Fickimile: $(49)$

Email:

Commission(s): +1

Telex:

WAYMAN PATRICK A PROF, GLEBE COTTAGE, GLEBE AVE, WICKLOW, IRELAND

Telephone: (353) 41169695 Ficsimile: (353) Telex:

Email:

Commixsion(s): $115,33,50$

WDOWIAK THOMAS J DR, PHYSICS DPT, UNIVERSITY OF ALABAMA, BIRMINGHAM AL 3529 4 , USA

Telephone: (1) $205934+736 \quad$ Ficsimile: (1) Telex: 888826 uabl) bhm

Email:

Commission(s): 15

WEAVER HAROLD F PROF, STSCI, HOMEWOOD CAMPUS, 3760 SAN MARTIN DR, BALTIMORE MD 21218, USA

Telephone: (1) 4103385004

Facsimile: (1)

Telex:

Email: weaverästsci.edu

Commiswion(s): $15,33,34,37$

WEAVER THOMAS A DR, LAWRENCE LIVERMORE LAB, L 17, BOX 808, LIVERMORE CA 94550, USA

Telephone: (1) $+15+23$ 1850

Facsimile: (1)

Telex:

Emiil:

Comminsion(s): 35,44

WEAVER WILLIAM BRUCE, MONTEREY INST RESEARCH, IN ASTRONOMY, 900 MAJOR SHERMAN LANE MONTEREY CA 93940, USA

Telcphonc: (1) +118 3753221 Facsimile: (1) 4086400164

Telex:

Email: 3303 pä,narpgs.bitnet/mira $a, n p s . n a v$, mi

Commission(s): 45

WEBB DAVID F, PHILLIPS LAB/GPSG, BOSTON COLLEGE, HANSCOM AFB MA 01731, USA

Telephone: (1) 617377 3970)

Fatsimile: (1) 6173773160 Telex:

Email: afglsc::webb

Commission(s): 10

WEBB JOHN, SCHOOL OF PIYYSICS, UNIV NEW SOUTH WALES, BOX 1, KENSINGTON NSW 2033, AUSTRALIA

Telephonc: (61)

Facsimile: $(6,1) 26,633+20$ Telex:

Email: jkwädedwin.phỵs.unisw.colu.au Commission(s):

WEBBER JOHN C DR, INTERFERONETRICS INC, 1SI LEESBURG PIKE, VIENNA VA 22180, USA

Telephone: (1) 703790185011

Ficsimile: (1)

Telex:

Ematil:

Commission(s): 
M 898

WEBBINK RONALD F DR, DPT OF ASTRONOMY, UNIVERSITY OF ILLINOIS, 1011 W SPRINGFIELD AVE URBANA IL 61801, USA

Telephone: (1) 217-333-9582

Facsimilu: (1)

Telex: 910-2+5-2+34 ast

Email:

Commission(s): $27,35,42$

WEBER JOSEPH, PHYSICS DPT, UNIVERSITY OF MARYLAND, COLLEGE PARK MD 20142, USA

Telephone: (1) 3014056081

Facsimile: (1) $3013149525 \quad$ Telex:

Email: jw116(a)umail.umd.edu

Commission(s): (19)

WEBER STEPHEN VANCE, LAWRENCE LIVERMORE LAB, L 477 , BOX 808, LIVERMORE CA 94550, USA

Telephone: (1) $+15+225433 \quad$ Ficsimile: (1)

Telex: 910-386-8339

Email:

Commission(s): 36

WEBROVA LUDMILA DR, ASTRONOMICAL INSTITUTE, CZECH ACADEMY SCIENCES, BOCNI II 1401 CZ 14131 PRAHA 4, CZECH R

Telephone: (42) $225 \$ 287 \quad$ Facsimile: (42)

Commission(s): 31

Telex: $122+86$

Email:

RONOMY CENTER, UKIRT, 665 KOMOHANA ST, HILO HI 96720, USA

WEBSTER ADRIAN S DR, JOINT ASTRONOMY CENTER, UKIRT, 665 KOMOHANA ST,
Telephone: (1) 8089613756
Facsimile: (1)

Email: $\quad$ Commission(s): 44,47

WEBSTER RACHEL, SCHOOL OF PHYSICS, UNIVERSITY OF MELBOURNE, PARKVILLE VIC 3152, AUSTRALIA

Telephone: (61) $33+75+5$ )

Facsimile: (61) $3347+783$

Telex:

Email: webster(ätauon.ph.unimell.edu.a Commission(s): 47

WEEDMAN DANIEL W PROF, DPT OF ASTRONOMY, PENNSYLVANIA STATE UNIV, 525 DAVEY LAB UNIVERSITY PARK PA 16802 , USA

Telephone: (1) $814865(1418$

Fatcsinile: (1)

Telex: $8+2510$

Email:

Commission(s): 28

WEEKES TREVOR C DR, FRED LAWRENCE WHIPPLE OBS, HARVARD-SMITHSONIAN CTR AMADO AZ 856450097 , USA

$\begin{array}{ll}\text { Telephone: (1) 602 629 6741 } & \text { Ficsimile: (1) } \\ \text { Email: } & \text { Commission(s): }\end{array}$

Email:

WEGNER GARY ALAN, DPT OF PHYSICS \& ASTRON, DARTMOUTH COLLEGE, WILDER LABORATORY HANOVER NH 03755 , USA

Telephonc: (1) 6036462359

Email:

Facsimile: (1)

Telex:

Commission(s): 29,31

WEHINGER PETER A DR, DPT OF PHYSICS, ARIZONA STATE UNIVERSITY, ASTRONOMY PROGRAM TEMPE AZ 85287, USA

Telephone: (1) 6112 965 4063 Ficsimile: (1)

Email: bitnet:wehinger/a;sucps $\quad$ Commission(s): $15,28,2^{9}$

Telex: $1+(1289$ halley asu ut

WEHLAU AMELIA DR, DPT OF ASTRONOMY, UNIV OF WESTERN ONTARIO, LONDON ON NGA 3K7, CANADA

Telephone: (1) 5196793186

Facsimile: (1) 5196613486

Telex: $06+713+$

Email: afwehlauänve.uwoca

Commision(s): 27,37

WEHLAU WILLIAM H PROF, DP'T OF ASTRONOMY, UNIV OF WESTERN ONTARIO, LONDON ON N6A 3K7, CANAD

Facsimile: (1) 51966120109

Telex:

Email: whwehlauaphobos.astro.uwo.ca Commission(s): 27,29,42

WEHRLE ANN ELIZABETH DR, CALTECH, MS 100) 22, IPAC, PASAdENA CA 91125, USA

Telephone: (1)

Facsimile: (1)

Telex:

Email: deimos:acw/acwacitueimoi.call Commission(s): 40,44

WEHRSE RAINER DR, INST F TIIEOR ASTROPIIYSIK, DER UNIVERSITAET, IM NEUENHEIMER FELD 561

D 69120 HEIDELBERG, GERMANY'

Telephone: $(49) 6221562837$

Facsimile: (49) $62215(1221$

Telex: 461515 unihd d

Email: b28(a)w.iwe.uni-heidlellecrg.de

Commission(s): $36 \mathrm{C}$

WEI MINGZHI, LICK OBSERVATORY, UNIVERSITY OF CAIIFORNIA, SANTA CRUZ CA 9EOG4, USA

Telephone: (1) $408+59$ 4911

Facsimile: (I) $40 \times+263115$

Telex:

Email: wmyunescloa.ucsc.edu

Commission(s): 40 
WEIDEMANN VOLKER PROF, INST THEOR PHYS \& STERNW, NEUE UNIV PHYSIK ZENTRUM OLSHAUSENSTR GEB N $61 \mathrm{C}$, D 24098 KIEL, GERMANY
Telephone: $(49)+31880+110$
Facsimile: (49)
Telex: 2927(06

Commistion(s): 05.36

WEIDENSCHILLING S J DR, PLANETARY SCIENCE INST, 620 N SIXTH AVE, TUCSON AZ 85705 8331, USA
Telephone: (1) 5206626300
Falcimile: (1) 5206228060 Telex:

Email: psikey::psikey/sjwappsi.edı

Commission(s): 15,16

WEIGELT GERD DR, MPI FUER RADIOASTRONOMIE, AUF DEM HUEGEL 69, D 53121 BONN, GERMANY

Telephone: (49) 228525243

Facsimile: (49) 228525229

Telex: $886+40$

Email: p561gwe(a)mpifr-bonn.mpg.de

Commission(s): 40

WEIGERT ALFRED PROF, HAMBURGER STERNWARTE, GOJENSBERGSWEG 112, D 21029 HAMBURG, GERMANY

Telephone: (49) $40722+4112$ Facsimile: (49)

Telex: 217884

Email:

Commission(s): 35,42

WEILER EDWARD J DR, NASA HEADQUARTERS, CODE SZ, 600 INDEPENDENCE AVE SW

WASHINGTON DC 20546, USA

Telephone: (1) $20235803+2$

Facsimile: (1)

Telex:

Email: eweiler@gm.ossat.h(y. Iassi.gov

Commission(s): $40,+2,44$

WEILER KURT W DR, NAVAL RESEARCH LABORATORY, CODE $+131,+555$ OVERLOOK AVE SW

WASHINGTON DC 20375 5000, USA

Telephone: (1) 2027670292

Facsintilc: (1)

Telex:

Email: span:11334::weiler

Commission(s): $28,34,40,44$

WEILL GILBERT M DR, SPOT IMAGE CORPORATION, 1897 PRESTON WHITE DRIVE, RESTON VA 22091 4326, USA Telephone: (1) 703 620 2200 Facsimile: (1)

Email: $\quad$ Commission(s):

Telex: 4993073

WEIMER THEOPHILE P F DR, OBSERVATOIRE DE PARIS, 61 AVE OBSERVATOIRE, F 75014 PARIS, FRANCE

Telephone: (33) $1+3201210$

Facsimile: (33)

Telex:

Email:

Commission(s): 16

WEINBERG J L DR, ISST SPACE ASTRONOMY LAB, 1031 NW GTH ST, SUITE B1, GAINESVILLE FL 32601, USA

Telephone: (1) 9043925450

Email: isstäfrecnet.ufl.edu

Facsimile: (1) $90+37250+2$

Commission(s): $22,4+$

Telex: 8108252308 spacela

WEINBERG STEVEN DR, ASTRONOMY DPT, UNIVERSITY OF TEXAS, RLM 15 308, AUSTIN TX 78712 1083, USA

Telephone: (1) $512471+394$

Facsimile: (1)

Telex: 910-87\-1305

Email:

Commission(s): 47

WEINBERGER RONALD DR, INSTITUT FUER ASTRONOMIE, TECHNIKERSTR 15, A 6020 INNSBRUCK, AUSTRIA

Tclephone: (43) 52227485251

Facsimile: $(+3) 52222185252$

Telex: 533808 unite a

Email: ronaldäast 1.uibl.ac.at

Commission(s):

WEIS EDWARD W DR, DPT OF ASTRONOMIY, VAN VLECK OBSERVATORY, WESLEYAN UNIVERSITY

MIDDLETOWN CT $06+57$, USA

Telephonc: (1) 2033479411

Facsimile: (1)

Commission(s): 26

Telex:

Email:

WEISBERG JOEL MARK, DPT PHYSICS \& ASTRONOMY, CARLETON COLLEGE, NORTHFIELD MN 55057, USA

Telephonc: (1) $507663+367$

Facsimile: (1)

Telex:

Email:

Commission(s):

WEISHEIT JON C DR, DPT SPACE PHYS \& ASTRON, RICE UNIVERSITY, BOX 1892, HOUSTON TX 77251 1892, USA

Telephone: (1)

Facsimile: (1)

Commission(s): 34,44

Telex:

Email:

$$
\text { Commiston(s): } 34,44
$$

WEISS ACHIM DR, MPI F PHYSIK \& ASTROPHYS, KARL SCHWARZSCHILDSTR 1

D 85740 GARCHING MUENCHEN, GERMANY

Telephonc: (49) 89329900

Email: acw (adgaipp1s
Telex: 524629 astro d 
WEISS NIGEL O DR, DPT APPLIED MATHS, \& THEORETICAL PHYSICS, SILVER STREET CAMBRIDGE CB3 9EW, UK

Telephone: (44) 1223351645

Email:

Facsimile: (4) $1223337918 \quad$ Telex: 81240

Commission(s): 12,35

WEISS WERNER W DR, INSTITUT FUER ASTRONOMIE, UNIVERSITAET WIEN, TUERKENSCHANZSTR 17 A 1180 WIEN, AUSTRIA

Telephone: $(+3) 14706800 / 7^{*} 6683$

Facsimile: $(43) 1470680(0)-15$

Telex: 116222 physi a

Email: weiss(a)avia.una.ac.at

Commission(s): $09,25,27,29$

WEISSKOPF MARTIN CH DR, NASA/MSFC, CODE ES 65, HUNTSVILLE AL 35812, USA

Telephone: (1) 2054533238

Facsinile: (1)

Telex:

Email:

Commission(s): 44

WEISSMAN PAUL ROBERT, JPL, MS 183 601, 4800 OAK GROVE DR, PASADENA CA 91109 , USA

Telephone: (1) 8183542636

Facsimile: (1)

Telex: $675+29$

Email:

Commission(s): 15,20

WEISTROP DONNA DR, DPT OF PHYSICS, UNIVERSITY OF NEVADA, 4505 S MARYLAND PARKWAY LAS VEGAS NV 89154 , USA

Telephone: (1)

Email: weistrop(änevada.edu

Fuesimile: (1)

Telex:

WELCH DOUGLAS L DR, DPT OF PHYSICS \& ASTRON, MCMASTER UNIVERSITY

HAMILTON ON L8S 4M1, CANADA

Telephone: (1) $4165259140 * 3186 \quad$ Facsimile: (I) $+165461252 \quad$ Telex: 061 8347

Email: welch(a)physun.physics.mcmaster. Commission(s): 27

WELCH GARY A DR, DPT OF ASTRONOMY, ST MARY'S UNIVERSITY, HALIFAX NS B3H 3C3, CANADA

Telephone: (1) $902+299780$

Facsimile: (1) 9024205561 Telex:

Email:

Commixsion(s): 28

WELCH WILLIAM J PROF, RADIO ASTRONOMY LAB, UNIVERSITY OF CALIFORNIA, GOI CAMPBELL HALL BERKELEY CA 9\$720, USA

Telephone: (1) $\$ 156426679$

Facsinile: (1)

Telex: 820181 uch ast ral

Email:

Commission(s): 40,51

WELLER CHARLES S DR, DAVID TAYLOR RES CENTER, CODE 1402, BETHESDA MD 20084 5000, USA

Telephone: (1) $301227127+$

Facsimile: (1)

Telex:

Email:

Commission(s): 49

WELLGATE G BERNARD MR, CANEHEATH HOUSE, ARLINGTON, POLEGATE BN26 6SJ, UK

Telephone: (44)

Facsimile: $(t+)$

Telex:

Emait:

Commission(s):

WELLINGTON KELVIN DR, CSIRO, DIV OF RADIOPHYSICS, BOX 76, EPPING NSW 2121, AUSTRALIA

Telephone: (61) $2372 \$ 375$

Facsimile: (61) $2372+400$

Telex: 26230 ad

Email: kwelling(a) r).csiro.au

Commission(s): $40,51 \mathrm{C}$

WELLMANN PETER PROF DR, INST ASTRON \& ASTROPHYSIK, UNIVERSITAETS STERNWARTE, SCHEINERSTR I D 81679 MUENCHEN, GERMANY

Telephone: (49) 89989021

Email:

Facsimile: (49) 8992209427 Telex:

Commission(s): $36,+2$

WELLS DONALD C DR, NRAO, 520 EDGEMONT RD, CHARLOTTESVILLE VA 22903, USA

Telephone: (1) 8042960277

Facsimile: (1) 8042960278 Telex:

Email: dwellsannaodu

Commission(s): $15 \mathrm{C}$

WELLS EDDIE NEIL, STSCI, HOMEWOOD CAMPUS, 370 SAN MARTIN DR, BALTIMORE MD 21218, USA

Telephone: (1) $\$ 10338+788$

Facsimile: (1) $+10338+767 \quad$ Telex:

Email: wells $\bar{a}$ stsci.edu

Commission(s): 15,16

WENDKER HEINRICH J PROF, HAMBURGER STERNWARTE, GOJENSBERGSWEG 112

D 21029 HAMBURG, GERMANY

Tclephonc: (49) 4072524112

Facsimile: (49)

Commission(s): 34,40

Telex: $21788+$ 
WENGER MARC, OBS DE STRASBOURG, 11 RUE UNIVERSITE, F G7WOU STRASBOURG, FRANCE

Telephone: (33) 88358219

Facsimile: (33) 88250160

Telex:

Email: simbad::wenger/wengeräsimbad.,

Commission(s): (15

WENIGER SCHAME DR, 23BIS RUE R SCHUMAN, F 9\$270 KREMLIN BICETRE, FRANCE

Telephone: (33)

Facsimile: (33)

Telcx:

Email:

Commission(s): 14,29

WENTZEL DONAT G DR, ASTRONOMY PROGRAM, UNIVERSITY OF MARYLAND, COLLEGE PARK MD 20742, USA

Telephone: (1) 301 t05 1518

Facsimile: (1) 301 31+9067

Telex:

Email: wentreläastro.umd.edu

Commission(s): $10,12,44,46 \mathrm{C}$

WENZEL W DR, ZNTRLINST FUER ASTROPHYS, STERNWARTE SONNEBERG, ROSA LUXEMBURG STR 17A

D 96575 SONNEBERG, GERMANY

Telephone: (49)

Facsimile: (49)

Telex:

Email:

Commission(s): 27

WERNER KLAUS, INST FUER ASTRONOMIE UND, ASTROPHYSIK, UNIVERSITAET KIEL, D 24098 KIEL, GERMANY

Telephone: $(49)+31880+1106$

Ficsimile: (49) $+31880+1(0)$

Telex:

Email: supats075/äastrophysik.uni-kicl.d100.dle

Commission(s): $36 \mathrm{C}$

WESEMAEL FRANCOIS DR, DPT DE PHYSIQUE, UNIVERSITE DE MONTREAL, CP GI28 SUCC A MONTREAL QC H3C 3J7, CANADA

Telephone: (1) 5143437355

Fucsimile: (1) $51+3+32071$

Telex: $05562+25$ udemphysas

Email:

Commission(s):

WESSELIUS PAUL R DR, SRON, BOX 800, NL 9700 AV GRONINGEN, NETHERLANDS

Telephone: (31) $5063+074$

Facximile: (31) $5063+033$

Telex:

Email: pauláguspace.rug.ni

Commission(s): $25,34,44,45$

WESSON PAULS DR, DPT OF PHYSICS, UNIVERSITY OF WATERLOO, WATERLOO ON N2L 3G1, CANADA

Telephone: (1) 5198851211

Facsimile: (1) 5197468115

Telex: 06955250

Emiail:

Commission(s): $21,47,51$

WEST DONALD K DR, NASA GSFC, CODE 684.1, GREENBELT MD 20771, USA

Telephone: (1) Facsimile: (1) Telex:

Email: $\quad$ Commission(s): 42

WEST RICHARD M DR, ESO, KARL SCHWARZSCHILDSTR 2, D 85740 GARCHING MUENCHEN, GERMANY

Telephone: (49) 89 $320106276 \quad$ Facsimile: (49) 893202362

Telex: $\$ 2828220$ eso d

Email: rwestiajeso.org

Commixsion(s): $06 \mathrm{P},(1) \mathrm{C}, 20,38 \mathrm{C}$

WEST ROBERT ALAN, JPL, MS 183 301, +800 OAK GROVE DR, PASADENA CA 91109, USA

Telephone: (1) $81835+0+79$ Ficsimile: (1) Telex; 675429)

Email: Commission(s): 16

WESTERGAARD NIELS J DR, DANISH SPACE RESEARCH INS, LUNDTOFTEVEJ 7, DK 2800 LYNGBY, DENMARK

Telephone: (45) $22882277 \quad$ Facsimile: (45)

Email:

Commission(s):

Telex: 37198 danru dk

WESTERHOUT GART DR, 81। WEST 38TH STR, BALTIMORE MD 212112203 , USA

Telephone: (1) +10 $235583+\quad$ Finsimile: (1) Telex:

Enail: $\quad$ Commision(s): $05,08,24,33,40$

WESTERLUND BENGT E PROF, ASTRONOMICAL OBSERVATORY, BOX 515, S 751 20 UPPSALA, SWEDEN

Telephone: (46) 18135157

Facsimile: (46)

Telex: $76012+$ univ uppss

Email:

Commission(s): $28,33,45$

WESTFOLD KEVIN C PROF, 7 BEAMSIEY ST, MALVERN, VICTORIA 3144, AUSTRALIA

Telephone: ( 61$)$

Facsimile: (61)

Telex:

Email:

Commixsion(s): t11,4t

WESTPHAL JAMES A PROF, CAL TECH, MS 170 25, PASADENA CA 91125 , USA

Telephone: (1) 2133564900

Facsimile: (1)

Telex:

Enail:

Comminission(s): $0 y,+t$ 
WETHERILL GEORGE W, DPT TERRESTR MAGNETISM, CARNEGIE INST WASHINGTON 52+1 BROAD BRANCH RD NW, WASHINGTON DC 20015, USA
Telephome: (1) 2029660863
Facsimile: (1)
Telex: $4+1)+27$ mign ui
Email: wetherillacciw.dnet.nasi.gov
Commission(s): $15,16,22,51$

WEYMANN RAY J PROF, CARNEGIE OBSERVATORIES, 813 SANTA BARBARA ST, PASADENA CA 91001, USA

Telephone: (1) 8185771122

Facsimile: (1) 8187958136

Telex: 1561318 ociw ut

Email:

Commission(s): 34

WHEELER J CRAIG PROF, ASTRONOMY DPT, UNIVERSITY OF TEXAS, RLM 15 308, AUSTIN TX 78712 1083, USA

Telephone: (1) $512+71+461$

Facsimile: (1)

Telex: 910-87ł-1351

Email:

Commission(s): $35,42 \mathrm{C}, 4+$

WHEELER JOHN A DR, DPT OF PHYSICS, PRINCETON UNIVERSITY, JADWIN HALL, PRINCETON NJ O85\$4, USA

Telephone: (1) $609258+400$

Facsimile: (1) $609258112+$

Telex: 4993512

Email: dwnsamso.anu.o\%..au

Commission(s): 44,47

WHIPPLE ARTHUR L DR, MCDONALD OBSERVATORY, UNIVERSITY OF TEXAS, AUSTIN TX 78712 1351, USA

Telephone: (1) $512+716332$

Facsimile: (1)

Telex:

Email: bitnet:asag105 a uta 3081

Commission(s): 07,20

WHIPPLE FRED L DR, CENTER FOR ASTROPHYSICS, HCO/SAO, 60 GARDEN ST, CAMBRIDGE MA 02138, USA

Telephone: (1) $617+957200$

Facsimile: (1)

Telex:

Email:

Commission(s): $15,216,22$

WHITAKER EWEN A, LUNAR \& PLANETARY LAB, UNIVERSITY OF ARIZONA, TUCSON AZ 85721, USA

Telephone: (1) 5206212888

Facsimile: (1)

Telcx: 910-952-11+3

Email:

Commission(s): 16,41

WHITE GLENN J, DPT OF PHYSICS, QUEEN MARY/WESTFIELD COLL, MILE END RD, LONDON E1 4NS, UK Telephone: (4t) $1719804811 * 4145 \quad$ Facsimile: $(44)$

Email:

Commission(s): 34

Telex: 893750

WHITE GRAEME LINDSAY DR, CSIRO, DIV OF RADIOPHYYICS, BOX 76, EPPING NSW 2121, AUSTRALIA

Telephone: (61) $28680222^{\star}+20$

Email:

Facsimile: (61) 28680310

Telex: 26230 astro ail

WHITE NATHANIEL M DR, LOWELL OBSERVATORY, 1400 W MARS HILL RD, BOX 1149, FLAGSTAFF AZ 86001, US Telephone: (1) 6027743358 Facsimile: (1) Telex:

Email:

Commission(s): 25

WHITE ORAN R DR, HIGH ALTITUDE OBSERVATORY, NCAR, BOX 3010, BOULDER CO 80307 3000, USA

Telephone: (1) $303+971000$

Facsimile: (1) $303+971568 \quad$ Telex:

Email: whiterăhao.ucar.edu

Commission(s):

WHITE R STEPHEN PROF, IGPP, UNIVERSITY OF CALIFORNIA, RIVERSIDE CA 92521, USA

Tclephone: (1) $7147874503 \quad$ Facsimile: (1)

Telex:

Email:

Commission(s):

WHITE RAYMOND E DR, STEWARD OBSERVATORY, UNIVERSITY OF ARIZONA, TUCSON AZ 85721, USA

Telephonc: (1) 5206216528

Facsimile: (1)

Telex: 467175

Email: nhiteajas.arizona.cdu

Commission(s): 33,37

WHITE RAYMOND EDWIN III, DPT PHYSICS \& ASTRONOMY, UNIVERSITY OF ALABAMA, BOX 870324 TUSCALOOSA AL 35487 (0324, USA

Telephone: (1) 2053481640

Facsimile: (1) 2053485051

Telex:

Email: whiteamerkin.astr.uit.edu

Commission(s):

WHITE RICHARD E, DPT OF ASTRONOMY, SMITH COLLEGE, CLARK SCIENCE CENTER

NORTHAMPTON MA 01063, USA

Telephone: (1) $41358+2701$

Facsimile: (l)

Telex:

Email:

Commission(s): 
WHITE RICHARD L, STSCI, HOMEWOOD CAMPUS, 3700 SAN MARTIN DR, BALTIMORE MD 21218, USA

Telephone: (1) $301338+797$

Facsimile: (1)

Telex:

Email:

Commission(s): 34,36

WHITE SIMON DAVID MANION, INSTITUTE OF ASTRONOMY, THE OBSERVATORIES, MADINGLEY RD

CAMBRIDGE CB3 OHA, UK

Telephone: (4) 1223337515

Email: swhite(âast.star.camm.ac.uk

Facsimile: (4) $1223337523 \quad$ Telex: 817297 astrong

Commission(s): 28,47

WHITELOCK PATRICIA ANN DR, SAAO, BOX 9, OBSERVATORY 7935, SOUTH AFRICA

Telephone: $(27)+70025 \quad$ Facsimile: (27) Telex: 5720309

Email:

Commission(s): $27,33,34,46$

WHITEOAK J B DR, CSIRO AUSTR TELE NTL FAC, PAUL WILD OBSERVATORY, LOCKED BAG 194 NARRABRI NSW 2390, AUSTRALIA

Telephone: (61) 67904051

Facsimile: (61) $6790+1090$

Telex: 26230

Email: jwhiteoa(a)atnf.csiro.au

Commission(s): 33,34,40P,50)

WHITFORD ALBERT E PROF, LICK OBSERVATORY, UNIVERSITY OF CALIFORNIA, SANTA CRUZ CA 950G4, USA

Telephone: (1) $408+292149 \quad$ Facsimile: (1)

Email:

Commission(s): 28

Telex:

WHITMORE BRADLEY C, STSCI, HOMEWOOD CAMPUS, 3701 SAN MARTIN DR, BALTIMORE MD 21218, USA

Telephone: (1) $301338+713$

Facsimilc: (1)

Telex:

Email:

Conmmission(s): 28

WHITNEY CHARLES A PROF, CENTER FOR ASTROPHYSICS, HCO/SAO, 60 GARDEN ST

CAMBRIDGE MA 02138, USA

$\begin{array}{ll}\text { Telephonc: (1) } 617+957+51 & \text { Facsimile: (1) } \\ \text { Email: } & \text { Commission(s): }\end{array}$

Email:

WHITROW GERALD JAMES PROF, 41 HOME PARK RD, WIMBLEDON, LONDON SW19 7HS, UK

Telephone: ( +4$) 1819+7343467$

Facsimile: (4+)

Telex:

Email:

Commission(s): 41,47

WHITTET DOUGLAS C B DR, DPT OF PHYSICS, RENSSELAER POLYTECHN INST, TROY NY 12180 3590, USA Telephone: (1) 5182766310 Facsimile: (1) $51827666080 \quad$ Telex:

Email:

Commission(s): 33,34

WHITTLE D MARK DR, UNIVERSITY STATION, UNIVERSITY OF VIRGINIA, BOX 3818 CHARLOTTESVILLE VA 22903, USA

Telephone: (1) 86+92+ 4900 Facsimile: (1)

Enail: dmw8fásirginia.edu

Commission(s):

Telex: 9109970174 nrao

WHITWORTH ANTHONY PETER, DPT APPLIED MATHS/ASTRON, UNIVERSITY COLLEGE

BOX 78, CARDIFF CF1 1XL, UK

Telephone: (4t) $1222+4211 \quad$ Facsimile: (4t)

Email:

Commisvion(s): 34

Telex: 498635 ulibefg

WICKRAMASINGHE D T DR, AUSTRALIAN NTL UNIVERSITY, DPT OF APPLIED MATHS, BOX 4 CANBERRA ACT 2600, AUSTRALIA

Telephone: (61)

Facsimile: (61)

Telex:

Email:

Commission(s):

WICKRAMASINGHE N C PROF, DPT APPLIED MATHS/ASTRON, UNIVERSITY COLLEGE, BOX 78 CARDIFF CF1 1XL, UK

Telcphone: $(+t) 1222+\$ 211$

Facsimile: (4)

Commission(s): $34,36,40$

Telex: 498635 ulibefe

Email:

ERONOMIE, BP 3, F 91371 VERRIERES LE BUIS, FRANCE

WIDEMANN THOMAS, SEIRVICE D'AERONOMIE, BP 3, F91371 VERRIERES L
Telephone: (33) $164+75256$
Fatesimile: (33) 169202999

Commission(s): 
$\mathrm{M} 904$

WIDING KENNETH G DR, NAVAL RESEARCH LABORATORY, CODE 7144, 4555 OVERLOOK AVE SW WASHINGTON DC 20375 5I(H), USA

Telephone: (1) 2027672605

Facsimile: (1)

Telex:

Email:

Commission(s):

WIEDLING TOR DR, OSTRA VILLAVAGEN 15, S 61136 NYKOPING, SWEDEN

Telephone: (46) Facsimile: (46) Telex:

Email:

Commission(s):

WIEHR EBERHARD DR, UNIVERSITAETS STERNWARTE, GOETTINGEN, GEISMARLANDSTR 11

D 37083 GOETTINGEN, GERMANY

Telephone: (49) $551395053 \quad$ Facsimile: (49) Telex: 96753

Email:

Commission(s): 10

WIELEBINSKI RICHARD PROF, MPI FUER RADIOASTRONOMIE, AUF DEM HUEGEL 69, D 53121 BONN, GERMANY

Telephone: (49) 228525300

Facsimile: (49) 228525436

Telex: $886+40$

Email:

Commission(s): $25,28 \mathrm{C}, 33,411,51$

WIELEN ROLAND PROF DR, ASTRON RECHEN INSTITUTE, MOENCHHOFSTR 12-It

D 69120 HEIDELBERG, GERMANY

Telephone: (49) $6221+9026$

Facsimile: $(\$ 9)$

Tclex; 461336 arihd d

Email:

Commission(s): $04,05 \mathrm{C}, 08,28,33,37$

WIESE WOLFGANG L DR, NTL BUREAU OF SATNDARDS, DIV $8+2$ RM A267 BLDG 22

GAITHERSBURG MD 20899, USA

Telephone: (1) 3019753201

Facsimile: (1) 3019753038

Telex: 898493

Emati:

Commission(s): $1+\mathrm{C}$

WIITA PAUL JOSEPH, DPT PHYSICS \& ASTRONOMY, GEORGIA STATE UNIVERSITY, ATLANTA GA 30303 3083, US

Telephone: (1) 4046582932

Facsimile: (1)

Telex:

Email:

Commission(s): 28

WIJNBERGEN JAN DR, LAB VOOR RUIMTEONDERZOEK, HOOGBOUW WSN, BOX 800 NL 9700 AV GRONINGEN, NETHERLANDS

Telephonc: (31) 50 $116660 \quad$ Facsimile: (31)

Telex: $\mathbf{5 3 5 7 2}$ stars nI

Email:

Commission(s):

WIKLIND TOMMY, ONSALA SPACE OBSERVATORY, $S+3992$ ONSALA, SWEDEN

Telephone: (46) 317725537

Facsimile: (46) 31772 5590 Telex:

Email: tommyäoso.chalmers.sc

Commission(s):

WILCOCK WILLIAM L PROF, SCHOOL OF PHYSICAL $\$$, MOLECULAR SCIENCES, UNIV COLLEGE OF N WALES BANGOR GWYNEDD LL57 2UW, UK

Telephone: (t+) $12+8351151$

Facsimile: (4t)

Telex: 61100

Email:

Commission(s): 09

WILD JOHN PAUL DR, CSIRO, LIMESTONE AVE, BOX 225, DICKSON ACT 26012, AUSTRALIA

Telephone: $(61) 62+8+5 \% 5$

Facsimile: (61)

Telex:

Email:

Commission(s): $1(0,40.49$

WILD PAUL PROF, ASTRONOMISCHES INSTITUT, UNIVERSITAET BERN, SIDLERSTRASSE S CH 3012 BERN, SWITZERLAND

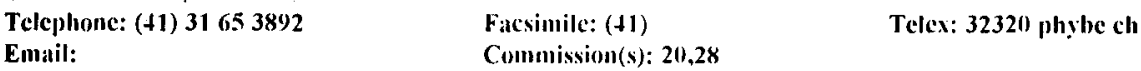

WILDEY ROBERT L PROF DR, ASTROPHYSICAL OBSERVATORY, N ARIZONA UNIVERSITY, FLAGSTAFF AZ 86011 USA

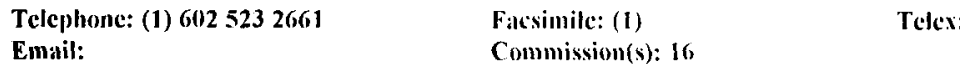

WILKENING LAUREL L DR, ADMIN BLDG 601, UNIVERSITY OF ARIZONA, TUCSON AZ 85721, USA

Telephone: (1) 5206263513

Facsimile: (1)

Telcx:

Email:

Commission(s): 15 
WILKES BELINDA J, CENTER FOR ASTROPHYSICS, HCO/SAO, 60 GARDEN ST, CAMBRIDGE MA 02138, USA

Telephone: (1) 617 t95 7268

Enail:

Commixsion(s):

Telex: $921+28$

WILKINS GEORGE A DR, WINDWARD, IIGIER BROOK MEADOW, SIDFORD, SIDMOUTH DEVON EXIO 9SS, UK Telephone: (tt) 1395 579 6+1 Facsimile: (4t)

Telex:

Email: g.a.wilkinsäu,uk.ac.exeter

Commission(s): $144,05 C, 19,31$

WILKINSON ALTHEA, DPT OF ASTRONONY, UNIVERSITY OF MANCHESTER, MANCHESTER M13 9PL, UK

Telephone: (4t) 1612737121

Facsimile: (4t)

Commission(s): 28

Telex:

Email:

WILKINSON DAVID T, DPT OF PHYSICS, PRINCETON UNIVERSITY, JADWIN HALL, PRINCETON NJ O8544, USA

Telephome: (1) $6199+52+406$

Facsimile: (1)

Telex:

Email:

Commission(s): $\$ 7$

WILKINSON PETER N DR, NRAL, JODRELL BANK, MACCLESFIELD SK11 9DL, UK

Telephone: (4) $147771321 \quad$ Facsimile: (4) Telex: 36149

Email:

Commission(s): 40

WILL CLIFFORD M DR, DPT OF PIIYSICS, WASIING'TON UNIVERSITY, ST LOUIS MO 63130, USA

Telephonc: (1) $31+88962+4$

Facsimile: (1)

Telcx:

Email:

Commission(s): 44,47

WILLIAMON RICHARD M, FERNBANK SCIENCE CENTER, 156 HEATON PARK DRIVE, 156 HEATON PARK DRIVE, ATLANTA GA 30307, USA

Telephone: (1) $+10+378+313$

Email:

Facsimile: (1)

Telex:

Commission(s): $27,+2,46$

WILLIAMS BARBARA A, DPT OF PHYSICS, UNIVERSITY OF DELAWARE, NEWARK DE 19716, USA

Telcphone: (1) $302+516526$

Facsimile: (1)

Telex:

Email:

Commission(s): 28

WILLIAMS CAROL A, DPT OF MATIEMIATICS, UNIVERSITY OF S FLORIDA, TAMPA FL 33620, USA

Telephone: (1) $81397+26+3$

Facsimile: (1)

Telex:

Email:

Commission(s): $144,07,24$

WILLIAMS DAVID A PROF, DPT OF MATHENATICS, UMIST, BOX 88, MANCHESTER MG0 1QD, UK

Telephone: (4) $1612363311 \quad$ Facsimile: (4)

Telex: 6660104

Email:

Commission(s): 34

WILLIAMS GLEN A DR, DPT OF PIIYSICS, CENTRAL MICHIGAN UNIV, MT PLEASANT MI 48858, USA

Telephone: (1) $51777+3365$

Facsimile: (1)

Telex:

Email: 32usq̣svajcmuvm

Commission(s): +2

WILLIAMS IWAN P PROF, ASTRONONIY UNIT, QUEEN MARY/WESTFIELD COLL, MILE END RD

LONDON E1 +NS, UK

$\begin{array}{lll}\text { Telephone: (4t) } 1719755452 & \text { Facsimile: (4t) } 1819833522 & \text { Telex: 893750 }\end{array}$

Email: i.p.williamsa,gmm.ac.uk

Commission(s): $15,16,211,22 P, 51$

WILLIAMS JAMES G DR. JPL, MS 26, 700, +800) OAK GROVE DR, PASADENA CA 91109, USA

Telephone: (1) $81835+6466$

Fatcimile: (1)

Commission(s): $14,16,19,20$

Telex: $9105883269 \mathrm{jpl}$

Email:

SICS, ALBION COLLEGE, ALBION MI +9224, USA

WILLIAMS JOIN A DR, DPT OF PIIYSICS, ALBION COLLEGE, ALBION MI 4922
Telephonc: (1) 5176295511
Facsimile: (1)

Email: $\quad$ Commission(s): 45

WILLIAMS PEREDUR M DR, ROYAL OBSERVATORY, BLACKFORD HILL, EDINBURGH EHO 3HJ, UK

Telephonc: (4t) $1316673321 \quad$ Facsimile: $(4+)$

Em:iil: $\quad$ Commission(s): 29)

Telex: 72383 rocding

WILLIAMS ROBERT E DR, CERRO TOLOLO, INTERAMERICAN OBSERVATORY, CASILLA 603, LA SERENA, CHILE Telephone: (56) 51213352

Email:

Facsimile: (56) $51212466 * 3+2$

Telex: 645227 aura ct

Commission(s): $28,34,42$ 
WILLIAMS THEODORE B DR, DPT PIIYSICS \& ASTRONOMY, RUTGERS UNIVERSITY, BOX 849 PISCATAWAY NJ 08854 (084\%, USA

Telephone: (1) 2019322516

Facsimile: (1)

Telex:

Email:

Commission(s): 28

WILLIS ALLAN J DR, DPT PHYSICS \& ASTRONOMY, UNIVERSITY COLLEGE LONDON, GOWER ST LONDON WC1E GBT, UK

Telephone: (4t) 1713877050

Facsimile: $(4+)$

Telex: 28722

Email:

Commission(s): $34,4 t$

WILLIS ANTHONY GORDON DR, DOMINION RADIO ASTROPHYS, OBSERVATORY, BOX 248

PENTICTON BC V2A 6K3, CANADA

Telephone: (1) $604+932277$

Facsimile: (1) $604+9337767$

Telex: $14+888127$

Email:

Commission(s): 40

WILLMER CHRISTOPHER N A, OBSERVATORIO NACIONAL, RUA GENERAL JOSE, CRISTINO 77 20921 030 RIO DE JANEIRO, BRAZIL.

Telephone: (55) 215896504

Facsimile: (55) 215800332

Telex:

Email: chaworon.br

Commission(s):

WILLMORE A PETER PROF, DPT OF SPACE RESEARCH, UNIVERSITY OF BIRMINGHAM, BOX 363 BIRMINGHAM B15 2TT, UK

Telephone: (44) $121+72$ 1301

Facsimile: (44)

Commission(s):

Telex: 338938 spaphy gg

Email:

WILLNER STEVEN PAUL DR, CENTER FOR ASTROPHYSICS, HCO/SAO, GO GARDEN ST CAMBRIDGE MA 02138, USA

Telephone: (1) $617+957123$

Facsimile: (1)

Email:

Commission(s): $34.4 t$

Telex: $921+28$ satellite cam

WILLS BEVERLEY J DR, ASTRONOMY DPT, UNIVERSITY OF TEXAS, RLM 15 308, AUSTIN TX 78712 1083, USA

Telephone: (1) $512+713+2+$

Facsimile: (1)

Telex: 910-87+-1351

Email:

Commission(s): $28,+11$

WILLS DEREK DR, ASTRONOMY DPT, UNIVERSITY OF TEXAS, RLM 15 308, AUSTIN TX 78712 1083, USA

Telephone: (1) $512+71+461$

Facsimile: (1)

Telex: $910-874-1351$

Email:

Commmission(s): 28,41

WILLSON LEE ANNE DR, DPT OF PHYSICS, IOWA STATE UNIVERSITY, AMES IA 50011, USA

Telephone: (1) 5152946765

Facsionile: (1)

Telex: 911-52(1-1157

Email:

Commission(s): $27,35,36, \mathrm{C}$

WILLSON ROBERT FREDERICK, DPT OF PHYSICS, TUFTS UNIVERSITY, MEDFORD MD (12155, USA

Telephone: (1) 6176285000

Facsimile: (1)

Telex:

Email:

Commission(s): 40,51

WILLSTROP RODERICK V DR, INSTITUTE OF ASTRONOMY, THE OBSERVATORIES, MADINGLEY RD CAMBRIDGE CB3 OHA, UK

Telephone: (44) 1223337548

Email:
Facsimile: (4t) 1223337523

Commission(s): 25,30
Telex: 817297 astron

WILSON ALBERT G DR, BOX 1871, SEBASTOPOL CA 95\$73, USA

Telephone: (1)

Email:

Facsimile: (1)

Commission(s): 28,47

Telex:
WILSON ANDREW S DR, ASTRONONIY PROGRAM, UNIVERSITY OF MARYLAND, COLLEGE PARK MD 207\$2, USA Telepthone: (1) $301+546061$

Email:

Telex: 7108260352

Commission(s): $4(1,47$

WILSON BRIAN G PROF, UNIVERSITY OF QUEENSLAND, 55 WALCOTT STREET, ST LUCIA QLD 4067 , AUSTRALIA

Telephone: (61) 73772200

Facsimile: (61)

Commission(s):

Telex: H11315 uniqgld ala

Email:

WILSON CURTIS A, ST JOHN'S COLLEGE, BOX 2881, ANNAPOLIS MD 21404, USA

Telephonc: (1) 3012632371

Facsimile: (1)

Telex:

Email:

Commission(s): +1 
WILSON JAMES R DR, 737 SOUTH M, LIVERMORE CA 94550, USA

Telephone: (1) $+15+22$ 16.59

Email:

Facsimile: (1)

Commission(s): $4 t$
Telex:

WILSON LIONEL DR, ENV SCIENCE DPT, LANCASTER UNIVERSITY, LANCASTER LA1 \&YQ, UK

Telephone: $(+4) 152+652011^{*}+1075$

Facsimile: (44)

Commission(s): 27

Email:

Telex: 6.5111 lqneulg

WILSON MICHAEL JOHN DR. BPT OF APPL MATHEMATICS, UNIVERSITY OF LEEDS, LEEDS LS2 9JTT, UK

Telephone: (t4)

Facsimile: (44)

Telex:

Em:til:

Commixsion(s):

WILSON P DR, GEOFORSCHUNGS ZENTRUM, TELEGRAFENBERG A17, D 14407 POTSDAM, GERMANY

Telephone: (49)

Facsimile: $(40)$

Telex:

Email:

Commission(s): 19C,21

WILSON PETER R PROF, DPT OF APPLIED MATHS, UNIVERSITY OF SYDNEY, SYDNEY NSW 2006, AUSTRALIA

Telephone: (61) 26922222

Facsimite: $(61) 26602903$

Telex:

Email:

Commission(s): 10,12,36

WILSON RICHARD, ROYAL GREENWICH OBS, MADINGLEY RD, CAMBRIDGE CB3 IEZ, UK

Telephone: (t+) $122337+74 t$

Facsimile: (4t) $122337+700$ Telex:

Email: rwwajnail.ast.cam.ac.uk

Commission(s):

WILSON ROBERT E PROF, DPT OF ASTRONOMY, UNIVERSITY OF FLORIDA, 211 SSRB

GAINESVILLE FL 32611, USA

Telephonc: (1) 910+392 1182

Facsimilc: (1)

Telex:

Email:

Commission(s): 35,42

WILSON ROBERT PROF SIR, DPT PHYSICS \& ASTRONOMY, UNIVERSITY COLLEGE LONDON, GOWER ST LONDON WC1E GBT, UK

Teleplone: (\$t) $171380715 t$

Em:iil:

\author{
Ficsimile: $(+t)$ \\ Commission(s): 14,4
}

Telex: 28722

WILSON ROBERT W DR, AT \& BELL LABORATORIES, HOII L 23\%, BOX 400, HOLMDEL NJ (17733, USA

Telephone: (1) 20194938133 Facsimile: (1) Telex:

Email: $\quad$ Commission(s): 34,40

WILSON S J, DPT OF MATIEMATICS, NTL, UNIVERSITY SINGAPORE, KENT RIDGE, SINGAPORE O511, SINGAPOR Telephone: $(65)$

Email:

Facsimile: (65)

Commission(s): 36

Telex:

WILSON THOMAS L DR, MPI FUER RADIOASTRONOMIE, AUF DEM HUEGEL (\%), D 53121 BONN, GERMANY

Telephone: (49) $228525378 \quad$ Filesimile: (49) $228525229 \quad$ Telex: 886440

Emait:

Commission(s): $34,40,51$

WILSON WILLIAM J DR, JPL, MS I68 327, 4860 OAK GROVE DR, PASADENA CA 91109, USA

Telephone: (1) $81835+5690$

Facsimile: (1)

Telex:

Email:

Commiscion(s): 40

WINCKLER JOHN R PROF, SCHOOL OF PHYS \& ASTRON, UNIVERSITY OF MINNESOTA, 116 CHURCH ST SE MINNEAPOLIS MN 5.5.5.5, USA

$\begin{array}{ll}\text { Telephone: (1) } 6123734688 & \text { Facsimile: (1) } \\ \text { Email: } & \text { Commission(s): }\end{array}$

WINDHORST ROGIER A DR, DPT OF PIIYSICS. ARIZONA STATE UNIVERSITY, TEMPE AZ 85287 1504, USA

Telephone: (1) 60129657143

Facsimile: (1)

Telex: 1561058

Email:

Commission(s): $199,28,411,47$

WING ROBERT F PROF, DPT OF ASTRONONY, OIIO STATE UNIVERSITY, 174 W 18TH AVE

COLUMBUS OH +32101106, USA

Telephone: (1) $614+227876$

Facimile: (1)

Telex:

Email: tst718 a,olstmwsal

Commission(s): 27,29,45 
WINGET DONALD E, ASTRONOMY DPT, UNIVERSITY OF TEXAS, RLM 15 308, AUSTIN TX 78712 1083, USA Telephone: (1) $512+71+461$

Fassimile: (1)

Telex:

Email:

Conmomission(s):

WINIARSKI MACIEJ, ASTRONOMICAL, OBSERVATORY, JAGIELLONIAN UNIVERSITY, UL ORLA I71 PL $302+4$ KRAKOW, POLAND

Telephone: (48)

Email:
Facsimile: (48) 12378053

Commission(s): 25
Telex: 32 6203 ujioa pl

WINK JOERN ERHARD DR, IRAM, 300 RUE DE LA PISCINE, F 38406 S MARTIN HERES CD, FRANCE

Telephone: (33) $76+23383$

Email:

Telex:

Commission(s): 40

WINKLER CHRISTOPH DR, ESA/ESTEC, SSD, BOX 299, NL 2200 AG NOORDWIJK, NETHERLANDS

Telephone: (31) 171983591 Facsimile: (31) $17198+690$

Telex: 39098

Email: cwinkleräjestec2.estec.esa.nl

Commission(s): t

WINKLER GERNOT M R DR, US NAVAL OBSERVATORY, TIME SERVICE DPT, 3450 MASSACHUSETTS AVE NW WASHINGTON DC 20392 5100, USA

Telephonc: (1) 2026531520

Email:

Facsimile: (1) 20126.5315017

Telex: 7108221970 navolesy

WINKLER KARL-HEINZ A DR, LOS ALAMOS NATIONAL LAB, MS B218X DOT, BOX IG63

LOS ALAMOS NM 87545, USA

Telephone: (1)

Email:

Facsimile: (1)

Telex:

Commixsion(s): 35

WINKLER PAUL FRANK DR, DPT OF PHYSICS, MIDDLEBURY COLLEGE, MIDDLEBURY VT O5753, USA

Telephone: (1) 8023883711

Facsinile: (1)

Telex: 353249

Enail:

Commission(s):

WINNBERG ANDERS DR, ONSALA SPACE OBSERVATORY, CHALMERS UNIV OF TECH, S 4992 ONSALA, SWEDEN

Telephone: (46) 317725527

Facsimile: (46) $317725590 \quad$ Telex:

Email: andersiagoso.chalmers.se

Commission(s): 34,40

WINNEWISSER GISBERT DR, I PHYSIKALISCHE INSTITUT, UNIVERSITAET KOELN, UNIVERSITATSSTR I4 D 5000 KOELN +1, GERMANY

Telephonc: (49) $211+703567$

Email:

Facsinule: (49)

Telex:

Commission(s): $14,34,40$

WIRAMIHARDJA SUHARDJA DR, BOSSCHA OBSERVATORY, LEMBANG 40391, INDONESIA

Telephone: (62) 2296001

Facsimile: (62) $22+416694$

Telex: $2832+$ bi

Email:

Commission(s):

WITHBROE GEORGE L DR, CENTER FOR ASTROPHYSICS, HCO/SAO, GI GARDEN ST, CAMBRIDGE MA 02138, USA

Telephone: (1) $617+957+38$

Facsimile: (1)

Telex:

Email:

Commission(s):

WITT ADOLF N DR, DPT PHYSICS \& ASTRONOMY, UNIVERSITY OF TOLEDO, 280I W BANCROFT ST

TOLEDO OH $\$ 36(16$, USA

Telephone: (1) $\$ 195372709$

Email:

Facsimide: (1)

Telex: $810 \$ 421633$

Commission(s): 21,34

WITTEN LOUIS PROF, DPT OF PIIYSICS, UNIVERSITY OF CINCINNATI, 210 BRAUNSTEIN ML 11, CINCINNATI OH 452210111 , USA

Telephone: (1) 5134756492

Facsimile: (1)

Telex:

Email:

Commission(s):

WITTMANN AXEL D DR, UNIVERSITAETS STERNWARTE, GOETTINGEN, GEISMARLANDSTR 11

D 37083 GOETTINGEN, GERMANY

Telephone: (49) $551395(142 \quad$ Facsimile: (49)

Email:

Commission(s): 10,12

Telex: 96753

WITZEL ARNO DR, MPI FUER RADIOASTRONONIE, AUF DEM HUEGEL 69, D 53121 BONN, GERMANY

Telephone: (49) 228525211

Facsimile: $(49)$

Commission(s): 41

Telex: $886+40$

Email: 
WIYANTO PAULUS, DPT OF ASTRONOMY, ITB, GANESHA 10, BANDUNG 40132, INDONESIA

Telephone: (62)

Facsimile: (62)

Telex:

Email:

Commission(s):

WKHARIN A S DR, MAIN ASTRONOMICAL OBS, UKRAINIAN ACAD OF SCIENCE, GOLOSEEVO

252127 KIEV, UKRAINE

Telephone: $(7) 04+66+765$

Facsimile: (7)

Commission(s): $05,(18$

Telex: 132517 nebo

Email:

WLERICK GERARD DR, OBSERVATOIRE DE PARIS, SECTION DE MEUDON, F 92195 MEUDON PPL CDX, FRANCE

Telephone: (33) 145072240

Facsimile: (33)

Telex:

Email:

Commission(s): 09,28

WNUK EDWIN, ASTRONOMICAL OBSERVATORY, A MICKIEWICZ UNIVERSITY, UL SLONECZNA 36

PL 60286 POZNAN, POLAND

Teleplone: (48)

Facsimile: (48)

Telex:

Email:

Commission(s): 07

WOAN GRAHAM, MULLARD RADIO ASTRON OBS, CAVENDISH LABORATORY, MADINGLEY RD

CAMBRIDGE CB3 OHE, UK

Telephone: (\$t) $12233372+7$

Email: grahanüümrao.cam.ac.uk

Facsimile: (4t) $122335+599 \quad$ Telex:

Commission(s):

WOEHL HUBERTUS DR, KIEPENHEUER INSTITUT, FUER SONNENPHYSIK, SCHOENECKSTR G

D 791(1 $\downarrow$ FREIBURG BREISGAU, GERMANY

Telephone: (49) $7613286 t$

Facsimile: (49)

Commission(s): $09,10,12,36$

Telex: 7721552 kis d

Email:

RNWARTE, KOENIGSTUHL, D 69117 HEIDELBERG, GERMANY

WOLF BERNHARD PH D, LANDESSTERNWARTE, KOENIGSTUHL, D 69117 HEI
Telephone: (49) 6221509213 Facsimile: (49) 6221509 2012 Telex:

Email: bwolfahp2.uni-heidelberg.te $\quad$ Commission(s): 29

WOLF MAREK, DPT ASTRONOMY/ASTROPHYS, CHARLES UNIVERSITY, SVEDSKA 8, CZ 150 O0 PRAHA 5, CZECH Telephone: $(+2) 2540395$

Email: wolf(â)earn.cvut.č

Facsimile: (42) 2299272

Telex:

Commission(s):

WOLF RAINER E A DR, MPI FUER ASTRONOMIE, KOENIGSTUHL, D 69117 HEIDELBERG, GERMANY

Telephone: (\$9) $62215281 \quad$ Facsimile: (\$9)

Email:

Commission(s):

Telex: $\$ 61789$ mpia d

WOLFE ARTHUR M PROF, CASS, UCSD, C 011 , LA JOLLA CA 92093 0216, USA

Telcphonc: (1) 619 5347435

Email: awolferaucsd

Facsimile: (1) 619 \$3+6316 Telex:

Commission(s):

WOLFENDALE ARNOLD W PROF, DPT OF PHYSICS, UNIVERSITY OF DURHAM, SOUTH RD, DURHAM DH1 3LE, U Telcphonc: (4) $19137+2160$

Facsimile: (\$4) $19137+3749$

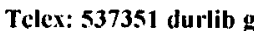

Email:

Commission(s): $4+\mathrm{C}$

WOLFF SIDNEY C DR, KITT PEAK NTL OBS, BOX 26732, 950 N CHERRY AVE, TUCSON AZ 85726 6732, USA

Telephone: (1) 5203275511

Facsimile: (1)

Commission(s): 29,36

Telex: 666-484 aura noao

Email:

WOLFIRE MARK GUY, NASA AMES RESEARCH CTR, MS 245 3, MOFFETT FIELD CA 94035, USA

Telephone: (1) 4156040321

Facsiutile: (1) +156046779

Telex:

Email: wolfire âcygnus.arc.nasil.gov

Commission(s): 34

WOLFSON C JACOB, LOCKHEED PALO ALTO RES LB, DPT 9130 BLDG 256, 3251 HANOVER ST, PALO ALTO CA $9+3041191$, USA

Telephone: (1) $415+242855$

Facsimile: (1) $415+2439 \%$

Telex:

Email:

Commission(s):

WOLFSON RICHARD DR, DPT OF PHYSICS, MIDDLEBURY COLLEGE, MIDDLEBURY VT 05753, USA

Telephone: (1) 8023883711

Email: wolfson ämidd.bitnct

Facsimile: (1)

Commission(s): 10

Telex: 
WOLSTENCROFT RAMON D DR, ROYAL OBSERVATORY, BLACKFORD HILL, EDINBURGH EH9 3HJ, UK

Telephone: (44) 1316673321

Facsimile: $(4+)$

Telex: 72383

Email:

Commission(s): $21,34,44,51$

WOLSZCZAN ALEXANDER DR, ARECIBO OBSERVATORY, BOX 995, ARECIBO PR 00613, USA

Telephone: (1) 8098782612

Facsimile: (1)

Telex: 385638

Email:

Commission(s): 34,40

WOLTER ANNA DR, OSS ASTRONOMICO DI BRERA, VIA BRERA 28, I 20121 MILANO, ITALY

Telephone: (39) 2874444

Facsimile: (39) $272001600 \quad$ Telex:

Email: astmib::wolter

Commission(s): 4

WOLTJER LODEWIJK PROF, OHP, F 04870 S MICHEL OBS, FRANCE

Telephone: (33) 92766662

Facsimile: (33) $92766295 / 41227883551$

Email:

Commission(s): $10,33,34,40,+4 \mathrm{C},+7, \mathrm{EC}$

Telex: 410690 ohp f

WOO JONG OK, KOREAN NTL UNIVERSITY, OF EDUCATION, CHUNGWON-GUN, CHUNGBUK 320 23, KOREA R
$\begin{array}{ll}\text { Telephone: (82) } 431603712 & \text { Facsimile: }(82) \\ \text { Email: } & \text { Commission(s): } 25,46\end{array}$

WOOD DOUGLAS O S, NRAO, BOX 0, SOCORRO NM 87801 0387, USA

Telephone: (1) $5058357398 \quad$ Facsinile: (1) $5058357027 \quad$ Telex:

Email: dwood@nrao.edu Commission(s): 34,40

WOOD F BRADSHAW PROF, DPT OF ASTRONOMY, UNIVERSITY OF FLORIDA, 211 SSRB

GAINESVILLE FL 32611, USA

Telephone: (1) 9043922059

Facsimile: (1) Telex:

Email:

Commission(s): 38,42

WOOD II H J DR, ASTRONOMY DPT, INDIANA UNIVERSITY, SWAIN WEST 319, BLOOMINGTON IN 47405, USA

Telephone: (1)

Facsimile: (1)

Telex:

Email:

Commission(s): 29

WOOD JANET H DR, DPT OF PHYSICS, UNIVERSITY OF KEELE, KEELE ST5 5BG, UK

Telephone: (44) $1782621111 \quad$ Facsimile: (t4)

Telex:

Email: jhw@uk.ac.kcele.ph.starlink Commission(s): 42

WOOD JOHN A DR, CENTER FOR ASTROPHYSICS, HCO/SAO, 60 GARDEN ST, CAMBRIDGE MA 02138, USA

Telephone: (1) $6174957278 \quad$ Facsimile: (1)

Telex: 921428 satellite cam

Email:

Commission(s): $15,16,22$

WOOD PETER R DR, MOUNT STROMLO \& SIDING, SPRING OBSERVATORIES, PRIVATE BAG

WODEN PO ACT 2606, AUSTRALIA

Telephone: (61) 62881111

Facsimilc: (61) $62+90233$

Telex: 62270 canopus aa

Email:

Commission(s): 27,35

WOOD ROGER DR, SATELLITE LASER RANGER GP, HERSTMONCEUX CASTLE, HAILSHAM BN27 1RP

EAST SUSSEX, UK

$\begin{array}{lll}\text { Telephone: (44) } 1323833171 * 3391 & \text { Facsimile: (44) } & \text { Telex: } 87451 \text { rgobsy g } \\ \text { Email: } & \text { Commission(s): } & \end{array}$

WOODSWORTH ANDREW W.DR, HERZBERG INST ASTROPHYS, DOMINION ASTROPHYS OBS

5071 W SAANICH RD, VICTORLA BC V8X 4 M 6, CANADA

Telephone: (1) 6043880024

Facsimile: (1) $60+3630045$

Telex: 0497295

Email:

Commission(s): 40

WOODWARD PAUL R DR, DPT OF ASTRONOMY, UNIVERSITY OF MINNESOTA, 116 CHURCH ST SE MINNEAPOLIS MN 55455, USA

Telephone: (1)

Facsimile: (1)

Telex:

Email:

Commission(s): 33,34

WOOLF NEVILLE J, STEWARD OBSERVATORY, UNIVERSITY OF ARIZONA, TUCSON AZ 85721, USA

Telephone: (1)

Facsimile: (1)

Telex:

Email:

Commission(s): 34,50 
WOOLFSON MICHAEL M PROF, DPT OF PHYSICS, UNIVERSITY OF YORK, HESLINGTON YORK YO1 5DD, UK Telephone: (4t) 190459861

Facsimile: $(+4)$

Commission(s): $15,16,21,22$

Telex: 57933 yorkulg

Email:

WOOLSEY E G, 1909 LAUDER DRIVE, OTTAWA ON K2A 1A9, CANADA
Telephone: (1)

Email: $\quad$ Commission(s):

Telex:

WOOSLEY S E PROF, LICK OBSERVATORY, UNIVERSITY OF CALIFORNIA, SANTA CRUZ CA 95064, USA

Telephone: (1) $4(184292976 \quad$ Facsimile: (I) Telex:

Email: woosley(âhelios.ucsc.edu Comnission(s): 28,35

WOOTTEN HENRY ALWYN, NRAO, S20 EDGEMONT RD, CHARLOTTESVILLE VA 22903, USA

Telephone: (1) 804 2960329 Facsimile: (1) 80+2960278 Telex:

Email: awootten ấnracodu Commission(s): 34,40

WORDEN SIMON P DR, 6757 N 27TH STREET, ARLINGTON VA 22213, USA

Telephone: (1) Facsimile: (1) Telex:

Email: $\quad$ Commission(s): 09,12

WORLEY CHARLES E DR, US NAVAL OBSERVATORY, ASTRON \& ASTROPHYSICS DIV

3450 MASSACHUSETTS AVE NW, WASHINGTON DC 203925100 , USA

Telephone: (1) 2026531588 Facsimile: (1) $202653094+\quad$ Telex:

Email: eqboddraco.usno.navy.mil Commission(s): 05,24,26P

WORRALL DIANA MARY, CENTER FOR ASTROPHYSICS, HCO/SAO, 60 GARDEN ST, CAMBRIDGE MA 02138, USA

Telephone: (1) $6174957139 \quad$ Facsimile: (I) Telex: 921428 satellite cam

Email: $\quad$ Commission(s): 28,44

WORRALL GORDON DR, BIRDSWOOD, EARDISLEY HEREFDS, UK

Telephone: (4t) Facsimile: (4t) Telex:

Ematil: $\quad$ Commission(s):

WORSWICK SUSAN, ROYAL GREENWICH OBS, MADINGLEY RD, CAMBRIDGE CB3 (IEZ, UK

Telephonc: (H) 122337 4004) Facsimile: (H) $122337+700 \quad$ Telex: $265451 / 265871 \mathrm{monrefg}$

Email: $\quad$ Commission(s): (0)

WOSZCZYK ANDRZEJ PROF, INSTITUTE OF ASTRONOMY, N COPERNICUS UNIVERSITY, UL CHOPINA 12/18

PL 87 100 TORUN, POLAND

Telephone: (48) $26018 \quad$ Facsimile: (48) Telex: 00552234 astr pl

Email:

Commission(s): $15,16 \mathrm{C}, 50$

WOUTERLOOT JAN GERARD A, I PHYSIKALISCHE INSTITUT, ZUELPICHERSTR 77, D 50937 KOELN, GERMANY

Facsimile: (49) $221+705162 \quad$ Telex: 8882291 unik d

Email: waterlootäghl.uni-kocln.de Commission(s): 33,34

WRAMDEMARK STIG S O DR, LUND OBSERVATORY, BOX $43, \mathrm{~S} 22100$ LUND, SWEDEN

Telephone: $(46) 46107313$

Facsimile: $(46)$

Telex: 33199 obsnot $s$

Email:

Commission(s): $25,33,37$

WRAY JAMES D DR, 21200 TODD VALLEY RD, TRLR 54, FORESTHILL CA 95631 9511, USA

Telephone: (1)

Facsimile: (1)

Telex:

Email:

Commission(s): 44

WRIGHT ALAN E DR, AAO, PRIVATE BAG, BOX 276, PARKES NSW 2870, AUSTRALIA

Telephone: (61) 68611732

Facsimile: (61) 68611730

Telex: 63999 qasar

Email: awrightia atnf.csiro.au

Commission(s): $05,40,51$

WRIGHT ANDREW, DPT OF MATHEMATICS, UNIVERSITY OF ST ANDREWS, ST ANDREWS, FIFE KY16 9SS, UK

Telephone: $(4+) 133+463736$

Facsimile: $(44) 133+4637+8$

Telex: $9312110846 \mathrm{sag}$

Email:

Commission(s):

WRIGHT EDWARD L DR, DPT OF ASTRONOMY, UNIVERSITY OF CALIFORNIA, LOS ANGELES CA 90024, USA

Telephone: (1) 2138255755

Facsimile: (1)

Email: bonnic::wright

Commission(s): 34,47

Telex: 
WRIGHT FRANCES W DR, DPT OF ASTRONOMY, HARVARD UNIVERSITY, GO GARDEN ST CAMBRIDGE MA 02138, USA

Telephone: (1) 6174952647

Ficsimile: (1)

Telex:

Email:

Commission(s): 27

WRIGHT HELEN GREUTER, THOMAS HOUSE APT 517, 1330 MASSACHUSETTS AVE, WASHINGTON DC 20005, USA

Telephone: (1)

Facsimile: (1)

Telex:

Email:

Commission(s): +1

WRIGHT JAMES P DR, NTL SCIENCE FOUNDATION, DIV ASTRONOMICAL SCIENCES, 1800 G ST NW

WASHINGTON DC 20550, USA

Telephone: (1) 2023577639

Facsimile: (1)

Telex:

Email:

Commission(s):

WRIGHT MELVYN C H DR, RADIO ASTRONOMY LAB, UNIVERSITY OF CALIFORNIA, GO1 CAMPBELL HALL, BERKELEY CA 9\$720, USA

Telephone: (1) $\$ 156+20420$

Facsimile: (1)

Telex:

Email:

Commission(s):

WRIXON GERARD T DR, NTL MICROELECTRON RES CTR, UNIVERSITY COLLEGE CORK, CORK, IRELAND

Telephone: (353) 121508375

Facsimile: $(353)$

Telex: 26050

Email:

Commission(s):

WROBEL JOAN MARIE DR, NRAO, BOX 0, SOCORRO-NM 878110387 , USA

Telephone: (1) 5058357000

Facsimile: (1) $5058357027 \quad$ Telex: 9109881710

Email: jwrobeláanranodu

Commission(s): 28,40

WROBLEWSKI HERBERT DR, DPT DE ASTRONOMIA, UNIVERSIDAD DE CHILE, CASILLA 36 D, SANTIAGO, CHILE

Telephone: (56) $2229+101$

Facsimile: $(56)$

Telex: $\$+1)$ (10)

Email:

Commission(s): 20,2t

WU BIN, INSTITUTE OF GEODESY $\&$, GEOPHYSICS, XU DONG LU, WUCHANG HUBEI 430077, CHINA PR

Telephone: (86) 276813855

Email:

Facsimile: (86) 277811242 Telex;

Commission(s): 19

WU CHI CHAO DR, STSCU/CSC, HOMEWOOD CAMPUS, 3701 SAN MARTIN DR, BALTIMORE MD 21218, USA

Telephone: (1) $301338+770$

Facsimile: (1)

Telex: u.s.al.

Email:

Commission(s): $34,4+$

WU GUICHEN, SHAANXI ASTRONOMICAL OBS, BOX 18, LINTONG, XIAN 710600, CHINA PR

Telephone: (86) $293232255 \quad$ Facsimile: (86) 9237313 496 $\quad$ Telex: $70121 \mathrm{csan} \mathrm{cn}$

Email: Commission(s): 31

WU HSIN-HENG DR, DPT OF PHYSICS, NTL CENTRAL UNIVERSITY, CHUNG LI TAIWAN, CHINA R

Telephone: (886)

Email:

Facsimile: (886)

Telex:

WU HUAI-WEI, SHANGHAI OBSERVATORY, CAS, 80 NANDAN RD, SHANGHAI 200(130, CHINA PR

Telephone: (86) 21386191

Email:

Telex: 33164 shao cn

WU LIAN-DA, PURPLE MOUNTAIN OBSERV, CAS, NANJING, CHINA PR

Telephone: (86) 2532893

Facsimile: (86) $25301+59$

Telex: $3+14+$ pmonj en

Email:

Commission(s): 07

WU LIN-XIANG, DPT OF GEOPHYSICS, BEIJING UNIVERSITY, BEIJING 100071, CHINA PR

Telephone: (86)

Facsimile: $(86)$

Telex:

Email:

Commission(s): $09,10,12$

WU MING-CHAN, YUNNAN OBSERVATORY, CAS, BOX 110 , KUNMING 72946 YUNNAN, CHINA PR

Telephone: (86) 8712035

Facsimile: $(86)$

Telex: 64040 yuobs en

Email:

Commission(s): 10,50

WU NAILONG DR, STSCI, HOMEWOOD CAMPUS, 3700 SAN MARTIN DR, BALTIMORE MD 21218, USA

Telephone: (1) $\$ 105166864$

Enıail: mailongrástsci.colu
Facsimile: (1) $+105168720 \quad$ Telex:

Commission(s): 40 
WU SHENGYIN, BEIJING ASTRONOMICAL OBS, CAS, W SUBURB, BEIJING 100080, CHINA PR

Telephone: (86)

Facsimile: (86)

Commission(s): 40

Telex: 22040 badas en

Email:

WU SHI TSAN DR, SCHOOL OF ENGINEERING, UNIVERSITY OF ALABAMA, HUNTSVILLE AL 35899, USA

Telephone: (1) 2058956413

Facsimilc: (1)

Telex:

Email:

Commission(s): 10,49

WU SHOU-XIAN, SHAANXI OBSERVATORY, CAS, LINTONG XIAN, SHAANXI, CHINA PR

Telephone: (86) 3355951

Facsimile: (86)

Telex: 70121 csao cn

Email:

Commission(s): 19,31

WU XIANG-PING, BEIJING ASTRONOMICAL OBS, CAS, BEIJING 100080, CHINA PR

Telephone: (86) 12546089

Email: wxp)(abano1.bao.ac.cn

Facsimile: (86) $125-1085$

Telex: $220+0$ bao cn

Commission(s): 47

WU XINJI, DPT OF GEOPHYSICS, BEIJING UNIVERSITY, BEIJING 100071, CHINA PR

Telejhonc: (86) $1282471 * 3929$

Facsimile: (86)

Commission(s): 40

Telex: 22239 pkuni en

Email:

WU YUEFANG, DPT OF GEOPHYSICS, BEIJING UNIVERSITY, BEIJING 100871, CHINA PR

Telephone: (86) $12559461 * 2963$

Facsimile: (86) 12561085

Telex: 22239 pkuni cn

Email:

Commission(s): 40

WU ZHIREN DR, SHANGHAI SCIENTIFICAL \&, TECH EDU PUBLISHING HOUSE, 393 GUAN SHEN YAUN RD SHANGHAI 200233, CHINA PR

Telephone: (86) 21365791

Facsimilc: (86)

Commission(s): 65

Telex:

Email:

WUELSER JEAN PIERRE, INSTITUTE FOR ASTRONOMY, UNIVERSITY OF HAWAII, 2680 WOODLAWN DRIVE HONOLULU HI 96822, USA

Telephone: (1) 8089568306

Email: wuclscräalkoko.ifa.hawaii.edı

Facsinile: (1) $8089569402 \quad$ Telex:

Commission(s):

WUENSCH JOHANN JAKOB, UNIVERSITAETS STERNWARTE, AUF DEM HUEGAL 71, D 53121 BONN, GERMANY

Telephonc: (49) 228733 649

Facsinile: (49) $228733672 \quad$ Telcs:

Email: wuensch (a):astro.uni-bonn.de Commission(s): 199

WUNNER GUENTER, LEHRSTUHL F THEORET ASTRO, PHYSIK DER UNIV TUEBINGEN

AUF DER MORGENSTELLE 12 C, D 72076 TUEBINGEN, GERMANY

Telephone: (49) $7071292487 \quad$ Facsimile: (49)

Email: $\quad$ Commission(s): 14,44

Telcx: 7262714 ait d

WYCKOFF SUSAN DR, DPT OF PHYSICS, ARIZONA STATE UNIVERSITY, ASTRONOMY PROGRAM TEMPE AZ 85287, USA

Telephonc: (1) 6029653561

Facsimile: (1)

Commission(s): $15,29,45$

Telex: 140289 halley asu ut

Email: wyckoffayasycos.bitnct

WYLLER ARNE A PROF, BOX 5501, SANTA FE NM 875012-55(1)1, USA

Telephone: (1) 505473584 !

Facsimile: (1) $505+7358+9$

Telex:

Email:

Commission(s): 09,12,36

WYNNE CHARLES G PROF, INSTITUTE OF ASTRONOMY, THE OBSERVATORIES, MADINGLEY RD, CAMBRIDGE CB3 OHA, UK

Telephonc: (44) 1223337548

Facsimile: (4t) 1223337523

Tclex: 817297 astron $g$

Email:

Commission(s): 09

WYNN-WILLIAMS C G DR, INSTITUTE FOR ASTRONOMY, UNIVERSITYY OF HAWAII, 2680 WOODLAWN DR HONOLULU HI 96822 , USA

Telephone: (1) 8089568807

Facsimile: (1) 8089882790

Telex:

Email:

Commission(s): 28,34 
WYSE ROSEMARY F DR, DPT PHYSICS \& ASTRONOMY, JOHNS HOPKINS UNIVERSITY, CHARLES \& 34TH ST BALTIMORE MD 21218 , USA

Telephone: (1) 4105165392

Facsimile: (1) +11516 8260)

Telex:

Email: jhmail::wyser::wyse

Commission(s): 33

WYTRZYSZCZAK IWONA, ASTRONOMICAL OBSERVATORY, A MICKIEWICZ UNIVERSITY, UL SLONECZNA 36 PL 60 286 POZNAN, POLAND

Telephone: (48) $61679670 \quad$ Facsimile: (48) $61536536 \quad$ Telex:

Email: iwona(applpuam11.amu.cou.pl Commission(s): (14,07

XANTHOPOULOS B C DR, DPT OF PHYSICS, UNIVERSITY OF CRETE, BOX 1527, GR 711 11 IRAKLION, GREECE

Telephone: (30) $81235576 \quad$ Facsimile: (30)

Email:

Commission(s): 47

Telex: 262728

XI ZE-ZONG, INST HISTORY NAT SCIENCE, 1 GONG YUAN WEST RD, BEIJING, CHINA PR

$\begin{array}{ll}\text { Telephone: (86) } 1557180 & \text { Facsimile: (86) } \\ \text { Email: } & \text { Commission(s): }+1\end{array}$

XIA XIAOYANG DR, DPT OF PHYSICS, TIANJIN NORMAL UNIVERSITY, TIANJIN 300074, CHINA PR

Telephone: (86) 22716989

Facsimile: (86)

Telex:

Email:

Commission(s): 28

XIA YI-FEI, DPT OF ASTRONOMY, NANJING UNIVERSITY, NANJING, CHINA PR

$\begin{array}{lll}\text { Telephone: }(86) 2534651 * 2882 & \text { Facsimile: (86) } & \text { Telex: } 34151 \text { prenu cn } \\ \text { Email: } & \text { Commission(s): } 08 & \end{array}$

XIA ZHIGUO DR, YUNNAN OBSERVATORY, CAS, BOX 110 , KUNMING 72946 YUNNAN, CHINA PR

Telephone: (86) 8712035 Facsimile: $(86)$ Telex:

Email: $\quad$ Commission(s): 40

XIAN DING-ZHANG, PURPLE MOUNTAIN OBSERV, CAS, NANJING, CHINA PR

Telephone: (86) $2537609 \quad$ Facsimile: (86) $25301459 \quad$ Telex: $341+4$ pmonj cn

Email:

Commission(s): 04

XIANG DELIN, PURPLE MOUNTAIN OBSERV, CAS, NANJING, ChINA PR

Telephone: (86) $2533738 \quad$ Ficsimile: (86) $25301459 \quad$ Telex: 34144 pmonj cn

Email:

Commission(s): 33,34

XIANG SHOUPING, ASTROPHYSICS DIVISION, UNIV SCIENCE \& TECHNOLOGY, HEFEI 230026 ANHUI, CHINA PR

Telephone: (86) 551331134

Facsimile: $(86)$

Commission(s): 47

Telex:

Email:

XIAO NAI-YUAN, DPT OF ASTRONOMY, NANJING UNIVERSITY, NANJING, CHINA PR

Telephone: $(86) 2534651 * 2882 \quad$ Facsimile: $(86)$

Email: Commission(s): 19

Telex: $3+151$ prenu cn

XIE GUANG-ZHONG, YUNNAN OBSERVATORY, CAS, BOX 110, KUNMING 72946 YUNNAN, CHINA PR

Telephone: (86) $8712035 \quad$ Facsimile: (86)

Email:

Commission(s):

Telex: 64040 yuobs $\mathrm{cn}$

XIE LIANGYUN, INSTITUTE, OF GEODEDY \&, GEOPHYSICS, XU DONG LU, WUCHANG HUBEI 43077, CHINA PR Telephone: (86)

Facsimile: $(86)$

Commission(s): 08 Telex:

Email:

XIONG DA-RUN, PURPLE MOUNTAIN OBSERV, CAS, NANJING, CHINA PR

Telephone: (86) $25+2817 \quad$ Facsimile: (86) $25301+59$ Telex: 34144 pmonj cn

Email:

Commixion(s): 27,35

XIRADAKI EVANGELIA DR, DPT OF ASTROPHYSICS, NTL UNIVERSITY OF ATHENS, PANEPISTIMIOPOLIS

GR 15783 ATHENS, GREECE

Telephone: (30) 17235 122/9628 306

Facsimile: (30)

Commission(s): 37

Telex:

Email:

XU AO-AO, DPT OF ASTRONOMY, NANJING UNIVERSITY, NANJING, CHINA PR

Telephone: $(86)$

Facsinile: (86)

Commission(s): 10

Telex: $3+151$ pre nu cn

Email: 
XU BANG-XIN, DPT OF ASTRONOMY, NANJING UNIVERSITY, NANJING, CHINA PR

Telephone: (86)

Facsimile: (86)

Telex:

Email:

Commiswion(s): 08,31

XU JIA-YAN, SHAANXI OBSERVATORY, CAS, LINTONG XIAN, SHAANXI, CHINA PR

Telephone: (86) 332255

Facsimile: (86) Telex:

Email:

Commission(s): $08 \mathrm{C}, 10$

XU JI-HONG DR, URUMQI ASTRONOMICAL STAT, CAS, XINJIANG 830011, CHINA PR

Telephone: (86) 335757

Facsimile: $(\mathbf{8 6})$

Telex: 79152 kwtwz $\mathrm{cn}$

Email: 8301011

Commission(s): 07

XU PEI-YUAN, INST ELECTRONIC PHYSICS, SUST, JIA DING, SHANGHAI, CHINA PR

Telcphone: (86) 21951602

Facsimile: (86)

Telex:

Email:

Commission(s): 40

XU PINXIN, PURPLE MOUNTAIN OBSERV, CAS, NANJING, CHINA PR

Telcphone: (86) 2532893

Facsimile: (86) 25301450

Telex: $3+1+4$ pmonj en

Email:

Commission(s): 07,22

XU TONG-QI, SHANGHAI OBSERVATORY, CAS, 8I NANDAN RD, SHANGHAI 200030, CHINA PR

Telephone: (86) 21386191

Facsimile: $(86)$

Commission(s): (08,19)

Telex: $3316+$ shao en

Email:

XU ZHENTAO, PURPLE MOUNTAIN OBSERV, CAS, NANJING, CHINA PR

Telephone: (86) 2531096

Facsimile: (86) $25301+50$

Telex: $3+1+4$ pmonj en

Ematil:

Commission(s): 10,41

XU ZHI-CAI, PURPLE MOUNTAIN OBSERV, CAS, NANJING, CHINA PR

Telephonc: $(86) 25+6700$

Facsimile: (86) $25301+59$

Telex: $3+1+4$ pmonj en

Email:

Commission(s): 40

YABUSHITA SHIN A PROF, DPT APPLIED MATHS \& PHYS, KYOTO UNIVERSITY, KITASHIRAKAWA SAKYO KU KYOTO 606, JAPAN

Telephone: $(81) 757512111 \quad$ Facsimile: $(81) \quad$ Telex:

Email:

Commission(s): $15,20,34$

YABUUTI KIYOSHI PROF, 20 TANAKA HIGASKI, HINOKUCH MACHI, KYOTO 606, JAPAN

Telephone: $(\mathbf{8 1})$

Facsimile: (81)

Telex:

Email:

Commission(s): +1

YAHIL AMOS DR, DPT OF EARTH \& SPACE SCI, ASTRONOMY PROGRAM, SUNY AT STONY BROOK STONY BROOK NY $1179+21101$, USA

Telephone: (1) $5162+66545 \quad$ Facsimile: (1)

Telex: 510-228-7767

Email:

Commission(s):

YAKOVKIN N A DR, ASTRONOMICAL OBSERVATORY, KIEV STATE UNIVERSITY, OBSERVATORNAYA UL 3 252053 KIEV, UKRAINE

Telephone: (7)

Facsimile: (7)

Telex:

Email:

Cammission(s): 11

YAKOVLEV DMITRY, DPT THEORET ASTROPHYSICS, IOFFE PHYSICAL TECHN INST 19+021 ST PETERSBURG, RUSSIA

Telephone: (7) 812

Facsimile: (7) 8122471017

Telex:

Email: yak\%astro.pti.sph.suamain.ioffe.rssi. ru

Commission(s):

YALLOP BERNARD D DR, ROYAL GREENWICH OBS, MADINGLEY RD, CAMBRIDGE CB3 OEZ, UK

Telephone: (44) $122337+735$

Email: bdy âmail.ast.cam.ac.uk

Facsimile: (4) 1223374700

Telex: 817235 ingostl' $g$

YAMAGATA TOMOHIKO DR, TEXTBOOK DIV, MINSTRY OF EDU SCI \& CULT, KASUMIGASEKI CHIYODA

TOKYO 100, JAPAN

Telephone: $(81) 33581+211 * 2+13$

Email: vamagatakomega.mtkillato.ac.jp
Facsimile: (81) 335920409

Commission(s): 28,33
Telex: 
YAMAGUCHI SHICHIRO, FACULTY OF ENGINEERING, GIFU UNIVERSITY, YANAGIDO, GIFU 501 11, JAPAN Telephone: (81) 582301111

Facsimile: $(81)$

Telcx:

Email:

Commission(s):

YAMAKOSHI KAZUO, INST COSMIC RAY RESEARCH, UNIVERSITY OF TOKYO, MIDORICHO TANASHI TOKYO 188, JAPAN

Telephonc: (81) $42+61+131$

Email:

Facsimile: (81)

Commission(s): 21,22

Telex: 02822371 icrtu j

YAMAMOTO TETSUO DR, ISAS, 3-1-1 YOSHINODAI, SAGAMIHARA, KANAGAWA 229, JAPAN

Telephone: (81) $427513911 \quad$ Facsimile: (81) Telex: 24550 spacetky j

Email:

Commission(s): 21

YAMASAKI ATSUMA DR, DPT OF GEOSCIENCE, NATIONAL DEFENSE ACADEMY, HASHIRIMIZU YOKOSUKA 239, JAPAN

Telephone: (81) 468413810

Email:

Facsimile: $(81)+68+459012 \quad$ Telex:

Commission(s): $42 \mathrm{C}$

YAMASHITA KOJUN DR, ISAS, 3-1-1 YOSHINODAI, SAGAMIHARA, KANAGAWA 229, JAPAN

Telephone: (81) $\$ 27513911$

Facsimile: (81)

Commission(s): 21,4

Telex:

Email:

YAMASHITA TAKUYA, NTL ASTRONOMICAL OBS, 211 OSAWA, MITAKA, TOKYO 181, JAPAN

Telephone: (81) $4223+3710$

Facsimile: $(81)+223+3608$

Telex:

Email: oyamash(âcl.mtlinao.ac.jp Commission(s): 34

YAMASHITA YASUMASA PROF, TOKYO ASTRONOMICAL OBS, NAOJ, OSAWA MITAKA, TOKYO 181, JAPAN

Telephone: (81)

Facsimile: (81)

Commission(s): $25,29,45$

Telex: 28223177 tantk $\mathrm{i}$

Email:

YAMAZAKI AKIRA DR, HYDROGRAPHIC DPT, GEODESY \& GEOPHYSICS DIV, TSUKIJI 5 CHUO KU TOKYO 104, JAPAN

Telephonc: (81) 35413811

Facsimile: $(\mathbf{8 1})$

Commission(s): 04,08

Telex: 02522222 jahyd j

Email:

YAN HAOJIAN, SHANGHAI OBSERVATORY, CAS, 80 NANDAN RD, SHANGHAI 200030, CHINA PR

Telephone: (86) 214386191

Facsimile: (86) 214384618

Telex:

Email:

Commission(s): 08

YAN JUN, PURPLE MOUNTAIN OBS, ACADEMIA SINICA, NANJING 210008, CHINA PR

Tclephonc: (86) 254432817

Facsimilc: (86) 253301459

Telex: $3+14+$ pmonj en

Email:

Commission(s): 34

YAN LIN-SHAN, SHANGHAI OBSERVATORY, CAS, 80 NANDAN RD, SHANGHAI 200030, CHINA PR

Telephone: (86) 21386191

Facsimile: (86)

Commission(s): 26

Telex: $3316+$ shao cn

Email:

YANG FUMIN, SHANGHAI OBSERVATORY, CAS, 80 NANDAN RD, SHANGHAI 200030, CHINA PR
Telephone: (86) 21386191
Facsimile: (86)

Email:

Commission(s): 19

YANG Ji, PURPLE MOUNTIAN OBS, ACADEMICA SINICA, NANJING 210008 , CHINA PR

Telephone: (86) 254432817

Facsimile: (86) $2533(1459$

Telex: $3 \$ 1+4$ pmoni cn

Email:

Commission(s): $3+$

YANG JIAN, PURPLE MOUNTAIN OBSERV, CAS, NANJING, CHINA PR

Telephone: (86) 25 \$6700

Facsimile: (86) $25301+59$

Telex: $3+1+4$ pmonj en

Email:

Commission(s): 40

YANG KE-JUN, SHAANXI ASTRONOMICAL OBS, ACADEMIA SINCICASKA, BOX 18 LINGTON XIAN 710600, CHINA PR

Telephone: (1)

Facsimile: (1)

Telex:

Email:

Commission(s): 31 
YANG LAN-TIAN, DPT OF PHYSICS, HUAZHONG NORMAL UNIV, WUHAN, CHINA PR

Telephonc: (86) $75601 * 3(0) / 401$

Facsimile: (86)

Telex:

Email:

Commission(s): $\downarrow 4,+7$

YANG SHI JIE, PURPLE MOUN'TAIN OBSERV, CAS, NANJING, CHINA PR

Telephone: (86) $25+6700$

Facsimile: $(86) 25301+50$

Telex: $3+1+4$ pmonj cn

Email:

Commission(s): 09

YANG STEPHENSON L S, DPT PHYSICS \& ASTRONOMY, UNIVERSITY OF VICTORIA

VICTORIA BC V8W 3P6, CANADA

Telephonc: (1) 601721 8655

Facsimile: (1) 604 $7217715 \quad$ Telex:

Email: vangääotter.physs.univical

Commission(s): 30

YANG TING-GAO, SHAANXI ASTRONOMICAL POBS, BOX 18, LINTONG, WIAN, CHINA PR

Telephonc: (86)

Facsimile: (86)

Telex:

Email:

Commission(s): 24

YANKULOVA IVANKA DR, DPT OF ASTRONOMY, UNIVERSITY OF SOFIA, ANTON IVANOV ST 5

BG 1126 SOFIA, BULGARIA

Telephonc: (359) 2544852

Facsimile: (359)

Telex:

Email:

Commission(s):

YANOVITSKIJ EDGARD G DR, MAIN ASTRONOMICAL OBS, UKRAINIAN ACAD OF SCIENCE, GOLOSEEVO 252127 KIEV, UKRAINE

Telephone: (7) 044663110

Faesimile: (7)

Telex: 131406 sky su

Email:

Commission(s): 36

YAO BAO-AN, SHANGHAI OBSERVATORY, CAS, 80 NANDAN RD, SHANGHAI 200030, CHINA PR

Telephone: (86) 21386191

Facsimile: $(80)$

Telex: 33164 shat en

Email:

Commission(s): 27

YAO JIN-XING, PURPLE MOUNTAIN OBSERV, CAS, NANJING, CHINA PR

Telephone: $(86) 25+6700$

Facsimile: (86) $25301+59$

Telex: $3+1+4$ pmonj en

Email:

Commission(s): 10

YAO ZHENG-QIU, DPT OF SCIENTIFIC COOP, SSRG, BOX $4+70$, DAMASCUS, SYRIA

Telephone: $(963)$

Facsimile: $(963)$

Telex:

Email:

Commission(s): 09

YAPLEE B S, 8 CREST VIEW CT, ROCKVILLE MD 20854, USA

$\begin{array}{ll}\text { Telephone: (1) } 3017620935 & \text { Facsimile: (1) } \\ \text { Email: } & \text { Commission(s): }\end{array}$

YAROV-YAROVOJ M S DR, MATHEMATICS DPT, MVTU, VTORAYA BAUMANSKAYA 5, 107005 MOSCOW, RUSSIA

Telephonc: (7) 952670392

Facsimile: (7)

Telex: 111572

Email:

Commission(s): 07

YASUDA HARUO PROF DR, TOKYO ASTRONOMICAL OBS, NAOJ, OSAWA MITAKA, TOKYO 181, JAPAN

Telephone: (81)

Facsimile: $(\mathbf{8 1})$

Telex:

Email:

Commission(s): 08

YATSKIV YA S DR, MAIN ASTRONOMICAL OBS, UKRAINIAN ACAD OF SCIENCE, GOLOSEEVO

252127 KIEV, UKRAINE

Telephone: (7) $044663110 \quad$ Facsimile: (7) $0442246387 \quad$ Telex: 131406 sky su

Email: yatskiv\%gato.kiev.ua(a) relay.ussr Commission(s): 08,19

YAU KEVIN K C DR, DPT OF PHYSICS, UNIVERSITY OF DURHAM, SOUTH RD, DURHAM DH1 3LE, UK

Telephone: (44) $19137 \$ 2153$

Facsimile: (4) 1913743749

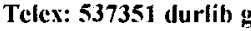

Email: yauouk.ac.dur.star

Commission(s): 41

YAUN KUO-CHUAN, TAIPEI OBSERVATORY, YUAN SHAN, TAIPEI 104 TAIWAN, CHINA R

Telephone: (886) 25947917

Facsimile: (886) 25910763

Telex:

Email:

Commission(s): 
YAVNEL ALEXANDER A DR, METEORITE COMMITTEE, ACADEMY OF SCIENCES, ULIANOVOJ M UL 3 K 1 117313 MOSCOW, RUSSIA

Telephone: (7) 951377538

Email:

\author{
Facsimile: (7) Telex: \\ Commission(s): 15,22
}

YE BINXUN, YUNNAN OBSERVATORY, CAS, BOX 110, KUNMING 72946 YUNNAN, CHINA PR

Telephone: (86) $8712035 \quad$ Facsimile: (86) Tclex: 64040 yuobs cn

Email:

Commission(s): 09

YE SHI-HUI, PURPLE MOUNTAIN OBSERV, CAS, NANJING, CHINA PR

Telephone: (86) $2546700 \quad$ Facsimile: (86) $25301459 \quad$ Telex: 34144 pmonj cn

Email:

Commission(s): 10

YE SHU-HUA, SHANGHAI OBSERVATORY, CAS, 80 NANDAN RD, SHANGHAI 200030, CHINA PR

Telephone: (86) $21386191 \quad$ Facsimile: (86) $21384618 \quad$ Telex: 33164 shao cn

Email:

Commission(s): 08,19,31C,38,40C,51

YE WENWEI, INST OF SEISMOLOGY, STATE SEISMO BUREAU, XIAO HONG SHAN WUHAN

HUBEI 230026 ANHUI, CHINA PR

Telephone: (86) 813401

Facsimile: (86)

Telex:

Email:

Commission(s):

YEE HOWARD K C DR, DPT OF ASTRONOMY, UNIVERSITY OF TORONTO, 60 ST GEORGE ST TORONTO ON MSS 1A1, CANADA

Telephone: (1) 4169784833

Facsimile: (1) $4169783921 \quad$ Telex: 06986766

Email: hyee@utorphys.bitnet

Commission(s):

YEH TYAN DR, HIGH ALTITUDE OBSERVATORY, NCAR, BOX 3000, BOULDER CO 80307 3000, USA

Telephone: (1) 3034975401

Facsimile: (1) $3034971568 \quad$ Telex:

Email:

Commission(s): 10,49

YEIVIN Y PROF, DPT OF PHYSICS \& ASTRON, TEL AVIV UNIVERSITY, RAMAT AVIV, TEL AVIV 69978, ISRAEL

Telephone: (972)

Facsimile: (972) Telex:

Email:

Commission(s):

YEN JUI-LIN PROF, DPT OF ELECTRICAL ENGIN, UNIVERSITY OF TORONTO, 60 ST GEORGE ST TORONTO ON M5S 1A4, CANADA

$\begin{array}{lll}\text { Telephone: (1) } 4169788756 & \text { Facsimile: (1) } 4169783921 & \text { Telex: } 06986766\end{array}$

Email:

Commission(s):

YEOMANS DONALD K DR, JPL, MS 301150 G, 4800 OAK GROVE DR, PASADENA CA 91109, USA

Telephone: (1) $8183542127 \quad$ Facsimile: (1) 8183931159

Telex: $675429 \mathrm{jpl}$ comm psd

Email: donald.k.ycomans(ajpl.nasa.gov Commission(s): 15,20P,22,41

YERSHOV VLADIMIR N, PULKOVO OBSERVATORY, PULKOVAKOYE SHOSSE 65 1, NASTAVNIKOV AVE 13 101 ST PETERSBURG 196140, RUSSIA

Telephone: (7) 812123449

Email: yersh@gao.spb.su

Facsimile: (7) 8123151701

Commission(s):

Telex: 121351 pulob su

YI ZHAO-HUA, DPT OF ASTRONOMY, NANJING UNIVERSITY, NANJING, CHINA PR

Telephone: (86) 2546700

Facsimile: (86)

Telex:

Email:

Commission(s): 07

YILMAZ FATMA DR, UNIVERSITY OBSERVATORY, ISTANBUL UNIVERSITY, 34452 ISTANBUL, TURKEY

Telephone: (90)

Facsimile: (90)

Telex:

Email:

Commission(s):

YILMAZ NIHAL DR, DPT OF ASTRONOMY, ANKARA UNIVERSITYA, FEN FAKULTESI, 06100 BESEVLER, TURKEY Telephone: $(90) 41236550$

Facsimile: (90)

Telex:

Email:

Commission(s):

YIN JI-SHENG, BEIJING ASTRONOMICAL OBS, CAS, W SUBURB, BEIJING 100080, CHINA PR

Telephone: (86) 1281203

Facsimile: (86)

Commission(s): 25

relex: 22040 baloas cn

Email: 
YIN QI-FENG, NRAO, 520 EDGENIONT RI), CHARLOTTESVILLE VA 22\%(13, USA

Telephone: (1) 8012960267 Fatcsimile: (1) Telex:

Email: ayuna,urao.edu

Commission(s): 40

YODER CHARLES F, JPL, MS 183 15(1), 4800 OAK GROVE DR, PASADENA CA 91109, USA

Telephone: (1) $81835+244+\quad$ Facsimile: (1) Telex: $617-5+29$

Email:

Commission(s): 16

YOKOSAWA MASAYOSHI DR, DPT OF PHYSICS, IBARAKI UNIVERSITY, 2-1-1 BUNKYO MITO, IBARAKI 310, JAPA

Telephone: (81) 292261621

Fatesimile: (81)

Telex:

Email:

Commission(s):

YOKOYAMA JUN'ICHI, YITP, KYOTO UNIVERSITY, GOKANOSHO, UJI G11, JAPAN

Telepbone: (81) $774317+31 \quad$ Facsimile: (81) $77+336226$ Telex:

$\begin{array}{ll}\text { Email: yokoyama(ayisun 1.yukawa.kyoto-u.ac.ij) Commission(s): } 47 & \end{array}$

YOKOYAMA KOICHI DR, INTL LATITUDE OBSERVATORY, NAOJ, HOSHIGAOKA MIZUSAWA SHI

IWATE (23, JAPAN

Telephone: (81) $1972+7111 \quad$ Facsimile: $(81) \quad$ Telex: 837628

Email:

Commission(s): 19

YOKOYAMA TADASHI DR, UNIVERSIDADE ESTADUAL, PAULISTA, CP 178, 13500 RIO CLARO, BRAZIL.

Telephone: (55) 19534 11122 Facsimile: (55) Telex: 011-31870

Email: $\quad$ Commission(s): 07

YONEYAMA TADAOKI DR, 2-1-16 IIIBARIGAOKA-KITA, HOYA-SH, TOKYO 202, JAPAN

Telephone: (81) Facsimile: (81) Telex:

Email: $\quad$ Commission(s):

YORK DONALD G DR, ASTRONOMY \& ASTROPHYS CTR, UNIVERSITY OF CHICAGO, 5640 S ELLIS AVE

CHICAGO IL 60637, USA

Telephone: (1) 3129628930

Facsimile: (1)

Telex: 910-221-5617

Email:

Comumission(s): 34

YORKE HAROLD W DR, INST F ASTRONOMIE \&, ASTROPHYSIK, AM HUBLAND, D 97074 WUERZBURG, GERMAN Telephone: (49)

Facsimile: (t)

Telex:

Email;

Commission(s): $34,35,36$

YOSHIDA ATSUMASA, INST PIIYSICAL \& CHEM RES, RIKEN, 2 HIROSAWA, WAKO SAITAMA 351 01, JAPAN

Telephone: $(81)+8+621111 * 3226 \quad$ Facsimile: $(81)+8+62+640$

Telex: 02962818 riken $j$

Email: ayoshida(äpostman.riken,go, jp) Commission(s): +4

YOSHIDA HARUO, TOKYO ASTRONOMICAL OBS, NAOJ, OSAWA MITAKA, TOKYO 181, JAPAN

Facsimile: $(81)+42+13793$

Telex: 02822307 taomtk

Email: yoshida(ac1.mtk.nao.ac.jp Commission(s): (07

YOSHIDA JUNZO PROF, DPT OF PHYSICS, KYOTO SANGYO UNIVERSITY, KAMIGAMO, KYOTO G03, JAPAN

Telephone: (81) $757012151 \quad$ Facsimile: (81)

Em:til:

Commission(s): 07

Telex: $5+22661$ ksuj

YOSHIDA SHIGEOMI, KISO OBSERVATORY, INSTITUTE OF ASTRONOMY, MITAKE KISO, NAGANO 397 01, JAPAN

Telephone: (81) $26+523360$

Facsimile: (81) 264523361

Telex:

Email: yoshida(a)kiso.iano.s.u-tolivo.ac.jp Commission(s): 34

YOSHI YUZURU DR, TOKYO ASTRONOMICAL OBS, NAOJ, OSAWA MITAKA, TOKYO 181, JAPAN

Telephone: $(81)+22325111$

Email: yoshii(a)top.mtk.nato.ac.jp Commission(s): 33C, +7

Telex: 2822307 taomtk j

YOSHIKAWA MAKOTO, COMMUNICATIONS RESEARCH, LABORATORY, 893 1 HIRAI KASHIMA

IBARAKI 314, JAPAN

Telephone: (81) 299 847150

Facsimile: (81) $2998+7160$

Telex:

Email: makoto(a)crl.go.jp)

Commission(s): 20

YOSHIMURA HIROKAZU DR, DPT OF ASTRONOMY, UNIVERSITY OF TOKYO, BUNKYO KU, TOKYO 113, JAPAN

Telephone: (81) 38122111

Facsimile: $(81)$

Telex: 33659 utrosci

Email:

Commission(s): 10,12 
M 920

YOSHIMURA MOTOHIKO DR, DPT OF PHYSICS, TOHOKU UNIVERSITY, ARAMAKI, SENDAI 980, JAPAN

Telephone: (81) $222221800 \quad$ Facsimile: (81) $222251891 \quad$ Telex:

Email: $\quad$ Commission(s): 47

YOSHINO KOUICHI, HARVARD SMITHSONIAN CFA, MS 50, 60 GARDEN ST, CAMBRIDGE MA 02138, USA

Telephone: (1) 6174952796 Facsimile: (1) $6174957455 \quad$ Telex:

Email: yoshino@cfa.harvard.edu

Commission(s): 14

YOSHIZAWA MASANORI DR, TOKYO ASTRONOMICAL OBS, NAOJ, OSAWA MITAKA, TOKYO 181, JAPAN

Telephone: (81) 422325111

Facsimile: (81) 422321924

Telex: 2822307 taomtk

Email:

Commission(s): 08C

YOSS KENNETH M DR, DPT OF ASTRONOMY, UNIVERSITY OF ILLINOIS, 1011 W SPRINGFIELD AVE URBANA IL 61801, USA

Telephone: (1) 2173333295

Facsimile: (1)

Commission(s): 30,45

Telex:

Email:

YOU JLAN-QI, PURPLE MOUNTAIN OBSERV, CAS, NANJING, CHINA PR
Telephone: (86) $2546700 \quad$ Facsimile: (86) $25301459 \quad$ Telex: 34144 pmonj cn

Email:

Commission(s): 10,12

YOU JUNHAN, ASTROPHYSICS DIVISION, UNIV SCIENCE \& TECHNOLOGY, HEIFI 230026 ANHUI, CHINA PR

Telephone: (86) 551331134

Facsimile: (86)

Telex: 90028 ustc en

Email:

Commission(s): 44

YOUNG ANDREW T DR, DPT OF ASTRONOMY, SAN DIEGO STATE UNIV, SAN DIEGO CA 92182, USA

Telephone: (1) 6195945817

Facsimile: (1)

Telex:

Email: aty@mintaka.sdsu.cdu

Commission(s): $16,25 \mathrm{C}$

YOUNG ARTHUR DR, DPT OF ASTRONOMY, SAN DIEGO STATE UNIV, SAN DIEGO CA 92182, USA

Telephone: (1) 6192656167

Facsimile: (1)

Telex:

Email: young@minaka.sdsu.edu

Commission(s):

YOUNG JUDITH SHARN, FIVE COLLEGE, RADIO ASTRONOMY OBSERV, B619 LEDERLE GRAD RES TWR AMHERST MA 01003, USA

Telephone: (1) 4135450789

Email:

Facsimile: (1)

Commission(s): 28

Telex: 95-5491

YOUNG LOUISE GRAY DR, DPT OF ASTRONOMY, SAN DIEGO STATE UNIV, SAN DIEGO CA 92182, USA

Telephone: (1) 6192878890

Facsimile: (1)

Telex:

Email:

Commission(s): 14,16

YOUNIS SAAD M, SARC, SCIENTIFIC RES COUNCIL, BOX 2441, JADIRIYAH BAGHDAD, IRAQ

Telephone: (964) 17765127

Facsimile: $(964)$

Commission(s): $24,33,34,40$

Telex: 2187 bathilmi ik

Email:

YOUSEF SHAHINAZ M DR, DPT OF ASTRONOMY, FACULTY OF SCIENCES, CAIRO UNIVERSITY, GEZA, EGYPT

Telephone: (20)

Facsimile: (20)

Telex:

Email:

Commission(s):

YOUSSEF NAHED H PROF, DPT OF ASTRONOMY, FACULTY OF SCIENCES, CAIRO UNIVERSITY, GEZA, EGYPT

Telephone: (20) 586041

Facsimilc: (20)

Telex:

Email:

Commission(s): 12

YU KYUNG-LOH PROF, DPT OF ASTRONOMYY 55, SEOUL NTL UNIVERSITY, KWANAK-KU, SEOUL 151-742,

KOREA R

Telephone: $(\mathbf{8 2})$

Facsimile: (82)

Commission(s): 08

Telex:

Email:

YU XIN ALFRED DR, DPT OF APLLIED MATHS, HONG KONG POLYTECHNIC, HUNG HOM

KOWLOON, HONG KONG

Telephone: (852) 37666951

Facsimile: (852) 33629045

Telex:

Email:

Commission(s): 
YU YAN DR, DPT OF RADIATION ONCOLOGY, UNIVERSITY OF ROCHESTER, 601 ELMWOOD AVE BOX 647 ROCHESTER NY $146+2$, USA

Telephone: (1)

Facsimile: (1)

Telex:

Email: yuäudeneb.mps.ohio-stitte.edu

Commission(s): 14

YUAN CHI PROF, DPT OF PHYSICS, CITY COLLEGE OF NY, 138 ST CONVENE AVE, NEW YORK NY 10031, USA

Telephonc: (1) 2126906823

Facsimile: (1)

Telex:

Email:

Commission(s): 33

YUASA MANABU DR, DPT OF MATH \& PHYSICS, KINKI UNIVERSITY, HIGASHI, OSAKA 577, JAPAN

Telcphone: (81)

Facsimile: (81)

Telex:

Email:

Commission(s): 07,20

YUE ZENG-YUAN, DPT OF GEOPHYSICS, BEIJING UNIVERSITY, BEIJING 100071, CHINA PR

Telephone: (86)

Facsimile: (86)

Telex:

Email:

Commission(s):

YULDASHBAEV TAIMAS S, ASTRONOMICAL INSTITUTE, UZBEK ACADEMY OF SCIENCES 700052 TASHKENT, UZBEKISTAN

Telephonc: (7)

Facsinile: (7)

Telex:

Email:

Commission(s):

YUMI SHIGERU PROF DR, KEYAKIDAI 1-12-2, KIYAMACHO, MIYAKIGUN, SAGA 841 02, JAPAN

Telephone: (81)

Facsimile: (81)

Telex:

Email:

Commission(s): 19

YUN HONG-SIK PROF, DPT OF ASTRONOMY, SEOUL NTL UNIVERSITY, KWANAK KU, SEOUL 151, KOREA R

Telephone: (82) $28806621 / 22$

Facsimile: (82) $28871+35$

Telex:

Email:

Commission(s): 111,12

YUNGELSON LEV R, INST OF ASTRONOMY, ACADEMY OF SCIENCES, PYATNITSKAYA UL 48 109017 MOSCOW, RUSSIA

Telephone: (7) 952315461

Email:

Facsimile: (7)

Telex: $\$ 12623$ sestp su

Commission(s): 35

ZABRISKIE F R PROF, RD 1, ALEXANDRIA PA 16611, USA

Telephone: (1) $814669+483$

Facsimile: (1)

Telex:

Email:

Commission(s):

ZACHARIADIS THEODOSIOS DR, RES CENTER FOR ASTRONOMY, ACADEMY OF ATHENS

14 ANAGNOSTOPOULOU ST, GR 10673 ATHENS, GREECE

Telephone: (30) 13613589

Facsimile: (30)

Telex:

Email: exaka20agrathun 1

Commission(s): 10

ZACHARIAS NORBERT DR, US NAVAL OBSERVATORY, 3450 MASSACHUSETTS AVE NW

WASHINGTON DC 203925400 , USA

Telephone: (1)

Facsimile: (1)

Telex:

Email: nzäpyxis.usno.navy.mil

Commission(s): 24

ZACHAROV IGOR DR, ASTRONOMICAL INSTITUTE, CZECH ACADEMY OF SCIENCES ONDREJOV OBSERVATORY, CZ 251 65 ONDREJOV, CZECH R

Telephone: (42) 20+857239/7111 Facsimile: (+2) 204851611

Email: astsun(ajesearn Commission(s): (19)

Telex: 121579

ZACHILAS LOUKAS DR, DPT OF CHEMISTRY, UNIVERSITY OF CRETE, BOX 1527

GR 71409 IRAKLION CRETE, GREECE

Telephonc: (30) 81212453

Email: zachilasagtalos.cc.uch.gr

Facsimile: (30) 81238468

Telex:

Commission(s): 33

ZADUNAISKY PEDRO E PROF, UNIVERSIDAD BUENOS AIRES, FAC CIENCIAS EXACTAS MATH CIUDAD UNIVERSITARIA PABI, $1+28$ BUENOS AIRES, ARGENTINA

Telephone: (54)

Facsimile: (54)

Telex:

Email:

Commission(s): 20 
ZAFIROPOULOS BASIL DR, DPT OF PHYSICS, UNIVERSITY OF PATRAS, GR 261 10 RION, GREECE

Telephone: (30) $61991973 \quad$ Falesimile: (30) 6199 1909) Telex: 312447 unpa gi"

Email:

Commission(s): 07

ZAHN JEAN-PAUL DR, OBSERVATUIRE DE PARIS, SECTION DE MEUDON, F 92195 MEUDON PPL CDX, FRANCE

Telephone: (33) 145077804

Enuil: zahua mesiob.obspm.ti

Facsimile: (33) 145077878 Telex:

Commission(s): $35 \mathrm{VP}, 36$

ZAITSEV VALERII V DR, INST OF APPLIED PHYSICS, ACADEMY OF SCIENCES, ULYANOV UL 4G 603600 N NOVGOROD, RUSSIA

Telephone: (7)

Facsimile: (7)

Telex:

Email:

Commission(s): 40

ZAKHOZHAJ VOLODIMIR, TIMUROVTSEV ST 70 APT 58, 310204 KHARKOV, UKRAINE

Telephone: (7) $057263+630$

Facsimile: (7)

Telex:

Email: akh(öastron.kharlioviua

Commission(s):

ZAMBON GIULIO DR, IM OBEREN BODEN 72, CH 8149 ZUERICH, SWITZERLAND

Telephone: (41)

Facsimile: (41)

Telex:

Email:

Commission(s):

ZAMBRANO ALEJANDRO DR, REAL INST Y OBSERVATORIO, DE LA ARMADA, CECILIO PUJAZON S/N E 11110 SAN FERNANDO, SPAIN

Telephone: $(34) 56883548 \quad$ Fatesimile: $(34) 56809302 \quad$ Telex: 76108 iome

Email:

Commission(s): 114

ZAMORANI GIOVANNI, IST DI RADIOASTRONOMIA, CNR, VIA IRNERIO 46, 1 40126 BOLOGNA, ITALY

Telephone: (39) 51232856

Facsimile: (39)

Commission(s): 44,47

Telex: $21166+$ infin bo $\mathrm{i}$

Email:

ZAMORANO JAIME DR, DPT DE ASTROFISICA, FAC DE FISICA, UNIVERSIDAD COMPLUTENSE

E 28040 MADRID, SPAJN

Telephone: $(34) 1+495316$

Email:
Facsimile: $(34)$

Commission(s): 28
Telex: $\$ 7272$

ZAMPIERI LUCA, SISSA, VIA BEIRUT 2-t, $13+1113$ TRIESTE, ITALY

Telephone: (39) 40 378 7522

Email: zampieri(a)tsmi19.sissit.it

Facsimile: (39) 403787528

Commission(s): 12

Telex:

ZANDER RODOLPHE DR, INSTITUT D'ASTROPHYSIQUE, UNIVERSITE DE LIEGE, AVE COINTE 5 B 4000 COINTE-LIEGE, BELGIUM

Telephone: $(32)+1529981$

Facsimile: (32) 41527474

Telex: +1264 astrlg b

Email:

Commission(s):

ZANINETTI LORENZO, IST DI FISICA GENERALE, CORSO D AZEGLIO 46, I 10125 TORINO, ITALY

Telcphone: (39) 11657694

Facsimile: (39)

Telex: 211041 infinto $i$

Email:

Commission(s):

ZAPPALA ROSARIO ALDO DR, IST DI ASTRONOMIA, CITTA UNIVERSITARIA, VIA A DORIA 6 I 95125 CATANIA, ITALY

Telephone: (39) $330533^{*} 493$

Email:

Facsimile: (39)

Telex: 970359 astret $i$

Commission(s): 10

ZAPPALA VINCENZO PROF, OSS ASTRONOMICO DI TORINO, ST OSSERVATORIO 20 I 10025 PINO TORINESE, ITALY

Telephone: (39) 114619035

Email: zappala(ato.astro.it

Facsimile: (39) $11+619030$

Telex: 213236 to astr $i$

Commission(s): 15VP,20

ZARE KHALIL DR, WRW 115 C, UNIVERSITY OF TEXAS, AUSTIN TX 78712, USA

Telcphone: (1) $512+71+23+$

Facsimile: (1) $512+713788 \quad$ Telex:

Email: aozy757(a)emx.cc.utexas.edu

Commission(s): 177

ZARNECKI JAN CHARLES DR, UNIT FOR SPACE SCIENCES, UNIVERSITY OF KENT, CANTERBURY CT2 7NR, UK Telephone: (4) $122776+000$

Facsimile: (4t) 1227762616

Email: jcrajuk.ac.ukc

Commision(s): $15,+4$

Telex: $965+49$ ulilib 
ZARRO DOMINIC M DR, NASA GSFC, CODE 682, GREENBELT MD 20771, USA

Telephonc: (1) $301286+689 \quad$ Facsimile: (1) 3012861617 Telex:

Email: 15833::zarro Commission(s): 12

ZASOV ANATOLE V DR, STERNBERG STATE ASTR INST, UNIVERSITETSKIJ PROSP 13, 119899 MOSCOW, RUSSIA

Telephone: (7)

Facsimile: (7)

Telex:

Email:

Commission(s): $28 \mathrm{C}$

ZAVATTI FRANCO, DPT DI ASTRONOMIA, UNIVERSITA DI BOLOGNA, VIA ZAMBONI 33, I 40126 BOLOGNA, ITAL

Telephone: (39) 51222956

Fatesimile: (39)

Commission(s): 28

Telex: 211604 infubo $\mathrm{i}$

Email:

ZAYER IGOR, LOCKHEED PALO ALTO RES LB, DPT 91 30 BLDG 252, 3251 HANOVER ST, PALO ALTO CA 94304 , US Telephone: (1) $+15+2+35+5$ Facsimile: (1) $+15+2+399+\quad$ Telex:

Email: izayer(a)solar.standford.edu

Commission(s):

ZDANAVICIUS KAZIMERAS DR, INST THEORETICAL PHYSICS, \& ASTRONOMY, GOSTAUTO 12

VILNIUS 2600, LITHUANIA

Telephone: (370) 2612898

Facsimile: $(370) 2224696$

Telex: $2611+1 \mathrm{Im} 1 \mathrm{su}$

Email: astrorajitpa.ft.ly

Commission(s): 45

ZEALEY WILLIAM J DR, DPT OF PHY'SICS, UNIVERSITY OF WOLLONGONG, BOX $11+4$

WOLLONGONG NSW 2500, AUSTRALIA

Telephone: (61) $42270555 \quad$ Facsimile: (61) Telex: 29022 ala

Email:

Commission(s): (19,34,46

ZEILIK MICHAEL II DR, DPT PHYSICS \& ASTRONOMY, UNIVERSITY OF NEW MEXICO, 800 YALE BLVD NE, ALBUQUERQUE NM 87131, USA

Telephone: (1) $505277+4+2$

Facsimile: (1)

Telex:

Email:

Commission(s): $34,+2,46$

ZEILINGEK WERNER W DR, ESO, KARL SCIIWARZSCHILDSTR 2, D 85740 GARCHING MUENCHEN, GERMANY

Telephone: (49) 89 $320062+7$

Email: werner(a)dgaesosı.bitnet Facsimile: (49) $8932006+80 \quad$ Telex: 5282820 eo d

Commission(s): 28

ZEIPPEN CLAUDE DR, OBSERVATOIRE DE PARIS, SECTION DE MEUDON, F 92195 MEUDON PPL CDX, FRANCE Telcphonc: (33) $145077+43$ Facsimile: (33)

Commission(s): 14

Telex: 201571

Email:

ZEKL HANS WILHELM, TON BELLER GMBH, BURGSTR 22, D 6140 BENSHEIM 3, GERMANY

Telephone: (49) $625173001 \quad$ Facsimile: (49) Telex: 468352

Email:

Commission(s):

ZELENKA ANTOINE DR, DACHSLENBERGSTR 56, CH 8180 BUELACH, SWITZERLAND

Telephone: (41) $18005156 \quad$ Facsimile: (41) Telex:

Email: $\quad$ Commission(s): 10,12

ZELLNER BENJAMIN H DR, DPT OF PHYSICS, GEORGIA SOUTHERN UNIV, LANDRUM BOX 8031

STATESBORO GA 30460$)$, USA

Telephone: (1) 9126810080

Facsimile: (1) $9126810471 \quad$ Telex:

Email: zellnerougsvms2.cc.gas.ou.celu Commission(s): 15

ZEL'MANOV A L DR, STERNBERG STATE ASTR INST, UNIVERSITETSKIJ PROSP 13, 119899 MOSCOW, RUSSIA

Telephone: (7)

Facsimile: (7)

Commission(s): 47

Telex:

Email:

ZENG QIN DR, PURPLE MOUNTAIN OBS, CAS, NANJING 2100018 , CHINA PR

Telephonc: (86) 25308516

Facsimile: (86) $25301+59$

Telex: $3+1+4$ pmonj en

Email:

Commission(s): 14,34

ZENSUS J-ANTON DR, NRAO, BOX (1, SOCORRO NM 87801 0387, USA

Telephone: (1) 5058357348

Facsimile: (1) 50583571127

Telex: 910-988-1710

Email: bitnet:azensus a,nrao.edu

Commission(s): 40 
ZERULL REINER H DR, BEREICH EXTRATERR PHYSIK, RUHR UNIVERSITAET BOCHUM D 46047 BOCHUM, GERMANY

Telephonc: (49) $23+7004576$

Facsimilc: (49)

Telcx: 0825860

Email:

Commission(s): 21

ZHAGAR YOURI H DR, ASTRONOMICAL OBSERVATORY, LATVIAN STATE UNIVERSITY, RAINIS BUL 19 2260198 RIGA, LATVIA

$\begin{array}{ll}\text { Telephonc: (371) } 132223149 & \text { Facsimile: (371) } \\ \text { Email: } & \text { Commission(s): }\end{array}$

Telex: 161171 tema su

ZHAI DI-SHENG, BEIJING ASTRONOMICAL OBS, CAS, W SUBURB, BEIJING 100080, CHINA PR

Telephone: (86) 1281698

Facsimile: (86)

Email:

Commission(s): $42 \mathrm{C}$

Telex: 22040 baoas en

ZHAI ZAOCHENG, SHANGHAI OBSERVATORY, CAS, 80 NANDAN RD, SHANGHAI 200030, CHINA PR

Telephone: (86) 21386191

Facsimile: (86)

Telex: 33164 shio $\mathrm{cn}$

Email:

Commission(s): 31

ZHANF SHOUZHONG DR, 414 WEST $120 \mathrm{ST}$, APT 401 , NEW YORK NY 100127, USA

Telephone: (1) 2126664689

Facsimile: (1)

Telex:

Email:

Commission(s): +1

ZHANG BAI-RONG, YUNNAN OBSERVATORY, CAS, BOX 110, KUNMING 729 46 YUNNAN, CHINA PR

Telephone: (86) 8712035

Facsimile: $(86)$

Telex: $610+10$ yubsen

Email:

Commission(s): 10,50

ZHANG BIN, DPT OF GEOPHYSICS, BEIJING UNIVERSITY, BEIJING 100071, CHINA PR

Telephone: (86)

Facsimile: (86)

Telex:

Email:

Commission(s): 33

ZHANG CHENG-YUE, STSCI, HOMEWOOD CAMPUS, 37010 SAN MARTIN DR, BALTIMORE MD 21218, USA

Telephone: (1) $\$ 105166864$

Facsimile: (1) +105168981 Telex:

Email: thangastsci.cdu

Commission(s): $3+$

ZHANG ER-HO DR, ASTRONOMY DPT, UNIVERSITY OF TEXAS, RLM 15 220, AUSTIN TX 78712 1083, USA

Telephone: (1) $512+71+462$

Facsimile: (1)

Telex:

Email:

Commission(s): 42

ZHANG FU JUN, SHANGHAI OBSERVATORY, CAS, 81 NANDAN RD, SHANGHAI 201030, CHINA PR

Telephone: (86) 21386191

Facsimile: (86)

Commission(s): 40

Telex:

Email:

ZHANG GUO-DONG, BEIJING ASTRONOMICAL, OBS, CAS, W SUBURB, BEIJING 100080, CHINA PR

Telephone: (86)

Facsimile: $(86)$

Telex:

Email:

Commission(s): 19

ZHANG HE-QI, PURPLE MOUNTAIN OBSERV, CAS, NANJING, CHINA PR

Telephone: $(86) 25+6700$

Facsimile: (86) $25301+59$

Telex: $3+1+4$ pmonj cn

Email:

Commission(s): $10,4+$

ZHANG HUI, SHAANXI OBSERVATORY, CAS, LINTONG XIAN, SHAANXI, CHINA PR

Telephone: (86) 332255

Fatcsimile: (86)

Comminssion(s): 188

Telex: 70121 csalo cn

Email:

ZHANG JIA-LU, ASTROPHYSICS DIVISION, UNIV SCIENCE \& TECHNOLOGY, HEFEI 230026 ANHUI, CHINA PR

Telephone: (86) 551331134

Facsimile: $(86)$

Commission(s): 44,47

Telex: 90028 uste cn

Email:

ZHANG JIA-XIANG, PURPLE MOUNTAIN OBSERV, CAS, NANJING, CHINA PR
Telephome: (86) $25+6700$
Facsimile: (86) $25301+59$

Email:

Commission(s): $20 \mathrm{C}$

ZHANG JINTONG, INSTITUTE OF GEODESY \&, GEOPHYSICS, XU DONG LU, WUCHANG HUBEI 430077, CHINA PR

Telephone: (86)

Facsimilc: (86)

Email:

Commission(s): $31,+2$

Telex: 
ZHANG PEIYU, PURPLE MOUNTAIN OBSERV, CAS, NANJING, CIIINA PR

$\begin{array}{ll}\text { Telephone: (86) } 2537521 & \text { Facsimile: (N6) } 25301459 \\ \text { Email: } & \text { Commission(s): } 41\end{array}$

ZHANG XIU ZHONG, SHANGIIAJ OBSERVATORY, CAS, \&I NANDAN RD, SHANGHAI 200030, CHINA PR Telephone: (86) 21386191 Facsimile: (86) $2138+618 \quad$ Telex: $3316+$ shato cn

Email:

Commission(s): (0)

ZHANG YOUYI, PURPLE MOUNTAIN OBSERV, CAS, NANJING, CHINA PR

Telephone: (86) $2546700 \quad$ Facsimile: (86) $25301+59$ Telex: $341+4$ pmonicn

Email: $\quad$ Commission(s): (0)

ZHANG ZHENG-PAN, CRESS, YORK UNIVERSITY, 4700 KEELE ST, NORTH YORK ON M3J 1P3, CANADA

Telephone: (1) +1651214011

Facsimile: (1) $+16512(079) \quad$ Telex:

Email: sheng(astpl.ists.ca

Commission(s): (17

ZHANG ZHEN-DA, DPT OF ASTRONOMY, NANJING UNIVERSITY, NANJING, CHINA PR

Telephone: (86) 25 34651*2882 Facsimile: $(86) \quad$ Telex: 34151 prenu cn

Email:

Commission(s): 10

ZHANG ZHEN-JIU, DPT OF PHYSICS, HUAZIIONG NORMAL UNIV, WUHAN, CHINA PR

Telephonc: $(86) 75601$

Facsimile: $(86)$

Telex: 6908

Email:

Commission(s): 44,47

ZHAO GANG, SHANGHAI OBSERVATORY, CAS, SI NANDAN RD, SHANGHAI, CHINA PR

Telephone: (86) $1125782 \% 6 \quad$ Facsimile: (86) 1112561085 Telex: 33164 shato cn

Email: galanowbont.lano.ac.co Commission(s): 29C,31

ZHAO GANG, BEIJING ASTRONOMICAL OBS, CAS, BEIJING 100080, CHINA PR

Telephone: (86) I $25782 \% 9$ Facsimile: (86) 12561085 Telex:

Email:

Commission(s):

ZHAO JUN HUI, HARVARD SMITHSONIAN CFA, MS 78, 60 GARDEN ST, CAMBRIDGE MA 02138, USA

Telephone: (1) $617+957294$

Facsimile: (1) 617 \$96 $7554 \quad$ Telex:

Email: jratoacfitharvard.edu

Comtmission(s): 40

ZHAO JUN-LIANG, SHANGHAI OBSERVATORY, CAS, , NO NANDAN RD, SHANGHAI 200130, CHINA PR

Telephone: (86) 2138619

Finsimile: $(86)$

Telex: 33164 shino cn

Email:

Commission(s): $33.37 \mathrm{C}$

ZHAO MING, SHANGHAI OBSERVATORY, CAS, 8I NANDAN RD, SHANGHAI 2000130, CHINA PR

Tclephone: (86) $21386191 \quad$ Facsimile: (86)

Email: $\quad$ Commission(s): 19

Telex: $3316+$ shato cn

ZHAO REN-YANG, BEIJING ASTRONOMICAL OBS, CAS, W SUBURB, BEIJING IUMOBI, CHINA PR

Telephone: (86)

Fic:simile: (86)

Email:

Commision(s): 10

Telex:

ZHAO YONGHENG, BEIJING ASTRONONICAL OBS, CAS, BEIJING 1000180, CHINA PR

Telephone: (86) $12578296 \quad$ Facsimile: (86) 12561085 Telex: 22040 bao cn

Email: yacua bao01.bao.accen Commission(s):

ZHARKOV VLADIMIR N DR, INST PIIYSICS OF THE EARTH, ACADEMY OF SCIENCES, GRUZINSKAYA 10 $1233+2$ MOSCOW, RUSSIA

Telephone: (7) $2545251 \quad$ Ficsimile: (7) Telex: 411196 ifzan

Email: $\quad$ Commission(s): 16

ZHARKOVA VATENINA DR, DPT PIISICS \& ASTRONOMY, UNIVERSITY OF GLASGOW, GLASGOW G12 8QQ, UK Telephone: (H) $1+13398855 x+153$ Facsimile: (4t) $1+1334 \%(129$ Telex:

Email: valiurlowarasolar.stanford.edu

Commission(s): 111

ZHDANOV VALERY, ASTRONOMIC, OBSERVATORY, KIEV UNIVERSITY, OBSERVATORNA STR 3 $25+053$ KIEV 53, UKRAINE

$\begin{array}{lll}\text { Telephone: (7) } 40+45131492 / 2162691 & \text { Facsimile: (7) } & \text { Telex: } \\ \text { Email: nokuaggluk.apcorg. } & \text { Commission(s): } 17\end{array}$ 
ZHEKOV SVETOZAR A DR, OSS ASTRONOMICO, DI ARCETRI, LARGO E FERMI 5,150125 FLORENCE, ITALY

Telephone: (39) 5527521 Facsimile: (39)

Telex: 23351 ikiban bg

Email:

Commisvion(s):

ZHELEZNIAKOV VLADIMIR V, INST OF APPLIED PHYSICS, ACADEMY OF SCIENCES, ULYANOV UL I6 G03600 N NOVGOROD, RUSSIA

Telephone: (7)

Facsimile: (7)

Telex:

Email:

Commisvion(s): 40

ZHELYAZKOV IVAN DR, FAC OF PUYSICS, LINIVERSITY OF SOFIA, 5 JAMES BOURCHIER BD BG 1126 SOFIA, BULGARIA

Telephone: (359) $262561 / 6+1$

Emait:
Facsimite: $(359) 2689085$

Commixsion(s): 10
Telex: 23296 suko r bg

ZHENG DA-WEI, SHANGHAI OBSERVATORY, CAS, 80 NANDAN RD, SHANGHAI 20M(130, CHINA PR

Telephone: (86) 21386191

Facsimile: $(86)$

Commission(s): 19

ZHENG JIA-QING, TURKU UNIVERSITY OBS, TUORLA, SF 21500 PIIKKIO, FINLAND

Telephone: (358) 212435822 Facsimile: (358) 212433767 Telex:

Email: zhengasara.utu.fi

Commission(s): 117

ZHENG WEI, CTR FOR ASTROPHYSCS SCI, JOHNS HOPKINS UNIVERSITY, BALTIMORE MD 21218 2695, USA

Telephone: (1) $+105165+59$

Fatesimile: (1) +105168260) Telex:

Email: zhengapha.jhu.edu

Commission(s): $28,+4$

ZHENG XUE-TANG, DPT OF APPIALI PHYSICS, EAST CIINA INSTITUTE OF, TECHNOLOGY

NANJING 210014 , CHINA PR

Telephone: (86)

Fatcsimile: ( 86$)$

Telex:

Email:

Commission(s): 117

ZHENG YING, PURPLE MOUNTAIN OBSERV, CAS, NANJING, CHINA PR

Telephone: (X6) $25+67010$

Facsimile: (\$6) $25301+59$

Telex: $3 \$ 1+4$ pmonj cn

Email:

Commission(s): 31

ZHENG YI-JIA, BEIJING ASTRONOMICAL OBS, CAS, W SUBURB, BEIJING IUOOBO, CHINA PR

Telephone: (86)

Facsimile: (86)

Commission(s): 40

Telex: 22040 baobs en

Email:

ZHEVAKIN S A PROF DR, RADIOPIIYSICAL, RESEARCH, INSTITUTE, LYADOV UL 25/14, G03G00 N NOVGOROD, RUSSIA

Telephone: (7) 366751

Facsimile: (7)

Commission(s): 35

Telex:

Email:

ZHILYAEV BORIS, MAIN ASTRONOMICAL OBS, UKRAINIAN ACAD OF SCIENCE, GOLOSEEVO 252127 KIEV, UKRAINE

Telephone: (7) $0+4266+760$

Email: manasagluk.apcory

Facsimile: (7) $11+42662147$

Tolex: 131406 sliy su

ZHOU BIFANG DR, NANJING ASTRONOMIICAL, INSTRUNENT RESEARCII CTR, IR2 BANCANG NANJING $2100+2$, CHINA PR

Telephone: (86) 25646191

Facsimile: $(86)$

Enail:

Commission(s): 100

Telex: 341125 issats co

ZHOU DAOQI, DP'T OF GEOPIIYSICS, BEIJING UNIVERSITY, BEIJING 1OUI71, CHINA PR

Telephone: (86) $1282471 * 3888 \quad$ Filesimile: $(86)$

Email:

Commission(s): 10,12,42

Telcx: 22239 pliuni

ZHOU HONG-NAN, DPT OF ASTRONONI, NANJING UNIVERSITY, NANJING, CHINA PR

Telephonc: $(86) 2534651 * 2882$

Facsimile: $(86)$

Commision(s): 177,42

Telex: $3+151$ prenu en

Email:

ZHOU TI-JIAN, DPT OF GEOPIIYSICS, BEIJING UNIVERSITY, BEIJING 100071, CIIINA PR
Telephone: (86) $1282471 \times 3888$
Eatsimile: $(86)$

Email:

Commisxion(s): 40 
ZHOU YOU-YUAN, ASTROPIIYSICS DIVISION, UNIV SCIENCE \& TECHNOLOGY, HEFEI 230026 ANHUI, CHINA PR

Telephonc: (86) 551331134

Facsimile: ( 86$)$

Telex: 90028 uste en

Emiail:

Commission(s): 28.47

ZHOU ZHEN-PU, PURPLE MOINTAIN OBSERV, CAS, NANJING, CIINA PR

Telephone: (86) 2533738

Facsimile: (86) $253111+59$

Telex: $3+11+$ pmonji en

Email:

Commission(s): 34

ZHU CI-SHENG, DPT OF ASTRONONIY, NANJING UNIVERSITY, NANJING, CHINA PR

Telephone: $(80) 2537551$ *2882

Facsimile: $(86)$

Commission(s): +2

Telex: $3+151$ prenu en

Email:

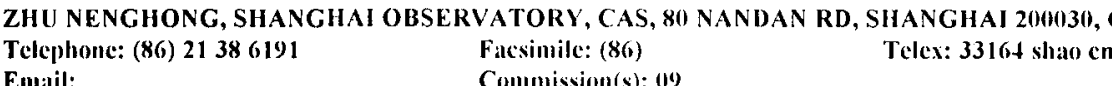

SHANGHAI 200030, CHINA PR

Email:

Commission(s): (19)

ZHU SHI-CHANG, DPT OF PIIYSICS, SHANGHAI TEACHERS UNIV, IO GILLIN RD, SHANGHAI, CHINA PR

Telephone: (86) $2138+301$

Facsimile: (86)

Telex: 9016

Email:

Commission(s): 47

ZHU WEN-YAO, SHANGHAI OBSERVATORY, CAS, 80 NANDAN RD, SHANGHAI 200030, CHINA PR

Telephone: (86) $21386191 \quad$ Facsimile: (86)

Email:

Commission(s): 07

Telex: $3316+$ shato cn

ZHU XINGFENG, ASTROPHYSICS DIVISION, LNIV SCIENCE \& TECHNOLOGY, HEFEI 230126 ANHUI, CHINA PR

Telephone: (86) $551331134 \quad$ Facsimile: (86)

Email:

Commission(s): 47

Telex: 90028 uste en

ZHU YAOZHONG DR, INSTITUTE OF GEODESY \&, GEOPIIYSICS, XU DONG LU

WUCHANG HUBEI +30077, CHINA PR

Telephone: $(86) 551813401 \quad$ Facsimile: $(86)$ Telex:

Emati:

Commission(s): 19)

ZHU YONG-HE, BEIJING ASTIRONONIICAL OBS, CAS, W SUBURB, BEIJING IOUIOUO, CHINA PR

Telephone: (86) 1281698

Facsimile: (86)

Telex: 22040 baobs en

Emait:

Commission(s): 19

ZHUANG QIXIANG, BONDAR-CLEGG N CO LTD, NTL RESEARCH COUNCIL, 5420 CANOTEC ROAD

OTTAWA ON K1J 8X5, CANADA

Telphone: (1) 613 749 2220 Facsinile: (1) Telex:

Email:

Commission(s): 31

ZHUANG WEIFENG, BEIJING ASTRONONICAL, OBS, CAS, W SUBURB, BEIJING IOOIIO, CHINA PR

Telephone: (86)

Facsimile: ( 86$)$

Telex:

Eniail:

Commission(s): +1

ZHUGZIIDA YUZEF D DR, IZNIIRAN, ACADENY OF SCIENCES, $1+20 \% 2$ TROITSK, RUSSIA

Telephone: (7)

Ficsimile: (7) Telex:

Email:

Commission(s): 10,12

ZICKGRAF FRANZ JOSEF, LANDESSTERNWARTE, KOENIGSTUHL, D 69117 HEIDELBERG, GERMANY

Telephone: (49) 6221509223

Ficnimile: (49) $62215(19) 2012$

Telex:

Email: frickgra,mail.lsw.umi-heidellserg.de

Commission(s):

ZIEBA STANISLAW DR, ASTRONONICAL OBSERVATORY, JAGIELLONIAN UNIVERSITY, UL ORLA 171

PL $3024+$ KRAKOW, POLAND

Tclephon: (48) $122238556 / 221877$

Facsimile: (48) 123780.53

Telex: 326203 ujoa pl

Email:

Commission(s): 411,4 ?

ZIEGLER HARALD, CADLAB, UNIVERSITAET PADERBORN, POSTFACH 1621, D 33046, PADERBORN, GERMANY Telephone: (49) $525128+175$ Filcsimile: (49) $52512891+0$ Telex:

Email: hamaldacadab.de

Comminion(s): 28 
ZIKIDES MICHAEL C DR, DPT OF ASTRONOMY, NTL UNIVERSITY OF ATIIENS, PANEPISTIMIOPOLIS GR 15771 ZOGRAFOS, GREECE

Telephone: (30) $172+341+$

Facsimile: (3i)

Telex:

Email:

Commission(s):

ZIMMERMANN HELMUT DR, UNIV STERNWARTE JENA, SCHILLERGAESSCHEN 2, D (17745 JENA, GERMANY

Telephonc: (49) 7827122

Facsimile: (49)

Telex:

Email:

Commission(s): 34,46

ZINN ROBERT J DR, DPT OF ASTRONOMY, YALE UNIVERSITY, BOX 6666, NEW HAVEN CT 06520, USA

Telephone: (1) $203+363+60$

Facsimile: (1)

Telex:

Email:

Commission(s): 28,37

ZINNECKER HANS, ASTRONOMISCHES INSTITUT, DER UNIVER WUERZBURG, AM HUBLAND

D $9707+$ WUERZBURG, GERNANY

Telephone: (\$9) 9318885031

Facsimile: (49) $9318884613 \quad$ Telex:

Email: hansa astro.uni-wueraburg.de

Commission(s): 26VP

ZIOLKOWSKI JANUSZ DR, COPERNICUS ASTRON CENTER, POLISH ACAD OF SCIENCES, UL BARTYCKA 18 PL 100 716 WARSAW, POLAND

Telephonc: $(48)$

Email:

Facsimile: (48)

Commission(s): $35,+2$

Telex:

ZIOLKOWSKI KRZYSZTOF DR, SPACE RESEARCH CENTER, POLISH ACAD OF SCIENCES, UL ORDONA 21 PL 01237 WARSAW, POLAND

Telephonc: $(\$ 8) 22+100+1$

Facsimile: $(48)$

Telex:

Email:

Commission(s): 20

ZIRIN HAROLD DR, CALTECH, NS $26+33$, PASADENA CA 91125, USA

Telephonc: (1) 8183563857

Facsimile: (1) Telex:

Email:

Commission(s): 10,12,14

ZIRKER JACK B DR, AIR FORCE GEOPHYSICS LAB, NTL SOLAR OBSERVATORY, SUNSPOT NM 88349, USA

Telephone: (1) $505+3+1390$

Facsimile: (1)

Commission(s): 12

Telex:

Email:

ZITELLI VALENTINA DR, DPT DI ASIRONONIIA, UNIVERSITA DI BOLOGNA, CP 596, 1 40100 BOLOGNA, ITALY

Telephonc: (39) $51259301 \quad$ Facsimile: (39)

Email: spant:37929

Commission(s):

Tclex: $52063+$ infin i

ZIURYS LUCY MARIE, DPT OF CIIENISTRY, ARIZONA STATE UNIVERSITY, TEMPE AZ 85287 1604, USA

Telephonc: (1) 6029657278

Facsimile: (1) $6029652747 \quad$ Telex:

Email: ziurvsaasuchm.Iit.istu.cilu

Commission(s):

ZIZNOVSKY JOZEF DR, ASTRONOMICAL. INSTITUTE, SLOVAK ACADEMY SCIENCES

SK 059 6I TATRANSKA LOMNI, SLONAK R

Telephone: (42) $969967866 / 7 / 8 \quad$ Facsinile: $(42) 969967656 \quad$ Telex:

Emaii: rigala,ta3.sk Commission(s): 25

ZLOBEC PAOLO DR, OAT, BOX SUCC TRIESTE S, VIA TIEPOLO 11, 134131 TRIESTE, ITALY

Telephone: (39) $40793921 \quad$ Facsimile: (39) Telex: 461137 oat $\mathrm{i}$

Emitil:

Commission( $): 16,411$

ZLOTNIK ELENA YA DR, INST OF APPLIED PHYSICS, ACADENY OF SCIENCES, ULYANOV UL UG 603600 N NOVGOROD, RUSSIA

Telephone: (7) 788312363519

Facsimile: (7) $788312362681 \quad$ Telex: 412580 fïrik su

Email:

Commission(s): 40

ZOLA STANISLAW, ASTRONONIIAI, OBSERVATORY, JAGIELLONIAN UNIVERSITY, UL ORLA 171

PL 30 2H4 KRAKOW, POLAND

Telephone: $(48) 12251497$

Email: zolata,oa.uj.edu.pl

Facsimile: (48) $12251318 \quad$ Telex:

Commission(s): 27,42

ZOMBECK MARTIN V DR, CENTHR FOR ASTROPIIYSICS, IICO/SAO, G) GARDEN ST, CAMBRIDGE MA 02138, USA Telephone: (1) $617+957227$

Facsimile: (1)

Telex: $921+28$ sattellite cam

Email:

Commiscion(s): 44 
ZOREC JUAN DR, INSTITU'T D'ASTROPIIYSIQUE, YSBIS BD ARAGO, F 750I4 PARIS, FRANCE

Telephone: (33) $1+4328121$

Facsimile: (33) 1 +4 328001 Telex:

Email:

Commission(s): 29

ZOSIMOVICH IRINA D, INSTITU'TE: OF IISTORY, UKRAINIAN ACAD OF SCIENCE, KIROV UL 4

252001 KIEV, UKRAINE

Telephone: (7) 044 29 $0272 \quad$ Facsimile: (7) Telex:

Email:

Commission(s): +1

ZOU HUI-CHENG, SHANGHAI OBSERVATORY, CAS, 80 NANDAN RD, SHANGHAI 200030, CHINA PR

Telephone: (86) $21386191 \quad$ Facsimile: (86) Telex: $3316+$ shat enen

Email:

Commission(s): 44

ZOU YI-XIN, BEIJING ASTRONOMICAL OBS, CAS, W SUBURB, BEIJING 100080, CHINA PR

Telephone: (86) 1281261

Facsimile: (86)

Commission(s): 10

Telex: 22040

Email:

ZOU ZHEN-LONG, BEIJING ASTRONOMICAL OBS, CAS, W SUBURB, BEIJING 100080, CHINA PR

Telephone: (86)

Facsimile: (86)

Telex: $220+0$ batoas en

Email;

Commission(s): 47

ZSOLDOS ENDRE DR, KONKOLY OBSERVATORY, THEGE U 13/17, BOX 67, H 1525 BUDAPEST, HUNGARY

Telephone: (36) $1175586(2 / 175+122$

Facsimile: (36) 11569641

Commission(s): 27

Telex: $227+60$ lionob h

Email: psoldos a ogyalla.konoly.hu

Commission(s): 27

ZUCCARELLO FRANCESCA, IST DI ASTRONOMIA, CITTA UNIVERSITARIA, VIA A DORIA G I 95125 CATANIA, ITALY

Tekphonc: (39) $330533 \quad$ Falesimile: (39)

Email:

Commission(s):

Telex: 970359 astret $i$

ZUCKERMAN BEN M DR, DPT OF ASTRONONY, UNIVERSITY OF CALIFORNIA, LOS ANGELES CA 90024, USA

Telephonc: (1) $2138259338 \quad$ Filesimile: (1)

Email: $\quad$ Commission(s): $27,34,40,51$

Telex: $9103+27597$

ZUIDERWIJK EDWARDUS J, ROYAL GREENWICH OBS, MADINGLEY RD, CAMBRIDGE CB3 UEZ, UK

Telephone: (4t) $122337+868 \quad$ Facsimile: (4t) 1223374700 Telex:

Email:

Commisvion(s): 42,47

ZVERKO JURAJ DR, ASTRONOMICAL, INSTITUTE. SLOVAK ACADEMY SCIENCES

SK 059 G0 TATRANSKA LOMNI, SLOVAK' R

Telephone: $(+2) 969967866 / 7 / 8$ Facsimile: $(+2) 969967656 \quad$ Telex:

Email: merlioatu3.sk Commission(s): 29

ZVOLANKOVA JUDITA, ASTRONOMICAL INSTITUTE, SLOVAK ACADEMY SCIENCES, DUBRAVSKA 9

SK $8+2$ 28 BRATISLAVA, SLOVAKR

Telephone: (42) 7375157 Facsimile: (42)

Email: $\quad$ Commission(s): 22

Telex: 93373 seis

ZWAAN CORNELIS PIROF DR. STERIRKUNDIG INSTITUTE, BOX MUOH, NL 35I8 TA UTRECHT, NETHERLANDS

Telephone: (31) 30535223

Facsimile: (31) 30535201

Telex: 41048 fylut $n$ l

Email: awanualys.ruu.nl

Commission(s): 11, 12,36

ZWITTER TOMAZ, ASTRONONICAL OBSERVATORY, INIVERSITY OF E KARDELJ, JADRANSKA I9

LJUBLJANA, SLOVENIA

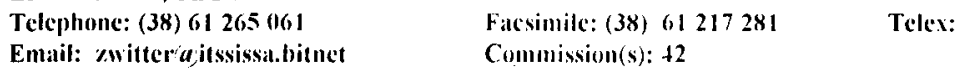

ZYLKA ROBERT, MPI FUER RADIOASTRONOMIE, AUF DEMI IIUEAL 69, D 53121 BONN, GERMANY

Telephone: (49) 228525376 Facsimile: (49) 228525229 Telex:

Email: ryllkita,mpiti-bonn.mpgede

Commission(s): +11 